\title{
Annual Energy Review 2011
}

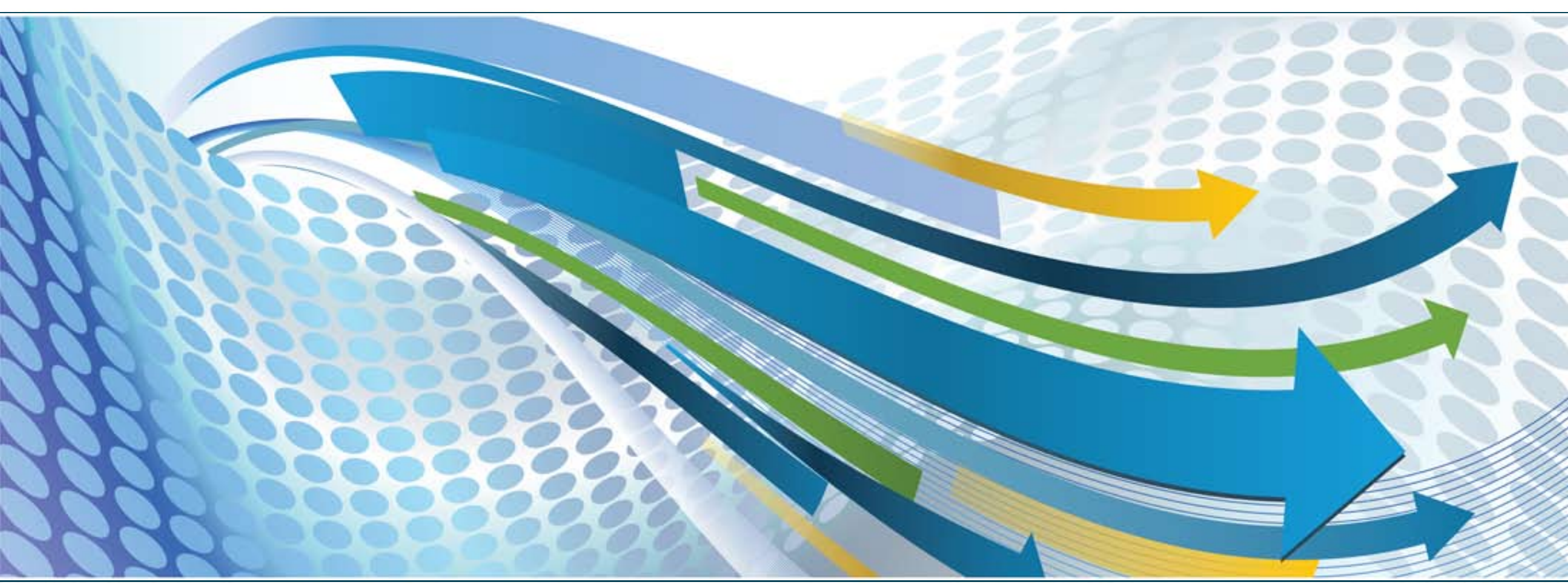




\section{Annual Energy Review 2011}

The Annual Energy Review (AER) is the U.S. Energy Information Administration's (EIA) primary report of annual historical energy statistics. For many series, data begin with the year 1949. Included are statistics on total energy production, consumption, trade, and energy prices; overviews of petroleum, natural gas, coal, electricity, nuclear energy, and renewable energy; financial and environment indicators; and data unit conversions.

Publication of this report is required under Public Law 95-91 (Department of Energy Organization Act), Section 205(c), and is in keeping with responsibilities given to the EIA under Section 205(a)(2), which states:

"The Administrator shall be responsible for carrying out a central, comprehensive, and unified energy data and information program which will collect, evaluate, assemble, analyze, and disseminate data and information....”

The AER is intended for use by Members of Congress, Federal and State agencies, energy analysts, and the general public. EIA welcomes suggestions from readers regarding the content of the AER and other EIA publications.

\section{Important Notes About the Data}

Data Displayed: For tables beginning in 1949, some early years (usually 1951-1954, 1956-1959, 1961-1964, 1966-1969, and 1971-1974) are not shown on the tables in the printed report or the Portable Document Format (PDF) files; however, all years of data are shown in the Excel formats and the HyperText Markup Language (HTML) files.

Comprehensive Changes: Most AER 2011 tables and figures carry a new year of data (usually 2011), which are often preliminary and likely to be revised next year, and revisions to much of the 2010 data, which are now final in many cases.

Monthly Data: The emphasis of the AER is on long-term trends. Analysts may wish to use the data in this report in conjunction with EIA's monthly releases that offer updates to the most recent years' data. In particular, see the Monthly Energy Review at http://www.eia.gov/totalenergy/data/monthly for statistics that include updates to many of the annual series in this report.

\section{Ordering Information}

This and other EIA publications may be purchased from the U.S. Government Printing Office:

$\begin{array}{lll}\text { - Internet } & \text { U.S. Government Online Bookstore } \\ \text { - Phone } & \text { DC Metro Area: 202-512-1800 } \\ & \text { Toll-Free: 866-512-1800 } \\ & \text { 7:00 a.m.-8:00 p.m., Eastern time, M-F } \\ \text { - } & \text { Fax } & \text { 202-512-2104 } \\ \text { - Mail } & \text { Superintendent of Documents } \\ & \begin{array}{l}\text { P.O. Box 371954 } \\ \text { Pittsburgh, PA 15250-7954 }\end{array}\end{array}$

For additional information see http://bookstore.gpo.gov/index.jsp.

Complimentary copies are available to certain groups, such as public and academic libraries; Federal, State, local, and foreign governments; EIA survey respondents; and the media. For further information and answers to questions on energy statistics, contact:
Office of Communications, EI-40
Forrestal Building, Room 1E-210
1000 Independence Avenue, S.W.
Washington, DC 20585
Telephone: 202-586-8800
Fax: 202-586-0114
Email: infoctr@eia.gov
Website: http://www.eia.gov

GPO Stock No: 061-003-01161-6

\section{Electronic Access}

The AER is available on EIA's website in a variety of formats at: http://www.eia.gov/totalenergy/data/annual.

- $\quad$ Full report and sections: PDF files

- $\quad$ Report tables: Excel, HTML, and PDF files

- Table data (unrounded): Excel files

- Graphs: PDF files

Note: In many cases, PDF files display selected annual data; Excel and HTML files display all years of data available. Excel files display the greatest data precision available. 


\title{
Annual Energy Review 2011
}

\section{September 2012}

\author{
U.S. Energy Information Administration \\ Office of Energy Statistics \\ U.S. Department of Energy \\ Washington, DC 20585
}

This report was prepared by the U.S. Energy Information Administration (EIA), the statistical and analytical agency within the U.S. Department of Energy. By law, EIA's data, analyses, and forecasts are independent of approval by any other officer or employee of the United States Government. The views in this report therefore should not be construed as representing those of the Department of Energy or other Federal agencies. 


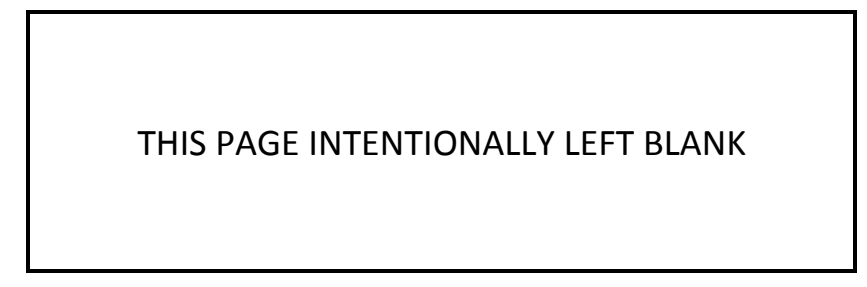




\section{Contacts}

The Annual Energy Review (AER) is prepared by the U.S. Energy Information Administration, Office of Survey Development and Statistical Integration, Integrated Energy Statistics Team, under the direction of Barbara T. Fichman, 202-586-5737 (barbara.fichman@eia.gov). Questions and comments about the AER may be referred to Ryan Repice, 202-586-5828 (ryan.repice@eia.gov), the Office of Communications, 202-586-8800 (infoctr@eia.gov), or the following subject specialists:

1. Energy Overview. . . . . . . . . . . . . . . . . . . . . . . . . Ryan Repice

2. Energy Consumption by Sector. ................. Ryan Repice Manufacturing Energy Consumption Survey............ Robert Adler Residential Energy Consumption Survey.............. James Berry

Commercial Buildings Energy Consumption Survey. ........ . Joelle Michaels

3. Financial Indicators. ...................... Ryan Repice

\section{Energy Resources}

Petroleum and Natural Gas. .................... Steven G. Grape

Coal. ............................... Nicholas Paduano

Uranium............................ Doug Bonnar

Renewable Energy.$\ldots \ldots \ldots \ldots \ldots \ldots \ldots \ldots \ldots$ Christopher R. Namovicz

5. Petroleum and Other Liquids. . . . . . . . . . . . . . . Jennifer Barrick

Prices................................ Marlana Anderson

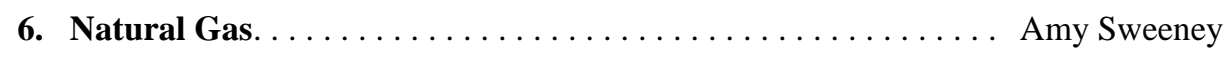

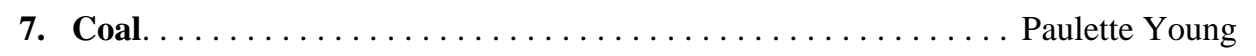

8. Electricity .............................. Channele Wirman

9. Nuclear Energy . . . . . . . . . . . . . . . . . . . . . . . . . . . . Michael Mobilia

10. Renewable Energy $\ldots \ldots \ldots \ldots \ldots \ldots \ldots \ldots \ldots \ldots \ldots \ldots$ Gwendolyn Bredehoeft

\section{Environment}

Greenhouse Gases......................... Perry Lindstrom

Environmental Equipment..................... Natalie Ko

\begin{abstract}
ryan.repice@eia.gov
ryan.repice@eia.gov

robert.adler@eia.gov

james.berry@eia.gov

joelle.michaels@eia.gov

ryan.repice@eia.gov
\end{abstract}

steven.grape@eia.gov

nicholas.paduano@eia.gov

douglas.bonnar@eia.gov

christopher.namovicz@eia.gov

jennifer.barrick@eia.gov

marlana.anderson@eia.gov

amy.sweeney@eia.gov

paulette.young@eia.gov

channele.wirman@eia.gov

michael.mobilia@eia.gov

gwendolyn.bredehoeft@eia.gov

perry.lindstrom@eia.gov

natalie.ko@eia.gov
202-586-5828

202-586-5828

202-586-1134

202-586-5543

202-586-8952

202-586-5828

202-586-1868

202-287-6326

202-586-1085

202-586-7120

202-586-6254

202-586-2970

202-586-2627

202-586-1719

202-586-5356

202-287-6318

202-586-5847

202-586-0934

202-586-3139 


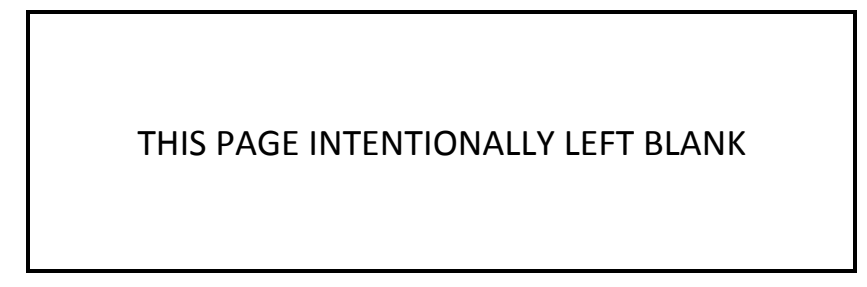




\section{Preface}

This thirtieth edition of the Annual Energy Review (AER) presents the U.S. Energy Information Administration's (EIA) most comprehensive look at integrated energy statistics. The summary statistics on the Nation's energy production, consumption, trade, stocks, and prices cover all major energy commodities and all energyconsuming sectors of the U.S. economy from 1949 through 2011. The AER is EIA's historical record of energy statistics and, because the coverage spans six decades, the statistics in this report are well-suited to long-term trend analysis.

The AER is a companion report to EIA's Monthly Energy Review (MER), and it covers most MER series over a much longer time span. Numerous additional series are included in the AER. The additional series are available because EIA's surveys, on which both reports are largely based, provide more extensive coverage of annual statistics than of monthly statistics.

AER statistics for recent years, particularly 2011, are more likely than statistics for earlier years to be revised by EIA as new information becomes available. The latest edition of the MER, therefore, is the recommended source for the most recent statistics for many of the series reported in the AER.

For the most part, fuel-specific statistics in the AER are expressed in physical units, such as barrels, cubic feet, short tons, and kilowatthours. Summary statistics in
Sections 1 and 2, however, are expressed in British thermal units (Btu), which allows different fuels to be compared and integrated summary statistics, such as the U.S. consumption of primary energy, to be calculated.

The AER emphasizes domestic energy statistics but also covers trade statistics. For example, statistics on petroleum imports by country of origin have been included in Section 5, "Petroleum," in order to give a complete picture of petroleum statistics.

Publication of the AER each year is in keeping with responsibilities given EIA in Section 205(a)(2) of the Department of Energy Organization Act, Public Law 95-91. The report is intended for use by Members of Congress, Federal and State agencies, energy analysts, and the general public. EIA welcomes suggestions from readers regarding its energy statistics. To make a suggestion or to obtain specific information regarding the contents of the AER, readers may contact any of the subject specialists listed as contacts on the preceding page.

Printed copies of the Annual Energy Review 2011 may be obtained by contacting the U.S. Government Printing Office or EIA's Office of Communications, as listed on the inside front cover of this report. The information in this report is also available electronically at http://www.eia.gov/totalenergy/data/annual/. 


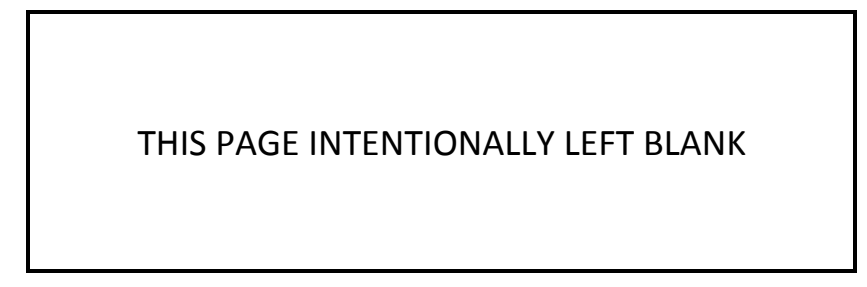




\section{Contents}

\section{Sections}

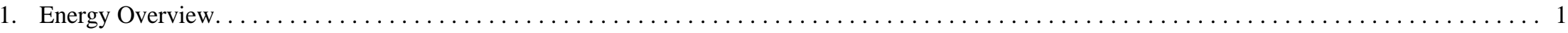

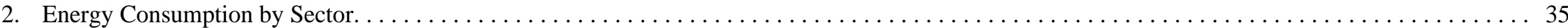

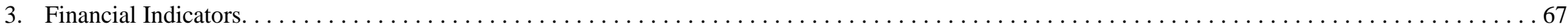

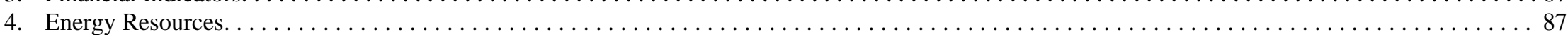

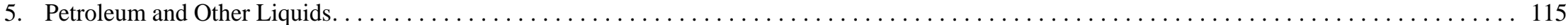

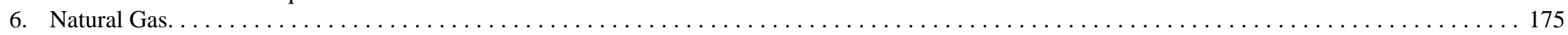

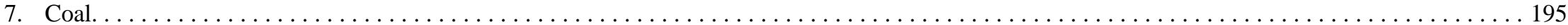

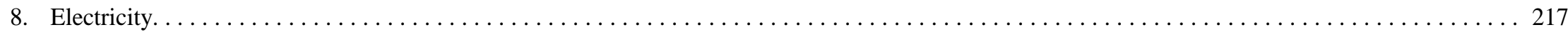

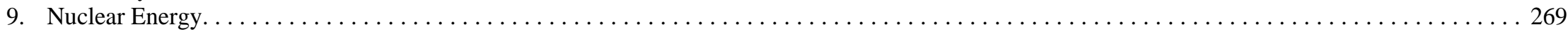

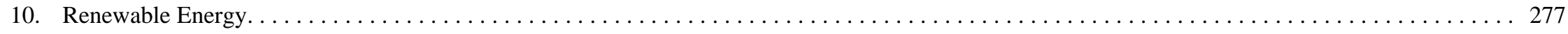

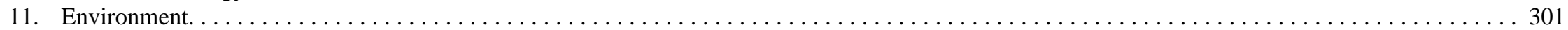

\section{Appendices}

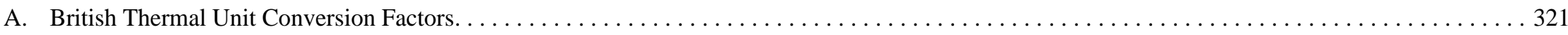

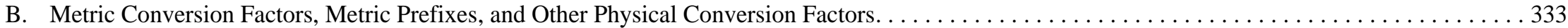

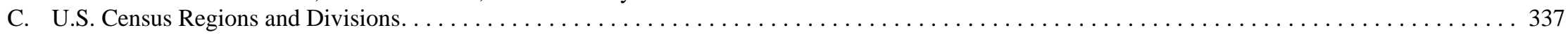

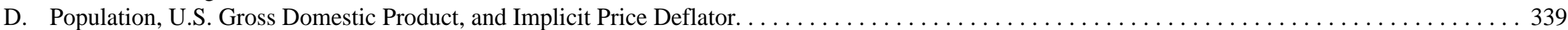

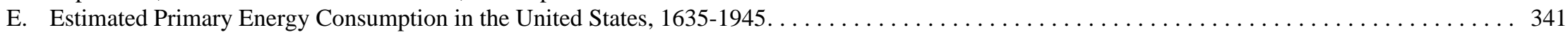

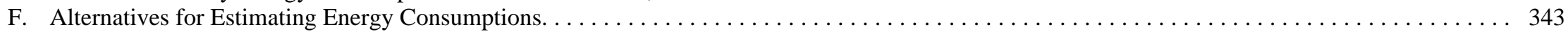

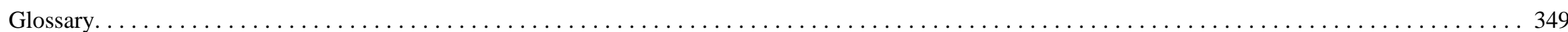

\section{Flow Diagrams}

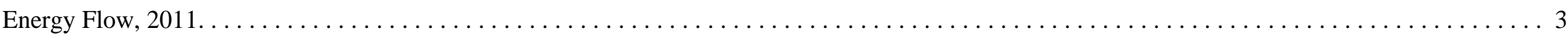

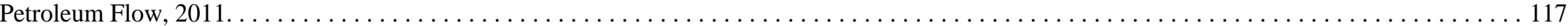

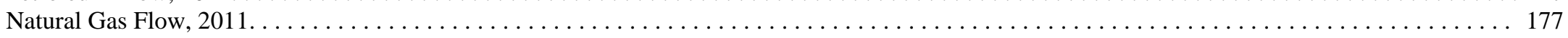

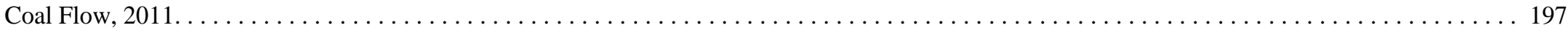

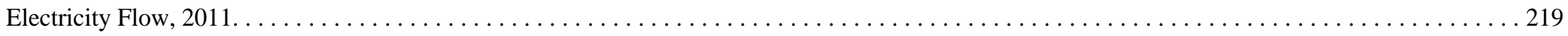


Tables

\section{Energy Overview}

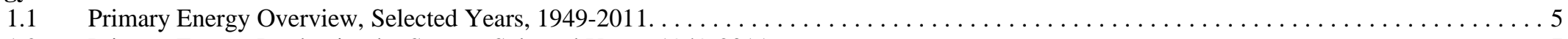

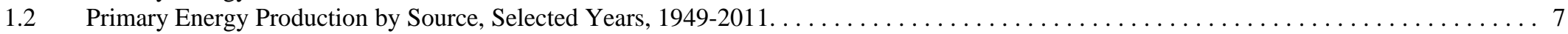

1.3 Primary Energy Consumption Estimates by Source, Selected Years, 1949-2011 . . . . . . . . . . . . . . . . . . . . . . 9

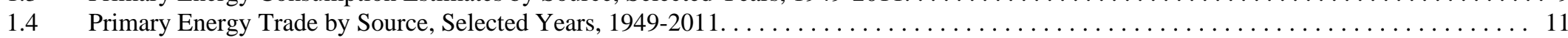

1.5 Energy Consumption, Expenditures, and Emissions Indicators Estimates, Selected Years, 1949-2011 . . . . . . . . . . . . . . . . . . . . . . . . . 13

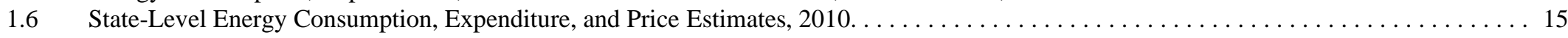

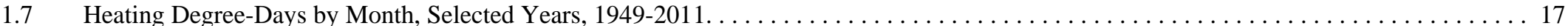

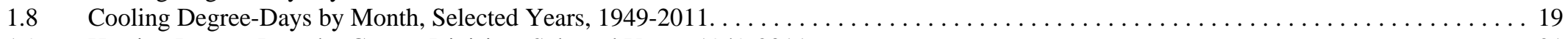

$1.9 \quad$ Heating Degree-Days by Census Division, Selected Years, 1949-2011 . . . . . . . . . . . . . . . . . . . . . . . . . . . . . . . . 21

1.10 Cooling Degree-Days by Census Division, Selected Years, 1949-2011. . . . . . . . . . . . . . . . . . . . . . . . . . 23

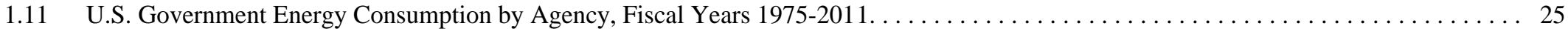

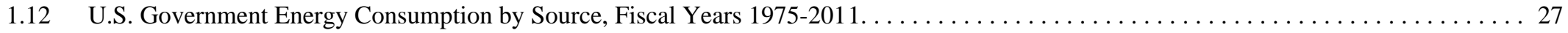

1.13 U.S. Government Energy Consumption by Agency and Source, Fiscal Years 2003, 2010, and 2011 . . . . . . . . . . . . . . . . . . . . . . . 29

1.14 Sales of Fossil Fuels Produced on Federal and American Indian Lands, Fiscal Years, 2003-2011 . . . . . . . . . . . . . . . . . . . . . . . . . . 31

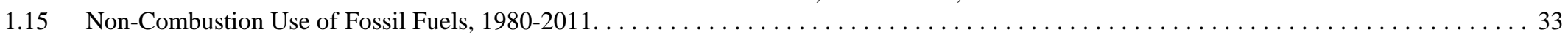

\section{Energy Consumption by Sector}

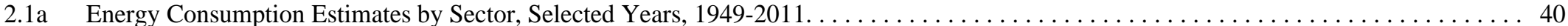

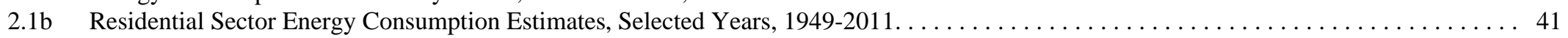

2.1c Commercial Sector Energy Consumption Estimates, Selected Years, 1949-2011 . . . . . . . . . . . . . . . . . . . . . . . . . . . . . . . . . 42

2.1d Industrial Sector Energy Consumption Estimates, Selected Years, 1949-2011. . . . . . . . . . . . . . . . . . . . . . . . . 43

2.1e Transportation Sector Energy Consumption Estimates, Selected Years, 1949-2011 . . . . . . . . . . . . . . . . . . . . . . . . 44

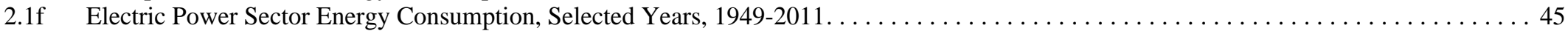

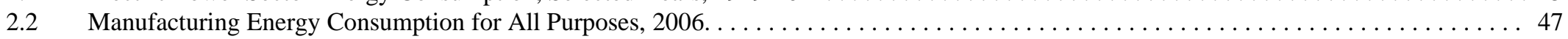

2.3 Manufacturing Energy Consumption for Heat, Power, and Electricity Generation by End Use, 2006. . . . . . . . . . . . . . . . . . . . . . . . . . 49

$2.4 \quad$ Household Energy Consumption by Census Region, Selected Years, 1978-2005. . . . . . . . . . . . . . . . . . . . . 51

2.5 Household Energy Consumption and Expenditures by End Use and Energy Source, Selected Years, 1978- 2005. . . . . . . . . . . . . . . . . . . . 53

2.6 Household End Uses: Fuel Types, Appliances, and Electronics, Selected Years, 1978-2009. . . . . . . . . . . . . . . . . . . . 5 55

2.7 Type of Heating in Occupied Housing Units, Selected Years, 1950-2009. . . . . . . . . . . . . . . . . . . . . . . . . . . . . . . . . . . . . . 57

2.8 Motor Vehicle Mileage, Fuel Consumption, and Fuel Economy, Selected Years, 1949-2010 . . . . . . . . . . . . . . . . . . . . . . 59

2.9 Commercial Buildings Consumption by Energy Source, Selected Years, 1979-2003. . . . . . . . . . . . . . . . . . . . . . . . 61

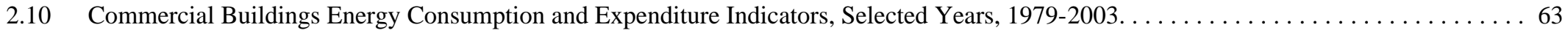

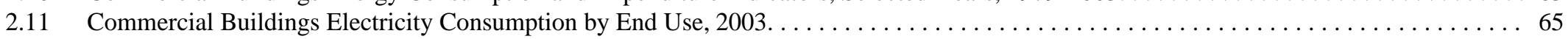

\section{Financial Indicators}

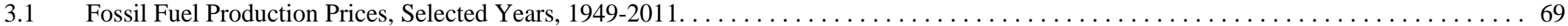

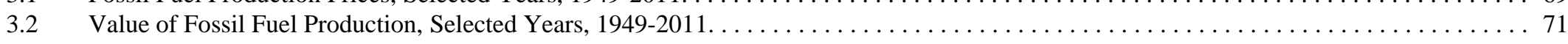

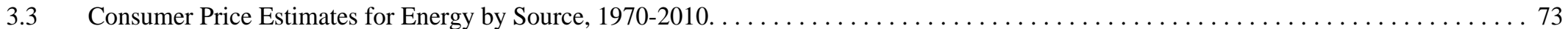

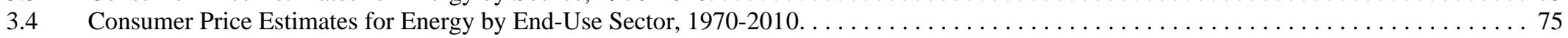

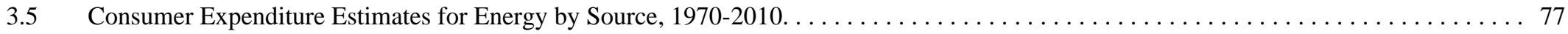

3.6 Consumer Expenditure Estimates for Energy by End-Use Sector, 1970-2010 . . . . . . . . . . . . . . . . . . . . . . . . . . . . 79 


\section{Tables}

\section{Financial Indicators-Continued}

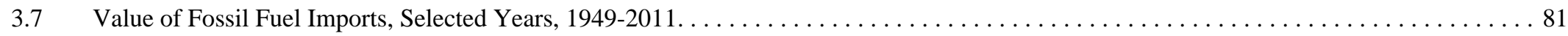

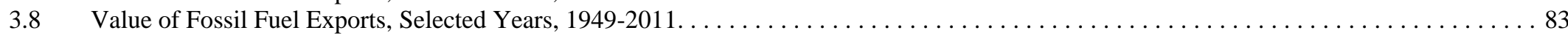

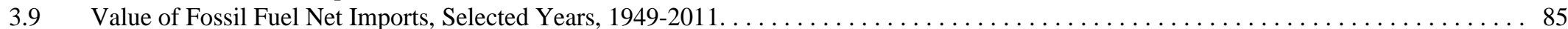

\section{Energy Resources}

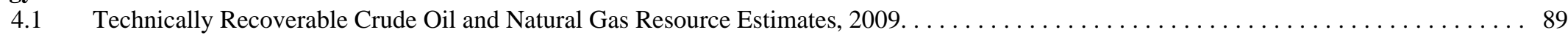

$4.2 \quad$ Crude Oil and Natural Gas Cumulative Production and Proved Reserves, $1977-2010 \ldots \ldots \ldots \ldots \ldots \ldots \ldots \ldots \ldots \ldots \ldots \ldots \ldots \ldots \ldots$

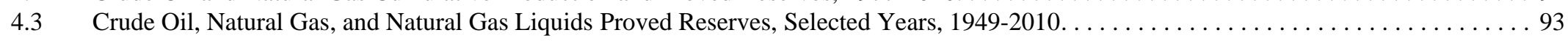

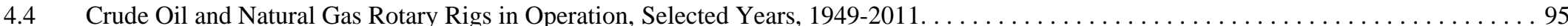

$4.5 \quad$ Crude Oil and Natural Gas Exploratory and Development Wells, Selected Years, $1949-2010 \ldots \ldots \ldots \ldots \ldots \ldots \ldots \ldots \ldots \ldots \ldots \ldots$

$4.6 \quad$ Crude Oil and Natural Gas Exploratory Wells, Selected Years, $1949-2010 \ldots \ldots \ldots \ldots \ldots \ldots \ldots \ldots \ldots \ldots \ldots \ldots \ldots \ldots \ldots \ldots \ldots \ldots$

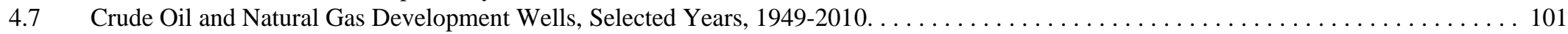

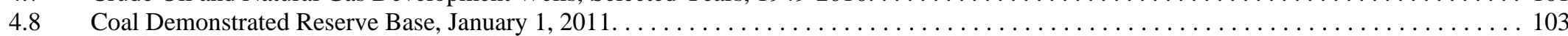

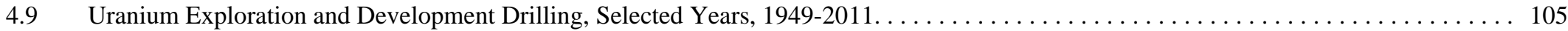

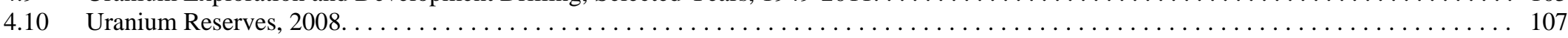

\section{Petroleum and Other Liquids}

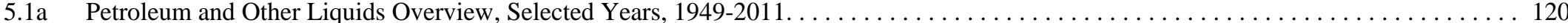

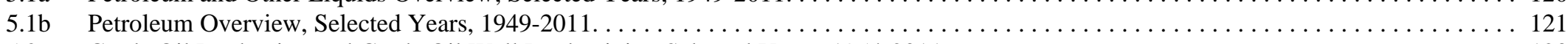

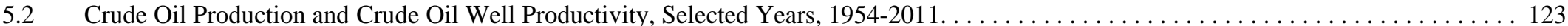

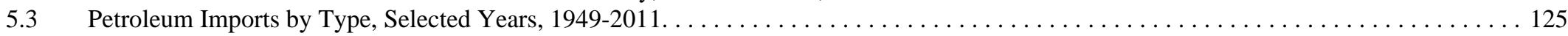

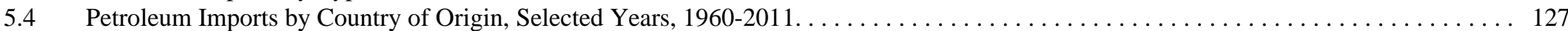

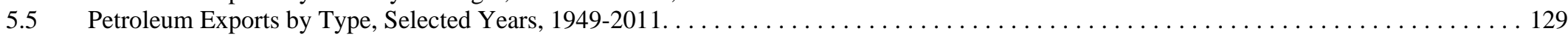

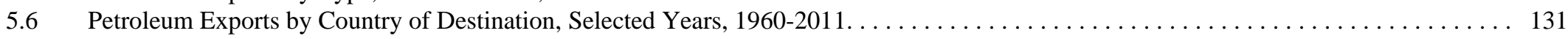

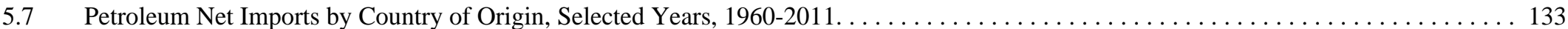

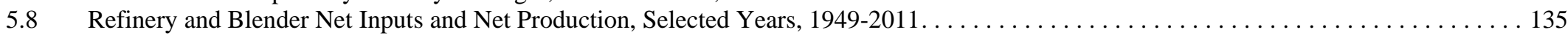

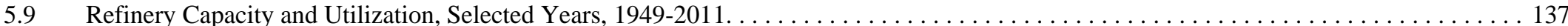

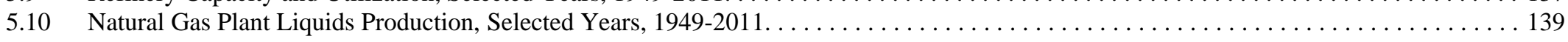

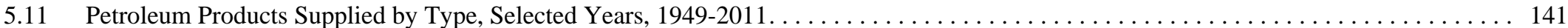

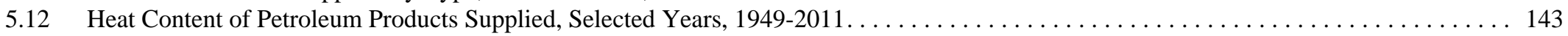

5.13a Petroleum Consumption Estimates: Residential and Commercial Sectors, Selected Years, 1949-2011 . . . . . . . . . . . . . . . . . . . . . 146

5.13b Petroleum Consumption Estimates: Industrial Sector, Selected Years, 1949-2011 . . . . . . . . . . . . . . . . . . . . . . . . . . . . . 147

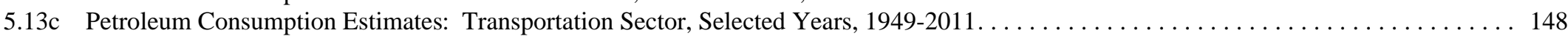

5.13d Petroleum Consumption Estimates: Electric Power Sector, Selected Years, 1949-2011. . . . . . . . . . . . . . . . . . . . . . . . . . 149

5.14a $\quad$ Heat Content of Petroleum Consumption Estimates: Residential and Commercial Sectors, Selected Years, $1949-2011 \ldots \ldots \ldots \ldots \ldots \ldots \ldots$

5.14b Heat Content of Petroleum Consumption Estimates: Industrial Sector, Selected Years, 1949-2011. . . . . . . . . . . . . . . . . . . . . . . 152

5.14 Heat Content of Petroleum Consumption Estimates: Transportation and Electric Power Sectors, Selected Years, 1949-2011 . . . . . . . . 153

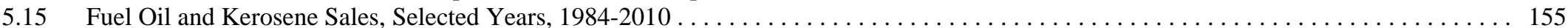

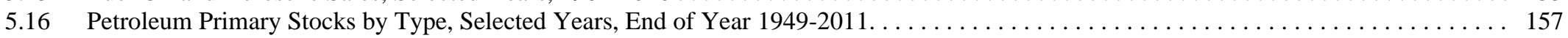

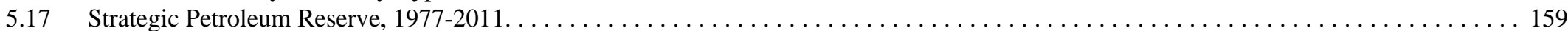


Tables

5. Petroleum and Other Liquids-Continued

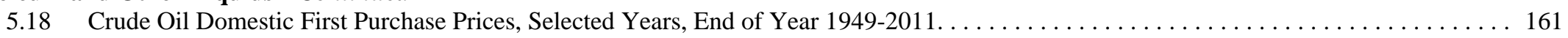

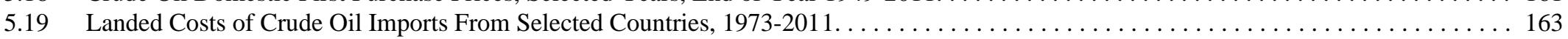

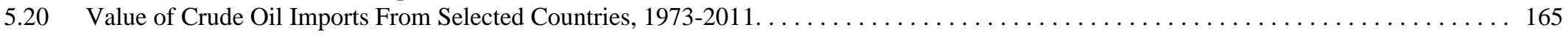

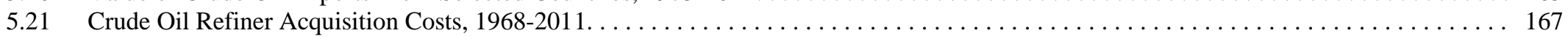

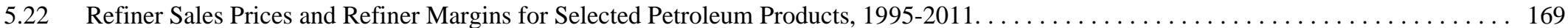

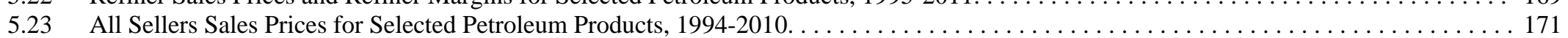

5.24 Retail Motor Gasoline and On-Highway Diesel Fuel Prices, Selected Years, 1949-2011 . . . . . . . . . . . . . . . . . . . . . . . . . . . . 173

\section{Natural Gas}

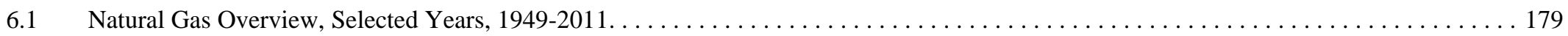

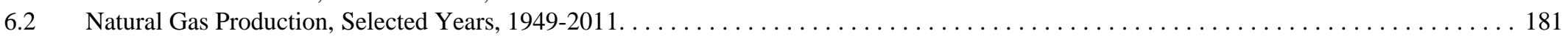

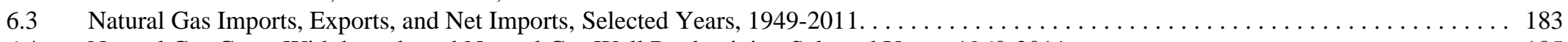

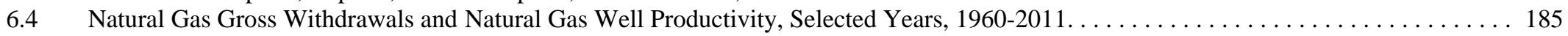

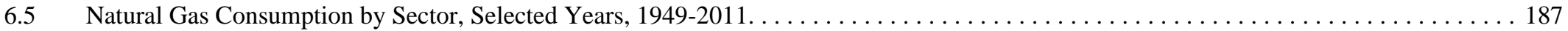

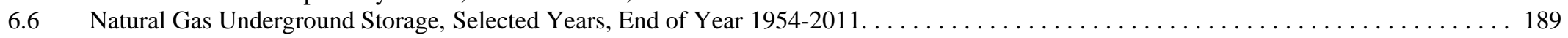

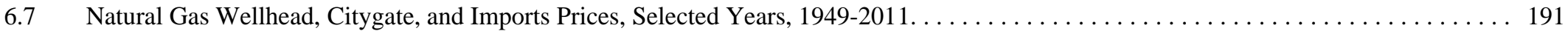

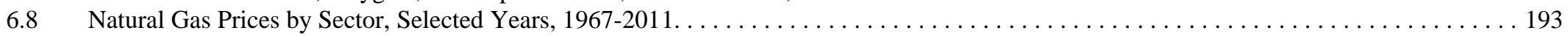

7. Coal

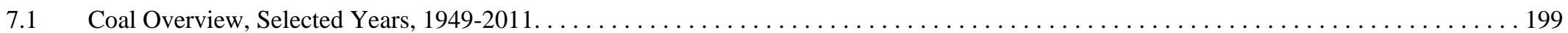

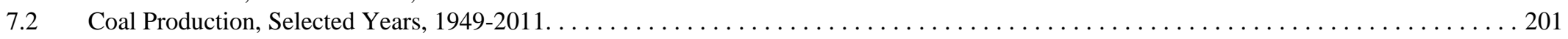

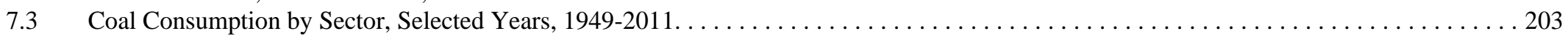

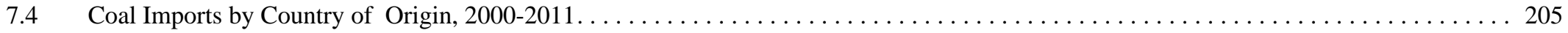

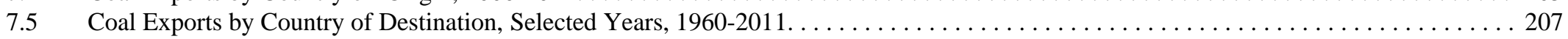

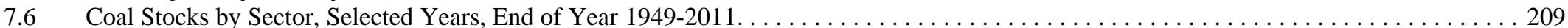

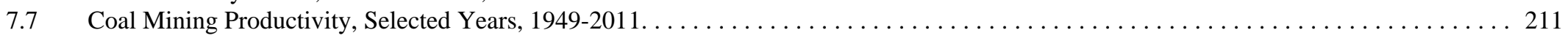

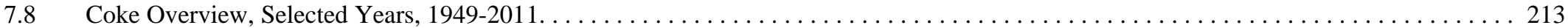

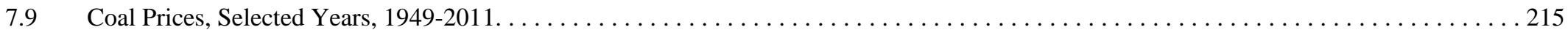

\section{Electricity}

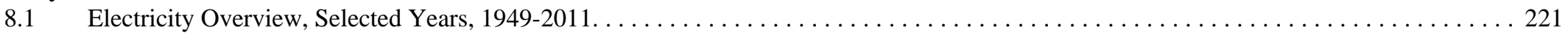

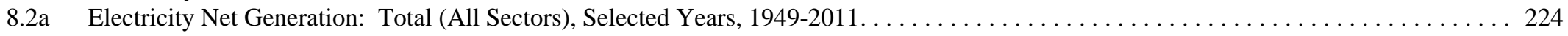

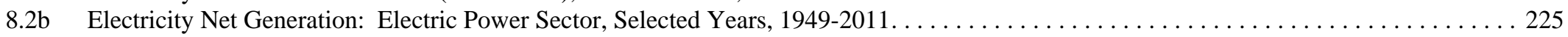

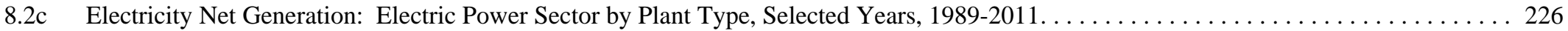

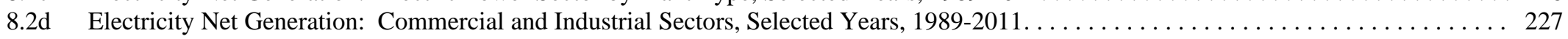

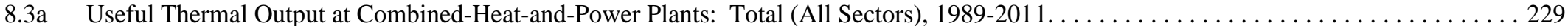

8.3b Useful Thermal Output at Combined-Heat-and-Power Plants: Electric Power Sector, 1989-2011 . . . . . . . . . . . . . . . . . . . 230

8.3c Useful Thermal Output at Combined-Heat-and-Power Plants: Commercial and Industrial Sectors, Selected Years, 1989-2011 . . . . . . . . 231

8.4a Consumption for Electricity Generation by Energy Source: Total (All Sectors), Selected Years, $1949-2011 \ldots \ldots \ldots \ldots \ldots \ldots \ldots \ldots 233$

8.4b Consumption for Electricity Generation by Energy Source: Electric Power Sector, Selected Years, $1949-2011 \ldots \ldots \ldots \ldots \ldots \ldots \ldots$. . . . . .

8.4c Consumption for Electricity Generation by Energy Source: Commercial and Industrial Sectors, Selected Years, 1989-2011 . . . . . . . . 235 


\section{Tables}

\section{Electricity-Continued}

8.5a Consumption of Combustible Fuels for Electricity Generation: Total (All Sectors), Selected Years, 1949-2011 . . . . . . . . . . . 238

8.5b Consumption of Combustible Fuels for Electricity Generation: Electric Power Sector, Selected Years, 1949-2011 . . . . . . . . . . . 239

8.5c Consumption of Combustible Fuels for Electricity Generation: Electric Power Sector by Plant Type, Selected Years, 1989-2011 . . . . . . 240

8.5d Consumption of Combustible Fuels for Electricity Generation: Commercial and Industrial Sectors, Selected Years, 1989-2011 . . . . . . . . 241

8.6a Estimated Consumption of Combustible Fuels for Useful Thermal Output at Combined-Heat-and-Power Plants:

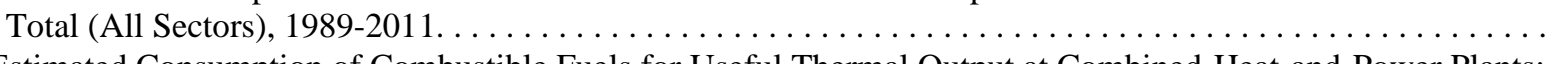

8.6b Estimated Consumption of Combustible Fuels for Useful Thermal Output at Combined-Heat-and-Power Plants:

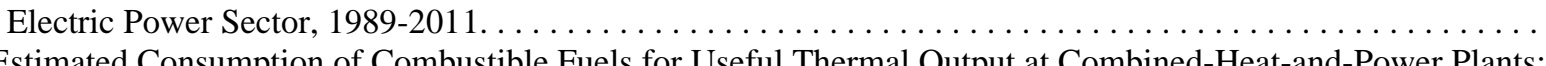

8.6c Estimated Consumption of Combustible Fuels for Useful Thermal Output at Combined-Heat-and-Power Plants:

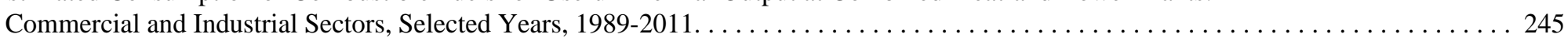

8.7a Consumption of Combustible Fuels for Electricity Generation and Useful Thermal Output: Total (All Sectors), 1989-2011 . . . . . . . . . 247

8.7b Consumption of Combustible Fuels for Electricity Generation and Useful Thermal Output: Electric Power Sector, 1989-2011 . . . . . . . 248

8.7c Consumption of Combustible Fuels for Electricity Generation and Useful Thermal Output:

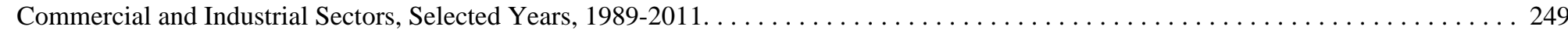

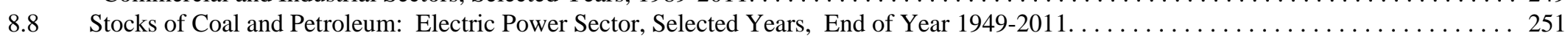

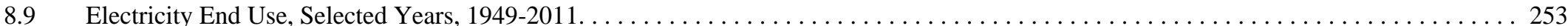

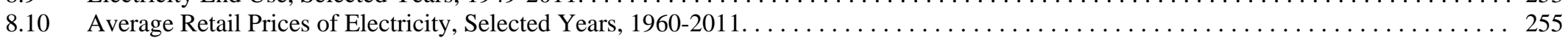

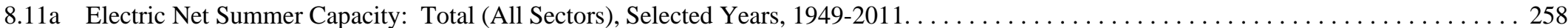

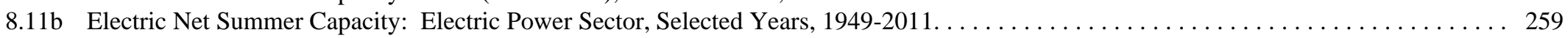

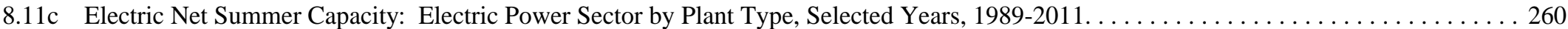

8.11d Electric Net Summer Capacity: Commercial and Industrial Sectors , Selected Years, 1989-2011 . . . . . . . . . . . . . . . . . . . . . 261

8.12a Electric Noncoincident Peak Load and Capacity Margin: Summer Peak Period, 1986-2011 . . . . . . . . . . . . . . . . . . . . . . . . . . 264

8.12b Electric Noncoincident Peak Load and Capacity Margin, Winter Peak Period, 1986-2011 . . . . . . . . . . . . . . . . . . . . . . 265

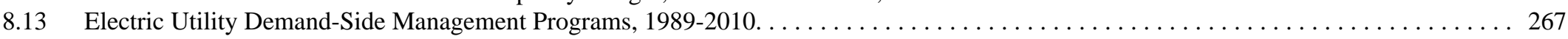

\section{Nuclear Energy}

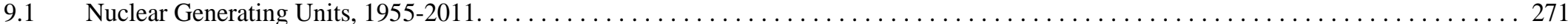

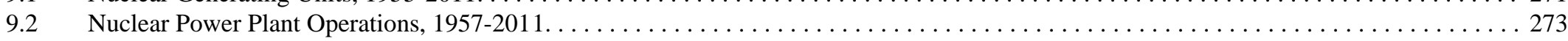

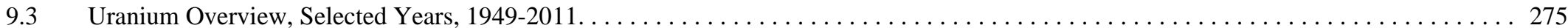

\section{Renewable Energy}

10.1 Renewable Energy Production and Consumption by Primary Energy Source, Selected Years, 1949-2011 . . . . . . . . . . . . . . 279

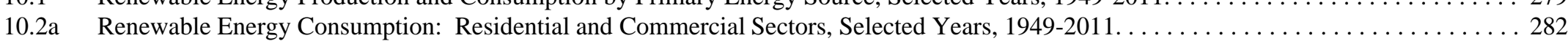

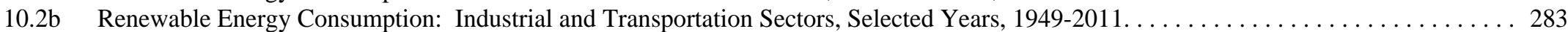

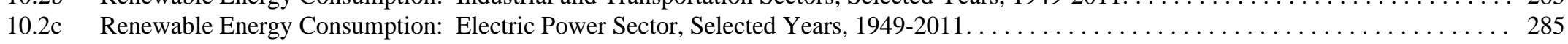

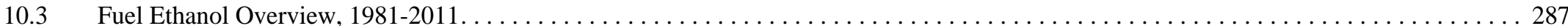

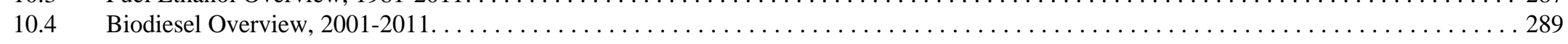

10.5 Estimated Number of Alternative-Fueled Vehicles in Use and Fuel Consumption, 1992-2010 . . . . . . . . . . . . . . . . . . . . . . . . 291

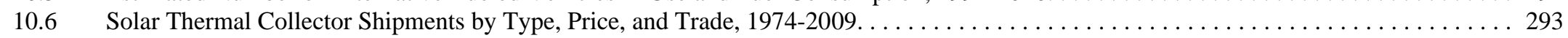




\section{Tables}

10. Renewable Energy-Continued

10.7 Solar Thermal Collector Shipments by Market Sector, End Use, and Type, 2001-2009. . . . . . . . . . . . . . . . . 295

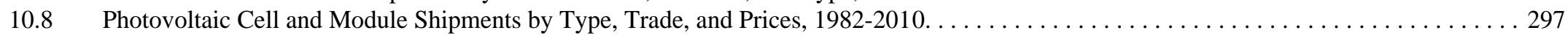

10.9 Photovoltaic Cell and Module Shipments by Sector and End Use, 1989-2010 . . . . . . . . . . . . . . . . . . . . . . . . . . . . 299

\section{Environment}

11.1 Carbon Dioxide Emissions From Energy Consumption by Source, Selected Years, 1949-2011 . . . . . . . . . . . . . . . . . . . . . . . 303

11.2a Carbon Dioxide Emissions From Energy Consumption: Residential Sector, Selected Years, 1949-2011 . . . . . . . . . . . . . . . . . . . . . . . . 305

11.2b Carbon Dioxide Emissions From Energy Consumption: Commercial Sector, Selected Years, 1949-2011. . . . . . . . . . . . . . . . . . . . . 306

11.2c Carbon Dioxide Emissions From Energy Consumption: Industrial Sector, Selected Years, 1949-2011 . . . . . . . . . . . . . . . . . . . . . . . . . 307

11.2d Carbon Dioxide Emissions From Energy Consumption: Transportation Sector, Selected Years, 1949-2011. . . . . . . . . . . . . . . . . . . . 308

11.2e Carbon Dioxide Emissions From Energy Consumption: Electric Power Sector, Selected Years, 1949-2011. . . . . . . . . . . . . . . . . . . . . . . 309

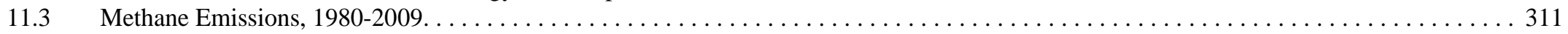

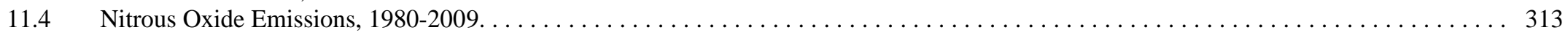

11.5a Emissions From Energy Consumption for Electricity Generation and Useful Thermal Output: Total (All Sectors), 1989-2010. . . . . . . . . . 315

11.5b Emissions From Energy Consumption for Electricity Generation and Useful Thermal Output: Electric Power Sector, 1989-2010. . . . . . . . 316

11.5c Emissions From Energy Consumption for Electricity Generation and Useful Thermal Output:

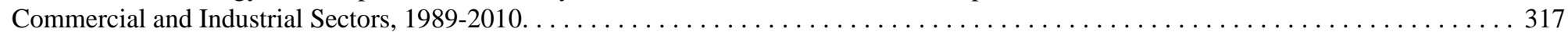

11.6 Installed Nameplate Capacity of Fossil-Fuel Steam-Electric Generators With Environmental Equipment, 1985-2010. . . . . . . . . . . . . . . . . 319

\section{Appendix A. British Thermal Unit Conversion Factors}

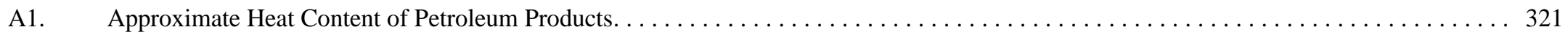

A2. $\quad$ Approximate Heat Content of Petroleum Production, Imports, and Exports, Selected Years, 1949-2011. . . . . . . . . . . . . . . . . . . 322

A3. Approximate Heat Content of Petroleum Consumption and Biofuels Production, Selected Years, 1949-2011. . . . . . . . . . . . . . . . . 323

A4. Approximate Heat Content of Natural Gas, Selected Years, 1949-2011. . . . . . . . . . . . . . . . . . . . . . . . . . . . . . . . 324

A5. Approximate Heat Content of Coal and Coal Coke, Selected Years, 1949-2011 . . . . . . . . . . . . . . . . . . . . . . . . . . . . 325

A6. Approximate Heat Rates for Electricity, and Heat Content of Electricity, Selected Years, 1949-2011 . . . . . . . . . . . . . . . . . . . . . . . . . 326

Appendix B. Metric Conversion Factors, Metric Prefixes, and Other Physical Conversion Factors

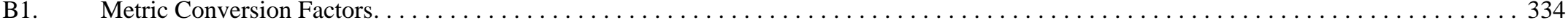

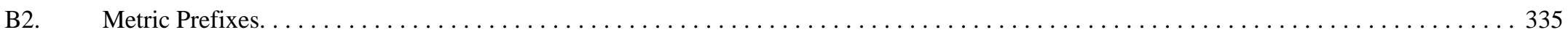

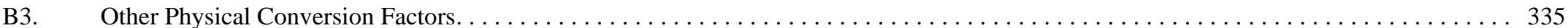

Appendix D. Population, U.S. Gross Domestic Product, and Implict Price Deflator

D1. Population, U.S. Gross Domestic Product, and Implicit Price Deflator, Selected Years, 1949-2011. . . . . . . . . . . . . . . . . . . . . . . . 339

Appendix E. Estimated Energy Consumption in the United States, 1635-1945

E1. $\quad$ Estimated Primary Energy Consumption in the United States, Selected Years, 1635-1945. 


\section{Tables}

Appendix F. Alternatives for Estimating Energy Consumption

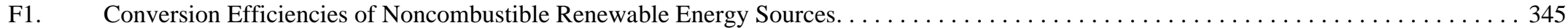

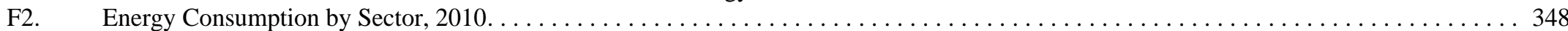

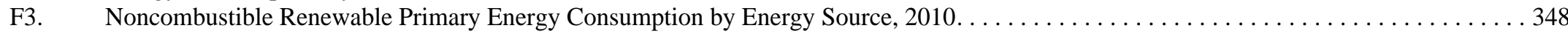




\section{Figures}

\section{Energy Overview}

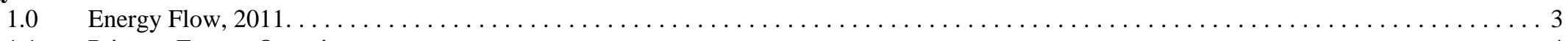

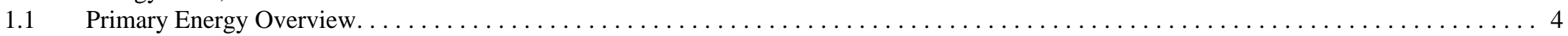

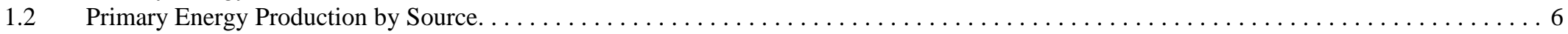

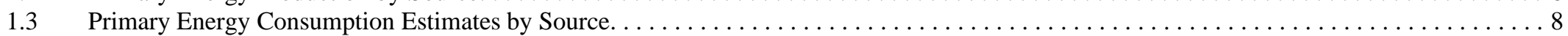

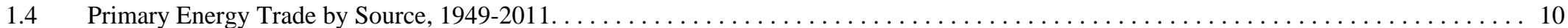

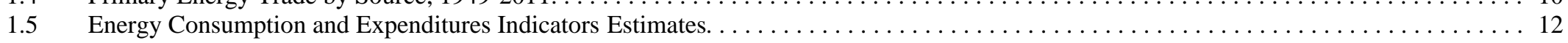

1.6 State-Level Energy Consumption Estimates and Estimated Consumption per Capita, 2010 . . . . . . . . . . . . . . . . . . . . . . . . . . . 14

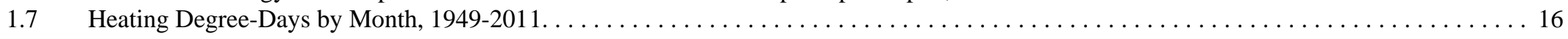

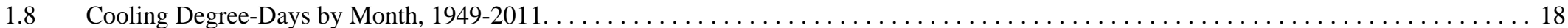

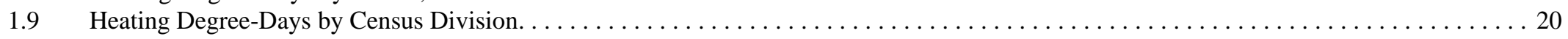

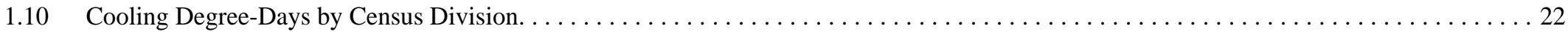

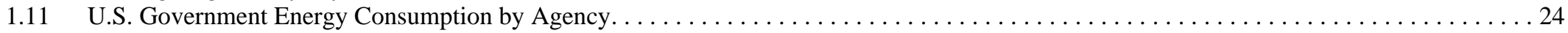

1.12 U.S. Government Energy Consumption by Source, Fiscal Years 1975-2011. . . . . . . . . . . . . . . . . . . . . . . . . . . . 26

1.13 U.S. Government Energy Consumption by Agency and Source, Fiscal Years 2003, 2010, and 2011 . . . . . . . . . . . . . . . . . . 28

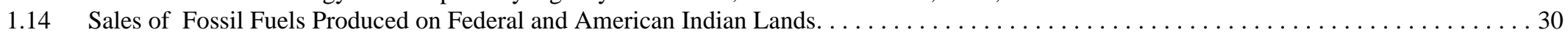

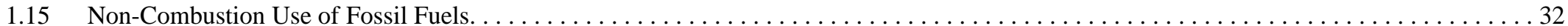

\section{Energy Consumption by Sector}

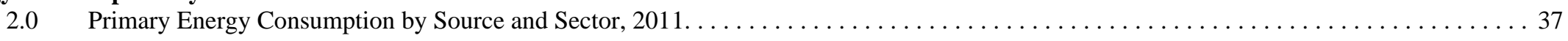

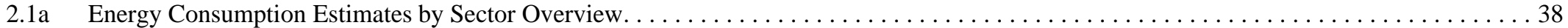

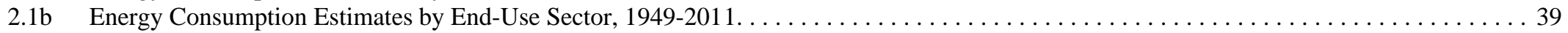

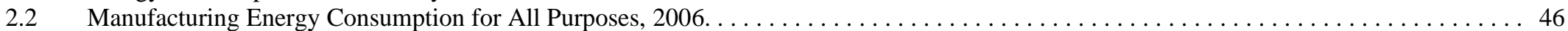

2.3 Manufacturing Energy Consumption for Heat, Power, and Electricity Generation, 2006. . . . . . . . . . . . . . . . . 48

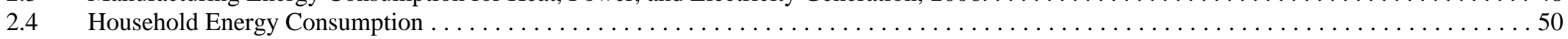

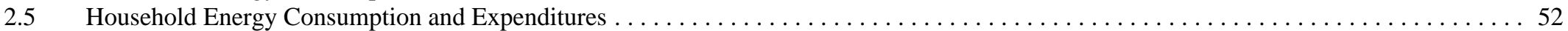

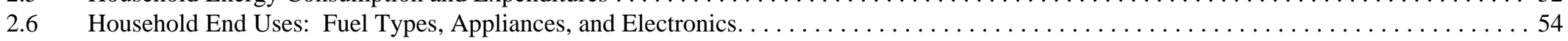

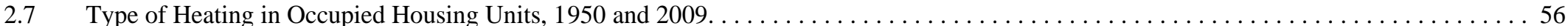

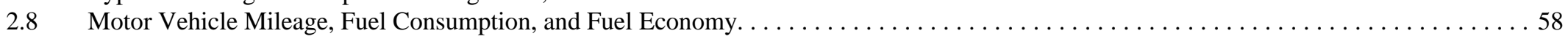

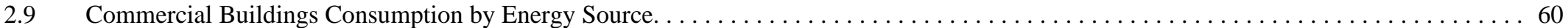

2.10 Commercial Buildings Energy Consumption and Expenditure Indicators, Selected Years, 1979-2003. . . . . . . . . . . . . . . . . . . . 62

2.11 Commercial Buildings Electricity Consumption by End Use, 2003. . . . . . . . . . . . . . . . . . . . . . . . . . . . . . . 64

\section{Financial Indicators}

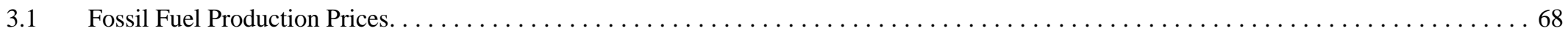

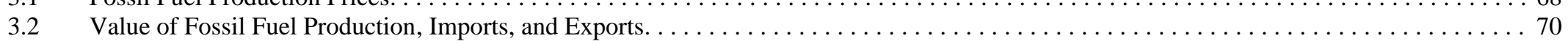

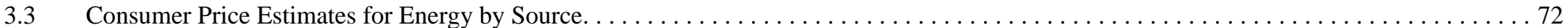

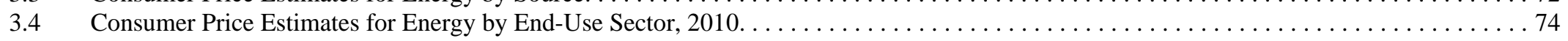

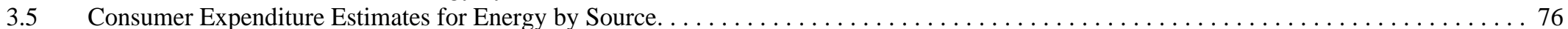

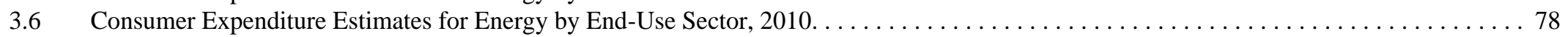

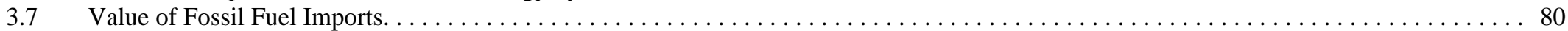

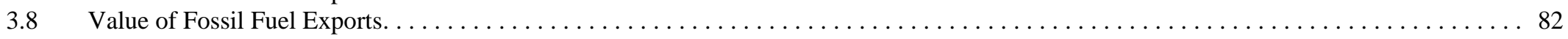




\section{Figures}

3. Financial Indicators-Continued

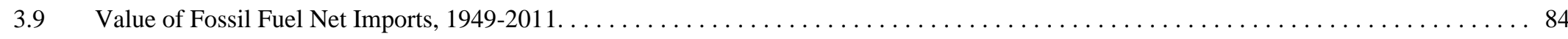

\section{Energy Resources}

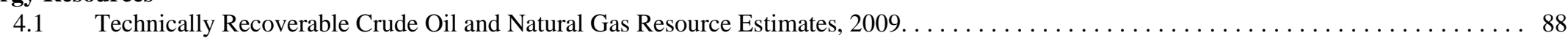

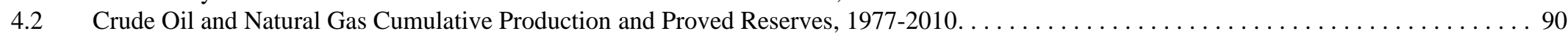

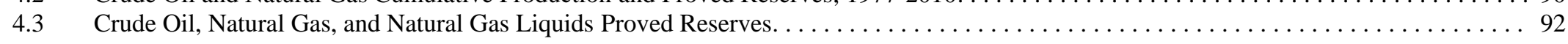

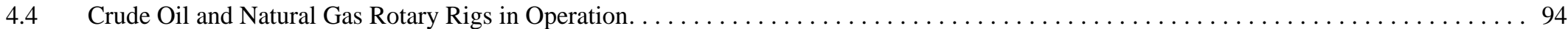

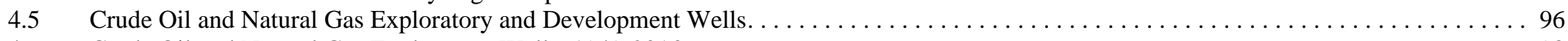

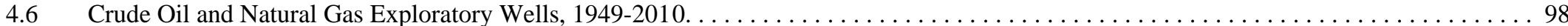

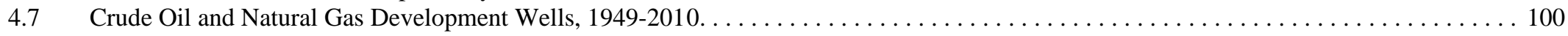

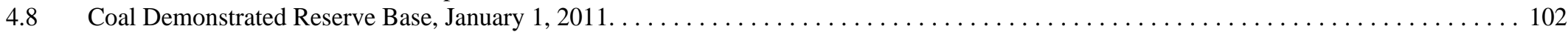

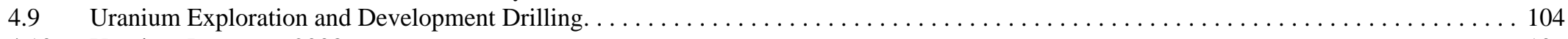

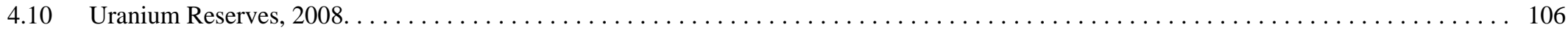

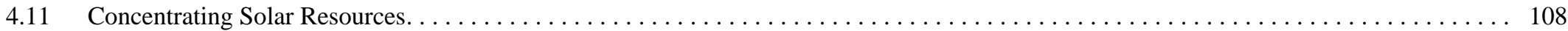

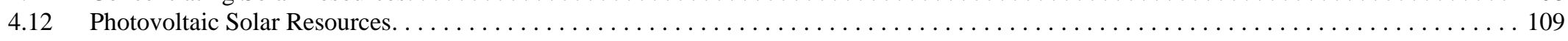

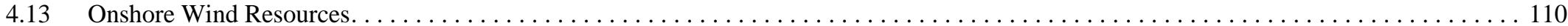

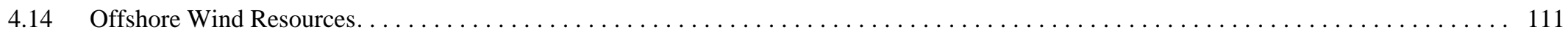

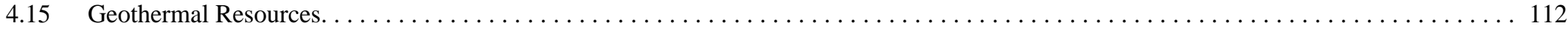

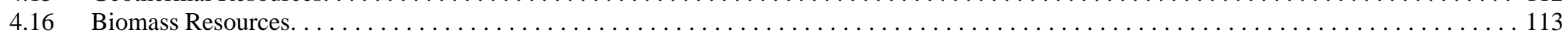

\section{Petroleum and Other Liquids}

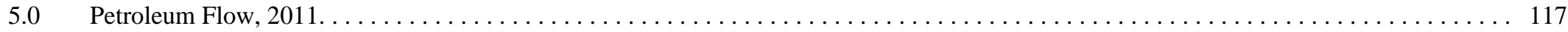

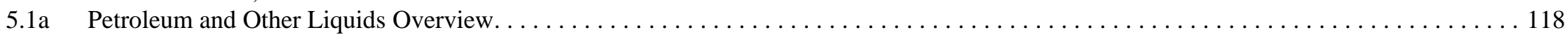

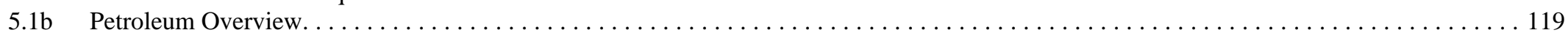

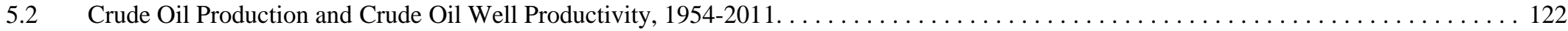

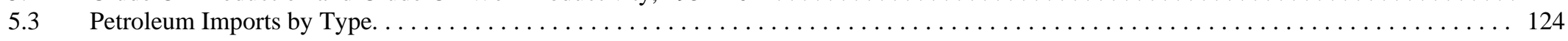

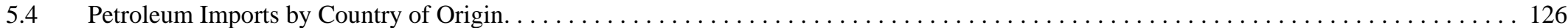

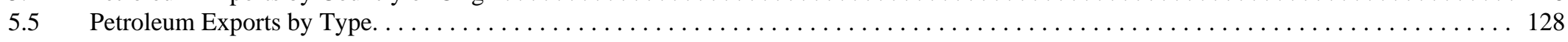

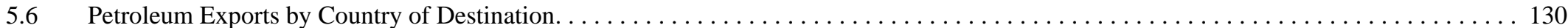

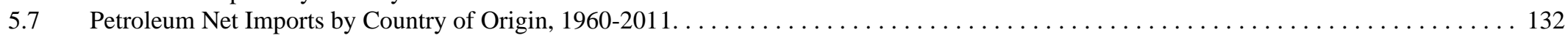

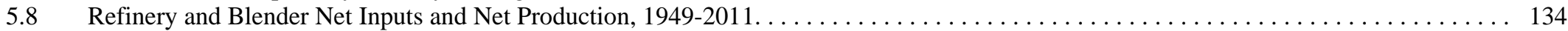

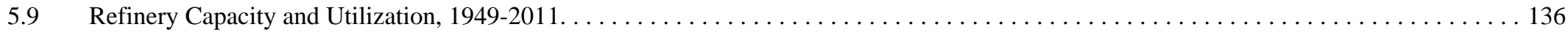

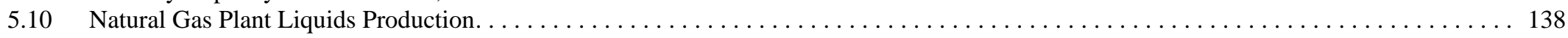

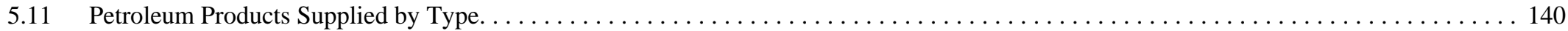

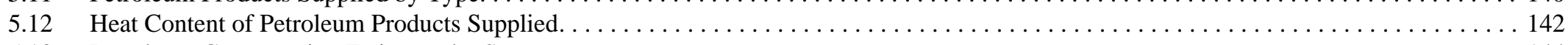

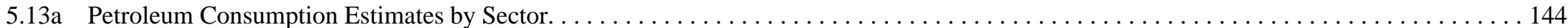

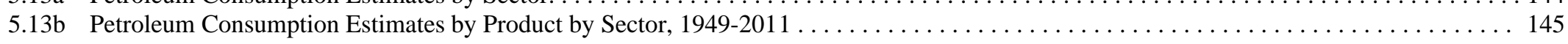

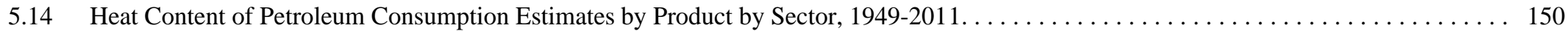

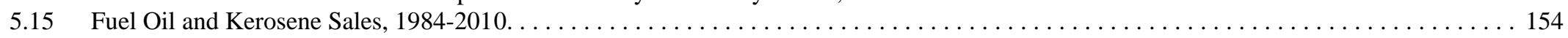




\section{Figures}

\section{Petroleum and Other Liquids-Continued}

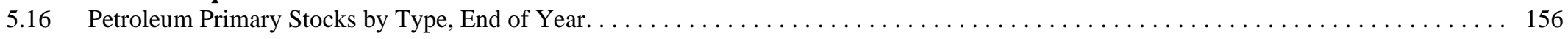

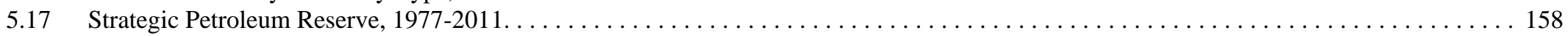

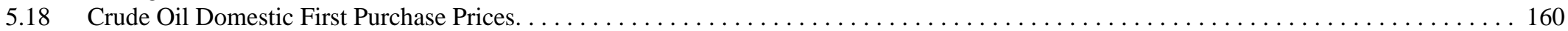

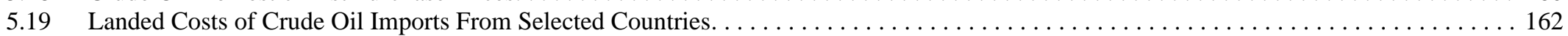

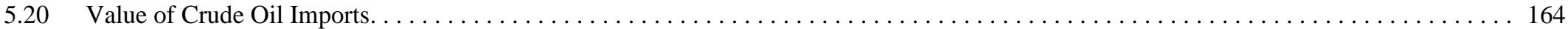

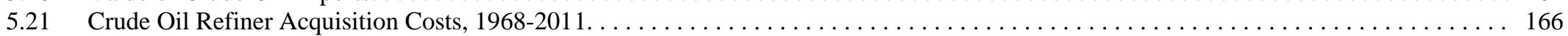

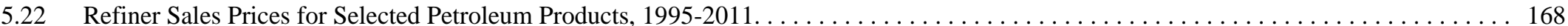

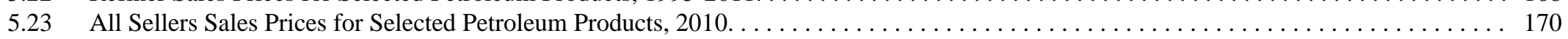

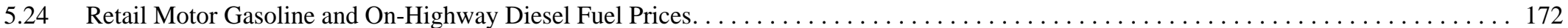

\section{Natural Gas}

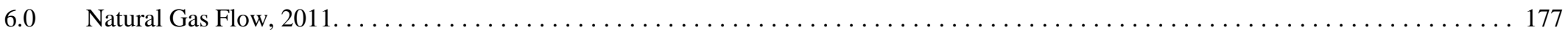

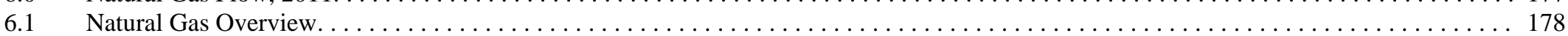

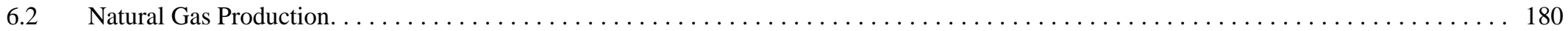

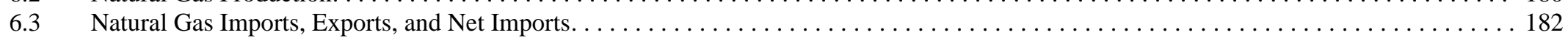

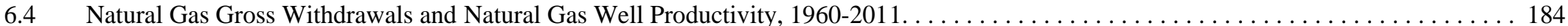

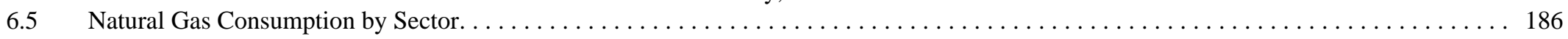

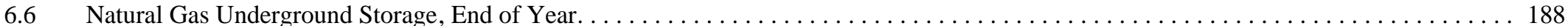

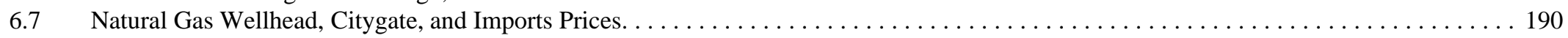

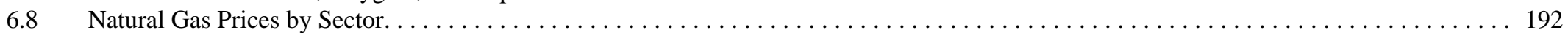

7. Coal

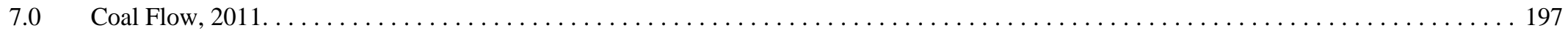

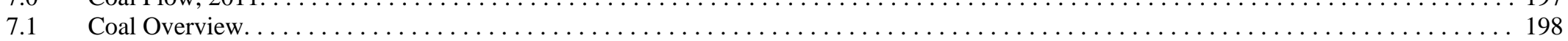

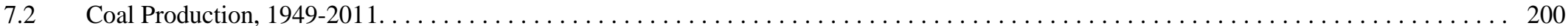

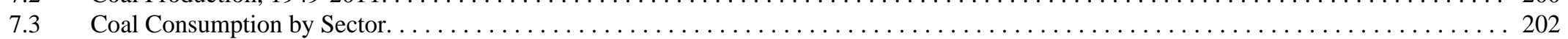

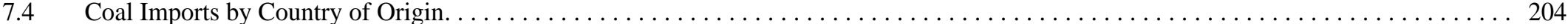

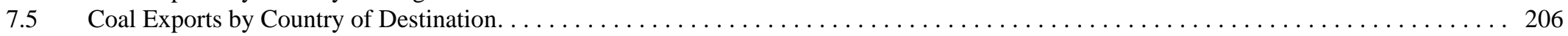

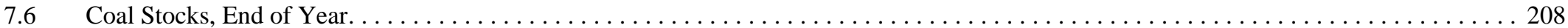

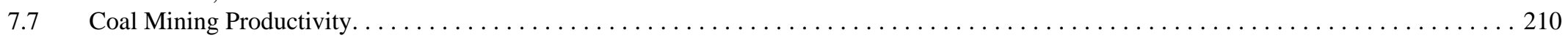

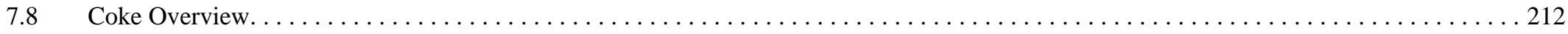

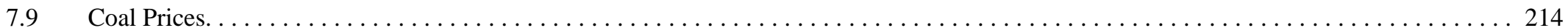

\section{Electricity}

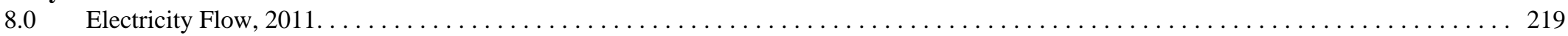

$8.1 \quad$ Electricity Overview. . . . . . . . .

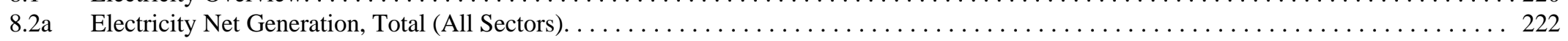

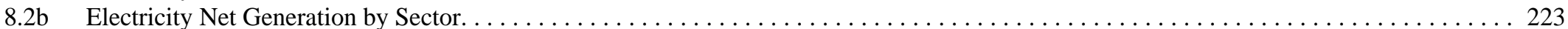

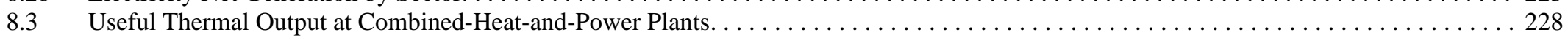

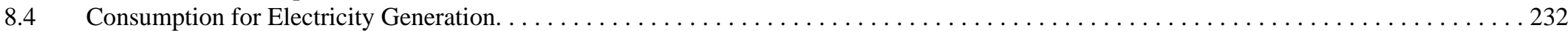

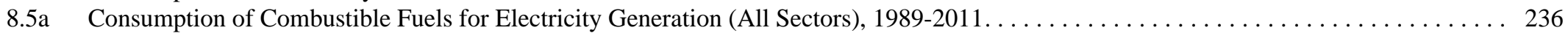




\section{Figures}

8. Electricity—Continued

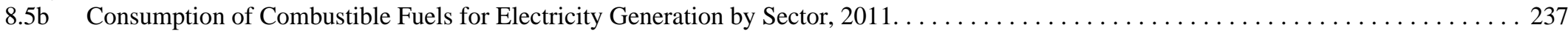
8.6 Estimated Consumption of Combustible Fuels for Useful Thermal Output at Combined-Heat-and-Power Plants by Sector, 1989-2011 . . . . 242
8.7 Consumption of Combustible Fuels for Electricity Generation and Useful Thermal Output, $1989-2011 \ldots \ldots \ldots \ldots \ldots \ldots \ldots \ldots \ldots \ldots$

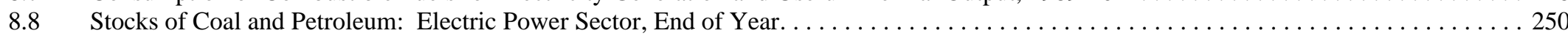

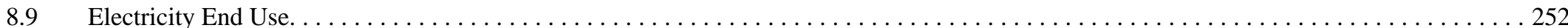

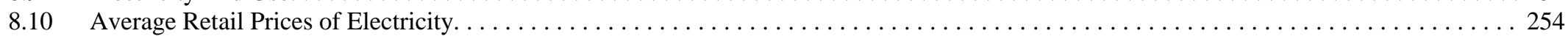

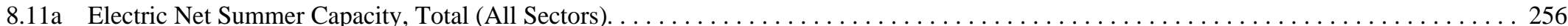

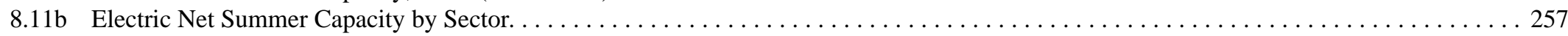
8.12a Electric Noncoincident Peak Load and Capacity Margin: Summer Peak Period . . . . . . . . . . . . . . . . . . . . . . . . . . . 262

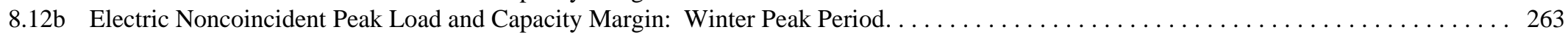

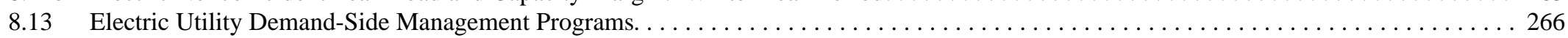

9. Nuclear Energy

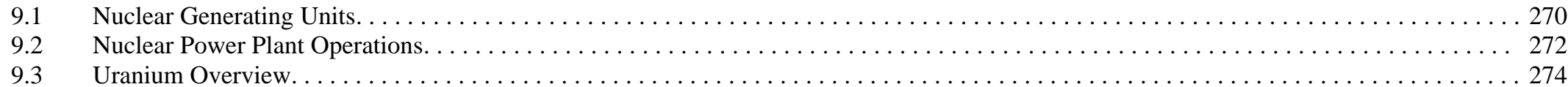

\section{Renewable Energy}

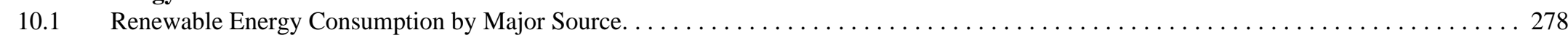

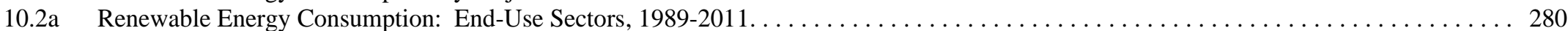

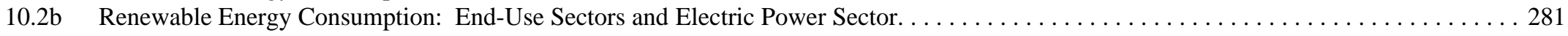

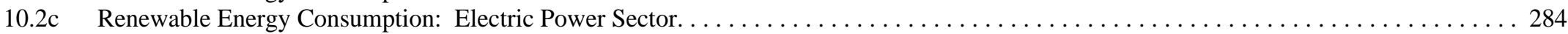

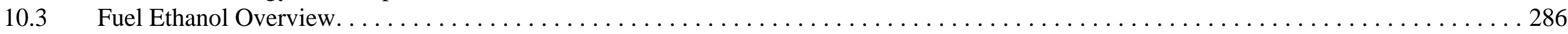

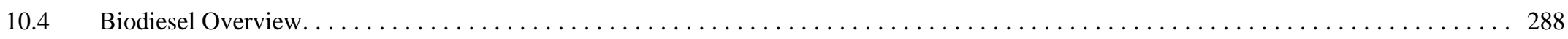

10.5 Estimated Number of Alternative-Fueled Vehicles in Use and Alternative Fuel Consumption. . . . . . . . . . . . . . . . . . . . 290

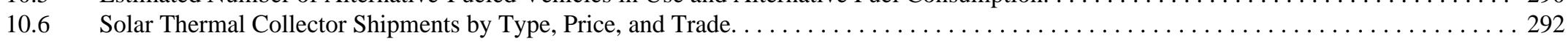

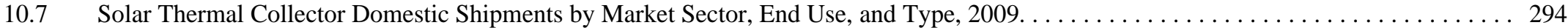

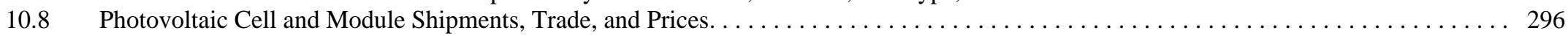

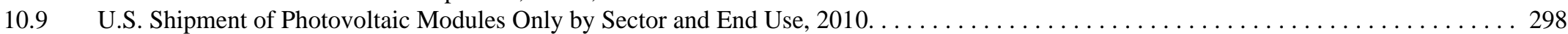

\section{Environment}

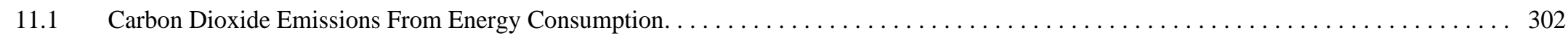

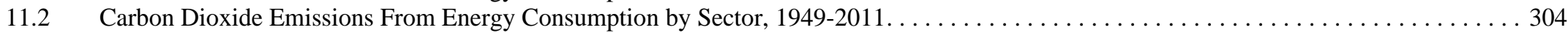

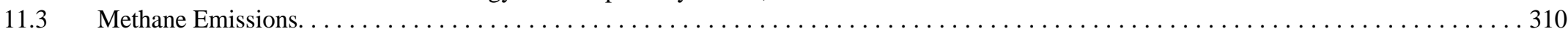

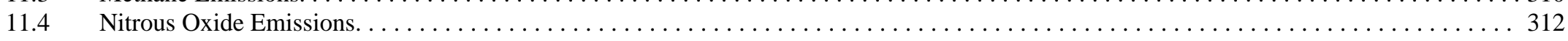

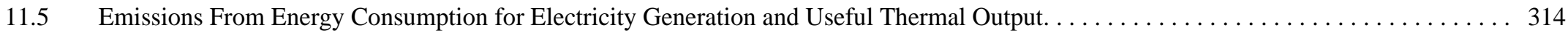

11.6 Installed Nameplate Capacity of Fossil-Fuel Steam-Electric Generators With Environmental Equipment. . . . . . . . . . . . . . . . 318

\section{Appendix C. U.S. Census Regions and Division}

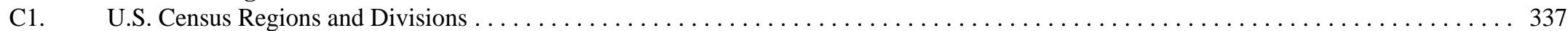




\section{Figures}

Appendix F. Alternatives for Estimating Energy Consumption

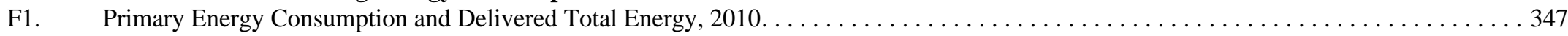




\section{Energy Overview}




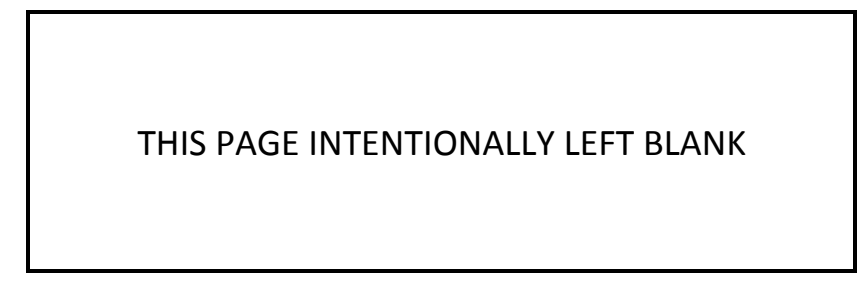


Figure 1.0 Energy Flow, 2011

(Quadrillion Btu)

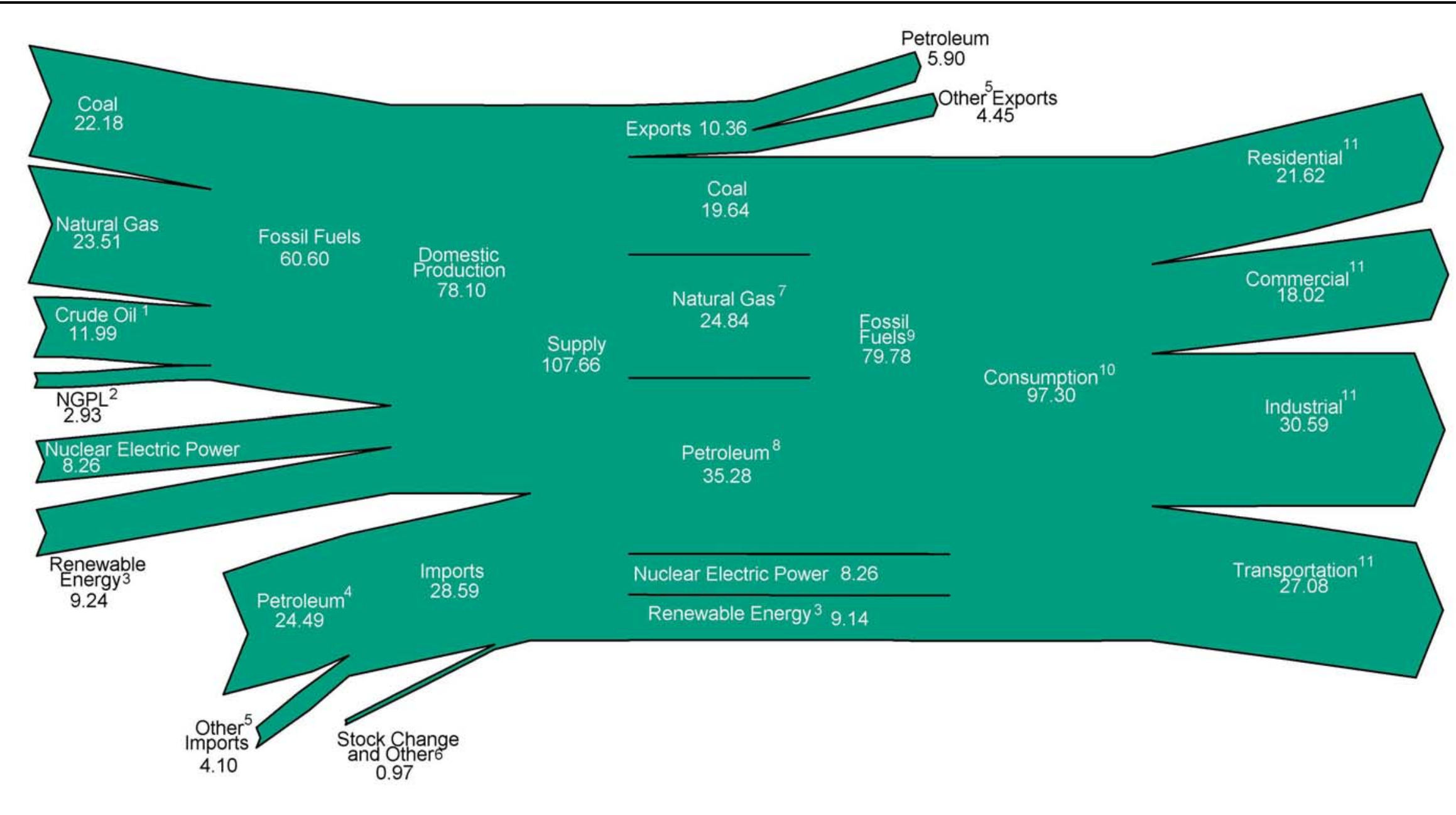

${ }^{1}$ Includes lease condensate.

${ }^{2}$ Natural gas plant liquids.

${ }^{3}$ Conventional hydroelectric power, biomass, geothermal, solar/photovoltaic, and wind.

${ }^{4}$ Crude oil and petroleum products. Includes imports into the Strategic Petroleum Reserve.

${ }^{5}$ Natural gas, coal, coal coke, biofuels, and electricity.

${ }^{6}$ Adjustments, losses, and unaccounted for.

${ }^{7}$ Natural gas only; excludes supplemental gaseous fuels.

${ }^{8}$ Petroleum products, including natural gas plant liquids, and crude oil burned as fuel.

\begin{abstract}
${ }^{9}$ Includes 0.01 quadrillion Btu of coal coke net imports.
${ }^{10}$ Includes 0.13 quadrillion Btu of electricity net imports.

${ }^{11}$ Total energy consumption, which is the sum of primary energy consumption, electricity retail sales, and electrical system energy losses. Losses are allocated to the end-use sectors in proportion to each sector's share of total electricity retail sales. See Note, "Electrical Systems Energy Losses," at end of Section 2.

Notes: - Data are preliminary. - Values are derived from source data prior to rounding for publication. - Totals may not equal sum of components due to independent rounding.
\end{abstract}

Sources: Tables 1.1, 1.2, 1.3, 1.4, and 2.1a. 


\section{Overview, 1949-2011}

$120-$

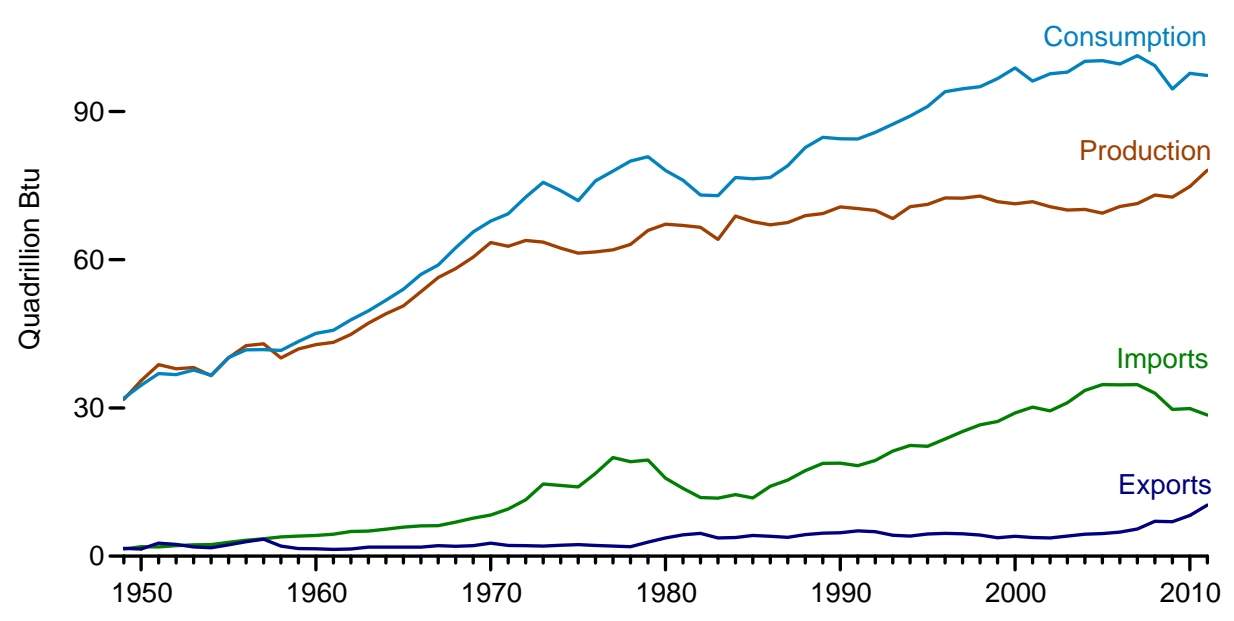

\section{Overview, 2011}

$120-$

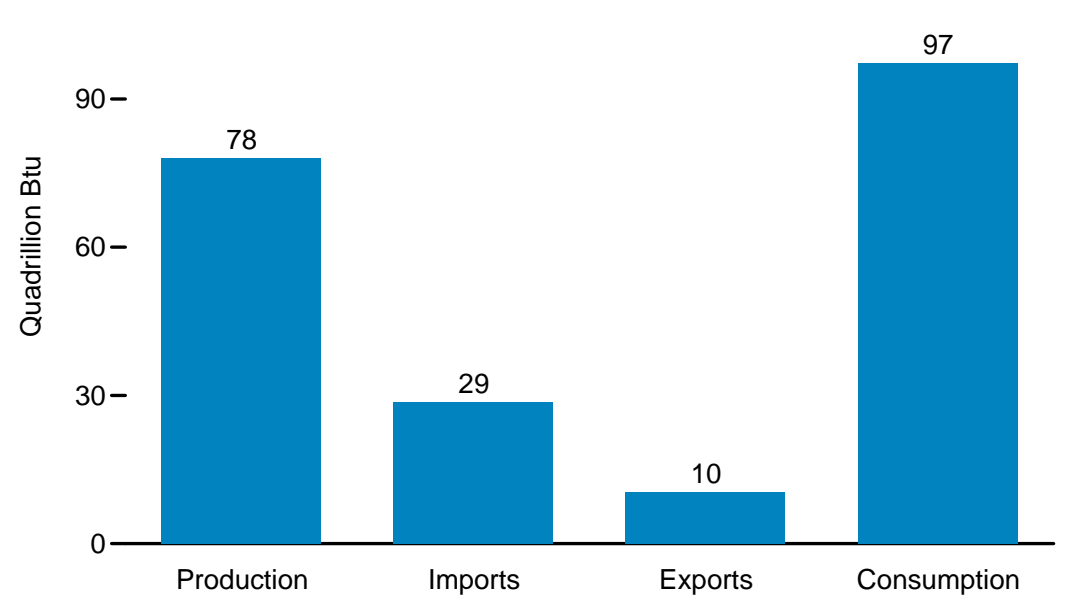

\section{Production and Consumption, 2011}

$120-$

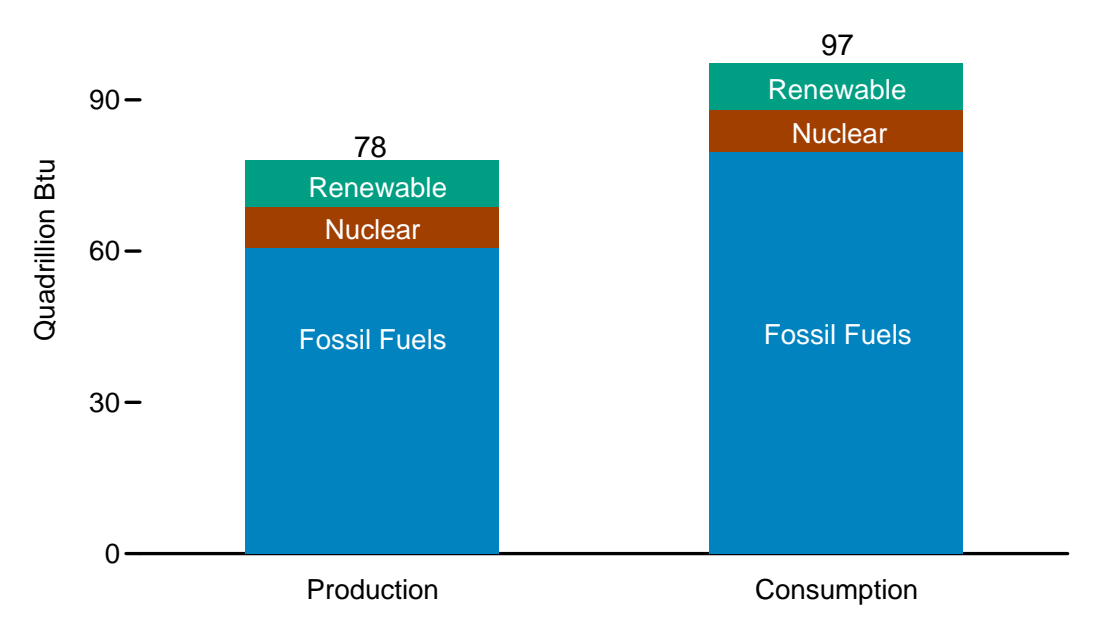

\section{Energy Flow, 2011}

(Quadrillion Btu)

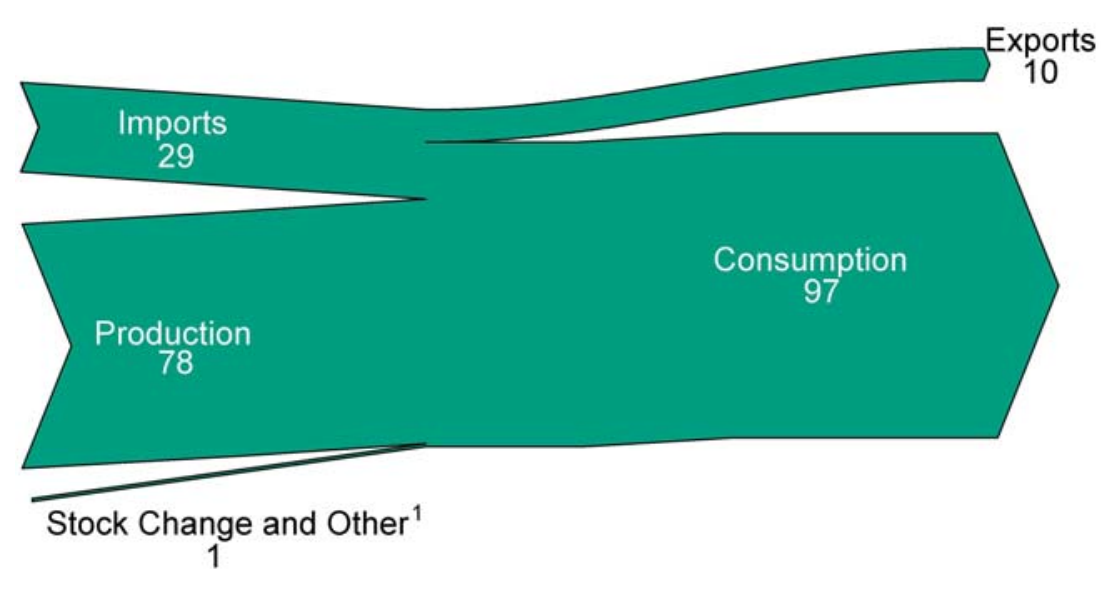


Table 1.1 Primary Energy Overview, Selected Years, 1949-2011

(Quadrillion Btu)

\begin{tabular}{|c|c|c|c|c|c|c|c|c|c|c|c|c|c|c|}
\hline \multirow[b]{3}{*}{ Year } & \multicolumn{4}{|c|}{ Production } & \multicolumn{5}{|c|}{ Trade } & \multirow{3}{*}{$\begin{array}{c}\text { Stock } \\
\text { Change } \\
\text { and } \\
\text { Other }{ }^{8}\end{array}$} & \multicolumn{4}{|c|}{ Consumption } \\
\hline & \multirow{2}{*}{$\begin{array}{l}\text { Fossil } \\
\text { Fuels }^{2} \\
\end{array}$} & \multirow{2}{*}{$\begin{array}{l}\text { Nuclear } \\
\text { Electric } \\
\text { Power }^{3}\end{array}$} & \multirow{2}{*}{$\begin{array}{c}\text { Renewable } \\
\text { Energy }^{4}\end{array}$} & \multirow[b]{2}{*}{ Total } & \multicolumn{2}{|c|}{ Imports } & \multicolumn{2}{|c|}{ Exports } & \multirow{2}{*}{\begin{tabular}{|c|} 
Net Imports ${ }^{1}$ \\
Total
\end{tabular}} & & \multirow{2}{*}{$\begin{array}{l}\text { Fossil } \\
\text { Fuels }{ }^{9}\end{array}$} & \multirow{2}{*}{$\begin{array}{l}\text { Nuclear } \\
\text { Electric } \\
\text { Power }^{3}\end{array}$} & \multirow{2}{*}{ 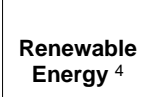 } & \multirow[b]{2}{*}{ Total ${ }^{1}$} \\
\hline & & & & & Petroleum ${ }^{5}$ & Total $^{6}$ & Coal & Total $^{7}$ & & & & & & \\
\hline 1949 & 28.748 & 0.000 & 2.974 & 31.722 & 1.427 & 1.448 & 0.877 & 1.592 & -0.144 & 0.403 & 29.002 & 0.000 & 2.974 & 31.982 \\
\hline 1950 & 32.563 & .000 & 2.978 & 35.540 & 1.886 & 1.913 & .786 & 1.465 & .448 & -1.372 & 31.632 & .000 & 2.978 & 34.616 \\
\hline 1955 & 37.364 & .000 & 2.784 & 40.148 & 2.752 & 2.790 & 1.465 & 2.286 & .504 & -.444 & 37.410 & .000 & 2.784 & 40.208 \\
\hline 1960 & 39.869 & .006 & 2.928 & 42.803 & 3.999 & 4.188 & 1.023 & 1.477 & 2.710 & -.427 & 42.137 & .006 & 2.928 & 45.086 \\
\hline 1965 & 47.235 & .043 & 3.396 & 50.674 & 5.402 & 5.892 & 1.376 & 1.829 & 4.063 & -.722 & 50.577 & .043 & 3.396 & 54.015 \\
\hline 1970 & 59.186 & .239 & 4.070 & 63.495 & 7.470 & 8.342 & 1.936 & 2.632 & 5.709 & -1.367 & 63.522 & .239 & 4.070 & 67.838 \\
\hline 1975 & 54.733 & 1.900 & 4.687 & 61.320 & 12.948 & 14.032 & 1.761 & 2.323 & 11.709 & -1.065 & 65.357 & 1.900 & 4.687 & 71.965 \\
\hline 1976 & 54.723 & 2.111 & 4.727 & 61.561 & 15.672 & 16.760 & 1.597 & 2.172 & 14.588 & -.175 & 69.107 & 2.111 & 4.727 & 75.975 \\
\hline 1977 & 55.101 & 2.702 & 4.209 & 62.012 & 18.756 & 19.948 & 1.442 & 2.052 & 17.896 & -1.946 & 70.991 & 2.702 & 4.209 & 77.961 \\
\hline 1978 & 55.074 & 3.024 & 5.005 & 63.104 & 17.824 & 19.106 & 1.078 & 1.920 & 17.186 & -.339 & 71.854 & 3.024 & 5.005 & 79.950 \\
\hline 1979 & 58.006 & 2.776 & 5.123 & 65.904 & 17.933 & 19.460 & 1.753 & 2.855 & 16.605 & -1.650 & 72.891 & 2.776 & 5.123 & 80.859 \\
\hline 1980 & 59.008 & 2.739 & 5.428 & 67.175 & 14.658 & 15.796 & 2.421 & 3.695 & 12.101 & -1.210 & 69.828 & 2.739 & 5.428 & 78.067 \\
\hline 1981 & 58.529 & 3.008 & 5.414 & 66.951 & 12.639 & 13.719 & 2.944 & 4.307 & 9.412 & -.257 & 67.571 & 3.008 & 5.414 & 76.106 \\
\hline 1982 & 57.458 & 3.131 & 5.980 & 66.569 & 10.777 & 11.861 & 2.787 & 4.608 & 7.253 & -.723 & 63.888 & 3.131 & 5.980 & 73.099 \\
\hline 1983 & 54.416 & 3.203 & 6.496 & 64.114 & 10.647 & 11.752 & 2.045 & 3.693 & 8.059 & .798 & 63.152 & 3.203 & 6.496 & 72.971 \\
\hline 1984 & 58.849 & 3.553 & 6.438 & 68.840 & 11.433 & 12.471 & 2.151 & 3.786 & 8.685 & -.892 & 66.506 & 3.553 & 6.438 & 76.632 \\
\hline 1985 & 57.539 & 4.076 & 6.084 & 67.698 & 10.609 & 11.781 & 2.438 & 4.196 & 7.584 & 1.110 & 66.093 & 4.076 & 6.084 & 76.392 \\
\hline 1986 & 56.575 & 4.380 & 6.111 & 67.066 & 13.201 & 14.151 & 2.248 & 4.021 & 10.130 & -.549 & 66.033 & 4.380 & 6.111 & 76.647 \\
\hline 1987 & 57.167 & 4.754 & 5.622 & 67.542 & 14.162 & 15.398 & 2.093 & 3.812 & 11.586 & -.074 & 68.521 & 4.754 & 5.622 & 79.054 \\
\hline 1988 & 57.875 & 5.587 & 5.457 & 68.919 & 15.747 & 17.296 & 2.499 & 4.366 & 12.929 & .861 & 71.557 & 5.587 & 5.457 & 82.709 \\
\hline 1989 & 57.483 & 5.602 & 6.235 & 69.320 & 17.162 & 18.766 & 2.637 & 4.661 & 14.105 & 1.361 & 72.911 & 5.602 & 6.235 & 84.786 \\
\hline 1990 & 58.560 & 6.104 & 6.041 & 70.705 & 17.117 & 18.817 & 2.772 & 4.752 & 14.065 & -.284 & 72.332 & 6.104 & 6.041 & 84.485 \\
\hline 1991 & 57.872 & 6.422 & 6.069 & 70.362 & 16.348 & 18.335 & 2.854 & 5.141 & 13.194 & .882 & 71.880 & 6.422 & 6.069 & 84.438 \\
\hline 1992 & 57.655 & 6.479 & 5.821 & $R_{69.956}$ & 16.968 & 19.372 & 2.682 & 4.937 & 14.435 & 1.392 & 73.396 & 6.479 & 5.821 & 85.783 \\
\hline 1993 & 55.822 & 6.410 & 6.083 & 68.315 & 18.510 & 21.273 & $\begin{array}{l}1.962 \\
\end{array}$ & 4.258 & 17.014 & 2.094 & 74.836 & 6.410 & 6.083 & 87.424 \\
\hline 1994 & 58.044 & 6.694 & 5.988 & 70.726 & 19.243 & 22.390 & 1.879 & 4.061 & 18.329 & .037 & 76.256 & 6.694 & 5.988 & 89.091 \\
\hline 1995 & 57.540 & 7.075 & 6.558 & 71.174 & 18.881 & 22.260 & 2.318 & 4.511 & 17.750 & 2.105 & 77.259 & 7.075 & 6.560 & 91.029 \\
\hline 1996 & 58.387 & 7.087 & 7.012 & 72.486 & 20.284 & 23.702 & 2.368 & 4.633 & 19.069 & 2.468 & 79.785 & 7.087 & 7.014 & 94.022 \\
\hline 1997 & 58.857 & 6.597 & 7.018 & 72.472 & 21.740 & 25.215 & 2.193 & 4.514 & 20.701 & $\begin{array}{l}1.429 \\
\text { S }\end{array}$ & 80.873 & 6.597 & 7.016 & 94.602 \\
\hline 1998 & 59.314 & 7.068 & 6.494 & 72.876 & 22.908 & 26.581 & 2.092 & 4.299 & 22.281 & -.140 & 81.369 & 7.068 & 6.493 & 95.018 \\
\hline 1999 & 57.614 & 7.610 & 6.517 & 71.742 & 23.133 & 27.252 & 1.525 & 3.715 & 23.537 & 1.373 & 82.427 & 7.610 & 6.516 & 96.652 \\
\hline 2000 & 57.366 & 7.862 & 6.104 & 71.332 & 24.531 & 28.973 & 1.528 & 4.006 & 24.967 & $\mathrm{R}_{2.515}$ & 84.731 & 7.862 & 6.106 & R98.814 \\
\hline 2001 & 58.541 & 8.029 & 5.164 & 71.735 & 25.398 & 30.157 & 1.265 & 3.771 & 26.386 & -1.953 & 82.902 & 8.029 & 5.163 & 96.168 \\
\hline 2002 & R56.837 & 8.145 & 5.734 & $R_{70.716}$ & 24.674 & 29.408 & $\begin{array}{l}1.032 \\
\end{array}$ & 3.669 & 25.739 & $\mathrm{R}_{1.190}$ & R83.699 & 8.145 & 5.729 & R97.645 \\
\hline 2003 & 56.099 & 7.959 & 5.982 & 70.040 & 26.219 & 31.061 & 1.117 & 4.054 & 27.007 & .931 & 84.014 & 7.959 & 5.983 & 97.978 \\
\hline 2004 & 55.895 & 8.222 & 6.070 & 70.188 & 28.197 & 33.544 & 1.253 & 4.434 & 29.110 & R. 864 & R85.819 & 8.222 & 6.082 & $\mathrm{R}_{1} 100.162$ \\
\hline 2005 & 55.038 & 8.161 & 6.229 & R69.428 & 29.248 & 34.709 & 1.273 & 4.560 & 30.149 & R. 705 & R85.794 & 8.161 & 6.242 & ${ }^{R} 100.282$ \\
\hline 2006 & 55.968 & 8.215 & $\mathrm{R}_{6.599}$ & R70.782 & 29.169 & 34.679 & 1.264 & 4.872 & 29.806 & R-. .599 & R84.702 & 8.215 & R6.649 & R99.629 \\
\hline 2007 & R56.409 & 8.455 & R6.509 & R71.373 & 28.781 & 34.703 & 1.507 & 5.482 & 29.221 & $\begin{array}{l}\text { R. } 702 \\
\text { n }\end{array}$ & R86.211 & 8.455 & $\mathrm{R}_{6.523}$ & $\begin{array}{r}R_{1} 101.296 \\
\end{array}$ \\
\hline 2008 & 57.482 & 8.427 & R7.202 & R73.111 & 27.685 & 32.992 & 2.071 & 7.060 & 25.932 & R. 231 & R83.549 & 8.427 & R7.186 & R99.275 \\
\hline 2009 & $R_{56.685}$ & 8.356 & $\mathrm{R}_{7.616}$ & ${ }^{R} 72.657$ & 25.082 & 29.706 & 1.515 & 6.965 & 22.741 & $\mathrm{R}_{-} .839$ & R78.488 & 8.356 & $R_{7.600}$ & R94.559 \\
\hline 2010 & $\mathrm{R}^{\mathrm{R}} 58.235$ & $\mathrm{R}_{8.434}$ & $\mathrm{R}_{8.136}$ & R74.806 & $\mathrm{R}_{25.371}$ & $\mathrm{R}_{29.877}$ & 2.101 & R8.234 & $\mathrm{R}_{21.643}$ & $\mathrm{R}_{1.273}$ & $\mathrm{R}_{81.109}$ & $\mathrm{R}_{8.434}$ & R8.090 & R97.722 \\
\hline $2011^{P}$ & 60.601 & 8.259 & 9.236 & 78.096 & 24.491 & 28.587 & 2.751 & 10.356 & 18.232 & .974 & 79.779 & 8.259 & 9.135 & 97.301 \\
\hline
\end{tabular}

1 Net imports equal imports minus exports. A minus sign indicates exports are greater than imports. 2 Coal, natural gas (dry), crude oil, and natural gas plant liquids.

3 Nuclear electricity net generation (converted to Btu using the nuclear heat rate-see Table A6). ${ }^{4}$ See Tables $10.1-10.2 \mathrm{C}$ for notes on series components and estimation; and see Note, "Renewable Energy Production and Consumption," at end of Section 10.

Crude oil and petroleum products. Includes imports into the Strategic Petroleum Reserve.

Also includes natural gas, coal, coal coke, fuel ethanol, biodiesel, and electricity.

Also includes natural gas, petroleum, coal coke, biodiesel, and electricity.

Calculated as consumption and exports minus production and imports. Includes petroleum stock change and adjustments; natural gas net storage withdrawals and balancing item; coal stock change,

${ }^{9}$ Coal, coal coke net imports, natural gas, and petroleum. For petroleum, product supplied is used as an approximation of petroleum consumption. See Note 1, "Petroleum Products Supplied and Petroleum Consumption," at end of Section 5.

10 Also includes electricity net imports.

$R=$ Revised. P=Preliminary.

Notes: • See "Primary Energy," "Primary Energy Production," and "Primary Energy Consumption" in

Glossary. - Totals may not equal sum of components due to independent rounding.
Web Pages: - See http://www.eia.gov/totalenergy/data/monthly/\#summary for updated monthly and annual data. - See http://www.eia.gov/totalenergy/data/annual/\#summary for all annual data beginning in

Sources: Tables 1.2, 1.3, and 1.4 


\section{By Source Category, 1949-2011}

$75-$

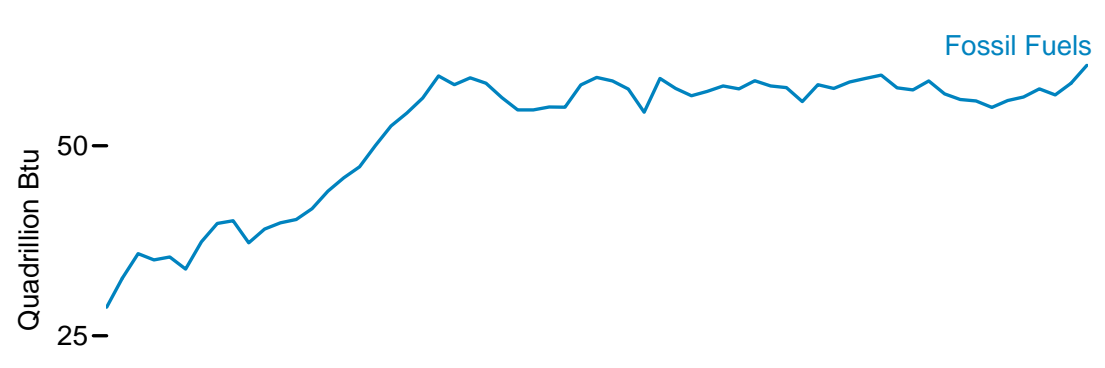

Nuclear Electric Power

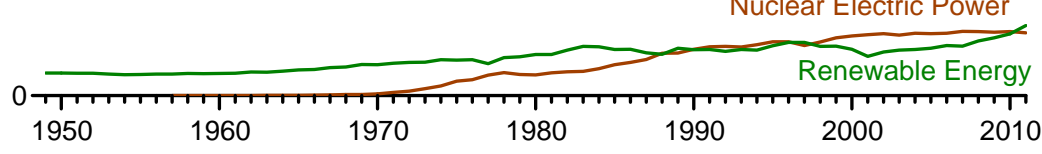

\section{By Source, 2011}

$30-$

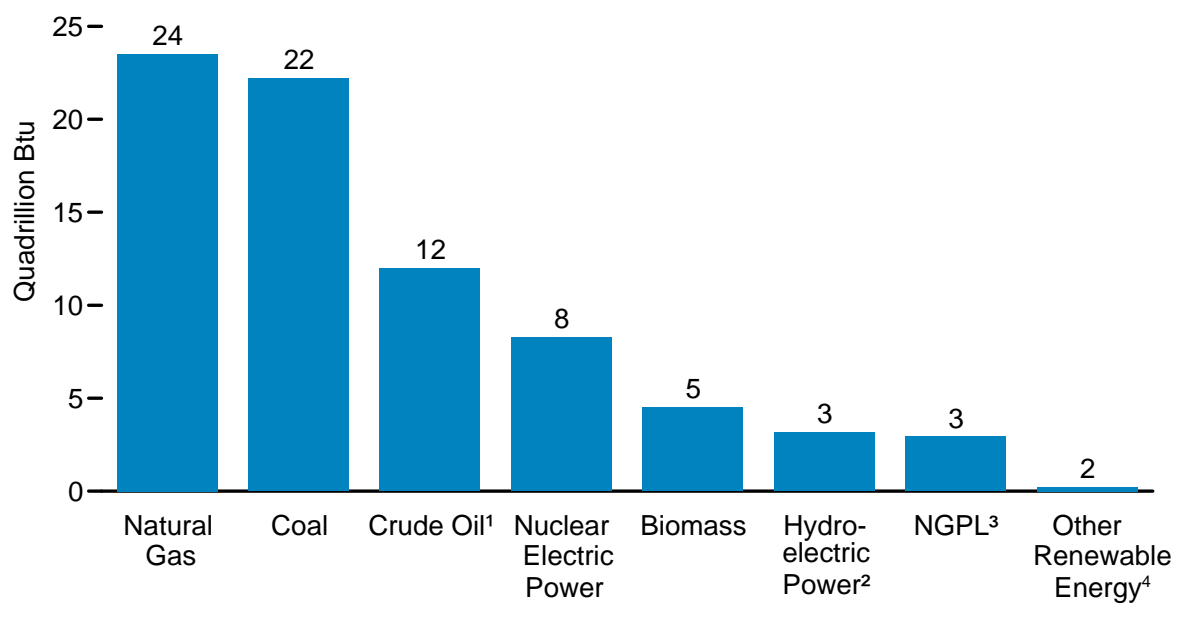

\section{By Source, 1949-2011}

$25-$

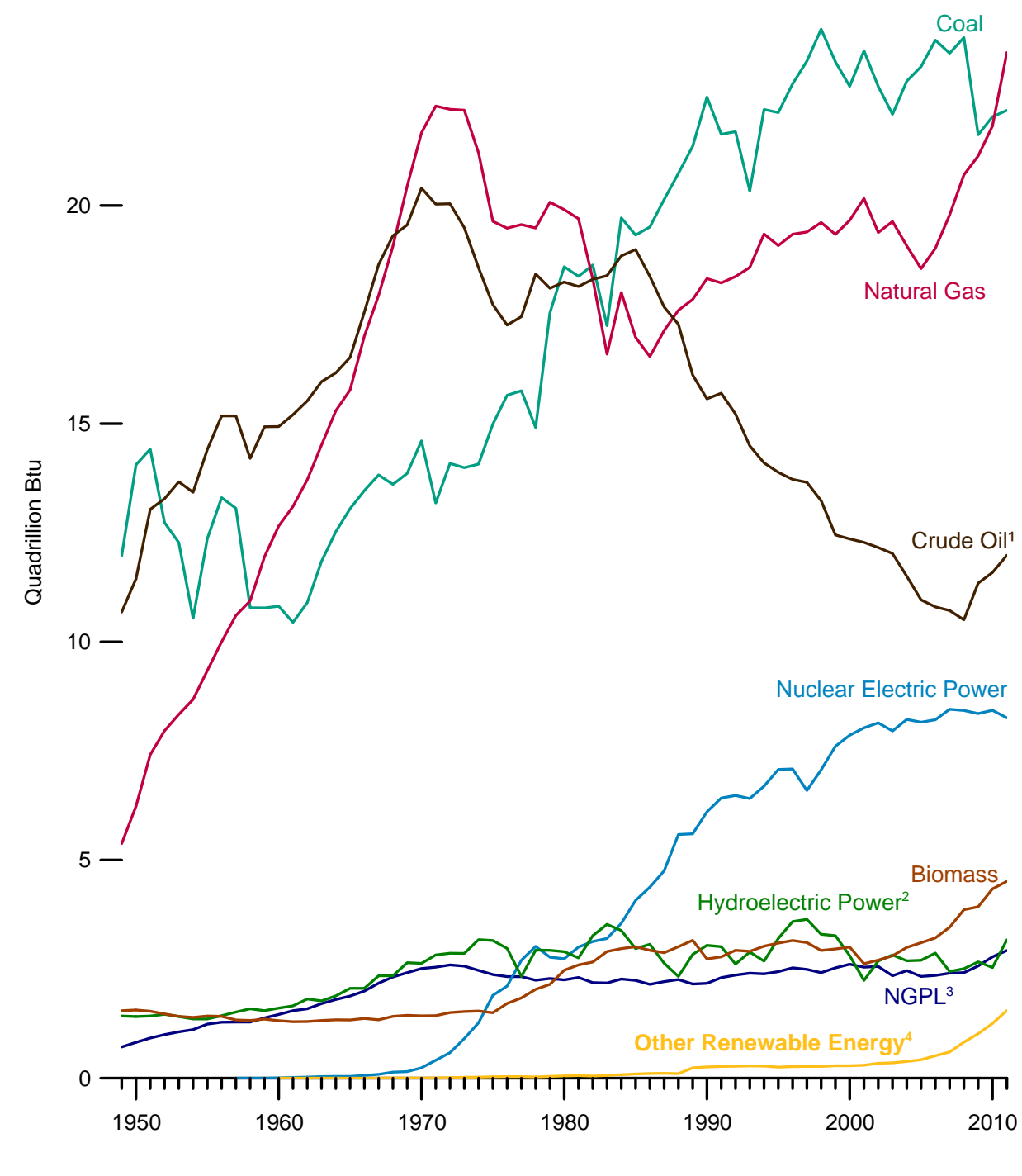

${ }^{4}$ Geothermal, solar/photovoltaic, and wind. Source: Table 1.2.

${ }^{1}$ Includes lease condensate.

${ }^{2}$ Conventional hydroelectric power.

${ }^{3}$ Natural gas plant liquids. 
Table 1.2 Primary Energy Production by Source, Selected Years, 1949-2011

(Quadrillion Btu)

\begin{tabular}{|c|c|c|c|c|c|c|c|c|c|c|c|c|c|}
\hline \multirow[b]{2}{*}{ Year } & \multicolumn{5}{|c|}{ Fossil Fuels } & \multirow[b]{2}{*}{$\begin{array}{l}\text { Nuclear } \\
\text { Electric } \\
\text { Power } 5\end{array}$} & \multicolumn{6}{|c|}{ Renewable Energy ${ }^{1}$} & \multirow[b]{2}{*}{ Total } \\
\hline & Coal $^{2}$ & $\begin{array}{c}\text { Natural } \\
\text { Gas } \\
\text { (Dry) }\end{array}$ & $\begin{array}{c}\text { Crude } \\
\text { Oil }^{3}\end{array}$ & NGPL 4 & Total & & $\begin{array}{l}\text { Hydro- } \\
\text { electric } \\
\text { Power } 6\end{array}$ & Geothermal 7 & Solar/PV 8 & Wind ${ }^{9}$ & Biomass 10 & Total & \\
\hline 1949 & 11.974 & 5.377 & 10.683 & 0.714 & 28.748 & 0.000 & 1.425 & NA & NA & NA & 1.549 & 2.974 & 31.722 \\
\hline 1950 & 14.060 & 6.233 & 11.447 & .823 & 32.563 & .000 & 1.415 & NA & NA & NA & 1.562 & 2.978 & 35.540 \\
\hline 1955 & 12.370 & 9.345 & 14.410 & 1.240 & 37.364 & .000 & 1.360 & NA & NA & NA & 1.424 & 2.784 & 40.148 \\
\hline 1960 & 10.817 & 12.656 & 14.935 & 1.461 & 39.869 & .006 & 1.608 & (s) & NA & NA & 1.320 & 2.928 & 42.803 \\
\hline 1965 & 13.055 & 15.775 & 16.521 & 1.883 & 47.235 & .043 & 2.059 & .002 & NA & NA & 1.335 & 3.396 & 50.674 \\
\hline 1970 & 14.607 & 21.666 & 20.401 & $\begin{array}{l}1.000 \\
2.512\end{array}$ & 59.186 & $\begin{array}{l}.045 \\
.239\end{array}$ & $\begin{array}{l}2.034 \\
2.634\end{array}$ & .006 & NA & NA & 1.431 & $\begin{array}{l}.090 \\
4.070\end{array}$ & 63.495 \\
\hline 1975 & 14.989 & 19.640 & 17.729 & 2.374 & 54.733 & 1.900 & 3.155 & .034 & NA & NA & 1.499 & 4.687 & 61.320 \\
\hline 1976 & 15.654 & 19.480 & $\begin{array}{l}17.262 \\
\text { 17. }\end{array}$ & $\begin{array}{l}2.327 \\
\end{array}$ & 54.723 & 2.111 & 2.976 & $\begin{array}{l}.038 \\
.038\end{array}$ & NA & NA & $\begin{array}{l}1.713 \\
\end{array}$ & 4.727 & 61.561 \\
\hline 1977 & 15.755 & 19.565 & 17.454 & 2.327 & 55.101 & 2.702 & 2.333 & .037 & NA & NA & 1.838 & 4.209 & 62.012 \\
\hline 1978 & 14.910 & 19.485 & 18.434 & 2.245 & 55.074 & 3.024 & 2.937 & .031 & NA & NA & 2.038 & 5.005 & 63.104 \\
\hline 1979 & 17.540 & 20.076 & 18.104 & 2.286 & 58.006 & 2.776 & 2.931 & .040 & NA & NA & 2.152 & 5.123 & 65.904 \\
\hline 1980 & 18.598 & 19.908 & 18.249 & 2.254 & 59.008 & 2.739 & 2.900 & .053 & NA & NA & 2.476 & 5.428 & 67.175 \\
\hline 1981 & 18.377 & 19.699 & 18.146 & 2.307 & 58.529 & 3.008 & 2.758 & .059 & NA & NA & 2.596 & 5.414 & 66.951 \\
\hline 1982 & 18.639 & 18.319 & 18.309 & 2.191 & 57.458 & 3.131 & 3.266 & .051 & NA & NA & 2.663 & 5.980 & 66.569 \\
\hline 1983 & 17.247 & 16.593 & 18.392 & 2.184 & 54.416 & 3.203 & 3.527 & .064 & NA & (s) & 2.904 & 6.496 & 64.114 \\
\hline 1984 & 19.719 & 18.008 & 18.848 & 2.274 & 58.849 & 3.553 & 3.386 & .081 & (s) & (s) & 2.971 & 6.438 & 68.840 \\
\hline $\begin{array}{r}1984 \\
1985\end{array}$ & 19.325 & $\begin{array}{l}10.000 \\
16.980\end{array}$ & $\begin{array}{l}10.040 \\
18.992\end{array}$ & $\begin{array}{l}2.274 \\
2.241\end{array}$ & $\begin{array}{l}50.049 \\
57.539\end{array}$ & $\begin{array}{l}.553 \\
4.076\end{array}$ & $\begin{array}{l}.306 \\
2.970\end{array}$ & .097 & $\begin{array}{l}\text { (s) } \\
\text { (s) }\end{array}$ & $\begin{array}{l}\text { (s) } \\
\text { (s) }\end{array}$ & 3.016 & $\begin{array}{l}.430 \\
6.084\end{array}$ & 67.698 \\
\hline 1986 & 19.509 & 16.541 & 18.376 & 2.149 & 56.575 & 4.380 & 3.071 & .108 & (s) & (s) & 2.932 & 6.111 & 67.066 \\
\hline 1987 & 20.141 & 17.136 & 17.675 & 2.215 & 57.167 & 4.754 & 2.635 & .112 & (s) & (s) & 2.875 & 5.622 & 67.542 \\
\hline 1988 & 20.738 & 17.599 & 17.279 & 2.260 & 57.875 & 5.587 & 2.334 & .106 & (s) & (s) & 3.016 & 5.457 & 68.919 \\
\hline 1989 & 221.360 & 17.847 & 16.117 & 2.158 & 57.483 & 5.602 & 2.837 & .162 & .055 & .022 & 3.159 & 6.235 & 69.320 \\
\hline 1990 & 22.488 & 18.326 & 15.571 & 2.175 & 58.560 & 6.104 & 3.046 & 171 & .059 & .029 & 2.735 & 6.041 & 70.705 \\
\hline 1991 & 21.636 & 18.229 & 15.701 & 2.306 & 57.872 & 6.422 & $\begin{array}{l}.040 \\
3.016\end{array}$ & .178 & .062 & .031 & 2.782 & $\begin{array}{l}0.041 \\
6.069\end{array}$ & 70.362 \\
\hline 1992 & 21.694 & 18.375 & 15.223 & 2.363 & 57.655 & 6.479 & 2.617 & .179 & .064 & .030 & 2.932 & 5.821 & R69.956 \\
\hline 1993 & 20.336 & 18.584 & 14.494 & 2.408 & 55.822 & 6.410 & 2.892 & .186 & .066 & .031 & 2.908 & 6.083 & 68.315 \\
\hline 1994 & 22.202 & 19.348 & 14.103 & 2.391 & 58.044 & 6.694 & 2.683 & .173 & .068 & .036 & 3.028 & 5.988 & 70.726 \\
\hline 1995 & 22.130 & 19.082 & 13.887 & 2.442 & 57.540 & $\begin{array}{l}7.075 \\
7.075\end{array}$ & $\begin{array}{l}3.000 \\
3.205\end{array}$ & .152 & .069 & .033 & $\begin{array}{l}3.0<0 \\
3.099\end{array}$ & 6.558 & 71.174 \\
\hline $\begin{array}{l}1995 \\
1996\end{array}$ & 22.790 & $\begin{array}{l}19.082 \\
19.344\end{array}$ & $\begin{array}{l}13.887 \\
13.723\end{array}$ & $\begin{array}{l}2.442 \\
2.530\end{array}$ & $\begin{array}{l}51.540 \\
58.387\end{array}$ & $\begin{array}{l}7.085 \\
7.087\end{array}$ & $\begin{array}{l}3.205 \\
3.590\end{array}$ & $\begin{array}{l}.152 \\
.163\end{array}$ & .070 & $\begin{array}{l}.033 \\
.033\end{array}$ & $\begin{array}{l}.099 \\
3.155\end{array}$ & $\begin{array}{l}.0558 \\
7.012\end{array}$ & 72.486 \\
\hline 1997 & 23.310 & 19.394 & 13.658 & 2.495 & 58.857 & 6.597 & $\begin{array}{l}3.640 \\
3.64\end{array}$ & .167 & .070 & .034 & 3.108 & 7.018 & 72.472 \\
\hline 1998 & 24.045 & 19.613 & 13.235 & $\begin{array}{l}2.4520 \\
\end{array}$ & 59.314 & $\begin{array}{l}.051 \\
7.068\end{array}$ & $\begin{array}{l}3.040 \\
3.297\end{array}$ & .168 & .069 & .031 & $\begin{array}{l}.100 \\
2.929\end{array}$ & 6.494 & 72.876 \\
\hline 1999 & 23.295 & 19.341 & 12.451 & 2.528 & 57.614 & 7.610 & 3.268 & .171 & .068 & .046 & 2.965 & 6.517 & 71.742 \\
\hline 2000 & 22.735 & 19.662 & 12.358 & 2.611 & 57.366 & 7.862 & 2.811 & .164 & $\begin{array}{r}.000 \\
\text { R.066 }\end{array}$ & .057 & 3.006 & $\begin{array}{l}6.104 \\
6.04\end{array}$ & 71.332 \\
\hline 2001 & 223.547 & 20.166 & $\begin{array}{r}2.350 \\
12.282\end{array}$ & 2.547 & 58.541 & $\begin{array}{l}1.002 \\
8.029\end{array}$ & 2.242 & $\begin{array}{l}.164 \\
.164\end{array}$ & $\begin{array}{l}.064 \\
\end{array}$ & .070 & $\begin{array}{l}.000 \\
2.624\end{array}$ & $\begin{array}{l}.1 .164 \\
5.164\end{array}$ & 71.735 \\
\hline 2002 & 22.732 & $R_{19.382}$ & 12.163 & 2.559 & R56.837 & 8.145 & $\begin{array}{l}2.689 \\
2.689\end{array}$ & .171 & $\begin{array}{l}.064 \\
.063\end{array}$ & .105 & $\begin{array}{l}2.024 \\
2.705\end{array}$ & $\begin{array}{l}5.104 \\
5.734\end{array}$ & R70.716 \\
\hline 2003 & 22.094 & 19.633 & 12.026 & $\begin{array}{l}2.359 \\
2.346\end{array}$ & 56.099 & $\begin{array}{l}0.145 \\
7.959\end{array}$ & $\begin{array}{l}2.009 \\
2.825\end{array}$ & .175 & $\begin{array}{l}.060 \\
.062\end{array}$ & .115 & 2.805 & $\begin{array}{l}5.104 \\
5.982\end{array}$ & 70.040 \\
\hline 2004 & 22.852 & 19.074 & 11.503 & 2.466 & 55.895 & 8.222 & 2.690 & .178 & .063 & .142 & 2.998 & 6.070 & 70.188 \\
\hline 2005 & 23.185 & 18.556 & 10.963 & 2.334 & 55.038 & 8.161 & 2.703 & .181 & .063 & 178 & 3.104 & 6.229 & R69.428 \\
\hline 2006 & 23.790 & $\begin{array}{l}19.050 \\
19.022\end{array}$ & 10.801 & $\begin{array}{l}2.004 \\
2.356\end{array}$ & 55.968 & $\begin{array}{l}.101 \\
8.215\end{array}$ & $\begin{array}{l}2.100 \\
2.869\end{array}$ & .181 & $\begin{array}{l}.060 \\
.068\end{array}$ & .264 & $\begin{array}{r}0.216 \\
\mathrm{R}_{3.216}\end{array}$ & $\begin{array}{r}0.259 \\
\mathrm{R} 6.599\end{array}$ & $\begin{array}{l}0 \\
\mathrm{R} 70.782\end{array}$ \\
\hline 2007 & 23.493 & $\begin{array}{r}19.022 \\
\mathrm{R}_{19}\end{array}$ & 10.721 & $\begin{array}{l}2.356 \\
2.409\end{array}$ & $\begin{array}{r}5.968 \\
\text { R56.409 }\end{array}$ & $\begin{array}{l}8.215 \\
8.455\end{array}$ & $\begin{array}{l}2.869 \\
2.446\end{array}$ & $\begin{array}{l}.181 \\
.186\end{array}$ & $\begin{array}{l}.068 \\
.076\end{array}$ & .341 & $\begin{array}{r}3.16 \\
\mathrm{R}_{3.461}\end{array}$ & $\begin{array}{r}0.599 \\
\mathrm{R} 6.509\end{array}$ & $R_{71.373}$ \\
\hline 2008 & 23.851 & 20.703 & 10.509 & $\begin{array}{l}2.4019 \\
\end{array}$ & 57.482 & $\begin{array}{l}8.4027 \\
8.427\end{array}$ & 2.511 & $\begin{array}{l}.192 \\
\end{array}$ & .089 & .546 & $\begin{array}{r}0.864 \\
R_{3.864}\end{array}$ & $\begin{array}{r}\text { R7.202 } \\
\text { R }\end{array}$ & R73.111 \\
\hline 2009 & $R_{21.624}$ & $\mathrm{R}_{21.139}$ & 11.348 & 2.574 & $\begin{array}{r}R_{56} 685 \\
\end{array}$ & $\begin{array}{l}8.356 \\
\end{array}$ & 2.669 & .200 & .098 & .721 & $\begin{array}{r}3.004 \\
R_{3} .928\end{array}$ & R7.616 & R72.657 \\
\hline 2010 & $R_{22.038}$ & R21.823 & $\mathrm{R}_{11.593}$ & 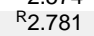 & R58.235 & R8.434 & R2.539 & R.208 & R. 126 & R.923 & R4.341 & R8.136 & R74.806 \\
\hline $2011^{\mathrm{P}}$ & 22.181 & 23.506 & 11.986 & 2.928 & 60.601 & 8.259 & 3.171 & .226 & .158 & $\begin{array}{r}1.168 \\
\end{array}$ & 4.511 & 9.236 & 78.096 \\
\hline
\end{tabular}

Most data are estimates. See Tables 10.1-10.2c for notes on series components and estimation; and see Note, "Renewable Energy Production and Consumption," at end of Section 10.

2 Beginning in 1989, includes waste coal supplied. Beginning in 2001, also includes a small amount of refuse recovery. See Table 7.1 .

Includes lease condensate.
Natural gas plant liquids.

Nuclear electricity net generation (converted to Btu using the nuclear heat rate-see Table A6). 6 Conventional hydroelectricity net generation (converted to Btu using the fossil-fuels heat rate-see Table A6).

Geothermal electricity net generation (converted to Btu using the fossil-fuels heat rate-see Table A6), and geothermal heat pump and direct use energy.

Solar thermal and photovoltaic (PV) electricity net generation (converted to Btu using the fossil-fuels

heat rate- - see Table A6), and solar thermal direct use energy.

(to

10 Wood and wood-derived fuels, biomass waste, and total biomass inputs to the production of fuel ethanol and biodiesel.

$R=$ Revised. P=Preliminary. NA=Not available. $(\mathrm{s})=$ Less than 0.0005 quadrillion Btu.
Notes: - See "Primary Energy Production" in Glossary. - Totals may not equal sum of components due to independent rounding.

Web Pages: - See http://www.eia.gov/totalenergy/data/monthly/\#summary for updated monthly and annual data. - See http://www.eia.gov/totalenergy/data/annual/\#summary for all annual data beginning in

Sources: Tables 5.1, 6.1, 7.1, 8.2a, 10.1, A2, A4 A5, and A6. 


\section{Figure 1.3 Primary Energy Consumption Estimates by Source}

\section{Total, 1949-2011}

$120-$

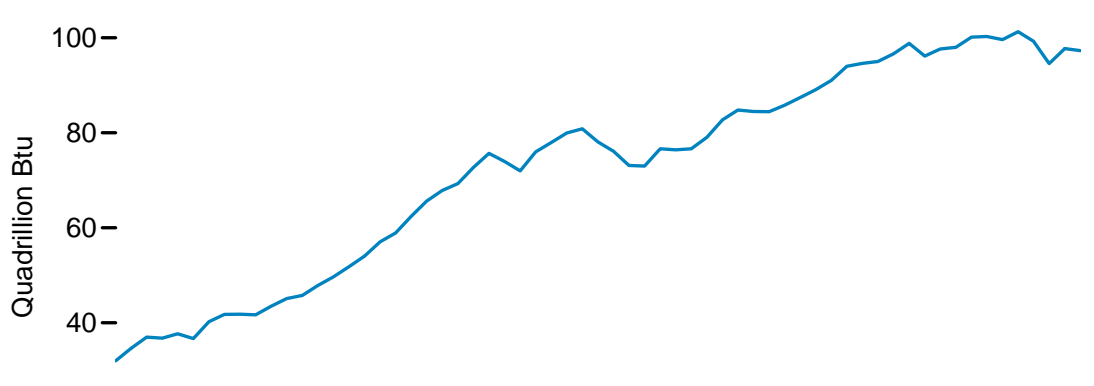

20-

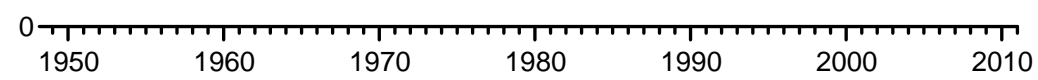

By Major Source, 2011

40-

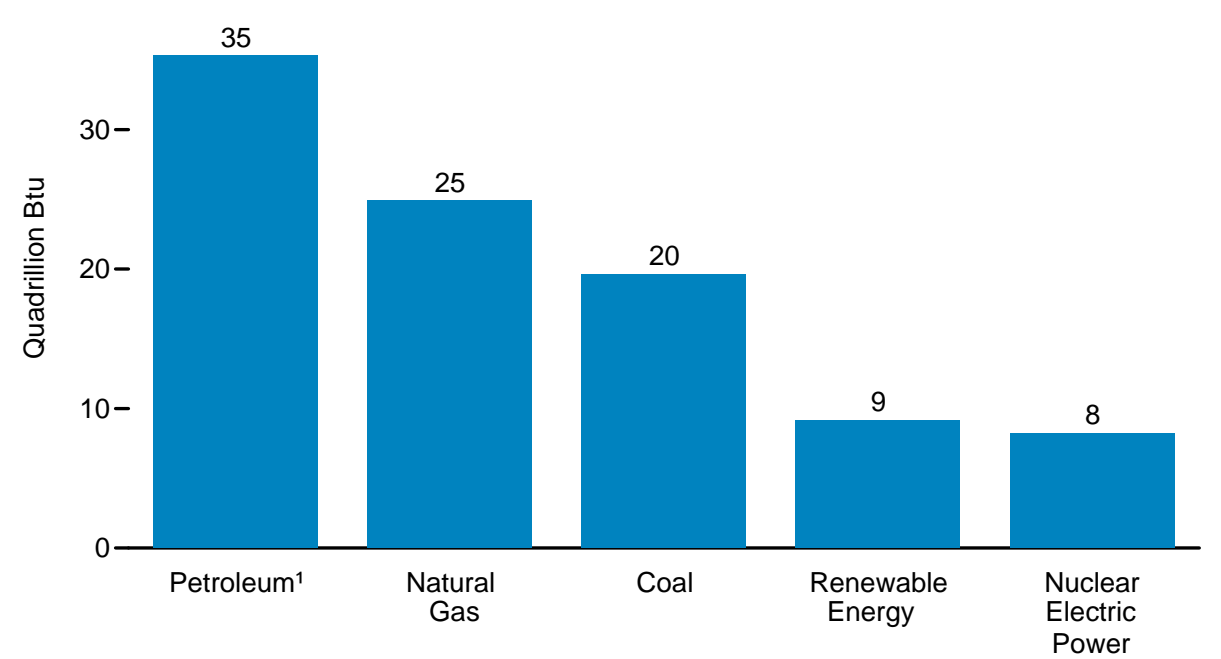

By Major Source, 1949-2011

$45-$

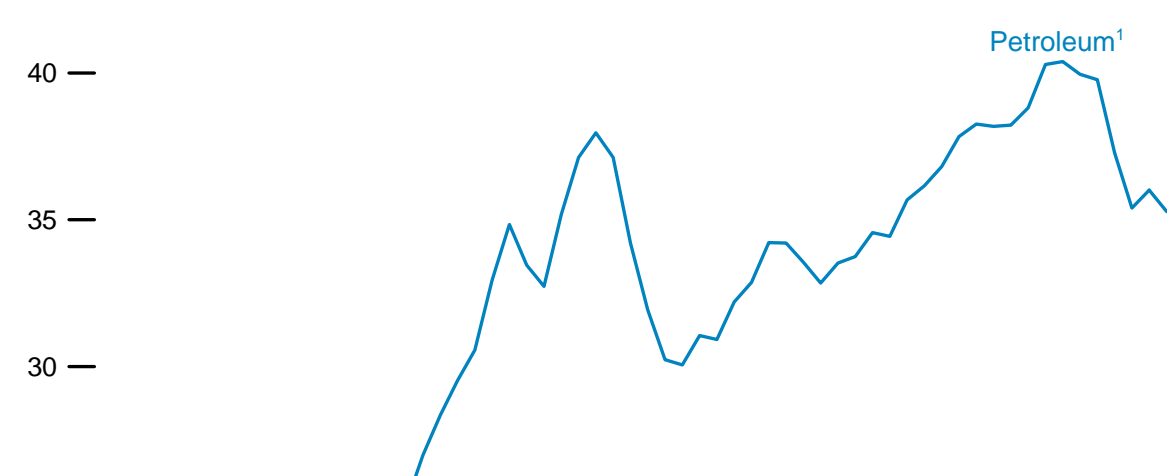

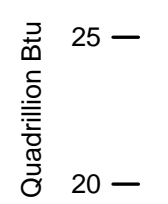
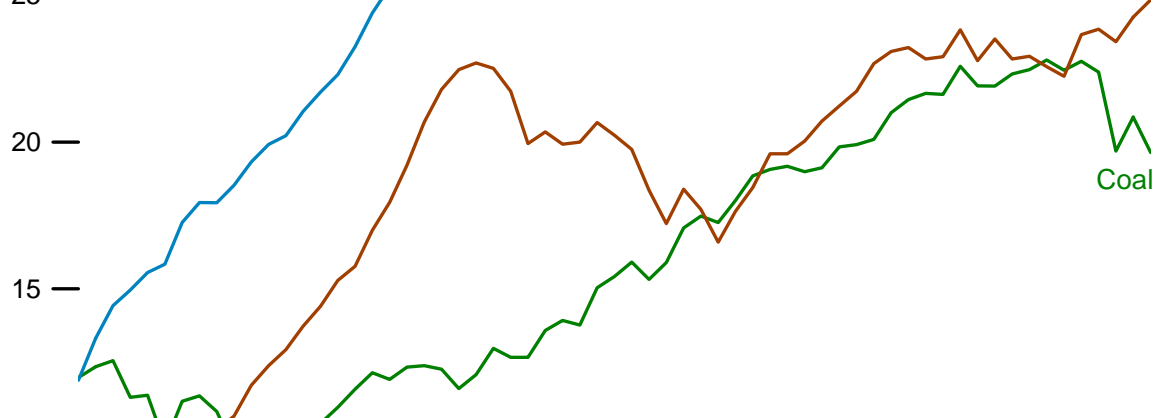

$10-$

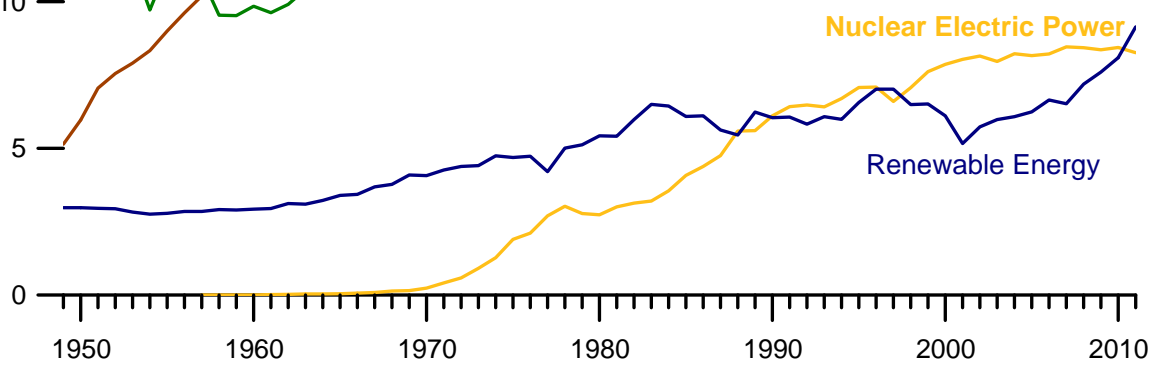

${ }^{1}$ Petroleum products supplied, including natural gas plant liquids and crude oil burned as fuel. Does not include biofuels that have been blended with petroleum-biofuels are included in "Renewable Energy." For petroleum, product supplied is used as an approximation of

Sources: Tables 1.2 and 1.3 . 
Table 1.3 Primary Energy Consumption Estimates by Source, Selected Years, 1949-2011

(Quadrillion Btu)

\begin{tabular}{|c|c|c|c|c|c|c|c|c|c|c|c|c|c|}
\hline \multirow[b]{3}{*}{ Year } & \multicolumn{5}{|c|}{ Fossil Fuels } & \multirow[b]{3}{*}{$\begin{array}{l}\text { Nuclear } \\
\text { Electric } \\
\text { Power }\end{array}$} & \multicolumn{5}{|c|}{ Renewable Energy ${ }^{1}$} & \multirow[b]{3}{*}{$\begin{array}{c}\text { Electricity } \\
\text { Net } \\
\text { Imports }{ }^{3}\end{array}$} & \multirow[b]{3}{*}{ Total } \\
\hline & \multirow[b]{2}{*}{ Coal } & \multirow{2}{*}{$\begin{array}{c}\text { Coal } \\
\text { Coke } \\
\text { Net } \\
\text { Imports }{ }^{3}\end{array}$} & \multirow[b]{2}{*}{$\begin{array}{l}\text { Natural } \\
\text { Gas }^{4}\end{array}$} & \multirow[b]{2}{*}{ Petroleum ${ }^{5}$} & \multirow[b]{2}{*}{ Total } & & \multicolumn{3}{|c|}{ Noncombustible ${ }^{2}$} & \multirow[b]{2}{*}{ Biomass $^{7}$} & \multirow[b]{2}{*}{ Total } & & \\
\hline & & & & & & & $\begin{array}{l}\text { Captured } \\
\text { Energy }{ }^{6}\end{array}$ & $\begin{array}{c}\text { Adjustment for } \\
\text { Fossil Fuel } \\
\text { Equivalence }\end{array}$ & Total 6,7 & & & & \\
\hline $\begin{array}{l}1949 \\
1950\end{array}$ & $\begin{array}{l}11.981 \\
12.347\end{array}$ & $\begin{array}{l}-0.007 \\
001\end{array}$ & $\begin{array}{l}5.145 \\
5.968\end{array}$ & $\begin{array}{l}11.883 \\
13.315\end{array}$ & $\begin{array}{l}29.002 \\
31.632\end{array}$ & $\begin{array}{r}0.000 \\
000\end{array}$ & $\begin{array}{r}0.323 \\
344\end{array}$ & $\begin{array}{l}1.101 \\
1.071\end{array}$ & $\begin{array}{l}1.425 \\
1.415\end{array}$ & $\begin{array}{l}1.549 \\
1562\end{array}$ & $\begin{array}{l}2.974 \\
2.978\end{array}$ & $\begin{array}{r}0.005 \\
006\end{array}$ & $\begin{array}{l}31.982 \\
34616\end{array}$ \\
\hline 1955 & 12.347 & $\begin{array}{l}.001 \\
-.010\end{array}$ & $\begin{array}{l}5.968 \\
8.998\end{array}$ & 17.255 & $\begin{array}{l}31.632 \\
37.410\end{array}$ & $\begin{array}{l}.000 \\
.000\end{array}$ & $\begin{array}{l}.344 \\
.397\end{array}$ & $\begin{array}{l}1.071 \\
.963\end{array}$ & $\begin{array}{l}1.415 \\
1.360\end{array}$ & $\begin{array}{l}1.562 \\
1.424\end{array}$ & $\begin{array}{l}2.9 / 8 \\
2.784\end{array}$ & $\begin{array}{l}.006 \\
.014\end{array}$ & $\begin{array}{l}34.616 \\
40.208\end{array}$ \\
\hline 1960 & 9.838 & -.006 & 12.385 & 19.919 & 42.137 & .006 & .510 & 1.098 & 1.608 & 1.320 & 2.928 & .015 & 45.086 \\
\hline 1965 & 11.581 & -.018 & 15.769 & 23.246 & 50.577 & .043 & .673 & 1.388 & 2.061 & 1.335 & 3.396 & (s) & 54.015 \\
\hline 1970 & 12.265 & $\begin{array}{l}-.058 \\
-.06\end{array}$ & 21.795 & 29.521 & 63.522 & .239 & .858 & 1.781 & 2.639 & $\begin{array}{l}1.431 \\
1.04\end{array}$ & 4.070 & $\begin{array}{l}.007 \\
\end{array}$ & 67.838 \\
\hline 1975 & 12.663 & .014 & 19.948 & 32.732 & 65.357 & 1.900 & $\begin{array}{r}.000 \\
1.045\end{array}$ & 2.143 & 3.188 & $\begin{array}{l}1.499 \\
1.49\end{array}$ & 4.687 & .021 & $\begin{array}{l}71.965 \\
\end{array}$ \\
\hline 1976 & 13.584 & (s) & 20.345 & 35.178 & 69.107 & 2.111 & .991 & 2.022 & 3.014 & $\begin{array}{l}1.713 \\
\text {. }\end{array}$ & 4.727 & .029 & 75.975 \\
\hline 1977 & 13.922 & .015 & 19.931 & 37.124 & 70.991 & 2.702 & .775 & 1.595 & 2.371 & 1.838 & 4.209 & .059 & 77.961 \\
\hline 1978 & 13.766 & .125 & 20.000 & 37.963 & 71.854 & 3.024 & .977 & 1.990 & 2.968 & 2.038 & 5.005 & .067 & 79.950 \\
\hline 1979 & 15.040 & .063 & 20.666 & 37.122 & 72.891 & 2.776 & .979 & 1.992 & 2.971 & 2.152 & 5.123 & .069 & 80.859 \\
\hline 1980 & 15.423 & $\begin{array}{l}-.035 \\
-.0\end{array}$ & 20.235 & 34.205 & 69.828 & 2.739 & .970 & 1.983 & 2.953 & 2.476 & 5.428 & .071 & 78.067 \\
\hline 1981 & 15.908 & $\begin{array}{l}-.016 \\
-.01\end{array}$ & 19.747 & 31.932 & 67.571 & 3.008 & .920 & $\begin{array}{l}1.898 \\
\text {. }\end{array}$ & 2.817 & 2.596 & 5.414 & .113 & 76.106 \\
\hline 1982 & 15.322 & -.022 & 18.356 & 30.232 & 63.888 & 3.131 & $\begin{array}{r}1.082 \\
\end{array}$ & 2.234 & 3.316 & 2.663 & 5.980 & .100 & 73.099 \\
\hline 1983 & 15.894 & $\begin{array}{l}-.016 \\
-.01\end{array}$ & 17.221 & 30.052 & 63.152 & 3.203 & 1.165 & 2.426 & 3.591 & 2.904 & 6.496 & .121 & 72.971 \\
\hline 1984 & 17.071 & -.011 & 18.394 & 31.053 & 66.506 & 3.553 & 1.133 & 2.334 & 3.467 & 2.971 & 6.438 & .135 & 76.632 \\
\hline 1985 & 17.478 & -.013 & 17.703 & 30.925 & 66.093 & 4.076 & 1.002 & 2.066 & 3.068 & 3.016 & 6.084 & .140 & 76.392 \\
\hline 1986 & 17.260 & -.017 & 16.591 & 32.198 & 66.033 & 4.380 & 1.038 & 2.141 & 3.179 & 2.932 & 6.111 & .122 & 76.647 \\
\hline 1987 & 18.008 & .009 & 17.640 & 32.864 & 68.521 & 4.754 & .900 & 1.847 & 2.747 & 2.875 & 5.622 & .158 & 79.054 \\
\hline 1988 & 18.846 & .040 & 18.448 & $\begin{array}{l}34.2204 \\
34.223\end{array}$ & 71.557 & 5.587 & .807 & $\begin{array}{l}1.041 \\
1.634\end{array}$ & 2.441 & 3.016 & 5.457 & $\begin{array}{l}.100 \\
.108\end{array}$ & $\begin{array}{l}8.054 \\
82.709\end{array}$ \\
\hline 1989 & 19.070 & .030 & 19.602 & 34.209 & 72.911 & 5.602 & $\begin{array}{r}.048 \\
1.048\end{array}$ & 2.028 & 3.076 & 3.159 & 6.235 & .037 & 84.786 \\
\hline 1990 & 19.173 & .005 & 19.603 & 33.552 & 72.332 & 6.104 & $\begin{array}{l}1.128 \\
\end{array}$ & 2.177 & 3.306 & 2.735 & 6.041 & .008 & 84.485 \\
\hline 1991 & 18.992 & .010 & 20.033 & 32.846 & 71.880 & 6.422 & 1.121 & 2.166 & 3.287 & 2.782 & 6.069 & .067 & 84.438 \\
\hline 1992 & 19.122 & .035 & 20.714 & 33.525 & 73.396 & 6.479 & 1.001 & 1.889 & 2.890 & 2.932 & 5.821 & .087 & 85.783 \\
\hline 1993 & 19.835 & .027 & 21.229 & 33.745 & 74.836 & 6.410 & 1.100 & 2.074 & 3.174 & 2.908 & 6.083 & .095 & 87.424 \\
\hline 1994 & 19.909 & .058 & 21.728 & 34.561 & $\begin{array}{l}76.256 \\
\end{array}$ & $\begin{array}{l}6.4104 \\
6.694\end{array}$ & $\begin{array}{l}1.030 \\
1.030\end{array}$ & $\begin{array}{l}1.930 \\
0\end{array}$ & 2.961 & 3.028 & 5.988 & .153 & $\begin{array}{l}89.091 \\
\end{array}$ \\
\hline 1995 & 20.089 & .061 & 22.671 & 34.438 & 77.259 & 7.075 & $\begin{array}{l}1.197 \\
\text { S }\end{array}$ & 2.262 & 3.459 & 3.101 & 6.560 & .134 & 91.029 \\
\hline 1996 & 21.002 & .023 & 23.085 & 35.675 & 79.785 & 7.087 & 1.326 & 2.530 & 3.857 & 3.157 & 7.014 & .137 & 94.022 \\
\hline 1997 & 21.445 & .046 & 23.223 & 36.159 & 80.873 & 6.597 & 1.360 & 2.550 & 3.910 & 3.105 & 7.016 & .116 & 94.602 \\
\hline 1998 & 21.656 & .067 & 22.830 & 36.816 & 81.369 & 7.068 & 1.247 & 2.318 & 3.565 & 2.927 & 6.493 & .088 & 95.018 \\
\hline 1999 & 21.623 & .058 & 22.909 & 37.838 & 82.427 & 7.610 & 1.240 & 2.312 & 3.552 & 2.963 & 6.516 & .099 & 96.652 \\
\hline 2000 & 22.580 & .065 & 23.824 & 38.262 & 84.731 & 7.862 & 1.090 & 2.008 & 3.098 & 3.008 & 6.106 & .115 & R98.814 \\
\hline 2001 & 21.914 & $\begin{array}{l}.029 \\
.029\end{array}$ & $\begin{array}{l}22.774 \\
22.773\end{array}$ & 38.186 & 82.902 & 8.029 & .893 & 1.647 & 2.540 & $\begin{array}{l}.000 \\
2.622\end{array}$ & 5.163 & .075 & 96.168 \\
\hline 2002 & 21.904 & .061 & $\mathrm{R}_{23.510}$ & 38.224 & R83.699 & 8.145 & $\begin{array}{l}.070 \\
1.070\end{array}$ & $\begin{array}{l}1.041 \\
1.959\end{array}$ & 3.029 & 2.701 & 5.729 & .072 & R97.645 \\
\hline 2003 & 22.321 & .051 & 22.831 & 38.811 & 84.014 & 7.959 & $\begin{array}{l}1.114 \\
\text {. }\end{array}$ & 2.062 & 3.176 & 2.807 & 5.983 & .022 & 97.978 \\
\hline 2004 & 22.466 & .138 & R22.923 & 40.292 & R85.819 & 8.222 & 1.103 & 1.969 & 3.073 & 3.010 & 6.082 & .039 & $R_{1} 100.162$ \\
\hline 2005 & 22.797 & .044 & R22.565 & 40.388 & R85.794 & 8.161 & 1.127 & 1.998 & 3.125 & R3.117 & 6.242 & .085 & $R_{1} 100.282$ \\
\hline 2006 & 22.447 & .061 & $R_{22.239}$ & 39.955 & R84.702 & 8.215 & 1.229 & 2.153 & 3.382 & $R_{3.267}$ & $R_{6.649}$ & .063 & R99.629 \\
\hline 2007 & 22.749 & .025 & $\mathrm{R}_{23.663}$ & 39.774 & $\begin{array}{r}R_{86.211}\end{array}$ & 8.455 & 1.125 & 1.924 & 3.048 & $R_{3.474}$ & $R_{6.523}$ & .107 & $R_{101.296}$ \\
\hline 2008 & 22.385 & $\begin{array}{l}.045 \\
.041\end{array}$ & $\begin{array}{l}R_{23} .003 \\
\end{array}$ & 37.280 & R83.549 & $\begin{array}{l}0.450 \\
8.427\end{array}$ & 1.238 & $\begin{array}{l}1.9<4 \\
2.099\end{array}$ & $\begin{array}{l}3.046 \\
3.338\end{array}$ & $\begin{array}{r}\text { R3.849 }\end{array}$ & $\begin{array}{r}0.525 \\
\text { R7.186 }\end{array}$ & $\begin{array}{l}.112 \\
.112\end{array}$ & $\begin{array}{l}\text { R99.275 }\end{array}$ \\
\hline 2009 & 19.692 & $\begin{array}{l}-.024 \\
-.02\end{array}$ & R23.416 & 35.403 & R78.488 & 8.356 & 1.382 & 2.306 & $\begin{array}{l}3.600 \\
3.688\end{array}$ & $\begin{array}{r}\mathrm{r} .045 \\
\mathrm{R} .912\end{array}$ & R7.600 & .116 & $\begin{array}{r}\text { R94.559 } \\
\end{array}$ \\
\hline 2010 & R20.850 & $\begin{array}{l}-.006 \\
-.006\end{array}$ & R24.256 & R36.010 & R81.109 & R8.434 & $\mathrm{R}_{1.440}$ & R2.355 & R3.796 & $\begin{array}{r}\mathrm{R} 4.294 \\
\end{array}$ & R8.090 & R. 089 & $\begin{array}{r}\text { R97.722 }\end{array}$ \\
\hline $2011^{P}$ & 19.643 & .011 & 24.843 & 35.283 & 79.779 & 8.259 & 1.785 & 2.939 & 4.724 & 4.411 & 9.135 & .127 & 97.301 \\
\hline
\end{tabular}

${ }^{1}$ Most data are estimates. See Note, "Renewable Energy Production and Consumption," at end of Section 10 .

2 Conventional hydroelectric power, geothermal, solar thermal, photovoltaic, and wind. See Note 1 "Noncombustible Renewable Energy," at end of section.

Net imports equal imports minus exports. A minus sign indicates exports are greater than imports. at end of Section 6 . Petroleum products supplied, including natural gas plant liquids and crude oil burned as fuel. Does petroleum, product supplied is used as an approximation - of petroleum consumption. See Note 1, petroleum, product supplied is used as an approximation of petroleum con

6 See Note 1, "Noncombustible Renewable Energy," at end of section. See Table 10.1 for a breakdown of individual sources.

$\mathrm{R}=$ Revised. $\mathrm{P}=$ Preliminary. $(\mathrm{s})=$ Less than 0.0005 and greater than -0.0005 quadrillion Btu.

Notes: - See "Primary Energy Consumption" in Glossary. - See Table E1 for estimated energy consumption for $1635-1945$. - See Note 3 , "Electricity Imports

Web Pages: - See http://www.eia.gov/totalenergy/data/monthly/\#summary for updated monthly and annual data. - See http://www.eia.gov/totalenergy/data/annual/\#summary for all annual data beginning in

Sources: Tables 5.12, 6.1, 7.1, 7.8, 8.1, 8.2a, 10.1, 10.3, A4, A5, and A6. 
Figure 1.4 Primary Energy Trade by Source, 1949-2011

\section{Energy Net Imports}

35-

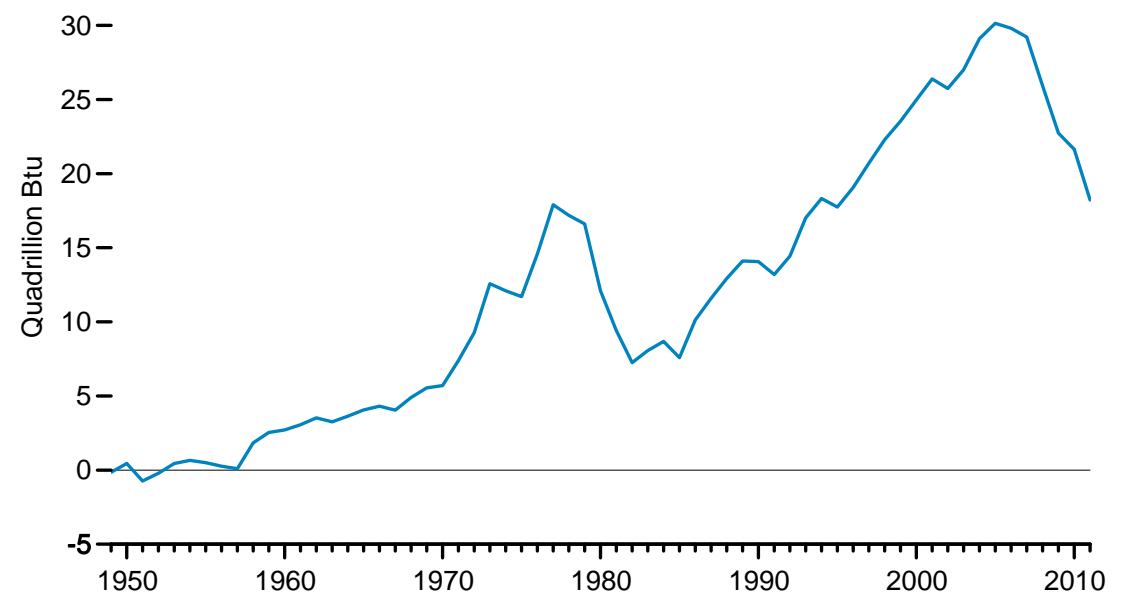

\section{Energy Imports}

$40-$

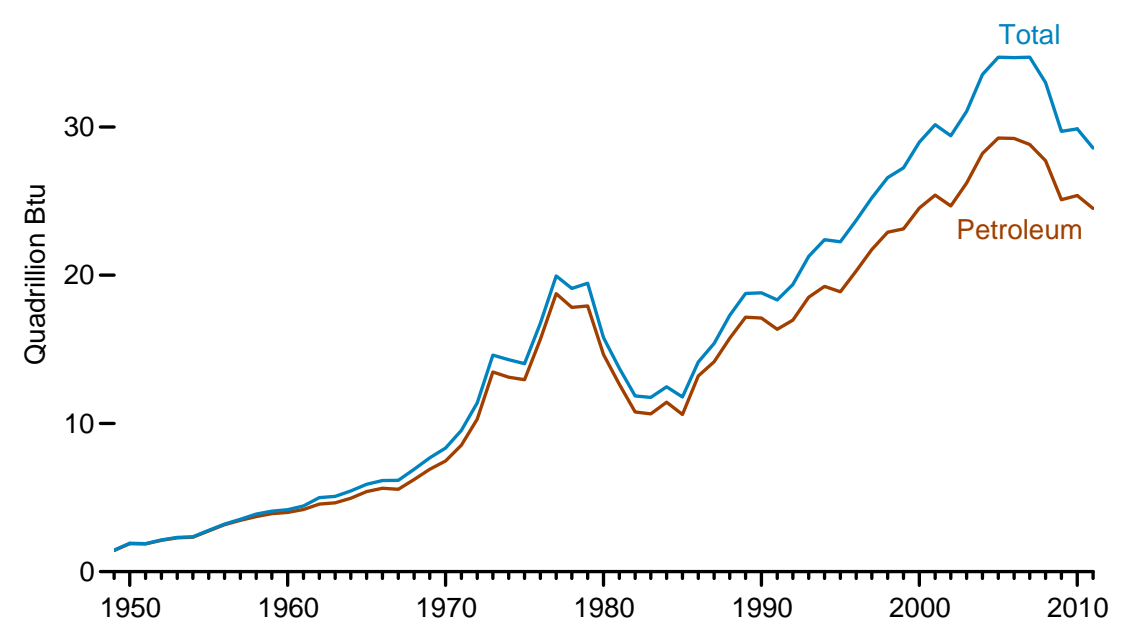

Imports and Exports, 2011

40-

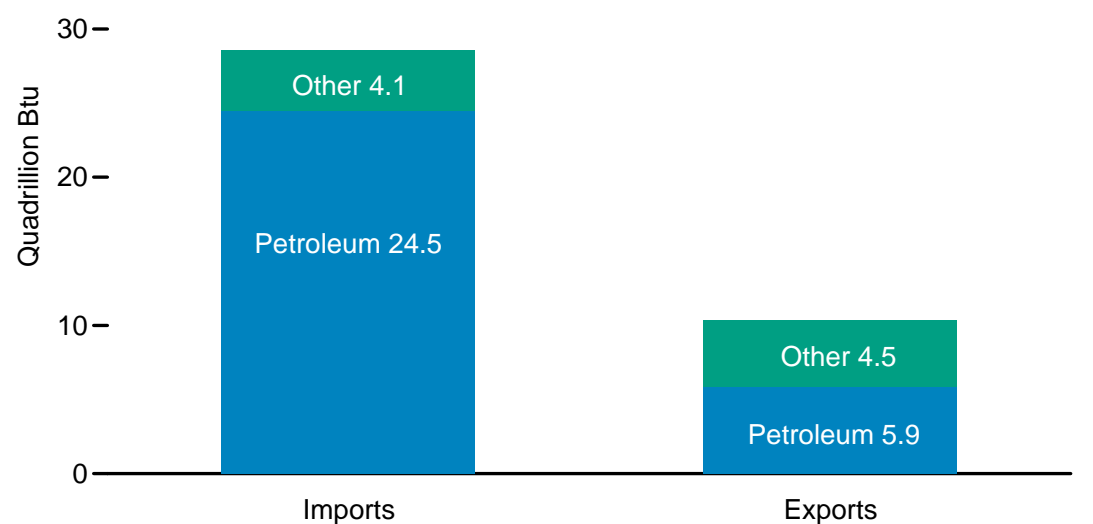

\section{Energy Exports}

12-

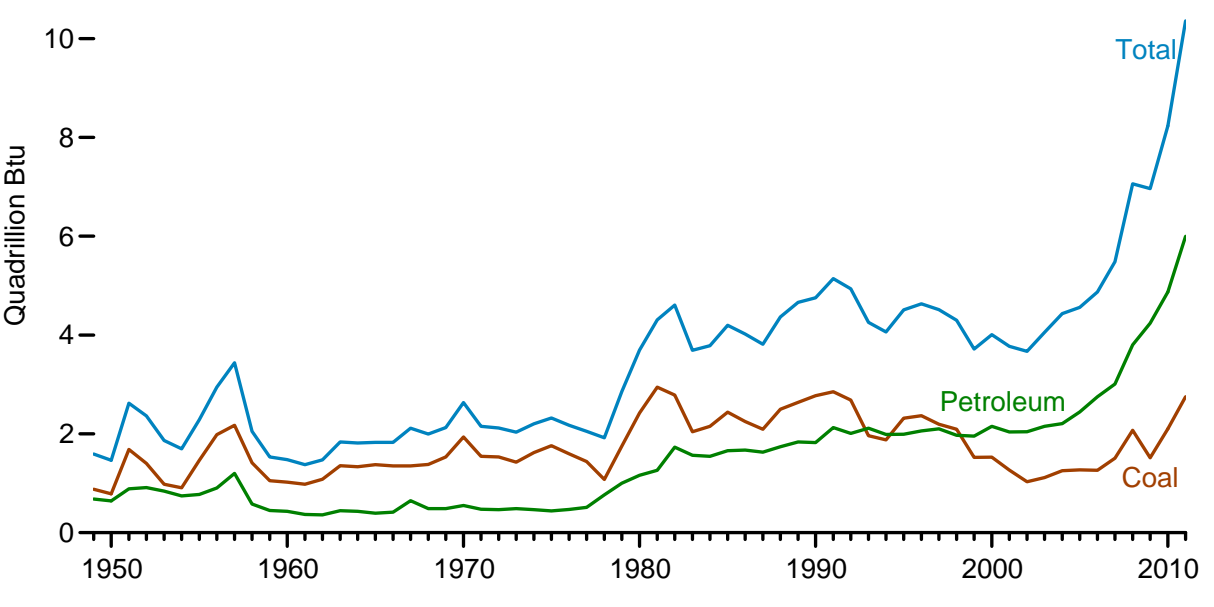


Table 1.4 Primary Energy Trade by Source, Selected Years, 1949-2011

(Quadrillion Btu)

\begin{tabular}{|c|c|c|c|c|c|c|c|c|c|c|c|c|c|c|c|c|c|c|c|}
\hline \multirow[b]{3}{*}{ Year } & \multicolumn{9}{|c|}{ Imports } & \multicolumn{9}{|c|}{ Exports } & \multirow{3}{*}{\begin{tabular}{|c|}
$\begin{array}{c}\text { Net } \\
\text { Imports }\end{array}$ \\
Total
\end{tabular}} \\
\hline & \multirow[b]{2}{*}{ Coal } & \multirow[b]{2}{*}{$\begin{array}{l}\text { Coal } \\
\text { Coke }\end{array}$} & \multirow[b]{2}{*}{$\begin{array}{c}\text { Natural } \\
\text { Gas }\end{array}$} & \multicolumn{3}{|c|}{ Petroleum } & \multirow[b]{2}{*}{$\begin{array}{c}\text { Bio- } \\
\text { fuels } 4\end{array}$} & \multirow[b]{2}{*}{$\begin{array}{l}\text { Elec- } \\
\text { tricity }\end{array}$} & \multirow[b]{2}{*}{ Total } & \multirow[b]{2}{*}{ Coal } & \multirow[b]{2}{*}{$\begin{array}{l}\text { Coal } \\
\text { Coke }\end{array}$} & \multirow[b]{2}{*}{$\begin{array}{l}\text { Natural } \\
\text { Gas }\end{array}$} & \multicolumn{3}{|c|}{ Petroleum } & \multirow[b]{2}{*}{$\begin{array}{c}\text { Bio- } \\
\text { fuels } 5\end{array}$} & & & \\
\hline & & & & $\begin{array}{c}\text { Crude } \\
\text { Oil }^{2}\end{array}$ & $\begin{array}{l}\text { Petroleum } \\
\text { Products }{ }^{3}\end{array}$ & Total & & & & & & & $\begin{array}{c}\text { Crude } \\
\text { Oil }^{2}\end{array}$ & $\begin{array}{l}\text { Petroleum } \\
\text { Products }{ }^{3}\end{array}$ & Total & & $\begin{array}{l}\text { Elec- } \\
\text { tricity }\end{array}$ & Total & \\
\hline 1949 & 0.008 & 0.007 & 0.000 & 0.915 & 0.513 & 1.427 & NA & 0.006 & 1.448 & 0.877 & 0.014 & 0.021 & 0.192 & 0.488 & 0.680 & NA & 0.001 & 1.592 & -0.144 \\
\hline 1950 & .009 & .011 & .000 & 1.056 & .830 & 1.886 & NA & .007 & 1.913 & .786 & .010 & .027 & .202 & .440 & .642 & NA & .001 & 1.465 & .448 \\
\hline 1955 & .008 & .003 & .011 & 1.691 & 1.061 & 2.752 & NA & .016 & 2.790 & 1.465 & .013 & .032 & .067 & .707 & .774 & NA & .002 & 2.286 & .504 \\
\hline 1960 & .007 & .003 & .161 & 2.196 & 1.802 & 3.999 & NA & .018 & 4.188 & 1.023 & .009 & .012 & .018 & .413 & .431 & NA & .003 & 1.477 & 2.710 \\
\hline 1965 & .005 & .002 & .471 & 2.654 & 2.748 & 5.402 & NA & .012 & 5.892 & 1.376 & .021 & .027 & .006 & .386 & .392 & NA & .013 & 1.829 & 4.063 \\
\hline 1970 & .001 & .004 & .846 & 2.814 & 4.656 & 7.470 & NA & .021 & 8.342 & 1.936 & .061 & .072 & .029 & .520 & .549 & NA & .014 & 2.632 & 5.709 \\
\hline 1975 & .024 & .045 & .978 & 8.721 & 4.227 & 12.948 & NA & .038 & 14.032 & 1.761 & .032 & .074 & .012 & .427 & .439 & NA & .017 & 2.323 & 11.709 \\
\hline 1976 & .030 & .033 & .988 & 11.239 & 4.434 & 15.672 & NA & .037 & 16.760 & 1.597 & .033 & .066 & .017 & .452 & .469 & NA & .008 & 2.172 & 14.588 \\
\hline 1977 & .041 & .045 & 1.037 & 14.027 & 4.728 & 18.756 & NA & .069 & 19.948 & 1.442 & .031 & .056 & .06 & .408 & .514 & NA & .009 & 2.052 & 17.896 \\
\hline 1978 & .074 & .142 & .995 & 13.460 & 4.364 & 17.824 & NA & .072 & 19.106 & 1.078 & .017 & .053 & .335 & .432 & .767 & NA & .005 & 1.920 & 17.186 \\
\hline 1979 & .051 & .099 & 1.300 & 13.825 & 4.108 & 17.933 & NA & .077 & 19.460 & 1.753 & .036 & .056 & .497 & .505 & 1.002 & NA & .007 & 2.855 & 16.605 \\
\hline 1980 & .030 & .016 & 1.006 & 11.195 & 3.463 & 14.658 & NA & .085 & 15.796 & 2.421 & .051 & .049 & .609 & .551 & 1.160 & NA & .014 & 3.695 & 12.101 \\
\hline 1981 & .026 & .013 & .917 & 9.336 & $\begin{array}{l}3.400 \\
3.303\end{array}$ & 12.639 & NA & .124 & 13.719 & 2.944 & .029 & .060 & .482 & .781 & 1.264 & NA & .010 & $\begin{array}{l}4.0307 \\
\end{array}$ & 9.412 \\
\hline 1982 & .019 & .003 & .950 & 7.418 & 3.360 & 10.777 & NA & .112 & 11.861 & 2.787 & .025 & .052 & .500 & 1.231 & $\begin{array}{l}1.732 \\
\text {. }\end{array}$ & NA & .012 & 4.608 & 7.253 \\
\hline 1983 & .032 & .001 & .940 & 7.079 & 3.568 & 10.647 & NA & .132 & 11.752 & 2.045 & .016 & .055 & .348 & 1.217 & 1.565 & NA & .011 & 3.693 & 8.059 \\
\hline 1984 & .032 & .014 & .847 & 7.302 & 4.131 & 11.433 & NA & .144 & 12.471 & 2.151 & .026 & .055 & .384 & 1.161 & 1.545 & NA & .009 & 3.786 & 8.685 \\
\hline 1985 & .049 & .014 & .952 & 6.814 & 3.796 & 10.609 & NA & .157 & 11.781 & 2.438 & .028 & .056 & $\begin{array}{l}.004 \\
.432\end{array}$ & $\begin{array}{l}1.101 \\
1.225\end{array}$ & $\begin{array}{l}1.657 \\
\end{array}$ & NA & .017 & $\begin{array}{l}4.196 \\
4.196\end{array}$ & 7.584 \\
\hline 1986 & .055 & .008 & .748 & 9.002 & 4.199 & 13.201 & NA & .139 & 14.151 & 2.248 & .025 & .062 & .326 & 1.344 & 1.670 & NA & .016 & 4.021 & 10.130 \\
\hline 1987 & .044 & .023 & .992 & 10.067 & 4.095 & 14.162 & NA & .178 & 15.398 & 2.093 & .014 & .055 & .319 & 1.311 & 1.630 & NA & .020 & 3.812 & 11.586 \\
\hline 1988 & .053 & .067 & 1.296 & 11.027 & 4.720 & 15.747 & NA & .133 & 17.296 & 2.499 & .027 & .075 & .329 & 1.412 & 1.741 & NA & .024 & 4.366 & 12.929 \\
\hline 1989 & .071 & .057 & 1.387 & 12.596 & 4.565 & 17.162 & NA & .089 & 18.766 & 2.637 & .027 & .109 & .300 & 1.536 & $\begin{array}{l}1.836 \\
\text {. }\end{array}$ & NA & .052 & 4.661 & 14.105 \\
\hline 1990 & .067 & .019 & 1.551 & 12.766 & 4.351 & 17.117 & NA & .063 & 18.817 & 2.772 & .014 & .087 & .230 & 1.594 & 1.824 & NA & .055 & 4.752 & 14.065 \\
\hline 1991 & .085 & .029 & 1.798 & 12.553 & 3.794 & 16.348 & NA & .075 & 18.335 & 2.854 & .020 & .132 & .246 & 1.882 & 2.128 & NA & .008 & 5.141 & 13.194 \\
\hline 1992 & .095 & .052 & 2.161 & 13.253 & 3.714 & 16.968 & NA & .096 & 19.372 & 2.682 & .017 & .220 & .188 & 1.819 & 2.008 & NA & .010 & 4.937 & 14.435 \\
\hline 1993 & .205 & .053 & 2.397 & 14.749 & $\begin{array}{l}3.760 \\
3.0\end{array}$ & 18.510 & .001 & .107 & 21.273 & 1.962 & .026 & .142 & .208 & 1.907 & 2.115 & NA & .012 & 4.258 & 17.014 \\
\hline 1994 & .222 & .083 & 2.682 & 15.340 & 3.904 & 19.243 & .001 & .160 & 22.390 & 1.879 & .024 & .164 & .209 & 1.779 & $\begin{array}{l}1.988 \\
\text {. }\end{array}$ & NA & .007 & 4.061 & 18.329 \\
\hline 1995 & .237 & .095 & 2.901 & 15.669 & 3.211 & 18.881 & .001 & .146 & 22.260 & 2.318 & .034 & .156 & .200 & 1.791 & 1.991 & NA & .012 & 4.511 & 17.750 \\
\hline 1996 & .203 & .063 & 3.002 & 16.341 & 3.943 & 20.284 & .001 & .148 & 23.702 & 2.368 & .040 & .155 & .233 & 1.825 & 2.059 & NA & .011 & 4.633 & 19.069 \\
\hline 1997 & .187 & .078 & $\begin{array}{l}3.006 \\
3.063\end{array}$ & 17.876 & $\begin{array}{l}3.064 \\
3.864\end{array}$ & 21.740 & $(\mathrm{~s})$ & .147 & 25.215 & $\begin{array}{l}2.000 \\
2.193\end{array}$ & .031 & .159 & .228 & $\begin{array}{l}1.020 \\
1.872\end{array}$ & $\begin{array}{l}2.00 \\
\end{array}$ & NA & .031 & $\begin{array}{l}4.050 \\
4.514\end{array}$ & 20.701 \\
\hline 1998 & .218 & .095 & 3.225 & 18.916 & 3.992 & 22.908 & (s) & .135 & 26.581 & 2.092 & .028 & .161 & .233 & 1.740 & 1.972 & NA & .047 & 4.299 & 22.281 \\
\hline 1999 & .227 & .080 & 3.664 & 18.935 & 4.198 & 23.133 & (s) & .147 & 27.252 & 1.525 & .022 & .164 & .250 & 1.705 & 1.955 & NA & .049 & 3.715 & 23.537 \\
\hline 2000 & .313 & .094 & 3.869 & 19.783 & 4.749 & 24.531 & (s) & .166 & 28.973 & 1.528 & .028 & .245 & .106 & 2.048 & 2.154 & NA & .051 & 4.006 & 24.967 \\
\hline 2001 & .495 & .063 & $\begin{array}{l}.005 \\
4.068\end{array}$ & 20.348 & 5.051 & 25.398 & .002 & .131 & 30.157 & 1.265 & .033 & .377 & .043 & $\begin{array}{l}1.040 \\
1.996\end{array}$ & $\begin{array}{l}2.039 \\
\end{array}$ & (s) & .056 & 3.771 & 26.386 \\
\hline 2002 & .422 & .080 & 4.104 & 19.920 & 4.754 & 24.674 & .002 & .125 & 29.408 & 1.032 & .020 & .520 & .019 & 2.023 & 2.042 & (s) & .054 & 3.669 & 25.739 \\
\hline 2003 & .626 & .068 & 4.042 & 21.060 & 5.159 & 26.219 & .002 & .104 & 31.061 & 1.117 & .018 & .686 & .026 & 2.124 & 2.151 & .001 & .082 & 4.054 & 27.007 \\
\hline 2004 & .682 & .170 & 4.365 & 22.082 & 6.114 & 28.197 & .013 & .117 & 33.544 & 1.253 & .033 & .862 & .057 & 2.151 & 2.208 & .001 & .078 & 4.434 & 29.110 \\
\hline 2005 & .762 & .088 & 4.450 & 22.091 & 7.157 & 29.248 & .012 & .150 & 34.709 & 1.273 & .043 & .735 & .067 & 2.374 & 2.442 & .001 & .065 & 4.560 & 30.149 \\
\hline 2006 & .906 & .101 & 4.291 & 22.085 & 7.084 & 29.169 & .066 & .146 & 34.679 & 1.264 & .040 & .730 & .052 & 2.699 & 2.751 & .004 & .083 & 4.872 & 29.806 \\
\hline 2007 & .909 & .061 & 4.723 & 21.914 & $\begin{array}{l}6.004 \\
6.868\end{array}$ & 28.781 & .054 & .175 & 34.703 & 1.507 & .036 & .830 & .058 & 2.949 & 3.007 & .035 & .069 & 5.482 & 29.221 \\
\hline 2008 & .855 & .089 & 4.084 & 21.448 & 6.237 & 27.685 & .084 & .195 & 32.992 & 2.071 & .049 & .972 & .061 & 3.739 & 3.800 & .086 & .083 & 7.060 & 25.932 \\
\hline 2009 & .566 & .009 & $\begin{array}{l}4.004 \\
3.845\end{array}$ & $\begin{array}{l}19.690 \\
\end{array}$ & 5.383 & 25.082 & .026 & .178 & 29.706 & 1.515 & .032 & $\begin{array}{r}.51< \\
1.082\end{array}$ & .093 & 4.147 & $\begin{array}{l}.000 \\
4.240\end{array}$ & .034 & $\begin{array}{l}.062 \\
.062\end{array}$ & 6.965 & 22.741 \\
\hline 2010 & .484 & .030 & $\mathrm{R}_{3.834}$ & $\mathrm{R}_{20.140}$ & $R_{5.231}$ & $\mathrm{R}_{25.371}$ & .004 & .154 & R29.877 & 2.101 & .036 & 1.147 & .088 & $R_{4.750}$ & $\mathrm{R}_{4.838}$ & R. 046 & R. .065 & $R_{8.234}$ & $\mathrm{R}_{21.643}$ \\
\hline $2011^{P}$ & .327 & .035 & 3.540 & 19.561 & 4.930 & 24.491 & .016 & .178 & 28.587 & 2.751 & .024 & $\begin{array}{l}1.521 \\
\text { S }\end{array}$ & .100 & 5.801 & 5.901 & .108 & .051 & 10.356 & 18.232 \\
\hline
\end{tabular}

1 Net imports equal imports minus exports. Minus sign indicates exports are greater than imports. 2 Crude oil and lease condensate. Imports data include imports into the Strategic Petroleum Reserve, which began in 1977

3 Petroleum products, unfinished oils, pentanes plus, and gasoline blending components. Does not include biofuels.

Fuel ethanol (minus denaturant) and biodiesel.

$R=$ Revised. $P=$ Preliminary. NA=Not available. $(s)=$ Less than 0.0005 quadrillion Btu.

Notes: - Includes trade between the United States (50 States and the District of Columbia) and its territories and possessions. - See "Primary Energy" in Glossary. - See Note 3, "Electricity Imports and Exports," at end of Section 8. - Totals may not equal sum of components due to independent rounding. annual data. - See http://www.eia.gov/totalenergy/data/annual//\#summary for all annual data beginning in

Sources: Tables 5.1b 5.3, 5.5, 6.1, 7.1, 7.8, 8.1, 10.3, 10.4, A2, A3, A4, A5, and A6. 


\section{Figure 1.5 Energy Consumption and Expenditures Indicators Estimates}

\section{Energy Consumption, 1949-2011}

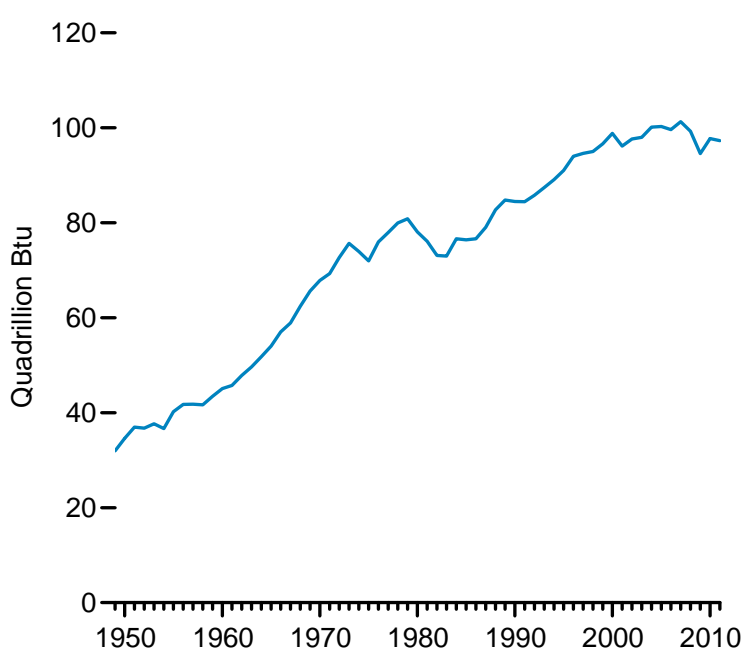

Energy Consumption per Capita, 1949-2011

$500-$

$400-$

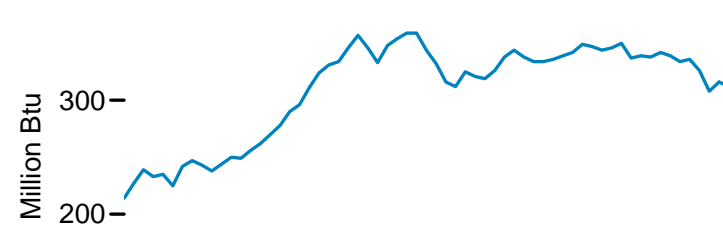

$100-$

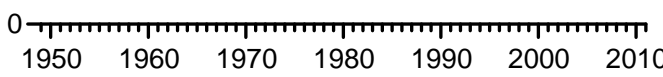

\section{Energy Expenditures, 1970-2010}

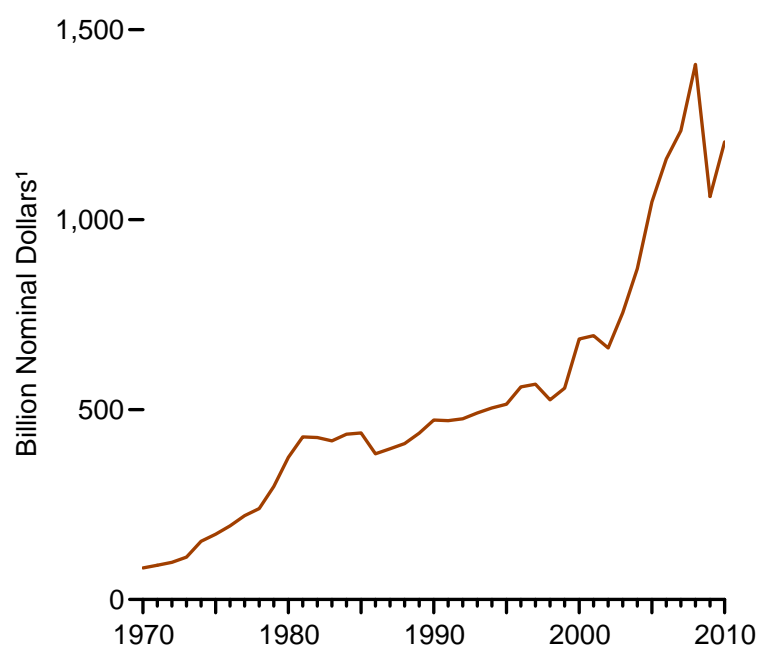

\section{Energy Expenditures per Capita,} 1970-2010

$5,000-$
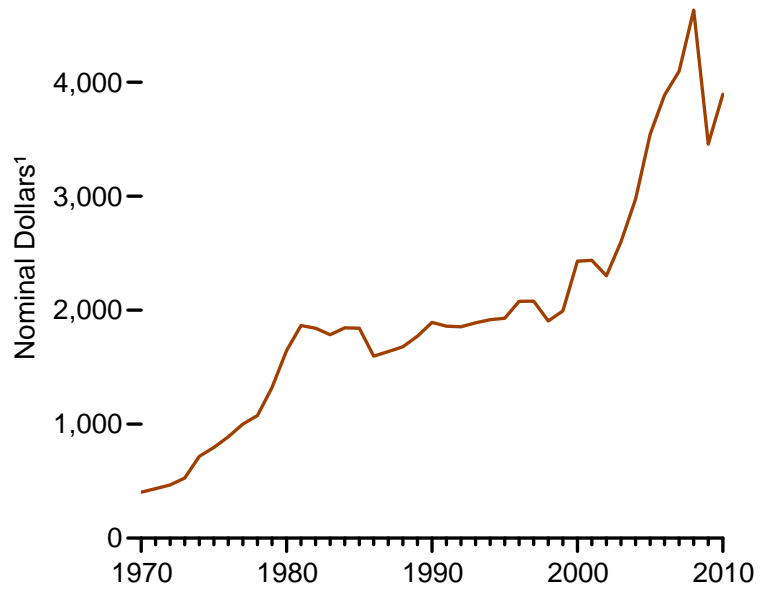

Energy Consumption per Real Dollar ${ }^{2}$ of

Gross Domestic Product, 1949-2011

20-

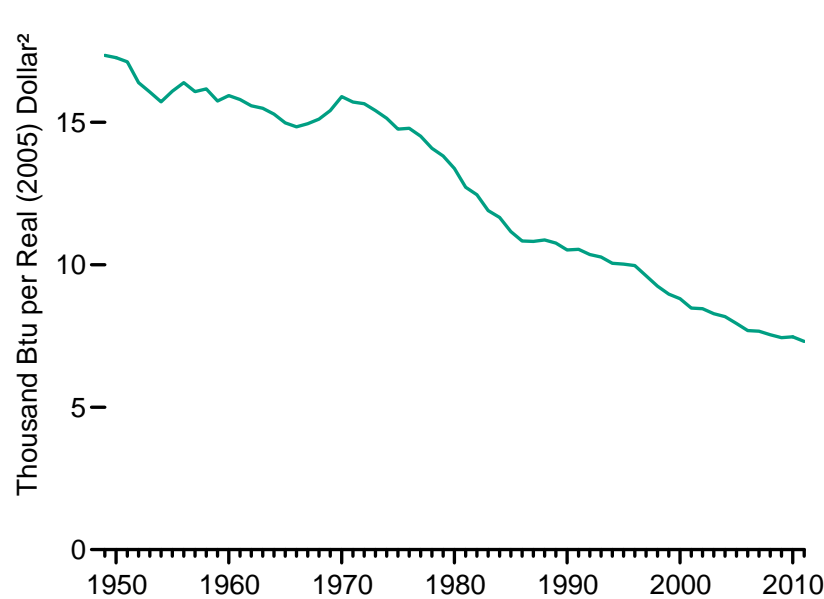

\section{Energy Expenditures as Share of Gross}

Domestic Product and Gross Output, ${ }^{3}$ 1987-2010

$12-$

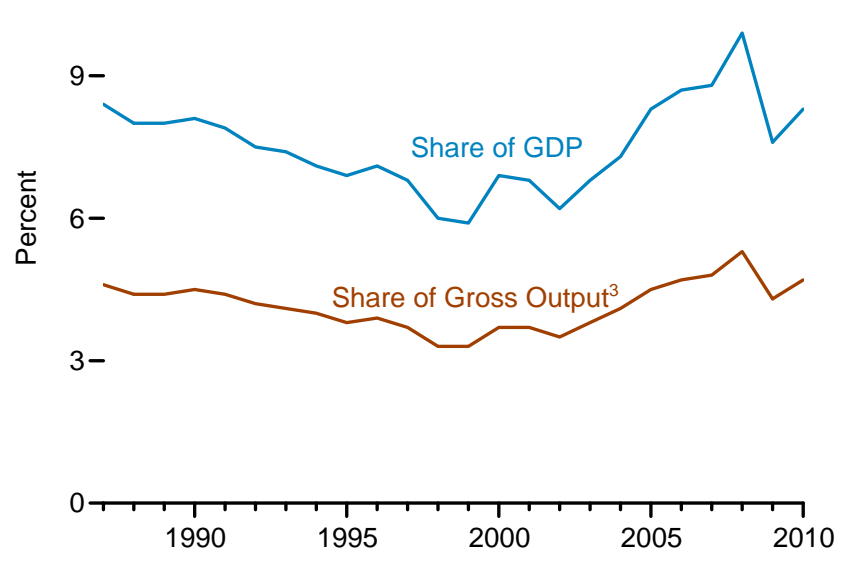

${ }^{1}$ See "Nominal Dollars" in Glossary.

2 In chained (2005) dollars, calculated by using gross domestic product implicit price deflators

in Table D1. See "Chained Dollars" in Glossary.

${ }^{3}$ Gross output is the value of gross domestic product (GDP) plus the value of intermediate inputs used to produce GDP.

Source: Table 1.5. 
Table 1.5 Energy Consumption, Expenditures, and Emissions Indicators Estimates, Selected Years, 1949-2011

\begin{tabular}{|c|c|c|c|c|c|c|c|c|c|c|c|}
\hline & $\begin{array}{c}\text { Energy } \\
\text { Consumption }\end{array}$ & $\begin{array}{c}\text { Energy } \\
\text { Consumption } \\
\text { per Capita }\end{array}$ & $\begin{array}{c}\text { Energy } \\
\text { Expenditures }\end{array}$ & $\begin{array}{c}\text { Energy } \\
\text { Expenditures }{ }^{1} \\
\text { per Capita }\end{array}$ & $\begin{array}{c}\text { Gross } \\
\text { Output }^{3}\end{array}$ & $\begin{array}{c}\text { Energy } \\
\text { Expenditures } \\
\text { as Share of } \\
\text { Gross Output }{ }^{3}\end{array}$ & $\begin{array}{c}\text { Gross } \\
\text { Domestic } \\
\text { Product (GDP) }\end{array}$ & $\begin{array}{c}\text { Energy } \\
\text { Expenditures }{ }^{1} \\
\text { as Share of GDP }\end{array}$ & $\begin{array}{c}\text { Gross } \\
\text { Domestic } \\
\text { Product (GDP) }\end{array}$ & $\begin{array}{c}\text { Energy } \\
\text { Consumption per } \\
\text { Real Dollar of GDP }\end{array}$ & $\begin{array}{l}\text { Carbon Dioxide } \\
\text { Emissions }{ }^{2} \text { per } \\
\text { Real Dollar of GDP }\end{array}$ \\
\hline Year & $\begin{array}{l}\text { Quadrillion } \\
\text { Btu }\end{array}$ & $\begin{array}{c}\text { Million } \\
\text { Btu }\end{array}$ & $\begin{array}{l}\text { Million } \\
\text { Nominal } \\
\text { Dollars }{ }^{4}\end{array}$ & $\begin{array}{l}\text { Nominal } \\
\text { Dollars } 4\end{array}$ & $\begin{array}{l}\text { Billion } \\
\text { Nominal } \\
\text { Dollars }{ }^{4}\end{array}$ & Percent & $\begin{array}{l}\text { Billion } \\
\text { Nominal } \\
\text { Dollars }{ }^{4}\end{array}$ & Percent & $\begin{array}{c}\text { Billion } \\
\text { Real } \\
\text { (2005) Dollars } 5\end{array}$ & $\begin{array}{l}\text { Thousand Btu } \\
\text { per Real } \\
\text { (2005) Dollar } 5\end{array}$ & $\begin{array}{c}\text { Metric Tons } \\
\text { Carbon Dioxide } \\
\text { per Million Real } \\
\text { (2005) Dollars }{ }^{5}\end{array}$ \\
\hline 1949 & 31.982 & 214 & NA & NA & NA & NA & 267.2 & NA & $\mathrm{R}_{1,843.1}$ & $\mathrm{R}_{17.35}$ & $\mathrm{R}_{1,197}$ \\
\hline 1950 & 34.616 & 227 & NA & NA & NA & NA & 293.7 & NA & $R_{2,004.2}$ & $\mathrm{R}_{17.27}$ & $R_{1,189}$ \\
\hline 1955 & 40.208 & 242 & NA & NA & NA & NA & 414.7 & NA & $\mathrm{R}_{2,498.2}$ & $R_{16.09}$ & $R_{1,075}$ \\
\hline 1960 & 45.086 & 250 & NA & NA & NA & NA & 526.4 & NA & $\mathrm{R}_{2,828.5}$ & $R_{15.94}$ & $R_{1,030}$ \\
\hline 1965 & 54.015 & 278 & NA & NA & NA & NA & 719.1 & NA & $\mathrm{R}_{3,607.0}$ & $R_{14.98}$ & R960 \\
\hline 1970 & 67.838 & 331 & 82,860 & 404 & NA & NA & $1,038.3$ & 8.0 & $\mathrm{R}_{4,266.3}$ & $\mathrm{R}_{15.90}$ & R9g9 \\
\hline 1975 & 71.965 & 333 & $\mathrm{R}_{171,837}$ & R796 & NA & NA & $1,637.7$ & 10.5 & $\mathrm{R}_{4,875.4}$ & $\mathrm{R}_{14.76}$ & $R_{910}$ \\
\hline 1976 & 75.975 & 348 & $R_{193,896}$ & 889 & NA & NA & $1,824.6$ & 10.6 & $\mathrm{R}_{5}, 136.9$ & $R_{14.79}$ & R916 \\
\hline 1977 & 77.961 & 354 & $R_{220,476}$ & 1,001 & NA & NA & $2,030.1$ & 10.9 & $R_{5}, 373.1$ & $R_{14.51}$ & R902 \\
\hline 1978 & 79.950 & 359 & $\mathrm{R}_{239,255}$ & $\mathrm{R}_{1,075}$ & NA & NA & $2,293.8$ & 10.4 & $\begin{array}{l}\text { R5,672.8 } \\
\mathrm{R}_{5}\end{array}$ & $R_{14.09}$ & R863 \\
\hline 1979 & 80.859 & 359 & R297,549 & 1,322 & NA & NA & $2,562.2$ & 11.6 & $\mathrm{R}_{5,850.1}$ & $R_{13.82}$ & R849 \\
\hline 1980 & 78.067 & 344 & $R_{374,347}$ & 1,647 & NA & NA & $2,788.1$ & 13.4 & $\mathrm{R}_{5,834.0}$ & $R_{13.38}$ & R818 \\
\hline 1981 & 76.106 & 332 & R 427,898 & $R_{1,865}$ & NA & NA & $3,126.8$ & 13.7 & $R_{5}, 982.1$ & $R_{12.72}$ & R776 \\
\hline 1982 & 73.099 & 316 & $\mathrm{R}_{426,479}$ & $\mathrm{R}_{1,841}$ & NA & NA & $3,253.2$ & 13.1 & $\mathrm{R}_{5,865.9}$ & $R_{12.46}$ & 751 \\
\hline 1983 & 72.971 & 312 & $\mathrm{R}_{417,476}$ & $\mathrm{R}_{1,786}$ & NA & NA & $3,534.6$ & 11.8 & $\mathrm{R}_{6,130.9}$ & $R_{11.90}$ & R715 \\
\hline 1984 & 76.632 & 325 & R 435,195 & 1,845 & NA & NA & $3,930.9$ & 11.1 & ${ }^{R} 6,571.5$ & $R_{11.66}$ & R702 \\
\hline 1985 & 76.392 & 321 & $\mathrm{R}_{438,347}$ & 1,842 & NA & NA & $4,217.5$ & 10.4 & $\mathrm{R}_{6,843.4}$ & $R_{11.16}$ & 672 \\
\hline 1986 & 76.647 & 319 & R 383,518 & 1,597 & NA & NA & $4,460.1$ & 8.6 & $\mathrm{R} 7,080.5$ & $R_{10.83}$ & $\mathrm{R}_{651}$ \\
\hline 1987 & 79.054 & 326 & R396,587 & $\mathrm{R}_{1,637}$ & $8,639.9$ & 4.6 & $\begin{array}{l}4,736.4 \\
4,736.1\end{array}$ & $\begin{array}{l}8.0 \\
8.4\end{array}$ & $\begin{array}{l}\text { R } 7,307.0 \\
\text { (n) }\end{array}$ & $\begin{array}{l}10.83 \\
R_{1}\end{array}$ & R652 \\
\hline 1988 & 82.709 & 338 & $\mathrm{R}_{410,515}$ & $\mathrm{R}_{1,679}$ & $9,359.5$ & 4.4 & $5,100.4$ & 8.0 & $\mathrm{R}_{7,607.4}$ & $\mathrm{R}_{10.87}$ & R655 \\
\hline 1989 & 84.786 & 344 & R 437,679 & 1,773 & $9,969.6$ & 4.4 & $5,482.1$ & 8.0 & $\mathrm{R}_{7,879.2}$ & $\mathrm{R}_{10.76}$ & 643 \\
\hline 1990 & 84.485 & 338 & $\mathrm{R}_{472,653}$ & 1,893 & $10,511.1$ & 4.5 & $5,800.5$ & 8.1 & $\mathrm{R}_{8,027.1}$ & 10.52 & R628 \\
\hline 1991 & 84.438 & 334 & $\mathrm{R}_{470,668}$ & 1,860 & $10,676.5$ & 4.4 & $5,992.1$ & 7.9 & $R_{8,008.3}$ & $R_{10.54}$ & $R_{624}$ \\
\hline 1992 & 85.783 & 334 & $\mathrm{R}_{475,644}$ & $R_{1,854}$ & $11,242.4$ & 4.2 & $6,342.3$ & 7.5 & $\mathrm{R}_{8,280.0}$ & $\mathrm{R}_{10.36}$ & 615 \\
\hline 1993 & 87.424 & 336 & R 491,231 & $R_{1,890}$ & $11,857.6$ & 4.1 & $6,667.4$ & 7.4 & $\mathrm{R}_{8,516.2}$ & $R_{10.27}$ & $R_{609}$ \\
\hline 1994 & 89.091 & 339 & 504,073 & 1,916 & $12,647.2$ & 4.0 & $7,085.2$ & 7.1 & $\mathrm{R}_{8,863.1}$ & $\mathrm{R}_{10.05}$ & 593 \\
\hline 1995 & 91.029 & 342 & 513,947 & 1,930 & $13,451.6$ & 3.8 & $7,414.7$ & 6.9 & $\mathrm{R}_{9,086.0}$ & $\mathrm{R}_{10.02}$ & $R_{585}$ \\
\hline 1996 & 94.022 & 349 & 559,890 & 2,078 & $14,259.9$ & 3.9 & $7,838.5$ & 7.1 & $\mathrm{R}_{9,425.8}$ & 9.97 & R584 \\
\hline 1997 & 94.602 & 347 & 566,714 & 2,079 & $15,160.5$ & $\begin{array}{l}3.7 \\
\text { (n) }\end{array}$ & $8,332.4$ & 6.8 & $R_{9,845.9}$ & $R_{9.61}$ & 566 \\
\hline 1998 & 95.018 & 344 & 525,515 & 1,905 & $15,987.4$ & 3.3 & $8,793.5$ & 6.0 & $\mathrm{R}_{10,274.7}$ & R9. 25 & 547 \\
\hline 1999 & 96.652 & 346 & R556,379 & 1,994 & 17.017 .4 & 3.3 & $9,353.5$ & 5.9 & $\mathrm{R}_{10.770 .7}$ & 8.97 & R528 \\
\hline 2000 & R98.814 & 350 & R685,902 & 2,431 & $18,305.7$ & $\begin{array}{l}3.0 \\
3.7\end{array}$ & $9,951.5$ & $\begin{array}{l}6.9 \\
6.9\end{array}$ & $\mathrm{R}_{11,216.4}$ & $\begin{array}{l}\mathrm{R} .31 \\
\mathrm{R} 8.81\end{array}$ & 523 \\
\hline 2001 & 96.168 & 337 & 694,484 & $\mathrm{R}_{2,437}$ & $18,576.5$ & 3.7 & $10,286.2$ & 6.8 & $R_{11,337.5}$ & 8.48 & 508 \\
\hline 2002 & R97.645 & $R_{339}$ & $\begin{array}{l}\text { R } 662,414 \\
\text { nat }\end{array}$ & $R_{2,303}$ & $18,874.2$ & 3.5 & $10,642.3$ & $\begin{array}{l}6.0 \\
6.2\end{array}$ & $R_{11,543.1}$ & 8.46 & 503 \\
\hline 2003 & 97.978 & 338 & 754,708 & $\mathrm{R}_{2,601}$ & $19,832.3$ & 3.8 & $\mathrm{R}_{11,142.2}$ & 6.8 & $R_{11,836.4}$ & $R_{8.28}$ & 495 \\
\hline 2004 & $\mathrm{R}_{100.162}$ & 342 & R871,097 & $\mathrm{R}_{2,975}$ & $21,267.7$ & 4.1 & $R_{11,853.3}$ & 7.3 & $R_{12,246.9}$ & $R_{8.18}$ & $\mathrm{R}_{488}$ \\
\hline 2005 & $\mathrm{R}_{100.282}$ & 339 & $\mathrm{R}_{1,046,897}$ & $\mathrm{R}_{3,543}$ & $23,046.9$ & 4.5 & $R_{12,623.0}$ & 8.3 & $R_{12,623.0}$ & R7.94 & R475 \\
\hline 2006 & R99.629 & 334 & $\mathrm{R}_{1,159,687}$ & $\mathrm{R}_{3,887}$ & $24,477.0$ & 4.7 & $R_{13,377.2}$ & 8.7 & $R_{12,958.5}$ & $\begin{array}{l}\mathrm{R} 7.69 \\
\end{array}$ & $\mathrm{R}_{457}$ \\
\hline 2007 & $R_{101.296}$ & 336 & $R_{1,234,282}$ & $\mathrm{R}_{4,097}$ & $25,819.7$ & 4.8 & $\mathrm{R}_{14,028.7}$ & 8.8 & $\mathrm{R}_{13,206.4}$ & R7.67 & $\mathrm{R}_{456}$ \\
\hline 2008 & R99.275 & 326 & $R_{1}, 408,845$ & $\mathrm{R}_{4,633}$ & $26,561.9$ & 5.3 & $R_{14,291.5}$ & R9. 9 & $R_{13,161.9}$ & R7.54 & R444 \\
\hline 2009 & R94.559 & 308 & $R_{1,061,220}$ & $R_{3,459}$ & $24,568.6$ & 4.3 & $R_{13,939.0}$ & R7.6 & $R_{12,703.1}$ & R7.44 & $R_{427}$ \\
\hline 2010 & $\begin{array}{l}\text { R97.722 } \\
\text { Res. }\end{array}$ & $R_{316}$ & $\mathrm{R}_{1,204,827}$ & $\mathrm{R}_{3,895}$ & $25,811.4$ & 4.7 & $R_{14,526.5}$ & $R_{8.3}$ & $\mathrm{R}_{13,088.0}$ & R7.47 & $\mathrm{R}_{429}$ \\
\hline $2011^{P}$ & 97.301 & 312 & NA & NA & NA & NA & $15,094.0$ & NA & $13,315.1$ & 7.31 & 412 \\
\hline
\end{tabular}

1 Expenditures include taxes where data are available.

Carbon dioxide emissions from energy consumption. See Table 11.1

3 Gross output is the value of GDP plus the value of intermediate inputs used to produce GDP.

See "Nominal Dollars" in Glossary.

In chained (2005) dollars. See "Chained Dollars" in Glossary.

Web Page: For all data beginning in 1949, see http://www.eia.gov/totalenergy/data/annual/\#summary. Sources: Energy Consumption: Table 1.3. Energy Expenditures: Table 3.5. Gross Domestic Product: Table D1. Population Data: Table D1. Gross Output: U.S. Department of Commerce, Bureau Dioxide Emissions: Table 11.1. Other Columns: Calculated by U.S. Energy Information Administration. 


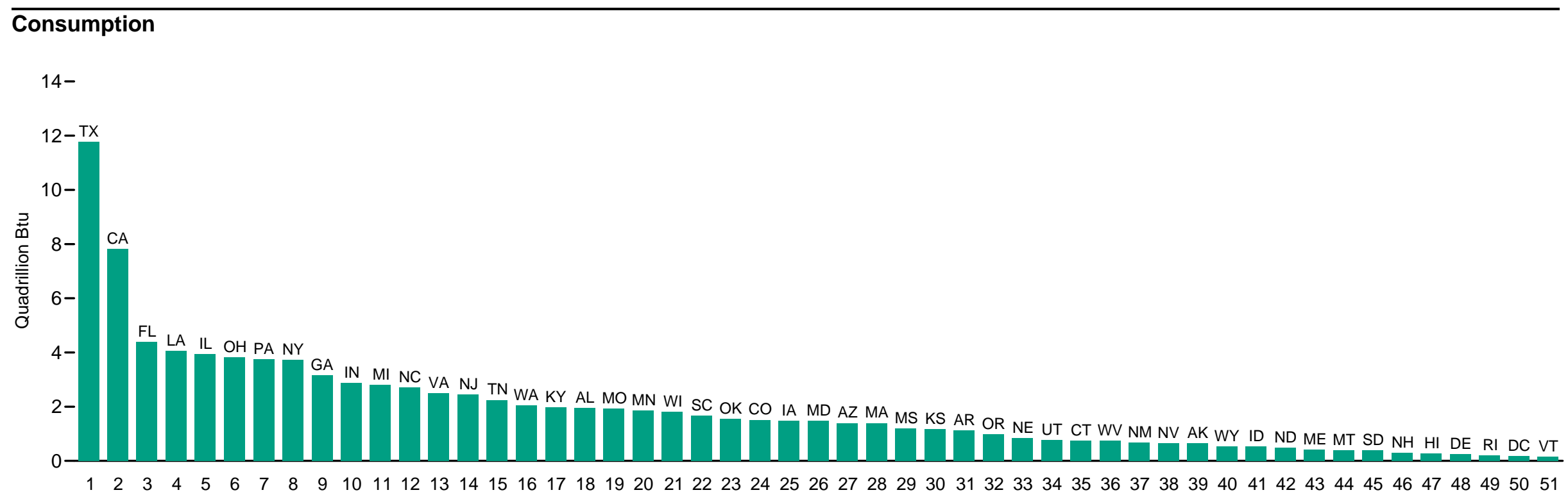

\section{Consumption per Capita}

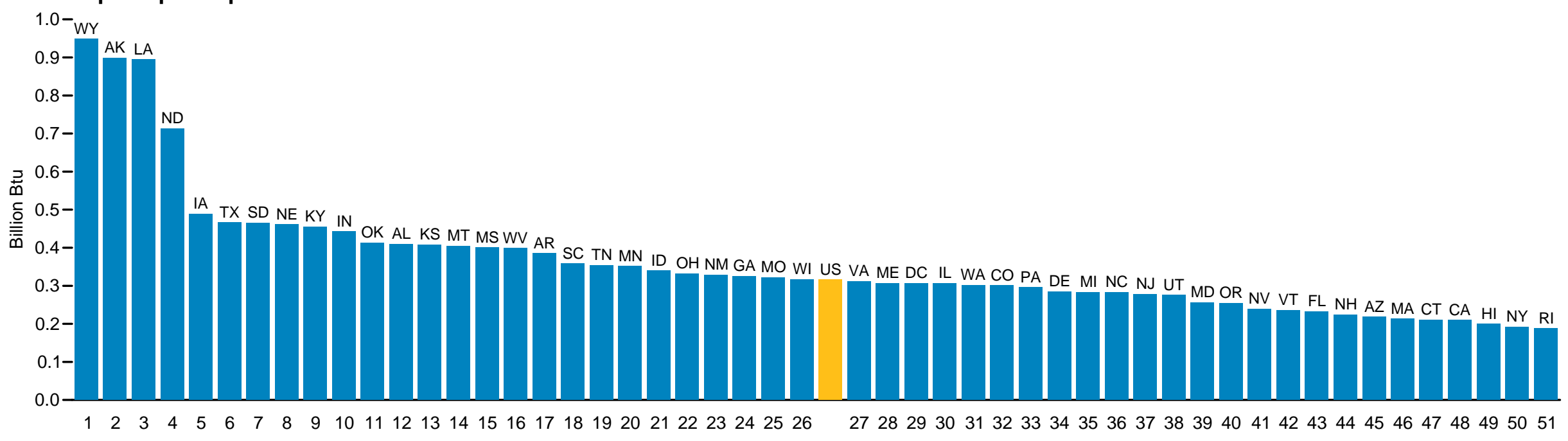


Table 1.6 State-Level Energy Consumption, Expenditure, and Price Estimates, 2010

\begin{tabular}{|c|c|c|c|c|c|c|c|c|c|c|}
\hline \multirow[b]{2}{*}{ Rank } & \multicolumn{2}{|c|}{ Consumption } & \multicolumn{2}{|c|}{ Consumption per Capita } & \multicolumn{2}{|c|}{ Expenditures ${ }^{1}$} & \multicolumn{2}{|c|}{ Expenditures ${ }^{1}$ per Capita } & \multicolumn{2}{|c|}{ Prices 1} \\
\hline & \multicolumn{2}{|c|}{ Trillion Btu } & \multicolumn{2}{|c|}{ Million Btu } & \multicolumn{2}{|c|}{ Million Dollars ${ }^{2}$} & \multicolumn{2}{|c|}{ Dollars ${ }^{2}$} & \multicolumn{2}{|c|}{ Dollars ${ }^{2}$ per Million Btu } \\
\hline 1 & Texas & $11,769.9$ & Wyoming & 948.1 & Texas & 137,532 & Alaska & 8,807 & Hawaii & 30.75 \\
\hline 2 & California & $7,825.7$ & Alaska & $\begin{array}{l}34.1 \\
898.5\end{array}$ & California & 117,003 & Louisiana & $\begin{array}{l}0,001 \\
8,661\end{array}$ & District of Columbia & 26.19 \\
\hline 3 & Florida & $4,381.9$ & Louisiana & 894.4 & New York & 61,619 & Wyoming & 7,904 & Connecticut & 25.63 \\
\hline 4 & Louisiana & $4,065.4$ & North Dakota & 712.6 & Florida & 60,172 & North Dakota & 6,740 & Vermont & 24.20 \\
\hline 5 & $\begin{array}{l}\text { Illinois } \\
\text { las }\end{array}$ & $3,936.7$ & lowa & 489.3 & Pennsylvania & 48,701 & Texas & 5,446 & New Hampshire & 23.87 \\
\hline 6 & Ohio & $3,833.7$ & Texas & $\begin{array}{l}409.3 \\
466.1\end{array}$ & Ohio & 45,081 & $\begin{array}{l}\text { lowa } \\
\text { lewas }\end{array}$ & 4,841 & Massachusetts & 23.32 \\
\hline 7 & Pennsylvania & $3,758.8$ & South Dakota & 464.9 & Illinois & 44,989 & Maine & 4,746 & Rhode Island & 23.12 \\
\hline 8 & New York & $3,728.4$ & $\begin{array}{l}\text { Nebraska } \\
\text { a a }\end{array}$ & 461.1 & Louisiana & 39,369 & South Dakota & 4,651 & $\begin{array}{l}\text { Delaware } \\
\text { lawe }\end{array}$ & 22.95 \\
\hline 9 & Georgia & $3,155.7$ & Kentucky & 454.7 & New Jersey & 37,362 & Montana & 4,610 & New York & 22.91 \\
\hline 10 & Indiana & $2,871.1$ & Indiana & 442.3 & Georgia & 37,338 & Kentucky & 4,526 & Maryland & 22.48 \\
\hline 11 & Michigan & $2,798.1$ & Oklahoma & 412.6 & Michigan & 34,540 & Alabama & 4,494 & Arizona & 21.78 \\
\hline 12 & North Carolina & $2,705.2$ & Alabama & $\begin{array}{l}412.0 \\
409.5\end{array}$ & North Carolina & $\begin{array}{l}34,940 \\
32,989\end{array}$ & Mississippi & $\begin{array}{r}4,494 \\
4,446\end{array}$ & Florida & 21.66 \\
\hline 13 & Virginia & $2,502.1$ & Kansas & 407.6 & Virginia & 29,826 & Nebraska & 4,421 & New Jersey & 20.91 \\
\hline 14 & New Jersey & $2,447.5$ & Montana & 405.1 & Indiana & 27,374 & Kansas & 4,357 & Nevada & 20.87 \\
\hline 15 & Tennessee & $2,250.6$ & Mississippi & 400.4 & Tennessee & 25,153 & Vermont & 4,344 & California & 20.66 \\
\hline 16 & Washington & $2,036.5$ & West Virginia & 398.4 & Massachusetts & 24,512 & Oklahoma & 4,268 & Alaska & 20.25 \\
\hline 17 & Kentucky & $1,976.5$ & Arkansas & $\begin{array}{l}385.3 \\
385.3\end{array}$ & $\begin{array}{l}\text { Washington } \\
\text { lon }\end{array}$ & 22,893 & West Virginia & 4,251 & North Carolina & 19.98 \\
\hline 18 & Alabama & $1,959.7$ & South Carolina & $\begin{array}{l}350.3 \\
358.3\end{array}$ & Missouri & $\begin{array}{l}22,895 \\
22,885\end{array}$ & New Jersey & $\begin{array}{l}4,246 \\
4,246\end{array}$ & Pennsylvania & 19.56 \\
\hline 19 & Missouri & $1,928.4$ & Tennessee & 354.0 & Maryland & 21,517 & Indiana & 4,217 & New Mexico & 19.40 \\
\hline 20 & Minnesota & $1,867.3$ & Minnesota & 351.6 & Alabama & 21,507 & Hawaii & 4,191 & Virginia & 18.91 \\
\hline 21 & Wisconsin & $1,800.1$ & $\begin{array}{l}\text { Idaho } \\
\text { Inta }\end{array}$ & 339.7 & Wisconsin & 21,483 & Arkansas & 4,128 & Oregon & 18.89 \\
\hline 22 & South Carolina & $1,661.6$ & Ohio & 332.3 & Minnesota & 20,869 & South Carolina & 4,034 & Maine & 18.78 \\
\hline 23 & Oklahoma & $1,551.6$ & New Mexico & $\begin{array}{l}329.2 \\
32.2\end{array}$ & Kentucky & 19,675 & District of Columbia & 4,033 & Missouri & 18.54 \\
\hline $\begin{array}{l}23 \\
24\end{array}$ & Colorado & $\begin{array}{l}1,551.6 \\
1,516.9\end{array}$ & Georgia & $\begin{array}{l}329.2 \\
324.9\end{array}$ & Arizona & 19,374 & $\begin{array}{l}\text { Delaware } \\
\text { Dalua }\end{array}$ & $\begin{array}{l}4,033 \\
4,019\end{array}$ & Tennessee & $\begin{array}{l}18.54 \\
18.33\end{array}$ \\
\hline 25 & lowa & $1,492.3$ & Missouri & 321.6 & South Carolina & 18,705 & Connecticut & 3,977 & South Carolina & 18.26 \\
\hline 26 & Maryland & $1,481.1$ & Wisconsin & 316.3 & Colorado & 16,751 & New Hampshire & 3,971 & Michigan & 18.22 \\
\hline 27 & Arizona & $1,399.6$ & Virginia & 311.8 & Oklahoma & 16,049 & Tennessee & 3,956 & Wisconsin & 18.22 \\
\hline 28 & Massachusetts & $1,396.9$ & Maine & $\begin{array}{l}306.8 \\
30.8\end{array}$ & lowa & 14,766 & Minnesota & 3,930 & Washington & 18.11 \\
\hline $\begin{array}{l}20 \\
29\end{array}$ & Mississippi & $1,189.2$ & District of Columbia & $\begin{array}{l}300.0 \\
306.6\end{array}$ & Connecticut & 14,221 & Ohio & $\begin{array}{l}3,907 \\
3,907\end{array}$ & Georgia & 17.96 \\
\hline 30 & Kansas & $1,165.3$ & Illinois & 306.5 & Mississippi & 13,206 & Georgia & 3,844 & Ohio & 17.93 \\
\hline 31 & Arkansas & $1,125.6$ & Washington & 302.0 & Oregon & 12,592 & Pennsylvania & $\begin{array}{l}3,044 \\
3,829\end{array}$ & Montana & 17.73 \\
\hline 32 & Oregon & 977.1 & Colorado & 300.5 & Kansas & 12,457 & Missouri & 3,817 & Kansas & 17.72 \\
\hline 33 & Nebraska & 843.8 & Pennsylvania & 295.6 & Arkansas & 12,061 & Wisconsin & 3,774 & Mississippi & 17.63 \\
\hline 34 & Utah & 763.7 & Delaware & 284.7 & Nevada & 9,294 & Massachusetts & 3,739 & Alabama & 17.49 \\
\hline $\begin{array}{l}34 \\
35\end{array}$ & Connecticut & 754.0 & Michigan & 283.3 & Utah & $\begin{array}{l},, 294 \\
8,332\end{array}$ & Maryland & $\begin{array}{l}3,719 \\
3,719\end{array}$ & $\begin{array}{l}\text { Texas } \\
\text { Texal }\end{array}$ & 17.46 \\
\hline 36 & West Virginia & 738.9 & North Carolina & 283.0 & Nebraska & 8,091 & Virginia & 3,717 & Colorado & 17.24 \\
\hline 37 & New Mexico & 680.1 & New Jersey & 278.1 & West Virginia & 7,882 & Idaho & 3,622 & Illinois & 17.17 \\
\hline 38 & Nevada & 646.1 & Utah & 275.2 & New Mexico & 7,435 & New Mexico & 3,599 & West Virginia & 17.09 \\
\hline 39 & Alaska & 641.7 & Maryland & 256.0 & Maine & 6,300 & Rhode Island & 3,506 & South Dakota & 16.92 \\
\hline 40 & Wyoming & 535.3 & Oregon & 254.6 & Alaska & 6,289 & Illinois & 3,503 & Kentucky & 16.89 \\
\hline 41 & $\begin{array}{l}\text { Idaho } \\
\text { Inty }\end{array}$ & 533.8 & Nevada & $\begin{array}{l}20.6 \\
238.9\end{array}$ & Hawaii & $\begin{array}{l}0,209 \\
5,714\end{array}$ & Michigan & $\begin{array}{l}3,005 \\
3,497\end{array}$ & Minnesota & $\begin{array}{l}16.09 \\
16.82\end{array}$ \\
\hline 42 & North Dakota & 480.7 & Vermont & 235.9 & $\begin{array}{l}\text { Idaho } \\
\text { Idaho }\end{array}$ & 5,691 & North Carolina & 3,451 & Oklahoma & 16.78 \\
\hline 43 & Maine & 407.3 & Florida & 232.6 & New Hampshire & 5,229 & Nevada & 3,437 & Arkansas & 16.76 \\
\hline 44 & Montana & 401.4 & New Hampshire & 224.4 & Montana & 4,568 & Washington & 3,395 & Idaho & 16.68 \\
\hline 45 & South Dakota & 379.6 & Arizona & 218.2 & North Dakota & 4,547 & $\begin{array}{l}\text { Colorado } \\
\text { Coll }\end{array}$ & 3,319 & Utah & 16.66 \\
\hline 46 & New Hampshire & 295.5 & Massachusetts & $\begin{array}{l}213.2 \\
213.1\end{array}$ & Wyoming & $\begin{array}{l}4,041 \\
4,462\end{array}$ & Oregon & $\begin{array}{l}3,281 \\
3,281\end{array}$ & Nebraska & 16.27 \\
\hline $\begin{array}{l}46 \\
47\end{array}$ & Hawaii & $\begin{array}{l}295.5 \\
272.2\end{array}$ & Connecticut & $\begin{array}{l}210.1 \\
210.9\end{array}$ & South Dakota & $\begin{array}{l}4,462 \\
3,798\end{array}$ & Florida & $\begin{array}{l}3,281 \\
3,194\end{array}$ & $\begin{array}{l}\text { Newa } \\
\text { lowa }\end{array}$ & $\begin{array}{l}1.27 \\
15.46\end{array}$ \\
\hline 48 & Delaware & 256.2 & California & 209.6 & Rhode Island & 3,690 & New York & 3,177 & Wyoming & 15.16 \\
\hline 49 & Rhode Island & 197.2 & Hawaii & 199.6 & Delaware & 3,616 & California & 3,134 & Indiana & 14.75 \\
\hline 50 & District of Columbia & 185.5 & New York & 192.2 & Vermont & 2.719 & Arizona & 3,021 & Louisiana & 14.73 \\
\hline \multirow{2}{*}{51} & Vermont & 147.6 & Rhode Island & 187.4 & District of Columbia & 2,439 & Utah & 3,002 & North Dakota & 13.73 \\
\hline & United States & $3,497,710.6$ & United States & 315.9 & United States & $5 \mathbf{1 , 2 0 4 , 8 2 7}$ & United States & 3,895 & United States & $\mathbf{1 8 . 7 3}$ \\
\hline
\end{tabular}

Prices and expenditures include taxes where data are available.

Prices are not adjusted for inflation. See "Nominal Dollars" in Glossary.

Includes -6.2 trillion Btu of coal coke net imports, which are not allocated to the States.

1) The U.S. consumplion value in this table does not match those in Tables 1.1 and 1.3 because it: 1) does not include biodiesel; and 2) is the sum of State values, which use State average heat contents to

5 Includes $\$ 158$ million for coal coke net imports, which are not allocated to the States.

Note: Rankings based on unrounded data.

Web Page: For related information, see http://www.eia.gov/state/seds/seds-data-complete.cfm.

Sources: Consumption: U.S. Energy Information Administration (EIA), "State Energy Data 2010: Consumption (June 2012), Tables C10 and C1T. Expenditures and Prices: EIA, "State Energy Data 2010: Prices and Expenditures" (June 2012), Table E15. "State Energy Data 2010" includes State-leve price and expenditure estimates are annul 1970 through 2010 . 
Figure 1.7 Heating Degree-Days by Month, 1949-2011

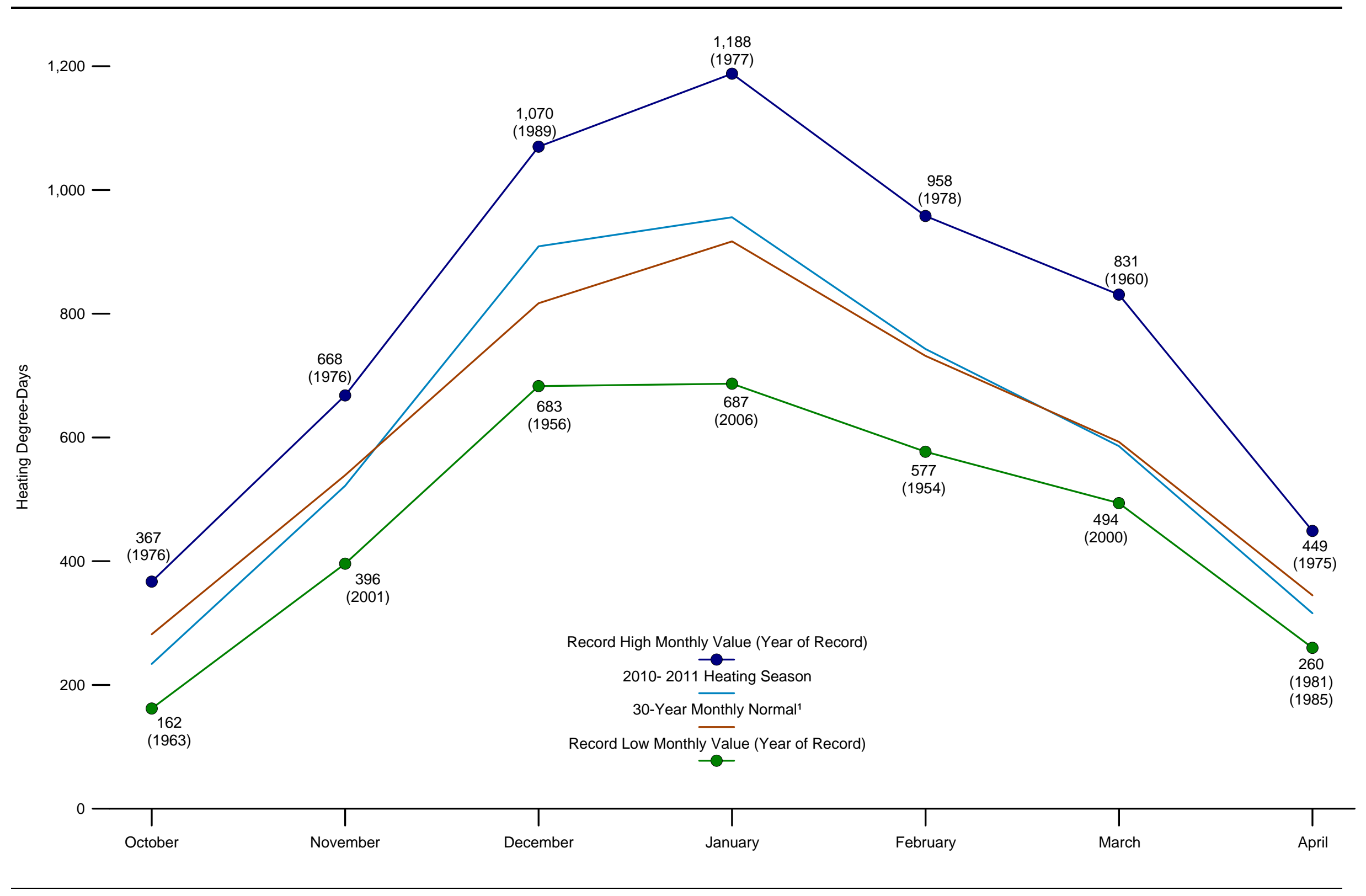

${ }^{1}$ Based on calculations of data from 1971 through 2000.

Source: Table 1.7. 
Table 1.7 Heating Degree-Days by Month, Selected Years, 1949-2011

\begin{tabular}{|c|c|c|c|c|c|c|c|c|c|c|c|c|c|}
\hline Year & January & February & March & April & May & June & July & August & September & October & November & December & Total \\
\hline 1949 & 858 & 701 & 611 & 330 & 128 & 21 & 7 & 9 & 94 & 209 & 503 & 763 & 4,234 \\
\hline 1950 & 761 & 721 & 693 & 412 & 162 & 40 & 11 & 18 & 85 & 196 & 565 & 872 & 4,536 \\
\hline 1955 & 927 & 759 & 600 & 272 & 121 & 48 & 9 & 6 & 56 & 237 & 600 & 886 & 4,521 \\
\hline 1960 & 884 & 780 & 831 & 278 & 160 & 33 & 7 & 11 & 48 & 254 & 502 & 936 & 4,724 \\
\hline 1965 & 907 & 780 & 738 & 355 & 114 & 48 & 11 & 14 & 78 & 271 & 494 & 739 & 4,549 \\
\hline 1970 & 1,063 & 758 & 685 & 344 & 120 & 31 & 4 & 9 & 55 & 253 & 541 & 801 & 4,664 \\
\hline 1975 & 821 & 742 & 686 & 449 & 117 & 37 & 5 & 13 & 100 & 235 & 462 & 805 & 4,472 \\
\hline 1976 & 974 & 609 & 544 & 309 & 178 & 28 & 8 & 19 & 81 & 367 & 668 & 941 & 4,726 \\
\hline 1977 & 1,188 & 751 & 529 & 270 & 119 & 38 & 6 & 13 & 59 & 295 & 493 & 844 & 4,605 \\
\hline 1978 & 1,061 & 958 & 677 & 350 & 157 & 31 & 7 & 11 & 59 & 283 & 517 & 847 & 4,958 \\
\hline 1979 & 1,079 & 950 & 575 & 364 & 148 & 37 & 6 & 15 & 58 & 271 & 528 & 750 & 4,781 \\
\hline 1980 & 887 & 831 & 680 & 338 & 142 & 49 & 5 & 10 & 54 & 316 & 564 & 831 & 4,707 \\
\hline 1981 & 984 & 689 & 620 & 260 & 165 & 25 & 6 & 11 & 76 & 327 & 504 & 845 & 4,512 \\
\hline 1982 & 1,067 & 776 & 620 & 408 & 114 & 62 & 7 & 19 & 75 & 264 & 515 & 692 & 4,619 \\
\hline 1983 & 874 & 706 & 588 & 421 & 189 & 35 & 6 & 5 & 53 & 251 & 509 & 990 & 4,627 \\
\hline 1984 & 1,000 & 645 & 704 & 371 & 172 & 28 & 7 & 7 & 88 & 223 & 565 & 704 & 4,514 \\
\hline 1985 & 1,057 & 807 & 557 & 260 & 123 & 47 & 5 & 17 & 69 & 243 & 506 & 951 & 4,642 \\
\hline 1986 & 859 & 734 & 542 & 295 & 123 & 30 & 9 & 18 & 76 & 258 & 558 & 793 & 4,295 \\
\hline 1987 & 920 & 714 & 573 & 309 & 107 & 20 & 8 & 13 & 61 & 345 & 491 & 773 & 4,334 \\
\hline 1988 & 1,004 & 778 & 594 & 344 & 134 & 30 & 3 & 5 & 72 & 352 & 506 & 831 & 4,653 \\
\hline 1989 & 789 & 832 & 603 & 344 & 163 & 32 & 5 & 14 & 73 & 259 & 542 & 1,070 & 4,726 \\
\hline 1990 & 728 & 655 & 535 & 321 & 184 & 29 & 6 & 10 & 56 & 246 & 457 & 789 & 4,016 \\
\hline 1991 & 921 & 639 & 564 & 287 & 98 & 30 & 6 & 7 & 69 & 242 & 586 & 751 & 4,200 \\
\hline 1992 & 852 & 644 & 603 & 345 & 152 & 46 & 14 & 24 & 74 & 301 & 564 & 822 & 4,441 \\
\hline 1993 & 860 & 827 & 664 & 368 & 128 & 38 & 11 & 9 & 89 & 302 & 580 & 824 & 4,700 \\
\hline 1994 & 1,031 & 813 & 594 & 293 & 174 & 21 & 6 & 16 & 65 & 268 & 479 & 723 & 4,483 \\
\hline 1995 & 847 & 750 & 556 & 375 & 174 & 31 & 4 & 7 & 77 & 233 & 605 & 872 & 4,531 \\
\hline 1996 & 945 & 748 & 713 & 360 & 165 & 27 & 8 & 9 & 72 & 276 & 630 & 760 & 4,713 \\
\hline 1997 & 932 & 672 & 552 & 406 & 198 & 31 & 7 & 16 & 63 & 273 & 592 & 800 & 4,542 \\
\hline 1998 & 765 & 623 & 596 & 331 & 109 & 41 & 4 & 5 & 33 & 245 & 482 & 717 & 3,951 \\
\hline 1999 & 861 & 647 & 645 & 319 & 139 & 31 & 5 & 12 & 62 & 275 & 413 & 760 & 4,169 \\
\hline 2000 & 886 & 643 & 494 & 341 & 115 & 29 & 12 & 12 & 69 & 244 & 610 & 1,005 & 4,460 \\
\hline 2001 & 935 & 725 & 669 & 302 & 115 & 29 & 8 & 6 & 69 & 260 & 396 & 689 & 4,203 \\
\hline 2002 & 776 & 669 & 622 & 281 & 184 & 23 & 3 & 8 & 37 & 298 & 560 & 812 & 4,273 \\
\hline 2003 & 944 & 801 & 572 & 344 & 165 & 41 & 4 & 5 & 62 & 260 & 477 & 784 & 4,459 \\
\hline 2004 & 968 & 766 & 495 & 303 & 107 & 37 & 7 & 20 & 47 & 251 & 487 & 802 & 4,290 \\
\hline 2005 & 859 & 676 & 648 & 305 & 186 & 25 & 3 & 6 & 39 & 236 & 466 & 866 & 4,315 \\
\hline 2006 & 687 & 731 & 600 & 264 & 137 & 23 & 2 & 9 & 82 & 304 & 467 & 690 & 3,996 \\
\hline 2007 & 841 & 853 & 502 & 372 & 111 & 24 & 5 & 7 & 44 & 175 & 521 & 800 & 4,255 \\
\hline 2008 & 892 & 741 & 617 & 319 & 183 & 26 & 5 & 13 & 52 & 281 & 534 & 831 & 4,494 \\
\hline 2009 & 969 & 705 & 583 & 330 & 132 & 40 & 14 & 12 & 60 & 330 & 441 & 877 & $\begin{array}{l}4,493 \\
4\end{array}$ \\
\hline 2010 & 940 & 820 & 552 & 263 & 132 & 27 & 5 & 7 & 50 & 234 & 522 & 909 & 4,461 \\
\hline $2011^{P}$ & 956 & 743 & 586 & 316 & 166 & 35 & 4 & 6 & 67 & 259 & 469 & 713 & 4,320 \\
\hline Normal $^{1}$ & 917 & 732 & 593 & 345 & 159 & 39 & 9 & 15 & 77 & 282 & 539 & 817 & 4,524 \\
\hline
\end{tabular}

1 Based on calculations of data from 1971 through 2000.

P=Preliminary

Notes: - This table excludes Alaska and Hawaii. - Degree-days are relative measurements of outdoor air temperature. Heating degree-days are deviations below the mean daily temperature of $65^{\circ} \mathrm{F}$. For example, a weather station recording a mean daily temperature of $40^{\circ} \mathrm{F}$ would report 25 heating degree-days. - Temperature information recorded by weather stations is used to calculate State-wide degree-day averages based on resident State population. Beginning in July 2001, data are weighted by the 2000 population. The population-weighted State figures are aggregated into Census divisions and the national average.
Web Pages: - See http://www.eia.gov/totalenergy/data/annual/\#summary for all data beginning in 1949. - For current data, see http://www.eia.gov/totalenergy/data/monthly/\#summary.

Sources: - 1949-2010-U.S. Department of Commerce, National Oceanic and Atmospheric Administration (NOAA), National Climatic Data Center, Asheville, North Carolina, Historical Climatology Series 5-1. Data are compiled from about 8,000 weather stations. - 2011 and Normal-U.S. Department of Commerce, NOAA, National Weather Service Climate Prediction Center, Camp Springs, Maryland, Degree Days Statistics. The data are based on mean daily temperatures recorded at about 200 major weather stations around the country. 


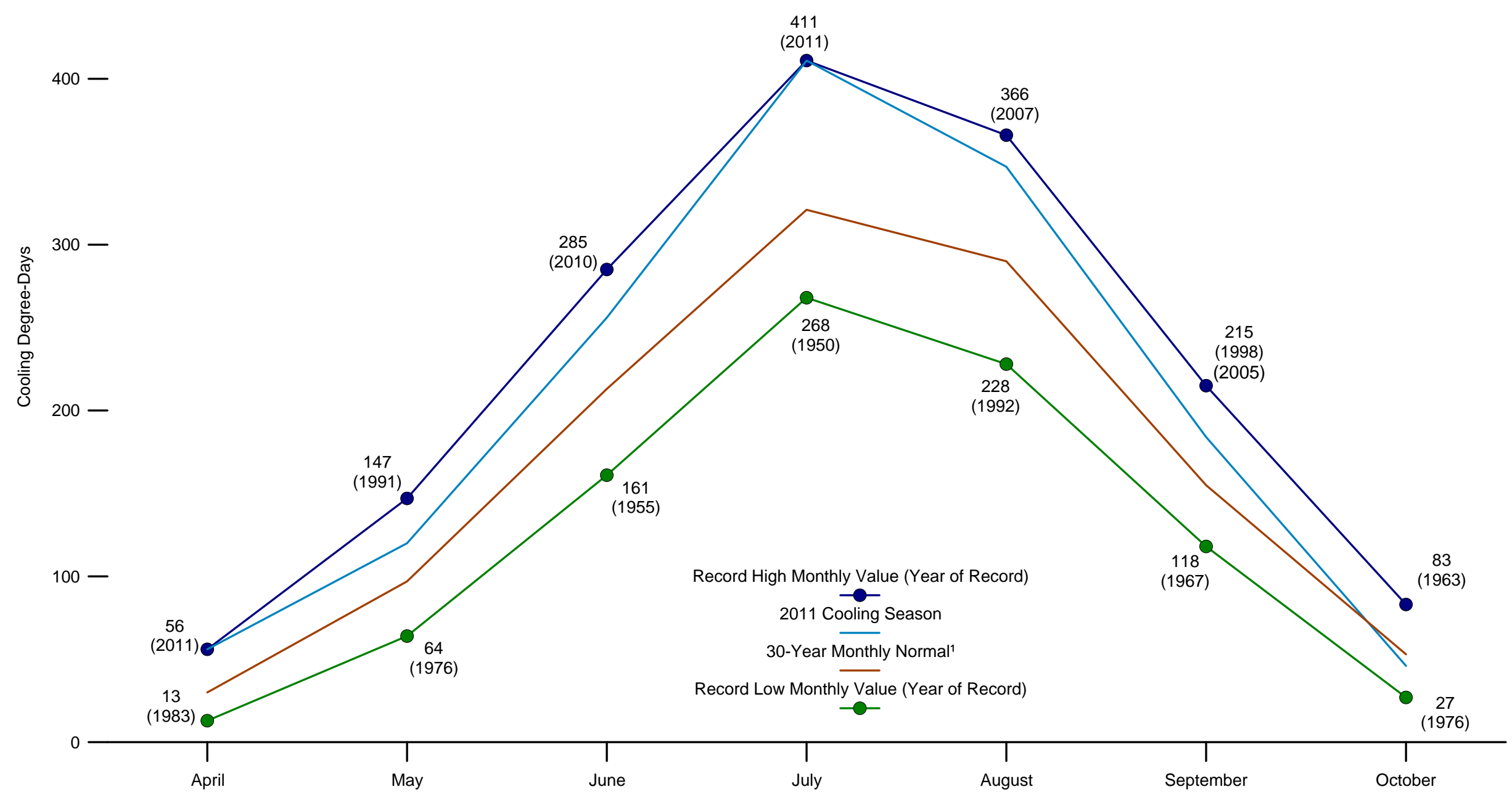


Table 1.8 Cooling Degree-Days by Month, Selected Years, 1949-2011

\begin{tabular}{|c|c|c|c|c|c|c|c|c|c|c|c|c|c|}
\hline Year & January & February & March & April & May & June & July & August & September & October & November & December & Total \\
\hline 1949 & 16 & 14 & 14 & 27 & 110 & 253 & 367 & 294 & 131 & 70 & 12 & 10 & 1,318 \\
\hline 1950 & 27 & 12 & 13 & 21 & 105 & 201 & 268 & 244 & 128 & 78 & 9 & 4 & 1,110 \\
\hline 1955 & 6 & 7 & 20 & 45 & 121 & 161 & 381 & 355 & 182 & 50 & 10 & 6 & 1,344 \\
\hline 1960 & 7 & 4 & 6 & 37 & 76 & 215 & 301 & 302 & 181 & 59 & 15 & 3 & 1,206 \\
\hline 1965 & 9 & 7 & 10 & 42 & 125 & 179 & 280 & 273 & 155 & 48 & 19 & 6 & 1,153 \\
\hline 1970 & 3 & 4 & 10 & 36 & 104 & 201 & 323 & 313 & 185 & 48 & 6 & 9 & 1,242 \\
\hline 1975 & 14 & 11 & 14 & 24 & 117 & 203 & 301 & 296 & 120 & 55 & 12 & 5 & 1,172 \\
\hline 1976 & 5 & 11 & 23 & 27 & 64 & 208 & 282 & 243 & 127 & 27 & 8 & 4 & 1,029 \\
\hline 1977 & 2 & 5 & 21 & 35 & 121 & 212 & 351 & 293 & 180 & 44 & 15 & 6 & 1,285 \\
\hline 1978 & 3 & 1 & 10 & 31 & 93 & 218 & 310 & 300 & 180 & 52 & 19 & 9 & 1,226 \\
\hline 1979 & 4 & 4 & 13 & 32 & 82 & 187 & 295 & 266 & 160 & 53 & 11 & 6 & 1,113 \\
\hline 1980 & 9 & 4 & 13 & 23 & 95 & 199 & 374 & 347 & 192 & 42 & 10 & 5 & 1,313 \\
\hline 1981 & 3 & 6 & 10 & 52 & 75 & 257 & 333 & 275 & 138 & 43 & 12 & 5 & 1,209 \\
\hline 1982 & 6 & 10 & 21 & 26 & 115 & 165 & 318 & 262 & 140 & 47 & 15 & 11 & 1,136 \\
\hline 1983 & 6 & 5 & 9 & 13 & 72 & 193 & 353 & 362 & 172 & 58 & 12 & 5 & 1,260 \\
\hline 1984 & 5 & 6 & 14 & 24 & 92 & 233 & 291 & 312 & 143 & 70 & 9 & 15 & 1,214 \\
\hline 1985 & 3 & 5 & 22 & 39 & 108 & 193 & 313 & 269 & 145 & 68 & 25 & 4 & 1,194 \\
\hline 1986 & 8 & 10 & 17 & 33 & 106 & 231 & 340 & 259 & 161 & 52 & 23 & 9 & 1,249 \\
\hline 1987 & 5 & 7 & 13 & 23 & 127 & 244 & 334 & 298 & 156 & 40 & 14 & 8 & 1,269 \\
\hline 1988 & 5 & 5 & 13 & 28 & 89 & 218 & 359 & 348 & 149 & 45 & 18 & 6 & 1,283 \\
\hline 1989 & 15 & 7 & 19 & 36 & 88 & 208 & 312 & 266 & 138 & 49 & 16 & 2 & 1,156 \\
\hline 1990 & 15 & 14 & 21 & 29 & 86 & 234 & 316 & 291 & 172 & 57 & 16 & 9 & 1,260 \\
\hline 1991 & 10 & 9 & 19 & 42 & 147 & 235 & 336 & 305 & 149 & 62 & 8 & 9 & 1,331 \\
\hline 1992 & 6 & 10 & 15 & 29 & 77 & 170 & 286 & 228 & 150 & 49 & 13 & 7 & 1,040 \\
\hline 1993 & 13 & 5 & 11 & 19 & 91 & 207 & 347 & 317 & 146 & 47 & 11 & 4 & 1,218 \\
\hline 1994 & 7 & 9 & 18 & 37 & 76 & 262 & 328 & 263 & 141 & 50 & 20 & 9 & 1,220 \\
\hline 1995 & 7 & 7 & 18 & 29 & 91 & 202 & 348 & 363 & 150 & 61 & 12 & 5 & 1,293 \\
\hline 1996 & 7 & 6 & 8 & 26 & 116 & 226 & 299 & 287 & 139 & 45 & 14 & 7 & 1,180 \\
\hline 1997 & 8 & 11 & 31 & 19 & 81 & 189 & 315 & 268 & 171 & 48 & 10 & 5 & 1,156 \\
\hline 1998 & 12 & 7 & 10 & 23 & 135 & 228 & 350 & 337 & 215 & 62 & 20 & 11 & 1,410 \\
\hline 1999 & 12 & 11 & 12 & 40 & 94 & 219 & 374 & 305 & 152 & 55 & 17 & 6 & 1,297 \\
\hline 2000 & 10 & 10 & 25 & 28 & 131 & 221 & 284 & 302 & 156 & 50 & 8 & 4 & 1,229 \\
\hline 2001 & 3 & 12 & 11 & 37 & 114 & 220 & 302 & 333 & 138 & 46 & 18 & 11 & 1,245 \\
\hline 2002 & 8 & 6 & 17 & 53 & 92 & 243 & 370 & 332 & 202 & 57 & 11 & 5 & 1,396 \\
\hline 2003 & 5 & 7 & 24 & 30 & 110 & 187 & 336 & 345 & 156 & 65 & 21 & 4 & 1,290 \\
\hline 2004 & 6 & 6 & 28 & 29 & 138 & 208 & 299 & 252 & 177 & 67 & 17 & 5 & 1,232 \\
\hline 2005 & 10 & 7 & 12 & 24 & 82 & 250 & 367 & 351 & 215 & 55 & 20 & 4 & 1,397 \\
\hline 2006 & 13 & 5 & 18 & 53 & 109 & 236 & 388 & 337 & 138 & 46 & 14 & 11 & 1,368 \\
\hline 2007 & 10 & 5 & 29 & 23 & 119 & 236 & 310 & 366 & 191 & 82 & 16 & 12 & 1,399 \\
\hline 2008 & 7 & 11 & 17 & 31 & 91 & 264 & 334 & 283 & 171 & 48 & 12 & 8 & 1,277 \\
\hline 2009 & 7 & 7 & 17 & 29 & 117 & 222 & 284 & 307 & 169 & 47 & 16 & 7 & 1,229 \\
\hline 2010 & 3 & 2 & 7 & 34 & 126 & 285 & 380 & 356 & 195 & 55 & 13 & 1 & 1,457 \\
\hline $2011^{\mathrm{P}}$ & 3 & 10 & 20 & 56 & 120 & 256 & 411 & 347 & 184 & 46 & 16 & 8 & 1,477 \\
\hline Normal ${ }^{1}$ & R 9 & 8 & 18 & $\mathrm{R}_{30}$ & R97 & $R_{213}$ & $R_{321}$ & $R_{290}$ & $R_{155}$ & $R_{53}$ & $\mathrm{R}_{15}$ & $\mathrm{R}_{7}$ & $\mathrm{R}_{1,216}$ \\
\hline
\end{tabular}

1 Based on calculations of data from 1971 through 2000.

$\mathrm{R}=$ Revised. $\mathrm{P}=$ Preliminary.

Notes: - This table excludes Alaska and Hawaii. - Degree-days are relative measurements of outdoor air temperature. Cooling degree-days are deviations above the mean daily temperature of $65^{\circ} \mathrm{F}$. For example, a weather station recording a mean daily temperature of $78^{\circ} \mathrm{F}$ would report 13 cooling degree-days. - Temperature information recorded by weather stations is used to calculate State-wide degree-day averages based on resident State population. Beginning in 2002, data are weighted by the 2000 population. The population-weighted State figures are aggregated into Census divisions and the national average.
Web Pages: • See http://www.eia.gov/totalenergy/data/annual/\#summary for all data beginning in 1949. - For current data, see http://www.eia.gov/totalenergy/data/monthly/\#summary.

Sources: - 1949-2010-U.S. Department of Commerce, National Oceanic and Atmospheric Administration (NOAA), National Climatic Data Center, Asheville, North Carolina, Historical Climatology Series 5-2. Data are compiled from about 8,000 weather stations. - 2011 and Normal-U.S. Department of Commerce, NOAA, National Weather Service Climate Prediction Center, Camp Springs, Maryland, Degree Days Statistics. The data are based on mean daily temperatures recorded at about 200 major weather stations around the country. 


\section{Figure 1.9 Heating Degree-Days by Census Division}

\section{U.S. ${ }^{1}$ Heating Degree-Days, 1949-2011}

$6,000-$

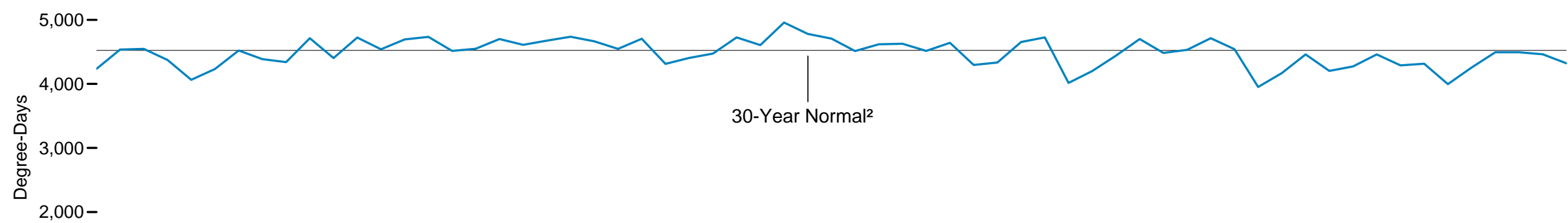

$1,000-$

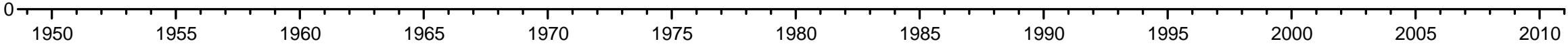

\section{Heating Degree-Days by Census Division, 2011}

$8,000-$

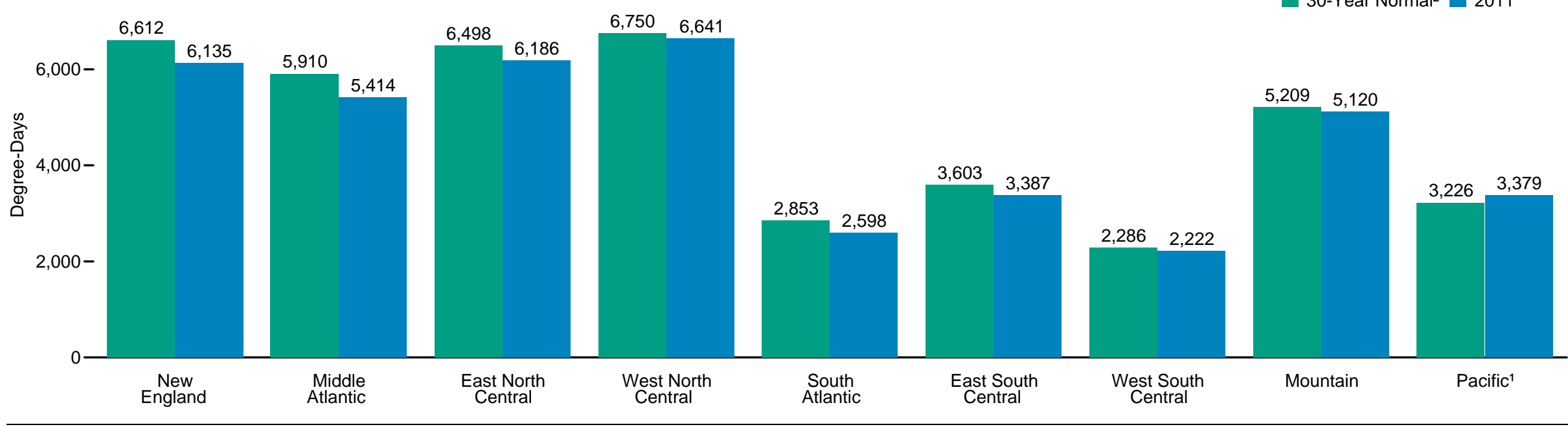

${ }^{1}$ Excludes Alaska and Hawaii.

${ }^{2}$ Based on calculations of data from 1971 through 2000.

Note: See Appendix C for map of Census divisions. Source: Table 1.9. 
Table 1.9 Heating Degree-Days by Census Division, Selected Years, 1949-2011

\begin{tabular}{|c|c|c|c|c|c|c|c|c|c|c|}
\hline Year & $\begin{array}{c}\text { New } \\
\text { England }\end{array}$ & $\begin{array}{c}\text { Middle } \\
\text { Atlantic }\end{array}$ & $\begin{array}{l}\text { East North } \\
\text { Central }\end{array}$ & $\begin{array}{l}\text { West North } \\
\text { Central }\end{array}$ & $\begin{array}{c}\text { South } \\
\text { Atlantic }\end{array}$ & $\begin{array}{l}\text { East South } \\
\text { Central }\end{array}$ & $\begin{array}{c}\text { West South } \\
\text { Central }\end{array}$ & Mountain & Pacific ${ }^{1}$ & $\begin{array}{l}\text { United } \\
\text { States }{ }^{1}\end{array}$ \\
\hline 1949 & 5,829 & 5,091 & 5,801 & 6,479 & 2,367 & 2,942 & 2,133 & 5,483 & 3,729 & 4,234 \\
\hline 1950 & 6,470 & 5,765 & 6,619 & 7,136 & 2,713 & 3,315 & 1,974 & 4,930 & 3,355 & 4,536 \\
\hline 1955 & 6,577 & 5,708 & 6,101 & 6,630 & 2,786 & 3,314 & 2,083 & 5,517 & 3,723 & 4,521 \\
\hline 1960 & 6,561 & 5,901 & 6,544 & 6,884 & 3,147 & 3,958 & 2,551 & 5,328 & 3,309 & 4,724 \\
\hline 1965 & 6,825 & 5,933 & 6,284 & 6,646 & 2,830 & 3,374 & 2,078 & 5,318 & 3,378 & 4,549 \\
\hline 1970 & 6,839 & 5,943 & 6,455 & 6,835 & 2,997 & 3,685 & 2,396 & 5,436 & 3,257 & 4,664 \\
\hline 1975 & 6,362 & 5,477 & 6,169 & 6,678 & 2,640 & 3,336 & 2,187 & 5,693 & 3,623 & 4,472 \\
\hline 1976 & 6,839 & 6,097 & 6,768 & 6,670 & 3,040 & 3,881 & 2,446 & 5,303 & 3,115 & 4,726 \\
\hline 1977 & 6,579 & 5,889 & 6,538 & 6,506 & 3,047 & 3,812 & 2,330 & 5,060 & 3,135 & 4,605 \\
\hline 1978 & 7,061 & 6,330 & 7,095 & 7,324 & 3,187 & 4,062 & 2,764 & 5,370 & 3,168 & 4,958 \\
\hline 1979 & 6,348 & 5,851 & 6,921 & 7,369 & 2,977 & 3,900 & 2,694 & 5,564 & 3,202 & 4,781 \\
\hline 1980 & 6,900 & 6,143 & 6,792 & 6,652 & 3,099 & 3,855 & 2,378 & 5,052 & 2,986 & 4,707 \\
\hline 1981 & 6,612 & 5,989 & 6,446 & 6,115 & 3,177 & 3,757 & 2,162 & 4,671 & 2,841 & 4,512 \\
\hline 1982 & 6,697 & 5,866 & 6,542 & 7,000 & 2,721 & 3,357 & 2,227 & 5,544 & 3,449 & 4,619 \\
\hline 1983 & 6,305 & 5,733 & 6,423 & 6,901 & 3,057 & 3,892 & 2,672 & 5,359 & 3,073 & 4,627 \\
\hline 1984 & 6,442 & 5,777 & 6,418 & 6,582 & 2,791 & 3,451 & 2,194 & 5,592 & 3,149 & 4,514 \\
\hline 1985 & 6,571 & 5,660 & 6,546 & 7,119 & 2,736 & 3,602 & 2,466 & 5,676 & 3,441 & 4,642 \\
\hline 1986 & 6,517 & 5,665 & 6,150 & 6,231 & 2,686 & 3,294 & 2,058 & 4,870 & 2,807 & 4,295 \\
\hline 1987 & 6,546 & 5,699 & 5,810 & 5,712 & 2,937 & 3,466 & 2,292 & 5,153 & 3,013 & 4,334 \\
\hline 1988 & 6,715 & 6,088 & 6,590 & 6,634 & 3,122 & 3,800 & 2,346 & 5,148 & 2,975 & 4,653 \\
\hline 1989 & 6,887 & 6,134 & 6,834 & 6,996 & 2,944 & 3,713 & 2,439 & 5,173 & 3,061 & 4,726 \\
\hline 1990 & 5,848 & 4,998 & 5,681 & 6,011 & 2,230 & 2,929 & 1,944 & 5,146 & 3,148 & 4,016 \\
\hline 1991 & 5,960 & 5,177 & 5,906 & 6,319 & 2,503 & 3,211 & 2,178 & 5,259 & 3,109 & 4,200 \\
\hline 1992 & 6,844 & 5,964 & 6,297 & 6,262 & 2,852 & 3,498 & 2,145 & 5,054 & 2,763 & 4,441 \\
\hline 1993 & 6,728 & 5,948 & 6,646 & 7,168 & 2,981 & 3,768 & 2,489 & 5,514 & 3,052 & 4,700 \\
\hline 1994 & 6,672 & 5,934 & 6,378 & 6,509 & 2,724 & 3,394 & 2,108 & 5,002 & 3,155 & 4,483 \\
\hline 1995 & 6,559 & 5,831 & 6,664 & 6,804 & 2,967 & 3,626 & 2,145 & 4,953 & 2,784 & 4,531 \\
\hline 1996 & 6,679 & 5,986 & 6,947 & 7,345 & 3,106 & 3,782 & 2,285 & 5,011 & 2,860 & 4,713 \\
\hline 1997 & 6,661 & 5,809 & 6,617 & 6,761 & 2,845 & 3,664 & 2,418 & 5,188 & 2,754 & 4,542 \\
\hline 1998 & 5,680 & 4,812 & 5,278 & 5,774 & 2,429 & 3,025 & 2,021 & 5,059 & 3,255 & 3,951 \\
\hline 1999 & 5,952 & 5,351 & 5,946 & 5,921 & 2,652 & 3,142 & 1,835 & 4,768 & 3,158 & 4,169 \\
\hline 2000 & 6,489 & 5,774 & 6,284 & 6,456 & 2,959 & 3,548 & 2,194 & 4,881 & 3,012 & 4,460 \\
\hline 2001 & 6,055 & 5,323 & 5,824 & 6,184 & 2,641 & 3,312 & 2,187 & 4,895 & 3,136 & 4,203 \\
\hline 2002 & 6,099 & 5,372 & 6,122 & 6,465 & 2,671 & 3,420 & 2,307 & 5,018 & 3,132 & 4,273 \\
\hline 2003 & 6,851 & 6,090 & 6,528 & 6,539 & 2,891 & 3,503 & 2,230 & 4,605 & 2,918 & 4,459 \\
\hline 2004 & 6,612 & 5,749 & 6,199 & 6,290 & 2,748 & 3,289 & 2,088 & 4,844 & 2,925 & 4,290 \\
\hline 2005 & 6,551 & 5,804 & 6,241 & 6,202 & 2,844 & 3,402 & 2,051 & 4,759 & 2,959 & 4,315 \\
\hline 2006 & 5,809 & 5,050 & 5,712 & 5,799 & 2,535 & 3,239 & 1,863 & 4,778 & 3,116 & 3,996 \\
\hline 2007 & 6,501 & 5,623 & 6,096 & 6,374 & 2,584 & 3,213 & 2,156 & 4,830 & 3,113 & 4,255 \\
\hline 2008 & 6,395 & 5,643 & 6,696 & 7,112 & 2,782 & 3,641 & 2,178 & 5,114 & 3,186 & 4,494 \\
\hline 2009 & 6,646 & 5,799 & 6,540 & 6,837 & 2,879 & 3,588 & 2,212 & 5,016 & 3,150 & 4,493 \\
\hline 2010 & 5,942 & 5,455 & 6,207 & 6,584 & 3,219 & 3,994 & 2,521 & 4,954 & 3,171 & 4,461 \\
\hline $2011^{P}$ & 6,135 & 5,414 & 6,186 & 6,641 & 2,598 & 3,387 & 2,222 & 5,120 & 3,379 & 4,320 \\
\hline Normal $^{2}$ & 6,612 & 5,910 & 6,498 & 6,750 & 2,853 & 3,603 & 2,286 & 5,209 & 3,226 & 4,524 \\
\hline
\end{tabular}

Excludes Alaska and Hawaii.

Based on calculations of data from 1971 through 2000.

$P=$ Preliminary

Notes: - Degree-days are relative measurements of outdoor air temperature. Heating degree-days are deviations below the mean daily temperature of $65^{\circ} \mathrm{F}$. For example, a weather station recording a mean daily temperature of $40^{\circ} \mathrm{F}$ would report 25 heating degree-days. - Temperature information recorded by weather stations is used to calculate State-wide degree-day averages based on resident State population. Beginning in July 2001, data are weighted by the 2000 population. The population-weighted State figures are aggregated into Census divisions and the national average. - See Appendix $\mathrm{C}$ for map of Census

divisions

Web Pages: • See http://www.eia.gov/totalenergy/data/annual/\#summary for all data beginning in 1949. - For current data, see http://www.eia.gov/totalenergy/data/monthly/\#summary.

Sources: - 1949-2010-U.S. Department of Commerce, National Oceanic and Atmospheric Administration (NOAA), National Climatic Data Center, Asheville, North Carolina, Historical Climatology Series 5-1. Data are compiled from about 8,000 weather stations. - 2011 and Normal-U.S. Department of Commerce, NOAA, National Weather Service Climate Prediction Center, Camp Springs, Maryland, emperatures recorded at about 200 major weather stations around the country. 


\section{Figure 1.10 Cooling Degree-Days by Census Division}

\section{U.S. ${ }^{1}$ Cooling Degree-Days, 1949-2011}

$2,000-$

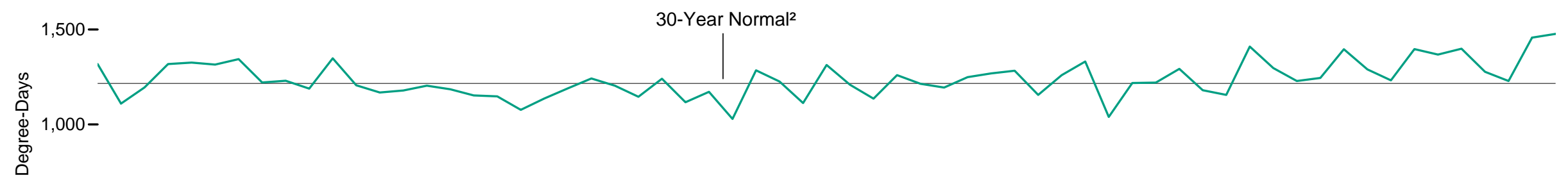

$500-$

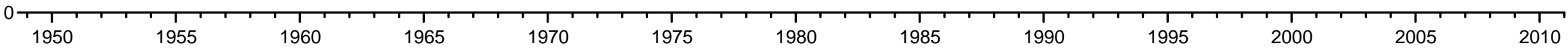

\section{Cooling Degree-Days by Census Division, 2011}

$4,000-$

30-Year Normal ${ }^{2} \square 2011$

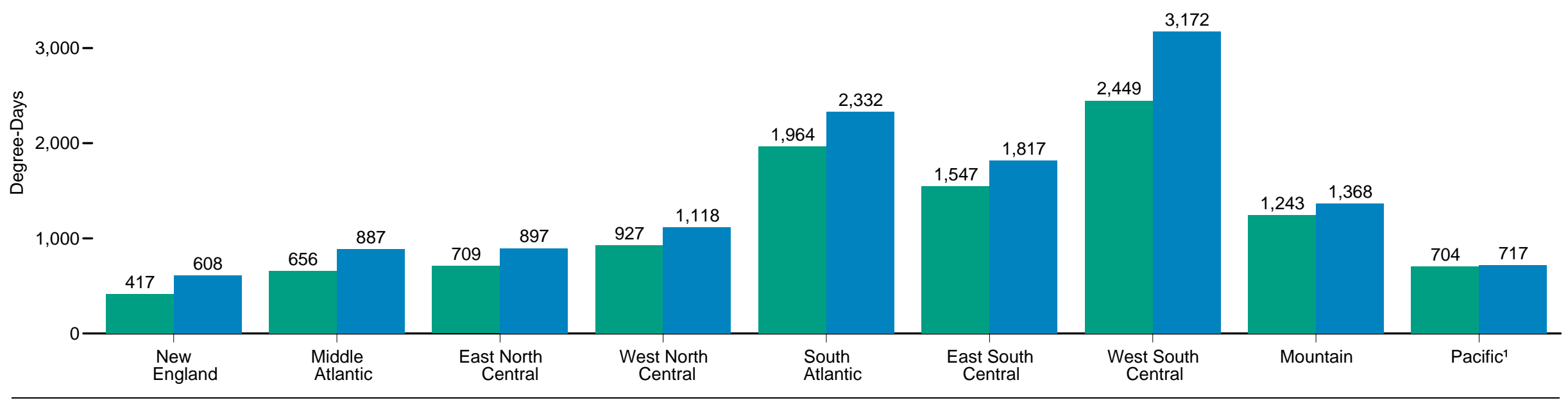

${ }^{1}$ Excludes Alaska and Hawaii.

${ }^{2}$ Based on calculations of data from 1971 through 2000.

Note: See Appendix C for map of Census divisions. Source: Table 1.10 
Table 1.10 Cooling Degree-Days by Census Division, Selected Years, 1949-2011

\begin{tabular}{|c|c|c|c|c|c|c|c|c|c|c|}
\hline Year & $\begin{array}{c}\text { New } \\
\text { England }\end{array}$ & $\begin{array}{c}\text { Middle } \\
\text { Atlantic }\end{array}$ & $\begin{array}{l}\text { East North } \\
\text { Central }\end{array}$ & $\begin{array}{l}\text { West North } \\
\text { Central }\end{array}$ & $\begin{array}{c}\text { South } \\
\text { Atlantic }\end{array}$ & $\begin{array}{l}\text { East South } \\
\text { Central }\end{array}$ & $\begin{array}{l}\text { West South } \\
\text { Central }\end{array}$ & Mountain & Pacific ${ }^{1}$ & $\begin{array}{l}\text { United } \\
\text { States }{ }^{1}\end{array}$ \\
\hline 1949 & 654 & 901 & 949 & 1,038 & 2,128 & 1,776 & 2,510 & 1,198 & 593 & 1,318 \\
\hline 1950 & 353 & 542 & 602 & 729 & 1,919 & 1,568 & 2,473 & 1,120 & 597 & 1,110 \\
\hline 1955 & 602 & 934 & 1,043 & 1,238 & 2,045 & 1,791 & 2,643 & 1,124 & 560 & 1,344 \\
\hline 1960 & 368 & 640 & 722 & 961 & 1,926 & 1,613 & 2,492 & 1,308 & 770 & 1,206 \\
\hline 1965 & 352 & 638 & 688 & 914 & 1,931 & 1,634 & 2,579 & 961 & 542 & 1,153 \\
\hline 1970 & 479 & 779 & 827 & 1,066 & 2,007 & $\begin{array}{l}1,662 \\
1,62\end{array}$ & 2,375 & $\begin{array}{r}., 163 \\
\end{array}$ & 689 & 1,242 \\
\hline 1975 & 467 & 708 & 788 & 1,003 & 2,011 & 1,520 & 2,261 & 1,031 & 547 & 1,172 \\
\hline 1976 & 402 & 597 & 619 & 939 & 1,675 & 1,232 & 2,035 & 1,058 & 620 & 1,029 \\
\hline 1977 & 407 & 689 & 823 & 1,122 & 2,020 & 1,808 & 2,720 & 1,256 & 715 & 1,285 \\
\hline 1978 & 378 & 615 & 741 & 1,027 & 1,972 & 1,685 & 2,638 & 1,174 & 738 & 1,226 \\
\hline 1979 & 434 & 588 & 618 & 871 & 1,833 & 1,412 & 2,242 & 1,164 & 770 & 1,113 \\
\hline 1980 & 487 & 793 & 816 & 1,217 & 2,075 & 1,834 & 2,734 & $\begin{array}{l}1,104 \\
1,202\end{array}$ & 658 & 1,313 \\
\hline 1981 & 436 & 657 & 658 & 924 & 1,889 & 1,576 & 2,498 & 1,331 & 876 & 1,209 \\
\hline 1982 & 321 & 541 & 643 & 859 & 1,958 & 1,537 & 2,502 & 1,121 & 619 & 1,136 \\
\hline 1983 & 538 & 799 & 934 & 1,178 & 1,925 & 1,579 & 2,288 & 1,174 & 776 & 1,260 \\
\hline 1984 & 468 & 649 & 724 & 955 & 1,865 & 1,508 & 2,469 & 1,190 & 956 & 1,214 \\
\hline 1985 & 372 & 627 & 643 & 830 & 2,004 & 1,596 & 2,599 & 1,210 & 737 & 1,194 \\
\hline 1986 & 301 & 626 & 738 & 1,021 & 2,149 & 1,792 & 2,618 & 1,188 & 664 & 1,249 \\
\hline 1987 & 406 & 729 & 918 & 1,115 & 2,067 & 1,718 & 2,368 & 1,196 & 706 & 1,269 \\
\hline 1988 & 545 & 782 & 975 & 1,230 & 1,923 & 1,582 & 2,422 & 1,320 & 729 & 1,283 \\
\hline 1989 & 426 & 658 & 652 & 864 & 1,977 & 1,417 & 2,295 & 1,330 & 685 & 1,156 \\
\hline 1990 & 477 & 656 & 647 & 983 & 2,143 & 1,622 & 2,579 & 1,294 & 827 & 1,260 \\
\hline 1991 & 511 & 854 & 959 & 1,125 & 2,197 & 1,758 & 2,499 & 1,182 & 672 & 1,331 \\
\hline 1992 & 276 & 460 & 449 & 637 & 1,777 & 1,293 & 2,201 & 1,206 & 905 & 1,040 \\
\hline 1993 & 486 & 764 & 735 & 817 & 2,092 & 1,622 & 2,369 & 1,113 & 708 & 1,218 \\
\hline 1994 & 548 & 722 & 664 & 887 & 2,005 & 1,448 & 2,422 & 1,436 & 801 & 1,220 \\
\hline 1995 & 507 & 803 & 921 & 985 & 2,081 & 1,671 & 2,448 & 1,234 & 754 & 1,293 \\
\hline 1996 & 400 & 623 & 629 & 821 & 1,867 & 1,474 & 2,515 & 1,381 & 856 & 1,180 \\
\hline 1997 & 395 & 586 & 574 & 873 & 1,886 & $\begin{array}{l}1,393 \\
\end{array}$ & 2,361 & 1,335 & 921 & $\begin{array}{l}1,100 \\
1,156\end{array}$ \\
\hline 1998 & 505 & 788 & 889 & 1,138 & 2,277 & 1,928 & 3,026 & 1,271 & 732 & 1,410 \\
\hline 1999 & 631 & 882 & 855 & 970 & 2,024 & 1,733 & 2,645 & 1,242 & 635 & 1,297 \\
\hline 2000 & 317 & 542 & 658 & 1,023 & 1,929 & 1,736 & 2,787 & 1,488 & 756 & 1,229 \\
\hline 2001 & 519 & 722 & 744 & $\begin{array}{l}1,028 \\
\text {. }\end{array}$ & 1,891 & 1,535 & 2,565 & 1,498 & 794 & 1,245 \\
\hline 2002 & 570 & 863 & 933 & 1,087 & 2,209 & 1,808 & 2,545 & 1,543 & 739 & 1,396 \\
\hline 2003 & 522 & 685 & 645 & 946 & 2,007 & 1,494 & 2,522 & 1,639 & 941 & 1,290 \\
\hline 2004 & 402 & 670 & 604 & 752 & 2,037 & $\begin{array}{l}1,449 \\
1,549\end{array}$ & 2,485 & 1,376 & 823 & 1,232 \\
\hline 2005 & 642 & 990 & 960 & 1,094 & 2,081 & 1,696 & 2,636 & 1,457 & 728 & 1,397 \\
\hline 2006 & 528 & 778 & 752 & 1,079 & 2,037 & 1,670 & 2,776 & 1,586 & 916 & 1,368 \\
\hline 2007 & 484 & 788 & 900 & 1,135 & 2,212 & 1,927 & 2,488 & 1,663 & 811 & 1,399 \\
\hline 2008 & 497 & 745 & 698 & 847 & 1,987 & 1,560 & 2,494 & $\begin{array}{l}1,004 \\
\end{array}$ & 868 & 1,277 \\
\hline 2009 & 362 & 587 & 547 & 720 & 2,025 & 1,497 & 2,570 & 1,504 & 884 & 1,229 \\
\hline 2010 & 657 & 997 & 975 & 1,123 & 2,267 & 2,004 & 2,750 & 1,450 & 655 & 1,457 \\
\hline $2011^{\mathrm{P}}$ & 608 & 887 & 897 & 1,118 & $\begin{array}{l}2,201 \\
2,332\end{array}$ & $\begin{array}{l}2,004 \\
1,817\end{array}$ & 3,172 & 1,368 & 717 & $\begin{array}{l}1,401 \\
1,477\end{array}$ \\
\hline Normal $^{2}$ & ${ }^{R} 417$ & $\mathrm{R}^{\mathrm{R}} 656$ & $\mathrm{R}_{709}$ & R 927 & $R_{1,964}$ & $\mathrm{R}_{1,547}$ & $\mathrm{R}_{2,449}$ & $R_{1,243}$ & $R^{R} 704$ & $R_{1,216}$ \\
\hline
\end{tabular}

1 Excludes Alaska and Hawaii.

2 Based on calculations of data from 1971 through 2000.

$\mathrm{R}=$ Revised. P=Preliminary.

Notes: - Degree-days are relative measurements of outdoor air temperature. Cooling degree-days are deviations above the mean daily temperature of $65^{\circ} \mathrm{F}$. For example, a weather station recording a mean daily temperature of $78^{\circ} \mathrm{F}$ would report 13 cooling degree-days. - Temperature information recorded by weather stations is used to calculate State-wide degree-day averages based on resident State population. Beginning in 2002, data are weighted by the 2000 population. The population-weighted State figures are aggregated into Census divisions and the national average. - See Appendix C for map of Census

divisions.

Web Pages: • See http://www.eia.gov/totalenergy/data/annual/\#summary for all data beginning in 1949. - For current data, see http://www.eia.gov/totalenergy/data/monthly/\#summary.

Sources: - 1949-2010-U.S. Department of Commerce, National Oceanic and Atmospheric Administration (NOAA), National Climatic Data Center, Asheville, North Carolina, Historical Climatology Series 5-2. Data are compiled from about 8,000 weather stations. - 2011 and Normal-U.S. Department of Commerce, NOAA, National Weather Service Climate Prediction Center, Camp Springs, Maryland, Degree Days Statistics. The data are the sum of monthly values and are based on mean daily emperatures recorded at about 200 major weather stations around the country. 
Figure 1.11 U.S. Government Energy Consumption by Agency

\section{Total and U.S. Department of Defense, Fiscal Years}

1975-2011

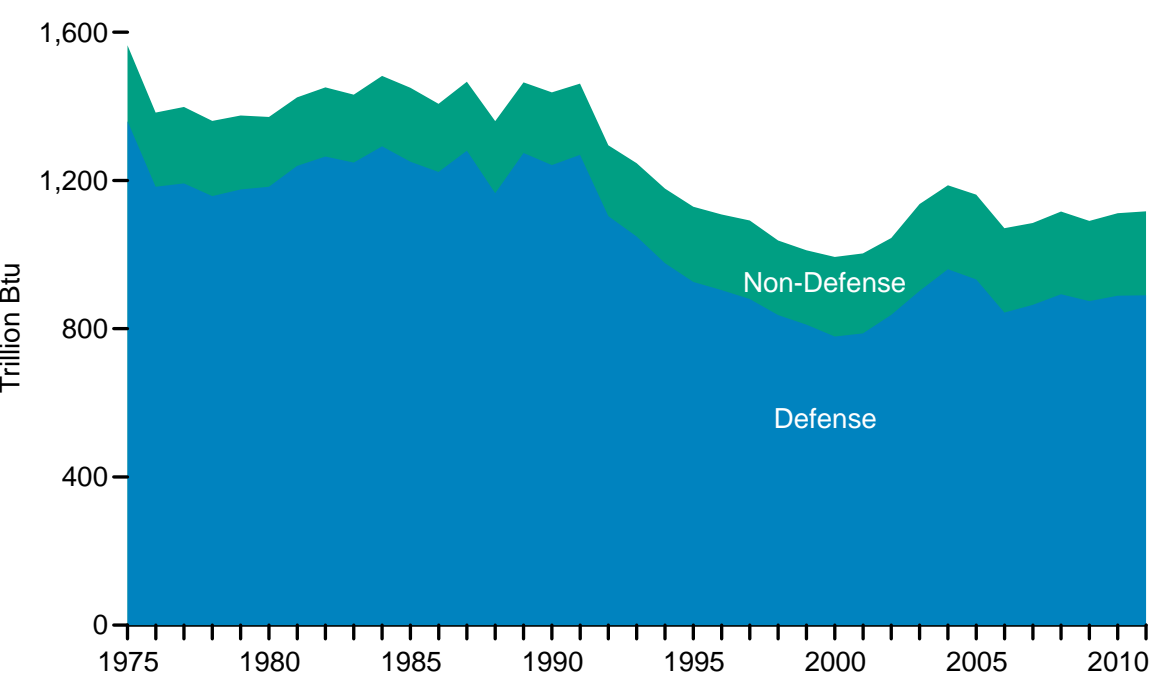

U.S. Department of Defense and Non-Defense Agencies, Fiscal Years 1975-2011

$1,600-$

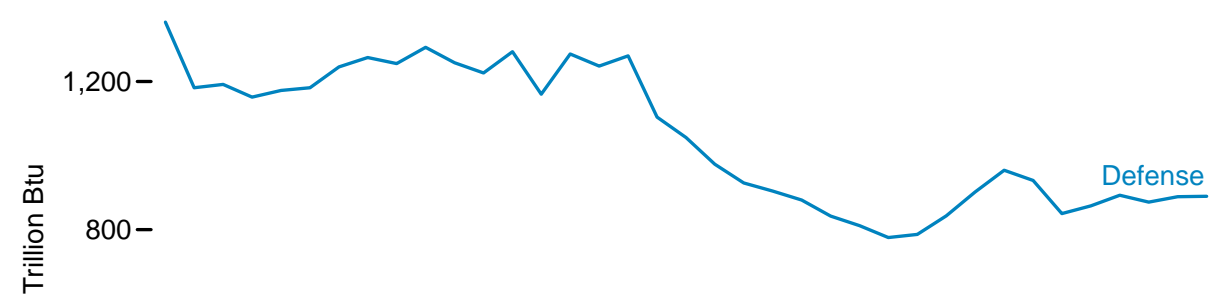

$400-$

Non-Defense

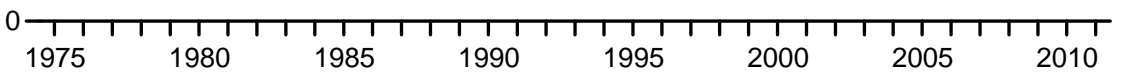

Non-Defense Agencies, Fiscal Year 2011 $50-$
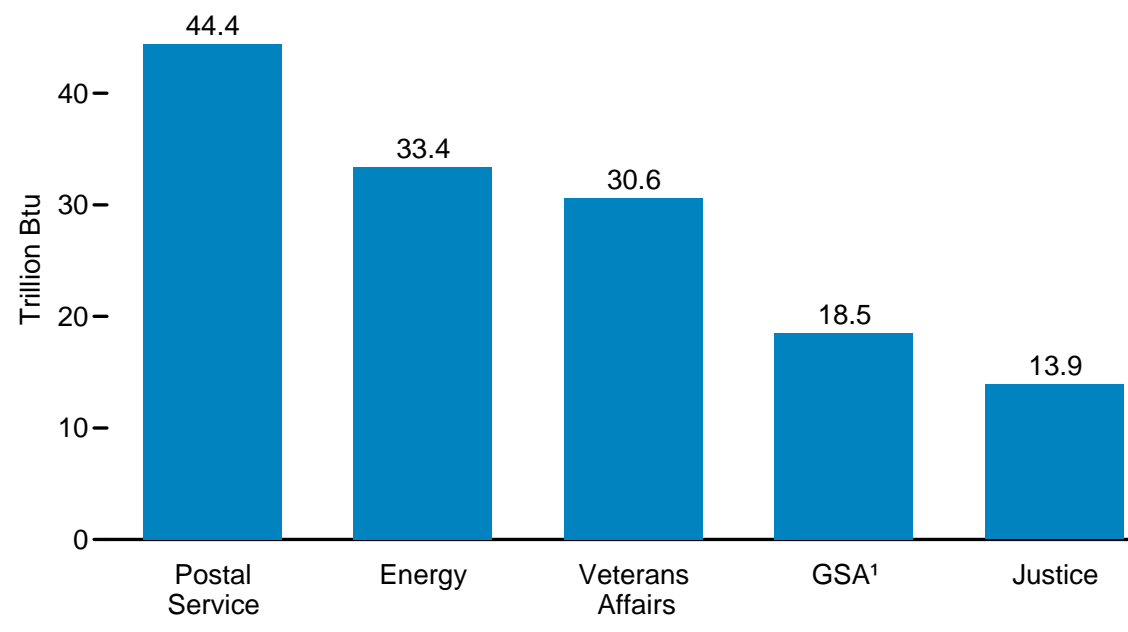

${ }^{1}$ General Services Administration.

${ }^{2}$ Health and Human Services.

${ }^{3}$ National Aeronautics and Space Administration.

${ }^{4}$ See Table 1.11 for list of agencies.

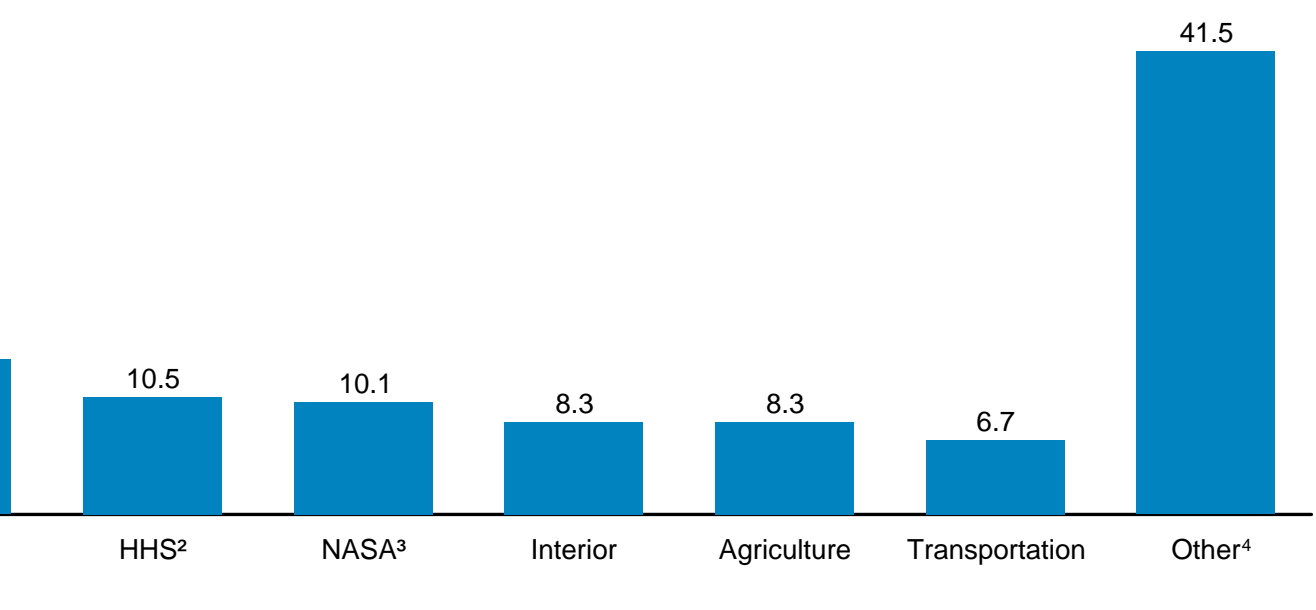

Note: The U.S. Government's fiscal year was October 1 through September 30, except in 1975 and 1976 when it was July 1 through June 30.

Source: Table 1.11. 
Table 1.11 U.S. Government Energy Consumption by Agency, Fiscal Years 1975-2011

(Trillion Btu)

\begin{tabular}{|c|c|c|c|c|c|c|c|c|c|c|c|c|c|}
\hline Year & Agriculture & Defense & Energy & GSA 1 & HHS ${ }^{2}$ & Interior & Justice & NASA ${ }^{3}$ & $\begin{array}{l}\text { Postal } \\
\text { Service }\end{array}$ & $\begin{array}{c}\text { Trans- } \\
\text { portation }\end{array}$ & $\begin{array}{c}\text { Veterans } \\
\text { Affairs }\end{array}$ & Other ${ }^{4}$ & Total \\
\hline 1975 & 9.5 & $1,360.2$ & 50.4 & 22.3 & 6.5 & 9.4 & 5.9 & 13.4 & 30.5 & 19.3 & 27.1 & 10.5 & $1,565.0$ \\
\hline 1976 & 9.3 & $1,183.3$ & 50.3 & 20.6 & 6.7 & 9.4 & 5.7 & 12.4 & 30.0 & 19.5 & 25.0 & 11.2 & $1,383.4$ \\
\hline 1977 & 8.9 & $1,192.3$ & 51.6 & 20.4 & 6.9 & 9.5 & 5.9 & 12.0 & 32.7 & 20.4 & 25.9 & 11.9 & $1,398.5$ \\
\hline 1978 & 9.1 & $1,157.8$ & 50.1 & 20.4 & 6.5 & 9.2 & 5.9 & 11.2 & 30.9 & 20.6 & 26.8 & 12.4 & $1,360.9$ \\
\hline 1979 & 9.2 & $1,175.8$ & 49.6 & 19.6 & 6.4 & 10.4 & 6.4 & 11.1 & 29.3 & 19.6 & 25.7 & 12.3 & $1,375.4$ \\
\hline 1980 & 8.6 & $1,183.1$ & 47.4 & 18.1 & 6.0 & 8.5 & 5.7 & 10.4 & 27.2 & 19.2 & 24.8 & 12.3 & $1,371.2$ \\
\hline 1981 & 7.9 & $1,239.5$ & 47.3 & 18.0 & 6.7 & 7.6 & 5.4 & 10.0 & 27.9 & 18.8 & 24.0 & 11.1 & $1,424.2$ \\
\hline 1982 & 7.6 & $1,264.5$ & 49.0 & 18.1 & 6.4 & 7.4 & 5.8 & 10.1 & 27.5 & 19.1 & 24.2 & 11.6 & $1,451.4$ \\
\hline 1983 & 7.4 & $1,248.3$ & 49.5 & 16.1 & 6.2 & 7.7 & 5.5 & 10.3 & 26.5 & 19.4 & 24.1 & 10.8 & $1,431.8$ \\
\hline 1984 & 7.9 & $1,292.1$ & 51.6 & 16.2 & 6.4 & 8.4 & 6.4 & 10.6 & 27.7 & 19.8 & 24.6 & 10.7 & $1,482.5$ \\
\hline 1985 & 8.4 & $1,250.6$ & 52.2 & 20.7 & 6.0 & 7.8 & 8.2 & 10.9 & 27.8 & 19.6 & 25.1 & 13.1 & $1,450.3$ \\
\hline 1986 & 6.8 & $1,222.8$ & 46.9 & 14.0 & 6.2 & 6.9 & 8.6 & 11.2 & 28.0 & 19.4 & 25.0 & 10.8 & $1,406.7$ \\
\hline 1987 & 7.3 & $1,280.5$ & 48.5 & 13.1 & 6.6 & 6.6 & 8.1 & 11.3 & 28.5 & 19.0 & 24.9 & 11.9 & $1,466.3$ \\
\hline 1988 & 7.8 & $1,165.8$ & 49.9 & 12.4 & 6.4 & 7.0 & 9.4 & 11.3 & 29.6 & 18.7 & 26.3 & 15.8 & $1,360.3$ \\
\hline 1989 & 8.7 & $1,274.4$ & 44.2 & 12.7 & 6.7 & 7.1 & 7.7 & 12.4 & 30.3 & 18.5 & 26.2 & 15.6 & $1,464.7$ \\
\hline 1990 & 9.6 & $1,241.7$ & 43.5 & 17.5 & 7.1 & 7.4 & 7.0 & 12.4 & 30.6 & 19.0 & 24.9 & 17.5 & $1,438.0$ \\
\hline 1991 & 9.6 & $1,269.3$ & 42.1 & 14.0 & 6.2 & 7.1 & 8.0 & 12.5 & 30.8 & 19.0 & 25.1 & 18.1 & $1,461.7$ \\
\hline 1992 & 9.1 & $1,104.0$ & 44.3 & 13.8 & 6.8 & 7.0 & 7.5 & 12.6 & 31.7 & 17.0 & 25.3 & 15.7 & $1,294.8$ \\
\hline 1993 & 9.3 & $1,048.8$ & 43.4 & 14.1 & 7.2 & 7.5 & 9.1 & 12.4 & 33.7 & 19.4 & 25.7 & 16.2 & $1,246.8$ \\
\hline 1994 & 9.4 & 977.0 & 42.1 & 14.0 & 7.5 & 7.9 & 10.3 & 12.6 & 35.0 & 19.8 & 25.6 & 17.1 & $1,178.2$ \\
\hline 1995 & 9.0 & 926.0 & 47.3 & 13.7 & 6.1 & 6.4 & 10.2 & 12.4 & 36.2 & 18.7 & 25.4 & 17.9 & $1,129.3$ \\
\hline 1996 & 9.1 & 904.5 & 44.6 & 14.5 & 6.6 & 4.3 & 12.1 & 11.5 & 36.4 & 19.6 & 26.8 & 18.5 & $1,108.5$ \\
\hline 1997 & 7.4 & 880.0 & 43.1 & 14.4 & 7.9 & 6.6 & 12.0 & 12.0 & 40.8 & 19.1 & 27.3 & 21.6 & $1,092.0$ \\
\hline 1998 & 7.9 & 837.1 & 31.5 & 14.1 & 7.4 & 6.4 & 15.8 & 11.7 & 39.5 & 18.5 & 27.6 & 20.3 & $1,037.9$ \\
\hline 1999 & 7.8 & 810.7 & 27.0 & 14.4 & 7.1 & 7.5 & 15.4 & 11.4 & 39.8 & 22.6 & 27.5 & 20.6 & $1,011.6$ \\
\hline 2000 & 7.4 & 779.1 & 30.5 & 17.6 & 8.0 & 7.8 & 19.7 & 11.1 & 43.3 & 21.2 & 27.0 & 21.0 & 993.8 \\
\hline 2001 & 7.4 & 787.2 & 31.1 & 18.4 & 8.5 & 9.5 & 19.7 & 10.9 & 43.4 & 17.8 & 27.7 & 21.4 & $1,003.0$ \\
\hline 2002 & 7.2 & 837.5 & 30.7 & 17.5 & 8.0 & 8.2 & 17.7 & 10.7 & 41.6 & 18.3 & 27.7 & 19.8 & $1,044.8$ \\
\hline 2003 & 7.7 & 902.3 & 31.6 & 19.6 & 10.1 & 8.2 & 22.7 & 10.8 & 50.9 & 5.6 & 30.5 & 36.2 & $1,136.3$ \\
\hline 2004 & 7.0 & 960.7 & 31.4 & 18.3 & 8.8 & 8.7 & 17.5 & 9.9 & 50.5 & 5.2 & 29.9 & 39.2 & $1,187.0$ \\
\hline 2005 & 7.5 & 933.2 & 29.6 & 18.4 & 9.6 & 8.6 & 18.8 & 10.3 & 53.5 & 5.0 & 30.0 & 37.2 & $1,161.6$ \\
\hline 2006 & 6.8 & 843.7 & 32.9 & 18.2 & 9.3 & 8.1 & 23.5 & 10.2 & 51.8 & 4.6 & 29.3 & 33.2 & $1,071.5$ \\
\hline 2007 & 6.8 & 864.6 & 31.5 & 19.1 & 9.9 & 7.5 & 20.7 & 10.6 & 45.8 & 5.6 & 30.0 & 33.2 & $1,085.3$ \\
\hline 2008 & 6.5 & 893.0 & 31.5 & 18.8 & 10.5 & 7.9 & 18.9 & 10.2 & 47.0 & 6.4 & 28.9 & 36.6 & $1,116.2$ \\
\hline 2009 & 6.6 & R874.3 & 31.1 & 18.6 & 10.8 & 7.9 & 16.5 & 10.2 & 44.2 & 4.3 & 29.9 & $R_{36.5}$ & $\mathrm{R}_{1,090.9}$ \\
\hline 2010 & 6.8 & R889.3 & $\mathrm{R}_{31.7}$ & 18.8 & 10.3 & 8.3 & $\mathrm{R}_{15.7}$ & 10.1 & $\mathrm{R}_{42.3}$ & 5.7 & 30.2 & R 42.8 & $\mathrm{R}_{1,112.0}$ \\
\hline $2011^{P}$ & 8.3 & 890.3 & 33.4 & 18.5 & 10.5 & 8.3 & 13.9 & 10.1 & 44.4 & 6.7 & 30.6 & 41.5 & $1,116.6$ \\
\hline
\end{tabular}

1 General Services Administration.

2 Health and Human Services.

3 National Aeronautics and Space Administration.

4 Includes National Archives and Records Administration, U.S. Department of Commerce, Tennessee Valley Authority, U.S. Department of Labor, National Science Foundation, Federal Trade Commission, Federal Communications Commission, Environmental Protection Agency, U.S. Department of Homeland Security, U.S. Department of Housing and Urban Development, Railroad Retirement Board, Equal Employment Opportunity Commission, Nuclear Regulatory Commission, U.S. Department of State, U.S. Department of the Treasury, Small Business Administration, Office of Personnel Management, Central Intelligence Agency, Consumer Product Safety Commission, Social Security Administration, U.S. Court Services and Offender Supervision Agency, Federal Housing Finance Agency, National Labor Relations Board, Securities and Exchange Commission, National Capital Planning Commission, Office of Special Counsel, and Peace Corps and Broadcasting Board of Governors.
Notes: - For 1975 and 1976, the U.S. Government's fiscal year was July 1 through June 30. Beginning in 1977, the U.S. Government's fiscal year is October 1 through September 30 (for example, fiscal year 2011 is October 2010 through September 2011). - Data in this table are developed using the following conversion factors (which in most cases are different from those in Tables A1-A6)-coal: 24.580 million Btu/short ton; natural gas: 1,031 Btu/cubic foot; aviation gasoline: 5.250 million Btu/barrel; fuel oil: 5.8254 million Btu/barrel; jet fuel: 5.460 million Btu/barrel; liquefied petroleum gases: 4.011 million Btu/barrel; motor gasoline: 5.250 million Btu/barrel; electricity: 3,412 Btu/kilowatthour; and purchased steam: 1,000 Btu/pound. - Data include energy consumed at foreign installations and in foreign operations, including aviation and ocean bunkering, primarily by the U.S. Department of Defense. U.S. Government energy use for electricity generation and uranium enrichment is excluded. - Totals may not equal sum of components due to independent rounding.

Web Page: See http://www1.eere.energy.gov/femp/regulations/facility reporting.html for related information

Source: U.S. Department of Energy, Office of Energy Efficiency and Renewable Energy, Federal Energy Management Program. 


\section{Total U.S. Government Energy Consumption}

1.8

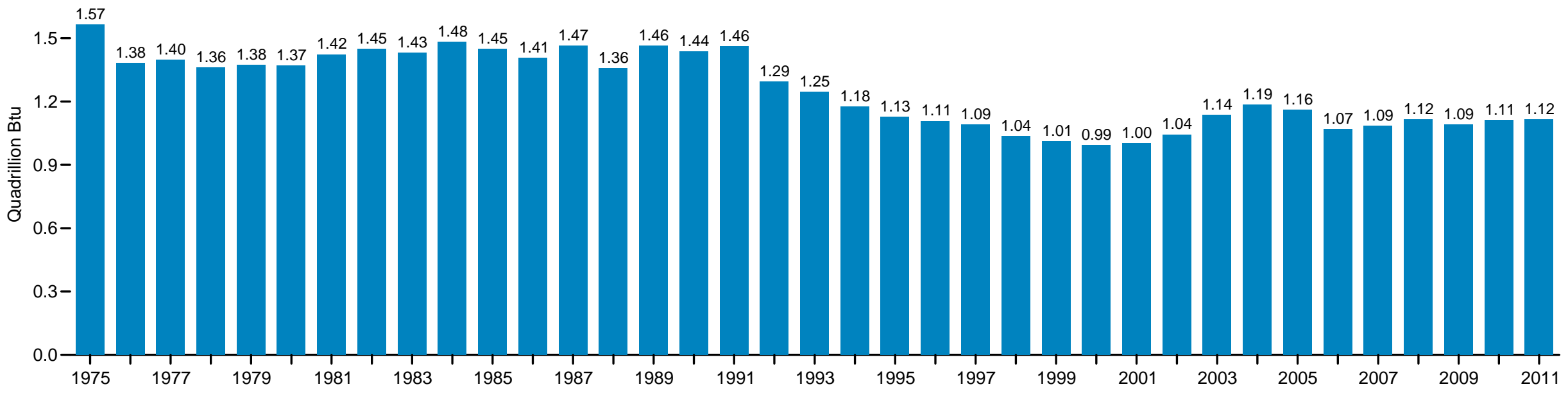

By Major Energy Source

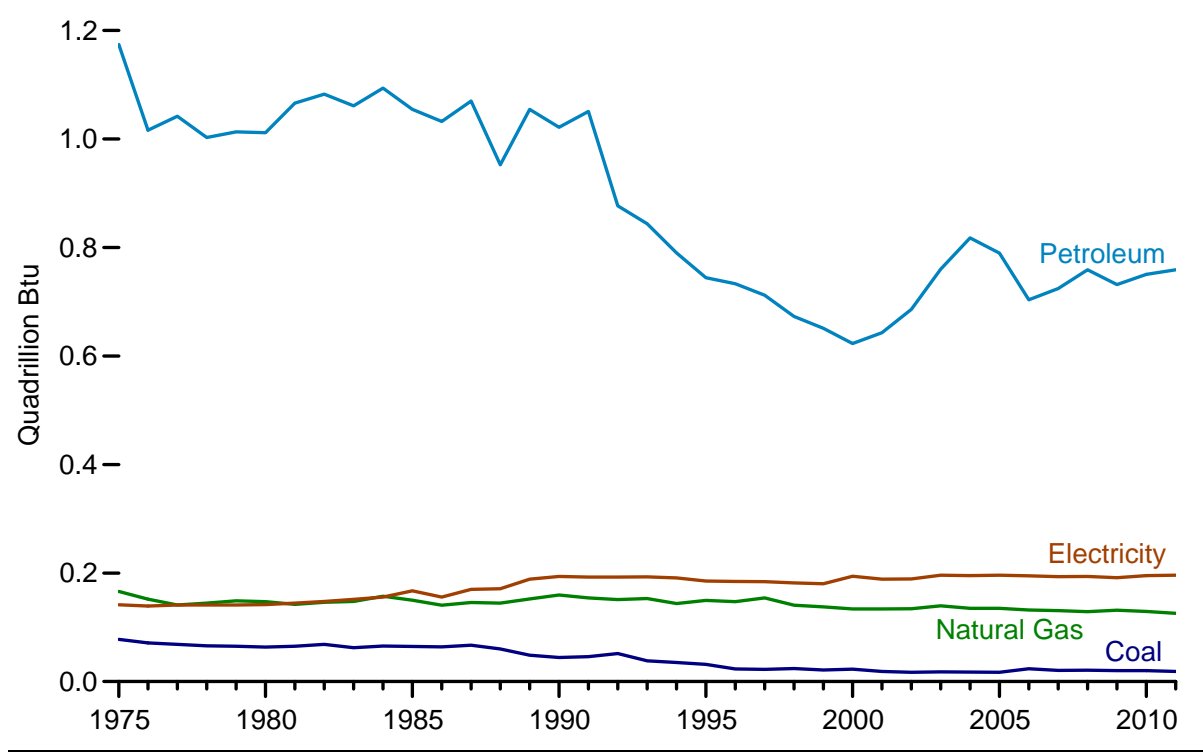

${ }^{1}$ Distillate fuel oil and residual fuel oil.

${ }^{2}$ Includes ethanol blended into motor gasoline.

Note: U.S. Government's fiscal year was October 1 through September 30, except in 1975
By Selected Petroleum Product

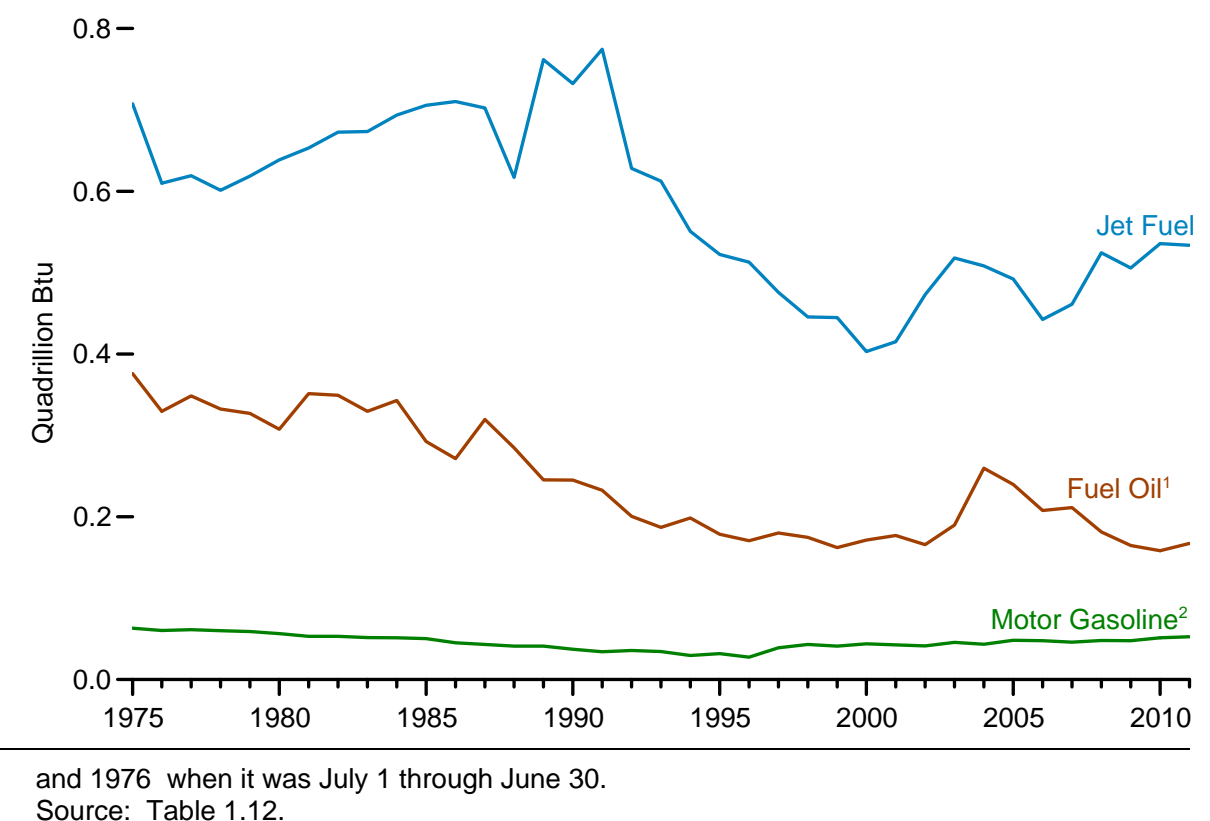


Table 1.12 U.S. Government Energy Consumption by Source, Fiscal Years 1975-2011

(Trillion Btu)

\begin{tabular}{|c|c|c|c|c|c|c|c|c|c|c|c|}
\hline \multirow[b]{2}{*}{ Year } & \multirow[b]{2}{*}{ Coal } & \multirow[b]{2}{*}{$\begin{array}{c}\text { Natural } \\
\text { Gas } 1\end{array}$} & \multicolumn{6}{|c|}{ Petroleum } & \multirow[b]{2}{*}{ Electricity } & \multirow{2}{*}{$\begin{array}{l}\text { Purchased } \\
\text { Steam } \\
\text { and Other } 6\end{array}$} & \multirow[b]{2}{*}{ Total } \\
\hline & & & $\begin{array}{l}\text { Aviation } \\
\text { Gasoline }\end{array}$ & Fuel Oil ${ }^{2}$ & Jet Fuel & $\begin{array}{c}\text { LPG }^{3} \\
\text { and Other } 4\end{array}$ & $\begin{array}{c}\text { Motor } \\
\text { Gasoline }\end{array}$ & Total & & & \\
\hline 1975 & 77.9 & 166.2 & 22.0 & 376.0 & 707.4 & 5.6 & 63.2 & $1,174.2$ & 141.5 & 5.1 & $1,565.0$ \\
\hline 1976 & 71.3 & 151.8 & 11.6 & 329.7 & 610.0 & 4.7 & 60.4 & $1,016.4$ & 139.3 & 4.6 & $1,383.4$ \\
\hline 1977 & 68.4 & 141.2 & 8.8 & 348.5 & 619.2 & 4.1 & 61.4 & $1,042.1$ & 141.1 & 5.7 & $1,398.5$ \\
\hline 1978 & 66.0 & 144.7 & 6.2 & 332.3 & 601.1 & 3.0 & 60.1 & $1,002.9$ & 141.0 & 6.4 & $1,360.9$ \\
\hline 1979 & 65.1 & 148.9 & 4.7 & 327.1 & 618.6 & 3.7 & 59.1 & $1,013.1$ & 141.2 & 7.1 & $1,375.4$ \\
\hline 1980 & 63.5 & 147.3 & 4.9 & 307.7 & 638.7 & 4.0 & 56.5 & $1,011.8$ & 141.9 & 6.8 & $1,371.2$ \\
\hline 1981 & 65.1 & 142.2 & 4.6 & 351.3 & 653.3 & 3.7 & 53.2 & $1,066.2$ & 144.5 & 6.2 & $1,424.2$ \\
\hline 1982 & 68.6 & 146.2 & 3.6 & 349.4 & 672.7 & 3.9 & 53.1 & $1,082.8$ & 147.5 & 6.2 & $1,451.4$ \\
\hline 1983 & 62.4 & 147.8 & 2.6 & 329.5 & 673.4 & 4.0 & 51.6 & $1,061.1$ & 151.5 & 9.0 & $1,431.8$ \\
\hline 1984 & 65.3 & 157.4 & 1.9 & 342.9 & 693.7 & 4.1 & 51.2 & $1,093.8$ & 155.9 & 10.1 & $1,482.5$ \\
\hline 1985 & 64.8 & 149.9 & 1.9 & 292.6 & 705.7 & 4.0 & 50.4 & $1,054.6$ & 167.2 & 13.9 & $1,450.3$ \\
\hline 1986 & 63.8 & 140.9 & 1.4 & 271.6 & 710.2 & 3.9 & 45.3 & $1,032.4$ & 155.8 & 13.7 & $1,406.7$ \\
\hline 1987 & 67.0 & 145.6 & 1.0 & 319.5 & 702.3 & 4.0 & 43.1 & $1,069.9$ & 169.9 & 13.9 & $1,466.3$ \\
\hline 1988 & 60.2 & 144.6 & 6.0 & 284.8 & 617.2 & 3.2 & 41.2 & 952.4 & 171.2 & 32.0 & $1,360.3$ \\
\hline 1989 & 48.7 & 152.4 & .8 & 245.3 & 761.7 & 5.7 & 41.1 & $1,054.5$ & 188.6 & 20.6 & $1,464.7$ \\
\hline 1990 & 44.3 & 159.4 & .5 & 245.2 & 732.4 & 6.4 & 37.2 & $1,021.7$ & 193.6 & 19.1 & $1,438.0$ \\
\hline 1991 & 45.9 & 154.1 & .4 & 232.6 & 774.5 & 9.0 & 34.1 & $1,050.7$ & 192.7 & 18.3 & $1,461.7$ \\
\hline 1992 & 51.7 & 151.2 & 1.0 & 200.6 & 628.2 & 11.4 & 35.6 & 876.8 & 192.5 & 22.5 & $1,294.8$ \\
\hline 1993 & 38.3 & 152.9 & .7 & 187.0 & 612.4 & 9.3 & 34.5 & 843.9 & 193.1 & 18.6 & $1,246.8$ \\
\hline 1994 & 35.0 & 143.9 & .6 & 198.5 & 550.7 & 10.9 & 29.5 & 790.2 & 190.9 & 18.2 & $1,178.2$ \\
\hline 1995 & 31.7 & 149.7 & .3 & 178.5 & 522.3 & 11.4 & 31.9 & 744.4 & 185.3 & 18.2 & $1,129.3$ \\
\hline 1996 & 23.3 & 147.4 & .2 & 170.6 & 513.0 & 21.7 & 27.6 & 733.2 & 184.5 & 20.1 & $1,108.5$ \\
\hline 1997 & 22.5 & 154.0 & .3 & 180.1 & 475.7 & 17.2 & 39.0 & 712.2 & 184.0 & 19.2 & $1,092.0$ \\
\hline 1998 & 23.9 & 140.7 & .2 & 174.6 & 445.5 & 9.4 & 43.1 & 672.8 & 181.8 & 18.8 & $1,037.9$ \\
\hline 1999 & 21.2 & 137.6 & .1 & 162.2 & 444.7 & 2.9 & 41.1 & 650.9 & 180.4 & 21.5 & $1,011.6$ \\
\hline 2000 & 22.7 & 134.0 & .2 & 171.4 & 403.1 & 4.3 & 43.9 & 622.9 & 194.0 & 20.2 & 993.8 \\
\hline 2001 & 18.8 & 133.9 & .2 & 177.0 & 415.2 & 7.9 & 42.5 & 642.9 & 188.8 & 18.6 & $1,003.0$ \\
\hline 2002 & 16.9 & 134.1 & .2 & 165.7 & 472.9 & 6.0 & 41.3 & 686.1 & 189.1 & 18.5 & $1,044.8$ \\
\hline 2003 & 17.7 & 139.7 & .3 & 189.8 & 517.9 & 6.6 & 45.7 & 760.3 & 196.1 & 22.5 & $1,136.3$ \\
\hline 2004 & 17.4 & 134.8 & .2 & 259.8 & 508.2 & 6.0 & 43.5 & 817.8 & 195.4 & 21.6 & $1,187.0$ \\
\hline 2005 & 17.1 & 135.1 & .4 & 239.8 & 492.2 & 9.0 & 48.2 & 789.6 & 195.9 & 23.9 & $1,161.6$ \\
\hline 2006 & 23.5 & 132.0 & .6 & 207.8 & 442.6 & 4.7 & 47.8 & 703.5 & 194.9 & 17.7 & $1,071.5$ \\
\hline 2007 & 20.4 & 130.8 & .4 & 211.4 & 461.1 & 5.6 & 46.0 & 724.5 & 193.2 & 16.4 & $1,085.3$ \\
\hline 2008 & 20.8 & 128.9 & .4 & 181.4 & 524.3 & 4.6 & 48.1 & 758.8 & 193.6 & 14.1 & $1,116.2$ \\
\hline 2009 & 20.3 & $\mathrm{R}_{131.4}$ & .3 & $\mathrm{R}_{164.8}$ & 505.6 & $\mathrm{R}_{13.3}$ & R 47.7 & R731.7 & $\mathrm{R}_{191.5}$ & $\mathrm{R}_{16.1}$ & $\mathrm{R}_{1,090.9}$ \\
\hline 2010 & 20.1 & $R_{129.3}$ & .4 & $\mathrm{R}_{158.4}$ & 535.8 & 4.8 & R51.3 & $\mathrm{R}_{750.7}$ & $\mathrm{R}_{195.1}$ & $\mathrm{R}_{16.8}$ & $\mathrm{R}_{1,112.0}$ \\
\hline $2011^{P}$ & 18.6 & 125.7 & .9 & 167.3 & 533.6 & 4.7 & 52.5 & 759.0 & 195.9 & 17.4 & $1,116.6$ \\
\hline
\end{tabular}

Natural gas, plus a small amount of supplemental gaseous fuels.

Distillate fuel oil and residual fuel oil.

Liquefied petroleum gases.

${ }_{4}$ Other types of fuel used in vehicles and equipment, primarily alternative fuels like methanol, ethanol, compressed natural gas, and biodiesel.

Includes ethanol blended into motor gasoline.

"Other" is chilled water, renewable energy, and other fuels reported as used in facilities.

R=Revised. $P=$ Preliminary.

Notes: - For 1975 and 1976, the U.S. Government's fiscal year was July 1 through June 30. Beginning in 1977, the U.S. Government's fiscal year is October 1 through September 30 (for example, fiscal year 2011 is October 2010 through September 2011). - Data in this table are developed using the following
conversion factors (which in most cases are different from those in Tables A1-A6)-coal: 24.580 million

Btu/short ton; natural gas: 1,031 Btu/cubic foot; aviation gasoline: 5.250 million Btu/barrel; fuel oil: 5.8254 million Btu/barrel; jet fuel. 5.460 million Btu/barrel; liquefied petroleum gases: 4.011 million Btu/barrel; motor gasoline: 5.250 million Btu/barrel, electricity: 3,412 Btu/kilowatthour, and purchased steam: 1,000 Btu/pound. - Data include energy consumed at foreign installations and in foreign operations, including aviation and ocean bunkering, primarily by the U.S. Department of Defense. U.S. Government energy use for electricity generation and uranium enrichment is excluded. - Totals may not equal sum of components

due to independent rounding.
Web Page: See http://www1.eere.energy.gov/femp/regulations/facility_reporting.html for related information.

Source: U.S. Department of Energy, Office of Energy Efficiency and Renewable Energy, Federal Energy Management Program. 


\section{By Agency}

800-

Department of Defense

All Other Agencies

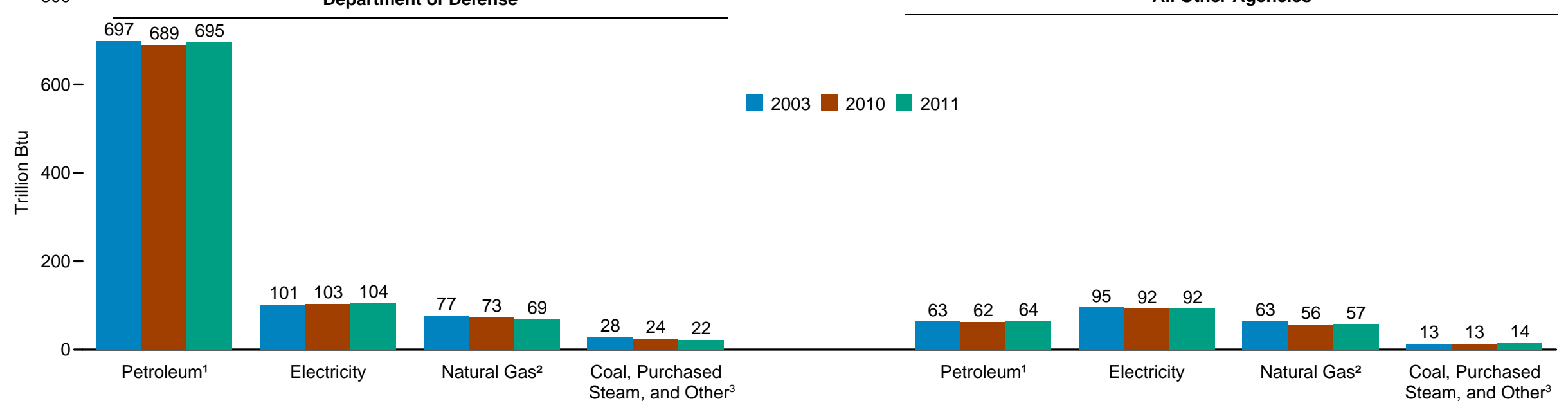

\section{By Source}

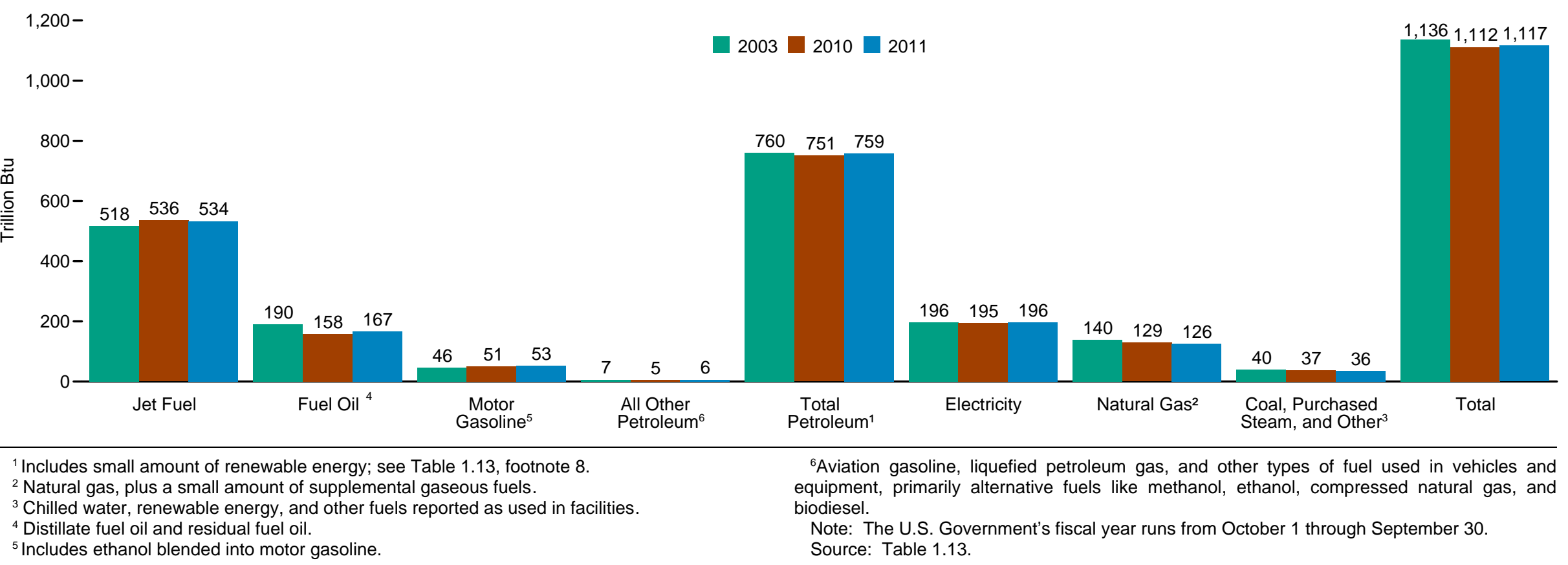


Table 1.13 U.S. Government Energy Consumption by Agency and Source, Fiscal Years 2003, 2010, and 2011 (Trillion Btu)

\begin{tabular}{|c|c|c|c|c|c|c|c|c|c|c|c|c|c|}
\hline $\begin{array}{l}\text { Resource } \\
\text { and Fiscal Years }\end{array}$ & Agriculture & Defense & Energy & GSA 1 & HHS $^{2}$ & Interior & Justice & NASA $^{3}$ & $\begin{array}{l}\text { Postal } \\
\text { Service }\end{array}$ & $\begin{array}{c}\text { Trans- } \\
\text { portation }\end{array}$ & $\begin{array}{l}\text { Veterans } \\
\text { Affairs }\end{array}$ & Other ${ }^{4}$ & Total \\
\hline \multicolumn{14}{|l|}{ Coal } \\
\hline 2003 & (s) & 15.4 & 2.0 & 0.0 & (s) & (s) & 0.0 & 0.0 & 0.0 & 0.0 & 0.2 & 0.0 & 17.7 \\
\hline 2010 & (s) & 15.5 & 4.5 & .0 & 0.0 & 0.0 & .0 & .0 & (s) & .0 & .1 & .0 & 20.1 \\
\hline 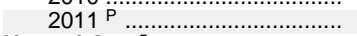 & 0.0 & 14.3 & 4.2 & .0 & .0 & .0 & & .0 & & .0 & .1 & .0 & 18.6 \\
\hline \multicolumn{14}{|l|}{ Natural Gas ${ }^{5}$} \\
\hline 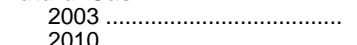 & $\begin{array}{l}1.4 \\
1.4\end{array}$ & $\begin{array}{l}76.6 \\
729\end{array}$ & $\begin{array}{l}7.0 \\
7.1\end{array}$ & $\begin{array}{l}7.6 \\
7.0\end{array}$ & 3.7 & 1.3 & $\begin{array}{l}8.6 \\
6.8\end{array}$ & 2.9 & $\begin{array}{r}10.4 \\
45\end{array}$ & .7 & 15.6 & $\begin{array}{r}4.2 \\
R 4.9\end{array}$ & $\begin{array}{rl} & 139.7 \\
R & 1293\end{array}$ \\
\hline $2011 \mathrm{P}$ & 1.7 & 68.6 & 7.4 & 7.1 & $\begin{array}{l}5.9 \\
5.9\end{array}$ & $\begin{array}{l}1.1 \\
1.2\end{array}$ & $\begin{array}{l}0.8 \\
4.2\end{array}$ & $\begin{array}{l}2.6 \\
2.5\end{array}$ & $\begin{array}{l}4.5 \\
6.3\end{array}$ & $\begin{array}{l}.3 \\
.8\end{array}$ & $\begin{array}{l}4.9 \\
15.1\end{array}$ & 5.0 & 125.7 \\
\hline \multicolumn{14}{|l|}{ Petroleum } \\
\hline 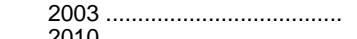 & 3.3 & 697.1 & 3.0 & .2 & 1.5 & $\begin{array}{l}4.4 \\
3.8\end{array}$ & 6.5 & 1.4 & 18.2 & 1.6 & 2.8 & 20.3 & 760.3 \\
\hline 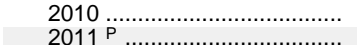 & $\begin{array}{l}3.2 \\
4.6\end{array}$ & $\begin{array}{l}688.8 \\
695.3\end{array}-100$ & $\begin{array}{l}2.8 \\
3.6\end{array}$ & .2 & $\begin{array}{r}.8 \\
9\end{array}$ & $\begin{array}{l}3.8 \\
3.7\end{array}$ & $\begin{array}{l}3.4 \\
4.1\end{array}$ & $\begin{array}{l}1.2 \\
1.1\end{array}$ & $\begin{array}{l}19.4 \\
20.5\end{array}$ & $\begin{array}{l}1.5 \\
1.1\end{array}$ & $\begin{array}{l}2.1 \\
2.3\end{array}$ & 21.4 & \\
\hline \multicolumn{14}{|l|}{ Aviation Gasoline } \\
\hline 2003 & (s) & (s) & (s) & .0 & .0 & (s) & .1 & (s) & .0 & (s) & .0 & (s) & .3 \\
\hline 2010 & (s) & .2 & .0 & .0 & .0 & (s) & .1 & (s) & .0 & (s) & .0 & (s) & .4 \\
\hline \multirow{2}{*}{\multicolumn{14}{|c|}{ Fuel Oil 6 . }} \\
\hline & & & & & & & & & & & & & \\
\hline 2003 & $\begin{array}{l}.4 \\
.6\end{array}$ & $\begin{array}{l}166.5 \\
138.2\end{array}$ & $\begin{array}{l}2.0 \\
1.6\end{array}$ & .1 & .9 & $\begin{array}{l}1.2 \\
1.3\end{array}$ & $\begin{array}{l}.4 \\
3\end{array}$ & .4 & $\begin{array}{l}5.1 \\
4.6\end{array}$ & .3 & $\begin{array}{l}1.9 \\
1.1\end{array}$ & $\begin{array}{r}10.7 \\
\mathrm{R} 95\end{array}$ & $\begin{aligned} & 189.8 \\
\text { R } & 158.4\end{aligned}$ \\
\hline $2011^{\mathrm{P}}$ & .6 & 146.1 & $\begin{array}{l}1.0 \\
2.4\end{array}$ & .1 & .6 & 1.4 & .3 & .2 & $\begin{array}{l}4.0 \\
4.9\end{array}$ & .1 & 1.1 & 9.5 & 167.3 \\
\hline \multicolumn{14}{|l|}{ Jet Fuel } \\
\hline 2003 & $\begin{array}{l}.0 \\
.0\end{array}$ & $\begin{array}{l}509.9 \\
529.0\end{array}$ & (s) & .0 & .0 & $\begin{array}{l}.1 \\
(\mathrm{~s})\end{array}$ & $\begin{aligned} 1.5 \\
2\end{aligned}$ & .6 & .0 & .6 & .0 & 5.2 & $\begin{array}{l}517.9 \\
5358\end{array}$ \\
\hline $2011 \mathrm{P}$ & .9 & 526.7 & .2 & .0 & .0 & $\begin{array}{l}\text { (s) } \\
\text { (s) }(x)\end{array}$ & .6 & .7 & .0 & .5 & .0 & 4.0 & 533.6 \\
\hline \multicolumn{14}{|l|}{ LPG $^{7}$ and Other 8} \\
\hline $2003 \ldots \ldots \ldots \ldots \ldots \ldots \ldots \ldots \ldots \ldots \ldots \ldots$ & .7 & $\begin{array}{l}4.2 \\
2.7\end{array}$ & .1 & $\begin{array}{l}\text { (s) } \\
\text { (s) }\end{array}$ & .1 & .7 & (s) & .1 & .2 & $\left(\begin{array}{l}1 \\
(\mathrm{~s})\end{array}\right.$ & (s) & .3 & 6.6 \\
\hline 2010 p 201. & .4 & 2.6 & .4 & $\begin{array}{l}\text { (s) } \\
\text { (s) }\end{array}$ & .1 & .2 & .12 & .1 & .3 & (s) & .1 & .3 & $\begin{array}{l}4.8 \\
4.7\end{array}$ \\
\hline \multicolumn{14}{|l|}{ Motor Gasoline ${ }^{9}$} \\
\hline 2003 & 2.2 & 16.5 & .9 & .1 & .5 & 2.4 & 4.5 & .2 & 12.9 & .7 & .9 & 4.1 & 45.7 \\
\hline 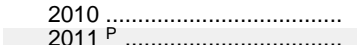 & 2.2 & 18.6 & .6 & .1 & .2 & 2.1 & 2.8 & .1 & 14.5 & .6 & .9 & R 8.5 & 51.3 \\
\hline 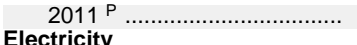 & 2.6 & 19.7 & .7 & .1 & .2 & 2.0 & 3.0 & .1 & 15.4 & .4 & 1.1 & 7.3 & 52.5 \\
\hline \multicolumn{14}{|l|}{ Electricity } \\
\hline $2003 \ldots \ldots \ldots \ldots \ldots \ldots \ldots \ldots \ldots \ldots \ldots \ldots \ldots \ldots \ldots \ldots \ldots$ & 2.6 & 101.1 & 18.0 & 10.0 & 3.6 & 2.4 & 7.0 & 5.8 & 21.7 & 3.2 & 10.2 & 10.5 & 196.1 \\
\hline 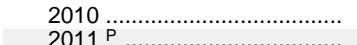 & 1.9 & $\begin{array}{l}\mathrm{R}_{103.2} \\
104.1\end{array}$ & $\begin{array}{r}\mathrm{R} 17.1 \\
17.9\end{array}$ & 9.9 & $\begin{array}{l}3.4 \\
3.5\end{array}$ & $\begin{array}{l}\mathrm{R}_{2} .7 \\
2.7\end{array}$ & $\begin{array}{l}5.4 \\
5.3\end{array}$ & $\begin{array}{r}\text { R } 5.4 \\
5.4\end{array}$ & 17.8 & $\begin{array}{r}\mathrm{R} 4.0 \\
4.1\end{array}$ & $\begin{array}{r}\mathrm{R} 11.1 \\
11.4\end{array}$ & $\begin{array}{r}\mathrm{R} 13.3 \\
13.4\end{array}$ & R 195.1 \\
\hline \multicolumn{14}{|l|}{ Purchased Steam and Other 10} \\
\hline 2003 & 3 & 12.2 & 1.6 & 1.8 & 1.3 & .1 & .7 & .8 & .7 & (s) & 1.7 & 1.2 & 22.5 \\
\hline $2010 \ldots \ldots \ldots \ldots \ldots \ldots \ldots \ldots \ldots \ldots .$. & .3 & R 8.8 & R .2 & 1.8 & .1 & .8 & .1 & R .8 & .5 & $\mathrm{R}(\mathrm{s})$ & 2.0 & R 1.4 & 16.8 \\
\hline \multirow{2}{*}{\multicolumn{10}{|c|}{ Total Energy }} & .7 & 1.7 & 1.5 & \\
\hline & 77 & 902.3 & & & 10.1 & & 22.7 & & & & & & 11363 \\
\hline 2010 & 6.8 & R889.3 & $\begin{array}{ll}\mathrm{R} 31.7 \\
\mathrm{R}\end{array}$ & 18.8 & 10.3 & $\begin{array}{l}8.2 \\
8.3\end{array}$ & $\mathrm{R} 15.7$ & 10.1 & $\mathrm{R} 42.3$ & $\begin{array}{l}5.6 \\
5.7\end{array}$ & $\begin{array}{l}30.5 \\
30.2\end{array}$ & $\begin{aligned} 30.2 \\
\mathrm{R} 42.8\end{aligned}$ & $\begin{aligned} 1,112.3 \\
R_{1}, 1120\end{aligned}$ \\
\hline $2011 \mathrm{P}$ & 8.3 & 890.3 & 33.4 & 18.5 & 10.5 & 8.3 & 13.9 & 10.1 & 44.4 & 6.7 & 30.6 & 41.5 & $1,116.6$ \\
\hline
\end{tabular}

1 General Services Administration.

2 Health and Human Services.

3 National Aeronautics and Space Administration

Includes National Archives and Records Administration, U.S. Department of Commerce, Tennessee

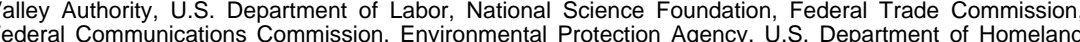
Security, U.S. Department of Housing and Urban Development, Railroad Retirement Board, Equal Employment Opportunity Commission, Nuclear Regulatory Commission, U.S. Department of State, U.S. Department of the Treasury, Office of Personnel Management, Consumer Product Safety Commission, Central Intelligence Agency, Social Security Administration, U.S. Information Agency (Internationa Supervision Agency, Federal Housing Finance Agency, National Labor Relations Board, Small Business Administration, Securities and Exchange Commission, National Capital Planning Commission, Office of Special Counsel, and Peace Corps and Broadcasting Board of Governors.

Natural gas, plus a small amount of supplemental gaseous fuels.

Distillate fuel oil and residual fuel oil.

8 Other types of fuel used in ve compressed natural gas, and biodiesel.

Includes ethanol blended into motor gasoline.

Chiled facilities.

Notes: - Beginning in 1977, the U.S. Government's fiscal year is October 1 through September 30 (for example, fiscal year 2011 is October 2010 through September 2011). - Data in this table are developed using the following conversion factors (which in most cases are different from those in Tables A1-A6) -coal: 24.580 million Btu/short ton; natural gas: 1,031 Btu/cubic foot; aviation gasoline: 5.250 million Btu/barrel; fuel oil: 5.8254 million Btu/barrel; jet fuel: 5.460 million Btu/barrel; liquefied petroleum gand purchased steam: 1000 Btu/pound. Data include energy consumed at foreign installations and in foreign operations, including aviation and ocean bunkering primarily by the U.S. Department of Defense. U.S. Government energy use for electricity generation and uranium enrichment is excluded. • Totals may

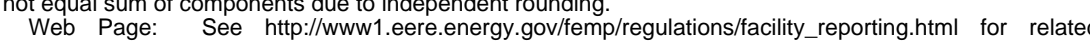

information.
Source: U.S. Department of Energy, Office of Energy Efficiency and Renewable Energy, Federal Energy Management Program. 
Figure 1.14 Sales of Fossil Fuels Produced on Federal and American Indian Lands

\section{Total, Fiscal Years ${ }^{1}$ 2003-2011}

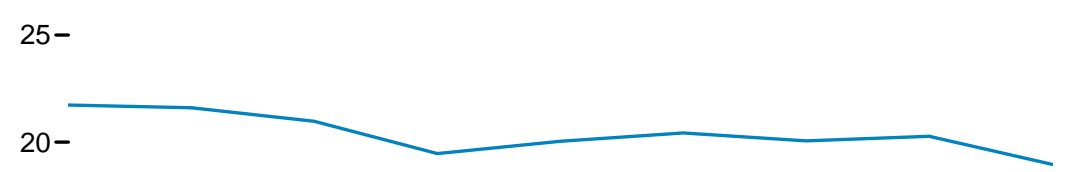

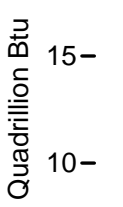

$5-$

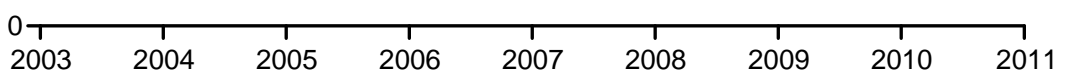

By Source, Fiscal Years $^{1}$ 2003-2011

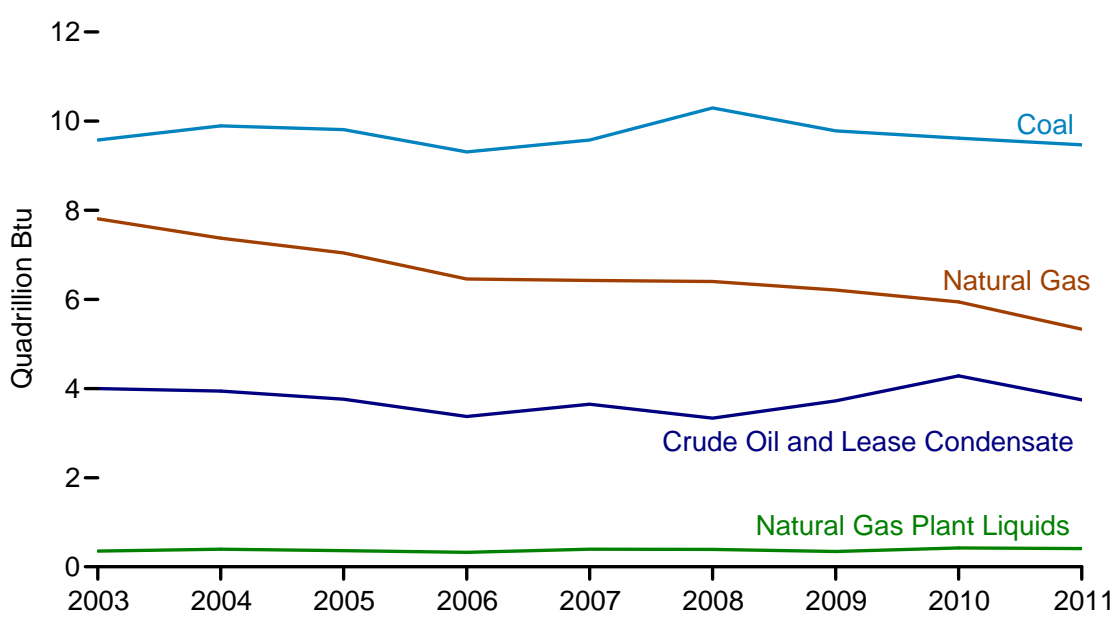

Federal and American Indian Lands Fossil Fuels Sales as Share of Total U.S. Fossil Fuels Production, Fiscal Years ${ }^{1}$ 2003-2011

45-

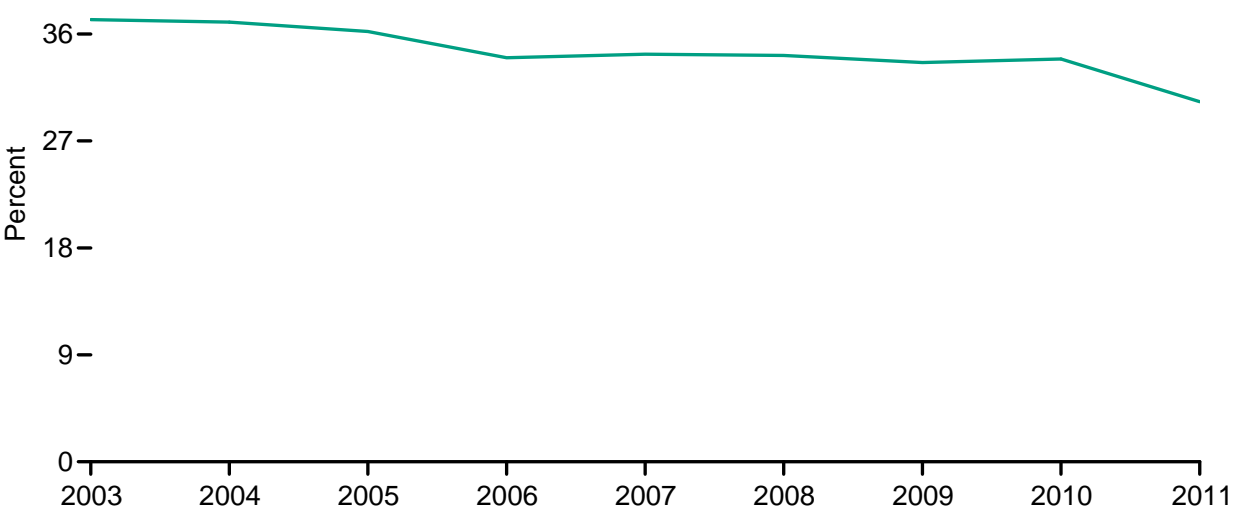

Federal and American Indian Lands Fossil Fuels Sales as Share of Total U.S. Fossil Fuels Production, By Source, Fiscal Year 2011

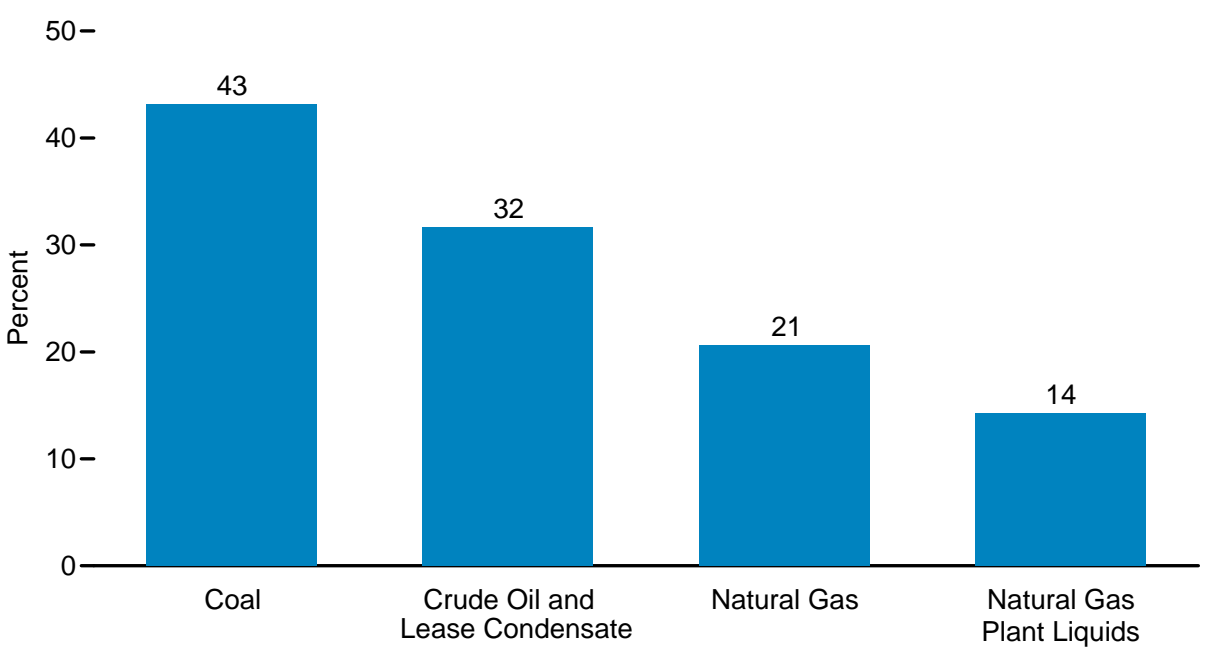

1 The U.S. Government's fiscal year runs from October 1 through September 30. The fiscal year is designated by the calendar year in which it ends.

Source: Table 1.14 
Table 1.14 Sales of Fossil Fuels Produced on Federal and American Indian Lands, Fiscal Years 2003-2011

\begin{tabular}{|c|c|c|c|c|c|c|c|c|c|c|c|c|c|c|}
\hline \multirow[b]{3}{*}{$\begin{array}{l}\text { Fiscal } \\
\text { Year }{ }^{7}\end{array}$} & \multicolumn{3}{|c|}{$\begin{array}{l}\text { Crude Oil and } \\
\text { Lease Condensate }\end{array}$} & \multicolumn{3}{|c|}{$\begin{array}{l}\text { Natural Gas } \\
\text { Plant Liquids }{ }^{1}\end{array}$} & \multicolumn{3}{|c|}{ Natural Gas 2} & \multicolumn{3}{|c|}{ Coal $^{3}$} & \multicolumn{2}{|c|}{$\begin{array}{c}\text { Total } \\
\text { Fossil Fuels }{ }^{4}\end{array}$} \\
\hline & \multicolumn{2}{|c|}{ Sales 5,6} & \multirow{2}{*}{$\begin{array}{c}\begin{array}{c}\text { Sales as } \\
\text { Share of } \\
\text { Total U.S. } \\
\text { Production }\end{array} \\
\text { Percent }\end{array}$} & \multicolumn{2}{|c|}{ Sales 5,6} & \multirow{2}{*}{$\begin{array}{c}\begin{array}{c}\text { Sales as } \\
\text { Share of } \\
\text { Total U.S. } \\
\text { Production }\end{array} \\
\text { Percent }\end{array}$} & \multicolumn{2}{|c|}{ Sales 5,6} & \multirow{2}{*}{$\begin{array}{c}\begin{array}{c}\text { Sales as } \\
\text { Share of } \\
\text { Total U.S. } \\
\text { Production }\end{array} \\
\text { Percent }\end{array}$} & \multicolumn{2}{|c|}{ Sales ${ }^{5,6}$} & \multirow{2}{*}{\begin{tabular}{|c|}
$\begin{array}{c}\text { Sales as } \\
\text { Share of } \\
\text { Total U.S. } \\
\text { Production }\end{array}$ \\
Percent
\end{tabular}} & \multirow{2}{*}{$\begin{array}{c}\text { Sales }^{5,6} \\
\begin{array}{c}\text { Quadrillion } \\
\text { Btu }\end{array}\end{array}$} & \multirow{2}{*}{$\begin{array}{c}\begin{array}{c}\text { Sales as } \\
\text { Share of } \\
\text { Total U.S. } \\
\text { Production }\end{array} \\
\text { Percent }\end{array}$} \\
\hline & $\begin{array}{l}\text { Million } \\
\text { Barrels }\end{array}$ & $\begin{array}{c}\text { Quadrillion } \\
\text { Btu }\end{array}$ & & $\begin{array}{l}\text { Million } \\
\text { Barrels }\end{array}$ & $\begin{array}{c}\text { Quadrillion } \\
\text { Btu }\end{array}$ & & $\begin{array}{l}\text { Trillion } \\
\text { Cubic Feet }\end{array}$ & $\begin{array}{c}\text { Quadrillion } \\
\text { Btu }\end{array}$ & & $\begin{array}{c}\text { Million } \\
\text { Short Tons }\end{array}$ & $\begin{array}{c}\text { Quadrillion } \\
\text { Btu }\end{array}$ & & & \\
\hline 2003 & $R_{689}$ & $\mathrm{R}_{4.00}$ & $R_{33} 3$ & R94 & $\mathrm{R}_{0} 0.35$ & $\mathrm{R}_{14.9}$ & $\mathrm{R}_{7.08}$ & $\mathrm{R}_{7.81}$ & R35.5 & $\mathrm{R}_{466}$ & R9. 58 & $\mathrm{R}_{43} .3$ & $\mathrm{R}_{21.74}$ & $\mathrm{R}_{37.2}$ \\
\hline 2004 & $R_{680}$ & $R_{3.94}$ & R33.8 & $R_{105}$ & R.39 & $R_{16.0}$ & $\mathrm{R}_{6} 6.68$ & R7.38 & $R_{34.0}$ & R484 & R9.89 & R 43.9 & $R_{21.60}$ & R37.0 \\
\hline 2005 & $R_{649}$ & $R_{3.76}$ & $R_{33.4}$ & R98 & .36 & $R_{15.0}$ & $\mathrm{R}_{6.38}$ & $\mathrm{R}_{7.04}$ & $R_{33.3}$ & $\begin{array}{r}R_{482} \\
\text { n }\end{array}$ & R9.81 & $R_{42.6}$ & $R_{20.98}$ & $\mathrm{R}_{36.2}$ \\
\hline 2006 & $\begin{array}{r}040 \\
\text { R582 }\end{array}$ & R3.37 & $\begin{array}{r}0.4 \\
\text { R31.8 }\end{array}$ & R87 & $\begin{array}{r}.00 \\
\text { R.32 }\end{array}$ & $R_{14.2}$ & $\begin{array}{r}.38 \\
\text { R5.85 }\end{array}$ & $\begin{array}{r}\text { R6.46 } \\
\text { R }\end{array}$ & $\begin{array}{r}30.3 \\
\text { R30.8 }\end{array}$ & $\begin{array}{r}482 \\
\text { R } 458\end{array}$ & $\begin{array}{r}\text { R9. } \\
\text { R1 }\end{array}$ & $\begin{array}{r}439.7 \\
R_{3}\end{array}$ & $\mathrm{R}_{19.46}$ & $\begin{array}{r}3.2 \\
R_{34.0}\end{array}$ \\
\hline 2007 & $R_{629}$ & $\mathrm{R}_{3.65}$ & R33.9 & $\mathrm{R}_{107}$ & R. 40 & R16.6 & R5.83 & $\mathrm{R}_{6.42}$ & $\mathrm{R}_{29.2}$ & R471 & R9.57 & $\mathrm{R}_{41.0}$ & $\mathrm{R}_{20.04}$ & $\mathrm{R}_{34.3}$ \\
\hline 2008 & R575 & $\mathrm{R}_{3.34}$ & $R_{31.5}$ & $\mathrm{R}_{106}$ & R. .39 & $\mathrm{R}_{15.9}$ & 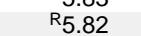 & $\mathrm{R}^{\mathrm{R}} 6.40$ & $\mathrm{R}_{27.7}$ & R509 & $\mathrm{R}_{10.30}$ & $\mathrm{R}_{43.8}$ & $\mathrm{R}_{20.43}$ & $R_{34.2}$ \\
\hline 2009 & R642 & $\begin{array}{r}\text { r.ju } \\
\mathrm{R}_{3.72}\end{array}$ & R33.7 & R93 & $\begin{array}{r}.05 \\
\text { R. } 34\end{array}$ & $\begin{array}{r}\text { R13.8 } \\
R_{13}\end{array}$ & $\begin{array}{r}.02 \\
\mathrm{R} 5.64\end{array}$ & $\begin{array}{r}\mathrm{R} \\
\mathrm{R} 6.21\end{array}$ & $\begin{array}{r}26.1 \\
\mathrm{R}_{2}\end{array}$ & $\begin{array}{r}\text { R } \\
\text { R }\end{array}$ & R9.78 & $\begin{array}{r}4.0 \\
R_{43.8}\end{array}$ & $\begin{array}{r}20.05 \\
R_{20}\end{array}$ & $\begin{array}{r}3.2 \\
R_{3} 3.6\end{array}$ \\
\hline 2010 & $\begin{array}{r}\text { R739 } \\
\text {. }\end{array}$ & $\begin{array}{r}R_{4} .29 \\
\end{array}$ & R37.1 & $R_{115}$ & R. 42 & $\mathrm{R}_{15.4}$ & $\begin{array}{l}\text { R5. } \\
\text { R }\end{array}$ & $\begin{array}{l}\text { R5. } \\
\text { R }\end{array}$ & $\begin{array}{l}24.1 \\
R_{2}\end{array}$ & R478 & R9.62 & $\begin{array}{r}4.0 \\
\mathrm{R}_{4}\end{array}$ & R20.27 & R33.9 \\
\hline $2011^{P}$ & 646 & 3.74 & 31.7 & 111 & .41 & 14.3 & 4.86 & 5.33 & 20.6 & 470 & 9.47 & 43.2 & 18.95 & 30.3 \\
\hline
\end{tabular}

1 Includes those quantities for which royalties were paid based on the value of the natural gas plant gas plant liquids were produced; however, the royalties paid were based on the value of natural gas processed. These lattr quanilies ari however, with natural gas.

2 Sales and production volumes are for marketed production. See "Natural Gas Marketed Production" in Glossary.

${ }^{3}$ Excludes waste coal. See "Waste Coal" in Glossary.

4 The sum of crude oil and lease condensate, natural gas plant liquids, natural gas, and coal.

5 Sales of fossil fuels produced in offshore and onshore areas the Federal Government owns or administers, including American Indian lands.

${ }^{6}$ Sales volumes are reported for the fiscal year in which the sales occurred as opposed to the date of the royalty payment. Volumes include fossil fuels for which royalties were paid, as well as those amounts exempt from royalty payments, such as additions to the Strategic Petroleum Reserve.

7 The U.S. Government's fiscal year runs from October 1 through September 30. The fiscal year is designated by the calendar year in which it ends.

$\mathrm{R}=$ Revised. P=Preliminary.

Note: Totals may not equal sum of components due to independent rounding.

Sources: Sales, Physical Data (Columns 1, 4, 7, and 10): U.S. Department of the Interior, Office of Natural Resources Revenue (ONRR), data for "Sales Year" (as opposed to "Accounting Year") revenue and non-revenue sales volumes (as of Feb. 6, 2012). For natural gas, the ONRR "Gas" data have been adjusted to remove nitrogen (using unpublished ONRR data). See http://www. onrr.gov/ONRRWebStats/Disbursements_Royalties.aspx?report=AllReportedRoyaltyRevenues\&yeartype=
FY\&year=2011\&datetype=PY and http://www.onrr.gov/ONRRWebStats/Disbursements_Royalties.aspx? eport=AllNonRevenueVolumesByCategoryAndCommodity\&yeartype $=F Y \& y e a r=2011 \&$ datetype $=P Y$.

Sales, Btu Data (Columns 2, 5, 8, 11, and 13): Calculated by the U.S. Energy Information Administration (EIA). Monthly estimates of the ONRR physical sales data are created by dividing the fiscal-year values by 12. These monthly estimates are converted to Btu using the appropriate heat content factors in Appendix A (crude oil and natural gas plants liquids production factors in Table A2; natural gas marketed production factors in Table A4; and coal production factors in Table A5). For the individual fuels, fiscal-year Btu estimates are calculated by summing the October-September monthly Btu values for each fiscal year. For total fossil fuels, the fiscal-year Btu estimates are the sum of the fiscal-year Btu values for crude oil and lease condensate, natural gas plant liquids, natural gas, and coal. Sales As Share of Total U.S. Production: Calculated by EIA by dividing fiscal-year Btu data for sales by fiscal-year Btu data for total U.S. production, then multiplying by 100 . For crude oil and lease condensate total U.S. production, monthly values from the Monthly Energy Review (MER) (May 2012), Table 3.1, are converted to Btu using the crude oil production factors in Table A2. For natural gas plant liquids total U.S. production, monthly values from the MER (May 2012). Table 3.1, are converted to Btu using the natural gas plant liquids production factors in Table A2. For natural gas total U.S. marketed production, monthly values from the MER (May 2012) Table 4.1, are converted to Btu using the natural gas marketed production factors in Table A4. For coal total U.S. production monthly values from the MER (May 2012), Table 6.1, are converted to Btu using the coal production factors in Table A5. For the individual fuels fiscal-year total u. calculated by summing the October-September monthly Btu values for each fiscal year. For fossil fuels total U.S. production, the fiscal-year Btu values are the sum of the fiscal-year total U.S. production Btu values for crude oil and lease condensate, natural gas plant liquids, natural gas, and coal. 


\section{Figure 1.15 Non-Combustion Use of Fossil Fuels}

\section{Total, 1980-2011}

$8-$

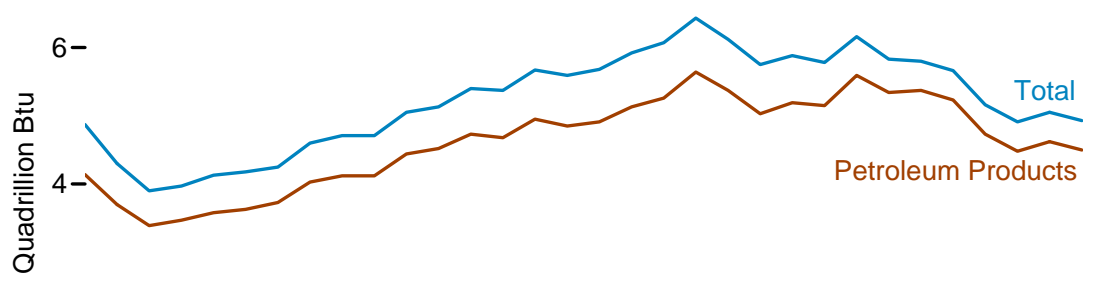

2-

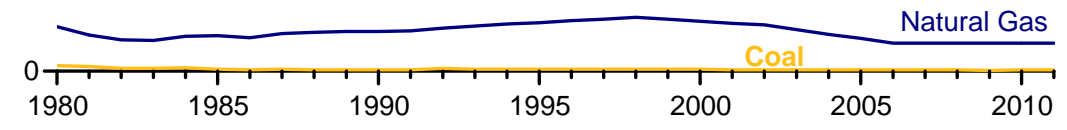

By Fuel, 2011

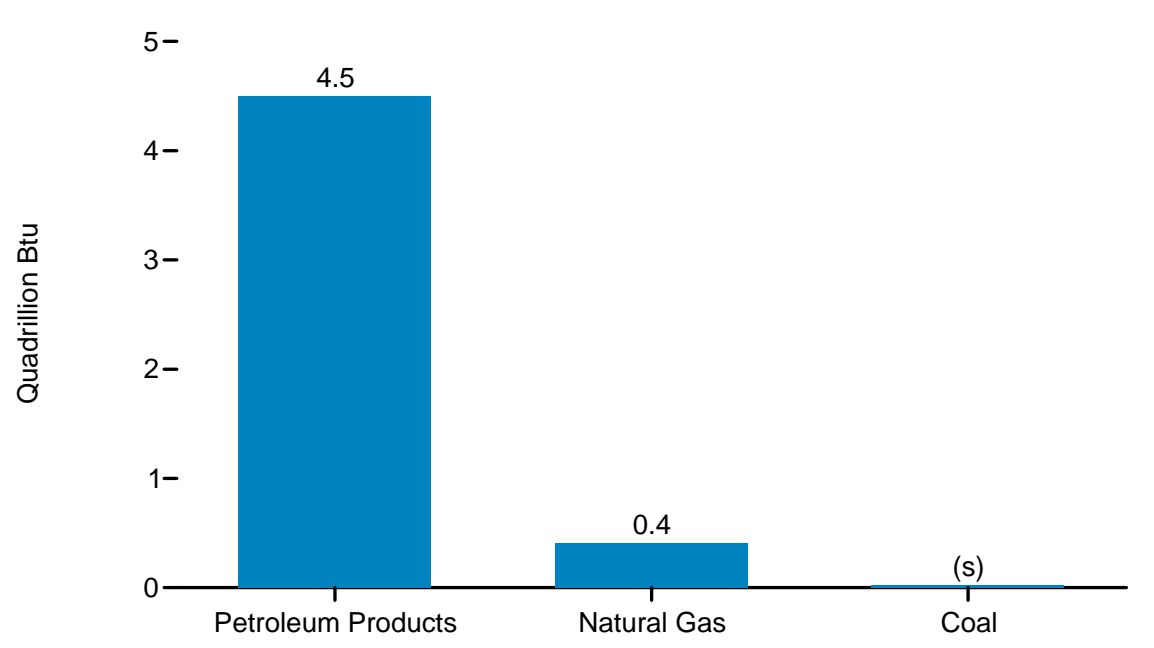

As Share of Total Energy Consumption, 1980-2011

$9-$

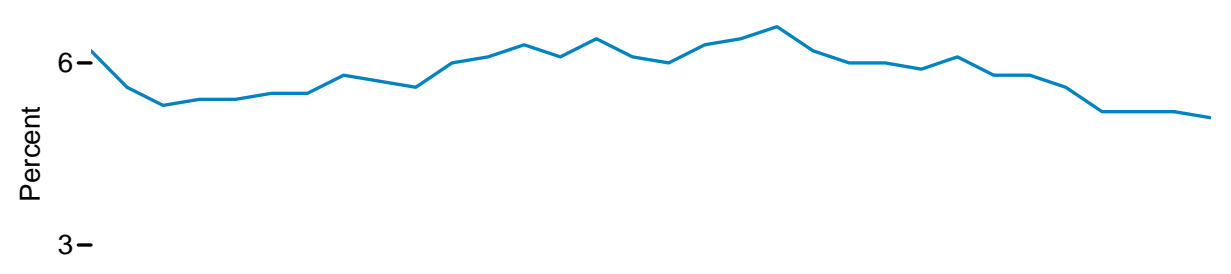

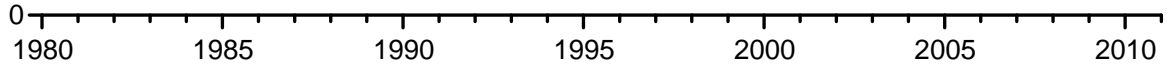

By Petroleum Product, 2011

$2.4-$

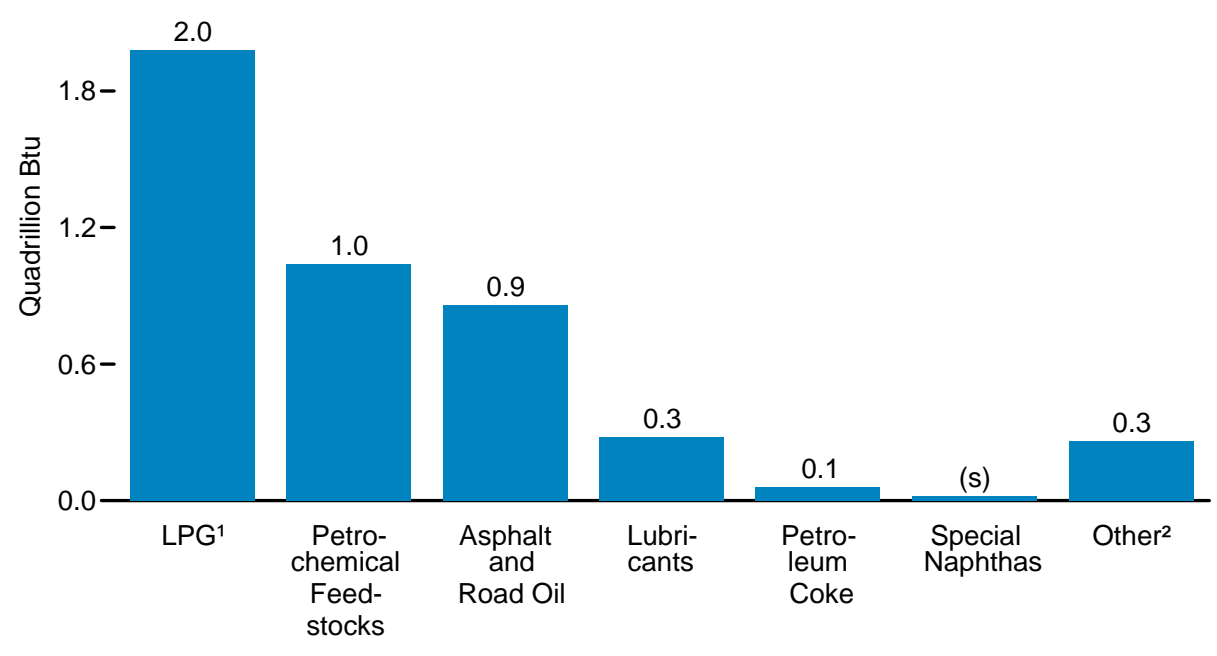

(s)=Less than 0.05 quadrillion Btu.

Note: See Note 2, "Non-Combustion Use of Fossil Fuels" at end of section

Source: Table 1.15 .

${ }^{2}$ Distillate fuel oil, residual fuel oil, waxes, and miscellaneous products. 
Table 1.15 Non-Combustion Use of Fossil Fuels, Selected Years, 1980-2011

\begin{tabular}{|c|c|c|c|c|c|c|c|c|c|c|c|c|}
\hline \multirow[b]{2}{*}{ Year } & \multicolumn{8}{|c|}{ Petroleum Products } & \multirow[b]{2}{*}{$\begin{array}{l}\text { Natural } \\
\text { Gas }{ }^{4}\end{array}$} & \multirow[b]{2}{*}{ Coal } & \multirow[b]{2}{*}{ Total } & \multirow[b]{2}{*}{$\begin{array}{l}\text { Percent of } \\
\text { Total Energy } \\
\text { Consumption }\end{array}$} \\
\hline & $\begin{array}{l}\text { Asphalt } \\
\text { and } \\
\text { Road Oil }\end{array}$ & $\begin{array}{l}\text { Liquefied } \\
\text { Petroleum } \\
\text { Gases }{ }^{1}\end{array}$ & Lubricants & $\begin{array}{c}\text { Petro- } \\
\text { chemical } \\
\text { Feedstocks }{ }^{2}\end{array}$ & $\begin{array}{l}\text { Petroleum } \\
\text { Coke }\end{array}$ & $\begin{array}{l}\text { Special } \\
\text { Naphthas }\end{array}$ & Other ${ }^{3}$ & Total & & & & \\
\hline & \multicolumn{12}{|c|}{ Physical Units 5} \\
\hline 1980 & 145 & 230 & 58 & 253 & $R_{14}$ & 37 & 58 & R795 & 639 & 2.4 & -- & -- \\
\hline 1985 & 156 & R278 & 53 & 144 & $R_{16}$ & 30 & 41 & R719 & 500 & $\begin{array}{l}2.4 \\
1.1\end{array}$ & -- & -- \\
\hline $\begin{array}{l}1990 \\
1991\end{array}$ & 176 & R373 & $\begin{array}{l}60 \\
63\end{array}$ & 199 & 20 & 20 & 39 & $\begin{array}{l}\text { R887 } \\
8892\end{array}$ & R567 & .6 & -- & $=-$ \\
\hline $\begin{array}{l}1991 \\
1992\end{array}$ & $\begin{array}{l}162 \\
166\end{array}$ & $\begin{array}{l}\text { R426 } \\
\text { R448 }\end{array}$ & $\begin{array}{l}53 \\
54\end{array}$ & $\begin{array}{l}203 \\
214\end{array}$ & $\begin{array}{r}17 \\
R 28\end{array}$ & $\begin{array}{l}17 \\
20\end{array}$ & $\begin{array}{l}44 \\
35\end{array}$ & $\begin{array}{l}\text { R922 } \\
\text { R966 }\end{array}$ & $\begin{array}{r}573 \\
\mathrm{R} 606\end{array}$ & $\begin{array}{r}.6 \\
12\end{array}$ & $\begin{array}{l}-- \\
--\end{array}$ & $\begin{array}{l}-- \\
--\end{array}$ \\
\hline 1993 & 174 & R436 & 55 & 216 & $\mathrm{R} 18$ & 20 & $\begin{array}{l}55 \\
35\end{array}$ & R955 & $\mathrm{R}_{640}$ & $\begin{array}{l}.2 \\
.9\end{array}$ & -- & -- \\
\hline 1994 & 176 & R483 & 58 & $\begin{array}{l}224 \\
224\end{array}$ & $\mathrm{R}_{21}$ & 15 & $\begin{array}{l}55 \\
35\end{array}$ & $R_{1,013}$ & 673 & .9 & -- & -- \\
\hline 1995 & 178 & R479 & 57 & 215 & $\mathrm{R}_{20}$ & 13 & 33 & R996 & $\begin{array}{l}\text { R } 695 \\
\text { R }\end{array}$ & .9 & -- & $-\overline{-}$ \\
\hline 1996 & 177 & R502 & 55 & 217 & $\mathrm{R}_{20}$ & 14 & 33 & $\mathrm{R}_{1,019}$ & R718 & .9 & -- & -- \\
\hline 1997 & 184 & R501 & 58 & 250 & $R_{15}$ & 14 & 34 & $\mathrm{R}_{1}, 056$ & R740 & .9 & -- & -- \\
\hline 1998 & 190 & R485 & 61 & 252 & 25 & 20 & $\begin{array}{l}34 \\
39\end{array}$ & $\mathrm{R}_{1}, 073$ & 762 & .8 & -- & -- \\
\hline 1999 & 200 & R566 & 62 & 238 & 36 & 28 & 37 & $\mathrm{R}_{1}, 166$ & R736 & .8 & -- & -- \\
\hline 2000 & 192 & R545 & 61 & 243 & 16 & $\begin{array}{l}20 \\
19\end{array}$ & 38 & $R_{1}, 114$ & R710 & .8 & -- & -- \\
\hline 2001 & 189 & R492 & 56 & 214 & 29 & 15 & 39 & $R_{1}, 034$ & R683 & .7 & -- & -- \\
\hline 2002 & 187 & R526 & 55 & 229 & 24 & 20 & 38 & $\mathrm{R}_{1}, 078$ & 657 & .7 & -- & -- \\
\hline 2003 & 184 & R511 & 51 & 247 & 20 & 15 & 36 & $\mathrm{R}_{1}, 064$ & R592 & .7 & -- & -- \\
\hline 2004 & 196 & R536 & 52 & 287 & 36 & 10 & 34 & $\mathrm{R}_{1,151}$ & R528 & .7 & -- & -- \\
\hline 2005 & 199 & R498 & 51 & 266 & 31 & 12 & 34 & $\mathrm{R}_{1,092}$ & R463 & .7 & -- & -- \\
\hline 2006 & R190 & R521 & 42 & 265 & 35 & 13 & 41 & $\mathrm{R} 1,108$ & R398 & .6 & -- & -- \\
\hline 2007 & 180 & R526 & 52 & 242 & 33 & 15 & 40 & $\mathrm{R}_{1}, 089$ & R398 & .6 & -- & -- \\
\hline 2008 & 152 & R484 & 48 & 210 & 37 & 16 & 41 & R989 & R398 & .6 & -- & -- \\
\hline 2009 & $\mathrm{R} 132$ & R532 & 43 & 185 & R30 & 9 & 41 & R972 & R398 & .4 & -- & -- \\
\hline \multirow{3}{*}{$2011^{P}$} & 132 & R581 & 48 & R196 & $R_{10}$ & 5 & 43 & $\mathrm{R}_{1,015}$ & R398 & .6 & -- & -- \\
\hline & 130 & 575 & 46 & 187 & 11 & 4 & 44 & 996 & 398 & .6 & -- & -- \\
\hline & \multicolumn{12}{|c|}{ Quadrillion Btu } \\
\hline 1980 & 0.96 & 0.78 & 0.35 & 1.43 & $\mathrm{R}_{0} 0.09$ & 0.19 & 0.34 & R4.14 & 0.65 & 0.08 & R4.87 & $\mathrm{R}_{6.2}$ \\
\hline 1985 & 1.03 & R. 96 & .32 & .82 & R. 10 & .16 & .24 & 3.63 & .52 & .03 & 4.18 & 5.5 \\
\hline 1990 & 1.17 & R1.33 & .36 & 1.12 & .12 & .11 & .23 & R4.44 & R. .58 & .02 & R5.05 & R6.0 \\
\hline 1991 & 1.08 & R1.52 & .32 & 1.15 & .11 & .09 & .26 & R4.52 & .59 & .02 & R5.13 & R6.1 \\
\hline 1992 & 1.10 & R1.61 & .33 & 1.20 & .17 & .10 & .21 & R4.73 & R. 63 & .04 & $\begin{array}{l}85.40 \\
85.07\end{array}$ & R6.3 \\
\hline 1993 & 1.15 & $\begin{array}{l}81.55 \\
81.70\end{array}$ & .34 & 1.22 & $\begin{array}{l}\text { R. } 11 \\
\end{array}$ & .10 & .20 & R4.68 & R.66 & .03 & R5.37 & R6.1 \\
\hline 1994 & $\begin{array}{l}1.17 \\
1.18\end{array}$ & $\begin{array}{l}\text { R.1.75 } \\
\text { R1 } 72\end{array}$ & $\begin{array}{l}.35 \\
35\end{array}$ & 1.26 & R.13 & .08 & .20 & 4.95 & .69 & .03 & 5.67 & 6.4 \\
\hline $\begin{array}{l}1995 \\
1996\end{array}$ & $\begin{array}{l}1.18 \\
1.18\end{array}$ & $\begin{array}{l}\mathrm{R} 1.72 \\
\mathrm{R} 1 \mathrm{R}\end{array}$ & .35 & $\begin{array}{l}1.21 \\
1\end{array}$ & $\begin{array}{l}\text { R.12 } \\
\text { R.12 }\end{array}$ & .07 & .20 & $\begin{array}{l}\text { R4.85 } \\
{ }^{2}\end{array}$ & R.71 & .03 & $\begin{array}{l}85.59 \\
\end{array}$ & ${ }^{R 6} 6.1$ \\
\hline $\begin{array}{l}1996 \\
1997\end{array}$ & $\begin{array}{l}1.18 \\
122\end{array}$ & $\begin{array}{l}\text { R1.80 } \\
\text { R1 } 80\end{array}$ & $\begin{array}{l}.34 \\
34\end{array}$ & 1.21 & & .07 & .20 & $\begin{array}{l}4.91 \\
\end{array}$ & $\begin{array}{l}\text { R. } 74 \\
\text { R.74 }\end{array}$ & .03 & $\begin{array}{l}55.68 \\
05\end{array}$ & R6.0 \\
\hline 1998 & $\begin{array}{l}1.22 \\
1.26\end{array}$ & $\begin{array}{r}1.80 \\
1.73\end{array}$ & $\begin{array}{l}.35 \\
37\end{array}$ & $\begin{array}{l}1.40 \\
1.40\end{array}$ & $\begin{array}{l}\text { R.09 } \\
.15\end{array}$ & $\begin{array}{l}.07 \\
11\end{array}$ & .20 & $\begin{array}{l}55.13 \\
\text { R5.26 }\end{array}$ & $\begin{array}{r}.76 \\
79\end{array}$ & $\begin{array}{l}.03 \\
.03\end{array}$ & $\begin{array}{l}\text { R5.92 } \\
\text { R6 }\end{array}$ & $\begin{array}{r}6.3 \\
R_{6} .4\end{array}$ \\
\hline 1999 & 1.32 & $\mathrm{R}_{2.04}$ & .37 & 1.30 & .22 & .15 & .22 & $\begin{array}{r}\mathrm{R} 5.60 \\
\end{array}$ & R. 76 & $\begin{array}{l}.03 \\
.03\end{array}$ & $\mathrm{R}_{6.43}$ & $\begin{array}{r}0.4 \\
\mathrm{R} 6.6\end{array}$ \\
\hline 2000 & 1.28 & $\mathrm{R}_{1.96}$ & .37 & 1.35 & .10 & .10 & .22 & R5.37 & R. .73 & .03 & $\mathrm{R}_{6.12}$ & $\mathrm{R}_{6.2}$ \\
\hline 2001 & 1.26 & R1.76 & .34 & 1.19 & .17 & .08 & .23 & R5.03 & R. .70 & .02 & R5.75 & $\mathrm{R}_{6.0}$ \\
\hline 2002 & 1.24 & R1.87 & .33 & 1.27 & .15 & .10 & 22 & R5.19 & .68 & .02 & R5.88 & R6.0 \\
\hline 2003 & 1.22 & $\mathrm{R}_{1} .83$ & .31 & 1.37 & .12 & .08 & .21 & R5.15 & R. 61 & .02 & $\begin{array}{r}R 5.78 \\
\end{array}$ & R5.9 \\
\hline 2004 & 1.30 & $\mathrm{R}_{1} .92$ & .31 & 1.59 & .22 & .05 & .20 & R5.59 & R.54 & .02 & R6.16 & 6.1 \\
\hline 2005 & 1.32 & $\mathrm{R}_{1.78}$ & .31 & 1.47 & .19 & .06 & .20 & R5.34 & R. 48 & .02 & R5.83 & 5.8 \\
\hline 2006 & 1.26 & R1.85 & .25 & 1.48 & .21 & .07 & .24 & R5.37 & R. 41 & .02 & R5.80 & 5.8 \\
\hline 2007 & 1.20 & R1.86 & .31 & 1.35 & .20 & .08 & .24 & R5.23 & R.41 & .02 & R5.66 & $\begin{array}{r}5.6 \\
85\end{array}$ \\
\hline 2008 & 1.01 & R1.70 & .29 & 1.17 & .23 & .08 & .24 & R4.73 & R. 41 & .02 & R5.16 & R5. \\
\hline 2009 & .87 & R1.85 & .26 & 1.03 & .18 & .05 & .24 & R4.48 & R. 41 & .01 & 4.91 & 5.2 \\
\hline 2010 & .88 & $\mathrm{R}_{2.02}$ & .29 & 1.09 & R. 06 & .03 & .25 & R4.62 & R. 41 & .02 & R5.05 & R5.2 \\
\hline $2011^{P}$ & .86 & 1.98 & .28 & 1.04 & .06 & .02 & .26 & 4.50 & .41 & .02 & 4.93 & 5.1 \\
\hline
\end{tabular}

${ }_{1}^{1}$ Liquefied petroleum gases and pentanes plus are aggregated to avoid disclosure of proprietary information.
2 Includes still gas not burned as refinery fuel.

Distillate fuel oil, residual fuel oil, waxes, and miscellaneous products.

4 U.S. Energy Information Administration (EIA) has altered the methodology for the natural gas estimates. The estimates are linearly interpolated between Manufacturing Energy Consumption Survey 5 Petroleum - million barrels; natural gas - billion cubic feet; and coal-million short tons.

R=Revised. P=Preliminary. $--=$ Not applicable.

Notes: - Estimates of consumption for non-combustion use shown in this table are included in total energy consumption (see Table 1.3). - See Note 2, "Non-Combustion Use of Fossil Fuels," at end of
section. - Because of changes in methodology, data series may be revised annually. • Estimates of

non-combustion use in this table are considered industrial uses with the exception of approximately half of he lubricants which are considered transportation use. - Totals may not equal sum of components due to Web Pages: • See http://www.eia.gov/totalenergy/data/annual/\#summary for all data beginning in 1980 (For related information, see http://www.ela.gov/environment.

Sources. Petroleum Products: - 1980-EIA, Energy Data Reports, Petroleum Statement, Annual and Sales or Liquelfed Manufactures, Hydrocarbon, Coal, and Coke Materials Consumed. - 1981 forward-U.S. Department of Commerce. Coal: - 1980 forward-EIA estimates based on the methodology underlying the nonfue emissions calculations in ElA's Emissions of Greenhouse Gases in the United States 2008. Percent of 


\section{Energy Overview}

Note 1. Noncombustible Renewable Energy. Noncombustible renewable energy is the sum of hydroelectric power, geothermal, solar/PV, and wind. In Table 1.3, total primary consumption of noncombustible renewable energy is reported as the sum of "Captured Energy” and the "Adjustment for Fossil Fuel Equivalence."

Captured energy represents the energy from noncombustible renewable resources that is actually "captured" for final use. It includes the electricity generated from noncombustible resources (i.e., net generation from Table 8.2a converted to Btu using the energy conversion factor of $3,412 \mathrm{Btu} / \mathrm{kWh}$ ) and the direct consumption of noncombustible renewable energy. Direct consumption of noncombustible renewable energy includes: solar thermal direct use energy, residential and commercial self-generated photovoltaic energy, geothermal energy from heat pumps, and direct use of geothermal energy.

The adjustment for fossil-fuel equivalence represents the energy losses that would have occurred if electricity from noncombustible renewable resources had been generated using the average fossil-fuel mix in a given year. The fossil-fuel equivalent value is determined by converting electricity generation to Btu using the average fossil-fuel heat rate from Table A6. The "Adjustment for Fossil Fuel Equivalence" is then calculated as the difference between the fossil-fuel equivalent value of electricity generated and "captured" electricity generation.

For more information, see Appendix F.

Note 2. Non-Combustion Use of Fossil Fuels. Most fossil fuels consumed in the United States and elsewhere are combusted to produce heat and power. However, some are used directly for non-combustion use as construction materials, lubricants, chemical feedstocks, solvents, and waxes. For example, asphalt and road oil are used for roofing and paving; liquefied petroleum gases are used to create intermediate products that are used in making plastics; lubricants, including motor oil and greases, are used in vehicles and various industrial processes; petrochemical feedstocks are used to make plastics, synthetic fabrics, and related products; and natural gas is used to make nitrogenous fertilizers and as feedstock in the chemical industry. Estimates of non-combustion use of fossil fuels are based on the methodology underlying the nonfuel emissions calculations in EIA's "Emissions of Greenhouse Gases in the United States," Chapter 2, at http://www.eia.gov/environment/emissions/ghg_report/. 


\section{Energy Consumption by Sector}




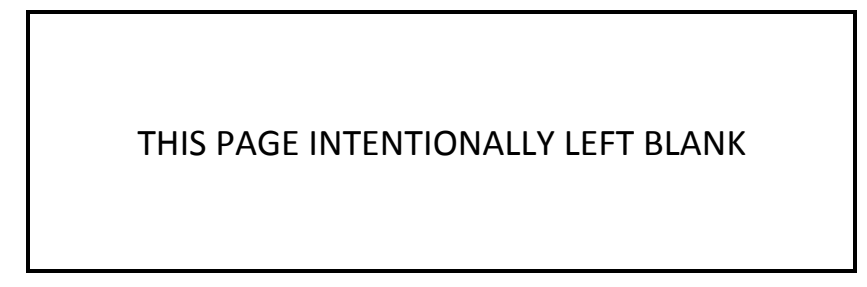


Figure 2.0 Primary Energy Consumption by Source and Sector, 2011

(Quadrillion Btu)

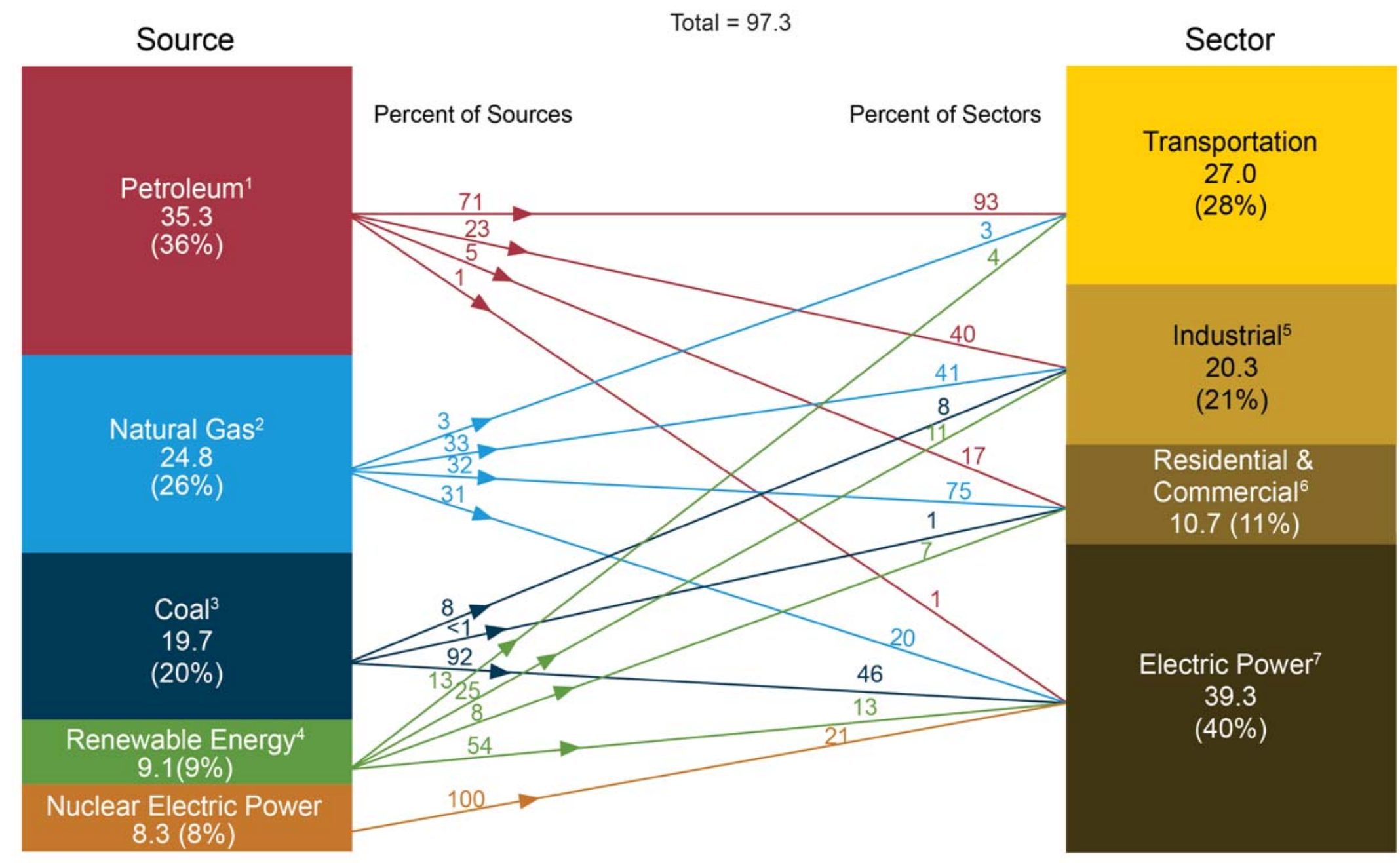

${ }^{1}$ Does not include biofuels that have been blended with petroleum—biofuels are included in "Renewable Energy."

${ }^{2}$ Excludes supplemental gaseous fuels.

${ }^{3}$ Includes less than 0.1 quadrillion Btu of coal coke net imports.

${ }^{4}$ Conventional hydroelectric power, geothermal, solar/photovoltaic, wind, and biomass.

${ }^{5}$ Includes industrial combined-heat-and-power (CHP) and industrial electricity-only plants

${ }^{6}$ Includes commercial combined-heat-and-power (CHP) and commercial electricity-only

${ }^{7}$ Electricity-only and combined-heat-and-power (CHP) plants whose primary business is to sell electricity, or electricity and heat, to the public. Includes 0.1 quadrillion Btu of electricity net imports not shown under "Source."

Notes: Primary energy in the form that it is first accounted for in a statistical energy balance before any transformation to secondary or tertiary forms of energy (for example, coal is used to generate electricity). - Sum of components may not equal total due to independent rounding.

Sources: U.S. Energy Information Administration, Annual Energy Review 2011, Tables 1.3 plants.

$2.1 \mathrm{~b}-2.1 \mathrm{f}, 10.3$, and 10.4 
Figure 2.1a Energy Consumption Estimates by Sector Overview

\section{Total Consumption by End-Use Sector, 1949-2011}

$40-$

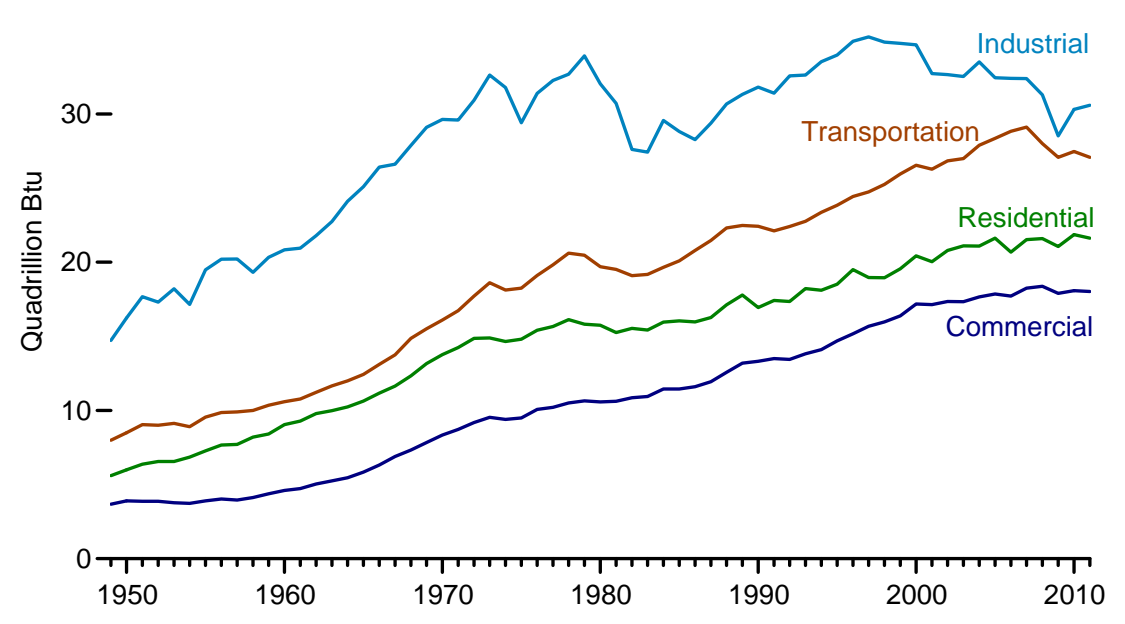

Primary and Total Consumption by Sector, 2011

$50-$

Primary Consumption $\square$ Total Consumption

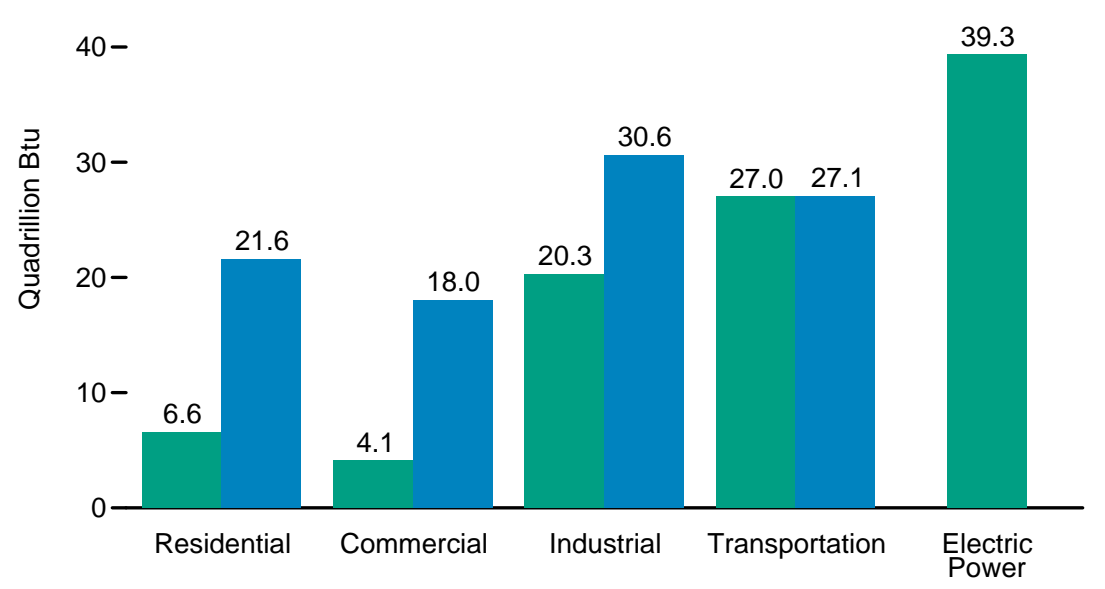

\section{End-Use Sector Shares of Total Consumption, 2011}

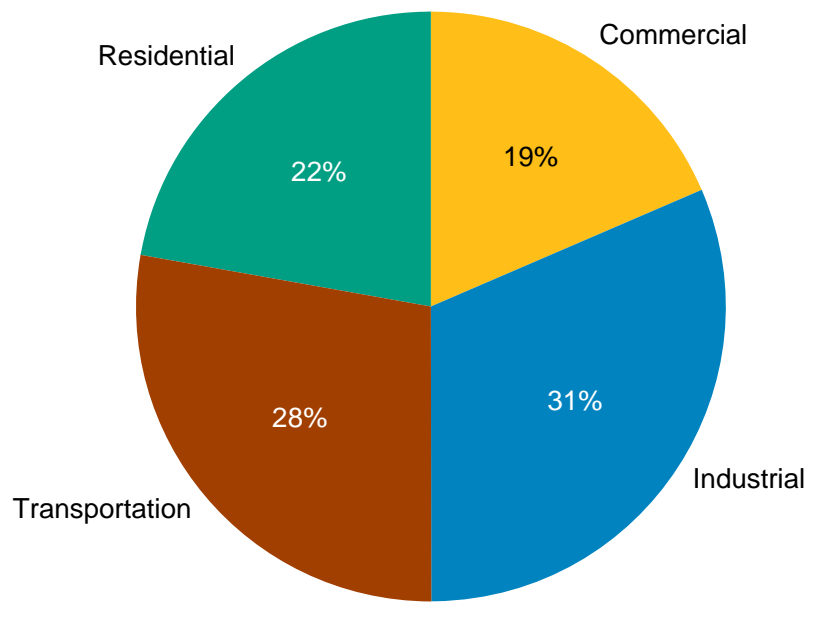

Electric Power Sector, 1949-2011

25-

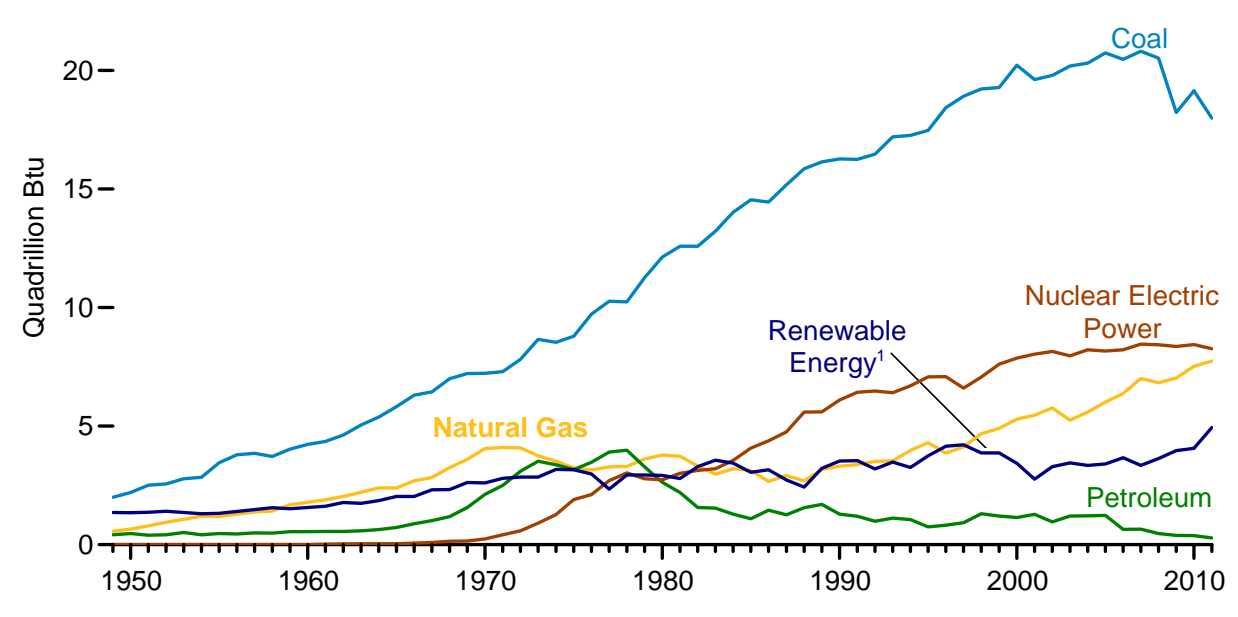

${ }^{1}$ Conventional hydroelectric power, geothermal, solar/photovoltaic, wind, and biomass. Note: - See "Primary Energy Consumption" in Glossary. • Sum of components may not Sources: Tables 2.1a and 2.1f. equal 100 percent due to independent rounding. 


\section{Figure 2.1b Energy Consumption Estimates by End-Use Sector, 1949-2011}

\section{Residential, By Major Source}

12-

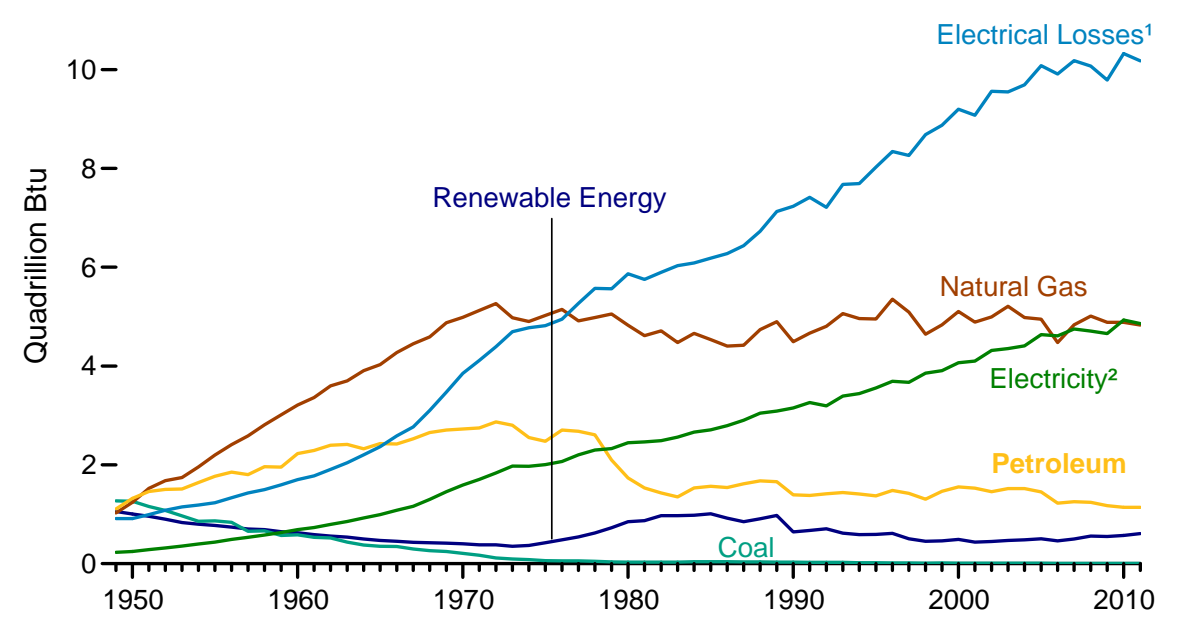

\section{Industrial, By Major Source}

$12-$

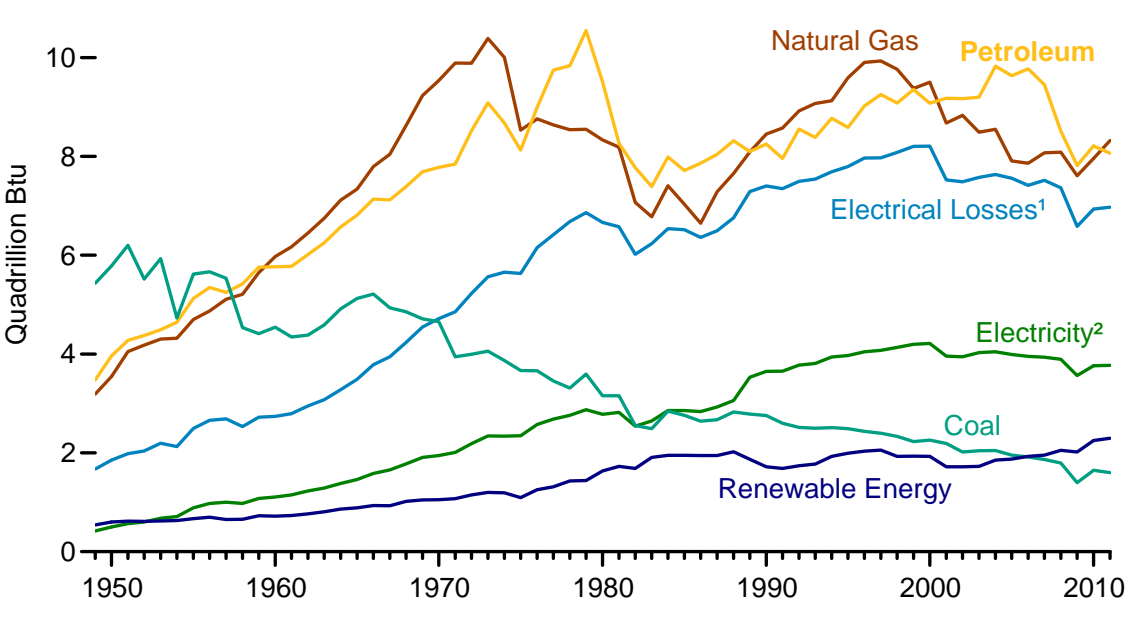

${ }^{1}$ Electrical system energy losses associated with the generation, transmission, and distribution of energy in the form of electricity.

\section{Commercial, By Major Source}

$12-$

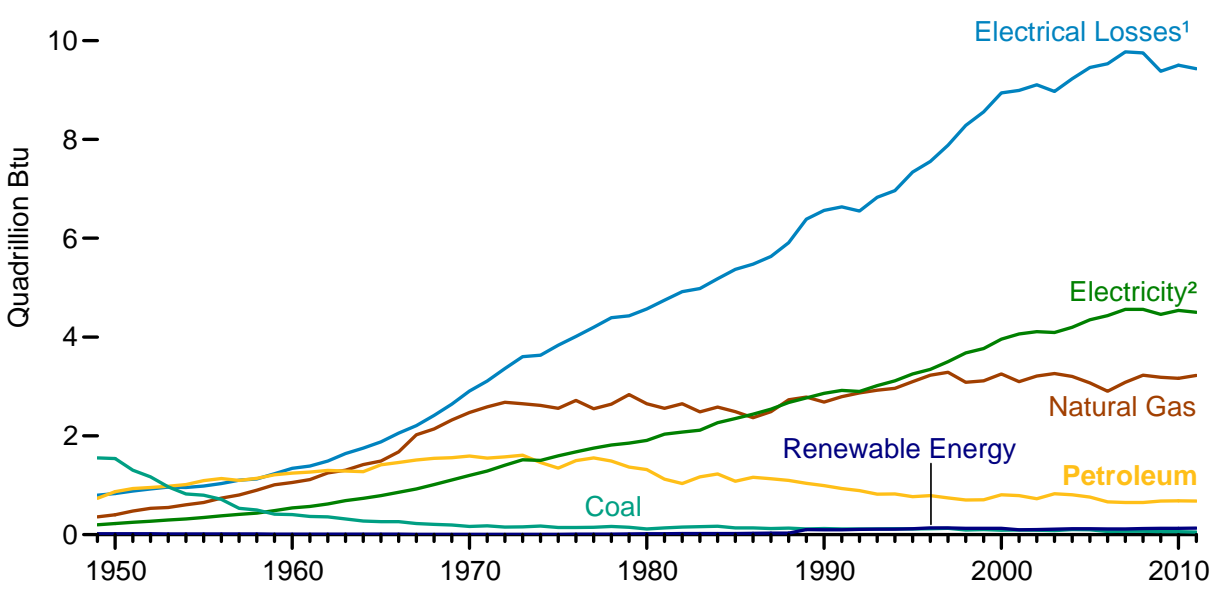

\section{Transportation, By Major Source}

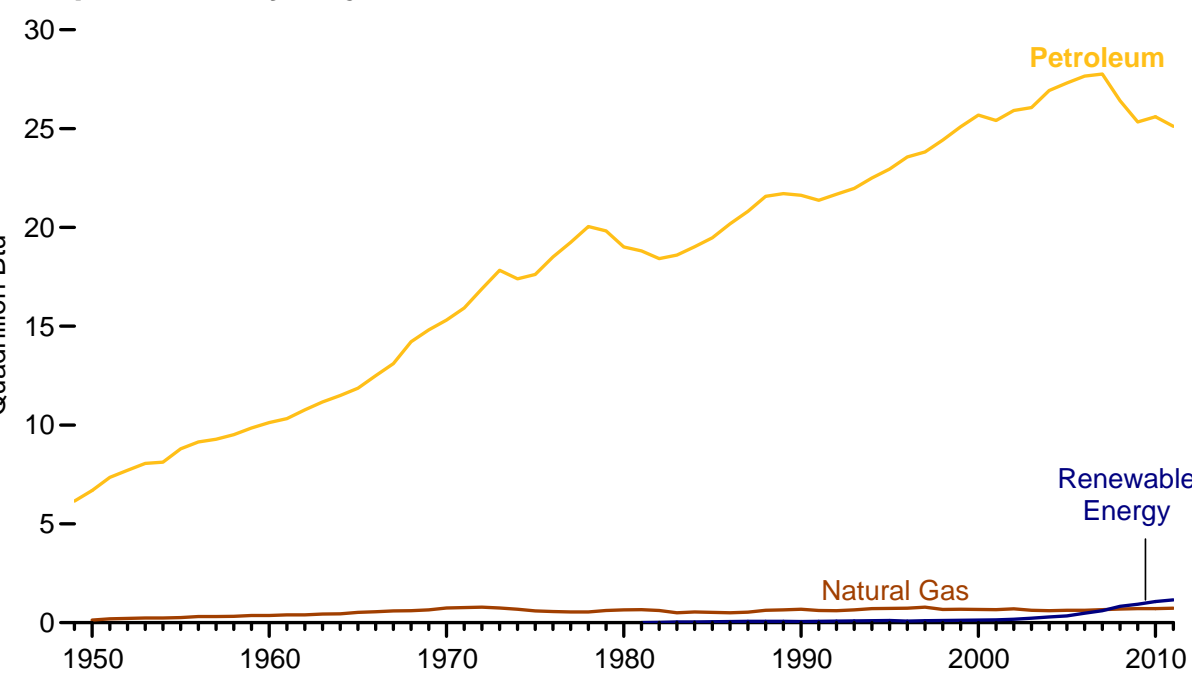

${ }^{2}$ Electricity retail sales.

Sources: Tables 2.1b-2.1e. 
Table 2.1a Energy Consumption Estimates by Sector, Selected Years, 1949-2011

(Trillion Btu)

\begin{tabular}{|c|c|c|c|c|c|c|c|c|c|c|c|}
\hline \multirow[b]{3}{*}{ Year } & \multicolumn{8}{|c|}{ End-Use Sectors } & \multirow{3}{*}{$\begin{array}{c}\begin{array}{c}\text { Electric } \\
\text { Power } \\
\text { Sector }{ }^{3,4}\end{array} \\
\text { Primary } 5\end{array}$} & \multirow{3}{*}{$\begin{array}{l}\text { Balancing } \\
\text { Item } 7\end{array}$} & \multirow{3}{*}{$\begin{array}{c}\text { Total } \\
\text { Primary }\end{array}$} \\
\hline & \multicolumn{2}{|c|}{ Residential } & \multicolumn{2}{|c|}{ Commercial ${ }^{1}$} & \multicolumn{2}{|c|}{ Industrial 2} & \multicolumn{2}{|c|}{ Transportation } & & & \\
\hline & Primary ${ }^{5}$ & Total 6 & Primary ${ }^{5}$ & Total 6 & Primary ${ }^{5}$ & Total 6 & Primary ${ }^{5}$ & Total 6 & & & \\
\hline 1949 & 4,460 & 5,599 & 2,669 & 3,669 & 12,633 & 14,724 & 7,880 & 7,990 & 4,339 & (s) & 31,982 \\
\hline 1950 & 4,829 & 5,989 & 2,834 & 3,893 & 13,890 & 16,241 & 8,383 & 8,492 & 4,679 & (s) & 34,616 \\
\hline 1955 & 5,608 & 7,278 & 2,561 & 3,895 & 16,103 & 19,485 & 9,474 & 9,550 & 6,461 & (s) & 40,208 \\
\hline 1960 & 6,651 & 9,039 & 2,723 & 4,609 & 16,996 & 20,842 & 10,560 & 10,596 & 8,158 & (s) & 45,086 \\
\hline 1965 & 7,279 & 10,639 & 3,177 & 5,845 & 20,148 & 25,098 & 12,399 & 12,432 & 11,012 & (s) & 54,015 \\
\hline 1970 & 8,322 & 13,766 & 4,237 & 8,346 & 22,964 & 29,628 & 16,062 & 16,098 & 16,253 & (s) & 67,838 \\
\hline 1975 & 7,990 & 14,813 & 4,059 & 9,492 & 21,434 & 29,413 & 18,210 & 18,245 & 20,270 & 1 & 71,965 \\
\hline 1976 & 8,391 & 15,410 & 4,371 & 10,063 & 22,665 & 31,393 & 19,067 & 19,101 & 21,473 & 8 & 75,975 \\
\hline 1977 & 8,194 & 15,662 & 4,258 & 10,208 & 23,165 & 32,263 & 19,786 & 19,822 & 22,551 & 7 & 77,961 \\
\hline 1978 & 8,260 & 16,132 & 4,309 & 10,512 & 23,244 & 32,688 & 20,583 & 20,617 & 23,553 & 2 & 79,950 \\
\hline 1979 & 7,919 & 15,813 & 4,366 & 10,648 & 24,192 & 33,925 & 20,437 & 20,472 & 23,943 & 2 & 80,859 \\
\hline 1980 & 7,439 & 15,753 & 4,105 & 10,578 & 22,595 & 32,039 & 19,659 & 19,697 & 24,269 & -1 & 78,067 \\
\hline 1981 & 7,045 & 15,262 & 3,837 & 10,616 & 21,318 & 30,712 & 19,478 & 19,514 & 24,425 & 3 & 76,106 \\
\hline 1982 & 7,147 & 15,531 & 3,864 & 10,860 & 19,053 & 27,614 & 19,052 & 19,089 & 23,979 & 4 & 73,099 \\
\hline 1983 & 6,832 & 15,425 & 3,840 & 10,938 & 18,548 & 27,428 & 19,134 & 19,177 & 24,614 & 3 & 72,971 \\
\hline $\begin{array}{l}1984 \\
1984\end{array}$ & $\begin{array}{l}7,211 \\
7,211\end{array}$ & 15,960 & $\begin{array}{l}4,001 \\
4,001\end{array}$ & 11,444 & 20,174 & 29,570 & $\begin{array}{r}19,609 \\
\end{array}$ & 19,656 & $\begin{array}{l}25,635 \\
\end{array}$ & 3 & 76,632 \\
\hline 1985 & 7,148 & 16,041 & 3,732 & 11,451 & 19,443 & 28,816 & 20,041 & 20,088 & 26,032 & -4 & 76,392 \\
\hline 1986 & 6,906 & 15,975 & 3,693 & 11,606 & 19,078 & 28,274 & 20,740 & 20,789 & 26,227 & 3 & 76,647 \\
\hline 1987 & 6,923 & 16,263 & 3,774 & 11,946 & 19,953 & 29,379 & 21,419 & 21,469 & 26,988 & -3 & 79,054 \\
\hline 1988 & 7,357 & 17,133 & 3,994 & 12,578 & 20,862 & 30,677 & 22,267 & 22,318 & 28,227 & 3 & 82,709 \\
\hline 1989 & 7,567 & 17,786 & 4,043 & 13,193 & 20,874 & 31,320 & 22,424 & 22,478 & $\begin{array}{r}429,869 \\
\end{array}$ & 9 & 84,786 \\
\hline 1990 & 6,557 & 16,945 & 3,896 & 13,320 & 21,180 & 31,810 & 22,366 & 22,420 & 30,495 & -9 & 84,485 \\
\hline 1991 & 6,747 & 17,420 & 3,945 & 13,500 & 20,824 & 31,399 & 22,065 & 22,118 & 30,856 & 1 & 84,438 \\
\hline 1992 & 6,950 & 17,356 & 3,991 & 13,441 & 21,756 & 32,571 & 22,363 & 22,415 & 30,723 & (s) & 85,783 \\
\hline 1993 & 7,146 & 18,218 & 3,973 & 13,820 & 21,753 & 32,629 & 22,715 & 22,768 & 31,847 & -10 & 87,424 \\
\hline 1994 & 6,978 & 18,112 & 4,016 & 14,098 & 22,393 & 33,521 & 23,311 & 23,366 & 32,399 & -6 & 89,091 \\
\hline 1995 & 6,936 & 18,519 & 4,101 & 14,690 & 22,719 & 33,971 & 23,791 & 23,846 & 33,479 & 3 & 91,029 \\
\hline 1996 & $\mathrm{R}_{7,467}$ & 19,504 & 4,273 & 15,172 & 23,410 & 34,904 & 24,383 & 24,437 & 34,485 & 4 & 94,022 \\
\hline 1997 & 7,033 & 18,965 & 4,295 & 15,681 & 23,686 & 35,200 & 24,695 & 24,750 & 34,886 & 6 & 94,602 \\
\hline 1998 & 6,413 & 18,955 & 4,005 & 15,968 & 23,177 & 34,843 & 25,201 & 25,256 & 36,225 & -3 & 95,018 \\
\hline 1999 & 6,775 & 19,557 & 4,053 & 16,376 & 22,950 & 34,764 & 25,891 & 25,949 & 36,976 & 6 & 96,652 \\
\hline 2000 & 7,159 & 20,425 & 4,278 & 17,175 & 22,824 & 34,664 & 26,489 & 26,548 & 38,062 & 2 & R98, 814 \\
\hline 2001 & 6,868 & 20,042 & 4,084 & 17,137 & 21,794 & 32,720 & 26,213 & 26,275 & 37,215 & -6 & 96,168 \\
\hline 2002 & $\mathrm{R}_{6,912}$ & $\mathrm{R}_{20,791}$ & $\mathrm{R}_{4,132}$ & $R_{17,345}$ & $\mathrm{R}_{21,799}$ & $R_{32,662}$ & $\mathrm{R}_{26,781}$ & $\mathrm{R}_{26,842}$ & 38,016 & 5 & R97,645 \\
\hline 2003 & 7,211 & 21,110 & 4,283 & 17,343 & 21,503 & 32,532 & 26,920 & 26,994 & 38,062 & -1 & 97,978 \\
\hline 2004 & 6,993 & 21,093 & 4,232 & 17,659 & $\mathrm{R}_{22,412}$ & $R_{33,520}$ & 27,817 & 27,895 & 38,713 & -6 & $\mathrm{R}_{1} 00,162$ \\
\hline 2005 & 6,909 & 21,626 & 4,051 & $R_{17,857}$ & $\mathrm{R}_{21,411}$ & $R_{32,446}$ & 28,272 & 28,353 & 39,638 & (s) & $\mathrm{R}_{1} 00,282$ \\
\hline 2006 & $\mathrm{R}_{6,168}$ & $\mathrm{R}_{20,688}$ & $R_{3,747}$ & $\mathrm{R}_{17,711}$ & $\mathrm{R}_{21,536}$ & $R_{32,401}$ & 28,751 & 28,830 & 39,428 & (s) & R99,629 \\
\hline 2007 & ${ }^{R} 6,598$ & $R_{21,531}$ & $\mathrm{R}_{3,922}$ & $\mathrm{R}_{18,255}$ & $\mathrm{R}_{21,370}$ & $\mathrm{R}_{32,394}$ & $\mathrm{R}_{29,029}$ & $\mathrm{R}_{29,117}$ & 40,377 & $R_{-1}$ & $\mathrm{R}_{101,296}$ \\
\hline 2008 & 6,817 & 21,596 & 4,073 & 18,381 & $\mathrm{R}_{20,480}$ & $R_{31,290}$ & 27,925 & 28,008 & 39,978 & (s) & R99,275 \\
\hline 2009 & 6,619 & $R_{21,064}$ & 4,061 & 17,899 & $\mathrm{R}_{18,813}$ & $R_{28,525}$ & $\mathrm{R}_{26,989}$ & $\mathrm{R}_{27,071}$ & 38,077 & (s) & R94,559 \\
\hline 2010 & $\mathrm{R}_{6,603}$ & $\mathrm{R}_{21,862}$ & $\mathrm{R}_{4,039}$ & $\mathrm{R}_{18,078}$ & $\mathrm{R}_{20,062}$ & R30,309 & R27,384 & R27,466 & R 39,626 & R8 & $\begin{array}{r}\text { R97,722 } \\
\end{array}$ \\
\hline $2011^{P}$ & 6,585 & 21,619 & 4,090 & 18,021 & 20,291 & 30,592 & 26,999 & 27,079 & 39,346 & -9 & 97,301 \\
\hline
\end{tabular}

Commercial sector, including commercial combined-heat-and-power (CHP) and commercial

including industrial combined-heat-and-power (CHP) and industrial electricity-only 3 Electricity-only and combined-heat-and-power (CHP) plants within the NAICS 22 category whose primary business is to sell electricity, or electricity and heat, to the public.

${ }^{4}$ Through 1988, data are for electric utilities only; beginning in 1989, data are for electric utilities and independent power producers.

See "Primary Energy Consumption" in Glossary.

Total energy consumption in the end-use sectors consists of primary energy consumption, electricity retail sales, and electrical system energy losses. See Note, "Electrical System Energy Losses," at end of
7 A balancing item. The sum of primary consumption in the five energy-use sectors equals the sum of 8 Primary energy consumption total. See Table 1.3 .

$\mathrm{R}=$ Revised. P=Preliminary. ( $\mathrm{s}$ =Less than 0.5 trillion Btu and greater than -0.5 trillion Btu.

Notes: • See Note 2, "Classification of Power Plants Into Energy-Use Sectors," at end of Section 8. - Totals may not equal sum of components due to independent rounding.

Web Pages: - See http://www.eia.gov/totalenergy/data/monthly/\#consumption for updated monthly and annual data. - See http://www.eia.gov/totalenergy/data/annual/\#consumption for all annual data beginning Sources: Tables 1.3 and $2.1 \mathrm{~b}-2.1 \mathrm{f}$ 
Table 2.1b Residential Sector Energy Consumption Estimates, Selected Years, 1949-2011

(Trillion Btu)

\begin{tabular}{|c|c|c|c|c|c|c|c|c|c|c|c|c|}
\hline \multirow[b]{3}{*}{ Year } & \multicolumn{9}{|c|}{ Primary Consumption ${ }^{1}$} & \multirow{3}{*}{$\begin{array}{c}\text { Electricity } \\
\text { Retail } \\
\text { Sales }^{8}\end{array}$} & \multirow{3}{*}{$\begin{array}{l}\text { Electrical } \\
\text { System } \\
\text { Energy } \\
\text { Losses } 9\end{array}$} & \multirow[b]{3}{*}{ Total } \\
\hline & \multicolumn{4}{|c|}{ Fossil Fuels } & \multicolumn{4}{|c|}{ Renewable Energy ${ }^{2}$} & \multirow{2}{*}{$\begin{array}{c}\text { Total } \\
\text { Primary }\end{array}$} & & & \\
\hline & Coal & Natural Gas ${ }^{3}$ & Petroleum ${ }^{4}$ & Total & Geothermal ${ }^{5}$ & Solar/PV 6 & Biomass $^{7}$ & Total & & & & \\
\hline 1949 & 1,272 & 1,027 & 1,106 & 3,405 & NA & NA & 1,055 & 1,055 & 4,460 & 228 & 911 & 5,599 \\
\hline 1950 & 1,261 & 1,240 & 1,322 & 3,824 & NA & NA & 1,006 & 1,006 & 4,829 & 246 & 913 & 5,989 \\
\hline 1955 & 867 & 2,198 & 1,767 & 4,833 & NA & NA & 775 & 775 & 5,608 & 438 & 1,232 & 7,278 \\
\hline 1960 & 585 & 3,212 & 2,227 & 6,024 & NA & NA & 627 & 627 & 6,651 & 687 & 1,701 & 9,039 \\
\hline 1965 & 352 & 4,028 & 2,432 & $\begin{array}{l}6,811 \\
6,81\end{array}$ & NA & NA & 468 & 468 & 7,279 & 993 & 2,367 & $\begin{array}{r}10,639 \\
\end{array}$ \\
\hline 1970 & 209 & 4,987 & 2,725 & 7,922 & NA & NA & 401 & 401 & 8,322 & 1,591 & 3,852 & 13,766 \\
\hline 1975 & 63 & 5,023 & 2,479 & 7,564 & NA & NA & 425 & 425 & 7,990 & 2,007 & 4,817 & 14,813 \\
\hline 1976 & 59 & 5,147 & 2,703 & 7,910 & NA & NA & 482 & 482 & 8,391 & 2,069 & 4,950 & 15,410 \\
\hline 1977 & 57 & 4,913 & 2,681 & 7,652 & NA & NA & 542 & 542 & 8,194 & 2,202 & 5,267 & 15,662 \\
\hline 1978 & 49 & 4,981 & 2,607 & 7,638 & NA & NA & 622 & 622 & 8,260 & 2,301 & 5,571 & 16,132 \\
\hline 1979 & 37 & 5,055 & $\begin{array}{l}2,099 \\
2,099\end{array}$ & $\begin{array}{l}7,190 \\
7,1\end{array}$ & NA & NA & 728 & 728 & $\begin{array}{l}7,919 \\
7,90\end{array}$ & 2,330 & 5,564 & 15,813 \\
\hline 1980 & 31 & 4,825 & 1,734 & 6,589 & NA & NA & 850 & 850 & 7,439 & 2,448 & 5,866 & 15,753 \\
\hline 1981 & 30 & 4,614 & 1,531 & 6,175 & NA & NA & 870 & 870 & 7,045 & 2,464 & 5,752 & 15,262 \\
\hline 1982 & 32 & 4,711 & 1,434 & 6,177 & NA & NA & 970 & 970 & 7,147 & 2,489 & 5,895 & 15,531 \\
\hline 1983 & 31 & 4,478 & 1,353 & 5,862 & NA & NA & 970 & 970 & 6,832 & 2,562 & 6,031 & 15,425 \\
\hline 1984 & 40 & 4,661 & 1,531 & $\begin{array}{l}6,231 \\
6,23\end{array}$ & NA & NA & 980 & 980 & 7,211 & 2,662 & $\begin{array}{l}6,087\end{array}$ & 15,960 \\
\hline $\begin{array}{l}1904 \\
1985\end{array}$ & $\begin{array}{l}40 \\
39\end{array}$ & $\begin{array}{l}4,001 \\
4,534\end{array}$ & 1,565 & $\begin{array}{l}0,201 \\
6,138\end{array}$ & NA & $N A$ & $\begin{array}{r}500 \\
1,010\end{array}$ & $\begin{array}{r}500 \\
1,010\end{array}$ & 7,148 & $\begin{array}{l}2,02 \\
2,709\end{array}$ & $\begin{array}{l}\text {, } \\
6,180\end{array}$ & $\begin{array}{l}16,041 \\
16,0\end{array}$ \\
\hline 1986 & 40 & 4,405 & 1,541 & 5,986 & NA & NA & 920 & 920 & 6,906 & 2,795 & 6,274 & 15,975 \\
\hline 1987 & 37 & 4,420 & 1,617 & 6,073 & NA & NA & 850 & 850 & 6,923 & 2,902 & 6,438 & 16,263 \\
\hline 1988 & 37 & 4,735 & 1,675 & 6,447 & NA & NA & 910 & 910 & 7,357 & 3,046 & 6,729 & 17,133 \\
\hline 1989 & 31 & 4,899 & 1,660 & 6,590 & 5 & 52 & 920 & 977 & 7,567 & 3,090 & 7,129 & 17,786 \\
\hline 1990 & 31 & 4,491 & 1,394 & 5,916 & 6 & 56 & 580 & 641 & 6,557 & 3,153 & 7,235 & 16.945 \\
\hline 1991 & 25 & 4,667 & 1,381 & $\begin{array}{l}6,073 \\
6\end{array}$ & 6 & 57 & 610 & 673 & 6,747 & 3,260 & 7,414 & 17,420 \\
\hline 1992 & 26 & 4,805 & 1,414 & 6,244 & 6 & $\mathrm{R}_{60}$ & 640 & 706 & 6,950 & 3,193 & 7,212 & 17,356 \\
\hline 1993 & 26 & 5,063 & 1,439 & 6,528 & 7 & 61 & 550 & 618 & 7,146 & 3,394 & 7,677 & 18,218 \\
\hline 1994 & 21 & 4,960 & 1,408 & 6,389 & 6 & 63 & 520 & 589 & 6,978 & 3,441 & 7,693 & 18,112 \\
\hline 1995 & 17 & 4,954 & 1,374 & $\begin{array}{l}6,345 \\
6,3\end{array}$ & 7 & 64 & 520 & 591 & 6,936 & 3,557 & $\begin{array}{l}8,026 \\
8\end{array}$ & 18,519 \\
\hline 1996 & 17 & $\begin{array}{l}4,934 \\
5,354\end{array}$ & $\begin{array}{r}1,484 \\
1,484\end{array}$ & $\begin{array}{l}0,045 \\
6,854\end{array}$ & 7 & $\begin{array}{l}04 \\
65\end{array}$ & 540 & 612 & $\begin{array}{r}\text { R7, } \\
\text { R } 4607\end{array}$ & $\begin{array}{l}5,501 \\
3,694\end{array}$ & $\begin{array}{l}\text {, U<0 } \\
8,344\end{array}$ & 19,504 \\
\hline 1997 & 16 & 5,093 & $\begin{array}{l}1,422 \\
\text { 1, }\end{array}$ & 6,531 & 8 & 64 & 430 & 502 & 7,033 & 3,671 & 8,261 & 18,965 \\
\hline 1998 & 12 & 4,646 & 1,304 & 5,962 & 8 & 64 & 380 & 452 & 6,413 & 3,856 & 8,686 & 18,955 \\
\hline 1999 & 14 & 4,835 & 1,465 & 6,314 & 9 & 63 & 390 & 461 & 6,775 & 3,906 & 8,875 & 19,557 \\
\hline 2000 & 11 & 5,105 & 1,554 & 6,670 & 9 & $\mathrm{R}_{61}$ & 420 & 489 & 7,159 & 4,069 & 9,197 & 20,425 \\
\hline 2001 & 12 & 4,889 & 1.529 & 6,430 & 9 & 59 & 370 & 438 & 6,868 & 4,100 & 9,074 & 20,042 \\
\hline 2002 & 12 & $\mathrm{R}_{4,995}$ & 1,457 & $\mathrm{R}_{6,464}$ & 10 & 57 & 380 & 448 & $\mathrm{R}_{6,912}$ & 4,317 & 9,562 & $\mathrm{R}_{20,791}$ \\
\hline 2003 & 12 & 5,209 & 1,519 & 6,741 & 13 & 57 & 400 & 470 & 7,211 & 4,353 & 9,546 & 21,110 \\
\hline 2004 & 11 & 4,981 & 1,520 & 6,513 & 14 & 57 & 410 & 481 & 6,993 & 4,408 & 9,691 & 21,093 \\
\hline 2005 & 8 & 4,946 & 1,451 & 6,406 & 16 & 58 & 430 & 504 & 6.909 & 4,638 & 10,079 & 21,626 \\
\hline 2006 & 6 & $\begin{array}{r}4,476 \\
\end{array}$ & 1,224 & 5,706 & 18 & 63 & R380 & $\begin{array}{r}\text { R462 } \\
\text { R }\end{array}$ & $R_{6,168}$ & 4,611 & 9,909 & $\begin{array}{r}R_{20}, 688 \\
\end{array}$ \\
\hline 2007 & 8 & $\begin{array}{r}4,810 \\
R_{4} 835\end{array}$ & $\begin{array}{l}1,254 \\
1,254\end{array}$ & $\mathrm{R}_{6,097}$ & 22 & 70 & R410 & $\begin{array}{l}\text { R502 } \\
\text { R }\end{array}$ & $\begin{array}{l}0,100 \\
R_{6,598}\end{array}$ & $\begin{array}{l}4,011 \\
4,750\end{array}$ & $\begin{array}{r}\text { 10,182 } \\
10,182\end{array}$ & $R_{21,531}$ \\
\hline 2008 & 8 & 5,010 & 1,243 & 6,261 & 26 & 80 & 450 & R557 & 6,817 & 4,708 & 10,071 & 21,596 \\
\hline 2009 & 8 & 4,883 & 1,176 & 6,067 & 33 & 89 & 430 & 552 & 6.619 & $\begin{array}{r}4,656 \\
\end{array}$ & 9,789 & $\begin{array}{r}R_{21}, 064\end{array}$ \\
\hline 2010 & 7 & $\mathrm{R}_{4,883}$ & $R_{1,142}$ & $R_{6,032}$ & 37 & $R_{114}$ & 420 & R571 & $\mathrm{R}_{6,603}$ & $R_{4} 4,933$ & $\begin{array}{r}\text { R } 10,326 \\
\end{array}$ & $\begin{array}{r}R_{21,84} \\
\end{array}$ \\
\hline $2011^{P}$ & 6 & 4,830 & 1,139 & 5,975 & 40 & 140 & 430 & 610 & 6,585 & 4,858 & 10,176 & 21,619 \\
\hline
\end{tabular}

1 See "Primary Energy Consumption" in Glossary.

8 Electricity retail sales to ultimate customers reported by electric utilities and, beginning in 1996, other 2 Data are estimates. See Table 10.2a for notes on series components.

${ }^{3}$ Natural gas only; excludes the estimated po

portion of supplemental gaseous fuels. See Note 1 ,

4 Based on petroleum product supplied. For petroleum, product supplied is used as an approximation of petroleum consumption. See Note 1, "Petroleum Products Supplied and Petroleum Consumption," at end of Section 5 .

Geothermal heat pump and direct use energy

Solar thermal direct use energy, and photovoltaic (PV) electricity net generation (converted to Btu using the fossil-fuels heat rate-see Table A6). Includes small amounts of distributed solar thermal and PV energy used in the commercial, industrial, and electric power sectors. 9 Total losses are calculated as the primary energy consumed by the electric power sector minus the
Tolitis energy content of electricity retail sales. Total losses are allocated to the end-use sectors in proportion to each sector's share of total electricity retail sales. See Note, "Electrical System Energy Losses," at end of section.

R=Revised. P=Preliminary. NA=Not available.

Note: Totals may not equal sum of components due to independent rounding.

Web Pages: - See http://www.eia.gov/totalenergy/data/monthly/\#consumption for updated monthly and annual data. - See http://www.eia.gov/totalenergy/data/annual/\#consumption for all annual data beginning

Sources: Tables 2.1f, 5.14a 6.5, 7.3, 8.9, 10.2a, A4, A5, and A6. 
Table 2.1c Commercial Sector Energy Consumption Estimates, Selected Years, 1949-2011

(Trillion Btu)

\begin{tabular}{|c|c|c|c|c|c|c|c|c|c|c|c|c|c|c|}
\hline \multirow[b]{3}{*}{ Year } & \multicolumn{11}{|c|}{ Primary Consumption 1} & \multirow{3}{*}{$\begin{array}{c}\text { Electricity } \\
\text { Retail } \\
\text { Sales } 11\end{array}$} & \multirow{3}{*}{$\begin{array}{l}\text { Electrical } \\
\text { System } \\
\text { Energy } \\
\text { Losses } 12\end{array}$} & \multirow[b]{3}{*}{ Total } \\
\hline & \multicolumn{4}{|c|}{ Fossil Fuels } & \multicolumn{6}{|c|}{ Renewable Energy ${ }^{2}$} & \multirow[b]{2}{*}{$\begin{array}{c}\text { Total } \\
\text { Primary }\end{array}$} & & & \\
\hline & Coal & Natural Gas ${ }^{3}$ & Petroleum 4,5 & Total & $\begin{array}{l}\text { Hydroelectric } \\
\text { Power }{ }^{6}\end{array}$ & Geothermal 7 & Solar/PV 8 & WInd ${ }^{9}$ & Biomass 10 & Total & & & & \\
\hline 1949 & 1,554 & 360 & 735 & 2,649 & NA & NA & NA & NA & 20 & 20 & 2,669 & 200 & 800 & 3,669 \\
\hline 1950 & 1,542 & 401 & 872 & 2,815 & NA & NA & NA & NA & 19 & 19 & 2,834 & 225 & 834 & $\begin{array}{l}5,003 \\
3,893\end{array}$ \\
\hline 1955 & 801 & 651 & 1,095 & 2,547 & NA & NA & NA & NA & 15 & 15 & 2,561 & 350 & 984 & 3,895 \\
\hline 1960 & 407 & 1,056 & 1,248 & 2,711 & NA & NA & NA & NA & 12 & 12 & 2,723 & 543 & 1,344 & 4,609 \\
\hline 1965 & 265 & 1,490 & 1,413 & 3,168 & NA & NA & NA & NA & 9 & 9 & 3,177 & & 1,880 & 5,845 \\
\hline 1970 & 165 & 2,473 & 1,592 & 4,229 & NA & NA & NA & NA & 8 & 8 & 4,237 & 1,201 & 2,908 & 8,346 \\
\hline 1975 & 147 & 2,558 & 1,346 & 4,051 & NA & NA & NA & NA & 8 & 8 & 4,059 & 1,598 & 3,835 & 9,492 \\
\hline 1976 & 144 & 2,718 & 1,500 & 4,362 & NA & NA & NA & NA & 9 & 9 & 4,371 & 1,678 & 4,014 & 10,063 \\
\hline 1977 & 148 & 2,548 & 1,552 & 4,248 & NA & NA & NA & NA & 10 & 10 & 4,258 & 1,754 & 4,196 & 10,208 \\
\hline 1978 & 165 & 2,643 & 1,490 & 4,297 & NA & NA & NA & NA & 12 & 12 & 4,309 & 1,813 & 4,390 & 10,512 \\
\hline 1979 & 149 & 2,836 & 1,367 & 4,352 & NA & NA & NA & NA & 14 & 14 & 4,366 & 1,854 & 4,428 & 10,648 \\
\hline 1980 & 115 & 2,651 & 1,318 & 4,084 & NA & NA & NA & NA & 21 & 21 & 4,105 & 1,906 & 4,567 & 10,578 \\
\hline 1981 & 137 & 2,557 & 1,122 & 3,816 & NA & NA & NA & NA & 21 & 21 & 3,837 & 2,033 & 4,746 & 10,616 \\
\hline 1982 & 155 & 2,650 & 1,037 & 3,842 & NA & NA & NA & NA & 22 & 22 & 3,864 & 2,077 & 4,919 & 10,860 \\
\hline 1983 & 162 & 2,486 & 1,170 & 3,818 & NA & NA & NA & NA & 22 & 22 & 3,840 & 2,116 & 4,982 & 10,938 \\
\hline 1984 & 169 & 2,582 & 1,227 & 3,978 & NA & NA & NA & NA & 22 & 22 & 4,001 & 2,264 & 5,179 & 11,444 \\
\hline 1985 & 137 & 2,488 & 1,083 & 3,708 & NA & NA & NA & NA & 24 & 24 & 3,732 & 2,351 & 5,368 & 11,451 \\
\hline 1986 & 135 & 2,367 & 1,162 & 3,665 & NA & NA & NA & NA & 27 & 27 & 3,693 & 2,439 & 5,475 & 11,606 \\
\hline 1987 & 125 & 2,489 & 1,131 & 3,745 & NA & NA & NA & NA & 30 & 30 & 3,774 & 2,539 & 5,633 & $\begin{array}{l}11,946 \\
\end{array}$ \\
\hline 1988 & 131 & 2,731 & 1,099 & 3,961 & NA & NA & $\mathrm{NA}$ & $\mathrm{NA}$ & 33 & 33 & $\begin{array}{l}3,994 \\
\end{array}$ & 2,675 & 5,909 & 12,578 \\
\hline 1989 & 115 & 2,785 & $\begin{array}{l}1,041 \\
\text { lo }\end{array}$ & $\begin{array}{l}3,941 \\
3,90\end{array}$ & 1 & 3 & INA & - & 99 & $\begin{array}{r}102 \\
102\end{array}$ & 4,043 & $\begin{array}{l}2,767 \\
2,7\end{array}$ & 6,384 & $\begin{array}{l}13,193 \\
13\end{array}$ \\
\hline 1990 & $\begin{array}{l}124 \\
124\end{array}$ & 2,682 & 991 & $\begin{array}{l}3,798 \\
3,74\end{array}$ & 1 & 3 & - & - & 94 & 98 & 3,896 & 2,860 & 6,564 & $\begin{array}{l}13,320 \\
\end{array}$ \\
\hline 1991 & $\begin{array}{l}116 \\
116\end{array}$ & $\begin{array}{l}2,002 \\
2,795\end{array}$ & 935 & $\begin{array}{l}3,846 \\
3,80\end{array}$ & 1 & 3 & $\overline{-}$ & $\overline{-}$ & $\begin{array}{l}94 \\
95\end{array}$ & $\begin{array}{r}90 \\
100\end{array}$ & $\begin{array}{l}\text {,os0 } \\
3,945\end{array}$ & $\begin{array}{l}\text { 2,o00 } \\
2,918\end{array}$ & $\begin{array}{l}\text {, J04 } \\
6,636\end{array}$ & 13,500 \\
\hline 1992 & $\begin{array}{l}117 \\
117\end{array}$ & 2,871 & 893 & $\begin{array}{l}3,840 \\
3,881\end{array}$ & 1 & 3 & $\overline{-}$ & $\overline{-}$ & 105 & 109 & $\begin{array}{l}\text {, } \\
3,945\end{array}$ & $\begin{array}{l}\text { 2,90 } \\
2,900\end{array}$ & $\begin{array}{l}6,030 \\
6,550\end{array}$ & 13,441 \\
\hline 1993 & 117 & $\begin{array}{l}2,811 \\
2,923\end{array}$ & 819 & $\begin{array}{l}3,801 \\
3,859\end{array}$ & 1 & 3 & $\overline{-}$ & $\overline{-}$ & 109 & 114 & $\begin{array}{l}3,971 \\
3,973\end{array}$ & $\begin{array}{l}\text {, } 3,019 \\
\text { ? }\end{array}$ & $\begin{array}{l}\begin{array}{l}6,050 \\
6,828\end{array}\end{array}$ & 13,820 \\
\hline 1994 & 118 & $\begin{array}{l}2,9<3 \\
2,962\end{array}$ & 825 & $\begin{array}{l}3,059 \\
3,905\end{array}$ & 1 & 4 & $\overline{-}$ & $\overline{-}$ & 106 & 112 & $\begin{array}{l}4,016 \\
4\end{array}$ & $\begin{array}{l}3,019 \\
3,116\end{array}$ & $\begin{array}{l}\begin{array}{l}6,0<0 \\
6,966\end{array}\end{array}$ & $\begin{array}{r}14,020 \\
14,098\end{array}$ \\
\hline 1995 & 117 & $\begin{array}{l}2,962 \\
3,096\end{array}$ & $\begin{array}{l}825 \\
769\end{array}$ & $\begin{array}{l}3,905 \\
3,982\end{array}$ & 1 & $\begin{array}{l}4 \\
5\end{array}$ & $\overline{-}$ & $\overline{-}$ & 113 & 118 & $\begin{array}{l}4,101 \\
4,10\end{array}$ & $\begin{array}{l}3,252 \\
3,1\end{array}$ & $\begin{array}{l}6,966 \\
7,338\end{array}$ & $\begin{array}{r}4,098 \\
14,690\end{array}$ \\
\hline 1996 & 122 & $\begin{array}{l}3,096 \\
3,226\end{array}$ & $\begin{array}{l}169 \\
790\end{array}$ & $\begin{array}{l}3,982 \\
4,138\end{array}$ & 1 & $\begin{array}{l}5 \\
5\end{array}$ & $\overline{-}$ & $\overline{-}$ & 129 & 135 & 4,273 & $\begin{array}{l}3,252 \\
3,344\end{array}$ & $\begin{array}{l}7,358 \\
7,555\end{array}$ & $\begin{array}{r}4,699 \\
15,172\end{array}$ \\
\hline 1997 & 129 & 3,285 & 743 & $\begin{array}{l}4,100 \\
4,157\end{array}$ & 1 & $\begin{array}{l}5 \\
6\end{array}$ & - & _- & 131 & 138 & 4,295 & $\begin{array}{l}\text {, J44 } \\
3,503\end{array}$ & $\begin{array}{l}7,853 \\
7,883\end{array}$ & 15,681 \\
\hline 1998 & 93 & 3,083 & 702 & 3.878 & 1 & 7 & - & - & 118 & 127 & 4.005 & 3,678 & 8,285 & 15,968 \\
\hline 1999 & 103 & 3,115 & 707 & 3,925 & 1 & 7 & - & - & 121 & 129 & 4,053 & $\begin{array}{l}3,766 \\
\end{array}$ & 8,557 & 16,376 \\
\hline 2000 & 92 & $\begin{array}{l}3,252 \\
3,2\end{array}$ & 807 & 4,150 & 1 & 8 & - & - & 119 & 128 & 4,278 & $\begin{array}{l}3,956 \\
3\end{array}$ & $\begin{array}{l}8,051 \\
8,942\end{array}$ & 17,175 \\
\hline 2001 & 97 & 3,097 & 790 & 3,984 & 1 & 8 & - & - & 92 & 101 & 4,084 & 4,062 & 8,990 & $\begin{array}{l}17,137 \\
\end{array}$ \\
\hline 2002 & 90 & $\mathrm{R}_{3,212}$ & 726 & $\mathrm{R}_{4,028}$ & (s) & 9 & - & - & 95 & 104 & $\begin{array}{r}\text { R4, } \\
R^{2}, 132\end{array}$ & $\begin{array}{l}4,110 \\
4,110\end{array}$ & 9,104 & R17,345 \\
\hline 2003 & 82 & 3,261 & 827 & 4,170 & 1 & 11 & - & - & 101 & 113 & 4,283 & 4,090 & $\begin{array}{l}8,104 \\
8,969\end{array}$ & 17,343 \\
\hline 2004 & 103 & $\begin{array}{l}3,201 \\
3,201\end{array}$ & 809 & 4,113 & 1 & 12 & $\overline{-}$ & - & 105 & 118 & $\begin{array}{r}4,203 \\
4,232\end{array}$ & $\begin{array}{l}4,090 \\
4,198\end{array}$ & $\begin{array}{l}\text {,9,229 } \\
9,229\end{array}$ & $\begin{array}{r}17,045 \\
17,659\end{array}$ \\
\hline 2005 & 97 & 3,073 & 761 & 3,932 & 1 & 14 & - & - & 105 & $\mathrm{R}_{120}$ & 4,051 & 4,351 & 9,455 & $\mathrm{R}_{17,857}$ \\
\hline 2006 & 65 & 2,902 & 663 & 3,629 & 1 & 14 & - & - & $\mathrm{R}_{103}$ & $\mathrm{R}_{118}$ & $\mathrm{R}_{3,747}$ & 4,435 & 9,529 & $\mathrm{R}_{17,711}$ \\
\hline 2007 & 70 & $\mathrm{R}_{3,085}$ & 649 & $\mathrm{R}_{3,805}$ & 1 & 14 & - & - & $R_{103}$ & 118 & $\mathrm{R}_{3,922}$ & 4,560 & 9,773 & $\mathrm{R}_{18,255}$ \\
\hline 2008 & 69 & 3,228 & 651 & 3,948 & 1 & 15 & (s) & - & 109 & 125 & 4,073 & 4,558 & 9,749 & 18,381 \\
\hline 2009 & 63 & 3,187 & 682 & 3,932 & 1 & 17 & (s) & (s) & 112 & 129 & 4,061 & 4,460 & 9,378 & 17,899 \\
\hline 2010 & $\mathrm{R}_{60}$ & $\mathrm{R}_{3,164}$ & R685 & $\mathrm{R}_{3}, 908$ & 1 & 19 & (s) & (s) & $\mathrm{R}_{111}$ & $\mathrm{R}_{130}$ & $R_{4,039}$ & $R_{4,539}$ & R9,501 & $\mathrm{R}_{18,078}$ \\
\hline $2011^{P}$ & 51 & 3,225 & 683 & 3,959 & 1 & 20 & $\begin{array}{l}\text { (s) } \\
\text { (s) }\end{array}$ & $\begin{array}{l}\text { (s) } \\
\text { (s) }\end{array}$ & 110 & 131 & 4,090 & 4,501 & 9,429 & 18,021 \\
\hline
\end{tabular}

1 See "Primary Energy Consumption" in Glossary.

a for notes on series components and estimation.

${ }_{3}$ Natural gas only; excludes the estimated portion of supplemental gaseous fuels. See Note 1 ,

"Supplemental Gaseous Fuels," at end of Section 6.

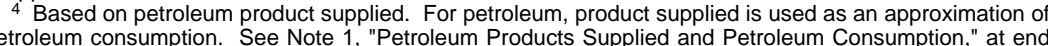
of Section 5 .

Does not include biofuels that have been blended with petroleum_-biofuels are included in "Biomass." 6 Conventional hydroelectricity net generation (converted to Btu using the fossil-fuels heat rate-see Table A6

Geothermal heat pump and direct use energy.

(P) electricity net generation (converted to Btu using the fossil-fuels heat rate-see Table A6) at commercial plants with capacity of 1 megawatt or greater.

Wind electricity net generation (converted to Btu using the fossil-fuels heat rate-see Table A6).

aricood and wood-derived fuels, municipal solid waste irom biogenic sources, land ingas, sludge waste,

includes non-renewable waste (municipal solid waste from non-biogenic sources, and tire-derived fuels). 11 Electricity retail sales to ultimate customers reported by electric utilities and, beginning in 1996, other 12 Total losses are caiders.

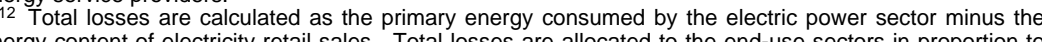
each sector's share of total electricity retail sales. See Note, "Electrical System Energy Losses," at end of section.

$R=$ Revised. $P=$ Preliminary. NA=Not available. $-=$ No data reported. $(s)=$ Less than 0.5 trillion Btu.

Notes: - The commercial sector includes commercial combined-heat-and-power (CHP) and commercial electricity-only plants. See Note 2, "Classification of Power Plants Into Energy-Use Sectors," at end o Web Pages: - See $\mathrm{http} \cdot / / \mathrm{ww}$.eia gov/totalenergy/data/monthly/\#consumption for up annual data. - See http://www.eia.gov/totalenergy/data/annual/\#consumption for all annual data beginning

Sources: Tables 2.1f, 5.14a, 6.5, 7.3, 8.9, 10.2a, A4, A5, and A6. 
Table 2.1d Industrial Sector Energy Consumption Estimates, Selected Years, 1949-2011

(Trillion Btu)

\begin{tabular}{|c|c|c|c|c|c|c|c|c|c|c|c|c|c|c|c|}
\hline \multirow[b]{3}{*}{ Year } & \multicolumn{12}{|c|}{ Primary Consumption 1} & \multirow{3}{*}{$\begin{array}{c}\text { Electricity } \\
\text { Retail } \\
\text { Sales }{ }^{11}\end{array}$} & \multirow{3}{*}{$\begin{array}{l}\text { Electrical } \\
\text { System } \\
\text { Energy } \\
\text { Losses } 12\end{array}$} & \multirow[b]{3}{*}{ Tota } \\
\hline & \multicolumn{5}{|c|}{ Fossil Fuels } & \multicolumn{6}{|c|}{ Renewable Energy ${ }^{2}$} & \multirow[b]{2}{*}{$\begin{array}{c}\text { Total } \\
\text { Primary }\end{array}$} & & & \\
\hline & Coal & $\begin{array}{l}\text { Coal Coke } \\
\text { Net Imports }\end{array}$ & $\underset{\text { Gas }^{3}}{\text { Natural }}$ & Petroleum 4,5 & Total & $\begin{array}{c}\text { Hydroelectric } \\
\text { Power }^{6}\end{array}$ & Geothermal 7 & Solar/PV 8 & Wind 9 & Biomass 10 & Total & & & & \\
\hline 1949 & 5,433 & -7 & 3,188 & 3,475 & 12,090 & 76 & NA & NA & NA & 468 & 544 & 12,633 & 418 & 1,672 & 14,724 \\
\hline 1950 & 5,781 & 1 & 3,546 & 3,960 & 13,288 & 69 & NA & NA & NA & 532 & 602 & 13,890 & 500 & 1,852 & 16,241 \\
\hline 1955 & 5,620 & -10 & 4,701 & 5,123 & 15,434 & 38 & & & NA & 631 & 669 & 16,103 & 887 & 2,495 & 19,485 \\
\hline 1960 & 4,543 & -6 & 5,973 & 5,766 & 16,277 & 39 & NA & NA & NA & 680 & 719 & 16,996 & 1,107 & 2,739 & 20,842 \\
\hline 1965 & 5,127 & -18 & 7,339 & 6,813 & 19,260 & 33 & NA & NA & NA & 855 & 888 & 20,148 & 1,463 & 3,487 & 25,098 \\
\hline 1970 & 4,656 & -58 & 9,536 & 7,776 & 21,911 & 34 & NA & NA & NA & 1,019 & 1,053 & 22,964 & 1,948 & 4,716 & 29,628 \\
\hline 1975 & 3,667 & 14 & 8,532 & 8,127 & 20,339 & 32 & NA & NA & NA & 1,063 & 1,096 & 21,434 & 2,346 & 5,632 & 29,413 \\
\hline 1976 & 3,661 & (s) & 8,762 & 8,990 & 21,412 & 33 & NA & NA & $\mathrm{NA}$ & 1,220 & 1,253 & 22,665 & 2,573 & 6,155 & 31,393 \\
\hline 1977 & 3,454 & 15 & 8,635 & 9,747 & 21,851 & 33 & NA & NA & NA & 1,281 & 1,314 & 23,165 & 2,682 & 6,416 & 32,263 \\
\hline 1978 & 3,314 & 125 & 8,539 & 9,835 & 21,812 & 32 & NA & NA & NA & 1,400 & 1,432 & 23,244 & 2,761 & 6,683 & 32,688 \\
\hline 1979 & 3,593 & 63 & 8,549 & 10,548 & 22,753 & 34 & NA & NA & NA & 1,405 & 1,439 & 24,192 & 2,873 & 6,860 & 33,925 \\
\hline 1980 & 3,155 & -35 & 8,333 & 9,509 & 20,962 & 33 & NA & NA & NA & 1,600 & 1,633 & 22,595 & 2,781 & 6,664 & 32,039 \\
\hline 1981 & 3,157 & -16 & 8,185 & 8,265 & 19,590 & 33 & NA & NA & NA & 1,695 & 1,728 & 21,318 & 2,817 & 6,576 & 30,712 \\
\hline 1982 & 2,552 & -22 & 7,068 & 7,772 & 17,370 & 33 & NA & NA & NA & 1,650 & 1,683 & 19,053 & 2,542 & 6,020 & 27,614 \\
\hline 1983 & 2,490 & -16 & 6,776 & 7,390 & 16,640 & 33 & NA & NA & NA & 1,874 & 1,908 & 18,548 & 2,648 & 6,232 & 27,428 \\
\hline 1984 & 2,842 & -11 & 7,405 & 7,987 & 18,222 & 33 & NA & NA & NA & 1,918 & 1,951 & 20,174 & 2,859 & 6,538 & 29,570 \\
\hline 1985 & 2,760 & -13 & 7,032 & 7,714 & 17,492 & 33 & NA & NA & NA & 1,918 & 1,951 & 19,443 & 2,855 & 6,518 & 28,816 \\
\hline 1986 & 2,641 & -17 & 6,646 & 7,860 & 17,130 & 33 & NA & NA & NA & 1,915 & 1,948 & 19,078 & 2,834 & 6,362 & 28,274 \\
\hline 1987 & 2,673 & 9 & 7,283 & 8,042 & 18,006 & 33 & NA & NA & NA & 1,914 & 1,947 & 19,953 & 2,928 & 6,497 & 29,379 \\
\hline 1988 & 2,828 & 40 & 7,655 & 8,317 & 18,840 & 33 & NA & $\mathrm{NA}$ & NA & 1,989 & 2,022 & 20,862 & 3,059 & 6,757 & 30,677 \\
\hline 1989 & 2,787 & 30 & 8,088 & 8,098 & 19,003 & 28 & 2 & - & - & 1,841 & 1,871 & 20,874 & 3,158 & 7,288 & 31,320 \\
\hline 1990 & 2,756 & 5 & 8,451 & 8,251 & 19,463 & 31 & 2 & - & - & 1,684 & 1,717 & 21,180 & 3,226 & 7,404 & 31,810 \\
\hline 1991 & 2,601 & 10 & 8,572 & 7,958 & 19,141 & 30 & 2 & - & - & 1,652 & 1,684 & 20,824 & 3,230 & 7,345 & 31,399 \\
\hline 1992 & 2,515 & 35 & 8,918 & 8,552 & 20,019 & 31 & 2 & - & - & 1,705 & 1,737 & 21,756 & 3,319 & 7,496 & 32,571 \\
\hline 1993 & 2,496 & 27 & 9,070 & 8,386 & 19,980 & 30 & 2 & - & - & 1,741 & 1,773 & 21,753 & 3,334 & 7,541 & 32,629 \\
\hline 1994 & 2,510 & 58 & 9,126 & 8,771 & 20,465 & 62 & 3 & - & - & 1,862 & 1,927 & 22,393 & 3,439 & 7,689 & 33,521 \\
\hline 1995 & 2,488 & 61 & 9,592 & 8,586 & 20,727 & 55 & 3 & - & - & 1,934 & 1,992 & 22,719 & 3,455 & 7,796 & 33,971 \\
\hline 1996 & 2,434 & 23 & 9,901 & 9,019 & 21,377 & 61 & 3 & - & - & 1,969 & 2,033 & 23,410 & 3,527 & 7,968 & 34,904 \\
\hline 1997 & 2,395 & 46 & 9,933 & 9,255 & 21,629 & 58 & 3 & - & - & 1,996 & 2,057 & 23,686 & 3,542 & 7,972 & 35,200 \\
\hline 1998 & 2,335 & 67 & 9,763 & 9,082 & 21,248 & 55 & 3 & - & - & 1,872 & 1,929 & 23,177 & 3,587 & 8,079 & 34,843 \\
\hline 1999 & 2,227 & 58 & 9,375 & 9,356 & 21,016 & 49 & 4 & - & - & $\begin{array}{l}1,882 \\
\end{array}$ & 1,934 & 22,950 & 3,611 & 8,203 & 34,764 \\
\hline 2000 & 2,256 & 65 & 9,500 & 9,075 & 20,896 & 42 & 4 & - & - & 1,881 & 1,928 & 22,824 & 3,631 & 8,208 & 34,664 \\
\hline 2001 & 2,192 & 29 & 8,676 & 9,178 & 20,075 & 33 & 5 & - & - & 1,681 & 1,719 & 21,794 & 3,400 & 7,526 & 32,720 \\
\hline 2002 & 2,019 & 61 & R8,832 & 9,168 & $\mathrm{R}_{20,079}$ & 39 & 5 & - & - & 1,676 & 1,720 & $\mathrm{R}_{21,799}$ & 3,379 & 7,484 & R32,662 \\
\hline 2003 & 2,041 & 51 & 8,488 & 9,197 & 19,777 & 43 & 3 & - & - & 1,679 & 1,726 & 21,503 & 3,454 & 7,575 & 32,532 \\
\hline 2004 & 2,047 & 138 & $R 8,550$ & 9,825 & $\mathrm{R}_{20,559}$ & 33 & 4 & - & - & 1,817 & 1,853 & $\mathrm{R} 22,412$ & 3.473 & 7,635 & R 33,520 \\
\hline 2005 & 1,954 & 44 & $\mathrm{R} 7,907$ & 9,633 & $\mathrm{R}_{19,538}$ & 32 & 4 & - & - & 1,837 & 1,873 & $\mathrm{R}_{21,411}$ & 3,477 & 7,557 & $\mathrm{R} 32,446$ \\
\hline 2006 & 1,914 & 61 & $R_{7,861}$ & 9,770 & $\mathrm{R}_{19,606}$ & 29 & 4 & - & - & 1,897 & 1,930 & $\mathrm{R}_{21,536}$ & 3.451 & 7.415 & R32,401 \\
\hline 2007 & 1,865 & 25 & $R_{8,074}$ & 9,451 & $\mathrm{R}_{19,414}$ & 16 & 5 & - & - & $R_{1}, 936$ & $R_{1}, 956$ & $\mathrm{R}_{21,370}$ & 3,507 & 7,517 & $R_{32,394}$ \\
\hline 2008 & 1,796 & 41 & $R 8,083$ & 8,511 & $\mathrm{R}_{18,431}$ & 17 & 5 & - & - & $R_{2,028}$ & $R_{2,049}$ & $\mathrm{R}_{20,480}$ & 3,444 & 7,365 & $\mathrm{R} 31,290$ \\
\hline 2009 & 1,396 & -24 & 7,609 & 7,816 & $\mathrm{R}_{16,797}$ & 18 & 4 & - & - & $\mathrm{R}_{1,994}$ & $\mathrm{R}_{2,016}$ & $\mathrm{R}_{18,813}$ & 3,130 & 6,582 & R28,525 \\
\hline 2010 & $\mathrm{R}_{1,649}$ & -6 & R7,959 & $R_{8,210}$ & $\mathrm{R} 17,812$ & 16 & 4 & $(\bar{s})$ & - & $R_{2,230}$ & $\mathrm{R}_{2,250}$ & $\begin{array}{l}10,062 \\
R_{20}\end{array}$ & $R_{3,313}$ & $R_{6,934}$ & $R_{30,309}$ \\
\hline $2011^{P}$ & 1,599 & 11 & 8,321 & 8,064 & 17,995 & 18 & $\begin{array}{l}4 \\
4\end{array}$ & (s) & (s) & 2,273 & 2,295 & 20,291 & 3,329 & $\begin{array}{l}0,973 \\
6,73\end{array}$ & 30,592 \\
\hline
\end{tabular}

See "Primary Energy Consumption" in Glossary.

Most data are estimates. See Table $10.2 \mathrm{~b}$ for notes on series components and estimation.

3 Natural gas only; excludes the estimated portion of supplemental gaseous fuels. See Note 1 Supplemental Gaseous Fuels," at end of Section 6.

etroleum consumption. See Note 1 "Petroleum Produm, product supplied is used as an approximation of

of Section 5.

5 Does not include biofuels that have been blended with petroleum-biofuels are included in "Biomass."
6 6 Conventional hydroelectricity net generation (cor
Table A6).
7 Geothermal heat pump and direct use energy.

8 Geothermal heat pump and direct use energy. Table A6) at industrial plants with capacity of 1 megawatt or greater.

9 Wind electricity net generation (converted to Btu using the fossil-fuels heat rate-see Table A6).

Wood and wood-derived fuels; municipal solid waste from biogenic sources, landfill gas, sludge waste,

agricultural byproducts, and other biomass; fuel ethanol (minus denaturant); and losses and co-products

(municipal solid waste from non-biogenic sources, and tire-derived fuels).

Electricity retail sales to ultimate customers reported by electric utilities and, beginning in 1996, other ${ }_{12}$ Total losses are calculated as the primary energy consumed by the electric power sector minus the energy content of electricity retail sales. Total losses are allocated to the end-use sectors in proportion to
each sector's share of total electricity retail sales. See Note, "Electrical System Energy Losses," at end of

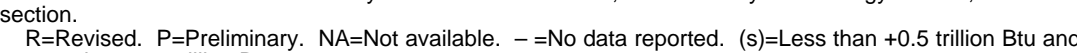

greater than -0.5 trillion Btu.
Notes: - The industrial sector includes industrial combined-heat-and-power (CHP) and industrial electricity-only plants. See Note 2, "Classification of Power Plants Into Energy-Use Sectors," at end of Web Pags: - See http:/www eia ov/totalenergy/ata/monthly/ttconsumption for un

annual data. - See http://www.eia.gov/totalenergy/data/annual/\#consumption for all annual data beginning

Sources: Tables 2.1f, 5.14b, 6.5, 7.3, 7.8, 8.9, 10.2b, A4, A5, and A6. 
Table 2.1e Transportation Sector Energy Consumption Estimates, Selected Years, 1949-2011

(Trillion Btu)

\begin{tabular}{|c|c|c|c|c|c|c|c|c|c|}
\hline \multirow[b]{3}{*}{ Year } & \multicolumn{6}{|c|}{ Primary Consumption 1} & \multirow{3}{*}{$\begin{array}{l}\text { Electricity } \\
\text { Retail } \\
\text { Sales }^{7}\end{array}$} & \multirow{3}{*}{$\begin{array}{c}\text { Electrical } \\
\text { System } \\
\text { Energy } \\
\text { Losses }^{8}\end{array}$} & \multirow[b]{3}{*}{ Total } \\
\hline & \multicolumn{4}{|c|}{ Fossil Fuels } & \multirow{2}{*}{\begin{tabular}{|c|} 
Renewable Energy $^{2}$ \\
Biomass $^{6}$
\end{tabular}} & \multirow{2}{*}{$\begin{array}{c}\text { Total } \\
\text { Primary }\end{array}$} & & & \\
\hline & Coal & Natural Gas ${ }^{3}$ & Petroleum 4,5 & Total & & & & & \\
\hline 1949 & 1,727 & NA & 6,152 & 7,880 & NA & 7,880 & 22 & 88 & 7,990 \\
\hline 1950 & 1,564 & 130 & 6,690 & 8,383 & NA & 8,383 & 23 & 86 & 8,492 \\
\hline 1955 & 421 & 254 & 8,799 & 9,474 & NA & 9,474 & 20 & 56 & 9,550 \\
\hline 1960 & 75 & 359 & 10,125 & 10,560 & NA & 10,560 & 10 & 26 & 10,596 \\
\hline 1965 & 16 & 517 & 11,866 & 12,399 & NA & 12,399 & 10 & 24 & 12,432 \\
\hline 1970 & 7 & 745 & 15,310 & 16,062 & NA & 16,062 & 11 & 26 & 16,098 \\
\hline 1975 & 1 & 595 & 17,615 & 18,210 & NA & 18,210 & 10 & 24 & 18,245 \\
\hline 1976 & (s) & 559 & 18,508 & 19,067 & NA & 19,067 & 10 & 24 & 19,101 \\
\hline 1977 & (s) & 543 & 19,243 & 19,786 & NA & 19,786 & 10 & 25 & 19,822 \\
\hline 1978 & $(9)$ & 539 & 20,044 & 20,583 & NA & 20,583 & 10 & 24 & 20,617 \\
\hline 1979 & (9) & 612 & 19,825 & 20,437 & NA & 20,437 & 10 & 24 & 20,472 \\
\hline 1980 & $\left({ }^{9}\right)$ & 650 & 19,009 & 19,659 & NA & 19,659 & 11 & 27 & 19,697 \\
\hline 1981 & $(9)$ & 658 & 18,813 & 19,471 & 7 & 19,478 & 11 & 25 & 19,514 \\
\hline 1982 & $\left({ }^{9}\right)$ & 612 & 18,422 & 19,034 & 18 & 19,052 & 11 & 26 & 19,089 \\
\hline 1983 & $\left({ }^{9}\right)$ & 505 & 18,595 & 19,100 & 34 & 19,134 & 13 & 30 & 19,177 \\
\hline 1984 & $(9)$ & 545 & 19,023 & 19,567 & 41 & 19,609 & 14 & 33 & 19,656 \\
\hline 1985 & $\left({ }^{9}\right)$ & 519 & 19,472 & 19,992 & 50 & 20,041 & 14 & 32 & 20,088 \\
\hline 1986 & $\left({ }^{\prime}\right)$ & 499 & 20,183 & 20,682 & 57 & 20,740 & 15 & 34 & 20,789 \\
\hline 1987 & $\left({ }^{9}\right)$ & 535 & 20,817 & 21,353 & 66 & 21,419 & 16 & 35 & 21,469 \\
\hline 1988 & $\left({ }^{9}\right)$ & 632 & 21,568 & 22,199 & 67 & 22,267 & 16 & 35 & 22,318 \\
\hline 1989 & $\left({ }^{\prime}\right)$ & 649 & 21,707 & 22,356 & 68 & 22,424 & 16 & 38 & 22,478 \\
\hline 1990 & $(9)$ & 680 & 21,626 & 22,306 & 60 & $\begin{array}{l}22,366 \\
\end{array}$ & 16 & 37 & 22,420 \\
\hline 1991 & $(9)$ & 620 & 21,374 & 21,995 & 70 & 22,065 & 16 & 37 & 22,118 \\
\hline 1992 & $\left({ }^{9}\right)$ & 608 & 21,675 & 22,283 & 80 & 22,363 & 16 & 36 & 22,415 \\
\hline 1993 & $\left({ }^{9}\right)$ & 645 & 21,977 & 22,621 & 94 & 22,715 & 16 & 37 & 22,768 \\
\hline 1994 & $\left(9^{\prime}\right)$ & 709 & 22,497 & 23,206 & 105 & 23,311 & 17 & 38 & 23,366 \\
\hline 1995 & $\left({ }^{9}\right)$ & 724 & 22,955 & 23,679 & 112 & 23,791 & 17 & 38 & 23,846 \\
\hline 1996 & $\left({ }^{\prime}\right)$ & 737 & 23,565 & 24,302 & 81 & 24,383 & 17 & 38 & 24,437 \\
\hline 1997 & $\left({ }^{\prime}\right)$ & 780 & 23,813 & 24,593 & 102 & 24,695 & 17 & 38 & 24,750 \\
\hline 1998 & $\left({ }^{9}\right)$ & 666 & 24,422 & 25,088 & 113 & 25,201 & 17 & 38 & 25,256 \\
\hline 1999 & $\left({ }^{9}\right)$ & 675 & 25,098 & 25,774 & 118 & 25,891 & 17 & 40 & 25,949 \\
\hline 2000 & $\left({ }^{9}\right)$ & 672 & 25,682 & 26,354 & 135 & 26,489 & 18 & 42 & 26,548 \\
\hline 2001 & $(9)$ & 658 & 25,412 & 26,070 & 142 & 26,213 & 20 & 43 & 26,275 \\
\hline 2002 & $\left({ }^{\prime}\right)$ & R699 & 25,913 & $\mathrm{R}_{26,612}$ & 170 & $\mathrm{R}_{26,781}$ & 19 & 42 & $\mathrm{R}_{26,842}$ \\
\hline 2003 & $\left({ }^{\prime}\right)$ & 627 & 26,063 & 26,690 & 230 & 26,920 & 23 & 51 & 26,994 \\
\hline 2004 & $\left({ }^{9}\right)$ & 602 & 26,925 & 27,527 & 290 & 27,817 & 25 & 54 & 27,895 \\
\hline 2005 & $\left({ }^{9}\right)$ & 624 & 27,309 & 27,933 & 339 & 28,272 & 26 & 56 & 28,353 \\
\hline 2006 & $(9)$ & 625 & 27,651 & 28,276 & 475 & $\begin{array}{l}28,751 \\
\end{array}$ & 25 & 54 & 28,830 \\
\hline 2007 & $\left(9^{\prime}\right)$ & R663 & 27,763 & $\mathrm{R}_{28,427}$ & 602 & $\begin{array}{r}R_{29,029} \\
\end{array}$ & 28 & 60 & $R_{29,117}$ \\
\hline 2008 & $\left({ }^{9}\right)$ & 692 & 26,407 & 27,099 & 826 & 27,925 & 26 & 56 & 28,008 \\
\hline 2009 & $\left({ }^{\prime}\right)$ & R715 & 25,339 & $\mathrm{R}_{26,054}$ & R935 & $\begin{array}{l}\mathrm{R}_{26,989}\end{array}$ & 27 & 56 & $\mathrm{R}_{27,071}$ \\
\hline 2010 & $\left({ }^{\prime}\right)$ & R716 & $\mathrm{R}_{25,595}$ & $\mathrm{R}_{26,310}$ & $\mathrm{R}_{1,074}$ & $\mathrm{R}_{27,384}$ & 26 & 55 & $\mathrm{R}_{27,466}$ \\
\hline $2011^{P}$ & $\left({ }^{\prime}\right)$ & 735 & 25,110 & 25,845 & 1,154 & 26,999 & 26 & 54 & 27,079 \\
\hline
\end{tabular}

1 See "Primary Energy Consumption" in Glossary.

Ther on series components.

3 Natural gas only; does not include supplemental gaseous fuels-see Note 1, "Supplemental Gaseous Fuels," at end of Section 6. Data are for natural gas consumed in the operation of pipelines (primarily in compressors) and small amounts consumed as vehicle fuel-see Table 6.5

Based on petroleum product supplied. For petroleum, product supplied is used as an approximation of petroleum consumption. See Note 1, "Petroleum Products Supplied and Petroleum Consumption," at end of Section 5.

Does not include biofuels that have been blended with petroleum—-biofuels are included in "Biomass." Fuel ethanol (minus denaturant) and biodiesel.

electric utilities and, beginning in 1996, other energy service providers.

8 Total losses are calculated as the primary energy consumed by the electric power sector minus the energy content of electricity retail sales. Total losses are allocated to the end-use sectors in proportion to

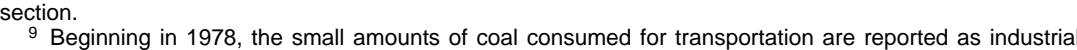
sector consumption.

$\mathrm{R}=$ Revised. $\mathrm{P}=$ Preliminary. $\mathrm{NA}=\mathrm{Not}$ available. $(\mathrm{s})=$ Less than 0.5 trillion Btu. Note: Totals may not equal sum of components due to independent rounding.

Web Pages: - See http://www.eia.gov/totalenergy/data/monthly/\#consumption for updated monthly and annual data. - See http://www.eia.gov/totalenergy/data/annual/\#consumption for all annual data beginning

Sources: Tables 2.1f, 5.14c, 6.5, 7.3, 8.9, 10.2b, A4, A5, and A6. 
Table 2.1f Electric Power Sector Energy Consumption, Selected Years, 1949-2011

(Trillion Btu)

\begin{tabular}{|c|c|c|c|c|c|c|c|c|c|c|c|c|c|}
\hline \multirow[b]{3}{*}{ Year } & \multicolumn{13}{|c|}{ Primary Consumption 1} \\
\hline & \multicolumn{4}{|c|}{ Fossil Fuels } & \multirow{2}{*}{$\begin{array}{l}\text { Nuclear } \\
\text { Electric } \\
\text { Power }^{5}\end{array}$} & \multicolumn{6}{|c|}{ Renewable Energy ${ }^{2}$} & \multirow{2}{*}{$\begin{array}{l}\text { Electricity } \\
\text { Net } \\
\text { Imports }\end{array}$} & \multirow[b]{2}{*}{$\begin{array}{c}\text { Total } \\
\text { Primary }\end{array}$} \\
\hline & Coal & $\begin{array}{c}\text { Natural } \\
\text { Gas }^{3}\end{array}$ & Petroleum ${ }^{4}$ & Total & & $\begin{array}{l}\text { Hydroelectric } \\
\text { Power }^{6}\end{array}$ & Geothermal $^{7}$ & Solar/PV ${ }^{8}$ & Wind $^{9}$ & Biomass ${ }^{10}$ & Total & & \\
\hline 1949 & 1,995 & 569 & 415 & 2,979 & 0 & 1,349 & NA & NA & NA & 6 & 1,355 & 5 & 4,339 \\
\hline 1950 & 2,199 & 651 & 472 & 3,322 & 0 & 1,346 & NA & NA & NA & 5 & 1,351 & 6 & 4,679 \\
\hline 1955 & 3,458 & 1,194 & 471 & 5,123 & 0 & 1,322 & NA & NA & NA & 3 & 1,325 & 14 & 6,461 \\
\hline 1960 & 4,228 & 1,785 & 553 & 6,565 & 6 & 1,569 & (s) & NA & NA & 2 & 1,571 & 15 & 8,158 \\
\hline 1965 & $\begin{array}{l}4,<20 \\
5,821\end{array}$ & 2,395 & 722 & $\begin{array}{l}0,000 \\
8,938\end{array}$ & 43 & 2,026 & (S) & NA & NA & 3 & 2,031 & (s) & $\begin{array}{r}0 \\
11,012\end{array}$ \\
\hline 1970 & 7,227 & 4,054 & 2,117 & 13,399 & 239 & 2,600 & 6 & NA & NA & 4 & 2,609 & $\begin{array}{c}101 \\
7\end{array}$ & 16,253 \\
\hline 1975 & 8,786 & 3,240 & 3,166 & 15,191 & 1,900 & 3,122 & 34 & NA & NA & 2 & 3,158 & 21 & 20,270 \\
\hline 1976 & 9,720 & 3,152 & 3,477 & 16,349 & 2,111 & 2,943 & 38 & NA & NA & 3 & 2,983 & 29 & 21,473 \\
\hline 1977 & 10,262 & 3,284 & 3,901 & 17,446 & 2,702 & 2,301 & 37 & NA & NA & 5 & 2,343 & 59 & 22,551 \\
\hline 1978 & 10,238 & 3,297 & 3,987 & 17,522 & 3,024 & 2,905 & 31 & NA & NA & 3 & 2,940 & 67 & 23,553 \\
\hline 1979 & 11,260 & 3,613 & 3,283 & 18,156 & 2,776 & 2,897 & 40 & NA & NA & 5 & 2,942 & 69 & 23,943 \\
\hline 1980 & 12,123 & 3,778 & 2,634 & 18,534 & 2,739 & 2,867 & 53 & NA & NA & 5 & 2,925 & 71 & 24,269 \\
\hline 1981 & 12,583 & 3,730 & 2,202 & 18,516 & 3,008 & 2,725 & 59 & NA & NA & 4 & 2,788 & 113 & 24,425 \\
\hline 1982 & 12,582 & 3,312 & 1,568 & 17,462 & 3,131 & 3,233 & 51 & NA & NA & 3 & 3,286 & 100 & 23,979 \\
\hline 1983 & 13,213 & 2,972 & 1,544 & 17,729 & 3,203 & 3,494 & 64 & NA & (s) & 4 & 3,562 & 121 & 24,614 \\
\hline 1984 & 14,019 & 3,199 & 1,286 & 18,504 & 3,553 & 3,353 & 81 & (s) & (s) & 9 & 3,443 & 135 & 25,635 \\
\hline 1985 & 14,542 & 3,135 & $\begin{array}{l}1,090 \\
1,20\end{array}$ & $\begin{array}{l}18,767 \\
18,767\end{array}$ & 4,076 & 2,937 & $\begin{array}{l}01 \\
97\end{array}$ & $\begin{array}{l}\text { (s) } \\
\text { (s) }\end{array}$ & $\begin{array}{l}\text { (s) } \\
\text { (s) }\end{array}$ & 14 & 3,049 & 140 & 26,032 \\
\hline 1986 & 14,444 & 2,670 & 1,452 & 18,566 & 4,380 & 3,038 & 108 & (s) & (s) & 12 & 3,158 & 122 & 26,227 \\
\hline 1987 & 15,173 & 2,916 & 1,257 & 19,346 & 4,754 & 2,602 & 112 & (s) & (s) & 15 & 2,729 & 158 & 26,988 \\
\hline 1988 & $\begin{array}{l}15,850 \\
\end{array}$ & $\begin{array}{l}2,693 \\
2,69\end{array}$ & $\begin{array}{l}1,563 \\
1,50\end{array}$ & 20,106 & 5,587 & 2,302 & 106 & $\begin{array}{l}\text { (s) } \\
\text { (s) }\end{array}$ & $\begin{array}{l}\text { (s) } \\
\text { (s) }\end{array}$ & 17 & $\begin{array}{r}2,425 \\
\end{array}$ & 108 & 28,227 \\
\hline $1989^{12}$ & 16,137 & 3,173 & 1,703 & 21,013 & 5,602 & 2,808 & 152 & 3 & 22 & 232 & 3,217 & 37 & 29,869 \\
\hline 1990 & 16,261 & 3,309 & 1,289 & 20,859 & 6,104 & 3,014 & 161 & 4 & 29 & 317 & 3,524 & 8 & 30,495 \\
\hline 1991 & 16,250 & 3,377 & 1,198 & 20,825 & 6,422 & 2,985 & 167 & 5 & 31 & 354 & 3,542 & 67 & 30,856 \\
\hline 1992 & 16,466 & 3,512 & 991 & 20,968 & 6,479 & 2,586 & 167 & 4 & 30 & 402 & 3,189 & 87 & 30,723 \\
\hline 1993 & 17,196 & 3,538 & 1,124 & 21,857 & 6,410 & 2,861 & 173 & 5 & 31 & 415 & 3,484 & 95 & 31,847 \\
\hline 1994 & 17,261 & 3,977 & 1,059 & 22,297 & 6,694 & 2,620 & 160 & 5 & 36 & 434 & 3,255 & 153 & 32,399 \\
\hline 1995 & 17,466 & 4,302 & 755 & 22,523 & 7,075 & 3,149 & 138 & 5 & 33 & 422 & 3,747 & 134 & 33,479 \\
\hline 1996 & 18,429 & 3,862 & 817 & 23,109 & 7,087 & 3,528 & 148 & 5 & 33 & 438 & 4,153 & 137 & 34,485 \\
\hline 1997 & 18,905 & $\begin{array}{l}4,126 \\
4,1\end{array}$ & 927 & 23,957 & $\begin{array}{l}6,597 \\
6,07\end{array}$ & 3,581 & 150 & 5 & 34 & 446 & $\begin{array}{l}4,216 \\
\text { 4 }\end{array}$ & 116 & 34,886 \\
\hline 1998 & 19,216 & 4,675 & 1,306 & 25,197 & 7,068 & 3,241 & 151 & 5 & 31 & 444 & 3,872 & 88 & 36,225 \\
\hline 1999 & 19,279 & 4,902 & 1,211 & 25,393 & 7,610 & 3,218 & 152 & 5 & 46 & 453 & 3,874 & 99 & 36,976 \\
\hline 2000 & 20,220 & 5,293 & 1,144 & 26,658 & 7,862 & 2,768 & 144 & 5 & 57 & 453 & 3,427 & 115 & 38,062 \\
\hline 2001 & 19,614 & 5,458 & 1,277 & 26,348 & 8,029 & 2,209 & 142 & 6 & 70 & 337 & 2,763 & 75 & 37,215 \\
\hline 2002 & 19,783 & 5,767 & 961 & 26,511 & 8,145 & 2,650 & 147 & 6 & 105 & 380 & 3,288 & 72 & 38,016 \\
\hline 2003 & 20,185 & 5,246 & 1,205 & 26,636 & 7,959 & 2,781 & 148 & 5 & 115 & 397 & 3,445 & 22 & 38,062 \\
\hline 2004 & 20,305 & 5,595 & 1,212 & 27,112 & 8,222 & 2,656 & 148 & 6 & 142 & 388 & 3,340 & 39 & 38,713 \\
\hline 2005 & 20,737 & 6,015 & 1,235 & 27,986 & 8,161 & 2,670 & 147 & 6 & 178 & 406 & 3,406 & 85 & 39,638 \\
\hline 2006 & 20,462 & 6,375 & 648 & 27,485 & 8,215 & 2.839 & 145 & 5 & 264 & 412 & 3,665 & 63 & 39,428 \\
\hline 2007 & 20,808 & 7,005 & 657 & 28,470 & 8.455 & 2,430 & 145 & 6 & 341 & 423 & 3,345 & 107 & 40,377 \\
\hline 2008 & 20,513 & 6,829 & 468 & 27,810 & 8.427 & 2,494 & 146 & 9 & 546 & 435 & 3,630 & 112 & 39,978 \\
\hline 2009 & 18,225 & 7,022 & 390 & 25,638 & 8,356 & 2,650 & 146 & 9 & 721 & 441 & 3,967 & 116 & 38,077 \\
\hline 2010 & 19,133 & R7, 527 & 378 & R27,039 & R8,434 & $R_{2}, 521$ & R148 & $\mathrm{R}_{12}$ & R923 & $\begin{array}{r}441 \\
\text { R459 }\end{array}$ & $\mathrm{R}_{4,064}$ & R89 & R39,626 \\
\hline $2011^{\mathrm{P}}$ & 17,986 & 7,740 & 288 & 26,014 & 8,259 & 3,153 & 163 & 18 & 1,168 & 444 & 4,945 & 127 & 39,346 \\
\hline
\end{tabular}

1 See "Primary Energy Consumption" in Glossary.
2 See Table 10.2c for notes on series components.

2 See Table 10.2c for notes on series components.
3 Natural gas only; excludes the estimated portion of supplemental gaseous fuels. See Note 1, ${ }^{3}$ Natural gas only; excludes the estimated portion
"Supplemental Gaseous Fuels," at end of Section 6.

4 Pee Table $5.14 \mathrm{c}$ for series components.
5 .

5 Nuclear electricity net generation (converted to Btu using the nuclear heat rate-see Table A6). ${ }^{6}$ Conventional hydroelectricity net generation (converted to Btu using the fossil-fuels heat rate-see Table A6).

Geothermal electricity net generation (converted to Btu using the fossil-fuels heat rate-see Table A6). 8 Solar thermal and photovoltaic (PV) electricity net generation (converted to Btu using the fossil-fuels heat rate-see Table A6).

Wossil-fuels heat rate-see Table A6).

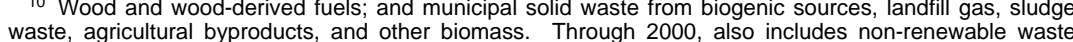

(municipal solid waste from non-biogenic sources, and tire-derived fuels).

12 Through 1988, data are for electric utilities only. Beginning in 1989, data are for electric utilities and independent power producers.

Notes: - Data are for fuels consumed to produce =Less than 0.5 trillion Btu. power sector comprises electricity-only and combined-heat-and-power (CHP) plants within the NAICS 22 category whose primary business is to sell electricity, or electricity and heat, to the public. - See Note 3 "Electricity Imports and Exports," at end of Section 8. - Totals may not equal sum of components due to independent rounding.

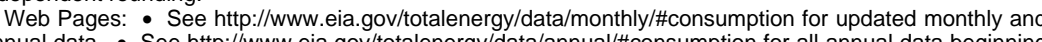
annual data.

Sources: Tables 5.14c, 6.5, 7.3, 8.1, 8.2b, 10.2c, A4, A5, and A6. 


\section{By Energy Source}

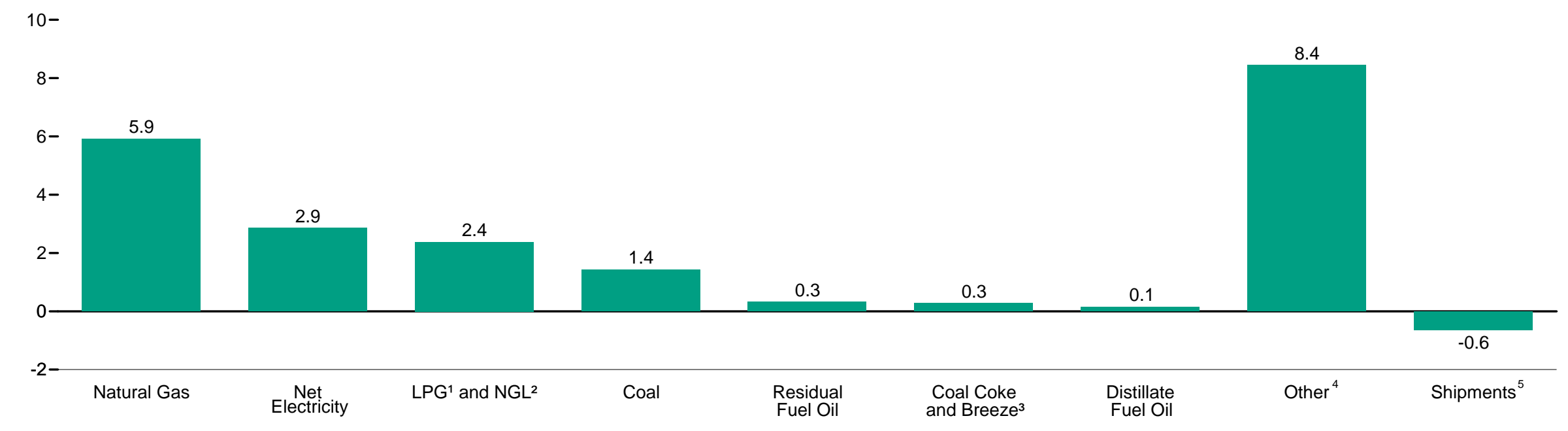

By North American Industry Classification System (NAICS) Code ${ }^{6}$

10-

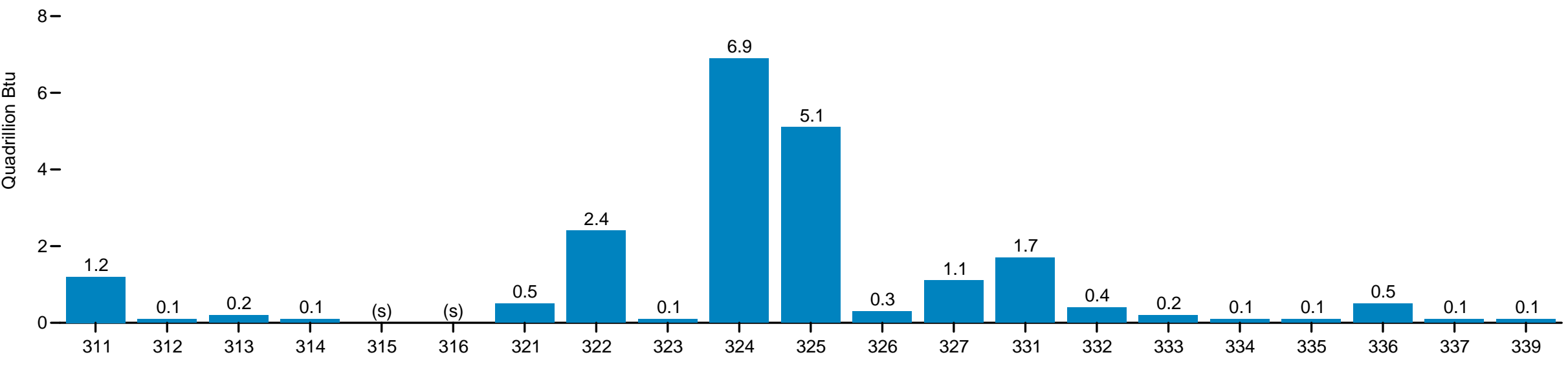

${ }^{1}$ Liquefied petroleum gases.

${ }^{2}$ Natural gas liquids.

${ }^{3}$ See "Breeze" in Glossary.

${ }^{4}$ Includes all other types of energy that respondents indicated were consumed or allocated.

${ }^{6}$ See Table 2.2 for Manufacturing Group titles of industries that correspond to the 3-digit

${ }^{5}$ Energy sources produced onsite from the use of other energy sources but sold or trans-

NAICS codes.

(s)=Less than 0.05 quadrillion Btu.

ferred to another entity.

Source: Table 2.2. 
Table 2.2 Manufacturing Energy Consumption for All Purposes, 2006

(Trillion Btu )

\begin{tabular}{|c|c|c|c|c|c|c|c|c|c|c|c|}
\hline $\begin{array}{l}\text { NAICS }{ }^{1} \\
\text { Code }\end{array}$ & Manufacturing Group & Coal & $\begin{array}{c}\text { Coal Coke } \\
\text { and } \\
\text { Breeze }^{2}\end{array}$ & Natural Gas & $\begin{array}{l}\text { Distillate } \\
\text { Fuel Oil }\end{array}$ & $\begin{array}{l}\mathrm{LPG}^{3} \\
\text { and } \\
\mathrm{NGL}^{4}\end{array}$ & $\begin{array}{c}\text { Residual } \\
\text { Fuel Oil }\end{array}$ & $\begin{array}{c}\text { Net } \\
\text { Electricity } 5\end{array}$ & Other 6 & $\begin{array}{l}\text { Shipments } \\
\text { of Energy } \\
\text { Sources } 7\end{array}$ & Total 8 \\
\hline 311 & Food & 147 & 1 & 638 & 16 & 3 & 26 & 251 & 105 & (s) & 1,186 \\
\hline 312 & Beverage and Tobacco Products ................................... & 20 & 0 & 41 & 1 & 1 & 3 & 30 & 11 & -0 & 107 \\
\hline 313 & Textile Mills & 32 & 0 & 65 & (s) & (s) & 2 & 66 & 12 & -0 & 178 \\
\hline 314 & Textile Product Mills & 3 & 0 & 46 & (s) & 1 & Q & 20 & (s) & -0 & 72 \\
\hline 315 & Apparel & 0 & 0 & 7 & (s) & (s) & $(\mathrm{s})$ & 7 & (s) & -0 & 14 \\
\hline 316 & Leather and Allied Products ………………………… & 0 & 0 & 1 & (s) & (s) & (s) & 1 & (s) & -0 & 3 \\
\hline 321 & 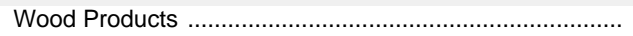 & Q & Q & 87 & 21 & 4 & 4 & 91 & 228 & -0 & 451 \\
\hline 322 & Paper & 221 & 0 & 474 & 13 & 5 & 91 & 247 & 1,302 & -0 & 2,354 \\
\hline 323 & 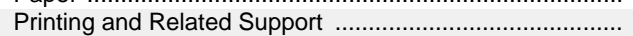 & 0 & 0 & 39 & (s) & 1 & (s) & 45 & (s) & -0 & 85 \\
\hline 324 & Petroleum and Coal Products .................................... & 102 & 1 & 849 & 33 & 29 & 58 & 137 & 5,744 & -89 & 6,864 \\
\hline 325 & 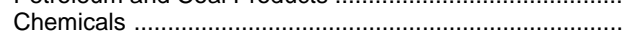 & 182 & 3 & 1,746 & 8 & 2,304 & 87 & 517 & 707 & -406 & 5,149 \\
\hline 326 & Plastics and Rubber Products ....................................... & Q & 0 & 128 & 3 & 5 & 9 & 182 & $(\mathrm{~s})$ & -0 & 337 \\
\hline 327 & 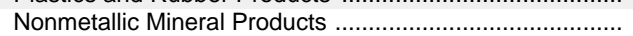 & 320 & 11 & 460 & 30 & 5 & 3 & 147 & 138 & -0 & 1,114 \\
\hline 331 & 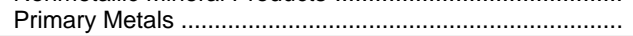 & 373 & 253 & 627 & 7 & 4 & 19 & 458 & 139 & -145 & 1,736 \\
\hline 332 & 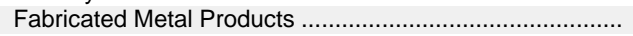 & 0 & Q & 240 & 2 & 5 & (s) & 143 & Q & -0 & 396 \\
\hline 333 & Machinery …ㄴ.. & 1 & 0 & 84 & 2 & 3 & $\mathrm{Q}$ & 111 & 2 & -0 & 204 \\
\hline 334 & Computer and Electronic Products ................................ & 0 & 0 & 45 & 1 & (s) & $(\mathrm{s})$ & 94 & 2 & -0 & 142 \\
\hline 335 & Electrical Equipment, Appliances, and Components ......... & (s) & 0 & 42 & Q & 1 & 0 & 44 & 21 & -5 & 103 \\
\hline 336 & 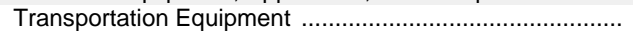 & 5 & $\mathrm{Q}$ & 249 & 3 & 5 & 7 & 195 & 13 & -0 & 477 \\
\hline 337 & Furniture and Related Products ................................. & 3 & 0 & 17 & Q & 1 & (s) & 32 & 8 & -0 & 61 \\
\hline 339 & Miscellaneous ………………………………………… & 0 & 0 & 25 & (s) & 1 & Q & 33 & $\mathrm{Q}$ & -0 & 66 \\
\hline - & Total Manufacturing & 1,433 & 272 & 5,911 & 143 & 2,376 & 314 & 2,851 & 8,443 & -645 & 21,098 \\
\hline
\end{tabular}

1 North American Industry Classification System (NAICS)

2 See "Breeze" in Glossary.

3 Liquefied petroleum gases.

4 Natural gas liquids.

5 "Net Electricity" is the sum of purchases, transfers in, and onsite generation from noncombustible renewable energy sources, minus quantities sold and transferred out; it excludes onsite generation from combustible fuels.

Includes all other types of energy that respondents indicated were consumed or allocated, such as asphalt and road oil, lubricants, naphtha less than 401 degrees Fahrenheit, other oils greater than or equal to 401 degrees Fahrenheit, special naphthas, waxes, and miscellaneous nonfuel products, which are nonfuel products assigned to the petroleum refining industry group (NAICS Code 324110).

7 Energy sources produced onsite from the use of other energy sources but sold or transferred to

another entity. Note that shipments of energy sources are subtracted from consumption.

${ }^{8}$ The sum of coal, coal coke and breeze, natural gas, distillate fuel oil, liquefied petroleum gases, natural gas liquids, residual fuel oil, net electricity, and other, minus shipments of energy sources.

(s)=Less than 0.5 trillion Btu and greater than -0.5 trillion Btu. Q=Data withheld because the relative standard error was greater than 50 percent.

Notes: - Data are estimates for the first use of energy for heat and power and as feedstocks or raw material inputs. "First use" is the consumption of energy that was originally produced offsite or was produced onsite from input materials not classified as energy. - Totals may not equal sum of components due to independent rounding.

Web Page: For related information, see http://www.eia.gov/emeu/mecs.

Source: U.S. Energy Information Administration, Form EIA-846, "2006 Manufacturing Energy Consumption Survey" and Form EIA-810, "Monthly Refinery Report" for 2006. 
Figure 2.3 Manufacturing Energy Consumption for Heat, Power, and Electricity Generation, 2006

\section{By Selected End Use ${ }^{1}$}

Process Heating

Machine Drive

Facility HVAC 2

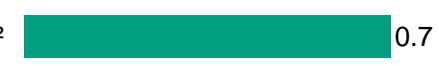

Process Cooling and Refrigeration

Electrochemical Processes

Facility Lighting
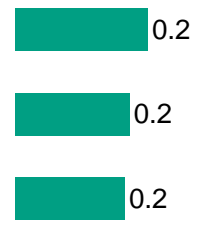

Conventional Electricity Generation (s)

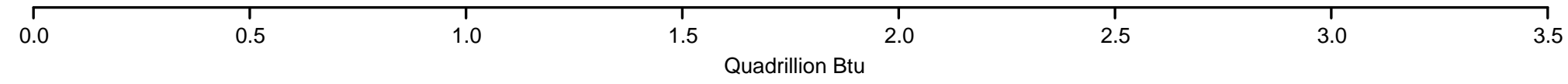

By Energy Source

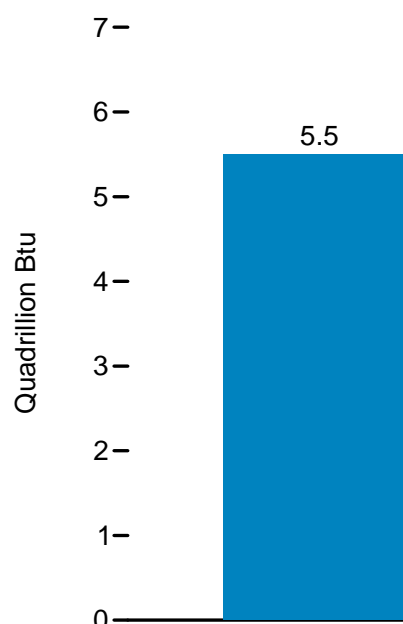

Natural Gas
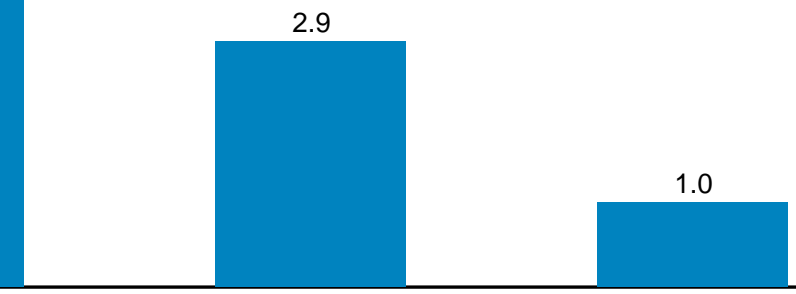

0.3

0.1

0.1

\begin{tabular}{l} 
Natural Gas Net Electricity \\
\hline${ }^{1}$ Excludes inputs of unallocated energy sources $(5,820$ trillion Btu). \\
${ }^{2}$ Heating, ventilation, and air conditioning. Excludes steam and hot water. \\
${ }^{3}$ Excludes coal coke and breeze. \\
${ }^{4}$ Liquefied petroleum gases.
\end{tabular}

Coal $^{3}$

Residual Fuel Oil Fuel Oil

Source: Table 2.3. 
Table 2.3 Manufacturing Energy Consumption for Heat, Power, and Electricity Generation by End Use, 2006

\begin{tabular}{|c|c|c|c|c|c|c|c|}
\hline \multirow[b]{2}{*}{ End-Use Category } & Net Electricity ${ }^{1}$ & Residual Fuel Oil & Distillate Fuel Oil & $\mathrm{LPG}^{2}$ and $\mathrm{NGL}^{3}$ & \multirow{2}{*}{$\frac{\text { Natural Gas }}{\text { Billion Cubic Feet }}$} & \multirow{2}{*}{$\frac{\text { Coal }^{4}}{\text { Million Short Tons }}$} & \multirow[b]{2}{*}{ Total ${ }^{5}$} \\
\hline & Million Kilowatthours & \multicolumn{3}{|c|}{ Million Barrels } & & & \\
\hline $\begin{array}{l}\text { Indirect End Use (Boiler Fuel) } \\
\text { Conventional Boiler Use }\end{array}$ & $\begin{array}{l}\mathbf{1 2 , 1 0 9} \\
12,109\end{array}$ & $\begin{array}{l}\mathbf{2 1} \\
11\end{array}$ & $\begin{array}{l}4 \\
3\end{array}$ & $\begin{array}{l}2 \\
2\end{array}$ & $\begin{array}{l}2,059 \\
1,245\end{array}$ & $\begin{array}{r}25 \\
6\end{array}$ & $\begin{array}{ll}-- \\
--\end{array}$ \\
\hline 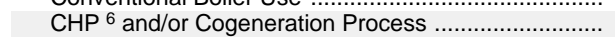 & -- & 10 & 1 & $(\mathrm{~s})$ & 814 & 19 & -- \\
\hline \multicolumn{8}{|l|}{ Direct End Use } \\
\hline All Process Uses & 657,810 & 10 & 9 & 10 & 2,709 & 19 & -- \\
\hline 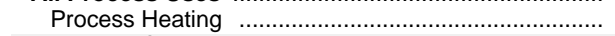 & 101,516 & 9 & 3 & 8 & 2,417 & 16 & -- \\
\hline Process Cooling and Refrigeration ................................... & 60,381 & $(\mathrm{~s})$ & (s) & (s) & 31 & (s) & -- \\
\hline Machine Drive .......................................................... & 422,408 & (s) & 4 & (s) & 126 & 3 & -- \\
\hline Electrochemical Processes ………………………......... & 60,323 & -- & -- & -- & -- & -- & -- \\
\hline 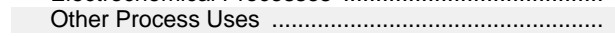 & 13,181 & (s) & 1 & 1 & 136 & (s) & -- \\
\hline 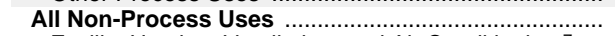 & 157,829 & 1 & 9 & 7 & 426 & (s) & -- \\
\hline Facility Heating, Ventilation, and Air Conditioning 7 ... & 77,768 & 1 & 1 & 1 & 367 & (s) & -- \\
\hline Facility Lighting & 58,013 & -- & -- & -- & -- & -- & -- \\
\hline Other Facility Support ……………………………. & 17,644 & (s) & (s) & (s) & 29 & (s) & -- \\
\hline Onsite Transportation ................................................... & 2,197 & -- & 6 & 5 & 3 & -- & -- \\
\hline Conventional Electricity Generation .................................. & -- & (s) & 1 & (s) & 19 & (s) & -- \\
\hline Other Non-Process Use .......................................... & 2,208 & (s) & 1 & (s) & 8 & (s) & -- \\
\hline End Use Not Reported & 7,634 & 8 & 1 & 1 & 164 & 2 & -- \\
\hline \multirow[t]{2}{*}{ Total } & 835,382 & 40 & 22 & 21 & 5,357 & 46 & -- \\
\hline & \multicolumn{7}{|c|}{ Trillion Btu } \\
\hline Indirect End Use (Boiler Fuel) & 41 & 133 & 23 & 8 & 2,119 & 547 & 2,871 \\
\hline 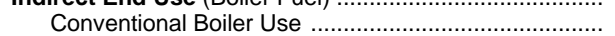 & 41 & 71 & 17 & 8 & 1,281 & 129 & 1,547 \\
\hline CHP 6 and/or Cogeneration Process ............................... & -- & 62 & 6 & 1 & 838 & 417 & 1,324 \\
\hline \multicolumn{8}{|l|}{ Direct End Use } \\
\hline All Process Uses & 2,244 & 62 & 52 & 39 & 2,788 & 412 & 5,597 \\
\hline 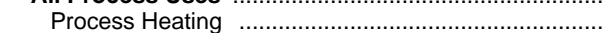 & 346 & 59 & 19 & 32 & 2,487 & 345 & 3,288 \\
\hline Process Cooling and Refrigeration .................................. & 206 & (s) & 1 & (s) & 32 & (s) & 239 \\
\hline 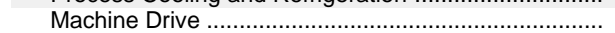 & 1,441 & 2 & 24 & 2 & 129 & 56 & 1,654 \\
\hline Electrochemical Processes ………………………..... & 206 & -- & -- & -- & -- & -- & 206 \\
\hline Other Process Uses & 45 & Q & 8 & 5 & 140 & 10 & 208 \\
\hline & 539 & 6 & 50 & 27 & 438 & 6 & 1,066 \\
\hline Facility Heating, Ventilation, and Air Conditioning $7 \ldots$ & 265 & 4 & 4 & 5 & 378 & 2 & 658 \\
\hline 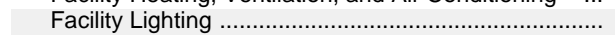 & 198 & -- & -- & -- & -- & -- & 198 \\
\hline 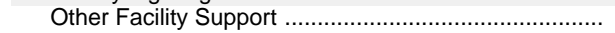 & 60 & 1 & (s) & (s) & 30 & (s) & 91 \\
\hline 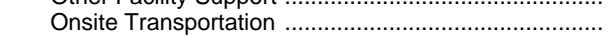 & 7 & -- & 35 & 20 & 3 & -- & 65 \\
\hline Conventional Electricity Generation .................................... & -- & (s) & 4 & (s) & 19 & 3 & 26 \\
\hline Other Non-Process Use …………………………….... & 8 & (s) & 6 & 1 & 8 & (s) & 23 \\
\hline End Use Not Reported & 26 & 49 & 4 & 5 & 168 & 52 & 304 \\
\hline Total & 2,850 & 251 & 129 & 79 & 5,512 & 1,016 & 9,838 \\
\hline \multicolumn{8}{|c|}{$\begin{array}{l}1 \text { "Net Electricity" is the sum of purchases, transfers in, and onsite generation from noncombustible } \\
\text { renewable energy sources, minus quantities sold and transferred out; it excludes onsite generation from } \\
\text { combustible fuels. } \\
2 \text { Liquefied petroleum gases. } \\
3 \text { Natural gas liquids. } \\
4 \text { Excludes coal coke and breeze. } \\
5 \text { Total of listed energy sources. Excludes inputs of unallocated energy sources }(5,820 \text { trillion Btu). } \\
6 \text { Combine-heat-and-power plants. } \\
7 \text { Excludes steam and hot water. } \\
--=\text { Not applicable. (s)=Estimate less than } 0.5 \text {. Q=Withheld because relative standard error is greater } \\
\text { than } 50 \text { percent. }\end{array}$} \\
\hline
\end{tabular}


Household Energy Consumpton by Census Region, Selected Years, 1978-2009¹

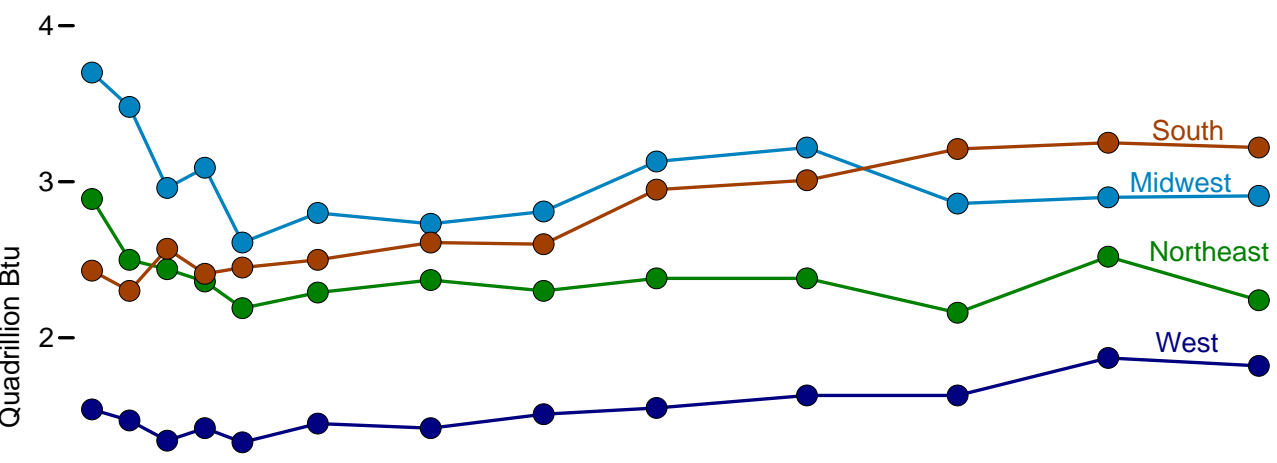

$1-$

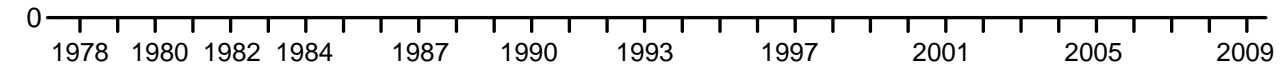

Energy Consumption per Household, Selected Years, 1978-2009¹

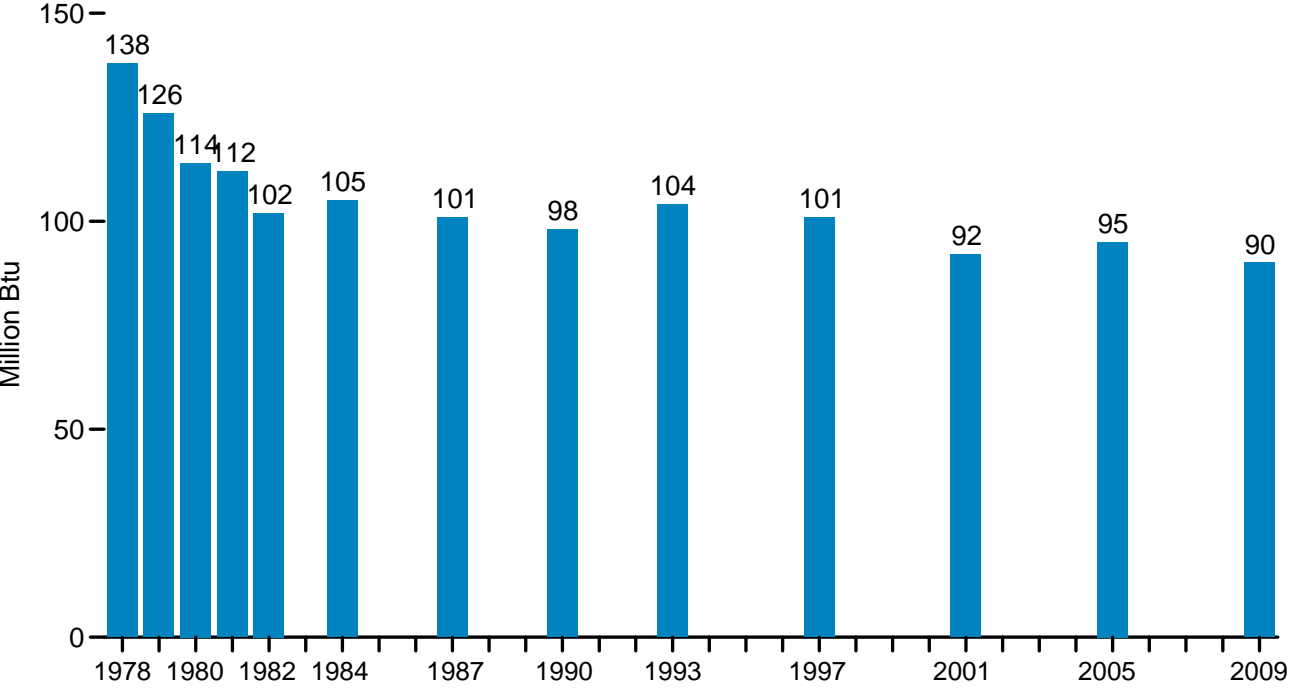

Household Energy Consumption by Source, 2009

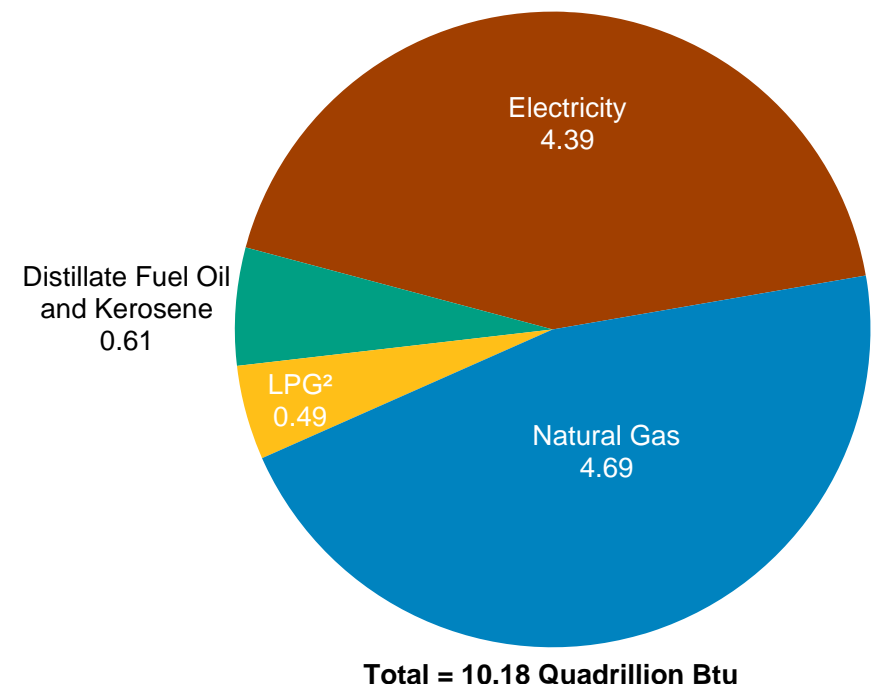

Energy Consumption per Household, by Census Region, 2009 $120-$

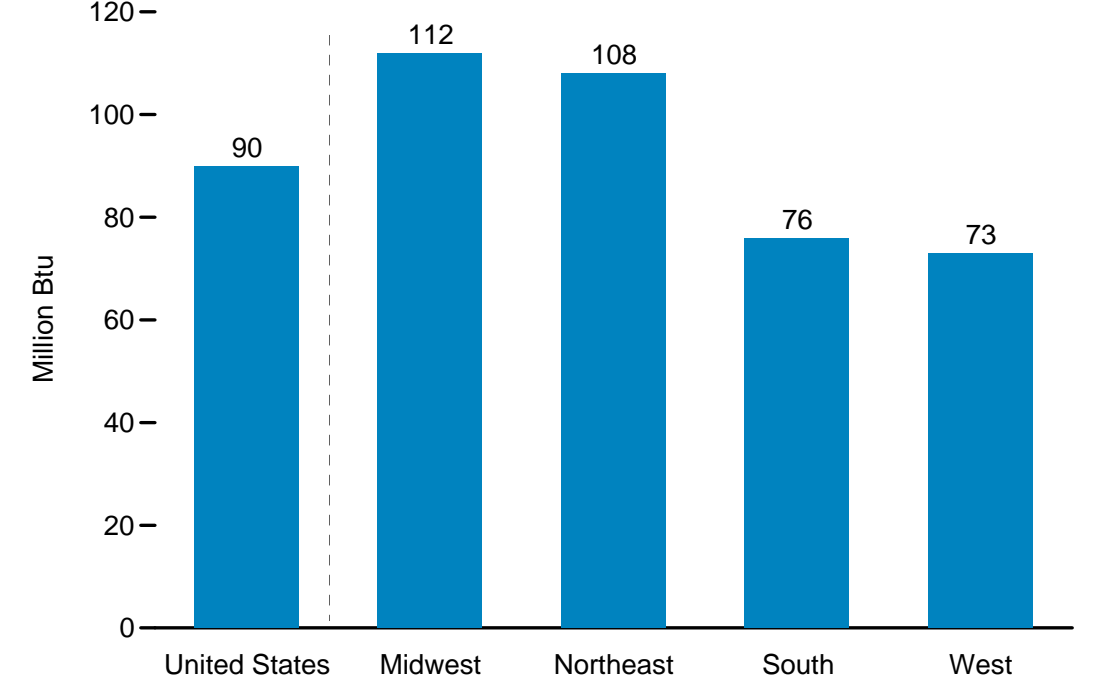

${ }^{1}$ For years not shown, there are no data available.

${ }^{2}$ Liquefied petroleum gases.

Notes: - Data include natural gas, electricity, distillate fuel oil, kerosene, and liquefied petroleum gases; data do not include wood. - Data for 1978-1984 are for April of the year shown through March of following year; data for 1987 forward are for the calendar year. - See Appendix $C$ for map of Census regions.

Source: Table 2.4 . 
Table 2.4 Household ' Energy Consumption by Census Region, Selected Years, 1978-2009

(Quadrillion Btu, Except as Noted)

\begin{tabular}{|c|c|c|c|c|c|c|c|c|c|c|c|c|c|}
\hline Census Region ${ }^{2}$ & 1978 & 1979 & 1980 & 1981 & 1982 & 1984 & 1987 & 1990 & 1993 & 1997 & 2001 & 2005 & 2009 \\
\hline United States Total (does not include wood) ....... & 10.56 & 9.74 & 9.32 & 9.29 & 8.58 & 9.04 & 9.13 & 9.22 & 10.01 & 10.25 & 9.86 & 10.55 & 10.18 \\
\hline Natural Gas & 5.58 & 5.31 & 4.97 & 5.27 & 4.74 & 4.98 & 4.83 & 4.86 & 5.27 & 5.28 & 4.84 & 4.79 & 4.69 \\
\hline Electricity ${ }^{3}$ & 2.47 & 2.42 & 2.48 & 2.42 & 2.35 & 2.48 & 2.76 & 3.03 & 3.28 & 3.54 & 3.89 & 4.35 & 4.39 \\
\hline Distillate Fuel Oil and Kerosene ............................ & 2.19 & 1.71 & 1.52 & 1.28 & 1.20 & 1.26 & 1.22 & 1.04 & 1.07 & 1.07 & .75 & .88 & .61 \\
\hline 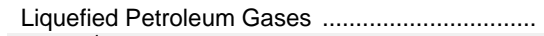 & .33 & .31 & .35 & .31 & .29 & .31 & .32 & .28 & .38 & .36 & .38 & .52 & .49 \\
\hline Wood ${ }^{4} \ldots \ldots \ldots$ & NA & NA & .85 & .87 & .97 & .98 & .85 & .58 & .55 & .43 & .37 & .43 & .50 \\
\hline Consumption per Household (million Btu) ${ }^{3} \ldots \ldots$ & 138 & 126 & 114 & 112 & 102 & 105 & 101 & 98 & 104 & 101 & 92 & 95 & 90 \\
\hline Northeast Total (does not include wood) .............. & 2.89 & 2.50 & 2.44 & 2.36 & 2.19 & 2.29 & 2.37 & 2.30 & 2.38 & 2.38 & 2.16 & 2.52 & 2.24 \\
\hline 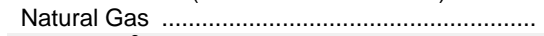 & 1.14 & 1.05 & .94 & 1.01 & .96 & .93 & 1.03 & 1.03 & 1.11 & 1.03 & .98 & 1.15 & 1.06 \\
\hline Electricity ${ }^{3}$ & .39 & .39 & .41 & .40 & .37 & .41 & .44 & .47 & .47 & .49 & .53 & .58 & .57 \\
\hline Distillate Fuel Oil and Kerosene .............................. & 1.32 & 1.03 & 1.07 & .93 & .83 & .93 & .87 & .78 & .78 & .84 & .60 & .72 & .52 \\
\hline Liquefied Petroleum Gases .................................. & .03 & .03 & .03 & .03 & .02 & .03 & .02 & .02 & .03 & .03 & .05 & .07 & .08 \\
\hline 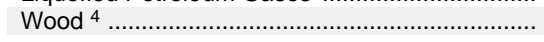 & NA & NA & .26 & .27 & .24 & .21 & .17 & .12 & .14 & .14 & .10 & .09 & .10 \\
\hline Consumption per Household (million Btu) ${ }^{3} \ldots \ldots .$. & 166 & 145 & 138 & 132 & 122 & 125 & 124 & 120 & 122 & 121 & 107 & 122 & 108 \\
\hline Midwest Total (does not include wood) ……........... & 3.70 & 3.48 & 2.96 & 3.09 & 2.61 & 2.80 & 2.73 & 2.81 & 3.13 & 3.22 & 2.86 & 2.91 & 2.91 \\
\hline 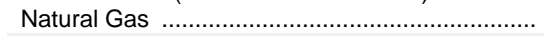 & 2.53 & 2.48 & 2.05 & 2.22 & 1.78 & 1.99 & 1.83 & 1.88 & 2.07 & 2.20 & 1.84 & 1.72 & 1.75 \\
\hline 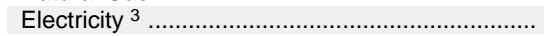 & .60 & .59 & .60 & .56 & .56 & .55 & .61 & .66 & .74 & .75 & .81 & .94 & .94 \\
\hline 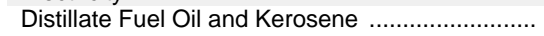 & .46 & .31 & .17 & .19 & .16 & .13 & .16 & .13 & .13 & .11 & .06 & .06 & .03 \\
\hline Liquefied Petroleum Gases ……..................... & .12 & .10 & .15 & .13 & .11 & .13 & .13 & 13 & .19 & .17 & .15 & .18 & .19 \\
\hline 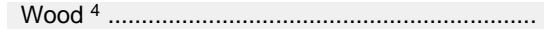 & NA & NA & .25 & .25 & .27 & .27 & .25 & .17 & .11 & .08 & .09 & .13 & .14 \\
\hline Consumption per Household (million Btu) ${ }^{3} \ldots \ldots$ & 180 & 168 & 141 & 146 & 122 & 129 & 123 & 122 & 134 & 134 & 117 & 113 & 112 \\
\hline South Total (does not include wood) ....................... & 2.43 & 2.30 & 2.57 & 2.41 & 2.45 & 2.50 & 2.61 & 2.60 & 2.95 & 3.01 & 3.21 & 3.25 & 3.22 \\
\hline Natural Gas & .96 & .91 & 1.12 & 1.15 & 1.14 & 1.15 & 1.09 & 1.03 & 1.18 & 1.13 & 1.13 & .94 & .94 \\
\hline Electricity ${ }^{3}$ & 1.00 & .97 & 1.06 & 1.01 & 1.01 & 1.06 & 1.22 & 1.36 & 1.51 & 1.67 & 1.89 & 2.07 & 2.09 \\
\hline 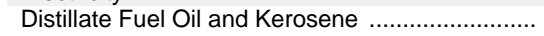 & .32 & .28 & .25 & .14 & .18 & .16 & .17 & .11 & .13 & .10 & .08 & .07 & .05 \\
\hline Liquefied Petroleum Gases ........................................ & .15 & .14 & .14 & .12 & .12 & .12 & .12 & .10 & .13 & .12 & .12 & .18 & .14 \\
\hline 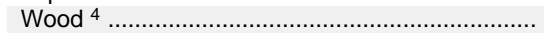 & NA & NA & .23 & .21 & .33 & .33 & .26 & .17 & .17 & .12 & .09 & .12 & .16 \\
\hline Consumption per Household (million Btu) ${ }^{3} \ldots \ldots$. & 99 & 92 & 95 & 87 & 87 & 85 & 84 & 81 & 88 & 84 & 83 & 80 & 76 \\
\hline West Total (does not include wood) ………............. & 1.54 & 1.47 & 1.34 & 1.42 & 1.33 & 1.45 & 1.42 & 1.51 & 1.55 & 1.63 & 1.63 & 1.87 & 1.82 \\
\hline 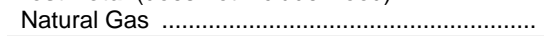 & .95 & .88 & .86 & .90 & .85 & .91 & .88 & .92 & .91 & .93 & .90 & .98 & .94 \\
\hline Electricity ${ }^{3}$ & .48 & .47 & .41 & .46 & .41 & .47 & .48 & .54 & .56 & .64 & .66 & .76 & .79 \\
\hline Distillate Fuel Oil and Kerosene ............................... & .09 & .09 & .04 & .03 & .03 & .04 & .02 & .02 & .03 & .03 & .02 & .03 & .01 \\
\hline Liquefied Petroleum Gases ………...................... & .03 & .04 & .04 & .04 & .04 & .03 & .05 & .03 & .04 & .04 & .06 & .10 & .08 \\
\hline Wood 4 . & NA & NA & .11 & .13 & .13 & .17 & .17 & .12 & .12 & .10 & .10 & .09 & .10 \\
\hline Consumption per Household (million Btu) ${ }^{3} \ldots \ldots .$. & 110 & 100 & 84 & 87 & 81 & 85 & 78 & 78 & 76 & 75 & 70 & 77 & 73 \\
\hline
\end{tabular}

1 Includes energy consumption in occupied primary housing units only, which differs from residential sector energy consumption.

2 See Appendix $C$ for map of Census regions.

Retail electricity. One kilowatthour $=3,412 \mathrm{Btu}$.

Wood is not included in the region and U.S. totals, or in the consumption-per-household data.

$\mathrm{NA}=$ Not available.
Notes: - Data are estimates, and are for major energy sources only. - For years not shown, there are

no data available. - Data for 1978-1984 are for April of year shown through March of following year; data for 1987 forward are for the calendar year. - Totals may not equal sum of components due to independent rounding.

Web Page: For related information, see http://www.eia.gov/consumption/residential/

Sources: - 1978 and 1979-U.S. Energy Information Administration (EIA), Form EIA-84, "Residential Energy Consumption Survey." • 1980 forward-EIA, Form EIA-457, "Residential Energy Consumption Survey." 


\section{Figure 2.5 Household Energy Consumption and Expenditures}

Household Energy Consumption by End Use, Selected Years, 1978-2005'

$8-$

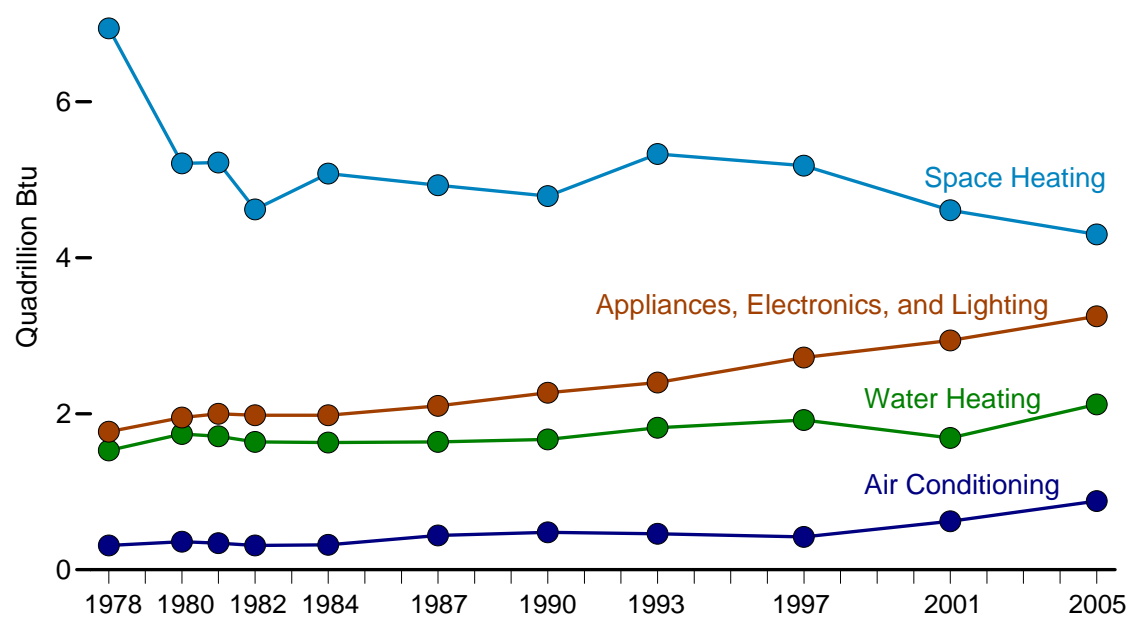

Household Energy Consumption for Space Heating by Fuel 2005

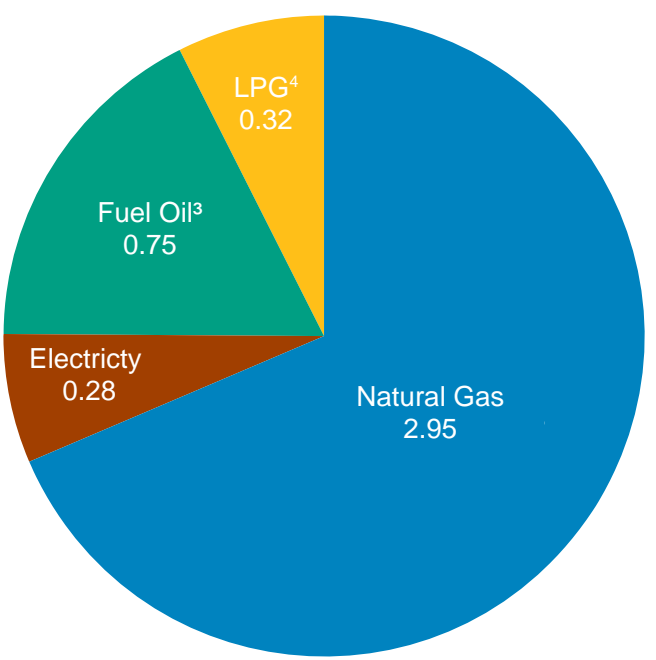

Total $=4.30$ Ouadrillion Btu
Household Energy Expenditures, Selected Years, 1978-2005

$250-$

$200-$

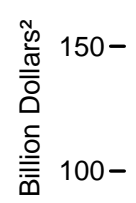

160

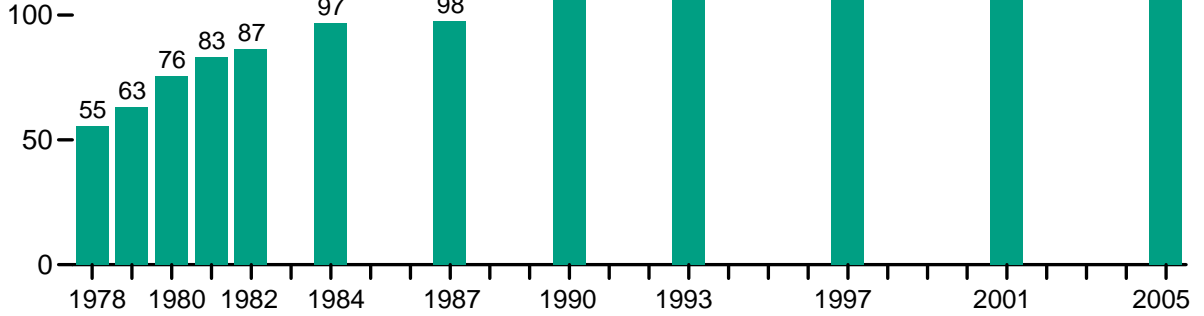

Appliances, Electronics, and Lighting Expenditures, Selected Years, 1978-2005'

$100-$

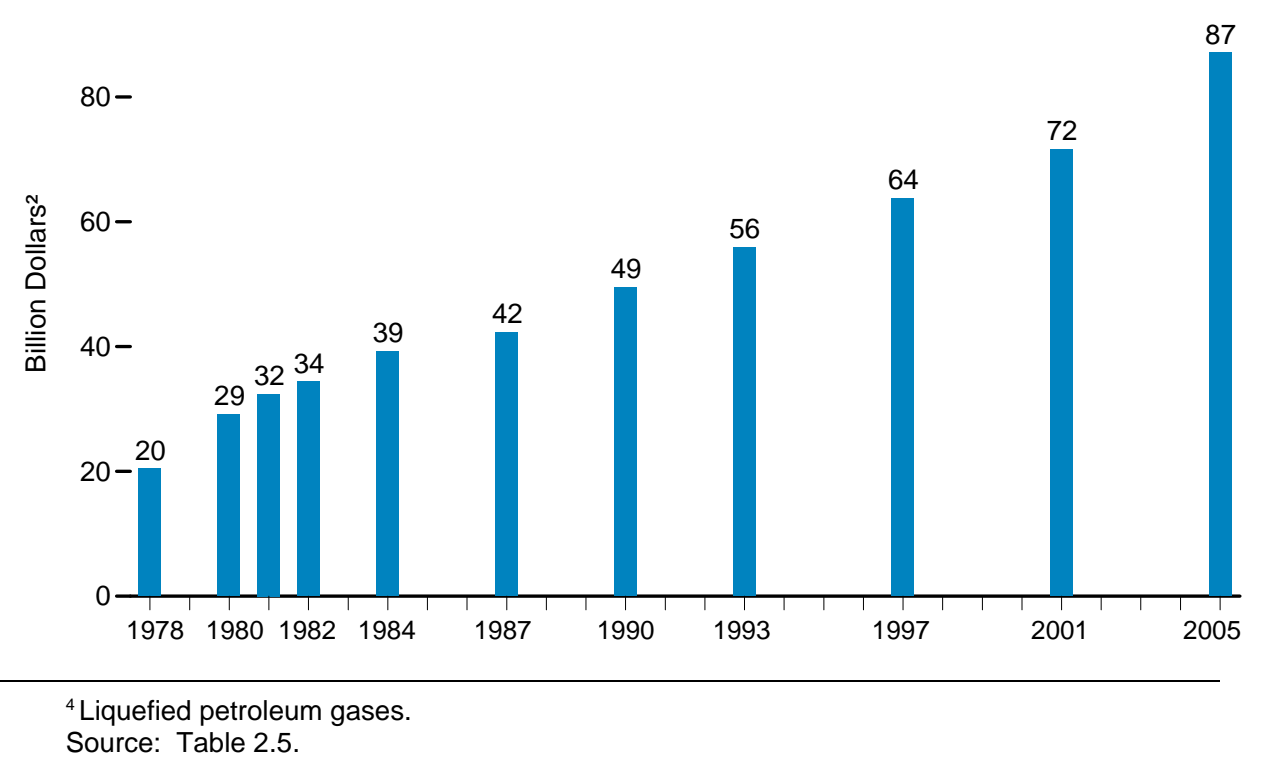

Source: Table 2.5 .

${ }^{1}$ For years not shown, there are no data available.

2 Prices are not adjusted for inflation. See "Nominal Dollars" in Glossary.

${ }^{3}$ Distillate fuel oil and kerosene. 
Table 2.5 Household ' Energy Consumption and Expenditures by End Use, Selected Years, 1978-2005

\begin{tabular}{|c|c|c|c|c|c|c|c|c|c|c|c|c|c|c|c|}
\hline \multirow[b]{2}{*}{ Year } & \multicolumn{5}{|c|}{ Space Heating } & \multirow{2}{*}{$\begin{array}{c}\begin{array}{c}\text { Air } \\
\text { Conditioning }\end{array} \\
\text { Electricity }{ }^{3}\end{array}$} & \multicolumn{5}{|c|}{ Water Heating } & \multicolumn{4}{|c|}{ Appliances, ${ }^{2}$ Electronics, and Lighting } \\
\hline & $\begin{array}{l}\text { Natural } \\
\text { Gas }\end{array}$ & $\begin{array}{l}\text { Elec- } \\
\text { tricity }^{3}\end{array}$ & $\begin{array}{l}\text { Fuel } \\
\text { Oil }{ }^{4}\end{array}$ & LPG 5 & Total & & $\begin{array}{l}\text { Natural } \\
\text { Gas }\end{array}$ & $\begin{array}{c}\text { Elec- } \\
\text { tricity }^{3}\end{array}$ & $\begin{array}{l}\text { Fuel } \\
\text { Oil }{ }^{4}\end{array}$ & LPG 5 & Total & $\begin{array}{l}\text { Natural } \\
\text { Gas }\end{array}$ & $\begin{array}{l}\text { Elec- } \\
\text { tricity }^{3}\end{array}$ & LPG 5 & Total \\
\hline & \multicolumn{15}{|c|}{ Consumption (quadrillion Btu) } \\
\hline 1978 & 4.26 & 0.40 & 2.05 & 0.23 & 6.94 & 0.31 & 1.04 & 0.29 & 0.14 & 0.06 & 1.53 & 0.28 & 1.46 & 0.03 & 1.77 \\
\hline 1980 & 3.41 & .27 & 1.30 & .23 & 5.21 & .36 & 1.15 & .30 & .22 & .07 & 1.74 & .36 & 1.54 & .05 & 1.95 \\
\hline 1981 & 3.69 & .26 & 1.06 & .21 & 5.22 & .34 & 1.13 & .30 & .22 & .06 & 1.71 & .43 & 1.52 & .05 & 2.00 \\
\hline 1982 & 3.14 & .25 & 1.04 & .19 & 4.62 & .31 & 1.15 & .28 & .15 & .06 & 1.64 & .43 & 1.50 & .05 & 1.98 \\
\hline 1984 & 3.51 & .25 & 1.11 & .21 & 5.08 & .32 & 1.10 & .32 & .15 & .06 & 1.63 & .35 & 1.59 & .04 & 1.98 \\
\hline 1987 & 3.38 & .28 & 1.05 & .22 & 4.93 & .44 & 1.10 & .31 & .17 & .06 & 1.64 & .34 & 1.72 & .04 & 2.10 \\
\hline 1990 & 3.37 & .30 & .93 & .19 & 4.79 & .48 & 1.16 & .34 & .11 & .06 & 1.67 & .33 & 1.91 & .03 & 2.27 \\
\hline 1993 & 3.67 & .41 & .95 & .30 & 5.33 & .46 & 1.31 & .34 & .12 & .05 & 1.82 & .29 & 2.08 & .03 & 2.40 \\
\hline 1997 & 3.61 & .40 & .91 & .26 & 5.18 & .42 & 1.29 & .39 & .16 & .08 & 1.92 & .37 & 2.33 & .02 & 2.72 \\
\hline 2001 & 3.32 & .39 & .62 & .28 & 4.61 & .62 & 1.15 & .36 & .13 & .05 & 1.69 & .37 & 2.52 & .05 & 2.94 \\
\hline \multirow[t]{2}{*}{2005} & 2.95 & .28 & .75 & .32 & 4.30 & .88 & 1.41 & .42 & .14 & .15 & 2.12 & .43 & 2.77 & .05 & 3.25 \\
\hline & \multicolumn{15}{|c|}{ Expenditures (billion nominal dollars ${ }^{6}$ ) } \\
\hline 1978 & 11.49 & 3.53 & 8.06 & 1.05 & 24.13 & 3.97 & 2.88 & 3.15 & 0.56 & 0.36 & 6.95 & 0.93 & 19.24 & 0.25 & 20.42 \\
\hline 1980 & 13.22 & 3.78 & 10.48 & 1.78 & 29.26 & 5.84 & 4.51 & 4.45 & 1.76 & .57 & 11.29 & 1.91 & 26.74 & .44 & 29.09 \\
\hline 1981 & 16.62 & 3.93 & 9.44 & 1.78 & 31.77 & 6.23 & 5.13 & 4.94 & 1.94 & .51 & 12.52 & 2.17 & 29.70 & .52 & 32.39 \\
\hline 1982 & 17.74 & 4.21 & 8.80 & 1.69 & 32.44 & 6.23 & 6.51 & 5.00 & 1.28 & .54 & 13.33 & 2.58 & 31.29 & .52 & 34.39 \\
\hline 1984 & 20.66 & 4.62 & 8.51 & 2.00 & 35.79 & 7.06 & 6.63 & 6.44 & 1.09 & .58 & 14.74 & 2.31 & 36.36 & .54 & 39.21 \\
\hline 1987 & 18.05 & 5.53 & 6.25 & 1.85 & 31.68 & 9.77 & 6.02 & 6.45 & .94 & .50 & 13.91 & 2.02 & 39.83 & .46 & 42.31 \\
\hline 1990 & 18.59 & 6.16 & 7.42 & 2.01 & 34.18 & 11.23 & 6.59 & 7.21 & .83 & .65 & 15.28 & 2.03 & 46.95 & .48 & 49.46 \\
\hline 1993 & 21.95 & 8.66 & 6.24 & 2.81 & 39.66 & 11.31 & 8.08 & 7.58 & .74 & .58 & 16.98 & 1.98 & 53.52 & .42 & 55.92 \\
\hline 1997 & 24.11 & 8.56 & 6.57 & 2.79 & 42.03 & 10.20 & 8.84 & 8.99 & 1.04 & .89 & 19.76 & 2.86 & 60.57 & .36 & 63.79 \\
\hline 2001 & 31.84 & 8.98 & 5.66 & 4.04 & 50.52 & 15.94 & 11.31 & 8.47 & 1.15 & .69 & 21.62 & 3.83 & 66.94 & .86 & 71.63 \\
\hline 2005 & 31.97 & 7.42 & 10.99 & 6.35 & 56.73 & 25.26 & 15.57 & 11.13 & 2.00 & 3.28 & 31.98 & 4.80 & 80.92 & 1.37 & 87.09 \\
\hline
\end{tabular}

1 Includes energy consumption in occupied primary housing units only, which differs from residential sector energy consumption.

2 Includes refrigerators.

Retail electricity. One kilowatthour $=3,412$ Btu.

4 Distillate fuel oil and kerosene.

6 Prices are not adjusted for inflation. See "Nominal Dollars" in Glossary.
Notes: • 2009 data for this table were not available in time for publication. - Data are estimates. - For years not shown, there are no data available. - Totals may not equal sum of components due to independent rounding.

Web Page: For related information, see http://www.eia.gov/consumption/residential/.

Sources: - 1978-U.S. Energy Information Administration (EIA), Form EIA-84, "Residential Energy Consumption Survey." • 1980 forward-EIA, Form ElA-457, "Residential Energy Consumption Survey." 
Figure 2.6 Household End Uses: Fuel Types, Appliances, and Electronics

Share of Households With Selected Appliances, 1980 and 2009

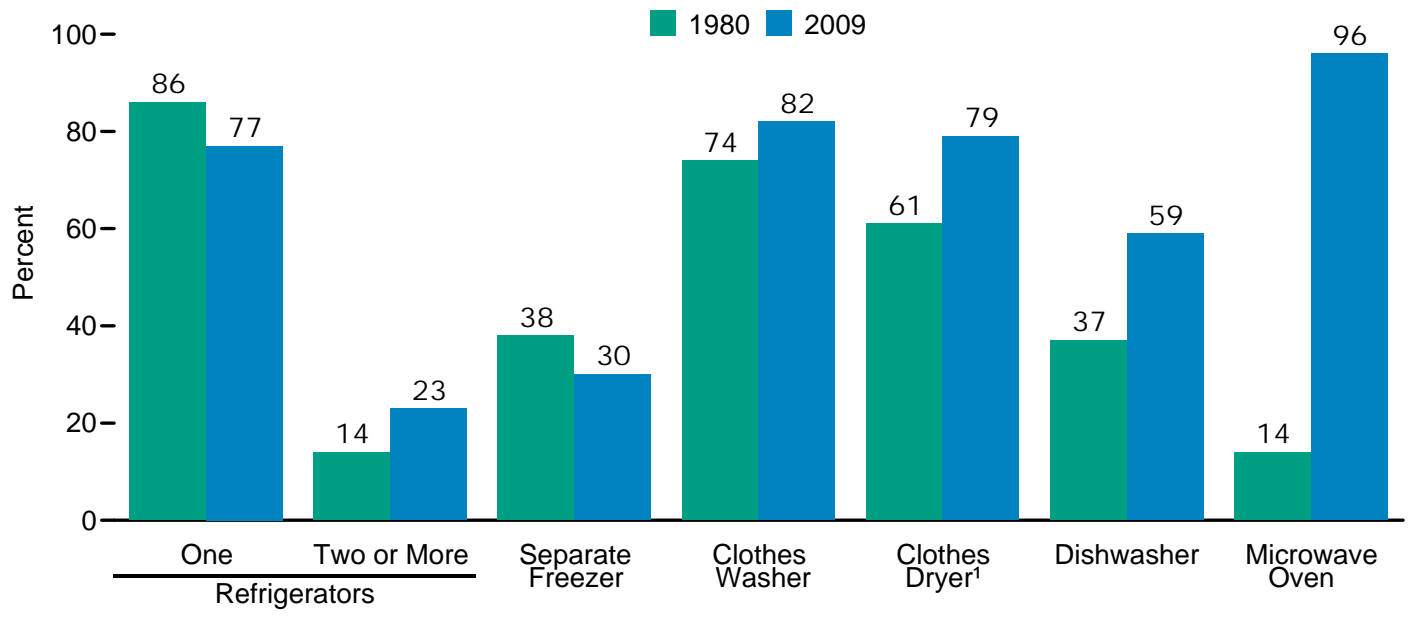

Share of Households With Selected Electronics, 1997 and 2009

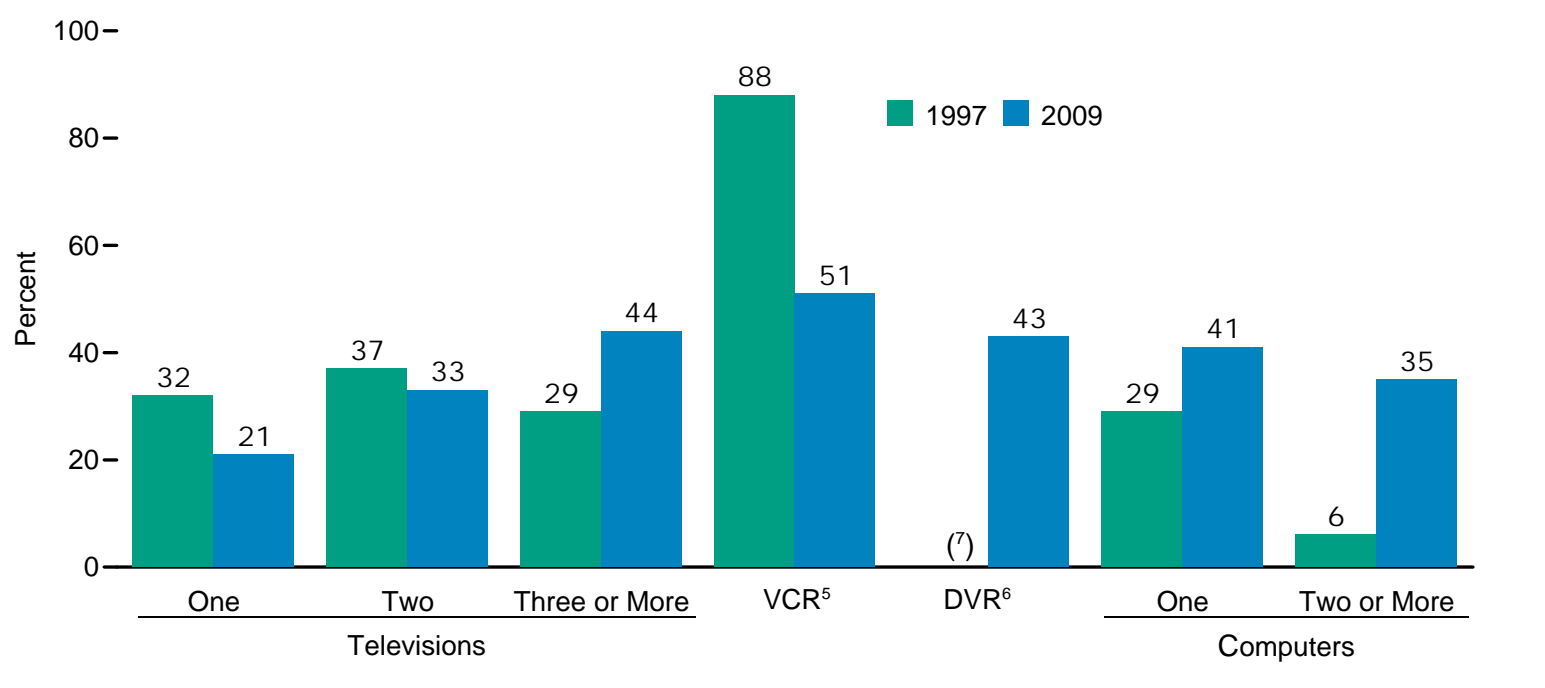

Space Heating by Main Fuel, 2009

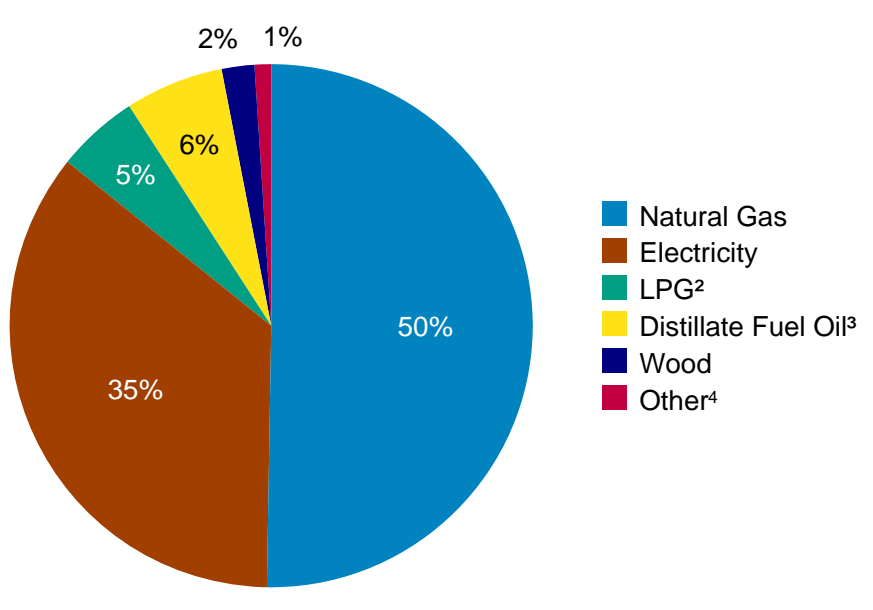

Air-Conditioning Equipment, 1980 and 2009

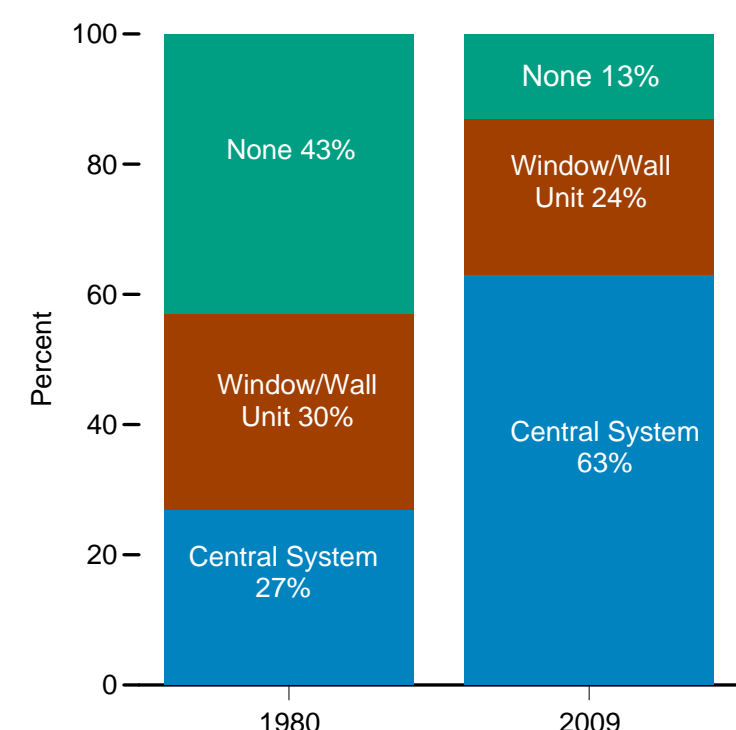

${ }^{1}$ Natural gas and electric.

${ }^{2}$ Liquefied petroleum gases.

${ }^{3}$ Includes kerosene.

${ }^{4} \mathrm{Coal}$, solar, other fuel, or no heating equipment. ${ }^{5}$ Video Cassette Recorder.
${ }^{6}$ Digital Video Recorder.

${ }^{7}$ Not collected in 1997.

Note: Total may not equal sum of components due to independent rounding.

Source: Table 2.6 . 
Table 2.6 Household End Uses: Fuel Types, Appliances, and Electronics, Selected Years, 1978-2009

\begin{tabular}{|c|c|c|c|c|c|c|c|c|c|c|c|c|c|c|}
\hline \multirow[b]{2}{*}{ Appliance } & \multicolumn{13}{|c|}{ Year } & \multirow{2}{*}{$\begin{array}{c}\text { Change } \\
1980 \text { to } 2009\end{array}$} \\
\hline & 1978 & 1979 & 1980 & 1981 & 1982 & 1984 & 1987 & 1990 & 1993 & 1997 & 2001 & 2005 & 2009 & \\
\hline \multirow[t]{2}{*}{ Total Households (millions) . } & 77 & 78 & 82 & 83 & 84 & 86 & 91 & 94 & 97 & 101 & 107 & 111 & 114 & 32 \\
\hline & \multicolumn{14}{|c|}{ Percent of Households } \\
\hline \multicolumn{15}{|l|}{ Space Heating - Main Fuel ${ }^{1}$} \\
\hline 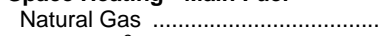 & 55 & 55 & 55 & 56 & 57 & 55 & 55 & 55 & 53 & 52 & 55 & 52 & 50 & -5 \\
\hline Electricity ${ }^{2}$ & 16 & 17 & 18 & 17 & 16 & 17 & 20 & 23 & 26 & 29 & 29 & 30 & 35 & 17 \\
\hline Liquefied Petroleum Gases ............. & 4 & 5 & 5 & 4 & 5 & 5 & 5 & 5 & 5 & 5 & 5 & 5 & 5 & 0 \\
\hline 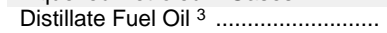 & 20 & 17 & 15 & 14 & 13 & 12 & 12 & 11 & 11 & 9 & 7 & 7 & 6 & -9 \\
\hline 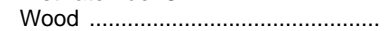 & 2 & 4 & 6 & 6 & 7 & 7 & 6 & 4 & 3 & 2 & 2 & 3 & 2 & -4 \\
\hline Other ${ }^{4}$ or No Equipment ...................... & 3 & 2 & 2 & 3 & 3 & 3 & 3 & 2 & 2 & 2 & 2 & 3 & 1 & -1 \\
\hline \multicolumn{15}{|l|}{ Air Conditioning - Equipment } \\
\hline Central System 5 & 23 & 24 & 27 & 27 & 28 & 30 & 34 & 39 & 44 & 47 & 55 & 59 & 63 & 36 \\
\hline 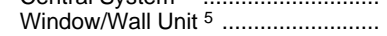 & 33 & 31 & 30 & 31 & 30 & 30 & 30 & 29 & 25 & 25 & 23 & 25 & 24 & -6 \\
\hline 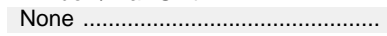 & 44 & 45 & 43 & 42 & 42 & 40 & 36 & 32 & 32 & 28 & 23 & 16 & 13 & -30 \\
\hline \multicolumn{15}{|l|}{ Water Heating - Main Fuel } \\
\hline 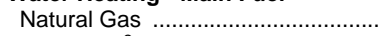 & 55 & 55 & 54 & 55 & 56 & 54 & 54 & 53 & 53 & 52 & 54 & 53 & 51 & -3 \\
\hline Electricity ${ }^{2}$ & 33 & 33 & 32 & 33 & 32 & 33 & 35 & 37 & 38 & 39 & 38 & 39 & 41 & 9 \\
\hline Liquefied Petroleum Gases ............. & 4 & 4 & 4 & 4 & 4 & 4 & 3 & 3 & 3 & 3 & 3 & 4 & 4 & 0 \\
\hline 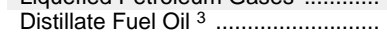 & 8 & 7 & 9 & 7 & 7 & 6 & 6 & 5 & 5 & 5 & 4 & 4 & 3 & -6 \\
\hline Other or No Water Heating ............... & 0 & 0 & 1 & 1 & 1 & 1 & 1 & 1 & 1 & 1 & 0 & 0 & 1 & 0 \\
\hline \multicolumn{15}{|l|}{ Appliances } \\
\hline 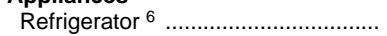 & 100 & NA & 100 & 100 & 100 & 100 & 100 & 100 & 100 & 100 & 100 & 100 & 100 & 0 \\
\hline 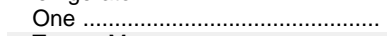 & 86 & NA & 86 & 87 & 86 & 88 & 86 & 84 & 85 & 85 & 83 & 78 & 77 & -9 \\
\hline Two or More & 14 & NA & 14 & 13 & 13 & 12 & 14 & 15 & 15 & 15 & 17 & 22 & 23 & 9 \\
\hline Separate Freezer ………………...... & 35 & NA & 38 & 38 & 37 & 37 & 34 & 34 & 35 & 33 & 32 & 32 & 30 & -8 \\
\hline 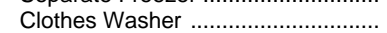 & 74 & NA & 74 & 73 & 71 & 73 & 75 & 76 & 77 & 77 & 79 & 83 & 82 & 8 \\
\hline Clothes Dryer ....…………………..... & 59 & NA & 61 & 61 & 60 & 62 & 66 & 69 & 70 & 71 & 74 & 79 & 79 & 18 \\
\hline 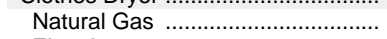 & 14 & NA & 14 & 16 & 15 & 16 & 15 & 16 & 14 & 15 & 16 & 17 & 15 & 1 \\
\hline 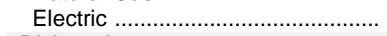 & 45 & NA & 47 & 45 & 45 & 46 & 51 & 53 & 57 & 55 & 57 & 61 & 63 & 16 \\
\hline 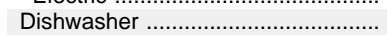 & 35 & NA & 37 & 37 & 36 & 38 & 43 & 45 & 45 & 50 & 53 & 58 & 59 & 22 \\
\hline 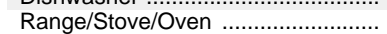 & 99 & NA & 99 & 100 & 99 & 99 & 99 & 100 & 100 & 99 & 100 & 99 & 99 & 0 \\
\hline Natural Gas ...………………... & 48 & NA & 46 & 46 & 47 & 46 & 43 & 42 & 33 & 35 & 35 & 35 & 34 & -12 \\
\hline 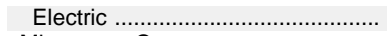 & 53 & NA & 57 & 56 & 56 & 57 & 60 & 59 & 63 & 62 & 62 & 62 & 60 & 3 \\
\hline 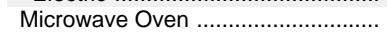 & 8 & NA & 14 & 17 & 21 & 34 & 61 & 79 & 84 & 83 & 86 & 88 & 96 & 82 \\
\hline \multicolumn{15}{|l|}{ Electronics } \\
\hline 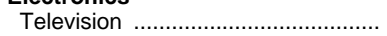 & NA & NA & 98 & 98 & 98 & 98 & 98 & 99 & 99 & 99 & 99 & 99 & 99 & 1 \\
\hline One & NA & NA & 47 & 51 & 49 & 46 & 40 & 35 & 34 & 32 & 27 & 21 & 21 & -26 \\
\hline 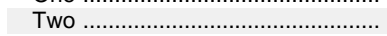 & NA & NA & 38 & 34 & 35 & 34 & 35 & 36 & 36 & 37 & 36 & 35 & 33 & -5 \\
\hline Three or More …… & NA & NA & 14 & 14 & 15 & 18 & 23 & 28 & 28 & 29 & 36 & 43 & 44 & 30 \\
\hline Video Cassette Recorder (VCR) .... & NA & NA & NA & NA & NA & NA & NA & NA & NA & 88 & 790 & 80 & 51 & NA \\
\hline Digital Video Recorder (DVR) .......... & NA & NA & NA & NA & NA & NA & NA & NA & NA & NA & NA & NA & 43 & NA \\
\hline Computer & NA & NA & NA & NA & NA & NA & NA & NA & NA & 35 & 56 & 68 & 76 & NA \\
\hline 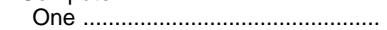 & NA & NA & NA & NA & NA & NA & NA & NA & NA & 29 & 42 & 45 & 41 & NA \\
\hline Two or More & NA & NA & NA & NA & NA & NA & NA & NA & NA & 6 & 15 & 23 & 35 & NA \\
\hline 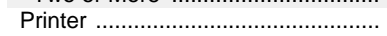 & NA & NA & NA & NA & NA & NA & NA & NA & 5 & 12 & 49 & 59 & 60 & NA \\
\hline
\end{tabular}

1 Includes households that have but do not use space heating equipment.

2 Retail (delivered) electricity.

3 Includes kerosene.

${ }_{5}$ Coal, solar, or other fuels.

System." Includes households that have but do not use air conditioning equipment.

Fewer than 0.5 percent of the households do not have a refrigerator.
The 2001 "Residential Energy Consumption Survey (RECS)" only had one question for VCRs and

DVD players.
NA=Not available.

Notes: - Data are estimates. - For years not shown, there are no data available. - Totals may not equal sum of components due to independent rounding.

(a)

Sources: • 1978 and 1979-U.S. Energy Information Administration (EIA), Form EIA-84, "RECS."

- 1980 forward-EIA, Form EIA-457, "RECS." 
Figure 2.7 Type of Heating in Occupied Housing Units, 1950 and 2009

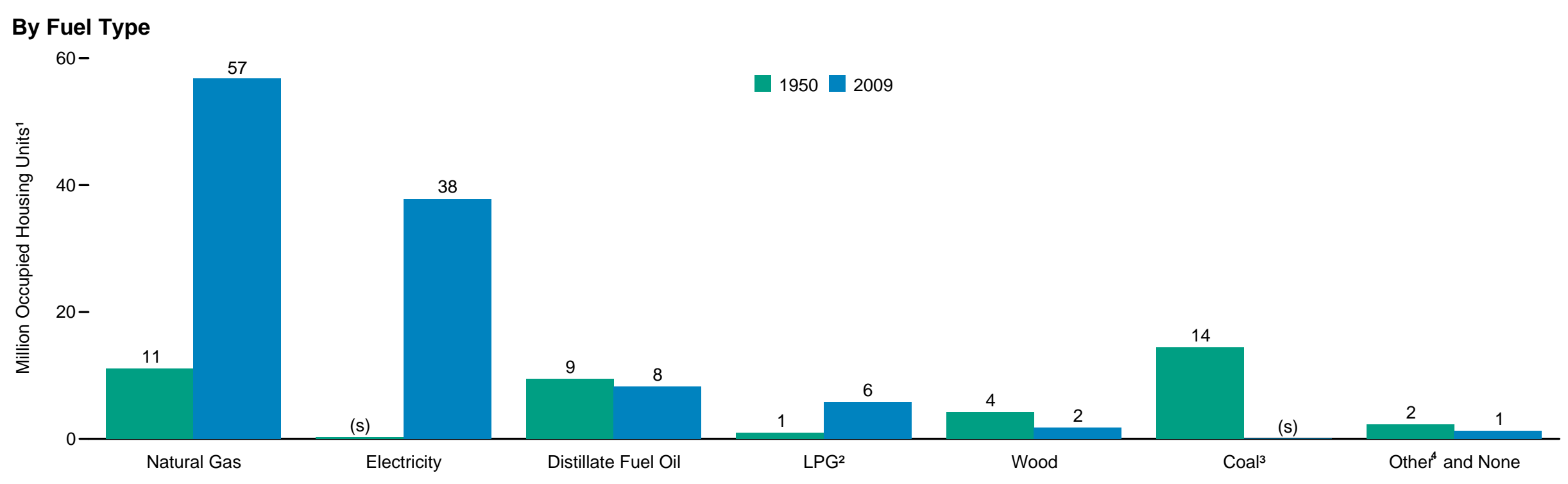

By Fuel Type, Share of Total

$60-$

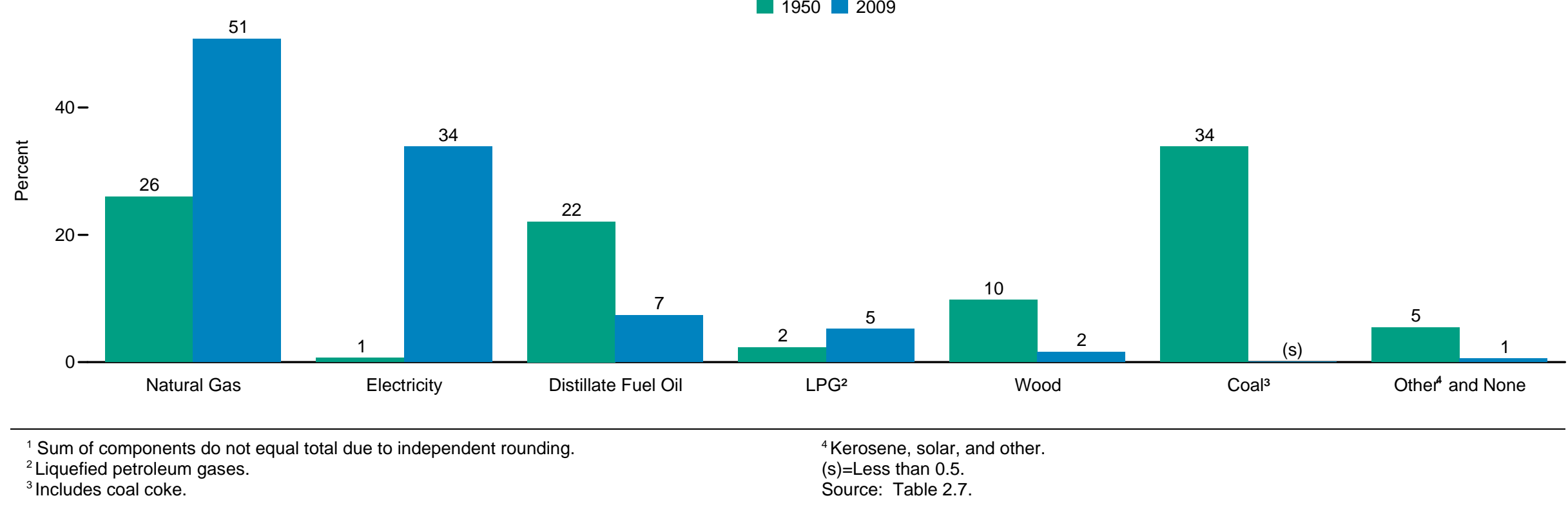


Table 2.7 Type of Heating in Occupied Housing Units, Selected Years, 1950-2009

\begin{tabular}{|c|c|c|c|c|c|c|c|c|c|c|c|}
\hline Year & Coal 1 & $\begin{array}{l}\text { Distillate } \\
\text { Fuel Oil }\end{array}$ & Kerosene & $\begin{array}{l}\text { Liquefied } \\
\text { Petroleum } \\
\text { Gases }\end{array}$ & $\begin{array}{c}\text { Natural } \\
\text { Gas }\end{array}$ & Electricity & Wood & Solar & Other $^{2}$ & None $^{3}$ & Total \\
\hline & \multicolumn{11}{|c|}{ Million Occupied Housing Units } \\
\hline 1950 & 14.48 & 9.46 & $\left({ }^{4}\right)$ & 0.98 & 11.12 & 0.28 & 4.17 & NA & 0.77 & 1.57 & 42.83 \\
\hline 1960 & 6.46 & 17.16 & $(4)$ & 2.69 & 22.85 & .93 & 2.24 & NA & .22 & .48 & 53.02 \\
\hline 1970 & 1.82 & 16.47 & $\left(4^{\prime}\right)$ & 3.81 & 35.01 & 4.88 & .79 & NA & .27 & .40 & 63.45 \\
\hline 1973 & .80 & 17.24 & $\left({ }^{4}\right)$ & 4.42 & 38.46 & 7.21 & .60 & NA & .15 & .45 & 69.34 \\
\hline 1975 & .57 & 16.30 & $\left({ }^{4}\right)$ & 4.15 & 40.93 & 9.17 & .85 & NA & .08 & .47 & 72.52 \\
\hline 1977 & .45 & 15.62 & .44 & 4.18 & 41.54 & 11.15 & 1.24 & NA & .15 & .51 & 75.28 \\
\hline 1979 & .36 & 15.30 & .41 & 4.13 & 43.32 & 13.24 & 1.14 & NA & .10 & .57 & 78.57 \\
\hline 1981 & .36 & 14.13 & .37 & 4.17 & 46.08 & 15.49 & 1.89 & NA & .10 & .59 & 83.18 \\
\hline $1983^{5}$ & .43 & 12.59 & .45 & 3.87 & 46.70 & 15.68 & 4.09 & NA & .16 & .68 & 84.64 \\
\hline 1985 & .45 & 12.44 & 1.06 & 3.58 & 45.33 & 18.36 & 6.25 & .05 & .37 & .53 & 88.43 \\
\hline 1987 & .41 & 12.74 & 1.08 & 3.66 & 45.96 & 20.61 & 5.45 & .05 & .28 & .66 & 90.89 \\
\hline 1989 & .34 & 12.47 & 1.07 & 3.66 & 47.40 & 23.06 & 4.59 & .04 & .40 & .66 & 93.68 \\
\hline 1991 & .32 & 11.47 & .99 & 3.88 & 47.02 & 23.71 & 4.44 & .03 & .41 & .86 & 93.15 \\
\hline 1993 & .30 & 11.17 & 1.02 & 3.92 & 47.67 & 25.11 & 4.10 & .03 & .50 & .91 & 94.73 \\
\hline 1995 & .21 & 10.98 & 1.06 & 4.25 & 49.20 & 26.77 & 3.53 & .02 & .64 & 1.04 & 97.69 \\
\hline 1997 & .18 & 10.10 & .75 & 5.40 & 51.05 & 29.20 & 1.79 & .03 & .36 & .62 & 99.49 \\
\hline 1999 & .17 & 10.03 & .72 & 5.91 & 52.37 & 31.14 & 1.70 & .02 & .21 & .54 & 102.80 \\
\hline $2001^{6}$ & .13 & 9.81 & .65 & 6.04 & 54.13 & 32.41 & 1.67 & .02 & .19 & .39 & 105.44 \\
\hline 2003 & .13 & 9.50 & .64 & 6.13 & 54.93 & 32.34 & 1.56 & .02 & .16 & .44 & 105.84 \\
\hline 2005 & .10 & 9.38 & .55 & 6.23 & 56.32 & 34.26 & 1.41 & .02 & .21 & .40 & 108.87 \\
\hline 2007 & .09 & 8.74 & .57 & 6.10 & 56.68 & 36.08 & 1.47 & .02 & .46 & .48 & 110.69 \\
\hline \multirow[t]{2}{*}{2009} & .10 & 8.21 & .60 & 5.82 & 56.81 & 37.85 & 1.78 & .01 & .24 & .38 & 111.81 \\
\hline & \multicolumn{11}{|c|}{ Percent } \\
\hline 1950 & 33.8 & 22.1 & $\left({ }^{4}\right)$ & 2.3 & 26.0 & 0.6 & 9.7 & NA & 1.8 & 3.7 & 100.0 \\
\hline 1960 & 12.2 & 32.4 & $\left({ }^{4}\right)$ & 5.1 & 43.1 & 1.8 & 4.2 & NA & .4 & .9 & 100.0 \\
\hline 1970 & 2.9 & 26.0 & $\left({ }^{4}\right)$ & 6.0 & 55.2 & 7.7 & 1.3 & NA & .4 & .6 & 100.0 \\
\hline 1973 & 1.2 & 24.9 & $\left({ }^{4}\right)$ & 6.4 & 55.5 & 10.4 & .9 & NA & .2 & .7 & 100.0 \\
\hline 1975 & .8 & 22.5 & $\left({ }^{4}\right)$ & 5.7 & 56.4 & 12.6 & 1.2 & NA & .1 & .6 & 100.0 \\
\hline 1977 & .6 & 20.7 & .6 & 5.6 & 55.2 & 14.8 & 1.6 & NA & .2 & .7 & 100.0 \\
\hline 1979 & .5 & 19.5 & .5 & 5.3 & 55.1 & 16.9 & 1.4 & NA & .1 & .7 & 100.0 \\
\hline 1981 & .4 & 17.0 & .4 & 5.0 & 55.4 & 18.6 & 2.3 & NA & .1 & .7 & 100.0 \\
\hline $1983^{5}$ & .5 & 14.9 & .5 & 4.6 & 55.2 & 18.5 & 4.8 & NA & .2 & .8 & 100.0 \\
\hline 1985 & .5 & 14.1 & 1.2 & 4.1 & 51.3 & 20.8 & 7.1 & .1 & .4 & .6 & 100.0 \\
\hline 1987 & .4 & 14.0 & 1.2 & 4.0 & 50.6 & 22.7 & 6.0 & .1 & .3 & .7 & 100.0 \\
\hline 1989 & .4 & 13.3 & 1.1 & 3.9 & 50.6 & 24.6 & 4.9 & (s) & .4 & .7 & 100.0 \\
\hline 1991 & .3 & 12.3 & 1.1 & 4.2 & 50.5 & 25.5 & 4.8 & (s) & .4 & .9 & 100.0 \\
\hline 1993 & .3 & 11.8 & 1.1 & 4.1 & 50.3 & 26.5 & 4.3 & (s) & .5 & 1.0 & 100.0 \\
\hline 1995 & .2 & 11.2 & 1.1 & 4.4 & 50.4 & 27.4 & 3.6 & (s) & .7 & 1.1 & 100.0 \\
\hline 1997 & .2 & 10.2 & .8 & 5.4 & 51.3 & 29.4 & 1.8 & (s) & .4 & .6 & 100.0 \\
\hline 1999 & .2 & 9.8 & .7 & 5.7 & 50.9 & 30.3 & 1.7 & (s) & .2 & .5 & 100.0 \\
\hline $2001^{6}$ & .1 & 9.3 & .6 & 5.7 & 51.3 & 30.7 & 1.6 & (s) & .2 & .4 & 100.0 \\
\hline 2003 & .1 & 9.0 & .6 & 5.8 & 51.9 & 30.6 & 1.5 & (s) & .1 & .4 & 100.0 \\
\hline 2005 & .1 & 8.6 & .5 & 5.7 & 51.7 & 31.5 & 1.3 & (s) & .2 & .4 & 100.0 \\
\hline 2007 & .1 & 7.9 & .5 & 5.5 & 51.2 & 32.6 & 1.3 & (s) & .4 & .4 & 100.0 \\
\hline 2009 & .1 & 7.3 & .5 & 5.2 & 50.8 & 33.9 & 1.6 & (s) & .2 & .3 & 100.0 \\
\hline
\end{tabular}

1 Includes coal coke.

2 Includes briquettes (made of pitch and sawdust), coal dust, waste material (such as corncobs), purchased steam, and other fuels not separately displayed.

In 1950 and 1960, also includes nonreporting units, which totaled 997 and 2,000 units, respectively.

4 Included in "Distillate Fuel Oil."
5 Beginning in 1983, the American Housing Survey for the United States has been a biennial survey.

${ }_{6}^{6}$ Beginning in 2001, data are consistent with the 2000 Census. For 2001 data consistent with the 1990 Census, see American Housing Survey for the United States: 200

Notes: - Includes mobile homes and individual housing units in apartment buildings. Housing units with more than one type of heating system are classified according to the principal type of heating system.

- Totals may not equal sum of components due to independent rounding.
Web Pages: - See http://www.eia.gov/totalenergy/data/annual/\#consumption for all data beginning in 1950. - For related information, see http://www.census.gov/hhes/www/ahs.html.

Sources: - 1950, 1960, and 1970-Bureau of the Census, Census of Population and Housing. - 1973-1981-Bureau of the Census, American Housing Survey for the United States, annual surveys, Table 2-5. 1983 forward-Bureau of the Census, American Housing Survey for the United States, 


\section{All Motor Vehicles, 1949-2010}

$175-$

$150-$

Fuel Economy ${ }^{1}$

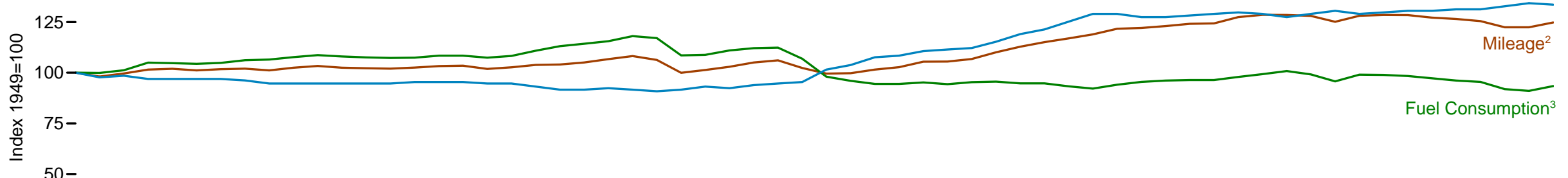

$50-$

$25-$

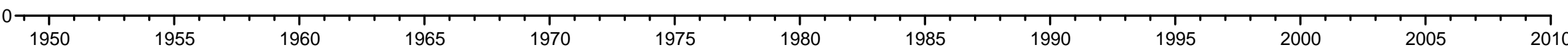

\section{Mileage, 1966-2010}

$32-$

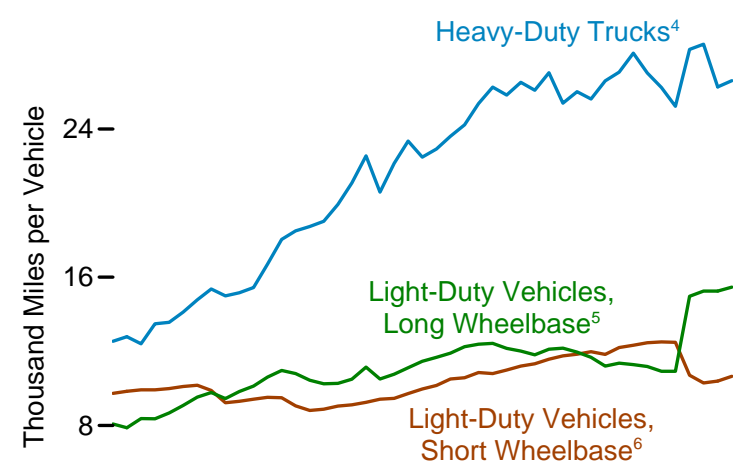

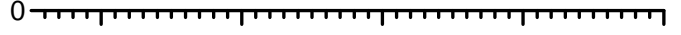

Fuel Consumption, 1966-2010

$5-$

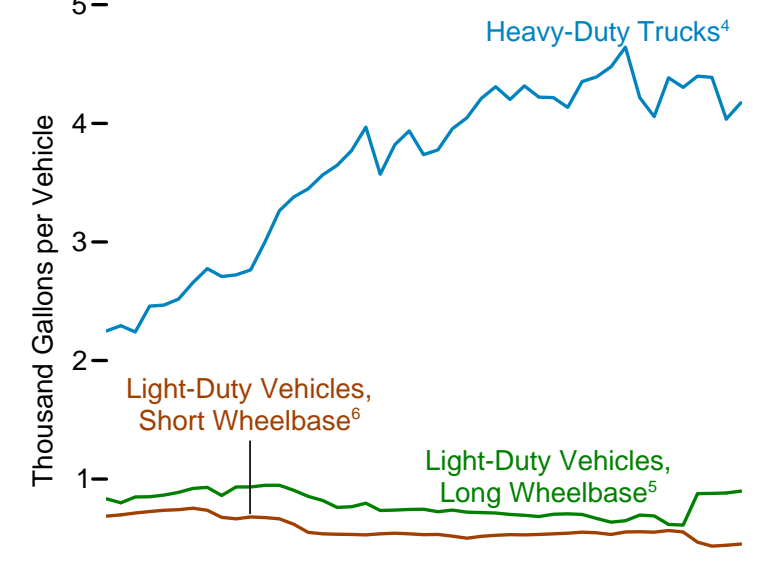

Fuel Economy, 1966-2010

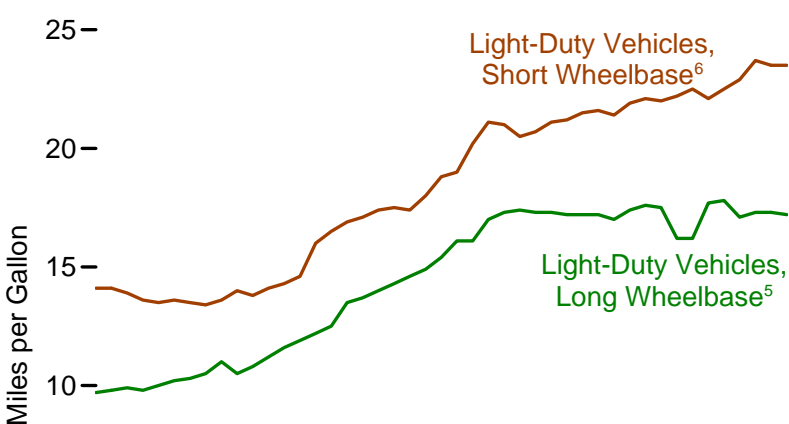

Heavy-Duty Trucks ${ }^{4}$

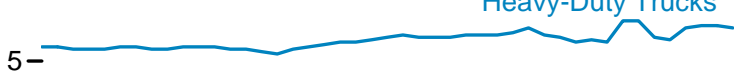

0 0

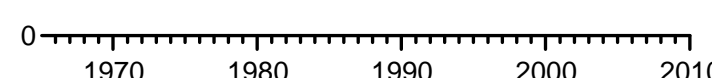

1970

1980

1990

$2000 \quad 2010$

${ }^{5}$ Through 2006, data are for vans, pickup trucks, sport utility vehicles, and a small number of trucks with 2 axles and 4 tires, such as step vans. Beginning in 2007, data are for large passenger cars, vans, pickup trucks, and sport utility vehicles with a wheelbase larger than 121 inches.

${ }^{6}$ Through 2006, data are for passenger cars (and, through 1989, for motorcycles). Beginning in 2007, data are for passenger cars, light trucks, vans, and sport utility vehicles with a wheelbase equal to or less than 121 inches.

Source: Table 2.8 . 
Table 2.8 Motor Vehicle Mileage, Fuel Consumption, and Fuel Economy, Selected Years, 1949-2010

\begin{tabular}{|c|c|c|c|c|c|c|c|c|c|c|c|c|}
\hline \multirow[b]{3}{*}{ Year } & \multicolumn{3}{|c|}{ Light-Duty Vehicles, Short Wheelbase ${ }^{1}$} & \multicolumn{3}{|c|}{ Light-Duty Vehicles, Long Wheelbase ${ }^{2}$} & \multicolumn{3}{|c|}{ Heavy-Duty Trucks ${ }^{3}$} & \multicolumn{3}{|c|}{ All Motor Vehicles 4} \\
\hline & Mileage & $\begin{array}{c}\text { Fuel } \\
\text { Consumption }\end{array}$ & $\begin{array}{c}\text { Fuel } \\
\text { Economy }\end{array}$ & Mileage & $\begin{array}{c}\text { Fuel } \\
\text { Consumption }\end{array}$ & $\begin{array}{c}\text { Fuel } \\
\text { Economy }\end{array}$ & Mileage & $\begin{array}{c}\text { Fuel } \\
\text { Consumption }\end{array}$ & $\begin{array}{c}\text { Fuel } \\
\text { Economy }\end{array}$ & Mileage & $\begin{array}{c}\text { Fuel } \\
\text { Consumption }\end{array}$ & $\begin{array}{c}\text { Fuel } \\
\text { Economy }\end{array}$ \\
\hline & $\begin{array}{l}\text { Miles per } \\
\text { Vehicle }\end{array}$ & $\begin{array}{l}\text { Gallons } \\
\text { per Vehicle }\end{array}$ & $\begin{array}{c}\text { Miles } \\
\text { per Gallon }\end{array}$ & $\begin{array}{l}\text { Miles per } \\
\text { Vehicle }\end{array}$ & $\begin{array}{l}\text { Gallons } \\
\text { per Vehicle }\end{array}$ & $\begin{array}{c}\text { Miles } \\
\text { per Gallon }\end{array}$ & $\begin{array}{l}\text { Miles per } \\
\text { vehicle }\end{array}$ & $\begin{array}{c}\text { Gallons } \\
\text { per vehicle }\end{array}$ & $\begin{array}{c}\text { Miles } \\
\text { per Gallon }\end{array}$ & $\begin{array}{l}\text { Miles per } \\
\text { Vehicle }\end{array}$ & $\begin{array}{l}\text { Gallons } \\
\text { per Vehicle }\end{array}$ & $\begin{array}{c}\text { Miles } \\
\text { per Gallon }\end{array}$ \\
\hline 1949 & 9,388 & 627 & 15.0 & $\left({ }^{5}\right)$ & $\left({ }^{5}\right)$ & $\left({ }^{5}\right)$ & 9,712 & 1,080 & 9.0 & 9,498 & 726 & 13.1 \\
\hline 1950 & 9,060 & 603 & 15.0 & $\left({ }^{5}\right)$ & $(5)$ & $\left(5^{5}\right)$ & 10,316 & 1,229 & 8.4 & 9,321 & 725 & 12.8 \\
\hline 1955 & 9,447 & 645 & 14.6 & $\left({ }^{5}\right)$ & $\left({ }^{5}\right)$ & $\left(5^{\prime}\right)$ & 10,576 & 1,293 & 8.2 & 9,661 & 761 & 12.7 \\
\hline 1960 & 9,518 & 668 & 14.3 & $\left({ }^{5}\right)$ & $\left({ }^{5}\right)$ & $\left(5^{\prime}\right)$ & 10,693 & 1,333 & 8.0 & 9,732 & 784 & 12.4 \\
\hline 1965 & 9,603 & 661 & 14.5 & $\left({ }^{5}\right)$ & $\left({ }^{5}\right)$ & $\left(5^{\prime}\right)$ & 10,851 & 1,387 & 7.8 & 9,826 & 787 & 12.5 \\
\hline 1970 & 9,989 & 737 & 13.5 & 8,676 & 866 & 10.0 & 13,565 & 2,467 & 5.5 & 9,976 & 830 & 12.0 \\
\hline 1975 & 9,309 & 665 & 14.0 & 9,829 & 934 & 10.5 & 15,167 & 2,722 & 5.6 & 9,627 & 790 & 12.2 \\
\hline 1976 & 9,418 & 681 & 13.8 & 10,127 & 934 & 10.8 & 15,438 & 2,764 & 5.6 & 9,774 & 806 & 12.1 \\
\hline 1977 & 9,517 & 676 & 14.1 & 10,607 & 947 & 11.2 & 16,700 & 3,002 & 5.6 & 9,978 & 814 & 12.3 \\
\hline 1978 & 9,500 & 665 & 14.3 & 10,968 & 948 & 11.6 & 18,045 & 3,263 & 5.5 & 10,077 & 816 & 12.4 \\
\hline 1979 & 9,062 & 620 & 14.6 & 10,802 & 905 & 11.9 & 18,502 & 3,380 & 5.5 & 9,722 & 776 & 12.5 \\
\hline 1980 & 8,813 & 551 & 16.0 & 10,437 & 854 & 12.2 & 18,736 & 3,447 & 5.4 & 9,458 & 712 & 13.3 \\
\hline 1981 & 8,873 & 538 & 16.5 & 10,244 & 819 & 12.5 & 19,016 & 3,565 & 5.3 & 9,477 & 697 & 13.6 \\
\hline 1982 & 9,050 & 535 & 16.9 & 10,276 & 762 & 13.5 & 19,931 & 3,647 & 5.5 & 9,644 & 686 & 14.1 \\
\hline 1983 & 9,118 & 534 & 17.1 & 10,497 & 767 & 13.7 & 21,083 & 3,769 & 5.6 & 9,760 & 686 & 14.2 \\
\hline 1984 & 9,248 & 530 & 17.4 & 11,151 & 797 & 14.0 & 22,550 & 3,967 & 5.7 & 10,017 & 691 & 14.5 \\
\hline 1985 & 9,419 & 538 & 17.5 & 10,506 & 735 & 14.3 & 20,597 & 3,570 & 5.8 & 10,020 & 685 & 14.6 \\
\hline 1986 & 9,464 & 543 & 17.4 & 10,764 & 738 & 14.6 & 22,143 & 3,821 & 5.8 & 10,143 & 692 & 14.7 \\
\hline 1987 & 9,720 & 539 & 18.0 & 11,114 & 744 & 14.9 & 23,349 & 3,937 & 5.9 & 10,453 & 694 & 15.1 \\
\hline 1988 & 9,972 & 531 & 18.8 & 11,465 & 745 & 15.4 & 22,485 & 3,736 & 6.0 & 10,721 & 688 & 15.6 \\
\hline 1989 & 10,157 & 533 & 19.0 & 11,676 & 724 & 16.1 & 22,926 & 3,776 & 6.1 & 10,932 & 688 & 15.9 \\
\hline 1990 & 10,504 & 520 & 20.2 & 11,902 & 738 & 16.1 & 23,603 & 3,953 & 6.0 & 11,107 & 677 & 16.4 \\
\hline 1991 & 10,571 & 501 & 21.1 & 12,245 & 721 & 17.0 & 24,229 & 4,047 & 6.0 & 11,294 & 669 & 16.9 \\
\hline 1992 & 10,857 & 517 & 21.0 & 12,381 & 717 & 17.3 & 25,373 & 4,210 & 6.0 & 11,558 & 683 & 16.9 \\
\hline 1993 & 10,804 & 527 & 20.5 & 12,430 & 714 & 17.4 & 26,262 & 4,309 & 6.1 & 11,595 & 693 & 16.7 \\
\hline 1994 & 10,992 & 531 & 20.7 & 12,156 & 701 & 17.3 & 25,838 & 4,202 & 6.1 & 11,683 & 698 & 16.7 \\
\hline 1995 & 11,203 & 530 & 21.1 & 12,018 & 694 & 17.3 & 26,514 & 4,315 & 6.1 & 11,793 & 700 & 16.8 \\
\hline 1996 & 11,330 & 534 & 21.2 & 11,811 & 685 & 17.2 & 26,092 & 4,221 & 6.2 & 11,813 & 700 & 16.9 \\
\hline 1997 & 11,581 & 539 & 21.5 & 12,115 & 703 & 17.2 & 27,032 & 4,218 & 6.4 & 12,107 & 711 & 17.0 \\
\hline 1998 & 11,754 & 544 & 21.6 & 12,173 & 707 & 17.2 & 25,397 & 4,135 & 6.1 & 12,211 & 721 & 16.9 \\
\hline 1999 & 11,848 & 553 & 21.4 & 11,957 & 701 & 17.0 & 26,014 & 4,352 & 6.0 & 12,206 & 732 & 16.7 \\
\hline 2000 & 11,976 & 547 & 21.9 & 11,672 & 669 & 17.4 & 25,617 & 4,391 & 5.8 & 12,164 & 720 & 16.9 \\
\hline 2001 & 11,831 & 534 & 22.1 & 11,204 & 636 & 17.6 & 26,602 & 4,477 & 5.9 & 11,887 & 695 & 17.1 \\
\hline 2002 & 12,202 & 555 & 22.0 & 11,364 & 650 & 17.5 & 27,071 & 4,642 & 5.8 & 12,171 & 719 & 16.9 \\
\hline 2003 & 12,325 & 556 & 22.2 & 11,287 & 697 & 16.2 & 28,093 & 4,215 & 6.7 & 12,208 & 718 & 17.0 \\
\hline 2004 & 12,460 & 553 & 22.5 & 11,184 & 690 & 16.2 & 27,023 & 4,057 & 6.7 & 12,200 & 714 & 17.1 \\
\hline 2005 & 12,510 & 567 & 22.1 & 10,920 & 617 & 17.7 & 26,235 & 4,385 & 6.0 & 12,082 & 706 & 17.1 \\
\hline 2006 & 12,485 & 554 & 22.5 & 10,920 & 612 & 17.8 & 25,231 & 4,304 & 5.9 & 12,017 & 698 & 17.2 \\
\hline 2007 & $1, \mathrm{R}_{10,710}$ & $1, \mathrm{R} 468$ & $1, \mathrm{R}_{22.9} .9$ & $2, \mathrm{R}_{14,970}$ & 2,R877 & $2, \mathrm{R}_{17.1}$ & $3, \mathrm{R}_{28,290}$ & $3, R_{4,398}$ & $3, \mathrm{R} 6.4$ & $\mathrm{R}_{11,915}$ & 693 & 17.2 \\
\hline 2008 & $R_{10,290}$ & R435 & $\mathrm{R}_{23.7}$ & $R_{15,256}$ & R880 & $R_{17.3}$ & $\mathrm{R}_{28,573}$ & $\mathrm{R}_{4,387}$ & $\mathrm{R}_{6.5}$ & $R_{11,631}$ & 667 & 17.4 \\
\hline 2009 & 10,391 & 442 & 23.5 & 15,252 & 882 & 17.3 & 26,274 & 4,037 & 6.5 & 11,631 & 661 & 17.6 \\
\hline $2010^{P}$ & 10,649 & 453 & 23.5 & 15,463 & 898 & 17.2 & 26,609 & 4,174 & 6.4 & 11,853 & 678 & 17.5 \\
\hline
\end{tabular}

1 Through 2006, data are for passenger cars (and, through 1989, for motorcycles). Beginning in 2007, data are for passenger cars, light trucks, vans, and sport utility vehicles with a wheelbase equal to or less than 121 inches.

Through 2006, data are for vans, pickup trucks, sport utility vehicles, and a small number of trucks with 2 axles and 4 tires, such as step vans. Beginning in 2007, data are for large passenger cars, vans, pickup trucks, and sport utility vehicles with a wheelbase larger than 121 inches.

Through 2006, data are for single-unit trucks with 2 axles and 6 or more tires, and combination trucks. Beginning in 2007, data are for single-unit trucks with 2 axles and 6 or more tires or a gross vehicle weight
rating exceeding 10,000 pounds, and combination trucks.

4 Includes buses and motorcycles, which are not separately displayed. 5 Included in "Heavy-Duty Trucks."

$\mathrm{R}=$ Revised. P=Preliminary.

Web Pages: - See http://www.eia.gov/totalenergy/data/annual/\#consumption for all data beginning in (249. - For related information, see http://www.fhwa.dot.gov/policyinformation/statistics.cfm

Sources: Light-Duty Vehicles, Short Wheelbase, 1990-1994: U.S. Department of Transportation Bureau of Transportation Statistics, National Transportation Statistics 1998, Table 4-13. All Other Data: VM-201A. 1995 toral Highway Administration (FHWA), Highway Stats, 


\section{Figure 2.9 Commercial Buildings Consumption by Energy Source}

\section{By Survey Year, 1979-2003}

$4-$
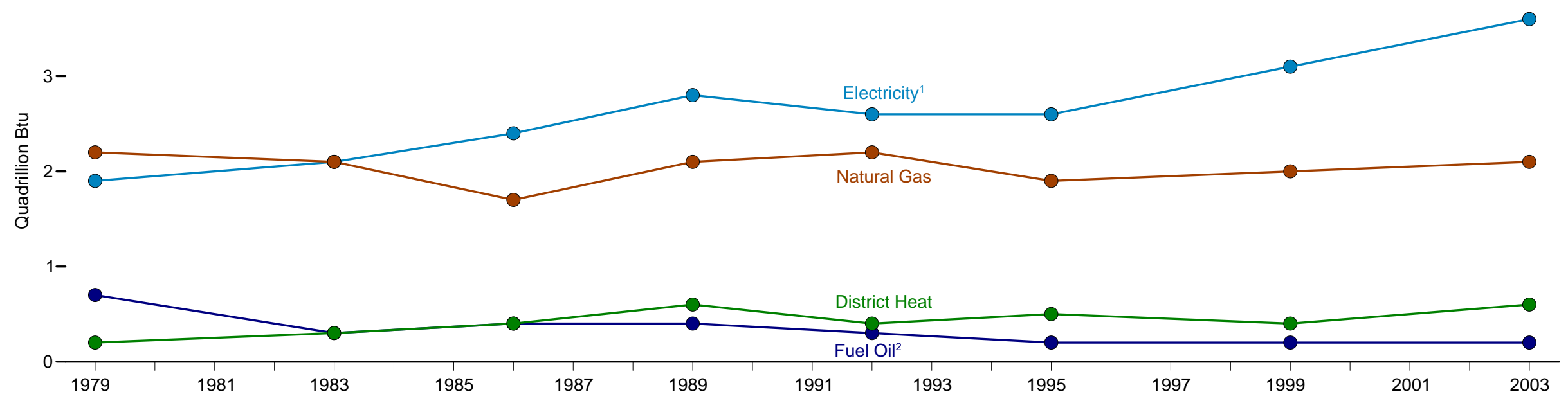

By Census Region, 2003

4-

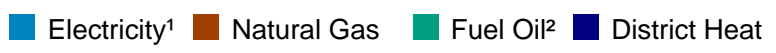

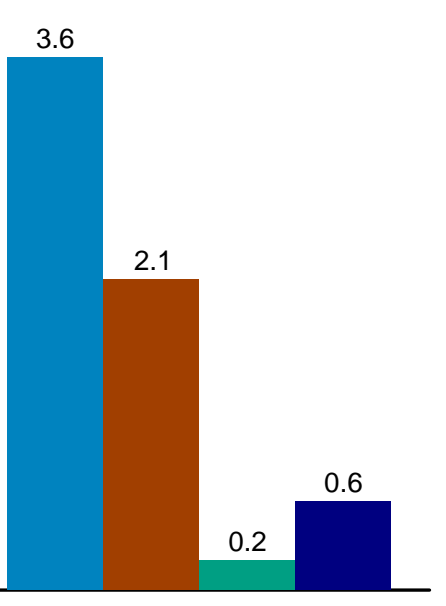

$3-$

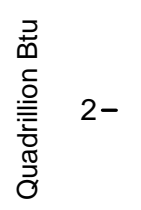

$1-$

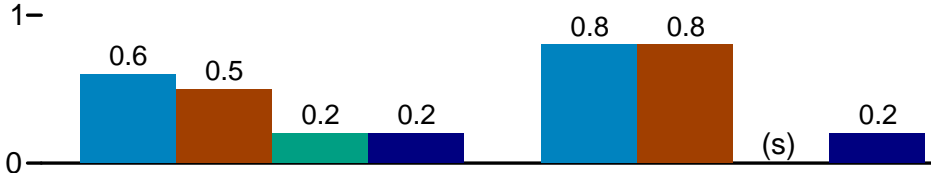

Northeast

Midwest

(s) Q

United States

${ }^{1}$ Electricity only; excludes electrical system energy losses.

${ }^{2}$ Distillate fuel oil, residual fuel oil, and kerosene.

(s)=Less than 0.05 quadrillion Btu.

$\mathrm{Q}=$ Data withheld because either the relative standard error was greater than 50 percent or fewer than 20 buildings were sampled.

Note: See Appendix $\mathrm{C}$ for map of Census regions.

Source: Table 2.9. 
Table 2.9 Commercial Buildings Consumption by Energy Source, Selected Years, 1979-2003

(Trillion Btu)

\begin{tabular}{|c|c|c|c|c|c|c|c|c|c|c|c|c|c|c|c|c|}
\hline \multirow[b]{2}{*}{$\begin{array}{l}\text { Energy Source } \\
\text { and Year }\end{array}$} & \multicolumn{3}{|c|}{ Square Footage Category } & \multicolumn{8}{|c|}{ Principal Building Activity } & \multicolumn{4}{|c|}{ Census Region ${ }^{1}$} & \multirow[b]{2}{*}{$\begin{array}{c}\text { All } \\
\text { Buildings }\end{array}$} \\
\hline & $\begin{array}{c}1,001 \text { to } \\
10,000\end{array}$ & $\begin{array}{c}10,001 \text { to } \\
100,000\end{array}$ & $\begin{array}{c}\text { Over } \\
100,000\end{array}$ & Education & $\begin{array}{l}\text { Food } \\
\text { Sales }\end{array}$ & $\begin{array}{l}\text { Food } \\
\text { Service }\end{array}$ & $\begin{array}{c}\text { Health } \\
\text { Care }\end{array}$ & Lodging & $\begin{array}{c}\text { Mercantile } \\
\text { and Service }\end{array}$ & Office & $\begin{array}{l}\text { All } \\
\text { Other }\end{array}$ & Northeast & Midwest & South & West & \\
\hline \multicolumn{17}{|l|}{ Major Sources ${ }^{2}$} \\
\hline $1979 \ldots \ldots \ldots \ldots \ldots$ & 1,255 & 2,202 & 1,508 & 511 & $\left({ }^{3}\right)$ & 336 & 469 & 278 & 894 & 861 & 1,616 & 1,217 & 1,826 & 1,395 & 526 & 4,965 \\
\hline $1983 \ldots \ldots \ldots \ldots \ldots$ & 1,242 & 1,935 & 1,646 & 480 & $\left({ }^{3}\right)$ & 414 & 463 & 362 & 812 & 1,018 & 1,274 & 858 & 1,821 & 1,462 & 682 & 4,823 \\
\hline & 1,273 & 2,008 & 1,696 & 633 & 147 & 247 & 456 & 299 & 985 & 1,008 & 1,202 & 1,037 & 1,585 & 1,459 & 896 & 4,977 \\
\hline 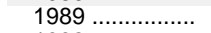 & 1,259 & 2,402 & 2,127 & 704 & 139 & 255 & 449 & 425 & 1,048 & 1,230 & 1,538 & 1,354 & 1,659 & 1,648 & 1,126 & 5,788 \\
\hline $1992 \ldots \ldots \ldots \ldots \ldots \ldots$ & 1,258 & 2,301 & 1,932 & 637 & 137 & 307 & 403 & 463 & 892 & 1,247 & 1,404 & 1,090 & 1,578 & 1,825 & 998 & 5,490 \\
\hline $1995^{4} \ldots \ldots \ldots \ldots \ldots$ & 1,332 & 2,152 & 1,838 & 614 & 137 & 332 & 561 & 461 & 973 & 1,019 & 1,225 & 1,035 & 1,497 & 1,684 & 1,106 & 5,321 \\
\hline 1999 ………............. & 1,381 & 2,300 & 2,053 & 649 & 201 & 447 & 515 & 450 & 1,145 & 1,089 & 1,237 & 1,116 & 1,509 & 1,961 & 1,147 & 5,733 \\
\hline $2003 \ldots \ldots \ldots \ldots \ldots . .$. & 1,248 & 2,553 & 2,721 & 820 & 251 & 427 & 594 & 510 & 1,333 & 1,134 & 1,455 & 1,396 & 1,799 & 2,265 & 1,063 & 6,523 \\
\hline \multicolumn{17}{|l|}{ Electricity ${ }^{5}$} \\
\hline 1979 .................... & 429 & 872 & 608 & 163 & $\left({ }^{3}\right)$ & 171 & 129 & 119 & 361 & 424 & 543 & 425 & 593 & 662 & 227 & 1,908 \\
\hline 1983 ………........ & 469 & 903 & 758 & 152 & $\left({ }^{3}\right)$ & 212 & 147 & 151 & 426 & 509 & 532 & 324 & 673 & 801 & 331 & 2,129 \\
\hline 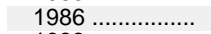 & 654 & 927 & 809 & 179 & 99 & 121 & 132 & 120 & 536 & 641 & 563 & 430 & 584 & 867 & 510 & 2,390 \\
\hline $1989 \ldots \ldots \ldots \ldots \ldots$ & 572 & 1,145 & 1,056 & 217 & 105 & 113 & 154 & 138 & 550 & 781 & 715 & 586 & 609 & 975 & 604 & 2,773 \\
\hline 1992 ................ & 586 & 991 & 1,033 & 235 & 113 & 138 & 138 & 189 & 444 & 704 & 649 & 419 & 622 & 1,002 & 566 & 2,609 \\
\hline $1995^{4} \ldots \ldots \ldots \ldots . . . .$. & 618 & 1,064 & 926 & 221 & 119 & 166 & 211 & 187 & 508 & 676 & 521 & 436 & 558 & 1,027 & 587 & 2,608 \\
\hline 1999 & 698 & 1,235 & 1,164 & 257 & 165 & 216 & 232 & 196 & 659 & 767 & 606 & 543 & 662 & 1,247 & 645 & 3,098 \\
\hline $2003 \ldots \ldots \ldots \ldots \ldots$ & 685 & 1,405 & 1,469 & 371 & 208 & 217 & 248 & 235 & 883 & 719 & 679 & 587 & 799 & 1,542 & 631 & 3,559 \\
\hline \multicolumn{17}{|l|}{ Natural Gas } \\
\hline $1979 \ldots \ldots \ldots \ldots \ldots . .$. & 646 & 996 & 532 & 214 & $\left({ }^{3}\right)$ & 145 & 221 & 115 & 422 & 272 & 784 & 443 & 1,007 & 470 & 255 & 2,174 \\
\hline $1983 \ldots \ldots \ldots \ldots \ldots \ldots$ & 684 & 809 & 597 & 246 & $\left({ }^{3}\right)$ & 188 & 218 & 170 & 327 & 365 & 576 & 278 & 978 & 523 & 311 & 2,091 \\
\hline 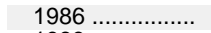 & 485 & 715 & 523 & 254 & 45 & 114 & 205 & 105 & 332 & 258 & 409 & 244 & 742 & 426 & 311 & 1,723 \\
\hline 1989 & 568 & 836 & 670 & 323 & 27 & 128 & 186 & 187 & 417 & 238 & 566 & 353 & 831 & 498 & 391 & 2,073 \\
\hline 1992 & 572 & 1,017 & 586 & 291 & 24 & 157 & 189 & 193 & 381 & 388 & 552 & 354 & 747 & 697 & 376 & 2,174 \\
\hline $1995^{4}$............... & 535 & 830 & 580 & 245 & 18 & 158 & 258 & 213 & 395 & 239 & 420 & 297 & 750 & 528 & 371 & 1,946 \\
\hline 1999 & 604 & 803 & 616 & 227 & 31 & 216 & 217 & 181 & 446 & 219 & 486 & 299 & 709 & 618 & 396 & 2,023 \\
\hline $2003 \ldots \ldots \ldots \ldots \ldots$ & 482 & 909 & 709 & 268 & 39 & 203 & 243 & 215 & 403 & 269 & 460 & 462 & 751 & 527 & 360 & 2,100 \\
\hline \multicolumn{17}{|l|}{ Fuel Oil ${ }^{6}$} \\
\hline 1979 & 177 & 272 & 231 & 107 & $\left({ }^{3}\right)$ & 15 & 97 & 20 & 103 & 107 & 232 & 285 & 133 & 237 & 26 & 681 \\
\hline 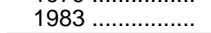 & 85 & 140 & 90 & 61 & $\left({ }^{3}\right)$ & Q & 28 & 18 & 43 & 75 & 79 & 172 & 28 & 104 & $Q$ & 314 \\
\hline 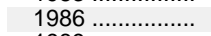 & 114 & 206 & 121 & 103 & Q & Q & $\mathrm{Q}$ & 20 & 105 & 39 & 130 & 270 & 63 & 86 & 23 & 442 \\
\hline 1989 & 101 & 170 & 86 & 71 & Q & $\mathrm{Q}$ & 17 & 10 & 76 & 43 & 122 & 237 & 61 & 50 & $Q$ & 357 \\
\hline $1992 \ldots \ldots \ldots \ldots \ldots$ & 86 & 111 & 75 & 62 & $Q$ & $\bar{Q}$ & 21 & 16 & 55 & 47 & 67 & 194 & 26 & 48 & $\mathrm{Q}$ & 272 \\
\hline $1995^{4} \ldots \ldots \ldots \ldots . .$. & 71 & 104 & 60 & 57 & $\mathrm{Q}$ & Q & 21 & $\mathrm{Q}$ & 49 & 28 & 70 & 168 & 16 & 45 & 7 & 235 \\
\hline 1999 & 29 & 73 & 60 & 48 & Q & Q & 19 & $\hat{Q}$ & 18 & 29 & 65 & 138 & 5 & 29 & 8 & 179 \\
\hline 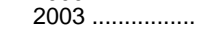 & 71 & 74 & 83 & 47 & $\mathrm{Q}$ & Q & 11 & 35 & 41 & 18 & 68 & 181 & 24 & 15 & 9 & 228 \\
\hline \multicolumn{17}{|l|}{ District Heat ${ }^{7}$} \\
\hline 1979 .................... & & 61 & & 27 & $\left({ }^{3}\right)$ & & 22 & & Q & 58 & 57 & 64 & 93 & Q & Q & 201 \\
\hline 1983 ........................ & $\hat{Q}$ & 83 & 202 & 21 & $\left({ }^{3}\right)$ & $\mathrm{Q}$ & 70 & 22 & $\bar{Q}$ & 68 & 87 & 84 & 141 & 34 & 30 & 289 \\
\hline 1986 ……............... & $\mathrm{Q}$ & 159 & 243 & 97 & $Q$ & $\mathrm{Q}$ & 80 & $\mathrm{Q}$ & 12 & 71 & 99 & 94 & 196 & 81 & 51 & 422 \\
\hline 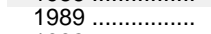 & 19 & 252 & 315 & Q & $\bar{Q}$ & $\hat{Q}$ & 92 & $\hat{Q}$ & $\mathrm{Q}$ & 167 & 134 & 179 & 159 & 126 & 121 & 585 \\
\hline $1992 \ldots \ldots \ldots \ldots \ldots$ & $\mathrm{Q}$ & 182 & 238 & 49 & NC & $\mathrm{Q}$ & 55 & 65 & Q & 109 & 135 & 123 & 183 & 78 & 51 & 435 \\
\hline $1995^{4}$ & Q & 154 & 271 & 91 & Q & $\mathrm{Q}$ & 70 & 57 & Q & 75 & 214 & 135 & 173 & 83 & Q & 533 \\
\hline 1999 ................. & Q & 158 & 213 & 117 & Q & $\mathrm{Q}$ & 46 & 68 & Q & 74 & 126 & 136 & 132 & 67 & 98 & 433 \\
\hline 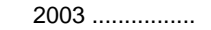 & Q & 165 & 460 & 134 & NC & $\mathrm{Q}$ & Q & Q & Q & 128 & 247 & 166 & 225 & 182 & Q & 636 \\
\hline
\end{tabular}

1 See Appendix $C$ for map of Census regions.

Includes electricity, natural gas, fuel oil, and district heat.

4 Beginning in 1995 , excludes commercial buildings at multi-building manufacturing facilities, and parking garages.

Electricity only; excludes electricity system energy losses.

Distillate fuel oil, residual fuel oil, and kerosene.

Through 1983, includes purchased steam only. Beginning in 1986, includes purchased and

non-purchased steam and hot water.
Q=Data withheld because either the relative standard error was greater than 50 percent or fewer than 20

buildings were sampled. $\mathrm{NC}=$ No cases in the sample

"Note: Data are estimates. Statistics for individual fuels are for all buildings using each fuel. Statistics for buildings using any of those fuels.

Web Page: For related information, see http://www.eia.gov/consumption/commercial/.

Sources: • 1979-U.S. Energy Information Administration (EIA), Form ElA-143, "Nonresidential Buildings Energy Consumption Survey." - 1983-EIA, Form EIA-788, "Nonresidential Buildings Energy Consumption Survey." 1 1986-EIA, Form EIA-871, "Nonresidential Buildings Energy Consumptio urvey." • 1989 forward-EIA, Form EIA-871A-F, "Commercial Buildings Energy Consumption Survey." 
Figure 2.10 Commercial Buildings Energy Consumption and Expenditure Indicators, Selected Years, 1979-2003

Buildings by Energy Source Used

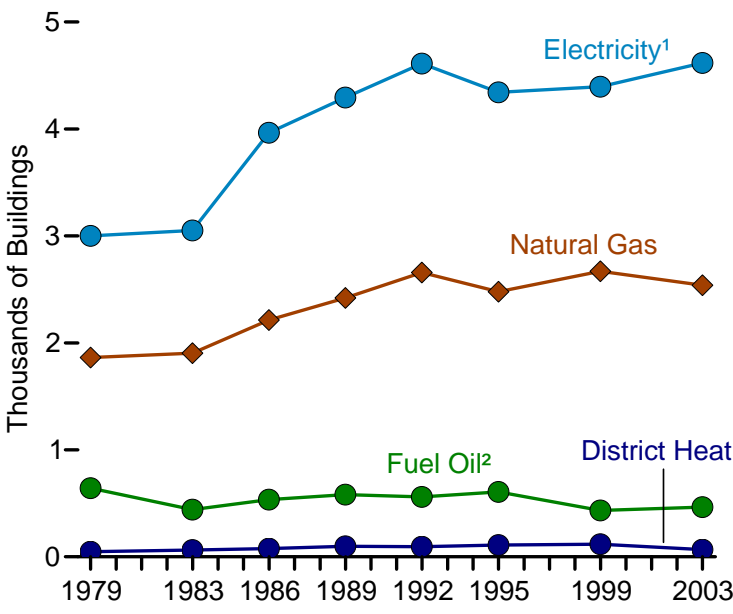

Square Footage per Building by Energy Source Used

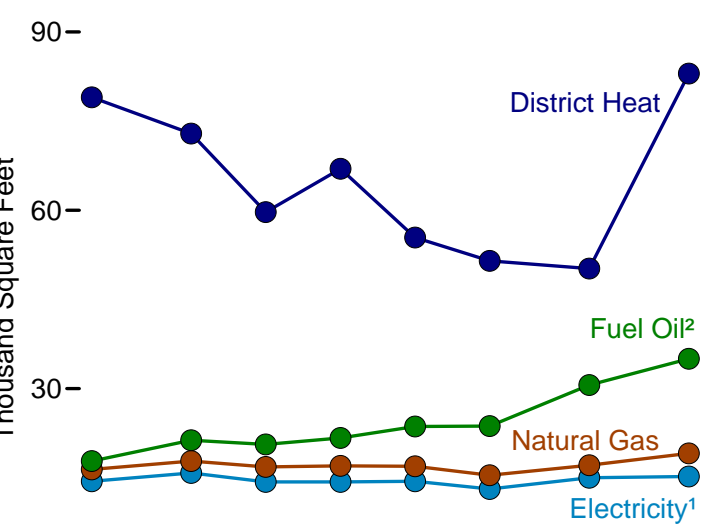

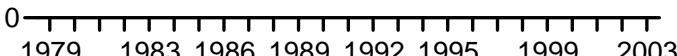

\section{Consumption}

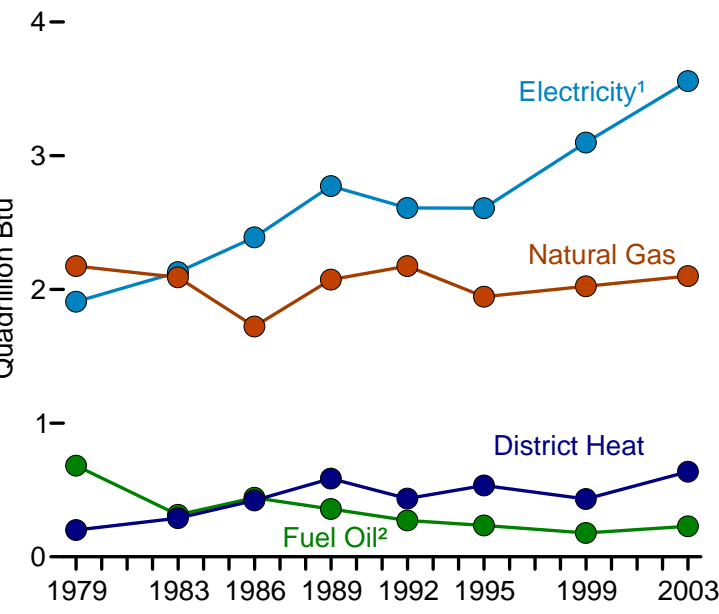

Expenditures

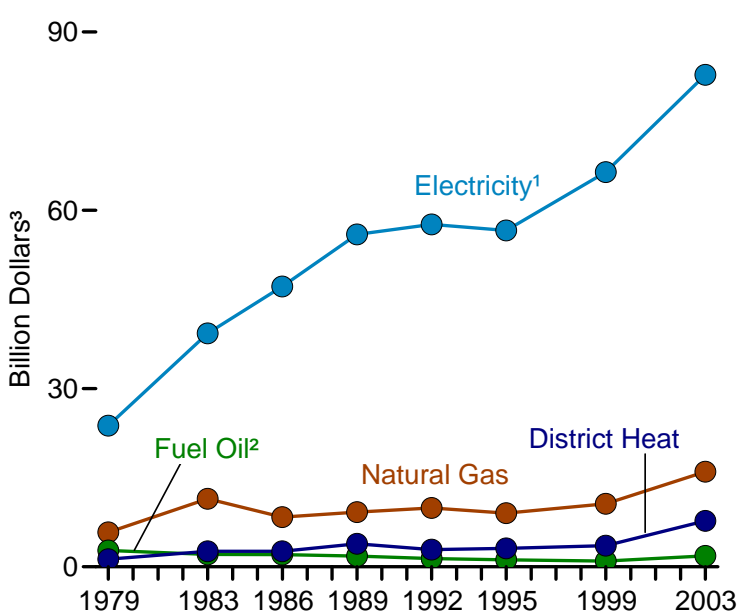

\section{Consumption per Square Foot}

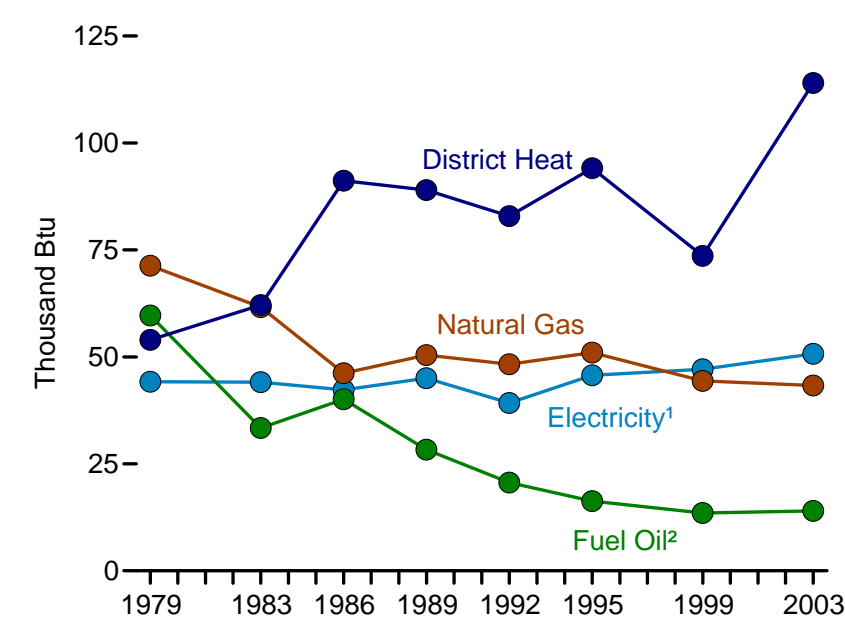

Expenditures Per Square Foot

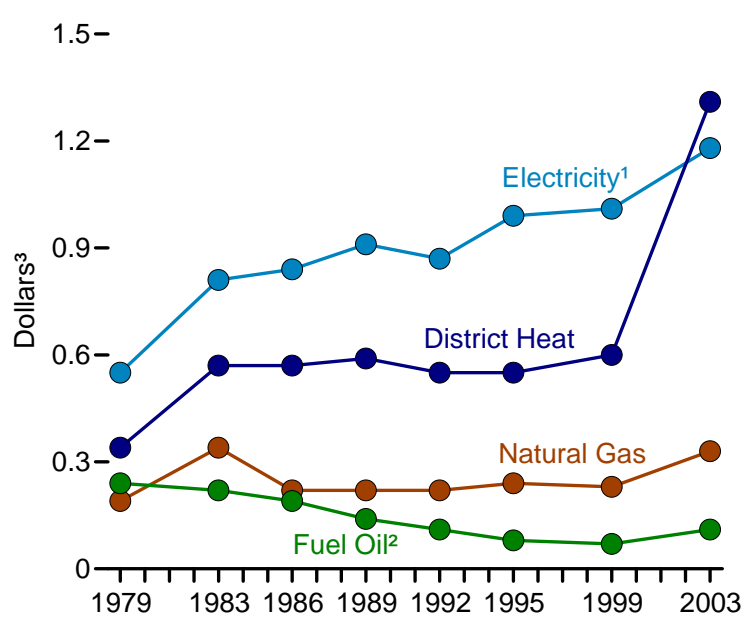

${ }^{3}$ Prices are not adjusted for inflation. See "Nominal Dollars" in Glossary. 
Table 2.10 Commercial Buildings Energy Consumption and Expenditure Indicators, Selected Years, $1979-2003$

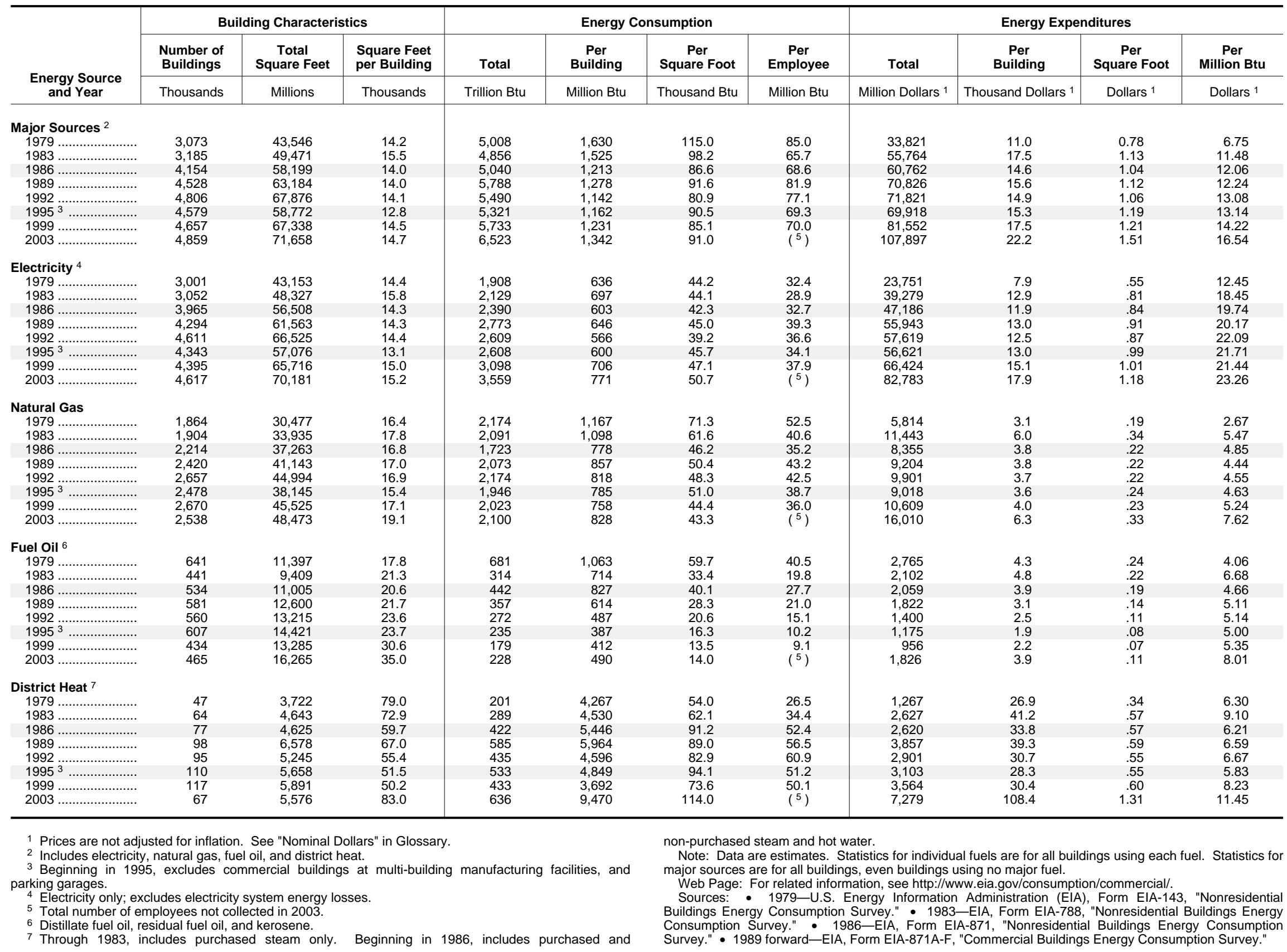




\section{By End Use}

$1,500-$

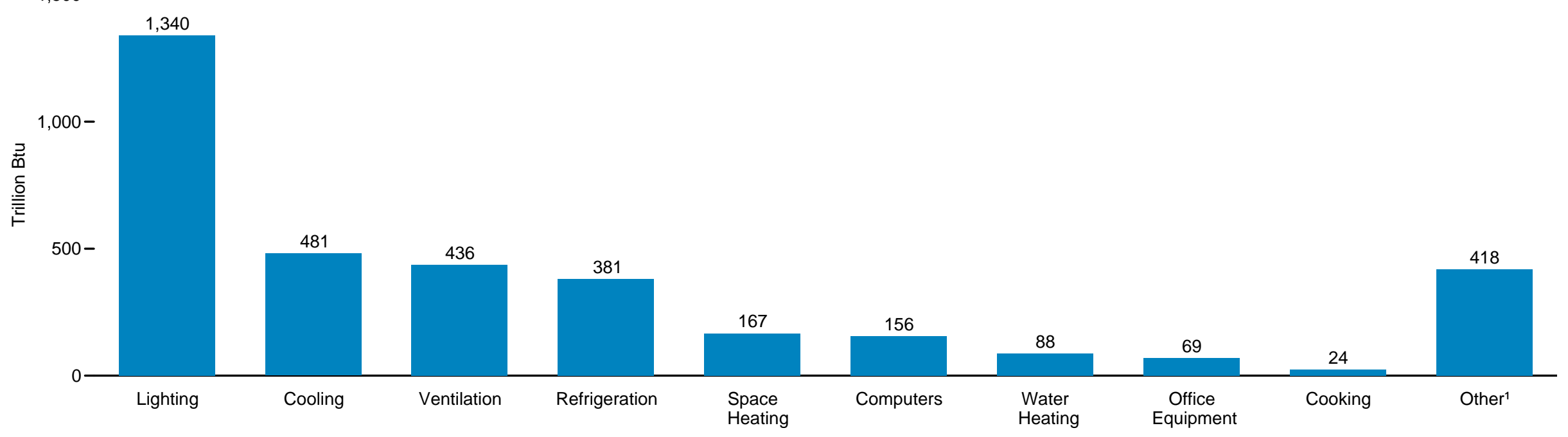

\section{By Principal Building Activity}

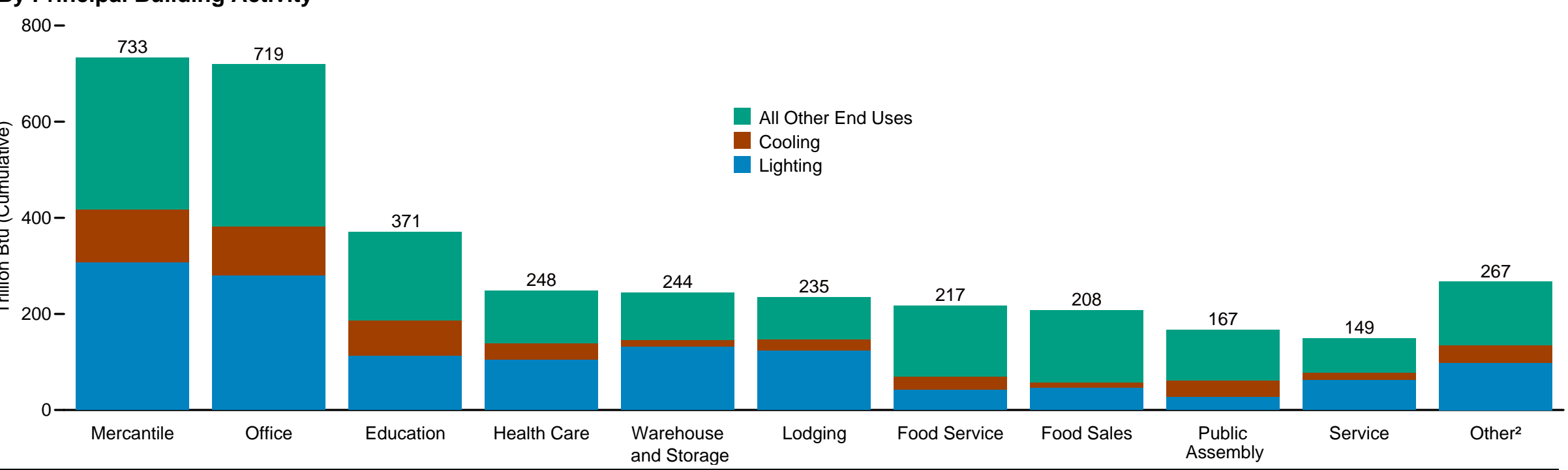

${ }^{1}$ Examples of "other" include medical, electronic, and testing equipment; conveyors, wrappers, hoists, and compactors; washers, disposals, dryers, and cleaning equipment; escalators, elevators, dumb waiters, and window washers; shop tools and electronic testing equipment; sign motors, time clocks, vending machines, phone equipment, and sprinkler controls; scoreboards, fire alarms, intercoms, television sets, radios, projectors, and door operators.

${ }^{2}$ Religious worship, public order and safety, vacant, and buildings that do not fit into any of the other named categories.

Note: Data are estimates for electricity consumption, excluding electrical system energy 
Table 2.11 Commercial Buildings Electricity Consumption by End Use, 2003

(Trillion Btu)

\begin{tabular}{|c|c|c|c|c|c|c|c|c|c|c|c|}
\hline End Use & $\begin{array}{l}\text { Space } \\
\text { Heating }\end{array}$ & Cooling & Ventilation & $\begin{array}{c}\text { Water } \\
\text { Heating }\end{array}$ & Lighting & Cooking & Refrigeration & $\begin{array}{c}\text { Office } \\
\text { Equipment }\end{array}$ & Computers & Other ${ }^{1}$ & Total \\
\hline All Buildings & 167 & 481 & 436 & 88 & 1,340 & 24 & 381 & 69 & 156 & 418 & 3,559 \\
\hline \multicolumn{12}{|l|}{ Principal Building Activity } \\
\hline 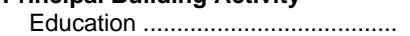 & 15 & 74 & 83 & 11 & 113 & 2 & 16 & 4 & 32 & 21 & 371 \\
\hline 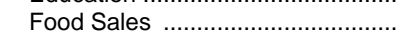 & 6 & 12 & 7 & $\mathrm{Q}$ & 46 & 2 & 119 & 2 & 2 & 10 & 208 \\
\hline 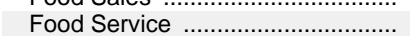 & 10 & 28 & 24 & 10 & 42 & 13 & 70 & 2 & 2 & 15 & 217 \\
\hline 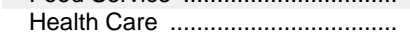 & 6 & 34 & 42 & 2 & 105 & 1 & 8 & 4 & 10 & 36 & 248 \\
\hline Inpatient ………………………........ & 3 & 25 & 38 & 2 & 76 & 1 & 4 & 2 & 7 & 21 & 178 \\
\hline 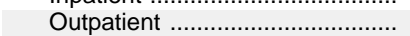 & 3 & 9 & 4 & $(\mathrm{~s})$ & 28 & (s) & 4 & 2 & 3 & 15 & 69 \\
\hline Lodging ……… & 14 & 24 & 14 & 12 & 124 & 2 & 12 & $\mathrm{Q}$ & 6 & 24 & 235 \\
\hline 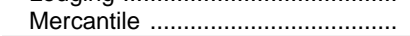 & 58 & 109 & 68 & 38 & 308 & 2 & 49 & 8 & 11 & 83 & 733 \\
\hline Retail (Other Than Mall) .............. & 6 & 25 & 16 & 2 & 111 & (s) & 22 & 3 & 4 & 22 & 211 \\
\hline Enclosed and Strip Malls .................. & 52 & 84 & 51 & 36 & 197 & $\begin{array}{r}101 \\
2\end{array}$ & 27 & 5 & 8 & 61 & 523 \\
\hline Office …… & 33 & 101 & 63 & 7 & 281 & 1 & 35 & 32 & 74 & 91 & 719 \\
\hline Public Assembly .................................. & 5 & 35 & 63 & (s) & 27 & (s) & 9 & $\mathrm{Q}$ & 3 & 23 & 167 \\
\hline Public Order and Safety ..................... & 2 & 8 & 10 & 3 & 18 & (s) & 3 & 1 & 2 & 10 & 57 \\
\hline 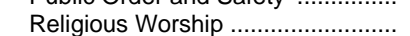 & 3 & 11 & 5 & (s) & 17 & (s) & 6 & (s) & 1 & 18 & 62 \\
\hline 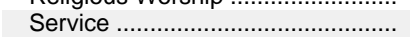 & 6 & 15 & 24 & (s) & 63 & Q & 9 & 1 & 3 & 28 & 149 \\
\hline Warehouse and Storage ................. & 5 & 13 & 20 & 2 & 132 & Q & 36 & 2 & 5 & 30 & 244 \\
\hline Other ${ }^{2}$ & 2 & 16 & 11 & $\mathrm{Q}$ & 59 & $\mathrm{Q}$ & 10 & $\mathrm{Q}$ & 5 & 22 & 133 \\
\hline Vacant ……1-1) & 1 & 2 & 1 & Q & 4 & Q & (s) & Q & (s) & 7 & 15 \\
\hline
\end{tabular}

1 Examples of "other" include medical, electronic, and testing equipment; conveyors, wrappers, hoists, and compactors; washers, disposals, dryers and cleaning equipment; escalators, elevators, dumb waiters, and window washers; shop tools and electronic testing equipment; sign motors, time clocks, vending machines, phone equipment, and sprinkler controls; scoreboards, fire alarms, intercoms, television sets, radios, projectors, and door operators.

Includes buildings that do not fit into any of the other named categories.

s)=Less than 0.5 trillion Btu. Q=Data withheld because either the relative standard error was greater

than 50 percent or fewer than 20 buildings were sampled.

Notes: - Data are estimates for electricity consumption, excluding electrical system energy losses. - One kilowatthour $=3,412 \mathrm{Btu}$.

Web Page: For related information, see http://www.eia.gov/consumption/commercia//.

Source: U.S. Energy Information Administration, "Commercial Buildings Energy Consumption Survey 2003," Table E3A. 


\section{Energy Consumption by Sector}

Note. Electrical System Energy Losses. Electrical system energy losses are calculated as the difference between total primary consumption by the electric power sector-see Table $2.1 \mathrm{f}$ - and the total energy content of electricity retail sales-see Tables 8.9 and A6. Most of these losses occur at steam-electric power plants (conventional and nuclear) in the conversion of heat energy into mechanical energy to turn electric generators. The loss is a thermodynamically necessary feature of the steam-electric cycle. Part of the energy input-to-output losses is a result of imputing fossil energy equivalent inputs for hydroelectric, geothermal, solar thermal, photovoltaic, and wind energy sources. In addition to conversion losses, other losses included power plant use of electricity, transmission and distribution of electricity from power plants to end-use consumers (also called "line losses"), and unaccounted for electricity. Total losses are allocated to the end-use sectors in proportion to each sector's share of total electricity sales. Overall, about two thirds of total energy input is lost in conversion. Currently, of electricity generated, approximately 5 percent is lost in plant use and 7 percent is lost in transmission and distribution. 


\section{Financial Indicators}




\section{Figure 3.1 Fossil Fuel Production Prices}

\section{Prices, 1949-2011}

20-

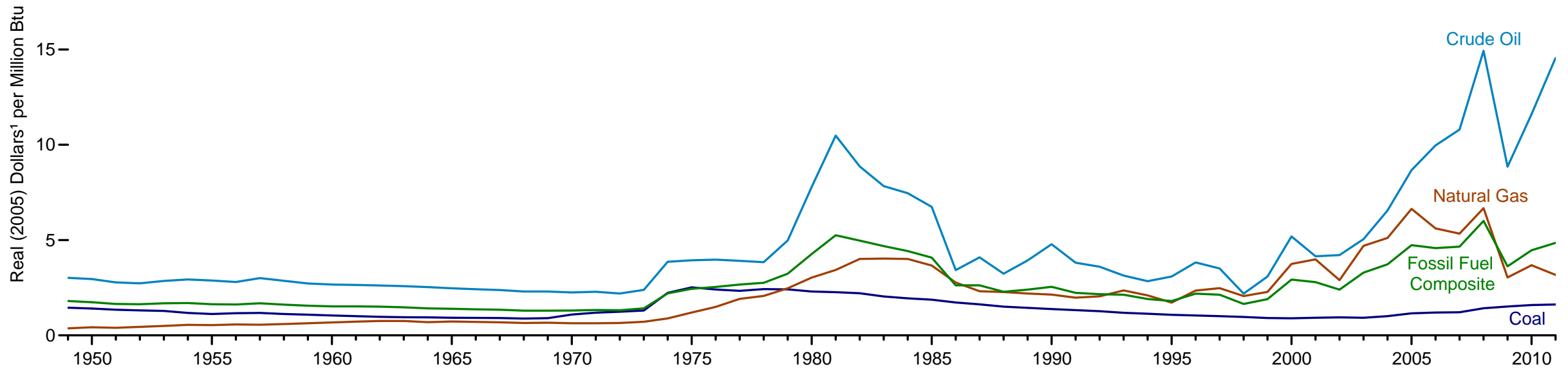

Fossil Fuel Composite Price, ${ }^{2}$ Change From Previous Year, 1950-2011

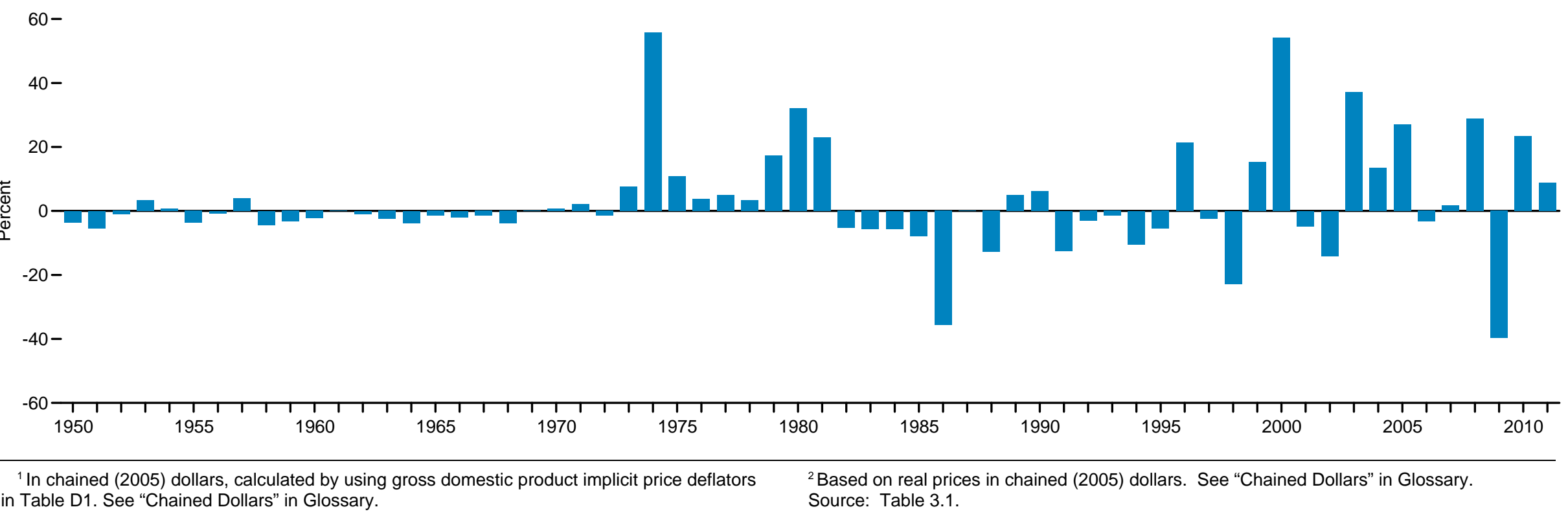


Table 3.1 Fossil Fuel Production Prices, Selected Years, 1949-2011

(Dollars per Million Btu)

\begin{tabular}{|c|c|c|c|c|c|c|c|c|c|}
\hline \multirow[b]{2}{*}{ Year } & \multicolumn{2}{|c|}{ Coal 1} & \multicolumn{2}{|c|}{ Natural Gas ${ }^{2}$} & \multicolumn{2}{|c|}{ Crude Oil ${ }^{3}$} & \multicolumn{3}{|c|}{ Fossil Fuel Composite ${ }^{4}$} \\
\hline & Nominal 5 & Real 6 & Nominal ${ }^{5}$ & Real ${ }^{6}$ & Nominal 5 & Real 6 & Nominal 5 & Real ${ }^{6}$ & $\begin{array}{c}\text { Percent } \\
\text { Change }^{7}\end{array}$ \\
\hline 1949 & 0.21 & $\begin{array}{l}1.45 \\
1.41\end{array}$ & 0.05 & 0.37 & 0.44 & $\begin{array}{r}3.02 \\
R 2.95\end{array}$ & 0.26 & $\begin{array}{l}1.81 \\
1.74\end{array}$ & -- \\
\hline 1955 & .19 & 1.12 & .09 & .54 & .48 & 2.88 & .27 & $R_{1.63}$ & -3.6 \\
\hline 1960 & .19 & 1.04 & .13 & .68 & .50 & 2.67 & .28 & 1.52 & -2.3 \\
\hline 1965 & .18 & .92 & .15 & .73 & .49 & $\mathrm{R}_{2.47}$ & .28 & 1.39 & -1.5 \\
\hline 1970 & .27 & 1.09 & .15 & .63 & .55 & $\mathrm{R}_{2.25}$ & .32 & 1.31 & .8 \\
\hline 1975 & .85 & 2.52 & .40 & 1.20 & 1.32 & 3.94 & .82 & 2.45 & 10.9 \\
\hline 1976 & .86 & 2.41 & .53 & $R_{1} .49$ & 1.41 & 3.98 & .90 & 2.54 & 3.8 \\
\hline 1977 & .88 & 2.34 & .72 & $\mathrm{R}_{1.91}$ & 1.48 & 3.91 & 1.01 & 2.67 & 5.1 \\
\hline 1978 & .98 & 2.43 & .84 & 2.07 & 1.55 & 3.84 & 1.12 & 2.76 & 3.4 \\
\hline 1979 & 1.06 & $\mathrm{R}_{2.41}$ & 1.08 & 2.47 & 2.18 & 4.98 & 1.42 & $R_{3.23}$ & 17.3 \\
\hline 1980 & 1.10 & 2.30 & 1.45 & 3.03 & 3.72 & $\mathrm{R}_{7.79}$ & 2.04 & $\mathrm{R}_{4.27}$ & 32.1 \\
\hline 1981 & 1.18 & $\mathrm{R}_{2.26}$ & 1.80 & R3.43 & 5.48 & $\mathrm{R}_{10.48}$ & $R_{2.74}$ & R5.25 & 22.9 \\
\hline 1982 & 1.23 & 2.21 & 2.22 & 4.01 & 4.92 & 8.87 & 2.76 & R4.97 & -5.3 \\
\hline 1983 & 1.18 & $\mathrm{R}_{2.04}$ & 2.32 & 4.03 & 4.52 & $\mathrm{R}_{7.83}$ & 2.70 & $\mathrm{R}_{4} .68$ & -5.8 \\
\hline 1984 & 1.16 & 1.95 & 2.40 & 4.01 & 4.46 & R7.46 & 2.65 & $R_{4.42}$ & -5.6 \\
\hline 1985 & 1.15 & 1.87 & 2.26 & $R_{3} .66$ & 4.15 & $R_{6.74}$ & 2.51 & 4.08 & -7.8 \\
\hline 1986 & 1.09 & $\mathrm{R}_{1.72}$ & 1.75 & $\mathrm{R}_{2.77}$ & 2.16 & $R_{3.42}$ & 1.65 & $\mathrm{R}_{2.62}$ & -35.6 \\
\hline 1987 & 1.05 & $\mathrm{R}_{1.62}$ & 1.50 & 2.32 & 2.66 & 4.10 & 1.70 & $\mathrm{R}_{2.62}$ & (s) \\
\hline 1988 & 1.01 & 1.51 & 1.52 & $\mathrm{R}_{2.27}$ & 2.17 & 3.24 & 1.53 & 2.29 & -12.8 \\
\hline 1989 & 1.00 & 1.44 & 1.53 & R2.19 & 2.73 & 3.93 & 1.67 & 2.40 & 5.0 \\
\hline 1990 & 1.00 & 1.38 & 1.55 & 2.14 & 3.45 & 4.78 & 1.84 & 2.55 & 6.2 \\
\hline 1991 & .99 & $\mathrm{R}_{1.32}$ & 1.48 & 1.98 & 2.85 & R3.81 & 1.67 & 2.23 & -12.5 \\
\hline 1992 & .97 & 1.27 & 1.57 & 2.05 & 2.76 & 3.60 & 1.66 & $\mathrm{R}_{2.16}$ & -3.1 \\
\hline 1993 & .93 & $\mathrm{R}_{1.18}$ & 1.84 & 2.36 & 2.46 & 3.14 & 1.67 & 2.13 & -1.4 \\
\hline 1994 & .91 & $\mathrm{R}_{1.13}$ & 1.67 & $\mathrm{R}_{2.09}$ & 2.27 & $R_{2.84}$ & 1.53 & 1.91 & -10.4 \\
\hline 1995 & .88 & 1.08 & 1.40 & 1.72 & 2.52 & 3.09 & 1.47 & $\mathrm{R}_{1.80}$ & -5.5 \\
\hline 1996 & .87 & 1.04 & 1.96 & $R_{2.35}$ & 3.18 & 3.83 & 1.82 & 2.19 & 21.3 \\
\hline 1997 & .85 & 1.01 & 2.10 & 2.48 & 2.97 & 3.51 & 1.81 & $\mathrm{R}_{2.13}$ & -2.5 \\
\hline 1998 & .83 & R. 96 & 1.77 & 2.07 & 1.87 & 2.19 & 1.41 & 1.65 & -22.8 \\
\hline 1999 & .79 & .91 & 1.98 & 2.28 & 2.68 & 3.09 & 1.65 & 1.90 & 15.4 \\
\hline 2000 & .80 & .90 & 3.32 & 3.75 & 4.61 & R5.19 & 2.60 & 2.93 & 54.2 \\
\hline 2001 & .84 & .92 & 3.62 & 3.99 & 3.77 & 4.15 & 2.53 & 2.79 & -4.8 \\
\hline 2002 & .87 & .94 & 2.67 & 2.90 & 3.88 & 4.21 & 2.21 & 2.40 & -14.1 \\
\hline 2003 & .87 & .93 & $R_{4.42}$ & $R_{4.70}$ & 4.75 & 5.05 & $\mathrm{R}_{3.10}$ & 3.29 & R37.2 \\
\hline 2004 & .98 & 1.01 & 4.95 & 5.11 & 6.34 & 6.55 & 3.61 & 3.73 & $\mathrm{R}_{13.4}$ \\
\hline 2005 & 1.16 & 1.16 & 6.64 & 6.64 & 8.67 & 8.67 & 4.74 & 4.74 & $\mathrm{R}_{27.0}$ \\
\hline 2006 & 1.24 & 1.20 & 5.79 & 5.61 & 10.29 & 9.97 & 4.73 & 4.58 & -3.3 \\
\hline 2007 & 1.29 & 1.21 & $\mathrm{R}_{5.67}$ & R5.34 & 11.47 & $\mathrm{R}_{10.80}$ & 4.95 & $R_{4} .66$ & $\mathrm{R}_{1.8}$ \\
\hline 2008 & 1.55 & 1.42 & 7.25 & 6.67 & 16.21 & 14.93 & 6.52 & 6.01 & $\mathrm{R}_{28.9}$ \\
\hline 2009 & 1.67 & 1.52 & 3.33 & 3.04 & 9.72 & R8.85 & 3.97 & 3.62 & -39.7 \\
\hline 2010 & 1.77 & $R_{1.59}$ & $R_{4} .08$ & $\mathrm{R}_{3} .68$ & 12.88 & $R_{11.61}$ & $\mathrm{R}_{4.96}$ & $R_{4.47}$ & $R_{23.4}$ \\
\hline $2011^{P}$ & 1.83 & 1.62 & 3.60 & 3.18 & 16.51 & 14.56 & 5.50 & 4.85 & 8.7 \\
\hline
\end{tabular}

1 Free-on-board (F.O.B.) rail/barge prices, which are the F.O.B. prices of coal at the point of first sale, excluding freight or shipping and insurance costs. See "Free on Board (F.O.B.)" in Glossary.

Wellhead prices (converted to dollars per million Btu using marketed production heat contents). See "Natural Gas Wellhead Price" in Glossary.

Dice "Crude Oil Domestic First Purchase Price" in Glossary.

Der Btu of each fossil fuel by the total Btu content of the production of lated value of total fossil fuel production by the accumulated Btu content of total fossilfuel production.
5 See "Nominal Dollars" in Glossary.

1n chained (2005) dollars, calculated by using gross domestic product implicit price deflators in Table 7 Based on real values.
B

R=Revised. P=Preliminary. $\quad--=$ Not applicable. (s)=Less than 0.05 percent and greater than -0.05 percent.

Web Page: For all data beginning in 1949, see http:/www.eia.gov/totalenergy/data/annual/\#financial.

Sources: Tables 5.18,6.7, 7.9, A2, A4, and A5. 


\section{Figure 3.2 Value of Fossil Fuel Production, Imports, and Exports}

\section{Overview, 1949-2011}

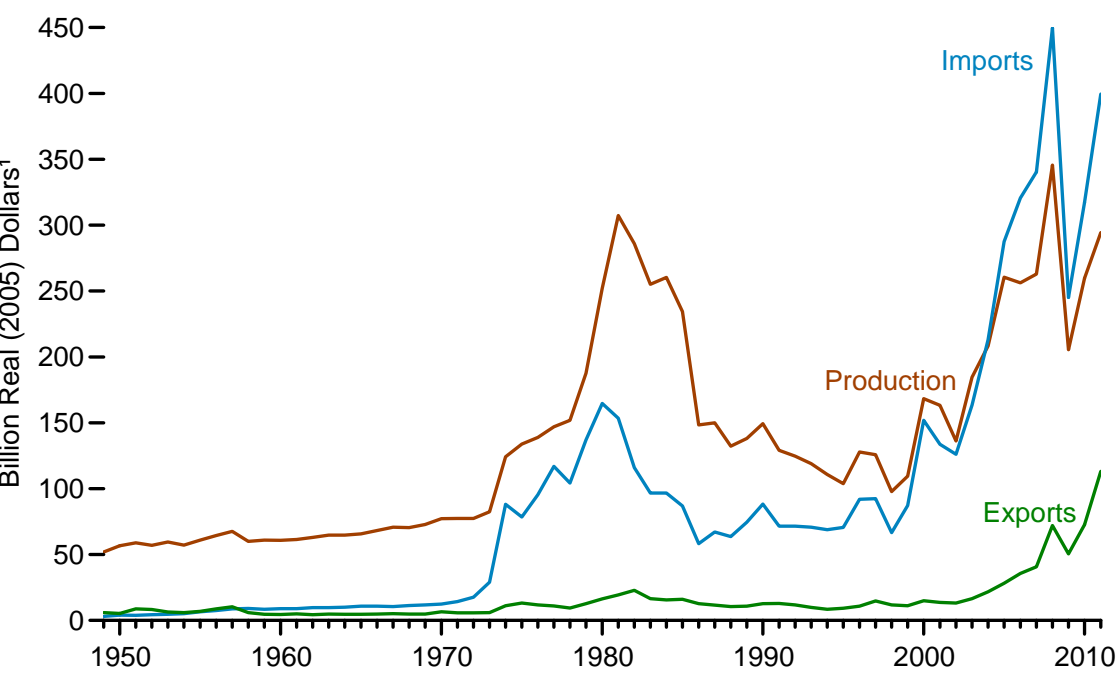

\section{Overview, 2011}

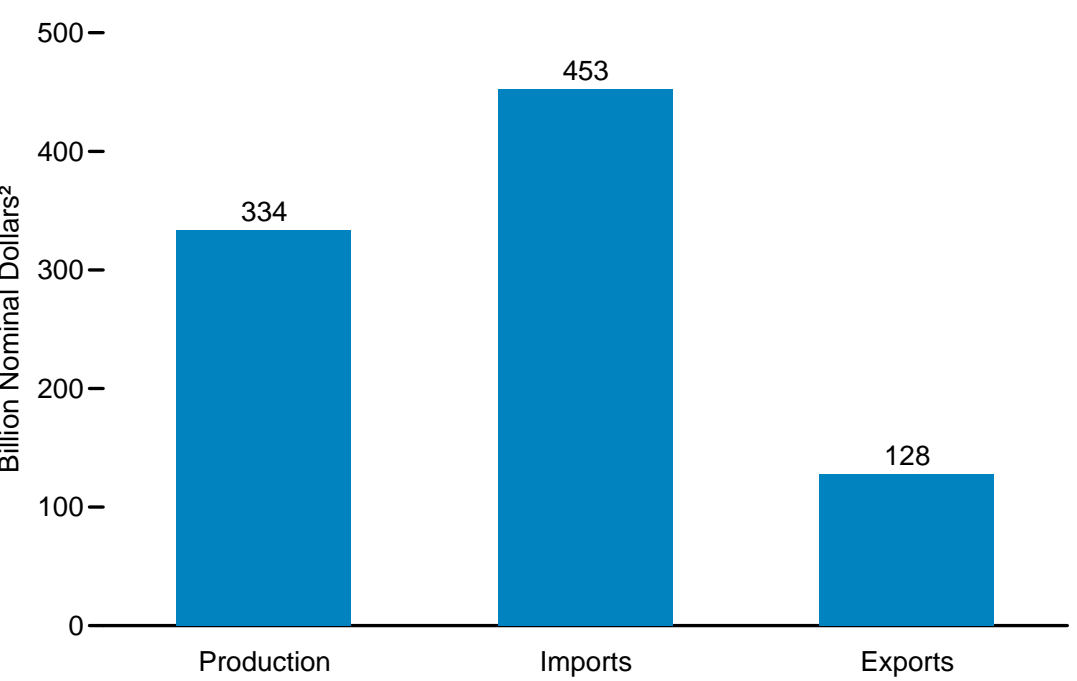

Production by Fuel, 1949-2011

$200-$

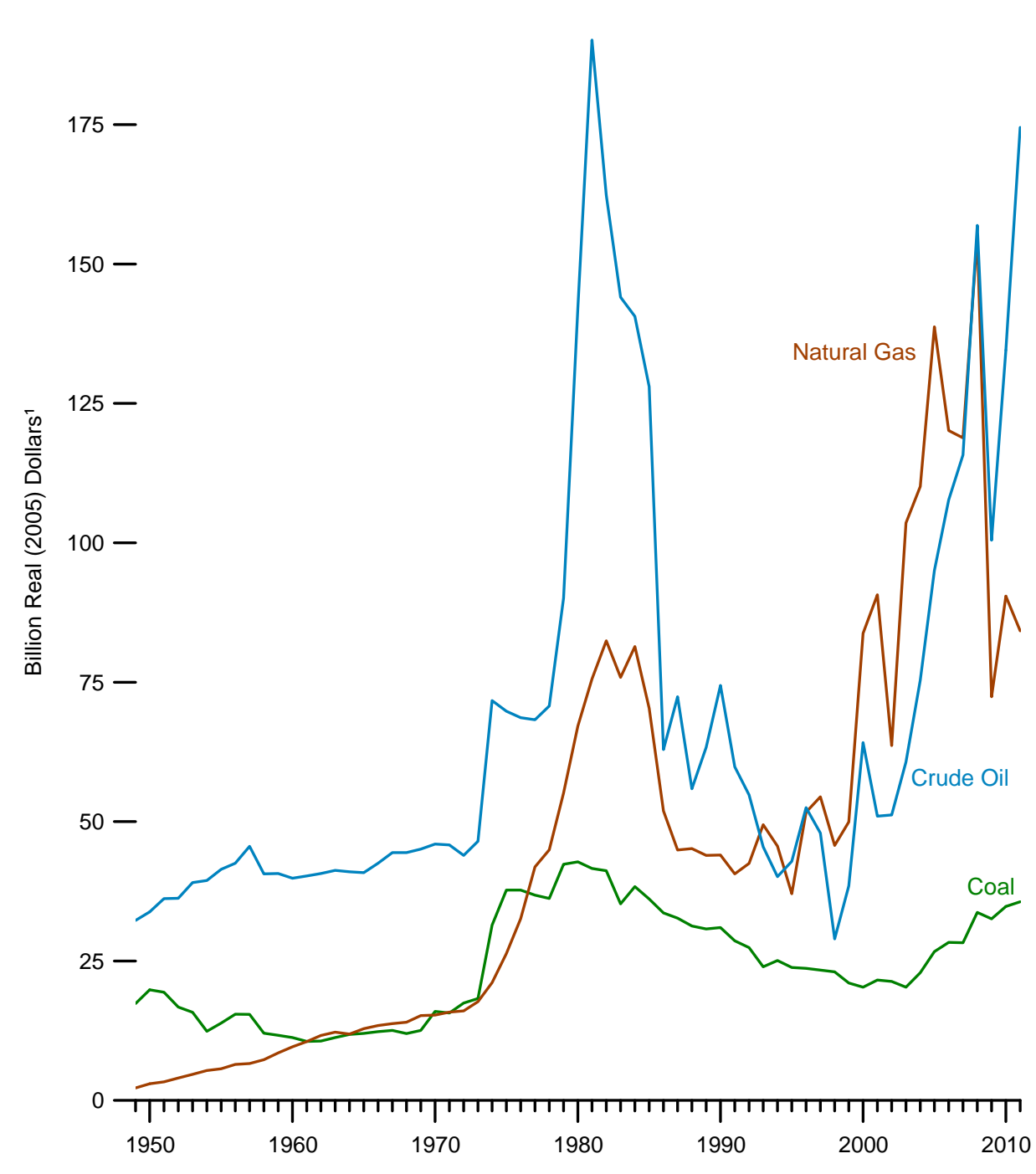

${ }^{2}$ See "Nominal Dollars" in Glossary. Sources: Tables 3.2, 3.7, and 3.8. 
Table 3.2 Value of Fossil Fuel Production, Selected Years, 1949-2011

(Billion Dollars)

\begin{tabular}{|c|c|c|c|c|c|c|c|c|}
\hline \multirow[b]{2}{*}{ Year } & \multicolumn{2}{|c|}{ Coal ${ }^{1}$} & \multicolumn{2}{|c|}{ Natural Gas ${ }^{2}$} & \multicolumn{2}{|c|}{ Crude Oil ${ }^{3,4}$} & \multicolumn{2}{|c|}{ Total } \\
\hline & Nominal 5 & Real 6 & Nominal 5 & Real 6 & Nominal 5 & Real $^{6}$ & Nominal 5 & Real 6 \\
\hline 1949 & 2.52 & $R_{17.37}$ & 0.33 & 2.24 & 4.68 & R32.27 & 7.52 & R51.88 \\
\hline 1950 & 2.91 & $R_{19.84}$ & .44 & 3.00 & 4.95 & $R_{33.80}$ & 8.30 & $R_{56.64}$ \\
\hline 1955 & 2.30 & $R_{13} .87$ & .94 & 5.67 & 6.88 & R41.45 & 10.12 & R60.99 \\
\hline 1960 & 2.10 & $R_{11.27}$ & 1.79 & 9.61 & 7.42 & R39.84 & 11.30 & R 60.72 \\
\hline 1965 & 2.40 & $R_{12.03}$ & 2.57 & $R_{12.87}$ & 8.15 & $\mathrm{R}_{40.86}$ & 13.11 & $\mathrm{R}_{65.76}$ \\
\hline 1970 & 3.88 & R15.96 & 3.73 & R15.31 & 11.19 & R 45.96 & 18.80 & R77.23 \\
\hline 1975 & 12.67 & $R_{37.71}$ & 8.85 & $\mathrm{R}_{26.34}$ & 23.45 & R 69.80 & 44.96 & $R_{133.85}$ \\
\hline 1976 & 13.40 & $R_{37.72}$ & 11.57 & $R_{32.58}$ & 24.37 & R68.63 & 49.34 & $\mathrm{R}_{138.92}$ \\
\hline 1977 & 13.91 & R36.81 & 15.82 & $\mathrm{R}_{41.87}$ & 25.79 & R68.26 & 55.52 & $\mathrm{R}_{146.94}$ \\
\hline 1978 & 14.65 & R36.23 & 18.18 & R44.95 & 28.60 & R70.74 & 61.43 & $\mathrm{R}_{151.92}$ \\
\hline 1979 & 18.55 & $\mathrm{R}_{42.36}$ & 24.16 & R55.15 & 39.45 & R90.08 & 82.16 & $\mathrm{R}_{187.59}$ \\
\hline 1980 & 20.45 & R42.79 & 32.09 & $R_{67.14}$ & 67.93 & $\mathrm{R}_{142.14}$ & 120.47 & $\mathrm{R}_{252.07}$ \\
\hline 1981 & 21.75 & $\mathrm{R}_{41.61}$ & 39.51 & R75.59 & 99.40 & $R_{190.16}$ & 160.66 & R307.36 \\
\hline 1982 & 22.84 & R41.18 & 45.71 & R82.42 & 90.03 & $R_{162.34}$ & 158.58 & R285.94 \\
\hline 1983 & 20.32 & $R_{35.24}$ & 43.73 & $R_{75.85}$ & 83.05 & $R_{144.05}$ & 147.10 & $\mathrm{R}_{255.15}$ \\
\hline 1984 & 22.94 & R38.36 & 48.69 & R81.40 & 84.10 & $R_{1} 140.60$ & 155.74 & $\mathrm{R}_{260.35}$ \\
\hline 1985 & 22.27 & R36.13 & 43.35 & R70.34 & 78.88 & $R_{128.00}$ & 144.50 & $\mathrm{R}_{234.47}$ \\
\hline 1986 & 21.18 & $\mathrm{R}_{33.62}$ & 32.71 & $R_{51.92}$ & 39.63 & R62.92 & 93.52 & $\mathrm{R}_{148.47}$ \\
\hline 1987 & 21.20 & $R_{32.70}$ & 29.11 & R44.91 & 46.93 & R72.40 & 97.24 & $\mathrm{R}_{150.02}$ \\
\hline 1988 & 20.97 & $R_{31.28}$ & 30.28 & R45.17 & 37.48 & R55.90 & 88.73 & $\mathrm{R}_{132.34}$ \\
\hline 1989 & 21.40 & $R_{30.76}$ & 30.58 & $\mathrm{R}_{43.95}$ & 44.07 & R63.34 & 96.05 & $\mathrm{R}_{138.05}$ \\
\hline 1990 & 22.39 & $R_{30.99}$ & 31.80 & $\mathrm{R}_{44.00}$ & 53.77 & R74.42 & 107.96 & $\mathrm{R}_{149.40}$ \\
\hline 1991 & 21.40 & $\mathrm{R}_{28.61}$ & 30.39 & $\mathrm{R}_{40.62}$ & 44.77 & R59.84 & 96.57 & $\mathrm{R}_{129.06}$ \\
\hline 1992 & 20.98 & $\mathrm{R}_{27.39}$ & 32.56 & $\mathrm{R}_{42.51}$ & 41.97 & $R_{54.79}$ & 95.50 & $\mathrm{R}_{124.68}$ \\
\hline 1993 & 18.77 & $R_{23.97}$ & 38.72 & R49.46 & 35.61 & R45.49 & 93.10 & $R_{118.92}$ \\
\hline 1994 & 20.06 & $\mathrm{R}_{25.09}$ & 36.46 & $\mathrm{R}_{45.61}$ & 32.07 & $\mathrm{R}_{40.12}$ & 88.59 & $\mathrm{R}_{110.83}$ \\
\hline 1995 & 19.45 & $R_{23.84}$ & 30.24 & $R_{37.05}$ & 35.00 & $R_{42.89}$ & 84.69 & $\mathrm{R}_{103.78}$ \\
\hline 1996 & 19.68 & $R_{23.67}$ & 42.99 & $R_{51.70}$ & 43.68 & R52.52 & 106.35 & $\mathrm{R}_{127.89}$ \\
\hline 1997 & 19.77 & $R_{23.36}$ & 46.09 & R54.46 & 40.57 & R47.94 & 106.43 & $\mathrm{R}_{125.77}$ \\
\hline 1998 & 19.75 & $R_{23.07}$ & 39.12 & $\mathrm{R}_{45.71}$ & 24.80 & $R_{28.98}$ & 83.68 & R97.77 \\
\hline 1999 & 18.30 & $R_{21.07}$ & 43.37 & $\mathrm{R}_{4} 4.94$ & 33.40 & $\mathrm{R}_{38.46}$ & 95.08 & $\mathrm{R}_{109.48}$ \\
\hline 2000 & 18.02 & $\mathrm{R}_{20.30}$ & 74.33 & R83.77 & 56.93 & $R_{64.17}$ & 149.27 & $\mathrm{R}_{168.25}$ \\
\hline 2001 & 19.60 & $\mathrm{R}_{21.60}$ & 82.28 & R90.69 & 46.25 & R50.97 & 148.13 & $\mathrm{R}_{163.27}$ \\
\hline 2002 & 19.68 & $\mathrm{R}_{21.34}$ & 58.66 & $\mathrm{R}_{63.63}$ & 47.21 & R51.20 & 125.54 & $\mathrm{R}_{136.17}$ \\
\hline 2003 & 19.13 & $R_{20.32}$ & 97.47 & $\mathrm{R}_{103.55}$ & 57.14 & R60.70 & 173.75 & $\mathrm{R}_{184.58}$ \\
\hline 2004 & 22.16 & 22.90 & 106.57 & $R_{110.10}$ & 72.93 & R75.35 & 201.66 & $\mathrm{R}_{208.35}$ \\
\hline 2005 & 26.69 & 26.69 & 138.74 & 138.74 & 95.03 & 95.03 & 260.46 & 260.46 \\
\hline 2006 & 29.25 & $\mathrm{R}_{28.34}$ & 124.03 & $\mathrm{R}_{120.15}$ & 111.16 & $\mathrm{R}_{1} 07.68$ & 264.44 & $\mathrm{R}_{256.16}$ \\
\hline 2007 & 30.04 & $R_{28.28}$ & 126.23 & $\mathrm{R}_{118.83}$ & 122.96 & $\mathrm{R}_{115.75}$ & 279.23 & $\mathrm{R}_{262.86}$ \\
\hline 2008 & 36.62 & $\mathrm{R}_{33.72}$ & $\mathrm{R}_{168.26}$ & $\mathrm{R}_{154.96}$ & 170.38 & $\mathrm{R}_{156.92}$ & R375.27 & R345.61 \\
\hline 2009 & 35.73 & $R_{32.56}$ & R79.45 & R72.40 & 110.25 & $\mathrm{R}_{1} 100.48$ & R225.43 & $\mathrm{R}_{205.44}$ \\
\hline 2010 & $R_{38.61}$ & $\mathrm{R}_{34.79}$ & $\mathrm{R}_{100.36}$ & $R_{90.42}$ & $R_{149.32}$ & $\mathrm{R}_{134.54}$ & $\mathrm{R}_{288.30}$ & $\mathrm{R}_{259.75}$ \\
\hline $2011^{P}$ & 40.39 & 35.63 & 95.47 & 84.22 & 197.83 & 174.51 & 333.69 & 294.36 \\
\hline
\end{tabular}

${ }^{1}$ Coal values are based on free-on-board (F.O.B.) rail/barge prices, which are the F.O.B. prices of coal at the point of first sale, excluding freight or shipping and insurance costs. See "Free on Board (F.O.B.)" in Glossary.

2 Natural gas values are for marketed production based on wellhead prices. See "Natural Gas Marketed Production" and "Natural Gas Wellhead Price" in Glossary.

3 Includes lease condensate.
4 Crude oil values are based on domestic first purchase prices. See "Crude Oil Domestic First

5 See "Nominal Dollars" in Glossary.

6 In chained (2005) dollars, calculated by using gross domestic product implicit price deflators in Table D1. See "Chained Dollars" in Glossary.

$R=$ Revised. $P=$ Preliminary.

Note: Totals may not equal sum of components due to independent rounding.

Web Page: For all data beginning in 1949, see http://www.eia.gov/totalenergy/data/annual/\#financial. Sources: Tables 5.1b, 5.18,6.2, 6.7, 7.2, and 7.9. 


\section{Figure 3.3 Consumer Price Estimates for Energy by Source}

\section{Total Energy, 1970-2010}

24-

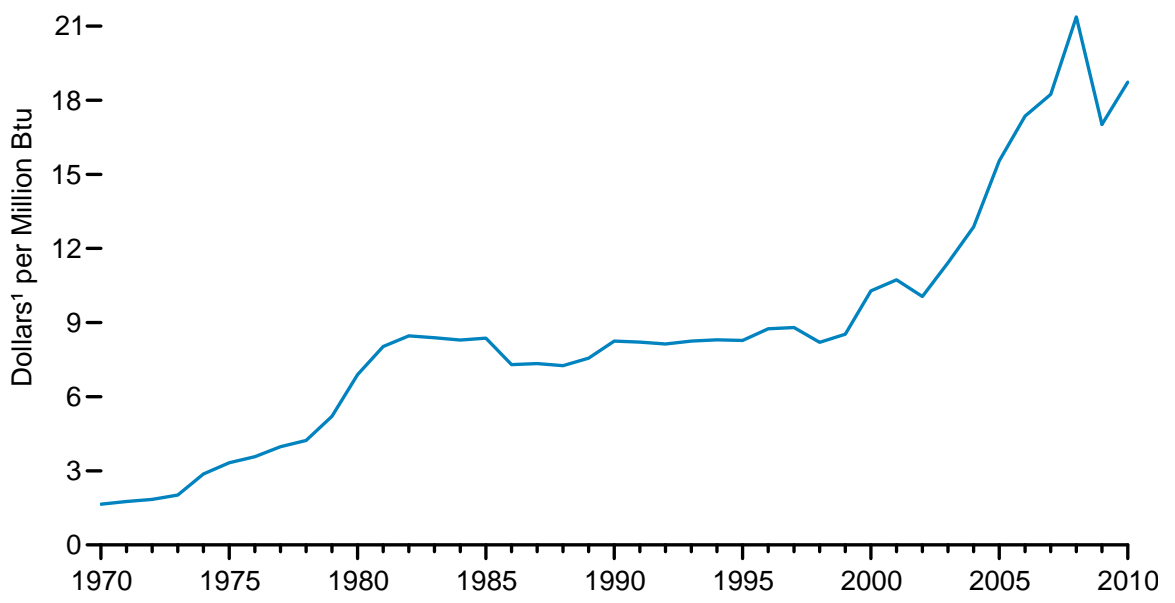

Prices $^{3}$ by Energy Type, Indexed, 1970-2010

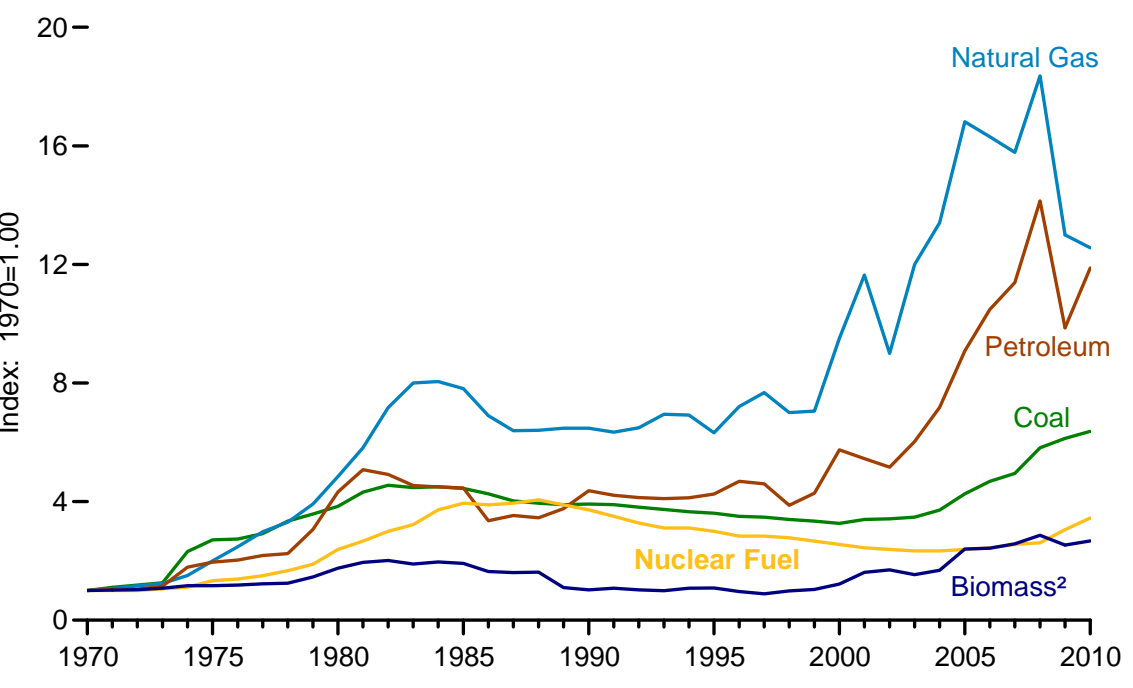

By Energy Type, 2010

35-

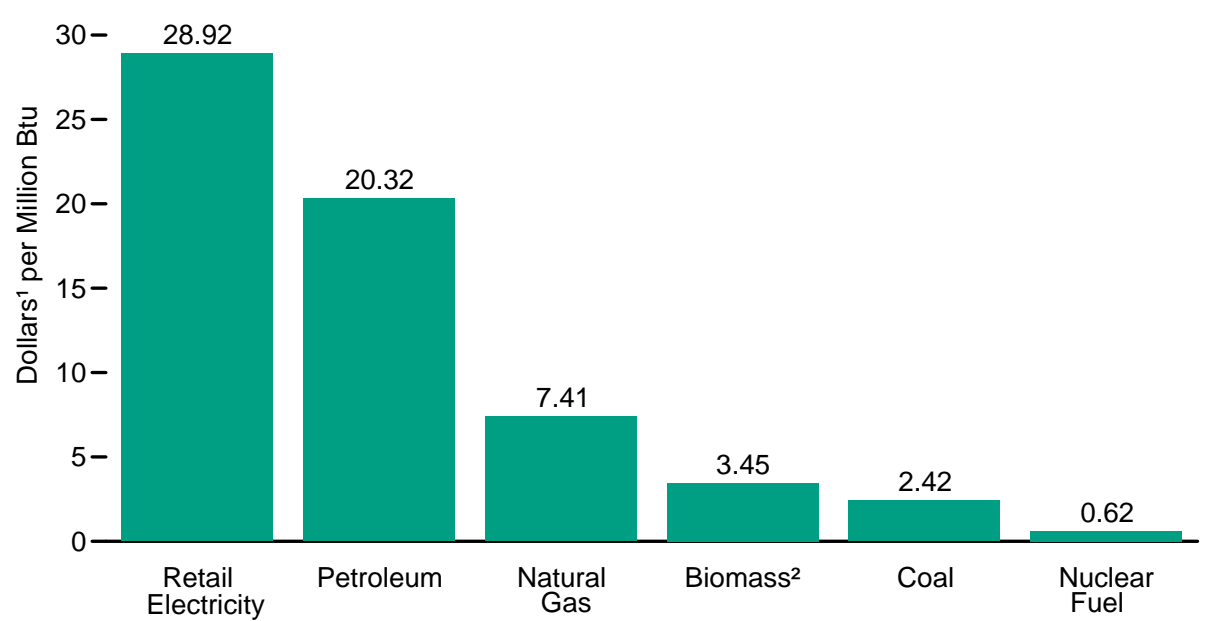

By Petroleum Product, 2010

$$
\text { 25- }
$$

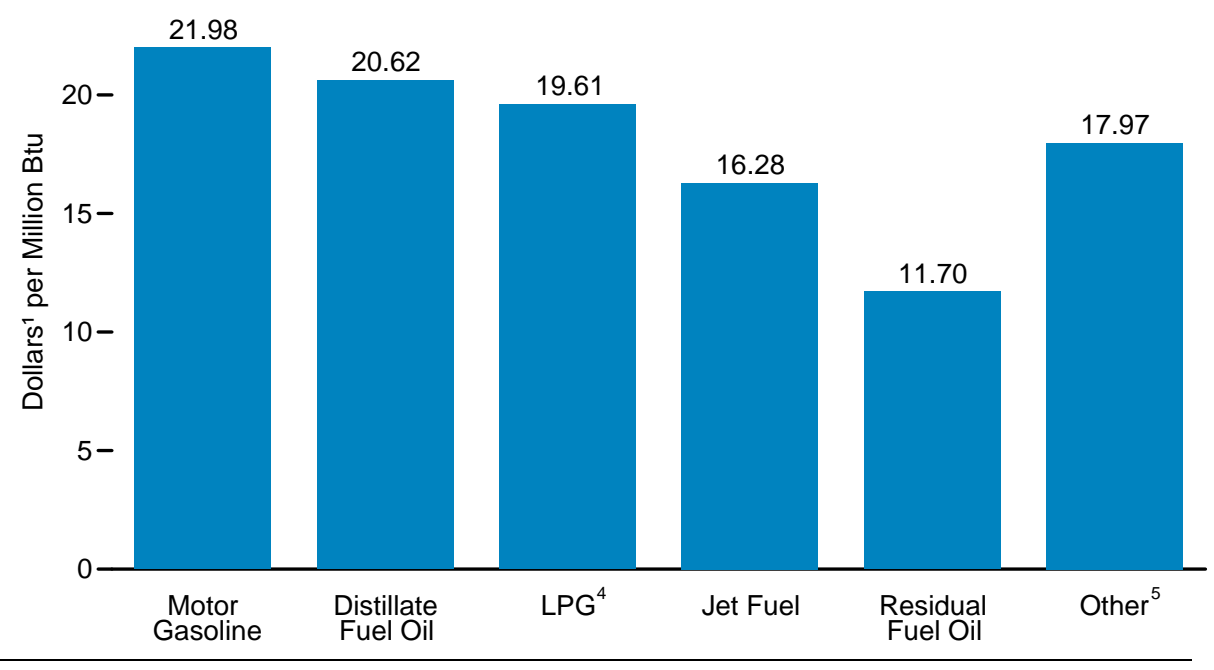

${ }^{5}$ Consumption-weighted average price for asphalt and road oil, aviation gasoline, kerosene, lubricants, petrochemical feedstocks, petroleum coke, special naphthas, waxes, and miscellaneous petroleum products.

Source: Table 3.3.

${ }^{1}$ Prices are not adjusted for inflation. See "Nominal Dollars" in Glossary.
2 Wood and wood-derived fuels, and biomass waste; excludes fuel ethanol and biodiesel. Prior to 2001, also includes non-biomass waste.

${ }^{3}$ Based on nominal dollars.

${ }^{4}$ Liquefied petroleum gases. 
Table 3.3 Consumer Price Estimates for Energy by Source, 1970-2010

(Dollars ${ }^{1}$ per Million Btu)

\begin{tabular}{|c|c|c|c|c|c|c|c|c|c|c|c|c|c|c|c|}
\hline \multirow[b]{3}{*}{ Year } & \multicolumn{12}{|c|}{ Primary Energy 2} & \multirow{3}{*}{$\begin{array}{c}\text { Electric } \\
\text { Power } \\
\text { Sector } 11,12\end{array}$} & \multirow[b]{3}{*}{$\begin{array}{c}\text { Retail } \\
\text { Electricity } 13\end{array}$} & \multirow[b]{3}{*}{$\begin{array}{c}\begin{array}{c}\text { Total } \\
\text { Energy }^{9,10,14}\end{array}\end{array}$} \\
\hline & \multirow[b]{2}{*}{ Coal } & \multirow[b]{2}{*}{$\begin{array}{c}\text { Natural } \\
\text { Gas }^{3}\end{array}$} & \multicolumn{7}{|c|}{ Petroleum } & \multirow[b]{2}{*}{$\begin{array}{c}\text { Nuclear } \\
\text { Fuel }\end{array}$} & \multirow[b]{2}{*}{ Biomass ${ }^{8}$} & \multirow[b]{2}{*}{ Total 9,10} & & & \\
\hline & & & $\begin{array}{l}\text { Distillate } \\
\text { Fuel Oil }\end{array}$ & $\begin{array}{c}\text { Jet } \\
\text { Fuel }{ }^{4}\end{array}$ & LPG 5 & $\begin{array}{c}\text { Motor } \\
\text { Gasoline }\end{array}$ & $\begin{array}{l}\text { Residual } \\
\text { Fuel Oil }\end{array}$ & Other ${ }^{7}$ & Total & & & & & & \\
\hline 1970 & 0.38 & 0.59 & 1.16 & 0.73 & 1.43 & 2.85 & 0.42 & 1.38 & 1.71 & 0.18 & 1.29 & 1.08 & 0.32 & 4.98 & 1.65 \\
\hline 1971 & $\begin{array}{l}.00 \\
.42\end{array}$ & .63 & 1.22 & .77 & 1.46 & 2.90 & .58 & 1.45 & 1.78 & $\begin{array}{l}.10 \\
.18\end{array}$ & 1.31 & 1.15 & .38 & $\begin{array}{l}4.90 \\
5.30\end{array}$ & 1.76 \\
\hline 1972 & $\begin{array}{l}.42 \\
.45\end{array}$ & $\begin{array}{l}.63 \\
.68\end{array}$ & $\begin{array}{l}1.22 \\
1.22\end{array}$ & .79 & $\begin{array}{l}1.46 \\
1.49\end{array}$ & $\begin{array}{l}2.90 \\
2.88\end{array}$ & $\begin{array}{l}.58 \\
.62\end{array}$ & $\begin{array}{l}1.45 \\
1.49\end{array}$ & 1.78 & $\begin{array}{l}.18 \\
.18\end{array}$ & $\begin{array}{l}1.31 \\
1.33\end{array}$ & $\begin{array}{l}1.15 \\
1.18\end{array}$ & $\begin{array}{l}.38 \\
.42\end{array}$ & $\begin{array}{l}5.30 \\
5.54\end{array}$ & 1.84 \\
\hline 1973 & $\begin{array}{l}.45 \\
.48\end{array}$ & $\begin{array}{l}.00 \\
.73\end{array}$ & 1.46 & 92 & R2. 00 & $\begin{array}{l}. .00 \\
3.10\end{array}$ & $\begin{array}{l}.02 \\
.75\end{array}$ & 1.49 & 1.97 & $\begin{array}{r}10 \\
19\end{array}$ & 1.00 & 1.10 & 47 & 586 & $\begin{array}{l}1.04 \\
2.02\end{array}$ \\
\hline 1974 & .88 & .89 & 2.44 & 1.58 & $\mathrm{R}_{2.80}$ & 4.32 & 1.82 & $\begin{array}{l}1.50 \\
2.60\end{array}$ & $\mathrm{R}_{3.06}$ & .20 & $\begin{array}{l}1.39 \\
1.50\end{array}$ & $\begin{array}{l}1.29 \\
1.94\end{array}$ & .87 & $\begin{array}{l}7.00 \\
7.42\end{array}$ & $\begin{array}{l}2.02 \\
2.87\end{array}$ \\
\hline 1975 & $\begin{array}{l}.00 \\
1.03\end{array}$ & 1.18 & 2.60 & 2.05 & $\mathrm{R}_{2.96}$ & 4.65 & $\begin{array}{l}1.02 \\
1.93\end{array}$ & 2.94 & $R_{3.35}$ & .24 & 1.50 & 2.19 & $\begin{array}{l}.01 \\
.97\end{array}$ & 8.61 & 3.33 \\
\hline 1976 & 1.04 & 1.46 & 2.77 & 2.25 & $\mathrm{R}_{3.20}$ & $\begin{array}{l}4.05 \\
4.84\end{array}$ & $\begin{array}{l}1.90 \\
1.90\end{array}$ & $\begin{array}{l}3.08 \\
\end{array}$ & $\mathrm{R}_{3.47}$ & .25 & 1.53 & 2.34 & 1.03 & 9.13 & 3.57 \\
\hline 1977 & 1.11 & 1.76 & 3.11 & 2.59 & $\mathrm{R}_{3} .66$ & 5.13 & 2.14 & 3.27 & 3.73 & .27 & 1.58 & 2.58 & 1.17 & 10.11 & 3.98 \\
\hline 1978 & 1.27 & 1.95 & 3.26 & 2.87 & R3.61 & 5.24 & 2.08 & 3.45 & 3.84 & .30 & 1.61 & R2.72 & 1.27 & 10.92 & 4.23 \\
\hline 1979 & 1.36 & 2.31 & 4.69 & 3.90 & $\mathrm{R}_{4} .50$ & 7.11 & 2.83 & 4.70 & 5.23 & .34 & 1.88 & 3.47 & 1.50 & 11.78 & 5.21 \\
\hline 1980 & 1.46 & 2.86 & 6.70 & 6.36 & $\begin{array}{l}\mathrm{R}_{5} .64 \\
\end{array}$ & 9.84 & 3.88 & 7.04 & 7.40 & .43 & 2.26 & 4.57 & 1.77 & 13.95 & 6.89 \\
\hline 1981 & 1.64 & 3.43 & 8.03 & 7.57 & $\mathrm{R}_{6.19}$ & $\begin{array}{r}10.94 \\
\end{array}$ & 4.91 & 8.67 & 8.68 & .48 & 2.52 & 5.25 & 2.04 & 16.14 & 8.03 \\
\hline 1982 & 1.73 & 4.23 & 7.78 & 7.23 & ${ }^{R} 6.68$ & 10.39 & 4.65 & $\begin{array}{l}.0 .01 \\
7.87\end{array}$ & $\begin{array}{r}\text { R8.00 } \\
\end{array}$ & .54 & 2.60 & R5.33 & $\begin{array}{l}2.04 \\
2.05\end{array}$ & $\begin{array}{l}18.14 \\
\end{array}$ & $\begin{array}{l}0.00 \\
8.46\end{array}$ \\
\hline 1983 & 1.70 & 4.72 & 7.32 & 6.53 & R7.20 & 9.12 & 4.50 & $\begin{array}{l}1.01 \\
7.60\end{array}$ & 7.77 & .58 & $\begin{array}{l}2.00 \\
2.44\end{array}$ & $\begin{array}{r}.00 \\
\text { R5.12 }\end{array}$ & 2.02 & $\begin{array}{l}10.10 \\
18.62\end{array}$ & $\begin{array}{l}0.40 \\
8.39\end{array}$ \\
\hline 1984 & 1.71 & 4.75 & 7.37 & 6.25 & $\mathrm{R}_{6} 6.95$ & 8.89 & 4.75 & $\begin{array}{l}7.00 \\
7.72\end{array}$ & 7.68 & .60 & $\begin{array}{l}2.44 \\
2.53\end{array}$ & 5.04 & $\begin{array}{l}2.02 \\
2.02\end{array}$ & $\begin{array}{l}10.02 \\
18.50\end{array}$ & $\begin{array}{r}0.09 \\
\text { R8.29 }\end{array}$ \\
\hline 1985 & 1.69 & 4.61 & 7.22 & 5.91 & $\begin{array}{r}\text { R } 6.63 \\
\end{array}$ & 9.01 & 4.30 & 7.55 & $\begin{array}{l}.08 \\
7.63\end{array}$ & .71 & $\begin{array}{l}2.05 \\
2.47\end{array}$ & $\begin{array}{r}3.04 \\
R_{4} .93\end{array}$ & $\begin{array}{l}2.02 \\
1.91\end{array}$ & $\begin{array}{l}10.00 \\
19.05\end{array}$ & $\begin{array}{l}8.29 \\
8.37\end{array}$ \\
\hline 1986 & $\begin{array}{l}1.062 \\
\end{array}$ & 4.07 & 5.68 & $\begin{array}{l}3.92 \\
\end{array}$ & $\mathrm{R}_{6.49}$ & 6.79 & 2.37 & $\begin{array}{l}.500 \\
5.80\end{array}$ & $\begin{array}{r}.03 \\
\mathrm{R} 5.74\end{array}$ & .70 & $\begin{array}{l}2.47 \\
2.12\end{array}$ & 3.97 & $\begin{array}{l}1.91 \\
1.60\end{array}$ & $\begin{array}{l}19.05 \\
19.05\end{array}$ & $\begin{array}{l}0.31 \\
7.30\end{array}$ \\
\hline $\begin{array}{l}1986 \\
1987\end{array}$ & $\begin{array}{l}1.62 \\
1.53\end{array}$ & $\begin{array}{l}4.07 \\
3.77\end{array}$ & $\begin{array}{l}5.68 \\
5.97\end{array}$ & $\begin{array}{l}3.92 \\
4.03\end{array}$ & $\begin{array}{r}.4 .41 \\
R_{6}\end{array}$ & $\begin{array}{l}.1 / 9 \\
7.23\end{array}$ & $\begin{array}{l}2.37 \\
2.86\end{array}$ & $\begin{array}{l}5.80 \\
5.63\end{array}$ & $\begin{array}{r}.14 \\
6.04\end{array}$ & .71 & $\begin{array}{l}2.12 \\
2.07\end{array}$ & $\begin{array}{l}3.97 \\
4.00\end{array}$ & $\begin{array}{l}1.60 \\
1.57\end{array}$ & $\begin{array}{l}19.05 \\
18.74\end{array}$ & $\begin{array}{l}7.30 \\
7.34\end{array}$ \\
\hline 1988 & $\begin{array}{l}1.05 \\
1.50\end{array}$ & 378 & $\begin{array}{l}5.91 \\
5.83\end{array}$ & $\begin{array}{l}4.05 \\
3.80\end{array}$ & R5.92 & $\begin{array}{l}1.25 \\
7.33\end{array}$ & $\begin{array}{l}2.00 \\
2.35\end{array}$ & $\begin{array}{l}.03 \\
5.26\end{array}$ & $\begin{array}{l}0.04 \\
5.91\end{array}$ & 73 & $\begin{array}{l}2.01 \\
2.09\end{array}$ & $\begin{array}{l}4.00 \\
389\end{array}$ & 1.59 & $\begin{array}{r}10.14 \\
18.68\end{array}$ & $\begin{array}{l}.34 \\
7.26\end{array}$ \\
\hline $\begin{array}{l}1980 \\
1989\end{array}$ & $\begin{array}{l}1.00 \\
1.48\end{array}$ & $\begin{array}{l}3.18 \\
3.82\end{array}$ & $\begin{array}{l}.03 \\
6.43\end{array}$ & $\begin{array}{l}3.00 \\
4.39\end{array}$ & $\begin{array}{r}.9 .98 \\
\mathrm{R}\end{array}$ & $\begin{array}{l}1.35 \\
8.02\end{array}$ & $\begin{array}{l}2.35 \\
2.72\end{array}$ & $\begin{array}{l}5.26 \\
5.50\end{array}$ & $\begin{array}{l}5.91 \\
6.43\end{array}$ & .73 & $\begin{array}{l}2.09 \\
1.42\end{array}$ & $\begin{array}{l}3.89 \\
4.07\end{array}$ & $\begin{array}{l}1.49 \\
151\end{array}$ & $\begin{array}{l}18.68 \\
1898\end{array}$ & $\begin{array}{r}7.26 \\
R 75\end{array}$ \\
\hline 1990 & $\begin{array}{l}1.40 \\
1.49\end{array}$ & $\begin{array}{l}.02 \\
3.82\end{array}$ & $\begin{array}{l}7.40 \\
7.68\end{array}$ & 5.68 & $\begin{array}{r}\mathrm{R} 6.00 \\
\end{array}$ & $\begin{array}{l}0.0< \\
9.12\end{array}$ & 3.17 & $\begin{array}{l}5.50 \\
5.82\end{array}$ & $\begin{array}{l}6.43 \\
7.47\end{array}$ & .67 & $\begin{array}{l}1.42 \\
1.32\end{array}$ & $\begin{array}{r}4.07 \\
\mathrm{R}_{4} .46\end{array}$ & $\begin{array}{l}1.51 \\
1.48\end{array}$ & $\begin{array}{l}18.98 \\
19.32\end{array}$ & $\begin{array}{r}7.56 \\
R_{8}\end{array}$ \\
\hline 1991 & $\begin{array}{l}1.49 \\
1.48\end{array}$ & $\begin{array}{l}3.82 \\
3.74\end{array}$ & $\begin{array}{l}7.68 \\
7.29\end{array}$ & $\begin{array}{l}.68 \\
4.83\end{array}$ & $\begin{array}{r}.83 \\
\mathrm{R} 6.87\end{array}$ & $\begin{array}{l}.112 \\
8.93\end{array}$ & $\begin{array}{l}3.11 \\
2.62\end{array}$ & $\begin{array}{l}5.82 \\
5.73\end{array}$ & $\begin{array}{r}\text { R7.4I } \\
\text { R }\end{array}$ & .63 & $\begin{array}{l}1.32 \\
1.39\end{array}$ & $\begin{array}{l}\mathrm{R}_{4.26}\end{array}$ & $\begin{array}{l}1.48 \\
1.40\end{array}$ & $\begin{array}{l}19.32 \\
19.84\end{array}$ & $\begin{array}{r}8.25 \\
\text { R8.21 }\end{array}$ \\
\hline 1992 & 1.45 & 3.83 & $\begin{array}{l}1.29 \\
7.09\end{array}$ & $\begin{array}{l}4.00 \\
4.52\end{array}$ & $\begin{array}{r}R_{6} \\
\end{array}$ & $\begin{array}{l}0.90 \\
8.96\end{array}$ & $\begin{array}{l}2.02 \\
2.28\end{array}$ & 5.51 & $\begin{array}{l}7.07 \\
\end{array}$ & $\begin{array}{l}.00 \\
.59\end{array}$ & $\begin{array}{l}1.05 \\
1.32\end{array}$ & 4.24 & $\begin{array}{l}1.40 \\
1.38\end{array}$ & $\begin{array}{r}19.04 \\
20.06\end{array}$ & 8.13 \\
\hline 1993 & 1.42 & 4.10 & 7.08 & 4.29 & $\mathrm{R}_{6} 6.27$ & 8.83 & 2.26 & 5.49 & $R_{7.01}$ & .56 & 1.28 & $R_{4.26}$ & 1.40 & 20.38 & 8.25 \\
\hline 1994 & 1.39 & 4.08 & 6.99 & 3.95 & 6.61 & 8.96 & 2.32 & 5.47 & 7.06 & .56 & 1.39 & 4.27 & 1.36 & 20.33 & 8.30 \\
\hline 1995 & $\begin{array}{l}1.37 \\
\text { S }\end{array}$ & 3.73 & 6.98 & 4.00 & 6.51 & 9.22 & 2.46 & 5.74 & 7.28 & .54 & 1.40 & 4.23 & 1.29 & 20.29 & 8.28 \\
\hline 1996 & 1.33 & 4.25 & 7.87 & $\begin{array}{l}4.00 \\
\end{array}$ & 7.98 & 9.85 & 2.80 & 6.20 & 8.01 & .51 & 1.25 & 4.63 & 1.35 & 20.16 & 8.75 \\
\hline 1997 & 1.32 & 4.53 & 7.66 & 4.53 & $\begin{array}{l}7.30 \\
\end{array}$ & 9.81 & 2.93 & 5.89 & 7.86 & .51 & 1.15 & 4.66 & 1.38 & 20.13 & 8.80 \\
\hline 1998 & $\begin{array}{l}1.02 \\
\end{array}$ & 4.13 & 6.57 & 3.35 & 5.95 & 8.45 & 2.15 & 5.02 & $\begin{array}{l}1.00 \\
6.63\end{array}$ & .50 & 1.27 & 4.08 & $\begin{array}{l}1.00 \\
1.32\end{array}$ & 19.80 & $\begin{array}{l}0.00 \\
8.20\end{array}$ \\
\hline 1999 & 1.27 & 4.16 & 7.19 & 4.01 & 6.60 & 9.31 & 2.51 & $\begin{array}{l}.02 \\
5.30\end{array}$ & $\begin{array}{l}.0 .00 \\
7.33\end{array}$ & .48 & 1.34 & $\begin{array}{l}4.00 \\
4.37\end{array}$ & $\begin{array}{l}1.02 \\
1.33\end{array}$ & 19.52 & $\begin{array}{l}0.20 \\
8.53\end{array}$ \\
\hline 2000 & 1.24 & $\begin{array}{r}4.61 \\
\text { R }\end{array}$ & 9.86 & 6.64 & 9.55 & $\begin{array}{r}11.89 \\
\end{array}$ & 4.32 & $\begin{array}{l}.300 \\
7.04\end{array}$ & 9.82 & $\begin{array}{l}40 \\
.46\end{array}$ & $\begin{array}{r}1.57 \\
R 1.57\end{array}$ & $\begin{array}{l}4.51 \\
5.70\end{array}$ & $\begin{array}{l}1.35 \\
1.71\end{array}$ & 20.03 & $\begin{array}{l}0.05 \\
1028\end{array}$ \\
\hline 2001 & $\begin{array}{l}1.24 \\
1.29\end{array}$ & 6.87 & $\begin{array}{l}5.00 \\
9.18\end{array}$ & $\begin{array}{l}0.04 \\
5.72\end{array}$ & $\begin{array}{r}.05 \\
\mathrm{Rg} .53\end{array}$ & 11.34 & $\begin{array}{l}4.0< \\
3.99\end{array}$ & $\begin{array}{l}1.04 \\
6.41\end{array}$ & $\begin{array}{l}9.82 \\
9.32\end{array}$ & $\begin{array}{l}46 \\
44\end{array}$ & 2.08 & $\begin{array}{l}5.10 \\
5.83\end{array}$ & 1.85 & $\begin{array}{l}20.03 \\
21.41\end{array}$ & 10.73 \\
\hline 2002 & $\begin{array}{l}1.29 \\
1.30\end{array}$ & $\begin{array}{l}6.81 \\
5.31\end{array}$ & $\begin{array}{l}.18 \\
8.64\end{array}$ & $\begin{array}{l}5.12 \\
5.33\end{array}$ & $\begin{array}{r}.53 \\
8.09\end{array}$ & $\begin{array}{l}11.30 \\
10.69\end{array}$ & $\begin{array}{l}3.99 \\
3.91\end{array}$ & $\begin{array}{l}6.41 \\
6.59\end{array}$ & $\begin{array}{l}9.32 \\
8.83\end{array}$ & $\begin{array}{l}.44 \\
.43\end{array}$ & $\begin{array}{l}2.08 \\
2.19\end{array}$ & $\begin{array}{l}5.83 \\
5.25\end{array}$ & $\begin{array}{l}1.85 \\
1.54\end{array}$ & $\begin{array}{l}21.41 \\
21.15\end{array}$ & 10.06 \\
\hline 2003 & $\begin{array}{l}1.00 \\
1.32\end{array}$ & 7.08 & $\begin{array}{r}0.04 \\
10.05\end{array}$ & 6.46 & $\begin{array}{l}0.09 \\
10.32\end{array}$ & 1234 & $\begin{array}{l}0.91 \\
4.75\end{array}$ & $\begin{array}{l}0.09 \\
762\end{array}$ & $\begin{array}{r}0.00 \\
1031\end{array}$ & $\begin{array}{l}40 \\
42\end{array}$ & $\begin{array}{l}2.19 \\
1.98\end{array}$ & 628 & $\begin{array}{l}1.04 \\
184\end{array}$ & 2185 & $\begin{array}{r}11.00 \\
11.42\end{array}$ \\
\hline 2004 & $\begin{array}{l}1.32 \\
1.41\end{array}$ & $\begin{array}{l}7.08 \\
7.91\end{array}$ & $\begin{array}{l}10.05 \\
12.23\end{array}$ & $\begin{array}{l}6.46 \\
8.93\end{array}$ & $\begin{array}{l}1.32 \\
12.24\end{array}$ & $\begin{array}{l}12.34 \\
14.67\end{array}$ & $\begin{array}{l}4.15 \\
4.92\end{array}$ & $\begin{array}{l}.62 \\
8.56\end{array}$ & $\begin{array}{l}10.31 \\
12.27\end{array}$ & $\begin{array}{l}.42 \\
.42\end{array}$ & $\begin{array}{l}7.98 \\
2.17\end{array}$ & $\begin{array}{l}6.28 \\
7.37\end{array}$ & $\begin{array}{l}1.84 \\
2.00\end{array}$ & $\begin{array}{l}21.85 \\
22.38\end{array}$ & $\begin{array}{l}11.42 \\
12.87\end{array}$ \\
\hline 2005 & $\begin{array}{l}1.41 \\
1.62\end{array}$ & 9.92 & 16.41 & $\begin{array}{r}.000 \\
12.86\end{array}$ & 14.58 & 17.89 & 6.65 & $\begin{array}{r}.0098 \\
\end{array}$ & 15.53 & .43 & 3.10 & 9.24 & 2.61 & 23.92 & 15.55 \\
\hline 2006 & $\begin{array}{l}1.02 \\
1.78\end{array}$ & 9.62 & $\begin{array}{l}1.455 \\
18.55\end{array}$ & $\begin{array}{l}1.00 \\
14.80\end{array}$ & $\begin{array}{l}1.00 \\
16.85\end{array}$ & 20.27 & $\begin{array}{l}.0 .05 \\
7.93\end{array}$ & $\begin{array}{l}1.90 \\
13.37\end{array}$ & $\begin{array}{l}1.00 \\
17.92\end{array}$ & $\begin{array}{l}.40 \\
.44\end{array}$ & $\begin{array}{r}.10 \\
R_{3} .13\end{array}$ & $\begin{array}{r}5.24 \\
10.21\end{array}$ & $\begin{array}{l}2.01 \\
2.48\end{array}$ & $\begin{array}{l}20.92 \\
26.15\end{array}$ & 17.36 \\
\hline 2007 & $\begin{array}{l}1.18 \\
1.88\end{array}$ & $\begin{array}{l}9.02 \\
9.31\end{array}$ & $\begin{array}{l}18.50 \\
19.87\end{array}$ & $\begin{array}{l}1.80 \\
16.01\end{array}$ & $\begin{array}{l}1.80 \\
18.76\end{array}$ & $\begin{array}{l}20.27 \\
22.01\end{array}$ & $\begin{array}{l}1.95 \\
8.57\end{array}$ & $\begin{array}{l}1.37 \\
14.94\end{array}$ & 1947 & $\begin{array}{r}.44 \\
46\end{array}$ & $\begin{array}{r}.13 \\
\text { R3.32 }\end{array}$ & $\mathrm{R} 10.74$ & $\begin{array}{l}2.48 \\
268\end{array}$ & $\begin{array}{l}20.15 \\
2684\end{array}$ & $\begin{array}{l}17.30 \\
1824\end{array}$ \\
\hline 2008 & $\begin{array}{l}1.08 \\
2.21\end{array}$ & $\begin{array}{r}.31 \\
10.83\end{array}$ & 26.33 & 22.56 & $\begin{array}{r}18.10 \\
23.35\end{array}$ & $\begin{array}{l}2.01 \\
25.53\end{array}$ & $\begin{array}{r}0.51 \\
12.64\end{array}$ & $\begin{array}{r}14.84 \\
18.83\end{array}$ & 24.18 & $\begin{array}{l}.40 \\
.47\end{array}$ & $\begin{array}{r}3.32 \\
\text { R3.69 }\end{array}$ & 12.93 & $\begin{array}{l}2.00 \\
3.21\end{array}$ & $\begin{array}{l}20.04 \\
28.64\end{array}$ & $\begin{array}{l}10.24 \\
21.37\end{array}$ \\
\hline 2009 & $\begin{array}{l}2.21 \\
2.33\end{array}$ & $\begin{array}{r}1.83 \\
\text { R7.67 }\end{array}$ & $\begin{array}{l}20.33 \\
16.98\end{array}$ & $\begin{array}{l}2.56 \\
12.61\end{array}$ & $\begin{array}{l}2.35 \\
16.38\end{array}$ & $\begin{array}{l}28.53 \\
18.51\end{array}$ & $\begin{array}{r}.64 \\
9.69\end{array}$ & $\begin{array}{r}18.30 \\
R 14.30\end{array}$ & $\begin{array}{r}24.18 \\
\text { R16.87 }\end{array}$ & .55 & $\begin{array}{r}\text { R3.69 } \\
\text { R }\end{array}$ & $\begin{array}{r}1.93 \\
9.37\end{array}$ & $\begin{array}{r}3.21 \\
R 2.45\end{array}$ & $\begin{array}{l}28.64 \\
28.90\end{array}$ & $\begin{array}{r}21.31 \\
\mathrm{R} 17.02\end{array}$ \\
\hline 2010 & $\begin{array}{l}2.33 \\
2.42\end{array}$ & 7.41 & $\begin{array}{l}16.98 \\
20.62\end{array}$ & $\begin{array}{l}12.61 \\
16.28\end{array}$ & $\begin{array}{l}10.38 \\
19.61\end{array}$ & $\begin{array}{l}18.51 \\
21.98\end{array}$ & $\begin{array}{r}9.69 \\
11.70\end{array}$ & $\begin{array}{l}17.90 \\
\end{array}$ & 20.32 & $\begin{array}{l}.55 \\
.62\end{array}$ & 3.45 & $\begin{array}{r}.37 \\
10.63\end{array}$ & $\begin{array}{r}2.45 \\
2.62\end{array}$ & $\begin{array}{l}28.90 \\
28.92\end{array}$ & 18.73 \\
\hline
\end{tabular}

1 Prices are not adjusted for inflation. See "Nominal Dollars" in Glossary.

Consumption-weighted average prices for all sectors, including the electric power sector.

Through 2004 , includes kerosene type and naphtha-type jet tue

jet fuel. Beginning in 2005, includes

5 Liquefied petroleum gases

Beginning in 1993, includes fuel ethanol blended into motor gasoline.

Consumption-weighted average price for asphalt and road oil, aviation gasoline, kerosene, lubricants, petrochemical feedstocks, petroleum coke, special naphthas, waxes, and miscellaneous petroleum

8 Wood and wood-derived fuels, and biomass waste; excludes fuel ethanol and biodiesel. Through

coal coke imports and exports, which are not separately displayed. In 2010, coal coke mports averaged 13.37 dollars per million Btu, and coal coke exports averaged 6.74 dollars per million Btu. includes electricity imports, which are not separately displayed. For 1981-1992, also includes fue

11.

Industry Classification System) 22 category whose primary business is to sell electricity, or electricity and heat, to the public. Through 1988, data are for electric utilities only; beginning in 1989, data are for electric utilities and independent power producers.

Consumption-weighted average electric power sector price for coal, natural gas, petroleum, nuclear ${ }_{13}$ Retail electricity prices paid by ultimate customers, reported by electric utilities and, beginning in 1996 other energy service providers.

Consumption-weighted average price for primary energy and retail electricity in the four end-use sectors (residential, commercial, industrial, and transportation); excludes energy in the electric power $\mathrm{R}=$ Revised

Notes: - Prices include taxes where data are available. - There are no direct fuel costs for hydroelectric, geothermal, wind, or solar energy.

Web Page: For related information, see http://www.eia.gov/state/seds/seds-data-complete.cfm. Source: U.S. Energy Information Administration, "State Energy Data 2010: Prices and Expenditures"
(June 2012) U. Table ET1. 
Figure 3.4 Consumer Price Estimates for Energy by End-Use Sector, 2010

\section{By Sector}

25-

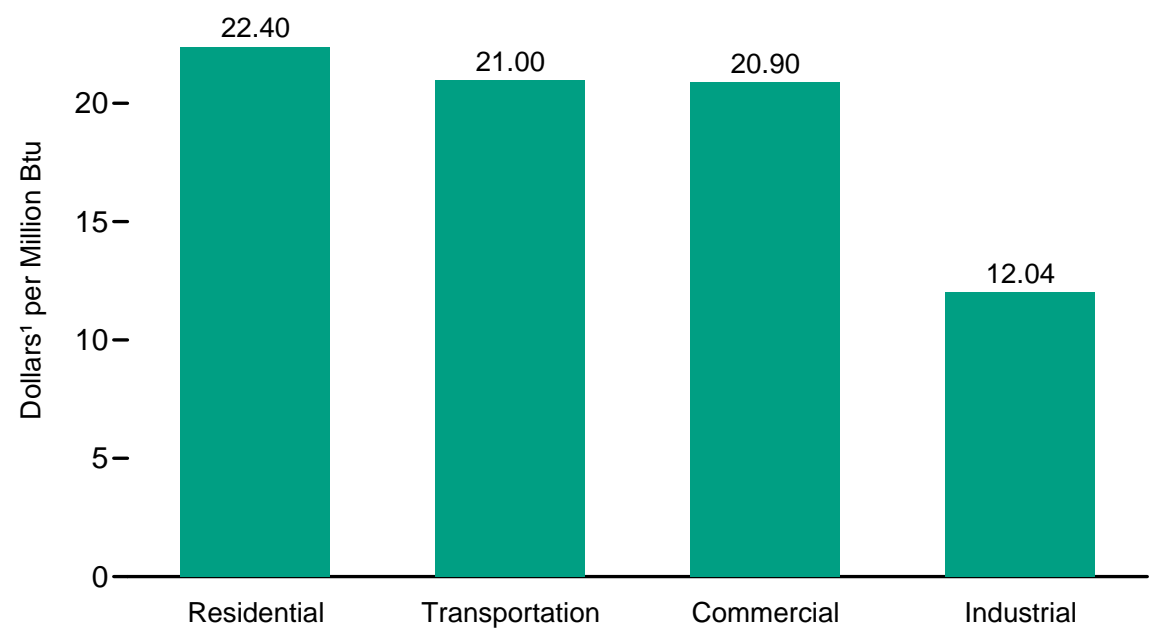

Commercial Sector by Major Source

$$
35-
$$

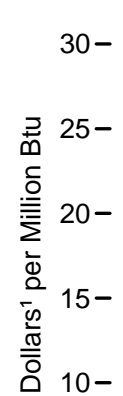

$30-$
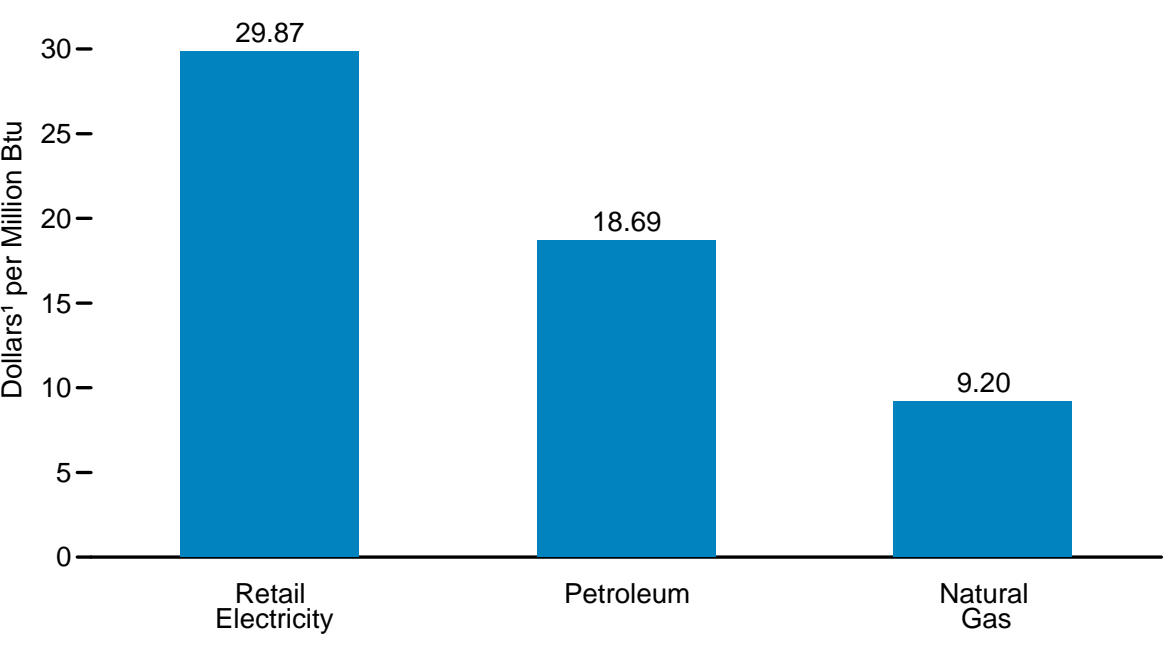

${ }^{1}$ Prices are not adjusted for inflation. See "Nominal Dollars" in Glossary.

${ }^{2}$ Wood and wood-derived fuels, and biomass waste; excludes fuel ethanol and biodiesel.

\section{Residential Sector by Major Source}

40-

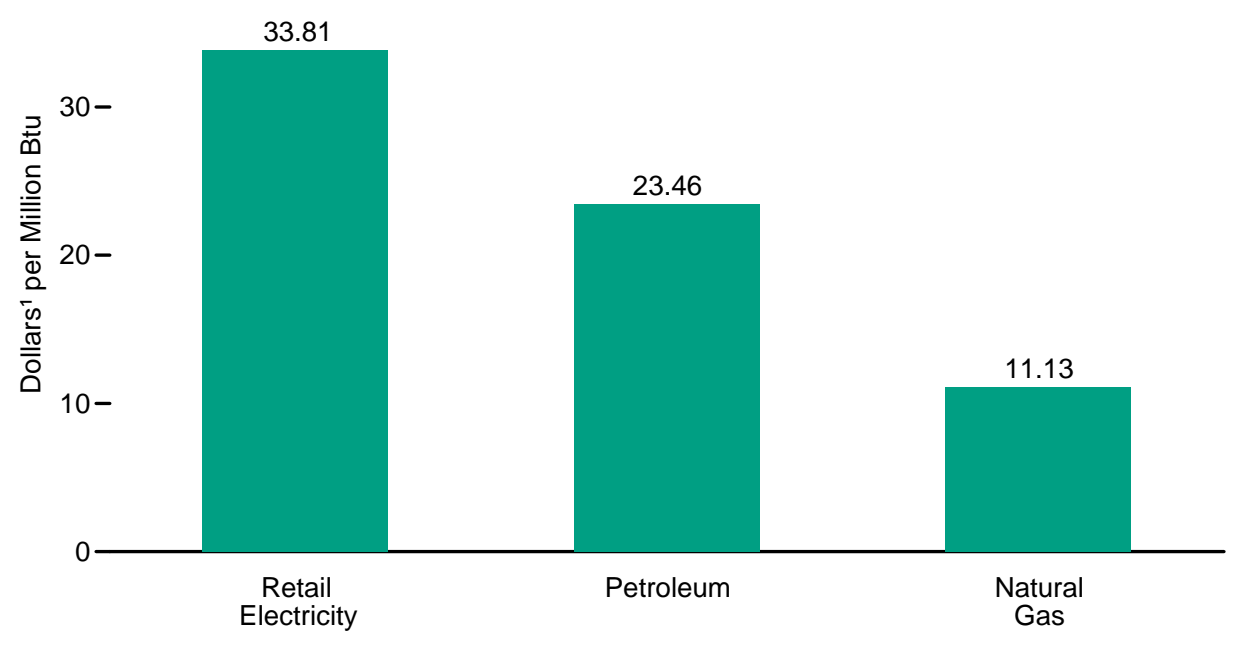

Industrial Sector by Major Source

25-

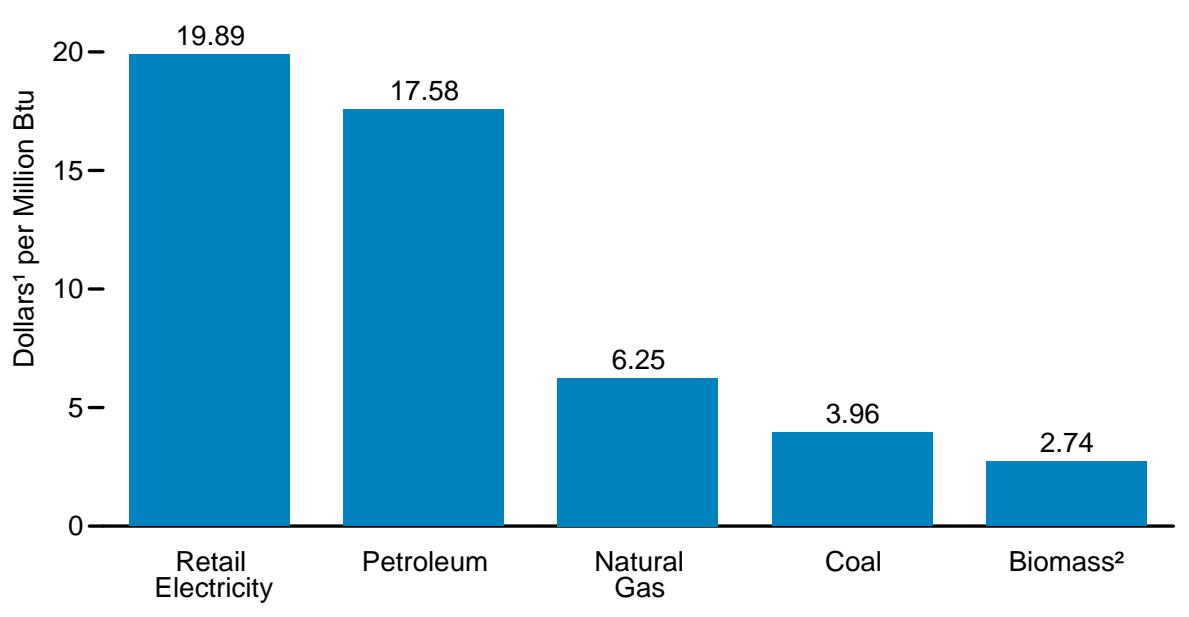

Notes: - Consumer prices are intended to represent prices paid by consumers. As such they include taxes where data are available. - There are no direct fuel costs for hydroelectric, geothermal, wind, or solar energy.

Source: Table 3.4. 
Table 3.4 Consumer Price Estimates for Energy by End-Use Sector, 1970-2010

(Dollars ${ }^{1}$ per Million Btu)

\begin{tabular}{|c|c|c|c|c|c|c|c|c|c|c|c|c|c|c|c|c|}
\hline \multirow[b]{2}{*}{ Year } & \multicolumn{4}{|c|}{ Residential } & \multicolumn{4}{|c|}{ Commercial } & \multicolumn{6}{|c|}{ Industrial } & \multicolumn{2}{|c|}{ Transportation } \\
\hline & $\begin{array}{c}\text { Natural } \\
\text { Gas }{ }^{2}\end{array}$ & Petroleum & $\begin{array}{c}\text { Retail } \\
\text { Electricity } 3\end{array}$ & Total 4 & $\begin{array}{c}\text { Natural } \\
\text { Gas }{ }^{2}\end{array}$ & Petroleum 5 & $\begin{array}{c}\text { Retail } \\
\text { Electricity } 3\end{array}$ & Total 6,7 & Coal & $\begin{array}{c}\text { Natural } \\
\text { Gas }{ }^{2}\end{array}$ & Petroleum 5 & Biomass 8 & $\begin{array}{c}\text { Retail } \\
\text { Electricity } 3\end{array}$ & Total 7,9 & Petroleum 5 & Total 7,10 \\
\hline 1970 & 1.06 & 1.54 & 6.51 & 2.10 & 0.75 & $\mathrm{R}_{0} 0.90$ & 6.09 & 1.97 & 0.45 & 0.38 & 0.98 & 1.59 & 2.99 & 0.84 & 2.31 & 2.31 \\
\hline 1971 & 1.12 & 1.59 & 6.80 & 2.24 & .80 & 1.02 & 6.44 & 2.15 & .50 & .41 & 1.05 & 1.59 & 3.22 & R. 93 & 2.37 & 2.37 \\
\hline 1972 & 1.18 & $\mathrm{R}_{1.61}$ & 7.09 & 2.37 & .86 & 1.05 & 6.71 & 2.32 & .55 & .46 & 1.05 & 1.59 & 3.40 & .99 & 2.38 & 2.38 \\
\hline 1973 & 1.26 & $\mathrm{R}_{2.09}$ & 7.44 & 2.71 & .91 & $R_{1.20}$ & 7.06 & 2.55 & .63 & .50 & 1.18 & 1.60 & 3.66 & 1.10 & 2.57 & 2.57 \\
\hline 1974 & 1.42 & 2.85 & 9.09 & 3.38 & 1.05 & $\mathrm{R}_{2.25}$ & 8.91 & $R_{3.40}$ & 1.22 & .67 & $\mathrm{R}_{2.25}$ & 1.60 & 4.95 & 1.78 & 3.70 & 3.70 \\
\hline 1975 & 1.67 & $R_{3.02}$ & 10.29 & 3.80 & 1.32 & $\mathrm{R}_{2.39}$ & 10.11 & $\mathrm{R}_{4.06}$ & 1.50 & .95 & $\mathrm{R}_{2.47}$ & 1.60 & 6.07 & $R_{2.21}$ & 4.02 & 4.02 \\
\hline 1976 & 1.94 & $R_{3.24}$ & 10.93 & $\mathrm{R}_{4.13}$ & 1.61 & $R_{2.49}$ & 10.82 & 4.38 & 1.50 & 1.21 & $\mathrm{R}_{2.58}$ & 1.60 & 6.48 & 2.43 & 4.20 & 4.21 \\
\hline 1977 & 2.30 & 3.64 & 11.87 & 4.76 & 2.00 & $\mathrm{R}_{2.84}$ & 11.99 & 5.12 & 1.56 & 1.48 & $\mathrm{R}_{2.85}$ & 1.59 & 7.33 & 2.78 & 4.47 & $\mathrm{R}_{4.47}$ \\
\hline 1978 & 2.52 & R3.77 & 12.63 & 5.13 & 2.20 & $R_{2.92}$ & 12.78 & R5.49 & 1.73 & 1.66 & $\mathrm{R}_{2.97}$ & 1.60 & 8.18 & $R_{3.04}$ & 4.59 & 4.59 \\
\hline 1979 & 2.92 & R5.32 & 13.60 & 5.99 & 2.69 & $\mathrm{R}_{4.14}$ & 13.72 & $\mathrm{R}_{6.27}$ & 1.75 & 1.96 & $\mathrm{R}_{4.00}$ & 1.60 & 8.94 & 3.63 & 6.19 & 6.19 \\
\hline 1980 & 3.60 & 7.24 & 15.71 & 7.46 & 3.32 & R5.62 & 16.06 & 7.83 & 1.87 & 2.52 & R5.76 & 1.67 & 10.81 & 4.71 & 8.60 & 8.61 \\
\hline 1981 & 4.19 & R8.65 & 18.17 & 8.82 & 3.91 & $R_{6.95}$ & 18.44 & R9.47 & 2.06 & 3.07 & R6.87 & 1.67 & 12.57 & 5.52 & 9.83 & 9.84 \\
\hline 1982 & 5.05 & ${ }^{R} 8.66$ & 20.11 & R9.77 & 4.70 & ${ }^{R} 6.62$ & 20.11 & $R_{10.34}$ & 2.09 & 3.80 & ${ }^{R} 6.53$ & 1.67 & 14.51 & $\mathrm{R}_{6} 6.06$ & 9.42 & 9.43 \\
\hline 1983 & 5.88 & R8.39 & 21.04 & 10.66 & 5.43 & $\mathrm{R}_{6.49}$ & 20.57 & $R_{10.91}$ & 1.91 & 4.10 & $\mathrm{R}_{6} 6.60$ & 1.67 & 14.54 & $\mathrm{R}_{6.22}$ & R8.43 & R8.44 \\
\hline 1984 & 5.95 & R8.44 & 20.96 & 10.66 & 5.40 & $R_{6.48}$ & 20.89 & $R_{11.12}$ & 1.91 & 4.13 & R6.58 & 1.67 & 14.16 & 6.12 & 8.25 & 8.26 \\
\hline 1985 & 5.94 & R8.14 & 21.66 & 10.91 & 5.34 & ${ }^{R} 6.46$ & 21.30 & $R_{11.63}$ & 1.90 & 3.87 & $\mathrm{R}^{\mathrm{R}} 6.32$ & 1.67 & 14.57 & $\mathrm{R}_{6} 6.04$ & 8.26 & 8.27 \\
\hline 1986 & 5.67 & R6.77 & 21.75 & 10.75 & 4.94 & $\mathrm{R}_{4.44}$ & 21.10 & $R_{11.20}$ & 1.80 & 3.20 & $\mathrm{R}_{4.93}$ & 1.65 & 14.45 & R5.37 & 6.21 & 6.22 \\
\hline 1987 & 5.39 & R6.59 & 21.82 & 10.71 & 4.64 & $\mathrm{R}_{4} .75$ & 20.44 & $R_{10.96}$ & 1.67 & 2.88 & $\mathrm{R}_{4} .98$ & 1.65 & 13.98 & 5.17 & 6.57 & 6.59 \\
\hline 1988 & 5.32 & R6.57 & 21.92 & 10.66 & 4.51 & $\mathrm{R}_{4.50}$ & 20.34 & $\mathrm{R}_{10.81}$ & 1.68 & 2.90 & $\mathrm{R}_{4.63}$ & 1.65 & 13.78 & R5.01 & 6.56 & 6.57 \\
\hline 1989 & 5.47 & $\mathrm{R}_{7} 7.54$ & 22.41 & 11.02 & 4.61 & $\mathrm{R}_{5.09}$ & 20.77 & $R_{11.25}$ & 1.68 & 2.93 & $\mathrm{R}_{4.70}$ & 1.20 & 13.85 & 4.92 & 7.17 & 7.18 \\
\hline 1990 & 5.63 & R8.69 & 22.96 & 11.87 & 4.70 & $\mathrm{R}_{6.04}$ & 21.20 & $R_{11.87}$ & 1.69 & 2.95 & R5.50 & .99 & 13.92 & R5.24 & 8.27 & 8.28 \\
\hline 1991 & 5.66 & R 8.48 & 23.57 & 12.08 & 4.69 & R5.59 & 21.73 & $R_{12.05}$ & 1.67 & 2.80 & R5.33 & 1.14 & 14.18 & $\mathrm{R}_{5.19}$ & 7.98 & 7.99 \\
\hline 1992 & 5.73 & R7.80 & 24.06 & $R_{11.97}$ & 4.75 & $\mathrm{R}_{5} .37$ & 22.15 & $R_{12.15}$ & 1.69 & 2.91 & $\mathrm{R}_{5} \mathrm{n}_{0} 02$ & 1.13 & 14.18 & R5.14 & 7.91 & R7.92 \\
\hline 1993 & 5.99 & R7.64 & 24.40 & $\mathrm{R}_{12.27}$ & 5.08 & $\mathrm{R}_{5.14}$ & 22.40 & $R_{12.55}$ & 1.63 & 3.12 & $\mathrm{R}_{4.95}$ & 1.12 & 14.22 & R5.17 & 7.87 & 7.88 \\
\hline 1994 & 6.23 & R7.57 & 24.57 & $\mathrm{R}_{12.60}$ & 5.35 & R5.05 & 22.35 & $\mathrm{R}_{12.72}$ & 1.62 & 3.09 & R5.06 & 1.15 & 14.00 & R5.16 & 7.91 & 7.92 \\
\hline 1995 & 5.89 & R7.49 & 24.63 & $R_{12.60}$ & 4.94 & $\mathrm{R}_{5.14}$ & 22.29 & $R_{12.62}$ & 1.63 & 2.80 & $\mathrm{R}_{5.23}$ & 1.21 & 13.68 & $\mathrm{R}_{4} 4.98$ & 8.08 & 8.09 \\
\hline 1996 & 6.16 & R8.63 & 24.50 & $\mathrm{R}_{12.69}$ & 5.26 & $\mathrm{R}_{6.17}$ & 22.17 & $\mathrm{R}_{12.76}$ & 1.62 & 3.30 & $\mathrm{R}_{6} 6.08$ & 1.01 & 13.49 & R5.41 & 8.76 & 8.77 \\
\hline 1997 & 6.75 & R8.61 & 24.71 & $\mathrm{R}_{13.25}$ & 5.67 & $\mathrm{R}_{6.10}$ & 22.03 & $R_{13} .03$ & 1.62 & 3.53 & R5.71 & 1.01 & 13.29 & R5.35 & 8.69 & 8.70 \\
\hline 1998 & 6.61 & R7.55 & 24.21 & $\mathrm{R}_{13.44}$ & 5.38 & $R_{5.09}$ & 21.48 & $R_{13.05}$ & 1.58 & 3.16 & $\mathrm{R}_{4.55}$ & 1.24 & 13.13 & 4.91 & 7.47 & 7.48 \\
\hline 1999 & 6.50 & R7.78 & 23.93 & $R_{13.15}$ & 5.22 & $\mathrm{R}_{5.56}$ & 21.01 & $R_{12.84}$ & 1.58 & 3.21 & R5.10 & 1.38 & 12.98 & R5.13 & 8.23 & 8.23 \\
\hline 2000 & R7.63 & $\mathrm{R}_{11.12}$ & 24.14 & $\mathrm{R}_{14.21}$ & $\mathrm{R}_{6.54}$ & R8.27 & 21.52 & $R_{13.90}$ & 1.55 & $\mathrm{R}_{4} .60$ & $\mathrm{R}_{7.35}$ & 1.43 & 13.60 & R6.43 & 10.71 & 10.72 \\
\hline 2001 & 9.42 & $R_{11.23}$ & 25.16 & $\mathrm{R}_{15.62}$ & 8.32 & R7.88 & 22.99 & $R_{15.54}$ & 1.63 & 5.71 & $\mathrm{R}_{6.81}$ & 1.95 & 14.78 & $\mathrm{R}_{6.90}$ & 10.20 & 10.21 \\
\hline 2002 & 7.69 & R9.99 & 24.75 & $R_{14.65}$ & 6.49 & ${ }^{R} 7.14$ & 22.81 & $R_{14.66}$ & 1.75 & $\mathrm{R}_{4.48}$ & $\mathrm{R}_{6.49}$ & 2.11 & 14.30 & $\mathrm{R}_{6} 6.31$ & 9.64 & 9.65 \\
\hline 2003 & 9.24 & $R_{11.85}$ & 25.56 & $\mathrm{R}_{15.81}$ & 8.07 & R8.62 & 23.54 & $R_{15.63}$ & 1.74 & 6.20 & $\mathrm{R}_{7.86}$ & 1.62 & 14.97 & R7.49 & 11.20 & 11.21 \\
\hline 2004 & 10.47 & $\mathrm{R}_{13.33}$ & 26.22 & $R_{17.01}$ & 9.19 & $\mathrm{R}_{10.16}$ & 23.95 & $R_{16.55}$ & 1.99 & 7.02 & R9.41 & 1.79 & 15.38 & R8.47 & 13.43 & 13.43 \\
\hline 2005 & 12.34 & $\mathrm{R}_{16.76}$ & 27.68 & $R_{19.16}$ & 10.98 & $\mathrm{R}_{13.55}$ & 25.40 & $R_{18.59}$ & 2.56 & 9.08 & $R_{11.99}$ & 2.73 & 16.77 & $\mathrm{R}_{10.41}$ & $R_{16.88}$ & 16.89 \\
\hline 2006 & 13.35 & $\mathrm{R}_{19.22}$ & 30.49 & $\mathrm{R}_{21.49}$ & 11.60 & $\mathrm{R}_{15.91}$ & 27.72 & $\mathrm{R}_{20.63}$ & 2.83 & R8.77 & $R_{14.32}$ & $\mathrm{R}_{2.65}$ & 18.02 & $\mathrm{R}_{11.39}$ & $\mathrm{R}_{19.12}$ & $\mathrm{R}_{19.12}$ \\
\hline 2007 & $R_{12.70}$ & $\mathrm{R}_{21.11}$ & 31.22 & $\mathrm{R}_{21.56}$ & $\mathrm{R}_{10.99}$ & $\mathrm{R}_{17.52}$ & 28.27 & $\mathrm{R}_{20.73}$ & 2.92 & R8.29 & $\mathrm{R}_{15.90}$ & $\mathrm{R}_{2.52}$ & 18.71 & $\mathrm{R}_{11.93}$ & 20.61 & 20.61 \\
\hline 2008 & 13.52 & $\mathrm{R}_{25.80}$ & 33.01 & $\mathrm{R}_{23.06}$ & 11.89 & $\mathrm{R}_{22.96}$ & 30.38 & $\mathrm{R}_{22.44}$ & 3.51 & 10.06 & $\mathrm{R}_{20.49}$ & $\mathrm{R}_{2.83}$ & 19.96 & $\mathrm{R}_{14.32}$ & 25.24 & 25.23 \\
\hline 2009 & 11.81 & $\mathrm{R}_{20.76}$ & 33.72 & $\mathrm{R}_{22.05}$ & $\mathrm{R}_{9.70}$ & $\mathrm{R}_{15.45}$ & 29.81 & $R_{20.67}$ & 3.87 & ${ }^{R} 6.46$ & $R_{13.87}$ & $\mathrm{R}_{2.62}$ & 20.00 & $R_{11.05}$ & 17.54 & 17.54 \\
\hline 2010 & 11.13 & 23.46 & 33.81 & 22.40 & 9.20 & 18.69 & 29.87 & 20.90 & 3.96 & 6.25 & 17.58 & 2.74 & 19.89 & 12.04 & 21.01 & 21.00 \\
\hline
\end{tabular}

1 Prices are not adjusted for inflation. See "Nominal Dollars" in Glossary.

Natural gas, plus a small amount of supplemental gaseous fuels.

${ }_{3}$ Retail electricity prices paid by ultimate customers, reported by electric utilities and, beginning in 1996, other energy service providers.

Includes coal, and wood and wood-derived fuels, which are not separately displayed.

Beginning in 1993, includes fuel ethanol blended into motor gasoline.

${ }^{6}$ Includes coal, wood and wood-derived fuels, and biomass waste, which are not separately displayed. Through 2000, also includes non-biomass waste.

dinto motor gasoline that is not included in the petroleum data for those years.

8 Wood and wood-derived fuels, and biomass waste; excludes fuel ethanol and biodiesel. Through 2000, also includes non-biomass waste.

Includes coal coke imports and exports, which are not separately displayed.

10 Includes coal, natural gas, and retail electricity, which are not separately displayed. R=Revised.

Notes: - Prices include taxes where data are available. - There are no direct fuel costs for hydroelectric, geothermal, wind, or solar energy.

列

Administration, "State Energy Data 2010: Prices and Expenditures" (June 2012), U.S. Tables ET3-ET6. 


\section{Figure 3.5 Consumer Expenditure Estimates for Energy by Source}

\section{Total Energy, 1970-2010}

$1,500-$

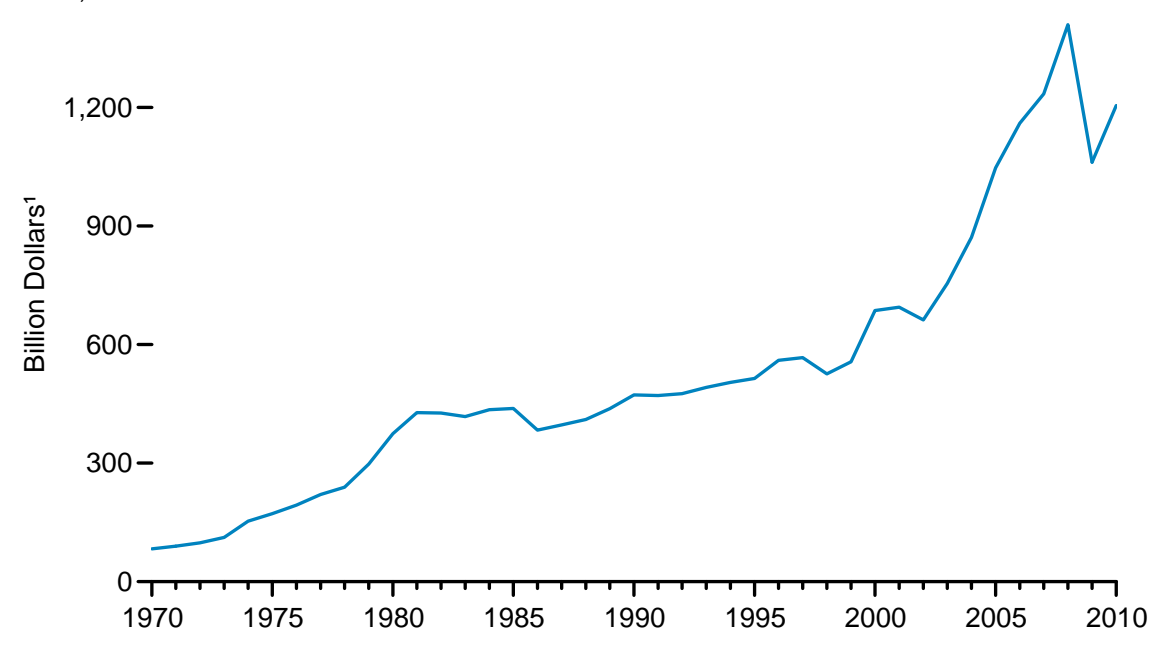

\section{Expenditures ${ }^{3}$ by Energy Type, Indexed, 1970-2010}

$25-$

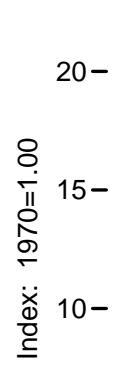

$5-$

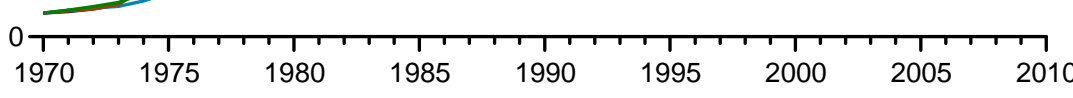

\section{By Energy Type, 2010}

$800-$

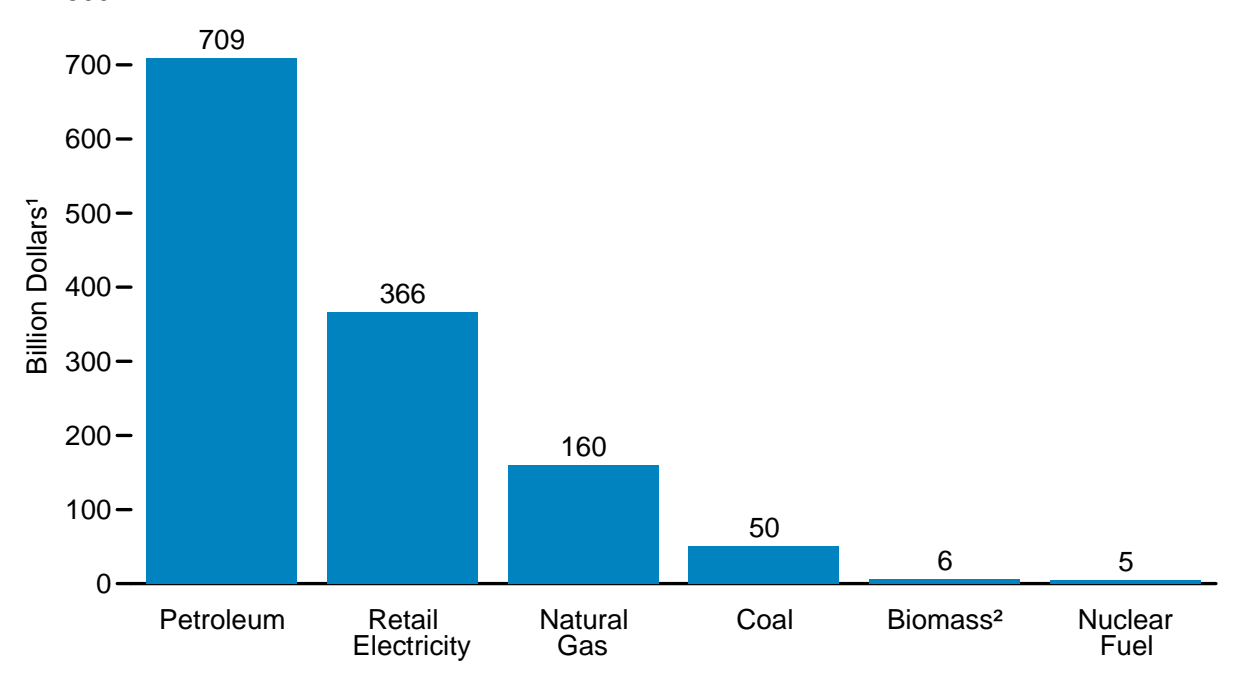

By Petroleum Product, 2010

$400-$

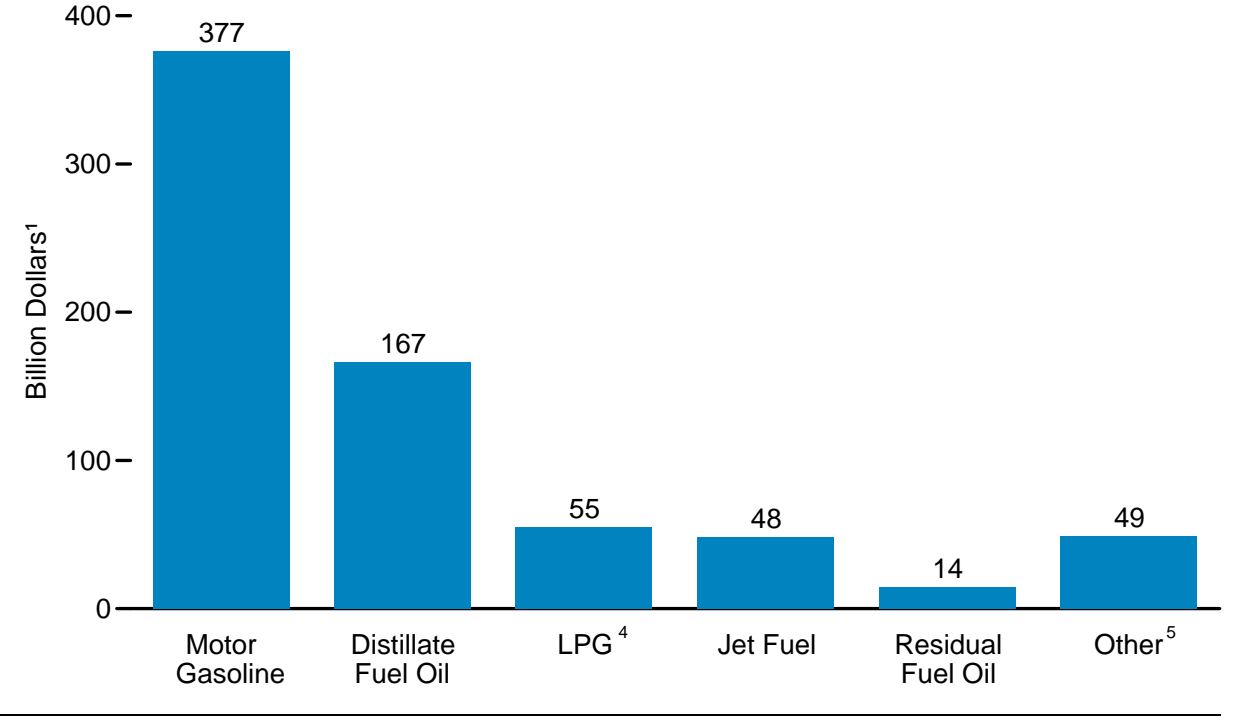

${ }^{5}$ Asphalt and road oil, aviation gasoline, kerosene, lubricants, petrochemical feedstocks, petroleum coke, special naphthas, waxes, and miscellaneous petroleum products. Source: Table 3.5.
${ }^{1}$ Prices are not adjusted for inflation. See "Nominal Dollars" in Glossary.

${ }^{2}$ Wood and wood-derived fuels, and biomass waste; excludes fuel ethanol and biodiesel.

${ }^{3}$ Based on nominal dollars.

${ }^{4}$ Liquefied petroleum gases. 
Table 3.5 Consumer Expenditure Estimates for Energy by Source, 1970-2010

(Million Dollars ${ }^{1}$ )

\begin{tabular}{|c|c|c|c|c|c|c|c|c|c|c|c|c|c|c|c|c|}
\hline \multirow[b]{3}{*}{ Year } & \multicolumn{13}{|c|}{ Primary Energy ${ }^{2}$} & \multirow[b]{3}{*}{$\begin{array}{c}\text { Electric } \\
\text { Power } \\
\text { Sector } 11,12\end{array}$} & \multirow[b]{3}{*}{\begin{tabular}{|c} 
Retail \\
Electricity 13
\end{tabular}} & \multirow[b]{3}{*}{$\begin{array}{c}\text { Total } \\
\text { Energy } 10,14\end{array}$} \\
\hline & \multirow[b]{2}{*}{ Coal } & \multirow[b]{2}{*}{$\begin{array}{c}\text { Coal Coke } \\
\text { Net } \\
\text { Imports }{ }^{3}\end{array}$} & \multirow[b]{2}{*}{$\begin{array}{l}\text { Natural } \\
\text { Gas }{ }^{4}\end{array}$} & \multicolumn{7}{|c|}{ Petroleum } & \multirow[b]{2}{*}{$\begin{array}{l}\text { Nuclear } \\
\text { Fuel }\end{array}$} & \multirow[b]{2}{*}{ Biomass ${ }^{9}$} & \multirow[b]{2}{*}{ Total ${ }^{10}$} & & & \\
\hline & & & & $\begin{array}{l}\text { Distillate } \\
\text { Fuel Oil }\end{array}$ & $\begin{array}{l}\text { Jet } \\
\text { Fuel } 5\end{array}$ & LPG 6 & $\begin{array}{c}\text { Motor } \\
\text { Gasoline }\end{array}$ & $\begin{array}{l}\text { Residual } \\
\text { Fuel Oil }\end{array}$ & Other ${ }^{8}$ & Total & & & & & & \\
\hline 1970 & 4,630 & -75 & 10,891 & 6,253 & 1,441 & 2,395 & 31,596 & 2,046 & 4,172 & 47,904 & 44 & 438 & 63,872 & $-4,357$ & 23,345 & 82,860 \\
\hline 1971 & 4,902 & -40 & 12,065 & 6,890 & 1,582 & 2,483 & 33,478 & 2,933 & 4,449 & 51,816 & 73 & 446 & 69,312 & $-5,491$ & 26,202 & 90,023 \\
\hline 1972 & 5,415 & -26 & 13,198 & 7,552 & 1,682 & 2,834 & 35,346 & 3,458 & 4,777 & 55,648 & 104 & 476 & 74,893 & $-6,551$ & 29,712 & 98,054 \\
\hline 1973 & 6,243 & 7 & 13,933 & 9,524 & 2,001 & $\mathrm{R}_{3,881}$ & 39,667 & 4,667 & 5,318 & R 65,057 & 177 & 502 & $R_{86,053}$ & $-7,952$ & 33,774 & $\mathrm{R}_{111,875}$ \\
\hline 1974 & 11,118 & 150 & 16,380 & 15,217 & 3,208 & $\mathrm{R}_{5,254}$ & 54,194 & 10,547 & 8,284 & $\begin{array}{l}\mathrm{R} 96,704 \\
\mathrm{R}\end{array}$ & 259 & 544 & $\mathrm{R}_{125,322}$ & $-14,558$ & 42,586 & $\mathrm{R}_{153,351}$ \\
\hline 1975 & 13,021 & 82 & 20,061 & 15,680 & 4,193 & $\mathrm{R}_{5,221}$ & 59,446 & 10,374 & 8,493 & $R_{103,407}$ & 448 & 534 & $R_{137,702}$ & $-16,545$ & 50,680 & R171,837 \\
\hline 1976 & 14,051 & 44 & 25,097 & 18,402 & 4,567 & $R_{5,992}$ & 64,977 & 11,648 & 9,925 & $\mathrm{R}_{115,512}$ & 520 & 622 & $R_{156,002}$ & $-19,078$ & 56,972 & $R_{193,896}$ \\
\hline 1977 & 15,416 & 67 & 29,602 & 22,004 & 5,517 & $R_{6,839}$ & 70,591 & 14,381 & 11,790 & $R_{131,121}$ & 743 & 694 & R177,966 & $-23,715$ & 66,225 & R220,476 \\
\hline 1978 & 17,551 & 362 & 33,185 & 23,587 & 6,205 & $\mathrm{R}_{6}, 646$ & 74,513 & 13,747 & 13,348 & $\mathrm{R}_{138,047}$ & 915 & 782 & $\mathrm{R}_{191,221}$ & $-26,125$ & 74,159 & $\mathrm{R} 239,255$ \\
\hline 1979 & 20,376 & 259 & 40,785 & 32,854 & 8,603 & R9,389 & 95,916 & 17,656 & 18,785 & $\mathrm{R}_{183,204}$ & 941 & 964 & R246,969 & $-31,472$ & 82,051 & R297,549 \\
\hline 1980 & 22,607 & -78 & 51,061 & 40,797 & 13,923 & $R_{10,926}$ & 124,408 & 21,573 & 26,049 & $\mathrm{R}_{237,676}$ & 1,189 & 1,232 & $\mathrm{R}_{314,279}$ & $-38,027$ & 98,095 & R374,347 \\
\hline 1981 & 26,159 & -31 & 60,544 & 48,200 & 15,607 & $R_{11}, 922$ & 138,138 & 22,668 & 28,571 & R265,106 & 1,436 & 1,429 & $\mathrm{R}_{355,717}$ & $-44,274$ & 116,455 & R 427,898 \\
\hline 1982 & 26,349 & -52 & 68,292 & 44,087 & 14,974 & $R_{12,972}$ & 130,305 & 17,632 & 22,447 & $\mathrm{R}_{242,418}$ & 1,684 & 1,510 & $R_{341,439}$ & $-42,354$ & 127,393 & $\mathrm{R}_{426,479}$ \\
\hline 1983 & 26,987 & -44 & 72,000 & 41,846 & 13,979 & $R_{14,147}$ & 115,803 & 14,099 & 21,573 & $\mathrm{R}_{221,446}$ & 1,859 & 1,517 & $\mathrm{R}_{325,311}$ & $-42,566$ & 134,731 & $\mathrm{R}_{417,476}$ \\
\hline 1984 & 29,025 & -22 & 77,169 & 44,668 & 15,097 & R14,197 & 114,429 & 14,410 & 22,812 & $\mathrm{R}_{225,612}$ & 2,384 & 1,608 & R337,460 & $-44,686$ & 142,420 & R435,195 \\
\hline 1985 & 29,678 & -34 & 72,938 & 43,972 & 14,747 & $\begin{array}{r}\mathrm{R} 13,752 \\
\mathrm{R}\end{array}$ & 118,048 & 11,493 & 22,088 & $\mathrm{R}_{224,100}$ & 2,878 & 1,597 & R 333,084 & $-43,970$ & 149,233 & R438,347 \\
\hline 1986 & $\begin{array}{l}29,018 \\
27,855\end{array}$ & $\begin{array}{l}-54 \\
-40\end{array}$ & $\begin{array}{l}12,930 \\
59,702\end{array}$ & $\begin{array}{l}45,113 \\
35,113\end{array}$ & 10,505 & $\mathrm{R}_{12,843}$ & $\begin{array}{r}11,048 \\
91,529\end{array}$ & 7,486 & $\begin{array}{l}22,080 \\
17,647\end{array}$ & $\begin{array}{l}\mathrm{R}_{175,122} \\
\text { R }\end{array}$ & $\begin{array}{l}2,078 \\
3,061\end{array}$ & 1,352 & $\begin{array}{r}\quad \\
R_{2}\end{array}$ & $\begin{array}{l}-43,91 \\
-37,0\end{array}$ & $\begin{array}{l}14,<33 \\
151,793\end{array}$ & $\begin{array}{r}\text { R } 383,518 \\
\end{array}$ \\
\hline 1987 & $\begin{array}{l}27,855 \\
27,532\end{array}$ & $\begin{array}{r}-40 \\
7\end{array}$ & $\begin{array}{l}59,02 \\
58,019\end{array}$ & $\begin{array}{l}35,115 \\
37,729\end{array}$ & $\begin{array}{l}10,405 \\
11,448\end{array}$ & $\begin{array}{l}12,843 \\
R_{12}, 969\end{array}$ & $\begin{array}{l}91,529 \\
99,864\end{array}$ & $\begin{array}{l}8,486 \\
8,062\end{array}$ & $\begin{array}{l}17,647 \\
17,687\end{array}$ & $\begin{array}{l}\mathrm{R}_{187,758} \\
\text { R }\end{array}$ & $\begin{array}{l}3,061 \\
3,378\end{array}$ & $\begin{array}{l}1,352 \\
1,300\end{array}$ & $\begin{array}{l}280,081 \\
R_{2}\end{array}$ & $\begin{array}{l}-3,0,016 \\
-38,182\end{array}$ & $\begin{array}{l}15, / 93 \\
154,685\end{array}$ & $\begin{array}{r}\text { R396,587 } \\
\text { R }\end{array}$ \\
\hline 1988 & 28,333 & 116 & 61,089 & 38,776 & 11,318 & $R_{12,906}$ & 103,323 & 7,259 & 16,779 & $R_{1} 90,360$ & 4,057 & 1,378 & R287,005 & $-38,553$ & 162,063 & R410,515 \\
\hline 1989 & 28,284 & 137 & 66,198 & 43,159 & 13,434 & $R_{12,266}$ & 112,720 & 8,357 & 17,060 & $R_{206,996}$ & 3,939 & 2,270 & $\mathrm{R}_{309,176}$ & $-40,829$ & 169,332 & R 437,679 \\
\hline 1990 & 28,602 & 22 & 65,278 & 49,335 & 17,784 & $\mathrm{R}_{13,840}$ & 126,558 & 8,721 & 19,255 & $R_{235,493}$ & 4,104 & 1,997 & $\mathrm{R}_{336,588}$ & $-40,626$ & 176,691 & R472,653 \\
\hline 1991 & 28,129 & 44 & 65,956 & 45,269 & 14,609 & $\mathrm{R}_{15,116}$ & 123,118 & 6,784 & 18,213 & $\mathrm{R}_{223,110}$ & 4,073 & $\begin{array}{l}1,991 \\
2,165\end{array}$ & $\mathrm{R}_{324,653}$ & $-38,752$ & $\begin{array}{l}184,767 \\
184\end{array}$ & R470,668 \\
\hline 1992 & 27,776 & 126 & 70,086 & 45,019 & 13,559 & $\mathrm{R}_{14,303}$ & 125,249 & 5,585 & 18,345 & $\mathrm{R}_{222,060}$ & 3,802 & 2,194 & $\mathrm{R}_{327,401}$ & $-38,663$ & 186,906 & $\mathrm{R}_{475,644}$ \\
\hline 1993 & 28,229 & 96 & 77,052 & 45,732 & 13,002 & $\mathrm{R}_{14,114}$ & 126,560 & 5,449 & 18,285 & $\mathrm{R}_{223,142}$ & 3,597 & 2,193 & $\mathrm{R}_{335,016}$ & $-40,317$ & 196,532 & $\mathrm{R}_{491,231}$ \\
\hline 1994 & 27,715 & 214 & 78,581 & 47,002 & 12,474 & 16,243 & 130,068 & 5,296 & 18,687 & 229,770 & 3,777 & 2,521 & 343,593 & $-40,352$ & 200,831 & 504,073 \\
\hline 1995 & 27,431 & 234 & 75,020 & 47,533 & 12,526 & 16,197 & 136,647 & 4,676 & 19,225 & 236,803 & 3,810 & 2,938 & 347,144 & $-39,073$ & 205,876 & 513,947 \\
\hline 1996 & 28,028 & 156 & 86,904 & 56,455 & 15,770 & 21,086 & 148,344 & 5,313 & 21,144 & 268,112 & 3,624 & 2,668 & 390,437 & $-41,652$ & 211,105 & 559,890 \\
\hline 1997 & 28,277 & 170 & 93,382 & 55,922 & 15,000 & 19,781 & 149,668 & 5,206 & 21,631 & 267,208 & 3,369 & 2,425 & 395,817 & $-42,947$ & 213,843 & 566,714 \\
\hline 1998 & 27,888 & 188 & 83,620 & 48,35 & 11,239 & 15,241 & 132,7 & 4,280 & 19,835 & 231,675 & 3,555 & 2,477 & 350,4 & $-43,311$ & 218,361 & 525,515 \\
\hline 1999 & 27,310 & 140 & 84,960 & 54,565 & 13,878 & 19,038 & 149,2 & 4,686 & 21,250 & 262,67 & 3,643 & $\mathrm{R}_{2,646}$ & R382,655 & $-44,689$ & 218,413 & R556,379 \\
\hline 2000 & 28,080 & 146 & 119,094 & 78,209 & 23,777 & 27,970 & 192,153 & 8,870 & 26,496 & 357,475 & 3,628 & $\mathrm{R}_{3}, 174$ & R514,379 & $-60,054$ & 231,577 & R685,902 \\
\hline 2001 & $\begin{array}{l}28,000 \\
28,202\end{array}$ & $\begin{array}{r}140 \\
82\end{array}$ & $\begin{array}{l}119,094 \\
139,388\end{array}$ & 75,035 & 19,602 & 25,543 & 185,752 & $\begin{array}{l}7,066 \\
7,266\end{array}$ & $\begin{array}{l}20,450 \\
23,097\end{array}$ & $\begin{array}{l}35,470 \\
336,294\end{array}$ & $\begin{array}{l}3,020 \\
3,524\end{array}$ & $\begin{array}{l}3,494 \\
3,47\end{array}$ & 513,673 & $\begin{array}{l}-00,054 \\
-64,672\end{array}$ & 245,483 & 694,484 \\
\hline 2002 & 28,511 & 180 & $R_{111,536}$ & 69,285 & 17,802 & 22,980 & 179,796 & 6,156 & 24,167 & 320,185 & 3,504 & 4,005 & $\begin{array}{l}\mathrm{R}_{469,045} \\
\text { (1) }\end{array}$ & $-54,230$ & 247,598 & 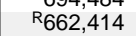 \\
\hline 2003 & $\begin{array}{l}28,511 \\
29,402\end{array}$ & $\begin{array}{l}180 \\
169\end{array}$ & $\begin{array}{l}141,536 \\
144.489\end{array}$ & $\begin{array}{l}6,285 \\
83,873\end{array}$ & $\begin{array}{l}11,802 \\
21,096\end{array}$ & $\begin{array}{l}22,980 \\
28,161\end{array}$ & $\begin{array}{l}1 / 7, / 96 \\
209,493\end{array}$ & $\begin{array}{l}0,156 \\
8,325\end{array}$ & $\begin{array}{l}24,16 / \\
28,061\end{array}$ & $\begin{array}{l}320,185 \\
379,010\end{array}$ & $\begin{array}{l}3,504 \\
3,362 \\
\end{array}$ & $\begin{array}{l}4,005 \\
3,599\end{array}$ & $\begin{array}{r}469,045 \\
561,401\end{array}$ & $\begin{array}{l}-54,230 \\
-64,685\end{array}$ & $\begin{array}{l}24,598 \\
257,992\end{array}$ & $\begin{array}{l}662,414 \\
754,708\end{array}$ \\
\hline 2004 & $\begin{array}{l}29,402 \\
31,764\end{array}$ & $\begin{array}{l}1,125 \\
\end{array}$ & 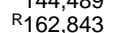 & $\begin{array}{r}83,813 \\
105,772\end{array}$ & 30,219 & $\begin{array}{l}28,101 \\
34.408\end{array}$ & $\begin{array}{l}254,4 \\
254,\end{array}$ & $\begin{array}{l}\text {,320 } \\
9,717\end{array}$ & $\begin{array}{l}20,001 \\
35,212\end{array}$ & 470,2 & $\begin{array}{l}3,502 \\
3,445\end{array}$ & $\begin{array}{l}3,599 \\
3,692\end{array}$ & $\begin{array}{r}501,401 \\
R 674.684\end{array}$ & $\begin{array}{l}-04,085 \\
-71,720\end{array}$ & 268,133 & R871,097 \\
\hline & $\begin{array}{l}31,104 \\
36,932\end{array}$ & & R200,356 & & & $\begin{array}{l}38,8 \\
38,7\end{array}$ & & 13,951 & 44,136 & & $\begin{array}{l}0,469 \\
3,469\end{array}$ & R5,897 & & & & $\mathrm{R}_{1,046,897}$ \\
\hline & & & & & & & & & & & & & & & & \\
\hline & & & & & & & & & & & & 57 & & & & \\
\hline & 49,438 & $\begin{array}{r}1,465 \\
\end{array}$ & & & & & 438 & 17,984 & 61,417 & & & $R_{6,9}$ & & & & \\
\hline & $\mathrm{R}_{45,825}$ & -42 & $\mathrm{R}_{159,362}$ & 131.050 & 36,353 & 43,466 & $\mathrm{R}_{317,082}$ & 11,310 & $\mathrm{R}_{39,268}$ & $\begin{array}{l}0 \\
\text { R } 578,529\end{array}$ & 4,560 & $\begin{array}{l}0,950 \\
R_{5}, 116\end{array}$ & $\begin{array}{r}1,100,04 l \\
R 795,506\end{array}$ & $\begin{array}{l}-110,723 \\
R_{-} 84.723\end{array}$ & & $\mathrm{R}_{1,061,220}$ \\
\hline 2010 & 50,407 & $\begin{array}{l}-4< \\
158\end{array}$ & 159,821 & 166,595 & 48,244 & $\begin{array}{l}45,400 \\
55,157\end{array}$ & 376,512 & 14,308 & $\begin{array}{l}48,600 \\
48,656\end{array}$ & 709,471 & $\begin{array}{l}4,234 \\
5,230\end{array}$ & 6,424 & 933,562 & $\begin{array}{l}-04,635 \\
-94,635\end{array}$ & $\begin{array}{l}300,400 \\
36,900\end{array}$ & $1,204,827$ \\
\hline
\end{tabular}

1 Prices are not adjusted for inflation. See "Nominal Dollars" in Glossary.

2 Expenditures by all sectors, including the electric power sector.

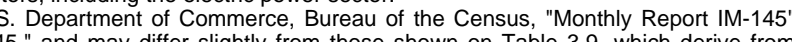
those shown on Table 3.9, which derive from Goods and Services, FT600 series.

5 Through 2004, includes kerosene-type and naphtha-type jet fuel. Beginning in 2005, includes kerosene-type jet fuel only.

Liquefied petroleum gases.

Asphalt and rad oil, aviation gasolie, kerosene, lubricants, petrochemical feedstocks, petroloum coke, special naphthas, waxes, and miscellaneous petroleum products.
9 . 2000 , also includes non-biomass waste.

10 Includes electricity imports, which are not separately displayed. For 1981-1992, also includes fuel ethanol blended into motor gasoline that is not included in the motor gasoline data for those years.
11 Electricity-only and combined-heat-and-power (CHP) plants within the NAICS (North American Industry

Classification System) 22 category whose primary business is to sell electricity, or electricity and heat, to the public. Through 1988, data are for electric utilities only; beginning in 1989, data are for electric utilities and 12 Expenditures by the electic

tric power sector for coal, natural gas, petroleum, nuclear fuel, wood, waste, and electricity imports. Values are negative so the columns will sum to the "Total Energy" column.
13 Retail electricity expenditures by ultimate customers, reported by electric utilities and, beginning in

1996 , other energy service providers.
14 Expenditures for primary energy and retail electricity by the four end-use sectors (residential commercial, industrial, and transportation); excludes expenditures for energy by the electric power secto

Notes: - Expenditures include taxes where data are available. - There are no direct fuel costs for independent rounding

Web Page: For related information, see http://www.eia.gov/state/seds/seds-data-complete.cfm.

Source: U.S. Energy Information Administration, "State Energy Data 2010: Prices and Expenditures" 
Figure 3.6 Consumer Expenditure Estimates for Energy by End-Use Sector, 2010

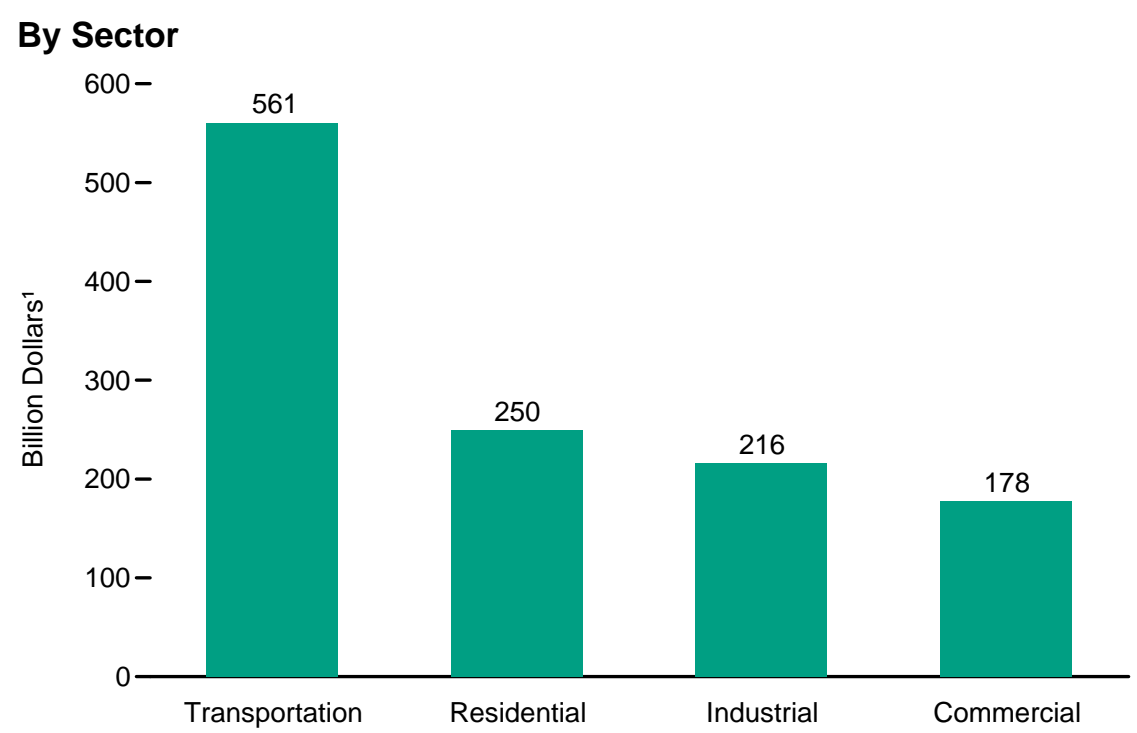

\section{Residential Sector by Major Source ${ }^{2}$}

$200-$

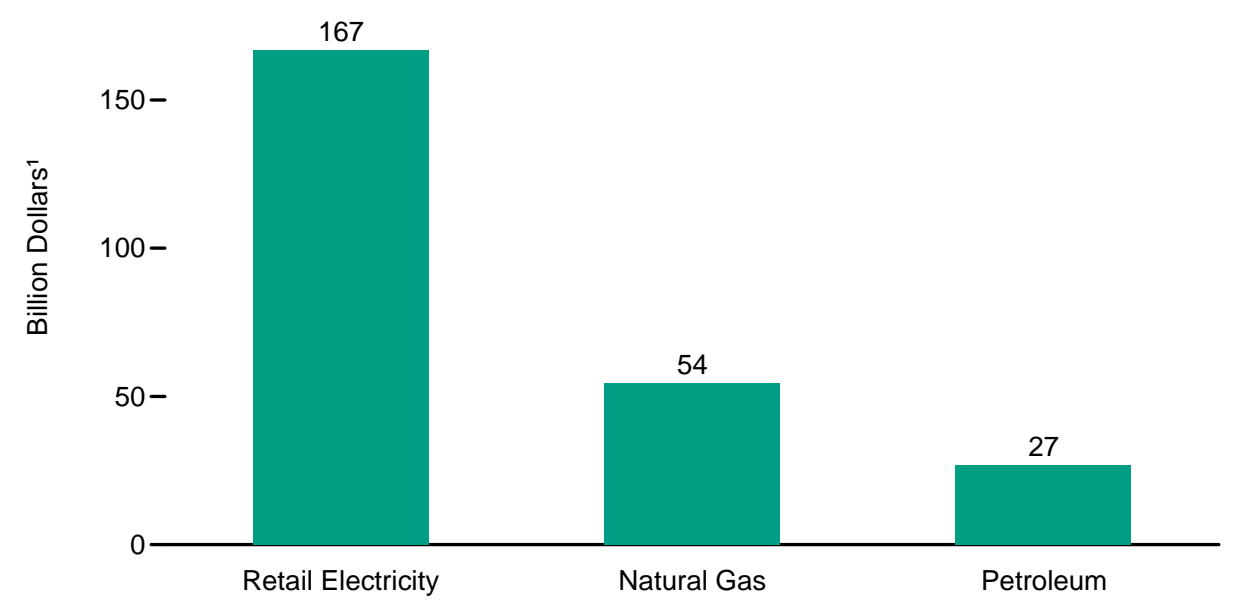

Commercial Sector by Major Source ${ }^{3}$

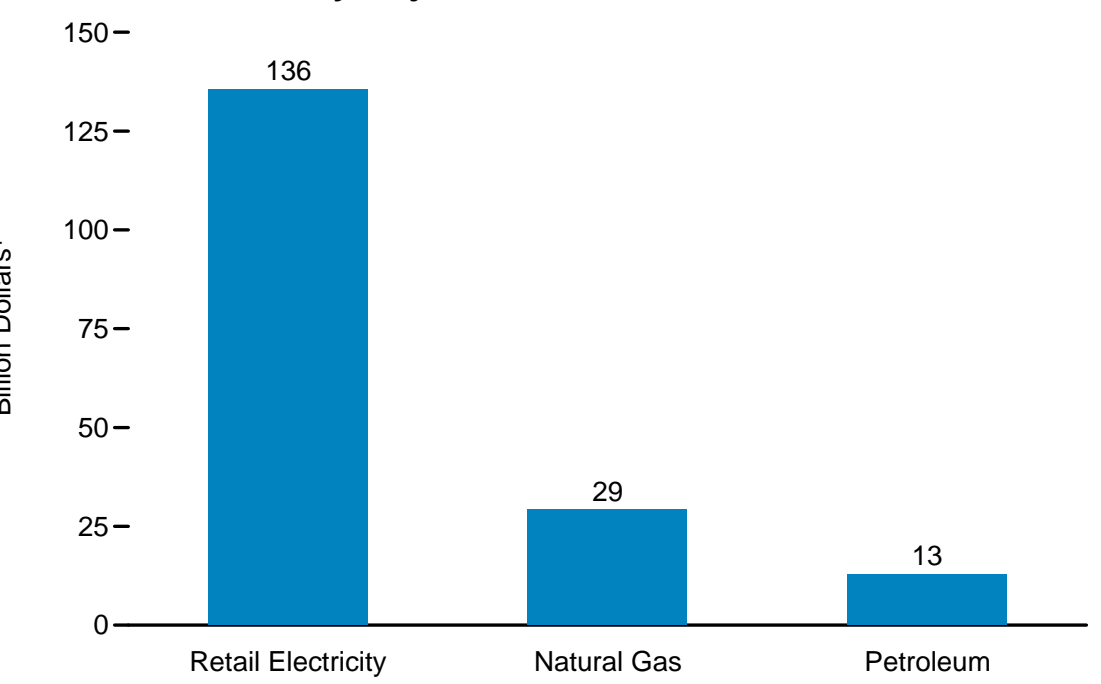

Industrial Sector by Major Source ${ }^{4}$

$$
125-
$$

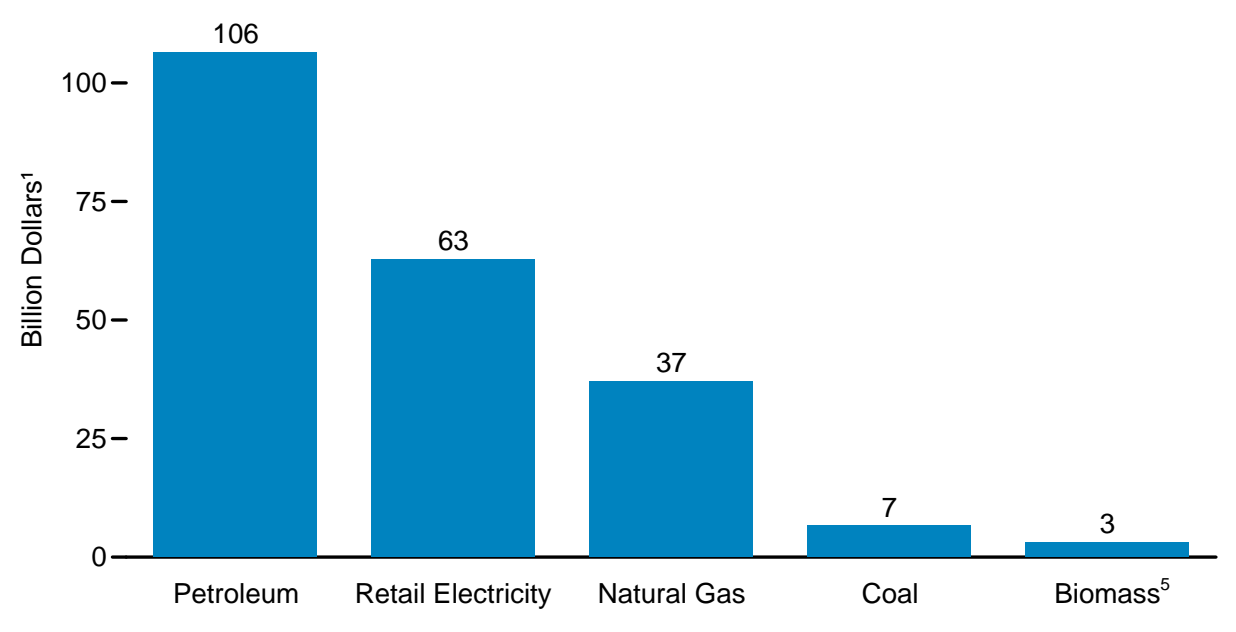

\footnotetext{
${ }^{1}$ Prices are not adjusted for inflation. See "Nominal Dollars" in Glossary.
2 Expenditures for coal and wood and wood-derived fuels are not displayed.

Expenditures for coal and wood and wood-derived fuels are not displayed.

${ }^{3}$ Expenditures for coal, wood and wood-derived fuels, and biomass waste are not displayed
${ }^{4}$ Expenditures for imports and exports of coal coke are not displayed.

${ }^{5}$ Wood and wood-derived fuels, and biomass waste; excludes fuel ethanol and biodiesel.
}

Notes: • Petroleum accounts for nearly all transportation sector expenditures. • There are no direct fuel costs for hydroelectric, geothermal, wind, or solar energy. • Totals may not equal the sum of components due to independent rounding. Source: Table 3.6. 
Table 3.6 Consumer Expenditure Estimates for Energy by End-Use Sector, 1970-2010

(Million Dollars ${ }^{1}$ )

\begin{tabular}{|c|c|c|c|c|c|c|c|c|c|c|c|c|c|c|c|c|}
\hline \multirow[b]{2}{*}{ Year } & \multicolumn{4}{|c|}{ Residential } & \multicolumn{4}{|c|}{ Commercial } & \multicolumn{6}{|c|}{ Industrial } & \multicolumn{2}{|c|}{ Transportation } \\
\hline & $\begin{array}{c}\text { Natural } \\
\text { Gas }^{2}\end{array}$ & Petroleum & $\begin{array}{c}\text { Retail } \\
\text { Electricity }{ }^{3}\end{array}$ & Total 4 & $\begin{array}{l}\text { Natural } \\
\text { Gas }^{2}\end{array}$ & Petroleum 5 & $\begin{array}{c}\text { Retail } \\
\text { Electricity } 3\end{array}$ & Total 6,7 & Coal & $\begin{array}{c}\text { Natural } \\
\text { Gas }{ }^{2}\end{array}$ & Petroleum 5 & Biomass ${ }^{8}$ & $\begin{array}{c}\text { Retail } \\
\text { Electricity } 3\end{array}$ & Total 7,9 & Petroleum 5 & Total 7,10 \\
\hline 1970 & 5,272 & 4,186 & 10,352 & 20,112 & 1,844 & 1,440 & 7,319 & 10,678 & 2,082 & 2,625 & 6,069 & 366 & 5,624 & 16,691 & 35,327 & 35,379 \\
\hline 1971 & 5,702 & 4,367 & 11,589 & 21,934 & 2,060 & 1,574 & 8,301 & 12,021 & 1,971 & 3,019 & 6,663 & 374 & 6,256 & 18,244 & 37,766 & 37,824 \\
\hline 1972 & 6,223 & 4,623 & 13,034 & 24,095 & 2,289 & 1,653 & 9,443 & 13,464 & 2,212 & 3,335 & 7,180 & 404 & 7,173 & 20,278 & 40,154 & 40,218 \\
\hline 1973 & 6,282 & $\mathrm{R}_{5,849}$ & 14,712 & $\mathrm{R}_{27,029}$ & 2,421 & 1,936 & 10,707 & 15,149 & 2,527 & 3,936 & 8,600 & 425 & 8,284 & 23,779 & 45,846 & 45,918 \\
\hline 1974 & 6,949 & $\mathrm{R}_{7,288}$ & 17,924 & $R_{32,459}$ & 2,741 & 3,290 & 13,373 & 19,579 & 4,704 & 4,971 & 15,408 & 421 & 11,184 & 36,837 & 64,368 & 64,476 \\
\hline 1975 & 8,410 & R7,486 & 20,644 & $\mathrm{R}_{36,835}$ & 3,385 & 3,219 & 16,157 & 22,956 & 5,498 & 5,844 & 15,544 & 386 & 13,760 & 41,113 & 70,813 & 70,933 \\
\hline 1976 & 9,992 & $R_{8,755}$ & 22,621 & $\mathrm{R}_{41,682}$ & 4,379 & 3,739 & 18,148 & 26,447 & 5,448 & 7.484 & 18,384 & 443 & 16,083 & 47,887 & 77,759 & 77,880 \\
\hline 1977 & 11,324 & R9,772 & 26,132 & $\begin{array}{r}\mathrm{R} 47,594 \\
\mathrm{r}\end{array}$ & 5,094 & 4,411 & 21,023 & 30,725 & 5,360 & 8,958 & 22,190 & 464 & 18,956 & 55,996 & 86,047 & 86,160 \\
\hline 1978 & 12,565 & $\mathrm{R} 9,831$ & 29,069 & R51,854 & 5,812 & 4,350 & 23,166 & 33,563 & 5,722 & 10,114 & 23,203 & 511 & 21,798 & 61,710 & 92,003 & 92,128 \\
\hline 1979 & 14,772 & $\mathrm{R}_{11,157}$ & 31,683 & $\begin{array}{r}\text { R58,149 } \\
\text { R5 } 14\end{array}$ & 7,623 & $\begin{array}{l}5,659 \\
5,650\end{array}$ & 25,433 & 38,944 & 6,247 & 12,110 & 33,705 & 512 & 24,797 & 77,630 & 122,688 & 122,826 \\
\hline 1980 & 17,497 & $R_{12,554}$ & 38,458 & R69,277 & 8,858 & 7,409 & 30,611 & 47,074 & 5,888 & 16,350 & 42,765 & 529 & 28,863 & 94,316 & 163,517 & 163,680 \\
\hline 1981 & 19,502 & $R_{13,250}$ & 44,780 & $\mathrm{R}_{78,486}$ & 10,085 & 7,794 & 37,484 & 55,629 & 6,441 & 20,432 & 47,171 & 558 & 34,007 & 108.581 & 184,946 & 185,203 \\
\hline 1982 & 23,987 & $\mathrm{R}_{12,413}$ & 50,045 & R87,506 & 12,565 & 6,865 & 41,759 & 61,503 & 5,301 & 20,504 & 41,841 & 540 & 35,364 & 103,503 & 173,553 & 173,968 \\
\hline 1983 & 26,564 & $R_{11,350}$ & 53,918 & R92,812 & 13,602 & 7,592 & 43,529 & 65,028 & 4,735 & 21,461 & 38,437 & 610 & 37,017 & 102,220 & 156,841 & 157.417 \\
\hline 1984 & 27,873 & $\mathrm{R}_{12,919}$ & 55,777 & R97,657 & 14,012 & 7,948 & 47,304 & 69,593 & 5,420 & 23,763 & 41,470 & 622 & 39,050 & 110,309 & $\begin{array}{l}156,979 \\
\text { 15, }\end{array}$ & 157,636 \\
\hline 1985 & 27,136 & $\mathrm{R}_{12,741}$ & 58,672 & R99,619 & 13,368 & 6,996 & 50,092 & 70,725 & 5,252 & 21,615 & 38,876 & 619 & 40,190 & 106,528 & 160,745 & 161,475 \\
\hline 1986 & 25,147 & $\mathrm{R}_{10,433}$ & 60,776 & $\begin{array}{l}\text { R97,173 } \\
\text { R97, }\end{array}$ & 11,770 & 5,166 & 51,449 & 68,637 & 4,745 & 16,479 & 30,567 & 639 & 39,271 & $\begin{array}{r}90,669 \\
91,669\end{array}$ & 125,353 & $\begin{array}{l}126,039 \\
\end{array}$ \\
\hline 1987 & $\begin{array}{l}23,926 \\
23,97\end{array}$ & $\mathrm{R}_{10,658}$ & 63,318 & R98,635 & 11,601 & 5,377 & 51,900 & $\begin{array}{l}09,098 \\
69,098\end{array}$ & 4,448 & 15,909 & $\begin{array}{l}31,0921 \\
31\end{array}$ & 636 & 39,109 & 91,210 & 136,807 & $\begin{array}{l}137,644 \\
137,64\end{array}$ \\
\hline 1988 & 25,332 & $R_{11,008}$ & 66,793 & $R_{103,907}$ & 12,377 & 4,950 & 54,411 & 71,969 & 4,744 & 17,257 & 29,123 & 662 & 40,507 & 92,418 & 141,382 & 142,221 \\
\hline 1989 & 26,951 & $R_{12,522}$ & 69,243 & $R_{109,553}$ & 12,908 & 5,293 & 57,460 & 75,911 & 4,650 & 18,770 & 28,561 & 1,323 & 42,255 & 95,705 & 155,591 & 156,510 \\
\hline 1990 & 25,439 & $R_{12,116}$ & 72,378 & $R_{110,905}$ & 12,681 & 5,986 & 60,627 & 79,605 & 4.636 & 19,348 & 34,132 & 906 & 43,358 & 102,411 & 178.852 & 179,732 \\
\hline 1991 & 26,508 & $\mathrm{R}_{11,703}$ & 76,828 & $R_{115,999}$ & 13,175 & 5,224 & 63,407 & 82,098 & $\begin{array}{l}4,030 \\
4,332\end{array}$ & $\begin{array}{l}18,912 \\
18,912\end{array}$ & 32,494 & 1,034 & 44,201 & 101,028 & 170,589 & $R_{171,543}$ \\
\hline 1992 & 27,599 & $R_{11,026}$ & 76,848 & $R_{116,394}$ & 13,685 & 4,796 & 64,233 & 83,007 & 4,245 & 20,553 & 32,208 & 1,079 & 45,474 & 103,699 & 171,482 & 172,544 \\
\hline 1993 & 30,533 & $\mathrm{R}_{10,990}$ & 82,814 & $R_{125,141}$ & 14,967 & 4,209 & 67,626 & 87,095 & 4,060 & 22,367 & 31,518 & 1,146 & $\begin{array}{l}45,726 \\
45,714\end{array}$ & 104,913 & 173,704 & $\begin{array}{l}174,082 \\
\end{array}$ \\
\hline 1994 & 31,028 & 10,656 & 84,552 & 126,962 & 15,927 & 4,162 & 69,637 & 90,010 & 4,060 & 22,556 & 33,612 & 1,279 & 46,257 & 107,978 & 178,724 & 179,123 \\
\hline 1995 & 29,362 & 10,289 & 87,610 & 127,961 & 15,383 & 3,956 & 72,481 & 92,106 & 4,068 & 21,487 & 34,177 & 1,699 & 45,402 & 107,067 & 186,411 & 186.813 \\
\hline 1996 & 33,219 & 12,796 & 90,503 & 137,340 & 17,106 & 4,879 & 74,121 & 96,414 & 3,943 & 26,167 & 40,853 & 1,432 & 46,102 & 118,654 & 207,078 & 207,483 \\
\hline 1997 & 34,590 & 12,239 & 90,704 & 138,201 & 18,755 & 4,531 & 77,153 & 100,758 & 3,887 & 28,411 & 39,886 & 1.435 & 45,610 & 119.400 & 207,940 & 208,353 \\
\hline 1998 & 30,875 & 9,852 & 93,360 & 134,602 & 16,667 & 3,575 & 78,999 & 99,492 & $\begin{array}{l}3,066 \\
3,566\end{array}$ & 24,515 & 32,143 & $\begin{array}{l}1,600 \\
1,600\end{array}$ & 45,634 & 107,647 & 183,368 & 183,775 \\
\hline 1999 & 31,577 & 11,397 & 93,482 & $R_{136,999}$ & 16,351 & 3,931 & 79,141 & 99,681 & 3,457 & 24,079 & 36,966 & 1,786 & 45,429 & 111,857 & 207,433 & 207,843 \\
\hline 2000 & 38,959 & 17,283 & 98,209 & $R_{155,299}$ & 21,339 & $\begin{array}{l}6,674 \\
6,601\end{array}$ & 85,129 & $\begin{array}{l}50,001 \\
113,423\end{array}$ & $\begin{array}{l}3,407 \\
3,507\end{array}$ & 34,624 & 52,066 & 1,888 & 47,859 & 140,090 & 276,642 & $\begin{array}{l}277,090 \\
277,00\end{array}$ \\
\hline 2001 & 46,189 & 17,169 & 103,158 & 167,241 & 25,879 & 6,225 & 93,402 & 125,790 & 3,572 & 38,597 & 47,173 & 2,216 & 48,519 & 140,158 & 260,785 & 261,295 \\
\hline 2002 & 38,490 & 14,549 & 106,834 & 160,542 & 20,926 & 5,187 & 93,763 & 120,164 & 3,526 & R31,199 & 45,685 & 2,592 & 46,606 & $\mathrm{R}_{129,788}$ & 251,441 & 251,919 \\
\hline 2003 & 48,278 & 18,010 & 111,249 & 178,374 & 26,411 & 7,137 & 96,263 & 130,132 & 3,552 & 41,168 & 54,228 & 1,935 & 49,962 & 151,015 & 294,544 & 295,188 \\
\hline 2004 & 52,265 & 20,264 & 115,577 & 189,080 & 29,518 & 8,233 & 100,546 & 138,694 & 4,064 & R47.464 & 71,052 & 1.919 & 51,491 & $\mathrm{R}_{177,114}$ & 365,526 & 366,208 \\
\hline 2005 & 61,196 & 24,320 & 128,393 & 215,186 & 33,838 & 10,331 & 110,522 & $\begin{array}{r}R_{155,165} \\
150\end{array}$ & $\begin{array}{l}5,004 \\
5,004\end{array}$ & R55,300 & 88,285 & 3.451 & 56.229 & $\begin{array}{r}R \\
R_{208.902}\end{array}$ & 466,785 & 467.644 \\
\hline 2006 & 59,834 & 23,531 & 140,582 & $R_{225,244}$ & 33,736 & 10,563 & 122,914 & $\mathrm{R}_{167,630}$ & 5,405 & R52,571 & 106,621 & $R_{3,509}$ & 59,764 & $\mathrm{R}_{228,377}$ & 537,500 & 538,436 \\
\hline 2007 & $\begin{array}{l}61,598 \\
61,58\end{array}$ & 26,468 & 148,295 & $\begin{array}{r}\text { R237,897 } \\
\text { R }\end{array}$ & 34,005 & 11,410 & 128,903 & $R_{174,798}$ & $\begin{array}{l}5,439 \\
5\end{array}$ & R51,126 & 113,491 & $R_{3,180}$ & 62,934 & $\mathrm{R}_{236,517}$ & 584,045 & 585,070 \\
\hline 2008 & 67,851 & $\begin{array}{l}20,400 \\
32,071\end{array}$ & $\begin{array}{l}150,433 \\
155,433\end{array}$ & 257,448 & $\begin{array}{l}38,476 \\
38,46\end{array}$ & 14,996 & 138,469 & $\mathrm{R}_{192,540}$ & $\begin{array}{l}6,290 \\
6,290\end{array}$ & R61,877 & 131,958 & $\mathrm{R}_{3,402}$ & $\begin{array}{l}0,904 \\
65,840\end{array}$ & $\mathrm{R}_{270,833}$ & 686,871 & 688,024 \\
\hline 2009 & R57,841 & 24,425 & 157,008 & $\mathrm{R}_{240,773}$ & 31,012 & $\mathrm{R}_{10,584}$ & 132,940 & $R_{175,023}$ & 5,388 & $\mathrm{R}_{36,302}$ & R80,591 & $R_{2,310}$ & 59,662 & $\mathrm{R}_{184,210}$ & $\mathrm{R}_{460,142}$ & $\begin{array}{r}000,0<4 \\
R_{461,214}\end{array}$ \\
\hline 2010 & 54,439 & 26,791 & $\begin{array}{l}166,782 \\
\end{array}$ & 249,739 & 29,183 & 12,863 & 135,559 & 178,128 & 6,591 & 36,993 & 106,495 & 3,190 & 62,745 & 216,172 & 559,758 & 560,787 \\
\hline
\end{tabular}

1 Prices are not adjusted for inflation. See "Nominal Dollars" in Glossary.

Natural gas, plus a small amount of supplemental gaseous fuels.

3 Retail electricity expenditures by ultimate customers, reported by electric utilities and, beginning in

1996, other energy service providers.

Includes coal and wood and wood-derived fuels, which are not separately displayed.

5 Beginning in 1993, includes fuel ethanol blended into motor gasoline.
6 Includes coal, wood and wood-derived fuels, and biomass waste, which are not separately displayed. Through 2000, also includes non-biomass waste.

For 1981-1992 includes fuel ethanol blended into motor gasoline that is not included in the petroleum ata for those years.

Wood and wood-derived fuels, and biomass waste; excludes fuel ethanol and biodiesel. Through

2000, also includes non-biomass waste.

Includes coal coke imports and exports, which are not separately displayed.

Includes coal, natural gas, and retail electricity, which are not separately displayed.

Notes: - Expenditures include taxes where data are available. - There are no direct fuel costs for hydroelectric, geothermal, wind, or solar energy. - Totals may not equal the sum of components due to independent rounding.

Web Page: For related information, see http://www.eia.gov/state/seds/seds-data-complete.cfm. Source: U.S. Energy Information Administration, "State Energy Data 2010: Prices and Expenditures"
(June 2012), U.S. Tables ET3-ET6. 


\section{Total, 1949-2011}

$500-$

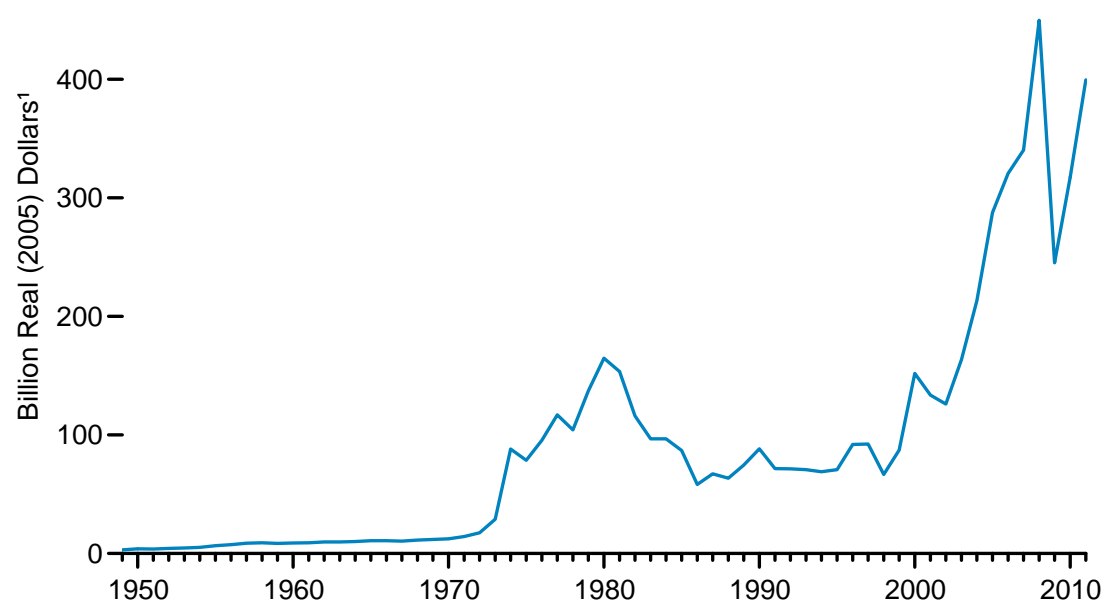

By Fuel, 2011

$$
\text { 400- }
$$

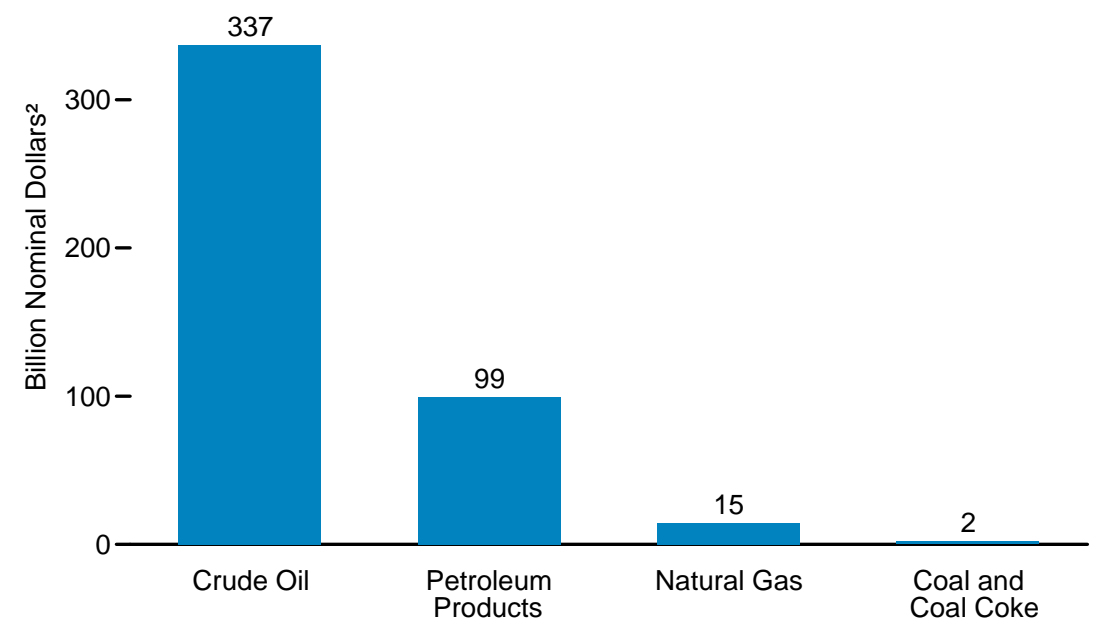

By Fuel, 1949-2011

$350-$

$300-$

$250-$

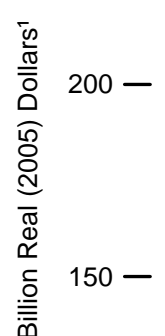

$100-$

$50-$
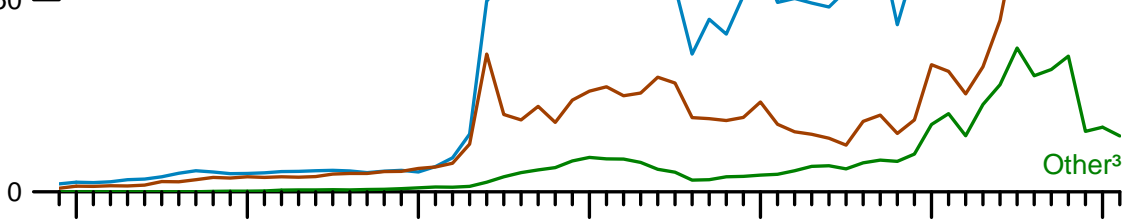

1960

1970

1980

1990

2000

2010

${ }^{3}$ Natural gas, coal, and coal coke. Source: Table 3.7.

${ }^{1}$ In chained (2005) dollars, calculated by using gross domestic product implicit price deflators in Table D1. See "Chained Dollars" in Glossary.

2 See "Nominal Dollars" in Glossary. 
Table 3.7 Value of Fossil Fuel Imports, Selected Years, 1949-2011

(Billion Dollars)

\begin{tabular}{|c|c|c|c|c|c|c|c|c|c|c|c|c|}
\hline \multirow[b]{2}{*}{ Year } & \multicolumn{2}{|c|}{ Coal } & \multicolumn{2}{|c|}{ Coal Coke } & \multicolumn{2}{|c|}{ Natural Gas } & \multicolumn{2}{|c|}{ Crude Oil ${ }^{1}$} & \multicolumn{2}{|c|}{ Petroleum Products 2} & \multicolumn{2}{|c|}{ Total } \\
\hline & Nominal $^{3}$ & Real ${ }^{4}$ & Nominal $^{3}$ & Real ${ }^{4}$ & Nominal $^{3}$ & Real ${ }^{4}$ & Nominal $^{3}$ & Real ${ }^{4}$ & Nominal $^{3}$ & Real ${ }^{4}$ & Nominal $^{3}$ & Real ${ }^{4}$ \\
\hline $\begin{array}{l}1949 \\
1950\end{array}$ & $\begin{array}{l}\text { (s) } \\
\text { (s) }\end{array}$ & $\begin{array}{l}0.02 \\
.02\end{array}$ & $\begin{array}{l}\text { (s) } \\
01\end{array}$ & $\begin{array}{l}0.03 \\
.04\end{array}$ & $\begin{array}{r}0.00 \\
.00\end{array}$ & $\begin{array}{l}0.00 \\
.00\end{array}$ & $\begin{array}{r}0.30 \\
.37\end{array}$ & $\begin{array}{l}2.10 \\
2.52\end{array}$ & $\begin{array}{r}0.14 \\
.21\end{array}$ & $\begin{array}{r}0.95 \\
R_{1} .46\end{array}$ & $\begin{array}{r}0.45 \\
59\end{array}$ & $\begin{array}{l}3.09 \\
4.04\end{array}$ \\
\hline 1955 & (s) & .02 & (s) & .01 & $(\mathrm{~s})$ & .01 & .65 & $\mathrm{R}_{3.94}$ & .44 & 2.66 & 1.10 & 6.64 \\
\hline 1960 & (s) & .01 & (s) & .01 & .03 & .15 & .90 & 4.81 & .73 & 3.93 & 1.66 & R8.91 \\
\hline 1965 & (s) & .01 & (s) & .01 & .11 & .53 & 1.12 & 5.62 & .92 & $\mathrm{R}_{4} .63$ & 2.15 & $\mathrm{R}_{10.79}$ \\
\hline 1970 & $\begin{array}{l}\text { (s) } \\
\text { (s) }\end{array}$ & (s) & (s) & .01 & .26 & 1.06 & 1.26 & 5.18 & 1.48 & $\mathrm{R}_{6.09}$ & 3.00 & $\mathrm{R}_{12.34}$ \\
\hline 1975 & . .02 & . .06 & . 16 & .47 & $\begin{array}{l}.26 \\
1.15\end{array}$ & $\begin{array}{l}1.00 \\
3.43\end{array}$ & $\begin{array}{r}1.20 \\
18.29\end{array}$ & $\begin{array}{r}0.10 \\
\text { R54.45 }\end{array}$ & $\begin{array}{l}.48 \\
6.77\end{array}$ & $R_{20.15}$ & $\begin{array}{l}3.00 \\
26.39\end{array}$ & $\begin{array}{l}\text { R78.56 } \\
\text { R }\end{array}$ \\
\hline 1976 & .02 & .05 & .11 & .31 & 1.66 & $\begin{array}{r}8.40 \\
\mathrm{R}_{4} .67\end{array}$ & 25.46 & $\begin{array}{l}\text { R71.67 } \\
\text { R }\end{array}$ & 6.65 & $\mathrm{R}_{18.73}$ & 33.90 & R95.43 \\
\hline 1977 & .04 & .10 & .13 & .35 & 2.00 & R5.29 & 33.59 & R88.91 & 8.42 & $\mathrm{R}_{22.28}$ & 44.18 & $R_{1} 16.93$ \\
\hline 1978 & .07 & .18 & .41 & 1.01 & 2.06 & 5.10 & 32.30 & R79.88 & 7.30 & $\mathrm{R}_{18.06}$ & 42.15 & $\mathrm{R}_{104.23}$ \\
\hline 1979 & .05 & .12 & .34 & .78 & 3.13 & 7.14 & 46.06 & $\mathrm{R}_{1} 105.16$ & 10.45 & $\mathrm{R}_{23.86}$ & 60.03 & R137.05 \\
\hline 1980 & .03 & .06 & .05 & .11 & 4.21 & 8.82 & 61.90 & $\mathrm{R}_{129.52}$ & 12.54 & $\mathrm{R}_{26.24}$ & 78.74 & $\mathrm{R}_{164.75}$ \\
\hline 1981 & .03 & .06 & .04 & .08 & 4.41 & R8.44 & 61.46 & $\mathrm{R}_{117.58}$ & 14.30 & $\begin{array}{l}\text { R27.35 } \\
\text { R }\end{array}$ & $\begin{array}{l}80.24 \\
80.74\end{array}$ & R153.51 \\
\hline 1982 & .02 & .04 & .01 & .02 & 4.69 & R8.46 & 45.72 & R82.45 & 13.86 & $\mathrm{R}_{25.00}$ & 64.31 & $R_{115.96}$ \\
\hline 1983 & .04 & .07 & (s) & (s) & 4.39 & $\mathrm{R}_{7.61}$ & 36.49 & R63.30 & 14.84 & $\mathrm{R}_{25.74}$ & 55.77 & R96.73 \\
\hline 1984 & .05 & .08 & .05 & .08 & 3.44 & 5.75 & 36.44 & R60.93 & 17.87 & $\mathrm{R}_{29.87}$ & 57.84 & R96.70 \\
\hline 1985 & .07 & .11 & .04 & .07 & 3.05 & 4.95 & 32.90 & R53.39 & 17.47 & $\mathrm{R}_{28.34}$ & 53.53 & R86.86 \\
\hline 1986 & .08 & .13 & .03 & .04 & $\begin{array}{l}3.05 \\
1.82\end{array}$ & $\begin{array}{l}4.90 \\
2.90\end{array}$ & 22.61 & R35.89 & 12.18 & $\begin{array}{r}2.04 \\
R_{1} .34\end{array}$ & $\begin{array}{l}5.53 \\
36.72\end{array}$ & $\begin{array}{r}0.00 \\
\text { R58.29 }\end{array}$ \\
\hline $\begin{array}{l}1900 \\
1987\end{array}$ & .06 & .09 & .05 & .08 & R1.94 & $\mathrm{R}_{2.99}$ & 29.13 & R44.09 & 12.37 & $R_{19.08}$ & $\begin{array}{l}43.54 \\
43.54\end{array}$ & R 67.17 \\
\hline 1988 & .06 & .10 & .19 & .29 & 2.38 & 3.55 & 27.55 & R41.09 & 12.43 & $R_{18.55}$ & 42.62 & R63.57 \\
\hline 1989 & .10 & .14 & .22 & .31 & 2.51 & $\mathrm{R}_{3.61}$ & 35.53 & R51.06 & 13.50 & $\mathrm{R}_{19.40}$ & $R_{51.86}$ & R74.53 \\
\hline 1990 & .09 & .13 & .07 & .10 & 2.97 & $\mathrm{R}_{4} .11$ & 43.78 & $\mathrm{R}_{60.59}$ & 16.90 & R23.39 & 63.83 & R88.32 \\
\hline 1991 & .11 & .15 & .09 & .12 & $\mathrm{R}_{3.25}$ & $\mathrm{R}_{4} .34$ & $\begin{array}{l}36.10 \\
36.90\end{array}$ & $R_{49.32}$ & 13.17 & $\mathrm{R}_{17.60}$ & $\begin{array}{r}0.00 \\
\text { R53.52 }\end{array}$ & R71.53 \\
\hline 1992 & .13 & .17 & .14 & .19 & $\mathrm{R}_{3.95}$ & R5.16 & 38.55 & R50.33 & 11.98 & $R_{15.65}$ & R54.76 & R71.49 \\
\hline 1993 & .25 & .32 & .17 & .21 & 4.77 & $\mathrm{R}_{6.09}$ & 38.47 & $\mathrm{R}_{49.14}$ & 11.74 & $R_{15.00}$ & 55.40 & R70.76 \\
\hline 1994 & .27 & .34 & .27 & .34 & $\mathrm{R}_{4.91}$ & 6.14 & 38.48 & $\mathrm{R}_{48.14}$ & 11.14 & $R_{13.94}$ & $R_{55.08}$ & $R_{68.90}$ \\
\hline 1995 & .32 & .40 & .33 & .40 & 4.23 & 5.19 & 42.81 & R52.46 & 9.95 & $R_{12.19}$ & 57.64 & R70.64 \\
\hline 1996 & .27 & .33 & .24 & .29 & 5.79 & $\mathrm{R}_{6.96}$ & 54.93 & $R_{66.06}$ & 15.27 & $R_{18.36}$ & $\begin{array}{r}\text { R7 } 76.54 \\
\end{array}$ & $\begin{array}{r}\text { R92.04 } \\
\text { P }\end{array}$ \\
\hline 1997 & .26 & .30 & .25 & .30 & 6.50 & $\begin{array}{l}0.90 \\
7.68\end{array}$ & $\begin{array}{l}54.23 \\
54.23\end{array}$ & $\begin{array}{l}\text { R64.08 } \\
\text { R }\end{array}$ & 516.93 & $5, R_{20.00}$ & 78.16 & R92.36 \\
\hline 1998 & .28 & .33 & .29 & .34 & 6.21 & 7.26 & 37.25 & $\mathrm{R}_{43.53}$ & 13.01 & $\mathrm{R}_{15.20}$ & 57.05 & R66.66 \\
\hline 1999 & .28 & .32 & .23 & .26 & 8.03 & R9. 25 & 50.89 & R58.60 & 16.28 & $R_{18.75}$ & 75.71 & R87.18 \\
\hline 2000 & .38 & .42 & .25 & .28 & 14.94 & $R_{16.84}$ & 89.88 & $\mathrm{R}_{1} 01.30$ & 29.38 & $R_{33.11}$ & 134.81 & $\mathrm{R}_{151.95}$ \\
\hline 2001 & .67 & .74 & .19 & .21 & $\begin{array}{l}17.62 \\
\end{array}$ & $\mathrm{R}_{19.42}$ & 74.29 & R81.89 & 28.45 & R31.36 & 121.23 & R133.62 \\
\hline 2002 & .60 & .65 & .24 & $\begin{array}{l}.<1 \\
\text { R. } 26\end{array}$ & $R_{12.65}$ & $\begin{array}{l}R_{13.42} \\
R_{13}\end{array}$ & 79.25 & $\begin{array}{l}\text { R85.05 } \\
\text { R }\end{array}$ & $\begin{array}{l}20.45 \\
23.52\end{array}$ & $R_{25.51}$ & $R_{116.26}$ & $\mathrm{R}_{126.11}$ \\
\hline 2003 & $\begin{array}{l}.00 \\
.79\end{array}$ & $\begin{array}{l}.05 \\
.84\end{array}$ & $\begin{array}{l}.24 \\
.24\end{array}$ & .25 & 20.39 & $\mathrm{R}_{21.66}$ & 101.80 & $\mathrm{R}_{108.14}$ & $\begin{array}{l}30.64 \\
30.64\end{array}$ & $R_{32.55}$ & 153.85 & $\mathrm{R}_{163.43}$ \\
\hline 2004 & 1.02 & $\begin{array}{l}.04 \\
1.06\end{array}$ & 1.23 & 1.27 & 24.74 & $R_{25.56}$ & 136.03 & $\mathrm{R}_{140.55}$ & 43.24 & R44.67 & 206.26 & $\mathrm{R}_{213.11}$ \\
\hline 2005 & 1.42 & 1.42 & .78 & .78 & 35.25 & 35.25 & 182.94 & 182.94 & 67.12 & 67.12 & 287.52 & 287.52 \\
\hline 2006 & 1.78 & 1.72 & .64 & .62 & 28.80 & $R_{27.90}$ & 225.16 & $\mathrm{R}_{218.11}$ & 74.56 & R72.22 & 330.93 & $\mathrm{R}_{320.57}$ \\
\hline 2007 & $\begin{array}{l}1.70 \\
1.73\end{array}$ & 1.63 & $\begin{array}{l}.04 \\
.48\end{array}$ & .02 & 31.65 & $\mathrm{R}_{29.80}$ & 245.77 & $\mathrm{R}_{231.36}$ & 81.85 & R77.05 & 361.48 & $\mathrm{R}_{340.29}$ \\
\hline 2008 & $\begin{array}{l}1.73 \\
2.05\end{array}$ & $\begin{array}{l}1.03 \\
1.88\end{array}$ & $\begin{array}{r}.48 \\
1.68\end{array}$ & $\begin{array}{r}.45 \\
1.54\end{array}$ & $\begin{array}{l}3.65 \\
34.66\end{array}$ & $\begin{array}{r}29.80 \\
\text { R31.92 }\end{array}$ & $\begin{array}{l}245.11 \\
353.54\end{array}$ & $\begin{array}{r}231.36 \\
\text { R325.59 }\end{array}$ & $\begin{array}{l}8.85 \\
96.31\end{array}$ & R88.70 & $\begin{array}{l}361.48 \\
488.23\end{array}$ & $\begin{array}{l}\text { R449.64 }\end{array}$ \\
\hline 2009 & $\begin{array}{l}2.05 \\
1.45\end{array}$ & $\begin{array}{l}1.00 \\
1.32\end{array}$ & $\begin{array}{l}.00 \\
.09\end{array}$ & $\begin{array}{l}.54 \\
.08\end{array}$ & $\begin{array}{l}34.00 \\
15.72\end{array}$ & $\mathrm{R}_{14.32}$ & $\begin{array}{l}353.04 \\
194.60\end{array}$ & $\mathrm{R}_{177.35}$ & 57.23 & R52.16 & $\begin{array}{l}400.20 \\
269.09\end{array}$ & $\begin{array}{r}\mathrm{R}_{245.04} \\
\end{array}$ \\
\hline 2010 & 1.39 & $\mathrm{R}_{1.25}$ & .40 & .36 & $\mathrm{R}_{16.91}$ & $\mathrm{R}_{15.23}$ & $\mathrm{R}_{260.11}$ & $\mathrm{R}_{234.35}$ & R73.36 & R66.09 & R352.16 & $\begin{array}{r}\text { R317.20 } \\
\text { R }\end{array}$ \\
\hline 2011 & $\mathrm{P}_{1.35}$ & $P_{1.19}$ & P. .55 & P.49 & $E_{14.61}$ & $E_{12.89}$ & P336.80 & $\mathrm{P}_{297.10}$ & P99.49 & P87.76 & P452.79 & P399.43 \\
\hline
\end{tabular}

1 Beginning in 1977, includes imports for the Strategic Petroleum Reserve. fuels.

3 See "Nominal Dollars" in Glossary.

4 In chained (2005) dollars, calculated by using gross domestic product implicit price deflators in Table D1. See "Chained Dollars" in Glossary.

There is a discontinuity in this time
commodity category "Other Mineral Fuels."

$\mathrm{R}=$ Revised. P=Preliminary. $\mathrm{E}=$ Estimate. (s)=Less than 0.005 billion.

Notes: - Includes value of imports into Puerto Rico from foreign countries; excludes receipts into the 50 States and the District of Columbia from the Virgin Islands and Puerto Rico. - Totals may not equal sum of components due to independent rounding.

Source: Coal and Coat Coke: Bureau of the

Natural Gas: • 1949-1962-Bureau of the Census, U.S. Imports of Merchandise for Consumption, 1964-1971-Bure Bureau of the Census, U.S. Imports of Merchandise for Consumption, FT125, and 1973-Federal Power Commission (FPC), Pipeline Imports and Exports of Natural Gas - Imports and Exports of LNG. - 1974-1977-FPC, United States Imports and Exports of Natural Gas, annual reports. - 1978-1981-U.S. Energy Information Administration (EIA), U.S. Imports and Exports of Natural Gas, annual reports. - 1982-2009-EIA, Natural Gas Monthly (NGM), monthly reports. - 2010-EIA, NGM (April 2012), Table 4. • 2011-EIA estimate based on volume and revenue data from U.S. Department of Energy, Otfice of Fossil Energy. Crude Oil and Petroleum Products: - 1949-1962-Bureau of the Census, U.S. Imports of Merchandise for Consumption, FT110. - 1963-Bureau of the Census, U.S. Imports of Merchandise for Consumption, FT125. - 1964-1988-Bureau of the Census, U.S. Imports for Consumption, FT135. • 1989 forward-Bureau of

Principal SITC Commodity Groupings," Annual Revisions and December 2011 issue. 


\section{Figure 3.8 Value of Fossil Fuel Exports}

\section{Total, 1949-2011}

$120-$

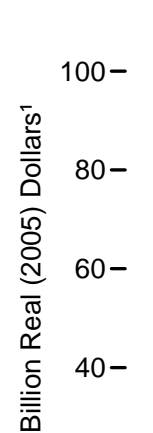

$\bar{m}$

$20-$

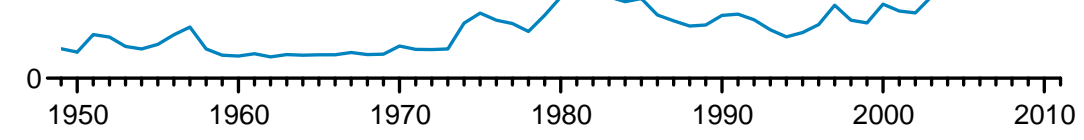

By Fuel, 2011

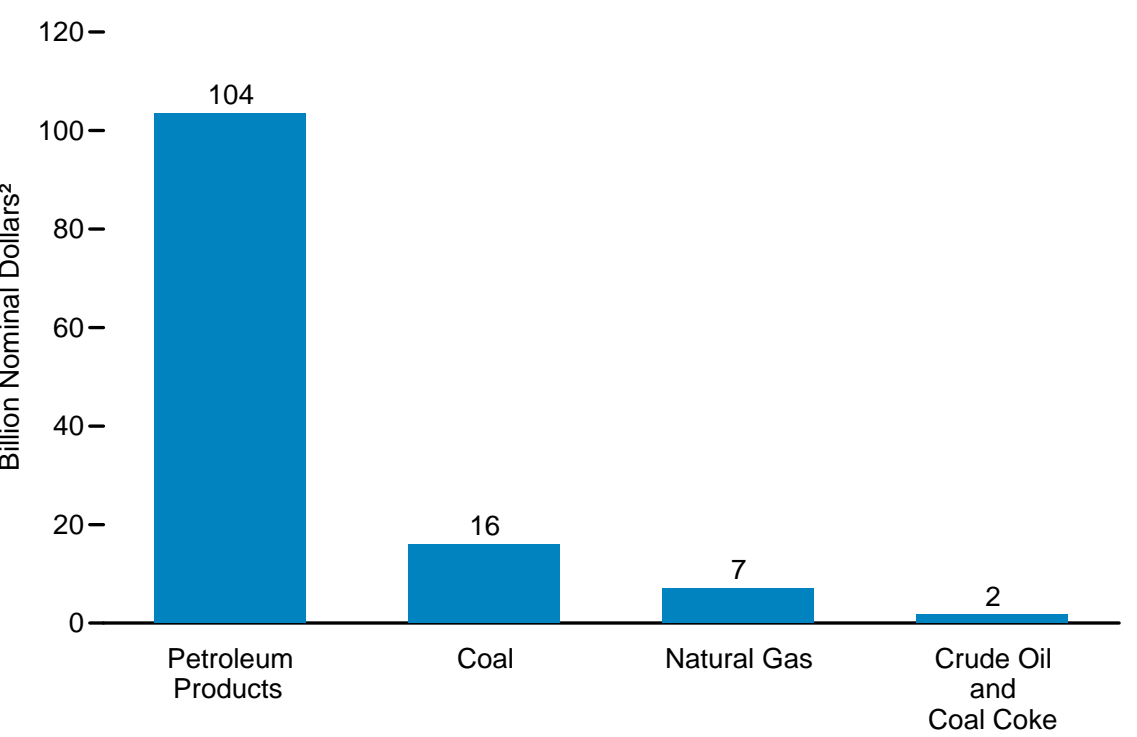

By Fuel, 1949-2011

$100-$

$90-$

$80-$

$70-$

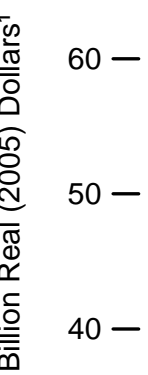

$30-$

$20-$

$10-$

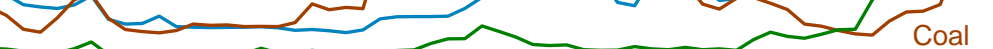

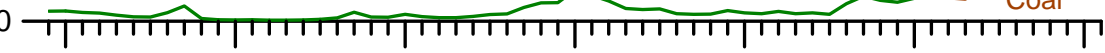

$\begin{array}{llllll}1950 & 1960 & 1970 & 1980 & 1990 & 2000\end{array}$

2010

${ }^{1}$ In chained (2005) dollars, calculated by using gross domestic product implicit price deflators in Table D1. See "Chained Dollars" in Glossary.

${ }^{3}$ Natural gas, crude oil, and coal coke

${ }^{2}$ See "Nominal Dollars" in Glossary. 
Table 3.8 Value of Fossil Fuel Exports, Selected Years, 1949-2011

(Billion Dollars)

\begin{tabular}{|c|c|c|c|c|c|c|c|c|c|c|c|c|}
\hline \multirow[b]{2}{*}{ Year } & \multicolumn{2}{|c|}{ Coal } & \multicolumn{2}{|c|}{ Coal Coke } & \multicolumn{2}{|c|}{ Natural Gas } & \multicolumn{2}{|c|}{ Crude Oil } & \multicolumn{2}{|c|}{ Petroleum Products 1} & \multicolumn{2}{|c|}{ Total } \\
\hline & Nominal $^{2}$ & Real $^{3}$ & Nominal $^{2}$ & Real $^{3}$ & Nominal $^{2}$ & Real $^{3}$ & Nominal $^{2}$ & Real $^{3}$ & Nominal $^{2}$ & Real $^{3}$ & Nominal $^{2}$ & Real $^{3}$ \\
\hline 1949 & 0.30 & 2.05 & 0.01 & 0.06 & (s) & 0.01 & 0.10 & 0.68 & 0.46 & $\mathrm{R}_{3.18}$ & 0.87 & $\mathrm{R}_{5} .98$ \\
\hline 1950 & .27 & 1.84 & .01 & .04 & (s) & .02 & .10 & .70 & .39 & 2.69 & .78 & R5.29 \\
\hline 1955 & .48 & 2.92 & .01 & .05 & .01 & .04 & .04 & .23 & .60 & 3.61 & 1.14 & $\mathrm{R}_{6} 6.85$ \\
\hline 1960 & .35 & 1.90 & .01 & .04 & $(\mathrm{~s})$ & .02 & .01 & .04 & .47 & $\begin{array}{l}2.01 \\
2.51\end{array}$ & .84 & 4.51 \\
\hline 1965 & .48 & 2.39 & .02 & .08 & .01 & .04 & $(\mathrm{~s})$ & .02 & .44 & 2.21 & .95 & 4.74 \\
\hline 1970 & .96 & 3.95 & .08 & .32 & .03 & .12 & .02 & .08 & .50 & 2.06 & 1.59 & $\mathrm{R}_{6} .53$ \\
\hline 1975 & 3.26 & R9.70 & .07 & .22 & .09 & .27 & (s) & (s) & 1.01 & 3.00 & 4.43 & $\mathrm{R}_{13.19}$ \\
\hline 1976 & 2.91 & $\mathrm{R}_{8.19}$ & .07 & .19 & .10 & .28 & .03 & .08 & 1.07 & $\begin{array}{l}3.01 \\
3.01\end{array}$ & 4.17 & $R_{11.75}$ \\
\hline 1977 & 2.66 & 7.03 & .07 & .19 & .11 & .28 & .21 & .55 & 1.14 & 3.01 & 4.18 & $R_{11.07}$ \\
\hline 1978 & 2.05 & 5.07 & .05 & .12 & .11 & .28 & .39 & .96 & 1.23 & 3.05 & 3.83 & R9.48 \\
\hline 1979 & 3.40 & 7.76 & .08 & .18 & .13 & .29 & .39 & .90 & 1.58 & 3.62 & 5.58 & $\mathrm{R}_{12.75}$ \\
\hline 1980 & 4.63 & R9.68 & .13 & .27 & .23 & .48 & .75 & 1.57 & $\begin{array}{l}2.12 \\
\text {. }\end{array}$ & 4.44 & 7.86 & $R_{16.44}$ \\
\hline 1981 & 5.92 & $R_{11.32}$ & .07 & .14 & .35 & .67 & .58 & $\begin{array}{l}1.10 \\
1.10\end{array}$ & 3.24 & $\begin{array}{r}R_{6.19}\end{array}$ & 10.16 & $R_{19.43}$ \\
\hline 1982 & 5.99 & $R_{10.80}$ & .06 & .11 & .30 & .54 & .47 & .85 & 5.86 & $\mathrm{R}_{10.57}$ & 12.68 & $R_{22.87}$ \\
\hline 1983 & 4.06 & 7.04 & .05 & .08 & .28 & .48 & .22 & .39 & 4.88 & $R_{8.46}$ & 9.48 & $\mathrm{R}_{16.45}$ \\
\hline 1984 & 4.13 & 6.91 & .07 & .12 & .27 & .45 & .19 & .31 & $\begin{array}{l}4.62 \\
4.62\end{array}$ & 7.72 & $\begin{array}{l}9.40 \\
9.27\end{array}$ & $R_{15.50}$ \\
\hline 1985 & 4.47 & 7.25 & .08 & .12 & .26 & .43 & .23 & .37 & 4.90 & $\mathrm{R}^{\mathrm{R}} 7.94$ & 9.93 & $\mathrm{R}_{16.11}$ \\
\hline 1986 & 3.93 & 6.24 & .07 & .10 & .17 & .27 & .12 & .19 & 3.77 & 5.98 & 8.05 & $\mathrm{R}_{12.78}$ \\
\hline 1987 & 3.40 & $\mathrm{R}_{5} .25$ & .05 & .07 & .17 & .26 & .13 & .19 & 3.80 & 5.86 & 7.54 & $\mathrm{R}_{11.63}$ \\
\hline 1988 & $\begin{array}{l}4.401 \\
4.01\end{array}$ & 5.99 & .08 & .12 & .20 & .30 & .08 & .12 & $\begin{array}{l}2.00 \\
2.72\end{array}$ & $\mathrm{R}_{4} .06$ & $\begin{array}{l}7.09 \\
\end{array}$ & $R_{10.58}$ \\
\hline 1989 & 4.29 & $\mathrm{R}_{6.16}$ & .08 & .12 & .27 & .39 & .21 & .30 & 2.65 & $\mathrm{R}_{3.81}$ & $\begin{array}{l}7.49 \\
\end{array}$ & R10.77 \\
\hline 1990 & 4.51 & $\mathrm{R}_{6.24}$ & .05 & .07 & .27 & .37 & .14 & .19 & 4.23 & 5.86 & 9.20 & $R_{12.73}$ \\
\hline 1991 & 4.62 & $\mathrm{R}_{6.17}$ & .05 & .07 & .33 & .45 & .03 & .04 & 4.65 & 6.22 & 9.69 & $\mathrm{R}_{12.95}$ \\
\hline 1992 & $\begin{array}{l}4.024 \\
4.24\end{array}$ & $\mathrm{R}_{5} .53$ & .04 & .06 & .49 & .64 & .03 & .04 & 4.27 & $\mathrm{R}_{5} .57$ & $\begin{array}{l}9.07 \\
9.07\end{array}$ & $R_{11.84}$ \\
\hline 1993 & $\begin{array}{l}3.09 \\
3.09\end{array}$ & R3.94 & .06 & .08 & .36 & .46 & .02 & .03 & 4.15 & 5.30 & 7.68 & 9.81 \\
\hline 1994 & 2.85 & $\mathrm{R}_{3.56}$ & .04 & .05 & .40 & .51 & .05 & .06 & 3.36 & 4.21 & $\mathrm{R}_{6.70}$ & R8.39 \\
\hline 1995 & 3.57 & 4.37 & .05 & .06 & .37 & .45 & .01 & .01 & 3.56 & $\mathrm{R}_{4} .36$ & 7.55 & R9.25 \\
\hline 1996 & $\begin{array}{l}3.69 \\
3.69\end{array}$ & $\mathrm{R}_{4.43}$ & .06 & .07 & .46 & .55 & .56 & .67 & 4.25 & 5.12 & 9.02 & $\mathrm{R}_{10.84}$ \\
\hline 1997 & 3.39 & $\mathrm{R}_{4} 4.00$ & .05 & .06 & .47 & .56 & 1.04 & 1.23 & 47.55 & 4,R8.92 & 12.51 & $\mathrm{R}_{14.78}$ \\
\hline 1998 & 3.04 & 3.55 & .04 & .05 & .39 & .46 & .90 & 1.05 & 5.68 & 6.64 & 10.04 & $R_{11.74}$ \\
\hline 1999 & 2.13 & 2.46 & .03 & .03 & .43 & .49 & .77 & .89 & 6.35 & 7.31 & 9.71 & $R_{11.18}$ \\
\hline 2000 & 2.04 & 2.30 & .05 & .06 & $\begin{array}{l}.40 \\
1.00\end{array}$ & 1.13 & .46 & .52 & 9.73 & 10.97 & 13.28 & $\mathrm{R}_{14.97}$ \\
\hline 2001 & 1.80 & 1.98 & .11 & .12 & 1.56 & $\mathrm{R}_{1.72}$ & .19 & .21 & 8.68 & R9. 57 & 12.34 & $\mathrm{R}_{13.60}$ \\
\hline 2002 & 1.60 & 1.74 & .06 & .07 & 1.76 & 1.91 & .09 & .10 & 8.65 & R9.38 & 12.17 & $R_{13.20}$ \\
\hline 2003 & 1.55 & 1.64 & .07 & .07 & 3.77 & 4.00 & .16 & .17 & 10.05 & 10.68 & 15.59 & $R_{16.56}$ \\
\hline 2004 & 2.60 & $\begin{array}{l}2.04 \\
2.68\end{array}$ & .11 & .11 & 5.20 & R5.37 & .28 & .29 & 12.85 & 13.28 & 21.04 & $R_{21.73}$ \\
\hline 2005 & 3.35 & $\begin{array}{l}3.00 \\
3.35\end{array}$ & .15 & .15 & 5.53 & 5.53 & $\begin{array}{l}.20 \\
.60\end{array}$ & .60 & 18.56 & 18.56 & 28.18 & 28.18 \\
\hline 2006 & 3.52 & 3.41 & .13 & .12 & 4.94 & 4.79 & .85 & .83 & 27.32 & 26.46 & 36.77 & 35.61 \\
\hline 2007 & 4.16 & 3.91 & .13 & .12 & 5.69 & R5.36 & 1.02 & R. .96 & 32.28 & $R_{30.39}$ & 43.27 & $\mathrm{R}_{40.74}$ \\
\hline 2008 & 7.96 & 7.33 & .21 & .19 & 8.26 & 7.61 & 2.27 & 2.09 & 59.43 & R54.73 & 78.13 & R71.96 \\
\hline 2009 & 5.99 & $\mathrm{R}_{5.46}$ & .14 & .12 & 4.79 & 4.37 & 1.62 & $\mathrm{R}_{1.47}$ & 42.89 & R39.09 & 55.43 & $\mathrm{R}_{50.52}$ \\
\hline 2010 & 9.84 & $R_{8.86}$ & .24 & .22 & $\mathrm{R}_{5} \mathrm{r} .71$ & $\begin{array}{r}4.01 \\
\mathrm{R}_{5} .14\end{array}$ & $\mathrm{R}_{1.37}$ & $\begin{array}{r}\mathrm{R}_{1} .23 \\
\end{array}$ & $\mathrm{R}_{63.41}$ & $\begin{array}{r}\text { R57.13 } \\
\text { R }\end{array}$ & R80.57 & 72.59 \\
\hline 2011 & $P_{15} .97$ & $P_{14.08}$ & P. 21 & Р.19 & E7.01 & $\mathrm{E}_{6.18}$ & $\mathrm{P} 1.46$ & $P_{1} .29$ & $\mathrm{P}_{103.54}$ & P91.34 & $\mathrm{P} 128.18$ & $P_{113.08}$ \\
\hline
\end{tabular}

1 Includes petroleum preparations, liquefied propane and butane, and, beginning in 1997, other mineral fuels.

See "Nominal Dollars" in Glossary

In chained (2005) dollars, calculated by using gross domestic product implicit price deflators in Table D1. See "Chained Dollars" in Glossary.

4 There is a discontinuity in this time series between 1996 and 1997 due to the addition of the commodity category "Other Mineral Fuels."

$R=$ Revised. $P=$ Preliminary. E=Estimate. (s)=Less than 0.005 billion

Notes: - Includes value of exports from Puerto Rico to foreign countries; excludes shipments from the 50 States and the District of Columbia to the Virgin Islands and Puerto Rico. - Totals may not equal sum of components due to independent rounding.
Sources: Coal and Coal Coke: Bureau of the Census, Foreign Trade Division, unpublished data. Natural Gas: - 1949-1971-Bureau of the Census, U.S. Exports, FT410 • 1972 and 1973-Federal Power Commission (FPC), Pipeline Imports and Exports of Natural Gas - Imports and Exports of LNG. 1978-1981-FPC, United States Imports and Exports of Natural Gas, annual reports. nnual reports. - 1982-2009-EIA, Natural Gas Monthly (NGM). Imports and Exports of Natural Gas, (April 2012), Table 5. - 2011-EIA estimate based on volume and revenue data from U.S. Department of Energy, Office of Fossil Energy. Crude Oil and Petroleum Products: - 1949-1988-Bureau of the Census, U.S. Exports, FT410. - 1989 forward-Bureau of the Census, Foreign Trade Division, U.S. Merchandise Trade, FT900, "Exports and Imports of Goods by Principal SITC Commodity Groupings,"
Annual Revisions and December 2011 issue. 


\section{Figure 3.9 Value of Fossil Fuel Net Imports, 1949-2011}

\section{Value of Fossil Fuel Net Imports}

400-

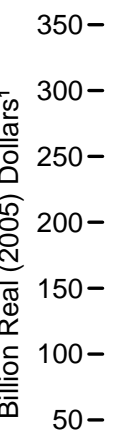

$$
0
$$

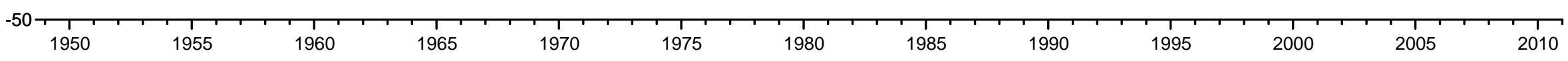

\section{Value of Fossil Fuel Net Imports by Fuel}

$$
400-
$$

$350-$

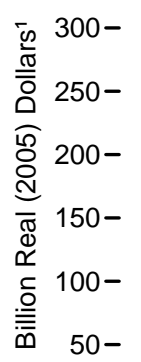

$$
\text { 50- }
$$

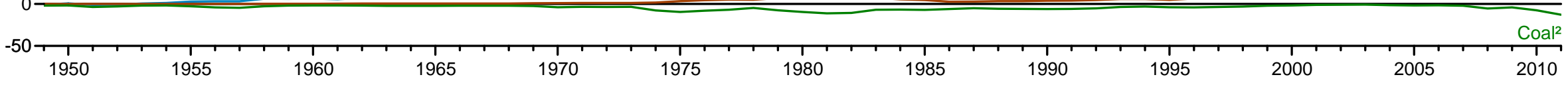

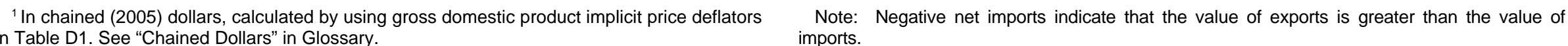
in Table D1. See "Chained Dollars" in Glossary.

${ }^{2}$ Includes small amounts of coal coke. Source: Table 3.9. 
Table 3.9 Value of Fossil Fuel Net Imports, Selected Years, 1949-2011

(Billion Dollars)

\begin{tabular}{|c|c|c|c|c|c|c|c|c|c|c|c|c|}
\hline \multirow[b]{2}{*}{ Year } & \multicolumn{2}{|c|}{ Coal } & \multicolumn{2}{|c|}{ Coal Coke } & \multicolumn{2}{|c|}{ Natural Gas } & \multicolumn{2}{|c|}{ Crude Oil } & \multicolumn{2}{|c|}{ Petroleum Products 1} & \multicolumn{2}{|c|}{ Total } \\
\hline & Nominal $^{2}$ & Real $^{3}$ & Nominal $^{2}$ & Real $^{3}$ & Nominal ${ }^{2}$ & Real $^{3}$ & Nominal ${ }^{2}$ & Real $^{3}$ & Nominal $^{2}$ & Real $^{3}$ & Nominal $^{2}$ & Real $^{3}$ \\
\hline 1949 & -0.29 & $R_{-}-2.03$ & (s) & -0.03 & (s) & -0.01 & 0.21 & 1.42 & -0.32 & -2.24 & -0.42 & -2.89 \\
\hline 1950 & -.27 & -1.82 & (s) & -.01 & (s) & -.02 & .27 & 1.82 & -.18 & -1.23 & -.18 & -1.26 \\
\hline 1955 & -.48 & $\mathrm{R}_{-2.90}$ & -.01 & -.04 & -.01 & -.03 & .62 & 3.71 & -.16 & -.95 & -.04 & -.22 \\
\hline 1960 & -.35 & -1.89 & -.01 & -.03 & .02 & .13 & .89 & 4.77 & .26 & 1.42 & .82 & 4.40 \\
\hline 1965 & -.48 & $\mathrm{R}_{-2.38}$ & -.01 & -.07 & .10 & .49 & 1.11 & $\mathrm{R}_{5.59}$ & .48 & 2.43 & 1.21 & $\mathrm{R}_{6.05}$ \\
\hline 1970 & -.96 & -3.95 & -.08 & -.31 & .23 & R. 93 & 1.24 & R5.10 & .98 & 4.03 & 1.41 & 5.81 \\
\hline 1975 & -3.24 & -9.64 & .08 & .24 & 1.06 & 3.16 & 18.29 & R54.45 & 5.76 & $\mathrm{R}_{17.15}$ & 21.96 & ${ }^{R} 65.36$ \\
\hline 1976 & -2.89 & $\mathrm{R}_{-8.14}$ & .04 & .12 & 1.56 & 4.39 & 25.43 & $\mathrm{R}_{71.59}$ & 5.58 & $\mathrm{R}_{15.71}$ & 29.72 & R83.68 \\
\hline 1977 & -2.62 & $\mathrm{R}_{-6.92}$ & .06 & .16 & 1.89 & $\mathrm{R}_{5.01}$ & 33.38 & R88.35 & 7.28 & R19.26 & 40.00 & $R_{105.86}$ \\
\hline 1978 & -1.98 & $\mathrm{R}_{-4.88}$ & .36 & .89 & 1.95 & 4.82 & 31.91 & R78.91 & 6.07 & $\mathrm{R}_{15.01}$ & 38.31 & R94.75 \\
\hline 1979 & -3.35 & $\mathrm{R}_{-7.64}$ & .26 & .59 & 3.00 & 6.85 & 45.66 & $\mathrm{R}_{104.26}$ & 8.87 & $\mathrm{R}_{20.24}$ & 54.44 & $\mathrm{R}_{124.30}$ \\
\hline 1980 & -4.60 & $\mathrm{R}_{-}^{\mathrm{R}}-9.62$ & -.08 & -.16 & 3.98 & 8.34 & 61.15 & $\mathrm{R}_{127.95}$ & 10.42 & $\mathrm{R}_{21.80}$ & 70.88 & $R_{148.31}$ \\
\hline 1981 & -5.89 & $\mathrm{R}_{-}-11.26$ & -.03 & -.06 & 4.06 & R7.77 & 60.88 & $R_{116.47}$ & 11.06 & $\mathrm{R}_{21.16}$ & 70.09 & $\mathrm{R}_{134.08}$ \\
\hline 1982 & -5.97 & $\mathrm{R}_{-10.76}$ & -.05 & -.09 & 4.39 & R7.92 & 45.25 & R81.60 & 8.00 & $\mathrm{R}_{14.43}$ & 51.63 & R93.09 \\
\hline 1983 & -4.01 & ${ }^{R}-6.96$ & -.04 & -.08 & 4.11 & 7.13 & 36.27 & ${ }^{\mathrm{R}} 62.91$ & 9.96 & $\mathrm{R}_{17.28}$ & 46.28 & $\mathrm{R}_{80.28}$ \\
\hline 1984 & -4.09 & $\mathrm{R}_{-6.83}$ & -.02 & -.04 & 3.17 & 5.30 & 36.26 & R 60.62 & 13.25 & $\mathrm{R}_{22.15}$ & 48.57 & $\mathrm{R}_{81.19}$ \\
\hline 1985 & -4.39 & $\mathrm{R}_{-7.13}$ & -.03 & $\mathrm{R}_{-.05}$ & $\mathrm{R}_{2.78}$ & 4.52 & 32.68 & R53.02 & 12.57 & $\mathrm{R}_{20.40}$ & 43.60 & R70.75 \\
\hline 1986 & -3.85 & ${ }^{\mathrm{R}}-6.11$ & -.04 & -.06 & 1.65 & 2.62 & 22.49 & $\mathrm{R}_{35.71}$ & 8.42 & $\mathrm{R}_{13.36}$ & 28.67 & $\mathrm{R}_{45.51}$ \\
\hline 1987 & -3.35 & -5.17 & .01 & .01 & $\mathrm{R}_{1.77}$ & $\mathrm{R}_{2.73}$ & 29.00 & $\mathrm{R}_{44.75}$ & 8.57 & $\mathrm{R}_{13.22}$ & 36.00 & $\mathrm{R}_{55.54}$ \\
\hline 1988 & -3.95 & -5.89 & .12 & .17 & 2.18 & 3.25 & 27.47 & R40.97 & 9.71 & $\mathrm{R}_{14.48}$ & 35.53 & R52.99 \\
\hline 1989 & -4.19 & ${ }^{R}-6.02$ & .14 & .20 & $\mathrm{R}_{2.25}$ & ${ }^{R} 3.23$ & 35.32 & $\mathrm{R}_{50.77}$ & 10.85 & $\mathrm{R}_{15.59}$ & ${ }^{\mathrm{R}} 44.36$ & ${ }^{\mathrm{R}} 63.76$ \\
\hline 1990 & -4.42 & $\mathrm{R}_{-6.11}$ & .02 & .03 & 2.71 & 3.75 & 43.65 & ${ }^{R} 60.40$ & 12.67 & $\mathrm{R}_{17.53}$ & 54.63 & R75.60 \\
\hline 1991 & -4.51 & $\mathrm{R}_{-6.02}$ & .04 & .06 & $\mathrm{R}_{2.91}$ & $R_{3.89}$ & 36.87 & $\mathrm{R}_{49.27}$ & 8.52 & $\mathrm{R}_{11.38}$ & $\mathrm{R}_{43.83}$ & R58.58 \\
\hline 1992 & -4.11 & -5.37 & .10 & .13 & 3.47 & $\mathrm{R}_{4.53}$ & 38.52 & ${ }^{R} 50.29$ & 7.72 & $\mathrm{R}_{10.07}$ & $\mathrm{R}_{45.69}$ & $R_{59.65}$ \\
\hline 1993 & -2.83 & -3.62 & .11 & .14 & 4.41 & R5.63 & 38.45 & $\mathrm{R}_{49.11}$ & 7.59 & 9.70 & 47.72 & ${ }^{R} 60.95$ \\
\hline 1994 & -2.58 & R-3.22 & .23 & .29 & 4.50 & 5.63 & 38.43 & R 48.07 & 7.78 & R9.73 & 48.37 & $\mathrm{R}_{60.51}$ \\
\hline 1995 & -3.24 & $\mathrm{R}_{-3.97}$ & .27 & .34 & 3.86 & $\mathrm{R}_{4.74}$ & 42.81 & $R_{52.46}$ & 6.39 & 7.83 & 50.09 & $\mathrm{R}_{61.38}$ \\
\hline 1996 & -3.41 & $\mathrm{R}_{-4.10}$ & .18 & .22 & 5.33 & ${ }^{R} 6.41$ & 54.37 & R65.38 & 11.01 & $\mathrm{R}_{13.24}$ & 67.49 & R81.15 \\
\hline 1997 & -3.13 & -3.70 & .20 & .23 & 6.02 & 7.12 & 53.19 & R62.85 & ${ }^{4} 9.37$ & $4, R_{11.08}$ & 65.65 & R77.58 \\
\hline 1998 & -2.75 & -3.22 & .25 & .29 & 5.82 & ${ }^{R} 6.80$ & 36.36 & $\mathrm{R}_{42.48}$ & 7.33 & $R_{8.57}$ & 47.00 & $R_{54.92}$ \\
\hline 1999 & -1.85 & -2.14 & .20 & .23 & 7.61 & 8.76 & 50.12 & R57.71 & 9.94 & $\mathrm{R}_{11.44}$ & 66.00 & R76.00 \\
\hline 2000 & -1.66 & -1.88 & .20 & .23 & 13.94 & $\mathrm{R}_{15.71}$ & 89.41 & $\mathrm{R}_{100.78}$ & 19.65 & $\mathrm{R}_{22.14}$ & 121.53 & $\mathrm{R}_{136.98}$ \\
\hline 2001 & -1.13 & -1.24 & .08 & .09 & 16.05 & $\mathrm{R}_{17.69}$ & 74.11 & R81.68 & 19.77 & $\mathrm{R}_{21.79}$ & 108.89 & $\mathrm{R}_{120.02}$ \\
\hline 2002 & -1.00 & -1.09 & .18 & .20 & $\mathrm{R}_{10.89}$ & $\mathrm{R}_{11.81}$ & 79.16 & R85.86 & 14.87 & $\mathrm{R}_{16.13}$ & $\mathrm{R}_{104.10}$ & $R_{112.91}$ \\
\hline 2003 & -.76 & -.81 & .17 & .18 & 16.62 & 17.66 & 101.64 & $R_{107.97}$ & 20.59 & $\mathrm{R}_{21.87}$ & 138.26 & $R_{146.87}$ \\
\hline 2004 & -1.57 & -1.63 & 1.12 & 1.16 & 19.54 & 20.19 & 135.75 & $\mathrm{R}_{140.26}$ & 30.38 & $\mathrm{R}_{31.39}$ & 185.23 & $R_{191.38}$ \\
\hline 2005 & -1.93 & -1.93 & .63 & .63 & 29.72 & 29.72 & 182.35 & 182.35 & 48.56 & 48.56 & 259.34 & 259.34 \\
\hline 2006 & -1.74 & -1.69 & .51 & .49 & 23.86 & $\mathrm{R}_{23.11}$ & 224.30 & $\mathrm{R}_{217.28}$ & 47.24 & $\mathrm{R}_{45.76}$ & 294.17 & $\mathrm{R}_{284.96}$ \\
\hline 2007 & -2.42 & -2.28 & .35 & .33 & 25.96 & $\mathrm{R}_{24.44}$ & 244.76 & $\mathrm{R}_{230.41}$ & 49.57 & ${ }^{\mathrm{R}} 46.67$ & 318.21 & $\mathrm{R}_{299.56}$ \\
\hline 2008 & -5.92 & -5.45 & 1.47 & 1.35 & 26.40 & $\mathrm{R}_{24.31}$ & 351.27 & $R_{323.50}$ & 36.89 & R33.97 & 410.10 & $R_{377.69}$ \\
\hline 2009 & -4.55 & $\mathrm{R}_{-4.14}$ & -.04 & -.04 & 10.92 & R9.96 & 192.99 & $R_{175.87}$ & 14.34 & $\mathrm{R}_{13.07}$ & 213.66 & $\mathrm{R}_{194.72}$ \\
\hline 2010 & -8.45 & $\mathrm{R}_{-7.61}$ & .16 & .14 & $\mathrm{R}_{11.20}$ & $\mathrm{R}_{10.09}$ & 258.74 & $R_{233.11}$ & R9.95 & R8.96 & $R_{271.60}$ & $R_{244.70}$ \\
\hline 2011 & $P_{-14.61}$ & $P_{-12.89}$ & P.34 & P. 30 & ${ }^{E} 7.60$ & ${ }^{E_{6}} 6.70$ & $P_{335.34}$ & $P_{295.81}$ & P-4.05 & ${ }^{P}-3.58$ & ${ }^{P_{324.61}}$ & $P_{286.35}$ \\
\hline
\end{tabular}

1 Includes petroleum preparations, liquefied propane and butane, and, beginning in 1997, other mineral fuels.

See "Nominal Dollars" in Glossary.

${ }^{3}$ In chained (2005) dollars, calculated by using gross domestic product implicit price deflators in Table D1. See "Chained Dollars" in Glossary.

4 There is a discontinuity in this time series between 1996 and 1997 due to the addition of the commodity category "Other Mineral Fuels."
$R=$ Revised. $P=$ Preliminary. E=Estimate. $(\mathrm{s})=$ Less than 0.005 billion and greater than -0.005 billion. Notes: - Net imports equal imports minus exports. Minus sign indicates that the value of exports is greater than the value of imports. - Totals may not equal sum of components due to independent rounding. - Data on this table may not equal data on Table 3.7 minus data on Table 3.8 due to independent rounding

Web Page: For all data beginning in 1949, see http://www.eia.gov/totalenergy/data/annual/\#financial. Sources: Tables 3.7 and 3.8. 


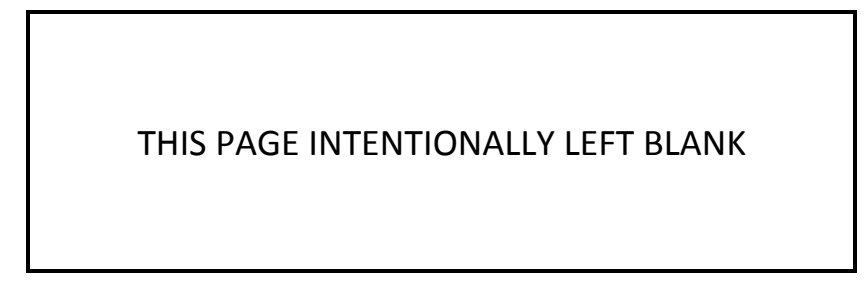




\section{Energy Resources}


Figure 4.1 Technically Recoverable Crude Oil and Natural Gas Resource Estimates, 2009

\section{Crude Oil and Lease Condensate, Total Technically} Recoverable Resources

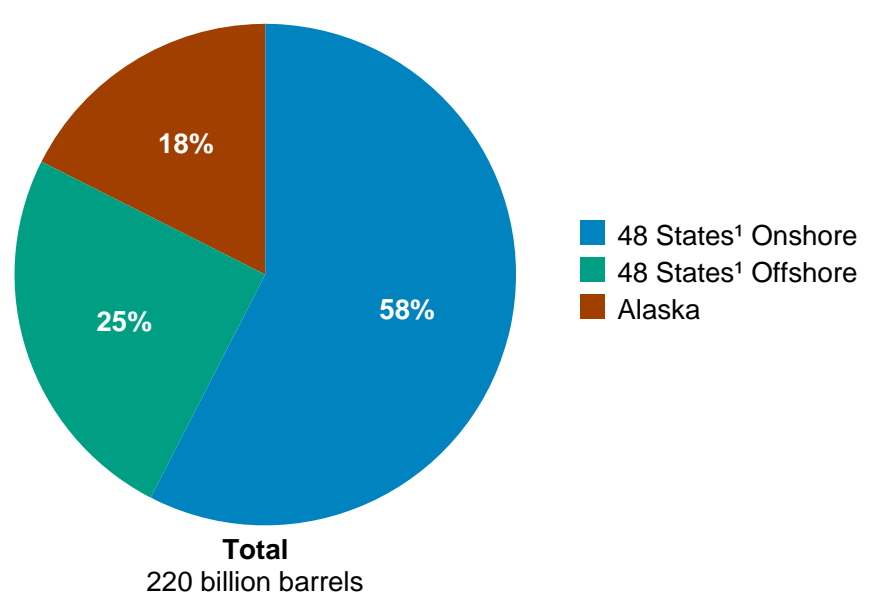

Crude Oil and Lease Condensate by Type

$250-$

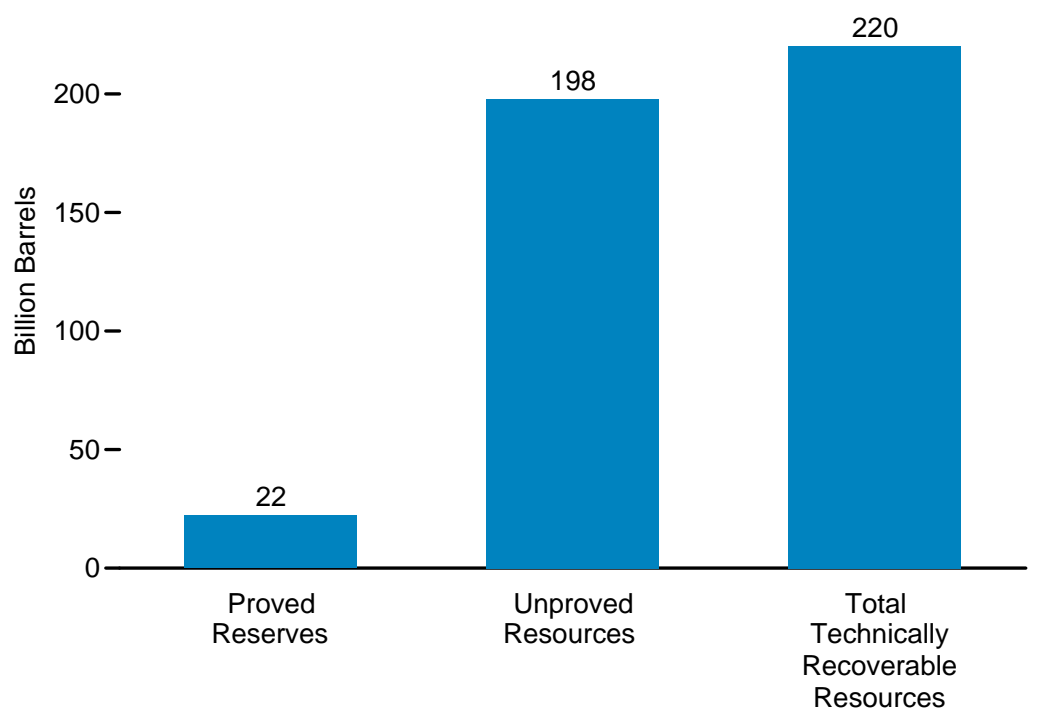

Dry Natural Gas, Total Technically Recoverable Resources

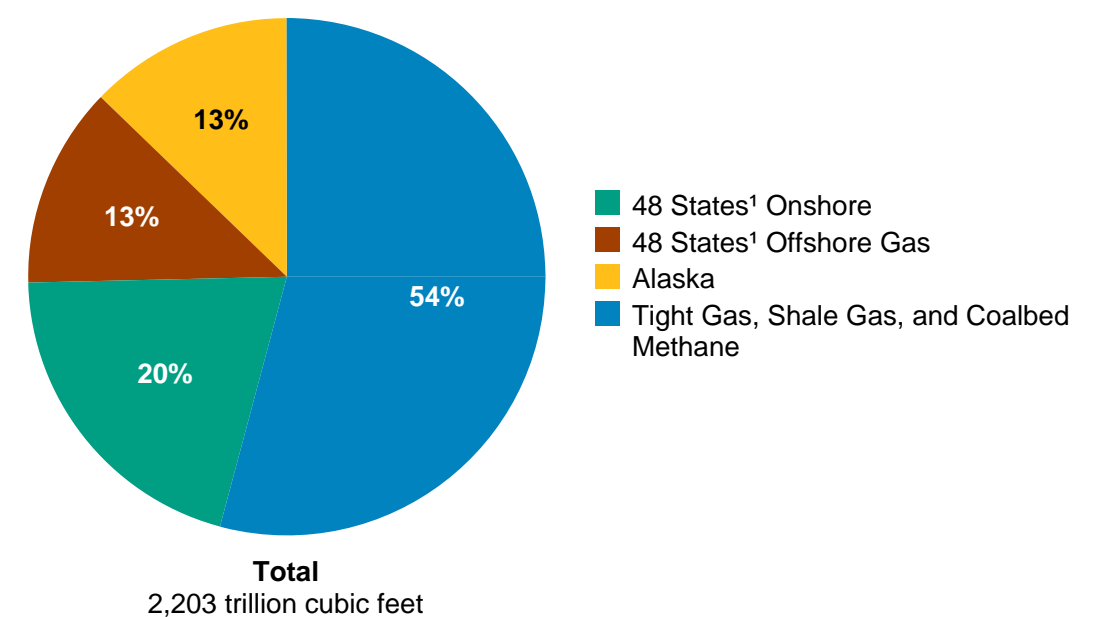

Dry Natural Gas by Type

$$
2,500-
$$

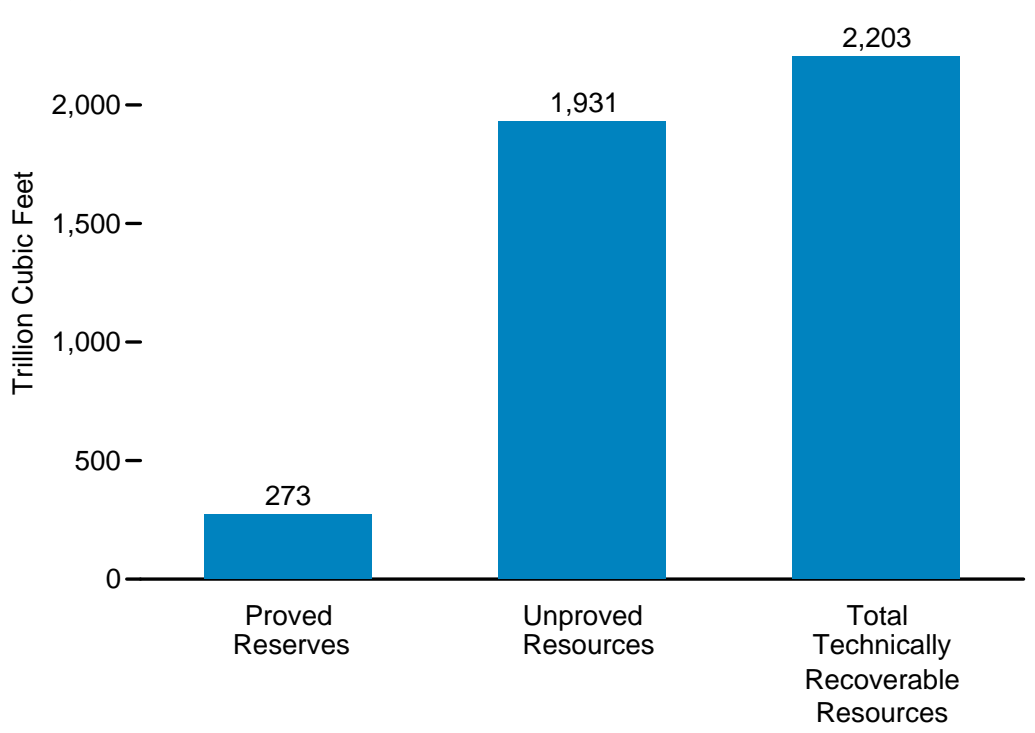

\footnotetext{
1 "48 States" is the United States excluding Alaska and Hawaii.

Note: Sum of components may not equal 100 percent due to independent rounding.
} 
Table 4.1 Technically Recoverable Crude Oil and Natural Gas Resource Estimates, 2009

\begin{tabular}{|c|c|c|c|}
\hline Region & Proved Reserves ${ }^{1}$ & Unproved Resources & $\begin{array}{l}\text { Total Technically } \\
\text { Recoverable Resources }\end{array}$ \\
\hline & \multicolumn{3}{|c|}{ Crude Oil and Lease Condensate (billion barrels) } \\
\hline 48 States $^{3}$ Onshore & 14.2 & 112.6 & 126.7 \\
\hline 48 States ${ }^{3}$ Offshore & 4.6 & 50.3 & 54.8 \\
\hline 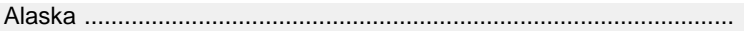 & 3.6 & 35.0 & 38.6 \\
\hline \multirow{2}{*}{ Total U.S. } & 22.3 & 197.9 & 220.2 \\
\hline & \multicolumn{3}{|c|}{ Dry Natural Gas ${ }^{4}$ (trillion cubic feet) } \\
\hline Conventionally Reservoired Fields ${ }^{5} \ldots \ldots \ldots$ & 105.5 & 904.0 & $1,009.5$ \\
\hline 48 States $^{3}$ Onshore Gas 6 & 81.4 & 369.7 & 451.1 \\
\hline 48 States $^{3}$ Offshore Gas ${ }^{7}$ & 15.0 & 262.6 & 277.6 \\
\hline Alaska & 9.1 & 271.7 & 280.8 \\
\hline 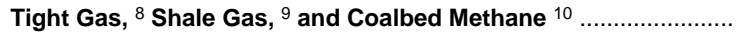 & 167.1 & $1,026.7$ & $1,193.8$ \\
\hline Total U.S. & 272.5 & $1,930.7$ & $2,203.3$ \\
\hline
\end{tabular}

1 See "Proved Reserves, Crude Oil," "Proved Reserves, Lease Condensate," and "Proved Reserves, Natural Gas" in Glossary.

2 "Technically recoverable" resources are those that are producible using current technology without reference to the economic viability thereof.

"48 States" is the United States excluding Alaska and Hawail.

Excludes natural gas plant liquids. See "Natural Gas, Dry" in Glossary.

5 Conventionally reservoired deposits are discrete subsurface accumulations of crude oil or natural gas usually defined, controlled, or limited by hydrocarbon/water contacts.

${ }^{6}$ Includes associated-dissolved (AD) natural gas that occurs in crude oil reservoirs either as free gas (associated) or as gas in solution with crude oil (dissolved gas).

Includes Federal offshore and State offshore waters (near-shore, shallow-water areas under State jurisdiction).

${ }^{8}$ Natural gas produced from a non-shale formation with extremely low permeability.
9 See "Shale Gas" in Glossary.

See "Coalbed Methane" in Glossary.

Notes: - See Tables 4.2 and 4.3 for more recent proved reserves data. - Data are at end of year. - Resources in areas where driling is officially prohibited are not included. Estimates of the resources in the Northern Allantic, Northern and Central Pacilic, and within a 50-mile buffer off the Mid and Southern Atlantic Outer Continental Shelf (OCS) are also excluded from the technically recoverable volumes. - Totals may not equal sum of components due to independent rounding.

Sources. Proved Reserves: U.S. Energy Information Administration (EIA), U.S. Crude Oil, Natural Gas, and Natural Gas Liquids Proved Reserves, 2010 (August 2012). Unproved Resources: U.S. Geological Survey National Oil and Gas Resource Assessment Team, with adjustments made to the shale gas data by EIA, Office of Energy Analysis. Total Technically Recoverable Resources: Calculated as the sum of proved reserves and unproved resources. 


\section{Figure 4.2 Crude Oil and Natural Gas Cumulative Production and Proved Reserves, 1977-2010}

\section{Crude Oil}

$250-$

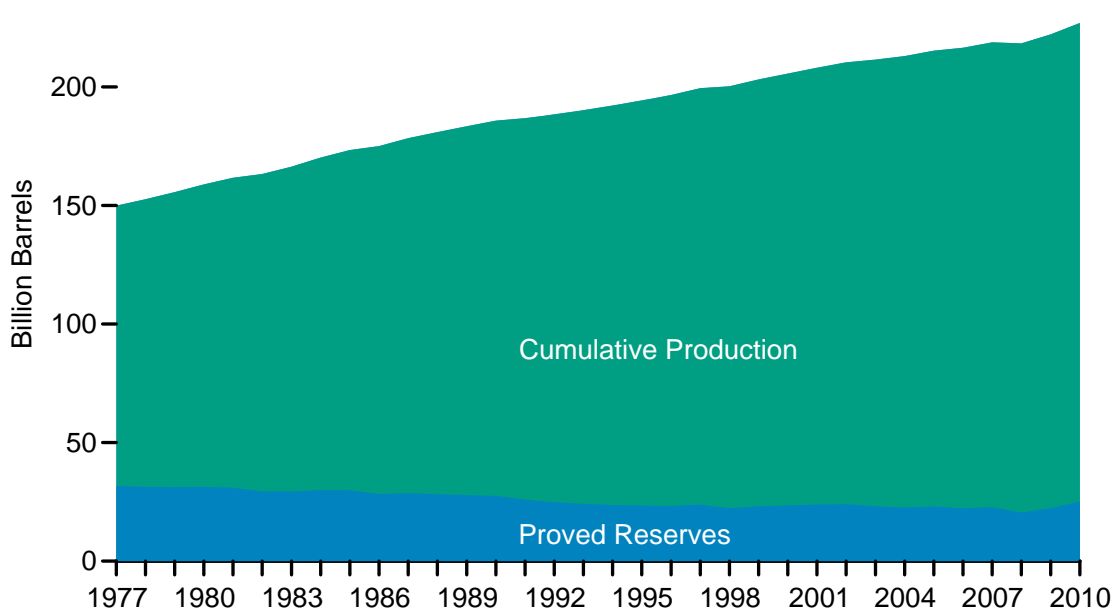

\section{Natural Gas (Dry)}

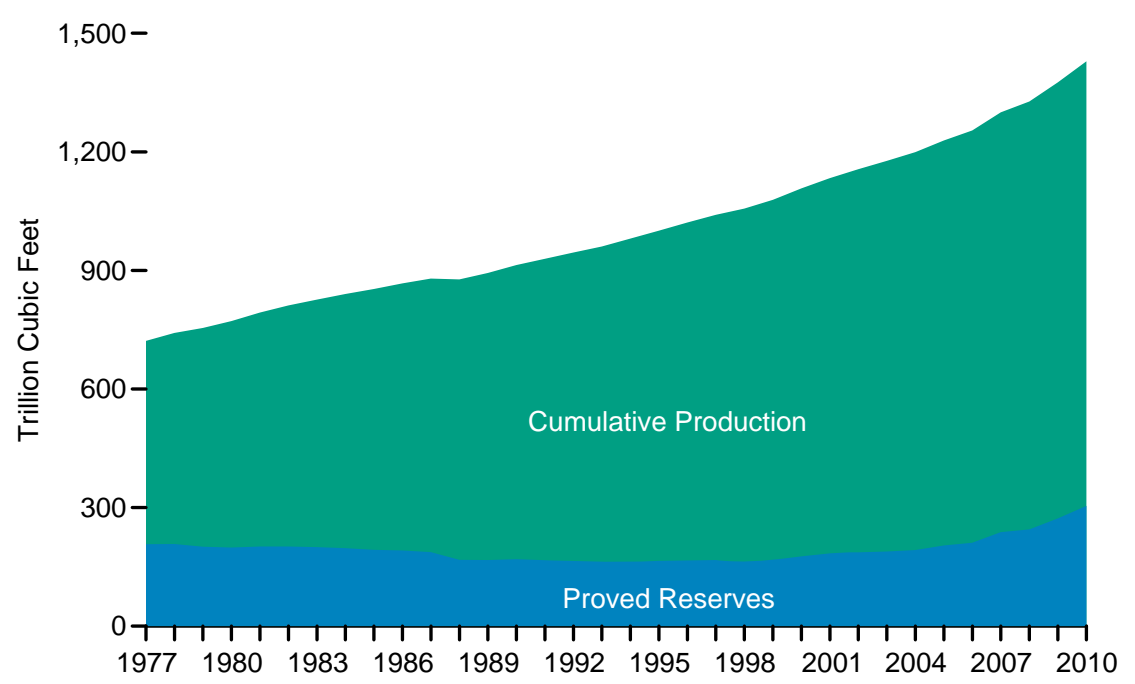

\section{Cumulative Production and Proved Reserves, Indexed}

$$
250-
$$

Natural Gas (Dry) Cumulative Production

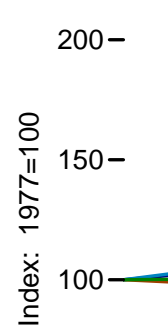

$50-$

\begin{tabular}{|c|c|c|c|c|c|c|c|c|c|c|c|}
\hline 0 T & 1 & 1 & 1 & 1 & 1 & 1 & 1 & 1 & 1 & 1 & $\neg$ \\
\hline 1977 & 1980 & 1983 & 1986 & 1989 & 1992 & 1995 & 1998 & 2001 & 2004 & 2007 & 2010 \\
\hline
\end{tabular}


Table 4.2 Crude Oil and Natural Gas Cumulative Production and Proved Reserves, 1977-2010

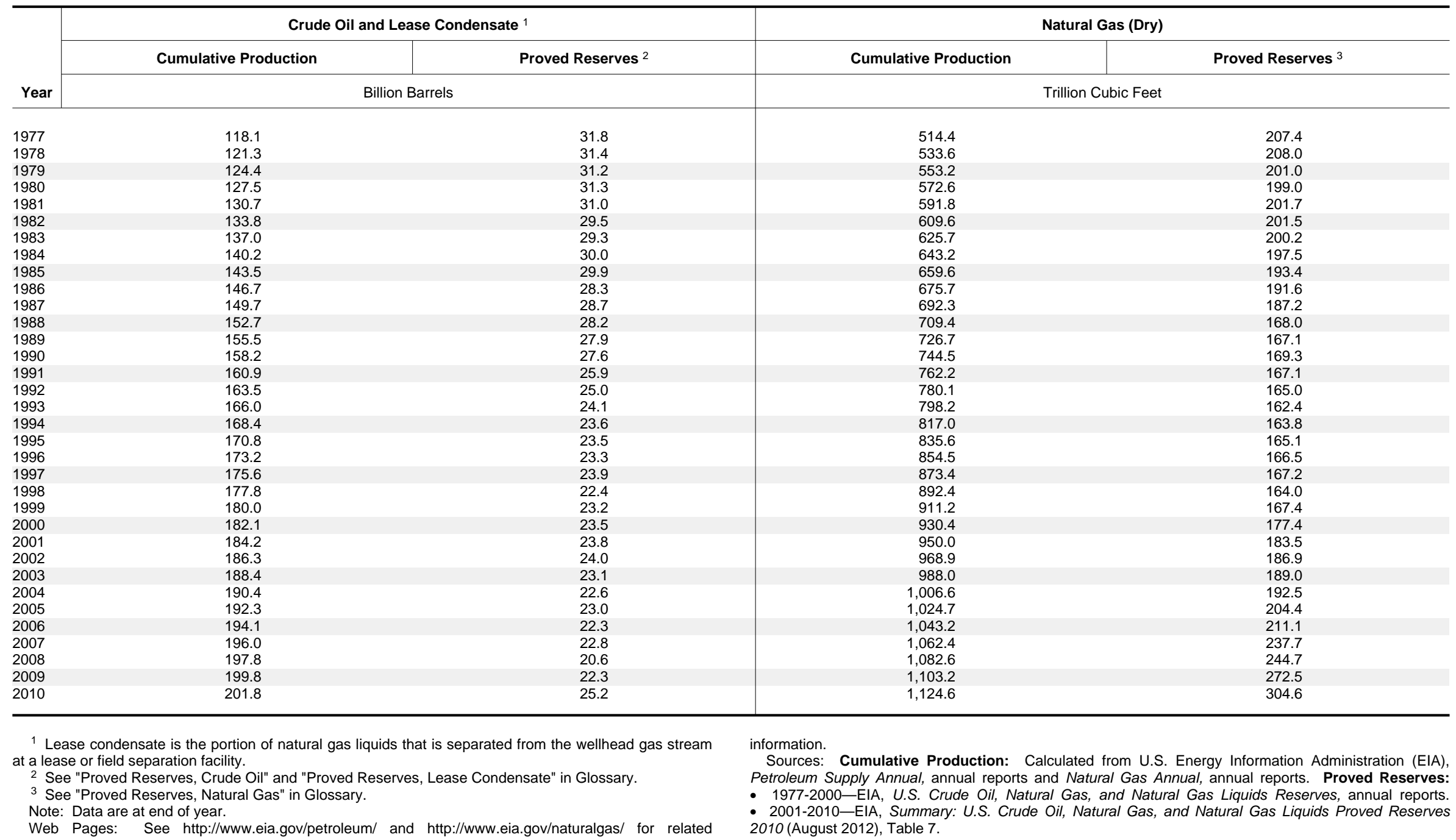




\section{Figure 4.3 Crude Oil, Natural Gas, and Natural Gas Liquids Proved Reserves}

\section{Total, 1949-2010}

$100-$

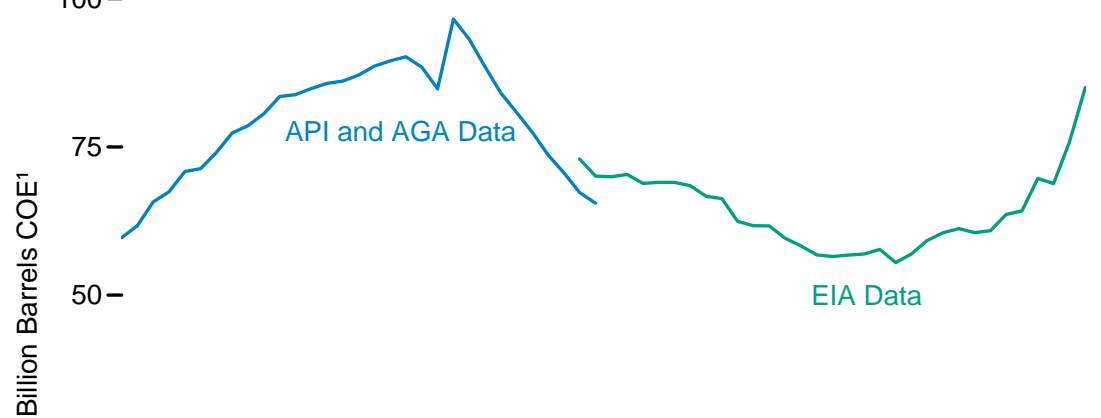

$25-$

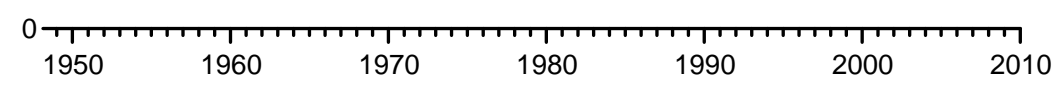

By Type, 2010

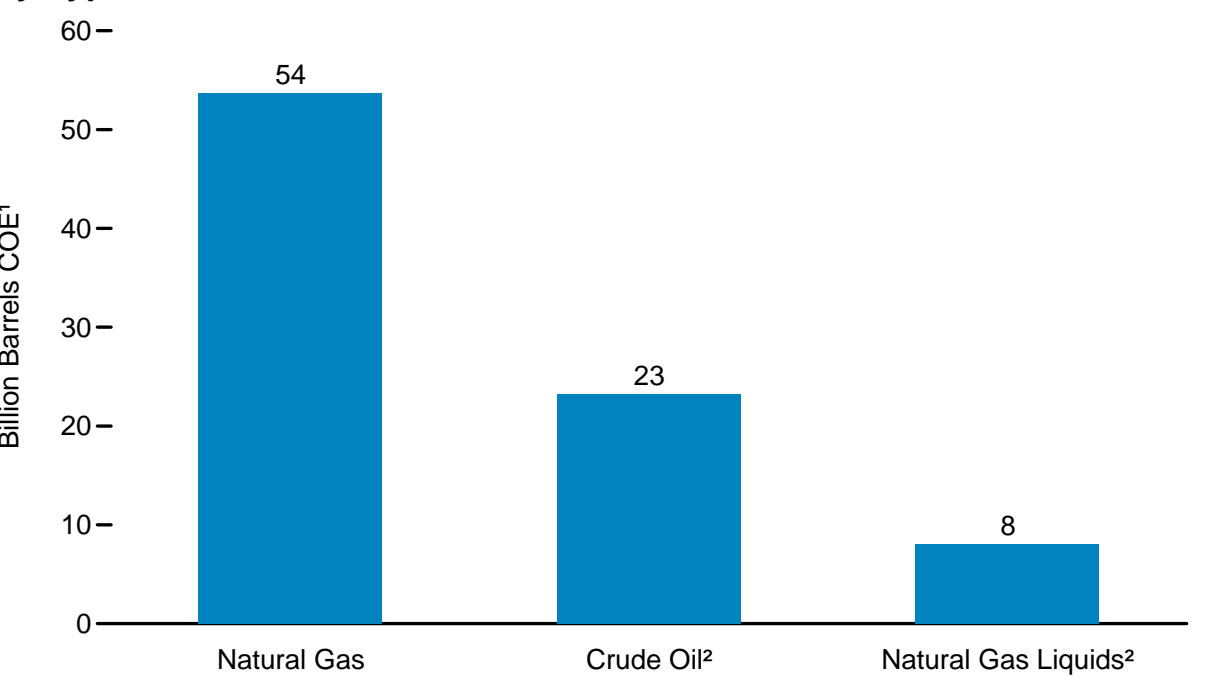

\section{Вy Type, 1949-2010}

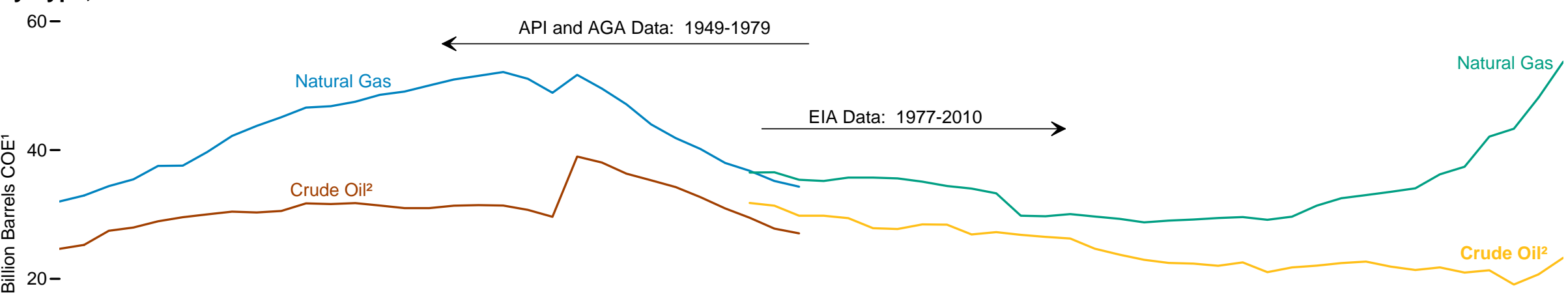

Natural Gas Liquids²

Natural Gas Liquids²

\begin{tabular}{|c|c|c|c|c|c|c|c|c|c|c|c|}
\hline $\begin{array}{c}1 \\
1950\end{array}$ & $\begin{array}{c}1955 \\
195\end{array}$ & $\begin{array}{l}1 \\
1960\end{array}$ & T' & ' & $\begin{array}{l} \\
1975\end{array}$ & $\begin{array}{l} \\
1980\end{array}$ & $\begin{array}{l}1985 \\
1985\end{array}$ & 'T' & $\begin{array}{c}1 \\
1995\end{array}$ & 'T & 2005 \\
\hline
\end{tabular}

${ }^{1} \mathrm{COE}=$ crude oil equivalent.

2 To the extent that lease condensate is measured or estimated it is included in "Natural Gas

Notes: - Data are at end of year. • API=American Petroleum Institute. AGA=American Gas Liquids"; otherwise, lease condensate is included in "Crude Oil."

Association. EIA=U.S. Energy Information Administration.

Source: Table 4.3. 
Table 4.3 Crude Oil, Natural Gas, and Natural Gas Liquids Proved Reserves, Selected Years, $1949-2010$

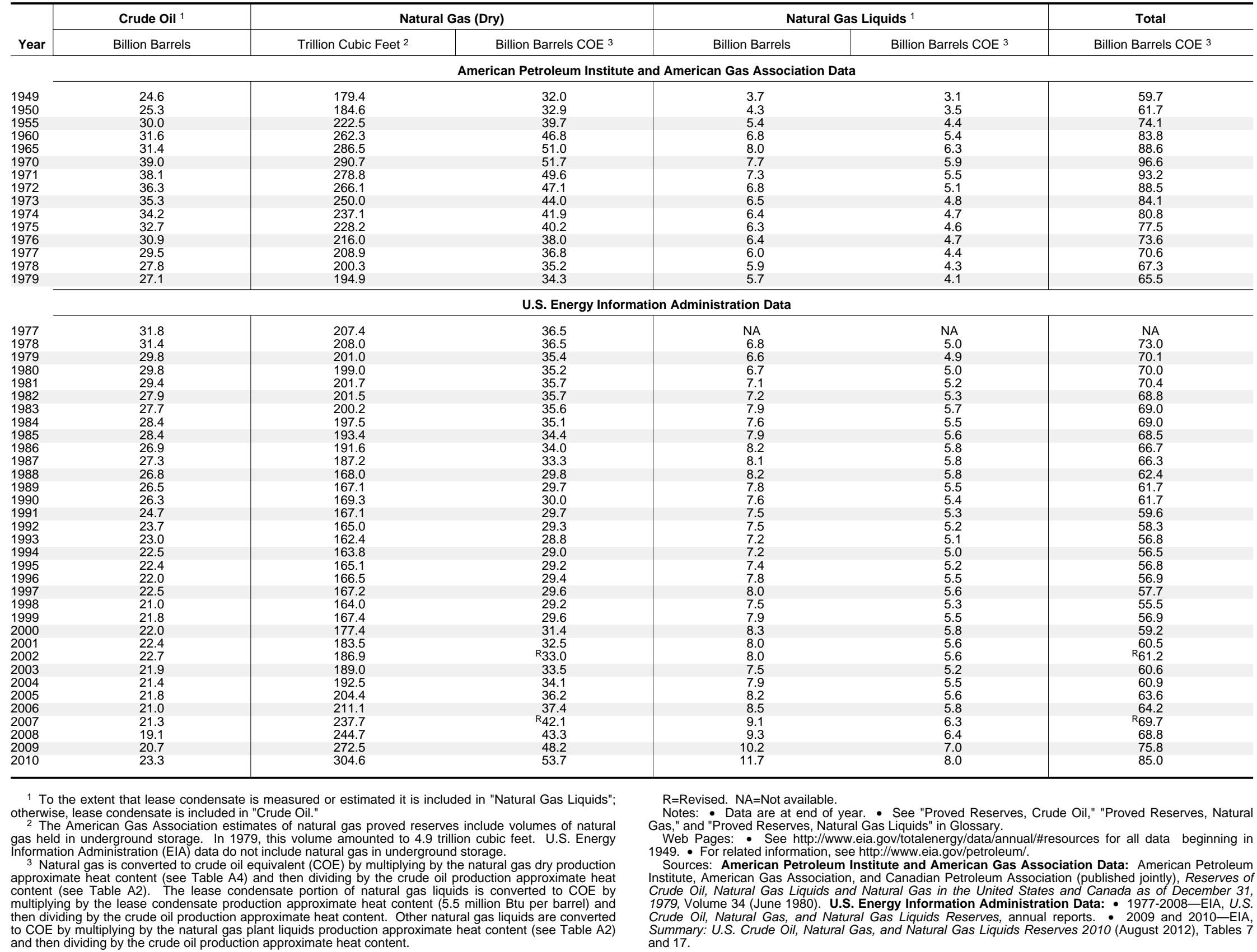


Figure 4.4 Crude Oil and Natural Gas Rotary Rigs in Operation

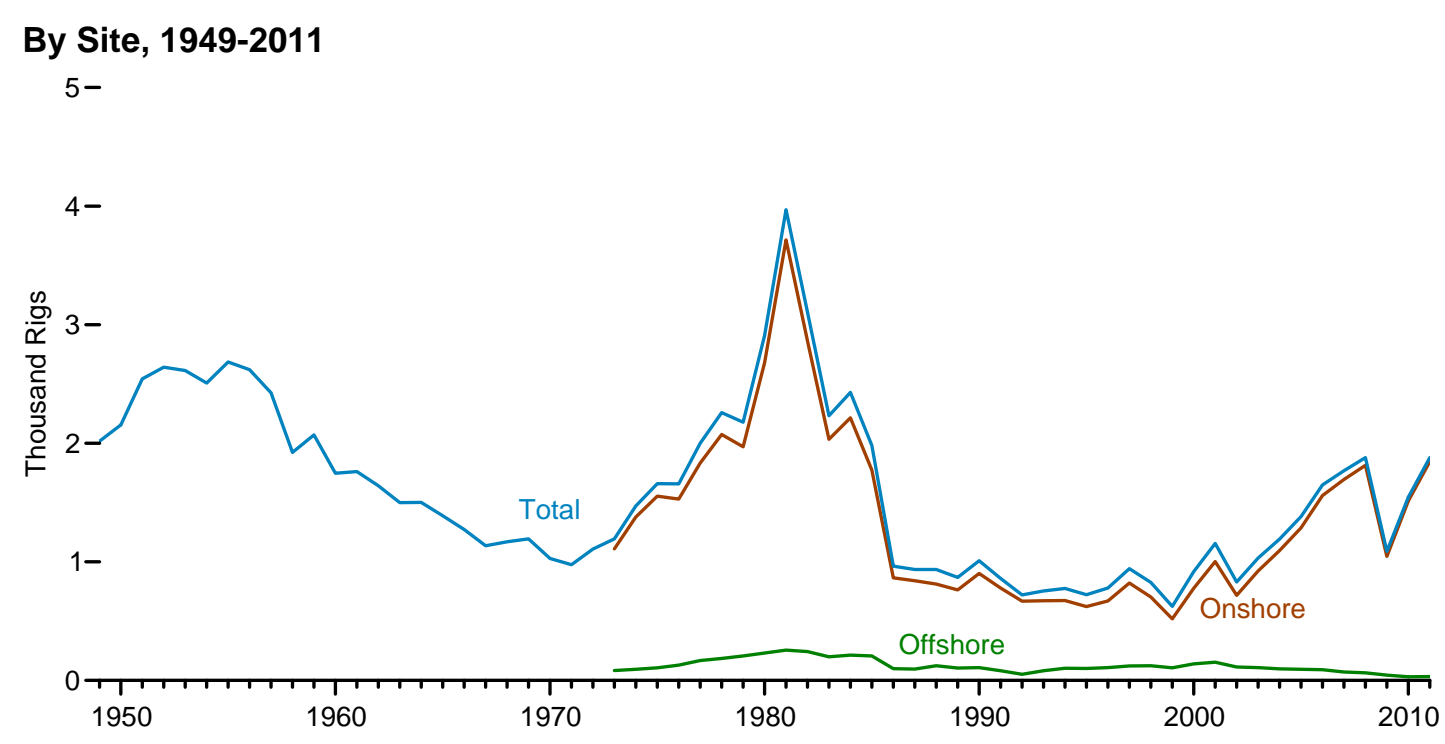

\section{By Site, 2011}

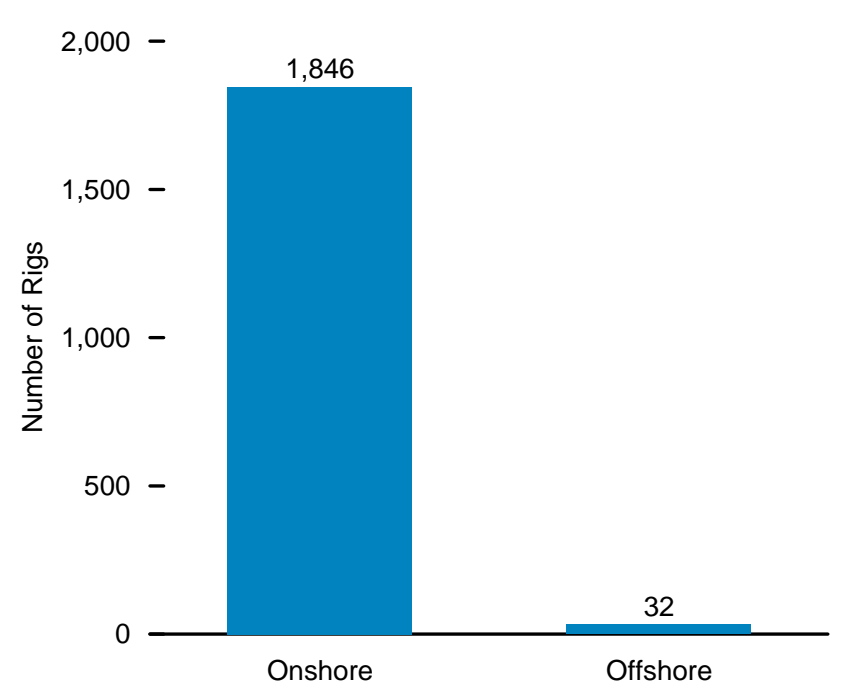

By Type, 1949-2011

5-

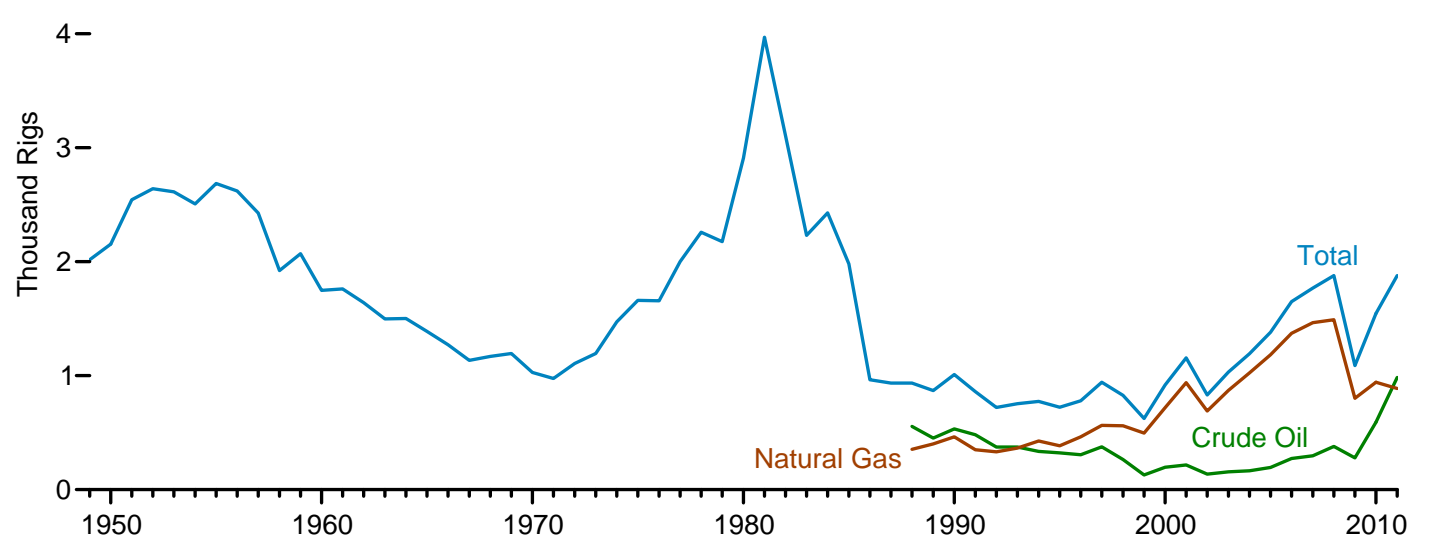

By Type, ${ }^{1} 2011$

1,250-

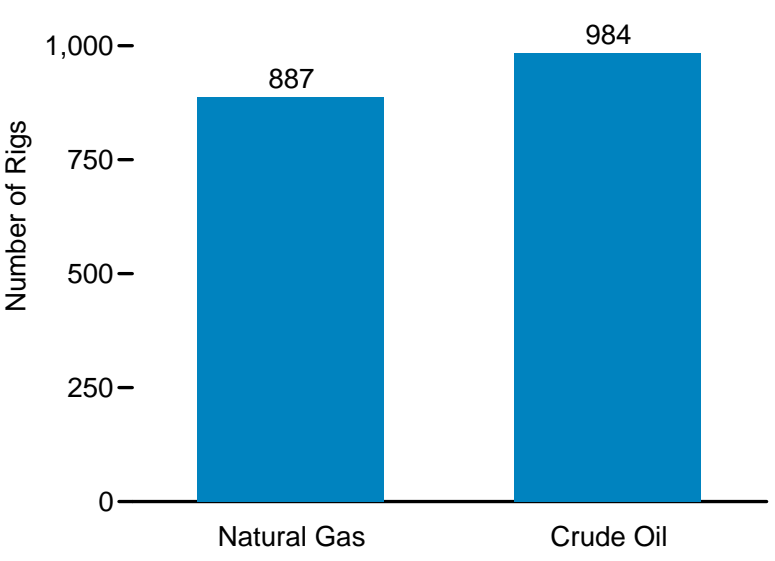
1 Rigs drilling for miscellaneous purposes, such as service wells, injection wells, and stra- $\quad$ Source: Table 4.4.
tigraphic tests, are not shown. 
Table 4.4 Crude Oil and Natural Gas Rotary Rigs in Operation, Selected Years, 1949-2011

(Number of Rigs)

\begin{tabular}{|c|c|c|c|c|c|}
\hline \multirow[b]{2}{*}{ Year } & \multicolumn{2}{|c|}{ By Site } & \multicolumn{2}{|c|}{ By Type } & \multirow[b]{2}{*}{ Total ${ }^{1}$} \\
\hline & Onshore & Offshore & Crude Oil & Natural Gas & \\
\hline 1949 & NA & NA & NA & NA & 2,017 \\
\hline 1950 & NA & NA & NA & NA & 2,154 \\
\hline 1955 & NA & NA & NA & NA & 2,686 \\
\hline 1960 & NA & NA & NA & NA & 1,748 \\
\hline 1965 & NA & NA & NA & NA & 1,388 \\
\hline 1970 & NA & NA & NA & NA & 1,028 \\
\hline 1975 & 1,554 & 106 & NA & NA & 1,660 \\
\hline 1976 & 1,529 & 129 & NA & NA & 1,658 \\
\hline 1977 & 1,834 & 167 & NA & NA & 2,001 \\
\hline 1978 & 2,074 & 185 & NA & NA & 2,259 \\
\hline 1979 & 1,970 & 207 & NA & NA & 2,177 \\
\hline 1980 & 2,678 & 231 & NA & NA & 2,909 \\
\hline 1981 & 3,714 & 256 & NA & NA & 3,970 \\
\hline 1982 & 2,862 & 243 & NA & NA & 3,105 \\
\hline 1983 & 2,033 & 199 & NA & NA & 2,232 \\
\hline 1984 & 2,215 & 213 & NA & NA & 2,428 \\
\hline 1985 & 1,774 & 206 & NA & NA & 1,980 \\
\hline 1986 & 865 & 99 & NA & NA & 964 \\
\hline 1987 & 841 & 95 & NA & NA & 936 \\
\hline 1988 & 813 & 123 & 554 & 354 & 936 \\
\hline 1989 & 764 & 105 & 453 & 401 & 869 \\
\hline 1990 & 902 & 108 & 532 & 464 & 1,010 \\
\hline 1991 & 779 & 81 & 482 & 351 & 860 \\
\hline 1992 & 669 & 52 & 373 & 331 & 721 \\
\hline 1993 & 672 & 82 & 373 & 364 & 754 \\
\hline 1994 & 673 & 102 & 335 & 427 & 775 \\
\hline 1995 & 622 & 101 & 323 & 385 & 723 \\
\hline 1996 & 671 & 108 & 306 & 464 & 779 \\
\hline 1997 & 821 & 122 & 376 & 564 & 943 \\
\hline 1998 & 703 & 123 & 264 & 560 & 827 \\
\hline 1999 & 519 & 106 & 128 & 496 & 625 \\
\hline 2000 & 778 & 140 & 197 & 720 & 918 \\
\hline 2001 & 1,003 & 153 & 217 & 939 & 1,156 \\
\hline 2002 & 717 & 113 & 137 & 691 & 830 \\
\hline 2003 & 924 & 108 & 157 & 872 & 1,032 \\
\hline 2004 & 1,095 & 97 & 165 & 1,025 & 1,192 \\
\hline 2005 & 1,287 & 94 & 194 & 1,184 & 1,381 \\
\hline 2006 & 1,559 & 90 & 274 & 1,372 & 1,649 \\
\hline 2007 & 1,695 & 72 & 297 & 1,466 & 1,768 \\
\hline 2008 & 1,814 & 65 & 379 & 1,491 & 1,879 \\
\hline 2009 & 1,046 & 44 & 278 & 801 & 1,089 \\
\hline 2010 & 1,514 & 31 & 591 & 943 & 1,546 \\
\hline 2011 & 1,846 & 32 & 984 & 887 & 1,879 \\
\hline
\end{tabular}

1 Sum of rigs drilling for crude oil, rigs drilling for natural gas, and other rigs (not shown) drilling for miscellaneous purposes, such as service wells, injection wells, and stratigraphic tests.

$\mathrm{NA}=$ Not available.

Notes. - Data are not for the exact calendar year but are an average for the 52 or 53 consecutive whole weeks that most nearly coincide with the calendar year. - Geographic coverage is the 50 States and the

District of Columbia. - Totals may not equal sum of components due to independent rounding.

Web Pages: - See http://www.eia.gov/totalenergy/data/monthly/\#crude for updated monthly and annual data. - See http://www.eia.gov/totalenergy/data/annual/\#resources for all annual data beginning in 1949. Source: Baker Hughes, Inc., Houston, TX, Rotary Rigs Running-By State, used with permission. See http://investor.shareholder.com/bhi/rig_counts/rc_index.cfm. 


\section{Figure 4.5 Crude Oil and Natural Gas Exploratory and Development Wells}

\section{Total Wells Drilled, 1949-2010}

$100-$

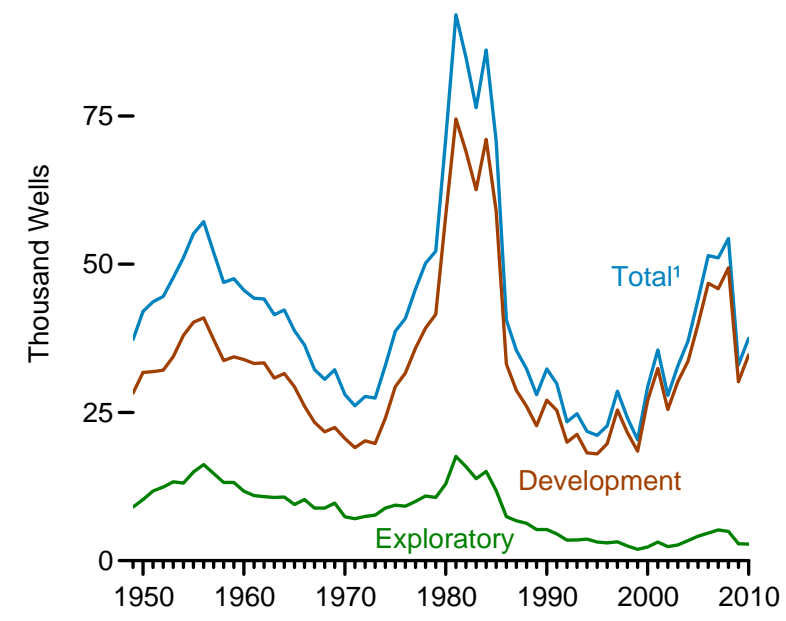

Wells Drilled, 2010

20-

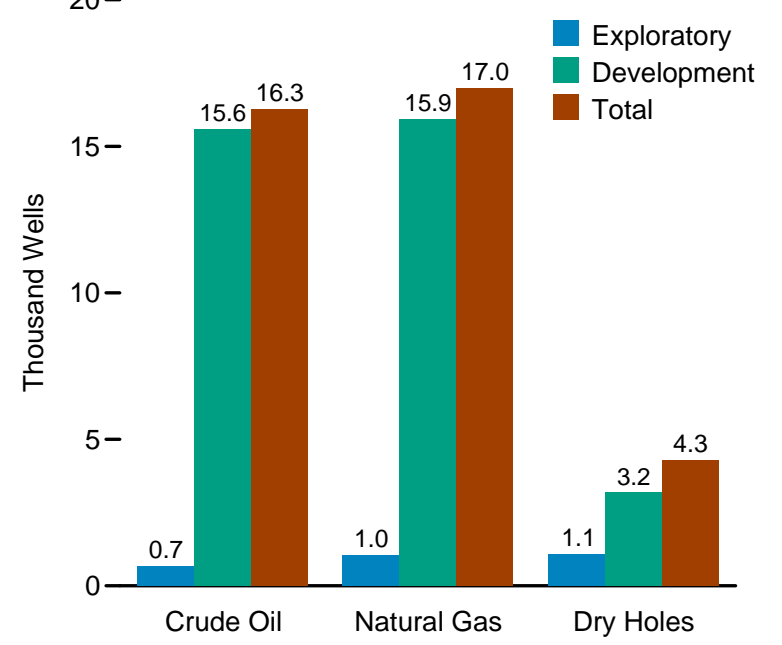

Total Wells Drilled by Type, 1949-2010

$50-$

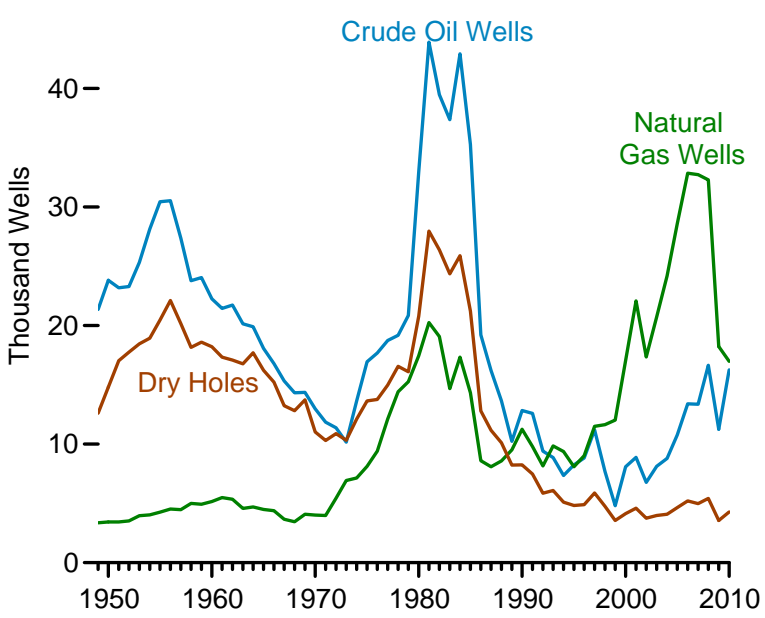

Footage Drilled, 2010

$200-$

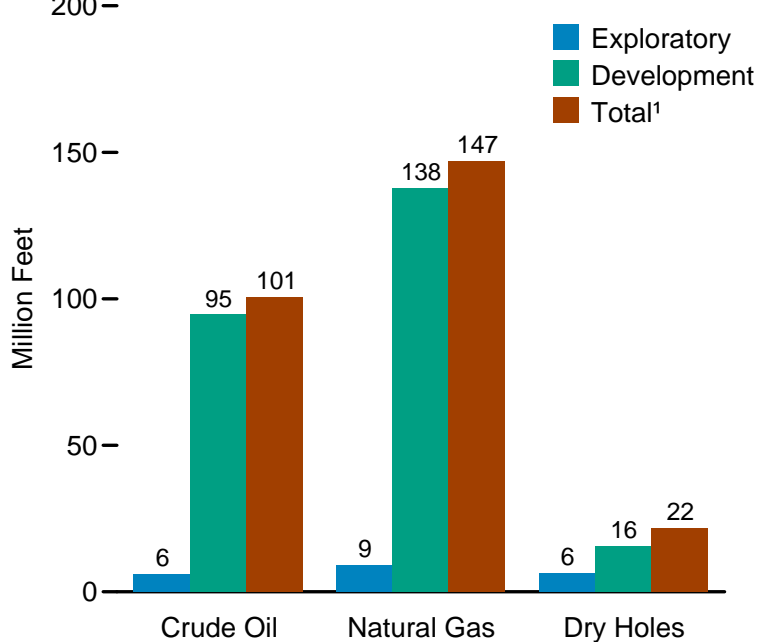

Successful Wells, 1949-2010

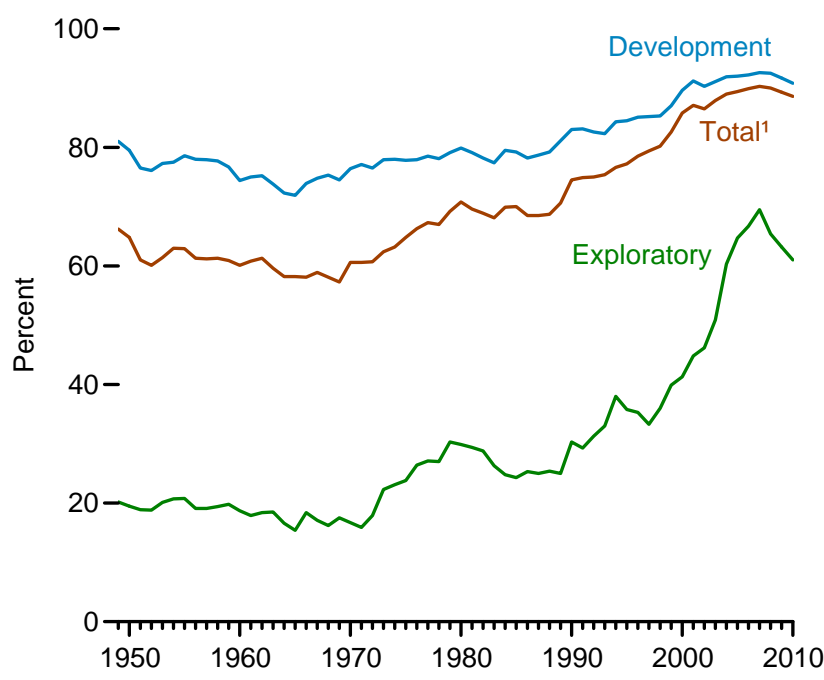

Average Depth, 2010

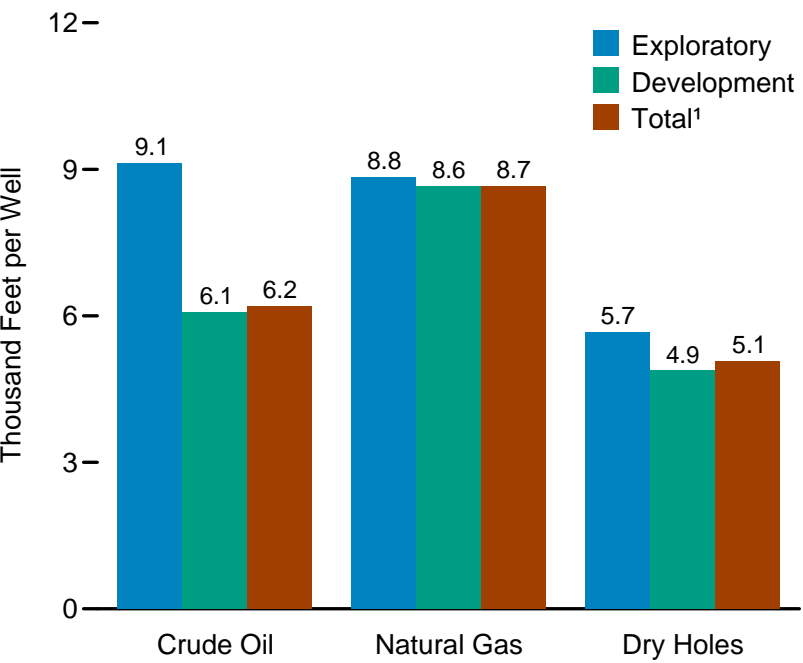

${ }^{1}$ Data are for exploratory and development wells combined.

Sources: Tables 4.5-4.7. 
Table 4.5 Crude Oil and Natural Gas Exploratory and Development Wells, Selected Years, 1949-2010

\begin{tabular}{|c|c|c|c|c|c|c|c|c|c|c|c|c|c|}
\hline \multirow[b]{3}{*}{ Year } & \multicolumn{4}{|c|}{ Wells Drilled } & \multirow{3}{*}{$\begin{array}{c}\begin{array}{c}\text { Successful } \\
\text { Wells }\end{array} \\
\text { Percent }\end{array}$} & \multicolumn{4}{|c|}{ Footage Drilled ${ }^{1}$} & \multicolumn{4}{|c|}{ Average Footage Drilled } \\
\hline & Crude Oil ${ }^{2}$ & Natural Gas ${ }^{3}$ & Dry Holes 4 & Total & & Crude Oil ${ }^{2}$ & Natural Gas ${ }^{3}$ & Dry Holes 4 & Total & Crude Oil ${ }^{2}$ & Natural Gas ${ }^{3}$ & Dry Holes 4 & Total \\
\hline & \multicolumn{4}{|c|}{ Number } & & \multicolumn{4}{|c|}{ Thousand Feet } & \multicolumn{4}{|c|}{ Feet per Well } \\
\hline 1949 & 21,352 & 3,363 & 12,597 & 37,312 & 66.2 & 79,428 & 12,437 & 43,754 & 135,619 & 3,720 & 3,698 & 3,473 & 3,635 \\
\hline 1950 & 23,812 & 3,439 & 14,799 & 42,050 & 64.8 & 92,695 & 13,685 & 50,977 & 157,358 & 3,893 & $\begin{array}{l}3,050 \\
3,979\end{array}$ & $\begin{array}{l}3,445 \\
3,445\end{array}$ & $\begin{array}{l}3,000 \\
3,742\end{array}$ \\
\hline 1955 & 30,432 & 4,266 & 20,452 & 55,150 & 62.9 & 121,148 & 19,930 & 85,103 & 226,182 & 3,981 & 4,672 & 4,161 & 4,101 \\
\hline 1960 & 22,258 & 5,149 & 18,212 & 45,619 & 60.1 & 86,568 & 28,246 & 77,361 & 192,176 & 3,889 & 5,486 & 4,248 & 4,213 \\
\hline 1965 & 18,065 & 4,482 & 16,226 & 38,773 & 58.2 & 73,322 & 24,931 & 76,629 & 174,882 & 4,059 & 5,562 & 4,723 & 4,510 \\
\hline 1970 & 12,968 & 4,011 & 11,031 & 28,010 & 60.6 & 56,859 & 23,623 & 58,074 & 138,556 & 4,385 & 5,860 & 5,265 & 4,943 \\
\hline 1975 & 16,948 & 8,127 & 13,646 & 38,721 & 64.8 & 66,819 & 44,454 & 69,220 & 180,494 & 3,943 & 5,470 & 5,073 & 4,661 \\
\hline 1976 & 17,688 & 9,409 & 13,758 & 40,855 & 66.3 & 68,892 & 49,113 & 68,977 & 186,982 & 3,895 & 5,220 & 5,014 & 4,577 \\
\hline 1977 & 18,745 & 12,122 & 14,985 & 45,852 & 67.3 & 75,451 & 63,686 & 76,728 & 215,866 & 4,025 & 5,254 & 5,120 & 4,708 \\
\hline 1978 & 19,181 & 14,413 & 16,551 & 50,145 & 67.0 & 77,041 & 75,841 & 85,788 & 238,669 & 4,017 & 5,262 & 5,183 & 4,760 \\
\hline 1979 & 20,851 & 15,254 & 16,099 & 52,204 & 69.2 & 82,688 & 80,468 & 81,642 & 244,798 & 3,966 & 5,275 & 5,071 & 4,689 \\
\hline 1980 & 32,959 & 17,461 & 20,785 & 71,205 & 70.8 & $\begin{array}{r}0,000 \\
125,262\end{array}$ & 92,106 & 99,575 & 316,943 & $\begin{array}{l}3,801 \\
3,801\end{array}$ & 5,275 & 4,791 & $\begin{array}{l}4,005 \\
4,451\end{array}$ \\
\hline 1981 & 43,887 & 20,250 & 27,953 & 92,090 & 69.6 & 172,167 & 108,353 & 134,934 & 415,454 & 3,923 & 5,351 & 4,827 & 4,511 \\
\hline 1982 & 39,459 & 19,076 & 26,379 & 84,914 & 68.9 & 149,674 & 107,149 & 123,746 & 380,569 & 3,793 & 5,617 & 4,691 & 4,482 \\
\hline 1983 & 37,366 & 14,684 & 24,355 & 76,405 & 68.1 & 136,849 & 78,108 & 105,222 & 320,179 & 3,662 & 5,319 & 4,320 & 4,191 \\
\hline 1984 & 42,906 & 17,338 & 25,884 & 86,128 & 69.9 & 162,653 & 91,480 & 119,860 & 373,993 & 3,791 & 5,276 & 4,631 & 4,342 \\
\hline 1985 & 35,261 & 14,324 & 21,211 & 70,796 & 70.0 & 137,728 & 76,293 & 100,388 & 314,409 & 3,906 & 5,326 & 4,733 & 4,441 \\
\hline 1986 & 19,213 & 8,599 & 12,799 & 40,611 & 68.5 & 76,825 & 45,039 & 60,961 & 182,825 & 3,999 & 5,238 & 4,763 & 4,502 \\
\hline 1987 & 16,210 & 8,096 & 11,167 & 35,473 & 68.5 & 66,358 & 42,584 & 53,588 & 162,530 & 4,094 & 5,260 & 4,799 & 4,582 \\
\hline 1988 & 13,646 & 8,578 & 10,119 & 32,343 & 68.7 & 58,639 & 45,363 & 52,517 & 156,519 & 4,297 & 5,288 & 5,190 & 4,839 \\
\hline 1989 & 10,230 & 9,522 & 8,236 & 27,988 & 70.6 & 43,266 & 49,081 & 42,099 & 134,446 & 4,229 & 5,154 & 5,112 & 4,804 \\
\hline 1990 & 12,839 & 11,246 & 8,245 & 32,330 & 74.5 & R56,591 & R57,028 & $\mathrm{R}_{42,433}$ & $\mathrm{R}_{156,052}$ & $\mathrm{R}_{4,408}$ & $\mathrm{R}_{5,071}$ & $R_{5,147}$ & $\mathrm{R}_{4,827}$ \\
\hline 1991 & 12,588 & 9,793 & 7,481 & 29,862 & $\begin{array}{l}74.9 \\
74.9\end{array}$ & $\begin{aligned} \\
R\end{aligned}$ & $R_{51,032}$ & $\mathrm{R}_{37,750}$ & $\mathrm{R}_{144,978}$ & $\begin{array}{r}4,460 \\
R_{4}\end{array}$ & $R_{5,211}$ & $\begin{array}{r}\text { R5,046 } \\
\text { R }\end{array}$ & $\mathrm{R}_{4,855}$ \\
\hline 1992 & 9,402 & 8,163 & 5,862 & 23,427 & 75.0 & $R_{45,748}$ & $\mathrm{R}_{44,727}$ & $\mathrm{R}_{29,451}$ & $\mathrm{R}_{119,926}$ & $\mathrm{R}_{4,866}$ & $R_{5,479}$ & $\mathrm{R}_{5,024}$ & $\mathrm{R}_{5,119}$ \\
\hline 1993 & 8,856 & 9,839 & 6,096 & 24,791 & 75.4 & $\mathrm{R}_{44,236}$ & $R_{58,240}$ & $\mathrm{R}_{31,018}$ & $\mathrm{R}_{133,494}$ & $\mathrm{R}_{4,995}$ & R5,919 & R5,088 & $R_{5}, 385$ \\
\hline 1994 & 7,348 & 9,375 & 5,096 & 21,819 & 76.6 & $R_{38,620}$ & $\mathrm{R}_{58,340}$ & $\mathrm{R}_{27,771}$ & $\mathrm{R}_{124,731}$ & $R_{5,256}$ & $R_{6,223}$ & $\mathrm{R}_{5,450}$ & $\mathrm{R}_{5,717}$ \\
\hline 1995 & 8,248 & 8,082 & 4,814 & 21,144 & 77.2 & $\mathrm{R}_{41,076}$ & R 49,746 & $\mathrm{R}_{26,349}$ & $\mathrm{R}_{117,171}$ & $\mathrm{R}_{4,980}$ & $\mathrm{R}_{6,155}$ & R5,473 & $\mathrm{R}_{5,542}$ \\
\hline 1996 & 8,836 & 9,027 & 4,890 & 22,753 & 78.5 & $R_{42,472}$ & R56,042 & $\mathrm{R}_{27,851}$ & $\mathrm{R}_{126,365}$ & $\mathrm{R}_{4,807}$ & $R_{6,208}$ & $R_{5,696}$ & $\mathrm{R}_{5,554}$ \\
\hline 1997 & 11,206 & 11,498 & 5,874 & 28,578 & 79.4 & $\mathrm{R}_{56,371}$ & $\mathrm{R}_{71,270}$ & $\mathrm{R}_{33,640}$ & $\mathrm{R}_{1} 61,281$ & $\mathrm{R}_{5,030}$ & $\mathrm{R}_{6,198}$ & R5,727 & $\mathrm{R}_{5,644}$ \\
\hline 1998 & 7,682 & 11,639 & 4,761 & 24,082 & 80.2 & $R_{38,579}$ & R 70,099 & $\mathrm{R}_{28,540}$ & $\mathrm{R}_{137,218}$ & $\begin{array}{r}\mathrm{R} 5,022 \\
\end{array}$ & $R_{6,023}$ & R5,995 & $\mathrm{R}_{5,698}$ \\
\hline 1999 & 4,805 & 12,027 & 3,550 & 20,382 & 82.6 & $\mathrm{R}_{22,024}$ & ${ }^{R} 60,217$ & $\mathrm{R}_{20,608}$ & $\mathrm{R}_{102,849}$ & 4,584 & $R_{5,007}$ & $\mathrm{R}_{5,805}$ & $\mathrm{R}_{5,046}$ \\
\hline 2000 & 8,090 & 17,051 & 4,146 & 29,287 & 85.8 & $\mathrm{R}_{36,745}$ & R83,618 & $\mathrm{R}_{24,076}$ & $R_{144,439}$ & $\begin{array}{r}R_{4,542} \\
\text { R }\end{array}$ & $\mathrm{R}_{4,904}$ & $\begin{array}{r}\mathrm{R} 5,807 \\
\mathrm{R}_{5}\end{array}$ & $\mathrm{R}_{4,932}$ \\
\hline 2001 & 8,888 & 22,072 & 4,598 & 35,558 & 87.1 & $\mathrm{R}_{43,172}$ & $\mathrm{R}_{110,734}$ & $\mathrm{R}_{26,221}$ & $\mathrm{R}_{180,127}$ & $\mathrm{R}_{4,857}$ & $\mathrm{R}_{5,017}$ & $\mathrm{R}_{5,703}$ & $\mathrm{R}_{5,066}$ \\
\hline 2002 & 6,775 & 17,342 & 3.754 & 27.871 & 86.5 & $\mathrm{R}_{30,892}$ & R93.041 & $R_{21}, 232$ & $\mathrm{R}_{145,165}$ & $\mathrm{R} 4,560$ & R5,365 & R5,656 & R5,208 \\
\hline 2003 & 8,129 & 20,722 & $\begin{array}{l}3,154 \\
3,982\end{array}$ & 32,833 & $\begin{array}{l}0.3 \\
87.9\end{array}$ & $\begin{array}{r}\text { R } 38,588 \\
\mathrm{R}_{3}, 588\end{array}$ & $\mathrm{R}_{115,916}$ & $\mathrm{R}_{22,744}$ & $\begin{array}{l}R_{177,248} \\
R_{177}\end{array}$ & $\begin{array}{l}\mathrm{R}_{4,747} \\
\text {. }\end{array}$ & $\begin{array}{l}\text { R5,594 } \\
\text { R }\end{array}$ & $\begin{array}{l}3,712 \\
R_{5}, 70\end{array}$ & $\begin{array}{r}\mathrm{R}, \mathrm{CU} \\
\mathrm{R}, 398\end{array}$ \\
\hline 2004 & 8,789 & 24,186 & 4,082 & 37,057 & 89.0 & $R_{42,109}$ & $\mathrm{R}_{138,449}$ & $\mathrm{R}_{23,714}$ & $\mathrm{R}_{204,272}$ & $\mathrm{R} 4,791$ & R5,724 & R5,809 & $\mathrm{R}_{5,512}$ \\
\hline 2005 & 10,779 & $\mathrm{R}_{28,590}$ & 4,653 & $\mathrm{R}_{44,022}$ & 89.4 & $R_{51,449}$ & $\mathrm{R}_{163,820}$ & $\mathrm{R}_{25,044}$ & $\mathrm{R}_{240,313}$ & $R_{4,773}$ & $R_{5,730}$ & $\mathrm{R}_{5,382}$ & $\mathrm{R}_{5,459}$ \\
\hline 2006 & $\mathrm{R}_{13,404}$ & $\begin{array}{r}2 \\
R_{32,838}\end{array}$ & $\begin{array}{r}4,000 \\
R_{5}, 206\end{array}$ & $\begin{array}{r}4,0<2 \\
R_{51,448}\end{array}$ & $\begin{array}{l}0.4 \\
89.9\end{array}$ & $\mathrm{R}_{63,340}$ & $\mathrm{R}_{191,646}$ & $\begin{array}{r}27,778 \\
R_{2}, 048\end{array}$ & $\begin{array}{r}\mathrm{R}_{282,764} \\
\text { (4) }\end{array}$ & $\mathrm{R}_{4,725}$ & $\begin{array}{l}\text { R5,836 } \\
\text { R }\end{array}$ & $\begin{array}{l}3,302 \\
R_{5}, 336\end{array}$ & $\mathrm{R}_{5}, 496$ \\
\hline 2007 & $\mathrm{R}_{13,361}$ & $R_{32,719}$ & $\begin{array}{r}\mathrm{R} \\
\mathrm{R}, 978\end{array}$ & R51,058 & 90.3 & $\mathrm{R}_{64,792}$ & $\mathrm{R}_{208,907}$ & $\mathrm{R}_{27,754}$ & $\mathrm{R}_{301,453}$ & $\mathrm{R}_{4,849}$ & $\mathrm{R}_{6,385}$ & R5,575 & $\mathrm{R}_{5,904}$ \\
\hline 2008 & $\mathrm{R}_{16,645}$ & R 32,274 & $\mathrm{R}_{5}, 428$ & $\mathrm{R}_{54,347}$ & R90.0 & R 82,646 & $\mathrm{R}_{223,224}$ & $\mathrm{R}_{28,572}$ & $\mathrm{R}_{334,442}$ & $R_{4,965}$ & $\mathrm{R}_{6,917}$ & $\mathrm{R}_{5,264}$ & $\mathrm{R}_{6,154}$ \\
\hline 2009 & $\mathrm{R}_{11,261}$ & $\mathrm{R}_{18,234}$ & $R_{3,552}$ & $\mathrm{R}_{33,047}$ & R89.3 & $\mathrm{R}_{62,771}$ & R156,200 & $\mathrm{R}_{20,520}$ & $\mathrm{R}_{239,491}$ & R5,574 & $R_{8,566}$ & R5,777 & $\mathrm{R}_{7,247}$ \\
\hline 2010 & $\mathrm{R}_{16,254}$ & $\mathrm{R}_{16,973}$ & $\mathrm{R}_{4,277}$ & $\mathrm{R}_{37,504}$ & R88.6 & $\mathrm{R}_{100,682}$ & $\mathrm{R}_{146,973}$ & $\mathrm{R}_{21,719}$ & $\mathrm{R}_{269,374}$ & $\mathrm{R}_{6,194}$ & $R_{8,659}$ & $\mathrm{R}_{5,078}$ & $\mathrm{R}_{7,183}$ \\
\hline
\end{tabular}

1 See "Footage Drilled" in Glossary.

2 See "Crude Oil Well" in Glossary.

3 See "Natural Gas Well" in Glosse
4 See "Dry Hole" in Glossary.

${ }^{4}$ See "Dry Hole" in Glossary.

$R=$ Revised.

Notes. - 2011 data for this table were not available in time for publication. - Data are estimates. - Data are for exploratory and development wells combined; see Table 4.6 for exploratory wells only, and - For 1949-1959, data represent wells completed in a given year. For 1960-1969, data are for well - For $1949-1959$, data represent wells completed in a given year. For 1960-1969, data are for well
completion reports received by the American Petroleum Institute during the reporting year. For 1970 forward, the data represent wells completed in a given year. The as-received well completion data for recent years are incomplete due to delays in the reporting of wells drilled. The U.S. Energy Information
Administration (EIA) therefore statistically imputes the missing data. - Totals may not equal sum of components due to independent rounding. Average depth may not equal average of components due to independent rounding.

Web Pages: - See http://www.eia.gov/totalenergy/data/monthly/\#crude for updated monthly and annual data. - See http://www.eia.gov/totalenergy/data/annual/\#resources for all annual data beginning in 1949. See $\mathrm{http}: / / \mathrm{ww}$.eia.gov/petroleum/ for related information.

Sources: - 1949-1965-Gulf Publishing Company, World Oil, "Forecast-Review" issue. - 1966-1969-American Petroleum Institute (API), Quarterly Review of Drilling Statistics for the United States, annual summaries and monthly reports. - 1970-1989_-EIA computations based on well reports Denver, CO. 


\section{Figure 4.6 Crude Oil and Natural Gas Exploratory Wells, 1949-2010}

\section{Exploratory Wells Drilled by Well Type}

$15-$

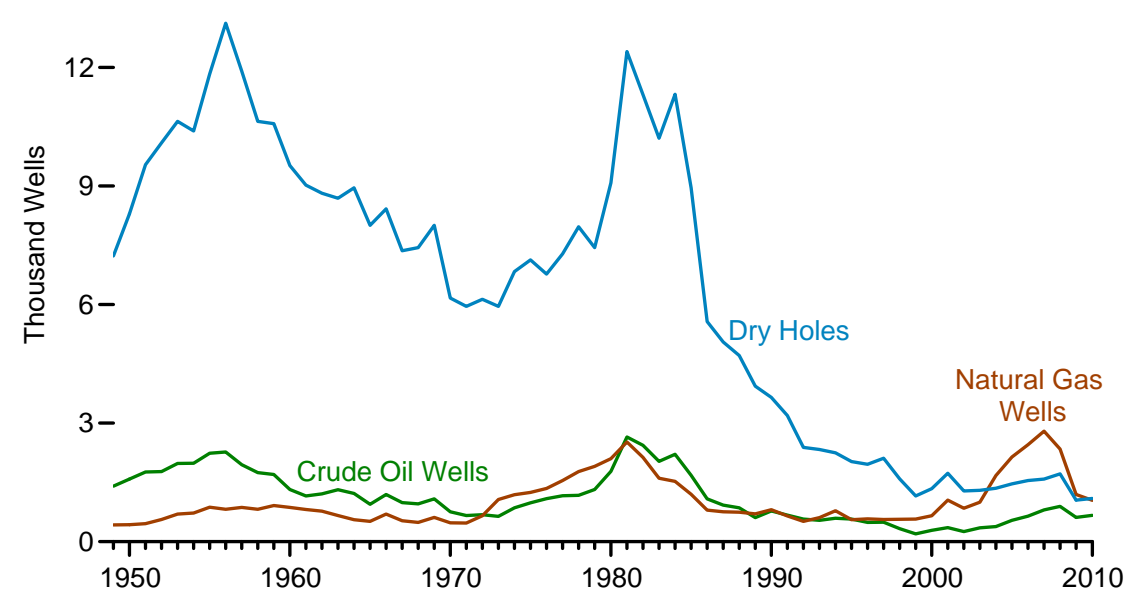

\section{Exploratory Wells Average Depth, All Wells}

$10-$

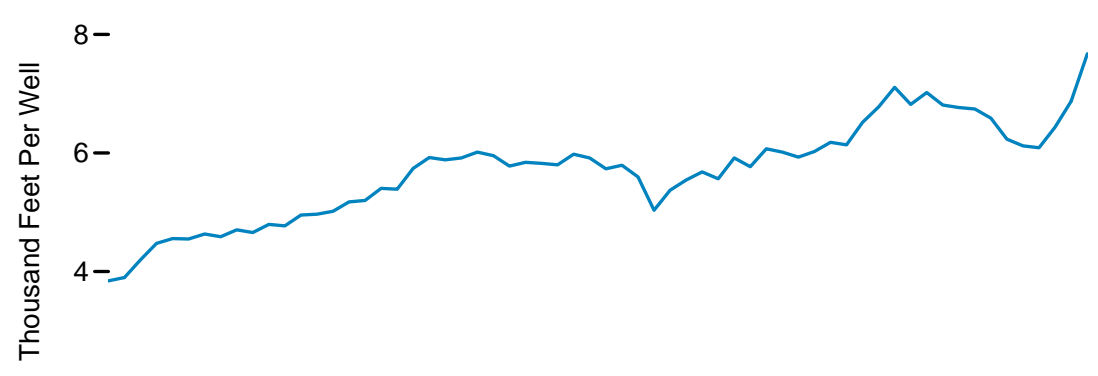

2-

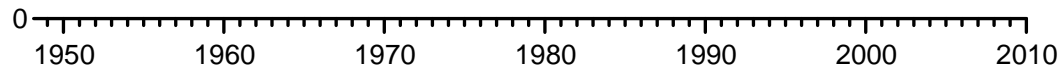

Exploratory Footage Drilled by Well Type

$80-$

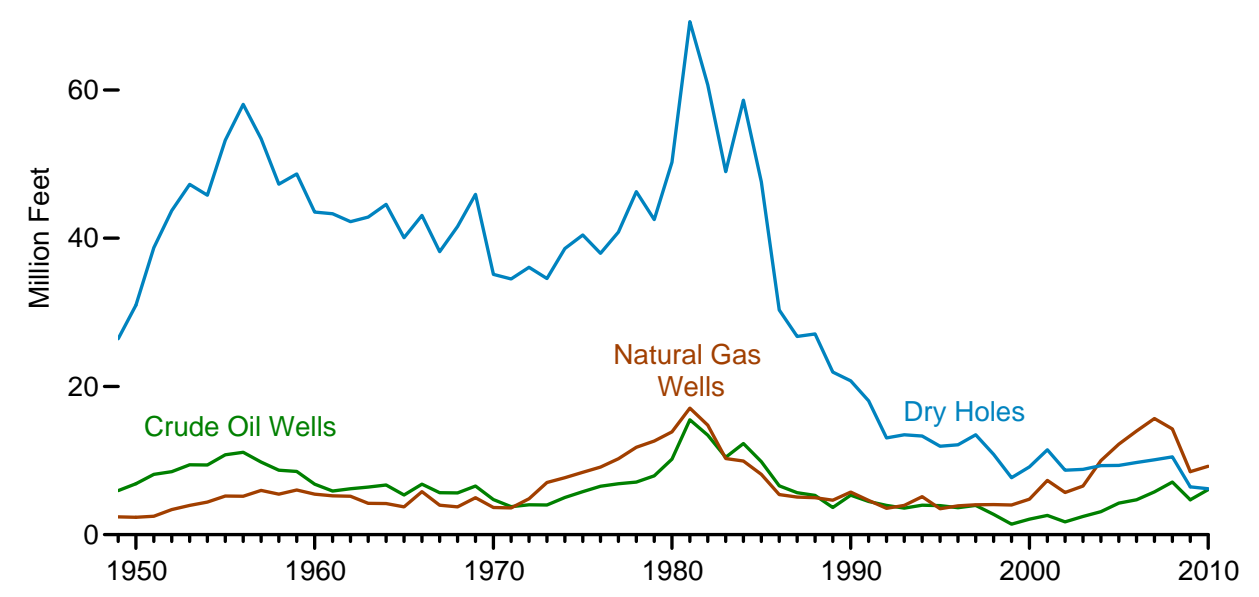

Exploratory Wells Average Depth by Well Type

$10-$

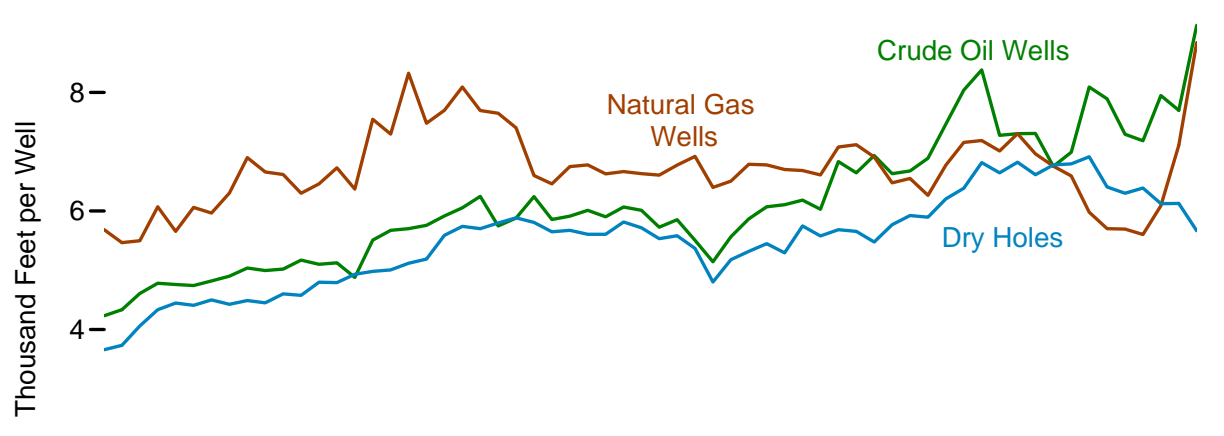

2-

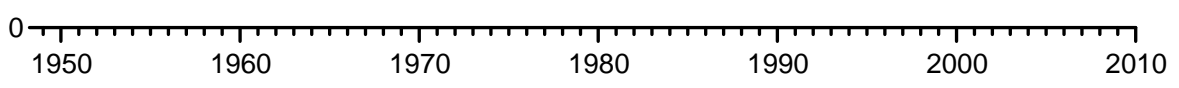

Note: These graphs depict exploratory wells only; see Figure 4.5 for all wells and Figure 4.7 Source: Table 4.6 . for development wells only. 
Table 4.6 Crude Oil and Natural Gas Exploratory Wells, Selected Years, 1949-2010

\begin{tabular}{|c|c|c|c|c|c|c|c|c|c|c|c|c|c|}
\hline \multirow[b]{3}{*}{ Year } & \multicolumn{4}{|c|}{ Wells Drilled } & \multirow{3}{*}{$\begin{array}{c}\begin{array}{c}\text { Successful } \\
\text { Wells }\end{array} \\
\text { Percent }\end{array}$} & \multicolumn{4}{|c|}{ Footage Drilled ${ }^{1}$} & \multicolumn{4}{|c|}{ Average Footage Drilled } \\
\hline & Crude Oil $^{2}$ & Natural Gas ${ }^{3}$ & Dry Holes ${ }^{4}$ & Total & & Crude Oil $^{2}$ & Natural Gas ${ }^{3}$ & Dry Holes ${ }^{4}$ & Total & Crude Oil $^{2}$ & Natural Gas ${ }^{3}$ & Dry Holes ${ }^{4}$ & Total \\
\hline & \multicolumn{4}{|c|}{ Number } & & \multicolumn{4}{|c|}{ Thousand Feet } & \multicolumn{4}{|c|}{ Feet per Well } \\
\hline 1949 & 1,406 & 424 & 7,228 & 9,058 & 20.2 & 5,950 & 2,409 & 26,439 & 34,798 & 4,232 & 5,682 & 3,658 & 3,842 \\
\hline 1950 & 1,583 & 431 & 8,292 & 10,306 & 19.5 & 6,862 & 2,356 & 30,957 & 40,175 & 4,335 & 5,466 & 3,733 & 3,898 \\
\hline 1955 & 2,236 & 874 & 11,832 & 14,942 & 20.8 & 10,774 & 5,212 & 53,220 & 69,206 & 4,819 & 5,964 & 4,498 & 4,632 \\
\hline 1960 & 1,321 & 868 & 9,515 & 11,704 & 18.7 & 6,829 & 5,466 & 43,535 & 55,831 & 5,170 & 6,298 & 4,575 & 4,770 \\
\hline 1965 & 946 & 515 & 8,005 & 9,466 & 15.4 & 5,366 & 3,757 & 40,081 & 49,204 & 5,672 & 7,295 & 5,007 & 5,198 \\
\hline 1970 & 757 & 477 & 6,162 & 7,396 & $\begin{array}{l}16.7 \\
16.7\end{array}$ & 4,729 & 3,678 & 35,123 & 43,530 & 6,247 & 7,695 & 5,700 & 5,885 \\
\hline 1975 & 982 & 1,248 & 7,129 & 9,359 & 23.8 & 5,806 & 8,422 & 40,448 & 54,677 & 5,913 & 6,748 & 5,674 & 5,842 \\
\hline 1976 & 1,086 & 1,346 & 6,772 & 9,204 & 26.4 & 6,527 & 9,121 & 37,969 & 53,617 & 6,010 & 6,777 & 5,607 & 5,825 \\
\hline 1977 & 1,164 & 1,548 & 7,283 & 9,995 & 27.1 & 6,870 & 10,255 & 40,823 & 57,949 & 5,902 & 6,625 & 5,605 & 5,798 \\
\hline 1978 & 1,171 & 1,771 & 7,965 & 10,907 & 27.0 & 7,105 & 11,798 & 46,295 & 65,197 & 6,067 & 6,662 & 5,812 & 5,978 \\
\hline 1979 & 1,321 & 1,907 & 7,437 & 10,665 & 30.3 & 7,941 & 12,643 & 42,512 & 63,096 & 6,011 & 6,630 & 5,716 & 5,916 \\
\hline 1980 & 1,777 & 2,099 & 9,081 & 12,957 & 29.9 & 10,177 & 13,862 & 50,249 & 74,288 & 5,727 & 6,604 & 5,533 & 5,733 \\
\hline 1981 & 2,651 & 2,522 & 12,400 & 17,573 & 29.4 & 15,515 & 17,079 & 69,214 & 101,808 & 5,853 & 6,772 & 5,582 & 5,793 \\
\hline 1982 & 2,437 & 2,133 & 11,307 & 15,877 & 28.8 & 13,413 & 14,763 & 60,680 & 88,856 & 5,504 & 6,921 & 5,367 & 5,597 \\
\hline 1983 & 2,030 & 1,605 & 10,206 & 13,841 & 26.3 & 10,437 & 10,264 & 48,989 & 69,690 & 5,141 & 6,395 & 4,800 & 5,035 \\
\hline 1984 & 2,209 & 1,528 & 11,321 & 15,058 & 24.8 & 12,294 & 9,935 & 58,624 & 80,853 & 5,565 & 6,502 & 5,178 & 5,369 \\
\hline 1985 & 1,680 & 1,200 & 8,954 & 11,834 & 24.3 & 9,854 & 8,144 & 47,604 & 65,602 & 5,865 & 6,787 & 5,317 & 5,544 \\
\hline 1986 & 1,084 & 797 & 5,567 & 7,448 & 25.3 & 6,579 & 5,401 & 30,325 & 42,305 & 6,069 & 6,777 & 5,447 & 5,680 \\
\hline 1987 & 926 & 756 & 5,052 & 6,734 & 25.0 & 5,652 & 5,064 & 26,746 & 37,462 & 6,104 & 6,698 & 5,294 & 5,563 \\
\hline 1988 & 855 & 747 & 4,711 & 6,313 & 25.4 & 5,286 & 4,992 & 27,079 & 37,357 & 6,182 & 6,683 & 5,748 & 5,917 \\
\hline 1989 & 607 & 706 & 3,934 & 5,247 & 25.0 & 3,659 & 4,664 & 21,947 & 30,270 & 6,028 & 6,606 & 5,579 & 5,769 \\
\hline 1990 & 778 & 811 & 3,652 & 5,241 & 30.3 & $\mathrm{R}_{5}, 316$ & $\begin{array}{r}\text { R5,740 } \\
\text { r }\end{array}$ & $\mathrm{R}_{20,761}$ & $\mathrm{R}_{31,817}$ & $R_{6,833}$ & R7,078 & $\mathrm{R}_{5,685}$ & $\mathrm{R}_{6,071}$ \\
\hline 1991 & 673 & 649 & 3,191 & 4,513 & 29.3 & 4,470 & $\mathrm{R}_{4,619}$ & 18,049 & $\mathrm{R}_{27,138}$ & 6,642 & $\mathrm{R}_{7,117}$ & 5,656 & 6,013 \\
\hline 1992 & 571 & 513 & 2,384 & 3,468 & 31.3 & $R_{3,959}$ & 3,544 & 13,058 & $R_{20,561}$ & $\mathrm{R}_{6,933}$ & 6,908 & 5,477 & $\mathrm{R}_{5,929}$ \\
\hline 1993 & 539 & 610 & 2,334 & 3,483 & 33.0 & $R_{3,572}$ & $R_{3,950}$ & $\mathrm{R}_{13,465}$ & $\mathrm{R}_{20,987}$ & $\mathrm{R}_{6,627}$ & $\mathrm{R}_{6,475}$ & $R_{5,769}$ & $\mathrm{R}_{6,026}$ \\
\hline 1994 & 595 & 782 & 2,247 & $\begin{array}{l}3,4024 \\
3,624\end{array}$ & 38.0 & 3,971 & $R_{5,121}$ & $\mathrm{R}_{13,306}$ & $\mathrm{R}_{22,398}$ & 6,674 & $R_{6,549}$ & 5,922 & $R_{6,180}$ \\
\hline 1995 & 570 & 558 & 2,024 & 3,152 & 35.8 & $\mathrm{R}_{3,927}$ & $R_{3,494}$ & $\mathrm{R}_{11,927}$ & $\mathrm{R}_{19,348}$ & $\begin{array}{r}R_{6,889}\end{array}$ & $R_{6,262}$ & 5,893 & $\mathrm{R}_{6,138}$ \\
\hline 1996 & 489 & 576 & 1,956 & 3,021 & 35.3 & $R_{3,650}$ & $R_{3,902}$ & 12,137 & $\mathrm{R}_{19,689}$ & $\mathrm{R}_{7,464}$ & $\mathrm{R}_{6,774}$ & 6,205 & $\mathrm{R}_{6,517}$ \\
\hline 1997 & 491 & 562 & 2,113 & 3,166 & 33.3 & $R_{3,946}$ & $\mathrm{R}_{4,022}$ & $\mathrm{R}_{13,485}$ & $\mathrm{R}_{21,453}$ & $\mathrm{R}_{8,037}$ & R7,157 & $\mathrm{R}_{6,382}$ & $\mathrm{R}_{6,776}$ \\
\hline 1998 & 327 & 566 & 1,590 & 2,483 & 36.0 & 2,740 & R 4,068 & $\mathrm{R}_{10,836}$ & $\mathrm{R}_{17,644}$ & $\begin{array}{r}0,357 \\
8,379\end{array}$ & R7,187 & $\begin{array}{l}0,002 \\
R_{6,815}\end{array}$ & $\begin{array}{r}0,106 \\
\mathrm{R} 7,106\end{array}$ \\
\hline 1999 & 197 & 570 & 1,157 & 1,924 & 39.9 & 1,433 & $R_{3,997}$ & 7,687 & $\mathrm{R}_{13,117}$ & 7,274 & $\mathrm{R}_{7,012}$ & 6,644 & $\mathrm{R}_{6,818}$ \\
\hline 2000 & 288 & 657 & 1,341 & 2,286 & 41.3 & 2,103 & $\mathrm{R}_{4,798}$ & $\mathrm{R}_{9,147}$ & $R_{16,048}$ & 7,302 & $R_{7,303}$ & $\mathrm{R}_{6,821}$ & $\mathrm{R}_{7,020}$ \\
\hline 2001 & 357 & 1,052 & 1,733 & 3,142 & 44.8 & $R_{2,608}$ & $R_{7,323}$ & $R_{11,458}$ & $\mathrm{R}_{21,389}$ & $R_{7,305}$ & $\mathrm{R}_{6,961}$ & $\mathrm{R}_{6,612}$ & $R_{6,807}$ \\
\hline 2002 & 258 & 844 & 1,282 & 2,384 & 46.2 & $R_{1,742}$ & $R_{5,701}$ & $\mathrm{R}_{8,687}$ & $\mathrm{R}_{16,130}$ & $R_{6,752}$ & $\mathrm{R}_{6,755}$ & $\mathrm{R}_{6,776}$ & ${ }^{R} 6,766$ \\
\hline 2003 & 350 & 997 & $\begin{array}{l}1,297 \\
\end{array}$ & 2,644 & 50.9 & $R_{2,446}$ & $R_{6,569}$ & $\mathrm{R}_{8,810}$ & $\mathrm{R}_{17,825}$ & $R_{6,989}$ & $\mathrm{R}_{6,589}$ & $\mathrm{R}_{6,793}$ & $\mathrm{R}_{6,742}$ \\
\hline 2004 & 383 & 1,671 & 1,350 & 3,404 & 60.3 & $\mathrm{R}_{3,098}$ & $\begin{array}{r}\mathrm{R} 9,988\end{array}$ & R9,331 & $\mathrm{R}_{22,417}$ & $\mathrm{R}_{8,089}$ & R5,977 & $\mathrm{R}_{6,912}$ & ${ }^{R} 6,585$ \\
\hline 2005 & 539 & $R_{2,141}$ & 1,462 & $\mathrm{R}_{4,142}$ & 64.7 & $\mathrm{R}_{4,252}$ & $\mathrm{R}_{12,208}$ & $R_{9,359}$ & $\mathrm{R}_{25,819}$ & $\mathrm{R}_{7,889}$ & $\mathrm{R}_{5,702}$ & $\mathrm{R}_{6,402}$ & $\mathrm{R}_{6,233}$ \\
\hline 2006 & $R_{646}$ & $\mathrm{R}_{2,456}$ & $R_{1,547}$ & $\begin{array}{r}\text { R4,649 } \\
\text { r }\end{array}$ & $\mathrm{R}_{66.7}$ & $\mathrm{R}_{4,710}$ & $\mathrm{R}_{13,987}$ & R9,745 & $\mathrm{R}_{28,442}$ & $R_{7,291}$ & R5,695 & $\mathrm{R}_{6,299}$ & ${ }^{R} 6,118$ \\
\hline 2007 & R806 & $R_{2,794}$ & $R_{1,582}$ & $\mathrm{R}_{5,182}$ & R69.5 & $\mathrm{R}_{5,790}$ & $\mathrm{R}_{15,658}$ & $\mathrm{R}_{10,102}$ & $\mathrm{R}_{31,550}$ & $\mathrm{R}_{7,184}$ & $\mathrm{R}_{5,604}$ & $\mathrm{R}_{6,386}$ & ${ }^{R} 6,088$ \\
\hline 2008 & R892 & $\mathrm{R}_{2,345}$ & $\mathrm{R}_{1,715}$ & $\mathrm{R}_{4,952}$ & $\mathrm{R}_{65.4}$ & $\mathrm{R} 7,088$ & $\mathrm{R}_{14,276}$ & $\mathrm{R}_{10,500}$ & $\mathrm{R}_{31,864}$ & R7,946 & $\mathrm{R}_{6,088}$ & $\mathrm{R}_{6,122}$ & $\mathrm{R}_{6,435}$ \\
\hline 2009 & $\mathrm{R}_{612}$ & $\mathrm{R}_{1,196}$ & $R_{1,052}$ & $R_{2,860}$ & $\mathrm{R}_{63.2}$ & $\mathrm{R}_{4,711}$ & $\mathrm{R}_{8,499}$ & $\mathrm{R}_{6,443}$ & $\mathrm{R}_{19,653}$ & $R_{7,698}$ & R7,106 & $\mathrm{R}_{6,125}$ & $\mathrm{R}_{6,872}$ \\
\hline 2010 & R668 & $R_{1,044}$ & $R_{1,093}$ & $\mathrm{R}_{2,805}$ & $\mathrm{R}_{61.0}$ & $\mathrm{R}_{6,099}$ & $R_{9,226}$ & $R_{6,192}$ & $\mathrm{R}_{21,517}$ & $R_{9,130}$ & $R_{8,837}$ & R5,665 & $\mathrm{R}_{7,671}$ \\
\hline
\end{tabular}

1 See "Footage Drilled" in Glossary

See "Crude Oil Well" in Glossary.

See "Natural Gas Well" in Glossary.

4 See "Dry Hole" in Glossary.

Notes: - 2011 data for this table were not available in time for publication. - Data are estimates. - Data are for exploratory wells only; see Table 4.5 for exploratory and development wells combined, and For 1960-1969, data are for well completion reports received by the American Petroleum Institute (API) during the reporting year. For 1970 forward, the data represent wells completed in a given year. The drilled. The U.S. Energy Information Administration (EIA) therefore statistically imputes the missing data. - Totals may not equal sum of components due to independent rounding. Average depth may not equal average of components due to independent rounding.

Web Pages: - See http://www.eia.gov/totalenergy/data/monthly/\#crude for updated monthly and annual data. - See http://www.eia.gov/totalenergy/data/annual/\#resources for all annual data beginning in 1949 . See http://www.eia.gov/petroleum/ for related information.

Sources: - 1949-1965-Gulf Publishing Company, World Oil, "Forecast-Review" issue - 1966-1969-American Petroleum Institute (API), Quarterly Review of Drilling Statistics for the United States, annual summaries and monthly reports. - 1970-1989_EIA computations based on well reports Denver, CO. 
Figure 4.7 Crude Oil and Natural Gas Development Wells, 1949-2010

\section{Development Wells Drilled by Well Type}

$50-$

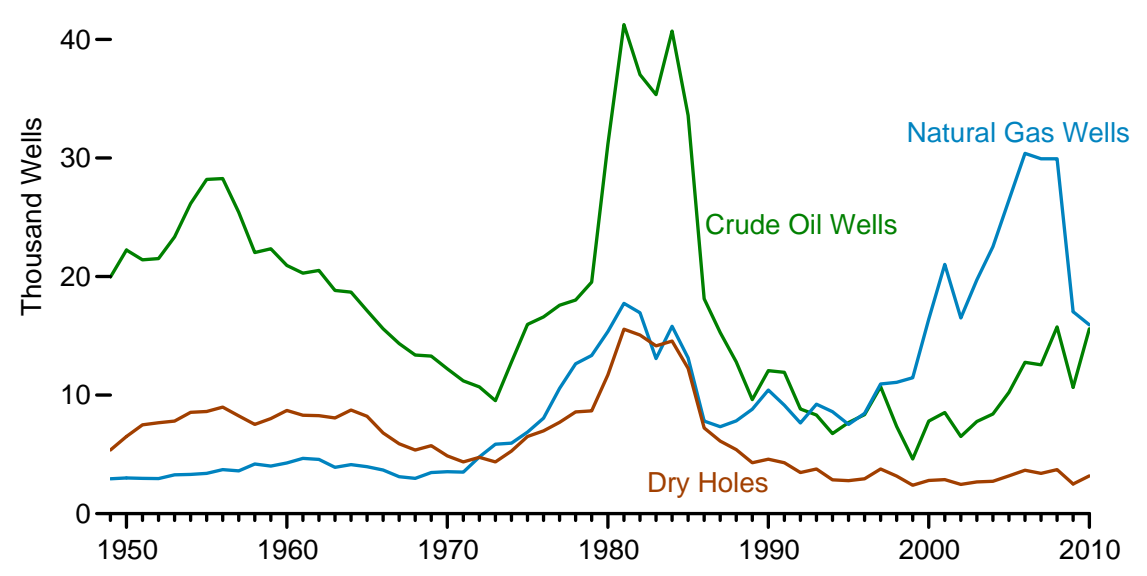

Development Wells Average Depth, All Wells

$10-$

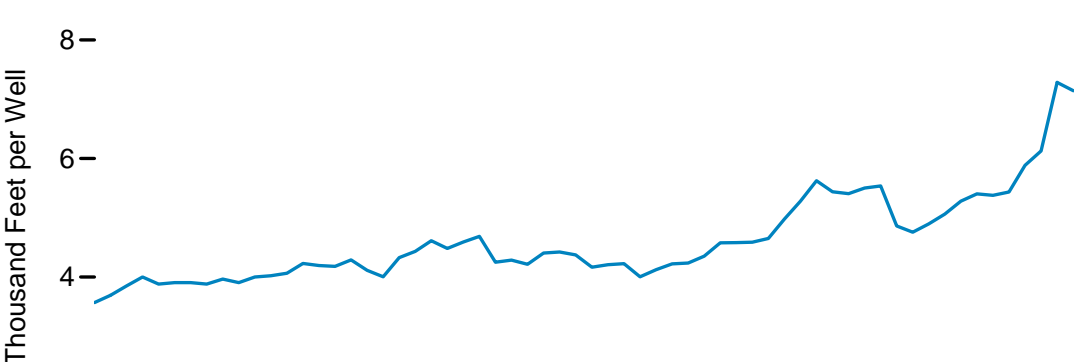

$2-$

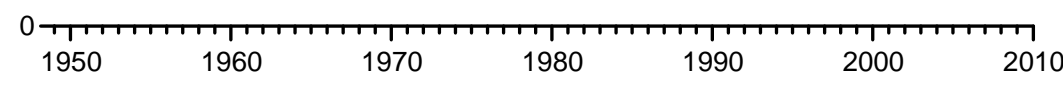

Development Footage Drilled by Well Type

250-

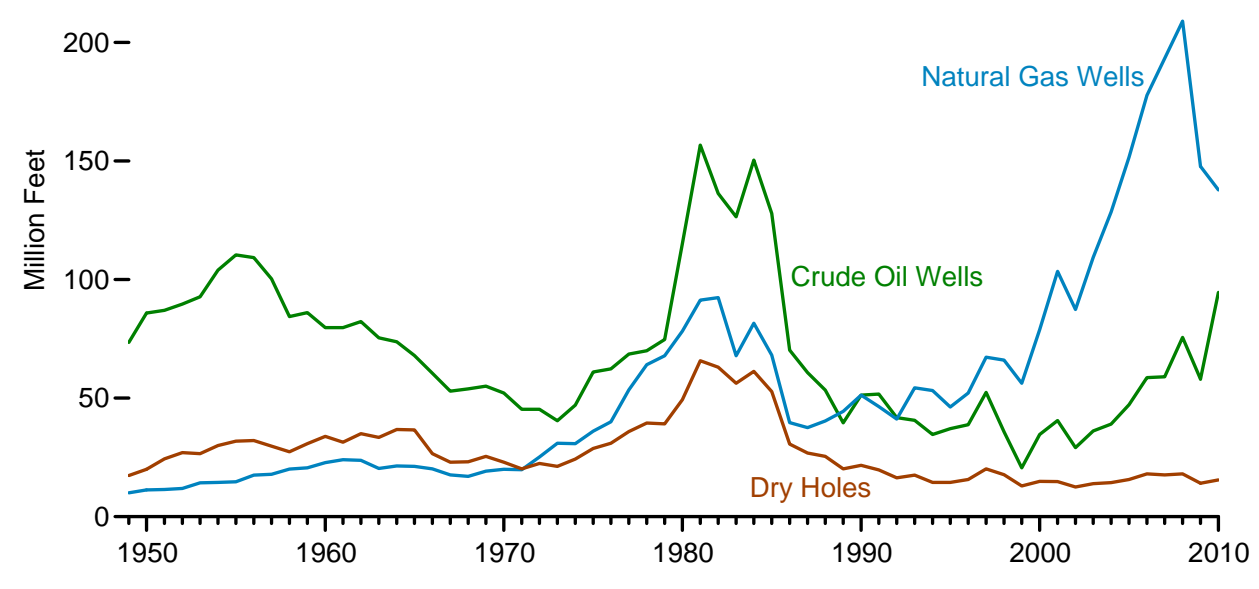

Development Wells Average Depth by Well Type

$10-$

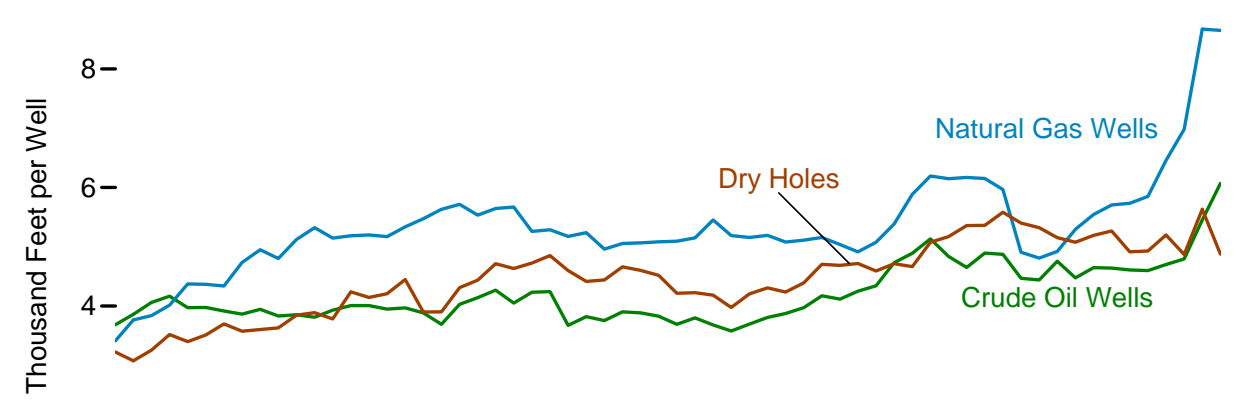

2-

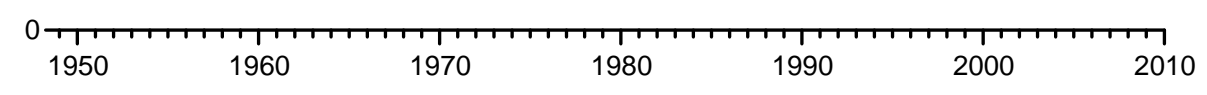

Note: These graphs depict development wells only; see Figure 4.5 for all wells and Figure Source: Table 4.7 . 
Table 4.7 Crude Oil and Natural Gas Development Wells, Selected Years, 1949-2010

\begin{tabular}{|c|c|c|c|c|c|c|c|c|c|c|c|c|c|}
\hline \multirow[b]{3}{*}{ Year } & \multicolumn{4}{|c|}{ Wells Drilled } & \multirow{2}{*}{$\begin{array}{l}\text { Successful } \\
\text { Wells }\end{array}$} & \multicolumn{4}{|c|}{ Footage Drilled ${ }^{1}$} & \multicolumn{4}{|c|}{ Average Footage Drilled } \\
\hline & Crude Oil 2 & Natural Gas ${ }^{3}$ & Dry Holes 4 & Total & & Crude Oil ${ }^{2}$ & Natural Gas ${ }^{3}$ & Dry Holes 4 & Total & Crude Oil ${ }^{2}$ & Natural Gas ${ }^{3}$ & Dry Holes ${ }^{4}$ & Total \\
\hline & \multicolumn{4}{|c|}{ Number } & Percent & \multicolumn{4}{|c|}{ Thousand Feet } & \multicolumn{4}{|c|}{ Feet per Well } \\
\hline 1949 & 19,946 & 2,939 & 5,369 & 28,254 & 81.0 & 73,478 & 10,028 & 17,315 & 100,821 & 3,684 & 3,412 & 3,225 & 3,568 \\
\hline 1950 & 22,229 & 3,008 & 6,507 & $\begin{array}{l}20,204 \\
31,744\end{array}$ & 79.5 & 85,833 & 11,329 & 20,020 & 117,183 & $\begin{array}{l}3,684 \\
3,861\end{array}$ & $\begin{array}{l}3,412 \\
3,766\end{array}$ & $\begin{array}{l}3,225 \\
3,077\end{array}$ & $\begin{array}{l}3,568 \\
3,691\end{array}$ \\
\hline 1955 & 28,196 & 3,392 & 8,620 & 40,208 & 78.6 & 110,374 & 14,718 & 31,883 & 156,976 & 3,915 & 4,339 & 3,699 & 3,904 \\
\hline 1960 & 20,937 & 4,281 & 8,697 & 33,915 & 74.4 & 79,739 & 22,780 & 33,826 & 136,345 & 3,809 & 5,321 & 3,889 & 4,020 \\
\hline 1965 & 17,119 & 3,967 & 8,221 & 29,307 & 71.9 & 67,956 & 21,174 & 36,548 & 125,678 & 3,970 & 5,337 & 4,446 & 4,288 \\
\hline 1970 & 12,211 & 3,534 & 4,869 & 20,614 & 76.4 & 52,130 & 19,945 & 22,951 & 95,026 & 4,269 & 5,644 & 4,714 & 4,610 \\
\hline 1975 & 15,966 & 6,879 & 6,517 & 29,362 & 77.8 & 61,013 & 36,032 & 28,772 & 125,817 & 3,821 & 5,238 & 4,415 & 4,285 \\
\hline 1976 & 16,602 & 8,063 & 6,986 & 31,651 & 77.9 & 62,365 & 39,992 & 31,008 & 133,365 & $\begin{array}{l}3,756 \\
3,756\end{array}$ & $\begin{array}{l}4,960 \\
4\end{array}$ & 4,439 & 4,214 \\
\hline 1977 & 17,581 & $\begin{array}{r}0,574 \\
10,5\end{array}$ & 7,702 & 35,857 & 78.5 & 68,581 & 53,431 & 35,905 & 157,917 & 3,901 & 5,053 & 4,662 & 4,404 \\
\hline 1978 & 18,010 & 12,642 & 8,586 & 39,238 & 78.1 & 69,936 & 64,043 & 39,493 & 173,472 & 3,883 & 5,066 & 4,600 & 4,421 \\
\hline 1979 & 19.530 & 13,347 & 8,662 & 41,539 & 79.1 & 74,747 & 67,825 & 39,130 & 181,702 & 3,827 & 5,082 & 4,517 & 4,374 \\
\hline 1980 & 31,182 & 15,362 & 11,704 & 58,248 & 79.9 & 115,085 & 78,244 & 49,326 & 242,655 & 3,691 & 5,093 & 4,214 & 4,166 \\
\hline 1981 & 41,236 & 17,728 & 15,553 & 74,517 & 79.1 & 156,652 & 91,274 & 65,720 & 313,646 & 3,799 & 5,149 & 4,226 & 4,209 \\
\hline 1982 & 37,022 & 16,943 & 15,072 & 69,037 & 78.2 & 136,261 & 92,386 & 63,066 & 291,713 & 3,681 & 5,453 & 4,184 & 4,225 \\
\hline 1983 & 35,336 & 13,079 & 14,149 & 62,564 & 77.4 & 126,412 & 67,844 & 56,233 & 250,489 & 3,577 & 5,187 & 3,974 & 4,004 \\
\hline 1984 & 40,697 & 15,810 & 14,563 & 71,070 & 79.5 & 150,359 & 81,545 & 61,236 & 293,140 & 3,695 & 5,158 & 4,205 & 4,125 \\
\hline 1985 & 33,581 & 13,124 & 12,257 & 58,962 & 79.2 & 127,874 & 68,149 & 52,784 & 248,807 & 3,808 & 5,193 & 4,306 & 4,220 \\
\hline 1986 & 18,129 & 7,802 & 7,232 & 33,163 & 78.2 & 70,246 & 39,638 & 30,636 & 140,520 & 3,875 & 5,080 & 4,236 & 4,237 \\
\hline 1987 & 15,284 & 7,340 & 6,115 & 28,739 & 78.7 & 60,706 & 37,520 & 26,842 & 125,068 & $\begin{array}{l}3,072 \\
3,972\end{array}$ & 5,112 & 4,390 & 4,352 \\
\hline 1988 & 12,791 & 7,831 & 5,408 & 26,030 & 79.2 & 53,353 & 40,371 & 25,438 & 119,162 & 4,171 & 5,155 & 4,704 & 4,578 \\
\hline 1989 & 9,623 & 8,816 & 4,302 & 22,741 & 81.1 & 39,607 & 44,417 & 20,152 & 104,176 & 4,116 & 5,038 & 4,684 & 4,581 \\
\hline 1990 & $\begin{array}{r}3,061 \\
12,01\end{array}$ & 10,435 & 4,593 & 27,089 & 83.0 & R51,275 & $R_{51,288}$ & $\mathrm{R}_{21,672}$ & $R_{124,235}$ & $\mathrm{R}_{4,251}$ & $\mathrm{R}_{4,915}$ & $\mathrm{R}_{4,718}$ & $\mathrm{R}_{4,586}$ \\
\hline 1991 & 11,915 & 9,144 & 4,290 & 25,349 & 83.1 & R51,726 & $\mathrm{R}_{46,413}$ & $\mathrm{R}_{19,701}$ & $\mathrm{R}_{117,840}$ & $\mathrm{R}_{4,341}$ & $\mathrm{R}_{5,076}$ & $\mathrm{R}_{4,592}$ & $R_{4,649}$ \\
\hline 1992 & 8,831 & 7,650 & 3,478 & 19,959 & 82.6 & $R_{41,789}$ & $\begin{array}{r}41,183 \\
R_{41,4}\end{array}$ & $\mathrm{R}_{16,393}$ & R99,365 & $\begin{array}{r}4,732 \\
R_{4}\end{array}$ & $\begin{array}{l}5,383 \\
R 5\end{array}$ & $\begin{array}{r}4,713 \\
\mathrm{R}_{4}, 713\end{array}$ & $\begin{array}{r}r, 049 \\
R_{4,978}\end{array}$ \\
\hline 1993 & 8,317 & 9,229 & 3,762 & 21,308 & 82.3 & $\mathrm{R}_{4}, 664$ & R54,290 & $R_{17,553}$ & $R_{112,507}$ & $R_{4}, 889$ & $\mathrm{R}_{5}, 883$ & $\mathrm{R}_{4}, 666$ & $\mathrm{R}_{5}, 280$ \\
\hline 1994 & 6,753 & 8,593 & 2,849 & 18,195 & 84.3 & R 34,649 & R53,219 & $R_{14,465}$ & $\mathrm{R}_{102,333}$ & R5,131 & $\mathrm{R}_{6,193}$ & R5,077 & $\mathrm{R}_{5,624}$ \\
\hline 1995 & 7,678 & 7,524 & $\begin{array}{l}2,790 \\
2,790\end{array}$ & $\begin{array}{l}17,992 \\
\end{array}$ & 84.5 & $\begin{array}{r}R 37,149 \\
R\end{array}$ & $\begin{array}{r}\mathrm{R}_{46,252} \\
\mathrm{R}\end{array}$ & $\mathrm{R}_{14,422}$ & R97,823 & $\mathrm{R}_{4,838}$ & $\mathrm{R}_{6,147}$ & $\begin{array}{r}\mathrm{R} 5,169 \\
\text { r }\end{array}$ & $\begin{array}{r}\mathrm{R}, 0<4 \\
\mathrm{R}_{5,437}\end{array}$ \\
\hline 1996 & 8,347 & 8,451 & 2,934 & $\begin{array}{l}19,732 \\
19,73\end{array}$ & 85.1 & $\mathrm{R}_{38,822}$ & $\begin{array}{r}\text { R52,140 } \\
\text { R }\end{array}$ & $\begin{array}{r}r, 414 \\
\mathrm{R}_{15,714}\end{array}$ & $\mathrm{R}_{106,676}$ & $\begin{array}{r}4,651 \\
R_{4}\end{array}$ & $\mathrm{R}_{6,170}$ & $\begin{array}{l}\mathrm{R} 5,356 \\
\mathrm{R}\end{array}$ & $\begin{array}{r}\mathrm{R}, 401 \\
\mathrm{R}_{5,406}\end{array}$ \\
\hline 1997 & $\begin{array}{l}0,715 \\
\end{array}$ & 10,936 & 3,761 & 25,412 & 85.2 & $\mathrm{R}_{52,425}$ & $\mathrm{R}_{67,248}$ & $R_{20,155}$ & $\mathrm{R}_{139,828}$ & $\mathrm{R}_{4,893}$ & $R_{6,149}$ & $R_{5,359}$ & $\mathrm{R}_{5,502}$ \\
\hline 1998 & 7,355 & 11,073 & $\begin{array}{l}3,171 \\
3,171\end{array}$ & $\begin{array}{l}21,599 \\
21,59\end{array}$ & 85.3 & $\mathrm{R}_{35,839}$ & $\mathrm{R}_{66,031}$ & $\mathrm{R}_{17,704}$ & $\mathrm{R}_{119,574}$ & $R_{4,873}$ & $\begin{array}{r}\text { R5,963 } \\
\text { R }\end{array}$ & $\begin{array}{r}\mathrm{R} 5,583 \\
\mathrm{R}\end{array}$ & $\begin{array}{r}\mathrm{R} \\
\mathrm{R} 5,536\end{array}$ \\
\hline 1999 & 4,608 & 11,457 & 2,393 & 18,458 & 87.0 & $\mathrm{R}_{20,591}$ & $\begin{array}{l}\text { R56,220 } \\
\mathrm{R}_{56,20}\end{array}$ & $\mathrm{R}_{12,921}$ & $\begin{array}{l}\text { R89,732 } \\
\text { Re }\end{array}$ & $\begin{array}{r}4,010 \\
4,469\end{array}$ & $\mathrm{R}_{4,907}$ & $\begin{array}{l}\text { r,00 } \\
\text { R5,399 }\end{array}$ & $\begin{array}{r}R_{4,861} \\
\text { r }\end{array}$ \\
\hline 2000 & 7,802 & 16,394 & 2,805 & 27,001 & 89.6 & $R_{34,642}$ & R78,820 & $R_{14,929}$ & $\mathrm{R}_{128,391}$ & $\mathrm{R}_{4,440}$ & $\mathrm{R}_{4,808}$ & 5,322 & $\mathrm{R}_{4,755}$ \\
\hline 2001 & 8.531 & 21.020 & 2865 & 32,416 & 91.2 & $\mathrm{R}_{40.564}$ & $\mathrm{R}_{103} 411$ & $R_{14.763}$ & $\mathrm{R}_{158,738}$ & $\mathrm{R}_{4}, 755$ & $\mathrm{R}_{4}, 920$ & R5, 153 & $R_{4} 897$ \\
\hline 2002 & 6,517 & 16,498 & 2,472 & 25,487 & 90.3 & $\mathrm{R}_{29,150}$ & R87,340 & $\mathrm{R}_{12,545}$ & $R_{129,035}$ & $\mathrm{R} 4,473$ & $\begin{array}{r}\text { R5,294 } \\
\text { R }\end{array}$ & R5,075 & $\begin{array}{r}\mathrm{R} 5,063 \\
\mathrm{R}\end{array}$ \\
\hline 2003 & 7,779 & 19,725 & 2,685 & $\begin{array}{l}20,401 \\
30,189\end{array}$ & 91.1 & $\mathrm{R}_{36,142}$ & $\begin{array}{l}\mathrm{R}_{109,347} \\
\text {. }\end{array}$ & $\begin{array}{r}13,045 \\
R_{13,934}\end{array}$ & $\begin{array}{r}R_{159,423} \\
\text { R }\end{array}$ & $\begin{array}{r}4,4 / 3 \\
\mathrm{R}_{4}, 646\end{array}$ & $\begin{array}{r}3,294 \\
\mathrm{R}_{5}, 544\end{array}$ & R5, 190 & $\begin{array}{r}\text { R5, } 281 \\
\text { R }\end{array}$ \\
\hline 2004 & 8,406 & 22,515 & 2,732 & 33,653 & 91.9 & $R_{39,011}$ & $\mathrm{R}_{128,461}$ & $\mathrm{R}_{14,383}$ & $\mathrm{R}_{181,855}$ & $\mathrm{R}_{4,641}$ & R5,706 & R5,265 & $\mathrm{R}_{5,404}$ \\
\hline 2005 & 10,240 & 26,449 & 3,191 & 39,880 & 92.0 & $R_{47,197}$ & $R_{151,612}$ & $R_{15,685}$ & $R_{214,494}$ & $\mathrm{R} 4,609$ & $\mathrm{R}_{5}, 732$ & $\mathrm{R}_{4}, 915$ & R5 378 \\
\hline 2006 & $\begin{array}{r}r, 758 \\
R_{1}, 758\end{array}$ & $\begin{array}{r}20,44 J \\
R_{30,382}\end{array}$ & $R_{3,659}$ & $\begin{array}{r}\text { R } 46,799 \\
\text { R }\end{array}$ & 92.2 & R58,630 & $\mathrm{R}_{177,659}$ & $\mathrm{R}_{18,033}$ & $\begin{array}{r}\mathrm{R} 254,322 \\
\end{array}$ & $\begin{array}{r}4,596 \\
R_{4}\end{array}$ & $\begin{array}{r}\mathrm{r}, 848 \\
\mathrm{R} 5,8 \mathrm{~s}\end{array}$ & $\mathrm{R}_{4,928}$ & $\begin{array}{r}\mathrm{R} \\
\mathrm{R} 5,434\end{array}$ \\
\hline 2007 & $\mathrm{R}_{12,555}$ & $\begin{array}{l}\mathrm{r} 29,002 \\
\mathrm{R} 29,925\end{array}$ & $\mathrm{R}_{3,396}$ & $\begin{array}{r}45,876 \\
\mathrm{R}_{4}\end{array}$ & R92.6 & $\begin{array}{r}\text { R5,0002 } \\
\text { R59,002 }\end{array}$ & $R_{193,249}$ & $\begin{array}{r}R \\
R_{17,652}\end{array}$ & $\begin{array}{r}\mathrm{R}_{269,903} \\
\text { rel }\end{array}$ & $\begin{array}{r}4,699 \\
\mathrm{R}_{4}, 690\end{array}$ & $\begin{array}{l}\mathrm{r}, 040 \\
\mathrm{R} 6,458\end{array}$ & $\begin{array}{r}4,198 \\
R 5,198\end{array}$ & $\begin{array}{r}\mathrm{R} 5,404 \\
\mathrm{R}, 883\end{array}$ \\
\hline 2008 & $\mathrm{R}_{15,753}$ & $\mathrm{R}_{29,929}$ & $R_{3,713}$ & R 49,395 & R92.5 & R75,558 & $\mathrm{R}_{208,948}$ & $\mathrm{R}_{18,072}$ & $\mathrm{R}_{302,578}$ & $\mathrm{R}_{4,796}$ & $\mathrm{R}_{6,981}$ & $\mathrm{R}_{4,867}$ & $\mathrm{R}_{6,126}$ \\
\hline 2009 & $\mathrm{R}_{10,649}$ & $\mathrm{R}_{17,038}$ & $R_{2,500}$ & R 30,187 & 91.7 & $\begin{array}{r}\text { R58,060 } \\
\text { R5, }\end{array}$ & $\begin{array}{r}\mathrm{R} 147,701 \\
\mathrm{R}\end{array}$ & $\mathrm{R}_{14,077}$ & $\begin{array}{r}\text { R219,838 } \\
\text { R }\end{array}$ & $\begin{array}{r}4,450 \\
R 5\end{array}$ & $\begin{array}{l}\text { R, } \\
\text { R } 6699\end{array}$ & $\begin{array}{r}4,001 \\
\mathrm{R} 5,631\end{array}$ & R7,283 \\
\hline 2010 & $\mathrm{R}_{15,586}$ & $\begin{array}{l}\mathrm{R}_{15,929} \\
\text {. }\end{array}$ & $\mathrm{R}_{3,184}$ & $R_{34,699}$ & R90.8 & R94,583 & $\mathrm{R}_{137,747}$ & $\mathrm{R}_{15,527}$ & $\mathrm{R}_{247,857}$ & $R_{6,068}$ & $\mathrm{R}_{8,648}$ & $\mathrm{R}_{4,877}$ & $\mathrm{R}_{7,143}$ \\
\hline
\end{tabular}

1 See "Footage Drilled" in Glossary

See "Crude Oil Well" in Glossary.

3 See "Natural Gas Well" in Gloss
4 See "Dry Hole" in Glossary.

R=Revised.

Notes: - 2011 data for this table were not available in time for publication. - Data are estimates. - Data are for development wells only; see Table 4.5 for exploratory and development wells combined, and Table 4.6 for exploratory wells only. - Service wells, stratigraphic tests, and core tests are excluded. - For 1949-1959, data represent wells completed in a given year. For 1960-1969, data are for well forward, the data represent wells completed in a given year. The as-received well completion data for recent years are incomplete due to delays in the reporting of wells drilled. The U.S. Energy Information Administration (EIA) therefore statistically imputes the missing data. - Totals may not equal sum of
components due to independent rounding. Average depth may not equal average of components due to independent rounding.

Web Pages: • See http://www.eia.gov/totalenergy/data/monthly/\#crude for updated monthly and annual data. - See http./www.eia.gov/totalenergy/data/annual/\#resources for all annual data beginning in 1949

- See http://www.eia.gov/petroleum/ for related information.

Sources: • 1949-1965-Gulf Publishing Company, World Oil, "Forecast-Review" issue. - 1966-1969-American Petroleum Institute (API), Quarterly Review of Drilling Statistics for the United States, annual summaries and monthly reports. - 1970-1989-EIA computations based on well reports submitted to the API. - 1990 forward-EIA computations based on well reports submitted to IHS, Inc.
Denver, CO. 
Figure 4.8 Coal Demonstrated Reserve Base, January 1, 2011

\section{By Key State}

140-

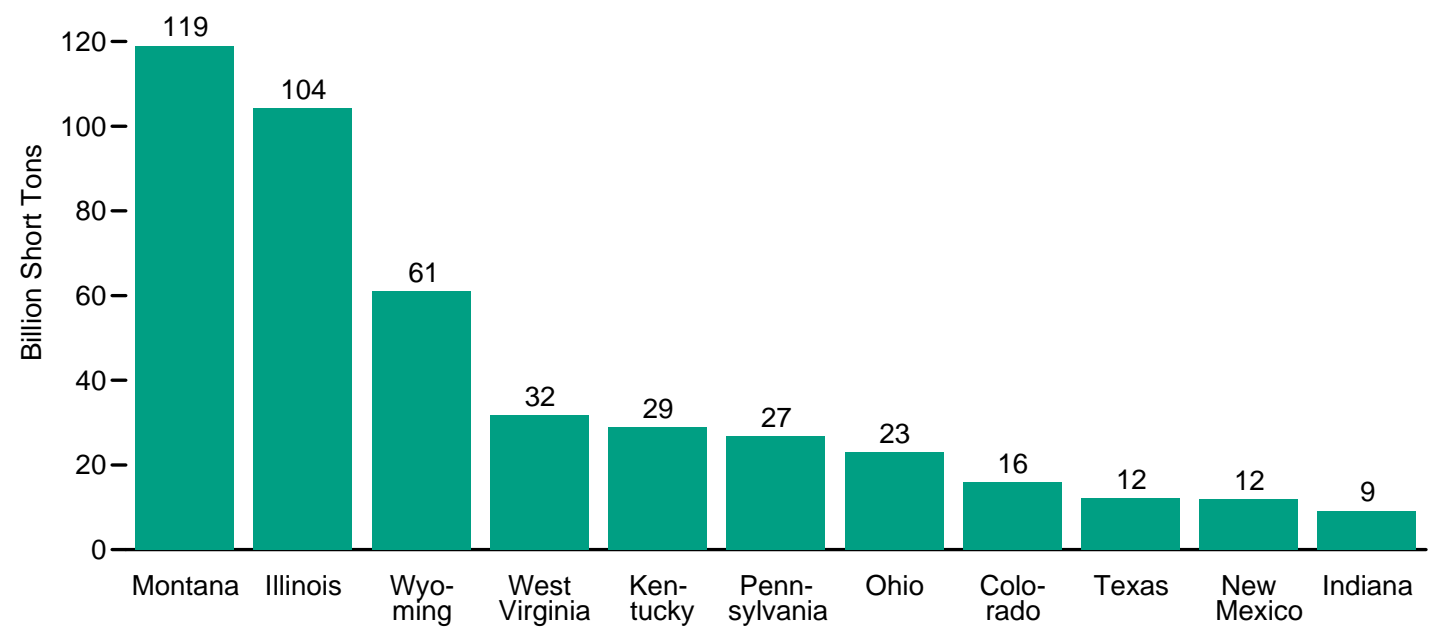

West and East of the Mississippi $300-$
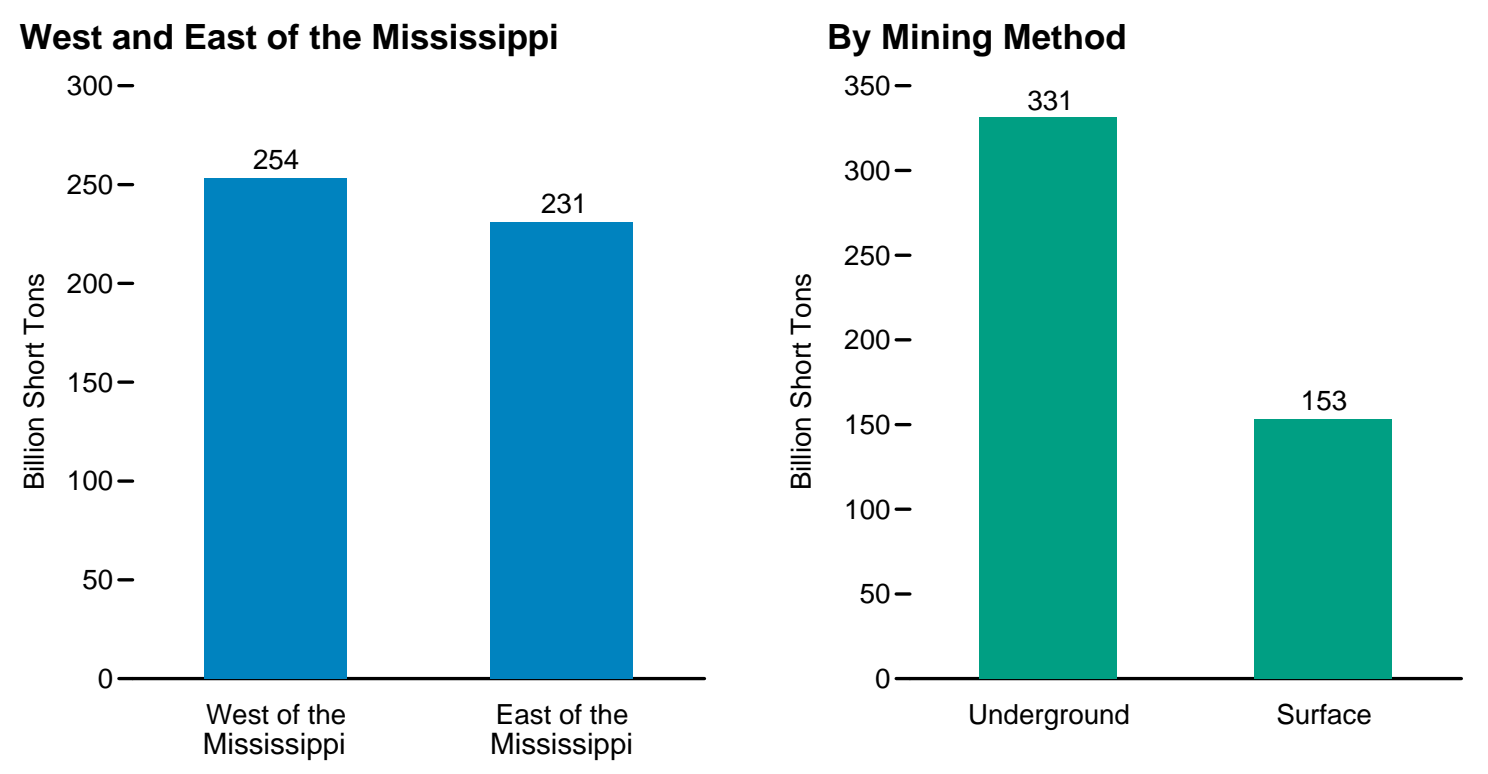

\section{By Region}

$350-$

$300-$

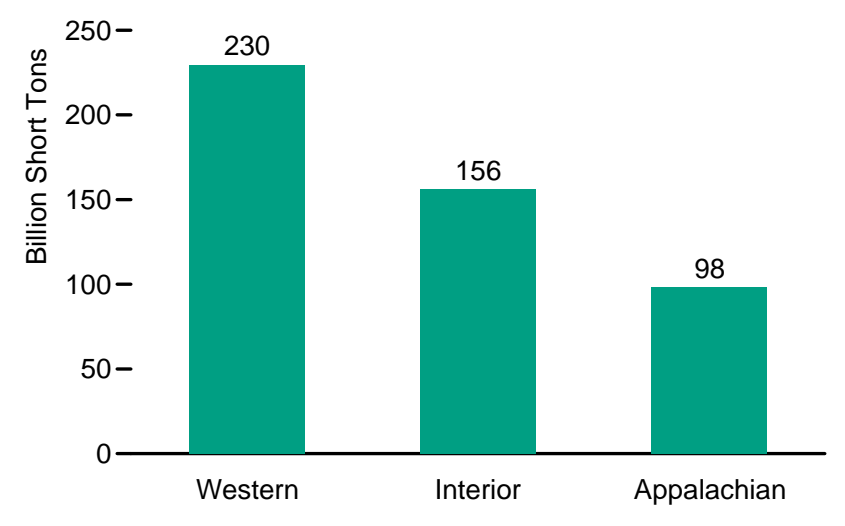

\section{By Rank}

300-

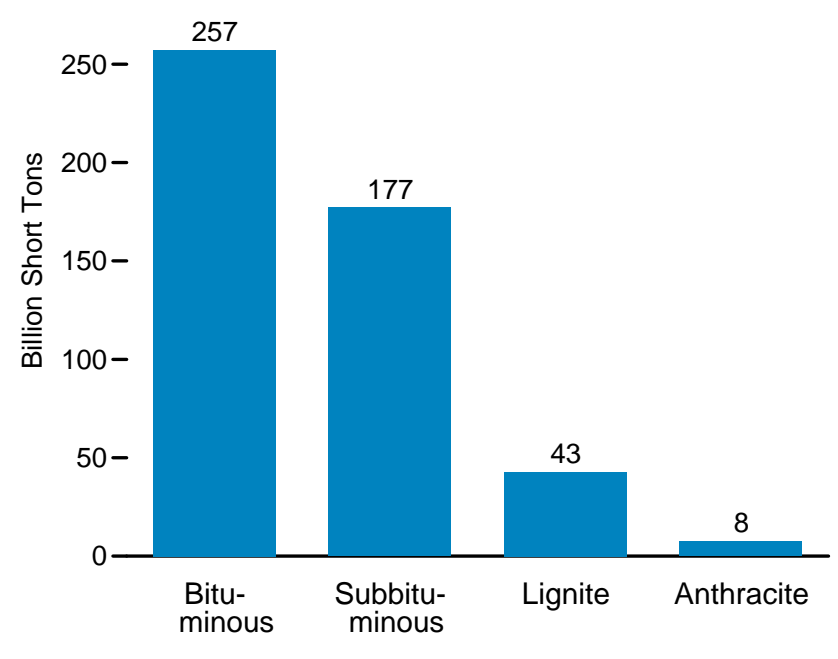

Source: Table 4.8. 
Table 4.8 Coal Demonstrated Reserve Base, January 1, 2011

(Billion Short Tons)

\begin{tabular}{|c|c|c|c|c|c|c|c|c|c|c|}
\hline \multirow[b]{2}{*}{ Region and State } & \multicolumn{2}{|c|}{ Anthracite } & \multicolumn{2}{|c|}{ Bituminous Coal } & \multicolumn{2}{|c|}{ Subbituminous Coal } & \multirow{2}{*}{$\begin{array}{c}\text { Lignite } \\
\text { Surface }{ }^{1}\end{array}$} & \multicolumn{3}{|c|}{ Total } \\
\hline & Underground & Surface & Underground & Surface & Underground & Surface & & Underground & Surface & Total \\
\hline Appalachian & 4.0 & 3.3 & 68.2 & 21.9 & 0.0 & 0.0 & 1.1 & 72.1 & 26.3 & 98.4 \\
\hline 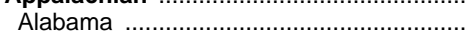 & .0 & .0 & .9 & 2.1 & .0 & .0 & 1.1 & .9 & 3.1 & 4.0 \\
\hline Kentucky, Eastern ...………………………. & .0 & .0 & .8 & 9.1 & .0 & .0 & .0 & .8 & 9.1 & 9.8 \\
\hline Ohio & .0 & .0 & 17.4 & 5.7 & .0 & .0 & .0 & 17.4 & 5.7 & 23.1 \\
\hline Pennsylvania …………………………...... & 3.8 & 3.3 & 18.9 & .8 & .0 & .0 & .0 & 22.7 & 4.2 & 26.9 \\
\hline 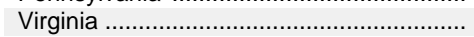 & .1 & .0 & .9 & .5 & .0 & .0 & .0 & 1.0 & .5 & 1.5 \\
\hline 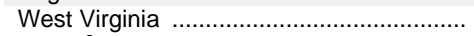 & .0 & .0 & 28.3 & 3.4 & .0 & .0 & .0 & 28.3 & 3.4 & 31.7 \\
\hline 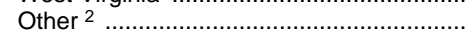 & .0 & .0 & 1.1 & .3 & .0 & .0 & .0 & 1.1 & .3 & 1.4 \\
\hline Interior & .1 & (s) & 116.6 & 27.1 & .0 & .0 & 12.6 & 116.7 & 39.6 & 156.4 \\
\hline 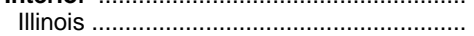 & .0 & .0 & 87.6 & 16.5 & .0 & .0 & .0 & 87.6 & 16.5 & 104.2 \\
\hline 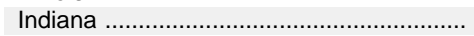 & .0 & .0 & 8.6 & .6 & .0 & .0 & .0 & 8.6 & .6 & 9.2 \\
\hline 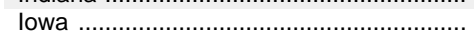 & .0 & .0 & 1.7 & .5 & .0 & .0 & .0 & 1.7 & .5 & 2.2 \\
\hline Kentucky, Western ……………………….... & .0 & .0 & 15.6 & 3.6 & .0 & .0 & .0 & 15.6 & 3.6 & 19.2 \\
\hline 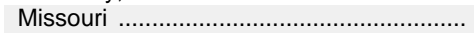 & .0 & .0 & 1.5 & 4.5 & .0 & .0 & .0 & 1.5 & 4.5 & 6.0 \\
\hline Oklahoma …………………………… & .0 & .0 & 1.2 & .3 & .0 & .0 & .0 & 1.2 & .3 & 1.5 \\
\hline 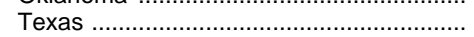 & .0 & .0 & .0 & .0 & .0 & .0 & 12.1 & .0 & 12.1 & 12.1 \\
\hline 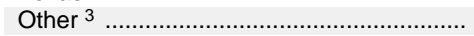 & .1 & (s) & .3 & 1.1 & .0 & .0 & 0.4 & .4 & 1.5 & 1.9 \\
\hline Western & (s) & .0 & 21.2 & 2.3 & 121.2 & 55.9 & 29.2 & 142.4 & 87.4 & 229.7 \\
\hline 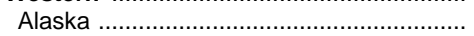 & .0 & .0 & .6 & .1 & 4.8 & .6 & $(\mathrm{~s})$ & 5.4 & .7 & 6.1 \\
\hline 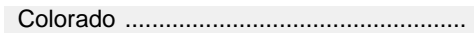 & (s) & .0 & 7.5 & .6 & 3.7 & .0 & 4.2 & 11.2 & 4.8 & 15.9 \\
\hline 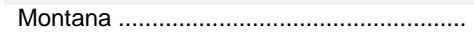 & .0 & .0 & 1.4 & .0 & 69.6 & 32.3 & 15.8 & 70.9 & 48.0 & 119.0 \\
\hline 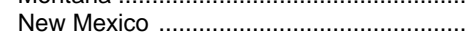 & (s) & .0 & 2.7 & .9 & 3.4 & 5.0 & .0 & 6.1 & 5.9 & 12.0 \\
\hline North Dakota …………………………... & .0 & .0 & .0 & .0 & .0 & .0 & 8.9 & .0 & 8.9 & 8.9 \\
\hline Utah & .0 & .0 & 4.9 & .3 & (s) & .0 & .0 & 4.9 & .3 & 5.2 \\
\hline 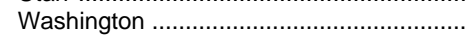 & .0 & .0 & .3 & .0 & 1.0 & .0 & (s) & 1.3 & $(\mathrm{~s})$ & 1.3 \\
\hline 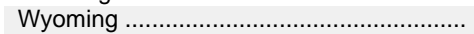 & .0 & .0 & 3.8 & .5 & 38.6 & 18.1 & .0 & 42.5 & 18.5 & 61.0 \\
\hline 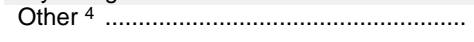 & .0 & .0 & (s) & .0 & (s) & $(\mathrm{s})$ & .4 & (s) & .4 & .4 \\
\hline U.S. Total & 4.1 & 3.4 & 206.0 & 51.2 & 121.1 & 55.9 & 42.8 & 331.2 & 153.3 & 484.5 \\
\hline States East of the Mississippi River .......... & 4.0 & 3.3 & 180.0 & 42.6 & .0 & .0 & 1.1 & 184.0 & 47.0 & 231.0 \\
\hline States West of the Mississippi River ......... & .1 & (s) & 25.9 & 8.6 & 121.1 & 55.9 & 41.7 & 147.2 & 106.3 & 253.5 \\
\hline
\end{tabular}

1 Lignite resources are not mined underground in the United States.

and on file, meeting minimum seam and depth criteria, and in the ground as of January 1, 2011. These coal resources are not totally recoverable. Net recoverability with current mining technologies ranges from 0 percent (in far northern Alaska) to more than 90 percent. Fifty-four percent of the demonstrated reserve Georgia, Maryland, North Carolina, and Tennessee.

3 Arkansas, Kansas, Louisiana, and Michigan.

4 Arizona, Idaho, Oregon, and South

Notes: - See U.S. Coal Reserves: 1997 Update on the Web Page for a description of the methodology used to produce these data. - Data represent remaining measured and indicated coal resources, analyzed base of coal in the United States is estimated to be recoverable. - Totals may not equal sum of components due to independent rounding.

Web Page: For related information, see http://www.eia.gov/coal/.

Source: U.S. Energy Information Administration, Coal Reserves Database. 


\section{Figure 4.9 Uranium Exploration and Development Drilling}

\section{Total Holes Drilled, 1958-2011 ${ }^{1}$}

$120-$

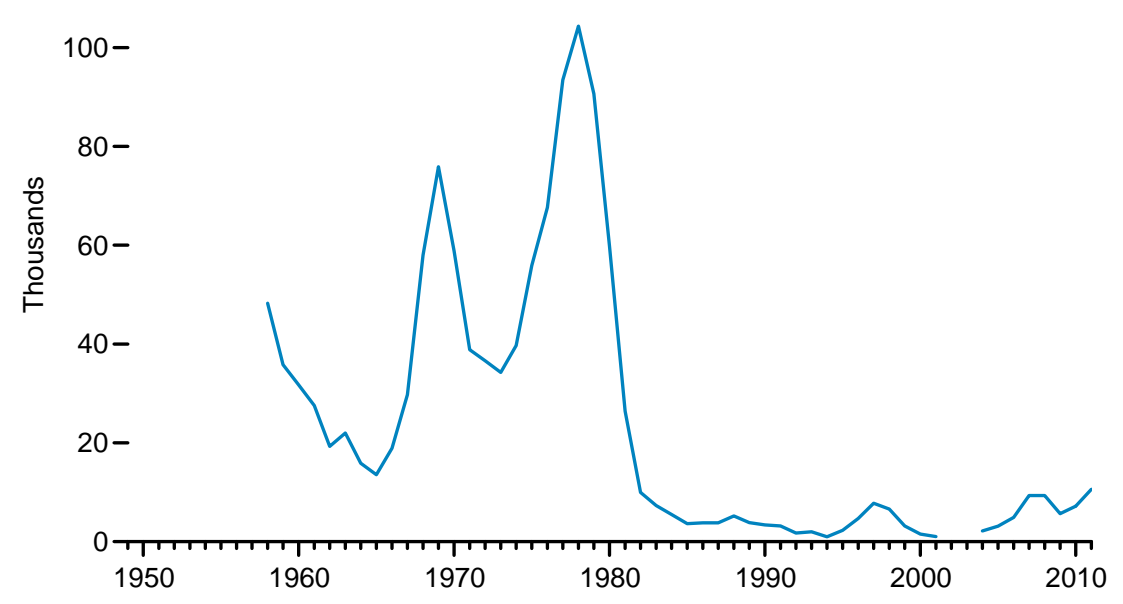

Total Footage Drilled, 1949-2011

$60-$

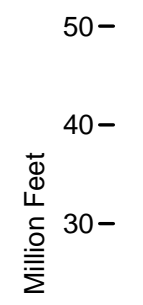

$20-$

20

$10-$

$10-$

$$
1950
$$

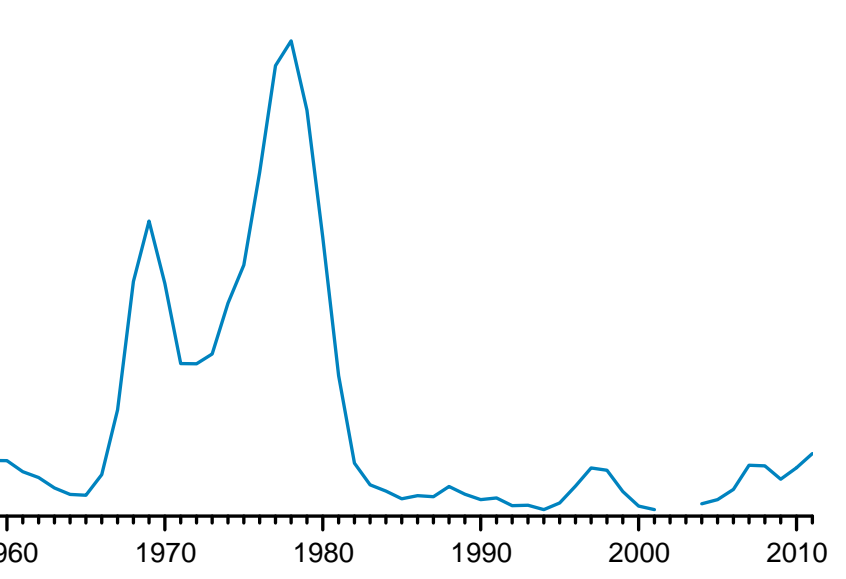

${ }^{1}$ In 2002 and 2003, data are withheld to avoid disclosure.

\section{Holes Drilled, Selected Years}

$30-$

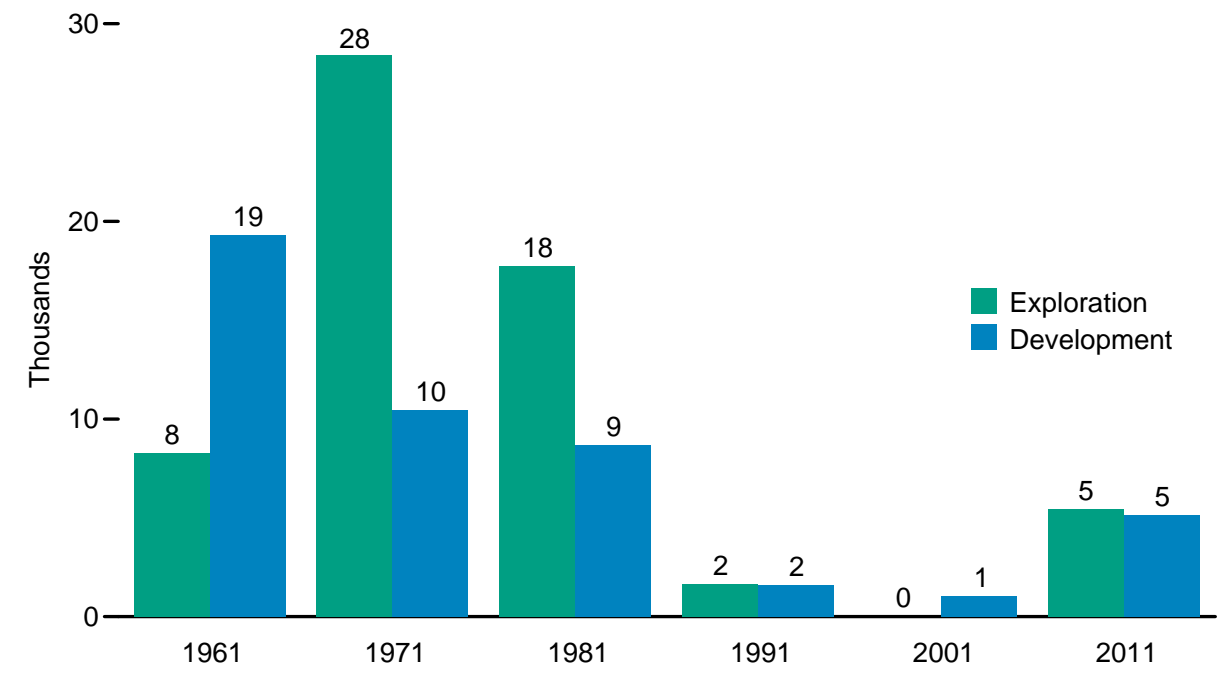

Footage Drilled, Selected Years

$12-$

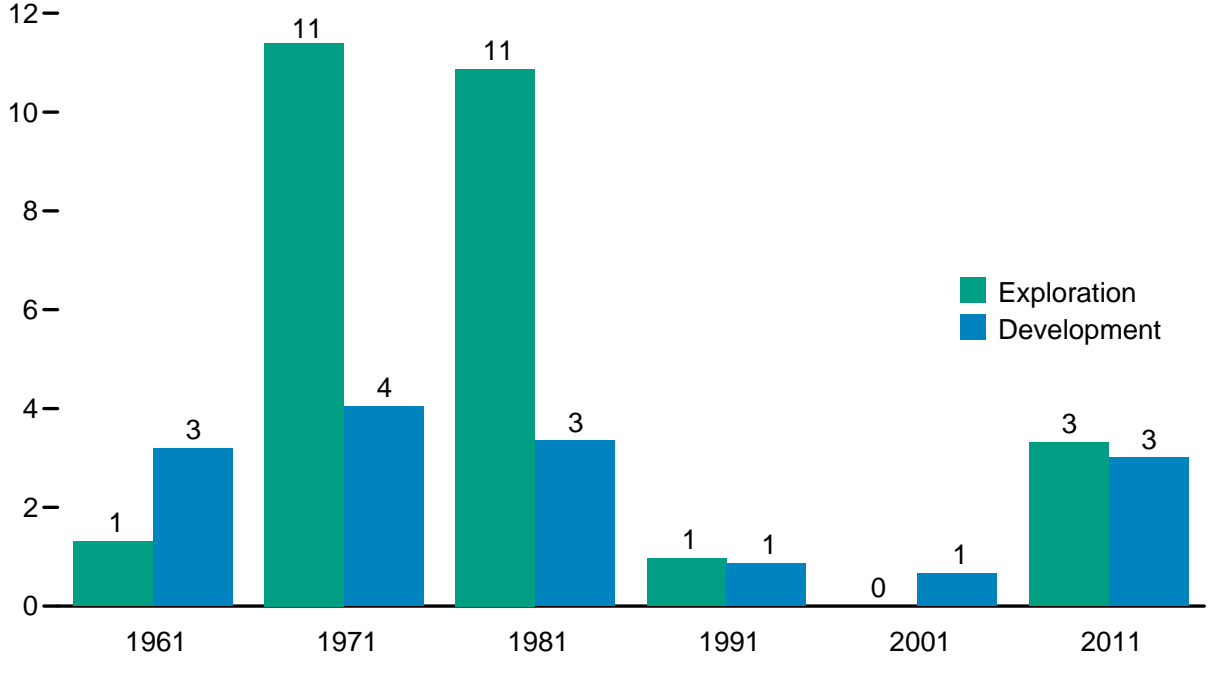


Table 4.9 Uranium Exploration and Development Drilling, Selected Years, 1949-2011

\begin{tabular}{|c|c|c|c|c|c|c|}
\hline \multirow[b]{3}{*}{ Year } & \multicolumn{2}{|c|}{ Exploration 1} & \multicolumn{2}{|c|}{ Development ${ }^{2}$} & \multicolumn{2}{|c|}{ Total } \\
\hline & Holes Drilled & Footage Drilled & Holes Drilled & Footage Drilled & Holes Drilled & Footage Drilled \\
\hline & Thousands & Million Feet & Thousands & Million Feet & Thousands & Million Feet \\
\hline 1949 & NA & 0.36 & NA & 0.05 & NA & 0.41 \\
\hline 1950 & NA & .57 & NA & .21 & NA & .78 \\
\hline 1955 & NA & 5.27 & NA & .76 & NA & 6.03 \\
\hline 1960 & 7.34 & 1.40 & 24.40 & 4.21 & 31.73 & 5.61 \\
\hline 1965 & 6.23 & 1.16 & 7.33 & .95 & 13.56 & 2.11 \\
\hline 1970 & 43.98 & 17.98 & 14.87 & 5.55 & 58.85 & 23.53 \\
\hline 1975 & 34.29 & 15.69 & 21.60 & 9.73 & 55.89 & 25.42 \\
\hline 1976 & 40.41 & 20.36 & 27.23 & 14.44 & 67.64 & 34.80 \\
\hline 1977 & 62.60 & 27.96 & 30.86 & 17.62 & 93.45 & 45.58 \\
\hline 1978 & 75.07 & 28.95 & 29.29 & 19.15 & 104.35 & 48.10 \\
\hline 1979 & 60.46 & 28.07 & 30.19 & 13.01 & 90.65 & 41.08 \\
\hline 1980 & 39.61 & 19.60 & 20.19 & 8.59 & 59.80 & 28.19 \\
\hline 1981 & 17.75 & 10.87 & 8.67 & 3.35 & 26.42 & 14.22 \\
\hline 1982 & 6.97 & 4.23 & 3.00 & 1.13 & 9.97 & 5.36 \\
\hline 1983 & 4.29 & 2.09 & 3.01 & 1.08 & 7.30 & 3.17 \\
\hline 1984 & 4.80 & 2.26 & .72 & .29 & 5.52 & 2.55 \\
\hline 1985 & 2.88 & 1.42 & .77 & .34 & 3.65 & 1.76 \\
\hline 1986 & 1.99 & 1.10 & 1.85 & .97 & 3.83 & 2.07 \\
\hline 1987 & 1.82 & 1.11 & 1.99 & .86 & 3.81 & 1.97 \\
\hline 1988 & 2.03 & 1.28 & 3.18 & 1.73 & 5.21 & 3.01 \\
\hline 1989 & 2.09 & 1.43 & 1.75 & .80 & 3.84 & 2.23 \\
\hline 1990 & 1.51 & .87 & 1.91 & .81 & 3.42 & 1.68 \\
\hline 1991 & 1.62 & .97 & 1.57 & .87 & 3.20 & 1.84 \\
\hline 1992 & .94 & .56 & .83 & .50 & 1.77 & 1.06 \\
\hline 1993 & .36 & .22 & 1.67 & .89 & 2.02 & 1.11 \\
\hline 1994 & .52 & .34 & .48 & .32 & 1.00 & .66 \\
\hline 1995 & .58 & .40 & 1.73 & .95 & 2.31 & 1.35 \\
\hline 1996 & 1.12 & $\begin{array}{l}.40 \\
.88\end{array}$ & 3.58 & 2.16 & 4.70 & 3.05 \\
\hline 1997 & $\begin{array}{l}1.94 \\
\end{array}$ & 1.33 & 5.86 & 3.56 & 7.79 & 4.88 \\
\hline 1998 & 1.37 & .89 & 5.23 & 3.75 & 6.60 & 4.64 \\
\hline 1999 & .27 & .18 & 2.91 & 2.33 & 3.18 & 2.50 \\
\hline 2000 & W & W & W & W & 1.55 & 1.02 \\
\hline 2001 & .00 & .00 & 1.02 & .66 & 1.02 & .66 \\
\hline 2002 & W & W & W & W & W & W \\
\hline 2003 & NA & NA & NA & NA & W & W \\
\hline 2004 & W & W & W & W & 2.19 & 1.25 \\
\hline 2005 & W & w & w & W & 3.14 & 1.67 \\
\hline 2006 & 1.47 & .82 & 3.43 & 1.89 & 4.90 & 2.71 \\
\hline 2007 & 4.35 & 2.20 & 5.00 & 2.95 & 9.35 & 5.15 \\
\hline 2008 & 5.20 & 2.54 & 4.16 & 2.55 & 9.36 & 5.09 \\
\hline 2009 & 1.79 & 1.05 & 3.89 & 2.69 & 5.68 & 3.74 \\
\hline 2010 & 2.44 & 1.46 & 4.77 & 3.44 & 7.21 & 4.90 \\
\hline 2011 & 5.44 & $\begin{array}{l}3.40 \\
3.32\end{array}$ & 5.16 & $\begin{array}{l}3.44 \\
3.00\end{array}$ & 10.60 & 6.33 \\
\hline
\end{tabular}

1 Includes surface drilling in search of new ore deposits or extensions of known deposits and drilling at the location of a discovery up to the time the company decides sufficient ore reserves are present to justify commercial exploitation.

2 Includes all surface drilling on an ore deposit to determine more precisely size, grade, and configuration subsequent to the time that commercial exploitation is deemed feasible.

$\mathrm{NA}=$ Not available. $\mathrm{W}=$ Value withheld to avoid disclosure of individual company data.

Note: Totals may not equal sum of components due to independent rounding.
Web Pages: - See http://www.eia.gov/totalenergy/data/annual/\#resources for all data beginning in 1949. • For related information, see http://www.eia.gov/nuclear/.

Sources: - 1949-1981-U.S. Department of Energy, Grand Junction Office, Statistical Data of the Uranium Industry, January 1, 1983, Report No. GJO-100 (1983), Table VIII-5. • 1982-2002-U.S. Energy Information Administration (EIA), Uranium Industry Annual, annual reports. • 2003-2005-EIA, "Domestic Uranium Production Report," annual reports. • 2006 forward-EIA, "2011 Domestic Uranium Production Report" (May 2012), Table 1. 
Figure 4.10 Uranium Reserves, 2008

\section{By State}

$500-$

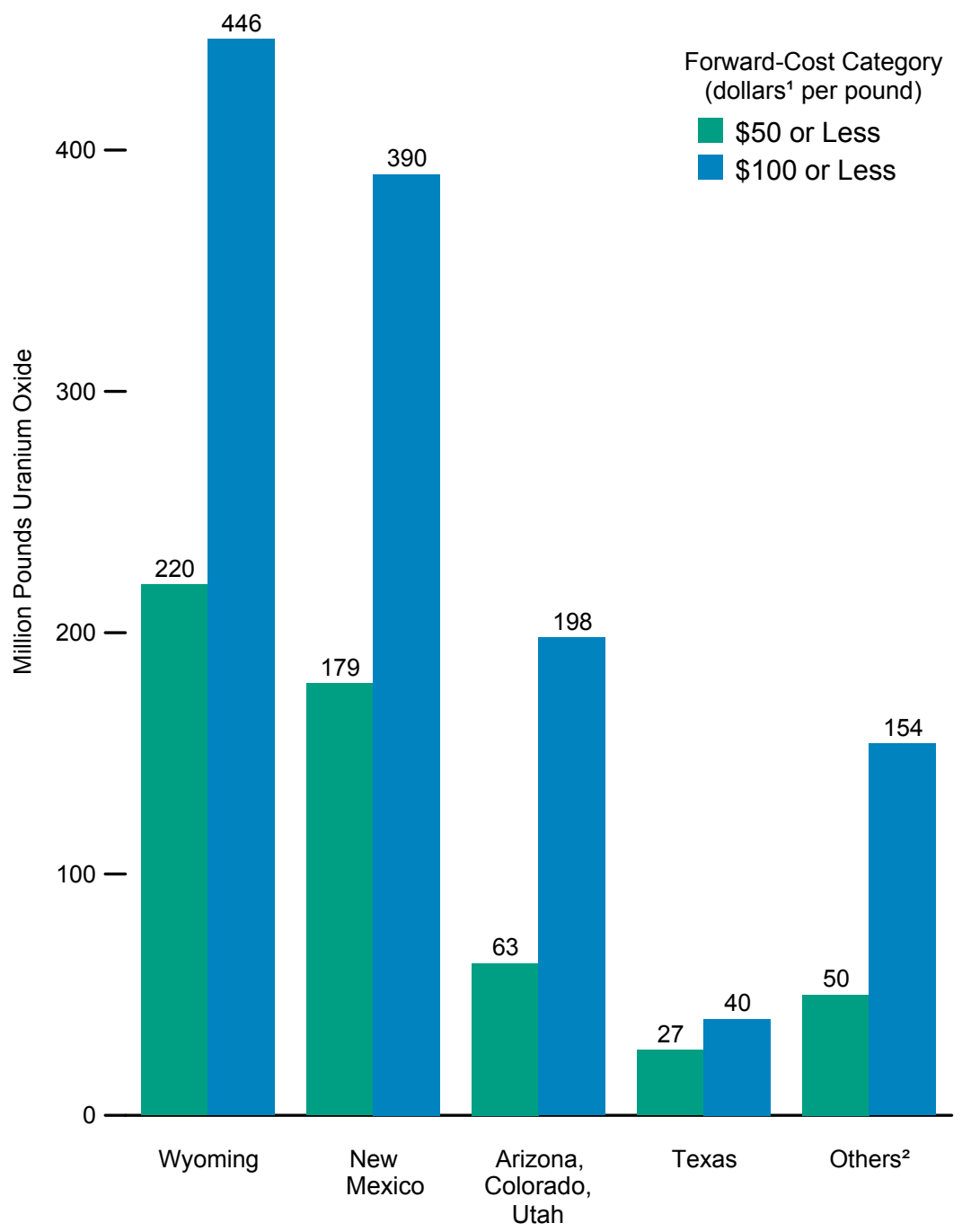

\section{Total Reserves}

$1,500-$

${ }^{1}$ See "Nominal Dollars" in Glossary.

${ }^{2}$ Alaska, California, Idaho, Montana, Nebraska, Nevada, North Dakota, Oregon, South Dakota, Virginia, and Washington.

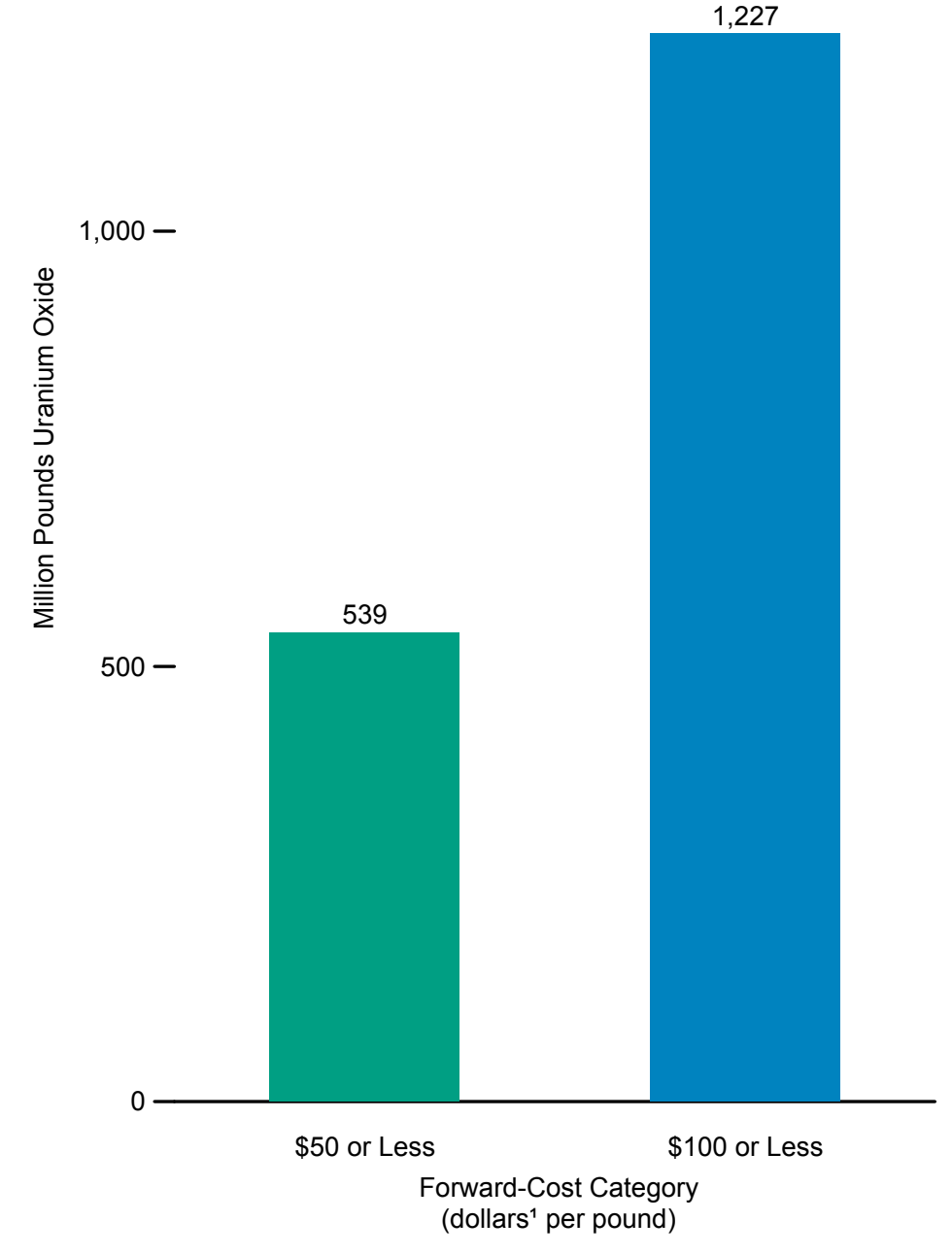

Notes: - See "Uranium Oxide" in Glossary. - Data are at end of year.

1,227

Source: Table 4.10 . 
Table 4.10 Uranium Reserves, ${ }^{1} 2008$

(Million Pounds Uranium Oxide)

\begin{tabular}{|c|c|c|}
\hline \multirow[b]{2}{*}{ State } & \multicolumn{2}{|c|}{ Forward-Cost ${ }^{2}$ Category (dollars ${ }^{3}$ per pound) } \\
\hline & $\$ 50$ or Less & $\$ 100$ or Less \\
\hline Total & 539 & 1,227 \\
\hline 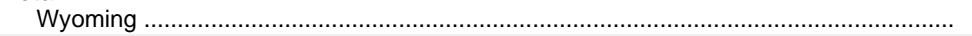 & 220 & 446 \\
\hline New Mexico …ㅅ․ & 179 & 390 \\
\hline Arizona, Colorado, Utah & 63 & 198 \\
\hline Texas & 27 & 40 \\
\hline 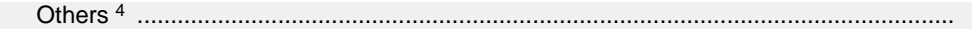 & 50 & 154 \\
\hline
\end{tabular}

1 The U.S. Energy Information Administration (EIA) category of uranium reserves is equivalent to the internationally reported category of "Reasonably Assured Resources" (RAR).

2 Forward costs include the costs for power and fuel, labor, materials, insurance, severance and ad valorem taxes, and applicable administrative costs. Past capital costs are considered "sunk" costs and mining of the individual deposits may or may not return such costs to investors. Sunk costs for such items as exploration and land acquisition are excluded as are the costs for income taxes, profit, and the cost of money. The forward costs used to estimate U.S. uranium ore reses are independent of the price at which uranium produced from the estimated reserves might be sold in the commercial market. Reserves values in forward-cost categories are cumulative; that is, the quantity at each level of forward cost includes all reserves at the lower cost in that category.

Prices are not adjusted for inflation. See "Nominal Dollars" in Glossary.

${ }^{4}$ Alaska, California, Idaho, Montana, Nebraska, Nevada, North Dakota, Oregon, South Dakota, Virginia, and Washington.

Notes: • Estimates are at end of year. • See "Uranium Oxide" in Glossary. • For updates, see http://www.eia.gov/cneaf/nuclear/page/reserves/ures.html.

Web Page: For related information, see http://www.eia.gov/nuclear/.

Sources: EIA, U.S. Uranium Reserves Estimates (July 2010), Table 1. 


\section{Figure 4.11 Concentrating Solar Resources}

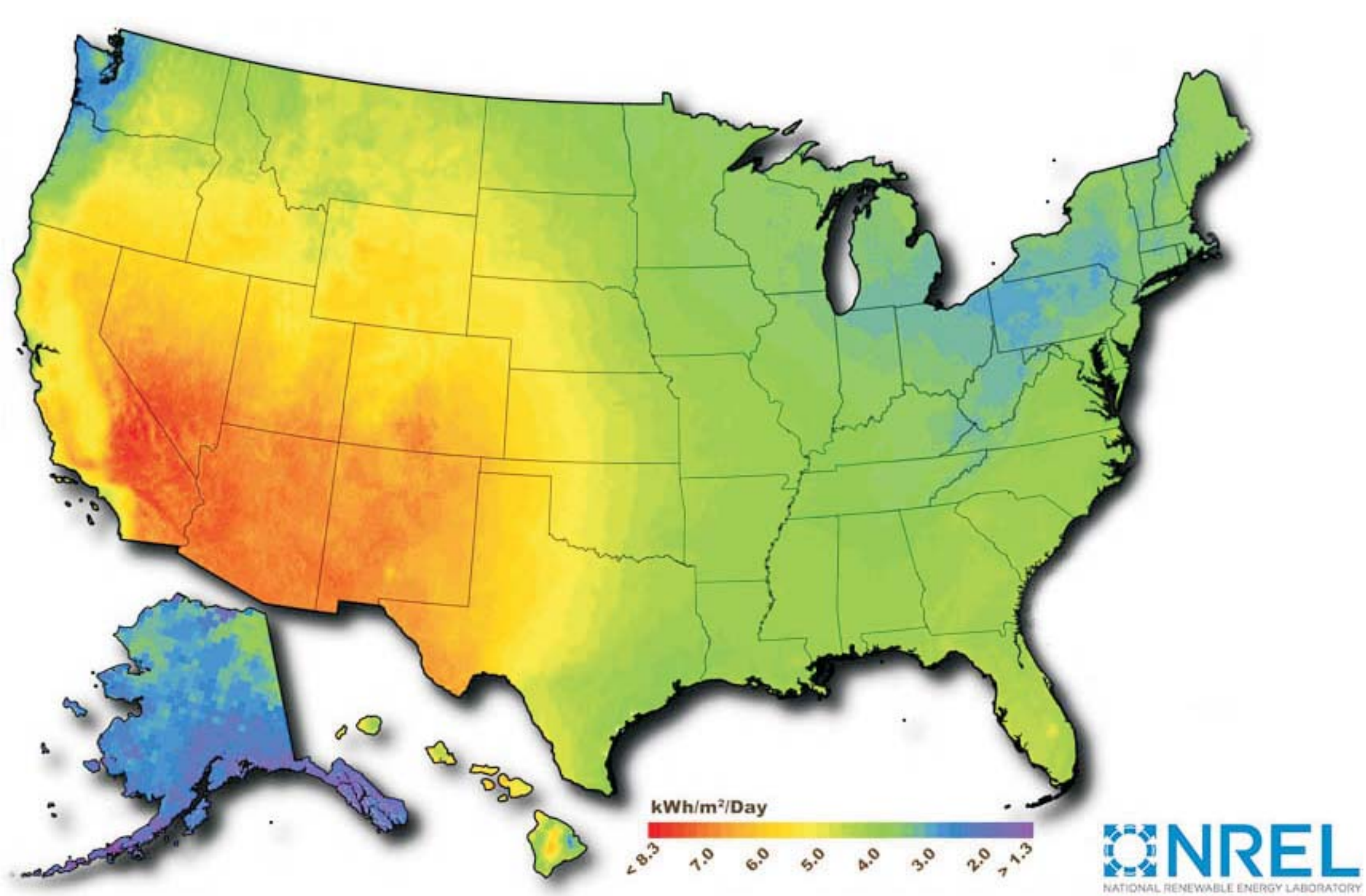

Notes: - Annual average direct normal solar resource data are shown. $\cdot \mathrm{kWh} / \mathrm{m}^{2} / \mathrm{Day}=$ kilowatthours per square meter per day.

Web Page: For related information, see http://www.nrel.gov/gis/maps.html.

Sources: This map was created by the National Renewable Energy Laboratory for the

Department of Energy (October 20, 2008). The data for Hawaii and the 48 contiguous States are a 10-kilometer $(\mathrm{km})$ satellite modeled dataset (SUNY/NREL, 2007) representing data from 1998-2005. The data for Alaska are a 40-km dataset produced by the Climatological Solar Radiation Model (NREL, 2003). 


\section{Figure 4.12 Photovoltaic Solar Resources}

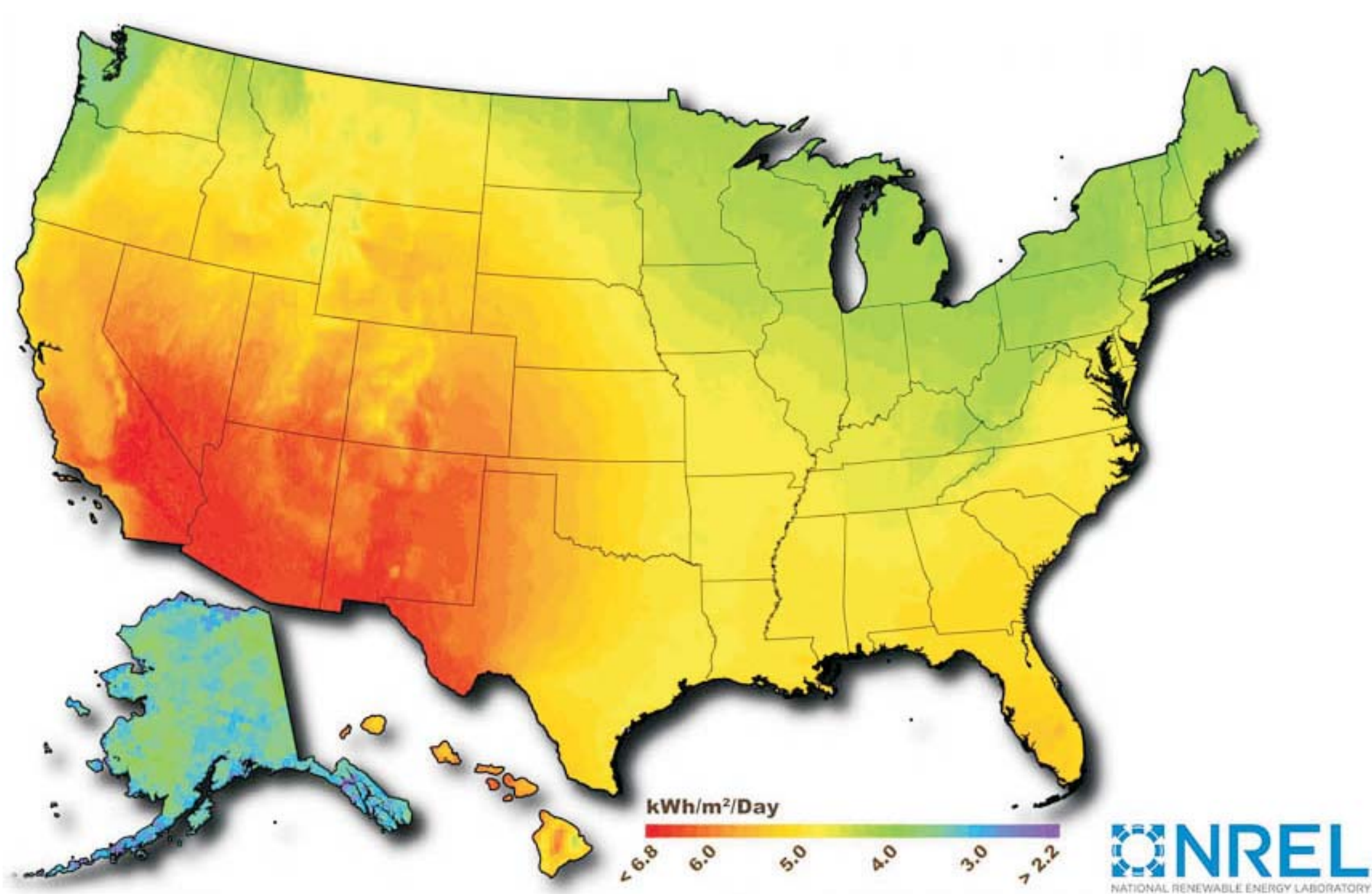

Notes: • Annual average solar resource data are shown for a tilt=latitude collector. • kWh/m²/Day = kilowatthours per square meter per day.

Web Page: For related information, see http://www.nrel.gov/gis/maps.html.
Sources: This map was created by the National Renewable Energy Laboratory for the Department of Energy (October 20,2008). The data for Hawaii and the 48 contiguous States are a 10-kilometer $(\mathrm{km})$ satellite modeled dataset (SUNY/NREL, 2007) representing data from 19982005. The data for Alaska are a 40-km dataset produced by the Climatological Solar Radiation Model (NREL, 2003). 


\section{Figure 4.13 Onshore Wind Resources}

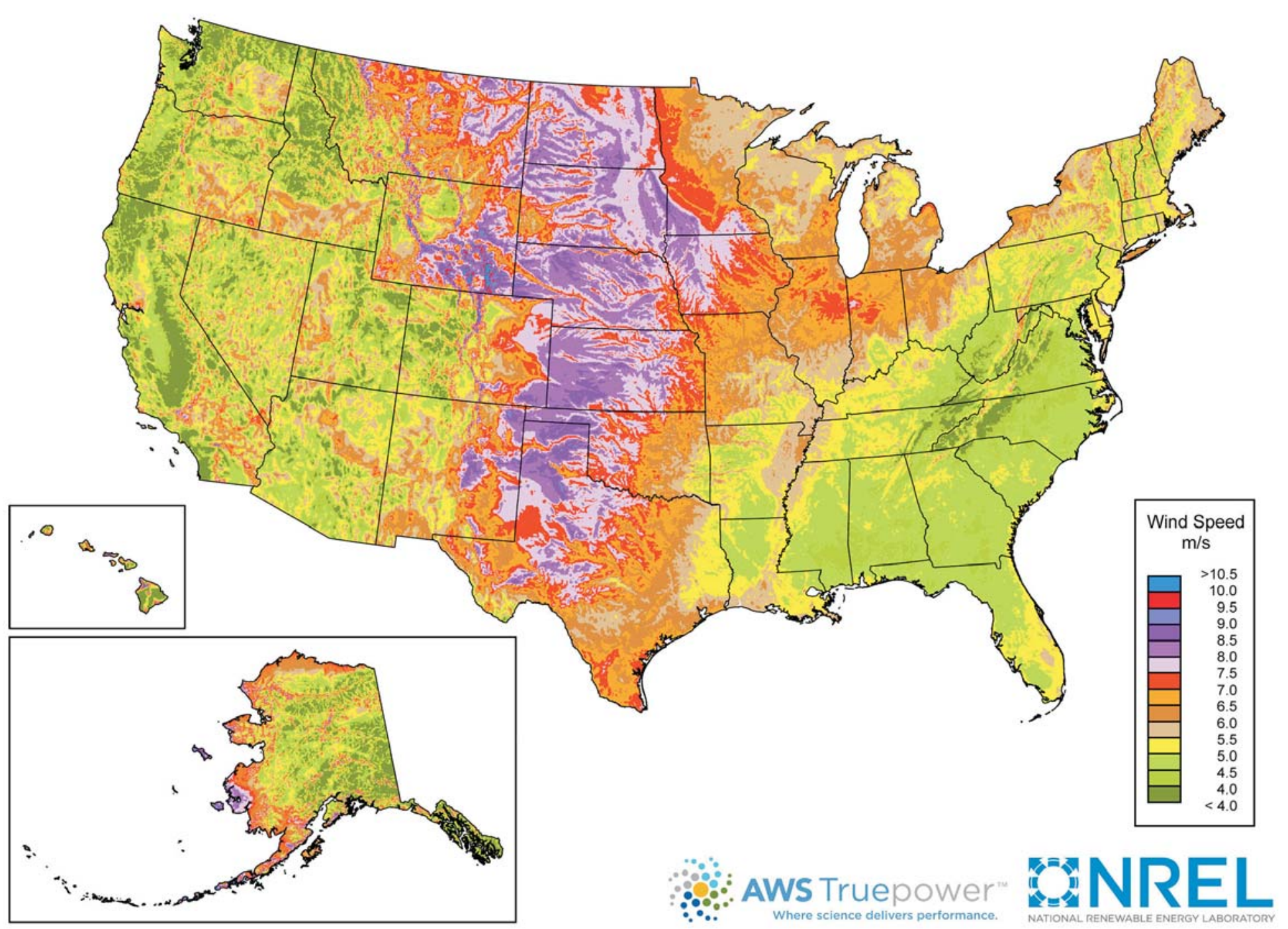

Notes: - Data are annual average wind speed at 80 meters. $\cdot \mathrm{m} / \mathrm{s}=$ meters per second. Web Page: For related information, see http://www.nrel.gov/gis/maps.html.

Sources: This map was created by the National Renewable Energy Laboratory for the Department of Energy (April 1, 2011). Wind resource estimates developed by AWS
Truepower, LLC for windNavigator ${ }^{\circ}$. See http://www.windnavigator.com and http://www.awstruepower.com. Spatial resolution of wind resource data: 2.5 kilometers. Projection: Albers Equal Area WGS84. 


\section{Figure 4.14 Offshore Wind Resources}

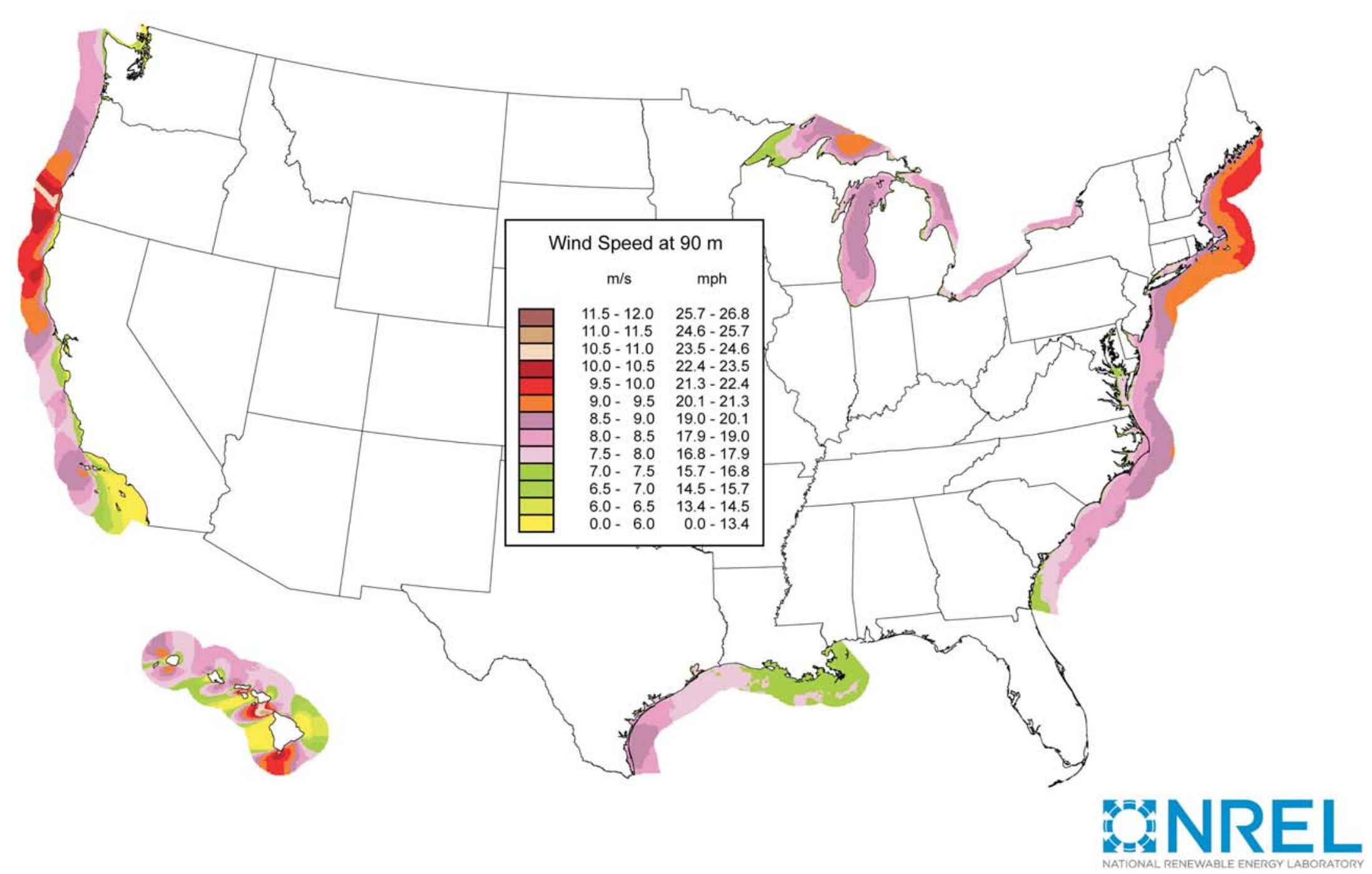

Notes: • Data are annual average wind speed at 90 meters. $\cdot \mathrm{m} / \mathrm{s}=$ meters per second

Web Page: For related information, see http://www.nrel.gov/gis/maps.html.

- $\mathrm{mph}=$ miles per hour.

Source: This map was created by the National Renewable Energy Laboratory for the

Department of Energy (January 10, 2011). 


\section{Figure 4.15 Geothermal Resources}

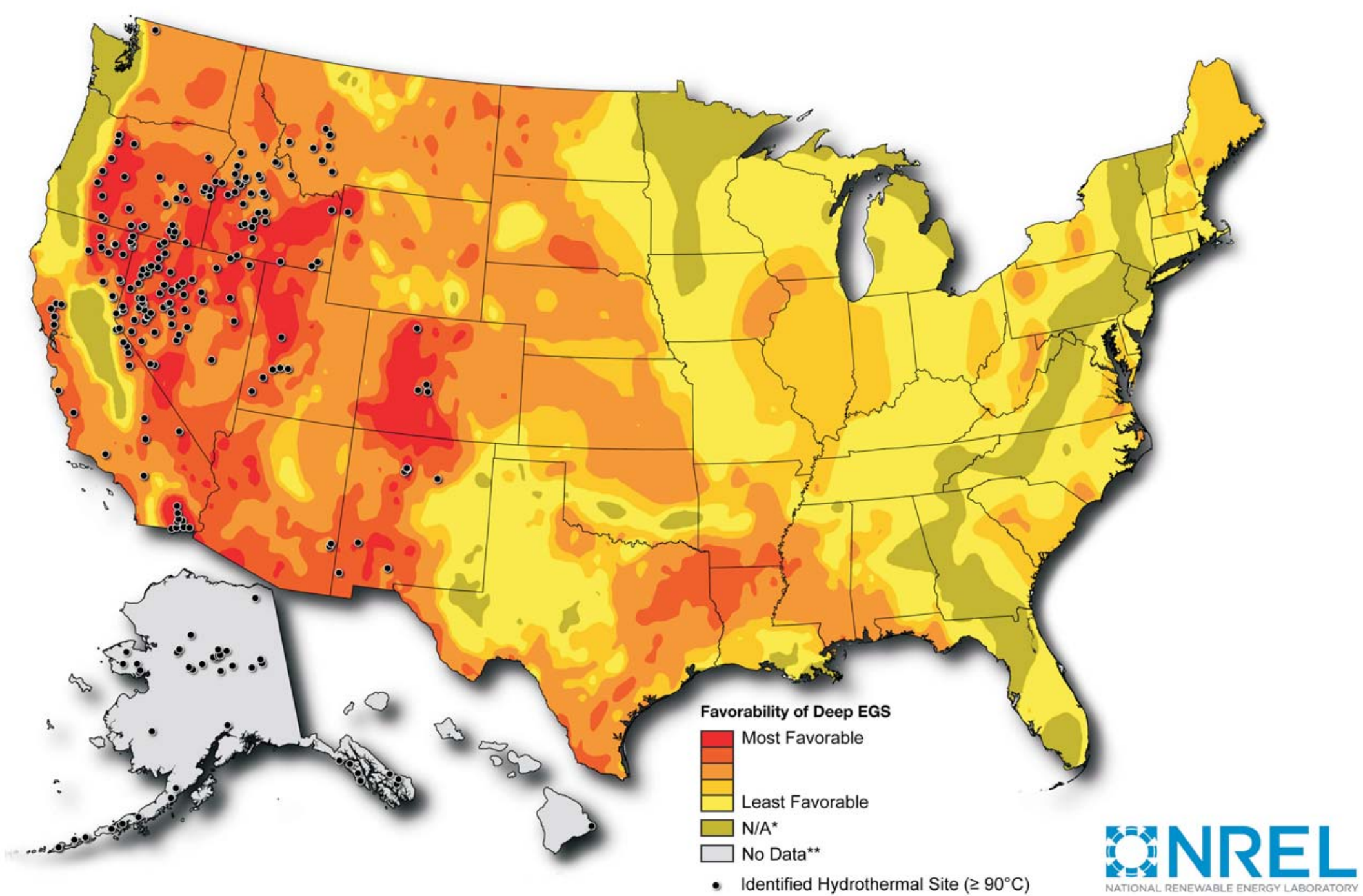

Notes: - Data are for locations of identified hydrothermal sites and favorability of deep enhanced geothermal systems (EGS). - Map does not include shallow EGS resources located near hydrothermal sites or USGS assessment of undiscovered hydrothermal resources. - *"N/A" regions have temperatures less than $150^{\circ} \mathrm{C}$ at 10 kilometers $(\mathrm{km})$ depth and were not assessed for deep EGS potential. • **Temperature at depth data for deep EGS in Alaska and Hawaii not available.

Web Page: For related information, see http://www.nrel.gov/gis/maps.html.
Sources: This map was created by the National Renewable Energy Laboratory for the Department of Energy (October 13, 2009). Source data for deep EGS includes temperature at depth from 3 to $10 \mathrm{~km}$ provided by Southern Methodist University Geothermal Laboratory (Blackwell \& Richards, 2010) and analyses (for regions with temperatures $\geq 150^{\circ} \mathrm{C}$ ) performed by NREL (2009). Source data for identified hydrothermal sites from USGS Assessment of Moderate- and High-Temperature Geothermal Resources of the United States (2008). 


\section{Figure 4.16 Biomass Resources}

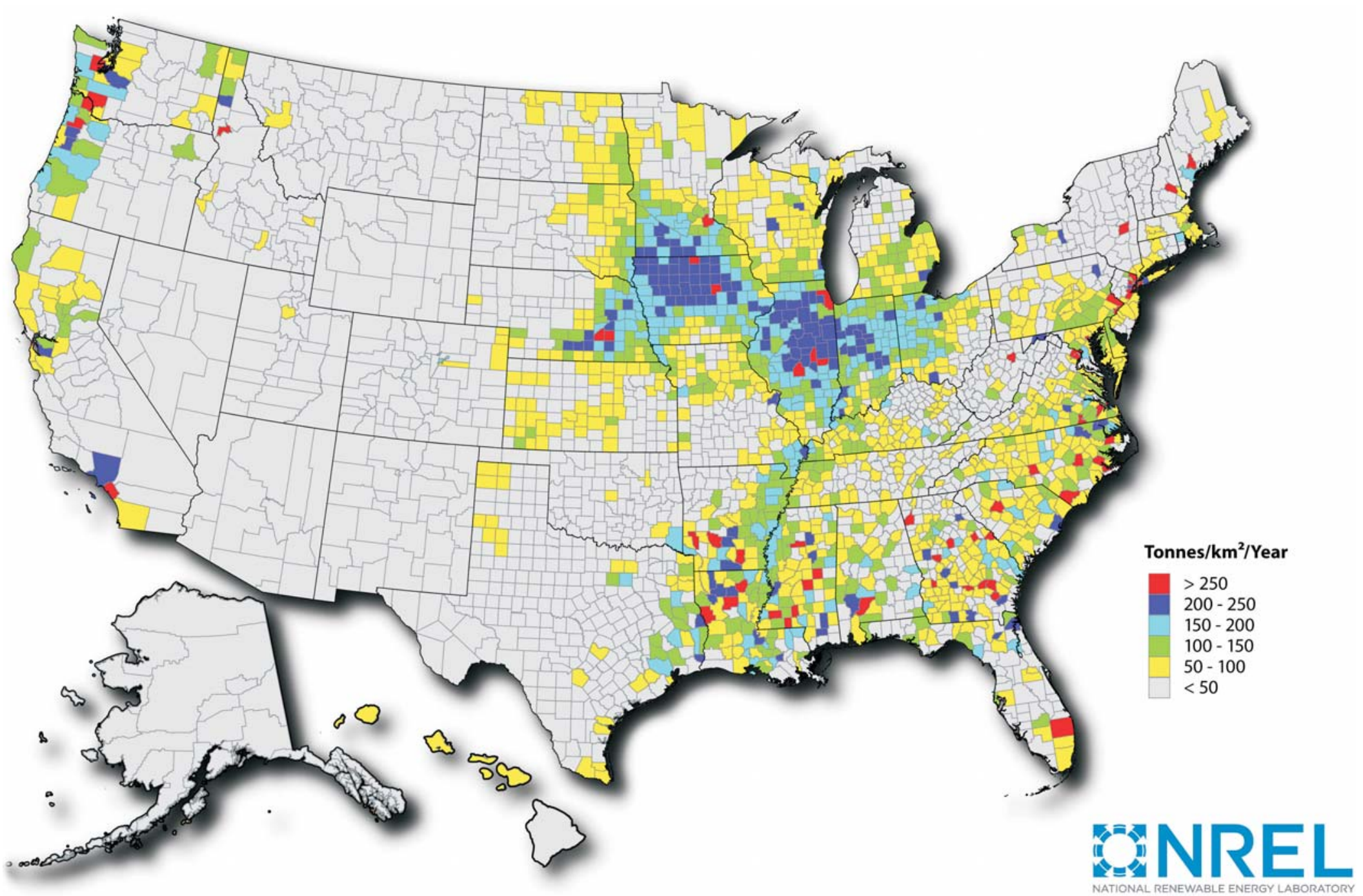

Notes: - Data are for total biomass per square kilometer. $\cdot \mathrm{km}^{2}=$ square kilometer. - This study estimates the biomass resources currently available in the United States by county. It includes the following feedstock categories: crop residues (5 year average: 2003-2007), forest and primary mill residues (2007), secondary mill and urban wood waste (2002), methane emissions from landfills (2008), domestic wastewater treatment (2007), and animal manure (2002). For more information on the data development, please refer to http://www.nrel.gov/docs/fy06osti/39181.pdf.
Although, the document contains the methodology for the development of an older assessment, the information is applicable to this assessment as well. The difference is only in the data's time period.

Web Page: For related information, see http://www.nrel.gov/gis/maps.html.

Source: This map was created by the National Renewable Energy Laboratory for the Department of Energy (September 23, 2009). 


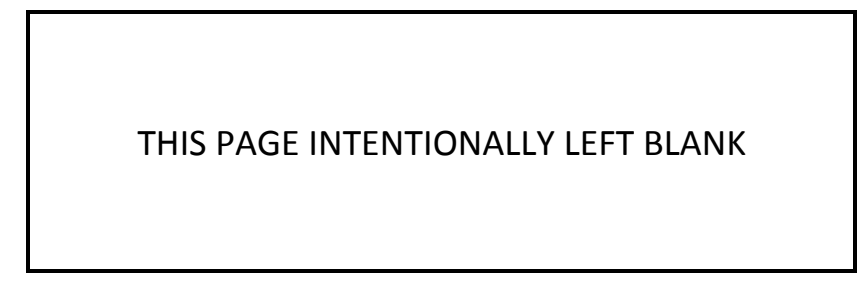




\section{Petroleum and Other Liquids}




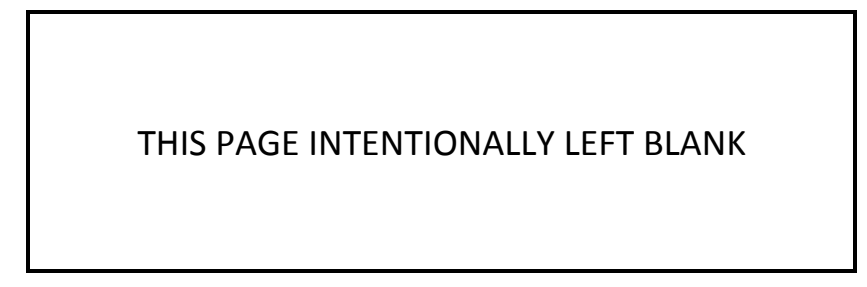


Figure 5.0. Petroleum Flow, 2011

(Million Barrels per Day)

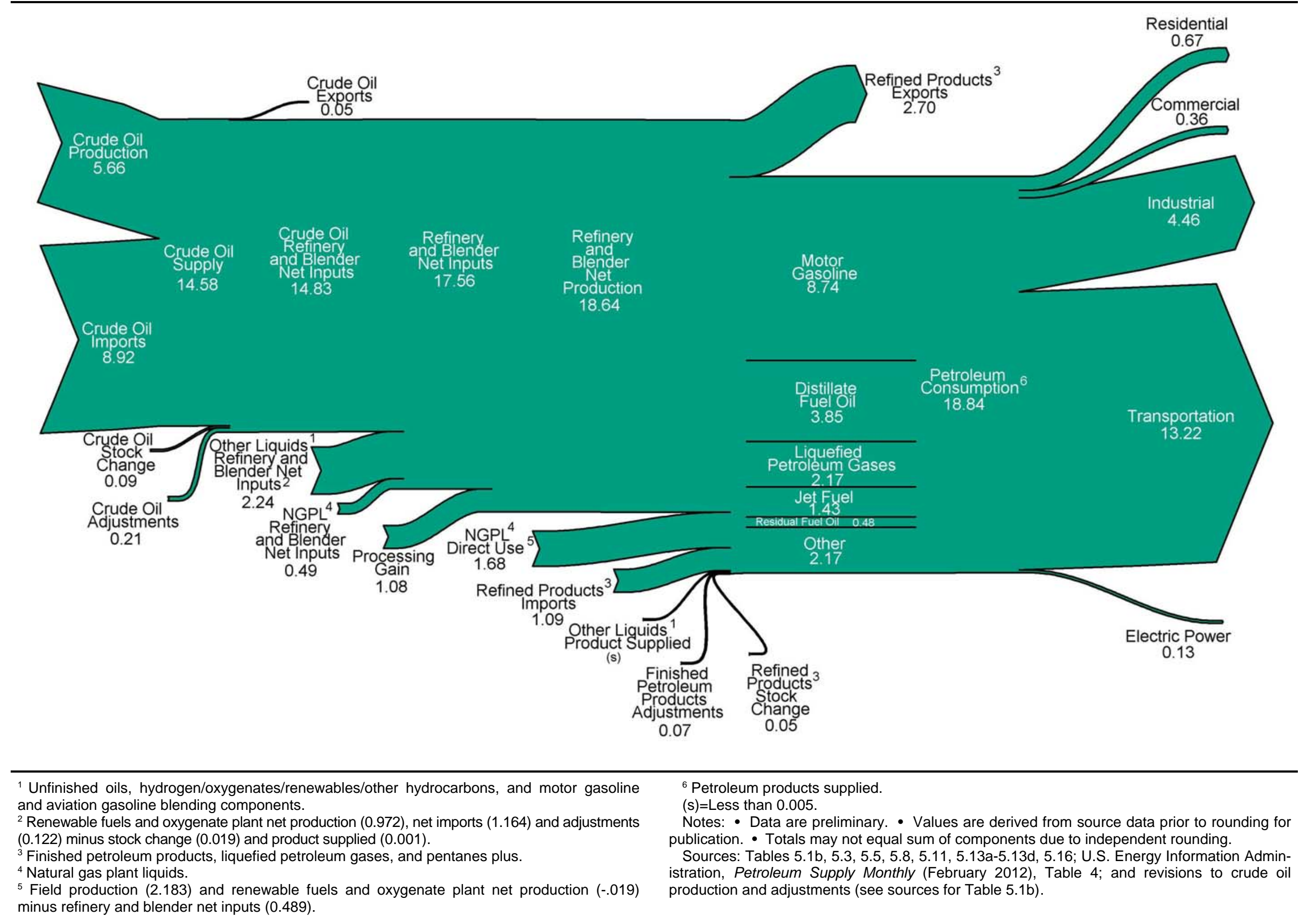


Figure 5.1a Petroleum and Other Liquids Overview

\section{Overview, 1949-2011}

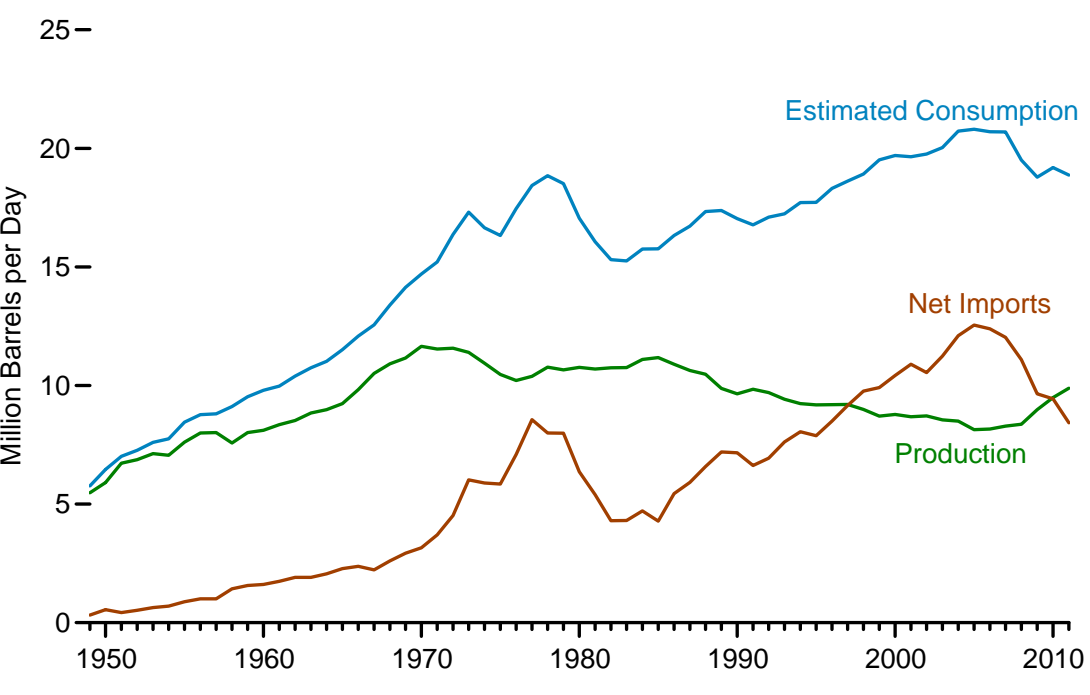

Production, 1949-2011
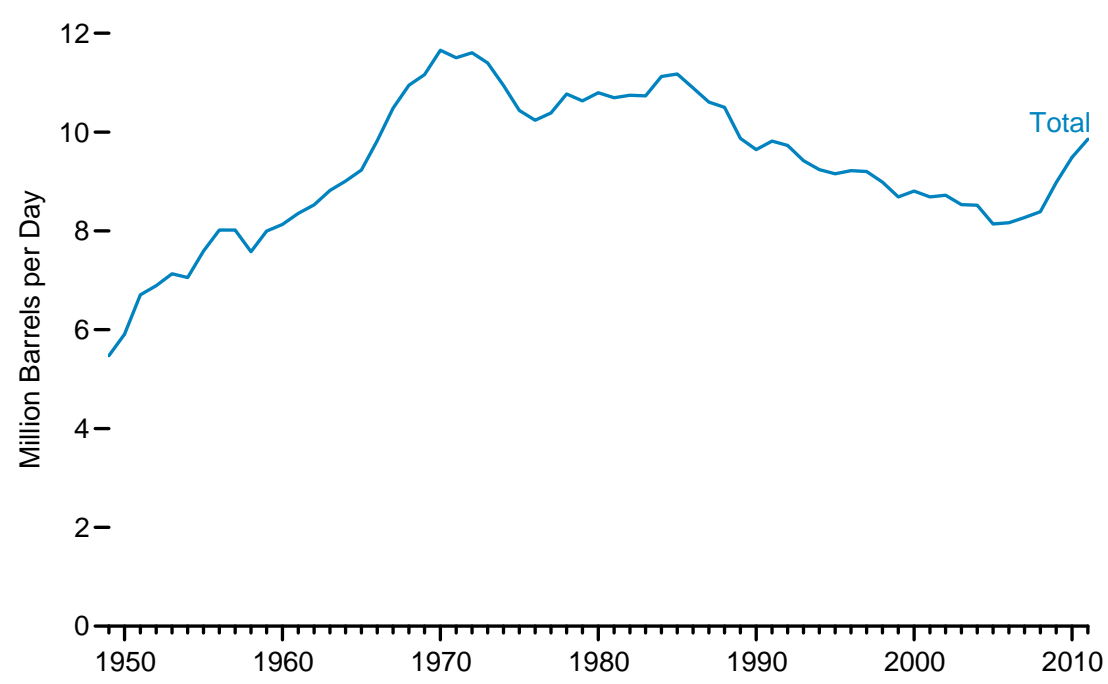

Overview, 2011

20-

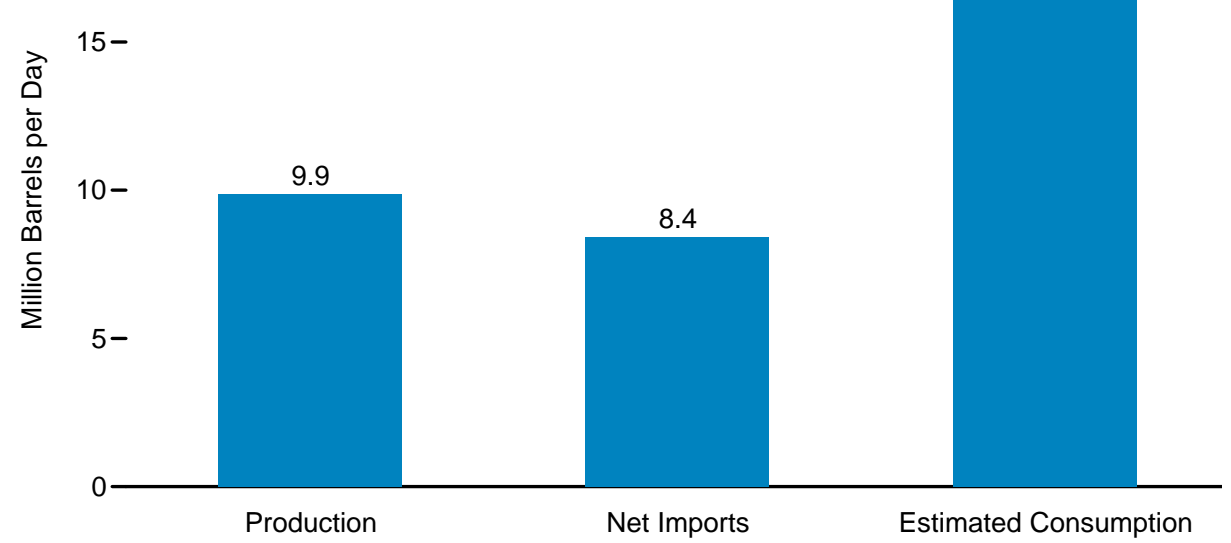

Production and Net Imports, Share of Estimated Consumption, 1949-2011

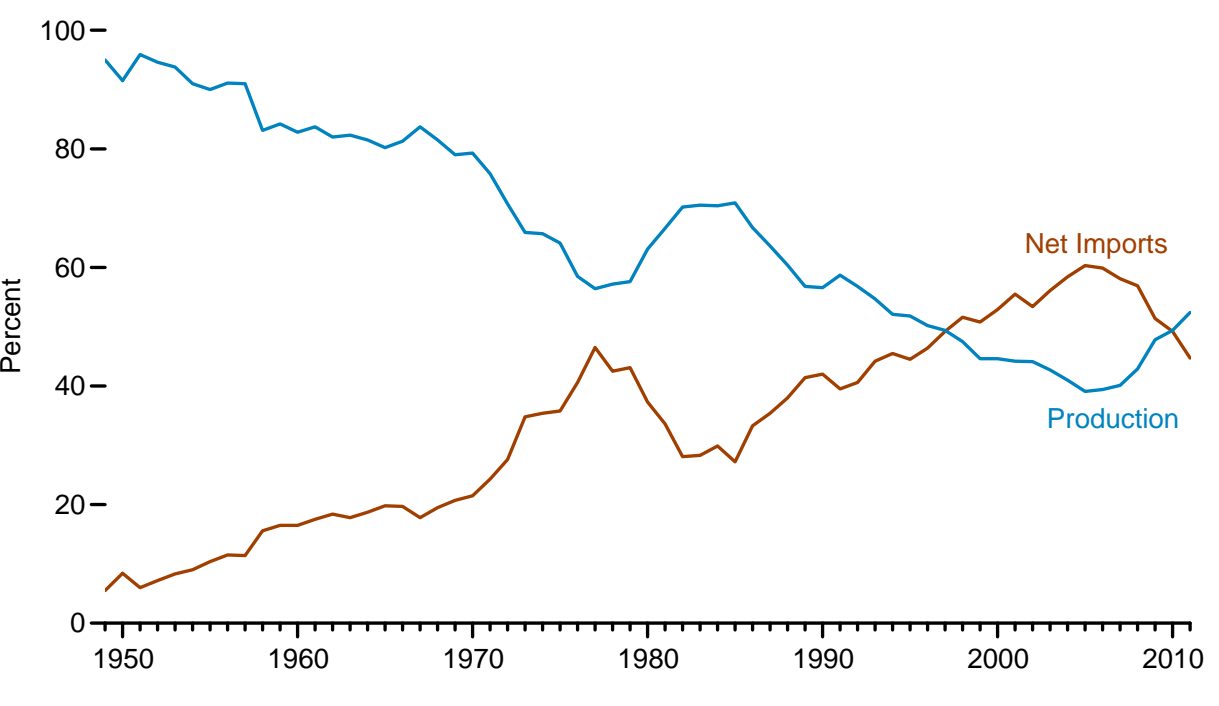

Source: Table 5.1a.

Note: Production includes production of crude oil (including lease condensate), natural gas plant liquids, fuel ethanol (minus denaturant), and biodiesel; and processing gain. 


\section{Figure 5.1b Petroleum Overview}

\section{Overview, 1949-2011}

25-

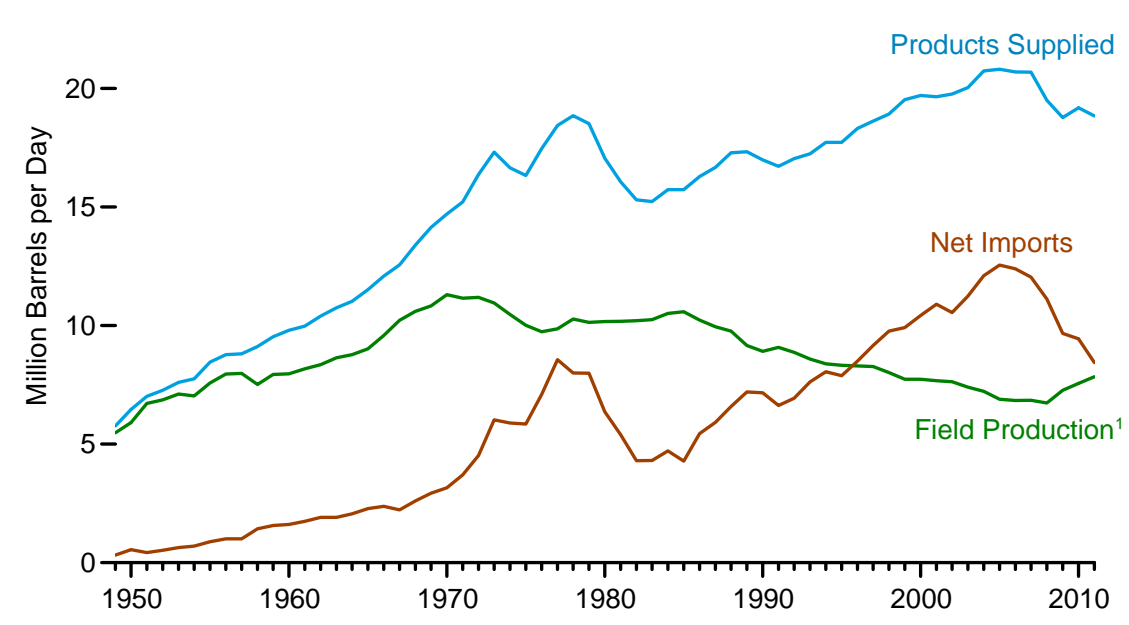

Crude Oil and Natural Gas Plant Liquids Field Production, 1949-2011 $10-$

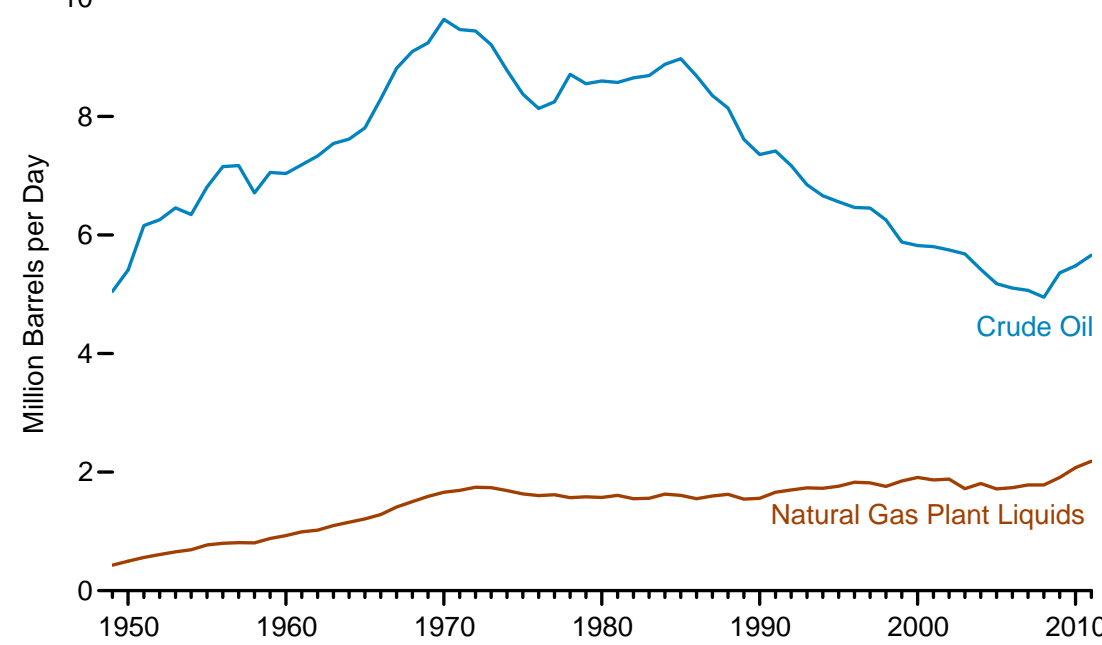

\section{Overview, 2011}

20-

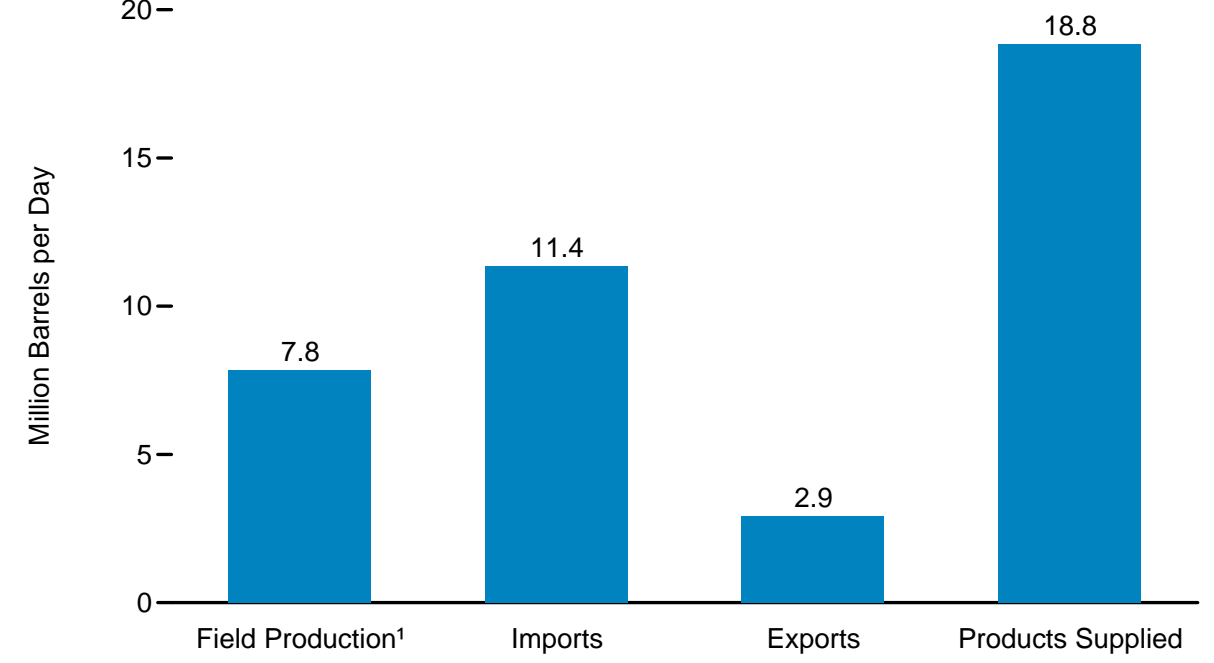

Trade, 1949-2011

12-

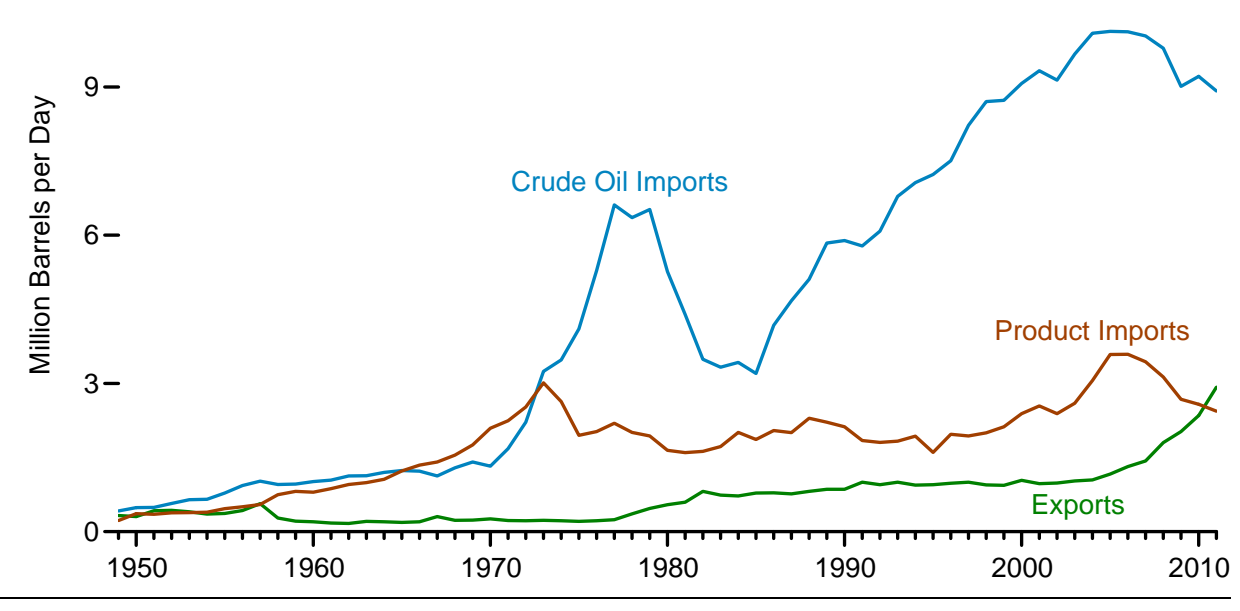

${ }^{1}$ Crude oil and natural gas plant liquids field production. Sources: Tables $5.1 \mathrm{~b}$.and 5.3 
Table 5.1a Petroleum and Other Liquids Overview, Selected Years, 1949-2011

\begin{tabular}{|c|c|c|c|c|c|c|}
\hline & Production ${ }^{1}$ & $\begin{array}{c}\text { Production } \\
\text { as Share of } \\
\text { Estimated Consumption }\end{array}$ & Net Imports ${ }^{2}$ & $\begin{array}{c}\text { Net Imports } \\
\text { as Share of } \\
\text { Estimated Consumption }\end{array}$ & $\begin{array}{c}\text { Balancing } \\
\text { Item }^{3}\end{array}$ & $\begin{array}{c}\text { Estimated } \\
\text { Consumption } 4\end{array}$ \\
\hline Year & Thousand Barrels per Day & Percent & Thousand Barrels per Day & Percent & \multicolumn{2}{|c|}{ Thousand Barrels per Day } \\
\hline 1949 & 5,475 & 95.0 & 318 & 5.5 & -30 & 5,763 \\
\hline 1950 & 5,908 & 91.5 & 545 & 8.4 & 5 & 6,458 \\
\hline 1955 & 7,611 & 90.0 & 880 & 10.4 & -37 & 8,455 \\
\hline 1960 & 8,110 & 82.8 & 1,613 & 16.5 & 74 & 9,797 \\
\hline 1965 & 9,234 & 80.2 & 2,281 & 19.8 & -2 & 11,512 \\
\hline 1970 & 11,656 & 79.3 & 3,161 & 21.5 & $-11 \overline{9}$ & 14,697 \\
\hline 1975 & 10,467 & 64.1 & 5,846 & 35.8 & 8 & 16,322 \\
\hline 1976 & 10,213 & 58.5 & 7,090 & 40.6 & 159 & 17,461 \\
\hline 1977 & 10,387 & 56.4 & 8,565 & 46.5 & -520 & 18,431 \\
\hline 1978 & 10,771 & 57.2 & 8,002 & 42.5 & 74 & 18,847 \\
\hline 1979 & 10,662 & 57.6 & 7,985 & 43.1 & -135 & 18,513 \\
\hline 1980 & 10,767 & 63.1 & 6,365 & 37.3 & -76 & 17,056 \\
\hline 1981 & 10,693 & 66.6 & 5,401 & 33.6 & -31 & 16,063 \\
\hline 1982 & 10,744 & 70.2 & 4,298 & 28.1 & 268 & 15,310 \\
\hline 1983 & 10,761 & 70.5 & 4,312 & 28.3 & 185 & 15,258 \\
\hline 1984 & 11,095 & 70.4 & 4,715 & 29.9 & -52 & 15,758 \\
\hline 1985 & 11,177 & 70.9 & 4,286 & 27.2 & 302 & 15,766 \\
\hline 1986 & 10,893 & 66.7 & 5,439 & 33.3 & -5 & 16,326 \\
\hline 1987 & 10,636 & 63.6 & 5,914 & 35.4 & 168 & 16,717 \\
\hline 1988 & 10,473 & 60.4 & 6,587 & 38.0 & 277 & 17,336 \\
\hline 1989 & 9,874 & 56.8 & 7,202 & 41.4 & 303 & 17,379 \\
\hline 1990 & 9,645 & 56.6 & 7,161 & 42.0 & 230 & 17,036 \\
\hline 1991 & 9,846 & 58.7 & 6,626 & 39.5 & 297 & 16,769 \\
\hline 1992 & 9,703 & 56.8 & 6,938 & 40.6 & 455 & 17,096 \\
\hline 1993 & 9,422 & 54.7 & 7,618 & 44.2 & 195 & 17,235 \\
\hline 1994 & 9,239 & 52.1 & 8,054 & 45.5 & 424 & 17,716 \\
\hline 1995 & 9,183 & 51.8 & 7,886 & 44.5 & 654 & 17,723 \\
\hline 1996 & 9,194 & 50.2 & 8,498 & 46.4 & 616 & 18,308 \\
\hline 1997 & 9,201 & 49.4 & 9,158 & 49.2 & 260 & 18,619 \\
\hline 1998 & 8,987 & 47.5 & 9,764 & 51.6 & 165 & 18,915 \\
\hline 1999 & 8,711 & 44.6 & 9,912 & 50.8 & 894 & 19,517 \\
\hline 2000 & 8,784 & 44.6 & 10,419 & 52.9 & 496 & 19,699 \\
\hline 2001 & 8,686 & 44.2 & 10,900 & 55.5 & 60 & 19,647 \\
\hline 2002 & 8,720 & 44.1 & 10,547 & 53.4 & 493 & 19,760 \\
\hline 2003 & 8,554 & 42.7 & 11,238 & 56.1 & 239 & 20,031 \\
\hline 2004 & 8,498 & 41.0 & 12,097 & 58.4 & 133 & 20,728 \\
\hline 2005 & 8,140 & 39.1 & 12,549 & 60.3 & 114 & 20,803 \\
\hline 2006 & 8,163 & 39.4 & 12,391 & 59.9 & 143 & 20,697 \\
\hline 2007 & 8,292 & 40.1 & 12,027 & 58.1 & 376 & 20,695 \\
\hline 2008 & 8,364 & 42.9 & 11,090 & 56.9 & 51 & 19,506 \\
\hline 2009 & $R_{8,981}$ & 47.8 & 9,654 & 51.4 & 154 & $\mathrm{R}_{18,789}$ \\
\hline 2010 & Rg, 490 & R49.4 & $\mathrm{Rg}, 435$ & 49.2 & R267 & $\mathrm{R}_{19,192}$ \\
\hline 2011 & E9,884 & ${ }_{52.4}$ & P8,432 & P 44.7 & $P_{561}$ & $P_{18,877}$ \\
\hline
\end{tabular}

1 Crude oil (including lease condensate) production; natural gas plant liquids production; and processing gain (refinery and blender net production minus refinery and blender net inputs). Beginning in 1981, also 2 Net imports (mis dena minus exports. Includes in 2001, also includes biodiesel production. Beginning in 1993 , also includes fuel ethanol (minus desaturant) net imports. Beginning in 2001 , includes biodiesel net imports. Beginning in 2009, also includes a small amount of other biofuels (such as bio-jet fuel and bio-ETBE) imports.

Includes petroleum and biofuels stock withdrawals (stock change multiplied by -1); petroleum adjustments; and biodiesel balancing item.

4 Includes estimated consumption of petroleum. Beginning in 1981, also includes estimated consumption of fuel ethanol minus denaturant. Beginning in 2001, also includes estimated consumption of biodiesel. Techniques used to estimate consumption vary depending on the product. Petroleum product supplied is used as an approximation of pertoleum consumpton, which is adusted to exclude biofuels in
Consumption," at end of section. Estimated consumption of fuel ethanol minus denaturant in 2011 is calculated as fuel ethanol refinery and blender net inputs minus fuel ethanol adjustments minus the amount f change; for other years, see sources in Table 10.4 .

$R=$ Revised. P=Preliminary. $E=$ Estimate.

Web Pages: - See http://www.eia.gov/totalenergy/data/monthly/\#petroleum for updated monthly and annual data. - See http://www.eia.gov/totalenergy/data/annual/\#petroleum for all annual data beginning in 949. - See http://www.eia.gov/petroleum/ and http://www.eia.gov/renewable/ for related information.

Sources: Production: Tables 5.1b, 10.3, and 10.4. Net Imports: Tables 5.1b, 10.3, and 10.4; and U.S. Energy Information Administration (EIA), Petroleum Supply Annual (PSA), Petroleum Supply Monthly (PSM), and earlier publications- see sources for Table 5.1b. Balancing He consumption minus production and net imports. Estimated Consumption: Tables 5.1b, 10.3, and 10.4 
Table 5.1b Petroleum Overview, Selected Years, 1949-2011

(Thousand Barrels per Day)

\begin{tabular}{|c|c|c|c|c|c|c|c|c|c|c|c|c|c|}
\hline \multirow[b]{3}{*}{ Year } & \multicolumn{5}{|c|}{ Field Production 1} & \multirow{3}{*}{$\begin{array}{c}\text { Renewable } \\
\text { Fuels } \\
\text { and } \\
\text { Oxygenates } 5\end{array}$} & \multirow{3}{*}{$\begin{array}{l}\text { Processing } \\
\text { Gain } 6\end{array}$} & \multicolumn{3}{|c|}{ Trade } & \multirow{3}{*}{$\begin{array}{c}\text { Stock } \\
\text { Change }{ }^{8,10}\end{array}$} & \multirow{3}{*}{$\begin{array}{l}\text { Adjust- } \\
\text { ments }{ }^{11}\end{array}$} & \multirow{3}{*}{$\begin{array}{l}\text { Petroleum } \\
\text { Products } \\
\text { Supplied }\end{array}$} \\
\hline & \multicolumn{3}{|c|}{ Crude Oil ${ }^{2}$} & \multirow{2}{*}{$\begin{array}{c}\text { Natural Gas } \\
\text { Plant Liquids }{ }^{4}\end{array}$} & \multirow[b]{2}{*}{ Total } & & & & & & & & \\
\hline & 48 States $^{3}$ & Alaska & Total & & & & & Imports 7,8 & Exports & $\begin{array}{c}\text { Net } \\
\text { Imports } 8,9\end{array}$ & & & \\
\hline 1949 & 5,046 & 0 & 5,046 & 430 & 5,477 & NA & -2 & 645 & 327 & 318 & -8 & -38 & 5,763 \\
\hline 1950 & $\begin{array}{l}5,407 \\
5,407\end{array}$ & 0 & $\begin{array}{l}5,040 \\
5,407\end{array}$ & 499 & $\begin{array}{l}5,406 \\
5,906\end{array}$ & NA & -2 & $\begin{array}{l}040 \\
850\end{array}$ & 305 & 545 & $\begin{array}{l}-0 \\
-56\end{array}$ & $\begin{array}{l}-50 \\
-51\end{array}$ & $\begin{array}{l}6,458 \\
6,103\end{array}$ \\
\hline 1955 & $\begin{array}{l}\text {, } \\
6,807\end{array}$ & 0 & $\begin{array}{l}\begin{array}{l}\text {, } \\
6,807\end{array} \\
\end{array}$ & 771 & $\begin{array}{l}7,906 \\
7,578\end{array}$ & NA & 34 & 1,248 & 368 & $\begin{array}{l}545 \\
880\end{array}$ & $\begin{array}{l}-50 \\
\text { (s) }\end{array}$ & $\begin{array}{l}-51 \\
-37 \\
-37\end{array}$ & $\begin{array}{l}8,458 \\
8,455\end{array}$ \\
\hline 1960 & 7,034 & 2 & 7,035 & 929 & 7,965 & NA & 146 & 1,815 & 202 & 1.613 & -83 -3 & -8 & 9,797 \\
\hline 1965 & 7,774 & 30 & 7,804 & 1.210 & 9,014 & NA & 220 & 2,468 & 187 & 2,281 & -8 & $\begin{array}{c}-0 \\
-10\end{array}$ & 11,512 \\
\hline 1970 & 9,408 & 229 & 9,637 & 1660 & 11,297 & NA & 359 & $\begin{array}{l}2,400 \\
3,419\end{array}$ & 259 & $\begin{array}{l}2,<01 \\
3.161\end{array}$ & 103 & -16 & 14.697 \\
\hline 1975 & $\begin{array}{l}8,400 \\
8,183\end{array}$ & 191 & 8,375 & 1,633 & 10,007 & NA & 460 & $\begin{array}{l}0,419 \\
6,056\end{array}$ & 209 & 5,846 & 32 & $\begin{array}{r}-10 \\
41\end{array}$ & $\begin{array}{l}1,091 \\
16.322\end{array}$ \\
\hline $\begin{array}{l}19 / 5 \\
1976\end{array}$ & $\begin{array}{l}8,183 \\
7,958\end{array}$ & $\begin{array}{l}191 \\
173\end{array}$ & $\begin{array}{l}8,3 / 5 \\
8,132\end{array}$ & $\begin{array}{l}1,633 \\
1,604\end{array}$ & $\begin{array}{r}10,001 \\
9,736\end{array}$ & $\begin{array}{l}\text { NA } \\
\text { NA }\end{array}$ & $\begin{array}{l}460 \\
477-1\end{array}$ & $\begin{array}{l}6,056 \\
7,313\end{array}$ & 223 & $\begin{array}{l}5,846 \\
7,090\end{array}$ & $\begin{array}{r}32 \\
-58\end{array}$ & $\begin{array}{c}41 \\
101\end{array}$ & $\begin{array}{l}16,322 \\
17,461\end{array}$ \\
\hline $\begin{array}{l}19 / 6 \\
1977\end{array}$ & $\begin{array}{l}7,958 \\
7,781\end{array}$ & $\begin{array}{l}1 / 3 \\
464\end{array}$ & $\begin{array}{l}8,132 \\
8,245\end{array}$ & $\begin{array}{l}1,604 \\
1,618\end{array}$ & $\begin{array}{l}9,366 \\
9,862\end{array}$ & $\begin{array}{l}\text { NA } \\
\text { NA }\end{array}$ & $\begin{array}{l}4 / 7 \\
524\end{array}$ & $\begin{array}{l}8,313 \\
8,807\end{array}$ & $\begin{array}{l}223 \\
243\end{array}$ & $\begin{array}{l}8,090 \\
8,565\end{array}$ & $\begin{array}{l}-58 \\
548\end{array}$ & $\begin{array}{r}101 \\
28\end{array}$ & $\begin{array}{l}1 /, 461 \\
18,431\end{array}$ \\
\hline 1978 & 7,478 & $\begin{array}{r}404 \\
1.229\end{array}$ & $\begin{array}{l}8,245 \\
8,707\end{array}$ & 1,567 & $\begin{array}{r}9,202 \\
10,275\end{array}$ & NA & $\begin{array}{l}324 \\
496\end{array}$ & 8,007 & $\begin{array}{l}243 \\
362\end{array}$ & $\begin{array}{l}-1002 \\
8,005\end{array}$ & $\begin{array}{l}540 \\
-94\end{array}$ & -20 & $\begin{array}{l}18,431 \\
18847\end{array}$ \\
\hline 1979 & $\begin{array}{l}7,418 \\
7,151\end{array}$ & $\begin{array}{l}1,229 \\
1,401\end{array}$ & 8,552 & 1,584 & $\begin{array}{l}10,135 \\
10,135\end{array}$ & NA & $\begin{array}{l}496 \\
527\end{array}$ & $\begin{array}{l}8,000 \\
8,456\end{array}$ & 471 & $\begin{array}{l}8,002 \\
7,985 \\
-10\end{array}$ & $\begin{array}{l}-94 \\
173\end{array}$ & 38 & $\begin{array}{l}18,847 \\
18.513\end{array}$ \\
\hline 1980 & 6,980 & $\begin{array}{l}1,617 \\
1,617\end{array}$ & $\begin{array}{l}8,597 \\
8,597\end{array}$ & $\begin{array}{l}1,504 \\
1,573\end{array}$ & 10,170 & NA & 597 & $\begin{array}{l}\text { l,450 } \\
6,900\end{array}$ & 544 & 6,365 & $\begin{array}{l}140 \\
140\end{array}$ & $\begin{array}{l}30 \\
64\end{array}$ & 17,056 \\
\hline 1981 & 6,962 & 1,609 & 8,572 & 1,609 & 10,180 & NA & 508 & 5,996 & 595 & $\begin{array}{l}5,401 \\
5\end{array}$ & $\begin{array}{l}140 \\
160\end{array}$ & $\begin{array}{r}04 \\
129\end{array}$ & 16,058 \\
\hline 1982 & 6,953 & 1,696 & 8,649 & 1,550 & 10,199 & NA & 531 & 5,113 & 815 & 4,298 & -147 & 121 & 15,296 \\
\hline 1983 & 6,974 & 1,714 & 8,688 & 1,559 & 10,246 & NA & 488 & 5,051 & 739 & 4,312 & -20 & 165 & 15,231 \\
\hline 1984 & 7,157 & 1,722 & 8,879 & 1,630 & 10,509 & NA & 553 & 5,437 & 722 & 4,715 & 280 & 228 & 15,726 \\
\hline 1985 & 7,146 & 1,825 & 8,971 & 1,609 & 10,581 & NA & 557 & 5,067 & 781 & 4,286 & -103 & 200 & 15,726 \\
\hline 1986 & 6,814 & 1,867 & 8,680 & 1,551 & 10,231 & NA & 616 & 6,224 & 785 & 5,439 & 202 & 197 & 16,281 \\
\hline 1987 & 6,387 & 1,962 & 8,349 & 1,595 & 9,944 & NA & 639 & 6,678 & 764 & 5,914 & 41 & 209 & 16,665 \\
\hline 1988 & 6,123 & 2,017 & 8,140 & $\begin{array}{l}1,625 \\
1,00\end{array}$ & 9,765 & NA & 655 & 7,402 & 815 & 6,587 & $\begin{array}{l}41 \\
-28\end{array}$ & 249 & 17,283 \\
\hline 1989 & 5,739 & 1,874 & 7,613 & 1,546 & 9,159 & $\mathrm{NA}$ & 661 & 8,061 & 859 & 7,202 & -43 & 260 & 17,325 \\
\hline 1990 & 5,582 & 1,773 & 7,355 & 1,559 & 8,914 & $\mathrm{NA}$ & 683 & $\begin{array}{l}8,018 \\
8,018\end{array}$ & 857 & 7,161 & 107 & 338 & 16,988 \\
\hline 1991 & 5,618 & 1,798 & 7,417 & 1,659 & 9,076 & NA & 715 & 7,627 & 1.001 & 6,626 & -10 & 287 & 16.714 \\
\hline 1992 & 5,457 & 1,714 & 7,171 & 1,697 & 8,868 & NA & 772 & $\begin{array}{l}7,021 \\
7,888\end{array}$ & 950 & $\begin{array}{l}6,938 \\
6,938\end{array}$ & -68 & $\begin{array}{l}381 \\
386\end{array}$ & 17,033 \\
\hline 1993 & 5,264 & 1,582 & 6,847 & 1,736 & 8,582 & NA & 766 & 8,620 & 1,003 & 7,618 & 151 & 422 & 17,237 \\
\hline 1994 & 5,103 & 1,559 & 6,662 & 1,727 & 8,388 & NA & 768 & 8,996 & 942 & 8.054 & 15 & 523 & 17,718 \\
\hline 1995 & 5,076 & 1.484 & 6,560 & 1,762 & 8,322 & NA & 774 & 8.835 & 949 & 7.886 & -246 & 496 & 17,725 \\
\hline 1996 & 5,071 & $\begin{array}{l}1,404 \\
1,393\end{array}$ & 6.465 & 1,830 & 8,295 & NA & 837 & 9,478 & 981 & 8.498 & -151 & 528 & 18,309 \\
\hline 1997 & 5,156 & 1,296 & 6.452 & 1.817 & 8,269 & NA & 850 & 10,162 & 1.003 & 9.158 & 143 & 487 & 18,620 \\
\hline 1998 & $\begin{array}{l}5,077 \\
5,076\end{array}$ & $\begin{array}{l}1,296 \\
1,175\end{array}$ & $\begin{array}{l}6,452 \\
6,252\end{array}$ & $\begin{array}{l}1,817 \\
1,759\end{array}$ & $\begin{array}{l}8,269 \\
8,011\end{array}$ & NA & $\begin{array}{l}850 \\
886\end{array}$ & 10,708 & $\begin{array}{l}1,003 \\
945\end{array}$ & 9,764 & $\begin{array}{l}143 \\
239\end{array}$ & $\begin{array}{l}481 \\
495\end{array}$ & $\begin{array}{l}18,620 \\
18,917\end{array}$ \\
\hline 1999 & $\begin{array}{l}4,077 \\
4,832\end{array}$ & $\begin{array}{l}1,050 \\
1,050\end{array}$ & $\begin{array}{l}\text {, } 5,852 \\
5,881\end{array}$ & $\begin{array}{r}1,1550 \\
1,850\end{array}$ & $\begin{array}{l}8,011 \\
7,731\end{array}$ & NA & $\begin{array}{l}886 \\
886\end{array}$ & $\begin{array}{r}10,082 \\
10,852\end{array}$ & $\begin{array}{l}945 \\
940\end{array}$ & $\begin{array}{l}9,164 \\
9,912\end{array}$ & $\begin{array}{l}239 \\
-422\end{array}$ & 567 & 19,519 \\
\hline 2000 & $\begin{array}{l}\begin{array}{l}4,832 \\
4,851\end{array} \\
\text { ? }\end{array}$ & I, & $\begin{array}{l}5,881 \\
5,822\end{array}$ & $\begin{array}{l}1,850 \\
1,911\end{array}$ & $\begin{array}{l}, 7,731 \\
7,733\end{array}$ & NA & $\begin{array}{l}886 \\
948\end{array}$ & $\begin{array}{l}10,852 \\
11,459\end{array}$ & $\begin{array}{r}940 \\
1.040\end{array}$ & $\begin{array}{r}9,912 \\
10,419\end{array}$ & $\begin{array}{r}-422 \\
-69\end{array}$ & $\begin{array}{l}567 \\
532\end{array}$ & $\begin{array}{l}19,719 \\
19,701\end{array}$ \\
\hline 2001 & $\begin{array}{l}4,851 \\
4,839\end{array}$ & 963 & $\begin{array}{l}5,822 \\
5,801\end{array}$ & $\begin{array}{l}1,911 \\
1,868\end{array}$ & $\begin{array}{l}, 7,33 \\
7,670\end{array}$ & $\begin{array}{l}\text { NA } \\
\text { NA }\end{array}$ & $\begin{array}{l}948 \\
903\end{array}$ & $\begin{array}{l}11,459 \\
11,871\end{array}$ & $\begin{array}{r}1,040 \\
971\end{array}$ & $\begin{array}{l}10,419 \\
10,900\end{array}$ & $\begin{array}{l}-69 \\
325\end{array}$ & $\begin{array}{l}532 \\
501\end{array}$ & $\begin{array}{l}19,01 \\
19,649\end{array}$ \\
\hline 2002 & $\begin{array}{l}4,039 \\
4,761\end{array}$ & 984 & $\begin{array}{l}5,001 \\
5,746\end{array}$ & 1,880 & 7,626 & NA & $\begin{array}{l}903 \\
957\end{array}$ & 11530 & 984 & 10,546 & $\begin{array}{l}3<5 \\
-105\end{array}$ & 527 & 19761 \\
\hline 2003 & $\begin{array}{l}4, / 61 \\
4,706\end{array}$ & $\begin{array}{l}984 \\
974\end{array}$ & $\begin{array}{l}5, / 46 \\
5,681\end{array}$ & $\begin{array}{l}1,880 \\
1,719\end{array}$ & $\begin{array}{l}, 626 \\
7,400\end{array}$ & $\begin{array}{l}\text { NA } \\
\text { NA }\end{array}$ & $\begin{array}{l}95 / \\
974\end{array}$ & $\begin{array}{l}11,530 \\
12,264\end{array}$ & $\begin{array}{r}984 \\
1.027\end{array}$ & $\begin{array}{l}10,546 \\
11,238\end{array}$ & $\begin{array}{r}-105 \\
56\end{array}$ & $\begin{array}{l}527 \\
478\end{array}$ & $\begin{array}{l}19,6161 \\
20.034\end{array}$ \\
\hline 2004 & $\begin{array}{l}4, / 06 \\
4.510\end{array}$ & $\begin{array}{l}9 / 4 \\
908\end{array}$ & $\begin{array}{l}5,681 \\
5,419\end{array}$ & $\begin{array}{l}1, / 19 \\
1,809\end{array}$ & $\begin{array}{l},, 400 \\
7,228\end{array}$ & $\begin{array}{l}\text { NA } \\
\text { NA }\end{array}$ & $\begin{array}{r}9 / 4 \\
1.051\end{array}$ & $\begin{array}{l}12,264 \\
13,145\end{array}$ & $\begin{array}{l}1,027 \\
1,048\end{array}$ & $\begin{array}{l}11,238 \\
12,097\end{array}$ & $\begin{array}{r}56 \\
209\end{array}$ & $\begin{array}{l}4 / 8 \\
564\end{array}$ & $\begin{array}{l}20,034 \\
20,731\end{array}$ \\
\hline 2005 & 4,314 & 864 & 5,178 & 1.717 & $\begin{array}{l}6.8205 \\
6.895\end{array}$ & NA & 989 & 13,714 & $\begin{array}{l}1,040 \\
1.165\end{array}$ & 12,549 & 145 & 513 & 20,802 \\
\hline 2006 & $\begin{array}{r}4,361 \\
4,361\end{array}$ & $\begin{array}{l}004 \\
741\end{array}$ & 5,102 & $\begin{array}{l}1,739 \\
1,79\end{array}$ & 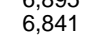 & NA & $\begin{array}{l}994 \\
994\end{array}$ & 13,707 & 1,317 & 12,390 & 60 & 522 & 20,687 \\
\hline 2007 & $\begin{array}{l}4,301 \\
4,342\end{array}$ & 722 & 5,064 & $\begin{array}{l}1,783 \\
1,1\end{array}$ & $\begin{array}{l}\text { 6,041 } \\
6,847\end{array}$ & NA & $\begin{array}{l}996 \\
996\end{array}$ & 13,468 & 1,433 & 12,036 & -148 & 653 & 20,680 \\
\hline 2008 & 4,268 & 683 & 4,950 & 1,784 & 6,734 & NA & 993 & $\begin{array}{r}12,400 \\
12,915\end{array}$ & $\begin{array}{l}1,435 \\
1.802\end{array}$ & 11,114 & $\begin{array}{r}-148 \\
195\end{array}$ & 852 & 19,498 \\
\hline 2009 & 4,715 & 645 & 5,361 & 1,910 & 7,270 & 746 & 979 & 11,691 & $\begin{array}{l}1,024 \\
2,024\end{array}$ & $\begin{array}{r}9,667 \\
\end{array}$ & 109 & 218 & 18,771 \\
\hline 2010 & $\mathrm{R}_{4,874}$ & R601 & R5,476 & $\mathrm{R}_{2,074}$ & $\mathrm{R} 7,550$ & R907 & $\mathrm{R}_{1,068}$ & $\mathrm{R}_{11,793}$ & $\mathrm{R}_{2,353}$ & $\mathrm{Rg}, 441$ & R49 & R264 & $\mathrm{R}_{19,180}$ \\
\hline 2011 & E 5,090 & E572 & $E_{5,662}$ & $P_{2,183}$ & $\mathrm{E} 7,844$ & P954 & $\mathrm{P}_{1,085}$ & $\mathrm{P}_{11,360}$ & $P_{2}, 924$ & P8,436 & $P_{-}-115$ & P402 & P18,835 \\
\hline
\end{tabular}

Crude oil production on leases, and natural gas liquids (liquefied petroleum gases, pentanes plus, and a small amount of finished petroleum products) production at natural gas processing plants. Excludes what was previously classiled as "Field Produclion "of finished molor gasoline, molor gasoline blending

2 Includes lease condensate.

United States excluding Alaska and Hawaii.

7 Refinery and blender net production minus refinery and blender net inputs. See Table 5.8 .

8 Beginning

9 Net imports equal imports minus exports.

10 A negative value indicates a decrease in stocks and a positive value indicates an increase. Includes crude oil stocks in the Strategic Petroleum Reserve, but excludes distillate fuel oil stocks in the Northeas

${ }_{11}$ An adjustment for crude oil, finished motor gasoline, motor gasoline blending components, fuel

ethanol, and distillate fuel oil. See EIA, Petroleum Supply Monthly (PSM), Appendix B.

$R=$ Revised. $P=$ Preliminary. $E=$ Estimate. $N A=$ Not available. $(S)=$ Less than 500 barrels per day and greater than -500 barrels per day

"Consumption," and Note 2 "Production and Product Supplied Statistics," at end of section. - Totals may

Web Pages: - See http://www.eia.gov/totalenergy/data/monthly/\#petroleum for updated monthly and annual data. - See http://www.eia.gov/totalenergy/data/annual/\#petroleum for all annual data beginning in 949. - See http://www.eia.gov/petroleum/ for related information.

Sources: • 1949-1975-Bureau of Mines, Mineral Industry Surveys, Petroleum Statement, Annual, Petroleum Statement Annual, annual reports. 1981-2009-EIA, Petroleum Supply Annual (PSA), annua reports. - 2010 and 2011-EIA, PSA, annual report; Petroleum Supply Monthly, monthly reports; and revisions to crude oil production, total field production, and adjustments, published in the Monthly Energy Review (May 2012), Table 3.1 (based on crude oil production data from: State govenment agencies, U.S. Department of the Interior, Bureau of Safety and Environmental Enforcement, and predecessor agencies 
Figure 5.2 Crude Oil Production and Crude Oil Well Productivity, 1954-2011

\section{Crude Oil Production by Location}

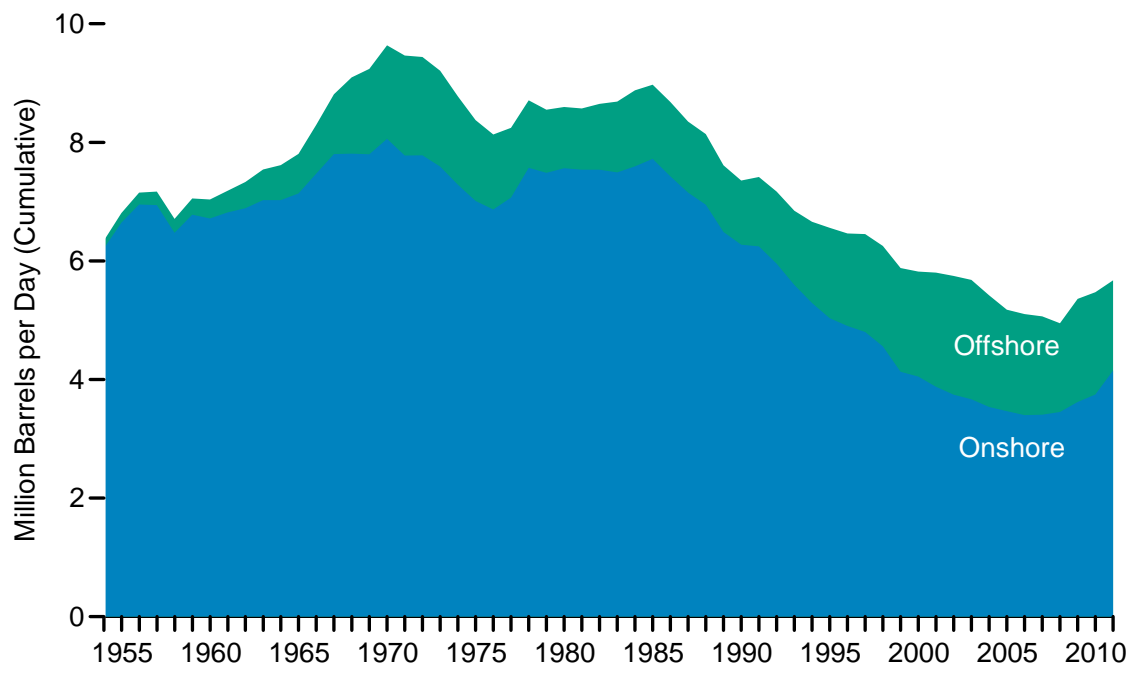

\section{Crude Oil Production, 48 States $^{1}$ and Alaska}

$$
10-
$$

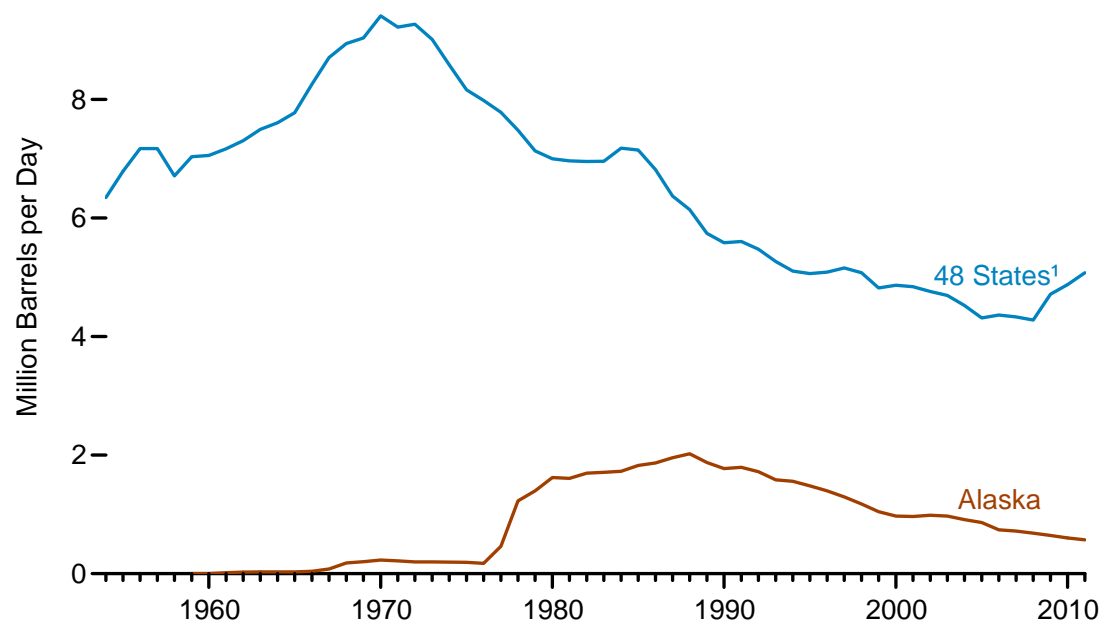

\section{Number of Producing Wells}

$800-$

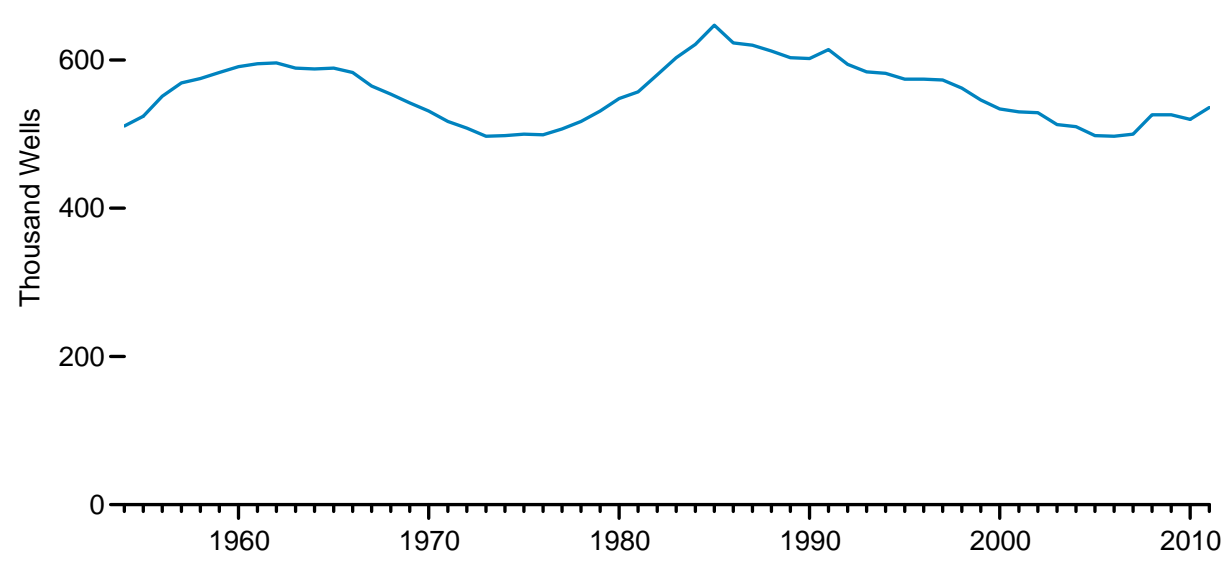

\section{Crude Oil Well Average Productivity}

20-

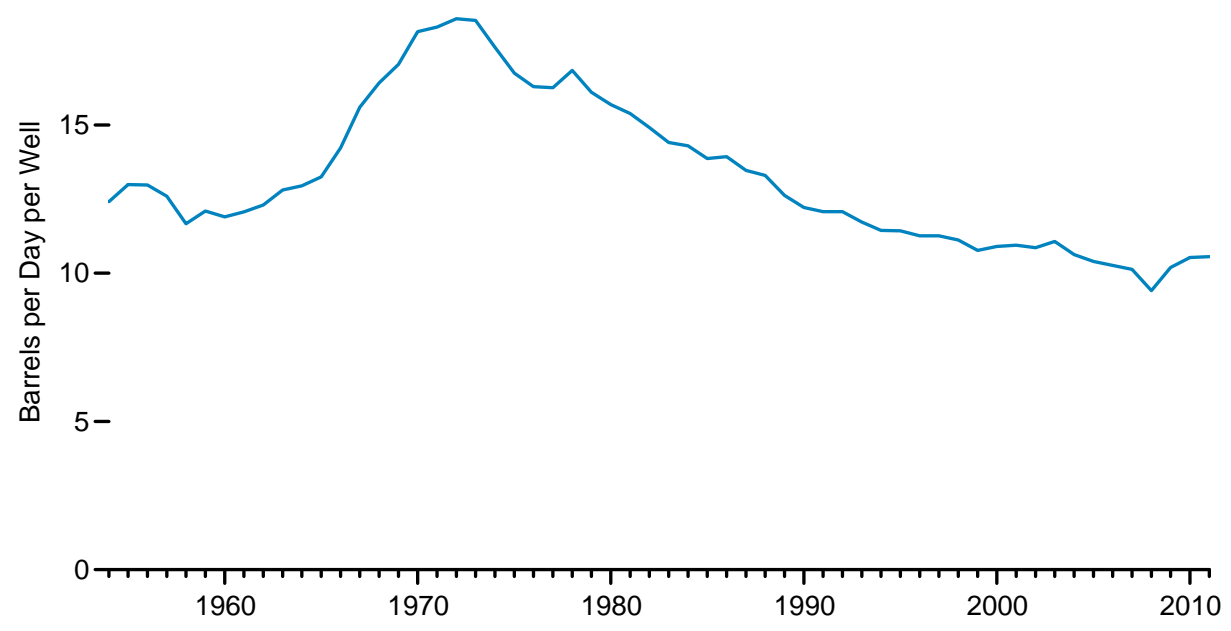


Table 5.2 Crude Oil Production and Crude Oil Well Productivity, Selected Years, 1954-2011

\begin{tabular}{|c|c|c|c|c|c|c|c|c|c|c|}
\hline \multirow[b]{4}{*}{ Year } & \multicolumn{8}{|c|}{ Crude Oil Production } & \multicolumn{2}{|c|}{ Crude Oil Well ${ }^{1}$ Productivity } \\
\hline & \multirow[b]{2}{*}{48 States $^{2}$} & \multirow[b]{2}{*}{ Alaska $^{3}$} & \multirow[b]{2}{*}{ Total } & \multirow[b]{2}{*}{ Onshore } & \multicolumn{3}{|c|}{ Offshore } & \multirow[b]{2}{*}{ Total } & \multirow{2}{*}{$\begin{array}{l}\text { Producing } \\
\text { Wells }{ }^{4}\end{array}$} & \multirow{2}{*}{$\begin{array}{c}\text { Average } \\
\text { Productivity } 5\end{array}$} \\
\hline & & & & & Federal & State & Total & & & \\
\hline & \multicolumn{3}{|c|}{ Thousand Barrels per Day } & \multicolumn{5}{|c|}{ Thousand Barrels per Day } & Thousands & $\begin{array}{c}\text { Barrels per Day } \\
\text { per Well }\end{array}$ \\
\hline 1954 & 6,342 & 0 & 6,342 & 6,209 & NA & NA & 133 & 6,342 & 511 & 12.4 \\
\hline 1955 & 6,807 & 0 & 6,807 & 6,645 & NA & NA & 162 & 6,807 & 524 & 13.0 \\
\hline 1960 & 7,034 & 2 & 7,035 & 6,716 & NA & NA & 319 & 7,035 & 591 & 11.9 \\
\hline 1965 & 7,774 & 30 & 7,804 & 7,140 & NA & NA & 665 & 7,804 & 589 & 13.2 \\
\hline 1970 & 9,408 & 229 & 9,637 & 8,060 & NA & NA & 1,577 & 9,637 & 531 & 18.1 \\
\hline 1975 & 8,183 & 191 & 8,375 & 7,012 & NA & NA & 1,362 & 8,375 & 500 & 16.8 \\
\hline 1976 & 7,958 & 173 & 8,132 & 6,868 & NA & NA & 1,264 & 8,132 & 499 & 16.3 \\
\hline 1977 & 7,781 & 464 & 8,245 & 7,069 & NA & NA & 1,176 & 8,245 & 507 & 16.3 \\
\hline 1978 & 7,478 & 1,229 & 8,707 & 7,571 & NA & NA & 1,136 & 8,707 & 517 & 16.8 \\
\hline 1979 & 7,151 & 1,401 & 8,552 & 7,485 & NA & NA & 1,067 & 8,552 & 531 & 16.1 \\
\hline 1980 & 6,980 & 1,617 & 8,597 & 7,562 & NA & NA & 1,034 & 8,597 & 548 & 15.7 \\
\hline 1981 & 6,962 & 1,609 & 8,572 & 7,537 & 773 & 261 & $\begin{array}{l}1,034 \\
\end{array}$ & 8,572 & 557 & 15.4 \\
\hline 1982 & 6,953 & $\begin{array}{l}1,696 \\
1,60\end{array}$ & 8,649 & $\begin{array}{l}7,538 \\
7,538\end{array}$ & 863 & 247 & $\begin{array}{l}1,110 \\
1,10\end{array}$ & $\begin{array}{l}8,649 \\
8,64\end{array}$ & 580 & $\begin{array}{l}14.9 \\
14.9\end{array}$ \\
\hline 1983 & 6,974 & 1,714 & 8,688 & 7,492 & 960 & 236 & 1,196 & 8,688 & 603 & 14.4 \\
\hline 1984 & 7,157 & $\begin{array}{l}1,722 \\
\end{array}$ & 8,879 & 7,596 & $\begin{array}{l}1,039 \\
\end{array}$ & 244 & 1,283 & 8,879 & 621 & 14.3 \\
\hline 1985 & 7,146 & 1,825 & 8,971 & 7,722 & 1,023 & 227 & 1,250 & 8,971 & 647 & 13.9 \\
\hline 1986 & 6,814 & 1,867 & 8,680 & 7,426 & 1,038 & 216 & 1,254 & 8,680 & 623 & 13.9 \\
\hline 1987 & 6,387 & 1,962 & 8,349 & 7,153 & 977 & 219 & 1,196 & 8,349 & 620 & 13.5 \\
\hline 1988 & $\begin{array}{l}\begin{array}{l}0,001 \\
6,123\end{array} \\
6\end{array}$ & $\begin{array}{l}2,902 \\
2,017\end{array}$ & $\begin{array}{l}8,040 \\
8,140\end{array}$ & $\begin{array}{l}6,949 \\
6\end{array}$ & 904 & 287 & 1,191 & $\begin{array}{l}8,045 \\
8,140\end{array}$ & 612 & $\begin{array}{l}13.5 \\
13.3\end{array}$ \\
\hline 1989 & 5,739 & 1,874 & 7,613 & 6,486 & 855 & 272 & 1,127 & 7,613 & 603 & 12.6 \\
\hline 1990 & 5,582 & 1,773 & 7,355 & 6,273 & 821 & 261 & 1,082 & 7,355 & 602 & 12.2 \\
\hline 1991 & 5,618 & 1,798 & 7,417 & 6,245 & 886 & 286 & 1,172 & 7,417 & 614 & 12.1 \\
\hline 1992 & 5,457 & 1,714 & 7,171 & 5,953 & 938 & 280 & 1,218 & 7,171 & 594 & 12.1 \\
\hline 1993 & 5,264 & 1,582 & 6,847 & R5,596 & 964 & 287 & $R_{1,250}$ & 6,847 & 584 & 11.7 \\
\hline 1994 & $\begin{array}{l}5,<64 \\
5,103\end{array}$ & $\begin{array}{r}1,582 \\
1,559\end{array}$ & $\begin{array}{l}0,047 \\
6,662\end{array}$ & 5,291 & $\begin{array}{r}964 \\
1,017\end{array}$ & 353 & 1,370 & $\begin{array}{l}6,047 \\
6,662\end{array}$ & $\begin{array}{l}584 \\
582\end{array}$ & 11.4 \\
\hline 1995 & 5,076 & 1,484 & 6,560 & 5,035 & 1,140 & 385 & 1,525 & 6,560 & 574 & 11.4 \\
\hline 1996 & 5,071 & 1,393 & 6,465 & 4,902 & 1,197 & 365 & 1,562 & 6,465 & 574 & 11.3 \\
\hline 1997 & 5,156 & 1,296 & 6,452 & 4,803 & 1,278 & 371 & 1,648 & 6,452 & 573 & 11.3 \\
\hline 1998 & 5,077 & 1,175 & $\begin{array}{l}6,252 \\
6,20\end{array}$ & 4,560 & 1,355 & 337 & $\begin{array}{l}1,692 \\
1,60\end{array}$ & 6,252 & 562 & 11.1 \\
\hline 1999 & $\begin{array}{l}4,832 \\
\end{array}$ & 1,050 & 5,881 & 4,132 & 1,462 & 288 & $\begin{array}{l}1,750 \\
1,75\end{array}$ & 5,881 & 546 & 10.8 \\
\hline 2000 & 4,851 & 970 & 5,822 & 4,049 & 1,525 & 248 & 1,773 & 5,822 & 534 & 10.9 \\
\hline 2001 & $\begin{array}{l}4,839 \\
4,80\end{array}$ & 963 & 5,801 & 3,879 & 1,621 & 302 & $\begin{array}{l}1,923 \\
\text { (1) }\end{array}$ & 5,801 & 530 & 10.9 \\
\hline 2002 & 4,761 & 984 & 5,746 & 3,743 & 1,637 & 365 & 2,003 & 5,746 & 529 & 10.9 \\
\hline 2003 & 4,706 & 974 & 5,681 & 3,668 & 1,641 & 371 & 2,012 & 5,681 & 513 & 11.1 \\
\hline 2004 & 4,510 & 908 & 5,419 & 3,536 & 1,527 & 356 & 1,883 & 5,419 & 510 & 10.6 \\
\hline 2005 & 4,314 & 864 & $\begin{array}{l}5,178 \\
5,419\end{array}$ & $\begin{array}{l}\text {, } 3000 \\
3,466\end{array}$ & $\begin{array}{l}1,354 \\
1,354\end{array}$ & 358 & $\begin{array}{r}\text {,.005 } \\
1,712\end{array}$ & 5,178 & 498 & 10.4 \\
\hline 2006 & 4,361 & 741 & 5,102 & 3,401 & 1,370 & 331 & 1,701 & 5,102 & 497 & 10.3 \\
\hline 2007 & 4,342 & 722 & 5,064 & $\begin{array}{l}3,407 \\
3,40\end{array}$ & 1,344 & 312 & 1,657 & 5,064 & 500 & 10.1 \\
\hline 2008 & 4,268 & 683 & 4,950 & 3,452 & 1,218 & 280 & 1,498 & 4,950 & 526 & 9.4 \\
\hline 2009 & 4,715 & 645 & 5,361 & 3,622 & 1,619 & 119 & 1,738 & 5,361 & 526 & 10.2 \\
\hline 2010 & $\mathrm{R}_{4,874}$ & $\mathrm{R}_{601}$ & $\mathrm{R}_{5,476}$ & $\mathrm{RE}_{3}, 744$ & $E_{1,609}$ & $E_{122}$ & $\mathrm{RE}_{1,732}$ & $\begin{array}{r}\text { R5,476 } \\
\text { R }\end{array}$ & $\begin{array}{l}\text { R520 } \\
\text { R5 }\end{array}$ & $\mathrm{R}_{10.5}$ \\
\hline 2011 & E5,090 & E572 & $E_{5,662}$ & $E_{4,178}$ & $E_{1,373}$ & $E_{111}$ & $\mathrm{E}_{1,484}$ & $E_{5,662}$ & P536 & $\mathrm{E}_{10.6}$ \\
\hline
\end{tabular}

1 See "Crude Oil Well" in Glossary.

2 United States excluding Alaska and Hawaii. Includes State onshore, State offshore, and Federal offshore production.

includes State onshore and State offshore production.

As of December 31 .
Through 1976, average productivity is based on the average number of producing wells. Beginning in 1977, average productivity is based on the number of wells producing at end of year.

$\mathrm{R}=$ Revised. P=Preliminary. $E=E s t i m a t e . ~ N A=N o t$ available.

Note: Totals may not equal sum of components due to independent rounding.

Web Pages: - See http://www.eia.gov/totalenergy/data/annual/\#petroleum for all data beginning in Sour For related information, see htt.//www.eia.gov/petroleum/.

Statement, Annual, annual reports; and U.S. Geological Survey, Outer Continental Shelf Statistics (June 1979). • 1976-1980-U.S. Energy Information Administration (EIA), Energy Data Reports, Petroleum and 2011-EIA, Monthly Energy Review (May 2012), Table 3.1; and crude oil production data from: State government agencies; U.S. Department of the Interior, Bureau of Safety and Environmental Enforcement, and predecessor agencies; and Form EIA-182, "Domestic Crude Oil First Purchase Report." Producing Wells: - 1954-1975-Bureau of Mines, Minerals Yearbook, "Crude Petroleum and Petroleum Products" chapter. - 1976-1980-EIA, Energy Data Reports, Petroleum Statement, Annual, annual reports. - 1981-1994-Independent Petroleum Association of America, The Oil Producing Industry in Your State. 1995 forward-Gulf Publishing Co., World Oil, February issues. Average Productivity: Calculated as total production divided by producing wells. 


\section{Figure 5.3 Petroleum Imports by Type}

\section{Total, 1949-2011}

$15-$

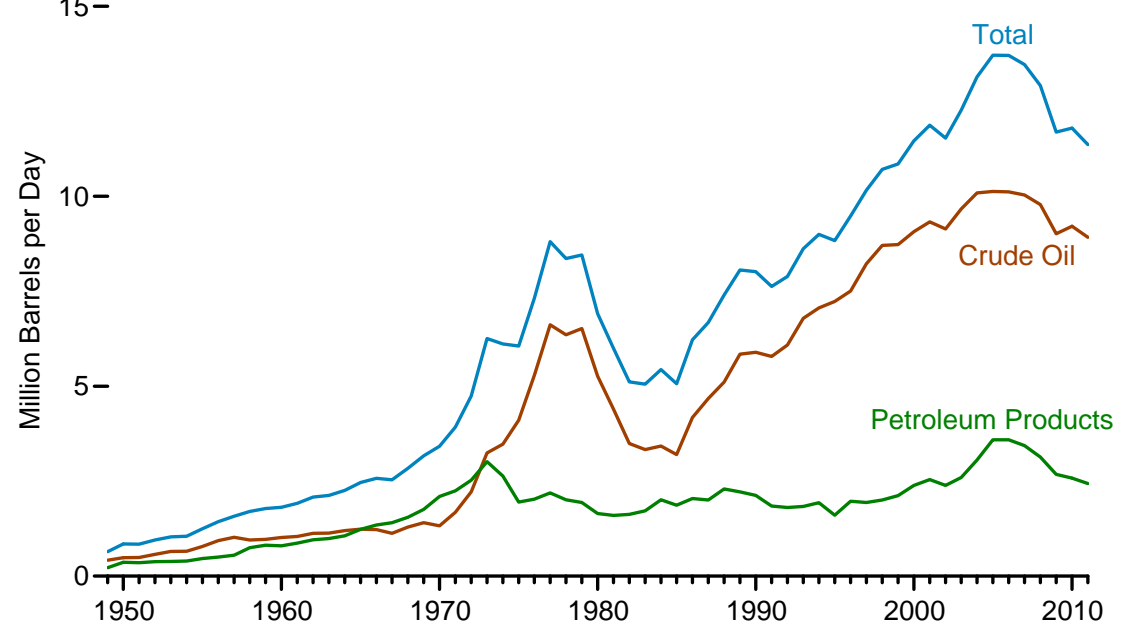

\section{By Selected Product, 1949-2011}

$2.0-$

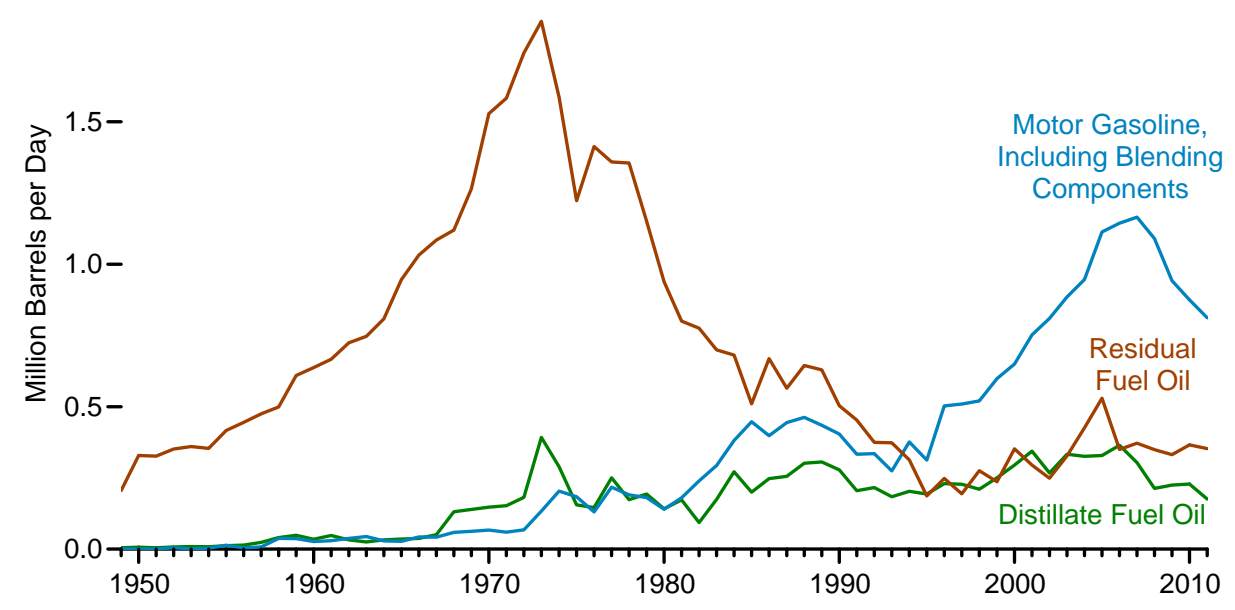

By Product, 2011

$800-$

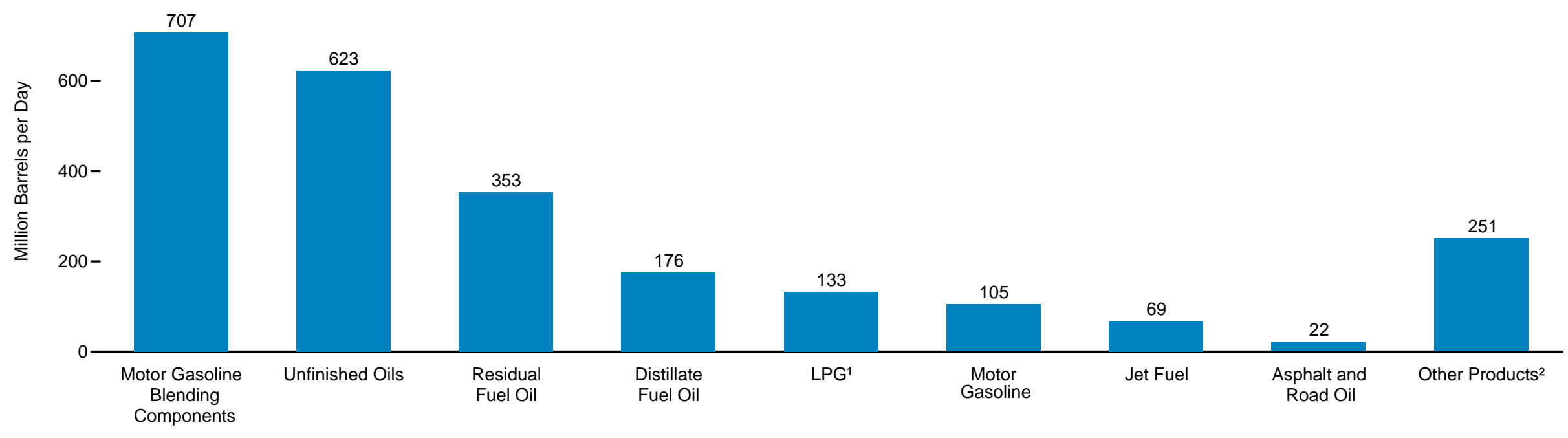

${ }^{1}$ Liquefied petroleum gases

${ }_{2}$ Aviation gasoline and blending components, kerosene, lubricants, naphtha-type jet fuel, pentanes plus, petrochemical feedstocks, petroleum coke, special naphthas, waxes, other hydrocarbons and oxygenates, and miscellaneous products. 
Table 5.3 Petroleum Imports by Type, Selected Years, 1949-2011

(Thousand Barrels per Day)

\begin{tabular}{|c|c|c|c|c|c|c|c|c|c|c|c|c|c|}
\hline \multirow[b]{3}{*}{ Year } & \multirow{3}{*}{$\begin{array}{l}\text { Crude } \\
\text { Oil } 1,2\end{array}$} & \multicolumn{11}{|c|}{ Petroleum Products } & \multirow[b]{3}{*}{$\begin{array}{c}\text { Total } \\
\text { Petroleum }\end{array}$} \\
\hline & & \multirow{2}{*}{$\begin{array}{l}\text { Asphalt and } \\
\text { Road Oil }\end{array}$} & \multirow{2}{*}{$\begin{array}{l}\text { Distillate } \\
\text { Fuel Oil }\end{array}$} & \multirow{2}{*}{$\begin{array}{l}\text { Jet } \\
\text { Fuel }^{3}\end{array}$} & \multicolumn{2}{|c|}{ Liquefied Petroleum Gases } & \multirow{2}{*}{$\begin{array}{c}\text { Motor } \\
\text { Gasoline }\end{array}$} & \multirow{2}{*}{$\begin{array}{l}\text { Motor Gasoline } \\
\text { Blending } \\
\text { Components }\end{array}$} & \multirow{2}{*}{$\begin{array}{l}\text { Residual } \\
\text { Fuel Oil }\end{array}$} & \multirow{2}{*}{$\begin{array}{l}\text { Unfinished } \\
\text { Oils }\end{array}$} & \multirow{2}{*}{$\begin{array}{c}\text { Other } \\
\text { Products }\end{array}$} & \multirow[b]{2}{*}{ Total } & \\
\hline & & & & & Propane 4 & Total & & & & & & & \\
\hline 1949 & 421 & 3 & 5 & $\left({ }^{3}\right)$ & 0 & 0 & 0 & 0 & 206 & 10 & 0 & 224 & 645 \\
\hline 1950 & 487 & 5 & 7 & $\left({ }^{3}\right)$ & 0 & 0 & (s) & $\left({ }^{7}\right)$ & 329 & 21 & 1 & 363 & 850 \\
\hline 1955 & 782 & 9 & 12 & $\left({ }^{3}\right)$ & 0 & 0 & 13 & $\left(7^{7}\right)$ & 417 & 15 & 0 & 466 & 1,248 \\
\hline 1960 & 1,015 & 17 & 35 & 34 & NA & 4 & 27 & $(7)$ & 637 & 45 & (s) & 799 & 1,815 \\
\hline 1965 & 1,238 & 17 & 36 & 81 & NA & 21 & 28 & $(7)$ & 946 & 92 & 10 & 1,229 & $\begin{array}{l}2,468 \\
2,46\end{array}$ \\
\hline 1970 & 1,324 & 17 & 147 & 144 & 26 & 52 & 67 & $(7)$ & 1,528 & 108 & 32 & 2,095 & 3,419 \\
\hline 1975 & 4,105 & 14 & 155 & 133 & 60 & 112 & 184 & $\left(7^{7}\right)$ & 1,223 & 36 & 95 & 1,951 & 6,056 \\
\hline 1976 & 5,287 & 11 & 146 & 76 & 68 & 130 & 131 & $\left(7^{\prime}\right)$ & 1,413 & 32 & 87 & 2,026 & 7,313 \\
\hline 1977 & 6,615 & 4 & 250 & 75 & 86 & 161 & 217 & (7) & 1,359 & 31 & 95 & 2,193 & 8,807 \\
\hline 1978 & 6,356 & 2 & 173 & 86 & 57 & 123 & 190 & $(7)$ & 1,355 & 27 & 50 & 2,008 & 8,363 \\
\hline 1979 & 6,519 & 4 & 193 & 78 & 88 & 217 & 181 & $(7)$ & 1,151 & 59 & 54 & 1,937 & 8,456 \\
\hline 1980 & 5,263 & 4 & 142 & 80 & 69 & 216 & 140 & $\left(7^{7}\right)$ & 939 & 55 & 72 & 1,646 & 6,909 \\
\hline 1981 & 4,396 & 4 & 173 & 38 & 70 & 244 & 157 & 24 & 800 & 112 & 48 & 1,599 & 5,996 \\
\hline 1982 & 3,488 & 5 & 93 & 29 & 63 & 226 & 197 & 42 & 776 & 174 & 84 & 1,625 & 5,113 \\
\hline 1983 & $\begin{array}{l}3,400 \\
3,329\end{array}$ & 7 & $\begin{array}{r}174 \\
\end{array}$ & 29 & 44 & 190 & 247 & 47 & 699 & 234 & $\begin{array}{l}94 \\
94\end{array}$ & $\begin{array}{l}1,722 \\
1,72\end{array}$ & 5,051 \\
\hline 1984 & 3,426 & 18 & 272 & 62 & 67 & 195 & 299 & 83 & 681 & 231 & 171 & 2,011 & 5,437 \\
\hline 1985 & 3,201 & 35 & 200 & 39 & 67 & 187 & 381 & 67 & 510 & 318 & 130 & 1,866 & 5,067 \\
\hline 1986 & 4,178 & 29 & 247 & 57 & 110 & 242 & 326 & 72 & 669 & 250 & 153 & 2,045 & 6,224 \\
\hline 1987 & 4,674 & 36 & 255 & 67 & 88 & 190 & 384 & 60 & 565 & 299 & 146 & 2,004 & 6,678 \\
\hline 1988 & 5,107 & 31 & 302 & 90 & $\begin{array}{r}00 \\
106\end{array}$ & 209 & 405 & 57 & 644 & 360 & 196 & $\begin{array}{l}\text { 2, } 295 \\
2,295\end{array}$ & $\begin{array}{l}7,402 \\
7,402\end{array}$ \\
\hline 1989 & 5,843 & 31 & 306 & 106 & 111 & 181 & 369 & 66 & 629 & 348 & 183 & 2,217 & 8,061 \\
\hline 1990 & 5,894 & 32 & 278 & 108 & 115 & 188 & 342 & 62 & 504 & 413 & 198 & 2,123 & 8,018 \\
\hline 1991 & 5,782 & 28 & 205 & 67 & 91 & 147 & 297 & 36 & 453 & 413 & 198 & 1,844 & 7,627 \\
\hline 1992 & 6,083 & 27 & 216 & 82 & 85 & 131 & 294 & 41 & 375 & 443 & 195 & 1,805 & 7,888 \\
\hline 1993 & 6,787 & 32 & 184 & 100 & 103 & 160 & 247 & 27 & 373 & 491 & 219 & 1,833 & 8,620 \\
\hline 1994 & $\begin{array}{l}l, r 01 \\
7,063\end{array}$ & 37 & 203 & 117 & 124 & 183 & 356 & 20 & 314 & 413 & 291 & $\begin{array}{l}, 050 \\
1,933\end{array}$ & $\begin{array}{l}\begin{array}{l}0,0<0 \\
8,996\end{array}\end{array}$ \\
\hline 1995 & 7,230 & 36 & 193 & 106 & $\begin{array}{l}102 \\
\text {. }\end{array}$ & 146 & 265 & 48 & 187 & 349 & 276 & 1,605 & 8,835 \\
\hline 1996 & 7,508 & 27 & 230 & 111 & 119 & 166 & 336 & 166 & 248 & 367 & 319 & 1,971 & 9.478 \\
\hline 1997 & 8,225 & 32 & 228 & 91 & 113 & 169 & 309 & 200 & 194 & 353 & 360 & 1,936 & 10,162 \\
\hline 1998 & 8,706 & 28 & 210 & 124 & 137 & 194 & 311 & 209 & 275 & 302 & 350 & 2,002 & 10,708 \\
\hline 1999 & 8,731 & 34 & 250 & 128 & 122 & 182 & 382 & 217 & 237 & 317 & 375 & $\begin{array}{l}2,122 \\
2,122\end{array}$ & 10,852 \\
\hline 2000 & 9,071 & 28 & 295 & 162 & 161 & 215 & 427 & 223 & 352 & 274 & 414 & 2,389 & 11,459 \\
\hline 2001 & 9,328 & 26 & 344 & 148 & 140 & 206 & 454 & 298 & 295 & 378 & 393 & 2,543 & $\begin{array}{l}11,871 \\
\text {. }\end{array}$ \\
\hline 2002 & 9,140 & 27 & 267 & 107 & 145 & 183 & 498 & 311 & 249 & 410 & 337 & 2,390 & 11,530 \\
\hline 2003 & 9,665 & 12 & 333 & 109 & 168 & 225 & 518 & 367 & 327 & 335 & 373 & 2,599 & 12,264 \\
\hline 2004 & $\begin{array}{r}10,088 \\
\end{array}$ & 43 & 325 & 127 & 209 & 263 & 496 & 451 & 426 & 490 & 436 & 3,057 & 13,145 \\
\hline 2005 & 10,126 & $\begin{array}{l}40 \\
43\end{array}$ & $\begin{array}{l}325 \\
329\end{array}$ & 190 & 233 & 328 & $\begin{array}{l}450 \\
603\end{array}$ & 510 & 530 & 582 & $\begin{array}{l}400 \\
473\end{array}$ & $\begin{array}{l}3,058 \\
3,588\end{array}$ & 13,714 \\
\hline 2006 & 10,118 & 50 & 365 & 186 & 228 & $\begin{array}{l}320 \\
332\end{array}$ & 475 & 669 & 350 & 689 & 473 & $\begin{array}{l}\text {, } 300 \\
3,589\end{array}$ & 13,707 \\
\hline 2007 & 10,031 & 40 & 304 & 217 & 182 & 247 & 413 & 753 & 372 & 717 & 375 & $\begin{array}{l}3,437 \\
3,43\end{array}$ & 13,468 \\
\hline 2008 & 9,783 & 25 & 213 & 103 & 185 & 253 & 302 & 789 & 349 & 763 & 337 & 3,132 & 12,915 \\
\hline 2009 & 9.013 & 22 & 225 & 81 & 147 & 182 & 223 & 719 & 331 & 677 & 217 & 2,678 & 11,691 \\
\hline 2010 & $\begin{array}{r}\mathrm{R}, 213 \\
\end{array}$ & 20 & R228 & $\begin{array}{l}\text { R } \\
\text { R98 }\end{array}$ & R121 & R153 & R134 & R741 & R366 & $\begin{array}{r}071 \\
\mathrm{R} 606\end{array}$ & $\mathrm{R}_{234}$ & $\begin{array}{r}2,010 \\
R_{2}, 580\end{array}$ & $\mathrm{R}_{11,793}$ \\
\hline $2011^{P}$ & 8,921 & 22 & 176 & 69 & 108 & 133 & 105 & 707 & 353 & 623 & 251 & 2,438 & 11,360 \\
\hline
\end{tabular}

1 Includes lease condensate.

Includes imports for the Strategic Petroleum Reserve, which began in 1977. See Table 5.17.

special naphthas. Beginning in 2005, also includes naphtha-type jet fuel. R=Revised. P-Prelimina fuel is included with kerosene in "Other Products." Beginning in 2005, naphtha-type jet fuel is included in "Other Products."

Finished motor gasoline. Through 1955, also includes naphtha-type jet fuel. Through 1963, also includes aviation gasoline and special naphthas. Through 1980, also includes motor gasoline blending

$c$
6 Aviation gasoline blending components, kerosene, lubricants, pentanes plus, petrochemical reedstocks, petroleum coke, waxes, other hydrocanbons and oxygenales, and miscellaneous products. 年 Web Pages: - See http://www.eia.gov/totalenergy/data/monthly/\#petroleum for updated monthly and annual data. - See http://www.eia.gov/totalenergy/data/annual/\#petroleum for all annual data beginning in Sources: - 1949-1975-Bureau of Mines, Mineral Industry Surveys, Petroleum Statement, Annual, annual reports. - 1976-1980-U.S. Energy Information Administration (EIA), Energy Data Reports, A Annual, annual reports. 1981-2010-ElA, Petroleum S Peports. • 2011-EIA, Petroleum Supply Monthly (February 2012). 
Figure 5.4 Petroleum Imports by Country of Origin

\section{Total, OPEC, and Non-OPEC, 1960-2011}

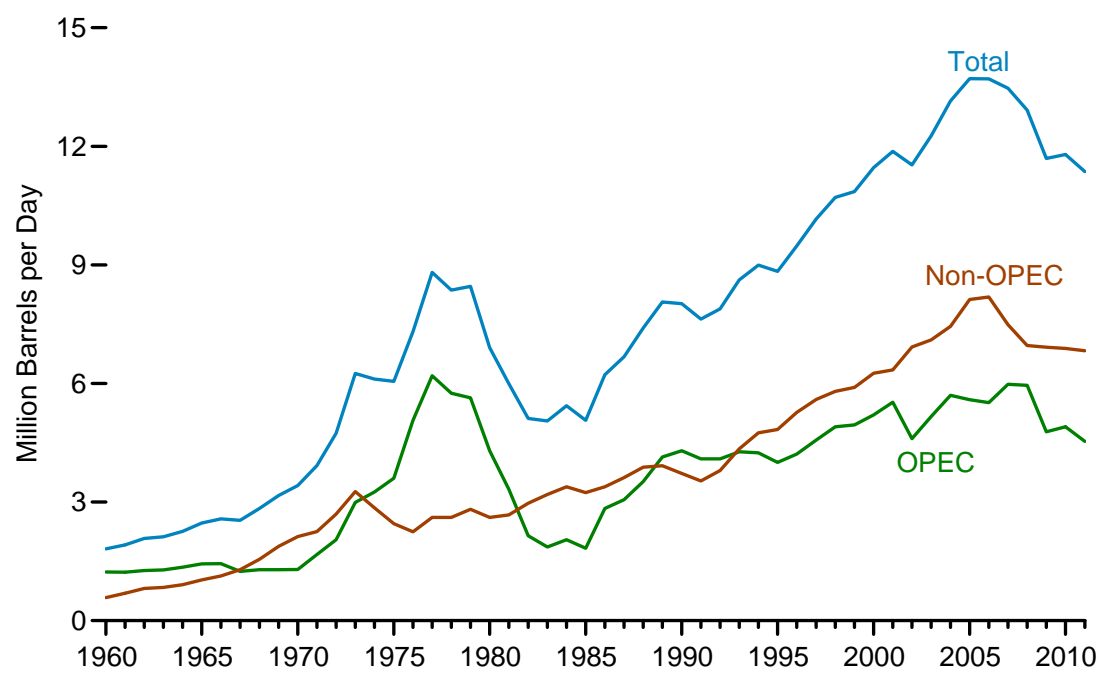

Selected OPEC Countries, 1960-2011

$2.0-$

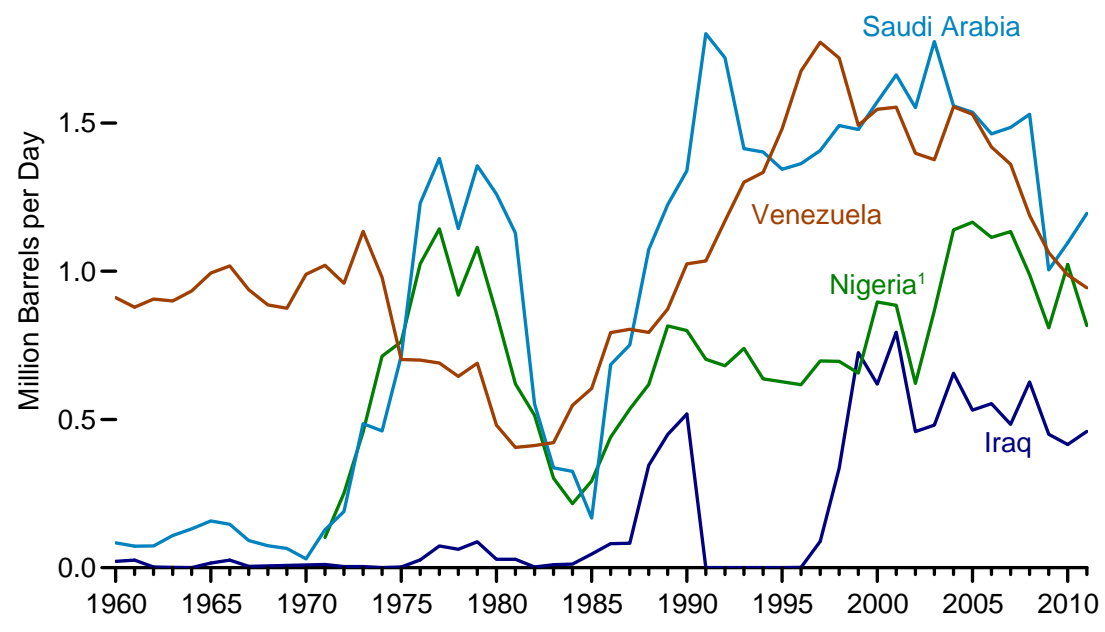

\section{Selected Countries, 2011}

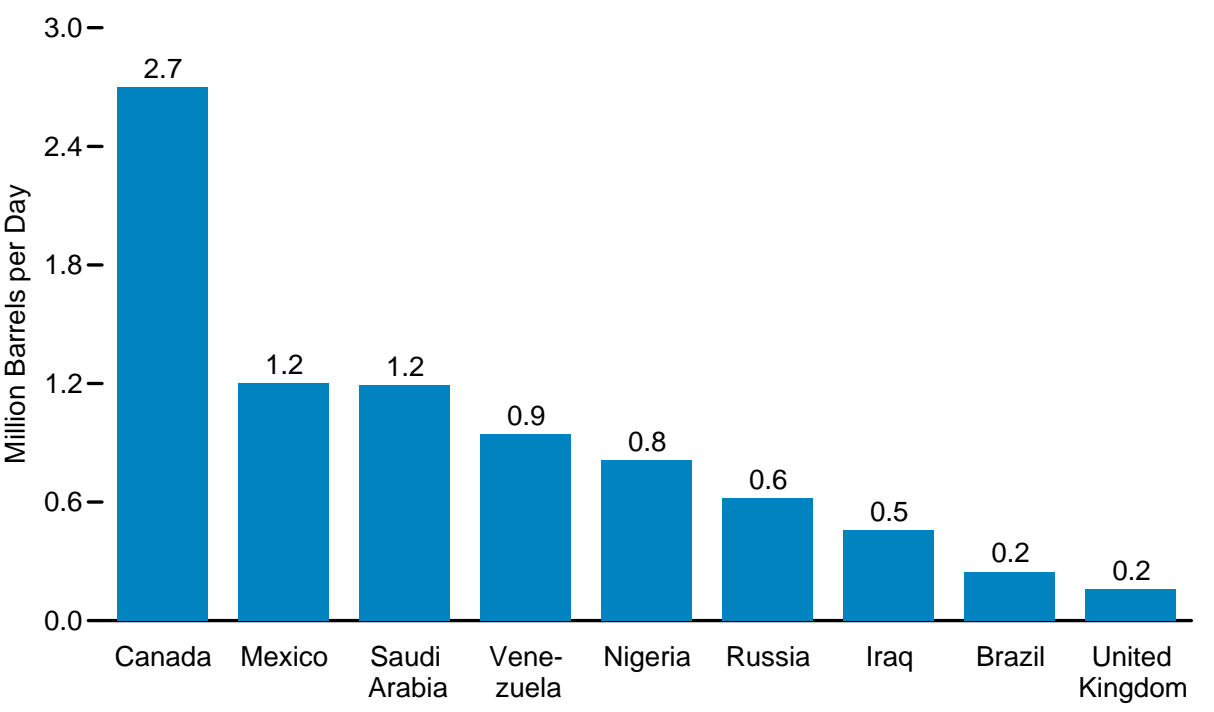

Selected Non-OPEC Countries, 1960-2011

$$
3.0-
$$

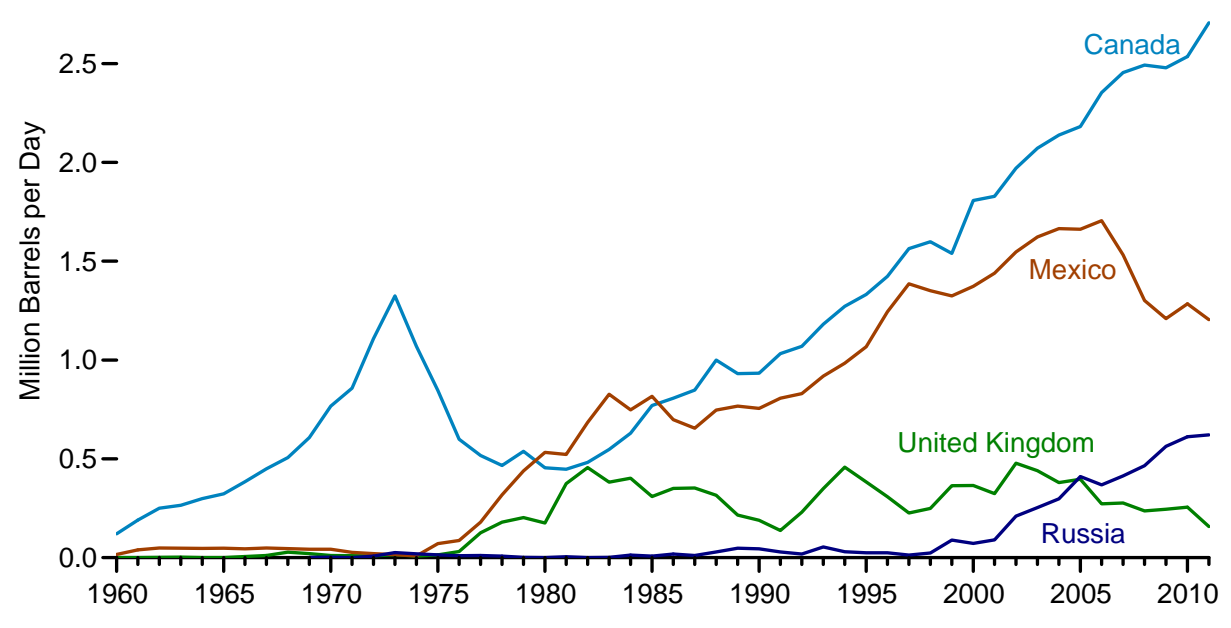

Note: OPEC=Organization of the Petroleum Exporting Countries. Source: Table 5.4. 
Table 5.4 Petroleum Imports by Country of Origin, Selected Years, 1960-2011

\begin{tabular}{|c|c|c|c|c|c|c|c|c|c|c|c|c|c|c|c|}
\hline \multirow[b]{3}{*}{ Year } & & \multicolumn{5}{|c|}{ Selected OPEC ${ }^{1}$ Countries } & \multicolumn{6}{|c|}{ Selected Non-OPEC ${ }^{1}$ Countries } & \multirow[b]{2}{*}{$\begin{array}{c}\text { Total } \\
\text { Imports }\end{array}$} & \multirow{2}{*}{$\begin{array}{l}\text { Imports From } \\
\text { Persian Gulf }{ }^{2} \\
\text { as Share of } \\
\text { Total Imports }\end{array}$} & \multirow{2}{*}{$\begin{array}{l}\text { Imports From } \\
\text { OPEC } 1 \\
\text { as Share of } \\
\text { Total Imports }\end{array}$} \\
\hline & $\begin{array}{c}\text { Persian } \\
\text { Gulf }^{2}\end{array}$ & Iraq & Nigeria & $\underset{\text { Arabia }^{3}}{\text { Saudi }}$ & Venezuela & $\begin{array}{c}\text { Total } \\
\text { OPEC }{ }^{4}\end{array}$ & Brazil & Canada & Mexico & Russia $^{5}$ & $\begin{array}{c}\text { United } \\
\text { Kingdom }\end{array}$ & $\begin{array}{c}\text { Total } \\
\text { Non-OPEC }\end{array}$ & & & \\
\hline & \multicolumn{13}{|c|}{ Thousand Barrels per Day } & \multicolumn{2}{|c|}{ Percent } \\
\hline 1960 & $\mathrm{RE}_{226}$ & 22 & $\left({ }^{6}\right)$ & 84 & 911 & 1,233 & 1 & 120 & 16 & 0 & (s) & 581 & 1,815 & $\mathrm{RE}_{17.9}$ & 68.0 \\
\hline 1965 & $\begin{array}{l}\mathrm{R} 359 \\
\mathrm{R}_{3510}-1\end{array}$ & 16 & $(6)$ & 158 & 994 & 1,439 & 0 & 323 & 48 & 0 & (s) & 1,029 & 2,468 & R14.5 & 58.3 \\
\hline 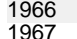 & $\begin{array}{l}\text { R319 } \\
\text { R203 }\end{array}$ & 26 & $\left(\begin{array}{l}6 \\
(6)\end{array}\right.$ & 147 & 1,018 & 1,444 & 0 & 384 & 45 & 0 & 6 & 1,129 & 2,573 & $\mathrm{R}_{12.4}$ & 56.1 \\
\hline 1968 & $\mathrm{R}_{218}$ & $\begin{array}{l}5 \\
0\end{array}$ & (6) & 74 & 886 & 1,287 & (s) & 506 & 45 & 0 & 28 & 1,290 & 2,840 & R7. & $\begin{array}{l}49.2 \\
453\end{array}$ \\
\hline 1969 & $\mathrm{R}_{193}$ & 0 & (6) & 65 & 875 & 1,286 & (3) & 608 & 43 & 2 & 20 & 1,879 & 3,166 & $\mathrm{R}_{6.1}$ & 40.6 \\
\hline 1970 & $\mathrm{R}_{184}$ & 0 & $\left(6^{\prime}\right)$ & 30 & 989 & 1,294 & 2 & 766 & 42 & 3 & 11 & 2126 & 3.419 & R5. 4 & 378 \\
\hline 1971 & R379 & 11 & 102 & 128 & 1,020 & 1,673 & 3 & 857 & 27 & 0 & 10 & 2,253 & 3.926 & $\mathrm{Rg} 7$ & 42.6 \\
\hline 1972 & 471 & 4 & 251 & 190 & 959 & 2,046 & 5 & 1,108 & 21 & 8 & 9 & 2,695 & 4,741 & 9.9 & 43.2 \\
\hline 1973 & 848 & 4 & 459 & 486 & 1.135 & 2993 & 9 & 1325 & 16 & 26 & 15 & 3.263 & 6.256 & 13.6 & 47.8 \\
\hline 1974 & $\begin{array}{r}1.039 \\
\end{array}$ & 0 & 713 & 461 & 979 & 3,256 & 2 & 1.070 & 8 & 20 & 8 & 2,856 & 6.112 & 17.0 & $\begin{array}{l}4.0 \\
533\end{array}$ \\
\hline 1975 & 1,165 & 2 & 762 & 715 & 702 & 3,601 & 5 & 846 & 71 & 14 & 14 & 2,454 & 6,056 & 19.2 & 59.5 \\
\hline 1976 & 1,840 & 26 & 1.025 & 1,230 & 700 & 5,066 & 0 & 599 & 87 & 11 & 31 & $\begin{array}{l}2,44 \\
2,247\end{array}$ & 7,313 & 25.2 & 69.3 \\
\hline 1977 & 2,448 & 74 & 1,143 & 1,380 & 690 & 6,193 & 0 & 517 & 179 & 12 & 126 & 2,614 & 8,807 & 27.8 & 70.3 \\
\hline 1978 & 2,219 & 62 & 919 & 1,144 & 646 & 5,751 & 0 & 467 & 318 & 8 & 180 & 2,612 & 8,363 & 26.5 & 68.8 \\
\hline 1979 & 2,069 & 88 & 1.080 & 1,356 & 690 & 5,637 & 1 & 538 & 439 & 1 & 202 & 2,819 & 8,456 & 24.5 & $\begin{array}{l}0.0 \\
66.7\end{array}$ \\
\hline 1980 & $\begin{array}{l}1,519 \\
\end{array}$ & $\begin{array}{l}00 \\
28\end{array}$ & $\begin{array}{l}, 000 \\
857\end{array}$ & $\begin{array}{l}1,260 \\
\end{array}$ & 481 & 4,300 & $\begin{array}{l}1 \\
3\end{array}$ & 455 & 533 & 1 & 176 & $\begin{array}{l}2,609 \\
2,60\end{array}$ & $\begin{array}{l}0,4009 \\
6,909\end{array}$ & 22.0 & 62.2 \\
\hline 1981 & 1,219 & (s) & 620 & 1,129 & 406 & 3,323 & 23 & 447 & 522 & 5 & 375 & 2,672 & 5,996 & 20.3 & 55.4 \\
\hline 1982 & 696 & 3 & 514 & 552 & 412 & 2,146 & 47 & 482 & 685 & 1 & 456 & 2,968 & 5,113 & 13.6 & 42.0 \\
\hline 1983 & 442 & 10 & 302 & 337 & 422 & $\begin{array}{l}1,862 \\
\end{array}$ & 41 & 547 & 826 & 1 & 382 & 3,189 & 5,051 & 8.8 & 36.9 \\
\hline 1984 & 506 & 12 & 216 & 325 & 548 & 2,049 & 60 & 630 & 748 & 13 & 402 & 3,388 & 5,437 & 9.3 & 37.7 \\
\hline 1985 & 311 & 46 & 293 & 168 & 605 & 1,830 & 61 & 770 & 816 & 8 & 310 & 3,237 & 5,067 & 6.1 & 36.1 \\
\hline 1986 & 912 & 81 & 440 & 685 & 793 & 2,837 & 50 & 807 & 699 & 18 & 350 & 3,387 & 6,224 & 14.7 & 45.6 \\
\hline 1987 & 1,077 & 83 & 535 & 751 & 804 & 3,060 & 84 & 848 & 655 & 11 & 352 & 3,617 & 6,678 & 16.1 & 45.8 \\
\hline 1988 & 1,541 & 345 & 618 & 1,073 & 794 & 3,520 & 98 & 999 & 747 & 29 & 315 & 3,882 & 7,402 & 20.8 & 47.6 \\
\hline 1989 & 1,861 & 449 & 815 & 1,224 & 873 & 4,140 & 82 & 931 & 767 & 48 & 215 & 3,921 & 8,061 & 23.1 & 51.4 \\
\hline 1990 & 1,966 & 518 & 800 & 1,339 & 1,025 & 4,296 & 49 & 934 & 755 & 45 & 189 & 3,721 & 8,018 & 24.5 & 53.6 \\
\hline 1991 & 1,845 & 0 & 703 & 1,802 & 1,035 & 4,092 & 22 & 1,033 & 807 & 29 & 138 & 3,535 & 7,627 & 24.2 & 53.7 \\
\hline 1992 & 1,778 & 0 & 681 & 1,720 & 1,170 & 4,092 & 20 & 1,069 & 830 & 18 & 230 & 3,796 & 7,888 & 22.5 & 51.9 \\
\hline 1993 & 1,782 & 0 & 740 & 1,414 & 1,300 & 4,273 & 33 & 1,181 & 919 & 55 & 350 & 4,347 & 8,620 & 20.7 & 49.6 \\
\hline 1994 & 1,728 & 0 & 637 & 1,402 & 1,334 & 4,247 & 31 & 1,272 & 984 & 30 & 458 & 4,749 & 8,996 & 19.2 & 47.2 \\
\hline 1995 & 1,573 & 0 & 627 & 1,344 & 1,480 & 4,002 & 8 & 1,332 & 1,068 & 25 & 383 & 4,833 & 8,835 & 17.8 & 45.3 \\
\hline 1996 & 1,604 & 1 & 617 & 1,363 & 1,676 & 4,211 & 9 & 1,424 & 1,244 & 25 & 308 & 5,267 & 9,478 & 16.9 & 44.4 \\
\hline 1997 & 1,755 & 89 & 698 & 1,407 & 1,773 & 4,569 & 5 & 1,563 & 1,385 & 13 & 226 & 5,593 & 10,162 & 17.3 & 45.0 \\
\hline 1998 & 2,136 & 336 & 696 & 1,491 & 1,719 & 4,905 & 26 & 1,598 & 1,351 & 24 & 250 & 5,803 & 10,708 & 19.9 & 45.8 \\
\hline 1999 & 2,464 & 725 & 657 & 1,478 & 1,493 & 4,953 & 26 & 1,539 & 1,324 & 89 & 365 & 5,899 & 10,852 & 22.7 & 45.6 \\
\hline 2000 & 2,488 & 620 & 896 & 1,572 & 1,546 & 5,203 & 51 & 1,807 & 1,373 & 72 & 366 & 6,257 & 11,459 & 21.7 & 45.4 \\
\hline 2001 & 2,761 & 795 & 885 & 1,662 & 1,553 & 5,528 & 82 & 1,828 & 1,440 & 90 & 324 & 6,343 & 11,871 & 23.3 & 46.6 \\
\hline 2002 & 2,269 & 459 & 621 & 1,552 & 1,398 & 4,605 & 116 & 1,971 & 1,547 & 210 & 478 & 6,925 & 11,530 & 19.7 & 39.9 \\
\hline 2003 & 2,501 & 481 & 867 & 1,774 & 1,376 & 5,162 & 108 & 2,072 & 1,623 & 254 & 440 & 7,103 & 12,264 & 20.4 & 42.1 \\
\hline 2004 & 2,493 & 656 & 1,140 & 1,558 & 1,554 & 5,701 & 104 & 2,138 & 1,665 & 298 & 380 & 7,444 & 13,145 & 19.0 & 43.4 \\
\hline 2005 & 2,334 & 531 & 1,166 & 1,537 & 1,529 & 5,587 & 156 & 2,181 & 1,662 & 410 & 396 & 8,127 & 13,714 & 17.0 & 40.7 \\
\hline 2006 & 2,211 & 553 & 1,114 & 1,463 & 1,419 & 5,517 & 193 & 2,353 & 1,705 & 369 & 272 & 8,190 & 13,707 & 16.1 & 40.2 \\
\hline 2007 & 2,163 & 484 & 1,134 & 1,485 & 1,361 & 5,980 & 200 & 2,455 & 1,532 & 414 & 277 & 7,489 & 13,468 & 16.1 & 44.4 \\
\hline 2008 & 2,370 & 627 & 988 & 1,529 & 1,189 & 5,954 & 258 & 2,493 & 1,302 & 465 & 236 & 6,961 & 12,915 & 18.4 & 46.1 \\
\hline 2009 & 1,689 & 450 & 809 & 1,004 & 1,063 & $\begin{array}{r}4,776 \\
\end{array}$ & 309 & 2,479 & 1,210 & 563 & 245 & 6,915 & 11,691 & 14.4 & 40.9 \\
\hline 2010 & $\mathrm{R}_{1,711}$ & $\mathrm{R}_{415}$ & $\mathrm{R}_{1,023}$ & $\mathrm{R}_{1,096}$ & R988 & R4,906 & $\mathrm{R}_{272}$ & $R_{2,535}$ & $\mathrm{R}_{1,284}$ & $\mathrm{R}_{612}$ & 256 & $\mathrm{R}_{6,887}$ & $\mathrm{R}_{11,793}$ & 14.5 & 41.6 \\
\hline $2011^{\mathrm{P}}$ & 1,862 & 460 & 817 & 1,195 & 944 & 4,534 & 249 & 2,706 & 1,205 & 621 & 158 & 6,825 & 11,360 & 16.4 & 39.9 \\
\hline
\end{tabular}

See "Organization of the Petroleum Exporting Countries (OPEC)" in Glossary.

2 Bahrain, Iran, Iraq, Kuwait, Qatar, Saudi Arabia, United Arab Emirates, and the Neutral Zone (between

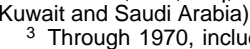

imports from the Neutral Zone. Beginning in 1971, includes imports

On this table, "Total OPEC" for all years includes Iran, Iraq, Kuwait, Saudi Arabia, Venezuela, and the Neutral Zone (between Kuwait and Saudi Arabia); beginning in 1961, also includes Qatar; beginning in 1962, also includes Libya; for 1962-2008, also includes Indonesia; beginning in 1967, also includes United Arab Emirates; beginning in 1969, also includes Algeria; beginning in 1971, also includes Nigeria; for 2007, on this table Ecuador is included in "Total Non-OPEC" for 2007): for 1975-1994, also includes Gabon; and beginning in 2007, also includes Angola. Data for all countries not included in "Total OPEC"

5 Through 1992, may include imports from republics other than Russia in the former U.S.S.R. See

6 Nigeria joined OPEC in 1971. For 1960-1970, Nigeria is included in "Total Non-OPEC."

$R=$ Revised. $P=$ Preliminary. $E=$ Estimate. ( $(s)=$ Less than 500 barrels per day.

Notes: - The country of origin for refined petroleum products may not be the country of origin for the fineries in the Caribben mports for the Strategic Petroleum Reserve, which began in 1977. - Totals may not equal sum of

Web Pages: - See http://www.eia.gov/totalenergy/data/monthly/\#petroleum for updated monthly and annual data. - See http://www.eia.gov/totalenergy/data/annual/\#petroleum for all annual data beginning Sources: - 1960-1975-Bureau of Mines, Minerals Yearbook, "Crude Petroleum and Petroleum Products" chapter. • 1976-1980-U.S. Energy Information Administration (EIA), Energy Data Reports, P.A.D. Districts Supply/Demand, Annual, annual reports. • 1981-2010-EIA, Petroleum Supply Annual, annual reports. - 2011-EIA, Petroleum Supply Monthly (February 2012). 


\section{Total, 1949-2011}

3,500-

$3,000-$

สิ $2,500-$

¿ั

$\frac{\infty}{d} 2,000-$

ฮั

을 $1,500-$

胥

옫 $1,000-$

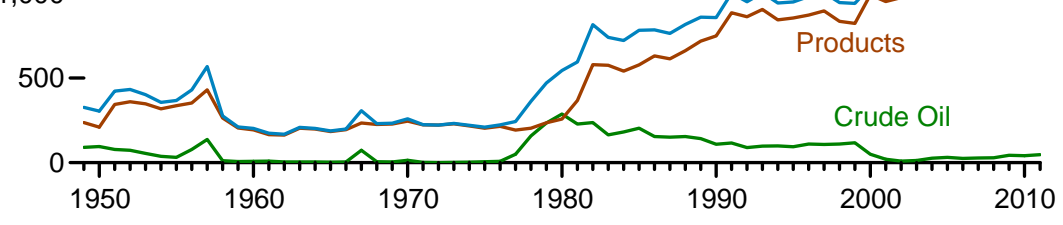

\section{By Selected Product, 1949-2011}

900-

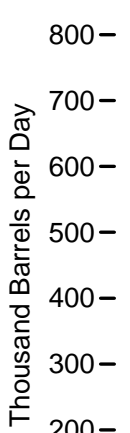

$800-$

๘

¿ $600-$

$500-$

$400-$

$300-$

$200-$

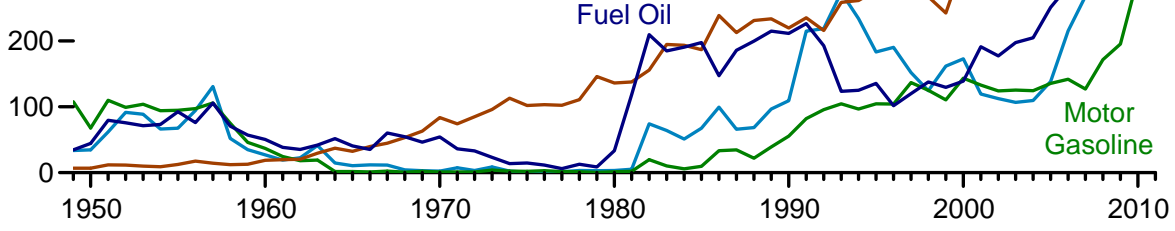

\section{By Product, 2011}

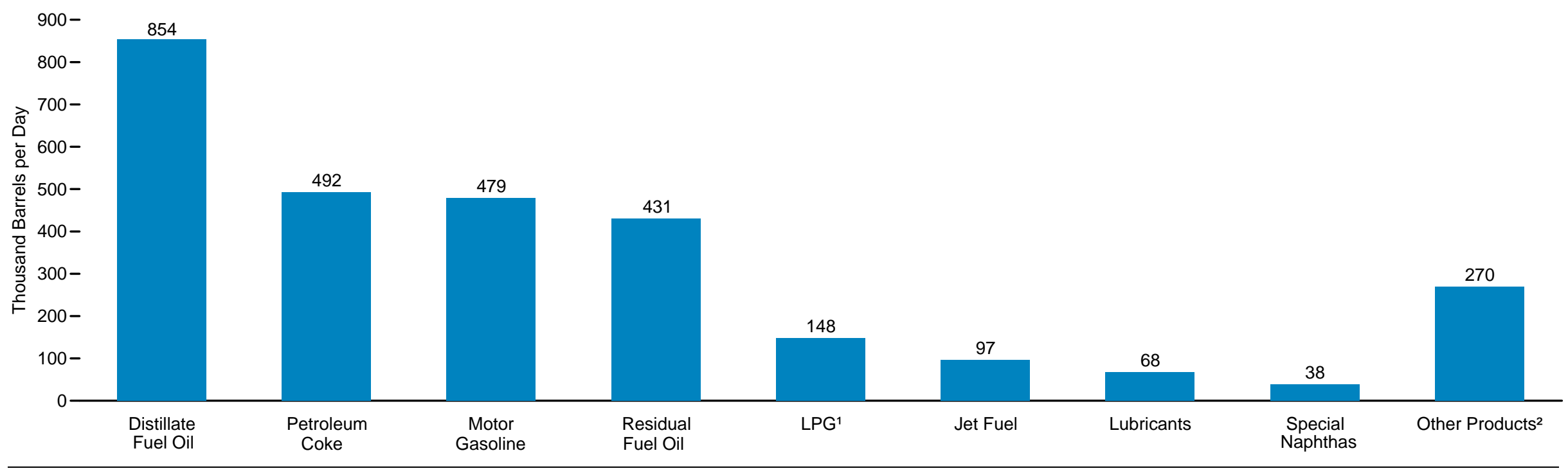

${ }^{1}$ Liquefied petroleum gases.

2 Asphalt and road oil, aviation gasoline, kerosene, motor gasoline blending components, naphtha-type jet fuel, pentanes plus, waxes, other hydrocarbons and oxygenates, and miscellaneous products.

Source: Table 5.5. 
Table 5.5 Petroleum Exports by Type, Selected Years, 1949-2011

(Thousand Barrels per Day)

\begin{tabular}{|c|c|c|c|c|c|c|c|c|c|c|c|c|c|c|}
\hline \multirow[b]{3}{*}{ Year } & \multirow{3}{*}{$\begin{array}{c}\text { Crude } \\
\text { Oil }^{1}\end{array}$} & \multicolumn{12}{|c|}{ Petroleum Products } & \multirow{3}{*}{$\begin{array}{c}\text { Total } \\
\text { Petroleum }\end{array}$} \\
\hline & & \multirow{2}{*}{$\begin{array}{l}\text { Distillate } \\
\text { Fuel Oil }\end{array}$} & \multirow{2}{*}{$\begin{array}{l}\text { Jet } \\
\text { Fuel }^{2}\end{array}$} & \multicolumn{2}{|c|}{ Liquefied Petroleum Gases } & \multirow[b]{2}{*}{ Lubricants } & \multirow{2}{*}{$\begin{array}{c}\text { Motor } \\
\text { Gasoline } 4\end{array}$} & \multirow{2}{*}{$\begin{array}{l}\text { Petroleum } \\
\text { Coke }\end{array}$} & \multirow{2}{*}{$\begin{array}{c}\text { Petrochemical } \\
\text { Feedstocks }\end{array}$} & \multirow{2}{*}{$\begin{array}{l}\text { Residual } \\
\text { Fuel Oil }\end{array}$} & \multirow{2}{*}{$\begin{array}{c}\text { Special } \\
\text { Naphthas }\end{array}$} & \multirow{2}{*}{$\begin{array}{c}\text { Other } \\
\text { Products } 5\end{array}$} & \multirow[b]{2}{*}{ Total } & \\
\hline & & & & Propane $^{3}$ & Total & & & & & & & & & \\
\hline 1949 & 91 & 34 & $\left({ }^{2}\right)$ & NA & 4 & 35 & 108 & 7 & 0 & 35 & NA & 15 & 236 & 327 \\
\hline 1950 & 95 & 35 & $\left({ }^{2}\right)$ & NA & 4 & 39 & 68 & 7 & 0 & 44 & NA & 12 & 210 & 305 \\
\hline 1955 & 32 & 67 & (s) & NA & 12 & 39 & 95 & 12 & 0 & 93 & NA & 18 & 336 & 368 \\
\hline 1960 & 8 & 27 & (s) & NA & 8 & 43 & 37 & 19 & 0 & 51 & NA & 9 & 193 & 202 \\
\hline 1965 & 3 & 10 & 3 & NA & 21 & 45 & 2 & 32 & 5 & 41 & 4 & 20 & 184 & 187 \\
\hline 1970 & 14 & 2 & 6 & 6 & 27 & 44 & 1 & 84 & 10 & 54 & 4 & 12 & 245 & 259 \\
\hline 1975 & 6 & 1 & 2 & 13 & 26 & 25 & 2 & 102 & 22 & 15 & 3 & 6 & 204 & 209 \\
\hline 1976 & 8 & 1 & 2 & 13 & 25 & 26 & 3 & 103 & 30 & 12 & 7 & 6 & 215 & 223 \\
\hline 1977 & 50 & 1 & 2 & 10 & 18 & 26 & 2 & 102 & 24 & 6 & 4 & 7 & 193 & 243 \\
\hline 1978 & 158 & 3 & 1 & 9 & 20 & 27 & 1 & 111 & 23 & 13 & 2 & 2 & 204 & 362 \\
\hline 1979 & 235 & 3 & 1 & 8 & 15 & 23 & (s) & 146 & 31 & 9 & 5 & 3 & 236 & 471 \\
\hline 1980 & 287 & 3 & 1 & 10 & 21 & 23 & 1 & 136 & 29 & 33 & 5 & 4 & 258 & 544 \\
\hline 1981 & 228 & 5 & 2 & 18 & 42 & 19 & 2 & 138 & 26 & 118 & 11 & 4 & 367 & 595 \\
\hline 1982 & 236 & 74 & 6 & 31 & 65 & 16 & 20 & 156 & 24 & 209 & 5 & 4 & 579 & 815 \\
\hline 1983 & 164 & 64 & 6 & 43 & 73 & 16 & 10 & 195 & 20 & 185 & 3 & 3 & 575 & 739 \\
\hline 1984 & 181 & 51 & 9 & 30 & 48 & 15 & 6 & 193 & 21 & 190 & 2 & 6 & 541 & 722 \\
\hline 1985 & 204 & 67 & 13 & 48 & 62 & 15 & 10 & 187 & 19 & 197 & 1 & 4 & 577 & 781 \\
\hline 1986 & 154 & 100 & 18 & 28 & 42 & 23 & 33 & 238 & 22 & 147 & 1 & 8 & 631 & 785 \\
\hline 1987 & 151 & 66 & 24 & 24 & 38 & 23 & 35 & 213 & 20 & 186 & 2 & 7 & 613 & 764 \\
\hline 1988 & 155 & 69 & 28 & 31 & 49 & 26 & 22 & 231 & 23 & 200 & 7 & 6 & 661 & 815 \\
\hline 1989 & 142 & 97 & 27 & 24 & 35 & 19 & 39 & 233 & 26 & 215 & 12 & 15 & 717 & 859 \\
\hline 1990 & 109 & 109 & 43 & 28 & 40 & 20 & 55 & 220 & 26 & 211 & 11 & 13 & 748 & 857 \\
\hline 1991 & 116 & 215 & 43 & 28 & 41 & 18 & 82 & 235 & 0 & 226 & 15 & 9 & 885 & 1,001 \\
\hline 1992 & 89 & 219 & 43 & 33 & 49 & 16 & 96 & 216 & 0 & 193 & 14 & 16 & 861 & 950 \\
\hline 1993 & 98 & 274 & 59 & 26 & 43 & 19 & 105 & 258 & 0 & 123 & 4 & 20 & 904 & 1,003 \\
\hline 1994 & 99 & 234 & 20 & 24 & 38 & 22 & 97 & 261 & 0 & 125 & 20 & 26 & 843 & 942 \\
\hline 1995 & 95 & 183 & 26 & 38 & 58 & 25 & 104 & 277 & 0 & 136 & 21 & 25 & 855 & 949 \\
\hline 1996 & 110 & 190 & 48 & 28 & 51 & 34 & 104 & 285 & 0 & 102 & 21 & 36 & 871 & 981 \\
\hline 1997 & 108 & 152 & 35 & 32 & 50 & 31 & 137 & 306 & 0 & 120 & 22 & 44 & 896 & 1,003 \\
\hline 1998 & 110 & 124 & 26 & 25 & 42 & 25 & 125 & 267 & 0 & 138 & 18 & 70 & 835 & 945 \\
\hline 1999 & 118 & 162 & 32 & 33 & 50 & 28 & 111 & 242 & 0 & 129 & 16 & 52 & 822 & 940 \\
\hline 2000 & 50 & 173 & 32 & 53 & 74 & 26 & 144 & 319 & 0 & 139 & 20 & 64 & 990 & 1,040 \\
\hline 2001 & 20 & 119 & 29 & 31 & 44 & 26 & 133 & 336 & 0 & 191 & 23 & 50 & 951 & 971 \\
\hline 2002 & 9 & 112 & 15 & 55 & 67 & 33 & 124 & 337 & 0 & 177 & 15 & 94 & 975 & 984 \\
\hline 2003 & 12 & 107 & 20 & 37 & 56 & 37 & 125 & 361 & 0 & 197 & 22 & 89 & 1,014 & 1,027 \\
\hline 2004 & 27 & 110 & 40 & 28 & 43 & 41 & 124 & 350 & 0 & 205 & 27 & 82 & 1,021 & 1,048 \\
\hline 2005 & 32 & 138 & 53 & 37 & 53 & 40 & 136 & 347 & 0 & 251 & 21 & 94 & 1,133 & 1,165 \\
\hline 2006 & 25 & 215 & 41 & 45 & 56 & 55 & 142 & 366 & 0 & 283 & 14 & 121 & 1,292 & 1,317 \\
\hline 2007 & 27 & 268 & 41 & 42 & 57 & 59 & 127 & 366 & 0 & 330 & 18 & 140 & 1,405 & 1,433 \\
\hline 2008 & 29 & 528 & 61 & 53 & 67 & 60 & 172 & 377 & 0 & 355 & 13 & 139 & 1,773 & 1,802 \\
\hline 2009 & 44 & 587 & 69 & 85 & 100 & 57 & 195 & 391 & 0 & 415 & 22 & 143 & 1,980 & 2,024 \\
\hline 2010 & 42 & 656 & 84 & 109 & 132 & 62 & 296 & 449 & 0 & 405 & 36 & $\mathrm{R}_{192}$ & $\mathrm{R}_{2,311}$ & $\mathrm{R}_{2,353}$ \\
\hline $2011^{P}$ & 47 & 854 & 97 & 124 & 148 & 68 & 479 & 492 & 0 & 431 & 38 & 270 & 2,877 & 2,924 \\
\hline
\end{tabular}

1 Includes lease condensate.

2 Through 1952, naphtha-type jet fuel is included in the products from which it was blended: gasoline, kerosene, and distillate fuel oil. Through 1964, kerosene-type jet fuel is included with kerosene in "Other Products." Beginning in 2005, naphtha-type jet fuel is included in "Other Products."

3 Includes propylene.

Finished motor gasoline. Through 1963, also includes aviation gasoline.

Asphalt and road oil, kerosene, motor gasoline blending components, pentanes plus, waxes, other hydrocarbons and oxygenates, and miscellaneous products. Through 1964, also includes kerosene-type jet fuel. Beginning in 1964, also includes aviation gasoline. Beginning in 2005, also includes naphtha-type

$R=$ Revised. $P=$ Preliminary. $N A=$ Not available. $(s)=$ Less than 500 barrels per day.

Notes: - Includes exports to U.S. possessions and territories. - Totals may not equal sum of components due to independent rounding.

Web Pages: - See http:/www.eia.gov/totalenergy/data/monthly/\#petroleum for updated monthly and annual data. - See http://www.eia.gov/totalenergy/data/annual/\#petroleum for all annual data beginning in 1949. - See http://www.eia.gov/petroleum/ for related information

Sources: - 1949-1975-Bureau of Mines, Mineral Industry Surveys, Petroleum Statement, Annual, annual reports. - 1976-1980-U.S. Energy Information Administration (EIA), Energy Data Reports, Petroleum Statement, Annual, annual reports. • 1981-2010-EIA, Petroleum Supply Annual, annua 


\section{Figure 5.6 Petroleum Exports by Country of Destination}

\section{Total Exports and Exports to Canada and Mexico, 1960-2011}

$3,500-$

3,000

๙ิ $2,500-$

¿ั

$\frac{\infty}{d} 2,000-$

离

일 $1,500-$

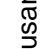

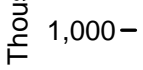

$500-$

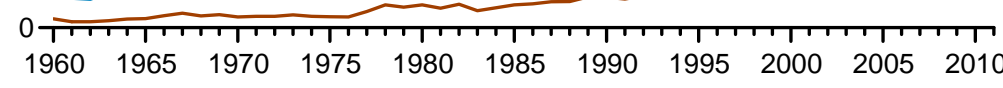

By Selected Country, 1960-2011

$600-$

$500-$

वे

告 $300-$

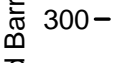

药 $200-$

$100-$

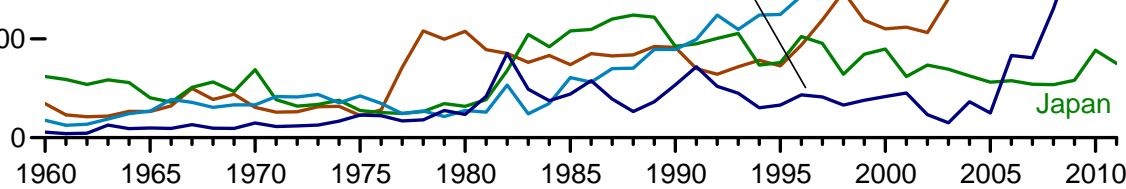

\section{By Selected Country, 2011}

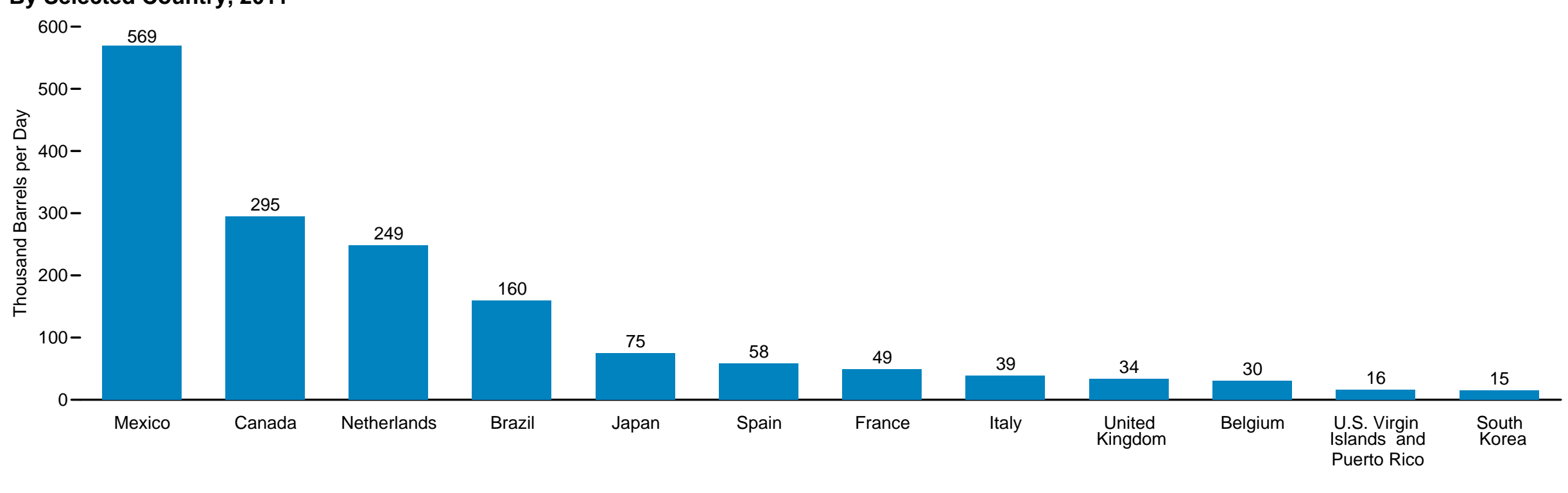

Source: Table 5.6. 
Table 5.6 Petroleum Exports by Country of Destination, Selected Years, 1960-2011

(Thousand Barrels per Day)

\begin{tabular}{|c|c|c|c|c|c|c|c|c|c|c|c|c|c|c|}
\hline Year & Belgium ${ }^{1}$ & Brazil & Canada & France & Italy & Japan & Mexico & $\begin{array}{l}\text { Nether- } \\
\text { lands }\end{array}$ & $\begin{array}{l}\text { South } \\
\text { Korea }\end{array}$ & Spain & $\begin{array}{c}\text { United } \\
\text { Kingdom }\end{array}$ & $\begin{array}{l}\text { U.S. Virgin } \\
\text { Islands and } \\
\text { Puerto Rico }\end{array}$ & Other & Total \\
\hline 1960 & 3 & 4 & 34 & 4 & 6 & 62 & 18 & 6 & NA & NA & 12 & 1 & 52 & 202 \\
\hline 1965 & 3 & 3 & 26 & 3 & 7 & 40 & 27 & 10 & NA & NA & 12 & 1 & 54 & 187 \\
\hline 1966 & 3 & 4 & 32 & 4 & 7 & 36 & 39 & 9 & NA & NA & 12 & 3 & 49 & 198 \\
\hline 1967 & 5 & 6 & 50 & 3 & 9 & 51 & 36 & 13 & NA & NA & 62 & 7 & 65 & 307 \\
\hline 1968 & 4 & 8 & 39 & 4 & 8 & 56 & 31 & 10 & NA & NA & 14 & 2 & 55 & 231 \\
\hline 1969 & 4 & 7 & 44 & 4 & 9 & 47 & 33 & 9 & NA & NA & 13 & 2 & 59 & 233 \\
\hline 1970 & 5 & 7 & 31 & 5 & 10 & 69 & 33 & 15 & NA & NA & 12 & 2 & 71 & 259 \\
\hline 1971 & 7 & 9 & 26 & 5 & 8 & 39 & 42 & 11 & NA & NA & 9 & 3 & 67 & 224 \\
\hline 1972 & 13 & 9 & 26 & 5 & 9 & 32 & 41 & 12 & NA & 4 & 10 & 4 & 59 & 222 \\
\hline 1973 & 15 & 8 & 31 & 5 & 9 & 34 & 44 & 13 & NA & 4 & 9 & 3 & 56 & 231 \\
\hline 1974 & 13 & 9 & 32 & 4 & 9 & 38 & 35 & 17 & NA & 4 & 6 & 6 & 48 & 221 \\
\hline 1975 & 9 & 6 & 22 & 6 & 10 & 27 & 42 & 23 & NA & 4 & 7 & 12 & 40 & 209 \\
\hline 1976 & 12 & 7 & 28 & 6 & 10 & 25 & 35 & 22 & NA & 4 & 13 & 22 & 39 & 223 \\
\hline 1977 & 16 & 6 & 71 & 9 & 10 & 25 & 24 & 17 & NA & 5 & 9 & 11 & 39 & 243 \\
\hline 1978 & 15 & 8 & 108 & 9 & 10 & 26 & 27 & 18 & NA & 5 & 7 & 86 & 42 & 362 \\
\hline 1979 & 19 & 7 & 100 & 13 & 15 & 34 & 21 & 28 & 2 & 9 & 7 & 170 & 45 & 471 \\
\hline 1980 & 20 & 4 & 108 & 11 & 14 & 32 & 28 & 23 & 2 & 8 & 7 & 220 & 70 & 544 \\
\hline 1981 & 12 & 1 & 89 & 15 & 22 & 38 & 26 & 42 & 10 & 18 & 5 & 220 & 97 & 595 \\
\hline 1982 & 17 & 8 & 85 & 24 & 32 & 68 & 53 & 85 & 28 & 24 & 14 & 212 & 165 & 815 \\
\hline 1983 & 22 & 2 & 76 & 23 & 35 & 104 & 24 & 49 & 15 & 34 & 8 & 144 & 202 & 739 \\
\hline 1984 & 21 & 1 & 83 & 18 & 39 & 92 & 35 & 37 & 17 & 29 & 14 & 152 & 182 & 722 \\
\hline 1985 & 26 & 3 & 74 & 11 & 30 & 108 & 61 & 44 & 27 & 28 & 14 & 162 & 193 & 781 \\
\hline 1986 & 30 & 3 & 85 & 11 & 39 & 110 & 56 & 58 & 12 & 39 & 8 & 113 & 222 & 785 \\
\hline 1987 & 17 & 2 & 83 & 12 & 42 & 120 & 70 & 39 & 25 & 31 & 6 & 136 & 179 & 764 \\
\hline 1988 & 25 & 3 & 84 & 12 & 29 & 124 & 70 & 26 & 24 & 36 & 9 & 147 & 226 & 815 \\
\hline 1989 & 23 & 5 & 92 & 11 & 37 & 122 & 89 & 36 & 17 & 28 & 9 & 141 & 249 & 859 \\
\hline 1990 & 20 & 2 & 91 & 17 & 48 & 92 & 89 & 54 & 60 & 33 & 11 & 101 & 240 & 857 \\
\hline 1991 & 22 & 13 & 70 & 27 & 55 & 95 & 99 & 72 & 66 & 23 & 13 & 117 & 330 & 1,001 \\
\hline 1992 & 22 & 20 & 64 & 9 & 38 & 100 & 124 & 52 & 80 & 21 & 12 & 95 & 315 & 950 \\
\hline 1993 & 21 & 16 & 72 & 8 & 34 & 105 & 110 & 45 & 74 & 30 & 10 & 108 & 370 & 1,003 \\
\hline 1994 & 26 & 15 & 78 & 11 & 35 & 74 & 124 & 30 & 66 & 30 & 10 & 104 & 338 & 942 \\
\hline 1995 & 21 & 16 & 73 & 11 & 46 & 76 & 125 & 33 & 57 & 38 & 14 & 123 & 317 & 949 \\
\hline 1996 & 27 & 29 & 94 & 18 & 32 & 102 & 143 & 43 & 60 & 34 & 9 & 72 & 318 & 981 \\
\hline 1997 & 21 & 15 & 119 & 11 & 30 & 95 & 207 & 41 & 50 & 42 & 12 & 18 & 340 & 1,003 \\
\hline 1998 & 14 & 18 & 148 & 8 & 30 & 64 & 235 & 33 & 33 & 30 & 11 & 4 & 317 & 945 \\
\hline 1999 & 11 & 27 & 119 & 7 & 25 & 84 & 261 & 38 & 49 & 26 & 9 & 8 & 276 & 940 \\
\hline 2000 & 14 & 28 & 110 & 10 & 34 & 90 & 358 & 42 & 20 & 40 & 10 & 10 & 277 & 1,040 \\
\hline 2001 & 16 & 23 & 112 & 13 & 33 & 62 & 274 & 45 & 14 & 51 & 13 & 4 & 312 & 971 \\
\hline 2002 & 19 & 26 & 106 & 12 & 29 & 74 & 254 & 23 & 11 & 54 & 12 & 9 & 354 & 984 \\
\hline 2003 & 13 & 27 & 141 & 9 & 39 & 69 & 228 & 15 & 10 & 39 & 6 & 9 & 421 & $\begin{array}{r}1,027 \\
\end{array}$ \\
\hline 2004 & 20 & 27 & 158 & 18 & 32 & 63 & 209 & 36 & 12 & 42 & 14 & 10 & 408 & 1,048 \\
\hline 2005 & 21 & 39 & 181 & 14 & 28 & 56 & 268 & 25 & 16 & 35 & 21 & 11 & 449 & 1,165 \\
\hline 2006 & 23 & 42 & 159 & 13 & 39 & 58 & 255 & 83 & 21 & 42 & 28 & 10 & 543 & 1,317 \\
\hline 2007 & 13 & 46 & 189 & 24 & 34 & 54 & 279 & 81 & 16 & 48 & 9 & 10 & 629 & 1,433 \\
\hline 2008 & 18 & 54 & 264 & 27 & 41 & 54 & 333 & 131 & 18 & 54 & 17 & 13 & 777 & 1,802 \\
\hline 2009 & 29 & 55 & 223 & 34 & 35 & 58 & 322 & 192 & 23 & 40 & 33 & 20 & 960 & 2,024 \\
\hline 2010 & 19 & $\mathrm{R}_{123}$ & $R_{233}$ & 36 & 37 & 88 & $\mathrm{R}_{448}$ & $\mathrm{R}_{165}$ & $\mathrm{R}_{13}$ & 36 & $R_{19}$ & 17 & $\mathrm{R}_{1,117}$ & $\mathrm{R}_{2,353}$ \\
\hline $2011^{P}$ & 30 & 160 & 295 & 49 & 39 & 75 & 569 & 249 & 15 & 58 & 34 & 16 & 1,335 & 2,924 \\
\hline
\end{tabular}

1 Through 2004, includes Luxembourg.

Sources: • 1960-1975-Bureau of Mines, Mineral Industry Surveys, Petroleum Statement, Annual, annual reports. - 1976-1980-U.S. Energy Information Administration (EIA), Energy Data Reports reports. • 2011-EIA, Petroleum Supply Monthly (February 2012).

Note: Totals may not equal sum of components due to independent rounding.

We

1960. - For related information, see http://www eia gov/petroleum/ 


\section{Figure 5.7 Petroleum Net Imports by Country of Origin, 1960-2011}

\section{Total, OPEC, and Non-OPEC}

$14-$

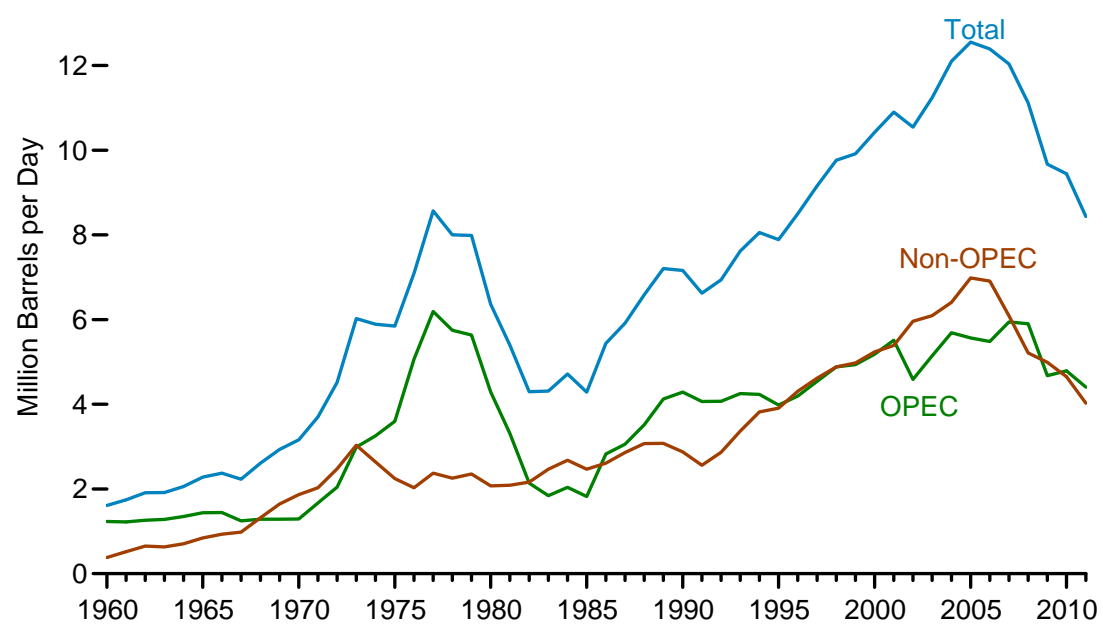

Total Net Imports as Share of Consumption $100-$

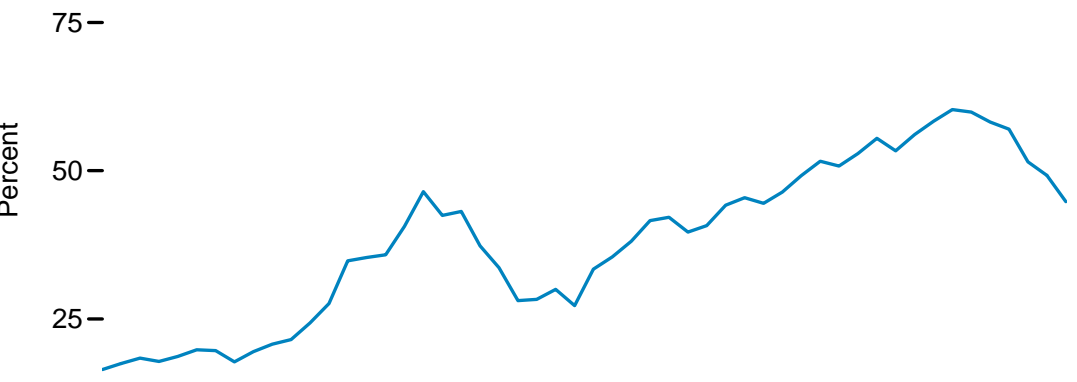

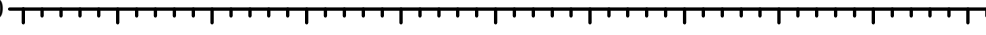

$\begin{array}{lllllllllll}1960 & 1965 & 1970 & 1975 & 1980 & 1985 & 1990 & 1995 & 2000 & 2005 & 2010\end{array}$

\section{By Selected Country}

4-

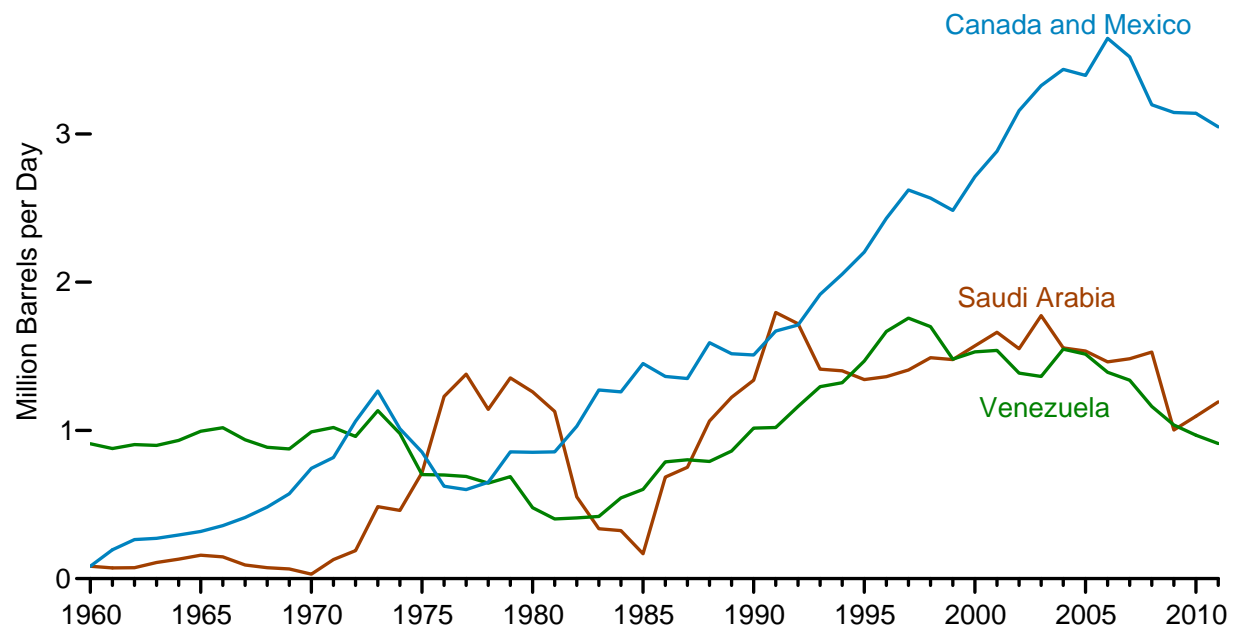

Net Imports From OPEC

$100-$
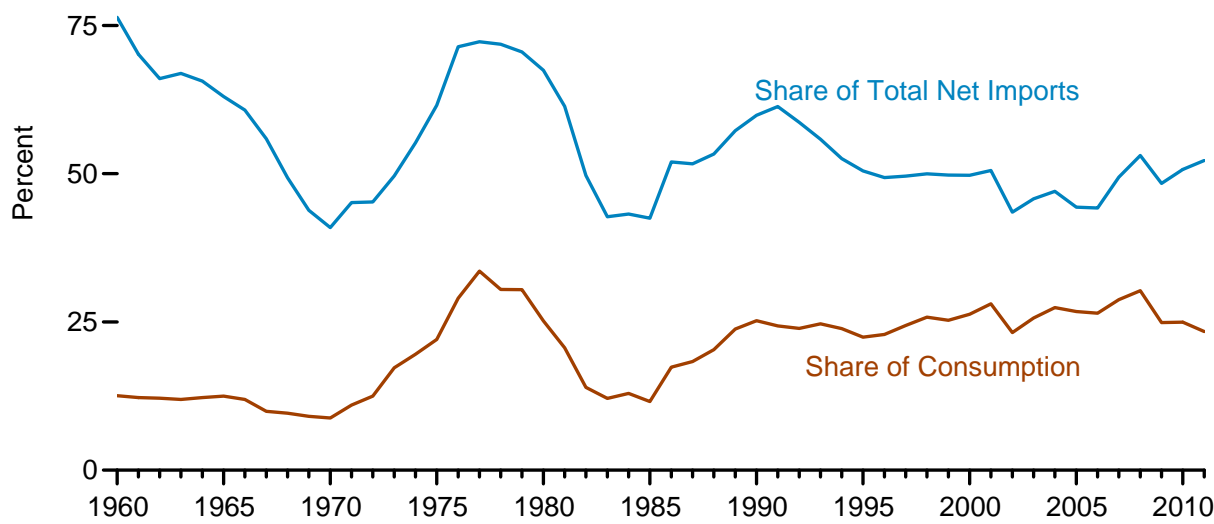
Table 5.7 Petroleum Net Imports by Country of Origin, Selected Years, 1960-2011

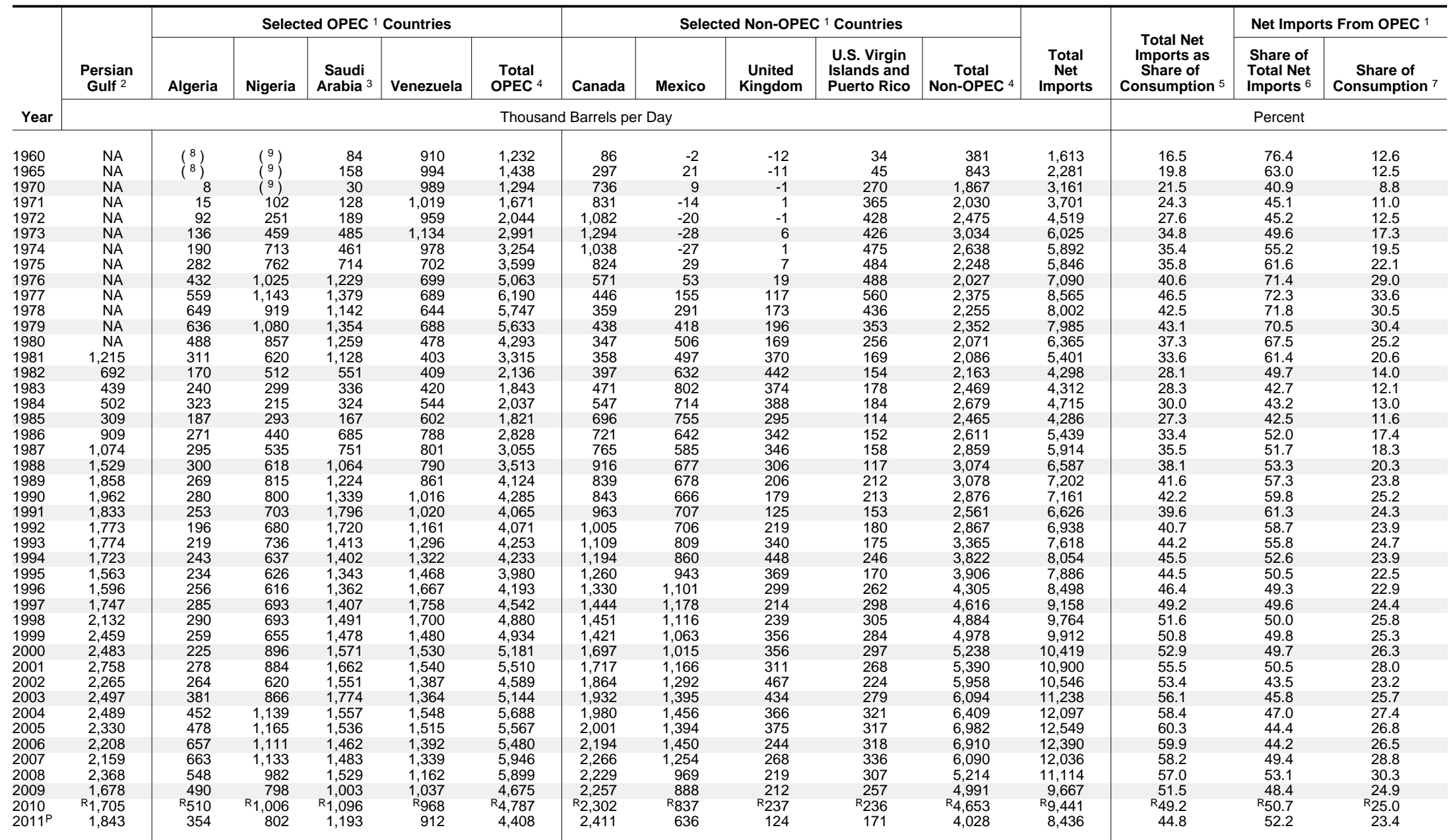

1 See "Organization of the Petroleum Exporting Countries (OPEC)" in Glossary.
2 Bahrain, Iran, Iraq, Kuwait, Qatar, Saudi Arabia, United Arab Emirates, and the Neutral Zone (between Kuwait and Saudi Arabia).

Through 1970, includes half the imports from the Neutral Zone. Beginning in 1971, includes imports from the Neutral Zone that are reported to U.S. Customs as originating in Saudi Arabia.

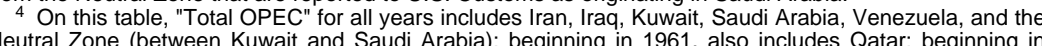
also includes Libya; for 1962-2008, also includes Indonesia: beginning in 1967, also includes Unitod Arab Emirates; beginning in 1969, also includes Algeria; beginning in 1971, also includes Nigeria; for 1973-1992 and beginning in 2008, also includes Ecuador (although Ecuador rejoined OPEC in November ( are included in "Total Non-OPEC."

(consumption).

Calculated by dividing net petroleum imports from OPEC countries by total net petroleum imports.

Calculated by dividing net petroleum imports from OPEC countries by total US petroleum product

supplied (consumption)

8 Algeria joined OPEC in 1969. For 1960-1968, Algeria is included in "Total Non-OPEC." 9 Nigeria joined OPEC in 1971 . For $1960-1970, N$
$R=$ Revised. P=Preliminary. NA $=$ Not available.

Notes: - The country of origin for refined petroleum products may not be the country of origin for the crude oil from which the refined products were produced. For example, refined products imported from
refineries in the Caribbean may have been produced from Middle East crude oil. for the Strategic Petroleum Reserve, which began in 1977. - Totals may not equal sum of components due to independent rounding.

1960. 960. - For related information, see http://www.eia.gov/petroleum/.

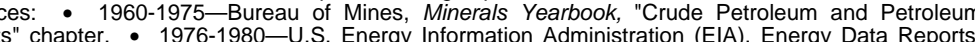
P.A.D. Districts Supply/Demand, Annual, annual reports. • 1981-2010-EIA, Petroleum Supply Annual, annual reports. - 2011-EIA, Petroleum Supply Monthly (February 2012) 
Figure 5.8 Refinery and Blender Net Inputs and Net Production, 1949-2011

\section{Refinery and Blender Net Inputs}

$16-$

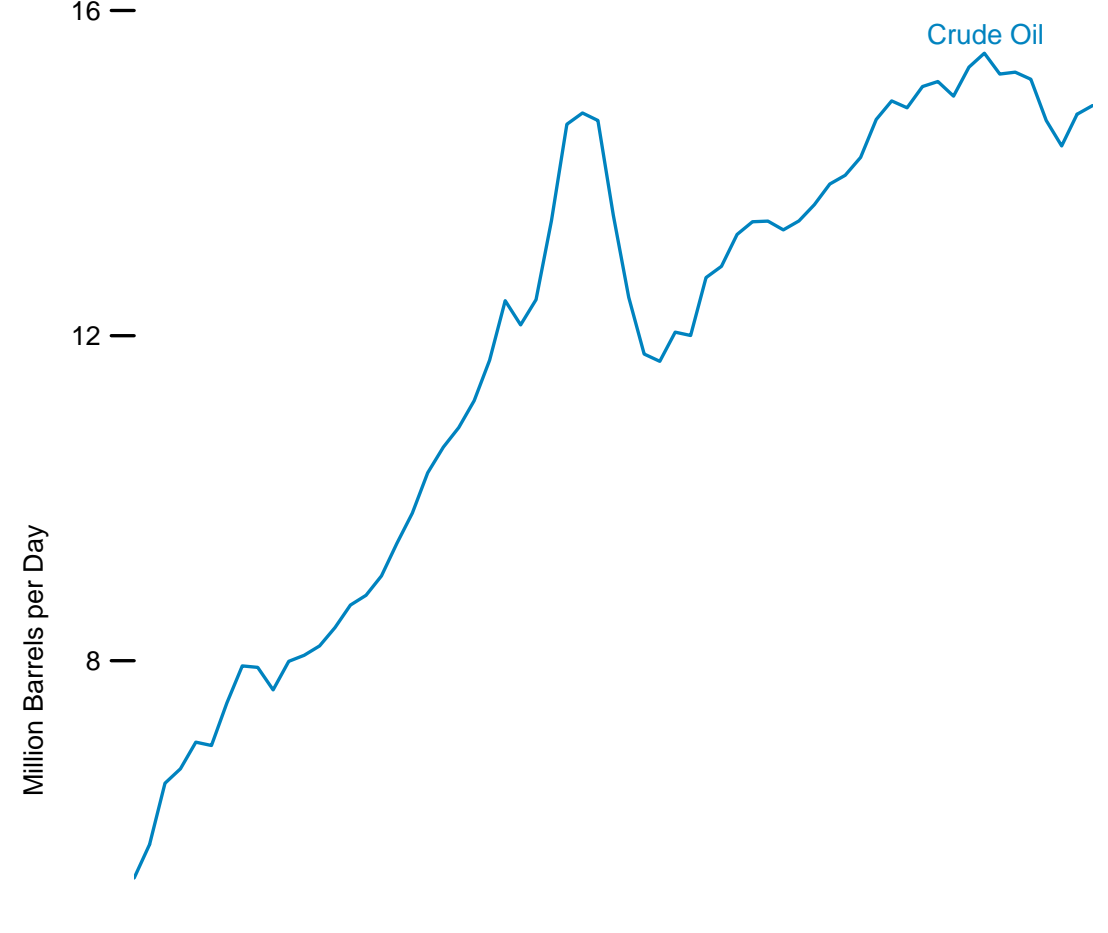

4-

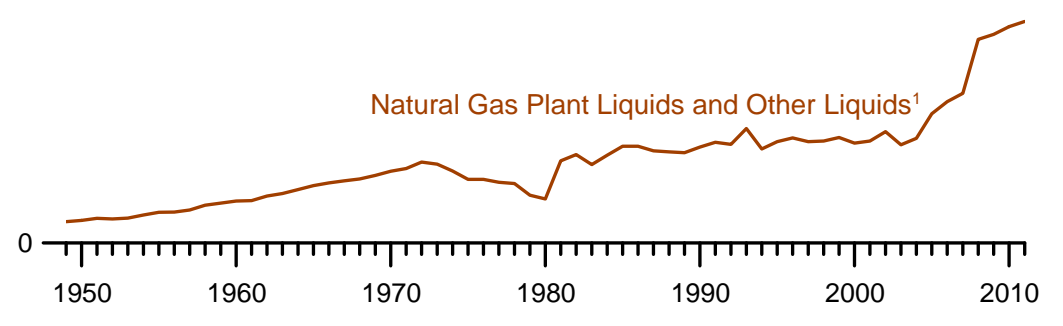

Refinery and Blender Net Production of Selected Products

$16-$

$12-$

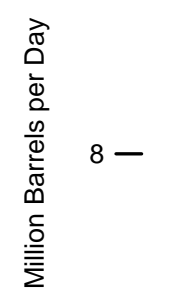

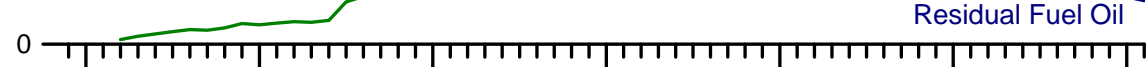

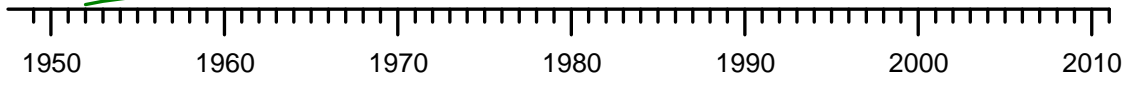


Table 5.8 Refinery and Blender Net Inputs and Net Production, Selected Years, 1949-2011

(Thousand Barrels per Day)

\begin{tabular}{|c|c|c|c|c|c|c|c|c|c|c|c|c|c|c|c|}
\hline \multirow[b]{2}{*}{ Year } & \multicolumn{4}{|c|}{ Refinery and Blender Net Inputs 1} & \multicolumn{10}{|c|}{ Refinery and Blender Net Production ${ }^{2}$} & \multirow[b]{2}{*}{$\begin{array}{c}\text { Processing } \\
\text { Gain }\end{array}$} \\
\hline & $\begin{array}{c}\text { Crude } \\
\text { Oil }^{3}\end{array}$ & $\begin{array}{l}\text { Natural Gas } \\
\text { Plant } \\
\text { Liquids }\end{array}$ & $\begin{array}{c}\text { Other } \\
\text { Liquids }{ }^{4}\end{array}$ & Total & $\begin{array}{l}\text { Asphalt } \\
\text { and } \\
\text { Road Oil }\end{array}$ & $\begin{array}{l}\text { Distillate } \\
\text { Fuel Oil }\end{array}$ & $\begin{array}{l}\text { Jet } \\
\text { Fuel }^{5}\end{array}$ & $\begin{array}{l}\text { Liquefied } \\
\text { Petroleum } \\
\text { Gases }\end{array}$ & $\begin{array}{c}\text { Motor } \\
\text { Gasoline }\end{array}$ & $\begin{array}{c}\text { Petroleum } \\
\text { Coke }\end{array}$ & $\begin{array}{c}\text { Residual } \\
\text { Fuel Oil }\end{array}$ & $\begin{array}{l}\text { Still } \\
\text { Gas }\end{array}$ & $\begin{array}{c}\text { Other } \\
\text { Products }{ }^{7}\end{array}$ & Total & \\
\hline 1949 & 5,327 & 234 & 28 & 5,588 & 155 & 934 & $\left({ }^{5}\right)$ & 64 & 2,572 & 46 & 1,164 & 226 & 425 & 5,587 & -2 \\
\hline 1950 & 5,739 & 259 & 19 & 6,018 & 179 & 1,093 & $\left(5^{\prime}\right)$ & 80 & 2,735 & 47 & 1,165 & 229 & 492 & 6,019 & 2 \\
\hline 1955 & 7,480 & 345 & 32 & 7,857 & 251 & 1,651 & 155 & 119 & 3,648 & 78 & 1,152 & 319 & 518 & 7,891 & 34 \\
\hline 1960 & 8,067 & 455 & 61 & 8,583 & 286 & 1,823 & 241 & 212 & 4,126 & 164 & 908 & 354 & 616 & 8,729 & 146 \\
\hline 1965 & 9,043 & 618 & 88 & 9,750 & 357 & 2,096 & 523 & 293 & 4,507 & 236 & 736 & 395 & 827 & 9,970 & 220 \\
\hline 1970 & 10,870 & 763 & 121 & 11,754 & 428 & 2,454 & 827 & 345 & 5,699 & 296 & 706 & 483 & 876 & 12,113 & 359 \\
\hline 1975 & 12,442 & 710 & 72 & 13,225 & 408 & 2,653 & 871 & 311 & 6,518 & 354 & 1,235 & 523 & 811 & 13,685 & 460 \\
\hline 1976 & 13,416 & 725 & 59 & 14,200 & 391 & 2,924 & 918 & 340 & 6,838 & 356 & 1,377 & 541 & 993 & 14,677 & 477 \\
\hline 1977 & 14,602 & 673 & 74 & 15,349 & 431 & 3,277 & 973 & 352 & 7,031 & 369 & 1,754 & 572 & 1,114 & 15,874 & 524 \\
\hline 1978 & $\begin{array}{r}14,002 \\
14,739\end{array}$ & 639 & $\begin{array}{l}14 \\
92\end{array}$ & 15,470 & 482 & $\begin{array}{l}3,167 \\
3,16\end{array}$ & 970 & 355 & $\begin{array}{l}7,167 \\
7,167\end{array}$ & 369 & $\begin{array}{l}1,667 \\
1,67\end{array}$ & 603 & $\begin{array}{l}1,186 \\
1\end{array}$ & $\begin{array}{r}15,966 \\
\end{array}$ & $\begin{array}{l}496 \\
496\end{array}$ \\
\hline 1979 & 14,648 & 510 & 78 & 15,236 & 467 & $\begin{array}{l}3,152 \\
3,152\end{array}$ & $\begin{array}{r}1,012 \\
\end{array}$ & 340 & 6,837 & 376 & 1,687 & 598 & 1,296 & 15,763 & 527 \\
\hline 1980 & 13,481 & 462 & 81 & 14,025 & 393 & 2,661 & 999 & 330 & 6,492 & 370 & 1,580 & 581 & 1,215 & 14,622 & 597 \\
\hline 1981 & 12,470 & 524 & 488 & 13,482 & 340 & 2,613 & 968 & 315 & 6,400 & 390 & 1,321 & 565 & 1,078 & 13,990 & 508 \\
\hline 1982 & 11,774 & 515 & 572 & 12,861 & 329 & 2,606 & 978 & 270 & 6,336 & 410 & 1,070 & 554 & 839 & 13,391 & 531 \\
\hline 1983 & 11,685 & 460 & 505 & 12,650 & 372 & 2,456 & $\begin{array}{r}1,022 \\
\end{array}$ & 328 & 6,338 & 420 & 852 & 550 & 801 & 13,138 & 488 \\
\hline 1984 & 12,044 & 500 & 581 & 13,126 & 386 & 2,680 & 1,132 & 363 & 6,453 & 439 & 891 & 559 & 776 & 13,679 & 553 \\
\hline 1985 & 12,002 & 509 & 681 & 13,192 & 401 & 2,686 & $\begin{array}{l}1,189 \\
1\end{array}$ & 391 & 6,419 & 455 & 882 & 584 & 743 & 13,750 & 557 \\
\hline 1986 & 12,716 & 479 & 711 & 13,906 & 410 & 2,796 & 1,293 & 417 & 6,752 & 506 & 889 & 641 & 818 & 14,522 & 616 \\
\hline 1987 & 12,854 & 466 & 667 & 13,987 & 434 & 2,729 & 1,343 & 449 & 6,841 & 512 & 885 & 643 & 791 & 14,626 & 639 \\
\hline 1988 & 13,246 & 511 & 610 & 14,367 & 443 & 2,857 & 1,370 & 499 & 6,956 & 544 & 926 & 670 & 758 & 15,022 & 655 \\
\hline 1989 & 13,401 & 499 & 613 & 14,513 & 424 & $\begin{array}{l}2,051 \\
2,899\end{array}$ & 1,403 & 554 & $\begin{array}{l}6,950 \\
6,963\end{array}$ & 542 & 954 & 681 & 755 & 15,175 & 661 \\
\hline 1990 & 13,409 & 467 & 713 & 14,589 & 449 & 2,925 & 1,488 & 499 & 6,959 & 552 & 950 & 673 & 778 & 15,272 & 683 \\
\hline 1991 & 13,301 & 472 & 768 & 14,541 & 430 & 2,962 & 1,438 & 536 & 6,975 & 568 & 934 & 651 & 761 & 15,256 & 715 \\
\hline 1992 & 13,411 & 469 & 745 & 14,626 & 419 & 2,974 & 1,399 & 607 & 7,058 & 596 & 892 & 659 & 796 & 15,398 & 772 \\
\hline 1993 & 13,613 & 491 & 917 & 15,021 & 451 & 3,132 & 1,422 & 592 & 7,304 & 619 & 835 & 653 & 780 & 15,787 & 766 \\
\hline 1994 & 13,866 & 465 & 691 & 15,023 & 451 & $\begin{array}{l}3,102 \\
3,205\end{array}$ & $\begin{array}{l}1,448 \\
1,42<\end{array}$ & 611 & $\begin{array}{l}7,004 \\
7,181\end{array}$ & 622 & 826 & 657 & 790 & 15,791 & 768 \\
\hline 1995 & 13,973 & 471 & 775 & 15,220 & 467 & 3,155 & 1,416 & 654 & 7,459 & 630 & 788 & 647 & 778 & 15,994 & 774 \\
\hline 1996 & 14,195 & 450 & 843 & 15,487 & 459 & 3,316 & 1,515 & 662 & 7,565 & 664 & 726 & 654 & 764 & 16,324 & 837 \\
\hline 1997 & 14,662 & 416 & 832 & 15,909 & 485 & 3,392 & 1,554 & 691 & 7,743 & 689 & 708 & 661 & 836 & 16,759 & 850 \\
\hline 1998 & 14,889 & 403 & 853 & 16,144 & 498 & 3,424 & 1,526 & 674 & $\begin{array}{l}7,892 \\
\text {. }\end{array}$ & 712 & 762 & 656 & 886 & 17,030 & 886 \\
\hline $\begin{array}{l}1999 \\
1999\end{array}$ & 14,804 & 372 & 927 & $\begin{array}{l}16,103 \\
\end{array}$ & 505 & $\begin{array}{l}\text {, } 3,394 \\
3,399\end{array}$ & 1,565 & 684 & $\begin{array}{l}7,056 \\
7,934\end{array}$ & 713 & 698 & 656 & 835 & $\begin{array}{l}16,989 \\
16,99\end{array}$ & 886 \\
\hline 2000 & 15,067 & 380 & 849 & 16,295 & 525 & 3,580 & 1,606 & 705 & 7,951 & 727 & 696 & 659 & 793 & 17,243 & 948 \\
\hline 2001 & 15,128 & 429 & 825 & 16,382 & 485 & 3,695 & 1,530 & 667 & 8,022 & 767 & 721 & 670 & 729 & 17,285 & 903 \\
\hline 2002 & 14,947 & 429 & 941 & 16,316 & 492 & 3,592 & 1,514 & 671 & 8,183 & 781 & 601 & 667 & 771 & 17,273 & 957 \\
\hline 2003 & 15,304 & 419 & 791 & 16,513 & 496 & 3,707 & 1,488 & 658 & 8,194 & 798 & 660 & 702 & 784 & 17,487 & 974 \\
\hline 2004 & 15,475 & 422 & 866 & 16,762 & 508 & 3,814 & 1,547 & 645 & 8,265 & 836 & 655 & 704 & 838 & 17,814 & $\begin{array}{r}1,051 \\
\end{array}$ \\
\hline 2005 & 15,220 & 441 & $\begin{array}{r}000 \\
1,149\end{array}$ & $\begin{array}{l}10,02 \\
16,811\end{array}$ & 512 & $\begin{array}{l}3,014 \\
3,954\end{array}$ & $\begin{array}{l}1,541 \\
1,546\end{array}$ & 573 & $\begin{array}{l}\text {, 2005 } \\
8,318\end{array}$ & 835 & 628 & 684 & $\begin{array}{l}000 \\
752\end{array}$ & 17,800 & $\begin{array}{r}1,051 \\
989\end{array}$ \\
\hline 2006 & 15,242 & 501 & $\begin{array}{l}1,238 \\
1,238\end{array}$ & $\begin{array}{l}16,981 \\
\text { 16, }\end{array}$ & 506 & $\begin{array}{l}4,934 \\
4,040\end{array}$ & $\begin{array}{r}1,040 \\
1,481\end{array}$ & 627 & $\begin{array}{l}8,364 \\
8,364\end{array}$ & 848 & 635 & $\begin{array}{l}004 \\
709\end{array}$ & 764 & 17,975 & 994 \\
\hline 2007 & 15,156 & 505 & $\begin{array}{l}1,337 \\
1,337\end{array}$ & $\begin{array}{l}16,999 \\
1699\end{array}$ & 456 & $\begin{array}{l}4,133 \\
4,133\end{array}$ & 1,448 & 655 & $\begin{array}{l}8,004 \\
8,358\end{array}$ & 823 & 673 & 697 & 752 & $\begin{array}{l}17,994 \\
\end{array}$ & 996 \\
\hline 2008 & 14,648 & 485 & 2,019 & 17,153 & 410 & 4,294 & 1,493 & 630 & 8,548 & 818 & 620 & 670 & 664 & 18,146 & 993 \\
\hline 2009 & 14,336 & 485 & 2,082 & 16,904 & 359 & 4.048 & 1,396 & 623 & 8.786 & 799 & 598 & 664 & 608 & 17,882 & 979 \\
\hline 2010 & $\begin{array}{l}\mathrm{R}_{14,720}\end{array}$ & 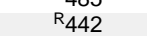 & $\begin{array}{l}R_{2,219}\end{array}$ & $\mathrm{R}_{17,385}$ & 378 & $\begin{array}{r}4,040 \\
R 4,223\end{array}$ & $\begin{array}{l}1,418 \\
1,490\end{array}$ & 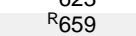 & $\begin{array}{r}\text { Rg, , } 059 \\
\text { ? }\end{array}$ & 812 & $\begin{array}{r}550 \\
\text { R585 }\end{array}$ & $\begin{array}{r}004 \\
R_{6} 672\end{array}$ & 647 & $\begin{array}{l}\mathrm{R} 18,452 \\
\mathrm{R}\end{array}$ & $R_{1,068}$ \\
\hline $2011^{\mathrm{P}}$ & 14,833 & 489 & 2,237 & 17,559 & 364 & 4,487 & 1,449 & 620 & 9,035 & 842 & 538 & 678 & 631 & 18,643 & 1,085 \\
\hline
\end{tabular}

1 See "Refinery and Blender Net Inputs" in Glossary.

See "Refinery and Blender Net Production" in Glossary.

(n)

4 Unfinished oils (net), other hydrocarbons, and hydrogen. Beginning in 1981, also includes aviation and motor gasoline blending components (net). Beginning in 1993, also includes oxygenates (net).

percent gasoline, 17 percent kerosene, and 12 percent distillate fuel oil. Through 1964, kerosene-type fuel is included with kerosene in "Other Products." Beginning in 2005, naphtha-type jet fuel is included in "Other Products."

Finished motor gasoline. Through 1963, also includes aviation gasoline and special naphthas. Beginning in 1993, also includes ethanol blended into motor gasolin

also includes kerosene-type jet fuel. Beginning in 1964, also includes aviation gasoline and special naphthas. Beginning in 2005 ,
R=Revised. P=Preliminary.

Note: Totals may not equal sum of components due to independent rounding

Web Pages: - See http://www.eia.gov/totalenergy/data/monthly/\#petroleum for updated monthly and annual data. - See http://www.eia.gov/totalenergy/data/annual/\#petroleum for all annual data beginning in 949. - See http://www.eia.gov/petroleum/ for related information.

Sources: - 1949-1975-Bureau of Mines, Mineral Industry Surveys, Petroleum Statement, Annual, annual reports. - 1976-1980-U.S. Energy Information Administration (EIA), Energy Data Reports, Petroleum Statement, Annual, annual reports. • 1981-2010-EIA, Petroleum Supply Annual, annual
reports. • 2011-EIA, Petroleum Supply Monthly (February 2012). 


\section{Figure 5.9 Refinery Capacity and Utilization, 1949-2011}

\section{Number of Operable Refineries}

$400-$

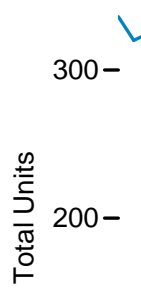

$100-$

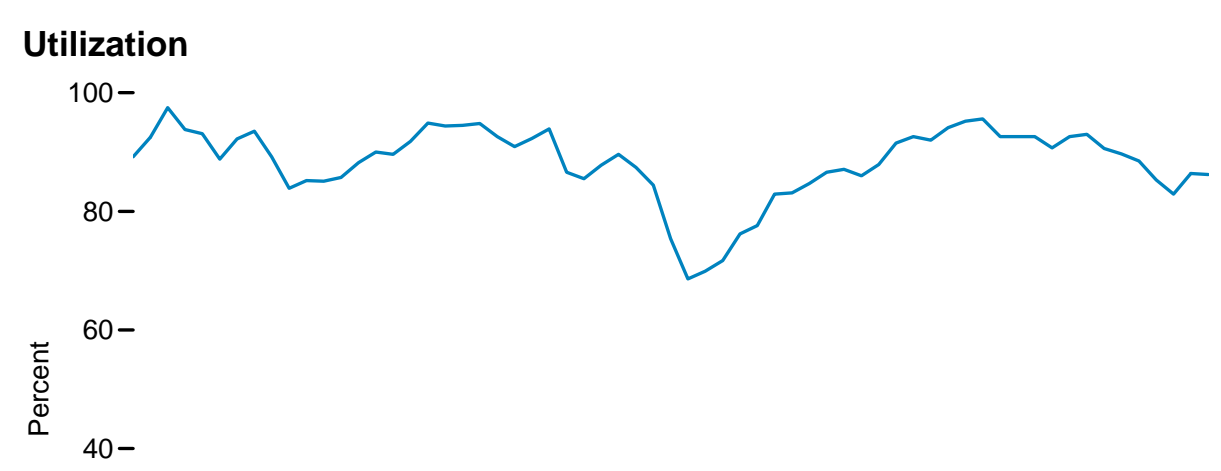

$20-$

\begin{tabular}{|c|c|c|c|c|c|c|}
\hline ידןד & Tा & 11 & 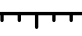 & rT & Tיד & Tाי \\
\hline 1950 & 1960 & 1970 & 1980 & 1990 & 2000 & 0010 \\
\hline
\end{tabular}

\section{Capacity}

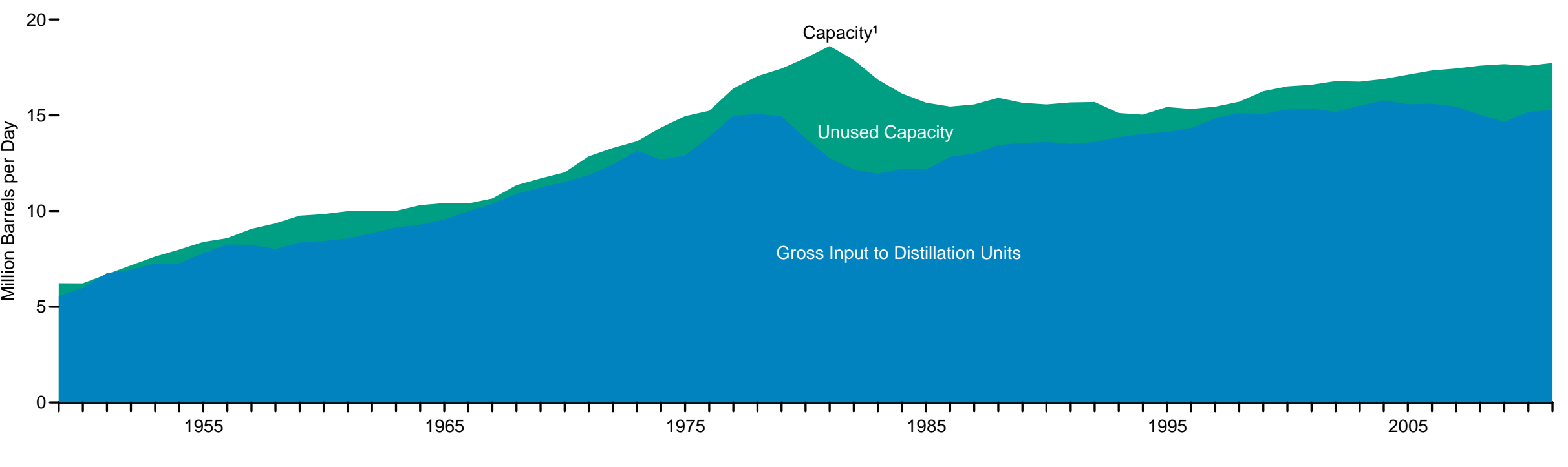


Table 5.9 Refinery Capacity and Utilization, Selected Years, 1949-2011

\begin{tabular}{|c|c|c|c|c|c|}
\hline \multirow[b]{3}{*}{ Year } & \multirow{2}{*}{$\begin{array}{c}\text { Operable } \\
\text { Refineries }{ }^{1}\end{array}$} & \multicolumn{2}{|c|}{ Operable Refineries Capacity } & \multirow{2}{*}{$\begin{array}{c}\text { Gross Input } \\
\text { to Distillation Units }\end{array}$} & \multirow[b]{2}{*}{ Utilization ${ }^{4}$} \\
\hline & & On January 1 & Annual Average 2 & & \\
\hline & Number & \multicolumn{2}{|c|}{ Thousand Barrels per Calendar Day } & Thousand Barrels per Day & Percent \\
\hline $\begin{array}{l}1949 \\
1950\end{array}$ & $\begin{array}{l}336 \\
320\end{array}$ & $\begin{array}{l}6,231 \\
6,223\end{array}$ & $\begin{array}{l}\text { NA } \\
\text { NA }\end{array}$ & $\begin{array}{l}5,556 \\
5,980\end{array}$ & $\begin{array}{l}89.2 \\
92.5\end{array}$ \\
\hline 1955 & 296 & 8,386 & NA & 7,820 & 92.2 \\
\hline 1960 & 309 & 9,843 & NA & 8,439 & 85.1 \\
\hline 1965 & 293 & 10,420 & NA & 9,557 & 91.8 \\
\hline 1970 & 276 & 12,021 & NA & 11,517 & 92.6 \\
\hline 1975 & 279 & 14,961 & NA & 12,902 & 85.5 \\
\hline 1976 & 276 & 15,237 & NA & 13,884 & 87.8 \\
\hline 1977 & 282 & 16,398 & NA & 14,982 & 89.6 \\
\hline 1978 & 296 & 17,048 & NA & 15,071 & 87.4 \\
\hline 1979 & 308 & 17,441 & NA & 14,955 & 84.4 \\
\hline 1980 & 319 & 17,988 & NA & 13,796 & 75.4 \\
\hline 1981 & 324 & 18,621 & 18,603 & 12,752 & 68.6 \\
\hline 1982 & 301 & 17,890 & 17,432 & 12,172 & 69.9 \\
\hline 1983 & 258 & 16,859 & 16,668 & 11,947 & 71.7 \\
\hline 1984 & 247 & 16,137 & 16,035 & 12,216 & 76.2 \\
\hline 1985 & 223 & 15,659 & 15,671 & 12,165 & 77.6 \\
\hline 1986 & 216 & 15,459 & 15,459 & 12,826 & 82.9 \\
\hline 1987 & 219 & 15,566 & 15,642 & 13,003 & 83.1 \\
\hline 1988 & 213 & 15,915 & 15,927 & 13,447 & R84.4 \\
\hline 1989 & 204 & 15,655 & 15,701 & 13,551 & R86.3 \\
\hline 1990 & 205 & 15,572 & 15,623 & 13,610 & 87.1 \\
\hline 1991 & 202 & 15,676 & 15,707 & 13,508 & 86.0 \\
\hline 1992 & 199 & 15,696 & 15,460 & 13,600 & 87.9 \\
\hline 1993 & 187 & 15,121 & 15,143 & 13,851 & 91.5 \\
\hline 1994 & 179 & 15,034 & 15,150 & 14,032 & 92.6 \\
\hline 1995 & 175 & 15,434 & 15,346 & 14,119 & 92.0 \\
\hline 1996 & 170 & 15,333 & 15,239 & 14,337 & 94.1 \\
\hline 1997 & 164 & 15,452 & 15,594 & 14,838 & 95.2 \\
\hline 1998 & 163 & 15,711 & 15,802 & 15,113 & 95.6 \\
\hline 1999 & 159 & 16,261 & 16,282 & 15,080 & 92.6 \\
\hline 2000 & 158 & 16,512 & 16,525 & 15,299 & 92.6 \\
\hline 2001 & 155 & 16,595 & 16,582 & 15,352 & 92.6 \\
\hline 2002 & 153 & 16,785 & 16,744 & 15,180 & 90.7 \\
\hline 2003 & 149 & 16,757 & 16,748 & 15,508 & 92.6 \\
\hline 2004 & 149 & 16,894 & 16,974 & 15,783 & 93.0 \\
\hline 2005 & 148 & 17,125 & 17,196 & 15,578 & 90.6 \\
\hline 2006 & 149 & 17,339 & 17,385 & 15,602 & 89.7 \\
\hline 2007 & 149 & 17,443 & 17,450 & 15,450 & 88.5 \\
\hline 2008 & 150 & 17,594 & 17,607 & 15,027 & 85.3 \\
\hline 2009 & 150 & 17,672 & 17,678 & 14,659 & 82.9 \\
\hline 2010 & 148 & 17,584 & $R_{17,575}$ & $\mathrm{R}_{15,177}$ & R86.4 \\
\hline $2011^{\mathrm{P}}$ & 148 & 17,736 & 17,726 & 15,283 & 86.2 \\
\hline
\end{tabular}

1 Through 1956, includes only those refineries in operation on January 1 ; beginning in 1957, includes all "operable" refineries on January 1 . See "Operable Refineries" in Glossary.

Average of monthly capacity data.

See Note 3, "Gross Input to Distillation Units," at end of section.

Through 1980, utilization is calculated by dividing gross input to distillation units by one-half of the sum of the current year's January 1 capacity and the following year's January 1 capacity. Beginning in 1981 (

Web Pages: - See http://www.eia.gov/totalenergy/data/annual/\#petroleum for all data beginning in
1949. • For related information, see http://www.eia.gov/petroleum/

Sources: - 1949-1977-Bureau of Mines, Information Circular, "Petroleum Refineries, Including Cracking Plants in the United States"; Minerals Yearbook, "Crude Petroleum and Petroleum Products" and "Natural Gas Liquids" chapters, and Mineral Industry Surveys, Petroleum Refineries, Annual, annual Refine. 1 in 1 . Refineries in the United States, annual reports. - 1981-2005-EIA, Petroleum Supply Annual (PSA) annul reports, and Form EIA 810 " "Monthy Refine

1; and Petroleum Supply Monthly (January-December 2011 issues), Table 30. 
Figure 5.10 Natural Gas Plant Liquids Production

\section{Total, 1949-2011}

2,500-

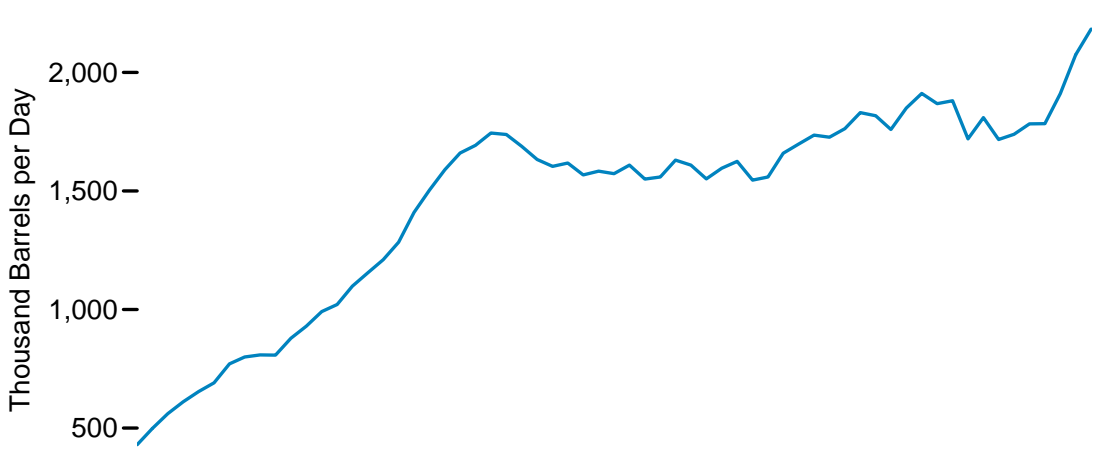

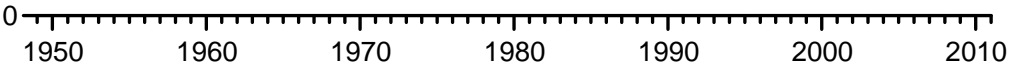

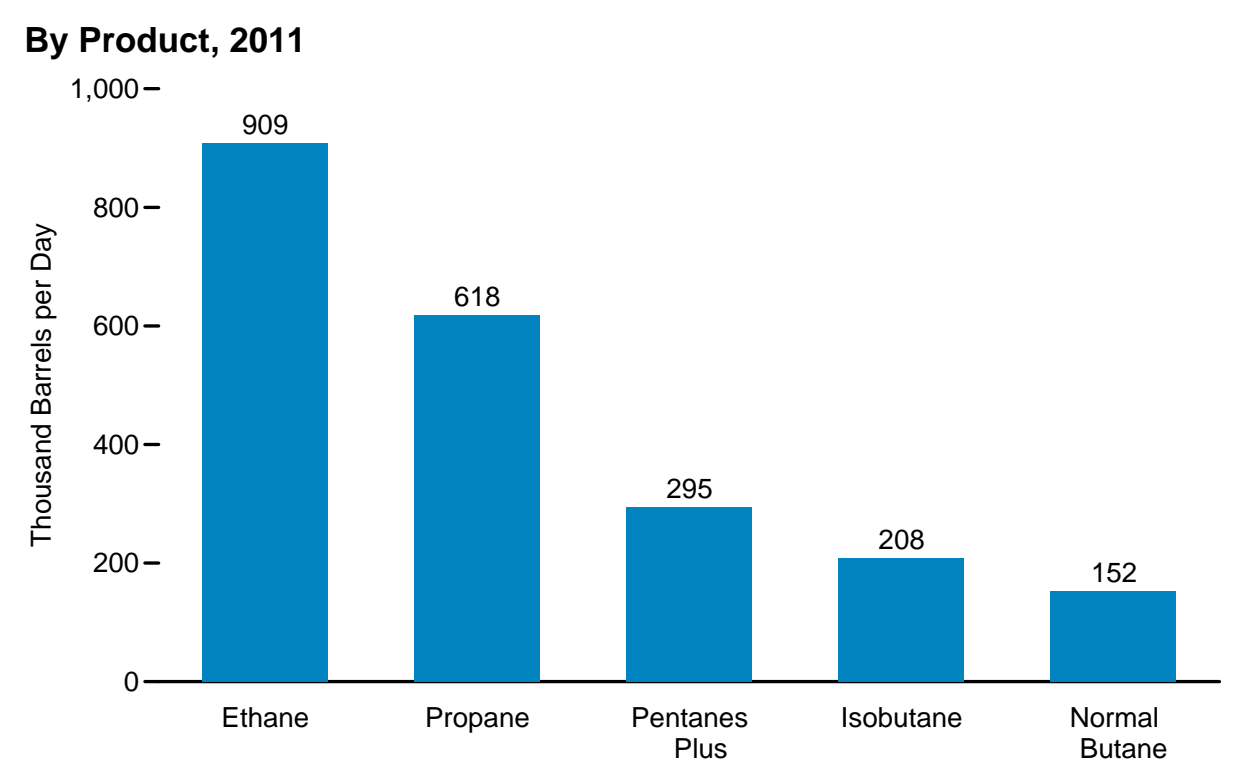

\section{By Selected Product, 1949-2011}

$1,200-$

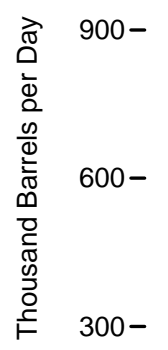

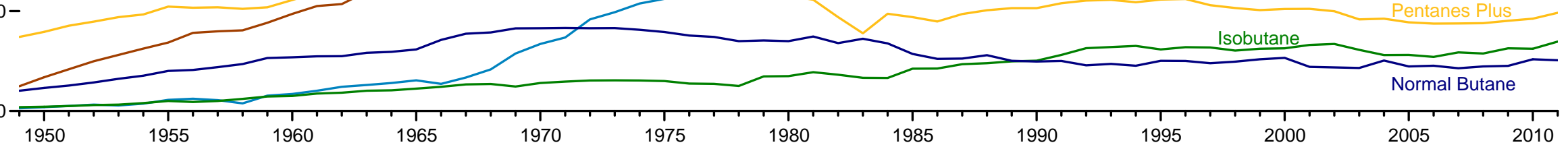

Source: Table 5.10. 
Table 5.10 Natural Gas Plant Liquids Production, Selected Years, 1949-2011

(Thousand Barrels per Day)

\begin{tabular}{|c|c|c|c|c|c|c|c|c|}
\hline \multirow[b]{2}{*}{ Year } & \multirow{2}{*}{$\begin{array}{l}\text { Finished } \\
\text { Petroleum } \\
\text { Products }{ }^{1}\end{array}$} & \multicolumn{5}{|c|}{ Liquefied Petroleum Gases } & \multirow[b]{2}{*}{$\begin{array}{l}\text { Pentanes } \\
\text { Plus }{ }^{4}\end{array}$} & \multirow[b]{2}{*}{ Total } \\
\hline & & Ethane $^{2}$ & Isobutane & $\begin{array}{l}\text { Normal } \\
\text { Butane }^{3}\end{array}$ & Propane 2,3 & Total & & \\
\hline 1949 & 53 & 8 & 11 & 61 & 74 & 155 & 223 & 430 \\
\hline 1950 & 66 & 12 & 13 & 69 & 101 & 195 & 238 & 499 \\
\hline 1955 & 68 & 34 & 30 & 120 & 205 & 390 & 313 & 771 \\
\hline 1960 & 47 & 51 & 45 & 161 & 291 & 549 & 333 & 929 \\
\hline 1965 & 41 & 92 & 67 & 185 & 390 & 734 & 434 & 1,210 \\
\hline 1970 & 25 & 201 & 84 & 248 & 561 & 1,095 & 540 & 1,660 \\
\hline 1975 & 7 & 337 & 90 & 237 & 552 & 1,217 & 409 & 1,633 \\
\hline 1976 & 6 & 365 & 82 & 227 & 521 & 1,195 & 403 & 1,604 \\
\hline 1977 & 5 & 397 & 81 & 223 & 513 & 1,214 & 399 & 1,618 \\
\hline 1978 & 3 & 406 & 75 & 210 & 491 & 1,182 & 382 & 1,567 \\
\hline 1979 & 26 & 400 & 104 & 212 & 500 & 1,216 & 342 & 1,584 \\
\hline 1980 & 23 & 396 & 105 & 210 & 494 & 1,205 & 345 & 1,573 \\
\hline 1981 & 18 & 397 & 117 & 224 & 519 & 1,256 & 334 & 1,609 \\
\hline 1982 & 11 & 426 & 109 & 204 & 519 & 1,258 & 282 & 1,550 \\
\hline 1983 & 12 & 456 & 100 & 217 & 541 & 1,314 & 233 & 1,559 \\
\hline 1984 & 4 & 505 & 99 & 203 & 527 & 1,334 & 292 & 1,630 \\
\hline 1985 & 14 & 493 & 127 & 171 & 521 & 1,313 & 282 & 1,609 \\
\hline 1986 & 4 & 485 & 128 & 157 & 508 & 1,277 & 269 & 1,551 \\
\hline 1987 & 4 & 499 & 141 & 157 & 503 & 1,300 & 291 & 1,595 \\
\hline 1988 & 4 & 501 & 144 & 167 & 506 & 1,319 & 302 & 1,625 \\
\hline 1989 & NA & 466 & 149 & 151 & 471 & 1,237 & 309 & 1,546 \\
\hline 1990 & NA & 477 & 151 & 149 & 474 & 1,250 & 309 & 1,559 \\
\hline 1991 & NA & 530 & 169 & 150 & 487 & 1,336 & 324 & 1,659 \\
\hline 1992 & NA & 541 & 189 & 137 & 499 & 1,365 & 332 & 1,697 \\
\hline 1993 & NA & 556 & 192 & 142 & 513 & 1,402 & 334 & 1,736 \\
\hline 1994 & NA & 559 & 195 & 136 & 510 & 1,400 & 326 & 1,727 \\
\hline 1995 & NA & 573 & 185 & 151 & 519 & 1,428 & 335 & 1,762 \\
\hline 1996 & NA & 627 & 192 & 150 & 525 & 1,494 & 336 & 1,830 \\
\hline 1997 & NA & 637 & 191 & 144 & 528 & 1,499 & 318 & 1,817 \\
\hline 1998 & NA & 607 & 181 & 148 & 513 & 1,450 & 309 & 1,759 \\
\hline 1999 & NA & 675 & 187 & 155 & 529 & 1,547 & 303 & 1,850 \\
\hline 2000 & NA & 717 & 188 & 160 & 539 & 1,605 & 306 & 1,911 \\
\hline 2001 & NA & 692 & 198 & 133 & 538 & 1,562 & 307 & 1,868 \\
\hline 2002 & NA & 700 & 201 & 131 & 549 & 1,581 & 300 & 1,880 \\
\hline 2003 & NA & 625 & 183 & 129 & 506 & 1,444 & 275 & 1,719 \\
\hline 2004 & NA & 686 & 168 & 152 & 526 & 1,532 & 277 & 1,809 \\
\hline 2005 & NA & 649 & 168 & 134 & 499 & 1,451 & 266 & 1,717 \\
\hline 2006 & NA & 676 & 163 & 136 & 501 & 1,476 & 263 & 1,739 \\
\hline 2007 & NA & 709 & 176 & 128 & 507 & 1,520 & 263 & 1,783 \\
\hline 2008 & NA & 701 & 173 & 134 & 512 & 1,520 & 264 & 1,784 \\
\hline 2009 & NA & 769 & 188 & 136 & 546 & 1,639 & 271 & 1,910 \\
\hline 2010 & NA & R869 & $\mathrm{R}_{187}$ & $\mathrm{R}_{155}$ & R586 & $R_{1}, 797$ & R277 & $\mathrm{R}_{2,074}$ \\
\hline $2011^{P}$ & NA & 909 & 208 & 152 & 618 & 1,888 & 295 & 2,183 \\
\hline
\end{tabular}

1 Motor gasoline, aviation gasoline, special naphthas, distillate fuel oil, and miscellaneous products.

2 Reported production of ethane-propane mixtures has been allocated 70 percent ethane and 30 percent propane.

Reported production of butane-propane mixtures has been allocated 60 percent butane and 40 percent propane.

Through 1983, "Pentanes Plus" was reported separately as natural gasoline, isopentane, and plant

R=Revised. P=Preliminary. NA=Not available.

Note: Totals may not equal sum of components due to independent rounding

Web Pages: - See http://www.eia.gov/totalenergy/data/annual/\#petroleum for all data beginning in Sourcs:

(Crude Petroleum and Petroleum Annul Anual, annual reports. • 1976-1980-U.S. Energy Information Ad Suvitration (EIA), Energy Data

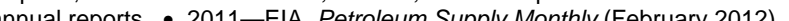




\section{Figure 5.11 Petroleum Products Supplied by Type}

\section{By Selected Product, 1949-2011}

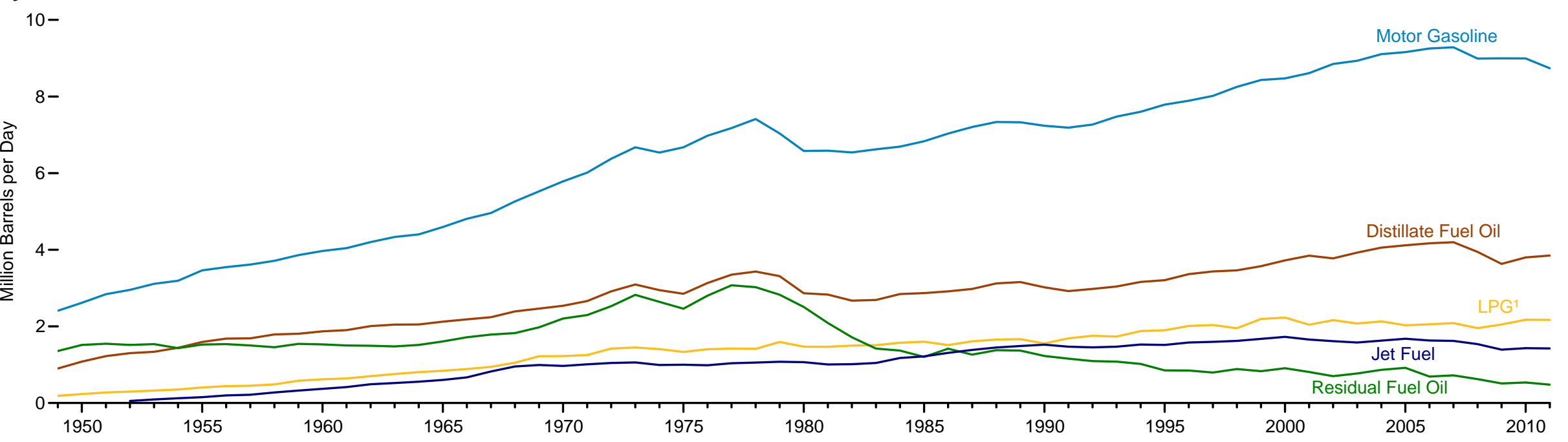

\section{By Product, 2011}

$10-$

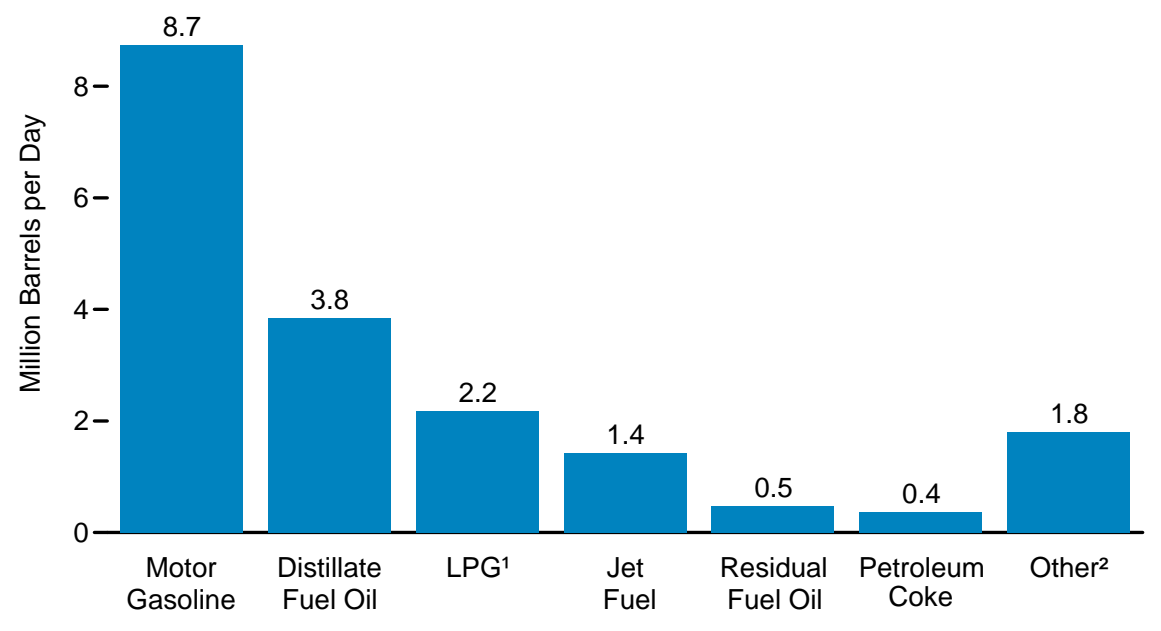

${ }^{1}$ Liquefied petroleum gases.

${ }^{2}$ Asphalt and road oil, aviation gasoline, kerosene, lubricants, naphtha-type jet fuel, pentanes plus, petrochemical feedstocks, special naphthas, still gas (refinery gas), waxes, miscellaneous products, and crude oil burned as fuel.

\section{Motor Gasoline's Share of Total Petroleum Products Supplied,} 1949-2011

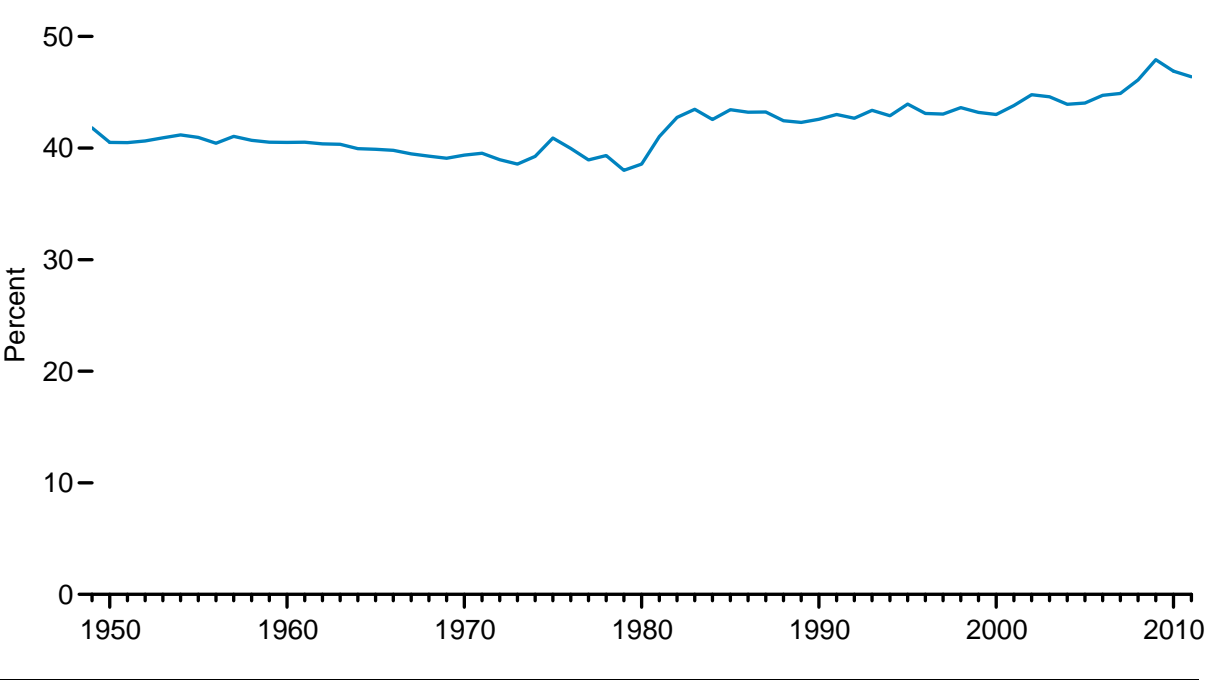

Source: 5.11 . 
Table 5.11 Petroleum Products Supplied by Type, Selected Years, 1949-2011

(Thousand Barrels per Day)

\begin{tabular}{|c|c|c|c|c|c|c|c|c|c|c|c|c|c|c|}
\hline \multirow[b]{2}{*}{ Year } & \multirow[b]{2}{*}{$\begin{array}{c}\text { Asphalt and } \\
\text { Road Oil }\end{array}$} & \multirow[b]{2}{*}{$\begin{array}{l}\text { Aviation } \\
\text { Gasoline }\end{array}$} & \multirow{2}{*}{$\begin{array}{l}\text { Distillate } \\
\text { Fuel Oil }{ }^{1}\end{array}$} & \multirow[b]{2}{*}{ Jet Fuel $^{2}$} & \multirow[b]{2}{*}{ Kerosene } & \multicolumn{2}{|c|}{ Liquefied Petroleum Gases } & \multirow[b]{2}{*}{ Lubricants } & \multirow{2}{*}{$\begin{array}{c}\text { Motor } \\
\text { Gasoline }{ }^{4}\end{array}$} & \multirow{2}{*}{$\begin{array}{l}\text { Petroleum } \\
\text { Coke }\end{array}$} & \multirow{2}{*}{$\begin{array}{l}\text { Residual } \\
\text { Fuel Oil }\end{array}$} & \multirow[b]{2}{*}{ Other ${ }^{5}$} & \multirow[b]{2}{*}{ Total } & \multirow{2}{*}{$\begin{array}{c}\text { Percentage } \\
\text { Change From } \\
\text { Previous Year } 6\end{array}$} \\
\hline & & & & & & Propane $^{3}$ & Total & & & & & & & \\
\hline 1949 & 157 & 93 & 902 & $\left({ }^{2}\right)$ & 281 & NA & 187 & 91 & 2,410 & 40 & 1,359 & 243 & 5,763 & -- \\
\hline 1950 & 180 & 108 & 1,082 & (2) & 323 & NA & 234 & 106 & 2,616 & 41 & 1,517 & 250 & 6,458 & 12.1 \\
\hline 1955 & 254 & 192 & 1,592 & 154 & 320 & NA & 404 & 116 & 3,463 & 67 & 1,526 & 366 & 8,455 & 9.0 \\
\hline 1960 & 302 & 161 & 1,872 & 371 & 271 & NA & 621 & 117 & 3,969 & 149 & 1,529 & 435 & 9,797 & 3.1 \\
\hline 1965 & 368 & 120 & 2,126 & 602 & 267 & NA & 841 & 129 & 4,593 & 202 & 1,608 & 657 & 11,512 & 4.2 \\
\hline 1970 & 447 & 55 & 2,540 & 967 & 263 & 776 & 1,224 & 136 & 5,785 & 212 & 2,204 & 866 & 14,697 & 4.0 \\
\hline 1975 & 419 & 39 & 2,851 & 1,001 & 159 & 783 & 1,333 & 137 & 6,675 & 247 & 2,462 & 1,001 & 16,322 & -2.0 \\
\hline 1976 & 411 & 37 & 3,133 & 987 & 169 & 830 & 1,404 & 152 & 6,978 & 243 & 2,801 & 1,145 & 17,461 & 7.3 \\
\hline 1977 & 436 & 38 & 3,352 & 1,039 & 175 & 821 & 1,422 & 160 & 7,177 & 268 & 3,071 & 1,294 & 18,431 & 5.3 \\
\hline 1978 & 479 & 39 & 3,432 & 1,057 & 175 & 778 & 1,413 & 172 & 7,412 & 256 & 3,023 & 1,391 & 18,847 & 2.3 \\
\hline 1979 & 476 & 38 & 3,311 & 1,076 & 188 & 849 & 1,592 & 180 & 7,034 & 246 & 2,826 & 1,546 & 18,513 & -1.8 \\
\hline 1980 & 396 & 35 & 2,866 & 1,068 & 158 & 754 & 1,469 & 159 & 6,579 & 237 & 2,508 & 1,581 & 17,056 & -7.6 \\
\hline 1981 & 342 & 31 & 2,829 & 1,007 & 127 & 773 & 1,466 & 153 & 6,588 & 252 & 2,088 & 1,176 & 16,058 & -6.1 \\
\hline 1982 & 342 & 25 & 2,671 & 1,013 & 129 & 798 & 1,499 & 140 & 6,539 & 248 & 1,716 & 973 & 15,296 & -4.7 \\
\hline 1983 & 373 & 26 & 2,690 & 1,046 & 127 & 751 & 1,509 & 146 & 6,622 & 229 & 1,421 & 1,042 & 15,231 & -.4 \\
\hline 1984 & 408 & 24 & 2,845 & 1,175 & 115 & 833 & 1,572 & 156 & 6,693 & 247 & 1,369 & 1,120 & 15,726 & 3.5 \\
\hline 1985 & 425 & 27 & 2,868 & 1,218 & 114 & 883 & 1,599 & 145 & 6,831 & 264 & $\begin{array}{l}1,202 \\
1,00\end{array}$ & $\begin{array}{l}1,032 \\
\text { S }\end{array}$ & $\begin{array}{l}15,726 \\
\end{array}$ & -.3 \\
\hline 1986 & 448 & 32 & 2,914 & 1,307 & 98 & 831 & 1,512 & 142 & 7,034 & 268 & 1,418 & 1,105 & 16,281 & 3.5 \\
\hline 1987 & 467 & 25 & 2,976 & 1,385 & 95 & 924 & 1,612 & 161 & 7,206 & 299 & 1,264 & 1,176 & 16,665 & 2.4 \\
\hline 1988 & 468 & 27 & 3,122 & 1,449 & 96 & 923 & 1,656 & 155 & 7,336 & 312 & 1,378 & 1,286 & 17,283 & 4.0 \\
\hline 1989 & 453 & 26 & 3,157 & $\begin{array}{l}1,489 \\
\text { S }\end{array}$ & 84 & 990 & 1,668 & 159 & 7,328 & 307 & 1,370 & 1,284 & 17,325 & (s) \\
\hline 1990 & 483 & 24 & 3,021 & 1,522 & 43 & 917 & 1,556 & 164 & 7,235 & 339 & 1,229 & 1,373 & 16,988 & -1.9 \\
\hline 1991 & 444 & 23 & 2,921 & 1,471 & 46 & 982 & 1,689 & 146 & 7,188 & 328 & 1,158 & 1,299 & 16,714 & -1.6 \\
\hline 1992 & 454 & 22 & 2,979 & 1,454 & 41 & 1,032 & 1,755 & 149 & 7,268 & 382 & 1,094 & 1,434 & 17,033 & 2.2 \\
\hline 1993 & 474 & 21 & 3,041 & 1,469 & 50 & 1,006 & 1,734 & 152 & 7,476 & 366 & 1,080 & 1,373 & 17,237 & .9 \\
\hline 1994 & 484 & 21 & 3,162 & 1,527 & 49 & 1,082 & $\begin{array}{l}1,880 \\
\text {. }\end{array}$ & 159 & 7,601 & 361 & $\begin{array}{l}1,021 \\
\text { S }\end{array}$ & 1,454 & 17,718 & 2.8 \\
\hline 1995 & 486 & 21 & 3,207 & 1,514 & 54 & 1,096 & 1,899 & 156 & 7,789 & 365 & 852 & 1,381 & 17,725 & (s) \\
\hline 1996 & 484 & 20 & 3,365 & 1,578 & 62 & 1,136 & 2,012 & 151 & 7,891 & 379 & 848 & 1,518 & 18,309 & 3.6 \\
\hline 1997 & 505 & 22 & 3,435 & 1,599 & 66 & 1,170 & 2,038 & 160 & 8,017 & 377 & 797 & 1,605 & 18,620 & 1.4 \\
\hline 1998 & 521 & 19 & 3,461 & 1,622 & 78 & 1,120 & 1,952 & 168 & 8,253 & 447 & 887 & 1,508 & 18,917 & 1.6 \\
\hline 1999 & 547 & 21 & 3,572 & 1,673 & 73 & 1,246 & 2,195 & 169 & 8.431 & 477 & 830 & 1,532 & 19.519 & 3.2 \\
\hline 2000 & 525 & 20 & 3,722 & 1,725 & 67 & 1,235 & 2,231 & 166 & 8,472 & 406 & 909 & 1,458 & $\begin{array}{l}19,701 \\
\end{array}$ & 1.2 \\
\hline 2001 & 519 & 19 & 3,847 & 1,655 & 72 & 1,142 & 2,044 & 153 & 8,610 & 437 & 811 & 1,481 & 19,649 & -.5 \\
\hline 2002 & 512 & 18 & 3,776 & 1,614 & 43 & 1,248 & 2,163 & 151 & 8,848 & 463 & 700 & 1,474 & 19,761 & .6 \\
\hline 2003 & 503 & 16 & 3,927 & 1,578 & 55 & 1,215 & 2,074 & 140 & 8,935 & 455 & 772 & 1,579 & 20,034 & 1.4 \\
\hline 2004 & 537 & 17 & 4,058 & 1,630 & 64 & 1,276 & 2,132 & 141 & 9,105 & 524 & 865 & 1,657 & 20,731 & 3.8 \\
\hline 2005 & 546 & 19 & 4,118 & 1,679 & 70 & 1,229 & 2,030 & 141 & 9,159 & 515 & 920 & 1,605 & 20,802 & .1 \\
\hline 2006 & 521 & 18 & 4,169 & 1,633 & 54 & 1,215 & 2,052 & 137 & 9,253 & 522 & 689 & 1,640 & 20,687 & -.6 \\
\hline 2007 & 494 & 17 & 4,196 & 1,622 & 32 & 1,235 & 2,085 & 142 & 9,286 & 490 & 723 & 1,593 & 20,680 & (s) \\
\hline 2008 & 417 & 15 & 3,945 & 1,539 & 14 & 1,154 & 1,954 & 131 & 8,989 & 464 & 622 & 1,408 & 19,498 & -5.5 \\
\hline 2009 & 360 & 14 & 3,631 & 1,393 & 18 & 1,160 & 2,051 & 118 & 8,997 & 427 & 511 & 1,251 & 18,771 & -4.0 \\
\hline 2010 & 362 & 15 & $\mathrm{R}_{3,800}$ & $\mathrm{R}_{1,432}$ & 20 & $\mathrm{R}_{1,160}$ & $\mathrm{R}_{2,173}$ & R131 & $\mathrm{R}_{8,993}$ & 376 & R535 & $\mathrm{R}_{1,343}$ & $\mathrm{R}_{19,180}$ & R2.2 \\
\hline $2011^{\mathrm{P}}$ & 355 & 15 & 3,849 & 1,425 & 12 & 1,138 & 2,171 & 124 & 8,736 & 367 & 480 & 1,300 & 18,835 & -1.8 \\
\hline
\end{tabular}

1 Beginning in 2009, includes renewable diesel fuel (including biodiesel) blended into distillate fuel oil. Through 1951, naphtha-type jet fuel is included in the products from which it was blended: in 1952, 71 naphtha-type jet fuel. Beginning in 1957, also includes kerosene-type jet fuel. Beginning in 2005, naphtha-type jet fuel is included in "Other."

3 Includes propylene.

${ }^{4}$ Finished motor gasoline. Through 1963, also includes special naphthas. Beginning in 1993, also includes ethanol blended into motor gasoline.

Pentanes plus, petrochemical feedstocks, still gas (refinery gas), waxes, and miscellaneous products. Beginning in 1964, also includes special naphthas. Beginning in 1981, also includes negative barrels per day of distillate and residual fuel oil reclassified as unfinished oils, and other products (from both primary and secondary supply) reclassified as gasoline blending components. Beginning in 1983, also includes

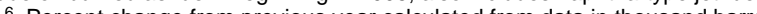

R=Revised. P=Preliminary. NA=Not available. $\quad--=$ Not applicable. $(\mathrm{s})=$ Less than 0.05 percent and greater than -0.05 percen

Notes: - For petroleum, product supplied is used as an approximation of petroleum consumption. See "Changes Affecting Petroleum Production and Product Supplied Statistics," at end of section. - Totals may not equal sum of components due to independent rounding.

Web Pages: - See http://www.eia.gov/totalenergy/data/monthly/\#petroleum for updated monthly and annual data. - See http://www.eia.gov/totalenergy/data/annual/\#pe Sources: - 1949-1975-Bureau of Mines, Mineral Industry Surveys, Petroleum Statement, Annual, annual reports. - 1976-1980-U.S. Energy Information Administration (EIA), Energy Data Reports, Petroleum Statement, Annual, annual repors. - 1981-2010-EIA, Petroleum Supply Annual, annua reports. • 2011-EIA, Petroleum Supply Monthly (February 2012). 


\section{Figure 5.12 Heat Content of Petroleum Products Supplied}

\section{Petroleum Products Supplied as Share}

of Total Energy Consumption, 1949-2011

$60-$

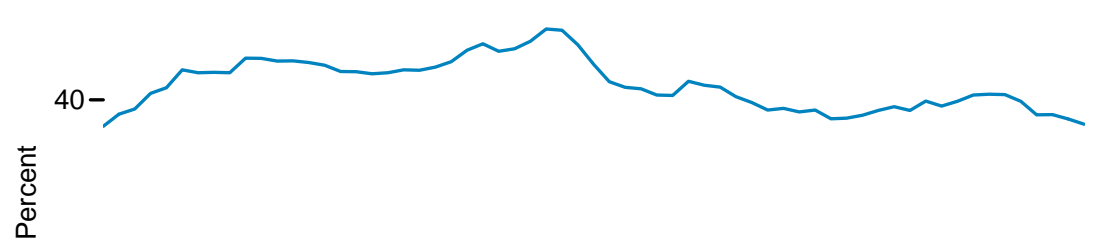

$20-$

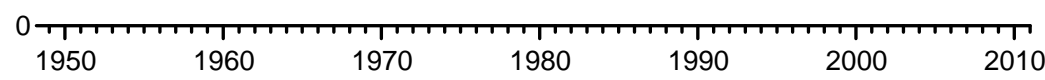

By Product, 2011

$$
20-
$$

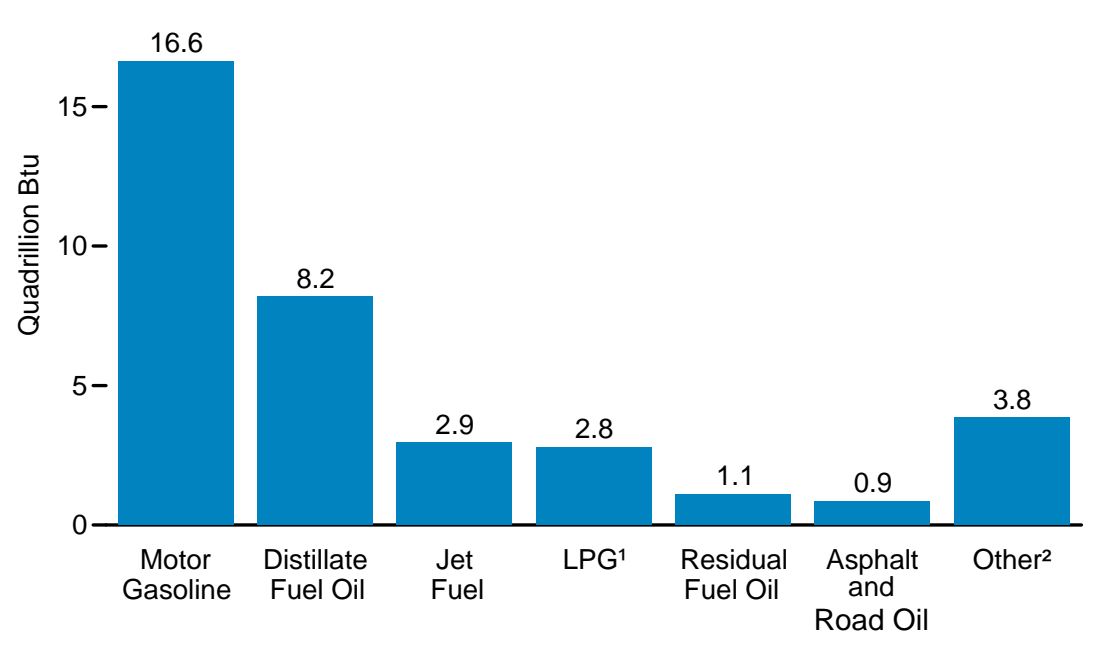

${ }^{1}$ Liquefied petroleum gases.

${ }^{2}$ Aviation gasoline, kerosene, lubricants, naphtha-type jet fuel, pentanes plus, petrochemical feedstocks, petroleum coke, special naphthas, still gas (refinery gas), waxes, miscellaneous products, and crude burned as fuel.
Total Petroleum and Motor Gasoline Product Supplied, 1949-2011

$$
50-
$$

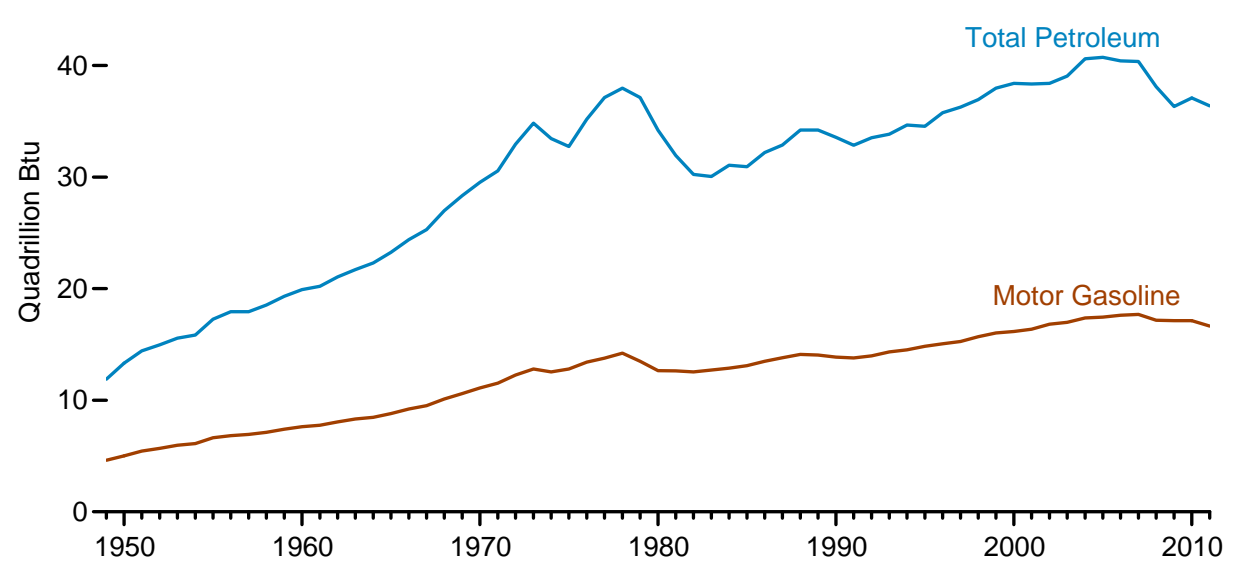

By Selected Product, 1949-2011

$$
\text { 20- }
$$

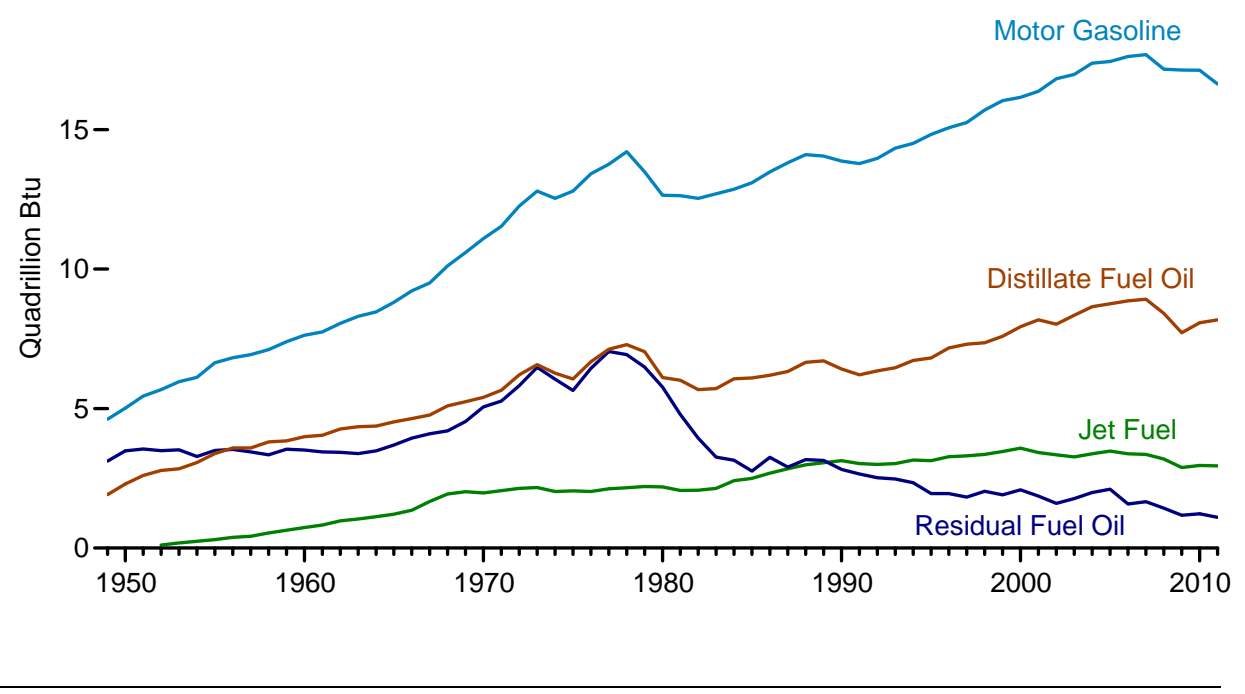

Sources: Tables 1.3 and 5.12 
Table 5.12 Heat Content of Petroleum Products Supplied, Selected Years, 1949-2011

(Trillion Btu)

\begin{tabular}{|c|c|c|c|c|c|c|c|c|c|c|c|c|c|c|}
\hline \multirow[b]{2}{*}{ Year } & \multirow{2}{*}{$\begin{array}{c}\text { Asphalt and } \\
\text { Road Oil }\end{array}$} & \multirow{2}{*}{$\begin{array}{l}\text { Aviation } \\
\text { Gasoline }\end{array}$} & \multirow{2}{*}{$\begin{array}{l}\text { Distillate } \\
\text { Fuel Oil }{ }^{1}\end{array}$} & \multirow[b]{2}{*}{ Jet Fuel $^{2}$} & \multirow[b]{2}{*}{ Kerosene } & \multicolumn{2}{|c|}{ Liquefied Petroleum Gases } & \multirow[b]{2}{*}{ Lubricants } & \multirow{2}{*}{$\begin{array}{c}\text { Motor } \\
\text { Gasoline }{ }^{4}\end{array}$} & \multirow{2}{*}{$\begin{array}{l}\text { Petroleum } \\
\text { Coke }\end{array}$} & \multirow{2}{*}{$\begin{array}{l}\text { Residual } \\
\text { Fuel Oil }\end{array}$} & \multirow[b]{2}{*}{ Other $^{5}$} & \multirow[b]{2}{*}{ Total } & \multirow{2}{*}{$\begin{array}{l}\text { Percentage } \\
\text { Change From } \\
\text { Previous Year }\end{array}$} \\
\hline & & & & & & Propane $^{3}$ & Total & & & & & & & \\
\hline 1949 & 380 & 172 & 1,918 & $\left({ }^{2}\right)$ & 582 & NA & 274 & 201 & 4,621 & 87 & 3,118 & 530 & 11,883 & -- \\
\hline 1950 & 435 & 199 & 2,300 & $\left({ }^{2}\right)$ & 668 & NA & 343 & 236 & 5,015 & 90 & 3,482 & 546 & 13,315 & 12.1 \\
\hline 1955 & 615 & 354 & 3,385 & 301 & 662 & NA & 592 & 258 & 6,640 & 147 & 3,502 & 798 & 17,255 & 8.9 \\
\hline 1960 & 734 & 298 & 3,992 & 739 & 563 & NA & 912 & 259 & 7,631 & 328 & 3,517 & 947 & 19,919 & 3.1 \\
\hline 1965 & 890 & 222 & 4,519 & 1,215 & 553 & NA & 1,232 & 286 & 8,806 & 444 & 3,691 & 1,390 & 23,246 & 4.2 \\
\hline 1970 & 1,082 & 100 & 5,401 & 1,973 & 544 & 1,086 & 1,689 & 301 & 11,091 & 465 & 5,057 & 1,817 & 29,521 & 4.2 \\
\hline 1975 & 1,014 & 71 & 6,061 & 2,047 & 329 & 1,097 & 1,807 & 304 & 12,798 & 542 & 5,649 & 2,109 & 32,732 & -2.2 \\
\hline 1976 & 998 & 67 & 6,679 & 2,026 & 351 & 1,166 & 1,907 & 338 & 13,415 & 537 & 6,445 & 2,413 & 35,178 & 7.5 \\
\hline 1977 & 1,056 & 70 & 7,126 & 2,126 & 363 & 1,150 & 1,908 & 354 & 13,760 & 589 & 7,047 & 2,724 & 37,124 & 5.5 \\
\hline 1978 & 1,160 & 71 & 7,296 & 2,164 & 363 & 1,089 & 1,892 & 380 & 14,211 & 562 & 6,936 & 2,928 & 37,963 & 2.3 \\
\hline 1979 & 1,153 & 70 & 7,039 & 2,204 & 389 & 1,189 & 2,138 & 397 & 13,487 & 541 & 6,485 & 3,217 & 37,122 & -2.2 \\
\hline 1980 & 962 & 64 & 6,110 & 2,190 & 329 & 1,059 & 1,976 & 354 & 12,648 & 522 & 5,772 & 3,278 & 34,205 & -7.9 \\
\hline 1981 & 828 & 56 & 6,014 & 2,062 & 263 & 1,082 & 1,949 & 339 & 12,631 & 553 & 4,791 & 2,446 & 31,932 & -6.6 \\
\hline 1982 & 829 & 47 & 5,679 & 2,072 & 266 & 1,117 & 1,978 & 309 & 12,538 & 545 & 3,939 & 2,030 & 30,232 & -5.3 \\
\hline 1983 & 904 & 48 & 5,720 & 2,141 & 263 & 1,051 & 1,990 & 324 & 12,697 & 503 & 3,260 & 2,202 & 30,052 & -.6 \\
\hline 1984 & 992 & 44 & 6,065 & 2,414 & 239 & 1,170 & 2,071 & 346 & 12,867 & 545 & 3,151 & 2,319 & 31,053 & 3.3 \\
\hline 1985 & 1,029 & 50 & 6,098 & 2,497 & 236 & 1,236 & 2,103 & 322 & 13,098 & 582 & 2,759 & 2,152 & 30,925 & -.4 \\
\hline 1986 & 1,086 & 59 & 6,196 & 2,682 & 203 & 1,163 & 2,010 & 315 & 13,487 & 590 & 3,255 & 2,315 & 32,198 & 4.1 \\
\hline 1987 & 1,130 & 46 & 6,328 & 2,843 & 196 & 1,294 & 2,152 & 356 & 13,816 & 657 & 2,901 & 2,439 & 32,864 & 2.1 \\
\hline 1988 & 1,136 & 49 & 6,655 & 2,982 & 200 & 1,296 & 2,213 & 343 & 14,105 & 687 & 3,170 & 2,682 & 34,223 & 4.1 \\
\hline 1989 & 1,096 & 48 & 6,712 & 3,059 & 174 & 1,387 & 2,243 & 352 & 14,050 & 676 & 3,144 & 2,656 & 34,209 & (s) \\
\hline 1990 & 1,170 & 45 & 6,422 & 3,129 & 88 & 1,284 & 2,059 & 362 & 13,872 & 745 & 2,820 & 2,839 & 33,552 & -1.9 \\
\hline 1991 & 1,077 & 42 & 6,210 & 3,025 & 96 & 1,374 & 2,228 & 324 & 13,781 & 722 & 2,657 & 2,685 & 32,846 & -2.1 \\
\hline 1992 & 1,102 & 41 & 6,351 & 3,001 & 86 & 1,449 & 2,328 & 330 & 13,973 & 843 & 2,518 & 2,951 & 33,525 & 2.1 \\
\hline 1993 & 1,149 & 38 & 6,466 & 3,028 & 103 & 1,409 & 2,282 & 337 & 14,335 & 804 & 2,479 & 2,822 & 33,842 & .9 \\
\hline 1994 & 1,173 & 38 & 6,723 & 3,154 & 101 & 1,515 & 2,494 & 352 & 14,511 & 793 & 2,342 & 2,988 & 34,670 & 2.4 \\
\hline 1995 & 1,178 & 40 & 6,818 & 3,132 & 112 & 1,534 & 2,512 & 346 & 14,825 & 802 & 1,955 & 2,837 & 34,556 & -.3 \\
\hline 1996 & 1,176 & 37 & 7,175 & 3,274 & 128 & 1,594 & 2,660 & 335 & 15,064 & 837 & 1,952 & 3,121 & 35,759 & 3.5 \\
\hline 1997 & 1,224 & 40 & 7,304 & 3,308 & 136 & 1,638 & 2,690 & 354 & 15,254 & 829 & 1,828 & 3,298 & 36,265 & 1.4 \\
\hline 1998 & 1,263 & 35 & 7,359 & 3,357 & 162 & 1,568 & 2,575 & 371 & 15,701 & 982 & 2,036 & 3,093 & 36,934 & 1.8 \\
\hline 1999 & 1,324 & 39 & 7,595 & 3,462 & 151 & 1,745 & 2,897 & 375 & 16,036 & 1,048 & 1,905 & 3,129 & 37,960 & 2.8 \\
\hline 2000 & 1,276 & 36 & 7,935 & 3,580 & 140 & 1,734 & 2,945 & 369 & 16,155 & 895 & 2,091 & 2,979 & 38,402 & 1.2 \\
\hline 2001 & 1,257 & 35 & 8,179 & 3,426 & 150 & 1,598 & 2,697 & 338 & 16,373 & 961 & 1,861 & 3,056 & 38,333 & -.2 \\
\hline 2002 & 1,240 & 34 & 8,028 & 3,340 & 90 & 1,747 & 2,852 & 334 & 16,819 & 1,018 & 1,605 & 3,040 & 38,400 & .2 \\
\hline 2003 & 1,220 & 30 & 8,349 & 3,265 & 113 & 1,701 & 2,748 & 309 & 16,981 & 1,000 & 1,772 & 3,264 & 39,051 & 1.7 \\
\hline 2004 & 1,304 & 31 & 8,652 & 3,383 & 133 & 1,791 & 2,824 & 313 & 17,379 & 1,156 & 1,990 & 3,428 & 40,593 & 3.9 \\
\hline 2005 & 1,323 & 35 & 8,755 & 3,475 & 144 & 1,721 & 2,682 & 312 & 17,444 & 1,133 & 2,111 & 3,318 & 40,732 & .3 \\
\hline 2006 & 1,261 & 33 & 8,864 & 3,379 & 111 & 1,701 & 2,700 & 303 & 17,622 & 1,148 & 1,581 & 3,416 & 40,420 & -.8 \\
\hline 2007 & 1,197 & 32 & 8,921 & 3,358 & 67 & 1,729 & 2,733 & 313 & 17,689 & 1,077 & 1,659 & 3,313 & 40,358 & -.2 \\
\hline 2008 & 1,012 & 28 & 8,411 & 3,193 & 30 & 1,620 & 2,574 & 291 & 17,168 & 1,022 & 1,432 & 2,941 & 38,101 & -5.6 \\
\hline 2009 & 873 & 27 & 7,720 & 2,883 & 36 & 1,624 & 2,664 & 262 & 17,135 & 938 & 1,173 & 2,611 & 36,321 & -4.7 \\
\hline 2010 & R878 & 27 & $\mathrm{R}_{8,080}$ & $R_{2,963}$ & 41 & $\mathrm{R}_{1,624}$ & $\mathrm{R}_{2,821}$ & $\mathrm{R}_{291}$ & $R_{17,127}$ & 826 & $\mathrm{R}_{1,228}$ & $\mathrm{R}_{2,800}$ & $\mathrm{R}_{37,082}$ & 2.1 \\
\hline $2011^{P}$ & 860 & 27 & 8,184 & 2,950 & 25 & 1,594 & 2,796 & 275 & 16,639 & 807 & 1,102 & 2,712 & 36,376 & -1.9 \\
\hline
\end{tabular}

1 Beginning in 2009, includes renewable diesel fuel (including biodiesel) blended into distillate fuel oil. 2 Through 1951, naphtha-type jet fuel is included in the products from which it was blended: in 1952, 71 percent gasoline, 17 percent kerosene, and 12 percent distillate fuel oil. Beginning in 1952, includes naphtha-type jet fuel. Beginning in 1957, also includes kerosene-type jet fuel. Beginning in 2005, naphtha-type jet fuel is included in "Other."

Finished motor gasoline. Through 1963, also includes special naphthas. Beginning in 1993, also includes ethanol blended into motor gasoline.

Pentanes plus, petrochemical feedstocks, still gas (refinery gas), waxes, and miscellaneous products. Beginning in 1964, also includes special naphthas. Beginning in 1981, also includes negative barrels per day of distillate and residual fuel oil reclassified as unfinished oils, and other products (from both primary
and secondary supply) reclassified as gasoline blending components. Beginning in 1983, also includes

crude oil burned as fuel. Beginning in 2005, also includes naphtha-type jet fuel.

$\mathrm{R}=$ Revised. $\mathrm{P}=$ Preliminary. NA=Not available. $--=$ Not applicable. $(\mathrm{s})=$ Less than 0.05 percent and greater than -0.05 percent.

Notes: - For petroleum, product supplied is used as an approximation of petroleum consumption. See "Note 1, "Petroleum Products Supplied and Petroleum Consumption," at end of section. • See Note 2, may not equal sum Petroleum Production and Product Supplied

We

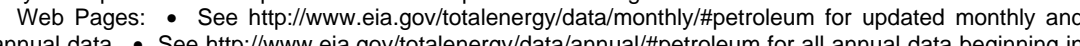
Sour 


\section{Figure 5.13a Petroleum Consumption Estimates by Sector}

\section{By Sector, 1949-2011}

$15-$

Transportation

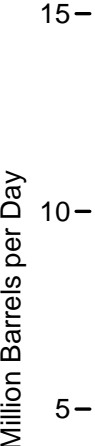

-

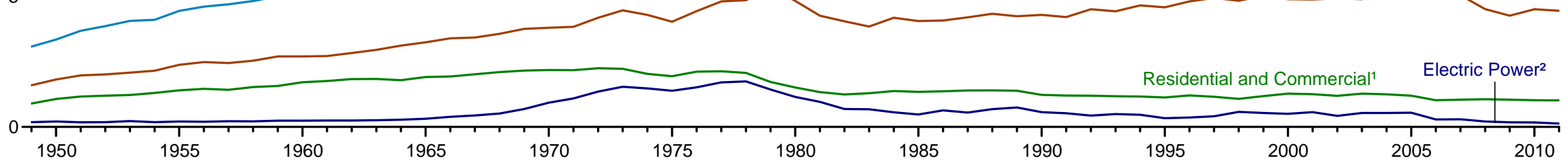

By Sector, 2011

$15-$

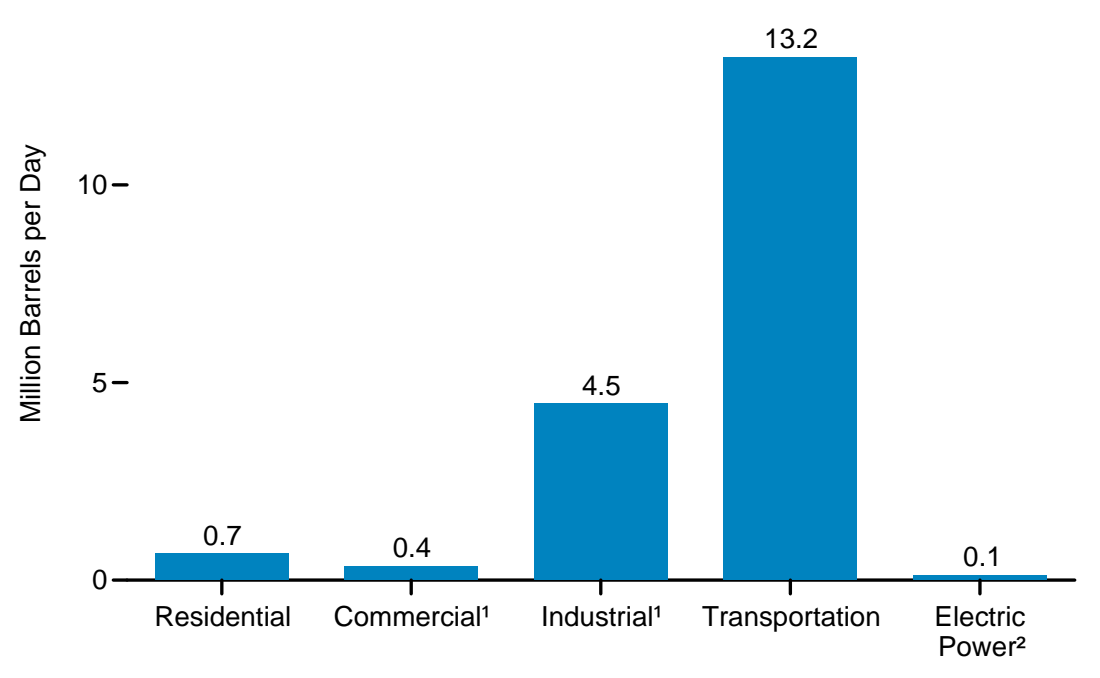

Sector Shares, 1949 and 2011

$75-$

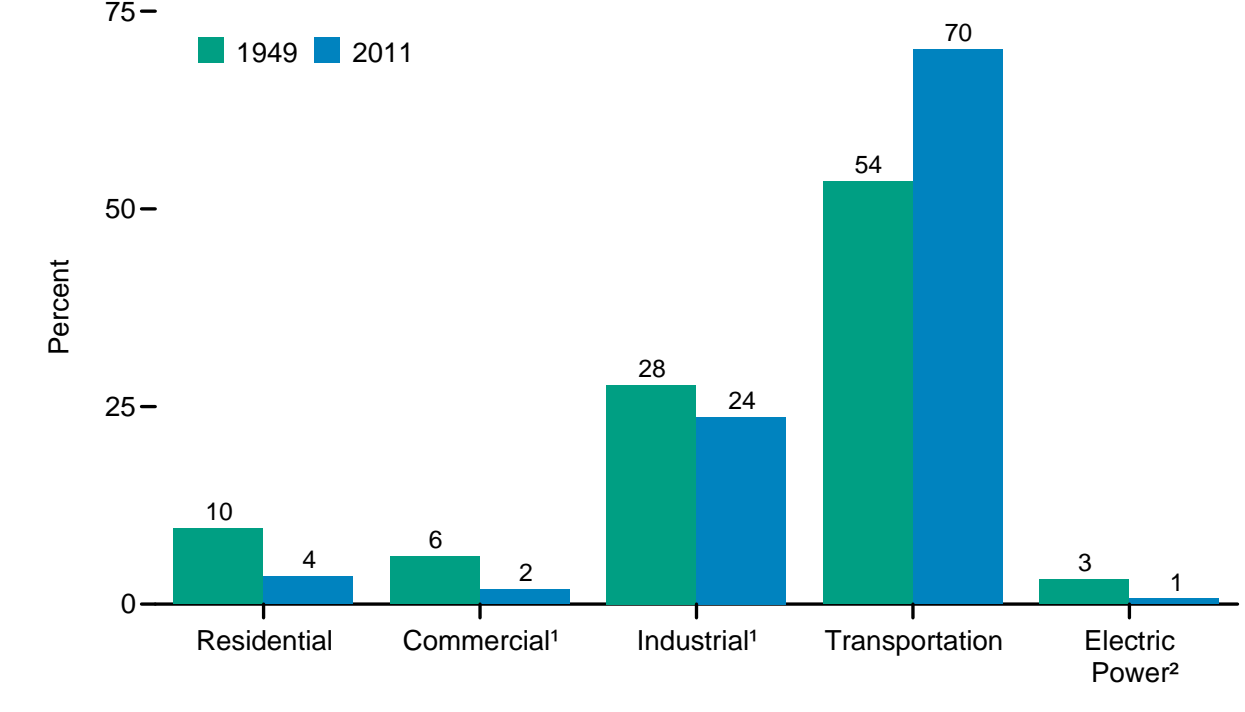

\footnotetext{
${ }^{1}$ Includes combined-heat-and-power plants and a small number of electricity-only plants

${ }^{2}$ Electricity-only and combined-heat-and-power plants whose primary business is to sell
}

Note: See related Figure $5.13 \mathrm{~b}$ electricity, or electricity and heat, to the public. 


\section{Figure 5.13b Petroleum Consumption Estimates by Product by Sector, 1949-2011}

\section{Residential and Commercial ${ }^{1}$ Sectors, Selected Products}

$1.5-$

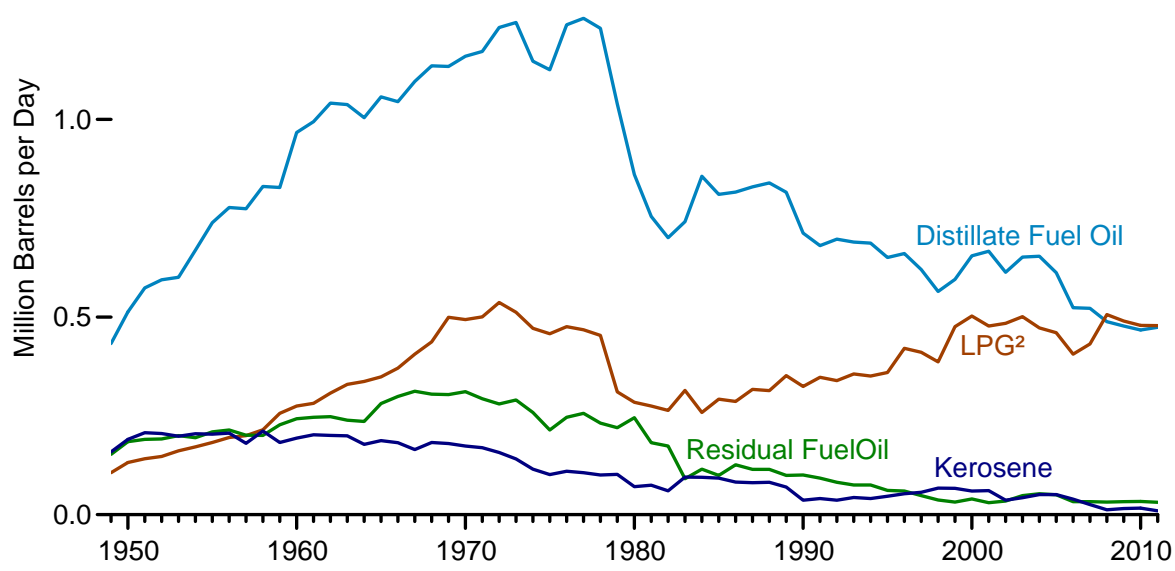

\section{Transportation Sector, Selected Products} $12-$

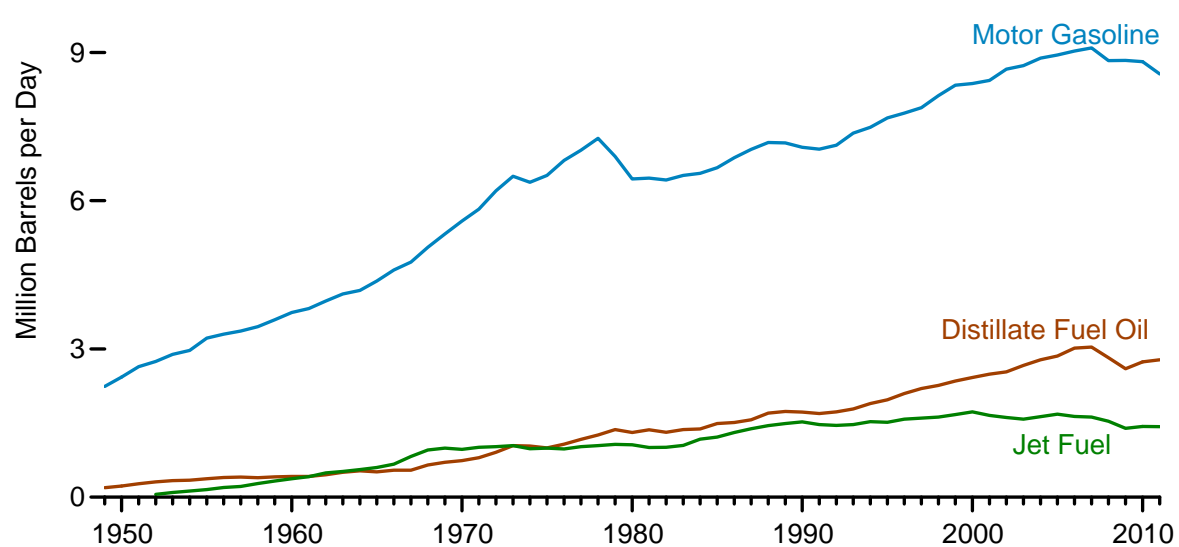

Industrial ${ }^{1}$ Sector, Selected Products

$2.0-$

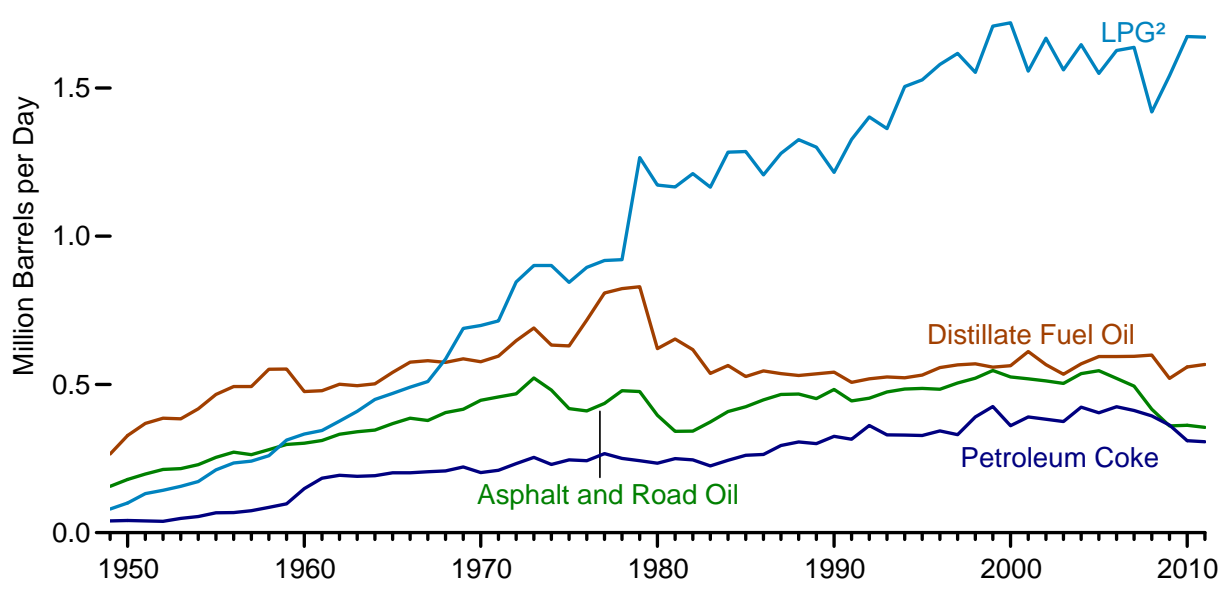

\section{Electric Power Sector} $2.0-$

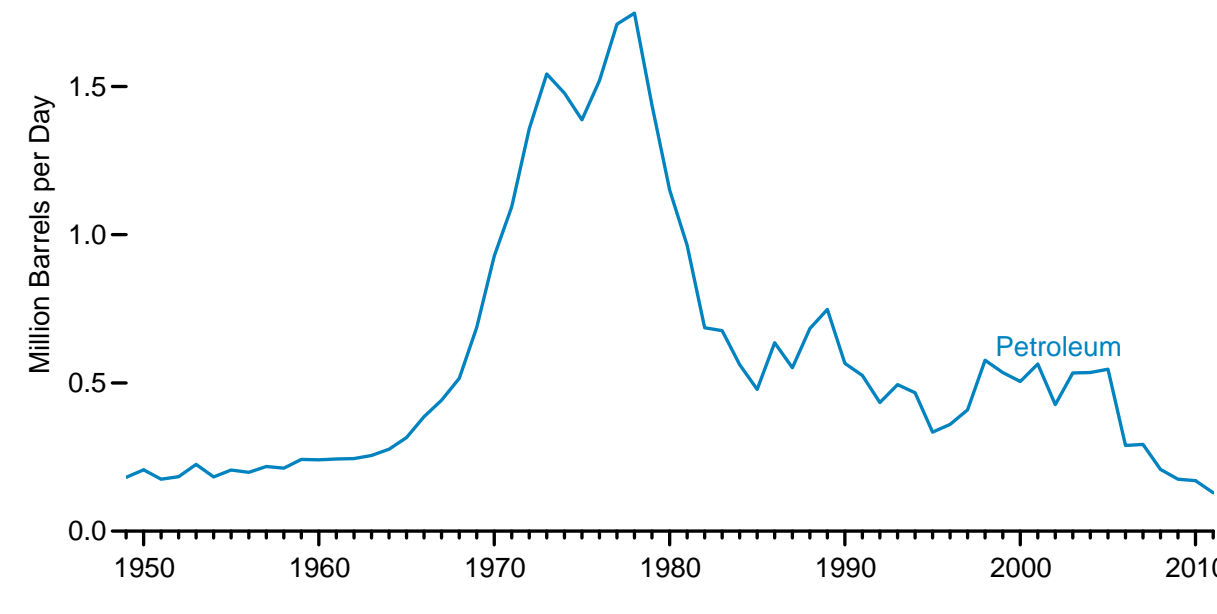

Note: See related Figure 5.13a.

Sources: Tables 5.13a-5.13d.

${ }^{1}$ Includes combined-heat-and-power plants and a small number of electricity-only plants.

${ }^{2}$ Liquefied petroleum gases.

${ }^{3}$ Electricity-only and combined-heat-and-power plants whose primary business is to sell electricity, or electricity and heat, to the public. 
Table 5.13a Petroleum Consumption Estimates: Residential and Commercial Sectors, Selected Years, 1949-2011

(Thousand Barrels per Day)

\begin{tabular}{|c|c|c|c|c|c|c|c|c|c|c|c|c|c|c|c|}
\hline \multirow[b]{3}{*}{ Year } & \multicolumn{4}{|c|}{ Residential Sector } & \multicolumn{11}{|c|}{ Commercial Sector } \\
\hline & \multirow{2}{*}{$\begin{array}{l}\text { Distillate } \\
\text { Fuel Oil }\end{array}$} & \multirow[b]{2}{*}{ Kerosene } & \multirow{2}{*}{$\begin{array}{l}\text { Liquefied } \\
\text { Petroleum } \\
\text { Gases }\end{array}$} & \multirow[b]{2}{*}{ Total } & \multicolumn{3}{|c|}{ Distillate Fuel Oil } & \multirow[b]{2}{*}{ Kerosene } & \multirow{2}{*}{$\begin{array}{l}\text { Liquefied } \\
\text { Petroleum } \\
\text { Gases }\end{array}$} & \multirow{2}{*}{$\begin{array}{c}\text { Motor } \\
\text { Gasoline }\end{array}$} & \multirow{2}{*}{$\begin{array}{l}\text { Petroleum } \\
\text { Coke }\end{array}$} & \multicolumn{3}{|c|}{ Residual Fuel Oil } & \multirow[b]{2}{*}{ Total } \\
\hline & & & & & CHP 1 & Other ${ }^{2}$ & Total & & & & & CHP 1 & Other ${ }^{2}$ & Total & \\
\hline 1949 & 329 & 140 & 84 & 553 & $\left({ }^{4}\right)$ & 104 & 104 & 19 & 22 & 48 & NA & $\left(4^{4}\right)$ & 153 & 153 & 346 \\
\hline 1950 & 390 & 168 & 104 & 662 & $(4)$ & 123 & 123 & 23 & 28 & 52 & NA & $\left({ }^{4}\right)$ & 185 & 185 & 411 \\
\hline 1955 & 562 & 179 & 144 & 885 & $\left({ }^{4}\right)$ & 177 & 177 & 24 & 38 & 69 & NA & $\left({ }^{4}\right)$ & 209 & 209 & 519 \\
\hline 1960 & 736 & 171 & 217 & 1,123 & $(4)$ & 232 & 232 & 23 & 58 & 35 & NA & $(4)$ & 243 & 243 & 590 \\
\hline 1965 & 805 & 161 & 275 & 1,242 & $(4)$ & 251 & 251 & 26 & 74 & 40 & NA & $(4)$ & 281 & 281 & 672 \\
\hline 1970 & 883 & 144 & 392 & 1,419 & $\left({ }^{4}\right)$ & 276 & 276 & 30 & 102 & 45 & NA & $(4)$ & 311 & 311 & 764 \\
\hline 1975 & 850 & 78 & 365 & 1,293 & $\left({ }^{4}\right)$ & 276 & 276 & 24 & 92 & 46 & NA & $\left({ }^{4}\right)$ & 214 & 214 & 653 \\
\hline 1976 & 932 & 89 & 379 & 1,400 & $\left({ }^{4}\right)$ & 308 & 308 & 21 & 97 & 50 & NA & $\left({ }^{4}\right)$ & 247 & 247 & 722 \\
\hline 1977 & 938 & 81 & 371 & 1,390 & $(4)$ & 318 & 318 & 25 & 96 & 52 & NA & $(4)$ & 256 & 256 & 748 \\
\hline 1978 & 917 & 74 & 360 & 1,351 & $\left({ }^{4}\right)$ & 313 & 313 & 26 & 94 & 56 & NA & $\left(4^{\prime}\right)$ & 232 & 232 & 721 \\
\hline 1979 & 765 & 64 & 243 & 1,072 & $(4)$ & 274 & 274 & 38 & 68 & 54 & NA & $(4)$ & 220 & 220 & 655 \\
\hline 1980 & 617 & 51 & 222 & 890 & $\left({ }^{4}\right)$ & 243 & 243 & 20 & 63 & 56 & NA & $\left({ }^{4}\right)$ & 245 & 245 & 626 \\
\hline 1981 & 540 & 41 & 213 & 794 & $\left(4^{\prime}\right)$ & 215 & 215 & 34 & 62 & 48 & NA & $\left(4^{\prime}\right)$ & 182 & 182 & 540 \\
\hline 1982 & 494 & 46 & 206 & 746 & $\left({ }^{4}\right)$ & 207 & 207 & 15 & 58 & 46 & NA & $\left(4^{4}\right)$ & 174 & 174 & 499 \\
\hline 1983 & 435 & 41 & 245 & 721 & $\left({ }^{4}\right)$ & 306 & 306 & 54 & 69 & 53 & NA & $\left(4^{\prime}\right)$ & 91 & 91 & 573 \\
\hline 1984 & 512 & 77 & 199 & 788 & $\left(4^{\prime}\right)$ & 345 & 345 & 17 & 59 & 56 & NA & $\left({ }^{4}\right)$ & 115 & 115 & 593 \\
\hline 1985 & 514 & 77 & 224 & 815 & $\left({ }^{4}\right)$ & 297 & 297 & 16 & 68 & 50 & NA & $\left({ }^{4}\right)$ & 99 & 99 & 530 \\
\hline 1986 & 523 & 59 & 220 & 801 & $(4)$ & 293 & 293 & 24 & 66 & 55 & NA & $(4)$ & 126 & 126 & 566 \\
\hline 1987 & 544 & 57 & 244 & 845 & $\left({ }^{4}\right)$ & 286 & 286 & 24 & 72 & 58 & NA & $\left({ }^{4}\right)$ & 114 & 114 & 554 \\
\hline 1988 & 558 & 69 & 243 & 870 & $\left(4^{4}\right)$ & 281 & 281 & 13 & 71 & 57 & NA & $\left({ }^{4}\right)$ & 115 & 115 & 537 \\
\hline 1989 & 546 & 57 & 273 & 876 & 3 & 267 & 270 & 13 & 78 & 53 & 0 & 2 & 97 & 99 & 514 \\
\hline 1990 & 460 & 31 & 252 & 742 & 3 & 249 & 252 & 6 & 73 & 58 & 0 & 3 & 97 & 100 & 489 \\
\hline 1991 & 438 & 35 & 270 & 743 & 2 & 241 & 243 & 6 & 77 & 44 & 0 & 2 & 91 & 92 & 463 \\
\hline 1992 & 460 & 31 & 263 & 754 & 1 & 236 & 238 & 5 & 76 & 41 & (s) & 2 & 80 & 82 & 443 \\
\hline 1993 & 458 & 37 & 278 & 773 & 2 & 230 & 232 & 7 & 78 & 15 & $\begin{array}{l}\text { (s) } \\
\text { (s) }\end{array}$ & 2 & 73 & 75 & 407 \\
\hline 1994 & 451 & 31 & 274 & 757 & 3 & 233 & 236 & 9 & 77 & 13 & (s) & 2 & 73 & 75 & 410 \\
\hline 1995 & 426 & 36 & 282 & 743 & 2 & 223 & 225 & 11 & 78 & 10 & (s) & 1 & 61 & 62 & 385 \\
\hline 1996 & 434 & 43 & 334 & 811 & 2 & 225 & 227 & 10 & 87 & 14 & (s) & 1 & 58 & 60 & 397 \\
\hline 1997 & 411 & 45 & 325 & 781 & 3 & 206 & 209 & 12 & 86 & 22 & (s) & 1 & 47 & 48 & 378 \\
\hline 1998 & 363 & 52 & 303 & 718 & 2 & 199 & 202 & 15 & 84 & 20 & (s) & 3 & 35 & 37 & 358 \\
\hline 1999 & 389 & 54 & 376 & 819 & 2 & 204 & 206 & 13 & 100 & 15 & (s) & 2 & 30 & 32 & 366 \\
\hline 2000 & 424 & 46 & 395 & 865 & 2 & 228 & 230 & 14 & 107 & 23 & (s) & 2 & 38 & 40 & 415 \\
\hline 2001 & 427 & 46 & 375 & 849 & 3 & 236 & 239 & 15 & 102 & 20 & (s) & 2 & 28 & 30 & 406 \\
\hline 2002 & 404 & 29 & 384 & 817 & 2 & 207 & 209 & 8 & 101 & 24 & (s) & 1 & 34 & 35 & 376 \\
\hline 2003 & 425 & 34 & 389 & 848 & 2 & 225 & 226 & 9 & 112 & 32 & (s) & 2 & 46 & 48 & 428 \\
\hline 2004 & 433 & 41 & 364 & 839 & 3 & 218 & 221 & 10 & 108 & 23 & (s) & 2 & 51 & 53 & 416 \\
\hline 2005 & 402 & 40 & 366 & 809 & 2 & 208 & 210 & 10 & 94 & 24 & (s) & 2 & 48 & 50 & 389 \\
\hline 2006 & 335 & 32 & 318 & 685 & 1 & 188 & 189 & 7 & 88 & 26 & (s) & 1 & 31 & 33 & 343 \\
\hline 2007 & 342 & 21 & 345 & 708 & 1 & 180 & 181 & 4 & 87 & 32 & (s) & 1 & 32 & 33 & $\begin{array}{l}430 \\
337\end{array}$ \\
\hline 2008 & 314 & 10 & 394 & 718 & 1 & 173 & 174 & 2 & 113 & 24 & (s) & 1 & 31 & 32 & 345 \\
\hline 2009 & 283 & 13 & 391 & 687 & 1 & 194 & 194 & 2 & 99 & 28 & (s) & 1 & 32 & 33 & 357 \\
\hline 2010 & $\mathrm{R}_{274}$ & $\mathrm{R}_{14}$ & R379 & $\mathrm{R}_{667}$ & 1 & $R_{192}$ & $\mathrm{R}_{193}$ & 2 & $R_{100}$ & $\mathrm{R}_{29}$ & (s) & (s) & $\mathrm{R}_{33}$ & R34 & $\mathrm{R}_{358}$ \\
\hline $2011^{P}$ & 278 & 9 & 378 & 665 & (s) & 196 & 196 & 1 & 100 & 28 & (s) & (s) & 31 & 32 & 357 \\
\hline
\end{tabular}

Commercial combined-heat-and-power (CHP) and a small number of commercial electricity-only plants. See Note 2, "Classification of Power Plants Into Energy-Use Sectors," at end of Section 8.

All commercial sector fuel use other than that in "CHP."

${ }^{3}$ Finished motor gasoline. Through 1963, also includes special naphthas. Beginning in 1993, also includes ethanol blended into motor gasoline.

Included in "Other."

R=Revised. P=Preliminary. NA=Not available. ( $(s)=$ Less than 500 barrels per day.

5.11. For petrotal prolut in Table

"Petroleum Products Supplied and Petroleum Consumption," at end of section. • Totals may not equal

sum of components due to independent rounding.
Web Pages: - See http://www.eia.gov/totalenergy/data/monthly/\#petroleum for updated monthly and annual data. - See $\mathrm{http}: / \mathrm{www}$.eia.gov/totalenergy/data/annual/\#petroleum for all annual data beginning in 1949. - See $h t t p: / / w w w . e i a . g o v / s t a t e s /$ seds.html for related information.

Sources: CHP and Petroleum Coke: Table 8.7c. All Other Data: - 1949-1959-Bureau of Mines, Mineral Industry Surveys, Petroleum Statement, Annual, annual reports, and U.S. Energy Information U.S. Tables CT4 and CT5. • 1973 forward-EIA, Monthly Energy Review (April 2012), Table 3.7a. 
Table 5.13b Petroleum Consumption Estimates: Industrial Sector, Selected Years, 1949-2011

(Thousand Barrels per Day)

\begin{tabular}{|c|c|c|c|c|c|c|c|c|c|c|c|c|c|c|c|c|}
\hline \multirow[b]{3}{*}{ Year } & \multicolumn{16}{|c|}{ Industrial Sector } \\
\hline & \multirow{2}{*}{$\begin{array}{c}\text { Asphalt } \\
\text { and } \\
\text { Road Oil }\end{array}$} & \multicolumn{3}{|c|}{ Distillate Fuel Oil } & \multirow[b]{2}{*}{ Kerosene } & \multirow{2}{*}{$\begin{array}{l}\text { Liquefied } \\
\text { Petroleum } \\
\text { Gases }\end{array}$} & \multirow[b]{2}{*}{ Lubricants } & \multirow{2}{*}{$\begin{array}{c}\text { Motor } \\
\text { Gasoline }^{3}\end{array}$} & \multicolumn{3}{|c|}{ Petroleum Coke } & \multicolumn{3}{|c|}{ Residual Fuel Oil } & \multirow{2}{*}{$\begin{array}{c}\text { Other } \\
\text { Petroleum } 4\end{array}$} & \multirow[b]{2}{*}{ Total } \\
\hline & & $\mathrm{CHP}^{1}$ & Other ${ }^{2}$ & Total & & & & & CHP 1 & Other ${ }^{2}$ & Total & $\mathrm{CHP}^{1}$ & Other ${ }^{2}$ & Total & & \\
\hline
\end{tabular}

\begin{tabular}{|c|c|c|c|}
\hline 1949 & 157 & $\left({ }^{5}\right)$ & 265 \\
\hline 1950 & 180 & $\left({ }^{5}\right)$ & 328 \\
\hline 1955 & 254 & (5) & 466 \\
\hline 1960 & 302 & $\left({ }^{5}\right)$ & 476 \\
\hline 1965 & 368 & $\left(5^{\prime}\right)$ & 541 \\
\hline 1970 & 447 & $(5)$ & 577 \\
\hline 1975 & 419 & $\left({ }^{5}\right)$ & 630 \\
\hline 1976 & 411 & $\left({ }^{5}\right)$ & 717 \\
\hline 1977 & 436 & (5) & 809 \\
\hline 1978 & 479 & $\left(5^{\prime}\right)$ & 823 \\
\hline 1979 & 476 & $(5)$ & 830 \\
\hline 1980 & 396 & $\left(5^{5}\right)$ & 621 \\
\hline 1981 & 342 & $\left({ }^{5}\right)$ & 653 \\
\hline 1982 & 342 & (5) & 617 \\
\hline 1983 & 373 & $\left(5^{5}\right)$ & 537 \\
\hline 1984 & 408 & $(5)$ & 564 \\
\hline 1985 & 425 & $\left(5^{\prime}\right)$ & 526 \\
\hline 1986 & 448 & $\left(5^{5}\right)$ & 546 \\
\hline 1987 & 467 & $(5)$ & 537 \\
\hline 1988 & 468 & $(5)$ & 530 \\
\hline 1989 & 453 & 5 & 531 \\
\hline 1990 & 483 & 7 & 534 \\
\hline 1991 & 444 & 12 & 495 \\
\hline 1992 & 454 & 10 & 509 \\
\hline 1993 & 474 & 10 & 515 \\
\hline 1994 & 484 & 10 & 513 \\
\hline 1995 & 486 & 6 & 526 \\
\hline 1996 & 484 & 8 & 549 \\
\hline 1997 & 505 & 8 & 558 \\
\hline 1998 & 521 & 16 & 554 \\
\hline 1999 & 547 & 16 & 542 \\
\hline 2000 & 525 & 10 & 553 \\
\hline 2001 & 519 & 9 & 602 \\
\hline 2002 & 512 & 6 & 561 \\
\hline 2003 & 503 & 10 & 525 \\
\hline 2004 & 537 & 9 & 561 \\
\hline 2005 & 546 & 11 & 583 \\
\hline 2006 & 521 & 5 & 589 \\
\hline 2007 & 494 & 4 & 591 \\
\hline 2008 & 417 & 3 & 596 \\
\hline 2009 & 360 & 7 & 514 \\
\hline 2010 & 362 & $R_{3}$ & R556 \\
\hline $2011^{\mathrm{P}}$ & 355 & 2 & 566 \\
\hline
\end{tabular}

\begin{tabular}{rrrrr}
265 & 123 & 80 & 36 & 121 \\
328 & 132 & 100 & 43 & 131 \\
466 & 116 & 212 & 47 & 17 \\
476 & 78 & 333 & 48 & 198 \\
541 & 80 & 470 & 62 & 17 \\
577 & 89 & 699 & 70 & 15 \\
630 & 58 & 844 & 68 & 11 \\
717 & 59 & 895 & 75 & 110 \\
809 & 69 & 918 & 82 & 102 \\
823 & 75 & 921 & 88 & 93 \\
830 & 86 & 1,266 & 92 & 84 \\
621 & 87 & 1,172 & 82 & 82 \\
653 & 52 & 1,166 & 79 & 83 \\
617 & 68 & 1,211 & 72 & 72 \\
537 & 32 & 1,166 & 75 & 5 \\
564 & 21 & 1,283 & 80 & 83 \\
526 & 21 & 1,285 & 75 & 114 \\
546 & 16 & 1,207 & 73 & 108 \\
537 & 14 & 1,279 & 83 & 107 \\
530 & 14 & 1,326 & 80 & 100 \\
536 & 14 & 1,300 & 82 & 104 \\
541 & 6 & 1,215 & 84 & 97 \\
507 & 5 & 1,326 & 75 & 101 \\
519 & 5 & 1,402 & 77 & 101 \\
525 & 6 & 1,363 & 78 & 94 \\
522 & 8 & 1,505 & 82 & 101 \\
532 & 7 & 1,527 & 80 & 105 \\
557 & 9 & 1,580 & 78 & 10 \\
566 & 9 & 1,617 & 82 & 111 \\
570 & 11 & 1,553 & 86 & 105 \\
558 & 6 & 1,709 & 87 & 80 \\
563 & 8 & 1,720 & 86 & 79 \\
611 & 11 & 1,557 & 79 & 155 \\
566 & 7 & 1,668 & 78 & 163 \\
534 & 12 & 1,561 & 72 & 171 \\
570 & 14 & 1,646 & 73 & 195 \\
594 & 19 & 1,549 & 72 & 187 \\
594 & 14 & 1,627 & 71 & 198 \\
595 & 6 & 1,637 & 73 & 161 \\
599 & 2 & 1,419 & 67 & 131 \\
521 & 2 & 1,541 & 61 & 128 \\
8559 & $\mathrm{R} 4$ & $\mathrm{R} 1,673$ & $\mathrm{R} 68$ & $\mathrm{R} 148$ \\
568 & 2 & 1,672 & 64 & 143 \\
& & & & \\
& & & & \\
\hline & & &
\end{tabular}

1 Industrial combined-heat-and-power (CHP) and a small number of industrial electricity-only plants. See Note 2, "Classification of Power Plants Into Energy-Use Sectors," at end of Section 8.

3 Finished motor gasoline. Through 1963, also includes special naphthas. Beginning in 1993, also includes ethanol blended into motor gasoline.
4 Pentanes plus, petrochemical feedstocks,

. Beginning in 1964, also includes special naphthas. Beginning in 1981, also includes negative barrels per day of distillate and residual fuel oil reclassified as unfinished oils, and other products (from both primary and secondary supply) reclassified as gasoline blending components. Beginning in 1983, also includes crude oil burned as fuel. Beginning in 2005, also includes naphtha-type jet fuel.

R=Revised. P=Preliminary

\begin{tabular}{|c|c|c|c|c|c|c|c|}
\hline$\left({ }^{5}\right)$ & 40 & 40 & $\left({ }^{5}\right)$ & 534 & 534 & 243 & 1,598 \\
\hline$\left({ }^{5}\right)$ & 41 & 41 & $\left({ }^{5}\right)$ & 617 & 617 & 250 & 1,822 \\
\hline$(5)$ & 67 & 67 & (5) & 686 & 686 & 366 & 2,387 \\
\hline$\left({ }^{5}\right)$ & 149 & 149 & $\left({ }^{5}\right)$ & 689 & 689 & 435 & 2,708 \\
\hline$\left({ }^{5}\right)$ & 202 & 202 & (5) & 689 & 689 & 657 & 3,247 \\
\hline$\left(5^{5}\right)$ & 203 & 203 & $\left({ }^{5}\right)$ & 708 & 708 & 866 & 3,808 \\
\hline$(5)$ & 246 & 246 & $\left(5^{5}\right)$ & 658 & 658 & 1,001 & 4,038 \\
\hline$\left(\begin{array}{l}5 \\
5\end{array}\right)$ & 242 & 242 & $\left({ }^{5}\right)$ & 792 & 792 & 1,145 & 4,447 \\
\hline$\left({ }^{5}\right)$ & 266 & 266 & $\left({ }^{5}\right)$ & 844 & 844 & 1,294 & 4,821 \\
\hline$\left({ }^{5}\right)$ & 250 & 250 & $\left({ }^{5}\right)$ & 748 & 748 & 1,391 & 4,867 \\
\hline$\left({ }^{5}\right)$ & 243 & 243 & $\left({ }^{5}\right)$ & 721 & 721 & 1,546 & 5,343 \\
\hline$(5)$ & 234 & 234 & (5) & 586 & 586 & 1,581 & 4,842 \\
\hline$\left(5^{5}\right)$ & 250 & 250 & $\left({ }^{5}\right)$ & 471 & 471 & 1,176 & 4,273 \\
\hline$\left({ }^{5}\right)$ & 246 & 246 & $\left({ }^{5}\right)$ & 456 & 456 & 973 & 4,058 \\
\hline$\left({ }^{5}\right)$ & 225 & 225 & $\left({ }^{5}\right)$ & 345 & 345 & 1,042 & 3,854 \\
\hline$\left({ }^{5}\right)$ & 244 & 244 & $\left({ }^{5}\right)$ & 386 & 386 & 1,120 & 4,191 \\
\hline (5) & 261 & 261 & (5) & 326 & 326 & 1,032 & 4,065 \\
\hline$\left(5^{5}\right)$ & 264 & 264 & $\left(5^{5}\right)$ & 321 & 321 & 1,105 & 4,087 \\
\hline$\left(\begin{array}{l}5 \\
5\end{array}\right)$ & 294 & 294 & $\left(\begin{array}{l}5 \\
5\end{array}\right)$ & 253 & 253 & 1,176 & 4,210 \\
\hline$\left({ }^{5}\right)$ & 306 & 306 & $\left({ }^{5}\right)$ & 237 & 237 & 1,286 & 4,347 \\
\hline 5 & 295 & 300 & 57 & 121 & 178 & 1,284 & 4,251 \\
\hline 25 & 300 & 325 & 63 & 116 & 179 & 1,373 & 4,304 \\
\hline 22 & 293 & 315 & 55 & 91 & 146 & 1,299 & 4,219 \\
\hline 26 & 336 & 362 & 59 & 109 & 168 & 1,434 & 4,522 \\
\hline 22 & 308 & 330 & 65 & 129 & 194 & 1,373 & 4,438 \\
\hline 25 & 304 & 329 & 69 & 113 & 183 & 1,454 & 4,667 \\
\hline 26 & 302 & 328 & 60 & 87 & 147 & 1,381 & 4,594 \\
\hline 27 & 317 & 343 & 66 & 80 & 146 & 1,518 & 4,819 \\
\hline 37 & 294 & 331 & 56 & 71 & 127 & 1,605 & 4,953 \\
\hline 29 & 362 & 390 & 60 & 40 & 100 & 1,508 & 4,844 \\
\hline 31 & 395 & 426 & 52 & 38 & 90 & 1,532 & 5,035 \\
\hline 19 & 342 & 361 & 48 & 57 & 105 & 1,458 & 4,903 \\
\hline 15 & 375 & 390 & 46 & 42 & 89 & 1,481 & 4,892 \\
\hline 21 & 362 & 383 & 37 & 46 & 83 & 1,474 & 4,934 \\
\hline 17 & 358 & 375 & 38 & 58 & 96 & 1,579 & 4,903 \\
\hline 18 & 405 & 423 & 46 & 62 & 108 & 1,657 & 5,222 \\
\hline 14 & 390 & 404 & 46 & 77 & 123 & 1,605 & 5,100 \\
\hline 21 & 404 & 425 & 33 & 71 & 104 & 1,640 & 5,193 \\
\hline 22 & 390 & 412 & 30 & 54 & 84 & 1,593 & 5,056 \\
\hline 16 & 377 & 394 & 14 & 73 & 86 & 1,408 & 4,523 \\
\hline 17 & 347 & 363 & 13 & 33 & 46 & 1,251 & 4,274 \\
\hline$R_{17}$ & R293 & 310 & ${ }^{R} 6$ & R46 & 52 & $\mathrm{R}_{1}, 343$ & $\mathrm{R}_{4,519}$ \\
\hline 17 & 289 & 307 & 5 & 44 & 49 & 1,300 & 4,460 \\
\hline
\end{tabular}

Notes: - For total petroleum consumption by all sectors, see petroleum products supplied data in Table "Petroleum Products Supplied and Petroleum Consumption," at end of section. - Totals may not equal sum of components due to independent rounding.

Web Pages: - See http://www.eia.gov/totalenergy/data/monthly/\#petroleum for updated monthly and annual data. - See http://www.eia.gov/totalenergy/data/annual/\#petroleum for all annual data beginning in 949. - See http://www.eia.gov/states/_seds.html for related information.

Sources: CHP: Table 8.7c. All Other Data: - 1949-1959-Bureau of Mines, Mineral Industry Surveys, Petroleum Statement, Annual, annual reports, and U.S. Energy Information Administration (EIA) estimates - 1960-1972-EIA, "State Energy Data 2010: Consumption" (June 2012), U.S. Table CT6. • 1973 
Table 5.13c Petroleum Consumption Estimates: Transportation Sector, Selected Years, 1949-2011

(Thousand Barrels per Day)

\begin{tabular}{|c|c|c|c|c|c|c|c|c|c|}
\hline \multirow[b]{3}{*}{ Year } & \multicolumn{9}{|c|}{ Transportation Sector } \\
\hline & \multirow{2}{*}{$\begin{array}{l}\text { Aviation } \\
\text { Gasoline }\end{array}$} & \multirow{2}{*}{$\begin{array}{l}\text { Distillate } \\
\text { Fuel Oil }^{1}\end{array}$} & \multicolumn{2}{|c|}{ Jet Fuel } & \multirow{2}{*}{$\begin{array}{l}\text { Liquefied } \\
\text { Petroleum Gases }\end{array}$} & \multirow[b]{2}{*}{ Lubricants } & \multirow{2}{*}{$\begin{array}{c}\text { Motor } \\
\text { Gasoline }\end{array}$} & \multirow{2}{*}{$\begin{array}{l}\text { Residual } \\
\text { Fuel Oil }\end{array}$} & \multirow[b]{2}{*}{ Total } \\
\hline & & & Kerosene Type & Total $^{2}$ & & & & & \\
\hline 1949 & 93 & 190 & 0 & $\left({ }^{2}\right)$ & 1 & 54 & 2,241 & 504 & 3,084 \\
\hline 1950 & 108 & 226 & 0 & $\left({ }^{2}\right)$ & 2 & 64 & 2,433 & 524 & 3,356 \\
\hline 1955 & 192 & 372 & 0 & 154 & 9 & 70 & 3,221 & 440 & 4,458 \\
\hline 1960 & 161 & 418 & 91 & 371 & 13 & 68 & 3,736 & 367 & 5,135 \\
\hline 1965 & 120 & 514 & 334 & 602 & 23 & 67 & 4,374 & 336 & 6,036 \\
\hline 1970 & 55 & 738 & 718 & 967 & 32 & 66 & 5,589 & 332 & 7,778 \\
\hline 1975 & 39 & 998 & 782 & 992 & 31 & 70 & 6,512 & 310 & 8,951 \\
\hline 1976 & 37 & 1,073 & 777 & 976 & 33 & 77 & 6,817 & 358 & 9,372 \\
\hline 1977 & 38 & 1,171 & 814 & 1,022 & 36 & 78 & 7,022 & 396 & 9,761 \\
\hline 1978 & 39 & 1,260 & 845 & 1,044 & 38 & 83 & 7,264 & 431 & 10,160 \\
\hline 1979 & 38 & 1,366 & 867 & 1,067 & 16 & 87 & 6,896 & 535 & 10,005 \\
\hline 1980 & 35 & 1,311 & 845 & 1,062 & 13 & 77 & 6,441 & 608 & 9,546 \\
\hline 1981 & 31 & 1,365 & 808 & 1,006 & 24 & 74 & 6,456 & 531 & 9,487 \\
\hline 1982 & 25 & 1,312 & 803 & 1,011 & 24 & 68 & 6,421 & 444 & 9,307 \\
\hline 1983 & 26 & 1,367 & 839 & 1,046 & 29 & 71 & 6,510 & 358 & 9,406 \\
\hline 1984 & 24 & 1,383 & 953 & 1,175 & 30 & 76 & 6,554 & 351 & 9,592 \\
\hline 1985 & 27 & 1,491 & 1,005 & 1,218 & 21 & 71 & 6,667 & 342 & 9,838 \\
\hline 1986 & 32 & 1,514 & 1,105 & 1,307 & 19 & 69 & 6,871 & 379 & 10,191 \\
\hline 1987 & 25 & 1,568 & 1,181 & 1,385 & 15 & 78 & 7,041 & 392 & 10,505 \\
\hline 1988 & 27 & 1,701 & 1,236 & $\begin{array}{l}1,449 \\
\text { S }\end{array}$ & 17 & 75 & 7,179 & 399 & 10,846 \\
\hline 1989 & 26 & 1,734 & 1,284 & 1,489 & 16 & 77 & 7,171 & 423 & 10,937 \\
\hline 1990 & 24 & 1,722 & 1,340 & 1,522 & 16 & 80 & 7,080 & 443 & 10,888 \\
\hline 1991 & 23 & 1,694 & 1,296 & 1,471 & 15 & 71 & 7,042 & 447 & 10,763 \\
\hline 1992 & 22 & 1,728 & 1,310 & 1,454 & 14 & 72 & 7,125 & 465 & 10,881 \\
\hline 1993 & 21 & 1,785 & 1,357 & 1,469 & 14 & 74 & 7,367 & 393 & 11,124 \\
\hline 1994 & 21 & 1,896 & 1,480 & 1,527 & 24 & 77 & 7,487 & 385 & 11,417 \\
\hline 1995 & 21 & 1,973 & 1,497 & 1,514 & 13 & 76 & 7,674 & 397 & 11,668 \\
\hline 1996 & 20 & $\begin{array}{l}2,096 \\
\end{array}$ & 1,575 & 1,578 & 11 & 73 & 7,772 & 370 & $\begin{array}{l}11,921 \\
\text { - }\end{array}$ \\
\hline 1997 & 22 & 2,198 & 1,598 & 1,599 & 10 & 78 & 7,883 & 310 & 12,099 \\
\hline 1998 & 19 & 2,263 & 1,623 & 1,622 & 13 & 81 & 8,128 & 294 & 12,420 \\
\hline 1999 & 21 & 2,352 & 1,675 & 1,673 & 10 & 82 & 8,336 & 290 & 12,765 \\
\hline 2000 & 20 & 2,422 & 1,725 & 1,725 & 8 & 81 & 8,370 & 386 & 13,012 \\
\hline 2001 & 19 & 2,489 & 1,656 & 1,655 & 10 & 74 & 8,435 & 255 & 12,938 \\
\hline 2002 & 18 & 2,536 & 1,621 & 1,614 & 10 & 73 & 8,662 & 295 & 13,208 \\
\hline 2003 & 16 & 2,665 & 1,578 & 1,578 & 12 & 68 & 8,733 & 249 & 13,321 \\
\hline 2004 & 17 & 2,783 & 1,630 & 1,630 & 14 & 69 & 8,887 & 321 & 13,720 \\
\hline 2005 & 19 & 2,858 & 1,679 & 1,679 & 20 & 68 & 8,948 & 365 & 13,957 \\
\hline 2006 & 18 & 3,017 & 1,633 & 1,633 & 20 & 67 & 9,029 & 395 & 14,178 \\
\hline 2007 & 17 & 3,037 & 1,622 & 1,622 & 16 & 69 & 9,093 & 433 & 14,287 \\
\hline 2008 & 15 & 2,824 & 1,539 & 1.539 & 29 & 64 & 8,834 & 400 & 13,704 \\
\hline 2009 & 14 & 2,600 & 1,393 & 1,393 & 20 & 57 & 8,840 & 353 & 13,279 \\
\hline 2010 & 15 & $R_{2,737}$ & $\mathrm{R}_{1,432}$ & $R_{1,432}$ & 21 & $\mathrm{R}_{64}$ & $\mathrm{R}_{8,816}$ & R382 & $R_{13,466}$ \\
\hline $2011^{P}$ & 15 & 2,779 & 1,425 & 1,425 & 21 & 60 & 8,565 & 359 & 13,223 \\
\hline
\end{tabular}

Beginning in 2009, includes renewable diesel fuel (including biodiesel) blended into distillate fuel oil. 2 Through 1951, naphtha-type jet fuel is included in the products from which jet fuel was blended: in 1952, 71 percent gasoline, 17 percent kerosene, and 12 percent distillate fuel oil. Beginning in 1952,
includes naphtha-type jet fuel. Beginning in 1957, also includes kerosene-type jet fuel. Beginning in 2005, includes kerosene-type jet fuel only.

Finished motor gasoline. Through 1963, also includes special naphthas. Beginning in 1993, also includes ethanol blended into motor gasoline.

$R=$ Revised. $P=$ Preliminary.

tion by all sectors, see petroleum products supplied data in Table

5.11. For petroleum, product supplied is used as an approximation of petroleum consumption. See Note 1 ,
"Petroleum Products Supplied and Petroleum Consumption," at end of section. • Totals may not equal sum of components due to independent rounding.

Web Pages: - See http://www.eia.gov/totalenergy/data/monthly/\#petroleum for updated monthly and annual data. - See http://www.eia.gov/totalenergy/data/annual/\#petroleum for all annual data beginning in 1949. - See http://www.eia.gov/states/_seds.html for related information.

Sources. - 1949-1959-Bureau of Mines, Mineral Industry Surveys, Petroleum Statement, Annual, annual reports, and U.S. Energy Information Administration (EIA) estimates. • 1960-1972-EIA, "State Energy Data 2010: Consumption" (June 2012), U.S. Table CT7. • 1973 forward-EIA, Monthly Energy
Review (April 2012), Table 3.7c. 
Table 5.13d Petroleum Consumption Estimates: Electric Power Sector, Selected Years, 1949-2011

(Thousand Barrels per Day)

\begin{tabular}{|c|c|c|c|c|c|c|c|c|c|c|c|c|}
\hline \multirow[b]{3}{*}{ Year } & \multicolumn{12}{|c|}{ Electric Power Sector ${ }^{1}$} \\
\hline & \multicolumn{4}{|c|}{ Electricity Only } & \multicolumn{4}{|c|}{ Combined Heat and Power (CHP) } & \multicolumn{4}{|c|}{ Total } \\
\hline & $\begin{array}{l}\text { Distillate } \\
\text { Fuel Oil }^{2}\end{array}$ & $\begin{array}{l}\text { Petroleum } \\
\text { Coke }\end{array}$ & $\begin{array}{l}\text { Residual } \\
\text { Fuel Oil }^{3}\end{array}$ & Total & $\begin{array}{l}\text { Distillate } \\
\text { Fuel Oil }^{2}\end{array}$ & $\begin{array}{l}\text { Petroleum } \\
\text { Coke }\end{array}$ & $\begin{array}{l}\text { Residual } \\
\text { Fuel Oil }^{3}\end{array}$ & Total & $\begin{array}{l}\text { Distillate } \\
\text { Fuel Oil }^{2}\end{array}$ & $\begin{array}{l}\text { Petroleum } \\
\text { Coke }\end{array}$ & $\begin{array}{l}\text { Residual } \\
\text { Fuel Oil }^{3}\end{array}$ & Total \\
\hline 1949 & 13 & NA & 169 & 182 & NA & NA & NA & NA & 13 & NA & 169 & 182 \\
\hline 1950 & 15 & NA & 192 & 207 & NA & NA & NA & NA & 15 & NA & 192 & 207 \\
\hline 1955 & 15 & NA & 191 & 206 & NA & NA & NA & NA & 15 & NA & 191 & 206 \\
\hline 1960 & 10 & NA & 231 & 241 & NA & NA & NA & NA & 10 & NA & 231 & 241 \\
\hline 1965 & 14 & NA & 302 & 316 & NA & NA & NA & NA & 14 & NA & 302 & 316 \\
\hline 1970 & 66 & 9 & 853 & 928 & NA & NA & NA & NA & 66 & 9 & 853 & 928 \\
\hline 1975 & 107 & 1 & 1,280 & 1,388 & NA & NA & NA & NA & 107 & 1 & 1,280 & 1,388 \\
\hline 1976 & 114 & 1 & $\begin{array}{l}1,405 \\
\end{array}$ & 1,520 & NA & NA & NA & NA & 114 & 1 & 1,405 & 1,520 \\
\hline 1977 & 134 & 1 & 1,575 & 1,710 & NA & NA & NA & NA & 134 & 1 & 1,575 & 1,710 \\
\hline 1978 & 130 & 5 & 1,612 & 1,747 & NA & NA & NA & NA & 130 & 5 & 1,612 & 1,747 \\
\hline 1979 & 84 & 4 & 1,350 & 1,437 & NA & NA & $N A$ & NA & 84 & 4 & 1,350 & 1,437 \\
\hline 1980 & 79 & 2 & 1,069 & 1,151 & NA & NA & NA & NA & 79 & 2 & 1,069 & 1,151 \\
\hline 1981 & 58 & 2 & 904 & 964 & NA & NA & NA & NA & 58 & 2 & 904 & 964 \\
\hline 1982 & 42 & 2 & 642 & 686 & NA & NA & NA & NA & 42 & 2 & 642 & 686 \\
\hline 1983 & 45 & 4 & 627 & 676 & NA & NA & NA & NA & 45 & 4 & 627 & 676 \\
\hline 1984 & 42 & 3 & 517 & 562 & NA & NA & NA & NA & 42 & 3 & 517 & 562 \\
\hline 1985 & 40 & 3 & 435 & 478 & NA & NA & NA & NA & 40 & 3 & 435 & 478 \\
\hline 1986 & 39 & 4 & 592 & 636 & NA & NA & NA & NA & 39 & 4 & 592 & 636 \\
\hline 1987 & 42 & 5 & 504 & 551 & NA & NA & NA & NA & 42 & 5 & 504 & 551 \\
\hline 1988 & 51 & 6 & 627 & 683 & NA & NA & NA & NA & 51 & 6 & 627 & 683 \\
\hline $1989^{4}$ & 70 & 7 & 663 & 740 & 2 & 0 & 6 & 8 & 72 & 7 & 669 & 748 \\
\hline 1990 & 41 & 14 & 497 & 551 & 4 & 0 & 10 & 15 & 45 & 14 & 507 & 566 \\
\hline 1991 & 38 & 13 & 469 & 520 & 1 & 0 & 4 & 5 & 39 & 13 & 473 & 526 \\
\hline 1992 & 33 & 18 & 371 & 422 & 2 & 2 & 8 & 12 & 34 & 20 & 379 & 434 \\
\hline 1993 & 37 & 21 & 409 & 467 & 4 & 15 & 9 & 27 & 41 & 36 & 418 & 494 \\
\hline 1994 & 46 & 16 & 369 & 431 & 11 & 15 & 10 & 36 & 56 & 32 & 379 & 467 \\
\hline 1995 & 44 & 15 & 237 & 296 & 7 & 22 & 9 & 38 & 51 & 37 & 247 & 334 \\
\hline 1996 & 47 & 14 & 263 & 325 & 4 & 22 & 10 & 36 & 51 & 36 & 273 & 360 \\
\hline 1997 & 48 & 23 & 301 & 373 & 4 & 23 & 10 & 37 & 52 & 46 & 311 & 410 \\
\hline 1998 & 61 & 30 & 448 & 539 & 3 & 26 & 8 & 37 & 64 & 56 & 456 & 576 \\
\hline 1999 & 63 & 26 & 409 & 497 & 3 & 25 & 9 & 38 & 66 & 51 & 418 & 535 \\
\hline 2000 & 77 & 20 & 370 & 466 & 6 & 25 & 8 & 39 & 82 & 45 & 378 & 505 \\
\hline 2001 & 76 & 25 & 430 & 531 & 4 & 22 & 7 & 33 & 80 & 47 & 437 & 564 \\
\hline 2002 & 59 & 54 & 281 & 394 & 1 & 26 & 6 & 33 & 60 & 80 & 287 & 427 \\
\hline 2003 & 71 & 66 & 373 & 510 & 5 & 14 & 6 & 24 & 76 & 79 & 379 & 534 \\
\hline 2004 & 49 & 83 & 376 & 509 & 3 & 17 & 6 & 26 & 52 & 101 & 382 & 535 \\
\hline 2005 & 51 & 94 & 376 & 521 & 3 & 17 & 6 & 26 & 54 & 111 & 382 & 547 \\
\hline 2006 & 34 & 82 & 151 & 267 & 1 & 15 & 6 & 22 & 35 & 97 & 157 & 289 \\
\hline 2007 & $\begin{array}{l}44 \\
40\end{array}$ & $\begin{array}{l}02 \\
65\end{array}$ & 167 & 272 & 2 & 13 & $\begin{array}{l}0 \\
6\end{array}$ & 21 & 42 & 78 & 173 & $\begin{array}{l}295 \\
293\end{array}$ \\
\hline 2008 & 33 & 58 & 99 & 189 & 2 & 12 & 6 & 19 & 34 & 70 & 104 & 209 \\
\hline 2009 & $\begin{array}{l}50 \\
32\end{array}$ & 50 & 73 & 154 & 1 & 13 & 6 & 21 & $\begin{array}{l}54 \\
33\end{array}$ & 63 & 79 & 175 \\
\hline 2010 & $\mathrm{R}_{37}$ & $\mathrm{R}_{61}$ & 62 & $\mathrm{R}_{159}$ & 1 & $\mathrm{R}_{4}$ & 6 & $\mathrm{R}_{11}$ & R38 & 65 & $R_{67}$ & 170 \\
\hline $2011^{P}$ & 28 & 56 & 35 & 119 & 1 & 5 & 6 & 11 & 29 & 60 & 41 & 130 \\
\hline
\end{tabular}

1 Electricity-only and combined-heat-and-power (CHP) plants within the NAICS 22 category whose primary business is to sell electricity, or electricity and heat, to the public. Electric utility CHP plants are included in "Electricity Only."

Fuel oil nos. 1, 2, and 4. For 1949-1979, data are for gas turbine and internal combustion plant use of petroleum. For $1980-2000$, electric utility data also include small amounts of kerosene and jet fuel.

${ }^{3}$ Fuel oil nos. 5 and 6. For 1949-1979, data are for steam plant use of petroleum. For 1980-2000

lectric utility data also include a small amount of fuel oll no. 4 .

4 Through 1988, data are for electric utilities only. Beginning in 1989, data are for electric utilities and R=Revised. P=Producers.

Notes: - For total petroleum consumption by all sectors, see petroleum products supplied data in Table 5.11. See Note 1, "Petroleum Products Supplied and Petroleum Consumption," at end of section. • See Tables $8.5 \mathrm{a}-8.5 \mathrm{~d}$ for the amount of petroleum used to produce electricity and Tables $8.6 \mathrm{a}-8.6 \mathrm{c}$ for the amount of petroleum used to produce useful thermal output. - Totals may not equal sum of components due to independent rounding.

Web Pages: - See http://www.eia.gov/totalenergy/data/monthly/\#petroleum for updated monthly and

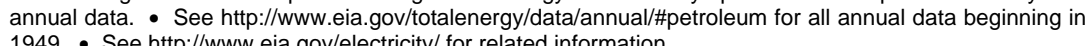

949. - See hitp://www.eia.gov/electricity/ for related information. 
Figure 5.14 Heat Content of Petroleum Consumption Estimates by Product by Sector, 1949-2011

\section{Residential and Commercial ${ }^{1}$ Sectors, Selected Products}

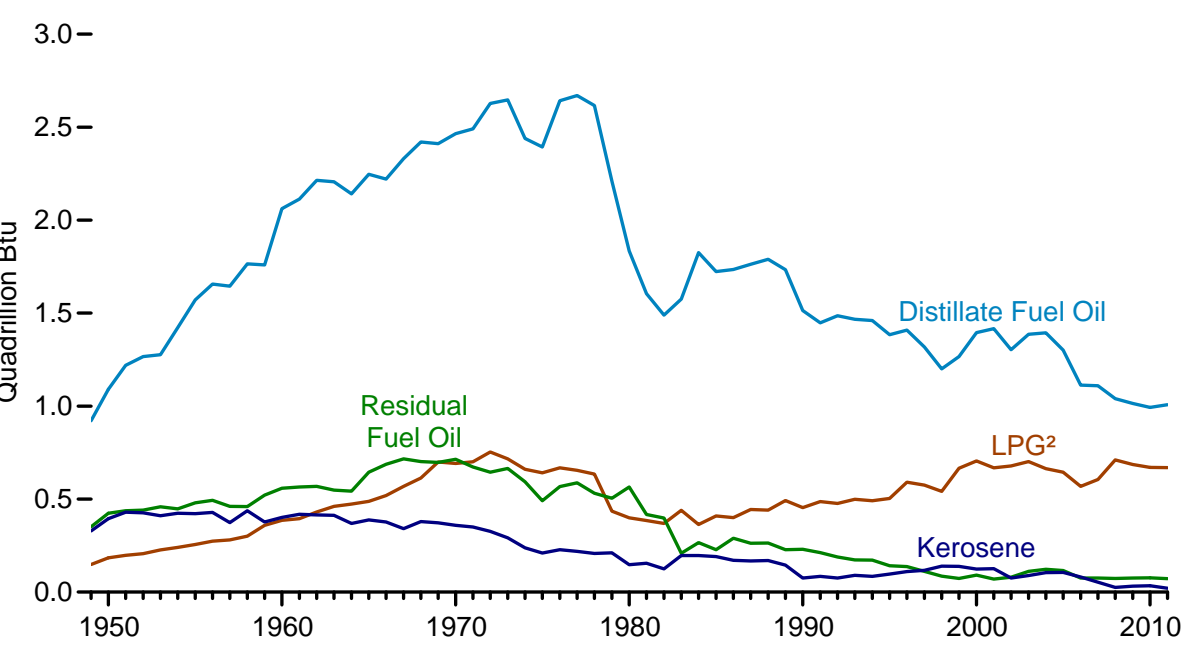

\section{Transportation Sector, Selected Products}

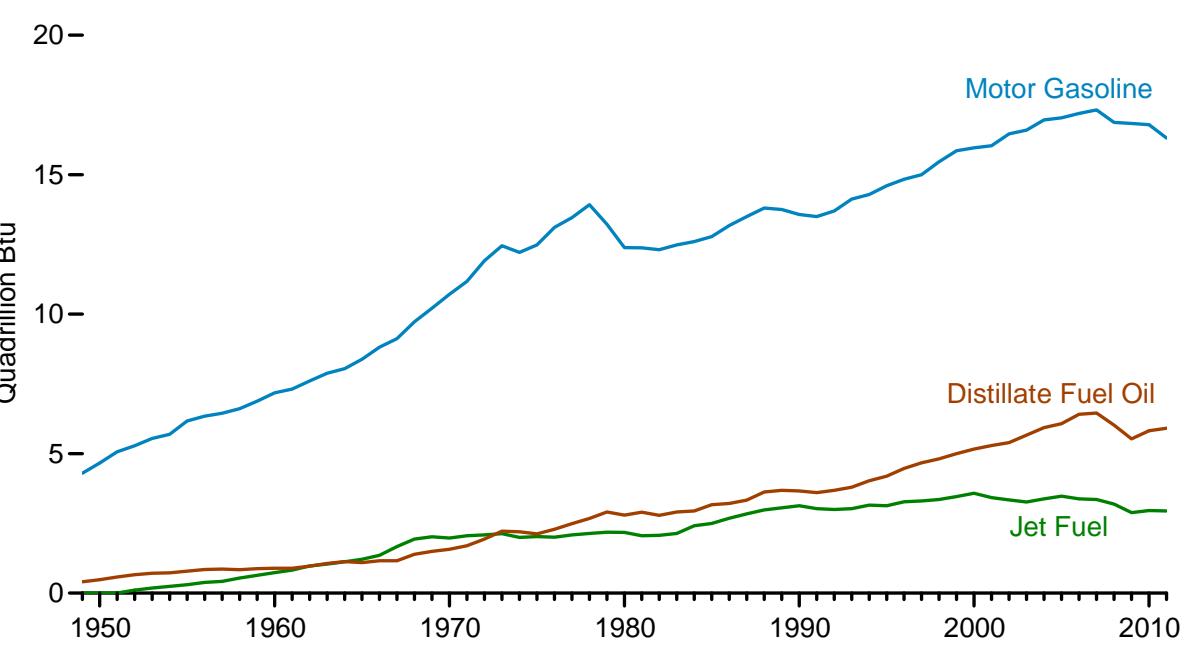

Industrial ${ }^{1}$ Sector, Selected Products

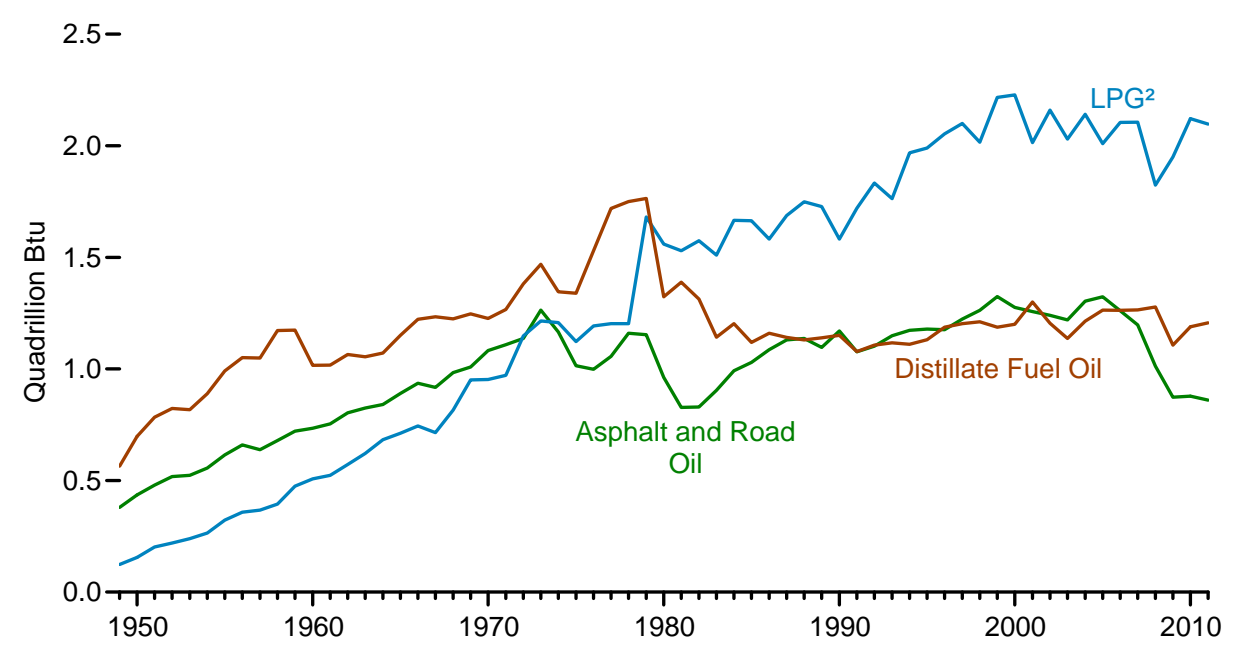

Electric Power Sector ${ }^{3}$

5-

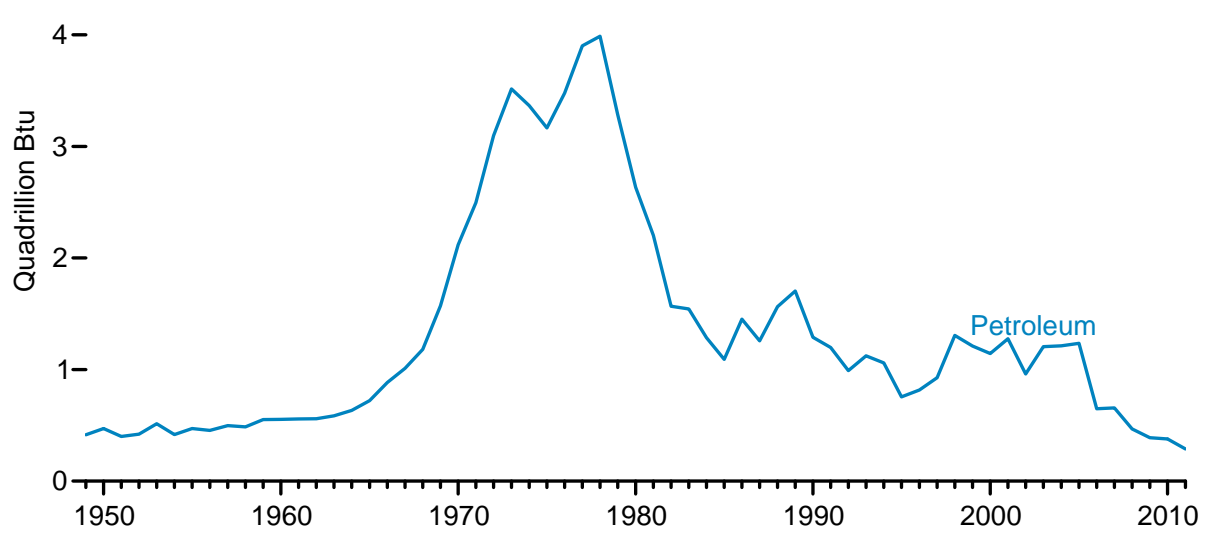

${ }^{1}$ Includes combined-heat-and-power plants and a small number of electricity-only plants. ${ }^{2}$ Liquefied petroleum gases.

${ }^{3}$ Electricity-only and combined-heat-and-power plants whose primary business is to sell electricity, or electricity and heat, to the public. Sources: Tables 5.14a-5.14c. 
Table 5.14a Heat Content of Petroleum Consumption Estimates: Residential and Commercial Sectors, Selected Years, 1949-2011 (Trillion Btu)

\begin{tabular}{|c|c|c|c|c|c|c|c|c|c|c|c|}
\hline \multirow[b]{2}{*}{ Year } & \multicolumn{4}{|c|}{ Residential Sector } & \multicolumn{7}{|c|}{ Commercial Sector } \\
\hline & $\begin{array}{l}\text { Distillate } \\
\text { Fuel Oil }\end{array}$ & Kerosene & $\begin{array}{l}\text { Liquefied } \\
\text { Petroleum } \\
\text { Gases }\end{array}$ & Total & $\begin{array}{l}\text { Distillate } \\
\text { Fuel Oil }\end{array}$ & Kerosene & $\begin{array}{l}\text { Liquefied } \\
\text { Petroleum } \\
\text { Gases }\end{array}$ & $\begin{array}{c}\text { Motor } \\
\text { Gasoline }\end{array}$ & $\begin{array}{l}\text { Petroleum } \\
\text { Coke }\end{array}$ & $\begin{array}{l}\text { Residual } \\
\text { Fuel Oil }\end{array}$ & Total \\
\hline 1949 & 700 & 289 & 117 & 1,106 & 221 & 39 & 31 & 92 & NA & 351 & 735 \\
\hline 1950 & 829 & 347 & 146 & 1,322 & 262 & 47 & 39 & 100 & NA & 424 & 872 \\
\hline 1955 & 1,194 & 371 & 202 & 1,767 & 377 & 51 & 54 & 133 & NA & 480 & 1,095 \\
\hline 1960 & 1,568 & 354 & 305 & 2,227 & 494 & 48 & 81 & 67 & NA & 559 & 1,248 \\
\hline 1965 & 1,713 & 334 & 385 & 2,432 & 534 & 54 & 103 & 77 & NA & 645 & 1,413 \\
\hline 1970 & 1,878 & 298 & 549 & 2,725 & 587 & 61 & 143 & 86 & NA & 714 & 1,592 \\
\hline 1975 & 1,807 & 161 & 512 & 2,479 & 587 & 49 & 129 & 89 & NA & 492 & 1,346 \\
\hline 1976 & 1,987 & 184 & 532 & 2,703 & 656 & 44 & 136 & 97 & NA & 567 & 1,500 \\
\hline 1977 & 1,994 & 167 & 520 & 2,681 & 676 & 52 & 135 & 101 & NA & 588 & 1,552 \\
\hline 1978 & 1,951 & 153 & 504 & 2,607 & 666 & 55 & 131 & 107 & NA & 532 & 1,490 \\
\hline 1979 & 1,626 & 133 & 340 & 2,099 & 584 & 78 & 95 & 104 & NA & 505 & 1,367 \\
\hline 1980 & 1,316 & 107 & 311 & 1,734 & 518 & 41 & 88 & 107 & NA & 565 & 1,318 \\
\hline 1981 & 1,147 & 85 & 299 & 1,531 & 457 & 69 & 87 & 92 & NA & 417 & 1,122 \\
\hline 1982 & 1,050 & 95 & 289 & 1,434 & 440 & 30 & 81 & 88 & NA & 399 & 1,037 \\
\hline 1983 & 924 & 85 & 344 & 1,353 & 651 & 111 & 96 & 102 & NA & 208 & 1,170 \\
\hline 1984 & 1,091 & 160 & 280 & 1,531 & 735 & 36 & 83 & 107 & NA & 266 & 1,227 \\
\hline 1985 & 1,092 & 159 & 314 & 1,565 & 631 & 33 & 95 & 96 & NA & 228 & 1,083 \\
\hline 1986 & 1,111 & 121 & 308 & 1,541 & 623 & 50 & 93 & 106 & NA & 290 & 1,162 \\
\hline 1987 & 1,156 & 119 & 342 & 1,617 & 607 & 49 & 102 & 111 & NA & 263 & 1,131 \\
\hline 1988 & 1,190 & 144 & 341 & 1,675 & 600 & 26 & 99 & 110 & NA & 264 & 1,099 \\
\hline 1989 & 1,160 & 117 & 383 & 1,660 & 574 & 28 & 109 & 102 & 0 & 228 & 1,041 \\
\hline 1990 & 978 & 64 & 352 & 1,394 & 536 & 12 & 102 & 111 & 0 & 230 & 991 \\
\hline 1991 & 930 & 72 & 378 & 1,381 & 517 & 12 & 108 & 85 & 0 & 212 & 935 \\
\hline 1992 & 980 & 65 & 369 & 1,414 & 507 & 11 & 107 & 80 & (s) & 189 & 893 \\
\hline 1993 & 974 & 76 & 390 & 1,439 & 493 & 14 & 109 & 30 & (s) & 173 & 819 \\
\hline 1994 & 960 & 65 & 384 & 1,408 & 501 & 19 & 107 & 25 & (s) & 172 & 825 \\
\hline 1995 & 905 & 74 & 395 & 1,374 & 479 & 22 & 109 & 18 & (s) & 141 & 769 \\
\hline 1996 & 926 & 89 & 469 & 1,484 & 483 & 21 & 122 & 27 & (s) & 137 & 790 \\
\hline 1997 & 874 & 93 & 455 & 1,422 & 444 & 25 & 120 & 43 & (s) & 111 & 743 \\
\hline 1998 & 772 & 108 & 424 & 1,304 & 429 & 31 & 118 & 39 & (s) & 85 & 702 \\
\hline 1999 & 828 & 111 & 526 & 1,465 & 438 & 27 & 140 & 28 & (s) & 73 & 707 \\
\hline 2000 & 905 & 95 & 555 & 1,554 & 491 & 30 & 150 & 45 & (s) & 92 & 807 \\
\hline 2001 & 908 & 95 & 526 & 1,529 & 508 & 31 & 143 & 37 & (s) & 70 & 790 \\
\hline 2002 & 860 & 60 & 537 & 1,457 & 444 & 16 & 141 & 45 & (s) & 80 & 726 \\
\hline 2003 & 905 & 70 & 544 & 1,519 & 481 & 19 & 157 & 60 & (s) & 111 & 828 \\
\hline 2004 & 924 & 85 & 512 & 1,520 & 470 & 20 & 152 & 45 & (s) & 122 & 810 \\
\hline 2005 & 854 & 84 & 513 & 1,451 & 447 & 22 & 131 & 46 & (s) & 116 & 762 \\
\hline 2006 & 712 & 66 & 446 & 1,224 & 401 & 15 & 123 & 49 & (s) & 75 & 664 \\
\hline 2007 & 726 & 44 & 484 & 1,254 & 384 & 9 & 121 & 61 & (s) & 75 & 651 \\
\hline 2008 & 669 & 21 & 553 & 1,243 & 372 & 4 & 158 & 46 & (s) & 73 & 653 \\
\hline 2009 & 602 & 28 & 547 & 1,176 & 413 & 4 & 139 & 53 & (s) & 76 & 685 \\
\hline 2010 & R583 & $\mathrm{R}_{29}$ & R530 & $\mathrm{R}_{1,142}$ & $\mathrm{R}_{410}$ & 5 & $R_{140}$ & R55 & (s) & R77 & R688 \\
\hline $2011^{P}$ & 592 & 18 & 530 & 1,139 & 417 & 3 & 140 & 54 & (s) & 73 & 686 \\
\hline
\end{tabular}

1 Finished motor gasoline. Through 1963, also includes special naphthas. Beginning in 1993, also includes ethanol blended into motor gasoline.

$R=$ Revised. P=Preliminary. NA=Not available. $(\mathrm{s})=$ Less than 0.5 trillion Btu.

Notes: - Data are estimates. - For total heat content of petroleum consumption by all sectors, see data for heat content of petroleum products supplied in Table 5.12. For petroleum, product supplied is used as
an approximation of petroleum consumption. See Note 1, "Petroleum Products Supplied and Petroleum
Consumption," at end of Section. - Totals may not equal sum of components due to independent rounding.

Web Pages: - See http://www.eia.gov/totalenergy/data/monthly/\#petroleum for updated monthly and annual data. - See http://www.eia.gov/totalenergy/data/annual/\#petroleum for all annual data beginning in - See http://www.eia.gov/states/_seds.html for related information. 
Table 5.14b Heat Content of Petroleum Consumption Estimates: Industrial Sector, Selected Years, 1949-2011

(Trillion Btu)

\begin{tabular}{|c|c|c|c|c|c|c|c|c|c|c|}
\hline \multirow[b]{2}{*}{ Year } & \multicolumn{10}{|c|}{ Industrial Sector } \\
\hline & $\begin{array}{l}\text { Asphalt } \\
\text { and } \\
\text { Road Oil }\end{array}$ & $\begin{array}{l}\text { Distillate } \\
\text { Fuel Oil }\end{array}$ & Kerosene & $\begin{array}{c}\text { Liquefied } \\
\text { Petroleum } \\
\text { Gases }\end{array}$ & Lubricants & $\begin{array}{c}\text { Motor } \\
\text { Gasoline } 1\end{array}$ & Petroleum Coke & $\begin{array}{l}\text { Residual } \\
\text { Fuel Oil }\end{array}$ & $\begin{array}{c}\text { Other } \\
\text { Petroleum } 2\end{array}$ & Total \\
\hline 1949 & 380 & 564 & 254 & 123 & 80 & 231 & 87 & 1,225 & 530 & 3,475 \\
\hline 1950 & 435 & 698 & 274 & 156 & 94 & 251 & 90 & 1,416 & 546 & 3,960 \\
\hline 1955 & 615 & 991 & 241 & 323 & 103 & 332 & 147 & 1,573 & 798 & 5,123 \\
\hline 1960 & 734 & 1,016 & 161 & 507 & 107 & 381 & 328 & 1,584 & 947 & 5,766 \\
\hline 1965 & 890 & 1,150 & 165 & 712 & 137 & 342 & 444 & 1,582 & 1,390 & 6,813 \\
\hline 1970 & 1,082 & 1,226 & 185 & 953 & 155 & 288 & 446 & 1,624 & 1,817 & 7,776 \\
\hline 1975 & 1,014 & 1,339 & 119 & 1,123 & 149 & 223 & 540 & 1,509 & $\begin{array}{l}2,109 \\
\end{array}$ & 8,127 \\
\hline 1976 & 998 & 1,530 & 123 & 1,192 & 166 & 211 & 535 & 1,822 & 2,413 & 8,990 \\
\hline 1977 & $\begin{array}{r}590 \\
1.056\end{array}$ & $\begin{array}{l}1,000 \\
1,719\end{array}$ & 143 & 1,203 & 182 & 196 & 586 & $\begin{array}{l}1,0<2 \\
1,937\end{array}$ & $\begin{array}{l}2,410 \\
2,724\end{array}$ & $\begin{array}{l}9,990 \\
9,747\end{array}$ \\
\hline 1978 & 1,160 & 1,750 & 156 & 1,203 & 195 & 178 & 550 & 1,716 & 2,928 & 9,835 \\
\hline 1979 & 1,153 & 1,764 & 177 & 1,681 & 204 & 162 & 533 & 1,655 & 3,217 & 10,548 \\
\hline 1980 & 962 & 1,324 & 181 & $\begin{array}{l}1,001 \\
1,559\end{array}$ & 182 & 158 & 516 & $\begin{array}{l}1,349 \\
1,340\end{array}$ & 3,278 & $\begin{array}{r}9,509 \\
9,509\end{array}$ \\
\hline 1981 & 828 & 1,389 & 108 & 1,530 & 175 & 160 & 549 & 1,081 & 2,446 & 8,265 \\
\hline 1982 & 829 & 1,313 & 141 & 1,575 & 159 & 138 & 541 & 1,047 & 2,030 & 7,772 \\
\hline 1983 & 904 & 1,142 & 66 & 1,510 & 167 & 112 & 495 & 791 & 2,202 & 7,390 \\
\hline 1984 & 992 & 1,203 & 43 & 1,666 & 178 & 160 & 538 & 889 & 2,319 & 7,987 \\
\hline 1985 & 1,029 & 1,119 & 44 & 1,664 & 166 & 218 & 575 & 748 & 2,152 & 7,714 \\
\hline 1986 & 1,086 & 1,160 & 32 & $\begin{array}{l}1,582 \\
\end{array}$ & 162 & 206 & 581 & 736 & 2,315 & 7,860 \\
\hline 1987 & 1,130 & 1,141 & 28 & 1,687 & 183 & 206 & 646 & 582 & 2,439 & 8,042 \\
\hline 1988 & 1,136 & 1,130 & 30 & 1,749 & 177 & 193 & 675 & 546 & 2,682 & 8,317 \\
\hline 1989 & 1,096 & 1,139 & 30 & 1,728 & 181 & 199 & 660 & 410 & $\begin{array}{l}2,656 \\
\end{array}$ & 8,098 \\
\hline 1990 & 1,170 & 1,150 & 12 & 1,582 & 186 & 185 & 714 & 411 & 2,839 & 8,251 \\
\hline 1991 & 1,077 & 1,078 & 11 & 1,720 & 167 & 193 & 693 & 334 & 2,685 & 7.958 \\
\hline 1992 & 1,102 & 1,107 & 10 & 1,833 & 170 & 194 & 798 & 387 & 2,951 & 8,552 \\
\hline 1993 & 1,149 & 1,117 & 13 & 1,763 & 173 & 180 & 725 & 446 & 2,822 & 8,388 \\
\hline 1994 & 1,173 & 1,111 & 17 & 1,969 & 181 & 192 & 723 & 419 & 2,988 & 8,773 \\
\hline 1995 & 1,178 & 1,131 & 15 & $\begin{array}{l}1,990 \\
\text { S }\end{array}$ & 178 & 200 & 721 & 337 & 2,837 & 8,588 \\
\hline 1996 & 1,176 & 1,187 & 18 & 2,054 & 173 & 200 & 757 & 335 & 3,121 & 9,020 \\
\hline 1997 & 1,224 & 1,203 & 19 & $\begin{array}{l}2,004 \\
2,100\end{array}$ & 182 & 212 & 727 & 291 & 3,298 & 9,256 \\
\hline 1998 & 1,263 & 1,211 & 22 & 2,016 & 191 & 199 & 858 & 230 & 3,093 & 9,083 \\
\hline 1999 & 1,324 & 1,187 & 13 & 2,217 & 193 & 152 & 936 & 207 & $\begin{array}{l}3,129 \\
\end{array}$ & 9,357 \\
\hline 2000 & 1,276 & 1,200 & 16 & 2,228 & 190 & 150 & 796 & 241 & 2,979 & 9,076 \\
\hline 2001 & 1,257 & 1,300 & 23 & 2,014 & 174 & 295 & 858 & 203 & 3,056 & 9,181 \\
\hline 2002 & 1.240 & 1,204 & 14 & 2,160 & 172 & 309 & 842 & 190 & $\begin{array}{l}3,040 \\
3,040\end{array}$ & 9,171 \\
\hline 2003 & 1,220 & 1,136 & 24 & 2,030 & 159 & 324 & 825 & 220 & 3.264 & 9,202 \\
\hline 2004 & 1,304 & 1,214 & 28 & 2,141 & 161 & 372 & 934 & 249 & 3,428 & 9,831 \\
\hline 2005 & $\begin{array}{l}1,004 \\
1,323\end{array}$ & $\begin{array}{l}1,264 \\
1,24\end{array}$ & $\begin{array}{l}<0 \\
39\end{array}$ & $\begin{array}{l}2,141 \\
2,009\end{array}$ & 160 & $\begin{array}{l}356 \\
356\end{array}$ & $\begin{array}{l}584 \\
889\end{array}$ & 281 & $\begin{array}{l}3,4<0 \\
3,318\end{array}$ & $\begin{array}{l}9,640 \\
9,640\end{array}$ \\
\hline 2006 & 1,261 & 1,263 & 30 & 2,104 & 156 & 376 & 934 & 239 & 3,416 & 9,780 \\
\hline 2007 & 1,197 & 1,265 & 13 & 2,106 & 161 & 306 & 906 & 193 & 3,313 & 9,461 \\
\hline 2008 & 1,012 & 1,277 & 4 & 1,823 & 150 & 250 & 868 & 199 & 2.941 & 8,523 \\
\hline 2009 & 873 & 1,107 & 4 & 1,950 & 135 & 244 & 799 & 106 & 2,611 & 7,829 \\
\hline 2010 & R878 & $R_{1,188}$ & R7 & $R_{2,121}$ & 149 & R281 & 682 & $\mathrm{R}_{120}$ & $R_{2,800}$ & $R_{8}, 227$ \\
\hline $2011^{P}$ & 860 & 1,207 & 4 & 2,097 & 141 & 273 & 674 & 113 & 2,712 & 8,081 \\
\hline
\end{tabular}

1 Finished motor gasoline. Through 1963, also includes special naphthas. Beginning in 1993, also includes ethanol blended into motor gasoline.

${ }^{2}$ Pentanes plus, petrochemical feedstocks, still gas (refinery gas), waxes, and miscellaneous products. Beginning in 1964, also includes special naphthas. Beginning in 1983, also includes crude oil burned as fuel.

$R=$ Revised. $P=$ Preliminary.

- For total heat content of petroleum consumption by all sectors, see data

an approximation of petroleum consumption. See Note 1, "Petroleum Products Supplied and Petroleum Consumption," at end of Section. - Totals may not equal sum of components due to independent rounding.

Web Pages: - See http://www.eia.gov/totalenergy/data/monthly/\#petroleum for updated monthly and

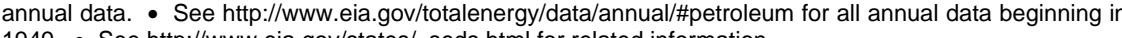
Sources: Tables 5.12, 5.13b, A1, and A3. 
Table 5.14c Heat Content of Petroleum Consumption Estimates: Transportation and Electric Power Sectors, Selected Years, 1949-2011 (Trillion Btu)

\begin{tabular}{|c|c|c|c|c|c|c|c|c|c|c|c|c|c|}
\hline \multirow[b]{3}{*}{ Year } & \multicolumn{9}{|c|}{ Transportation Sector } & \multicolumn{4}{|c|}{ Electric Power Sector ${ }^{1}$} \\
\hline & \multirow{2}{*}{$\begin{array}{l}\text { Aviation } \\
\text { Gasoline }\end{array}$} & \multirow[b]{2}{*}{$\begin{array}{l}\text { Distillate } \\
\text { Fuel Oil }{ }^{2}\end{array}$} & \multicolumn{2}{|c|}{ Jet Fuel } & \multirow{2}{*}{$\begin{array}{l}\text { Liquefied } \\
\text { Petroleum } \\
\text { Gases }\end{array}$} & \multirow[b]{2}{*}{ Lubricants } & \multirow[b]{2}{*}{$\begin{array}{c}\text { Motor } \\
\text { Gasoline }{ }^{4}\end{array}$} & \multirow[b]{2}{*}{$\begin{array}{l}\text { Residual } \\
\text { Fuel Oil }\end{array}$} & \multirow[b]{2}{*}{ Total } & \multirow{2}{*}{$\begin{array}{l}\text { Distillate } \\
\text { Fuel Oil }{ }^{5}\end{array}$} & \multirow[b]{2}{*}{$\begin{array}{l}\text { Petroleum } \\
\text { Coke }\end{array}$} & \multirow[b]{2}{*}{$\begin{array}{l}\text { Residual } \\
\text { Fuel Oil }{ }^{6}\end{array}$} & \multirow[b]{2}{*}{ Tota } \\
\hline & & & Kerosene Type & Total $^{3}$ & & & & & & & & & \\
\hline 1949 & 172 & 405 & 0 & 0 & 2 & 120 & 4,298 & 1,156 & 6,152 & 28 & NA & 387 & 415 \\
\hline 1950 & 199 & 480 & 0 & 0 & 3 & 141 & 4,664 & 1,201 & 6,690 & 32 & NA & 440 & 472 \\
\hline 1955 & 354 & 791 & 0 & 301 & 13 & 155 & 6,175 & 1,009 & 8,799 & 32 & NA & 439 & 471 \\
\hline 1960 & 298 & 892 & 188 & 739 & 19 & 152 & 7,183 & 844 & 10,125 & 22 & NA & 530 & 553 \\
\hline 1965 & 222 & 1,093 & 691 & 1,215 & 32 & 149 & 8,386 & 770 & 11,866 & 29 & NA & 693 & 722 \\
\hline 1970 & 100 & 1,569 & 1,486 & 1,973 & 44 & 147 & 10,716 & 761 & 15,310 & 141 & 19 & 1,958 & 2,117 \\
\hline 1975 & 71 & 2,121 & 1,619 & 2,029 & 43 & 155 & 12,485 & 711 & 17,615 & 226 & 2 & 2,937 & 3,166 \\
\hline 1976 & 67 & 2,288 & 1,613 & 2,002 & 47 & 172 & 13,107 & 824 & 18,508 & 243 & 2 & 3,232 & 3,477 \\
\hline 1977 & 70 & 2,489 & 1,684 & 2,090 & 50 & 172 & 13,464 & 908 & 19,243 & 283 & 3 & 3,614 & 3,901 \\
\hline 1978 & 71 & 2,679 & 1,750 & 2,138 & 54 & 184 & 13,927 & 990 & 20,044 & 276 & 12 & 3,699 & 3,987 \\
\hline 1979 & 70 & 2,905 & 1,795 & 2,186 & $\begin{array}{l}22 \\
22\end{array}$ & $\begin{array}{l}193 \\
193\end{array}$ & 13,221 & $\begin{array}{r}1,228 \\
\end{array}$ & 19,825 & 178 & 8 & $\begin{array}{l}3,097 \\
3,00\end{array}$ & 3,283 \\
\hline 1980 & 64 & 2,795 & 1,754 & 2,179 & 18 & 172 & 12,383 & 1,398 & 19,009 & 169 & 5 & 2,459 & 2,634 \\
\hline 1981 & 56 & 2,901 & 1,671 & 2,058 & 34 & 165 & 12,379 & 1,219 & 18,813 & 124 & 4 & 2,073 & 2,202 \\
\hline 1982 & 47 & 2,790 & 1,661 & 2,069 & 34 & 150 & 12,312 & 1,020 & 18,422 & 89 & 4 & 1,474 & 1,568 \\
\hline 1983 & 48 & 2,905 & 1,736 & 2,141 & 40 & 157 & 12,482 & 821 & 18,595 & 96 & 8 & 1,440 & 1,544 \\
\hline 1984 & 44 & 2,948 & $\begin{array}{l}1,977 \\
\text { a }\end{array}$ & 2,414 & 43 & 168 & 12,600 & 807 & 19,023 & 88 & 8 & 1,190 & 1,286 \\
\hline $\begin{array}{l}1904 \\
1985\end{array}$ & 50 & $\begin{array}{l}2,940 \\
3,170\end{array}$ & $\begin{array}{l}2,079 \\
2,019\end{array}$ & 2,497 & $\begin{array}{l}40 \\
30\end{array}$ & 156 & 12,784 & 786 & 19,472 & $\begin{array}{l}\circ 0 \\
85\end{array}$ & 7 & 998 & $\begin{array}{l}, 2000 \\
1,090\end{array}$ \\
\hline 1986 & 59 & 3,218 & 2,287 & 2,682 & 27 & 153 & 13,174 & 870 & 20,183 & 83 & 9 & 1,359 & 1,452 \\
\hline 1987 & 46 & 3,335 & 2,444 & 2,843 & 22 & 173 & 13,499 & 900 & 20,817 & 90 & 10 & 1,157 & 1,257 \\
\hline 1988 & 49 & 3,626 & 2,565 & 2,982 & 23 & 167 & 13,802 & 919 & 21,568 & 109 & 12 & 1,442 & 1,563 \\
\hline 1989 & 48 & 3,687 & 2,658 & 3,059 & 23 & 171 & 13,749 & 971 & 21,707 & 152 & 16 & 1,535 & 1,703 \\
\hline 1990 & 45 & $\begin{array}{l}3,001 \\
3,661\end{array}$ & 2,774 & $\begin{array}{l}3,129 \\
3,109\end{array}$ & 23 & 176 & 13,575 & 1,016 & 21,626 & 97 & 30 & 1,163 & $\begin{array}{l}1,289 \\
1,289\end{array}$ \\
\hline 1991 & 42 & 3,601 & 2,681 & 3,025 & 21 & 157 & 13,503 & 1,026 & 21,374 & 84 & 29 & 1,085 & 1,198 \\
\hline 1992 & 41 & 3,684 & 2,718 & 3,001 & 19 & 161 & 13,699 & 1,070 & 21,675 & 74 & 45 & 872 & 991 \\
\hline 1993 & 38 & 3,796 & 2,809 & 3,028 & 20 & 163 & 14,126 & 901 & 22,073 & 86 & 79 & 959 & 1,124 \\
\hline 1994 & 38 & 4,032 & 3,063 & 3,154 & 34 & 171 & 14,293 & 883 & 22,605 & 120 & 70 & 869 & $\begin{array}{l}1,059 \\
\text { n }\end{array}$ \\
\hline 1995 & 40 & 4,195 & 3,099 & 3,132 & 18 & 168 & 14,607 & 911 & 23,070 & 108 & 81 & 566 & 755 \\
\hline 1996 & $\begin{array}{l}40 \\
37\end{array}$ & 4,469 & $\begin{array}{l}3,268 \\
3,08\end{array}$ & $\begin{array}{l}3,102 \\
3,274\end{array}$ & 16 & 163 & $\begin{array}{r}14,8077 \\
14,837\end{array}$ & 851 & 23,648 & 109 & 80 & 628 & 817 \\
\hline 1997 & 40 & $\begin{array}{l}4,409 \\
4,672\end{array}$ & $\begin{array}{l}3,200 \\
3,307\end{array}$ & $\begin{array}{l}3,308 \\
3,3\end{array}$ & 14 & 172 & $\begin{array}{r}14,009 \\
14,999\end{array}$ & 712 & $\begin{array}{l}20,040 \\
23,918\end{array}$ & 111 & 102 & $\begin{array}{l}0<0 \\
715\end{array}$ & 927 \\
\hline 1998 & 35 & 4,812 & 3,359 & 3,357 & 18 & 180 & 15,463 & 674 & 24,538 & 136 & 124 & 1,047 & 1,306 \\
\hline 1999 & 39 & 5,001 & 3,466 & 3,462 & 14 & 182 & 15,855 & 665 & 25,219 & 140 & 112 & 959 & 1,211 \\
\hline 2000 & 36 & 5,165 & 3,580 & 3,580 & 12 & 179 & 15,960 & 888 & 25,820 & 175 & 99 & 871 & 1,144 \\
\hline 2001 & 35 & 5,292 & 3,427 & 3,426 & 14 & 164 & 16,041 & 586 & 25,557 & 171 & 103 & $\begin{array}{r}1,003 \\
\end{array}$ & 1,277 \\
\hline 2002 & 34 & 5,392 & 3,354 & 3,340 & 14 & 162 & 16,465 & 677 & 26,085 & 127 & 175 & $\begin{array}{l}, 650 \\
659\end{array}$ & 961 \\
\hline 2003 & 30 & 5,666 & 3,266 & 3,265 & 17 & 150 & 16,597 & 571 & 26,297 & 161 & 175 & 869 & 1,205 \\
\hline 2004 & 31 & 5,932 & 3,382 & 3,383 & 19 & 152 & 16,962 & 740 & 27,219 & 111 & 222 & 879 & 1,212 \\
\hline 2005 & 35 & 6,076 & 3,475 & 3,475 & 28 & 151 & 17,043 & 837 & 27,645 & 115 & 243 & 876 & 1.235 \\
\hline 2006 & 33 & 6,414 & $\begin{array}{l}3,379 \\
3,40\end{array}$ & 3,379 & 27 & 147 & 17,197 & 906 & 28,105 & 74 & 214 & 361 & 648 \\
\hline 2007 & $\begin{array}{l}50 \\
32\end{array}$ & $\begin{array}{l}0,414 \\
6,457\end{array}$ & $\begin{array}{l}0,358 \\
3,358\end{array}$ & $\begin{array}{l}3,358 \\
3,358\end{array}$ & 22 & $\begin{array}{l}151 \\
152\end{array}$ & 17,321 & 994 & $\begin{array}{l}20,105 \\
28,335\end{array}$ & $\begin{array}{l}84 \\
89\end{array}$ & 171 & 397 & $\begin{array}{l}040 \\
657\end{array}$ \\
\hline 2008 & 28 & 6,020 & 3,193 & 3,193 & 40 & 141 & $\begin{array}{l}16,872 \\
\end{array}$ & 920 & 27,214 & 73 & 154 & 240 & 468 \\
\hline 2009 & 27 & 5,528 & 2,883 & 2,883 & 28 & 127 & 16,837 & 810 & 26,240 & 70 & 139 & 181 & 390 \\
\hline 2010 & 27 & $\mathrm{R}_{5,818}$ & $R_{2,963}$ & $\mathrm{R}_{2,963}$ & 29 & $\mathrm{R}_{141}$ & $\mathrm{R}_{16,791}$ & R877 & $\mathrm{R}_{26,646}$ & 80 & $\mathrm{R}_{144}$ & $\mathrm{R}_{154}$ & 378 \\
\hline $2011^{P}$ & 27 & 5,908 & 2,950 & 2,950 & 29 & 133 & 16,312 & 823 & 26,182 & 62 & 132 & 94 & 288 \\
\hline
\end{tabular}

1 Electricity-only and combined-heat-and-power (CHP) plants within the NAICS 22 category whose primary business is to sell electricity, or electricity and heat, to the public. Through 1988, electric utilities only; beginning in 1989 , data are for electric utilities and independent power producers.
2 Beginning in 2009 , includes renewable diesel fuel (including biodiesel) blended into distillate fuel oil.

Through 1951, naphtha-type jet fuel is included in the products from which jet fuel was blended: 1952, 71 percent gasoline, 17 percent kerosene, and 12 percent distillate fuel oil. Beginning in 1952 , includes naphtha-type jet fuel. Beginning in 1957, also includes kerosene-type jet fuel. Beginning in 2005,

includes kerosene-type jet fuel only.
4 Finished motor gasoline. Through 1963, also includes special naphthas. Beginning in 1993, also includes ethanol blended into motor gasoline.

tor petroleum. For 1980-2000, electric utility data also include small amounts of kerosene and jet fuel.
6 Fuel oil nos. 5 and 6. For 1949-1979, data are for steam plant use of petroleum. For 1980-2000, electric utility data also include a small amount of fuel oil no. 4.

R=Revised. P=Preliminary. NA=Not available.
Notes: - Data for "Transportation Sector" are estimates. - For total heat content of petroleum consumption by all sectors, see data for heat content of petroleum products supplied in Table 5.12. For the transportation sector, petroleum product supplied is used as an approximation of petroleum consumption. See Note 1, "Petroleum Products Supplied and Petroleum Consumption," at end of Section. • Totals may not equal sum of components due to independent rounding.

Web Pages. - See http:/www.eia.gov/otalenergy/data/monthly/\#petroleum for updated monthly and annual data. - See http://www.eia.gov/totalenergy/data/annual/\#petroleum for all annual data beginning in Sources: Tables 5.13c, 5.13d, A1, and A3. 
Figure 5.15 Fuel Oil and Kerosene Sales, 1984-2010

\section{Total by Fuel}

$5-$

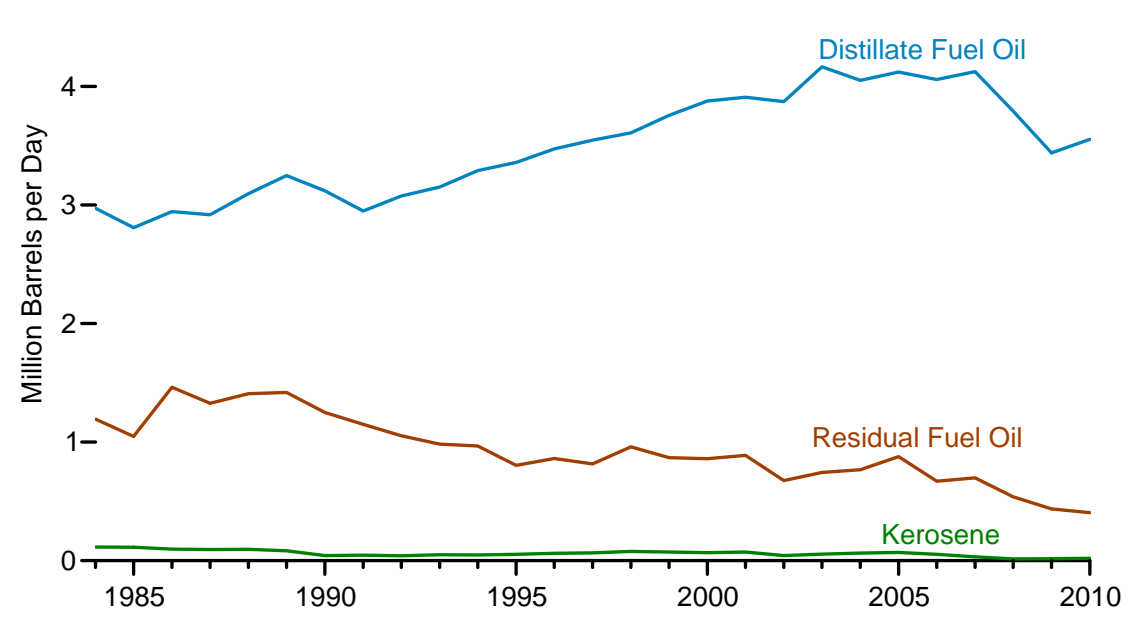

Residual Fuel Oil by Major End Use

$750-$

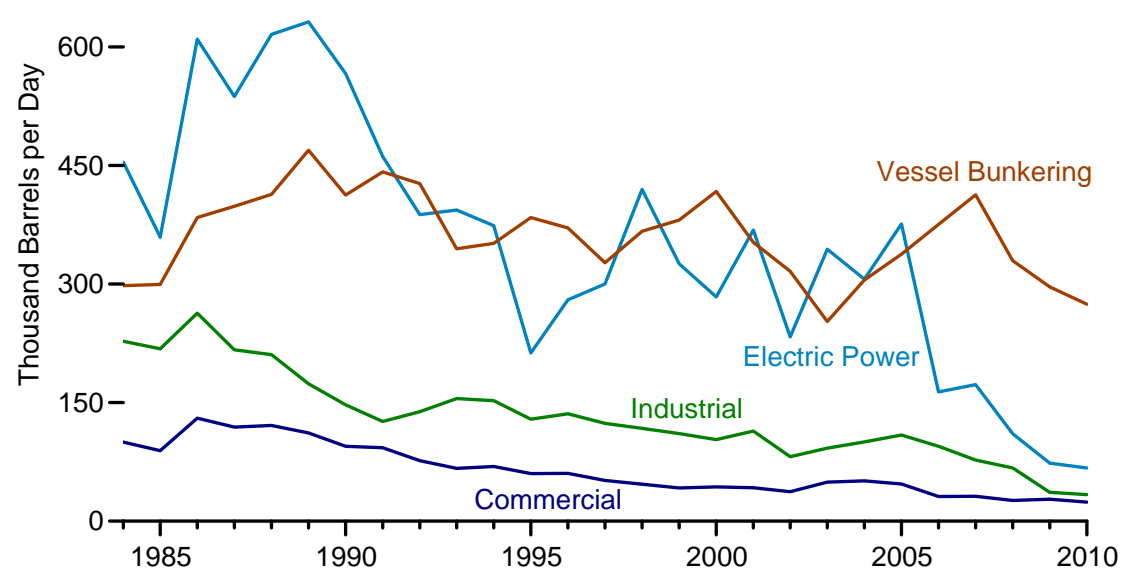

\section{Distillate Fuel Oil by Selected End Use}

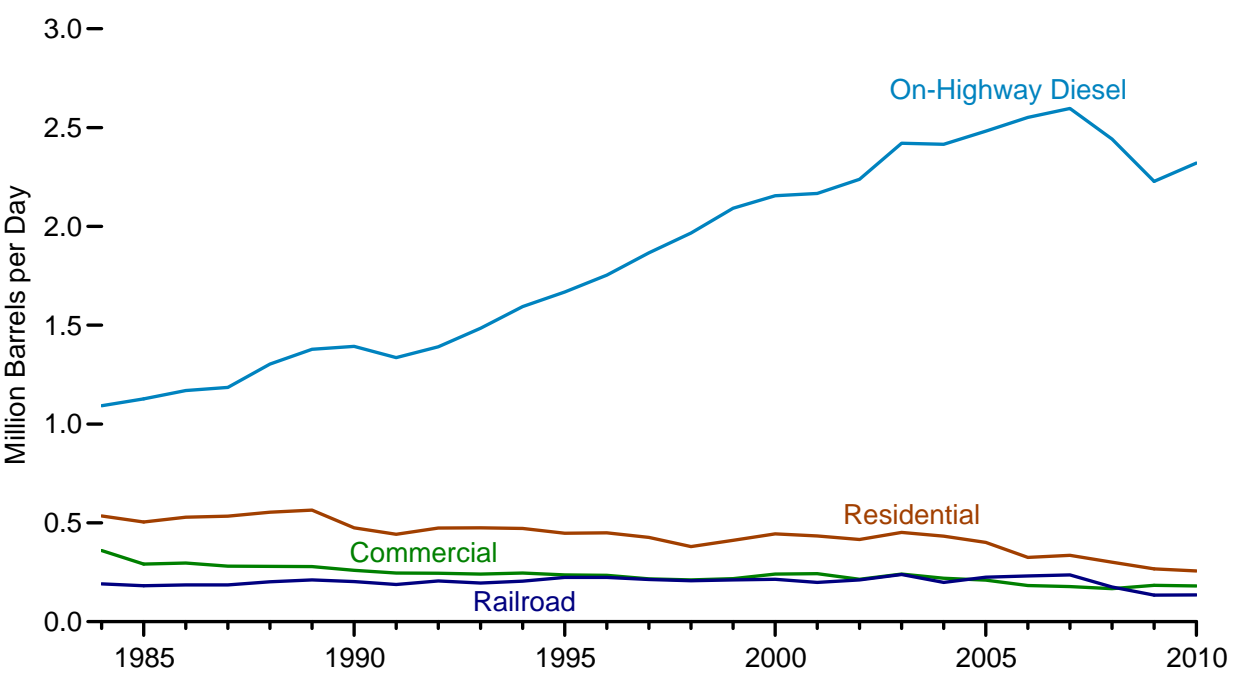

\section{Kerosene by Major End Use}

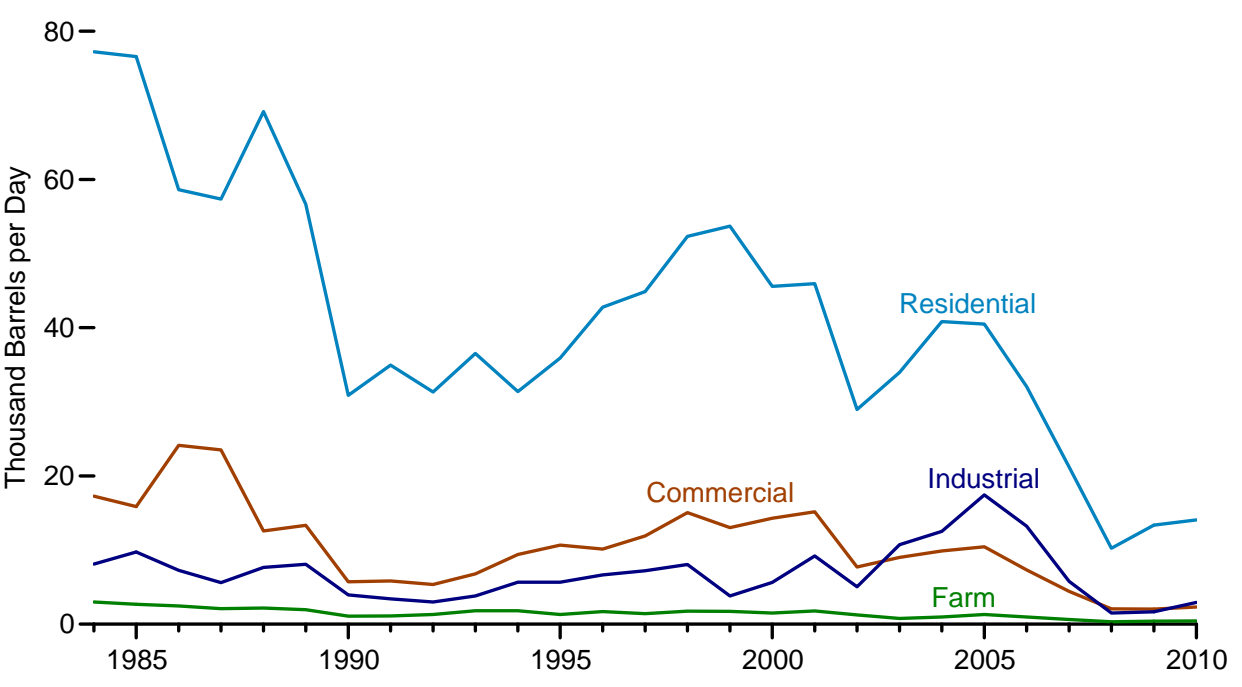

Source: Table 5.15. 
Table 5.15 Fuel Oil and Kerosene Sales, Selected Years, 1984-2010

(Thousand Barrels per Day)

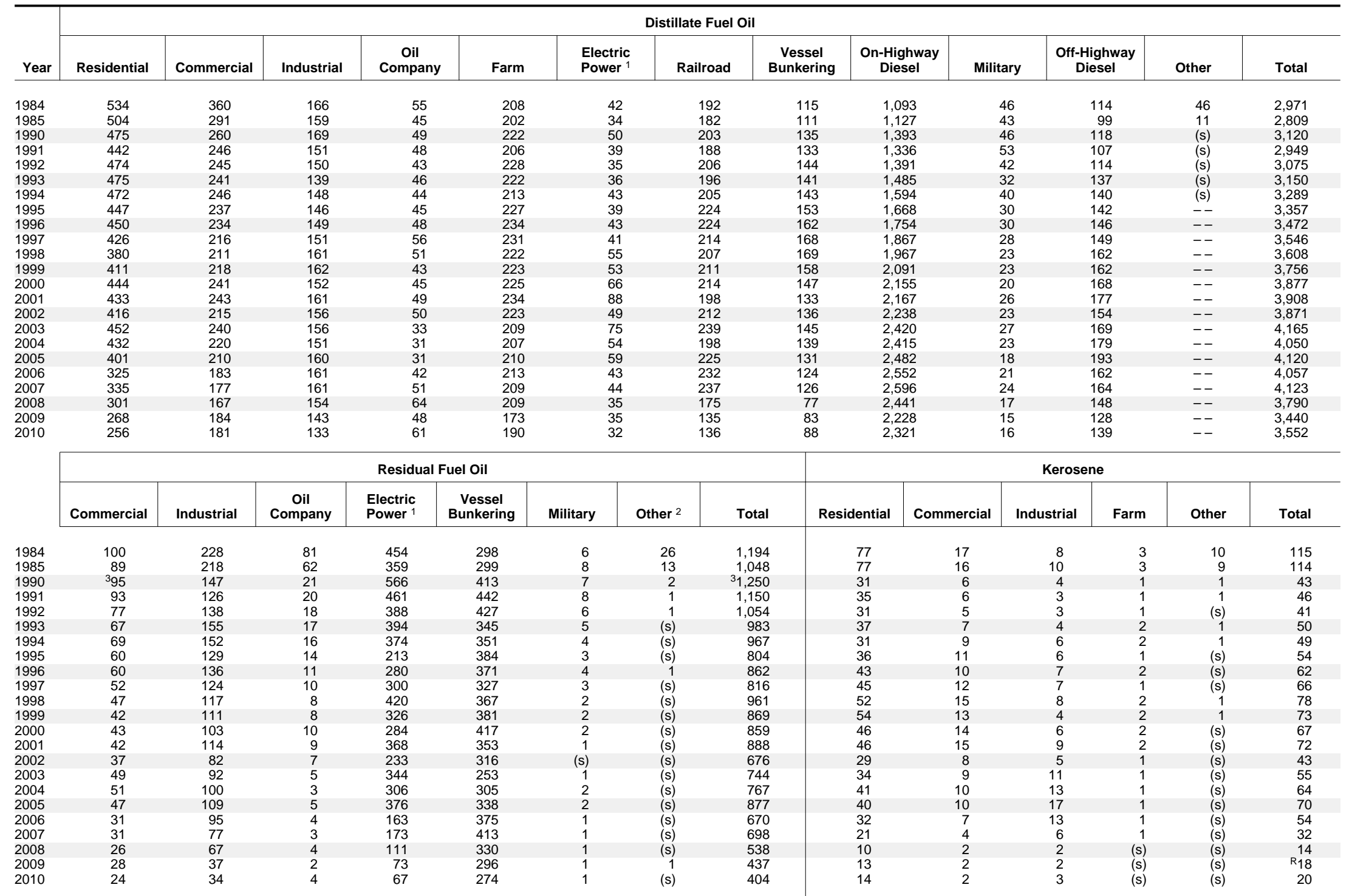

Electricity-only and combined-heat-and-power (CHP) plants within the NAICS 22 category whose primary business is to sell electricity, or electricity and heat, to the public.

Sales to railroads are included in "Other."

Value has been revised since publication in the reports cited after "Sources."

$--=$ Not applicable. $(\mathrm{s})=$ Less than 0.5 thousand barrels per day.

1984. - For related information, see http://www.eia.gov/petroleum/fueloilkerosene/.

Sources: • 1984-U.S. Energy Information Administration (EIA), Petroleum Marketing Annual 1988 October 1989), Tables A1-A3, and unpublished revision. - 1985-2004-EIA, Fuel OIl and Kerosene Sales, annual reports, Tables 1-3, and unpublished revisions. - 2005 forward-EIA, Fuel Oil and
Kerosene Sales 2010 (February 2012). 


\section{Figure 5.16 Petroleum Primary Stocks by Type, End of Year}

\section{Total Stocks and Strategic Petroleum Reserve (SPR) Stocks, 1949-2011}

2,000-

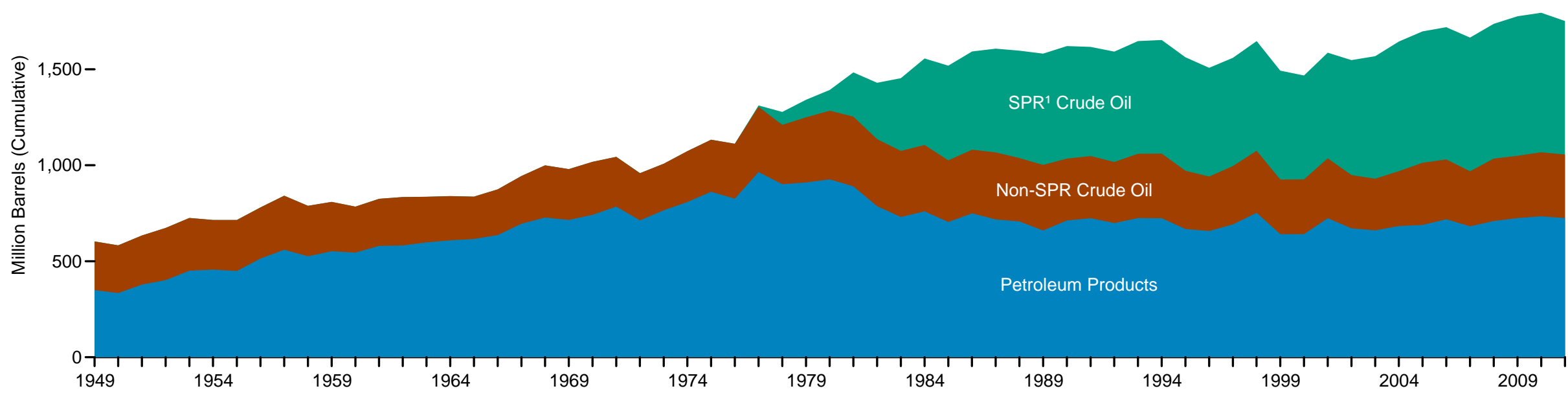

By Type, 2011

$1,200-$

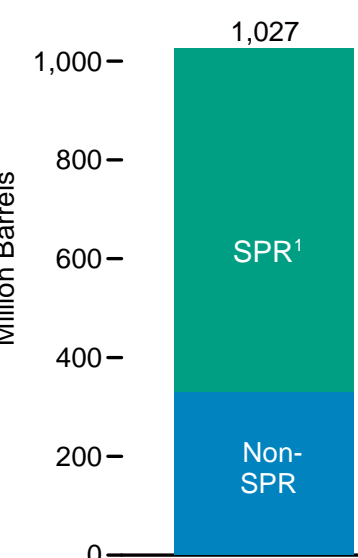

Crude Oil ${ }^{2}$

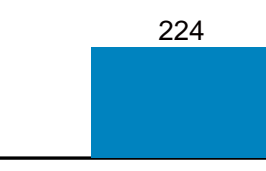

Motor Gasoline
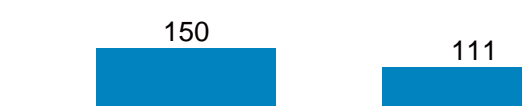

Distillate

Fuel Oil

Liquefied

Petroleum Gases

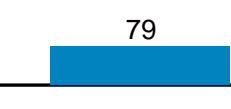

Unfinished

Oils

42

34

85

${ }^{1}$ Crude oil stocks in the SPR include non-U.S. stocks held under foreign or commercial storage agreements. See Figure 5.17 for additional information about the SPR.

${ }^{3}$ Asphalt and road oil, aviation gasoline and blending components, kerosene, lubricants, naphtha-type jet fuel, pentanes plus, petrochemical feedstocks, petroleum coke, special naphthas, waxes, other hydrocarbons and oxygenates, and miscellaneous products.

${ }^{2}$ Includes lease condensate and crude oil stored in the Strategic Petroleum Reserve (SPR). Source: Table 5.16 
Table 5.16 Petroleum Primary Stocks by Type, Selected Years, End of Year 1949-2011

(Million Barrels)

\begin{tabular}{|c|c|c|c|c|c|c|c|c|c|c|c|c|c|}
\hline \multirow[b]{3}{*}{ Year } & \multicolumn{3}{|c|}{ Crude Oil and Lease Condensate } & \multicolumn{9}{|c|}{ Petroleum Products } & \multirow{3}{*}{$\begin{array}{c}\text { Total } \\
\text { Petroleun }\end{array}$} \\
\hline & \multirow[b]{2}{*}{ SPR ${ }^{1}$} & \multirow[b]{2}{*}{ Non-SPR ${ }^{2,3}$} & \multirow[b]{2}{*}{ Total $^{3}$} & \multirow{2}{*}{$\begin{array}{l}\text { Distillate } \\
\text { Fuel Oil }{ }^{4}\end{array}$} & \multirow{2}{*}{$\begin{array}{l}\text { Jet } \\
\text { Fuel } 5\end{array}$} & \multicolumn{2}{|c|}{ Liquefied Petroleum Gases } & \multirow{2}{*}{$\begin{array}{c}\text { Motor } \\
\text { Gasoline } 7\end{array}$} & \multirow{2}{*}{$\begin{array}{c}\text { Residual } \\
\text { Fuel Oil }\end{array}$} & \multirow{2}{*}{$\begin{array}{l}\text { Unfinished } \\
\text { Oils }\end{array}$} & \multirow{2}{*}{$\begin{array}{c}\text { Other } \\
\text { Products } 8\end{array}$} & \multirow{2}{*}{$\begin{array}{c}\text { Total } \\
\text { Products }\end{array}$} & \\
\hline & & & & & & Propane 6 & Total & & & & & & \\
\hline 1949 & 0 & 253 & 253 & 75 & $\left({ }^{5}\right)$ & $\left({ }^{9}\right)$ & 1 & 110 & 60 & 66 & 37 & 350 & 603 \\
\hline 1950 & 0 & 248 & 248 & 72 & $(5)$ & $(9)$ & 2 & 116 & 41 & 70 & 34 & 334 & 583 \\
\hline 1955 & 0 & 266 & 266 & 111 & 3 & $\left({ }^{9}\right)$ & 7 & 165 & 39 & 68 & 55 & 449 & 715 \\
\hline 1960 & 0 & 240 & 240 & 138 & 7 & $\left({ }^{9}\right)$ & 23 & 195 & 45 & 62 & 76 & 545 & 785 \\
\hline 1965 & 0 & 220 & 220 & 155 & 19 & $\left({ }^{9}\right)$ & 30 & 175 & 56 & 89 & 92 & 616 & 836 \\
\hline 1970 & 0 & 276 & 276 & 195 & 28 & $\left({ }^{9}\right)$ & 67 & 209 & 54 & 99 & 89 & 741 & 1,018 \\
\hline 1975 & 0 & 271 & 271 & 209 & 30 & 82 & 125 & 235 & 74 & 106 & 82 & 862 & 1,133 \\
\hline 1976 & 0 & 285 & 285 & 186 & 32 & 74 & 116 & 231 & 72 & 110 & 78 & 826 & 1,112 \\
\hline 1977 & 7 & 340 & 348 & 250 & 35 & 81 & 136 & 258 & 90 & 113 & 82 & 964 & 1,312 \\
\hline 1978 & 67 & 309 & 376 & 216 & 34 & 87 & 132 & 238 & 90 & 109 & 82 & 901 & 1,278 \\
\hline 1979 & 91 & 339 & 430 & 229 & 39 & 64 & 111 & 237 & 96 & 118 & 82 & 911 & 1,341 \\
\hline 1980 & 108 & 358 & 466 & 205 & 42 & 65 & 120 & 261 & 92 & 124 & 82 & 926 & 1,392 \\
\hline 1981 & 230 & 363 & 594 & 192 & 41 & 76 & 135 & 253 & 78 & 111 & 80 & 890 & 1,484 \\
\hline 1982 & 294 & 350 & 644 & 179 & 37 & 54 & 94 & 235 & 66 & 105 & 70 & 786 & 1,430 \\
\hline 1983 & 379 & 344 & 723 & 140 & 39 & 48 & 101 & 222 & 49 & 108 & 72 & 731 & 1,454 \\
\hline 1984 & 451 & 345 & 796 & 161 & 42 & 58 & 101 & 243 & 53 & 94 & 67 & 760 & 1,556 \\
\hline 1985 & 493 & 321 & 814 & 144 & 40 & 39 & 74 & 223 & 50 & 107 & 67 & 705 & 1,519 \\
\hline 1986 & 512 & 331 & 843 & 155 & 50 & 63 & 103 & 233 & 47 & 94 & 68 & 750 & 1,593 \\
\hline 1987 & 541 & 349 & 890 & 134 & 50 & 48 & 97 & 226 & 47 & 93 & 70 & 718 & 1,607 \\
\hline 1988 & 560 & 330 & 890 & 124 & 44 & 50 & 97 & 228 & 45 & 100 & 70 & 707 & 1,597 \\
\hline 1989 & 580 & 341 & 921 & 106 & 41 & 32 & 80 & 213 & 44 & 106 & 70 & 660 & 1,581 \\
\hline 1990 & 586 & $\begin{array}{l}541 \\
323\end{array}$ & 908 & 132 & $\begin{array}{l}41 \\
52\end{array}$ & $\begin{array}{l}42 \\
49\end{array}$ & 98 & 220 & $\begin{array}{l}44 \\
49\end{array}$ & 99 & 63 & 712 & $\begin{array}{l}1,001 \\
1,621\end{array}$ \\
\hline 1991 & 569 & 325 & 893 & 144 & 49 & 48 & 92 & 219 & 50 & 98 & 72 & 724 & 1,617 \\
\hline 1992 & 575 & 318 & 893 & 141 & 43 & 39 & 89 & 216 & 43 & 95 & 73 & $\begin{array}{l}699 \\
\end{array}$ & $\begin{array}{l}1,592 \\
\end{array}$ \\
\hline 1993 & 587 & 335 & 922 & 141 & 40 & 51 & 106 & 226 & 44 & 88 & 78 & 725 & 1,647 \\
\hline 1994 & 592 & 337 & 929 & 145 & 47 & 46 & 99 & 215 & 42 & 91 & 84 & 724 & 1,653 \\
\hline 1995 & 592 & 303 & 895 & 130 & 40 & 43 & 93 & 202 & 37 & 86 & 79 & 668 & 1,563 \\
\hline 1996 & 566 & 284 & 850 & 127 & 40 & 43 & 86 & 195 & 46 & 88 & 76 & 658 & 1,507 \\
\hline 1997 & 563 & $\begin{array}{l}204 \\
305\end{array}$ & 868 & 138 & 44 & $\begin{array}{l}45 \\
44\end{array}$ & $\begin{array}{l}00 \\
89\end{array}$ & 210 & $\begin{array}{l}40 \\
40\end{array}$ & $\begin{array}{l}\circ 0 \\
89\end{array}$ & 81 & $\begin{array}{l}030 \\
692\end{array}$ & $\begin{array}{l}1,007 \\
1,560\end{array}$ \\
\hline 1998 & 571 & 324 & 895 & 156 & 45 & 65 & 115 & 216 & 45 & 91 & 85 & 752 & 1,647 \\
\hline 1999 & 567 & 284 & 852 & 125 & 41 & 43 & 89 & 193 & 36 & 86 & 70 & 641 & 1,493 \\
\hline 2000 & 541 & 286 & 826 & 118 & 45 & 41 & 83 & 196 & 36 & 87 & 77 & 641 & 1,468 \\
\hline 2001 & 550 & 312 & 862 & 145 & 42 & 66 & 121 & 210 & 41 & 88 & 78 & 724 & 1,586 \\
\hline 2002 & 599 & 278 & 877 & 134 & 39 & 53 & 106 & 209 & 31 & 76 & 76 & 671 & 1,548 \\
\hline 2003 & 638 & 269 & 907 & 137 & 39 & 50 & 94 & 207 & 38 & 76 & 71 & 661 & $\begin{array}{l}1,0408 \\
\end{array}$ \\
\hline 2004 & 676 & 286 & 961 & 126 & 40 & 55 & 104 & 218 & 42 & 81 & 72 & 683 & 1,645 \\
\hline 2005 & 685 & 324 & $\begin{array}{r}1,008 \\
\end{array}$ & 136 & 42 & 57 & 109 & 208 & 37 & 86 & 71 & 689 & 1,698 \\
\hline 2006 & 689 & 312 & $\begin{array}{l}1,001 \\
\end{array}$ & 144 & 39 & 62 & 113 & 212 & 42 & 84 & 85 & 719 & $\begin{array}{l}1,000 \\
\end{array}$ \\
\hline 2007 & 697 & 286 & 983 & 134 & 39 & 52 & 96 & 218 & 39 & 81 & 75 & 682 & 1,665 \\
\hline 2008 & 702 & 326 & 1,028 & 146 & 38 & 55 & 113 & 214 & 36 & 83 & 79 & 709 & 1.737 \\
\hline 2009 & 727 & 325 & $\begin{array}{l}1,0<20 \\
1,052\end{array}$ & 166 & 43 & 50 & 102 & 223 & 37 & 80 & 73 & 725 & 1,776 \\
\hline 2010 & 727 & $R_{333}$ & $R_{1,060}$ & $\begin{array}{l}160 \\
164\end{array}$ & $\begin{array}{l}45 \\
43\end{array}$ & $\begin{array}{l}50 \\
49\end{array}$ & $R_{108}$ & $\begin{array}{l}219 \\
219\end{array}$ & 41 & 81 & 77 & $\begin{array}{l}8<0 \\
\text { R734 }\end{array}$ & $\begin{array}{l}1,710 \\
1,794\end{array}$ \\
\hline $2011^{\mathrm{P}}$ & 696 & 331 & 1,027 & 150 & $\begin{array}{l}40 \\
42\end{array}$ & $\begin{array}{l}49 \\
55\end{array}$ & 111 & 224 & 34 & $\begin{array}{l}01 \\
79\end{array}$ & 85 & 725 & $\begin{array}{l}1,754 \\
1,751\end{array}$ \\
\hline
\end{tabular}

"SPR" is the Strategic Petroleum Reserve, which began in 1977. Crude oil stocks in the SPR include non-U.S. Stocks held under foreign or commercial storage agreements.
2 All crude oil and lease condensate stocks other than those in "SPR."

3 Beginning in 1981, includes stocks of Alaskan crude oil in transit.

4 Does not include stocks that are held in the Northeast Heating Oil Reserve.

5 Through 1951, naphtha-type jet fuel is included in the products from which it was blended: in 1952, 71 percent gasoline, 17 percent kerosene, and 12 percent distillate fuel oil. Through 1964, kerosene-type je fuel is included with kerosene in "Other Products." Beginning in 2005, naphtha-type jet fuel is included in "Other Products."

Finished motor gasoline, motor gasoline blending components, and gasohol. Through 1963, also

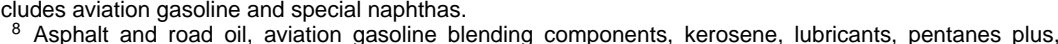

petrochemical feedstocks, petroleum coke, waxes, other hydrocarbons and oxygenates, and miscellaneous products. Through 1964, also includes kerosene-type jet fuel. Beginning in 1964, also includes aviation

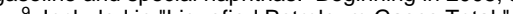

$R=$ Revised. $P=$ Preliminary.

Note: Totals may not equal sum of components due to independent rounding

Web Pages: - See http://www.eia.gov/totalenergy/data/monthly/\#petroleum for updated monthly and annual data. - See http://www.eia.gov/totalenergy/data/annual/\#petroleum for all annual data beginning in 1949. - See $\mathrm{http}: / / \mathrm{ww}$.eia.gov/petroleum/ for related information.

Sources: - 1949-1975-Bureau of Mines, Mineral Industry Surveys, Petroleum Statement, Annual, annual reports. - 1976-1980-U.S. Energy Information Administration (EIA), Energy Data Reports, Petroleum Statement, Annual, annual reports. - 1981-2010-EIA, Petroleum Supply Annual, annual 
Figure 5.17 Strategic Petroleum Reserve, 1977-2011

\section{End-of-Year Stocks in SPR}

$800-$

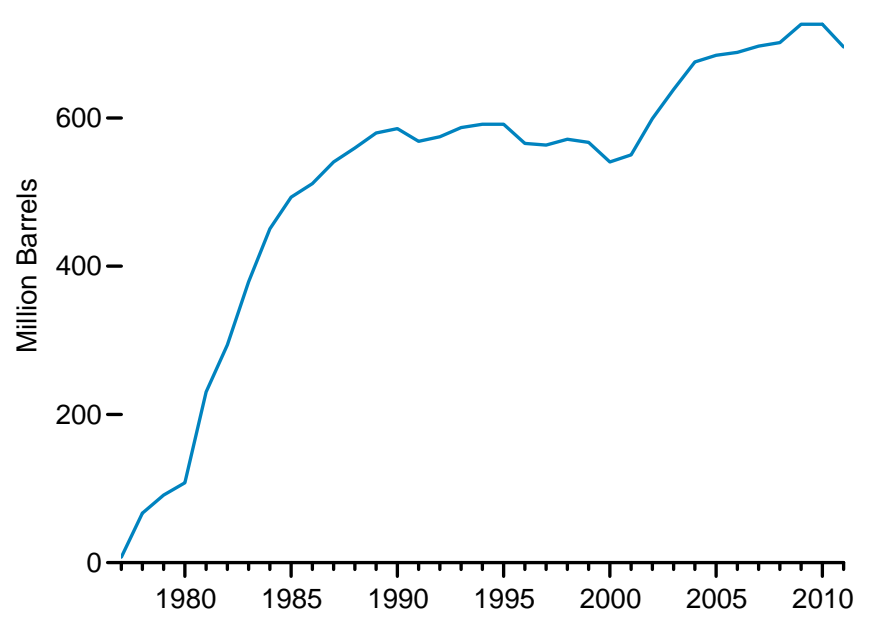

SPR as Share of Domestic Stocks

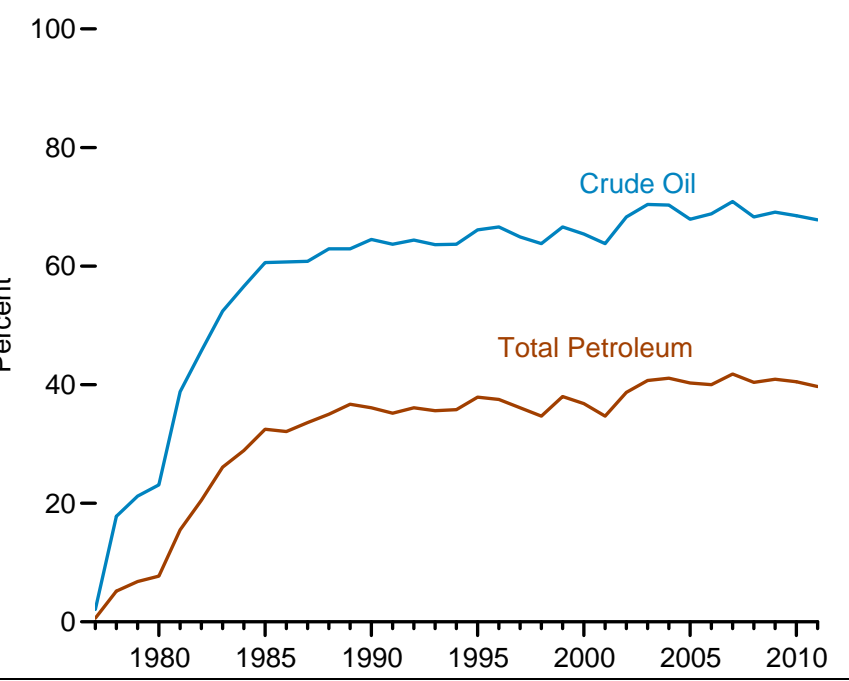

\section{Crude Oil Imports for SPR ${ }^{1}$}

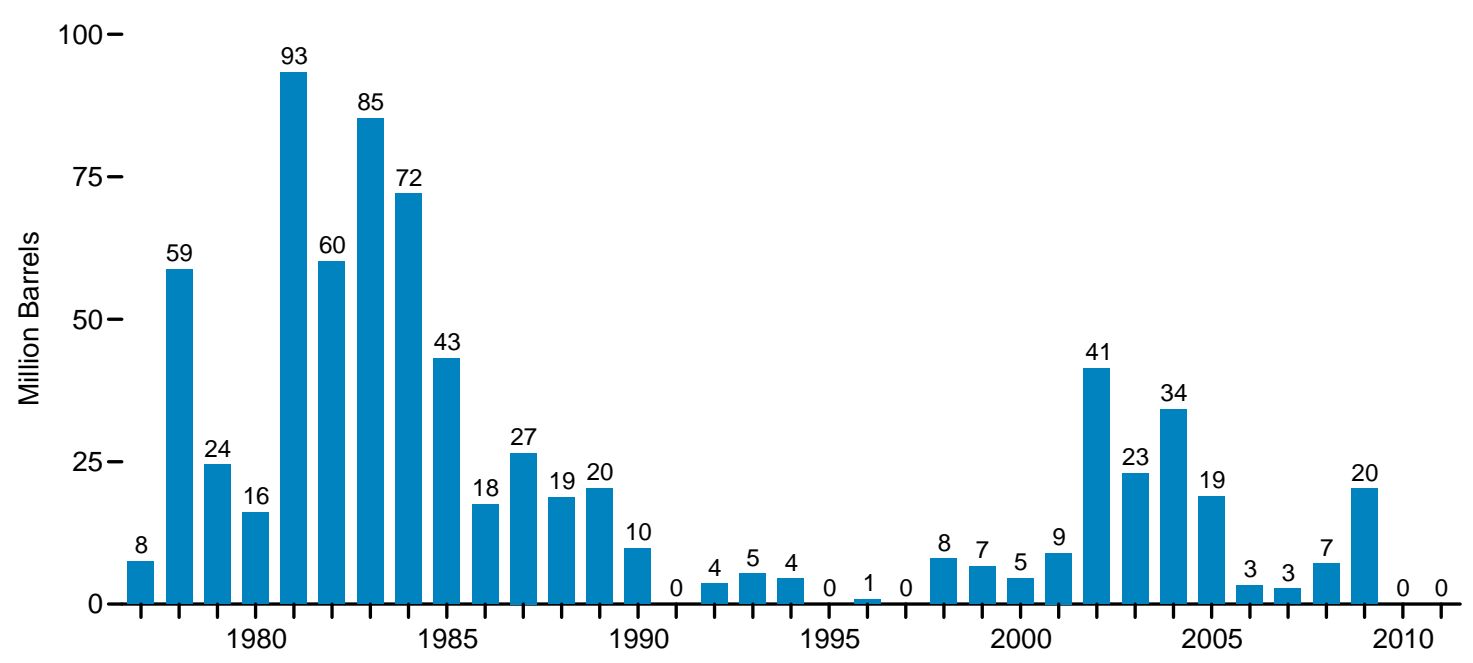

SPR Stocks as Days of Petroleum Net Imports ${ }^{2}$

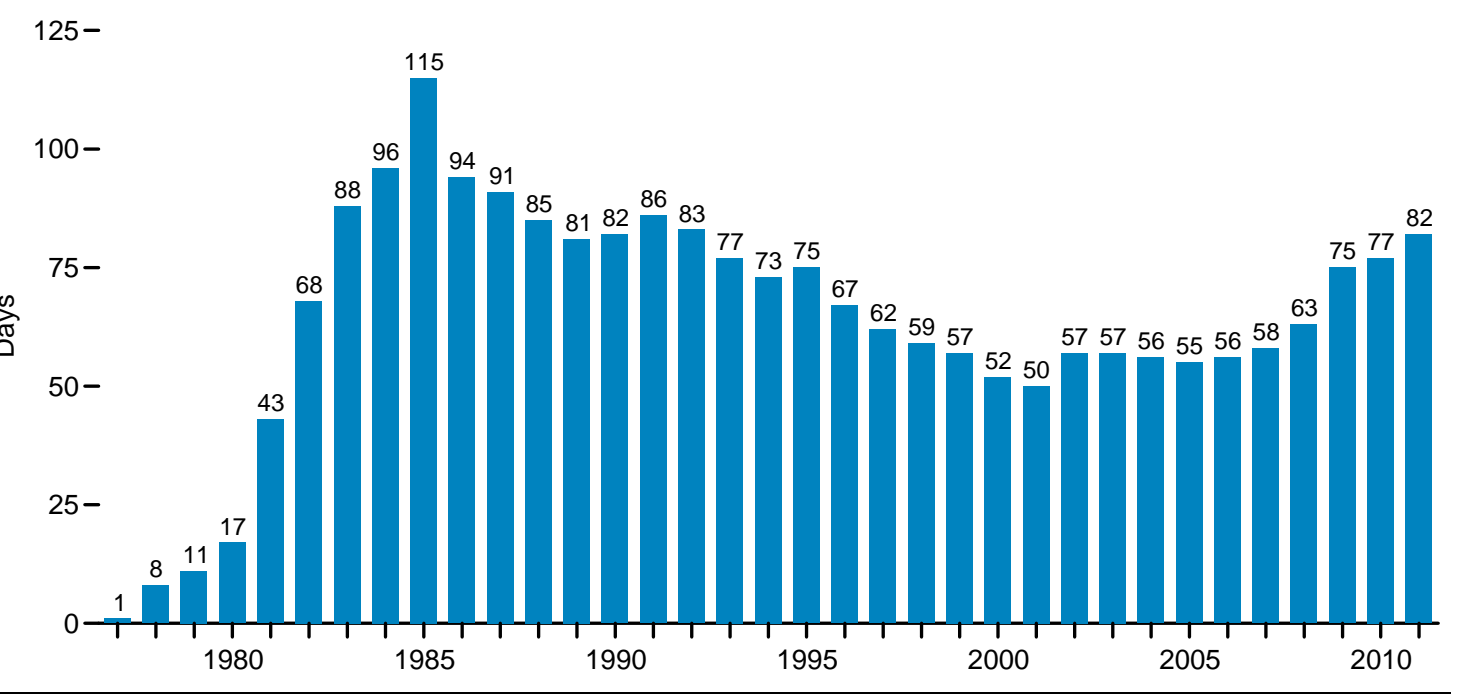

${ }^{1}$ Imported by SPR and imported by others for SPR.

${ }^{2}$ Derived by dividing end-of-year SPR stocks by annual average daily net imports of all

Note: SPR=Strategic Petroleum Reserve. petroleum.

Source: Table 5.17 
Table 5.17 Strategic Petroleum Reserve, 1977-2011

(Million Barrels, Except as Noted)

\begin{tabular}{|c|c|c|c|c|c|c|c|c|c|c|}
\hline \multirow[b]{2}{*}{ Year } & \multicolumn{2}{|c|}{ Foreign Crude Oil Receipts } & \multicolumn{2}{|c|}{ Domestic Crude Oil Receipts } & \multicolumn{2}{|c|}{ Withdrawals } & \multicolumn{3}{|c|}{ End-of-Year Stocks } & \multirow[b]{2}{*}{$\begin{array}{c}\text { Days of } \\
\text { Petroleum } \\
\text { Net Imports }{ }^{4}\end{array}$} \\
\hline & $\begin{array}{c}\text { Imported } \\
\text { by SPR }\end{array}$ & $\begin{array}{c}\text { Imported } \\
\text { by Others } 1,2\end{array}$ & Purchases & Exchanges ${ }^{2}$ & Sales & Exchanges & Quantity & $\begin{array}{l}\text { Percent of } \\
\text { Crude Oil }{ }^{3} \\
\text { Stocks }\end{array}$ & $\begin{array}{c}\text { Percent of } \\
\text { Total Petroleum } \\
\text { Stocks }\end{array}$ & \\
\hline 1977 & 7.54 & 0.00 & 50.37 & 0.00 & 0.00 & 0.00 & 7.46 & 2.1 & 0.6 & 1 \\
\hline 1978 & 58.80 & .00 & .00 & .00 & .00 & .00 & 66.86 & 17.8 & 5.2 & 8 \\
\hline 1979 & 24.43 & .00 & (s) & .00 & .00 & .00 & 91.19 & 21.2 & 6.8 & 11 \\
\hline 1980 & 16.07 & .00 & 1.30 & .00 & .00 & .00 & 107.80 & 23.1 & 7.7 & 17 \\
\hline 1981 & 93.30 & .00 & 28.79 & .00 & .00 & .00 & 230.34 & 38.8 & 15.5 & 43 \\
\hline 1982 & 60.19 & .00 & 3.79 & .00 & .00 & .00 & 293.83 & 45.7 & 20.5 & 68 \\
\hline 1983 & 85.29 & .00 & .42 & .00 & .00 & .00 & 379.09 & 52.4 & 26.1 & 88 \\
\hline 1984 & 72.04 & .00 & .05 & .00 & .00 & .00 & 450.51 & 56.6 & 28.9 & 96 \\
\hline 1985 & 43.12 & .00 & .17 & .00 & .00 & .00 & 493.32 & 60.6 & 32.5 & 115 \\
\hline 1986 & 17.56 & .00 & 1.21 & .00 & .00 & .00 & 511.57 & 60.7 & 32.1 & 94 \\
\hline 1987 & 26.52 & .00 & 2.69 & .00 & .00 & .00 & 540.65 & 60.8 & 33.6 & 91 \\
\hline 1988 & 18.76 & .00 & .01 & .00 & .00 & .00 & 559.52 & 62.9 & 35.0 & 85 \\
\hline 1989 & 20.35 & .00 & .00 & .00 & .00 & .00 & 579.86 & 62.9 & 36.7 & 81 \\
\hline 1990 & 9.77 & .00 & .00 & .00 & 3.91 & .00 & 585.69 & 64.5 & 36.1 & 82 \\
\hline 1991 & .00 & .00 & .00 & .00 & 17.22 & .00 & 568.51 & 63.7 & 35.2 & 86 \\
\hline 1992 & 3.59 & .00 & 2.60 & .00 & .00 & .00 & 574.72 & 64.4 & 36.1 & 83 \\
\hline 1993 & 5.37 & .00 & 6.96 & .00 & .00 & .00 & 587.08 & 63.6 & 35.6 & 77 \\
\hline 1994 & 4.49 & .00 & .11 & .00 & .00 & .00 & 591.67 & 63.7 & 35.8 & 73 \\
\hline 1995 & .00 & .00 & .00 & .00 & .00 & .00 & 591.64 & 66.1 & 37.9 & 75 \\
\hline 1996 & .00 & .90 & .00 & .00 & 25.82 & .90 & 565.82 & 66.6 & 37.5 & 67 \\
\hline 1997 & .00 & .00 & .00 & .00 & 2.33 & .00 & 563.43 & 64.9 & 36.1 & 62 \\
\hline 1998 & .00 & 7.98 & .00 & .00 & .00 & .00 & 571.41 & 63.8 & 34.7 & 59 \\
\hline 1999 & 3.04 & 3.60 & .00 & 1.42 & .00 & 10.75 & 567.24 & 66.6 & 38.0 & 57 \\
\hline 2000 & 3.01 & 1.50 & .00 & 2.29 & .00 & ${ }^{6} 33.35$ & 540.68 & 65.4 & 36.8 & 52 \\
\hline 2001 & 3.91 & 5.07 & .58 & .00 & .00 & .00 & 550.24 & 63.8 & 34.7 & 50 \\
\hline 2002 & 5.77 & 35.59 & .00 & 7.64 & .00 & .00 & 599.09 & 68.3 & 38.7 & 57 \\
\hline 2003 & .00 & 22.94 & .00 & 16.40 & .00 & .00 & 638.39 & 70.4 & 40.7 & 57 \\
\hline 2004 & .00 & 34.24 & .00 & 8.47 & .00 & 5.44 & 675.60 & 70.3 & 41.1 & 56 \\
\hline 2005 & .00 & 18.88 & .00 & 8.41 & 11.03 & 9.82 & 684.54 & 67.9 & 40.3 & 55 \\
\hline 2006 & .00 & 3.31 & .00 & 2.44 & .00 & 1.57 & 688.61 & 68.8 & 40.0 & 56 \\
\hline 2007 & .00 & 2.70 & .00 & 1.68 & .00 & .00 & 696.94 & 70.9 & 41.8 & 58 \\
\hline 2008 & .00 & 7.11 & .00 & 3.20 & .00 & 5.40 & 701.82 & 68.3 & 40.4 & 63 \\
\hline 2009 & .00 & 20.29 & .00 & 4.47 & .00 & .00 & 726.62 & 69.1 & 40.9 & 75 \\
\hline 2010 & .00 & .00 & .00 & .38 & .00 & .40 & 726.55 & R68.5 & 40.5 & 77 \\
\hline 2011 & .00 & .00 & .00 & .00 & 30.59 & .00 & 695.95 & 67.8 & 39.7 & 82 \\
\hline
\end{tabular}

1 Imported crude oil received represents volumes of imported crude oil received at SPR storage facilities for which the costs associated with the importation and delivery of crude oil are the responsibility of the commercial importer under contract to supply the SPR.

2 The values shown for 1998 and 1999 represent an exchange agreement in which SPR received approximately 8.5 million barrels of high quality oil in exchange for approximately 11 million barrels of lower quallty crude oil shipped from SPR during 1999 and 2000. Also, beginning in 1999, a portion of the crude Eil in-kind royalties from Federal leases in the Gulf or Mexico was transferred to the U.S. Department of Energy and exchanged wh commercial entities for crude oll was the SPR. Crude oil exchange barrels delivered to SPR could be either domestic or imported as long as the crude oil met the specification Receipts, Imported by Others," while exchange barrels of domestic crude oil are included in "Domestic Crude Oil Receipts, Exchanges."

3 Includes lease condensate stocks.

${ }_{4}$ Derived by dividing end-of-year SPR stocks by annual average daily net imports of all petroleum.

The quantity of domestic fuel oil which was in storage prior to injection of foreign crude oil.

6 Includes 30 million barrels released to increase heating oil stocks in exchange for a like quantity plus bonus percentage to be returned in 2001 and 2002, as well as additional barrels to create a Northeas Home Heating Oil Reserve.

Rillion barrels.

Note: "SPR" is the Strategic Petroleum Reserve-petroleum stocks maintained by the Federa Government for use during periods of major supply interruption.

(

Sources. Imported by SPR and End-ot-Year Stocks, Quantity: • 1977-1980-U.S. Energy Information Admits (Fobruary 2012). Department of Energy, Assistant Secretary for Fossil Energy, unpublished data. All Other Data: Calculated. 


\section{U.S. Average Prices, 1949-2011}

$120-$

$$
100-
$$

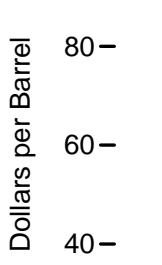

$40-$

$20-$

$$
0 \frac{1}{1950}
$$
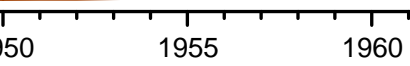

Nominal' Prices

$125-$

Alaska North Slope, California, and Texas 1977-2011

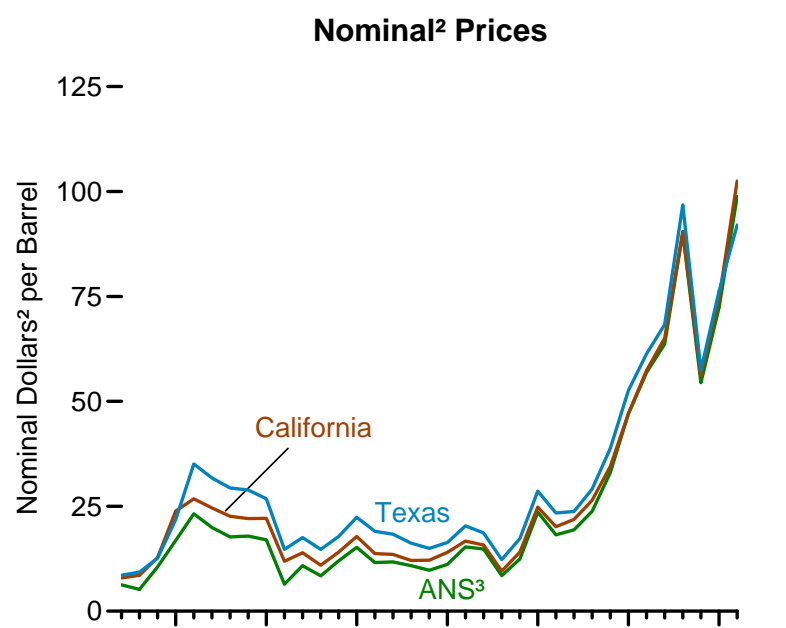

$\begin{array}{llllllll}1980 & 1985 & 1990 & 1995 & 2000 & 2005 & 2010\end{array}$

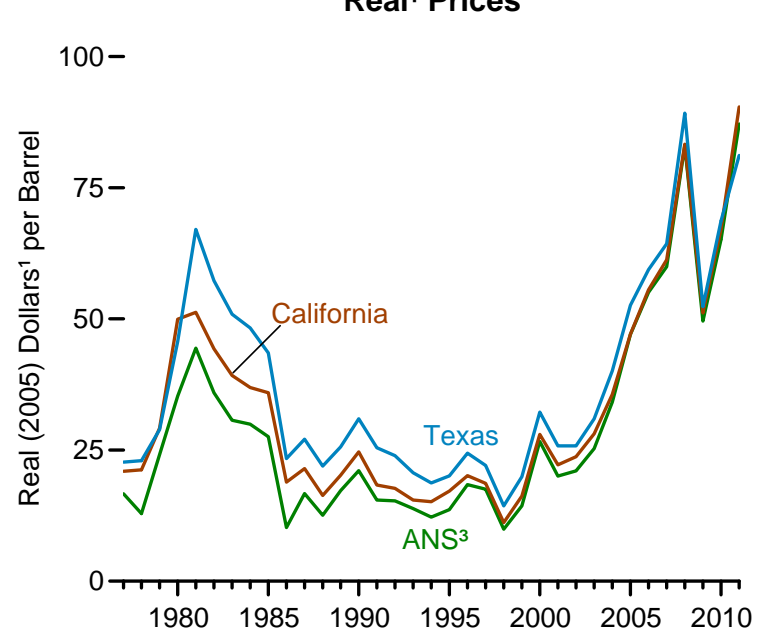

$600-$

Real ${ }^{1}$ Prices, Indexed 1977=100

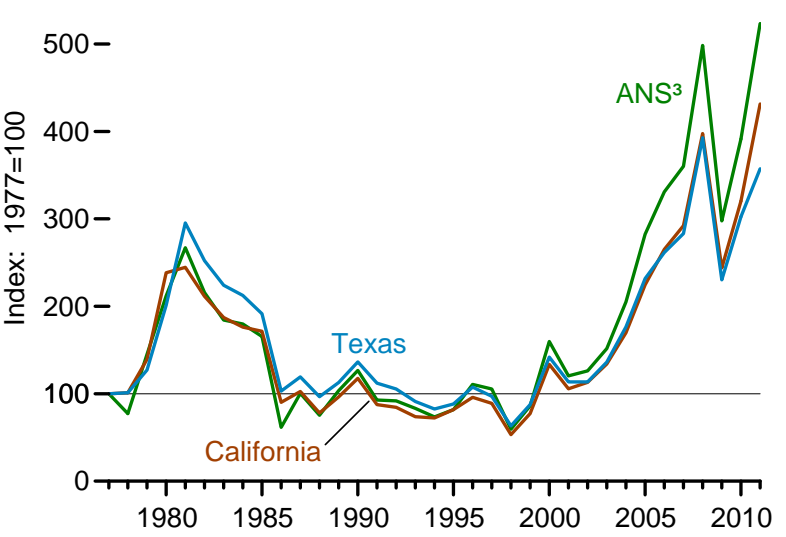

${ }^{1}$ In chained (2005) dollars, calculated by using gross domestic product implicit price defla- $\quad{ }^{3}$ Alaska North Slope. tors in Table D1. See "Chained Dollars" in Glossary.

2 See "Nominal Dollars" in Glossary. 
Table 5.18 Crude Oil Domestic First Purchase Prices, Selected Years, 1949-2011

(Dollars per Barrel)

\begin{tabular}{|c|c|c|c|c|c|c|c|c|}
\hline \multirow[b]{2}{*}{ Year } & \multicolumn{2}{|c|}{ Alaska North Slope } & \multicolumn{2}{|c|}{ California } & \multicolumn{2}{|c|}{ Texas } & \multicolumn{2}{|c|}{ U.S. Average } \\
\hline & Nominal 1 & Real $^{2}$ & Nominal 1 & Real $^{2}$ & Nominal 1 & Real $^{2}$ & Nominal 1 & Real $^{2}$ \\
\hline 1949 & -- & -- & NA & NA & NA & NA & 2.54 & $\mathrm{R}_{17.52}$ \\
\hline 1950 & -- & -- & NA & NA & NA & NA & 2.51 & $R_{17.13}$ \\
\hline 1955 & -- & -- & NA & NA & NA & NA & 2.77 & $R_{16.69}$ \\
\hline 1960 & NA & NA & NA & NA & NA & NA & 2.88 & $\mathrm{R}_{15.47}$ \\
\hline 1965 & NA & NA & NA & NA & NA & NA & 2.86 & $R_{14.35}$ \\
\hline 1970 & NA & NA & NA & NA & NA & NA & 3.18 & $R_{13.07}$ \\
\hline 1975 & NA & NA & NA & NA & NA & NA & 7.67 & $R_{22.83}$ \\
\hline 1976 & NA & NA & NA & NA & NA & NA & 8.19 & $R_{23.06}$ \\
\hline 1977 & ${ }^{3} 6.29$ & 3,R 16.65 & 7.92 & $\mathrm{R}_{20.96}$ & 8.58 & $R_{22.71}$ & 8.57 & $R_{22.68}$ \\
\hline 1978 & 5.21 & $\mathrm{R}_{12.88}$ & 8.58 & $\mathrm{R}_{21.22}$ & 9.29 & $R_{22.98}$ & 9.00 & $R_{22.26}$ \\
\hline 1979 & 10.57 & $R_{24.13}$ & 12.78 & $R_{29.18}$ & 12.65 & $R_{28.88}$ & 12.64 & $R_{28.86}$ \\
\hline 1980 & 16.87 & $R_{35.30}$ & 23.87 & $\mathrm{R}_{49.95}$ & 21.84 & $\mathrm{R}_{45.70}$ & 21.59 & $\mathrm{R}_{45.18}$ \\
\hline 1981 & 23.23 & R44.44 & 26.80 & R51.27 & 35.06 & R67.07 & 31.77 & R60.78 \\
\hline 1982 & 19.92 & $R_{35.92}$ & 24.58 & $\mathrm{R}_{44.32}$ & 31.77 & R57.29 & 28.52 & R51.43 \\
\hline 1983 & 17.69 & $R_{30.68}$ & 22.61 & $R_{39.22}$ & 29.35 & $R_{50.91}$ & 26.19 & $\mathrm{R}_{45.43}$ \\
\hline 1984 & 17.91 & R29.94 & 22.09 & R36.93 & 28.87 & R 48.26 & 25.88 & R43.27 \\
\hline 1985 & 16.98 & $\mathrm{R}_{27.55}$ & 22.14 & R35.93 & 26.80 & $\mathrm{R}_{43.49}$ & 24.09 & R39.09 \\
\hline 1986 & 6.45 & $R_{10.24}$ & 11.90 & $\mathrm{R}_{18.89}$ & 14.73 & $R_{23.38}$ & 12.51 & $\mathrm{R}_{19.86}$ \\
\hline 1987 & 10.83 & $\mathrm{R}_{16.71}$ & 13.92 & $\mathrm{R}_{21.48}$ & 17.55 & $\mathrm{R}_{27.08}$ & 15.40 & $\mathrm{R}_{23.76}$ \\
\hline 1988 & 8.43 & $R_{12.57}$ & 10.97 & $R_{16.36}$ & 14.71 & $R_{21.94}$ & 12.58 & $\mathrm{R}_{18.76}$ \\
\hline 1989 & 12.00 & $R_{17.25}$ & 14.06 & $\mathrm{R}_{20.21}$ & 17.81 & $R_{25.60}$ & 15.86 & $R_{22.79}$ \\
\hline 1990 & 15.23 & $R_{21.08}$ & 17.81 & $R_{24.65}$ & 22.37 & $R_{30.96}$ & 20.03 & $\mathrm{R}_{27.72}$ \\
\hline 1991 & 11.57 & $\mathrm{R}_{15.46}$ & 13.72 & $\mathrm{R}_{18.34}$ & 19.04 & $\mathrm{R}_{25.45}$ & 16.54 & $\mathrm{R}_{22.11}$ \\
\hline 1992 & 11.73 & $R_{15.31}$ & 13.55 & $R_{17.69}$ & 18.32 & $R_{23.92}$ & 15.99 & $\mathrm{R}_{20.88}$ \\
\hline 1993 & 10.84 & $\mathrm{R}_{13.85}$ & 12.11 & $\mathrm{R}_{15.47}$ & 16.19 & $\mathrm{R}_{20.68}$ & 14.25 & $\mathrm{R}_{18.20}$ \\
\hline 1994 & 9.77 & $\mathrm{R}_{12.22}$ & 12.12 & $R_{15.16}$ & 14.98 & $\mathrm{R}_{18.74}$ & 13.19 & $R_{16.50}$ \\
\hline 1995 & 11.12 & $R_{13.63}$ & 14.00 & $R_{17.16}$ & 16.38 & $\mathrm{R}_{20.07}$ & 14.62 & $R_{17.92}$ \\
\hline 1996 & 15.32 & $\mathrm{R}_{18.42}$ & 16.72 & $\mathrm{R}_{20.11}$ & 20.31 & R24.42 & 18.46 & $R_{22.20}$ \\
\hline 1997 & 14.84 & $R_{17.54}$ & 15.78 & $\mathrm{R}_{18.65}$ & 18.66 & $R_{22.05}$ & 17.23 & $\mathrm{R}_{20.36}$ \\
\hline 1998 & 8.47 & R9.90 & 9.55 & $\mathrm{R}_{11.16}$ & 12.28 & $R_{14.35}$ & 10.87 & $\mathrm{R}_{12.70}$ \\
\hline 1999 & 12.46 & $R_{14.35}$ & 14.08 & $R_{16.21}$ & $\begin{array}{l}17.29 \\
\text {. }\end{array}$ & $R_{19.91}$ & 15.56 & $R_{17.92}$ \\
\hline 2000 & 23.62 & $R_{26.62}$ & 24.82 & $\mathrm{R}_{27.97}$ & 28.60 & $R_{32.24}$ & 26.72 & $\mathrm{R}_{30.12}$ \\
\hline 2001 & 18.18 & $R_{20.04}$ & 20.11 & $\mathrm{R}_{22.17}$ & 23.41 & $\mathrm{R}_{25.80}$ & 21.84 & $\mathrm{R}_{24.07}$ \\
\hline 2002 & 19.37 & $\mathrm{R}_{21.01}$ & 21.87 & $R_{23.72}$ & 23.77 & $\mathrm{R}_{25.78}$ & 22.51 & $\mathrm{R}_{24.42}$ \\
\hline 2003 & 23.78 & $R_{25.26}$ & 26.43 & $R_{28.08}$ & 29.13 & $R_{30.94}$ & 27.56 & $R_{29.28}$ \\
\hline 2004 & 33.03 & 34.13 & 34.47 & $R_{35.61}$ & 38.79 & 40.08 & 36.77 & $R_{37.99}$ \\
\hline 2005 & 47.05 & 47.05 & 47.08 & 47.08 & 52.61 & 52.61 & 50.28 & 50.28 \\
\hline 2006 & 56.86 & R55.08 & 57.34 & R55.55 & 61.31 & R59.39 & 59.69 & R57.82 \\
\hline 2007 & 63.69 & R59.96 & 65.07 & $R_{61.26}$ & 68.30 & $\mathrm{R}_{64.30}$ & 66.52 & $R_{62.62}$ \\
\hline 2008 & 90.10 & $R_{82.98}$ & 90.47 & R83.32 & 96.85 & R89.20 & 94.04 & R86.61 \\
\hline 2009 & 54.41 & $R_{49.59}$ & 56.11 & R51.14 & 57.40 & R52.31 & 56.35 & R51.35 \\
\hline 2010 & 72.33 & $R_{65.17}$ & 74.51 & $R_{67.13}$ & 76.23 & $R_{68.68}$ & 74.71 & $\mathrm{R}_{67.31}$ \\
\hline 2011 & 98.79 & 87.15 & 102.50 & 90.42 & 91.99 & 81.15 & 95.73 & 84.45 \\
\hline
\end{tabular}

1 See "Nominal Dollars" in Glossary.

2 In chained (2005) dollars, calculated by using gross domestic product implicit price deflators in Table D1. See "Chained Dollars" in Glossary.

Average for July through December only.

$=$ Revised. NA $=$ Not available. $--=$ Not applicable.

Note: Prices are for the marketed first sales price of domestic crude oil. See Note 4, "Crude Oil Domestic First Purchase Prices," at end of section.

Web Pages: - See http://www.eia.gov/totalenergy/data/monthly/\#prices for updated monthly and annual

data. - See http://www.eia.gov/totalenergy/data/annual/\#petroleum for all annual data beginning in 1949. - See http://www.eia.gov/petroleum/ for related information.

Sources: - 1949-1973-Bureau of Mines, Minerals Yearbook, "Crude Petroleum and Petroleum Products" chapter. • 1974-January 1976-Federal Energy Administration (FEA), Form FEA-90, "Crude Petroleum Production Monthly Report." • February 1976-1977-FEA, Form FEA-P-124, "Domestic Crude Oil Purchaser's Monthly Report." • 1978-1984-U.S. Energy Information Administration (EIA), Petroleum Marketing Annual, annual reports. • 1985 forward-EIA, Petroleum Marketing Monthly (April 2012), Table 
Figure 5.19 Landed Costs of Crude Oil Imports From Selected Countries

\section{Total, 1973'-2011}

$120-$

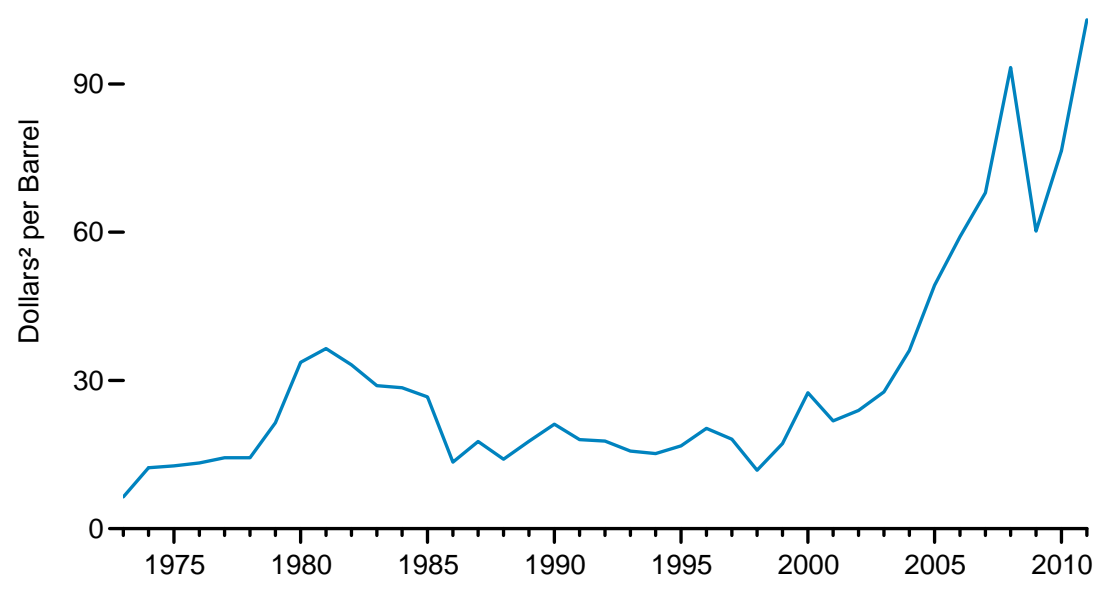

By Selected OPEC Country, 1973¹-2011

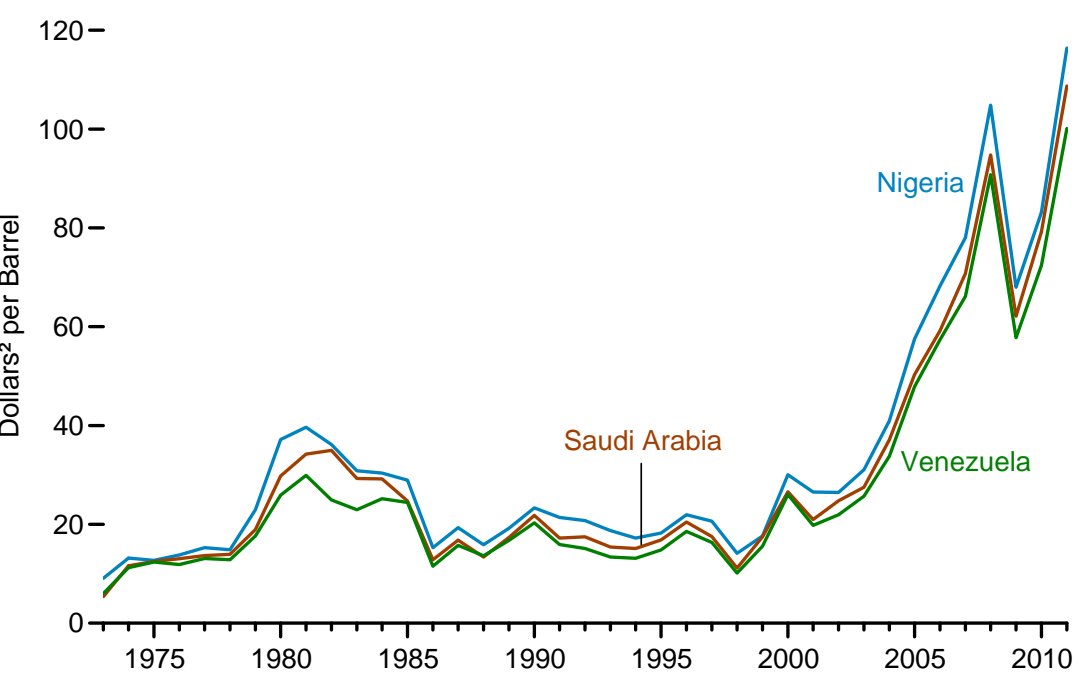

${ }^{1} 1973$ cost is based on October, November, and December data only.

2 Prices are not adjusted for inflation. See "Nominal Dollars" in Glossary.
By Selected Country, 2011

$150-$

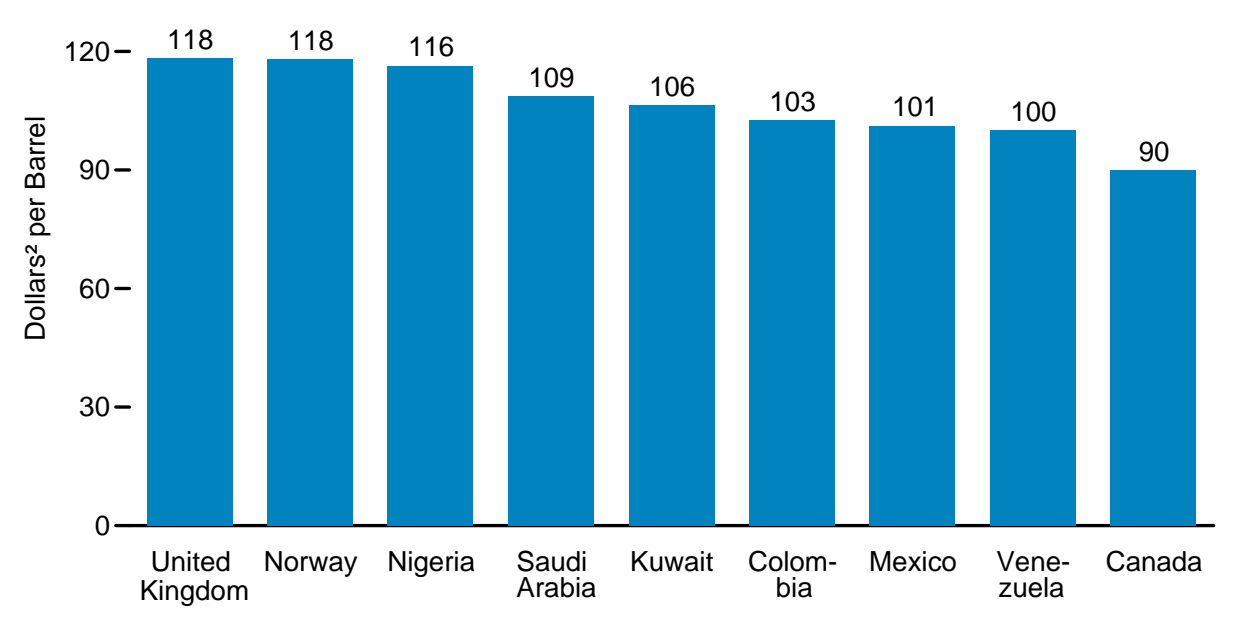

By Selected Non-OPEC Country, 1973¹-2011

$120-$

$100-$

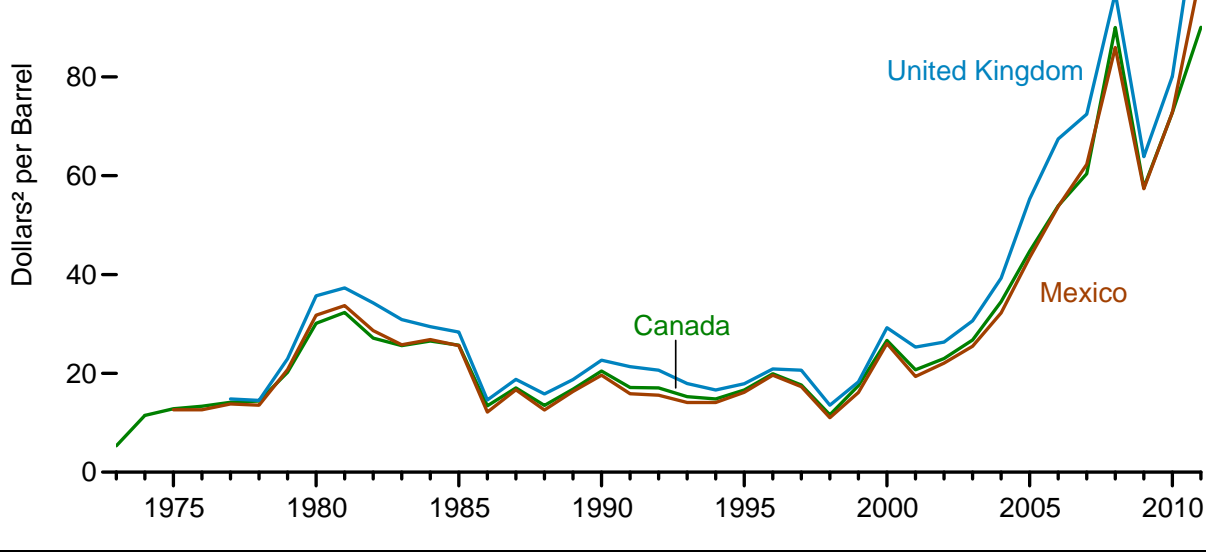

Note: OPEC=Organization of the Petroleum Exporting Countries. Source: Table 5.19. 
Table 5.19 Landed Costs of Crude Oil Imports From Selected Countries, 1973-2011

(Dollars ${ }^{1}$ per Barrel)

\begin{tabular}{|c|c|c|c|c|c|c|c|c|c|c|c|c|c|}
\hline \multirow[b]{2}{*}{ Year } & \multirow[b]{2}{*}{$\begin{array}{c}\text { Persian } \\
\text { Gulf }^{3}\end{array}$} & \multicolumn{5}{|c|}{ Selected OPEC ${ }^{2}$ Countries } & \multicolumn{6}{|c|}{ Selected Non-OPEC ${ }^{2}$ Countries } & \multirow[b]{2}{*}{ Total } \\
\hline & & Kuwait & Nigeria & $\begin{array}{c}\text { Saudi } \\
\text { Arabia }\end{array}$ & Venezuela & $\begin{array}{c}\text { Total } \\
\text { OPEC } 4\end{array}$ & Canada & Colombia & Mexico & Norway & $\begin{array}{c}\text { United } \\
\text { Kingdom }\end{array}$ & $\begin{array}{c}\text { Total } \\
\text { Non-OPEC } 4\end{array}$ & \\
\hline $1973^{5}$ & 5.91 & W & 9.08 & 5.37 & 5.99 & 6.85 & 5.33 & W & - & - & - & 5.64 & 6.41 \\
\hline 1974 & 12.21 & W & 13.16 & 11.63 & 11.25 & 12.49 & 11.48 & W & W & - & - & 11.81 & 12.32 \\
\hline 1975 & 12.64 & W & 12.70 & 12.50 & 12.36 & 12.70 & 12.84 & - & 12.61 & 12.80 & - & 12.70 & 12.70 \\
\hline 1976 & 13.03 & W & 13.81 & 13.06 & 11.89 & 13.32 & 13.36 & - & 12.64 & 13.74 & W & 13.35 & 13.32 \\
\hline 1977 & 13.85 & W & 15.29 & 13.69 & 13.11 & 14.35 & 14.13 & - & 13.82 & 14.93 & 14.83 & 14.42 & 14.36 \\
\hline 1978 & 14.01 & W & 14.88 & 13.94 & 12.84 & 14.34 & 14.41 & - & 13.56 & 14.68 & 14.53 & 14.38 & 14.35 \\
\hline 1979 & 20.42 & W & 22.97 & 18.95 & 17.65 & 21.29 & 20.22 & - & 20.77 & 22.55 & 22.97 & 22.10 & 21.45 \\
\hline 1980 & 30.59 & W & 37.15 & 29.80 & 25.92 & 33.56 & 30.11 & W & 31.77 & 36.82 & 35.68 & 33.99 & 33.67 \\
\hline 1981 & 34.61 & - & 39.66 & 34.20 & 29.91 & 36.60 & 32.32 & - & 33.70 & 38.70 & 37.29 & 36.14 & 36.47 \\
\hline 1982 & 34.94 & - & 36.16 & 34.99 & 24.93 & 34.81 & 27.15 & - & 28.63 & 34.70 & 34.25 & 31.47 & 33.18 \\
\hline 1983 & 29.37 & - & 30.85 & 29.27 & 22.94 & 29.84 & 25.63 & - & 25.78 & 30.72 & 30.87 & 28.08 & 28.93 \\
\hline 1984 & 29.07 & W & 30.36 & 29.20 & 25.19 & 29.06 & 26.56 & - & 26.85 & 30.05 & 29.45 & 28.14 & 28.54 \\
\hline 1985 & 25.50 & - & 28.96 & 24.72 & 24.43 & 26.86 & 25.71 & - & 25.63 & 28.32 & 28.36 & 26.53 & 26.67 \\
\hline 1986 & 12.92 & 11.70 & 15.29 & 12.84 & 11.52 & 13.46 & 13.43 & 12.85 & 12.17 & 15.98 & 14.63 & 13.52 & 13.49 \\
\hline 1987 & 17.47 & 18.14 & 19.32 & 16.81 & 15.76 & 17.64 & 17.04 & 18.43 & 16.69 & 19.10 & 18.78 & 17.66 & 17.65 \\
\hline 1988 & 13.51 & 12.84 & 15.88 & 13.37 & 13.66 & 14.18 & 13.50 & 14.47 & 12.58 & 15.43 & 15.82 & 13.96 & 14.08 \\
\hline 1989 & 17.37 & 16.90 & 19.19 & 17.34 & 16.78 & 17.78 & 16.81 & 18.10 & 16.35 & 19.06 & 18.74 & 17.54 & 17.68 \\
\hline 1990 & 20.55 & 17.01 & 23.33 & 21.82 & 20.31 & 21.23 & 20.48 & 22.34 & 19.64 & 21.11 & 22.65 & 20.98 & 21.13 \\
\hline 1991 & 17.34 & 18.48 & 21.39 & 17.22 & 15.92 & 18.08 & 17.16 & 19.55 & 15.89 & 21.44 & 21.37 & 17.93 & 18.02 \\
\hline 1992 & 17.58 & 16.99 & 20.78 & 17.48 & 15.13 & 17.81 & 17.04 & 18.46 & 15.60 & 20.90 & 20.63 & 17.67 & 17.75 \\
\hline 1993 & 15.26 & 14.23 & 18.73 & 15.40 & 13.39 & 15.68 & 15.27 & 16.54 & 14.11 & 18.99 & 17.92 & 15.78 & 15.72 \\
\hline 1994 & 15.00 & 14.49 & 17.21 & 15.11 & 13.12 & 15.08 & 14.83 & 15.80 & 14.09 & 17.09 & 16.64 & 15.29 & 15.18 \\
\hline 1995 & 16.78 & 16.47 & 18.25 & 16.84 & 14.81 & 16.61 & 16.65 & 17.45 & 16.19 & 18.06 & 17.91 & 16.95 & 16.78 \\
\hline 1996 & 20.45 & 20.32 & 21.95 & 20.49 & 18.59 & 20.14 & 19.94 & 22.02 & 19.64 & 21.34 & 20.88 & 20.47 & 20.31 \\
\hline 1997 & 17.44 & 17.03 & 20.64 & 17.52 & 16.35 & 17.73 & 17.63 & 19.71 & 17.30 & 20.26 & 20.64 & 18.45 & 18.11 \\
\hline 1998 & 11.18 & 11.00 & 14.14 & 11.16 & 10.16 & 11.46 & 11.62 & 13.26 & 11.04 & 13.83 & 13.55 & 12.22 & 11.84 \\
\hline 1999 & 17.37 & 16.77 & 17.63 & 17.48 & 15.58 & 16.94 & 17.54 & 18.09 & 16.12 & 19.06 & 18.26 & 17.51 & 17.23 \\
\hline 2000 & 26.77 & 26.28 & 30.04 & 26.58 & 26.05 & 27.29 & 26.69 & 29.68 & 26.03 & 30.13 & 29.26 & 27.80 & 27.53 \\
\hline 2001 & 20.73 & 19.66 & 26.55 & 20.98 & 19.81 & 21.52 & 20.72 & 25.88 & 19.37 & 25.77 & 25.32 & 22.17 & 21.82 \\
\hline 2002 & 24.13 & 23.04 & 26.45 & 24.77 & 21.93 & 23.83 & 22.98 & 25.28 & 22.09 & 26.60 & 26.35 & 23.97 & 23.91 \\
\hline 2003 & 27.54 & 26.82 & 31.07 & 27.50 & 25.70 & 27.70 & 26.76 & 30.55 & 25.48 & 30.51 & 30.62 & 27.68 & 27.69 \\
\hline 2004 & 36.53 & 35.89 & 40.95 & 37.11 & 33.79 & 36.84 & 34.51 & 39.03 & 32.25 & 39.92 & 39.28 & 35.29 & 36.07 \\
\hline 2005 & 49.68 & 48.36 & 57.55 & 50.31 & 47.87 & 51.36 & 44.73 & 53.42 & 43.47 & 56.23 & 55.28 & 47.31 & 49.29 \\
\hline 2006 & 58.92 & 57.64 & 68.26 & 59.19 & 57.37 & 61.21 & 53.90 & 62.13 & 53.76 & 64.39 & 67.44 & 57.14 & 59.11 \\
\hline 2007 & 69.83 & 66.01 & 78.01 & 70.78 & 66.13 & 71.14 & 60.38 & 70.91 & 62.31 & 71.66 & 72.47 & 63.96 & 67.97 \\
\hline 2008 & 93.59 & 86.35 & 104.83 & 94.75 & 90.76 & 95.49 & 90.00 & 93.43 & 85.97 & 104.13 & 96.95 & 90.59 & 93.33 \\
\hline 2009 & 62.15 & 61.12 & 68.01 & 62.14 & 57.78 & 61.90 & 57.60 & 58.50 & 57.35 & 59.80 & 63.87 & 58.58 & 60.23 \\
\hline 2010 & R78.58 & R75.91 & $R_{83.15}$ & R79.25 & 72.43 & R78.27 & 72.80 & 74.25 & 72.86 & 82.20 & 80.12 & R74.67 & R76.49 \\
\hline $2011^{P}$ & 108.01 & 106.33 & 116.40 & 108.74 & 100.14 & 107.83 & 90.03 & 102.53 & 101.22 & 118.09 & 118.35 & 98.76 & 102.98 \\
\hline
\end{tabular}

1 Prices are not adjusted for inflation. See "Nominal Dollars" in Glossary.

3 Sahrain, Iran, Iraq, Kuwait, Qatar, Saudi Arabia, United Arab Emirates, and the Neutral Zone (between Kuwait and Saudi Arabia).

${ }^{4}$ On this table, "Total OPEC" for all years includes Algeria, Iran, Iraq, Kuwait, Libya, Nigeria, Qatar, Saudi Arabia, United Arab Emirates, and Venezuela; for 1973-2008, also includes Indonesia; for 1973- 2 and be (although Gabon was a member of OPEC for only 1975-1994); and beginning in 2007, also includes Angola. Data for all countries not included in "Total OPEC" are included in "Total Non-OPEC."

(actober, November, and December data only.

$\mathrm{R}=$ Revised. $\mathrm{P}=$ Preliminary. $\quad-=$ No data reported. $\mathrm{W}=$ Value witheld to avoid disclosure of individual

company data.

Noles. - Data are for landed costs of crude oil imports only; they do not account for refined petroleum products imported into the United States. • See "Crude Oil Landed Cost" in Glossary. • Totals may no equal sum of components due to independent rounding.

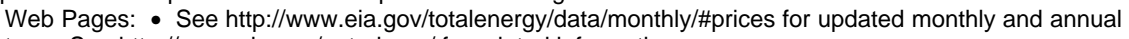
ata. - See http://www.eia.gov/petroleum/ for related information.

Sources: - 1973-Septemner 1977_Federal Energy Administration, Form FEA-F701-M-0, "Transfer "EEA-F701. "Torm "Trance Pricing Report." - Octor 1982-Jun 1984 EIA, Form EP-51. "M2-EIA, Form ERA-51, "Transtion Report." " July 1984 forrand EIA, Petroleum Marketing Monthly (April 2012), Table 22; and EIA, Form EIA-856, "Monthly Forign Crude Oil Acquisition Report." 


\section{Figure 5.20 Value of Crude Oil Imports}

\section{Total, 1973-2011}

$360-$

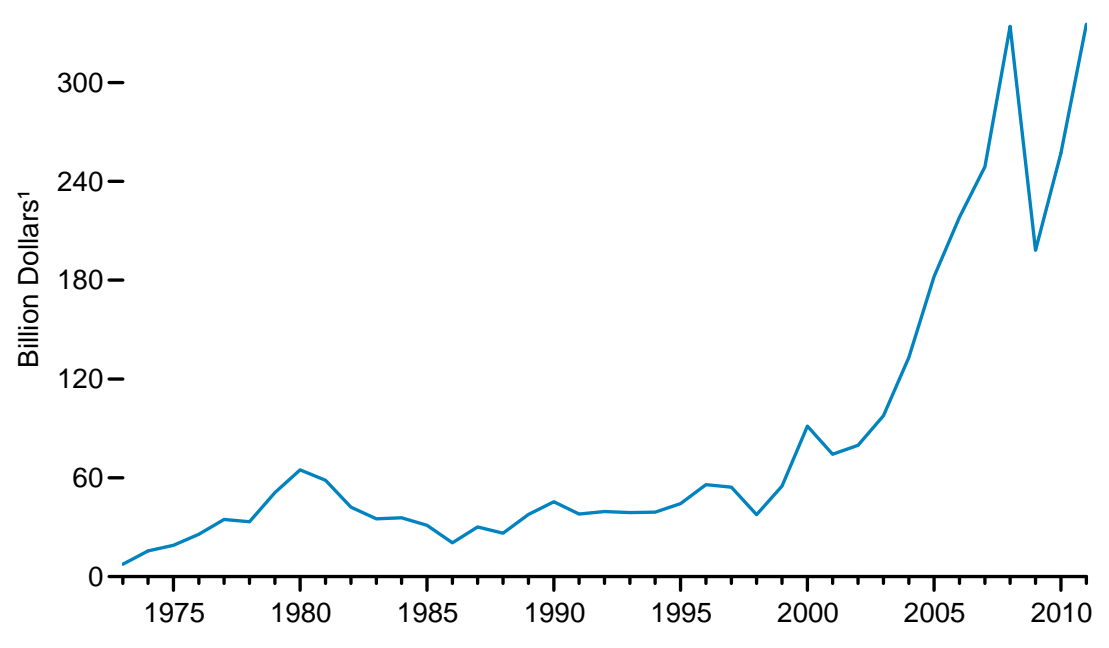

Totals, 2011

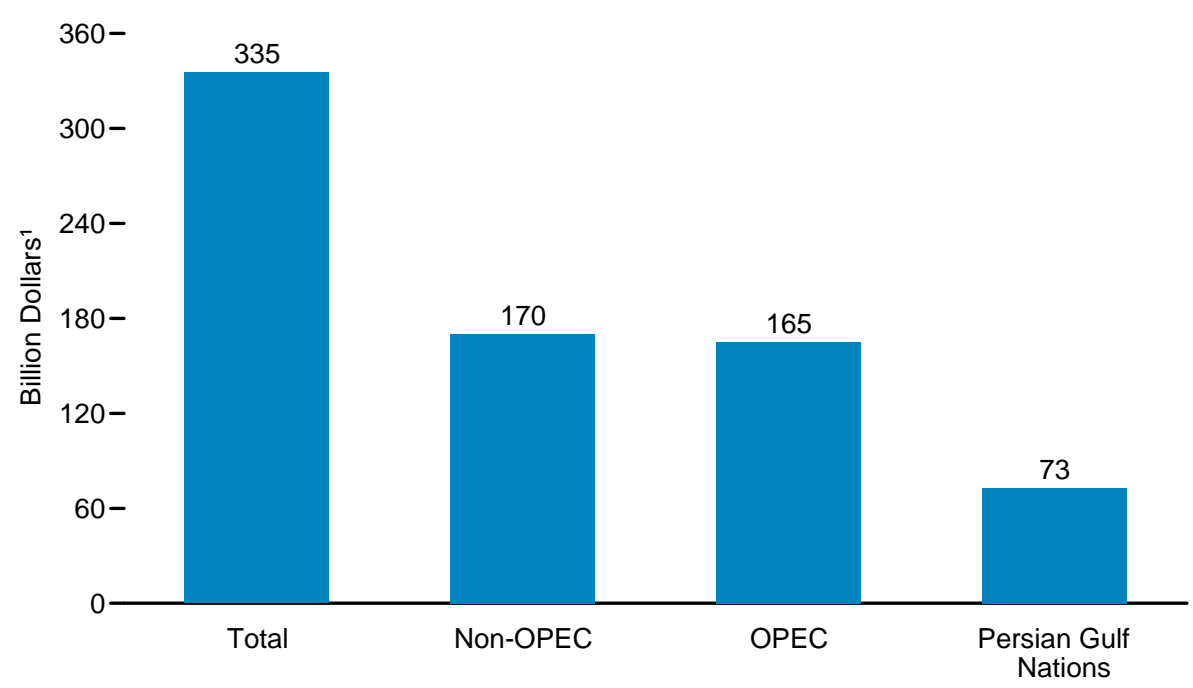

\section{By Selected Country, 2011}

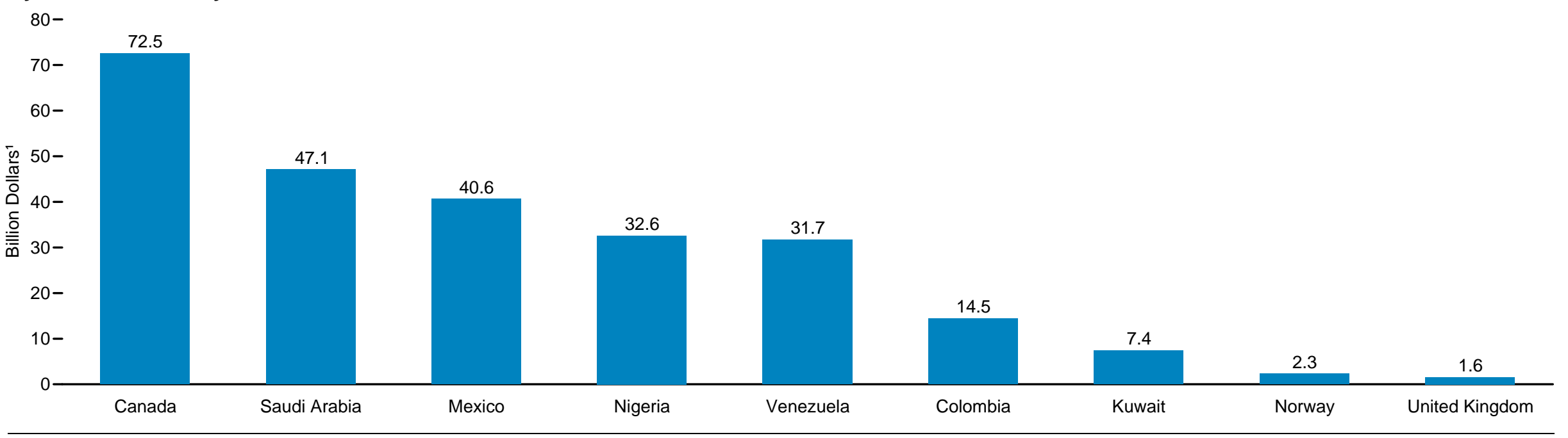

${ }^{1}$ Prices are not adjusted for inflation. See "Nominal Dollars" in Glossary

Source: Table 5.20 . 
Table 5.20 Value of Crude Oil Imports From Selected Countries, 1973-2011

(Billion Dollars ${ }^{1}$ )

\begin{tabular}{|c|c|c|c|c|c|c|c|c|c|c|c|c|c|}
\hline \multirow[b]{2}{*}{ Year } & \multirow[b]{2}{*}{$\begin{array}{c}\text { Persian } \\
\text { Gulf } 3\end{array}$} & \multicolumn{5}{|c|}{ Selected OPEC ${ }^{2}$ Countries } & \multicolumn{6}{|c|}{ Selected Non-OPEC ${ }^{2}$ Countries } & \multirow[b]{2}{*}{ Total $^{5}$} \\
\hline & & Kuwait & Nigeria & $\begin{array}{c}\text { Saudi } \\
\text { Arabia }\end{array}$ & Venezuela & $\begin{array}{c}\text { Total } \\
\text { OPEC } 4\end{array}$ & Canada & Colombia & Mexico & Norway & $\begin{array}{c}\text { United } \\
\text { Kingdom }\end{array}$ & $\begin{array}{c}\text { Total } \\
\text { Non-OPEC } 4\end{array}$ & \\
\hline 1973 & 1.7 & W & 1.5 & 0.9 & 0.8 & 5.2 & 1.9 & W & - & 0.0 & 0.0 & 2.4 & 7.6 \\
\hline 1974 & 4.4 & W & 3.3 & 1.9 & 1.3 & 11.6 & 3.3 & .0 & W & - & .0 & 4.1 & 15.6 \\
\hline 1975 & 5.2 & W & 3.5 & 3.2 & 1.8 & 14.9 & 2.8 & .0 & .3 & .1 & - & 4.1 & 19.0 \\
\hline 1976 & 8.7 & W & 5.1 & 5.8 & 1.0 & 22.2 & 1.8 & - & .4 & .2 & W & 3.6 & 25.8 \\
\hline 1977 & 12.2 & W & 6.3 & 6.9 & 1.2 & 29.6 & 1.4 & .0 & .9 & .3 & .5 & 5.1 & 34.7 \\
\hline 1978 & 11.3 & W & 4.9 & 5.8 & .8 & 27.1 & 1.3 & .0 & 1.6 & .6 & .9 & 6.2 & 33.3 \\
\hline 1979 & 15.3 & W & 9.0 & 9.3 & 1.9 & 39.7 & 2.0 & .0 & 3.3 & .6 & 1.7 & 11.3 & 51.0 \\
\hline 1980 & 16.9 & W & 11.4 & 13.6 & 1.5 & 47.5 & 2.2 & .0 & 5.9 & 1.9 & 2.3 & 17.4 & 64.9 \\
\hline 1981 & 15.1 & .0 & 8.8 & 13.9 & 1.6 & 39.0 & 1.9 & .0 & 5.8 & 1.6 & 5.0 & 19.5 & 58.5 \\
\hline 1982 & 8.4 & - & 6.7 & 6.8 & 1.4 & 22.0 & 2.1 & .0 & 6.7 & 1.3 & 5.5 & 20.2 & 42.2 \\
\hline 1983 & 4.3 & - & 3.4 & 3.4 & 1.4 & 16.1 & 2.6 & .0 & 7.2 & .7 & 4.1 & 19.1 & 35.2 \\
\hline 1984 & 4.8 & W & 2.3 & 3.3 & 2.3 & 16.1 & 3.3 & .0 & 6.5 & 1.2 & 4.1 & 19.7 & 35.8 \\
\hline 1985 & 2.3 & - & 3.0 & 1.2 & 2.7 & 12.9 & 4.4 & .0 & 6.7 & .3 & 2.9 & 18.3 & 31.2 \\
\hline 1986 & 3.8 & .1 & 2.4 & 2.9 & 1.8 & 10.4 & 2.8 & .3 & 2.8 & .3 & 1.7 & 10.2 & 20.6 \\
\hline 1987 & 6.0 & .5 & 3.7 & 3.9 & 2.8 & 15.5 & 3.8 & .8 & 3.7 & .5 & 2.1 & 14.7 & 30.1 \\
\hline 1988 & 6.7 & .4 & 3.5 & 4.4 & 2.2 & 14.0 & 3.4 & .6 & 3.1 & .3 & 1.5 & 12.3 & 26.3 \\
\hline 1989 & 11.0 & 1.0 & 5.6 & 7.1 & 3.0 & 21.9 & 3.9 & .9 & 4.3 & .9 & 1.1 & 15.8 & 37.7 \\
\hline 1990 & 13.5 & .5 & 6.7 & 9.5 & 4.9 & 27.2 & 4.8 & 1.1 & 4.9 & .7 & 1.3 & 18.2 & 45.5 \\
\hline 1991 & 11.0 & (s) & 5.3 & 10.7 & 3.9 & 22.3 & 4.7 & .9 & 4.4 & .6 & .8 & 15.7 & 38.0 \\
\hline 1992 & 10.5 & .2 & 5.1 & 10.2 & 4.6 & 22.2 & 5.0 & .7 & 4.5 & .9 & 1.5 & 17.3 & 39.5 \\
\hline 1993 & 9.1 & 1.8 & 4.9 & 7.2 & 4.9 & 20.7 & 5.0 & .9 & 4.4 & .9 & 2.0 & 18.3 & 38.9 \\
\hline 1994 & 8.8 & 1.6 & 3.9 & 7.2 & 5.0 & 19.7 & 5.3 & .8 & 4.8 & 1.2 & 2.4 & 19.4 & 39.1 \\
\hline 1995 & 9.1 & 1.3 & 4.1 & 7.7 & 6.2 & 21.6 & 6.3 & 1.3 & 6.1 & 1.7 & 2.2 & 22.6 & 44.3 \\
\hline 1996 & 11.1 & 1.8 & 4.8 & 9.4 & 8.9 & 25.3 & 7.8 & 1.8 & 8.7 & 2.3 & 1.6 & 30.5 & 55.8 \\
\hline 1997 & 10.4 & 1.6 & 5.2 & 8.3 & 8.3 & 24.4 & 7.7 & 1.9 & 8.6 & 2.1 & 1.3 & 29.9 & 54.4 \\
\hline 1998 & 8.3 & 1.2 & 3.6 & 5.7 & 5.1 & 17.4 & 5.4 & 1.7 & 5.3 & 1.1 & .8 & 20.2 & 37.6 \\
\hline 1999 & 15.0 & 1.5 & 4.0 & 8.8 & 6.5 & 26.1 & 7.5 & 3.0 & 7.4 & 1.8 & 1.9 & 28.8 & 54.9 \\
\hline 2000 & 23.6 & 2.5 & 9.6 & 14.8 & 11.7 & 45.4 & 13.2 & 3.5 & 12.5 & 3.3 & 3.1 & 46.0 & 91.4 \\
\hline 2001 & 20.2 & 1.7 & 8.2 & 12.3 & 9.3 & 38.1 & 10.3 & 2.5 & 9.9 & 2.6 & 2.3 & 36.2 & 74.3 \\
\hline 2002 & 19.5 & 1.8 & 5.7 & 13.7 & 9.6 & 35.5 & 12.1 & 2.2 & 12.1 & 3.4 & 3.9 & 44.3 & 79.8 \\
\hline 2003 & 24.4 & 2.0 & 9.4 & 17.3 & 11.1 & 46.3 & 15.1 & 1.8 & 14.6 & 2.0 & 4.0 & 51.4 & 97.7 \\
\hline 2004 & 32.1 & 3.2 & 16.2 & 20.3 & 16.0 & 68.0 & 20.4 & 2.0 & 18.9 & 2.1 & 3.4 & 65.2 & 133.2 \\
\hline 2005 & 40.0 & 4.0 & 22.6 & 26.5 & 21.7 & 90.3 & 26.7 & 3.0 & 24.7 & 2.4 & 4.5 & 91.9 & 182.2 \\
\hline 2006 & 46.5 & 3.8 & 25.8 & 30.7 & 23.9 & 106.9 & 35.5 & 3.2 & 30.9 & 2.3 & 3.2 & 111.4 & 218.3 \\
\hline 2007 & 53.9 & 4.2 & 30.9 & 37.4 & 27.7 & 139.9 & 41.6 & 3.6 & 32.0 & 1.5 & 2.7 & 109.0 & 248.9 \\
\hline 2008 & 80.1 & 6.5 & 35.4 & 52.1 & 34.5 & 189.2 & 64.4 & 6.1 & 37.3 & 1.1 & 2.8 & 145.0 & 334.2 \\
\hline 2009 & 37.6 & 4.0 & 19.3 & 22.2 & 20.1 & 98.4 & 40.8 & 5.4 & 22.9 & 1.3 & 2.4 & 99.7 & 198.1 \\
\hline 2010 & ${ }^{R} 48.6$ & 5.4 & $\mathrm{R}_{29.8}$ & $\mathrm{R}_{31.3}$ & 24.1 & $\mathrm{R}_{130.1}$ & 52.4 & 9.2 & $R_{30.6}$ & .8 & 3.5 & $\mathrm{R}_{127.2}$ & $\mathrm{R}_{257.2}$ \\
\hline $2011^{P}$ & 72.9 & 7.4 & 32.6 & 47.1 & 31.7 & 165.1 & 72.5 & 14.5 & 40.6 & 2.3 & 1.6 & 170.2 & 335.3 \\
\hline
\end{tabular}

Prices are not adjusted for inflation. See "Nominal Dollars" in Glossary.

See "Organization of the Petroleum Exporting Countries (OPEC)" in Glossary.

3 Bahrain, Iran, Iraq, Kuwait, Qatar, Saudi Arabia, United Arab Emirates, and the Neutral Zone (between Kuwait and Saudi Arabia)

"On this table, "Total OPEC" for all years includes Algeria, Iran, Iraq, Kuwait, Libya, Nigeria, Qatar, Saudi Arabia, United Arab Emirates, and Venezuela; for 1973-2008, also includes Indonesia; for 1973-1992 and beginning in 2008, also includes Ecuador (although Ecuador rejoined OPEC in November 2007, on this table Ecuador is included in "Tolal Non-OPEC" for 2007), for 1974-1995, also includes Gabon (although Gabon was a member of OPEC for only 1975-1994); and beginning in 2007, also includes Angola. Data for all countries not included in "Total OPEC" are included in "Total Non-OPEC."

Data shown here represent landed value; they differ from data in Table 3.7, which are data from U.S.

Customs that represent crude oil value at the port of loading.

$\mathrm{R}=$ Revised. $\mathrm{P}=$ Preliminary. $\quad-=$ No data reported. $(\mathrm{s})=$ Less than $\$ 0.05$ billion. $\mathrm{W}=\mathrm{Value}$ withheld to avoid disclosure of individual company data.

Notes: - Crude oil import volumes used to calculate values in this table are for the 50 States and the District of Columbia. - Totals may not equal sum of components due to independent rounding.

Web Page: For related information, see http://www.eia.gov/petroleum/.

Sources: Calculated by using prices on Table 5.19 and volume data from the following sources: - 1973-1975-U.S. Department of the Interior, Bureau of Mines, Petroleum Statement, Annual, annual reports. - 1976-1980-U.S. Energy Information Administration (EIA), Petroleum Statement, Annual, annual reports. • 1981-2010-EIA, Petroleum Supply Annual, annual reports. • 2011-EIA, Petroleum Supply Monthly (February 2012). 
Figure 5.21 Crude Oil Refiner Acquisition Costs, 1968-2011

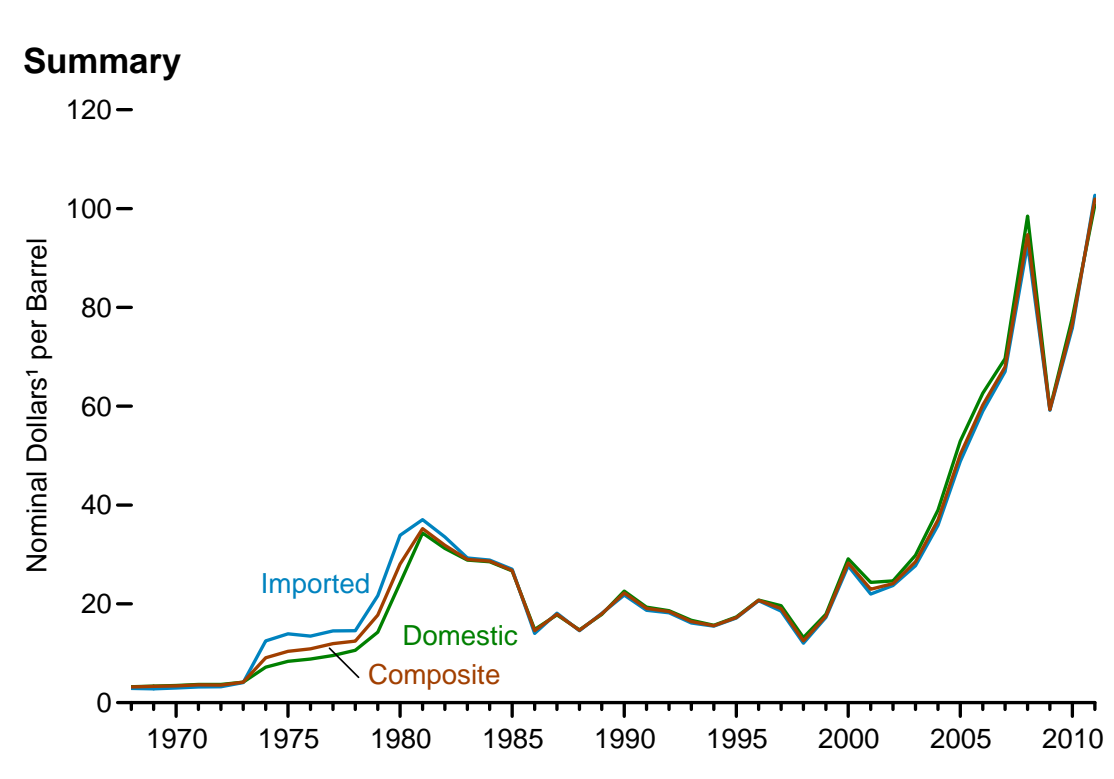

\section{Composite Costs}

$120-$

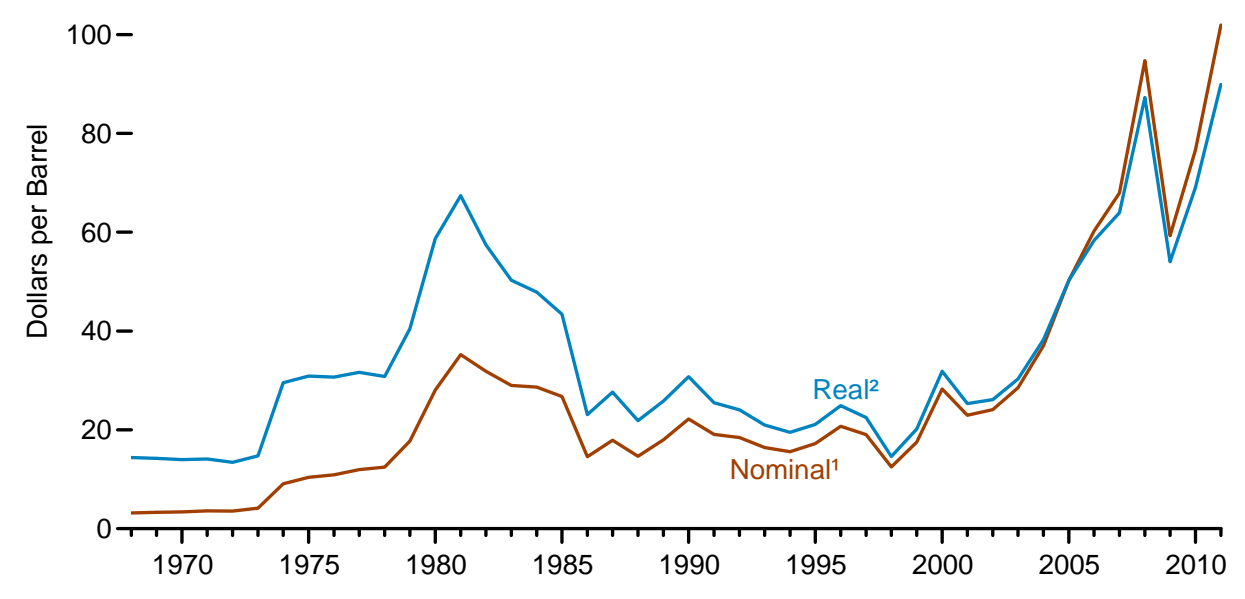

Domestic Costs

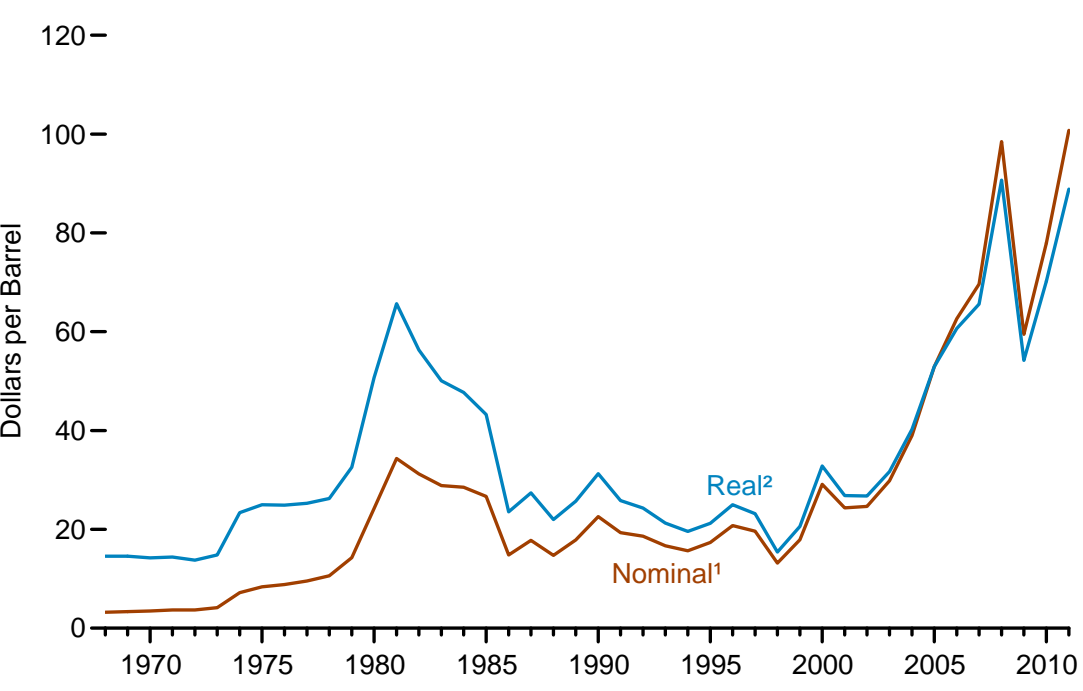

Imported Costs

$120-$

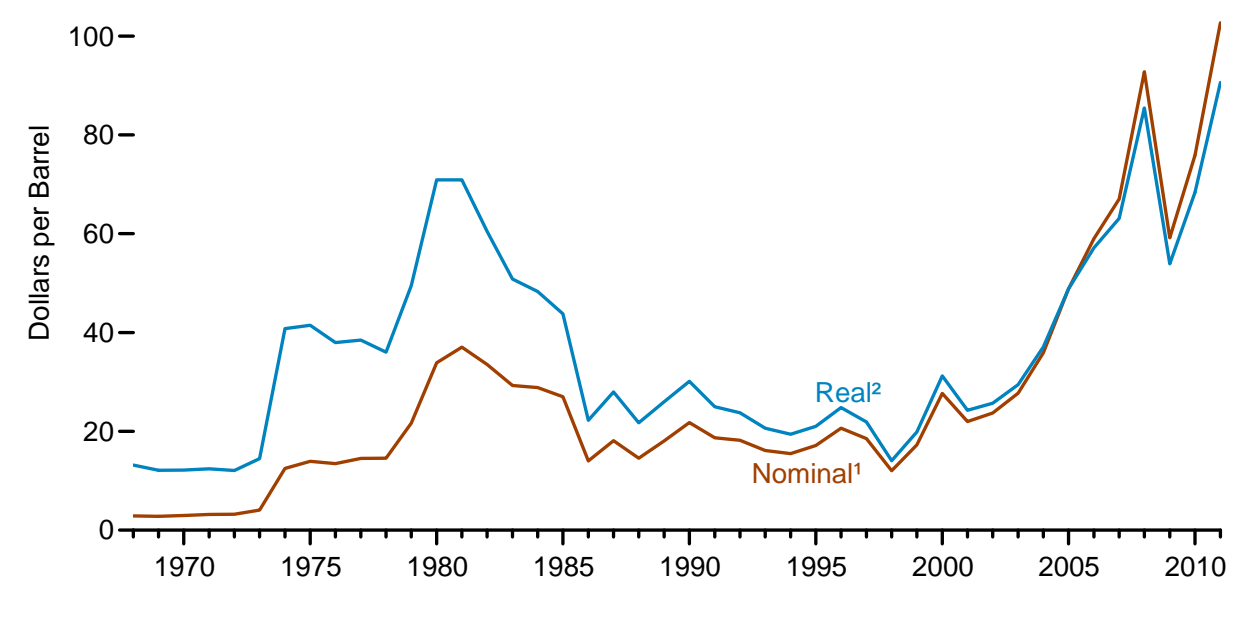

${ }^{1}$ See "Nominal Dollars" in Glossary.

2 In chained (2005) dollars, calculated by using gross domestic product implicit price defla-

Source: Table 5.21 . tors in Table D1. See "Chained Dollars" in Glossary. 
Table 5.21 Crude Oil Refiner Acquisition Costs, 1968-2011

(Dollars per Barrel)

\begin{tabular}{|c|c|c|c|c|c|c|}
\hline \multirow[b]{2}{*}{ Year } & \multicolumn{2}{|c|}{ Domestic } & \multicolumn{2}{|c|}{ Imported } & \multicolumn{2}{|c|}{ Composite } \\
\hline & Nominal 1 & Real $^{2}$ & Nominal 1 & Real $^{2}$ & Nominal 1 & Real $^{2}$ \\
\hline $1968 \mathrm{E}$ & 3.21 & $R_{14.57}$ & 2.90 & $\mathrm{R}_{13.16}$ & 3.17 & $R_{14.39}$ \\
\hline $1969 \mathrm{E}$ & 3.37 & $R_{14.58}$ & 2.80 & $R_{12.11}$ & 3.29 & $\mathrm{R}_{14.23}$ \\
\hline $1970^{\mathrm{E}}$ & 3.46 & $R_{14.22}$ & 2.96 & $R_{12.16}$ & 3.40 & $R_{13.97}$ \\
\hline $1971^{\mathrm{E}}$ & 3.68 & $R_{14.40}$ & 3.17 & $R_{12.41}$ & $\begin{array}{l}3.70 \\
3.60\end{array}$ & $R_{14.09}$ \\
\hline $1972^{\mathrm{E}}$ & 3.67 & $\mathrm{R}_{13.77}$ & 3.22 & $\mathrm{R}_{12.08}$ & 3.58 & $R_{13.43}$ \\
\hline $1973 \mathrm{E}$ & 4.17 & $R_{14.82}$ & 4.08 & $\mathrm{R}_{14.50}$ & 4.15 & $\mathrm{R}_{14.75}$ \\
\hline 1974 & 7.18 & $\mathrm{R}_{23.40}$ & 12.52 & $\mathrm{R}_{40.80}$ & 9.07 & $R_{29.55}$ \\
\hline 1975 & 8.39 & $\mathrm{R}_{24.98}$ & 13.93 & R41.47 & 10.38 & $\mathrm{R}_{30.90}$ \\
\hline 1976 & 8.84 & $\mathrm{R}_{24.89}$ & 13.48 & $R_{37.95}$ & 10.89 & $\mathrm{R}_{30.66}$ \\
\hline 1977 & 9.55 & $\mathrm{R}_{25.28}$ & 14.53 & $R_{38.46}$ & 11.96 & $R_{31.65}$ \\
\hline 1978 & 10.61 & $\mathrm{R}_{26.24}$ & 14.57 & R36.03 & 12.46 & R30.81 \\
\hline 1979 & 14.27 & $R_{32.58}$ & 21.67 & R 49.48 & 17.72 & $\mathrm{R}_{40.46}$ \\
\hline 1980 & 24.23 & R50.70 & 33.89 & R70.91 & 28.07 & R58.73 \\
\hline 1981 & 34.33 & $\mathrm{R}_{65.68}$ & 37.05 & R70.88 & 35.24 & $\mathrm{R}_{67.42}$ \\
\hline 1982 & 31.22 & $\begin{array}{r}8.00 \\
\text { R56.29 }\end{array}$ & 33.55 & R60.50 & 31.87 & $\begin{array}{l}\text { R57.47 } \\
\text { R }\end{array}$ \\
\hline 1983 & 28.87 & R50.08 & 29.30 & R50.82 & 28.99 & R50.28 \\
\hline 1984 & 28.53 & R 47.70 & 28.88 & R 48.28 & 28.63 & $R_{47.86}$ \\
\hline 1985 & 26.66 & R43.26 & 26.99 & R43.80 & 26.75 & $\mathrm{R}_{43.41}$ \\
\hline 1986 & 14.82 & $\mathrm{R}_{23.53}$ & 14.00 & $\mathrm{R}_{22.23}$ & 14.55 & $\mathrm{R}_{23.10}$ \\
\hline 1987 & 17.76 & $\mathrm{R}_{27.40}$ & 18.13 & $\mathrm{R}_{27.97}$ & 17.90 & $\mathrm{R}_{27.62}$ \\
\hline 1988 & 14.74 & $\mathrm{R}_{21.98}$ & 14.56 & $\mathrm{R}_{21.72}$ & 14.67 & $R_{21.88}$ \\
\hline 1989 & 17.87 & $\mathrm{R}_{25.68}$ & 18.08 & $R_{25.99}$ & 17.97 & $\mathrm{R}_{25.83}$ \\
\hline 1990 & 22.59 & $R_{31.26}$ & 21.76 & $R_{30.11}$ & 22.22 & $R_{30.75}$ \\
\hline 1991 & 19.33 & $\mathrm{R}_{25.83}$ & 18.70 & $R_{24.99}$ & 19.06 & $\mathrm{R}_{25.47}$ \\
\hline 1992 & 18.63 & $\mathrm{R}_{24.32}$ & 18.20 & $\mathrm{R}_{23.76}$ & 18.43 & $\mathrm{R}_{24.06}$ \\
\hline 1993 & 16.67 & $\mathrm{R}_{21.29}$ & 16.14 & $\mathrm{R}_{20.62}$ & 16.41 & $R_{20.96}$ \\
\hline 1994 & 15.67 & $R_{19.60}$ & 15.51 & $\mathrm{R}_{19.40}$ & 15.59 & $\mathrm{R}_{19.50}$ \\
\hline 1995 & 17.33 & $\mathrm{R}_{21.24}$ & 17.14 & $\mathrm{R}_{21.00}$ & 17.23 & $R_{21.11}$ \\
\hline 1996 & 20.77 & $\mathrm{R}_{24.98}$ & 20.64 & $\mathrm{R}_{24.82}$ & 20.71 & $R_{24.90}$ \\
\hline 1997 & 19.61 & $R_{23.17}$ & 18.53 & $R_{21.90}$ & 19.04 & $\mathrm{R}_{22.50}$ \\
\hline 1998 & 13.18 & $\mathrm{R}_{15.40}$ & 12.04 & $\mathrm{R}_{14.07}$ & 12.52 & $\mathrm{R}_{14.63}$ \\
\hline 1999 & 17.90 & $\mathrm{R}_{20.61}$ & 17.26 & $R_{19.88}$ & 17.51 & $R_{20.16}$ \\
\hline 2000 & 29.11 & R32.81 & 27.70 & $R_{31.22}$ & 28.26 & $R_{31.85}$ \\
\hline 2001 & 24.33 & $\mathrm{R}_{26.82}$ & 22.00 & $\mathrm{R}_{24.25}$ & 22.95 & $\mathrm{R}_{25.30}$ \\
\hline 2002 & 24.65 & $\mathrm{R}_{26.74}$ & 23.71 & $\begin{array}{r}R_{25.72}\end{array}$ & 24.10 & $\mathrm{R}_{26.14}$ \\
\hline 2003 & 29.82 & $R_{31.68}$ & 27.71 & $R_{29.44}$ & 28.53 & $R_{30.31}$ \\
\hline 2004 & 38.97 & $\mathrm{R}_{40.26}$ & 35.90 & $R_{37.09}$ & 36.98 & 38.21 \\
\hline 2005 & 52.94 & 52.94 & 48.86 & 48.86 & 50.24 & 50.24 \\
\hline 2006 & 62.62 & $R_{60.66}$ & 59.02 & R57.17 & 60.24 & R58.35 \\
\hline 2007 & 69.65 & $R_{65.57}$ & 67.04 & $R_{63.11}$ & 67.94 & $\mathrm{R}_{63.96}$ \\
\hline 2008 & 98.47 & R90.69 & 92.77 & R85.44 & 94.74 & R87.25 \\
\hline 2009 & 59.49 & $\begin{array}{r}\text { R54.22 } \\
\text { R }\end{array}$ & 59.17 & $\begin{array}{r}0.44 \\
\text { R53.92 }\end{array}$ & 59.29 & $\begin{array}{r}\text { R54.03 } \\
\text { R }\end{array}$ \\
\hline 2010 & 77.96 & R70.24 & 75.88 & R68.37 & 76.69 & R69.10 \\
\hline 2011 & 100.74 & 88.87 & 102.70 & 90.60 & 101.93 & 89.92 \\
\hline
\end{tabular}

See "Nominal Dollars" in Glossary.

2 In chained (2005) dollars, calculated by using gross domestic product implicit price deflators in Table D1. See "Chained Dollars" in Glossary.

$\mathrm{R}=$ Revised. E=Estimate.

Note: Costs are for crude oil to refiners, including transportation and other fees; they do not include crude oil purchased for the Strategic Petroleum Reserve. The cost for each category and for the composite is derived by dividing the sum of the total purchasing (acquisition) costs of all refiners by the total volume of Web Parch

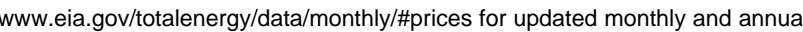
Wee http //www eia gov/petroleum/ for related information.

Sources: • 1968-1973-U.S. Energy Information Administration (EIA) estimates. The cost of domestic crude oil was derived by adding estimated transportation costs to the reported average domestic firs purchase value. The cost of imported crude oil was derived by adding an estimated ocean transport cost based on the published "Average Freight Rate Assessment to the average "Free Alongside Ship" value published by the U.S. Bureau of the Census. The composite cost was derived by weighting domestic costs and imported costs on the basis of quantities produced and imported. - 1974-January 1976-Federal Energy Administration (FEA), Form FEA-96, "Monthly Cost Allocation Report." • February Energy I forgy Intormation Administration (EIA), Petroleum Marketing A E 
Figure 5.22 Refiner Sales Prices for Selected Petroleum Products, 1995-2011

\section{To Resellers}

$3.50-$

$3.00-$

$2.50-$

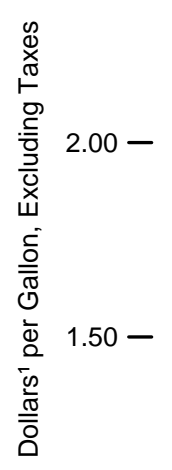

$1.00-$

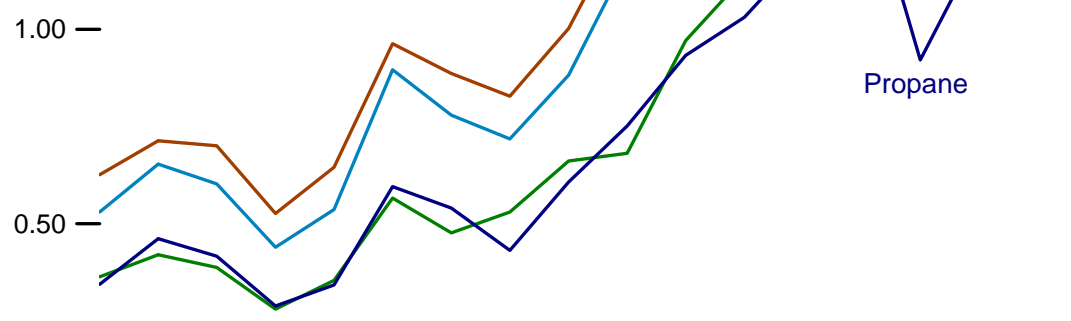

0.00

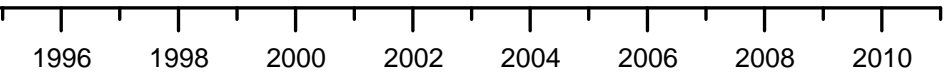

To End Users

$3.50-$

$3.00-$

$2.50-$

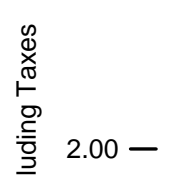

$2.00-$

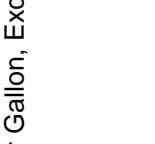

$1.50-$

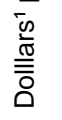

0.00

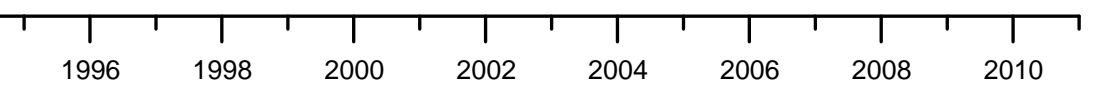

${ }^{1}$ Prices are not adjusted for inflation. See "Nominal Dollars" in Glossary.

Source: Table 5.22 
Table 5.22 Refiner Sales Prices and Refiner Margins for Selected Petroleum Products, 1995-2011

(Dollars ${ }^{1}$ per Gallon, Excluding Taxes)

\begin{tabular}{|c|c|c|c|c|c|c|c|c|c|c|c|c|c|c|c|c|c|}
\hline Product & 1995 & 1996 & 1997 & 1998 & 1999 & 2000 & 2001 & 2002 & 2003 & 2004 & 2005 & 2006 & 2007 & 2008 & 2009 & 2010 & 2011 \\
\hline \multicolumn{18}{|l|}{ Sales Prices to Resellers: ${ }^{2}$} \\
\hline Aviation Gasoline ...................... & 0.975 & 1.055 & 1.065 & 0.912 & 1.007 & 1.330 & 1.256 & 1.146 & 1.288 & 1.627 & 2.076 & 2.490 & 2.758 & 3.342 & 2.480 & 2.874 & 3.739 \\
\hline Motor Gasoline & .626 & .713 & .700 & .526 & .645 & .963 & .886 & .828 & 1.002 & 1.288 & 1.670 & 1.969 & 2.182 & 2.586 & 1.767 & 2.165 & 2.867 \\
\hline Unleaded Regular ………................ & .593 & .685 & .673 & .499 & .620 & .942 & .865 & .806 & .981 & 1.269 & 1.654 & 1.950 & 2.161 & 2.570 & 1.747 & 2.146 & 2.847 \\
\hline Unleaded Midgrade …………........... & .670 & .759 & .749 & .576 & .696 & 1.013 & .945 & .885 & 1.061 & 1.340 & 1.708 & 2.016 & 2.245 & 2.610 & 1.784 & 2.185 & 2.893 \\
\hline Unleaded Premium ……….............. & .722 & .803 & .792 & 617 & .726 & 1.055 & 980 & .928 & 1.113 & 1.408 & 1.789 & 2.117 & 2.357 & 2.746 & 1.958 & 2.346 & 3.049 \\
\hline Kerosene …………………… & .580 & .714 & .653 & .465 & .550 & .969 & .821 & .752 & .955 & 1.271 & 1.757 & 2.007 & 2.249 & 2.851 & 1.844 & 2.299 & 3.065 \\
\hline Jet Fuel, Kerosene-Type ................ & .539 & 646 & .613 & 450 & .533 & .880 & .763 & .716 & .871 & 1.208 & 1.723 & 1.961 & 2.171 & 3.020 & 1.719 & 2.185 & 3.014 \\
\hline No. 1 Distillate & .625 & .751 & .723 & .513 & .634 & 1.019 & .883 & .805 & 1.033 & 1.289 & 1.801 & 2.044 & 2.430 & 2.712 & 2.050 & 2.573 & 3.223 \\
\hline No. 2 Distillate & .530 & 653 & .602 & .439 & .536 & .896 & .779 & .718 & .882 & 1.178 & 1.720 & 1.991 & 2.190 & 2.970 & 1.707 & 2.208 & 3.025 \\
\hline No. 2 Fuel Oil ....................................... & .511 & .639 & .590 & .422 & .493 & .886 & .756 & .694 & .881 & 1.125 & 1.623 & 1.834 & 2.072 & 2.745 & 1.657 & 2.147 & 2.907 \\
\hline 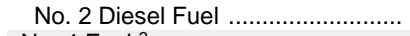 & .538 & 659 & .606 & 444 & .546 & .898 & .775 & .724 & .883 & 1.187 & 1.737 & 2.012 & 2.203 & 2.994 & 1.713 & 2.214 & 3.034 \\
\hline No. 4 Fuel $^{3}$ & .463 & .603 & .551 & 383 & .430 & .778 & 697 & .663 & .793 & 1.033 & 1.377 & 1.395 & 1.551 & 2.157 & 1.561 & w & 2.801 \\
\hline Residual Fuel Oil ............................... & .363 & .420 & .387 & .280 & .354 & .566 & .476 & .530 & .661 & .681 & .971 & 1.136 & 1.350 & 1.866 & 1.342 & 1.697 & 2.336 \\
\hline Sulfur $<=1 \%{ }^{4} \ldots \ldots \ldots \ldots \ldots \ldots$ & .383 & .456 & .415 & 299 & .382 & .627 & .523 & .546 & .728 & .764 & 1.115 & 1.202 & 1.406 & 1.918 & 1.337 & 1.756 & 2.389 \\
\hline Sulfur $>1 \%^{4}$ & .338 & 389 & .366 & .269 & .329 & .512 & .428 & .508 & .588 & .601 & .842 & 1.085 & 1.314 & 1.843 & 1.344 & 1.679 & 2.316 \\
\hline Propane (Consumer Grade) ............ & .344 & 461 & 416 & 288 & .342 & 595 & .540 & 431 & .607 & .751 & .933 & 1.031 & 1.194 & 1.437 & .921 & 1.212 & 1.467 \\
\hline \multicolumn{18}{|l|}{ Sales Prices to End Users: ${ }^{2}$} \\
\hline Aviation Gasoline ....................... & 1.005 & 1.116 & 1.128 & .975 & 1.059 & 1.306 & 1.323 & 1.288 & 1.493 & 1.819 & 2.231 & 2.682 & 2.849 & 3.273 & 2.442 & 3.028 & 3.803 \\
\hline Motor Gasoline ....…………….... & .765 & .847 & .839 & .673 & .781 & 1.106 & 1.032 & .947 & 1.156 & 1.435 & 1.829 & 2.128 & 2.345 & 2.775 & 1.888 & 2.301 & 3.050 \\
\hline Unleaded Regular .............................. & .717 & .807 & .798 & 630 & .742 & 1.073 & .997 & .912 & 1.123 & 1.404 & 1.802 & 2.099 & 2.315 & 2.748 & 1.856 & 2.270 & 3.021 \\
\hline Unleaded Midgrade …................... & .808 & 896 & .895 & .728 & .835 & 1.168 & 1.100 & 1.010 & 1.218 & 1.499 & 1.893 & 2.213 & 2.438 & 2.879 & 1.997 & 2.416 & 3.165 \\
\hline Unleaded Premium .......................... & .890 & .972 & .973 & .805 & .906 & 1.242 & 1.175 & 1.088 & 1.305 & 1.596 & 1.992 & 2.320 & 2.552 & 2.965 & 2.122 & 2.536 & 3.283 \\
\hline Kerosene ……............................. & .589 & .740 & .745 & .501 & .605 & 1.123 & 1.045 & .990 & 1.224 & 1.160 & 1.957 & 2.244 & 2.263 & 3.283 & 2.675 & 3.063 & 3.616 \\
\hline Jet Fuel, Kerosene-Type ................... & .540 & .651 & .613 & .452 & .543 & .899 & .775 & .721 & .872 & 1.207 & 1.735 & 1.998 & 2.165 & 3.052 & 1.704 & 2.201 & 3.054 \\
\hline 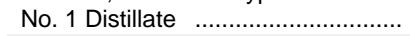 & .620 & .726 & .689 & .551 & .621 & .988 & .902 & .828 & 1.017 & 1.262 & 1.832 & 2.137 & 2.286 & 2.983 & 2.141 & 2.705 & 3.467 \\
\hline No. 2 Distillate & .560 & .680 & 642 & .492 & .580 & .934 & .840 & .759 & .942 & 1.235 & 1.777 & 2.091 & 2.266 & 3.143 & 1.840 & 2.318 & 3.119 \\
\hline No. 2 Fuel Oil ............ ………... & .562 & .673 & .636 & .482 & .558 & .927 & .829 & .737 & .933 & 1.173 & 1.705 & 1.982 & 2.241 & 2.986 & 1.962 & 2.462 & 3.193 \\
\hline No. 2 Diesel Fuel ............................ & .560 & 681 & .642 & .494 & .584 & .935 & .842 & .762 & .944 & 1.243 & 1.786 & 2.096 & 2.267 & 3.150 & 1.834 & 2.314 & 3.117 \\
\hline No. 4 Fuel ${ }^{3} \ldots \ldots \ldots \ldots \ldots \ldots \ldots$ & .505 & .603 & .565 & .428 & .474 & .769 & 679 & .657 & .856 & 1.017 & W & W & W & W & W & W & W \\
\hline Residual Fuel Oil ................................ & .392 & .455 & .423 & .305 & .374 & .602 & .531 & .569 & .698 & .739 & 1.048 & 1.218 & 1.374 & 1.964 & 1.341 & 1.713 & 2.401 \\
\hline Sulfur $<=1 \%^{4} \ldots \ldots \ldots \ldots \ldots \ldots \ldots$ & .436 & .526 & .488 & .354 & .405 & .708 & .642 & .640 & .804 & .835 & 1.168 & 1.342 & 1.436 & 2.144 & 1.413 & 1.920 & 2.736 \\
\hline Sulfur $>1 \%{ }^{4} \ldots \ldots \ldots \ldots \ldots \ldots \ldots \ldots$ & .377 & .433 & .403 & .287 & .362 & .566 & .492 & .544 & .651 & .692 & .974 & 1.173 & 1.350 & 1.889 & 1.306 & 1.619 & 2.257 \\
\hline Propane (Consumer Grade) ............. & .492 & .605 & .552 & .405 & .458 & .603 & .506 & .419 & .577 & .839 & 1.089 & 1.358 & 1.489 & 1.892 & 1.220 & 1.481 & 1.709 \\
\hline \multicolumn{18}{|l|}{ Refiner Margins ${ }^{5}$} \\
\hline 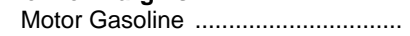 & .216 & .220 & .247 & .228 & .228 & 290 & .340 & .254 & .323 & .408 & .474 & .535 & .564 & .330 & .355 & .339 & .440 \\
\hline Jet Fuel, Kerosene-Type .................. & .129 & .153 & .160 & .152 & .116 & .207 & .217 & .142 & .192 & .328 & .527 & .527 & .553 & .764 & .307 & .359 & .587 \\
\hline No. 2 Distillate & .120 & .160 & .149 & .141 & .119 & .223 & .233 & .144 & .203 & .298 & .524 & .557 & .572 & .714 & .295 & .382 & .598 \\
\hline Residual Fuel Oil ................................... & -.048 & -.072 & -.066 & -.018 & -.063 & -107 & -.070 & -.044 & -.018 & -.199 &. .225 & -.298 & -.268 & -.390 & -.070 & -.129 & -.091 \\
\hline Composite $^{6} \ldots \ldots \ldots \ldots$ & .181 & .194 & .200 & .195 & .189 & .261 & .297 & .216 & .281 & .367 & .484 & .530 & .553 & .453 & .328 & .344 & .481 \\
\hline
\end{tabular}

1 Prices are not adjusted for inflation. See "Nominal Dollars" in Glossary.

Sales for resale (wholesale sales) are those made to purchasers who are other than ultimate consumers. Sales to end users are those made directly to the ultimate consumer, including bulk customers, such as agriculture, industry, and utilities, as well as residential and commercial customers.

Includes No. 4 fuel oil and No. 4 diesel fuel.

4 Sulfur content by weight.
5 In this table, refiner margin is the difference between the composite refiner acquisition price of crude oil (see Table 5.21) and the price to resellers.

${ }^{6}$ A volume weighted average of the refiner prices to resellers for aviation gasoline, kerosene-type jet fuel, kerosene, motor gasoline, distillate fuel nos. 1,2, and 4, and residual fuel oil.

$W=$ Value withheld to avoid disclosure of individual company data.

Web Pages: - See http://www.eia.gov/totalenergy/data/monthly/\#prices for updated monthly and annual

data. - See http://www.eia.gov/petroleum/ for related information.
Source: U.S. Energy Information Administration, Petroleum Marketing Monthly (April 2012), Tables 1, 2 , 4,6 , and 16 . 


\section{Motor Gasoline, Selected Grades}

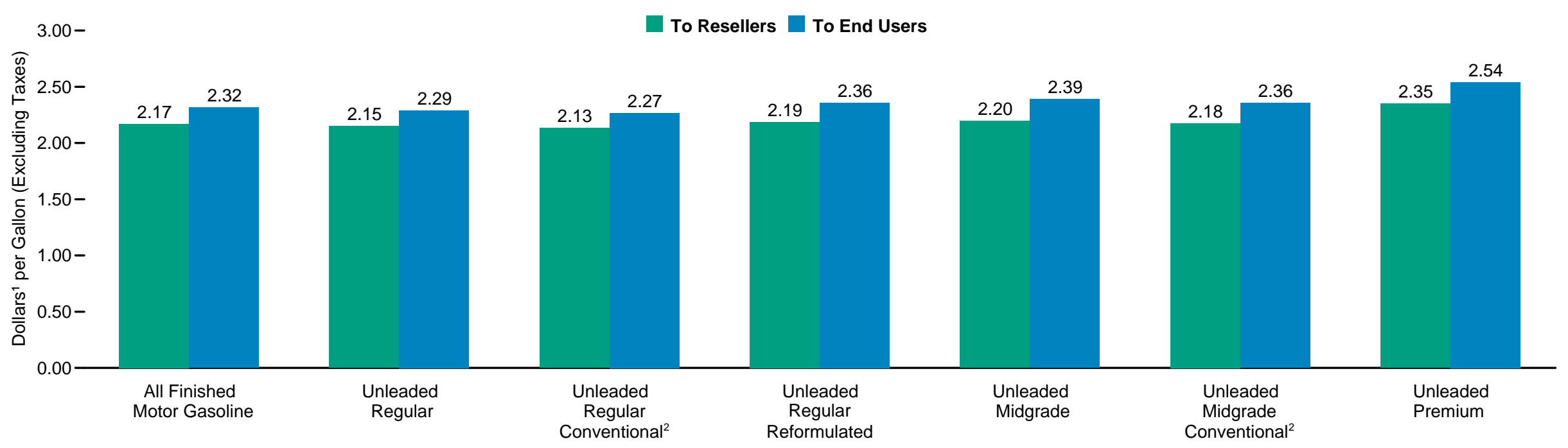

Distillate Fuel Oil, Residual Fuel Oil, and Propane

To Resellers To End Users

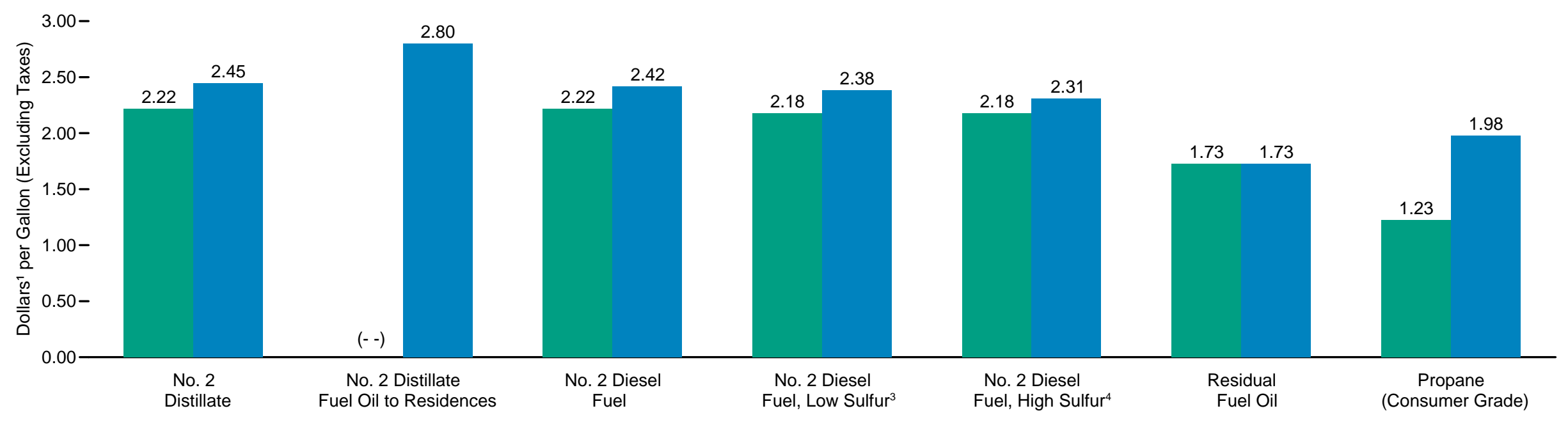

${ }^{1}$ Prices are not adjusted for inflation. See "Nominal Dollars" in Glossary.

${ }^{2}$ Includes oxygenated motor gasoline.

${ }^{3}>15$ and $<=500$ parts per million.

- - = Not applicable.

${ }^{4}>500$ parts per million.

Source: Table 5.23. 
Table 5.23 All Sellers Sales Prices for Selected Petroleum Products, 1994-2010 (Dollars ${ }^{1}$ per Gallon, Excluding Taxes)

\begin{tabular}{|c|c|c|c|c|c|c|c|c|c|c|c|c|c|c|c|c|c|}
\hline Product & 1994 & 1995 & 1996 & 1997 & 1998 & 1999 & 2000 & 2001 & 2002 & 2003 & 2004 & 2005 & 2006 & 2007 & 2008 & 2009 & 2010 \\
\hline \multicolumn{18}{|l|}{ Sales Prices to Resellers ${ }^{2}$} \\
\hline 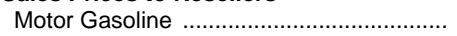 & 0.602 & 0.630 & 0.715 & 0.703 & 0.530 & 0.645 & 0.966 & 0.888 & 0.832 & 1.001 & 1.288 & 1.675 & 1.973 & 2.186 & 2.587 & 1.773 & 2.169 \\
\hline Unleaded Regular ........................................... & .571 & .599 & .689 & .677 & .504 & .621 & .946 & .868 & .813 & .982 & 1.271 & 1.659 & 1.956 & 2.165 & 2.570 & 1.753 & 2.151 \\
\hline 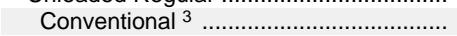 & .565 & .583 & .672 & .658 & .484 & .596 & .918 & .838 & .794 & .950 & 1.241 & 1.639 & 1.930 & 32.145 & 32.564 & 31.732 & 32.133 \\
\hline Oxygenated ${ }^{3}$ & .627 & .662 & .745 & .754 & .575 & .690 & 1.016 & .947 & .858 & 1.031 & 1.289 & 1.667 & 1.951 & $\left({ }^{3}\right)$ & $\left({ }^{3}\right)$ & $\left({ }^{3}\right)$ & $\left({ }^{3}\right)$ \\
\hline Reformulated ……………………… & .632 & .646 & .733 & .725 & .551 & .676 & 1.006 & .930 & .856 & 1.058 & 1.339 & 1.708 & 2.022 & 2.215 & 2.583 & 1.799 & 2.187 \\
\hline Unleaded Midgrade ……………………........... & .641 & .673 & .760 & .751 & .579 & .694 & 1.014 & .945 & .886 & 1.056 & 1.338 & 1.719 & 2.024 & 2.252 & 2.636 & 1.803 & 2.196 \\
\hline 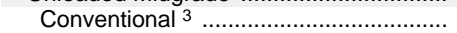 & .633 & .651 & .737 & .723 & .550 & .658 & .977 & .901 & .852 & 1.015 & 1.304 & 1.697 & 1.992 & 32.235 & 32.611 & 31.777 & 32.176 \\
\hline 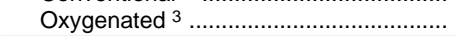 & .689 & .711 & .789 & .791 & .599 & .695 & 1.021 & .965 & .885 & 1.043 & 1.305 & 1.682 & 1.999 & $\left({ }^{3}\right)$ & $\left({ }^{3}\right)$ & $\left({ }^{3}\right)$ & $\left({ }^{3}\right)$ \\
\hline 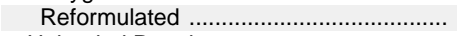 & .722 & .719 & .802 & .801 & 632 & .758 & 1.080 & 1.022 & .952 & 1.150 & 1.410 & 1.790 & 2.127 & 2.311 & 2.730 & 1.903 & 2.275 \\
\hline Unleaded Premium ................................... & .696 & .724 & .804 & .794 & .618 & .724 & 1.055 & .980 & .929 & 1.105 & 1.400 & 1.792 & 2.122 & 2.362 & 2.749 & 1.959 & 2.350 \\
\hline 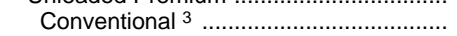 & .686 & .695 & .777 & .764 & .587 & .688 & 1.013 & .933 & .897 & 1.055 & 1.351 & 1.756 & 2.083 & 32.330 & 32.743 & 31.932 & 32.327 \\
\hline Oxygenated ${ }^{3}$ & .757 & .787 & .851 & .856 & .674 & .779 & 1.119 & 1.020 & .952 & 1.131 & 1.390 & 1.761 & 2.049 & $\left({ }^{3}\right)$ & $\left({ }^{3}\right)$ & $\left({ }^{3}\right)$ & $\left({ }^{3}\right)$ \\
\hline 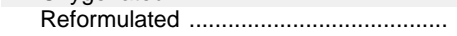 & .769 & .779 & .851 & .845 & .671 & .787 & 1.117 & 1.054 & .986 & 1.189 & 1.467 & 1.844 & 2.181 & 2.407 & 2.757 & 1.994 & 2.376 \\
\hline 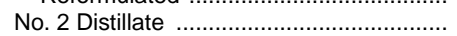 & .529 & .536 & .660 & .611 & .450 & .538 & .901 & .785 & .728 & .891 & 1.178 & 1.727 & 1.999 & 2.208 & 2.975 & 1.727 & 2.216 \\
\hline No. 2 Diesel Fuel ....................... & .538 & .546 & .667 & .616 & .454 & .552 & .904 & .791 & .735 & .891 & 1.191 & 1.754 & 2.029 & 2.230 & 3.013 & 1.730 & 2.219 \\
\hline Ultra-Low Sulfur $\left(<=15 \mathrm{ppm}{ }^{4}\right) \ldots \ldots \ldots \ldots \ldots$ & NA & NA & NA & NA & NA & NA & NA & NA & NA & NA & NA & NA & NA & 2.258 & 3.022 & 1.742 & 2.222 \\
\hline Low Sulfur $\left(>15\right.$ and $\left.<=500 \mathrm{ppm}^{4}\right) \ldots$ & .542 & .551 & .673 & .619 & .457 & .557 & .909 & .794 & .738 & .895 & 1.197 & 1.761 & 2.045 & 2.211 & 2.983 & 1.648 & 2.180 \\
\hline High Sulfur $\left(>500 \mathrm{ppm}^{4}\right)$........................ & .519 & .524 & .639 & .602 & .437 & .519 & .875 & .771 & .712 & .870 & 1.146 & 1.704 & 1.915 & 1.959 & 2.887 & 1.724 & 2.177 \\
\hline 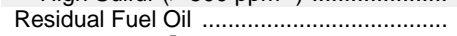 & .322 & .366 & .427 & .396 & .284 & .355 & .579 & .496 & .526 & .675 & .682 & 1.006 & 1.141 & 1.342 & 1.851 & 1.341 & 1.727 \\
\hline Sulfur $<=1 \%^{5}$ & .350 & .383 & .461 & .424 & .305 & .382 & .638 & .542 & .548 & .732 & .740 & 1.107 & 1.205 & 1.377 & 1.898 & 1.349 & 1.812 \\
\hline 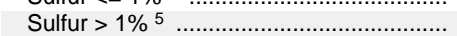 & .298 & .344 & .397 & .375 & .271 & .333 & .523 & .438 & .502 & .621 & .638 & .899 & 1.087 & 1.314 & 1.821 & 1.337 & 1.694 \\
\hline Propane (Consumer Grade) ....................... & .336 & .354 & .471 & .426 & .297 & .354 & .603 & .556 & .440 & .615 & .761 & .939 & 1.041 & 1.206 & 1.455 & .955 & 1.226 \\
\hline \multicolumn{18}{|l|}{ Sales Prices to End Users ${ }^{2}$} \\
\hline Motor Gasoline ....................................... & .729 & .761 & .843 & .831 & .660 & .762 & 1.091 & 1.022 & .943 & 1.135 & 1.423 & 1.828 & 2.121 & 2.338 & 2.772 & 1.893 & 2.316 \\
\hline Unleaded Regular ........................................... & .690 & .721 & .809 & .797 & .623 & .728 & 1.063 & .993 & .915 & 1.108 & 1.399 & 1.807 & 2.098 & 2.313 & 2.750 & 1.867 & 2.290 \\
\hline 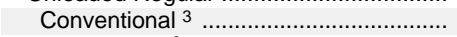 & .685 & .714 & .801 & .785 & .610 & .708 & 1.044 & .968 & .901 & 1.082 & 1.373 & 1.789 & 2.073 & 32.297 & 32.731 & ${ }^{3} 1.842$ & 32.267 \\
\hline Oxygenated ${ }^{3}$ & .737 & .773 & .861 & .887 & .694 & .782 & 1.118 & 1.059 & .964 & 1.142 & 1.414 & 1.768 & 2.076 & $(3)$ & $(3)$ & $(3)$ & $\left({ }^{3}\right)$ \\
\hline Reformulated …........ & .743 & .741 & .833 & .822 & .651 & .777 & 1.109 & 1.051 & .949 & 1.183 & 1.478 & 1.861 & 2.168 & 2.357 & 2.807 & 1.944 & 2.358 \\
\hline Unleaded Midgrade …………………….......... & .770 & .802 & .885 & .880 & .711 & .812 & 1.146 & 1.086 & 1.002 & 1.195 & 1.482 & 1.877 & 2.183 & 2.412 & 2.846 & 1.969 & 2.390 \\
\hline 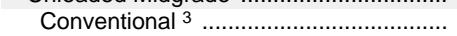 & .766 & .793 & .874 & .865 & 695 & .787 & 1.122 & 1.052 & .985 & 1.166 & 1.453 & 1.855 & 2.151 & 32.389 & 32.816 & 31.934 & 32.357 \\
\hline 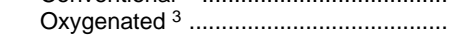 & .821 & .838 & .929 & .964 & .763 & .853 & 1.185 & 1.120 & 1.031 & 1.193 & 1.459 & 1.824 & 2.138 & $\left({ }^{3}\right)$ & $\left(3^{3}\right)$ & $\left(3^{3}\right)$ & $\left({ }^{3}\right)$ \\
\hline Reformulated …………………………......... & .851 & .829 & .916 & .915 & .748 & .869 & 1.197 & 1.156 & 1.042 & 1.277 & 1.569 & 1.945 & 2.273 & 2.476 & 2.936 & 2.077 & 2.494 \\
\hline Unleaded Premium .......................................... & .852 & .883 & .962 & .955 & .786 & .880 & 1.218 & 1.154 & 1.081 & 1.282 & 1.580 & 1.988 & 2.303 & 2.529 & 2.961 & 2.124 & 2.542 \\
\hline Conventional $^{3}$.................................... & .846 & .871 & .950 & .939 & .769 & .856 & 1.192 & 1.119 & 1.063 & 1.245 & 1.538 & 1.958 & 2.263 & 32.497 & 32.935 & 32.082 & 32.504 \\
\hline 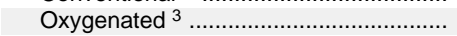 & .908 & .938 & 1.019 & 1.054 & .845 & .940 & 1.279 & 1.218 & 1.128 & 1.307 & 1.568 & 1.915 & 2.236 & $\left({ }^{3}\right)$ & $\left({ }^{3}\right)$ & $\left({ }^{3}\right)$ & $\left({ }^{3}\right)$ \\
\hline 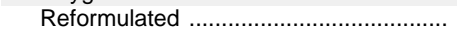 & .937 & .914 & .991 & .988 & .822 & .931 & 1.267 & 1.217 & 1.116 & 1.355 & 1.660 & 2.043 & 2.373 & 2.584 & 3.004 & 2.196 & 2.607 \\
\hline No. 2 Distillate ....……………………... & .675 & .673 & .793 & .753 & .599 & .678 & 1.044 & .948 & .874 & 1.058 & 1.339 & 1.895 & 2.176 & 2.365 & 3.222 & 1.964 & 2.449 \\
\hline No. 2 Distillate to Residences ${ }^{6}$............... & .884 & .867 & .989 & .984 & .852 & .876 & 1.311 & 1.250 & 1.129 & 1.355 & 1.548 & 2.052 & 2.365 & 2.592 & 3.219 & 2.386 & 2.798 \\
\hline No. 2 Diesel Fuel …………………. & .628 & .636 & .757 & .714 & .562 & .654 & 1.006 & .912 & .837 & 1.008 & 1.316 & 1.886 & 2.165 & 2.348 & 3.230 & 1.918 & 2.415 \\
\hline Ultra-Low Sulfur $\left(<=15 \mathrm{ppm}{ }^{4}\right) \ldots \ldots \ldots \ldots \ldots$ & NA & NA & NA & NA & NA & NA & NA & NA & NA & NA & NA & NA & NA & 2.386 & 3.233 & 1.922 & 2.423 \\
\hline Low Sulfur $\left(>15\right.$ and $\left.<=500 \mathrm{ppm}^{4}\right) \ldots .$. & .642 & .645 & .767 & .719 & .565 & .663 & 1.014 & .917 & .841 & 1.014 & 1.325 & 1.899 & 2.183 & 2.317 & 3.232 & 1.913 & 2.381 \\
\hline High Sulfur $\left(>500 \mathrm{ppm}{ }^{4}\right)$.......................... & .598 & .614 & .732 & .698 & .555 & .620 & .981 & .892 & .822 & .986 & 1.281 & 1.833 & 2.084 & 2.187 & 3.187 & 1.865 & 2.308 \\
\hline 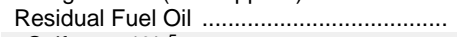 & .358 & .397 & .464 & .429 & .311 & .378 & .609 & .533 & .561 & .696 & .725 & 1.041 & 1.212 & 1.376 & 1.944 & 1.340 & 1.729 \\
\hline Sulfur $<=1 \%^{5}$ & .403 & .433 & .529 & .472 & .356 & .406 & .683 & .620 & .612 & .785 & .794 & 1.148 & 1.322 & 1.445 & 2.098 & 1.422 & 1.953 \\
\hline Sulfur $>1 \%^{5}$ & .327 & .376 & .430 & .407 & .292 & .366 & .576 & .498 & .540 & .651 & .687 & .985 & 1.175 & 1.354 & 1.896 & 1.312 & 1.662 \\
\hline 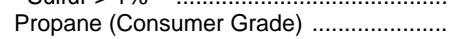 & .776 & .766 & .886 & .878 & .774 & .781 & 1.048 & 1.094 & .958 & 1.150 & 1.307 & 1.530 & 1.727 & 1.888 & 2.245 & 1.777 & 1.976 \\
\hline
\end{tabular}

1 Prices are not adjusted for inflation. See "Nominal Dollars" in Glossary.

2 Sales for resale (wholesale sales) are those made to purchasers who are other than ultimate consumers. Sales to end users are those made directly to the ultimate consumer, including bulk

customers, such as agriculture, industry, and utilties, as well as residential and commercial customers.

Beginning in 2007, oxygenated motor gasoline is included with conventional motor gasoline.

Parts per million.

${ }^{6}$ See Note 5, "Historical Residential Heating Oil Prices," at end of section for historical data.
$\mathrm{NA}=$ Not available.

Note: Data for this table are not available for 2011

Web Page: For related information, see http://www.eia.gov/petroleum/.

Sources: • 1994-2009-U.S. Energy Information Administration (EIA), Petroleum Marketing Annual, annual reports. • 2010-EIA, Petroleum Marketing Monthly (April 2011); EIA, Form EIA-782A "Reserters'; and EIA, Form EIA-782B, 


\section{Figure 5.24 Retail Motor Gasoline and On-Highway Diesel Fuel Prices}

\section{Motor Gasoline, All Grades, 1978-2011}

$4.00-$
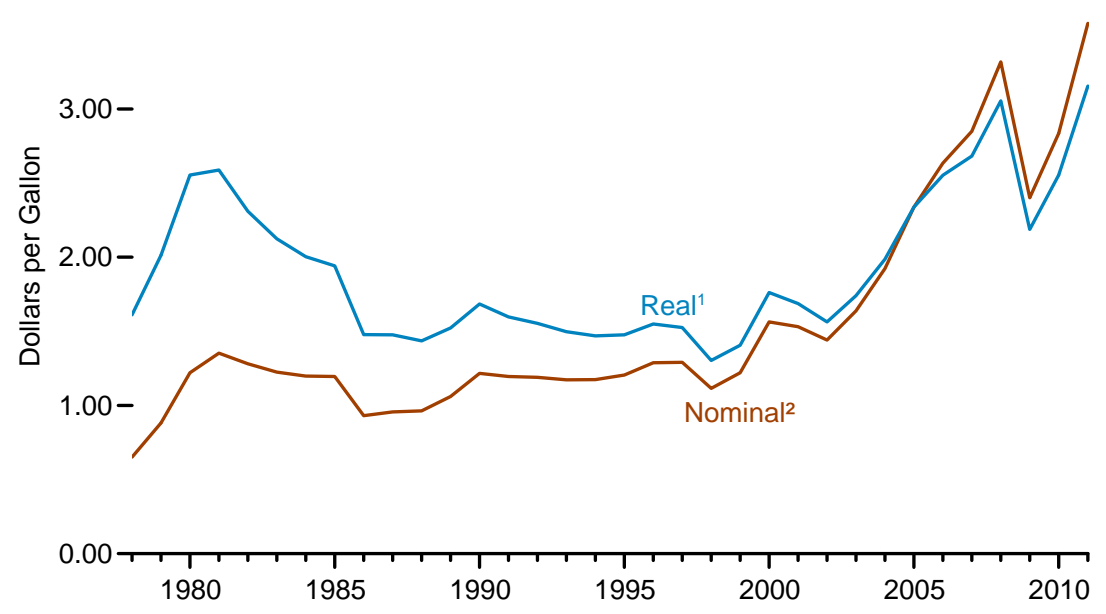

Motor Gasoline by Grade, 1949-2011

$4.00-$

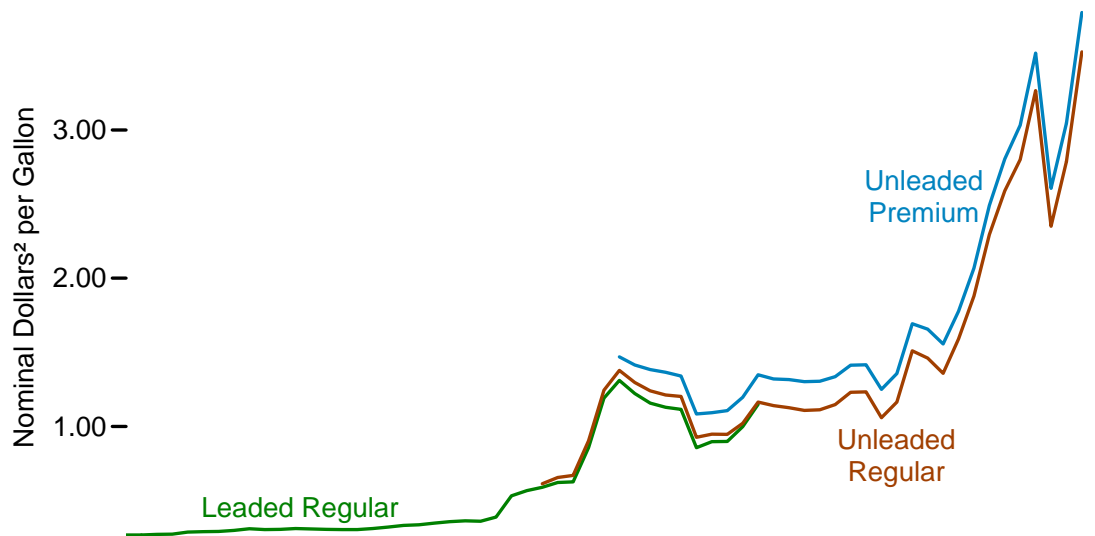

Regular Motor Gasoline by Area Type and On-Highway Diesel Fuel, 2011 $4.50-$

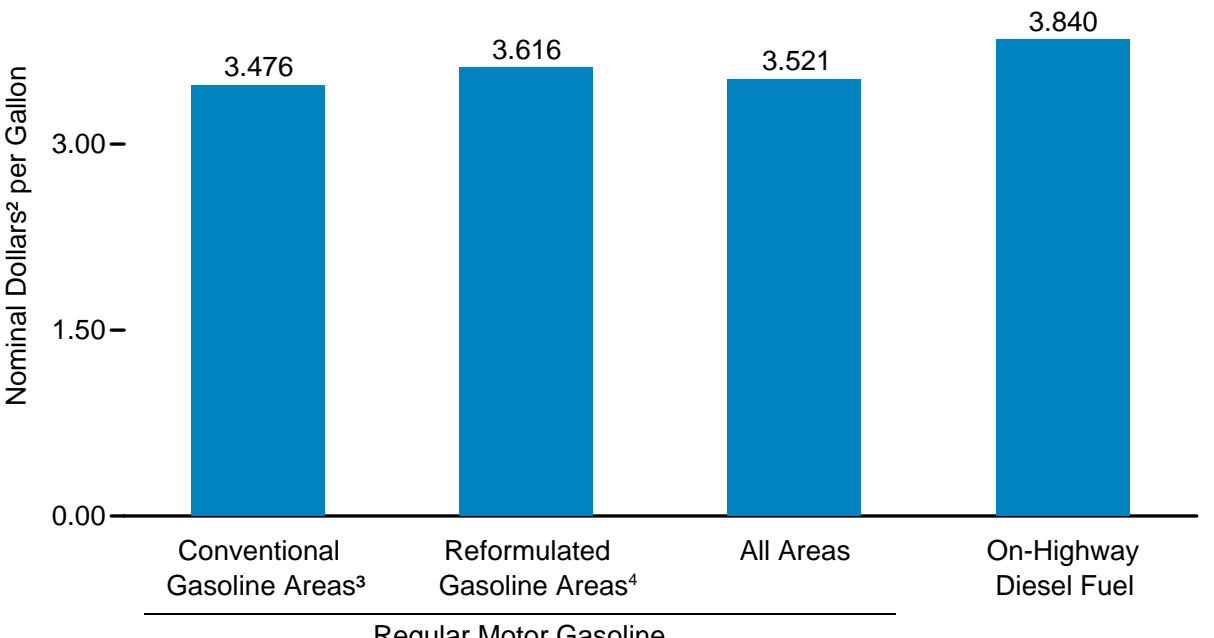

Motor Gasoline Price and Consumption, 1978-2011, Indexed to 1978 $2.5-$

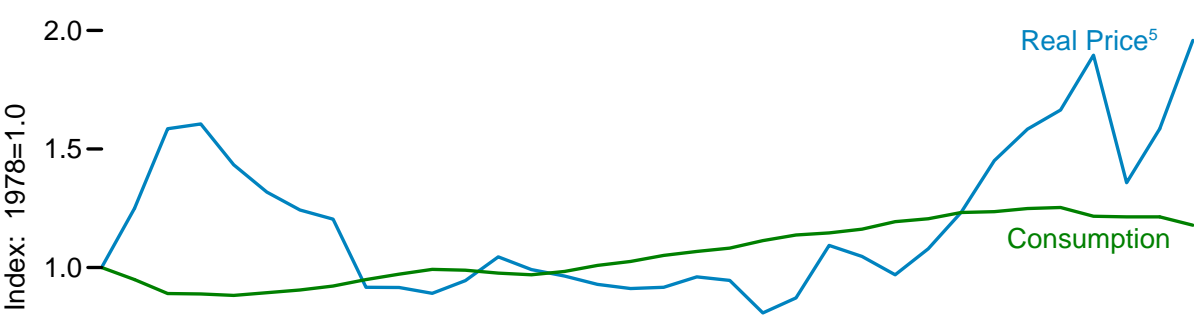

$0.5-$

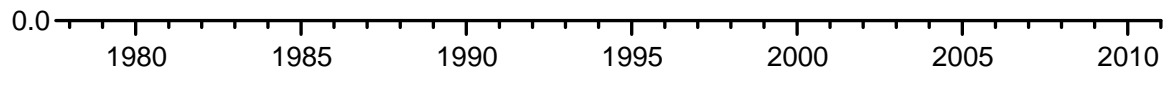

4 "Reformulated Gasoline Areas" are ozone nonattainment areas designated by the Environmental Protection Agency that require the use of reformulated gasoline.

${ }^{5}$ All grades, in chained (2005) dollars.

Sources: Tables 5.11 and 5.24
${ }^{1}$ In chained (2005) dollars, calculated by using gross domestic product implicit price defla-
ors in Table D1. See "Chained Dollars" in Glossary. ${ }^{2}$ See "Nominal Dollars" in Glossary.

${ }^{3}$ Any area that does not require the sale of reformulated gasoline. 
Table 5.24 Retail Motor Gasoline and On-Highway Diesel Fuel Prices, Selected Years, 1949-2011

(Dollars per Gallon)

\begin{tabular}{|c|c|c|c|c|c|c|c|c|c|c|c|c|}
\hline \multirow[b]{3}{*}{ Year } & \multicolumn{8}{|c|}{ Motor Gasoline by Grade } & \multicolumn{3}{|c|}{ Regular Motor Gasoline by Area Type } & \multirow{3}{*}{$\begin{array}{r}\begin{array}{r}\text { On-Highway } \\
\text { Diesel Fuel }\end{array} \\
\text { Nominal }^{5}\end{array}$} \\
\hline & \multicolumn{2}{|c|}{ Leaded Regular } & \multicolumn{2}{|c|}{ Unleaded Regular } & \multicolumn{2}{|c|}{ Unleaded Premium } & \multicolumn{2}{|c|}{ All Grades } & \multirow{2}{*}{$\begin{array}{c}\begin{array}{c}\text { Conventional } \\
\text { Gasoline Areas }\end{array}{ }^{1,2} \\
\text { Nominal }^{5}\end{array}$} & \multirow{2}{*}{$\begin{array}{c}\begin{array}{c}\text { Reformulated } \\
\text { Gasoline Areas }\end{array} \\
\text { Nominal } \\
\end{array}$} & \multirow{2}{*}{$\begin{array}{l}\text { All Areas } \\
\text { Nominal } 5\end{array}$} & \\
\hline & Nominal 5 & Real 6 & Nominal 5 & Real 6 & Nominal 5 & Real 6 & Nominal 5 & Real 6 & & & & \\
\hline 1949 & 0.268 & $\mathrm{R}_{1.848}$ & NA & NA & NA & NA & NA & NA & NA & NA & NA & NA \\
\hline 1950 & .268 & $R_{1.829}$ & NA & NA & NA & NA & NA & NA & NA & NA & NA & NA \\
\hline 1955 & .291 & $R_{1.753}$ & NA & NA & NA & NA & NA & NA & NA & NA & NA & NA \\
\hline 1960 & .311 & $R_{1} .671$ & NA & NA & NA & NA & NA & NA & NA & NA & NA & NA \\
\hline 1965 & .312 & $\mathrm{R}_{1.565}$ & NA & NA & NA & NA & NA & NA & NA & NA & NA & NA \\
\hline 1970 & .357 & $R_{1} .467$ & NA & NA & NA & NA & NA & NA & NA & NA & NA & NA \\
\hline 1975 & .567 & $R_{1.688}$ & NA & NA & NA & NA & NA & NA & NA & NA & NA & NA \\
\hline 1976 & .590 & $\mathrm{R}_{1} .661$ & .614 & $R_{1} .729$ & NA & NA & NA & NA & NA & NA & NA & NA \\
\hline 1977 & $\begin{array}{l}.590 \\
.622\end{array}$ & $\mathrm{R}_{1.646}$ & .656 & $\mathrm{R}_{1.736}$ & NA & NA & NA & NA & NA & NA & NA & NA \\
\hline 1978 & R. .627 & $\mathrm{R}_{1.551}$ & .670 & $\mathrm{R}_{1.657}$ & $\mathrm{NA}$ & NA & .652 & $\mathrm{R}_{1.612}$ & NA & NA & NA & NA \\
\hline 1979 & .857 & $R_{1.957}$ & .903 & $\mathrm{R}_{2.062}$ & $\mathrm{NA}$ & NA & .882 & $R_{2.014}$ & NA & NA & NA & NA \\
\hline 1980 & 1.191 & $\mathrm{R}_{2.492}$ & 1.245 & $\mathrm{R}_{2.605}$ & NA & NA & 1.221 & $\mathrm{R}_{2.555}$ & NA & NA & NA & NA \\
\hline 1981 & $\begin{array}{l}1.311 \\
0\end{array}$ & $\mathrm{R}_{2.508}$ & $\begin{array}{l}1.378 \\
\text {. }\end{array}$ & $\mathrm{R}_{2.636}$ & $\begin{array}{l}71.470 \\
7\end{array}$ & $7, R_{2.812}$ & $\begin{array}{l}1.353 \\
\end{array}$ & $\mathrm{R}_{2.588}$ & NA & NA & NA & NA \\
\hline 1982 & 1.222 & $\mathrm{R}_{2.203}$ & $\begin{array}{l}1.296 \\
\end{array}$ & $R_{2.337}$ & 1.415 & $\mathrm{R}_{2.551}$ & 1.281 & $R_{2.310}$ & NA & NA & NA & NA \\
\hline 1983 & $\begin{array}{r}1.22 \\
\mathrm{R}_{1} .158\end{array}$ & 2.009 & $\begin{array}{l}1.296 \\
1.241\end{array}$ & $\begin{array}{l}2.337 \\
R_{2} .153\end{array}$ & $\begin{array}{l}.415 \\
1.383\end{array}$ & $\begin{array}{l}2.551 \\
R_{2} .399\end{array}$ & 1.225 & $\begin{array}{r}2.125 \\
\text { R2. }\end{array}$ & NA & NA & NA & NA \\
\hline 1984 & $\begin{array}{l}1.129 \\
\end{array}$ & $\mathrm{R}_{1.887}$ & 1.212 & $\mathrm{R}_{2.026}$ & 1.366 & $\mathrm{R}_{2.284}$ & $\begin{array}{l}1.198 \\
0\end{array}$ & $\mathrm{R}_{2.003}$ & NA & NA & NA & NA \\
\hline 1985 & 1.115 & $\mathrm{R}_{1} .809$ & 1.202 & $\mathrm{R}_{1} .950$ & 1.340 & $\mathrm{R}_{2.174}$ & 1.196 & $\mathrm{R}_{1.941}$ & NA & NA & NA & NA \\
\hline 1986 & .857 & $R_{1} .361$ & .927 & $R_{1.472}$ & 1.085 & $R_{1.722}$ & .931 & $\mathrm{R}_{1.478}$ & NA & NA & NA & NA \\
\hline 1987 & $\begin{array}{l}.097 \\
.897\end{array}$ & $R_{1} .384$ & . 948 & $\mathrm{R}_{1.463}$ & 1.093 & $\mathrm{R}_{1.686}$ & .957 & $\begin{array}{r}1.476 \\
R_{1}\end{array}$ & NA & NA & NA & NA \\
\hline 1988 & $\begin{array}{r}.091 \\
R .900\end{array}$ & $\begin{array}{l}1.004 \\
1.342\end{array}$ & $\begin{array}{l}.940 \\
.946\end{array}$ & $\mathrm{R}_{1.411}$ & 1.107 & $R_{1.651}$ & R. 964 & 1.438 & NA & NA & NA & NA \\
\hline 1989 & R. .997 & $\mathrm{R}_{1.433}$ & $\mathrm{R}_{1} .022$ & 1.469 & 1.197 & $\mathrm{R}_{1.720}$ & $\begin{array}{l}1.064 \\
1.060\end{array}$ & $\begin{array}{r}1.450 \\
R\end{array}$ & NA & NA & NA & NA \\
\hline 1990 & 1.149 & $\mathrm{R}_{1.590}$ & 1.164 & $\mathrm{R}_{1.611}$ & 1.349 & $\mathrm{R}_{1.867}$ & 1.217 & $\mathrm{R}_{1.684}$ & NA & NA & NA & NA \\
\hline 1991 & NA & NA & 1.140 & $R_{1.524}$ & 1.321 & $\mathrm{R}_{1.765}$ & 1.196 & $R_{1} .598$ & 1.098 & NA & 1.098 & NA \\
\hline 1992 & NA & NA & $\begin{array}{l}1.127 \\
\end{array}$ & $R_{1.471}$ & 1.316 & $\mathrm{R}_{1.718}$ & $\begin{array}{l}1.190 \\
\text { S }\end{array}$ & $\mathrm{R}_{1.554}$ & 1.087 & NA & $\begin{array}{l}1.087 \\
\text {. }\end{array}$ & NA \\
\hline 1993 & NA & NA & 1.108 & $R_{1} 415$ & 1.302 & $R_{1} .663$ & 1.173 & $R_{1} .498$ & 21.067 & NA & 1.067 & NA \\
\hline 1994 & NA & NA & $\begin{array}{l}1.108 \\
1.112\end{array}$ & $\begin{array}{r}.415 \\
\mathrm{R}_{1} .391\end{array}$ & $\begin{array}{l}.302 \\
1.305\end{array}$ & $\begin{array}{l}1.663 \\
R_{1} .632\end{array}$ & $\begin{array}{l}1.173 \\
1.174\end{array}$ & $\begin{array}{r}1.498 \\
\mathrm{R}_{1} .469\end{array}$ & 21.072 & NA & $\begin{array}{l}1.06 / \\
1.075\end{array}$ & NA \\
\hline 1995 & NA & NA & $\begin{array}{l}1.147 \\
0\end{array}$ & $R_{1.406}$ & 1.336 & $R_{1.637}$ & 1.205 & $R_{1.477}$ & 21.103 & 41.163 & 1.111 & $\begin{array}{r}1.109 \\
\end{array}$ \\
\hline 1996 & NA & NA & 1.231 & $\mathrm{R}_{1.480}$ & 1.413 & $R_{1} .699$ & 1.288 & $\mathrm{R}_{1.549}$ & 21.192 & 41.242 & 1.199 & 1.235 \\
\hline 1997 & NA & NA & $\begin{array}{l}1.234 \\
1.20\end{array}$ & $R_{1.458}$ & 1.416 & $R_{1.673}$ & $\begin{array}{l}1.291 \\
\text { S }\end{array}$ & $\mathrm{R}_{1.525}$ & 21.189 & 41.252 & 1.199 & 1.198 \\
\hline 1998 & NA & NA & $\begin{array}{l}1.259 \\
1.059\end{array}$ & $\mathrm{R}_{1.237}$ & $\begin{array}{l}1.410 \\
1.250\end{array}$ & $R_{1.461}$ & 1.115 & $\begin{array}{r}\mathrm{R} \\
\mathrm{R} 1.303\end{array}$ & 21.017 & 41.078 & $\begin{array}{l}1.1530 \\
1.030\end{array}$ & $\begin{array}{l}1.044 \\
1.04\end{array}$ \\
\hline 1999 & NA & NA & $\begin{array}{l}1.059 \\
1.165\end{array}$ & $\mathrm{R}_{1.342}$ & $\begin{array}{l}1.250 \\
1.357\end{array}$ & $\mathrm{R}_{1.563}$ & 1.221 & $\mathrm{R}_{1.406}$ & 21.116 & 41.195 & $\begin{array}{l}1.030 \\
1.136\end{array}$ & $\begin{array}{l}1.044 \\
1.121\end{array}$ \\
\hline 2000 & NA & NA & $\begin{array}{l}1.105 \\
1.510\end{array}$ & $\mathrm{R}_{1.702}$ & $\begin{array}{l}1.351 \\
1.693\end{array}$ & $\begin{array}{l}1.503 \\
R_{1} .908\end{array}$ & 1.563 & $\begin{array}{l}1.7460 \\
\mathrm{R}_{1} .762\end{array}$ & 21.462 & 41.543 & $\begin{array}{l}1.136 \\
1.484\end{array}$ & 1.491 \\
\hline 2001 & NA & NA & 1.461 & $R_{1.610}$ & 1.657 & $R_{1.826}$ & 1.531 & $\mathrm{R}_{1.687}$ & 1.384 & 1.498 & 1.420 & 1.401 \\
\hline 2002 & NA & NA & 1.358 & $R_{1.473}$ & 1.556 & $R_{1.688}$ & 1.441 & $\mathrm{R}_{1.563}$ & 1.313 & 1.408 & 1.345 & 1.319 \\
\hline 2003 & NA & NA & $\begin{array}{l}1.591 \\
1.591\end{array}$ & $\mathrm{R}_{1.690}$ & 1.777 & 1.888 & 1.638 & $\mathrm{R}_{1.740}$ & $\begin{array}{l}1.516 \\
\text { lo }\end{array}$ & $\begin{array}{l}1.655 \\
1.655\end{array}$ & $\begin{array}{l}1.561 \\
1.561\end{array}$ & $\begin{array}{l}1.509 \\
\end{array}$ \\
\hline 2004 & NA & NA & 1.880 & $\mathrm{R}_{1.942}$ & 2.068 & $\begin{array}{l}1.000 \\
2.137\end{array}$ & $\begin{array}{l}1.030 \\
1.923\end{array}$ & 1.987 & $\begin{array}{l}1.510 \\
1.812\end{array}$ & $\begin{array}{l}1.053 \\
1.937\end{array}$ & $\begin{array}{l}1.501 \\
1.852\end{array}$ & $\begin{array}{l}1.509 \\
1.810\end{array}$ \\
\hline 2005 & NA & NA & 2.295 & 2.295 & 2.491 & 2.491 & 2.338 & 2.338 & 2.240 & 2.335 & 2.270 & 2.402 \\
\hline 2006 & NA & NA & 2.589 & $R_{2.508}$ & 2.805 & 2.717 & 2.635 & $R_{2.553}$ & 2.533 & 2.654 & 2.572 & 2.705 \\
\hline 2007 & NA & NA & 2.801 & $R_{2.637}$ & 3.033 & $R_{2.855}$ & 2.849 & $R_{2.682}$ & 2.767 & 2.857 & 2.796 & 2.885 \\
\hline 2008 & NA & NA & 3.266 & $\mathrm{R}_{3.008}$ & 3.519 & $\mathrm{R}_{3.241}$ & 3.317 & $\mathrm{R}_{3.055}$ & 3.213 & 3.314 & 3.246 & 3.803 \\
\hline 2009 & NA & NA & $\begin{array}{l}2.200 \\
2.350\end{array}$ & $\begin{array}{r}.000 \\
\text { R2.142 }\end{array}$ & 2.607 & $\begin{array}{r}r .376 \\
R_{2}\end{array}$ & $\begin{array}{l}2.401 \\
2.401\end{array}$ & $\begin{array}{r}\text { R2.188 } \\
\text { R }\end{array}$ & 2.315 & $\begin{array}{l}.0 .014 \\
2.433\end{array}$ & $\begin{array}{l}.240 \\
2.353\end{array}$ & $\begin{array}{l}2.005 \\
2.467\end{array}$ \\
\hline 2010 & NA & NA & $\begin{array}{l}2.350 \\
2.788\end{array}$ & $\mathrm{R}_{2.512}$ & $\begin{array}{l}3.001 \\
3.047\end{array}$ & $\mathrm{R}_{2.745}$ & $\begin{array}{l}2.4011 \\
2.836\end{array}$ & $\mathrm{R}_{2.555}$ & $\begin{array}{l}2.515 \\
2.742\end{array}$ & $\begin{array}{l}2.435 \\
2.864\end{array}$ & $\begin{array}{l}2.353 \\
2.782\end{array}$ & $\begin{array}{l}2.401 \\
2.992\end{array}$ \\
\hline 2011 & NA & NA & 3.527 & 3.111 & 3.792 & 3.345 & 3.577 & 3.155 & 3.476 & 3.616 & 3.521 & 3.840 \\
\hline
\end{tabular}

1 Any area that does not require the sale of reformulated gasoline.
2 For 1993-2000, data collected for oxygenated areas are included in "Conventional Gasoline Areas." 3 "Reformulated Gasoline Areas" are ozone nonattainment areas designated by the Environment Protection Agency that require the use of reformulated gasoline.

4 For 1995-2000, data collected for combined oxygenated and reformulated areas are included in "Reformulated Gasoline Areas."

See "Nominal Dollars" in Glossary.

${ }^{6}$ In chained (2005) dollars, calculated by using gross domestic product implicit price deflators in Table

D1. See "Chained Dollars" in Glossary.

on September through December data only.

Note: See "Motor Gasoline Grades," "Motor Gasoline, Conventional," "Motor Gasoline, Oxygenated," and "Motor Gasoline, Reformulated" in Glossary. Web Pages: - See http://www.eia.gov/totalenergy/data/monthly/\#prices for updated monthly and annual - See http://www.eia.gov/petroleum/ for related information.

Sources: Motor Gasoline by Grade: - 1949-1973-Platt's Oil Price Handbook and Oilmanac, 1974, 51st Edition. - 1974 forward-U.S. Energy Information Administration (EIA), annual averages of monthly data from the U.S. Department of Labor, Bureau of Labor Statistics, U.S. City Average Gasoline Prices. Regular Motor Gasoline by Area Type: EIA, weighted annual averages of data from "Weekly U.S. Retal "Weekly Retail On-Highway Diesel Prices." 


\section{Petroleum}

Note 1. Petroleum Products Supplied and Petroleum Consumption. Petroleum product supplied (see Table 5.11) is used as an approximation of petroleum consumption. Petroleum products supplied is calculated as field production plus renewable fuels and oxygenates production plus processing gain plus net imports minus stock change plus adjustments. Total products supplied include natural gas plant liquids, unfinished oils, aviation gasoline blending components, and finished petroleum products. Crude oil burned on leases and at pipeline stations was reported as product supplied for either distillate fuel oil or residual fuel oil until January 1983. From January 1983 through December 2010, crude oil product supplied was reported based on quantities reported on Form EIA-813 "Monthly Crude Oil Report." Beginning with data for January 2010, reporting crude oil used directly on Form EIA-813 was discontinued, and crude oil product supplied was assumed equal to zero. The sector allocation of product supplied in Tables 5.13a-5.13d for products used in more than one sector is derived from sales to ultimate consumers by refiners, marketers, distributors, and dealers (see U.S. Energy Information Administration (EIA) report Fuel Oil and Kerosene Sales) and from EIA electric power sector petroleum consumption data (see Tables 8.7b and 8.7c).

Note 2. Changes Affecting Petroleum Production and Product Supplied Statistics. Beginning in January 1981, several U.S. Energy Information Administration survey forms and calculation methodologies were changed to reflect new developments in refinery and blending plant practices and to improve data integrity. Those changes affect production and product supplied statistics for motor gasoline, distillate fuel oil, and residual fuel oil, and stocks of motor gasoline. On the basis of those changes, motor gasoline production during the last half of 1980 would have averaged 289 thousand barrels per day higher than that which was published on the old basis. Distillate and residual fuel oil production and product supplied for all of 1980 would have averaged, respectively, 105 thousand and 54 thousand barrels per day higher than the numbers that were published. A new adjustment was introduced for calculating finished motor gasoline product supplied beginning with data for January 1993. The new adjustment transferred product supplied for motor gasoline blending components and fuel ethanol to supply of finished motor gasoline. Applying the new gasoline adjustment method to gasoline product supplied data for 1992 increased the reported quantity 108 thousand barrels per day at the U.S. level from 7,268 thousand barrels per day to 7,376 thousand barrels per day.

Note 3. Gross Input to Distillation Units. The methods of deriving Gross Input to Distillation Units (GIDU) in this report are as follows: for 1949-1966, GIDU is estimated by summing annual crude oil runs to stills, net unfinished oil reruns at refineries, and shipments of natural gasoline and plant condensate from natural gas processing plants to refineries; for 1967-1973, GIDU is estimated by summing annual crude oil runs to stills, net unfinished oil reruns, and refinery input of natural gasoline and plant condensate; for 1974-1980, GIDU is published annual data; and for 1981 forward, GIDU is the sum of reported monthly data.

Note 4. Crude Oil Domestic First Purchase Prices. Crude oil domestic first purchase prices were derived as follows: for 1949-1973, weighted average domestic first purchase values as reported by State agencies and calculated by the Bureau of Mines; for 1974 and 1975, weighted averages of a sample survey of major first purchasers' purchases; for 1976 forward, weighted averages of all first purchasers' purchases.

Note 5. Historical Residential Heating Oil Prices. Residential heating oil prices for 1956 through 1987 were formerly published in the Annual Energy Review. Those data, in cents per gallon, are: 1956-15.2; 1957-16.0; 1958-15.1; 1959-15.3; 1960-15.0; 1961-15.6; 1962-15.6; 1963-16.0; 1964-16.1; 1965-16.0; 1966-16.4; 1967-16.9; 1968-17.4; 1969-17.8; 1970-18.5; $1971-19.6 ; 1972-19.7 ; 1973-22.8 ; 1974-36.0 ; 1975-37.7 ; 1976-40.6$; 1977-46.0; 1978-49.0; 1979-70.4; 1980-97.4; 1981-119.4; 1982-116.0; 1983-107.8; 1984-109.1; 1985-105.3; 1986-83.6; and 1987-80.3. The sources of these data are: 1956-1974—Bureau of Labor Statistics, "Retail Prices and Indexes of Fuels and Utilities for Residential Usage,” monthly; January 1975-September 1977-Federal Energy Administration, Form FEA-P112-M-1, “No. 2 Heating Oil Supply/Price Monitoring Report”; October 1977-December 1977-U.S. Energy Information Administration (EIA), Form EIA-9, "No. 2 Heating Oil Supply/Price Monitoring Report”; 1978 forward—EIA, Petroleum Marketing Annual, Table 15. 
6. Natural Gas 


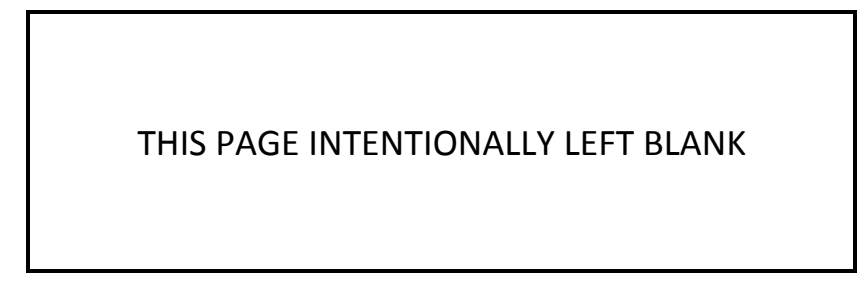




\section{Figure 6.0 Natural Gas Flow, 2011}

(Trillion Cubic Feet)

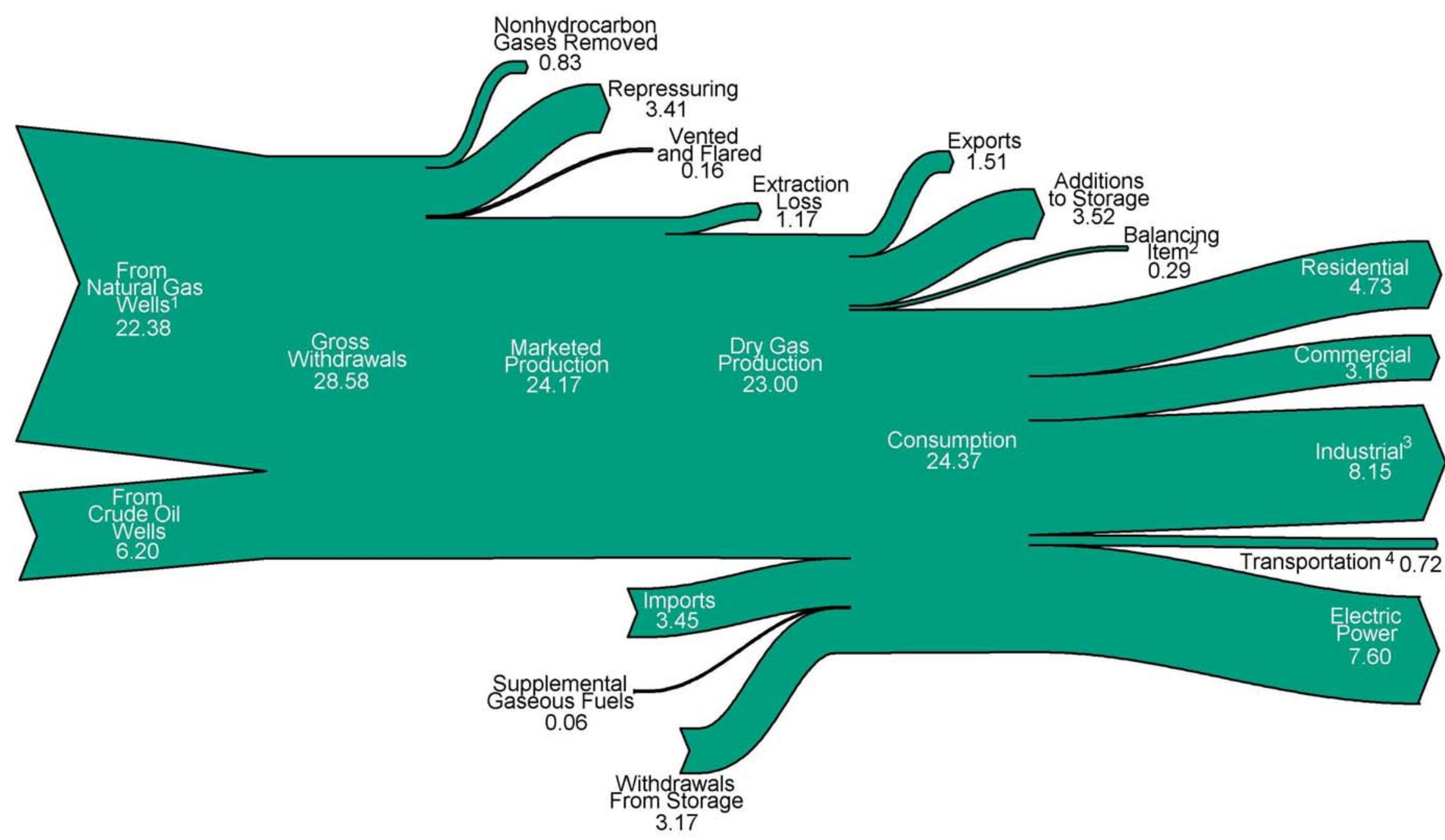

${ }^{1}$ Includes natural gas gross withdrawals from coalbed wells and shale gas wells.

${ }^{2}$ Quantities lost and imbalances in data due to differences among data sources.

${ }^{4}$ Natural gas consumed in the operation of pipelines (primarily in compressors), and as fuel in

${ }^{3}$ Lease and plant fuel, and other industrial. the delivery of natural gas to consumers; plus a small quantity used as vehicle fuel.

Notes: - Data are preliminary. - Values are derived from source data prior to rounding for publication. - Totals may not equal sum of components due to independent rounding.

Sources: Tables 6.1, 6.2, and 6.5. 


\section{Figure 6.1 Natural Gas Overview}

\section{Overview, 1949-2011}

$30-$
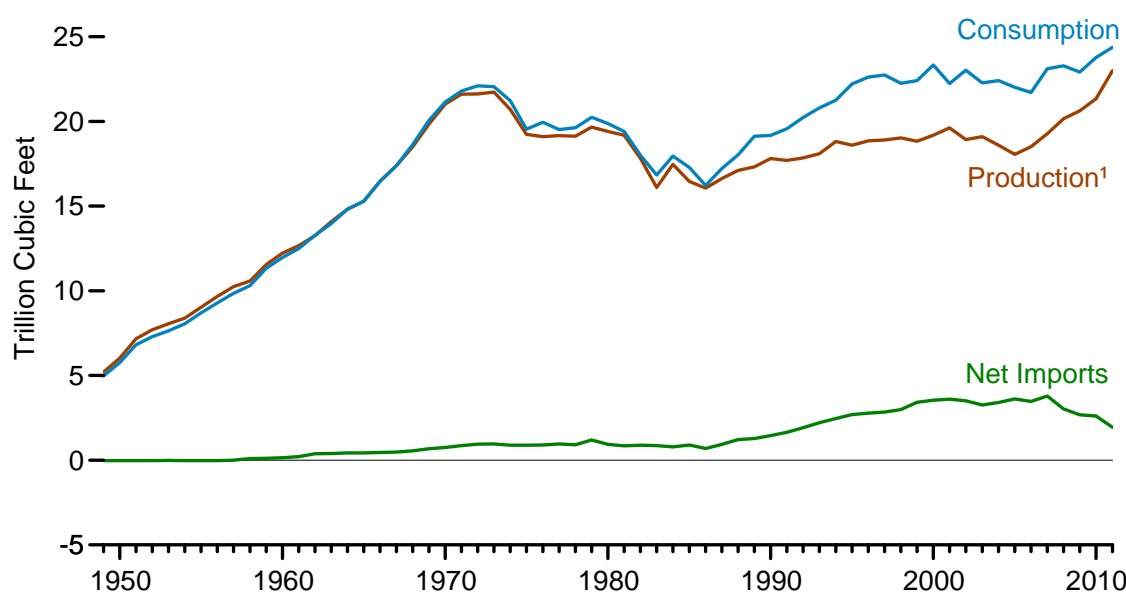

\section{Overview, 2011}

$30-$

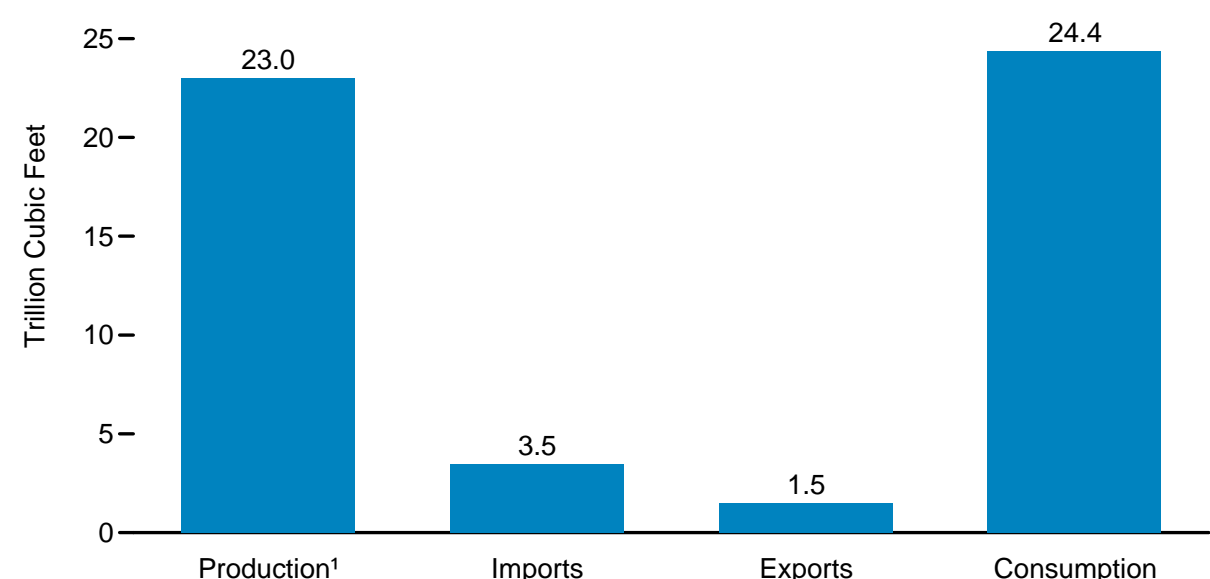

\section{Storage Additions and Withdrawals, 1949-2011}

$4-$

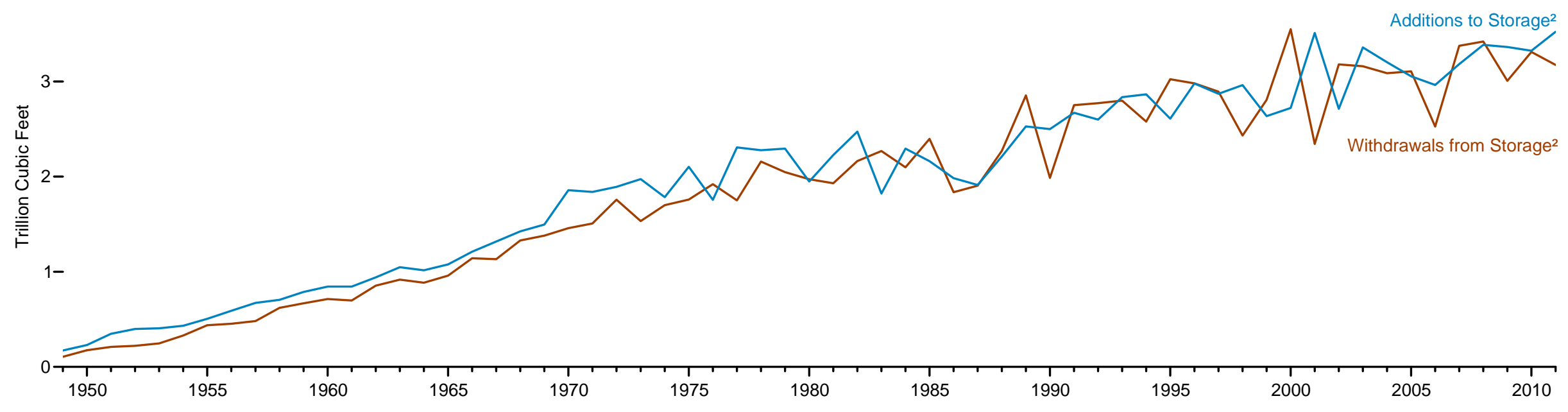

${ }^{1}$ Dry gas.

2 Underground storage. For 1980-2010, also includes liquefied natural gas in above-ground Source: Table 6.1. 
Table 6.1 Natural Gas Overview, Selected Years, 1949-2011

(Billion Cubic Feet)

\begin{tabular}{|c|c|c|c|c|c|c|c|c|c|c|}
\hline \multirow[b]{2}{*}{ Year } & \multirow{2}{*}{$\begin{array}{l}\text { Dry Gas } \\
\text { Production }\end{array}$} & \multirow{2}{*}{$\begin{array}{l}\text { Supplemental } \\
\text { Gaseous Fuels }\end{array}$} & \multicolumn{3}{|c|}{ Trade } & \multicolumn{3}{|c|}{ Storage ${ }^{1}$ Activity } & \multirow[b]{2}{*}{ Balancing Item ${ }^{5}$} & \multirow[b]{2}{*}{ Consumption 6} \\
\hline & & & Imports & Exports & Net Imports ${ }^{3}$ & Withdrawals & Additions & Net Withdrawals ${ }^{4}$ & & \\
\hline 1949 & 5,195 & NA & 0 & 20 & -20 & 106 & 172 & -66 & -139 & 4,971 \\
\hline 1950 & 6,022 & NA & 0 & 26 & -26 & 175 & 230 & -54 & -175 & 5,767 \\
\hline 1955 & 9,029 & NA & 11 & 31 & -20 & 437 & 505 & -68 & -247 & 8,694 \\
\hline 1960 & 12,228 & NA & 156 & 11 & 144 & 713 & 844 & -132 & -274 & 11,967 \\
\hline 1965 & 15,286 & NA & 456 & 26 & 430 & 960 & 1,078 & -118 & -319 & 15,280 \\
\hline 1970 & 21,014 & NA & 821 & 70 & 751 & 1,459 & 1,857 & -398 & -228 & 21,139 \\
\hline 1975 & 19,236 & NA & 953 & 73 & 880 & 1,760 & 2,104 & -344 & -235 & 19,538 \\
\hline 1976 & 19,098 & NA & 964 & 65 & 899 & 1,921 & 1,756 & 165 & -216 & 19,946 \\
\hline 1977 & 19,163 & NA & 1,011 & 56 & 955 & 1,750 & 2,307 & -557 & -41 & 19,521 \\
\hline 1978 & 19,122 & NA & 966 & 53 & 913 & 2,158 & 2,278 & -120 & -287 & 19,627 \\
\hline 1979 & 19,663 & NA & 1,253 & 56 & 1,198 & 2,047 & 2,295 & -248 & -372 & 20,241 \\
\hline 1980 & 19,403 & 155 & 985 & 49 & 936 & 1,972 & 1,949 & 23 & -640 & 19,877 \\
\hline 1981 & 19,181 & 176 & 904 & 59 & 845 & 1,930 & 2,228 & -297 & -500 & 19,404 \\
\hline 1982 & 17,820 & 145 & 933 & 52 & 882 & 2,164 & 2,472 & -308 & -537 & 18,001 \\
\hline 1983 & 16,094 & 132 & 918 & 55 & 864 & 2,270 & 1,822 & 447 & -703 & 16,835 \\
\hline 1984 & 17,466 & 110 & 843 & 55 & 788 & 2,098 & 2,295 & -197 & -217 & 17,951 \\
\hline 1985 & 16,454 & 126 & 950 & 55 & 894 & 2,397 & 2,163 & 235 & -428 & 17,281 \\
\hline 1986 & 16,059 & 113 & 750 & 61 & 689 & 1,837 & 1,984 & -147 & -493 & 16,221 \\
\hline 1987 & 16,621 & 101 & 993 & 54 & 939 & 1,905 & 1,911 & -6 & -444 & 17,211 \\
\hline 1988 & 17,103 & 101 & 1,294 & 74 & 1,220 & 2,270 & 2,211 & 59 & -453 & 18,030 \\
\hline 1989 & 17,311 & 107 & 1,382 & 107 & 1,275 & 2,854 & 2,528 & 326 & 101 & 719,119 \\
\hline 1990 & 17,810 & 123 & 1,532 & 86 & 1,447 & 1,986 & 2,499 & -513 & 307 & 719,174 \\
\hline 1991 & 17,698 & 113 & 1,773 & 129 & 1,644 & 2,752 & 2,672 & 80 & 27 & 719,562 \\
\hline 1992 & 17,840 & 118 & 2,138 & 216 & 1,921 & 2,772 & 2,599 & 173 & 176 & 720,228 \\
\hline 1993 & 18,095 & 119 & 2,350 & 140 & 2,210 & 2,799 & 2,835 & -36 & 401 & 20,790 \\
\hline 1994 & 18,821 & 111 & 2,624 & 162 & 2,462 & 2,579 & 2,865 & -286 & 139 & 21,247 \\
\hline 1995 & 18,599 & 110 & 2,841 & 154 & 2,687 & 3,025 & 2,610 & 415 & 396 & 22,207 \\
\hline 1996 & 18,854 & 109 & 2,937 & 153 & 2,784 & 2,981 & 2,979 & 2 & 860 & 22,609 \\
\hline 1997 & 18,902 & 103 & 2,994 & 157 & 2,837 & 2,894 & 2,870 & 24 & 871 & 22,737 \\
\hline 1998 & 19,024 & 102 & 3,152 & 159 & 2,993 & 2,432 & 2,961 & -530 & 657 & 22,246 \\
\hline 1999 & 18,832 & 98 & 3,586 & 163 & 3,422 & 2,808 & 2,636 & 172 & -119 & 22,405 \\
\hline 2000 & 19,182 & 90 & 3,782 & 244 & 3,538 & 3,550 & 2,721 & 829 & $R_{-306}$ & 23,333 \\
\hline 2001 & 19,616 & 86 & 3,977 & 373 & 3,604 & 2,344 & 3,510 & $-1,166$ & 99 & 22,239 \\
\hline 2002 & 18,928 & 68 & 4,015 & 516 & 3,499 & 3,180 & 2,713 & 467 & $\mathrm{R}_{65}$ & $\mathrm{R}_{23,027}$ \\
\hline 2003 & 19,099 & 68 & 3,944 & 680 & 3,264 & 3,161 & 3,358 & -197 & 44 & 22,277 \\
\hline 2004 & 18,591 & 60 & 4,259 & 854 & 3,404 & 3,088 & 3,202 & -114 & ${ }^{R} 461$ & $\mathrm{R}_{22,403}$ \\
\hline 2005 & 18,051 & 64 & 4,341 & 729 & 3,612 & 3,107 & 3,055 & 52 & $\mathrm{R}_{236}$ & $R_{22,014}$ \\
\hline 2006 & 18,504 & 66 & 4,186 & 724 & 3,462 & 2,527 & 2,963 & -436 & $\mathrm{R}_{103}$ & $\mathrm{R}_{21,699}$ \\
\hline 2007 & 19,266 & 63 & 4,608 & 822 & 3,785 & 3,375 & 3,183 & 192 & R-203 & $\mathrm{R}_{23,104}$ \\
\hline 2008 & 20,159 & 61 & 3,984 & 963 & 3,021 & 3,420 & 3,385 & 34 & $\mathrm{R}_{2}$ & R23,277 \\
\hline 2009 & $\mathrm{R}_{20,624}$ & 65 & 3,751 & 1,072 & 2,679 & 3,007 & 3,362 & -355 & $R_{-103}$ & $\mathrm{R}_{22,910}$ \\
\hline 2010 & $\mathrm{R}_{21,332}$ & $\mathrm{R}_{65}$ & $\mathrm{R}_{3,741}$ & $\mathrm{R}_{1,137}$ & $\mathrm{R}_{2,604}$ & $\mathrm{R}_{3,311}$ & $\mathrm{R}_{3,324}$ & $\mathrm{R}_{-13}$ & $\mathrm{R}-213$ & $\mathrm{R}_{23,775}$ \\
\hline 2011 & $\mathrm{E}_{23,000}$ & $P_{61}$ & $P_{3,453}$ & $P_{1,507}$ & $P_{1,946}$ & $P_{3,175}$ & $P_{3,523}$ & P-348 & $P_{-}-290$ & $P_{24,369}$ \\
\hline
\end{tabular}

1 Underground storage. For 1980-2010, also includes liquefied natural gas in above-ground tanks. 2 See Note 1, "Supplemental Gaseous Fuels," at end of section.

exports are greater than imports. ${ }^{4}$ Net withdrawals equal withdrawals minus additions. Minus sign indicates additions are greater than ${ }_{5}$ Quantities lost and imbalances in data due to differences among data sources. Since 1980, excludes intransit shipments that cross the U.S.-Canada border (i.e., natural gas delivered to its destination via the other country).

See Note 2, "Natural Gas Consumption," at end of section.

" both "Other Industrial" and "Electric Power Sector" on Table 6.5. See Note 3, "Natural Gas Consumption, R=Revised. Pr Preliminary.

Notes: - Beginning with 1965 , all volumes are shown on a pressure base of 14.73 p.s.i.a. at $60^{\circ} \mathrm{F}$. For prior years, the pressure base was 14.65 p.s.i.a. at $60^{\circ} \mathrm{F}$. - Totals may not equal sum of components due to independent rounding. annual data. - See http://www.eia.gov/totalenergy/data/annual/\#naturalgas for all annual data beginning in 1949. - See http://www.eia.gov/naturalgas/ for related information.

Sources: Dry Gas Production: Table 6.2. Supplemental Gaseous Fuels: • 1980-2006-U.S. Energy Information Administration (EIA), Natural Gas Annual (NGA), annual reports. • 2007 forward-EIA, Natural Gas Monthly (NGM) (March 2012), Table 1. Trade: Table 6.3. Storage Activity: - 1949-2010-EIA, NGA, annual reports. - 2011-EIA, NGM (March 2012), Table 8. Balancing Item: Calculated as consumption minus dry gas production, supplemental gaseous fuels, net imports, and net 


\section{Figure 6.2 Natural Gas Production}

\section{Gross Withdrawals and Dry Gas Production, 1949-2011}

$30-$

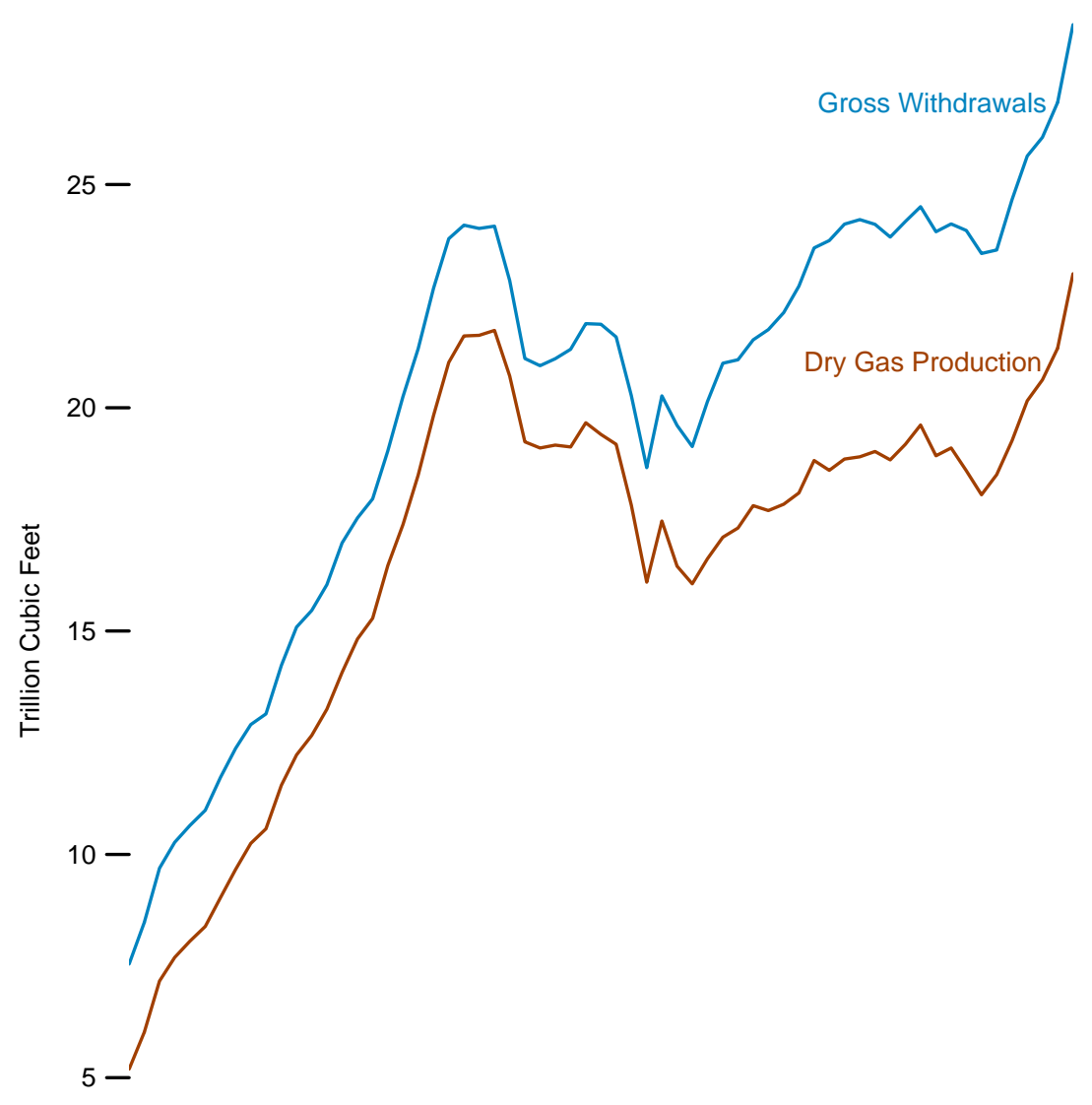

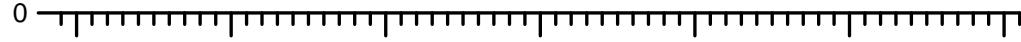

$\begin{array}{lllllll}1950 & 1960 & 1970 & 1980 & 1990 & 2000 & 2010\end{array}$

Production Flow, 2011

(Trillion Cubic Feet)

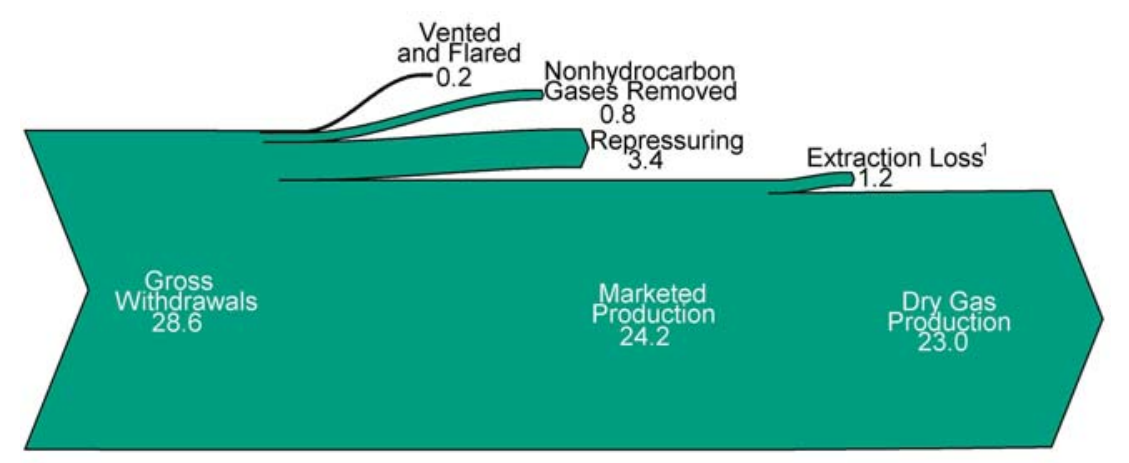

Gross Withdrawals by Well Type, 2011

30-

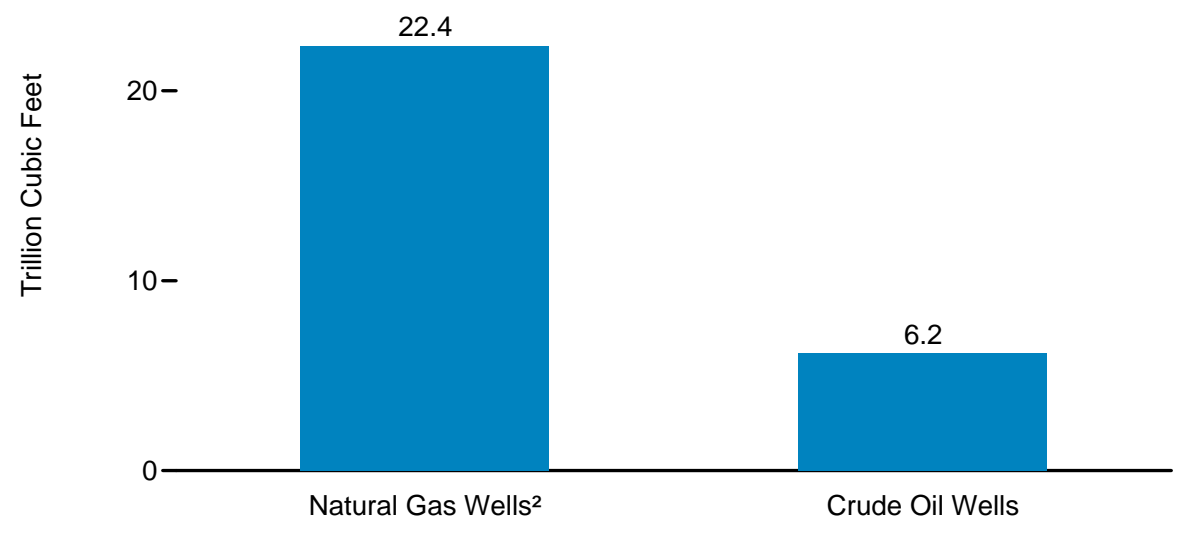

${ }^{1}$ Volume reduction resulting from the removal of natural gas plant liquids, which are transferred to petroleum supply.

${ }^{2}$ Includes natural gas gross withdrawals from coalbed wells and shale gas wells. Source: Table 6.2. 
Table 6.2 Natural Gas Production, Selected Years, 1949-2011

(Billion Cubic Feet)

\begin{tabular}{|c|c|c|c|c|c|c|c|c|c|c|c|}
\hline \multirow[b]{2}{*}{ Year } & \multicolumn{5}{|c|}{ Natural Gas Gross Withdrawals } & \multirow[b]{2}{*}{ Repressuring } & \multirow{2}{*}{$\begin{array}{l}\text { Nonhydrocarbon } \\
\text { Gases Removed }\end{array}$} & \multirow{2}{*}{$\begin{array}{l}\text { Vented } \\
\text { and Flared }\end{array}$} & \multirow{2}{*}{$\begin{array}{l}\text { Marketed } \\
\text { Production }\end{array}$} & \multirow{2}{*}{$\begin{array}{l}\text { Extraction } \\
\text { Loss }^{1}\end{array}$} & \multirow{2}{*}{$\begin{array}{l}\text { Dry Gas } \\
\text { Production }\end{array}$} \\
\hline & Natural Gas Wells & Crude Oil Wells & Coalbed Wells & Shale Gas Wells & Total & & & & & & \\
\hline 1949 & 4,986 & 2,561 & NA & NA & 7,547 & 1,273 & NA & 854 & 5,420 & 224 & 5,195 \\
\hline 1950 & 5,603 & 2,876 & NA & NA & 8,480 & 1,397 & NA & 801 & 6,282 & 260 & 6,022 \\
\hline 1955 & 7,842 & 3,878 & NA & NA & 11,720 & 1,541 & NA & 774 & 9,405 & 377 & 9,029 \\
\hline 1960 & 10,853 & 4,234 & NA & NA & 15,088 & 1,754 & NA & 563 & 12,771 & 543 & 12,228 \\
\hline 1965 & 13,524 & 4,440 & NA & NA & 17,963 & 1,604 & NA & 319 & 16,040 & 753 & 15,286 \\
\hline 1970 & 18,595 & 5,192 & NA & NA & 23,786 & 1,376 & NA & 489 & 21,921 & 906 & 21,014 \\
\hline 1975 & 17,380 & 3,723 & NA & NA & 21,104 & 861 & NA & 134 & 20,109 & 872 & 19,236 \\
\hline 1976 & 17,191 & 3,753 & NA & NA & 20,944 & 859 & NA & 132 & 19,952 & 854 & 19,098 \\
\hline 1977 & 17,416 & 3,681 & NA & NA & 21,097 & 935 & NA & 137 & 20,025 & 863 & 19,163 \\
\hline 1978 & 17,394 & 3,915 & NA & NA & 21,309 & 1,181 & NA & 153 & 19,974 & 852 & 19,122 \\
\hline 1979 & 18,034 & 3,849 & NA & NA & 21,883 & 1,245 & NA & 167 & 20,471 & 808 & 19,663 \\
\hline 1980 & 17,573 & 4,297 & NA & NA & 21,870 & 1,365 & 199 & 125 & 20,180 & 777 & 19,403 \\
\hline 1981 & 17,337 & 4,251 & NA & NA & 21,587 & 1,312 & 222 & 98 & 19,956 & 775 & 19,181 \\
\hline 1982 & 15,809 & 4,463 & NA & NA & 20,272 & 1,388 & 208 & 93 & 18,582 & 762 & 17,820 \\
\hline 1983 & 14,153 & 4,506 & NA & NA & 18,659 & 1,458 & 222 & 95 & 16,884 & 790 & 16,094 \\
\hline 1984 & 15,513 & 4,754 & NA & NA & 20,267 & 1,630 & 224 & 108 & 18,304 & 838 & 17,466 \\
\hline 1985 & 14,535 & 5,071 & NA & NA & 19,607 & 1,915 & 326 & 95 & 17,270 & 816 & 16,454 \\
\hline 1986 & 14,154 & 4,977 & NA & NA & 19,131 & 1,838 & 337 & 98 & 16,859 & 800 & 16,059 \\
\hline 1987 & 14,807 & 5,333 & NA & NA & 20,140 & 2,208 & 376 & 124 & 17,433 & 812 & 16,621 \\
\hline 1988 & 15,467 & 5,532 & NA & NA & 20,999 & 2,478 & 460 & 143 & 17,918 & 816 & 17,103 \\
\hline 1989 & 15,709 & 5,366 & NA & NA & 21,074 & 2,475 & 362 & 142 & 18,095 & 785 & 17,311 \\
\hline 1990 & 16,054 & 5,469 & NA & NA & 21,523 & 2,489 & 289 & 150 & 18,594 & 784 & 17,810 \\
\hline 1991 & 16,018 & 5,732 & NA & NA & 21,750 & 2,772 & 276 & 170 & 18,532 & 835 & 17,698 \\
\hline 1992 & 16,165 & 5,967 & NA & NA & 22,132 & 2,973 & 280 & 168 & 18,712 & 872 & 17,840 \\
\hline 1993 & 16,691 & 6,035 & NA & NA & 22,726 & 3,103 & 414 & 227 & 18,982 & 886 & 18,095 \\
\hline 1994 & 17,351 & 6,230 & NA & NA & 23,581 & 3,231 & 412 & 228 & 19,710 & 889 & 18,821 \\
\hline 1995 & 17,282 & 6,462 & NA & NA & 23,744 & 3,565 & 388 & 284 & 19,506 & 908 & 18,599 \\
\hline 1996 & 17,737 & 6,376 & NA & NA & 24,114 & 3,511 & 518 & 272 & 19,812 & 958 & 18,854 \\
\hline 1997 & 17,844 & 6,369 & NA & NA & 24,213 & 3,492 & 599 & 256 & 19,866 & 964 & 18,902 \\
\hline 1998 & 17,729 & 6,380 & NA & NA & 24,108 & 3,427 & 617 & 103 & 19,961 & 938 & 19,024 \\
\hline 1999 & 17,590 & 6,233 & NA & NA & 23,823 & 3,293 & 615 & 110 & 19,805 & 973 & 18,832 \\
\hline 2000 & 17,726 & 6,448 & NA & NA & 24,174 & 3,380 & 505 & 91 & 20,198 & 1,016 & 19,182 \\
\hline 2001 & 18,129 & 6,371 & NA & NA & 24,501 & 3,371 & 463 & 97 & 20,570 & 954 & 19,616 \\
\hline 2002 & 17,795 & 6,146 & NA & NA & 23,941 & 3,455 & 502 & 99 & 19,885 & 957 & 18,928 \\
\hline 2003 & 17,882 & 6,237 & NA & NA & 24,119 & 3,548 & 499 & 98 & 19,974 & 876 & 19,099 \\
\hline 2004 & 17,885 & 6,084 & NA & NA & 23,970 & 3,702 & 654 & 96 & 19,517 & 927 & 18,591 \\
\hline 2005 & 17,472 & 5,985 & NA & NA & 23,457 & 3,700 & 711 & 119 & 18,927 & 876 & 18,051 \\
\hline 2006 & 17,996 & 5,539 & NA & NA & 23,535 & 3,265 & 731 & 129 & 19,410 & 906 & 18,504 \\
\hline 2007 & 17,065 & 5,818 & 1,780 & NA & 24,664 & 3,663 & 661 & 143 & 20,196 & 930 & 19,266 \\
\hline 2008 & 15,618 & 5,747 & 1,986 & 2,284 & 25,636 & 3,639 & 719 & 167 & 21,112 & 953 & 20,159 \\
\hline 2009 & $\mathrm{R}_{14,885}$ & $\mathrm{R}_{5,812}$ & 1,977 & 3,384 & $\mathrm{R}_{26,057}$ & 3,522 & 722 & 165 & $\mathrm{R}_{21,648}$ & 1,024 & $\mathrm{R}_{20,624}$ \\
\hline 2010 & 2,R 20,841 & $R_{5,995}$ & $\left({ }^{2}\right)$ & $\left({ }^{2}\right)$ & $\mathrm{R}_{26,836}$ & $R_{3,432}$ & R837 & $\mathrm{R}_{166}$ & $\mathrm{R}_{22,402}$ & $R_{1,070}$ & $\mathrm{R}_{21,332}$ \\
\hline 2011 & E 22,378 & $E_{6,199}$ & $\left(2^{\prime}\right)$ & $\left({ }^{2}\right)$ & P28,576 & $E_{3,410}$ & $E_{831}$ & $E_{165}$ & $\mathrm{E}_{24,170}$ & $P_{1}, 169$ & $E_{23,000}$ \\
\hline
\end{tabular}

1 Volume reduction resulting from the removal of natural gas plant liquids, which are transferred to petroleum supply (see Tables 5.1b and 5.10)

Beginning in 2010, natural gas gross withdrawals from coalbed wells and shale gas wells are included "Natural Gas Wells."

Revised. $P=$ Preliminary. $E=E s t i m a t e . ~ N A=N o t$ available.

Notes: - Beginning with 1965 data, all volumes are shown on a pressure base of 14.73 p.s.i.a. at $60^{\circ} \mathrm{F}$ . Totals may not equal sum of components Web Pages: - See htp annual data. - See $\mathrm{http}: / / \mathrm{www}$.eia.gov/totalenergy/data/annual/\#naturalgas for all annual data beginning in 1949. - See http://www.eia.gov/naturalgas/ for related information.

Sources: Natural Gas Wells, Crude Oil Wells, Coalbed Wells, and Shale Gas Wells: 1949-1966-Bureau of Mines, Minerals Yearbook, "Natural Gas" chapter. • 1967-2010_U.S. Energy based lon privis (EIA), Nalural Gas Aithdrawals, Marked Production, Extraction Loss, Dry os proustion's data. Tolal Gross Withdrawals, Marketed Production, Extraction Loss, and Donty (Modu 2012). Tabl 1. All Other Data: 1949-2010 EIA NGA annual reports. N2011- GIA

estimates based on previous year's data. 
Figure 6.3 Natural Gas Imports, Exports, and Net Imports

\section{Trade Overview, 1949-2011}

$5-$

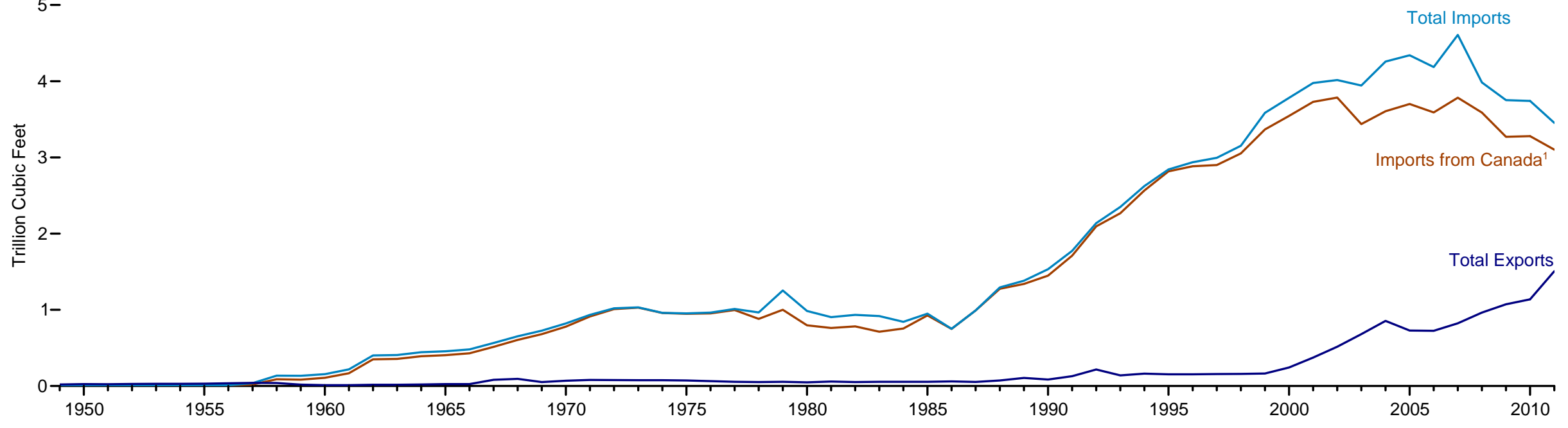

Trade, 2011

Net Imports as Share of Consumption, 1958-2011

$5-$ 20-
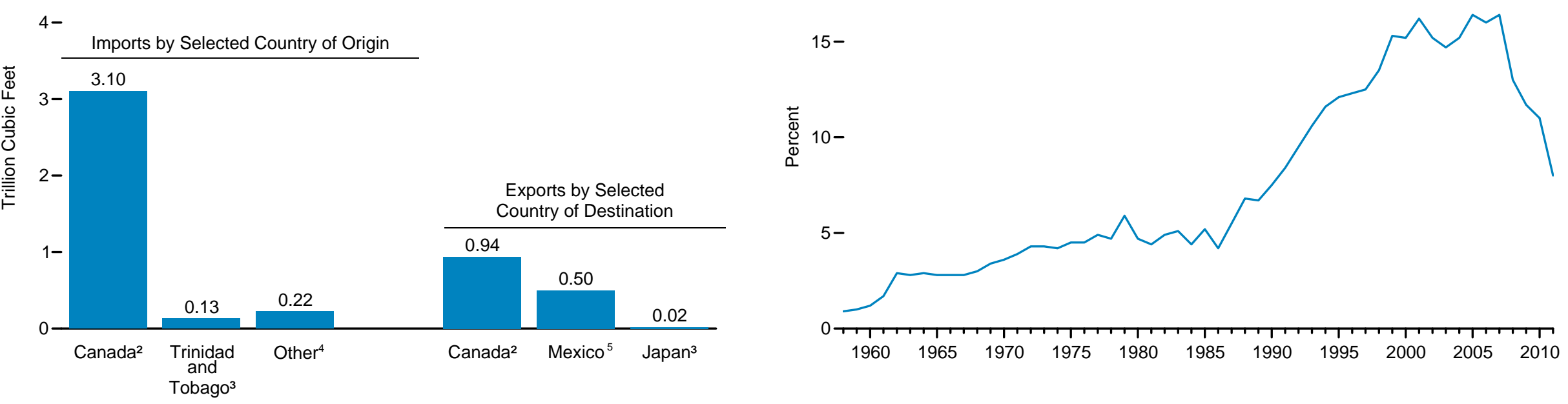

${ }^{1}$ By pipeline, except for very small amounts of liquefied natural gas in 1973, 1977, and 1981.
${ }^{2}$ By pipeline.

${ }^{4}$ By pipeline from Mexico, and as liquefied natural gas from Egypt, Nigeria, Norway, Qatar, Peru,

${ }^{3}$ As liquefied natural gas. and Yemen.

${ }^{5}$ By pipeline, except for very small amounts of liquefied natural gas.

Source: Table 6.3. 
Table 6.3 Natural Gas Imports, Exports, and Net Imports, Selected Years, 1949-2011

(Billion Cubic Feet, Except as Noted)

\begin{tabular}{|c|c|c|c|c|c|c|c|c|c|c|c|c|c|c|c|c|}
\hline \multirow[b]{2}{*}{ Year } & \multicolumn{9}{|c|}{ Imports by Country of Origin } & \multicolumn{5}{|c|}{ Exports by Country of Destination } & \multicolumn{2}{|c|}{ Net Imports 1} \\
\hline & Algeria $^{2}$ & Canada $^{3}$ & Egypt $^{2}$ & Mexico $^{3}$ & Nigeria 2 & Qatar ${ }^{2}$ & $\begin{array}{c}\text { Trinidad } \\
\text { and Tobago } 2\end{array}$ & Other 2,4 & Total & Canada $^{3}$ & Japan $^{2}$ & Mexico $^{3}$ & Other 2,5 & Total & Total & $\begin{array}{l}\text { Percent of U.S. } \\
\text { Consumption }\end{array}$ \\
\hline 1949 & 0 & 0 & 0 & 0 & 0 & 0 & 0 & 0 & 0 & (s) & 0 & 20 & 0 & 20 & -20 & $\left({ }^{6}\right)$ \\
\hline 1950 & 0 & 0 & 0 & 0 & 0 & 0 & 0 & 0 & 0 & 3 & 0 & 23 & 0 & 26 & -26 & $(6)$ \\
\hline 1955 & 0 & 11 & 0 & (s) & 0 & 0 & 0 & 0 & 11 & 11 & 0 & 20 & 0 & 31 & -20 & $\left({ }^{6}\right)$ \\
\hline 1960 & 0 & 109 & 0 & 47 & 0 & 0 & 0 & 0 & 156 & 6 & 0 & 6 & 0 & 11 & 144 & 1.2 \\
\hline 1965 & 0 & 405 & 0 & 52 & 0 & 0 & 0 & 0 & 456 & 18 & 0 & 8 & 0 & 26 & 430 & $\begin{array}{l}2.8 \\
\end{array}$ \\
\hline 1970 & 1 & 779 & 0 & 41 & 0 & 0 & 0 & 0 & 821 & 11 & 44 & 15 & 0 & 70 & 751 & 3.6 \\
\hline 1975 & 5 & 948 & 0 & 0 & 0 & 0 & 0 & 0 & 953 & 10 & 53 & 9 & 0 & 73 & 880 & 4.5 \\
\hline 1976 & 10 & 954 & 0 & 0 & 0 & 0 & 0 & 0 & 964 & 8 & 50 & 7 & 0 & 65 & 899 & 4.5 \\
\hline 1977 & 11 & 997 & 0 & 2 & 0 & 0 & 0 & 0 & $\begin{array}{r}504 \\
1,011\end{array}$ & (s) & 52 & 4 & 0 & 56 & 955 & $\begin{array}{l}4.5 \\
4.9\end{array}$ \\
\hline 1978 & 84 & 881 & 0 & 0 & 0 & 0 & 0 & 0 & 966 & (s) & 48 & 4 & 0 & 53 & 913 & 4.7 \\
\hline 1979 & 253 & 1,001 & 0 & 0 & 0 & 0 & 0 & 0 & 1,253 & (s) & 51 & 4 & 0 & 56 & 1,198 & 5.9 \\
\hline 1980 & 86 & 797 & 0 & 102 & 0 & 0 & 0 & 0 & 985 & (s) & 45 & 4 & 0 & 49 & 936 & 4.7 \\
\hline 1981 & 37 & 762 & 0 & 105 & 0 & 0 & 0 & 0 & 904 & (s) & 56 & 3 & 0 & 59 & 845 & 4.4 \\
\hline 1982 & 55 & 783 & 0 & 95 & 0 & 0 & 0 & 0 & 933 & (s) & 50 & 2 & 0 & 52 & 882 & 4.9 \\
\hline 1983 & 131 & 712 & 0 & 75 & 0 & 0 & 0 & 0 & 918 & (s) & 53 & 2 & 0 & 55 & 864 & 5.1 \\
\hline 1984 & 36 & 755 & 0 & 52 & 0 & 0 & 0 & 0 & 843 & (s) & 53 & 2 & 0 & 55 & 788 & 4.4 \\
\hline $\begin{array}{l}1984 \\
1985\end{array}$ & 24 & 926 & 0 & 0 & 0 & 0 & 0 & 0 & $\begin{array}{l}040 \\
950\end{array}$ & $\begin{array}{l}\text { (s) } \\
\text { (s) }\end{array}$ & 53 & 2 & 0 & 55 & 894 & $\begin{array}{l}.4 .4 \\
5.2\end{array}$ \\
\hline 1986 & 0 & 749 & 0 & 0 & 0 & 0 & 0 & 2 & 750 & (1) & 50 & 2 & 0 & 61 & 689 & 4.2 \\
\hline 1987 & 0 & 993 & 0 & 0 & 0 & 0 & 0 & 0 & 993 & 3 & 49 & 2 & 0 & 54 & 939 & 5.5 \\
\hline 1988 & 17 & 1,276 & 0 & 0 & 0 & 0 & 0 & 0 & 1,294 & 20 & 52 & 2 & 0 & 74 & 1,220 & 6.8 \\
\hline $\begin{array}{l}1980 \\
1989\end{array}$ & 42 & $\begin{array}{l}1,330 \\
1,330\end{array}$ & 0 & 0 & 0 & 0 & 0 & 0 & $\begin{array}{r}1,294 \\
1,382\end{array}$ & 38 & 51 & 17 & 0 & $\begin{array}{r}14 \\
107\end{array}$ & $\begin{array}{l}1,275 \\
1,20\end{array}$ & $\begin{array}{l}.0 \\
6.7\end{array}$ \\
\hline 1990 & 84 & 1,448 & 0 & 0 & 0 & 0 & 0 & 0 & 1,532 & 17 & 53 & 16 & 0 & 86 & $\begin{array}{l}1,447 \\
\end{array}$ & 7.5 \\
\hline 1991 & 64 & 1,710 & 0 & 0 & 0 & 0 & 0 & 0 & 1,773 & 15 & 54 & 60 & 0 & 129 & 1,644 & 8.4 \\
\hline 1992 & 43 & 2,094 & 0 & 0 & 0 & 0 & 0 & 0 & 2,138 & 68 & 53 & 96 & 0 & 216 & $\begin{array}{l}1,921 \\
\end{array}$ & 9.5 \\
\hline 1993 & 82 & 2,267 & 0 & 2 & 0 & 0 & 0 & 0 & 2350 & 45 & 56 & 40 & 0 & 140 & 2,210 & 106 \\
\hline 1994 & 51 & 2,566 & 0 & 7 & 0 & 0 & 0 & 0 & $\begin{array}{l}2,624 \\
\end{array}$ & 53 & 63 & 47 & 0 & 162 & 2,462 & 11.6 \\
\hline 1995 & 18 & 2,816 & 0 & 7 & 0 & 0 & 0 & 0 & 2,841 & 28 & 65 & 61 & 0 & 154 & 2,687 & 12.1 \\
\hline 1996 & 35 & 2,883 & 0 & 14 & 0 & 0 & 0 & 5 & 2,937 & 52 & 68 & 34 & 0 & 153 & 2,784 & 12.3 \\
\hline $\begin{array}{l}1996 \\
1997\end{array}$ & $\begin{array}{l}30 \\
66\end{array}$ & $\begin{array}{l}2,003 \\
2,899\end{array}$ & 0 & $\begin{array}{l}14 \\
17\end{array}$ & 0 & 0 & 0 & $\begin{array}{r}5 \\
12\end{array}$ & $\begin{array}{l}2,951 \\
2,994\end{array}$ & 56 & $\begin{array}{l}00 \\
62\end{array}$ & $\begin{array}{l}34 \\
38\end{array}$ & 0 & 157 & $\begin{array}{l}2,1,44 \\
2,837\end{array}$ & $\begin{array}{l}1.3 \\
12.5\end{array}$ \\
\hline 1998 & 69 & 3,052 & 0 & 15 & 0 & 0 & 0 & 17 & 3,152 & 40 & 66 & 53 & 0 & 159 & 2,993 & 13.5 \\
\hline 1999 & 76 & 3,368 & 0 & 55 & 0 & 20 & 51 & 17 & 3,586 & 39 & 64 & 61 & 0 & 163 & 3,422 & 15.3 \\
\hline 2000 & 47 & 3,544 & 0 & 12 & 13 & 46 & 99 & 21 & 3,782 & 73 & 66 & 106 & 0 & 244 & 3,538 & 15.2 \\
\hline 2001 & $\begin{array}{l}41 \\
65\end{array}$ & $\begin{array}{l}3,044 \\
3,729\end{array}$ & 0 & 10 & 38 & $\begin{array}{l}40 \\
23\end{array}$ & 98 & 14 & $\begin{array}{l}3,102 \\
3,977\end{array}$ & $\begin{array}{l}10 \\
167\end{array}$ & 66 & 141 & 0 & 373 & $\begin{array}{l}3,000 \\
3,604\end{array}$ & $\begin{array}{l}1.2 \\
16.2\end{array}$ \\
\hline 2002 & 27 & 3,785 & 0 & 2 & 8 & 35 & 151 & 8 & 4,015 & 189 & 63 & 263 & 0 & 516 & 3,499 & 15.2 \\
\hline 2003 & 53 & 3,437 & 0 & 0 & 50 & 14 & 378 & 11 & 3,944 & 271 & 66 & 343 & 0 & 680 & 3,264 & 14.7 \\
\hline 2004 & 120 & 3,607 & 0 & 0 & 12 & 12 & 462 & 46 & 4,259 & 395 & 62 & 397 & 0 & 854 & 3,404 & 15.2 \\
\hline 2005 & 97 & 3,700 & 73 & 9 & 8 & 3 & 439 & 11 & 4.341 & 358 & 65 & 305 & 0 & 729 & 3,612 & 16.4 \\
\hline 2006 & 17 & 3,590 & 120 & 13 & 57 & 0 & 389 & 0 & 4,186 & 341 & 61 & 322 & 0 & 724 & 3,462 & 16.0 \\
\hline 2007 & 77 & 3,783 & 115 & 54 & 95 & 18 & 448 & 18 & 4,608 & 482 & 47 & 292 & 2 & 822 & $\begin{array}{l}3,785 \\
3,785\end{array}$ & 16.4 \\
\hline 2008 & 0 & 3,589 & 55 & 43 & 12 & 3 & 267 & 15 & 3.984 & 559 & 39 & 365 & 0 & 963 & 3.021 & 13.0 \\
\hline 2009 & 0 & $\begin{array}{l}3,009 \\
3,271\end{array}$ & $\begin{array}{r}50 \\
160\end{array}$ & $\begin{array}{l}43 \\
28\end{array}$ & 13 & $\begin{array}{r}5 \\
13\end{array}$ & 236 & 29 & $\begin{array}{l}3,704 \\
3,751\end{array}$ & $\begin{array}{l}509 \\
701\end{array}$ & $\begin{array}{l}39 \\
31\end{array}$ & $\begin{array}{l}365 \\
338\end{array}$ & 3 & $\begin{array}{r}903 \\
1,072\end{array}$ & $\begin{array}{l}3,0<1 \\
2,679\end{array}$ & 11.7 \\
\hline 2010 & 0 & $\mathrm{R}_{3,280}$ & 73 & 30 & 42 & 46 & 190 & 81 & $\mathrm{R}_{3,741}$ & R739 & 33 & 333 & 32 & $R_{1,137}$ & $\mathrm{R}_{2,604}$ & $\mathrm{R}_{11.0}$ \\
\hline $2011^{P}$ & 0 & 3,102 & 35 & 3 & 2 & 91 & 129 & 92 & 3,453 & 937 & 18 & 500 & 52 & 1,507 & 1,946 & 8.0 \\
\hline
\end{tabular}

Net imports equal imports minus exports

As liquefied natural gas.

By pipeline, except for very small amounts of liquefied natural gas imported from Canada in 1973, 1977, and 1981, and exported to Mexico beginning in 1998 .

4 Australia in 1997-2001 and 2004; Brunei in 2002; Equatorial Guinea in 2007; Indonesia in 1986 and 2000; Malaysia in 1999 and 2002-2005; Norway in 2008 forward; Oman in 2000-2005; Peru in 2010 and 2011; United Arab Emirates in 1996-2000; Yemen in 2010 and 2011, and Other (unassigned) in 2004. Brazil in 2010 and 2011, Chile in 2011; China in 2011; India in 2010 and 2011, Russia in 2007; South Korea in 2009 forward; Spain in 2010 and 2011; and United Kingdom in 2010 and 2011. Not meaningful because there were net exports during this year.

Note: Totals may not equal sum of components due to independent rounding

Web Pages: - See http://www.eia.gov/totalenergy/data/monthly/\#naturalgas for updated monthly and annual data. - See http://www.eia.gov/totalenergy/data/annual/\#naturalgas for all annual data beginning in 1949. - See http://www.eia.gov/naturalgas/ for related information.

Sources: Percent of U.S. Consumption: Calculated by dividing natural gas net imports by total natural gas consumption-see Table 6.1. All Other Data: - 1949-1954-U.S. Energy Information Administration (EIA) estimates based on Bureau of Mines, Minerals Yearbook, "Natural Gas" chapter. " 1955-1971-EIA, Federal Power Commission, by telephone. - 1972-1987-EIA, Form FPC-14, "Annual Report for Importers and Exporters of Natural Gas." - 1988-2009-EIA, Natural Gas Annual, annual reports. 
Figure 6.4 Natural Gas Gross Withdrawals and Natural Gas Well Productivity, 1960-2011

\section{Gross Withdrawals by Location}

$35-$

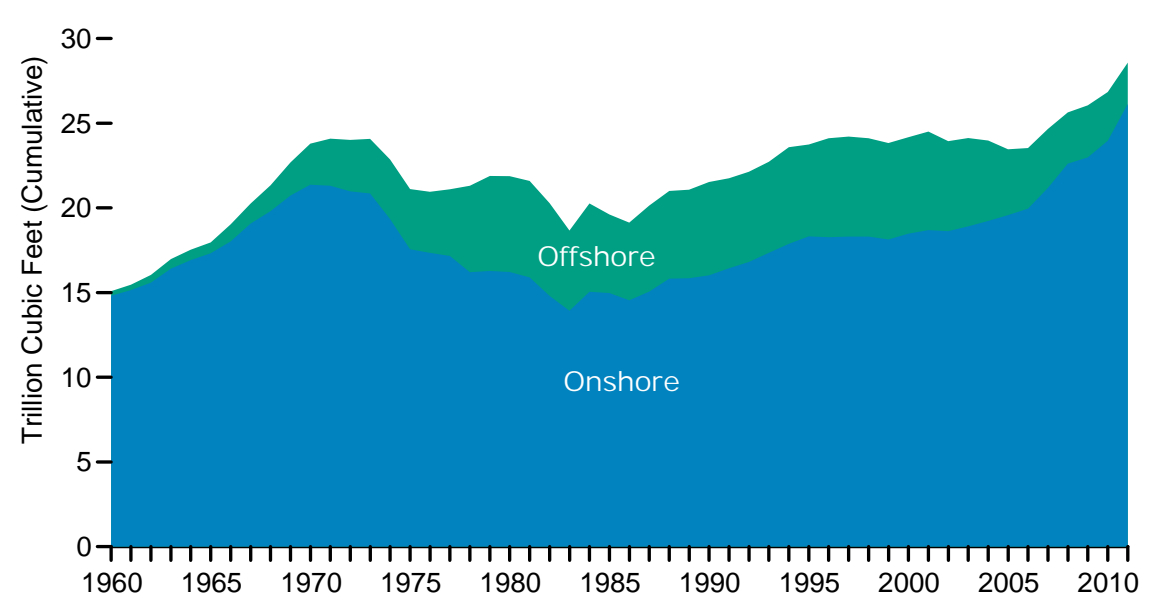

\section{Gross Withdrawals by State and Federal Gulf of Mexico}

$15-$
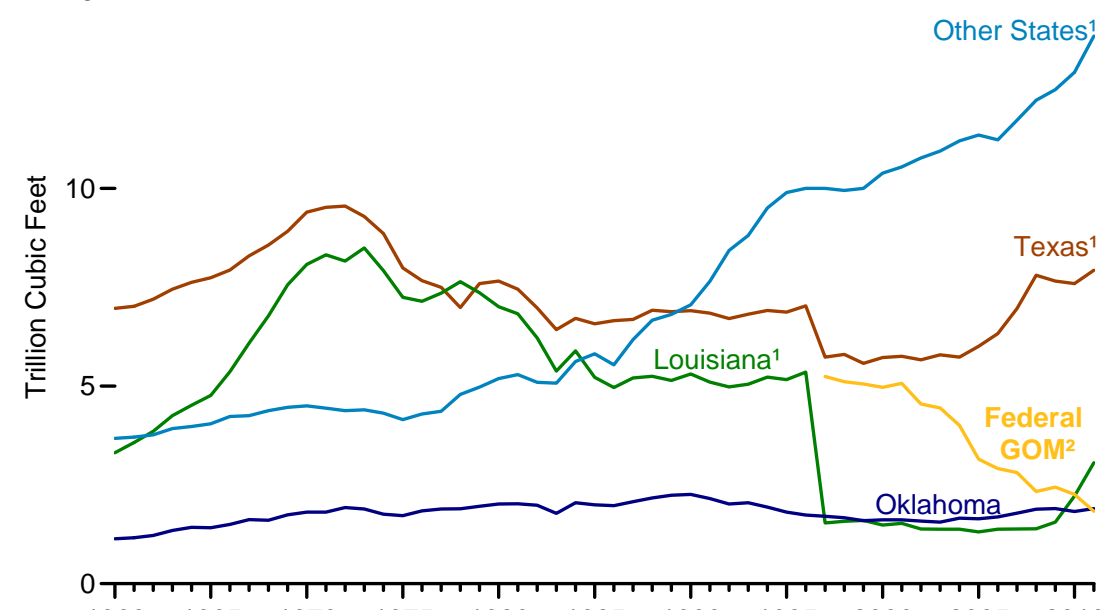

$\begin{array}{lllllllllll}1960 & 1965 & 1970 & 1975 & 1980 & 1985 & 1990 & 1995 & 2000 & 2005 & 2010\end{array}$

\section{Number of Producing Wells}

$600-$

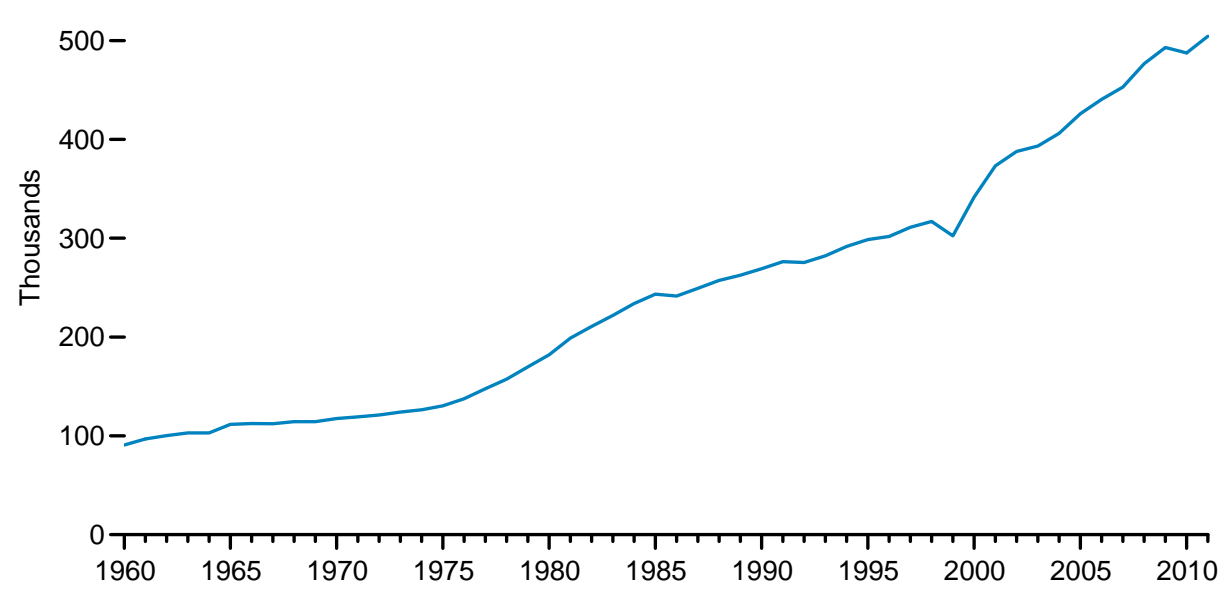

Natural Gas Well Average Productivity

$500-$
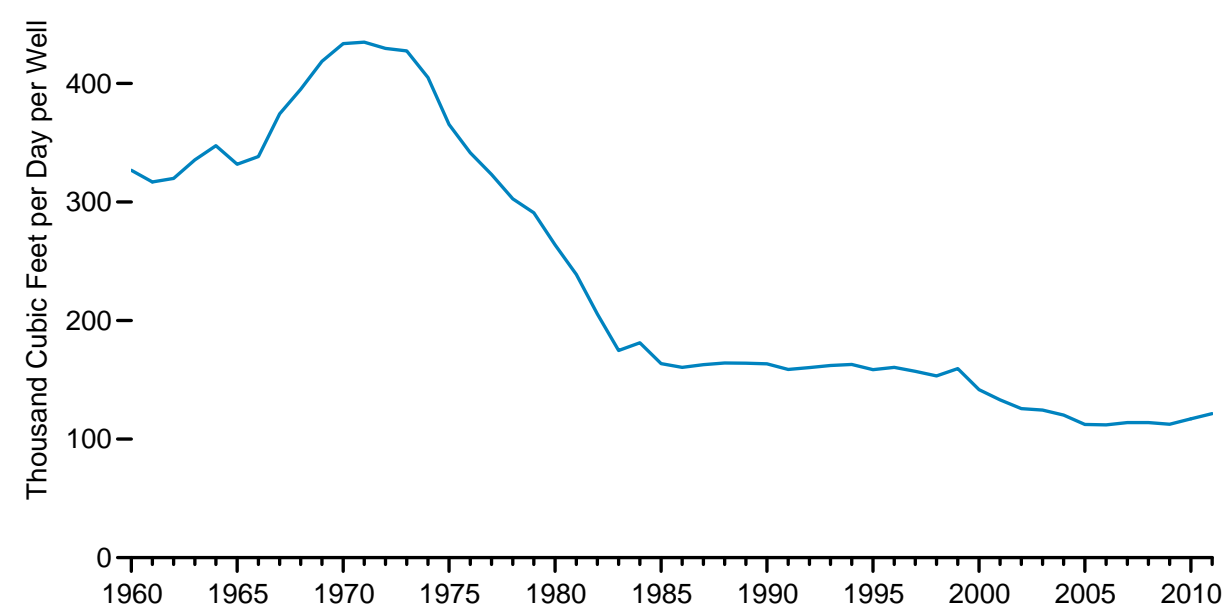

1 Through 1996, includes gross withdrawals in Federal offshore areas of the Gulf of Mexico; beginning in 1997, these are included in "Federal Gulf of Mexico." 
Table 6.4 Natural Gas Gross Withdrawals and Natural Gas Well Productivity, Selected Years, 1960-2011

\begin{tabular}{|c|c|c|c|c|c|c|c|c|c|c|c|c|c|c|}
\hline \multirow[b]{4}{*}{ Year } & \multicolumn{11}{|c|}{ Natural Gas Gross Withdrawals From Crude Oil, Natural Gas, Coalbed, and Shale Gas Wells } & \multicolumn{3}{|c|}{ Natural Gas Well Productivity } \\
\hline & \multirow[b]{2}{*}{ Texas $^{1}$} & \multirow[b]{2}{*}{ Louisiana ${ }^{1}$} & \multirow[b]{2}{*}{ Oklahoma } & \multirow{2}{*}{$\begin{array}{l}\text { Other } \\
\text { States }{ }^{1}\end{array}$} & \multirow{2}{*}{$\begin{array}{c}\text { Federal } \\
\text { Gulf of } \\
\text { Mexico }^{2}\end{array}$} & \multirow[b]{2}{*}{ Total } & \multirow[b]{2}{*}{ Onshore } & \multicolumn{3}{|c|}{ Offshore } & \multirow[b]{2}{*}{ Total } & \multirow{2}{*}{$\begin{array}{c}\text { Gross With- } \\
\text { drawals From } \\
\text { Natural Gas Wells }\end{array}$} & \multirow{2}{*}{$\begin{array}{c}\text { Producing } \\
\text { Wells }{ }^{4}\end{array}$} & \multirow{2}{*}{$\begin{array}{c}\text { Average } \\
\text { Productivity }\end{array}$} \\
\hline & & & & & & & & Federal & State & Total & & & & \\
\hline & \multicolumn{6}{|c|}{ Billion Cubic Feet } & & & on Cubic & & & Billion Cubic Feet & Thousands & $\begin{array}{l}\text { Thousand Cubic Feet } \\
\text { Per Day Per Well }\end{array}$ \\
\hline 1960 & 6,965 & 3,313 & 1,133 & 3,677 & $\left({ }^{2}\right)$ & 15,088 & 14,815 & 273 & NA & 273 & 15,088 & 10,853 & 91 & 326.7 \\
\hline 1965 & 7,741 & 4,764 & 1,414 & 4,044 & (2) & 17,963 & 17,318 & 646 & NA & 646 & 17,963 & 13,524 & 112 & 331.8 \\
\hline 1966 & 7,935 & 5,365 & 1,502 & 4,232 & (2) & 19,034 & 18,026 & 1,007 & NA & 1,007 & 19,034 & 13,894 & 112 & 338.4 \\
\hline 1967 & 8,292 & 6,087 & 1,621 & 4,252 & (2) & 20,252 & 19,065 & 1,187 & NA & 1,187 & 20,252 & 15,345 & 112 & 374.3 \\
\hline 1968 & 8,566 & 6,778 & 1,607 & 4,375 & (2) & 21,325 & 19,801 & 1,524 & NA & 1,524 & 21,325 & 16,540 & 114 & 395.1 \\
\hline 1969 & 8,915 & 7,561 & 1,742 & 4,462 & (2) & 22,679 & 20,725 & 1,954 & NA & 1,954 & 22,679 & 17,489 & 114 & 418.6 \\
\hline 1970 & 9,399 & 8,076 & 1,811 & 4,501 & (2) & 23,786 & 21,368 & 2,419 & NA & 2,419 & 23,786 & 18,595 & 117 & 433.6 \\
\hline 1971 & 9,519 & 8,319 & 1,809 & 4,442 & (2) & 24,088 & 21,311 & 2,777 & NA & 2,777 & 24,088 & 18,925 & 119 & 434.8 \\
\hline 1972 & 9,550 & 8,160 & 1,928 & 4,378 & (2) & 24,016 & 20,978 & 3,039 & NA & 3,039 & 24,016 & 19,043 & 121 & 429.4 \\
\hline 1973 & 9,290 & 8,491 & 1,890 & 4,396 & (2) & 24,067 & 20,856 & 3,212 & NA & 3,212 & 24,067 & 19,372 & 124 & 427.4 \\
\hline 1974 & 8,859 & 7,920 & 1,757 & 4,314 & (2) & 22,850 & 19,335 & 3,515 & NA & 3,515 & 22,850 & 18,669 & 126 & 404.9 \\
\hline 1975 & 7,989 & 7,242 & 1,721 & 4,152 & (2) & 21,104 & 17,555 & 3,549 & NA & 3,549 & 21,104 & 17,380 & 130 & 365.3 \\
\hline 1976 & 7,666 & 7,143 & 1,842 & 4,293 & (2) & 20,944 & 17,348 & 3,596 & NA & $\begin{array}{l}3,596 \\
3,59\end{array}$ & 20,944 & 17,191 & 138 & 341.5 \\
\hline 1977 & 7,496 & 7,351 & $\begin{array}{l}1,888 \\
\end{array}$ & 4,362 & (2) & 21,097 & 17,165 & 3,932 & NA & 3,932 & 21,097 & 17,416 & 148 & 323.1 \\
\hline 1978 & 6,988 & 7,639 & 1,892 & 4,790 & (2) & 21,309 & $\mathrm{R}_{16,197}$ & 4,356 & 756 & R5,111 & 21,309 & 17,394 & 157 & 302.7 \\
\hline 1979 & 7,594 & 7,359 & 1,958 & 4,973 & (2) & 21,883 & $\mathrm{R}_{16,280}$ & 4,822 & 781 & $\mathrm{R}_{5}, 603$ & 21,883 & 18,034 & 170 & 290.8 \\
\hline 1980 & 7,656 & 7,008 & 2,019 & 5,187 & (2) & 21,870 & $\mathrm{R}_{16,220}$ & 4,902 & 748 & R5,650 & 21,870 & 17,573 & 182 & 263.8 \\
\hline 1981 & 7,452 & 6,830 & 2,019 & 5,287 & (2) & 21,587 & $\mathrm{R}_{15,894}$ & 4,991 & 703 & R5,693 & 21,587 & 17,337 & 199 & 238.9 \\
\hline 1982 & 6,976 & 6,217 & 1,985 & 5,094 & (2) & 20,272 & $\mathrm{R}_{14,806}$ & 4,773 & 693 & R5,466 & 20,272 & 15,809 & 211 & 205.5 \\
\hline 1983 & 6,429 & 5,379 & 1,780 & 5,071 & (2) & 18,659 & $\mathrm{R}_{13,924}$ & 4,182 & 553 & $R_{4}, 735$ & 18,659 & 14,153 & 222 & 174.7 \\
\hline 1984 & 6,712 & 5,888 & 2,046 & 5,620 & (2) & 20,267 & $\mathrm{R}_{15,046}$ & 4,707 & 513 & $R 5,220$ & 20,267 & 15,513 & 234 & 181.2 \\
\hline 1985 & 6,577 & 5,218 & 1,993 & 5,818 & (2) & 19,607 & $\mathrm{R}_{14,975}$ & 4,186 & 446 & $\mathrm{R}_{4,632}$ & 19,607 & 14,535 & 243 & 163.6 \\
\hline 1986 & 6,656 & 4,965 & 1,972 & 5,538 & (2) & 19,131 & $\mathrm{R}_{14,542}$ & 4,186 & 403 & R4,589 & 19,131 & 14,154 & 242 & 160.6 \\
\hline 1987 & 6,688 & 5,205 & 2,073 & 6,174 & (2) & 20,140 & $\mathrm{R}_{15,062}$ & 4,672 & 406 & $\begin{array}{r}\text { R } 5,078 \\
\text {. }\end{array}$ & 20,140 & 14,807 & 249 & $\begin{array}{l}102.8 \\
\end{array}$ \\
\hline 1988 & 6,919 & 5,248 & 2,167 & 6,665 & (2) & 20,999 & $\mathrm{R}_{15,818}$ & 4,747 & 434 & R5, 181 & 20,999 & 15,467 & 257 & 164.3 \\
\hline 1989 & 6,881 & $\begin{array}{l},<40 \\
5,143\end{array}$ & 2,237 & $\begin{array}{l}6,000 \\
6,813\end{array}$ & (2) & 21,074 & $\begin{array}{l}\mathrm{R}_{15,810} \\
\end{array}$ & $\begin{array}{l}4,771 \\
4,771\end{array}$ & $\begin{array}{l}404 \\
460\end{array}$ & R5,231 & 21,074 & 15,709 & 262 & 164.0 \\
\hline 1990 & 6,907 & 5,303 & 2,258 & 7,054 & (2) & 21,523 & $\mathrm{R}_{16,013}$ & 5,047 & 463 & R5,509 & 21,523 & 16,054 & $R_{270}$ & $\mathrm{R}_{163.0}$ \\
\hline 1991 & 6,846 & 5,100 & 2,154 & 7,651 & (2) & 21,750 & $\mathrm{R}_{16,442}$ & 4,850 & $\begin{array}{l}400 \\
459\end{array}$ & R5,308 & 21,750 & 16,018 & R277 & $\begin{array}{r}158.0 \\
\text { R158.4 }\end{array}$ \\
\hline 1992 & 6,708 & 4,977 & 2,017 & 8,429 & (2) & 22,132 & $R_{16,808}$ & 4.772 & 552 & R5, 324 & 22,132 & 16,165 & R276 & R160.0 \\
\hline 1993 & 6,817 & 5,047 & 2,050 & 8,812 & $(2)$ & 22,726 & $R_{17,352}$ & 4,766 & 607 & R5,373 & 22,726 & 16,691 & 282 & 162.1 \\
\hline 1994 & 6,912 & 5,226 & 1,935 & 9,508 & (2) & 23,581 & $R_{17,880}$ & 4,996 & 704 & R5,701 & 23,581 & 17,351 & $\begin{array}{l}\text { Loc } \\
292\end{array}$ & 162.9 \\
\hline 1995 & 6,873 & 5,163 & 1,812 & 9,896 & (2) & 23,744 & $\mathrm{R}_{18,312}$ & $\begin{array}{r}4,950 \\
4,942\end{array}$ & 490 & R5, 432 & 23,744 & 17,282 & 299 & 158.6 \\
\hline 1996 & $\begin{array}{l}0,028 \\
7,028\end{array}$ & 5,351 & $\begin{array}{l}1,735 \\
1,735\end{array}$ & $\begin{array}{l}\text {,,o50 } \\
9,999\end{array}$ & (2) & $\begin{array}{l}25,114 \\
24,114\end{array}$ & $\mathrm{R}_{18,270}$ & 5,246 & 597 & R5,844 & $\begin{array}{l}24,114 \\
24,14\end{array}$ & 17,737 & 302 & $\begin{array}{l}100.6 \\
160.6\end{array}$ \\
\hline 1997 & $\begin{array}{l}15,730 \\
15,0\end{array}$ & 11,538 & 1,704 & 19,999 & 5,242 & $\begin{array}{l}24,213 \\
24,14\end{array}$ & $\mathrm{R}_{18,306}$ & 5,316 & 591 & $\begin{array}{r}\mathrm{R} 5,906 \\
\text { R }\end{array}$ & 24,213 & 17,844 & 311 & 157.2 \\
\hline 1998 & 5,799 & $\begin{array}{l}1,579 \\
1,579\end{array}$ & $\begin{array}{l}1,669 \\
1,669\end{array}$ & 9,950 & 5,110 & $\begin{array}{l}24,108 \\
24,10\end{array}$ & $\begin{array}{l}R_{18,308} \\
\text { R }\end{array}$ & $\begin{array}{l}5,185 \\
5,185\end{array}$ & 615 & R5,801 & 24,108 & $\begin{array}{l}17,729 \\
17,729\end{array}$ & 317 & 153.3 \\
\hline $\begin{array}{l}1990 \\
1999\end{array}$ & 5,575 & $\begin{array}{l}1,599 \\
\end{array}$ & $\begin{array}{l}1,009 \\
1,594\end{array}$ & $\begin{array}{r}5,002 \\
10,002\end{array}$ & 5,053 & $\begin{array}{l}23,823 \\
23,82\end{array}$ & $\begin{array}{l}\mathrm{R} 18,133 \\
\mathrm{R}\end{array}$ & 5,131 & 559 & $\begin{array}{l}\mathrm{R} 5,689 \\
\text { R }\end{array}$ & $\begin{array}{l}23,823 \\
23,10\end{array}$ & 17,590 & $\begin{array}{l}511 \\
302\end{array}$ & $\begin{array}{l}159.4 \\
\end{array}$ \\
\hline 2000 & $\begin{array}{l}5,5 / 5 \\
5,723\end{array}$ & $\begin{array}{l}1,599 \\
1,485\end{array}$ & $\begin{array}{l}1,594 \\
1,613\end{array}$ & $\begin{array}{l}10,022 \\
10,386\end{array}$ & 4,968 & $\begin{array}{l}25,174 \\
24,174\end{array}$ & R18,474 & 5,044 & 656 & $\begin{array}{l}\text { R5,699 } \\
\text { R5, }\end{array}$ & $\begin{array}{l}24,174 \\
24,0\end{array}$ & $\begin{array}{l}17,726 \\
17,50\end{array}$ & 342 & 141.7 \\
\hline 2001 & 5,752 & 1,525 & 1,615 & 10,542 & 5,066 & 24,501 & $\mathrm{R}_{18,685}$ & 5,137 & 679 & $\mathrm{R} 5,816$ & 24,501 & 18,129 & 373 & 133.1 \\
\hline 2002 & 5,661 & 1,382 & 1,582 & 10,769 & 4,548 & 23,941 & $\begin{array}{l}\mathrm{R}_{18,629} \\
\text { 18, }\end{array}$ & 4,615 & 697 & $\mathrm{R} 5,312$ & 23,941 & 17,795 & 388 & 125.7 \\
\hline 2003 & 5,791 & 1,378 & 1,558 & 10,944 & 4,447 & 24,119 & $\mathrm{R}_{18,903}$ & 4,505 & 710 & $\mathrm{R}_{5,216}$ & 24,119 & 17,882 & 393 & 124.6 \\
\hline 2004 & 5,734 & 1,377 & 1,656 & 11,202 & 4,001 & 23,970 & $\mathrm{R}_{19,233}$ & 4,055 & 681 & $\begin{array}{r}\mathrm{R} 4,736 \\
\text { R }\end{array}$ & 23,970 & 17,885 & 406 & 120.3 \\
\hline 2005 & 6,007 & 1,310 & 1,639 & 11,350 & 3,151 & 23,457 & $\mathrm{R}_{19,567}$ & 3,205 & 685 & $\mathrm{R}_{3,890}$ & 23,457 & 17,472 & 426 & 112.4 \\
\hline 2006 & 6,326 & 1,378 & 1,689 & 11,227 & 2,914 & 23,535 & $\mathrm{R}_{19,951}$ & 2,955 & 630 & $\mathrm{R} 3,584$ & 23,535 & 17,996 & 441 & 111.9 \\
\hline 2007 & 6,961 & 1,383 & 1,784 & 11,723 & 2,813 & 24,664 & $\mathrm{R}_{21,187}$ & 2,859 & 618 & $\mathrm{R} 3,477$ & 24,664 & 3,R 18,845 & 453 & $\mathrm{R}_{114.0}$ \\
\hline 2008 & 7,801 & 1,388 & 1,887 & 12,231 & 2,330 & 25,636 & R22,608 & 2,375 & 654 & $\mathrm{R}_{3,029}$ & 25,636 & R19,889 & 477 & R114.0 \\
\hline 2009 & 7,654 & 1,559 & $\begin{array}{l}R_{1}, 902 \\
\text {. }\end{array}$ & 12,499 & 2,444 & R26,057 & $\mathrm{R}_{22,985}$ & 2,485 & 587 & $\mathrm{R} 3,072$ & R26,057 & $\mathrm{R}_{20,245}$ & 493 & $\mathrm{R}_{112.5}$ \\
\hline 2010 & $\mathrm{R} 7,594$ & $R_{2,218}$ & 1,827 & $\mathrm{R}_{12,938}$ & 2,259 & R26,836 & $\mathrm{R}_{23,960}$ & 2,300 & 576 & $\begin{array}{r}\mathrm{R} 2,876 \\
\mathrm{r}\end{array}$ & R26,836 & $\mathrm{R}_{20,841}$ & R488 & $R_{117.1}$ \\
\hline 2011 & P7,931 & $P_{3,060}$ & $\mathrm{P}_{1,899}$ & $\mathrm{P}_{13,855}$ & $\mathrm{P}_{1,830}$ & P28,576 & $E_{26,152}$ & $\mathrm{E}_{1,868}$ & E556 & $E_{2,424}$ & P28,576 & $E_{22,378}$ & E504 & $E_{121.5}$ \\
\hline
\end{tabular}

1 Through 1996, includes gross withdrawals in Federal offshore areas of the Gulf of Mexico; beginning in 1997, these are included in "Federal Gulf of Mexico." 2 Gross withdrawals from Federal offshore areas of the Gulf of Mexico. Through 1996, these gross

withdrawals are included in "Texas," "Louisiana," and "Other States."
3 Beginning in 2007, includes natural gas gross withdrawals from coalbed wells, and beginning in 2008, from shale gas wells.

$R=$ Revised. $P=$ Preliminary. E=Estimate. NA=Not available.

Note: Totals may not equal sum of components due to independent rounding

Web Pages: - See http://www.eia.gov/totalenergy/data/annual/\#naturalgas for all data beginning in

1960. • For related information, see http://wwW.eia.gov/naturalgas/.
Sources: Total (Gross Withdrawals): • 1960-2006-U.S. Energy Information Administration (EIA),

Natural Gas Annual (NGA), annual reports. • 2007 forward-EIA, Natural Gas Monthly (March 2012), Table 1. Total (Offshore): • 1960-1981-U.S. Geological Survey.• 1982-1985-U.S. Minerals Management Service, Mineral Revenues-The 1989 Report on Receipts from Federal and Indian Leases,

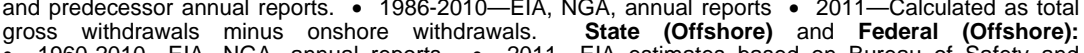
- 1960-2010-EIA, NGA, annual reports. - 2011-EIA estimates based on Bureau of Safety and withdrawals from natural gas wells divided by the number of producing wells, and then divided by the number of days in the year. All Other Data: - 1960-1966-Bureau of Mines, Natural Gas Production and Consumption. 1967-2010-EIA, NGA, annual reports and unpublished revisions. • 2011-EIA estimates based on previous year's data. 


\section{Figure 6.5 Natural Gas Consumption by Sector}

\section{By Sector, 1949-2011}

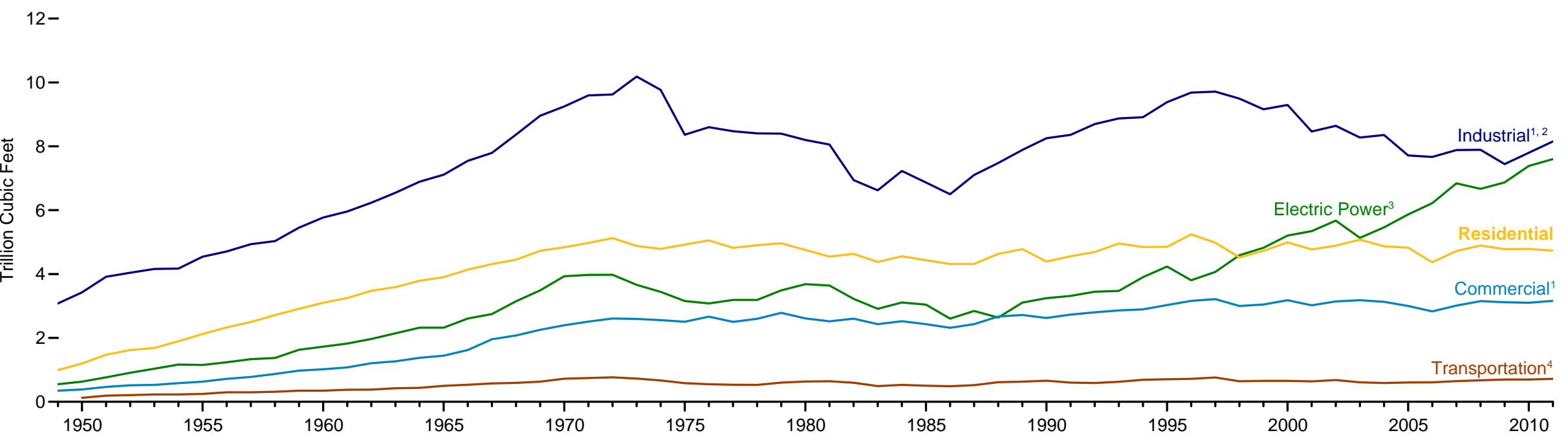

By Sector, 2011

$10-$

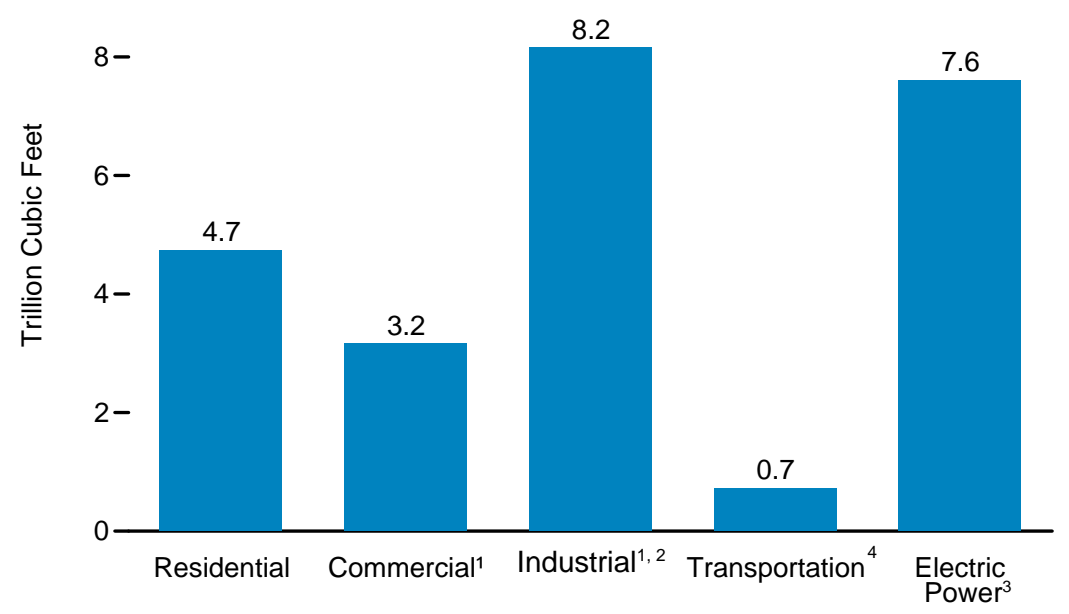

${ }^{1}$ Includes combined-heat-and-power plants and a small number of electricity-only plants.

${ }^{2}$ Lease and plant fuel, and other industrial.

${ }^{3}$ Electricity-only and combined-heat-and-power plants whose primary business is to sell elec-

tricity, or electricity and heat, to the public.
Sector Shares, 1950 and 2011

$75-$

$1950 \square 2011$

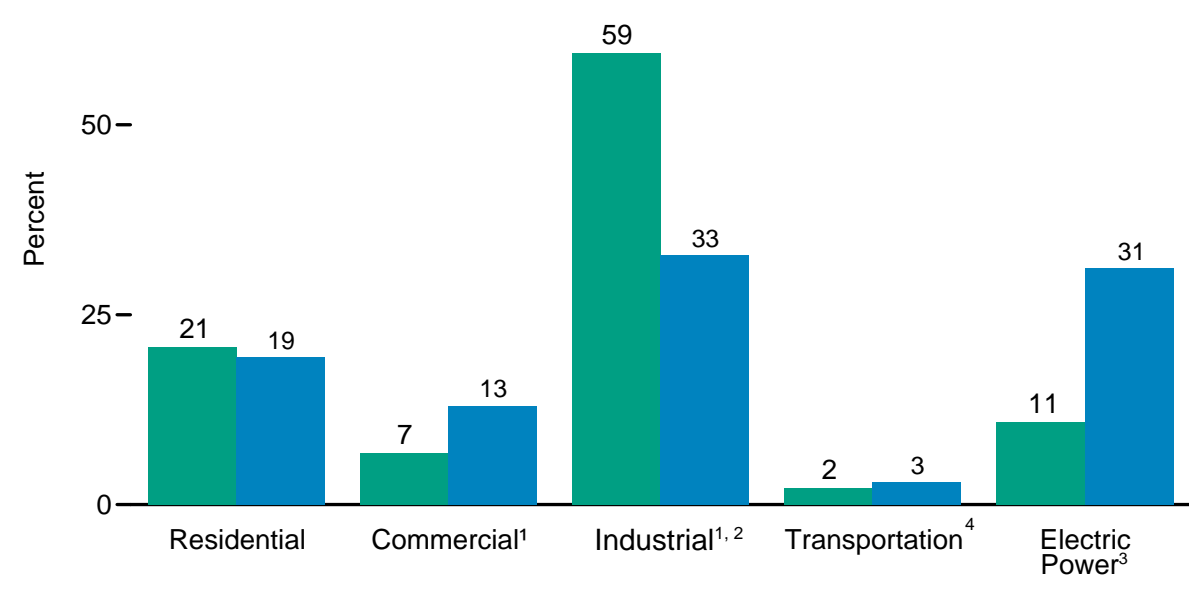

${ }^{4}$ Natural gas consumed in the operation of pipelines (primarily in compressors), and as fuel in the delivery of natural gas to consumers; plus a small quantity used as vehicle fuel.

Source: Table 6.5. 
Table 6.5 Natural Gas Consumption by Sector, Selected Years, 1949-2011

(Billion Cubic Feet)

\begin{tabular}{|c|c|c|c|c|c|c|c|c|c|c|c|c|c|c|c|c|}
\hline \multirow[b]{3}{*}{ Year } & \multirow{3}{*}{$\begin{array}{l}\text { Residential } \\
\text { Sector }\end{array}$} & \multicolumn{3}{|c|}{ Commercial Sector } & \multicolumn{5}{|c|}{ Industrial Sector } & \multicolumn{3}{|c|}{ Transportation Sector } & \multicolumn{3}{|c|}{ Electric Power Sector 1} & \multirow[b]{3}{*}{ Total } \\
\hline & & \multirow[b]{2}{*}{$\mathrm{CHP}^{2}$} & \multirow[b]{2}{*}{ Other ${ }^{3}$} & \multirow[b]{2}{*}{ Total } & \multirow{2}{*}{$\begin{array}{l}\text { Lease and } \\
\text { Plant Fuel }\end{array}$} & \multicolumn{3}{|c|}{ Other Industrial } & \multirow[b]{2}{*}{ Total } & \multirow{2}{*}{$\begin{array}{c}\text { Pipelines } \\
\text { and Dis- } \\
\text { tribution }^{7}\end{array}$} & \multirow{2}{*}{$\begin{array}{l}\text { Vehicle } \\
\text { Fuel }{ }^{8}\end{array}$} & \multirow[b]{2}{*}{ Total } & \multirow{2}{*}{$\begin{array}{l}\text { Electricity } \\
\text { Only }\end{array}$} & \multirow[b]{2}{*}{ CHP } & & \\
\hline & & & & & & $\mathrm{CHP}^{4}$ & Non-CHP 5 & Total & & & & & & & Total & \\
\hline 1949 & 993 & $\left({ }^{9}\right)$ & 348 & 348 & 835 & $(10)$ & 2,245 & 2,245 & 3,081 & NA & NA & NA & 550 & NA & 550 & 4.971 \\
\hline 1950 & $\begin{array}{r}993 \\
1,198\end{array}$ & (9) & $\begin{array}{l}348 \\
388\end{array}$ & $\begin{array}{l}340 \\
388\end{array}$ & $\begin{array}{l}835 \\
928\end{array}$ & (10) & $\begin{array}{l}2,<45 \\
2,498\end{array}$ & $\begin{array}{l}2,245 \\
2,498\end{array}$ & $\begin{array}{l}3,001 \\
3,426\end{array}$ & 126 & NA & 126 & 629 & NA & 629 & 5,767 \\
\hline 1955 & 2,124 & (9) & $\begin{array}{l}300 \\
629\end{array}$ & $\begin{array}{l}300 \\
629\end{array}$ & 1,131 & (10) & $\begin{array}{l}2,490 \\
3,411\end{array}$ & $\begin{array}{l}3,490 \\
3,411\end{array}$ & $\begin{array}{l}\quad, 4<0 \\
4,542\end{array}$ & 245 & NA & 245 & 1,153 & NA & 1,153 & 8,694 \\
\hline 1960 & 3,103 & (9) & 1,020 & 1,020 & 1,237 & (10) & 4,535 & $\begin{array}{l}4,535 \\
4,535\end{array}$ & 5,771 & 347 & NA & 347 & 1,725 & NA & 1,725 & 11,967 \\
\hline 1965 & 3,903 & (9) & 1,444 & 1,444 & $\begin{array}{l}1,156 \\
1,156\end{array}$ & $(10)$ & 5,955 & $\begin{array}{l}4,955 \\
5,955\end{array}$ & 7,112 & 501 & NA & 501 & 2,321 & NA & 2,321 & 15,280 \\
\hline 1970 & 4,837 & (9) & 2,399 & 2,399 & 1,399 & $(10)$ & 7,851 & 7,851 & 9,249 & 722 & NA & 722 & $\begin{array}{l}3,932 \\
3,921\end{array}$ & NA & 3,932 & 21,139 \\
\hline 1975 & 4,924 & $(9)$ & 2,508 & 2,508 & 1,396 & $(10)$ & 6,968 & $\begin{array}{l}6,968 \\
6,968\end{array}$ & 8,365 & 583 & NA & 583 & 3,158 & NA & 3,158 & 19,538 \\
\hline 1976 & 5,051 & (9) & 2,668 & 2,668 & 1,634 & (10) & $\begin{array}{l}6,964 \\
6,90\end{array}$ & $\begin{array}{l}6,964 \\
6\end{array}$ & 8,598 & 548 & NA & 548 & $\begin{array}{l}3,081 \\
3,1\end{array}$ & NA & 3,081 & 19,946 \\
\hline 1977 & 4,821 & (9) & 2,501 & 2,501 & 1,659 & (10) & 6,815 & 6,815 & 8,474 & 533 & NA & 533 & 3,191 & NA & 3,191 & 19,521 \\
\hline 1978 & 4,903 & $(9)$ & 2,601 & 2,601 & 1,648 & (10) & 6,757 & 6,757 & 8,405 & 530 & NA & 530 & 3,188 & NA & 3,188 & 19,627 \\
\hline 1979 & 4,965 & $(9)$ & 2,786 & 2,786 & 1,499 & $(10)$ & 6,899 & 6,899 & 8,398 & 601 & $\mathrm{NA}$ & 601 & 3,491 & $\mathrm{NA}$ & 3,491 & 20,241 \\
\hline 1980 & 4,752 & $(9)$ & 2,611 & 2,611 & 1,026 & $(10)$ & 7,172 & 7,172 & 8,198 & 635 & $\mathrm{NA}$ & 635 & 3,682 & $\mathrm{NA}$ & 3,682 & 19,877 \\
\hline 1981 & 4,546 & $(9)$ & 2,520 & 2,520 & 928 & $(10)$ & 7,128 & 7,128 & 8,055 & 642 & NA & 642 & 3,640 & NA & 3,640 & 19,404 \\
\hline 1982 & 4,633 & $(9)$ & 2,606 & 2,606 & 1,109 & $(10)$ & 5,831 & 5,831 & 6,941 & 596 & $\mathrm{NA}$ & 596 & 3,226 & NA & 3,226 & 18,001 \\
\hline 1983 & 4,381 & $(9)$ & 2,433 & 2,433 & 978 & $(10)$ & 5,643 & 5,643 & 6,621 & 490 & $\mathrm{NA}$ & 490 & 2,911 & NA & 2,911 & 16,835 \\
\hline 1984 & 4,555 & & 2,524 & 2,524 & 1,077 & $(10)$ & 6,154 & 6,154 & 7,231 & 529 & $\mathrm{NA}$ & 529 & 3,111 & NA & 3,111 & 17,951 \\
\hline 1985 & 4,433 & $(9)$ & 2,432 & 2,432 & 966 & $(10)$ & 5,901 & 5,901 & 6,867 & 504 & $\mathrm{NA}$ & 504 & 3,044 & $\mathrm{NA}$ & 3,044 & 17,281 \\
\hline 1986 & 4,314 & & 2,318 & 2,318 & & (10) & 5,579 & 5,579 & 6,502 & 485 & NA & 485 & 2,602 & NA & 2,602 & 16,221 \\
\hline 1987 & 4,315 & $(9)$ & 2,430 & 2,430 & 1,149 & $\left(\begin{array}{l}10 \\
(10)\end{array}\right.$ & 5,953 & 5,953 & 7,103 & 519 & NA & 519 & 2,844 & NA & 2,844 & 17,211 \\
\hline 1988 & 4,630 & $\left({ }^{9}\right)$ & 2,670 & 2,670 & 1,096 & $\left({ }^{10}\right)$ & 6,383 & 6,383 & 7,479 & 614 & NA & 614 & 2,636 & NA & 2,636 & 18,030 \\
\hline 1989 & 4,781 & 30 & 2,688 & 2,718 & 1,070 & 914 & 115,903 & ${ }^{11} 6,816$ & 7,886 & 629 & NA & 629 & 112,791 & 11315 & 113,105 & 1119,119 \\
\hline 1990 & 4,391 & 46 & 2,576 & 2,623 & 1,236 & 1,055 & $\begin{array}{l}115,963 \\
116\end{array}$ & $\begin{array}{l}117,018 \\
117\end{array}$ & 8,255 & 660 & (s) & 660 & $\begin{array}{l}112,794 \\
11\end{array}$ & 11451 & 113,245 & 1119,174 \\
\hline $\begin{array}{l}1991 \\
1992\end{array}$ & $\begin{array}{l}4,556 \\
4690\end{array}$ & $\begin{array}{l}52 \\
62\end{array}$ & $\begin{array}{l}2,676 \\
2,740\end{array}$ & $\begin{array}{r}2,729 \\
2803\end{array}$ & $\begin{array}{l}1,129 \\
1171\end{array}$ & $\begin{array}{l}1,061 \\
1107\end{array}$ & $\begin{array}{l}1116,170 \\
116420\end{array}$ & $\begin{array}{l}111,231 \\
117,527\end{array}$ & $\begin{array}{l}8,360 \\
8698\end{array}$ & $\begin{array}{l}601 \\
588\end{array}$ & (s) & $\begin{array}{l}602 \\
590\end{array}$ & $\begin{array}{l}112,822 \\
112,802\end{array}$ & $\begin{array}{l}11494 \\
11619\end{array}$ & $\begin{array}{l}113,316 \\
113\end{array}$ & $\begin{array}{l}1119,562 \\
1120228\end{array}$ \\
\hline 1993 & 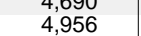 & $\begin{array}{l}62 \\
65\end{array}$ & 2,796 & $\begin{array}{l}2,803 \\
2,862\end{array}$ & 1,172 & 1,124 & 6,576 & 7,700 & $\begin{array}{l}8,698 \\
8,872\end{array}$ & $\begin{array}{l}624 \\
624\end{array}$ & 3 & $\begin{array}{l}590 \\
627\end{array}$ & 2,755 & 718 & $\begin{array}{l}3,448 \\
3,473\end{array}$ & 20,790 \\
\hline 1994 & 4,848 & 72 & 2,823 & 2,895 & 1,124 & 1,176 & 6,613 & 7,790 & 8,913 & 685 & 3 & 689 & 3,065 & 838 & 3,903 & 21,247 \\
\hline 1995 & 4,850 & 78 & 2,953 & 3,031 & 1,220 & 1,258 & 6,906 & 8,164 & 9,384 & 700 & 5 & 705 & 3,288 & 949 & 4,237 & 22,207 \\
\hline 1996 & 5,241 & 82 & 3,076 & 3,158 & 1,250 & 1,289 & 7,146 & 8,435 & 9,685 & 711 & 6 & 718 & 2,824 & 983 & 3,807 & 22,609 \\
\hline 1997 & 4,984 & 87 & 3,128 & 3,215 & 1,203 & 1,282 & 7,229 & 8,511 & 9,714 & 751 & 8 & 760 & 3,039 & 1,026 & 4,065 & 22,737 \\
\hline 1998 & 4,520 & 87 & 2,912 & 2,999 & 1,173 & 1,355 & 6,965 & 8,320 & 9,493 & 635 & 9 & 645 & 3,544 & 1,044 & 4,588 & 22,246 \\
\hline 1999 & 4,726 & 84 & 2,961 & 3,045 & 1,079 & 1,401 & 6,678 & 8,079 & 9,158 & 645 & 12 & 657 & 3,729 & 1,090 & 4,820 & 22,405 \\
\hline 2000 & 4,996 & 85 & 3,098 & 3,182 & 1,151 & 1,386 & 6,757 & 8,142 & 9,293 & 642 & 13 & 655 & 4,093 & 1,114 & 5,206 & 23,333 \\
\hline 2001 & 4,771 & 79 & 2,944 & 3,023 & 1,119 & 1,310 & 6,035 & 7,344 & 8,463 & 625 & 15 & 640 & 4,164 & 1,178 & 5,342 & 22,239 \\
\hline 2002 & 4,889 & 74 & 3,070 & 3,144 & 1,113 & 1,240 & $\mathrm{R}_{6,287}$ & $R 7,527$ & $R_{8,640}$ & 667 & 15 & 682 & 4,258 & 1,413 & 5,672 & $\mathrm{R}_{23,027}$ \\
\hline 2003 & 5,079 & 58 & 3,121 & 3,179 & 1,122 & 1,144 & 6,007 & 7,150 & 8,273 & 591 & 18 & 610 & 3,780 & 1,355 & 5,135 & 22,277 \\
\hline 2004 & 4,869 & 72 & 3,057 & 3,129 & 1,098 & 1,191 & $\mathrm{R}_{6,066}$ & R7,256 & $R_{8}, 354$ & 566 & 21 & 587 & 4,142 & 1,322 & 5,464 & $\mathrm{R}_{22,403}$ \\
\hline 2005 & 4,827 & 68 & 2,931 & 2,999 & 1,112 & 1,084 & R5,518 & $R_{6}, 601$ & R7,713 & 584 & 23 & 607 & 4,592 & 1,277 & 5,869 & $\mathrm{R}_{22,014}$ \\
\hline 2006 & 4,368 & 68 & 2,764 & 2,832 & 1,142 & 1,115 & $R_{5}, 412$ & $\mathrm{R}_{6,527}$ & R7,669 & 584 & 24 & 608 & 5,091 & 1,131 & 6,222 & $\mathrm{R}_{21,699}$ \\
\hline 2007 & 4,722 & 70 & 2,943 & 3,013 & 1,226 & 1,050 & R5,604 & R6,655 & R7,881 & 621 & 25 & 646 & 5,612 & 1,230 & 6,841 & R 23,104 \\
\hline 2008 & 4,892 & 66 & 3,086 & 3,153 & 1,220 & 955 & R5,715 & R6,670 & R7,890 & 648 & 26 & 674 & 5,520 & 1,148 & 6,668 & R 23,277 \\
\hline 2009 & R4,779 & 76 & 3,043 & 3,119 & 1,275 & 990 & $R_{5}, 178$ & 6,167 & $\mathrm{R} 7,443$ & R670 & $\mathrm{R}_{27}$ & R697 & 5,751 & 1,122 & 6,873 & $\mathrm{R}_{22,910}$ \\
\hline 2010 & $\begin{array}{r}R_{4}, 787 \\
4735\end{array}$ & $\begin{array}{l}\text { R86 } \\
81\end{array}$ & $\mathrm{R}_{3}, 016$ & $\mathrm{R}_{3,102}$ & $\begin{array}{r}R_{1}, 282 \\
1,383\end{array}$ & $\begin{array}{r}R_{1}, 029 \\
1,24\end{array}$ & R5,488 & ${ }^{R} 6,517$ & $\begin{array}{l}\text { R7,800 } \\
8152\end{array}$ & R669 & R31 & $\begin{array}{l}\text { R7700 } \\
710\end{array}$ & ${ }^{R} 6,239$ & $\mathrm{R}_{1,148}$ & R7,387 & $\begin{array}{l}R_{2} 23,775 \\
21360\end{array}$ \\
\hline & 4,735 & 81 & 3,080 & 3,161 & 1,383 & 1,024 & 5,746 & $6, / 69$ & 8,153 & 686 & & $/ 18$ & 6,440 & 1,162 & , 602 & 24,369 \\
\hline
\end{tabular}

Electricity-only and combined-heat-and-power (CHP) plants within the NAICS 22 category whose primary business is to sell electricity, or electricity and heat, to the public. Through 1988, data are for

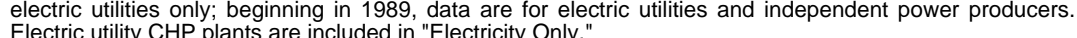

2 Commercial combined-heat-and-power (CHP) and a small number of commercial electricity-only

"Supplemental Gaseous Fuels," at end of section. - See Tables $8.5 \mathrm{a}-8.5 \mathrm{~d}$ for the amount of natural gas used to produce electricity and Tables $8.6 \mathrm{a}-8.6 \mathrm{c}$ for the amount of natural gas used to produce usef thermal output. - See Note 2, "Natural Gas Consumption," at end of section. - Beginning with 1965, al 14.65 p.s.i.a. at $60^{\circ} \mathrm{F}$. - Totals may not equal sum of components due to independent rounding. Web Pages: - See http://www.eia.gov/totalenergy/data/monthly/\#naturalgas for updated monthly and annual data. - See http://www.eia.gov/totalenergy/data/annual/\#naturalgas for all annual data beginning in 949. - See http://www.eia.gov/naturalgas/ for related information.
Sources: Residential, Commercial Total, Lease and Plant Fuel, Other Industrial Total, and Pipelines and Distribution: - 1949-2006-U.S. Energy Information Administration (EIA), Natural Gas Annual (NGA), annual reports and unpublished revisions. - 2007 forward-EIA, Natural Gas Monthly (NGM) (March 2012), Table 2. Commercial CHP and Industrial CHP: Table 8.7c. Vehicle Fuel: • 1990 and 1991-EIA, NGA 2000 (November 2001), Table 95. " 1992-1998-EIA, "Alternatives to Traditional Transportation Fuels 1999" (October 1999), Table 10, and "Alternatives to Traditional Transportation Fuels gasoling factor (see Table A3) and dividing by the natural gas end-use sectors conversion factor (see Table A4). Power Sector: Tables 8.5b, 8.5c, 8.6b, and 8.7b. All Other Data: Calculated.
Pol2), Table 2. Electric

4 Industrial combined-heat-and-power (CHP) and a small number of industrial electricity-only pla

5 All industrial sector fuel use other than that in "Lease and Plant Fuel" and "Ind
6 Natural gas consumed in the operation of pipelines, primarily in compressors.

8 Vehicle fuel data do not reflect revised data shown in Table 10.5. See Note 4, "Natural Gas Vehicle Fuel," at end of section.

10 Included in "Industrial Non-CHP." both "Other Industrial" and "Electric Power Sector." See Note 3, "Natural Gas Consumption, 1989-1992,"

at end of section.

Notes: - Data are for natural gas, plus a small amount of supplemental gaseous fuels. See Note 1 


\section{Figure 6.6 Natural Gas Underground Storage, End of Year}

Total (Base Gas and Working Gas) Underground Storage, 1954-2011

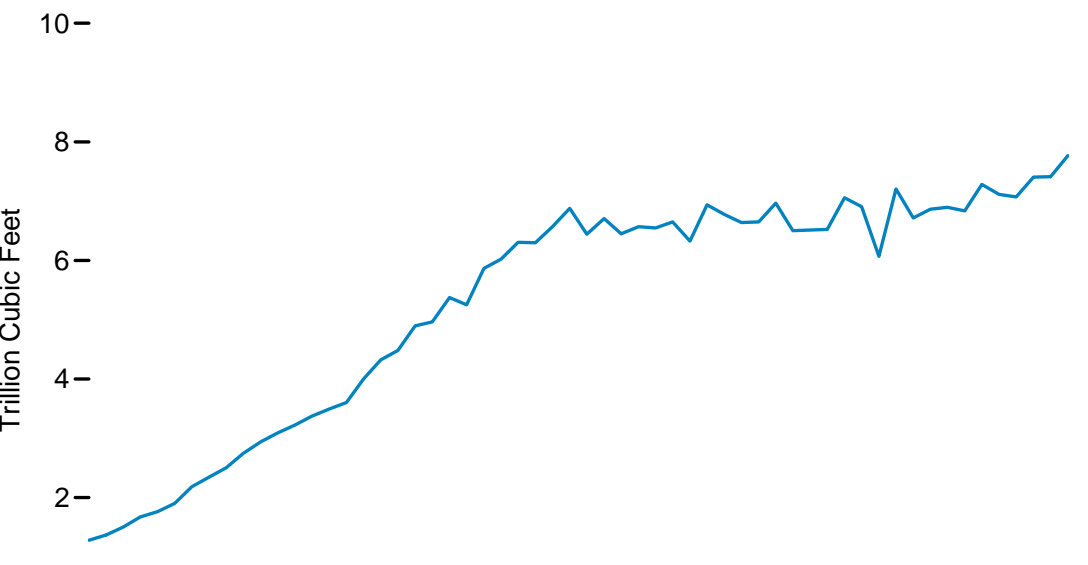

0

$\begin{array}{llllllllllll}1955 & 1960 & 1965 & 1970 & 1975 & 1980 & 1985 & 1990 & 1995 & 2000 & 2005 & 2010\end{array}$
Underground Storage Capacity, 1975-2011

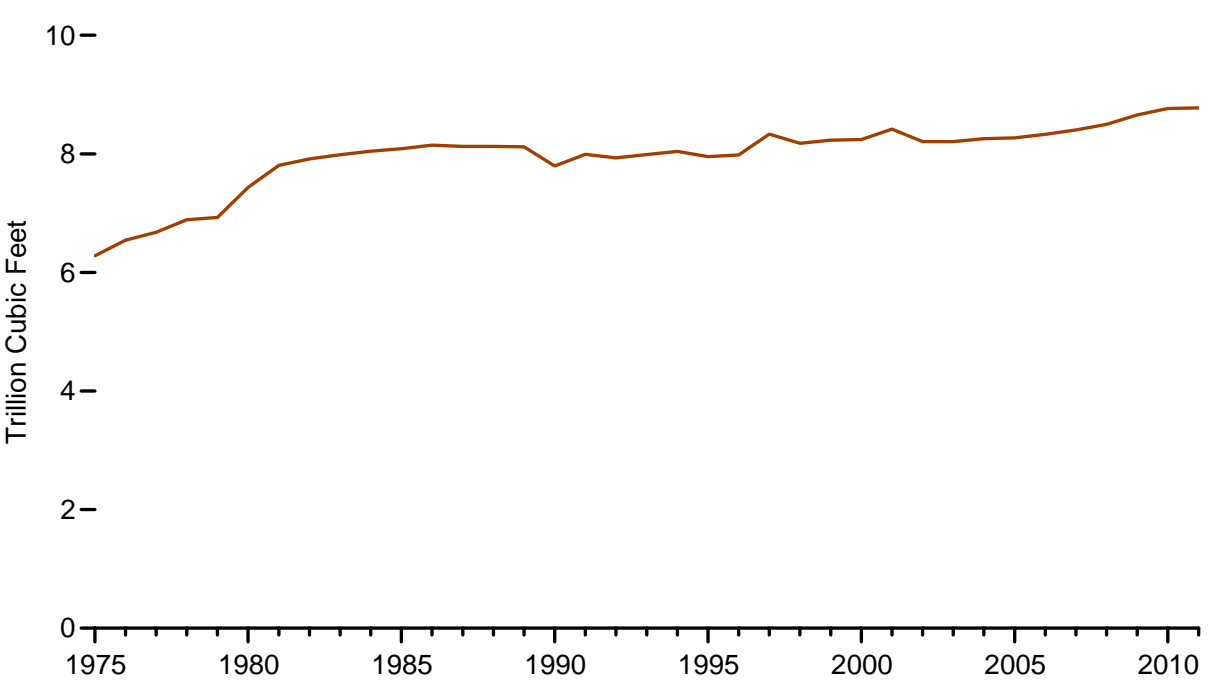

Base Gas and Working Gas in Underground Storage, 1954-2011

$5-$

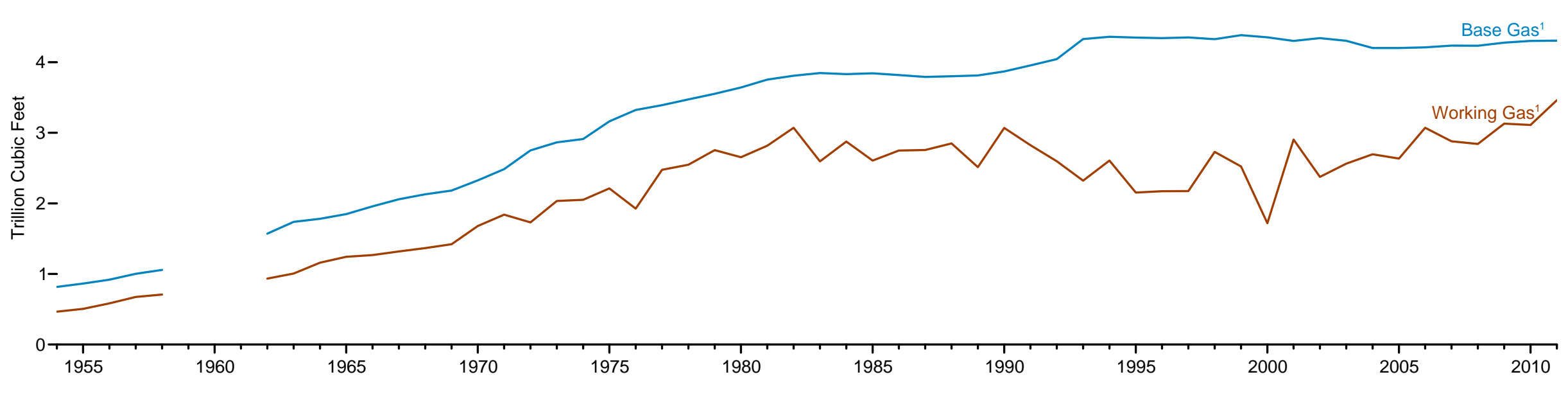

${ }^{1}$ Working-gas and base-gas data were not collected in 1959, 1960, and 1961. 
Table 6.6 Natural Gas Underground Storage, Selected Years, End of Year 1954-2011

(Billion Cubic Feet)

\begin{tabular}{|c|c|c|c|c|c|c|c|c|c|c|}
\hline \multirow[b]{3}{*}{ Year } & \multicolumn{9}{|c|}{ Natural Gas in Underground Storage } & \multirow{3}{*}{$\begin{array}{l}\text { Natural Gas } \\
\text { Underground } \\
\text { Storage } \\
\text { Capacity }\end{array}$} \\
\hline & \multicolumn{3}{|c|}{ Base Gas ${ }^{1}$} & \multicolumn{3}{|c|}{ Working Gas } & \multicolumn{3}{|c|}{ Total } & \\
\hline & Salt Caverns & $\begin{array}{l}\text { Other Than } \\
\text { Salt Caverns }\end{array}$ & Total & Salt Caverns & $\begin{array}{l}\text { Other Than } \\
\text { Salt Caverns }\end{array}$ & Total & Salt Caverns & $\begin{array}{l}\text { Other Than } \\
\text { Salt Caverns }\end{array}$ & Total & \\
\hline 1954 & NA & NA & 817 & NA & NA & 465 & NA & NA & 1,281 & NA \\
\hline 1955 & NA & NA & 863 & NA & NA & 505 & NA & NA & $\begin{array}{l}1,281 \\
1,368\end{array}$ & NA \\
\hline 1960 & NA & NA & NA & NA & NA & NA & NA & NA & 2,184 & NA \\
\hline 1961 & NA & NA & NA & NA & NA & NA & NA & NA & 2,344 & NA \\
\hline 1962 & NA & NA & 1,571 & NA & NA & 933 & NA & NA & 2,504 & NA \\
\hline 1963 & NA & NA & 1,738 & NA & NA & 1,007 & NA & NA & 2,745 & NA \\
\hline $\begin{array}{l}1964 \\
1965\end{array}$ & $\begin{array}{l}\text { NA } \\
\text { NA }\end{array}$ & NA & $\begin{array}{l}1,781 \\
1,848\end{array}$ & NA & NA & $\begin{array}{r}1,159 \\
1242\end{array}$ & $\begin{array}{l}\text { NA } \\
\text { NA }\end{array}$ & $\begin{array}{l}N A \\
N A\end{array}$ & $\begin{array}{l}2,940 \\
3\end{array}$ & $\begin{array}{l}\text { NA } \\
N A\end{array}$ \\
\hline $\begin{array}{l}1965 \\
1966\end{array}$ & NA & NA & $\begin{array}{l}1,848 \\
1,958\end{array}$ & NA & NA & $\begin{array}{l}1,242 \\
1,267\end{array}$ & $\begin{array}{l}\text { NA } \\
\text { NA }\end{array}$ & NA & $\begin{array}{l}3,090 \\
3,225\end{array}$ & NA \\
\hline 1967 & NA & NA & $\begin{array}{r}1,958 \\
2,058\end{array}$ & NA & NA & $\begin{array}{l}1,267 \\
1,318\end{array}$ & NA & NA & $\begin{array}{l}3,225 \\
3,376\end{array}$ & NA \\
\hline 1968 & NA & NA & $\begin{array}{l}2,050 \\
2,128\end{array}$ & NA & NA & $\begin{array}{l}1,318 \\
\end{array}$ & NA & NA & $\begin{array}{l}3,370 \\
3,495\end{array}$ & NA \\
\hline 1969 & NA & $\mathrm{NA}$ & 2,181 & NA & NA & 1,421 & NA & NA & 3,602 & NA \\
\hline 1970 & NA & NA & 2,326 & NA & NA & $\begin{array}{l}1,6<1 \\
\end{array}$ & NA & NA & $\begin{array}{l}3,000 \\
4,004\end{array}$ & NA \\
\hline 1971 & NA & NA & 2,485 & NA & NA & $\begin{array}{l}1,840 \\
\end{array}$ & NA & NA & $\begin{array}{r}4,325 \\
4,32\end{array}$ & NA \\
\hline 1972 & NA & $\mathrm{NA}$ & 2,751 & NA & NA & 1,729 & NA & NA & 4,480 & NA \\
\hline 1973 & NA & NA & 2,864 & NA & & 2,034 & $\mathrm{NA}$ & NA & 4,898 & NA \\
\hline 1974 & NA & NA & 2,912 & NA & NA & 2,050 & NA & NA & 4,962 & NA \\
\hline 1975 & NA & NA & 3,162 & NA & NA & 2,212 & NA & NA & 5,374 & 6,280 \\
\hline 1976 & NA & NA & 3,323 & NA & NA & 1,926 & NA & NA & 5,250 & 6,544 \\
\hline 1977 & NA & NA & 3,391 & NA & NA & 2,475 & NA & NA & 5,866 & 6,678 \\
\hline 1978 & NA & NA & 3,473 & NA & NA & 2,547 & NA & NA & 6,020 & 6,890 \\
\hline $\begin{array}{l}1979 \\
1980\end{array}$ & NA & NA & $\begin{array}{l}3,553 \\
3642\end{array}$ & NA & NA & $\begin{array}{l}2,753 \\
\end{array} 655$ & NA & NA & 6,306 & 6,929 \\
\hline $\begin{array}{l}1980 \\
1981\end{array}$ & $\begin{array}{l}\text { NA } \\
\text { NA }\end{array}$ & $\begin{array}{l}\text { NA } \\
\text { NA }\end{array}$ & $\begin{array}{l}3,642 \\
3,752\end{array}$ & $\begin{array}{l}\text { NA } \\
\text { NA }\end{array}$ & $\begin{array}{l}\text { NA } \\
\text { NA }\end{array}$ & $\begin{array}{l}2,6555 \\
2,817\end{array}$ & $\begin{array}{l}\text { NA } \\
\text { NA }\end{array}$ & $\begin{array}{l}\text { NA } \\
\text { NA }\end{array}$ & $\begin{array}{l}6,296 \\
6,569\end{array}$ & $\begin{array}{l}7,434 \\
7,805\end{array}$ \\
\hline 1982 & NA & NA & 3,808 & NA & NA & $\begin{array}{l}2,817 \\
3,071\end{array}$ & NA & NA & $\begin{array}{l}0,560 \\
6,879\end{array}$ & $\begin{array}{l}1,805 \\
7,915\end{array}$ \\
\hline 1983 & NA & NA & 3,847 & NA & NA & $\begin{array}{l}3,595 \\
2,50\end{array}$ & NA & NA & $\begin{array}{l}0,871 \\
6,442\end{array}$ & $\begin{array}{l}7,915 \\
7,985\end{array}$ \\
\hline 1984 & NA & $\mathrm{NA}$ & 3,830 & NA & NA & $\begin{array}{l}2,0956 \\
\end{array}$ & NA & NA & $\begin{array}{l}0,442 \\
6,706\end{array}$ & 8,043 \\
\hline 1985 & NA & $\mathrm{NA}$ & 3,842 & NA & NA & 2,607 & NA & NA & 6,448 & 8,087 \\
\hline 1986 & NA & NA & 3,819 & NA & NA & $\begin{array}{r}2,747 \\
2,740\end{array}$ & NA & NA & $\begin{array}{l}0,440 \\
6,567\end{array}$ & $\begin{array}{l}8,017 \\
8,145\end{array}$ \\
\hline 1987 & NA & NA & 3,792 & NA & NA & 2,756 & NA & NA & 6,548 & 8,124 \\
\hline 1988 & NA & NA & 3,800 & NA & NA & 2,850 & NA & NA & 6,650 & 8,124 \\
\hline 1989 & NA & NA & 3,812 & NA & NA & 2,513 & NA & NA & 6,325 & 8,120 \\
\hline 1990 & NA & NA & 3,868 & NA & NA & 3,068 & NA & NA & 6,936 & 7,794 \\
\hline 1991 & NA & NA & 3,954 & NA & NA & 2,824 & NA & NA & 6,778 & 7,993 \\
\hline 1992 & NA & NA & 4,044 & NA & NA & 2,597 & NA & NA & 6,641 & 7,932 \\
\hline 1993 & NA & NA & 4,327 & NA & NA & 2,322 & NA & NA & 6,649 & 7,989 \\
\hline $\begin{array}{l}1994 \\
1995\end{array}$ & 44 & 4,317 & 4,360 & 70 & 2,536 & 2,606 & 113 & 6,853 & 6,966 & 8,043 \\
\hline $\begin{array}{l}1995 \\
1996\end{array}$ & $\begin{array}{l}60 \\
64\end{array}$ & $\begin{array}{l}4,290 \\
4,27\end{array}$ & $\begin{array}{l}4,349 \\
4,341\end{array}$ & $\begin{array}{l}72 \\
85\end{array}$ & $\begin{array}{l}2,082 \\
2087\end{array}$ & $\begin{array}{r}2,153 \\
2,173\end{array}$ & $\begin{array}{l}131 \\
149\end{array}$ & $\begin{array}{l}6,371 \\
6364\end{array}$ & $\begin{array}{r}6,503 \\
6\end{array}$ & 7,953 \\
\hline 1997 & $\begin{array}{l}64 \\
67\end{array}$ & $\begin{array}{l}4,283 \\
4,283\end{array}$ & $\begin{array}{l}4,341 \\
4,350\end{array}$ & $\begin{array}{l}85 \\
83\end{array}$ & $\begin{array}{l}2,087 \\
2,092\end{array}$ & $\begin{array}{l}2,175 \\
2,175\end{array}$ & $\begin{array}{l}149 \\
150\end{array}$ & $\begin{array}{l}\text {,3,364 } \\
6,375\end{array}$ & $\begin{array}{l}0,51 \\
6,525\end{array}$ & $\begin{array}{l}1,980 \\
8,332\end{array}$ \\
\hline 1998 & 67 & $\begin{array}{l}4,200 \\
4,259\end{array}$ & 4,326 & $\begin{array}{l}00 \\
104\end{array}$ & $\begin{array}{l}2,092 \\
2,626\end{array}$ & $\begin{array}{l}2,175 \\
2,730\end{array}$ & 171 & $\begin{array}{l}6,384 \\
6,884\end{array}$ & $\begin{array}{l}0,525 \\
7,056\end{array}$ & $\begin{array}{l}8,352 \\
8,179\end{array}$ \\
\hline 1999 & 69 & 4,314 & 4,383 & 100 & $\begin{array}{l}2,0<0 \\
2,423\end{array}$ & $\begin{array}{l}2,730 \\
2,523\end{array}$ & 169 & $\begin{array}{l}0,04 \\
6,738\end{array}$ & $\begin{array}{l}1,050 \\
6,906\end{array}$ & 8,229 \\
\hline 2000 & 70 & 4,282 & 4,352 & 72 & $\begin{array}{l}<, 4<0 \\
1,647\end{array}$ & $\begin{array}{l}2,0<0 \\
1,719\end{array}$ & 142 & 5,929 & $\begin{array}{l}0,9071 \\
6,071\end{array}$ & $\begin{array}{l}0,249 \\
8,241\end{array}$ \\
\hline 2001 & 77 & 4,224 & 4,301 & 115 & $\begin{array}{r}1,041 \\
2,789\end{array}$ & 2,904 & 191 & 7,013 & 7,204 & $\begin{array}{l}0,415 \\
8,415\end{array}$ \\
\hline 2002 & 75 & 4,265 & 4,340 & 102 & 2,273 & 2,375 & 177 & 6,539 & 6,715 & 8,207 \\
\hline 2003 & 76 & 4,227 & 4,303 & 125 & 2,438 & 2,563 & 201 & 6,665 & 6,866 & 8,206 \\
\hline 2004 & 72 & 4,129 & 4,201 & 98 & 2,598 & 2,696 & 170 & 6,727 & 6,897 & 8,255 \\
\hline 2005 & 78 & 4,122 & 4,200 & 123 & 2,513 & 2,635 & 201 & 6,635 & 6,835 & 8,268 \\
\hline 2006 & 77 & 4,134 & 4,211 & 144 & 2,926 & 3,070 & 222 & 7,059 & 7,281 & 8,330 \\
\hline 2007 & 80 & 4,154 & 4,234 & 123 & 2,756 & 2,879 & 203 & 6,910 & 7,113 & 8,402 \\
\hline 2008 & 86 & 4,146 & 4,232 & 154 & 2,686 & 2,840 & 240 & 6,832 & 7,073 & 8,499 \\
\hline $\begin{array}{l}2009 \\
2010\end{array}$ & $\begin{array}{l}116 \\
135\end{array}$ & $\begin{array}{r}4,161 \\
R_{4} 166\end{array}$ & $\begin{array}{r}4,277 \\
R_{4}, 301\end{array}$ & $\begin{array}{r}186 \\
R 220\end{array}$ & $\begin{array}{r}2,944 \\
R_{2}^{2} 891\end{array}$ & $\begin{array}{r}3,130 \\
R_{3}, 111\end{array}$ & $\begin{array}{r}302 \\
R_{355}\end{array}$ & $\begin{array}{r}7,105 \\
\text { R7 }\end{array}$ & $\begin{array}{l}7,407 \\
7412\end{array}$ & $\begin{array}{r}8,656 \\
R 8764\end{array}$ \\
\hline $2011^{P}$ & 142 & $\begin{array}{r}4,163 \\
4\end{array}$ & $\begin{array}{r}4,305 \\
4,305\end{array}$ & 308 & 3,154 & 3,462 & 451 & 7,317 & 7,767 & 8,776 \\
\hline
\end{tabular}

1 Includes native gas.

Depleted fields, aquifers, and other types of storage not using salt formations

Includes both active and inactive fields.

Notes: - Beginning with 1965 , all volumes are shown on a pressure base of $14.73 \mathrm{ps}$. .a. at $60^{\circ} \mathrm{F}$. For prior years, the pressure base was 14.65 p.s.i.a. at $60^{\circ} \mathrm{F}$. - Totals may not equal sum of components due

to independent rounding.

1954. - See http://www.eia.gov/naturalgas/ for related information.
Sources: - 1954-1974-American Gas Association, Gas Facts. - 1975-1978-Federal Energy Administration, Form FEA-G318-M-O, "Underground "Gas Storage Report," and Federal Powe Administration (EIA), Form EIA-191, "Underground Gas Storage Report," and Federal Energy Regulatory Commission, Form FERC-8, "Underground Gas Storage Report." 1985-2009-EIA, Natural Gas Monthly (NGM), monthly reports, and Natural Gas Annual, annual reports. - 2010 and 2011 - EIA, NGM (March 2012), Tables 8, 10, and 11, and Form EIA-191M, "Monthly Underground Gas Storage Report." 
Figure 6.7 Natural Gas Wellhead, Citygate, and Imports Prices

Wellhead, Citygate, and Imports, 2011

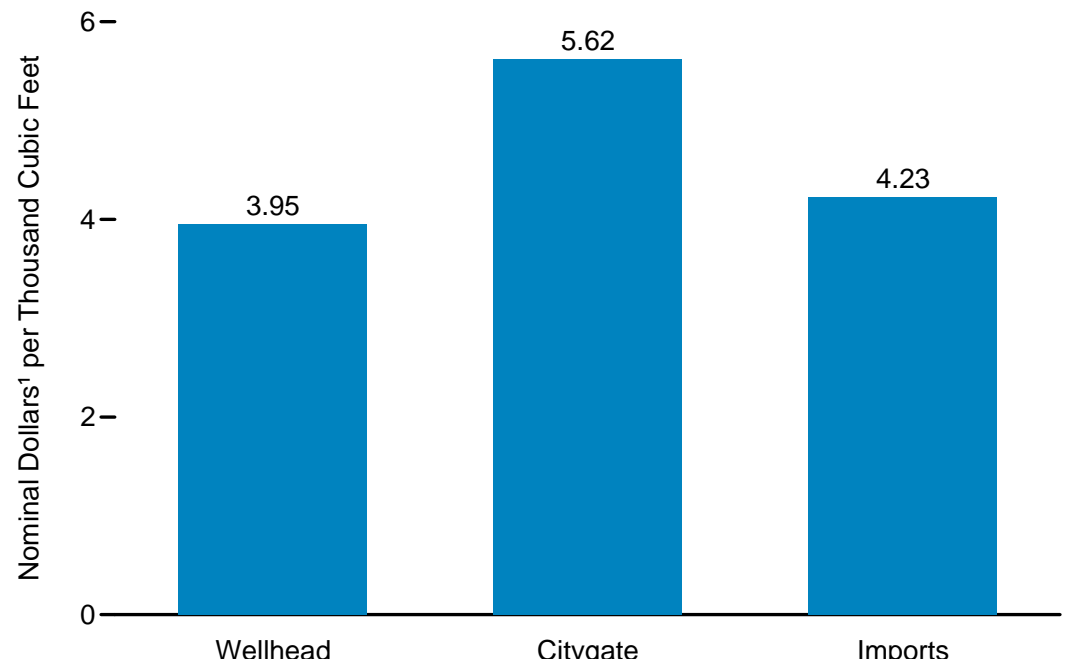

Wellhead, 1949-2011

10-
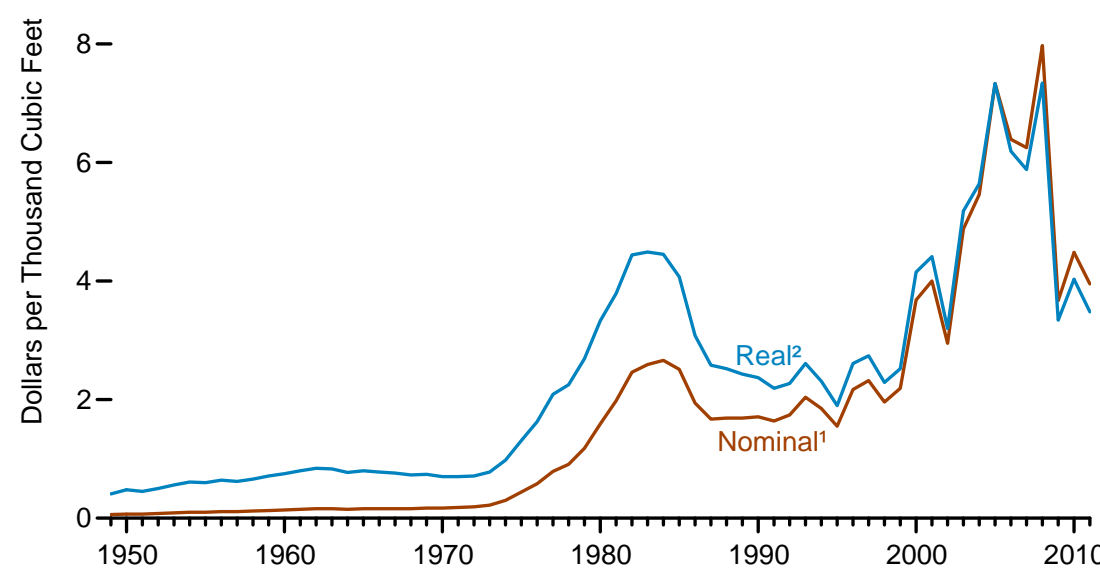

Wellhead, Citygate, and Imports, 1949-2011

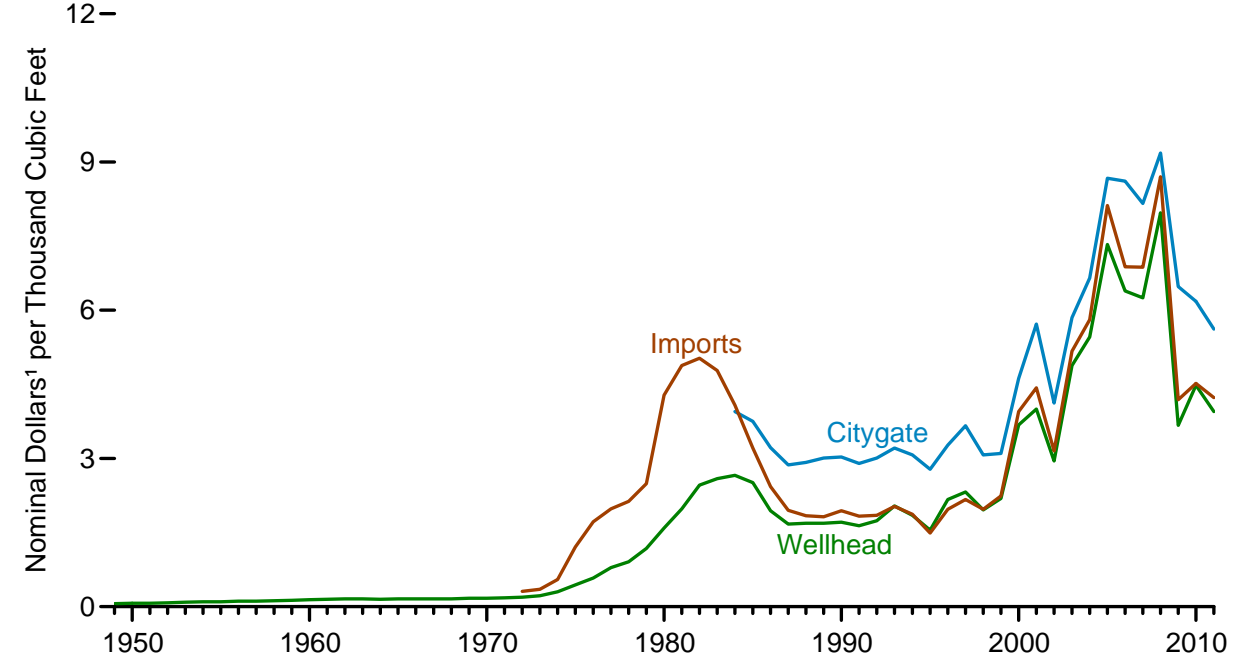

Imports, 1972-2011

12-

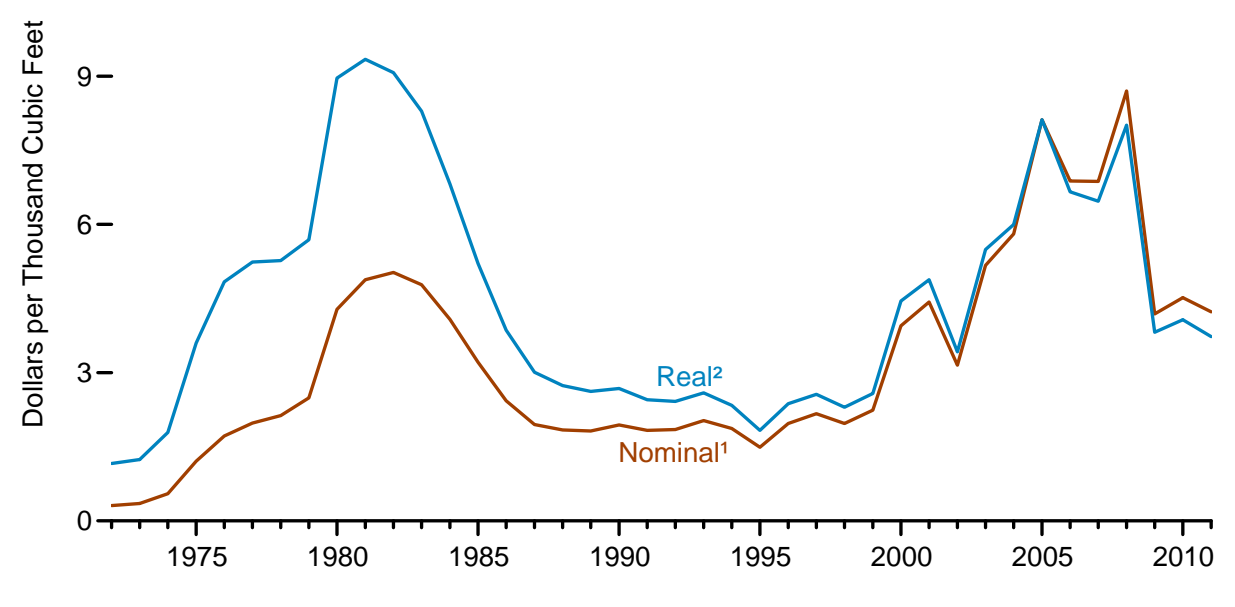

${ }^{1}$ See "Nominal Dollars" in Glossary.

2 In chained (2005) dollars, calculated by using gross domestic product implicit price deflators

Source: Table 6.7. 
Table 6.7 Natural Gas Wellhead, Citygate, and Imports Prices, Selected Years, 1949-2011

(Dollars per Thousand Cubic Feet)

\begin{tabular}{|c|c|c|c|c|c|c|}
\hline \multirow[b]{2}{*}{ Year } & \multicolumn{2}{|c|}{ Wellhead ${ }^{1}$} & \multicolumn{2}{|c|}{ Citygate $^{2}$} & \multicolumn{2}{|c|}{ Imports } \\
\hline & Nominal $^{3}$ & Real 4 & Nominal $^{3}$ & Real 4 & Nominal $^{3}$ & Real 4 \\
\hline 1949 & 0.06 & 0.41 & NA & NA & NA & NA \\
\hline 1950 & .07 & .48 & NA & NA & NA & NA \\
\hline 1955 & .10 & .60 & NA & NA & NA & NA \\
\hline 1960 & .14 & .75 & NA & NA & NA & NA \\
\hline 1965 & .16 & .80 & NA & NA & NA & NA \\
\hline 1970 & .17 & .70 & NA & NA & NA & NA \\
\hline 1975 & .44 & 1.31 & NA & NA & 1.21 & $R_{3} .60$ \\
\hline 1976 & .58 & 1.63 & NA & NA & 1.72 & $\mathrm{R}_{4.84}$ \\
\hline 1977 & .79 & 2.09 & NA & NA & 1.98 & 5.24 \\
\hline 1978 & .91 & 2.25 & NA & NA & 2.13 & 5.27 \\
\hline 1979 & 1.18 & $\mathrm{R}_{2.69}$ & NA & NA & 2.49 & 5.69 \\
\hline 1980 & 1.59 & 3.33 & NA & NA & 4.28 & 8.96 \\
\hline 1981 & 1.98 & 3.79 & NA & NA & 4.88 & 9.34 \\
\hline 1982 & 2.46 & 4.44 & NA & NA & 5.03 & R9.07 \\
\hline 1983 & 2.59 & $\mathrm{R}_{4} 4.49$ & NA & NA & 4.78 & R8.29 \\
\hline 1984 & 2.66 & 4.45 & 3.95 & $R_{6.60}$ & 4.08 & $\mathrm{R}_{6.82}$ \\
\hline 1985 & 2.51 & $\mathrm{R}_{4} 4.07$ & 3.75 & $\mathrm{R}_{6.08}$ & 3.21 & 5.21 \\
\hline 1986 & 1.94 & 3.08 & 3.22 & $\mathrm{R}_{5.11}$ & 2.43 & 3.86 \\
\hline 1987 & 1.67 & 2.58 & 2.87 & 4.43 & 1.95 & 3.01 \\
\hline 1988 & 1.69 & 2.52 & 2.92 & 4.36 & 1.84 & $\mathrm{R}_{2.74}$ \\
\hline 1989 & 1.69 & 2.43 & 3.01 & 4.33 & 1.82 & 2.62 \\
\hline 1990 & 1.71 & 2.37 & 3.03 & $\mathrm{R}_{4.19}$ & 1.94 & $\mathrm{R}_{2.68}$ \\
\hline 1991 & 1.64 & 2.19 & 2.90 & 3.88 & 1.83 & 2.45 \\
\hline 1992 & 1.74 & 2.27 & 3.01 & 3.93 & 1.85 & 2.42 \\
\hline 1993 & 2.04 & 2.61 & 3.21 & 4.10 & 2.03 & $R_{2.59}$ \\
\hline 1994 & 1.85 & $\mathrm{R}_{2.31}$ & 3.07 & 3.84 & 1.87 & 2.34 \\
\hline 1995 & 1.55 & 1.90 & 2.78 & 3.41 & 1.49 & 1.83 \\
\hline 1996 & 2.17 & 2.61 & 3.27 & $R_{3.93}$ & 1.97 & 2.37 \\
\hline 1997 & 2.32 & 2.74 & 3.66 & $\mathrm{R}_{4} 4.32$ & 2.17 & $\mathrm{R}_{2.56}$ \\
\hline 1998 & 1.96 & 2.29 & 3.07 & 3.59 & 1.97 & 2.30 \\
\hline 1999 & 2.19 & 2.52 & 3.10 & 3.57 & 2.24 & 2.58 \\
\hline 2000 & 3.68 & 4.15 & 4.62 & 5.21 & 3.95 & $\mathrm{R}_{4} .45$ \\
\hline 2001 & 4.00 & 4.41 & 5.72 & $\mathrm{R}_{6.30}$ & 4.43 & $\mathrm{R}_{4} 4.88$ \\
\hline 2002 & 2.95 & 3.20 & 4.12 & 4.47 & 3.15 & 3.42 \\
\hline 2003 & 4.88 & $\mathrm{R}_{5.18}$ & 5.85 & $\mathrm{R}_{6.21}$ & 5.17 & 5.49 \\
\hline 2004 & 5.46 & 5.64 & 6.65 & 6.87 & 5.81 & 6.00 \\
\hline 2005 & 7.33 & 7.33 & 8.67 & 8.67 & 8.12 & 8.12 \\
\hline 2006 & 6.39 & 6.19 & 8.61 & 8.34 & 6.88 & 6.66 \\
\hline 2007 & 6.25 & 5.88 & 8.16 & 7.68 & 6.87 & $\mathrm{R}_{6.47}$ \\
\hline 2008 & 7.97 & 7.34 & 9.18 & 8.45 & 8.70 & 8.01 \\
\hline 2009 & 3.67 & 3.34 & $\mathrm{R}_{6.48}$ & R5.91 & 4.19 & 3.82 \\
\hline 2010 & $\mathrm{R}_{4.48}$ & $\mathrm{R}_{4.04}$ & $\mathrm{R}_{6.18}$ & 5.57 & 4.52 & $\mathrm{R}_{4.07}$ \\
\hline 2011 & E3.95 & E3.48 & ${ }^{P} 5.62$ & P4.96 & $\mathrm{P}_{4} 4.23$ & $P_{3.73}$ \\
\hline
\end{tabular}

1 See "Natural Gas Wellhead Price" in Glossary.

See "Citygate" in Glossary.

See "Nominal Dollars" in Glossary.

${ }^{4}$ In chained (2005) dollars, calculated by using gross domestic product implicit price deflators in Table D1. See "Chained Dollars" in Glossary.

R=Revised. $P=$ Preliminary. $E=$ Estimate. $N A=$ Not available Web Pages: - See http://www.eia.gov/totalenergy/data/monthly/\#prices for updated monthly and annual
data. - See http://www.eia.gov/totalenergy/data/annual/\#naturalgas for all annual data beginning in 1949.
- See http://www.eia.gov/naturalgas/ for related information. Gas Annual (NGA), annual reports. - 2007 forward-EIA, Natural Gas Monthly (NGM) (March 2012), Table 3. Imports: - 1972 and 1973-Federal Power Commission (FPC), Pipeline Imports and Exports of Natural Gas-Imports and Exports of LNG. • 1974-1976-FPC, Uniled Slates Imports and Exports of Natural Gas, annual reports. • 1977-2009-EIA, NGA, annual reports. • 2010 and 2011-EIA, NGM (March 2012), Tables 3 and 4 . 


\section{Figure 6.8 Natural Gas Prices by Sector}

\section{Nominal ${ }^{1}$ Prices, 2011}

$12-$

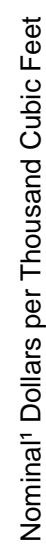
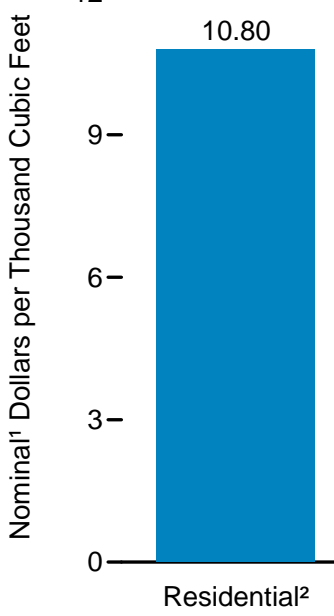

(1)

Commercial $^{3}$

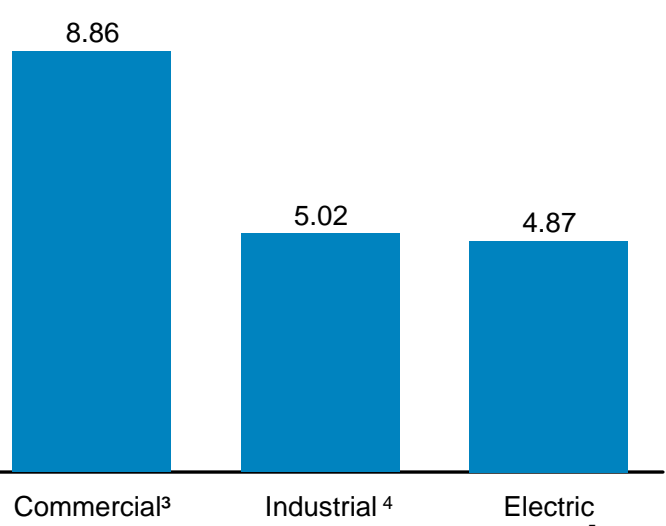

Industrial 4

\section{Real ${ }^{6}$ Prices, Indexed, 1980-2011}

$2.00-$

1.50- - Electric Power
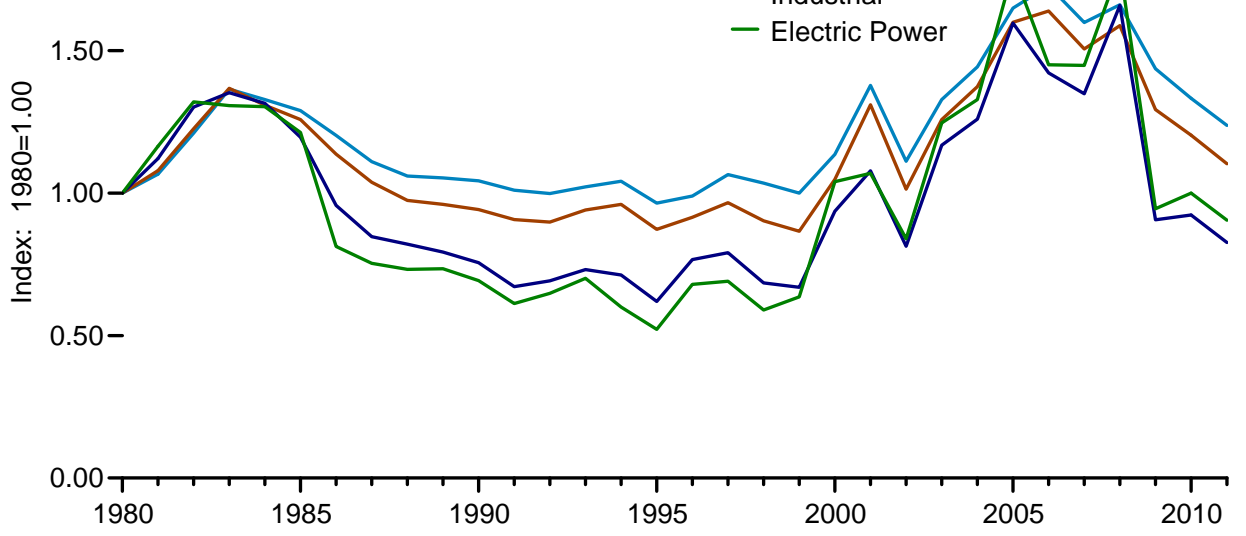

$0.50-$

- Residential

- Commercial

- Industrial

\section{Real ${ }^{6}$ Prices, 1967-2011}

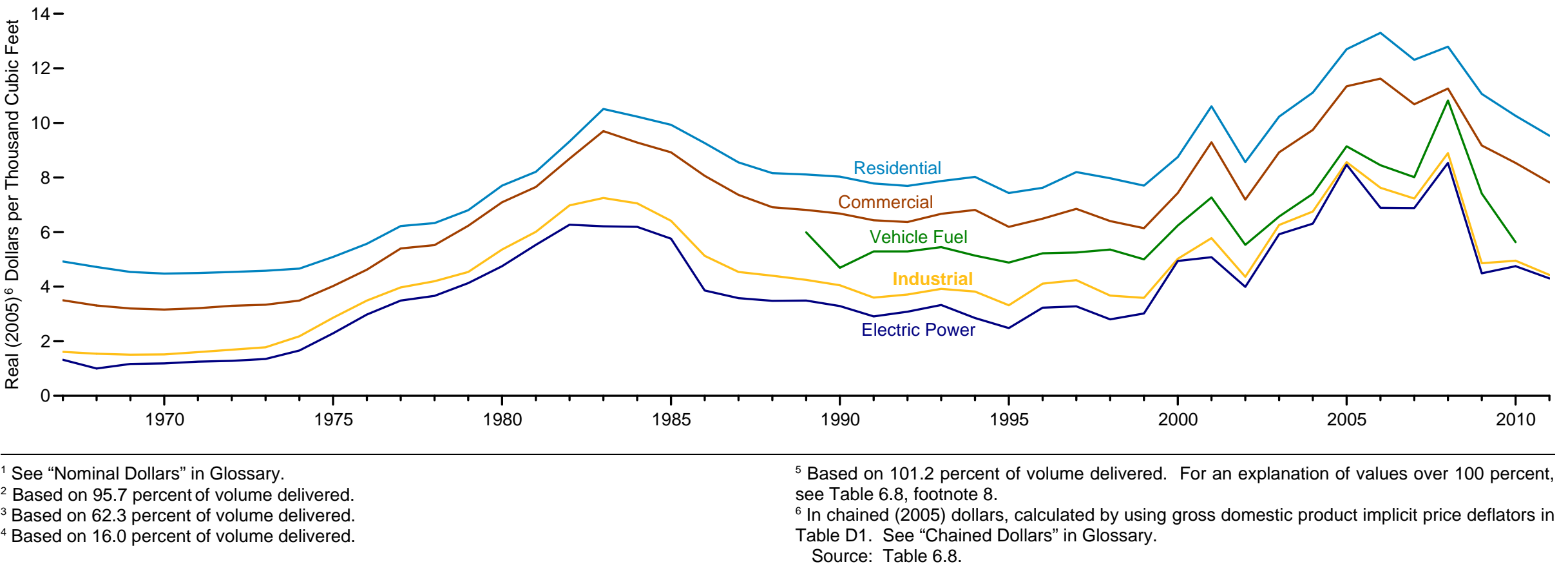

Electric
Power $^{5}$

Power 
Table 6.8 Natural Gas Prices by Sector, Selected Years, 1967-2011

(Dollars per Thousand Cubic Feet, Except as Noted)

\begin{tabular}{|c|c|c|c|c|c|c|c|c|c|c|c|c|c|c|}
\hline \multirow[b]{3}{*}{ Year } & \multicolumn{3}{|c|}{ Residential Sector } & \multicolumn{3}{|c|}{ Commercial Sector ${ }^{1}$} & \multicolumn{3}{|c|}{ Industrial Sector ${ }^{2}$} & \multirow{2}{*}{\multicolumn{2}{|c|}{$\begin{array}{c}\text { Transportation Sector } \\
\text { Vehicle Fuel }{ }^{4} \text { Prices }\end{array}$}} & \multicolumn{3}{|c|}{ Electric Power Sector ${ }^{3}$} \\
\hline & \multicolumn{2}{|c|}{ Prices } & \multirow{2}{*}{$\begin{array}{l}\text { Percentage } \\
\text { of } \\
\text { Sector }{ }^{7}\end{array}$} & \multicolumn{2}{|c|}{ Prices } & \multirow{2}{*}{$\begin{array}{c}\text { Percentage } \\
\text { of } \\
\text { Sector }{ }^{7}\end{array}$} & \multicolumn{2}{|c|}{ Prices } & \multirow{2}{*}{$\begin{array}{c}\text { Percentage } \\
\text { of } \\
\text { Sector } 7\end{array}$} & & & \multicolumn{2}{|c|}{ Prices } & \multirow{2}{*}{$\begin{array}{c}\text { Percentage } \\
\text { of } \\
\text { Sector } 7,8\end{array}$} \\
\hline & Nominal 5 & Real 6 & & Nominal 5 & Real 6 & & Nominal 5 & Real ${ }^{6}$ & & Nominal 5 & Real 6 & Nominal 5 & Real 6 & \\
\hline 1967 & 1.04 & R4.92 & NA & 0.74 & 3.50 & NA & 0.34 & 1.61 & NA & NA & NA & 0.28 & $\mathrm{R}_{1.32}$ & NA \\
\hline 1970 & $\begin{array}{l}1.04 \\
1.09\end{array}$ & 4.48 & NA & 0.77 & R3.16 & NA & .37 & $\begin{array}{l}1.01 \\
1.52\end{array}$ & NA & NA & NA & $\begin{array}{l}0.20 \\
.29\end{array}$ & 1.19 & NA \\
\hline 1975 & 1.71 & $\begin{array}{l}4.40 \\
5.09\end{array}$ & NA & 1.35 & $\begin{array}{l}4.10 \\
4.02\end{array}$ & NA & $\begin{array}{l}.51 \\
.96\end{array}$ & 2.86 & NA & NA & NA & .77 & 2.29 & 96.1 \\
\hline 1976 & 1.98 & R5.57 & NA & 1.64 & 4.62 & NA & 1.24 & 3.49 & NA & NA & NA & 1.06 & $\mathrm{R} 2.98$ & 96.2 \\
\hline 1977 & 2.35 & R6.22 & NA & 2.04 & 5.40 & NA & 1.50 & 3.97 & NA & NA & NA & 1.32 & R3.49 & $\begin{array}{l}96.2 \\
97.1\end{array}$ \\
\hline 1978 & $\begin{array}{l}2.35 \\
2.56\end{array}$ & $\begin{array}{r}.22 \\
\mathrm{R} 6.33\end{array}$ & NA & $\begin{array}{l}2.04 \\
2.23\end{array}$ & $\begin{array}{l}5.40 \\
5.52\end{array}$ & NA & $\begin{array}{l}1.50 \\
1.70\end{array}$ & $\begin{array}{r}3.97 \\
R 4.20\end{array}$ & NA & NA & NA & $\begin{array}{l}1.32 \\
1.48\end{array}$ & 3.66 & 98.0 \\
\hline 1979 & 2.98 & R6.80 & NA & 2.73 & R6.23 & NA & $\begin{array}{l}1.99 \\
1.96\end{array}$ & $\mathrm{R}_{4} .54$ & NA & NA & NA & 1.81 & $\begin{array}{r}r .00 \\
R 4.13\end{array}$ & 96.1 \\
\hline 1980 & 3.68 & R7.70 & NA & 3.39 & R7.09 & NA & 2.56 & 5.36 & NA & NA & NA & 2.27 & 4.75 & 96.9 \\
\hline 1981 & 4.29 & 8.21 & NA & 4.00 & R7.65 & NA & 3.14 & 6.01 & NA & NA & NA & 2.89 & 5.53 & 97.6 \\
\hline 1982 & 5.17 & R9.32 & NA & 4.82 & R8.69 & NA & 3.87 & 6.98 & 85.1 & NA & NA & 3.48 & R6.27 & 92.6 \\
\hline 1983 & 6.06 & $\mathrm{R}_{10.51}$ & NA & 5.59 & 9.70 & NA & 4.18 & R7.25 & 80.7 & NA & NA & 3.58 & 6.21 & 93.9 \\
\hline 1984 & 6.12 & $\mathrm{R}_{10.23}$ & NA & 5.55 & R9.28 & NA & 4.22 & R7.05 & 74.7 & NA & NA & 3.70 & 6.19 & 94.4 \\
\hline 1985 & 6.12 & R9. 93 & NA & 5.50 & R8.92 & NA & 3.95 & 6.41 & 68.8 & NA & NA & 3.55 & R5.76 & 94.0 \\
\hline 1986 & 5.83 & 9.26 & NA & 5.08 & R8.06 & NA & 3.23 & 5.13 & 59.8 & NA & NA & 2.43 & 3.86 & 91.7 \\
\hline 1987 & 5.54 & 8.55 & NA & 4.77 & R7.36 & 93.1 & 2.94 & 4.54 & 47.4 & NA & NA & 2.32 & 3.58 & 91.6 \\
\hline 1988 & 5.47 & R8.16 & NA & 4.63 & 6.91 & 90.7 & 2.95 & 4.40 & 42.6 & NA & NA & 2.33 & 3.48 & 89.6 \\
\hline 1989 & 5.64 & 8.11 & 99.9 & 4.74 & R6.81 & 89.1 & 2.96 & R4.25 & 36.9 & 4.17 & R5.99 & 2.43 & R3.49 & 79.6 \\
\hline 1990 & 5.80 & 8.03 & 99.2 & 4.83 & R6.68 & 86.6 & 2.93 & R4.05 & 35.2 & 3.39 & R4.69 & 2.38 & R3.29 & 76.8 \\
\hline 1991 & 5.82 & 7.78 & 99.2 & 4.81 & 6.43 & 85.1 & 2.69 & 3.60 & 32.7 & 3.96 & R5.29 & 2.18 & R2.91 & 79.3 \\
\hline 1992 & 5.89 & R7.69 & 99.1 & 4.88 & R6.37 & 83.2 & 2.84 & 3.71 & 30.3 & 4.05 & 5.29 & 2.36 & 3.08 & 76.5 \\
\hline 1993 & 6.16 & 7.87 & 99.1 & 5.22 & 6.67 & 83.9 & 3.07 & 3.92 & 29.7 & 4.27 & R5.45 & 2.61 & R3.33 & 74.1 \\
\hline 1994 & 6.41 & R8.02 & 99.1 & 5.44 & 6.81 & 79.3 & 3.05 & 3.82 & 25.5 & 4.11 & R5.14 & 2.28 & 2.85 & 73.4 \\
\hline 1995 & 6.06 & 7.43 & 99.0 & 5.05 & 6.19 & 76.7 & 2.71 & 3.32 & 24.5 & 3.98 & 4.88 & 2.02 & 2.48 & 71.4 \\
\hline 1996 & 6.34 & R7.62 & 99.0 & 5.40 & R6.49 & 77.6 & 3.42 & R4.11 & 19.4 & 4.34 & $\begin{array}{l}5.00 \\
5.22\end{array}$ & 2.69 & R3.23 & 68.4 \\
\hline 1997 & 6.94 & R8.20 & 98.8 & 5.80 & R6.85 & 70.8 & $\begin{array}{l}3.42 \\
3.59\end{array}$ & R4.24 & 18.1 & $\begin{array}{l}4.04 \\
4.44\end{array}$ & 5.25 & 2.78 & R3.28 & $\begin{array}{l}60.4 \\
68.0\end{array}$ \\
\hline 1998 & 6.82 & R7.97 & 97.7 & 5.48 & R6.40 & 67.0 & $\begin{array}{l}3.14 \\
3.14\end{array}$ & 3.67 & $\begin{array}{l}16.1 \\
16.1\end{array}$ & $\begin{array}{l}4.44 \\
4.59\end{array}$ & R5.36 & 2.40 & $\mathrm{R}_{2.80}$ & $\begin{array}{l}60.0 \\
63.7\end{array}$ \\
\hline 1999 & 6.69 & R7.70 & 95.2 & 5.33 & 6.14 & 66.1 & $\begin{array}{l}3.14 \\
3.12\end{array}$ & $\begin{array}{r}5.07 \\
R 3.59\end{array}$ & $\begin{array}{l}18.1 \\
18.8\end{array}$ & $\begin{array}{l}4.39 \\
4.34\end{array}$ & 5.00 & $\begin{array}{l}2.40 \\
2.62\end{array}$ & 3.02 & 58.3 \\
\hline 2000 & 7.76 & 8.75 & 92.6 & 6.59 & 7.43 & 63.9 & 4.45 & 5.02 & $\begin{array}{l}19.0 \\
19.8\end{array}$ & $\begin{array}{l}4.54 \\
5.54\end{array}$ & $\begin{array}{r}5.00 \\
R 6.24\end{array}$ & $\begin{array}{l}2.02 \\
4.38\end{array}$ & $\begin{array}{l}3.02 \\
4.94\end{array}$ & $\begin{array}{l}50.5 \\
50.5\end{array}$ \\
\hline 2001 & 9.63 & $\begin{array}{r}R_{1} 0.61 \\
\end{array}$ & 92.4 & $\begin{array}{l}0.59 \\
8.43\end{array}$ & $\begin{array}{r}\text { R9. } \\
\text { R9.29 }\end{array}$ & $\begin{array}{l}0.9 \\
66.0\end{array}$ & $\begin{array}{l}4.45 \\
5.24\end{array}$ & 5.78 & 20.8 & $\begin{array}{l}5.34 \\
6.60\end{array}$ & $\begin{array}{l}0.24 \\
\text { R7.27 }\end{array}$ & $\begin{array}{l}4.00 \\
4.61\end{array}$ & $\begin{array}{r}4.94 \\
\text { R5.08 }\end{array}$ & 40.2 \\
\hline 2002 & 7.89 & R8.56 & 97.9 & $\begin{array}{l}0.630 \\
6.63\end{array}$ & R7.19 & 77.4 & $\begin{array}{l}. .02 \\
4.02\end{array}$ & 4.36 & 22.7 & 5.10 & R5.53 & 33.68 & $\begin{array}{l}33.99 \\
3\end{array}$ & $\begin{array}{l}383.9 \\
383.9\end{array}$ \\
\hline 2003 & 9.63 & 10.23 & 97.5 & 8.40 & R8.92 & 78.2 & 5.89 & 6.26 & 22.1 & 6.19 & R6.57 & 5.57 & 5.92 & 91.2 \\
\hline 2004 & 10.75 & 11.11 & 97.7 & 9.43 & 9.74 & 78.0 & 6.53 & 6.75 & R23.6 & 7.16 & 7.40 & 6.11 & 6.31 & 89.8 \\
\hline 2005 & 12.70 & 12.70 & R98.1 & 11.34 & 11.34 & 82.1 & 8.5 & 8.56 & R24.0 & 9.14 & 9.14 & 8.47 & 8.47 & 91.3 \\
\hline 2006 & 13.73 & 13.30 & 98.1 & 12.00 & 11.62 & 80.8 & 7.87 & 7.6 & 23.4 & 8.72 & 8.4 & 7.11 & 6.89 & 93.4 \\
\hline 2007 & 13.08 & 12.31 & 98.0 & 11.34 & R10.68 & 80.4 & 7.68 & 7.23 & 22.2 & 8.50 & R8.01 & 7.31 & 6.88 & 92.2 \\
\hline 2008 & 13.89 & 12.79 & 97.5 & 12.23 & 11.26 & 79.9 & 9.65 & R8.89 & 20.5 & 11.75 & 10.82 & 9.26 & 8.53 & 101.1 \\
\hline 2009 & $\begin{array}{r}12.14 \\
R_{11} .39\end{array}$ & $\begin{array}{l}\text { R11.06 } \\
\text { R10 }\end{array}$ & $\begin{array}{r}97.4 \\
R 97.4\end{array}$ & $\begin{array}{l}10.06 \\
09.17\end{array}$ & $\begin{array}{l}\text { R9.17 } \\
R 8.53\end{array}$ & $\begin{array}{r}77.8 \\
R 775\end{array}$ & $\begin{array}{r}5.33 \\
R 5.19\end{array}$ & $\begin{array}{r}4.86 \\
R 195\end{array}$ & $\begin{array}{l}18.8 \\
R 18\end{array}$ & 8.13 & $\begin{array}{r}7.41 \\
R 5.63\end{array}$ & 4.93 & R4.49 & 101.1 \\
\hline $\begin{array}{l}2010 \\
2011^{P}\end{array}$ & $\begin{array}{r}\mathrm{R} 11.39 \\
10.80\end{array}$ & $\begin{array}{r}{ }_{1}^{10.26} \\
9.53\end{array}$ & $\begin{array}{r}r 97.4 \\
95.7\end{array}$ & $\begin{array}{r}{ }^{9} 9.47 \\
8.86\end{array}$ & $\begin{array}{r}R 8.53 \\
7.82\end{array}$ & $\begin{array}{r}r 77.5 \\
62.3\end{array}$ & $\begin{array}{r}5.49 \\
5.02\end{array}$ & $\begin{array}{r}r_{4} 4.95 \\
4.43\end{array}$ & $\begin{array}{r}{ }^{R} 18.0 \\
16.0\end{array}$ & $\begin{array}{l}\mathrm{R}_{6} 6.25 \\
\text { NA }\end{array}$ & $\begin{array}{l}\text { R5.63 } \\
\text { NA }\end{array}$ & $\begin{array}{r}\text { R5.27 } \\
4.87\end{array}$ & $\begin{array}{l}4.75 \\
4.30\end{array}$ & $\begin{aligned} R_{1} 100.8 \\
1012\end{aligned}$ \\
\hline & & & & & & & & & & & & & & 101.2 \\
\hline
\end{tabular}

1 Commercial sector, including commercial combined-heat-and-power (CHP) and commercial electricity-only plants.
2 Industrial sector, including industrial combined-heat-and-power (CHP) and industrial electricity-only plants.

ectricity-only and combined-heat-and-power (CHP) plants within the NAICS 22 category whose primary business is to sell electricity, or electricity and heat, to the public. Through 2001, data are for electric utilities only; beginning in 2002 , data are for electric utilities and independent power producers. See (

stations that are used primarily or exclusively by fleet

See "Nominal Dollars" in Glossary.

In chained (2005) dollars, calculated by using gross domestic product implicit price deflators in Table D1. See "Chained Dollars" in Glossary.

8 The percentage of the sector's consumption in Table 6.5 for which price data are available. as consumption-this can occur when combined-heat-and-power (CHP) plants report fuel receipts related to non-electric generating activities.

$\mathrm{R}=$ Revised. P=Preliminary. $\mathrm{NA}=$ Not available.

Notes: - Prices are for natural gas, plus a small amount of supplemental gaseous fuels. - The averag

by the total quantity consumed. - Prices are intended to include all taxes. - See Note 2, "Classification of Web Pages: - See http://www.eia.gov/totalenergy/data/monthly/\#prices for updated monthly and annual data. - See http://www.eia.gov/totalenergy/data/annual/\#natur

- See http://www.eia.gov/naturalgas/ for related information. (EIA), Form EIA-176, "Annual Report of Natural and Supplemental Gas Supply and Disposition." - 2007 torward - EIA, Natural Gas Monthly (NGM) (March 2012), Table 3. Vehicle ruel: ElA, Natural Gas as the quantity of natural gas receipts by electric utilities reported on Form FERC-423, "Monthly Report of Cost and Quantity of Fuels for Electric Utility Plants" (and predecessor forms), divided by the quantity of natural gas consumed by the electric power sector (for 1973-1988, see Table 8.5b; for 1989-2001, see Table 8.7b). - 2002-2007-Calculated by EIA as the quantity of natural gas receipts by electric utilities and independent power producers reported on Forms FERC-423, "Monthly Report of Cost and Quantity of divided by the quantity of natural gas consumed by the electric power sector (see Table 8.76 ) . 2008 power producers reported on Form EIA-923, "Power Plant Operations Report," divided by the quantity of natural gas consumed by the electric power sector (see Table 8.7b). All Other Data: • 1967-2006-EIA,
NGA, annual reports. • 2007 forward-EIA, NGM (March 2012), Table 3. 


\section{Natural Gas}

Note 1. Supplemental Gaseous Fuels. Supplemental gaseous fuels are any substances that, introduced into or commingled with natural gas, increase the volume available for disposition. Such substances include, but are not limited to, propane-air, refinery gas, coke oven gas, still gas, manufactured gas, biomass gas, or air or inert gases added for British thermal unit (Btu) stabilization.

Annual data beginning with 1980 are from the U.S. Energy Information Administration (EIA), Natural Gas Annual. Unknown quantities of supplemental gaseous fuels are included in consumption data for 1979 and earlier years.

Although the total amount of supplemental gaseous fuels consumed is known for 1980 forward, the amount consumed by each energy-use sector is estimated by EIA These estimates are used to create natural gas (without supplemental gaseous fuels) data for Tables 1.3, 2.1b, 2.1c, 2.1d, and 2.1f (note: to avoid double-counting in these tables, supplemental gaseous fuels are accounted for in their primary energy category: "Coal," "Petroleum," or "Biomass"). It is assumed that supplemental gaseous fuels are commingled with natural gas consumed by the residential, commercial, other industrial, and electric power sectors, but are not commingled with natural gas used for lease and plant fuel, pipelines and distribution, or vehicle fuel. The estimated consumption of supplemental gaseous fuels by each sector (residential, commercial, other industrial, and electric power) is calculated as that sector's natural gas consumption (see Table 6.5) divided by the sum of natural gas consumption by the residential, commercial, other industrial, and electric power sectors (see Table 6.5), and then multiplied by total supplemental gaseous fuels consumption (see Table 6.1). For estimated sectoral consumption of supplemental gaseous fuels in Btu, the residential, commercial, and other industrial values in cubic feet are multiplied by the "End-Use Sectors" conversion factors (see Table A4), and the electric power values in cubic feet are multiplied by the "Electric Power Sector" conversion factors (see Table A4). Total supplemental gaseous fuels consumption in Btu is calculated as the sum of the Btu values for the sectors.

Note 2. Natural Gas Consumption. Natural gas consumption statistics are compiled from surveys of natural gas production, transmission, and distribution companies and from surveys of electric power generation. Consumption by sector from these surveys is compiled on a national and individual State basis and then balanced with national and individual State supply data. Included in the data are the following: Residential Sector-Consumption by private households for space heating, cooking, and other household uses; Commercial Sector-Consumption by nonmanufacturing establishments; government agencies; and, through 1995, those engaged in agriculture, forestry, and fishing. The commercial sector includes generators that produce electricity and/or useful thermal output primarily to support the activities of the above-mentioned commercialestablishments; Industrial Sector-Consumption by establishments engaged primarily in processing unfinished materials into another form of product (including mining; petroleum refining; manufacturing; and, beginning in 1996, agriculture, forestry, and fishing), and natural gas industry use for lease and plant fuel. The industrial sector includes generators that produce electricity and/or useful thermal output primarily to support the above-mentioned industrial activities; Transportation Sector-Natural gas transmission (pipeline) fuel, and natural gas delivered for use as vehicle fuel; and Electric Power Sector (electric utilities and independent power producers) - Consumption for electricity generation and useful thermal output at electricity-only and combined-heat-and-power (CHP) plants within the NAICS (North American Industry Classification System) 22 category whose primary business is to sell electricity, or electricity and heat, to the public.

Note 3. Natural Gas Consumption, 1989-1992. Prior to 1993, deliveries to nonutility generators were not separately collected from natural gas companies on Form EIA-176, “Annual Report of Natural and Supplemental Gas Supply and Disposition.” As a result, for 1989-1992, those volumes are probably included in both the industrial and electric power sectors and double-counted in total consumption. In 1993, 0.28 trillion cubic feet was reported as delivered to nonutility generators.

Note 4. Natural Gas Vehicle Fuel. In Table 6.5, for 1992 forward, natural gas vehicle fuel data do not reflect revised data shown in Table 10.5. These revisions, in million cubic feet, are: 1992-2,112; 1993-2,860; 1994-3,222; 1995-4,619; 1996-6,111; 1997-8,393; 1998-9,416; 1999-10,398; 2000-11,461; 2001-13,788; 2002-15,872; 2003-17,484; 2004-21,487; 2005-22,578; 2006-23,317; 2007-24,256; 2008-25,659; 2009-26,936; and $2010-28,297$.

Note 5. Coverage of Electric Power Sector Natural Gas Prices. For 1973-1982, data for electric power sector natural gas prices include all electric utility plants at which the generator nameplate capacity of all steam-electric units combined totaled 25 megawatts or greater. For 1974-1982, peaking units are also included and counted toward the 25-megawatt-or-greater total. For 1983-1990, data include all electric utility plants at which the generator nameplate capacity of all steam-electric units combined totaled 50 megawatts or greater. For 1991-2001, data include all electric utility plants at which the generator nameplate capacity of all steam-electric units and combined-cycle units together totaled 50 megawatts or greater. For 2002 forward, data include electric utility and independent power producer plants at which the total facility fossil-fueled nameplate generating capacity is 50 or more megawatts, regardless of unit type. 
7. Coal 


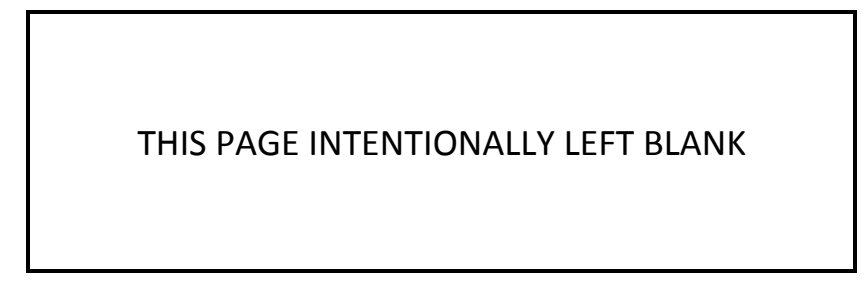


Figure 7.0 Coal Flow, 2011

(Million Short Tons)

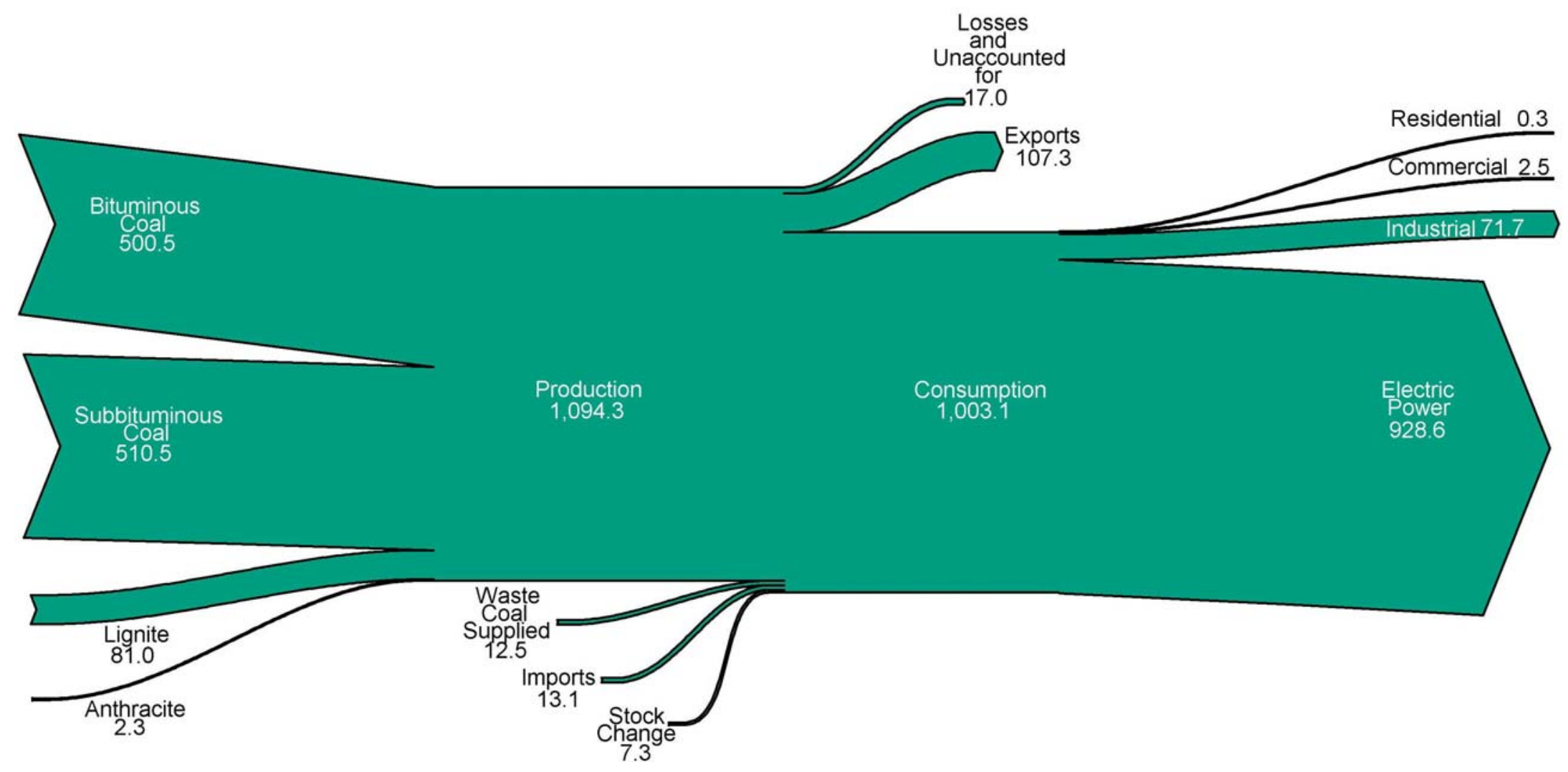

Notes: - Production categories are estimated; all data are preliminary. • Values are derived Sources: Tables 7.1, 7.2, and 7.3. from source data prior to rounding for publication. - Totals may not equal sum of components due to independent rounding. 


\section{Figure 7.1 Coal Overview}

\section{Overview, 1949-2011}

$1,500-$

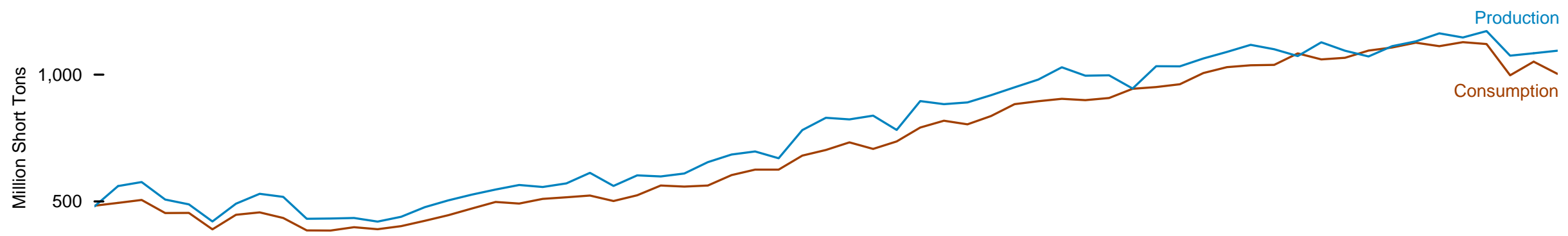

Net Exports

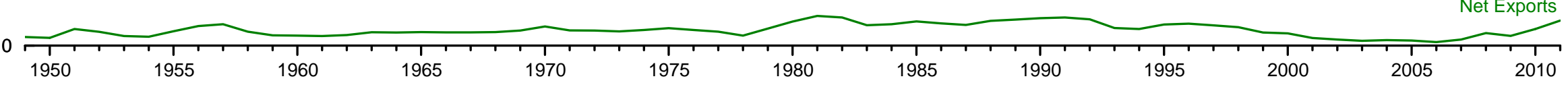

\section{Overview, 2011}

1,500 -

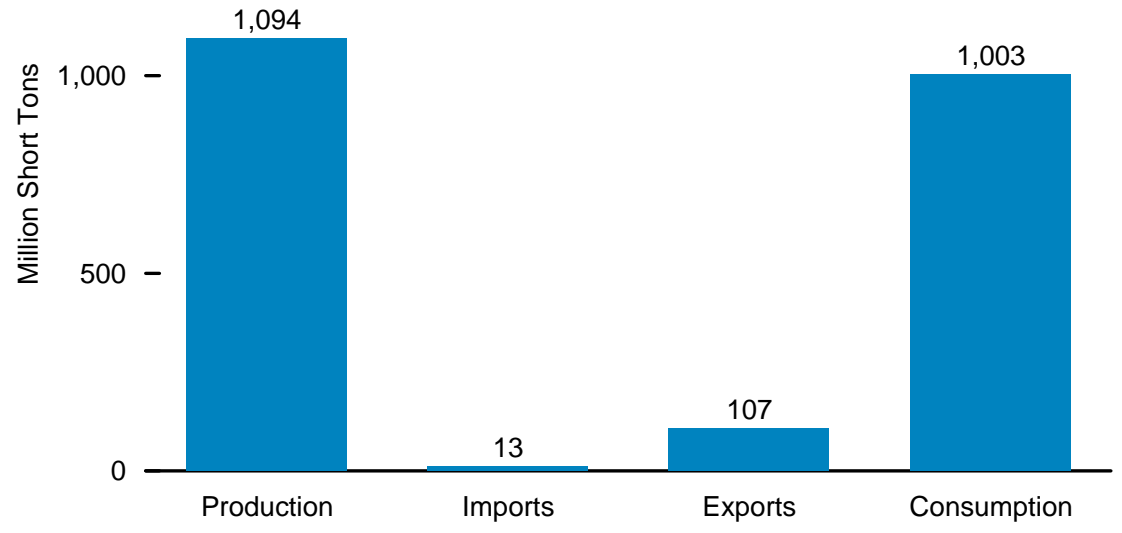

\section{Production as Share of Consumption by Major Source, 1949-2011}

$$
125-
$$

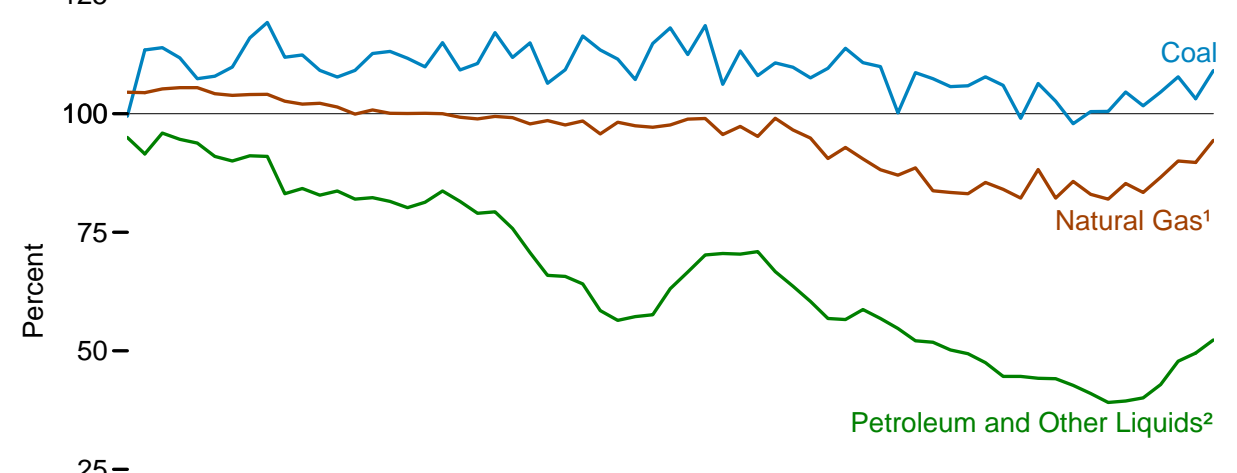

$25-$

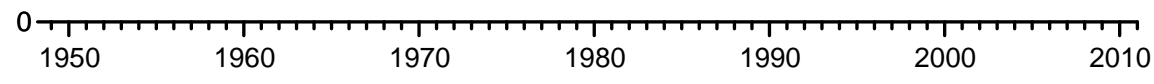

\footnotetext{
${ }^{1}$ Dry natural gas production as share of natural gas consumption.

2 Petroleum and other liquids production as share of petroleum and other liquids estimated consumption.
}

Sources: Tables 5.1a, 6.1, and 7.1 
Table 7.1 Coal Overview, Selected Years, 1949-2011

(Million Short Tons)

\begin{tabular}{|c|c|c|c|c|c|c|c|c|}
\hline \multirow[b]{2}{*}{ Year } & \multirow[b]{2}{*}{ Production ${ }^{1}$} & \multirow{2}{*}{$\begin{array}{l}\text { Waste Coal } \\
\text { Supplied }^{2}\end{array}$} & \multicolumn{3}{|c|}{ Trade } & \multirow[b]{2}{*}{ Stock Change 4,5} & \multirow{2}{*}{$\begin{array}{l}\text { Losses and } \\
\text { Unaccounted for }\end{array}$} & \multirow[b]{2}{*}{ Consumption } \\
\hline & & & Imports & Exports & Net Imports ${ }^{3}$ & & & \\
\hline $\begin{array}{l}1949 \\
1950\end{array}$ & $\begin{array}{l}480.6 \\
560.4\end{array}$ & $\begin{array}{l}\text { NA } \\
\text { NA }\end{array}$ & $\begin{array}{r}0.3 \\
4\end{array}$ & $\begin{array}{l}32.8 \\
294\end{array}$ & $\begin{array}{l}-32.5 \\
-290\end{array}$ & 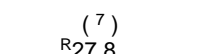 & $\begin{array}{r}7-35.1 \\
\end{array}$ & 483.2 \\
\hline 1955 & 490.8 & NA & $\begin{array}{l}.4 \\
.3\end{array}$ & 54.4 & -54.1 & $\mathrm{R}_{-4.0}$ & $\mathrm{R}_{-6.3} .3$ & 447.0 \\
\hline 1960 & 434.3 & NA & 3 & 38.0 & -37.7 & $R_{-3.2}$ & $\mathrm{R}_{1.7}$ & 398.1 \\
\hline 1965 & 527.0 & NA & .2 & 51.0 & -50.8 & $\mathrm{R}_{1.9}$ & $\mathrm{R}_{2.2}$ & 472.0 \\
\hline 1970 & 612.7 & NA & (s) & 71.7 & -71.7 & $\mathrm{R}_{11.1}$ & $\mathrm{R}_{6.6}$ & 523.2 \\
\hline 1975 & 654.6 & NA & .9 & 66.3 & -65.4 & 32.2 & -5.5 & 562.6 \\
\hline 1976 & 684.9 & NA & 1.2 & 60.0 & -58.8 & 8.5 & 13.8 & 603.8 \\
\hline 1977 & 697.2 & NA & 1.6 & 54.3 & -52.7 & 22.6 & -3.4 & 625.3 \\
\hline 1978 & 670.2 & NA & 3.0 & 40.7 & -37.8 & -4.9 & 12.1 & 625.2 \\
\hline 1979 & 781.1 & NA & 2.1 & 66.0 & -64.0 & 36.2 & .4 & 680.5 \\
\hline 1980 & 829.7 & NA & 1.2 & 91.7 & -90.5 & 25.6 & 10.8 & 702.7 \\
\hline 1981 & 823.8 & NA & 1.0 & 112.5 & -111.5 & -19.0 & -1.4 & 732.6 \\
\hline 1982 & 838.1 & NA & .7 & 106.3 & -105.5 & 22.6 & 3.1 & 706.9 \\
\hline 1983 & 782.1 & NA & 1.3 & 77.8 & -76.5 & -29.5 & -1.6 & 736.7 \\
\hline 1984 & 895.9 & NA & 1.3 & 81.5 & -80.2 & 28.7 & -4.3 & 791.3 \\
\hline 1985 & 883.6 & NA & 2.0 & 92.7 & -90.7 & -27.9 & 2.8 & 818.0 \\
\hline 1986 & 890.3 & NA & 2.2 & 85.5 & -83.3 & 4.0 & -1.2 & 804.2 \\
\hline 1987 & 918.8 & NA & 1.7 & 79.6 & -77.9 & 6.5 & -2.5 & 836.9 \\
\hline 1988 & 950.3 & NA & 2.1 & 95.0 & -92.9 & -24.9 & -1.3 & 883.6 \\
\hline 1989 & 980.7 & 1.4 & 2.9 & 100.8 & -98.0 & -13.7 & 2.9 & 895.0 \\
\hline 1990 & $1,029.1$ & $\begin{array}{l}3.4 \\
3.3\end{array}$ & 2.7 & 105.8 & -103.1 & 26.5 & -1.7 & 904.5 \\
\hline 1991 & 996.0 & 4.0 & 3.4 & 109.0 & -105.6 & -.9 & -3.9 & 899.2 \\
\hline 1992 & 997.5 & 6.3 & 3.8 & 102.5 & -98.7 & -3.0 & .5 & 907.7 \\
\hline 1993 & 945.4 & 8.1 & 8.2 & 74.5 & -66.3 & -51.9 & -4.9 & 944.1 \\
\hline 1994 & $1,033.5$ & 8.2 & 8.9 & 71.4 & -62.5 & 23.6 & 4.3 & 951.3 \\
\hline 1995 & $1,033.0$ & 8.6 & 9.5 & 88.5 & -79.1 & $\begin{array}{l}-.0 \\
-.3\end{array}$ & .6 & 962.1 \\
\hline 1996 & $1,063.9$ & 8.8 & 8.1 & 90.5 & -82.4 & -17.5 & 1.4 & $1,006.3$ \\
\hline 1997 & $1,089.9$ & 8.1 & 7.5 & 83.5 & -76.1 & -11.3 & 3.7 & $1,029.5$ \\
\hline 1998 & $1,117.5$ & 8.7 & 8.7 & 78.0 & -69.3 & 24.2 & -4.4 & $1,037.1$ \\
\hline 1999 & $1,100.4$ & 8.7 & 9.1 & 58.5 & -49.4 & 24.0 & -2.9 & $1,038.6$ \\
\hline 2000 & $1,073.6$ & 9.1 & 12.5 & 58.5 & -46.0 & -48.3 & .9 & $1,084.1$ \\
\hline 2001 & $11,127.7$ & 10.1 & 19.8 & 48.7 & -28.9 & 41.6 & 7.1 & $1,060.1$ \\
\hline 2002 & $1,094.3$ & 9.1 & 16.9 & 39.6 & -22.7 & 10.2 & 4.0 & $1,066.4$ \\
\hline 2003 & $1,071.8$ & 10.0 & 25.0 & 43.0 & -18.0 & -26.7 & -4.4 & $1,094.9$ \\
\hline 2004 & $1,112.1$ & 11.3 & 27.3 & 48.0 & -20.7 & -11.5 & 6.9 & $1,107.3$ \\
\hline 2005 & $1,131.5$ & 13.4 & 30.5 & 49.9 & -19.5 & -9.7 & 9.1 & $1,126.0$ \\
\hline 2006 & $1,162.7$ & $\begin{array}{l}14.4 \\
14.4\end{array}$ & 36.2 & 49.6 & -13.4 & $\begin{array}{r}-3.1 \\
42.6\end{array}$ & 8.8 & $1,112.3$ \\
\hline 2007 & $1,146.6$ & 14.1 & 36.3 & 59.2 & -22.8 & 5.8 & 4.1 & $1,128.0$ \\
\hline 2008 & $1,171.8$ & 14.1 & 34.2 & 81.5 & -47.3 & 12.4 & 5.7 & $1,120.5$ \\
\hline 2009 & $1,074.9$ & 13.7 & 22.6 & 59.1 & -36.5 & 39.7 & 15.0 & 997.5 \\
\hline 2010 & $\mathrm{R}_{1}, 084.4$ & $\mathrm{R}_{13.7}$ & 19.4 & 81.7 & -62.4 & $\mathrm{R}_{-11.2}$ & $\mathrm{R}_{-4.4}$ & $\mathrm{R}_{1,051.3}$ \\
\hline $2011^{P}$ & $1,094.3$ & 12.5 & 13.1 & 107.3 & -94.2 & -7.3 & 17.0 & $1,003.1$ \\
\hline
\end{tabular}

1 Beginning in 2001, includes a small amount of refuse recovery (coal recaptured from a refuse mine, and cleaned to reduce the concentration of noncombustible materials).

(n) bank or slurry dam, anthracite culm 1989, waste col supplied is counted as a supply-side item to balance thdustrial sectors. Beginning in included in "Consumption"

${ }^{3}$ Net imports equal imports minus exports. Minus sign indicates exports are greater than imports.

4 Through 1972, excludes coal stocks at producers and distributors. For 1980-2007, excludes coal

stocks in the residential and commercial sectors.

A negative value indicates a decrease in stocks and a positive value indicates an increase.

${ }^{6}$ The difference between calculated coal supply and disposition, due to coal quantities lost or to data reporting problems.

$\mathrm{R}=$ Revised. $\mathrm{P}=$ Preliminary. NA=Not available. $(\mathrm{s})=$ Less than 0.05 million short tons.

Notes: - See Note 1, "Coal Consumption," at end of section. - Totals may not equal sum of components due to independent rounding.

data - See http //www eia./Wov/totala.gov/totalenergy/data/monthly/\#coal for updated monthly and annual htp:// Sol annual data beginning in 1949. - See Sources: Production: Table 7.2. Waste Coal Supplied: • 1989-1997-U.S. Energy Information Administration (EIA), Form EIA-867, "Annual Nonutility Power Producer Report." • 1998-2000-EIA, Form EIA-860B, "Annual Electric Generator Report-Nonutility." • 2001-2004-EIA, Form EIA-906, "Power Plant Report," and Form EIA-3, "Quarterly Coal Consumption and Quality Report-Manufacturing Plants. - 2005 forward-EIA, Quarterly Coal Report (QCR) October-December 2011 (April 2012), Table ES-1. Imports: - 1949-2000-U.S. Department of Commerce, Bureau of the Census, "Monthly Report IM 145. - 2001 forward-Table 7.4. Exports: Table 7.5. Stock Change: Table 7.6. Losses and Unaccounted for: Calculated as the sum of production, imports, and waste coal supplied, minus exports, stock 
Figure 7.2 Coal Production, 1949-2011
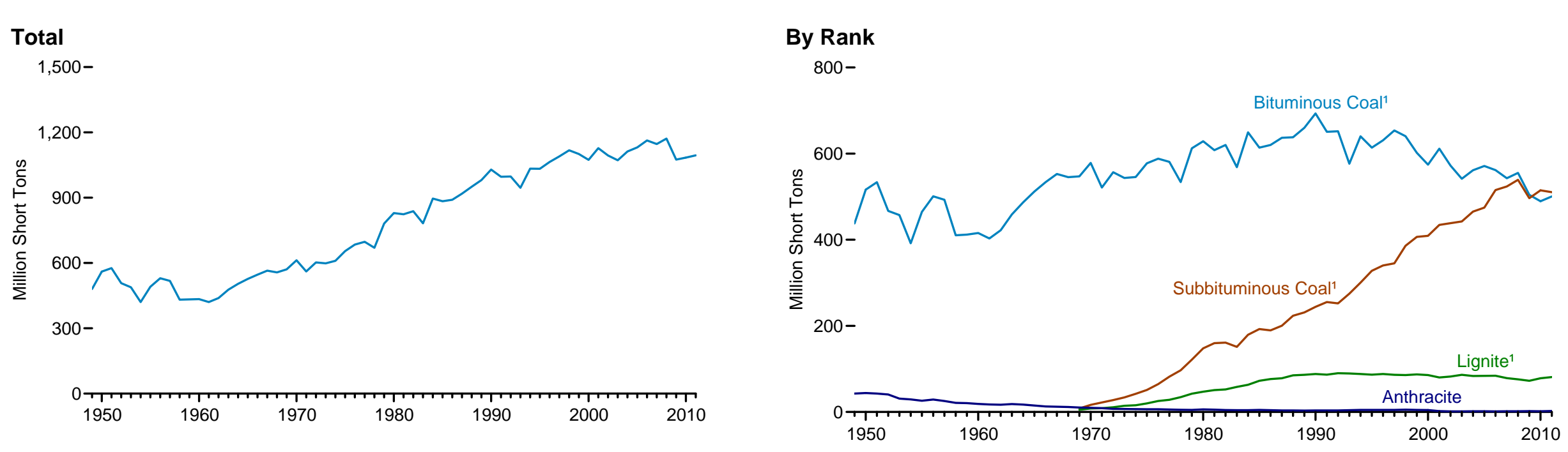

\section{By Mining Method}

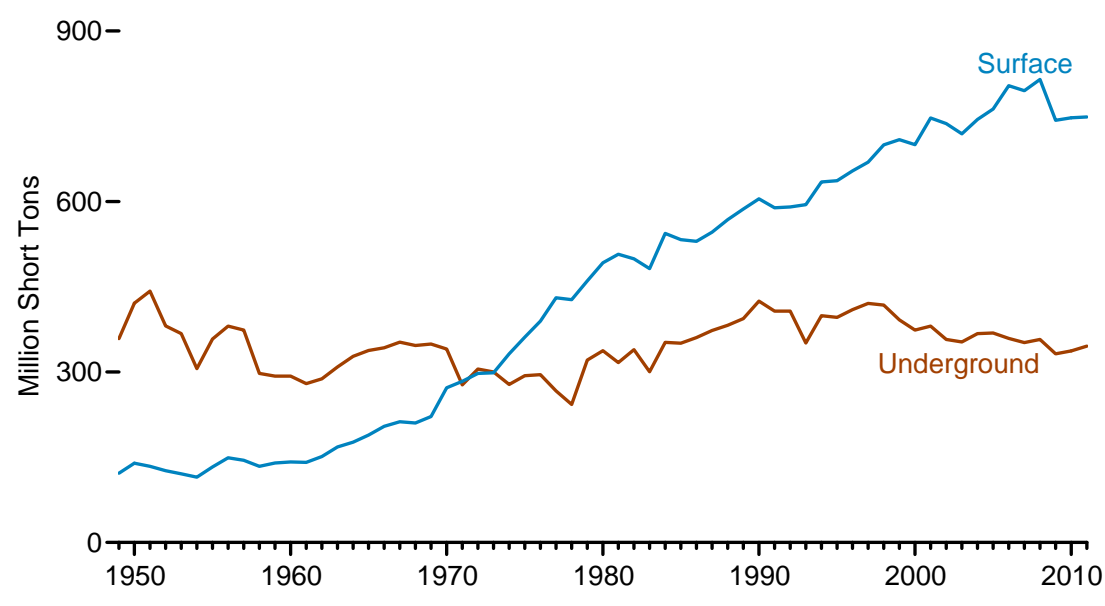

\section{By Location}

$800-$

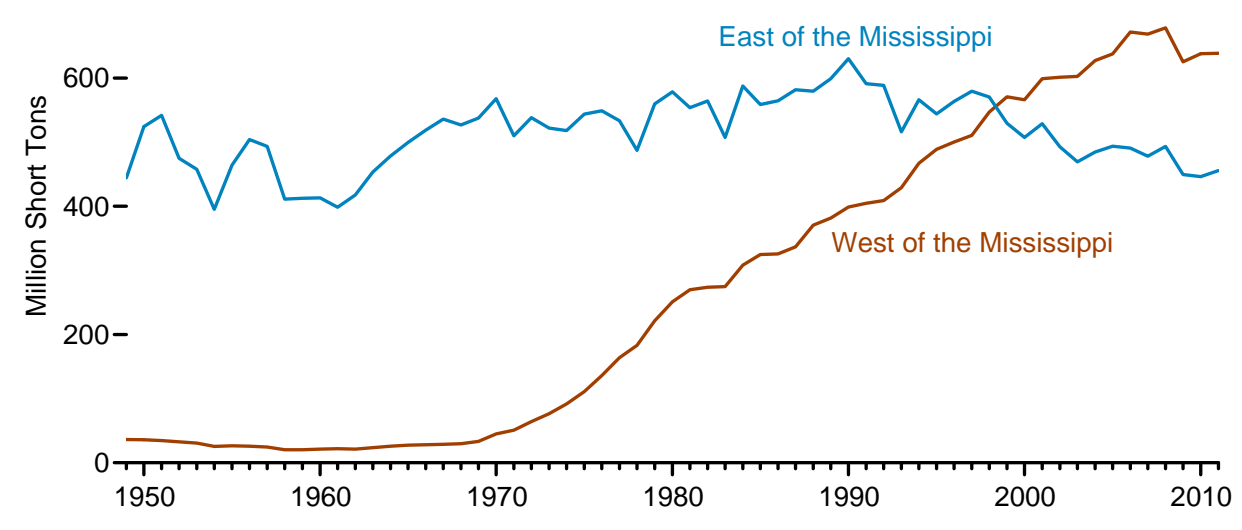

${ }^{1}$ Subbituminous coal and lignite are included in bituminous coal prior to 1969.

Source: Table 7.2. 
Table 7.2 Coal Production, Selected Years, 1949-2011

(Million Short Tons)

\begin{tabular}{|c|c|c|c|c|c|c|c|c|c|}
\hline \multirow[b]{2}{*}{ Year } & \multicolumn{4}{|c|}{ Rank } & \multicolumn{2}{|c|}{ Mining Method } & \multicolumn{2}{|c|}{ Location } & \multirow[b]{2}{*}{ Total 1} \\
\hline & $\begin{array}{l}\text { Bituminous } \\
\text { Coal } 1\end{array}$ & $\begin{array}{c}\text { Subbituminous } \\
\text { Coal }\end{array}$ & Lignite & Anthracite 1 & Underground & Surface 1 & $\begin{array}{l}\text { East of the } \\
\text { Mississippi } 1\end{array}$ & $\begin{array}{l}\text { West of the } \\
\text { Mississippi } 1\end{array}$ & \\
\hline 1949 & 437.9 & $\left({ }^{2}\right)$ & $\left({ }^{2}\right)$ & 42.7 & 358.9 & 121.7 & 444.2 & 36.4 & 480.6 \\
\hline 1950 & 516.3 & $\left({ }^{2}\right)$ & $\left({ }^{2}\right)$ & 44.1 & 421.0 & 139.4 & 524.4 & 36.0 & 560.4 \\
\hline 1955 & 464.6 & $(2)$ & $(2)$ & 26.2 & 358.0 & 132.9 & 464.2 & 26.6 & 490.8 \\
\hline 1960 & 415.5 & $\left(2^{\prime}\right)$ & $(2)$ & 18.8 & 292.6 & 141.7 & 413.0 & 21.3 & 434.3 \\
\hline 1965 & 512.1 & $\left({ }^{2}\right)$ & $\left({ }^{2}\right)$ & 14.9 & 338.0 & 189.0 & 499.5 & 27.4 & 527.0 \\
\hline 1970 & 578.5 & 16.4 & 8.0 & 9.7 & 340.5 & 272.1 & 567.8 & 44.9 & 612.7 \\
\hline 1975 & 577.5 & 51.1 & 19.8 & 6.2 & 293.5 & 361.2 & 543.7 & 110.9 & 654.6 \\
\hline 1976 & 588.4 & 64.8 & 25.5 & 6.2 & 295.5 & 389.4 & 548.8 & 136.1 & 684.9 \\
\hline 1977 & 581.0 & 82.1 & 28.2 & 5.9 & 266.6 & 430.6 & 533.3 & 163.9 & 697.2 \\
\hline 1978 & 534.0 & 96.8 & 34.4 & 5.0 & 242.8 & 427.4 & 487.2 & 183.0 & 670.2 \\
\hline 1979 & 612.3 & 121.5 & 42.5 & 4.8 & 320.9 & 460.2 & 559.7 & 221.4 & 781.1 \\
\hline 1980 & 628.8 & 147.7 & 47.2 & 6.1 & 337.5 & 492.2 & 578.7 & 251.0 & 829.7 \\
\hline 1981 & 608.0 & 159.7 & 50.7 & 5.4 & 316.5 & 507.3 & 553.9 & 269.9 & 823.8 \\
\hline 1982 & 620.2 & 160.9 & 52.4 & 4.6 & 339.2 & 499.0 & 564.3 & 273.9 & 838.1 \\
\hline 1983 & 568.6 & 151.0 & 58.3 & 4.1 & 300.4 & 481.7 & 507.4 & 274.7 & 782.1 \\
\hline 1984 & 649.5 & 179.2 & 63.1 & 4.2 & 352.1 & 543.9 & 587.6 & 308.3 & 895.9 \\
\hline 1985 & 613.9 & 192.7 & 72.4 & 4.7 & 350.8 & 532.8 & 558.7 & 324.9 & 883.6 \\
\hline 1986 & 620.1 & 189.6 & 76.4 & 4.3 & 360.4 & 529.9 & 564.4 & 325.9 & 890.3 \\
\hline 1987 & 636.6 & 200.2 & 78.4 & 3.6 & 372.9 & 545.9 & 581.9 & 336.8 & 918.8 \\
\hline 1988 & 638.1 & 223.5 & 85.1 & 3.6 & 382.2 & 568.1 & 579.6 & 370.7 & 950.3 \\
\hline 1989 & 659.8 & 231.2 & 86.4 & 3.3 & 393.8 & 586.9 & 599.0 & 381.7 & 980.7 \\
\hline 1990 & 693.2 & 244.3 & 88.1 & 3.5 & 424.5 & 604.5 & 630.2 & 398.9 & $1,029.1$ \\
\hline 1991 & 650.7 & 255.3 & 86.5 & 3.4 & 407.2 & 588.8 & 591.3 & 404.7 & 996.0 \\
\hline 1992 & 651.8 & 252.2 & 90.1 & 3.5 & 407.2 & 590.3 & 588.6 & 409.0 & 997.5 \\
\hline 1993 & 576.7 & 274.9 & 89.5 & 4.3 & 351.1 & 594.4 & 516.2 & 429.2 & 945.4 \\
\hline 1994 & 640.3 & 300.5 & 88.1 & 4.6 & 399.1 & 634.4 & 566.3 & 467.2 & $1,033.5$ \\
\hline 1995 & 613.8 & 328.0 & 86.5 & 4.7 & 396.2 & 636.7 & 544.2 & 488.7 & $1,033.0$ \\
\hline 1996 & 630.7 & 340.3 & 88.1 & 4.8 & 409.8 & 654.0 & 563.7 & 500.2 & $1,063.9$ \\
\hline 1997 & 653.8 & 345.1 & 86.3 & 4.7 & 420.7 & 669.3 & 579.4 & 510.6 & $1,089.9$ \\
\hline 1998 & 640.6 & 385.9 & 85.8 & 5.3 & 417.7 & 699.8 & 570.6 & 547.0 & $1,117.5$ \\
\hline 1999 & 601.7 & 406.7 & 87.2 & 4.8 & 391.8 & 708.6 & 529.6 & 570.8 & $1,100.4$ \\
\hline 2000 & 574.3 & 409.2 & 85.6 & 4.6 & 373.7 & 700.0 & 507.5 & 566.1 & $1,073.6$ \\
\hline 2001 & ${ }^{1} 611.3$ & 434.4 & 80.0 & 11.9 & 380.6 & ${ }^{1} 747.1$ & ${ }^{1} 528.8$ & ${ }^{1} 598.9$ & $11,127.7$ \\
\hline 2002 & 572.1 & 438.4 & 82.5 & 1.4 & 357.4 & 736.9 & 492.9 & 601.4 & $1,094.3$ \\
\hline 2003 & 541.5 & 442.6 & 86.4 & 1.3 & 352.8 & 719.0 & 469.2 & $\begin{array}{l}002.5 \\
602.5\end{array}$ & $\begin{array}{l}1,071.8 \\
1.071\end{array}$ \\
\hline 2004 & 561.5 & 465.4 & 83.5 & 1.7 & 367.6 & 744.5 & 484.8 & 627.3 & $1,112.1$ \\
\hline 2005 & 571.2 & 474.7 & 83.9 & 1.7 & 368.6 & 762.9 & 493.8 & 637.7 & $1,131.5$ \\
\hline 2006 & 561.6 & 515.3 & 84.2 & 1.5 & 359.0 & 803.7 & 490.8 & 672.0 & $1,162.7$ \\
\hline 2007 & 542.8 & 523.7 & 78.6 & 1.6 & 351.8 & 794.8 & 478.2 & 668.5 & $1,146.6$ \\
\hline 2008 & 555.3 & 539.1 & 75.7 & 1.7 & 357.1 & 814.7 & 493.3 & 678.5 & $1,171.8$ \\
\hline 2009 & 504.1 & 496.4 & 72.5 & 1.9 & 332.1 & 742.9 & 449.6 & 625.3 & $1,074.9$ \\
\hline 2010 & $\mathrm{R}_{489.5}$ & R514.8 & R78.2 & $\mathrm{R}_{1.8}$ & $R_{337.2}$ & R747.2 & $\mathrm{R}_{446.2}$ & $R_{638.2}$ & $R_{1}, 084.4$ \\
\hline 2011 & ${ }_{5} 500.5$ & E510.5 & E81.0 & $\mathrm{E}_{2.3}$ & $E_{345.5}$ & E748.8 & ${ }^{E} 455.8$ & $E_{638.5}$ & $\mathrm{P}_{1,094.3}$ \\
\hline
\end{tabular}

1 Beginning in 2001, includes a small amount of refuse recovery. 2 Included in "Bituminous Coal."

$\mathrm{R}=$ Revised. $\mathrm{P}=$ Preliminary. E=Estimate

Note. Totals may not equal sum of components due to independent rounding

Web Pages: • For all data beginning in 1949, see http://www.eia.gov/totalenergy/data/annual/\#coal. - For related information, see http://www.eia.gov/coal/

Sources: - 1949-1975-Bureau of Mines, Minerals Yearbook, "Coal-Bituminous and Lignite" and Data Reports, Coal-Bituminous and Lignite in 1976 and Coal-Pennsylvania Anthracite 1976. • 1977

and 1978-EIA, Energy Data Reports, Bituminous Coal and Lignite Production and Mine Operations-1977; 1978, Coal-Pennsylvania Anthracite 1977; 1978, and Coal Production, annual reports. - 1979 and 1980-EIA, Energy Data Reports, Weekly Coal Report and Coal Production, annual reports. - 1989-2000-EIA, Coal Industry Annual, annual reports. - 2001-2010-EIA, Annual Coal Report, annual reports "Coal Production Report"; and U.S. Department of Labor, Mine Safety and Health Administration, Form 7000-2, "Quarterly Mine Employment and Coal Production Report." 


\section{Figure 7.3 Coal Consumption by Sector}

\section{By Sector, 1949-2011}

$1,200-$

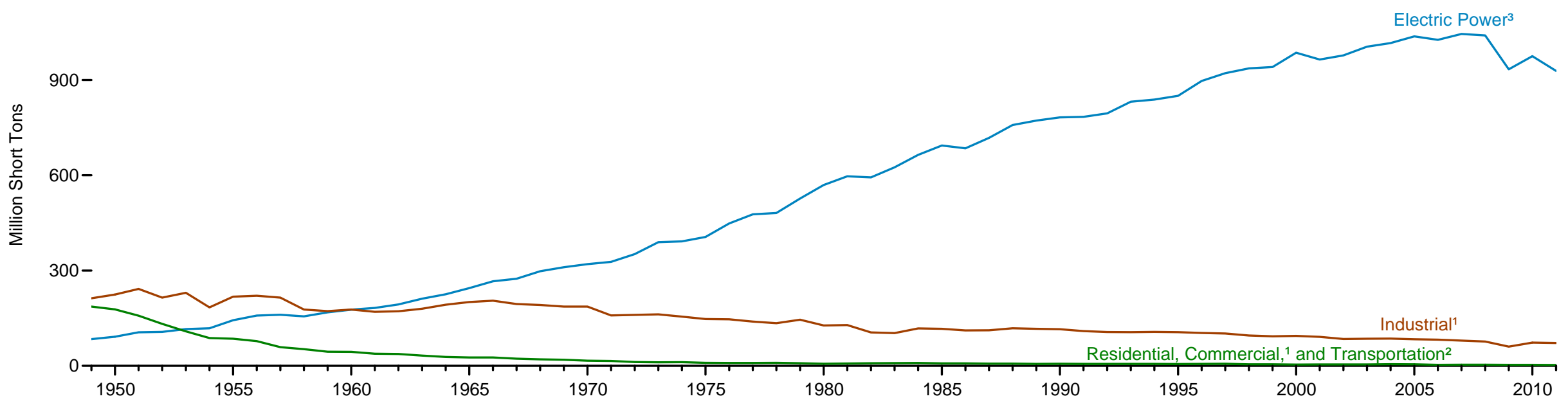

\section{By Sector, 2011}

$1,200-$

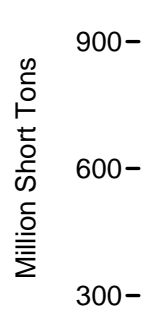

\section{Sector Shares, 1949 and 2011}

$100-$

$1949 \square 2011$

$80-$

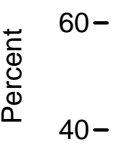

40-

$20-$

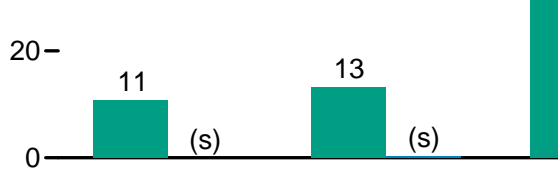

Residential

44

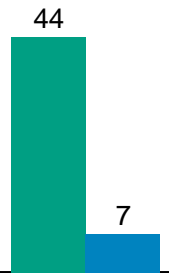

Industrial $^{1}$

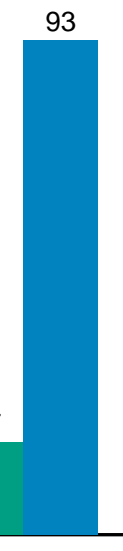

Electric Power $^{3}$

1 Includes combined-heat-and-power (CHP) plants and a small number of electricity-only
plants.

2For 1978 forward, small amounts of transportation sector use are included in "Industrial."
${ }^{3}$ Electricity-only and combined-heat-and-power (CHP) plants whose primary business is to sell electricity, or electricity and heat, to the public.

(s)=Less than 0.5 
Table 7.3 Coal Consumption by Sector, Selected Years, 1949-2011

(Million Short Tons)

\begin{tabular}{|c|c|c|c|c|c|c|c|c|c|c|c|c|c|c|}
\hline \multirow[b]{3}{*}{ Year } & \multirow{3}{*}{$\begin{array}{c}\text { Residential } \\
\text { Sector }^{1}\end{array}$} & \multicolumn{3}{|c|}{ Commercial Sector ${ }^{1}$} & \multicolumn{5}{|c|}{ Industrial Sector } & \multirow{3}{*}{$\begin{array}{c}\text { Transportation } \\
\text { Sector }\end{array}$} & \multicolumn{3}{|c|}{ Electric Power Sector ${ }^{2}$} & \multirow[b]{3}{*}{ Total } \\
\hline & & \multirow[b]{2}{*}{$\mathrm{CHP}^{3}$} & \multirow[b]{2}{*}{ Other 4} & \multirow[b]{2}{*}{ Total } & \multirow[b]{2}{*}{ Coke Plants } & \multicolumn{3}{|c|}{ Other Industrial } & \multirow[b]{2}{*}{ Total } & & \multirow{2}{*}{$\begin{array}{c}\text { Electricity } \\
\text { Only }\end{array}$} & \multirow[b]{2}{*}{ CHP } & \multirow[b]{2}{*}{ Total } & \\
\hline & & & & & & CHP 5 & Non-CHP 6 & Total & & & & & & \\
\hline 1949 & 52.4 & $\left({ }^{7}\right)$ & 64.1 & 64.1 & 91.4 & $\left({ }^{8}\right)$ & 121.2 & 121.2 & 212.6 & 70.2 & 84.0 & NA & 84.0 & 483.2 \\
\hline 1950 & 51.6 & $\left(7^{7}\right)$ & 63.0 & 63.0 & 104.0 & $\left({ }^{8}\right)$ & 120.6 & 120.6 & 224.6 & 63.0 & 91.9 & NA & 91.9 & 494.1 \\
\hline 1955 & 35.6 & $\left(7^{7}\right)$ & $\begin{array}{l}0.0 \\
32.9\end{array}$ & 32.9 & $\begin{array}{l}107.7 \\
\end{array}$ & $(8)$ & 110.1 & 110.1 & 217.8 & 17.0 & 143.8 & NA & $\begin{array}{r}143.8 \\
\end{array}$ & 447.0 \\
\hline 1960 & $\begin{array}{l}3.6 \\
24.2\end{array}$ & (7) & $\begin{array}{l}3.9 \\
16.8\end{array}$ & $\begin{array}{l}3.9 \\
16.8\end{array}$ & 81.4 & (8) & 96.0 & 96.0 & $\begin{array}{l}177.4 \\
\end{array}$ & 3.0 & 176.7 & NA & 176.7 & 398.1 \\
\hline 1965 & 14.6 & $\left(7^{7}\right)$ & 11.0 & 11.0 & 95.3 & $(8)$ & 105.6 & 105.6 & 200.8 & .7 & 244.8 & NA & 244.8 & 472.0 \\
\hline 1970 & 9.0 & $\left(7^{7}\right)$ & 7.1 & 7.1 & 96.5 & (8) & $\begin{array}{r}\quad 90.0 \\
90.2\end{array}$ & 90.2 & 186.6 & .3 & $\begin{array}{l}244.8 \\
320.2\end{array}$ & $\begin{array}{l}\text { NA } \\
\text { NA }\end{array}$ & $\begin{array}{l}244.8 \\
320.2\end{array}$ & $\begin{array}{l}4 / 2.0 \\
523.2\end{array}$ \\
\hline 1975 & 2.8 & $\left(7^{\prime}\right)$ & 6.6 & 6.6 & 83.6 & $(8)$ & 63.6 & 63.6 & 147.2 & (s) & 406.0 & NA & 406.0 & 562.6 \\
\hline 1976 & 2.6 & $\left(\begin{array}{l}7 \\
7\end{array}\right)$ & 6.3 & 6.3 & 84.7 & $\left(8^{\prime}\right)$ & 61.8 & 61.8 & 146.5 & (s) & 448.4 & NA & 448.4 & 603.8 \\
\hline 1977 & 2.5 & $\left(7^{7}\right)$ & 6.4 & 6.4 & 77.7 & $\left({ }^{8}\right)$ & 61.5 & 61.5 & 139.2 & (s) & 477.1 & NA & 477.1 & 625.3 \\
\hline 1978 & 2.2 & $\left(7^{7}\right)$ & 7.3 & 7.3 & 71.4 & $(8)$ & 63.1 & 63.1 & 134.5 & $\left({ }^{8}\right)$ & 481.2 & NA & 481.2 & 625.2 \\
\hline 1979 & 1.7 & $(7)$ & 6.7 & 6.7 & 77.4 & $(8)$ & 67.7 & 67.7 & 145.1 & $(8)$ & 527.1 & NA & 527.1 & 680.5 \\
\hline 1980 & 1.4 & (7) & 5.1 & 5.1 & 66.7 & (8) & 60.3 & 60.3 & 127.0 & (8) & 569.3 & NA & 569.3 & 702.7 \\
\hline $\begin{array}{l}1980 \\
1981\end{array}$ & $\begin{array}{l}1.4 \\
1.3\end{array}$ & (7) & $\begin{array}{l}5.1 \\
6.1\end{array}$ & $\begin{array}{l}5.1 \\
6.1\end{array}$ & $\begin{array}{l}6.1 \\
61.0\end{array}$ & (8) & $\begin{array}{l}6.3 \\
67.4\end{array}$ & $\begin{array}{l}6.3 \\
67.4\end{array}$ & 128.4 & (8) & $\begin{array}{l}56.3 \\
596.8\end{array}$ & NA & $\begin{array}{l}569.3 \\
596.8\end{array}$ & 732.6 \\
\hline 1982 & 1.4 & $(7)$ & $\begin{array}{l}.1 \\
6.8\end{array}$ & $\begin{array}{l}.1 \\
6.8\end{array}$ & 40.9 & (8) & $\begin{array}{l}04.1 \\
64.1\end{array}$ & 64.1 & 105.0 & (8) & 593.7 & NA & 593.7 & $\begin{array}{l}706.0 \\
706.9\end{array}$ \\
\hline 1983 & $\begin{array}{l}1.4 \\
1.4\end{array}$ & $\left(7^{\prime}\right)$ & $\begin{array}{l}7.0 \\
7.1\end{array}$ & $\begin{array}{l}.00 \\
7.1\end{array}$ & 37.0 & $(8)$ & 66.0 & 66.0 & 103.0 & (8) & 625.2 & NA & 625.2 & 736.7 \\
\hline 1984 & 1.7 & $\left(7^{\prime}\right)$ & 7.4 & 7.4 & 44.0 & (8) & 73.7 & 73.7 & 117.8 & $(8)$ & 664.4 & NA & 664.4 & 791.3 \\
\hline 1985 & 1.7 & $\left(7^{\prime}\right)$ & 6.1 & 6.1 & 41.1 & (8) & 75.4 & 75.4 & 116.4 & $\left({ }^{8}\right)$ & 693.8 & NA & 693.8 & 818.0 \\
\hline 1986 & 1.8 & $\left(7^{7}\right)$ & 5.9 & 5.9 & 35.9 & (8) & 75.6 & 75.6 & 111.5 & $(8)$ & 685.1 & NA & 685.1 & 804.2 \\
\hline 1987 & 1.6 & $\left(7^{\prime}\right)$ & 5.3 & 5.3 & 37.0 & (8) & 75.2 & 75.2 & 112.1 & $\left({ }^{8}\right)$ & 717.9 & NA & 717.9 & 836.9 \\
\hline 1988 & $\begin{array}{l}1.0 \\
1.6\end{array}$ & $(7)$ & $\begin{array}{l}5.0 \\
5.6\end{array}$ & $\begin{array}{l}5.0 \\
5.6\end{array}$ & 41.9 & (8) & 76.3 & 76.3 & 118.1 & (8) & 758.4 & NA & 758.4 & $\begin{array}{l}80.5 \\
883.6\end{array}$ \\
\hline $\begin{array}{l}1988 \\
1989\end{array}$ & $\begin{array}{l}1.6 \\
1.3\end{array}$ & 1.1 & $\begin{array}{l}5.6 \\
3.7\end{array}$ & $\begin{array}{l}.6 \\
4.9\end{array}$ & $\begin{array}{l}41.9 \\
40.5\end{array}$ & 24.9 & $\begin{array}{l}10.3 \\
51.3\end{array}$ & 76.1 & 116.6 & (8) & $\begin{array}{l}158.4 \\
767.4\end{array}$ & 4.8 & $\begin{array}{l}758.4 \\
772.2\end{array}$ & $\begin{array}{l}88.6 \\
895.0\end{array}$ \\
\hline 1990 & $\begin{array}{l}1.3 \\
1.3\end{array}$ & 1.2 & 4.2 & 5.4 & 38.9 & 27.8 & 48.5 & 76.3 & 115.2 & (8) & 774.2 & 8.4 & 782.6 & 904.5 \\
\hline 1991 & 1.1 & 1.2 & 3.8 & $\begin{array}{l}5.4 \\
5.0\end{array}$ & 33.9 & 27.0 & 48.4 & 75.4 & 109.3 & (8) & 773.2 & $\begin{array}{r}10.7 \\
\end{array}$ & 783.9 & 899.2 \\
\hline 1992 & 1.1 & 1.2 & 3.9 & 5.0 & 32.4 & 28.2 & 45.8 & 74.0 & 106.4 & (8) & 781.2 & 13.9 & 795.1 & 907.7 \\
\hline 1993 & 1.1 & 1.4 & 3.7 & 5.1 & 31.3 & 28.9 & 46.0 & 74.9 & 106.2 & $\left({ }^{8}\right)$ & 816.6 & 15.1 & 831.6 & 944.1 \\
\hline 1994 & .9 & 1.3 & 3.8 & 5.1 & 31.7 & 29.7 & 45.5 & 75.2 & 106.9 & $(8)$ & 821.2 & 17.1 & 838.4 & 951.3 \\
\hline 1995 & .8 & 1.4 & 3.6 & 5.1 & 33.0 & 29.4 & 43.7 & 73.1 & 106.1 & $(8)$ & 832.9 & 17.3 & 850.2 & 962.1 \\
\hline 1996 & .0 & $\begin{array}{l}1.4 \\
1.7\end{array}$ & 3.6 & 5.3 & 31.7 & 29.4 & 42.3 & 71.7 & 103.4 & $(8)$ & 878.8 & 18.1 & 896.9 & $1,006.3$ \\
\hline 1997 & .7 & 1.7 & $\begin{array}{l}.0 \\
4.0\end{array}$ & $\begin{array}{l}.35 \\
5.8\end{array}$ & 30.2 & $\begin{array}{l}29.4 \\
29.9\end{array}$ & $\begin{array}{l}41.5 \\
41.7\end{array}$ & 71.5 & $\begin{array}{l}101.4 \\
101.7\end{array}$ & (8) & $\begin{array}{l}01.0 \\
904.2\end{array}$ & $\begin{array}{l}17.1 \\
17.1\end{array}$ & $\begin{array}{l}890.9 \\
921.4\end{array}$ & $\begin{array}{l}1,000.3 \\
1,029.5\end{array}$ \\
\hline 1998 & .5 & 1.4 & 2.9 & 4.3 & 28.2 & 28.6 & 38.9 & 67.4 & 95.6 & (8) & 920.4 & 16.3 & 936.6 & $1,037.1$ \\
\hline 1999 & .6 & 1.5 & 2.8 & 4.3 & 28.1 & 27.8 & 37.0 & 64.7 & 92.8 & (8) & 924.7 & 16.2 & 940.9 & $1,038.6$ \\
\hline 2000 & .5 & 1.5 & 2.1 & 3.7 & 28.9 & 28.0 & 37.2 & 65.2 & 94.1 & $\left({ }^{8}\right)$ & 967.1 & $\begin{array}{l}10.4 \\
18.7\end{array}$ & 985.8 & $\begin{array}{l}1,030.1 \\
1.084 .1\end{array}$ \\
\hline 2001 & .5 & 1.4 & 2.4 & 3.9 & 26.1 & 25.8 & 39.5 & 65.3 & 91.3 & $(8)$ & 946.1 & 18.4 & 964.4 & $1,060.1$ \\
\hline 2002 & .5 & 1.4 & 2.5 & 3.9 & 23.7 & 26.2 & 34.5 & 60.7 & 84.4 & $(8)$ & 960.1 & 17.4 & 977.5 & $1,066.4$ \\
\hline 2003 & .6 & 1.8 & 1.9 & 3.7 & 24.2 & 24.8 & 36.4 & 61.3 & 85.5 & $(8)$ & 983.5 & 21.6 & 1.005 .1 & 1094.9 \\
\hline 2004 & $\begin{array}{l}.0 \\
.5\end{array}$ & $\begin{array}{l}1.0 \\
1.9\end{array}$ & 2.7 & $\begin{array}{l}.1 \\
4.6\end{array}$ & $\begin{array}{l}23.7 \\
23.7\end{array}$ & $\begin{array}{l}26.0 \\
26.6\end{array}$ & $\begin{array}{l}50.4 \\
35.6\end{array}$ & $\begin{array}{l}0.0 \\
62.2\end{array}$ & $\begin{array}{l}85.9 \\
85.9\end{array}$ & (8) & $\begin{array}{l}900.5 \\
994.8\end{array}$ & $\begin{array}{l}21.5 \\
21.5\end{array}$ & $1,016.3$ & $1,107.3$ \\
\hline 2005 & $\begin{array}{l}.5 \\
.4\end{array}$ & $\begin{array}{l}1.9 \\
1.9\end{array}$ & 2.4 & $\begin{array}{l}4.6 \\
4.3\end{array}$ & $\begin{array}{l}23.1 \\
23.4\end{array}$ & $\begin{array}{l}25.6 \\
25.9\end{array}$ & $\begin{array}{l}3.0 \\
34.5\end{array}$ & 60.3 & $\begin{array}{l}85.9 \\
83.8\end{array}$ & (8) & $\begin{array}{r}994.0 \\
1,015.6\end{array}$ & $\begin{array}{l}21.5 \\
21.8\end{array}$ & $\begin{array}{l}1,037.5 \\
1,037\end{array}$ & $1,126.0$ \\
\hline 2006 & .3 & 1.9 & $\begin{array}{l}1.1 \\
\text {. }\end{array}$ & 2.9 & 23.0 & 25.3 & 34.2 & 59.5 & 82.4 & $\left({ }^{8}\right)$ & $1,004.8$ & 21.9 & $1,026.6$ & $1,112.3$ \\
\hline 2007 & .4 & 1.9 & 1.2 & 3.2 & 22.7 & 22.5 & 34.1 & 56.6 & 79.3 & $(8)$ & $\begin{array}{l}1,022.8 \\
\end{array}$ & 22.3 & $\begin{array}{l}1,045.1 \\
\end{array}$ & $1,128.0$ \\
\hline 2008 & .4 & 2.0 & 1.1 & $\begin{array}{l}3.2 \\
3.2\end{array}$ & 22.1 & 21.9 & 32.5 & 54.4 & 76.5 & (8) & $\begin{array}{l}1,017.8 \\
\end{array}$ & 22.8 & $1,040.6$ & $1,120.5$ \\
\hline 2009 & .4 & 1.8 & 1.1 & 2.9 & 15.3 & 19.8 & 25.5 & 45.3 & 60.6 & $(8)$ & $\begin{array}{r}913.6 \\
913.6\end{array}$ & $\begin{array}{l}22.0 \\
20.1\end{array}$ & $\begin{array}{r}1,040.6 \\
933.6\end{array}$ & $\begin{array}{r}1<0.5 \\
997.5\end{array}$ \\
\hline 2010 & .3 & $R_{1.7}$ & 1.0 & 2.7 & 21.1 & $\mathrm{R}_{24.6}$ & $\mathrm{R}_{27.4}$ & R52.1 & R73.2 & $(8)$ & R954.5 & $\mathrm{R}_{20.5}$ & R975.1 & $R_{1}, 051.3$ \\
\hline $2011^{P}$ & .3 & 1.6 & .8 & 2.5 & 21.4 & 24.7 & 25.6 & 50.3 & 71.7 & $(8)$ & 909.6 & 18.9 & 928.6 & $1,003.1$ \\
\hline
\end{tabular}

See Note 2, "Residential and Commercial Coal Consumption Estimates," at end of section.

2 Electricity-only and combined-heat-and-power (CHP) plants within the NAICS 22 category whose primary business is to sell electricity, or electricity and heat, to the public. Through 1988, data are for electric utilities only; beginning in 1989, data are for electric utilities and independent power producers.

${ }^{3}$ Commeriat pombined heat-and

sumber of commercial electricity-only

5 Industrial combined-heat-and-power (CHP) and a small number of industrial electricity-only plants.

6 All industrial sector fuel use other than that in "Coke Plants" and "Industrial CHP."

8 Included in "Commercial Other."

$R=$ Revised. $P=$ Preliminary. NA=Not available. $(\mathrm{s})=$ Less than 0.05 million short tons.

Notes: - See Tables $8.5 \mathrm{a}-8.5 \mathrm{~d}$ for the amount of coal used to produce electricity and Tables $8.6 \mathrm{a}-8.6 \mathrm{c}$

for the amount of coal used to produce useful thermal output. - See Note 1, "Coal Consumption," at end of section. - See Note 2, "Classification of Power Plants Into Energy-Use Sectors," at end of Section 8. - Tolals may not equal sum of components due to independent rounding.

Web Pages: - See http://www.eia.gov/totalenergy/data/monthly/\#coal for updated monthly and annual data. - See http://www.eia.gov/totalenergy/data/annual/\#coal for all annual data beginning in 1949. - See Sources: Commercial CHP and Industrial

8.5c, 8.6b and 8.7b. All Other Data: - 1949-1975 "Coal—Bituminous and Lignite" and "Coal—Pennsylvania Anthracite" chapters. - 1976-U.S. Energy Information Administration (EIA), Energy Data Reports, Coal-Bituminous and Lignite in 1976 and Coal-Pennsylvania Anthracite 1976. - 1977 and 1978-EIA, Energy Data Reports, Coal-Pennsylvania Anthracite 1977, 1978, and Weekly Coal Report. 1979 and 1980 - ElA, Energy Data Report, Weekly Coal Report. I I 


\section{Figure 7.4 Coal Imports by Country of Origin}

\section{Total, $2000-2011$}

$40-$

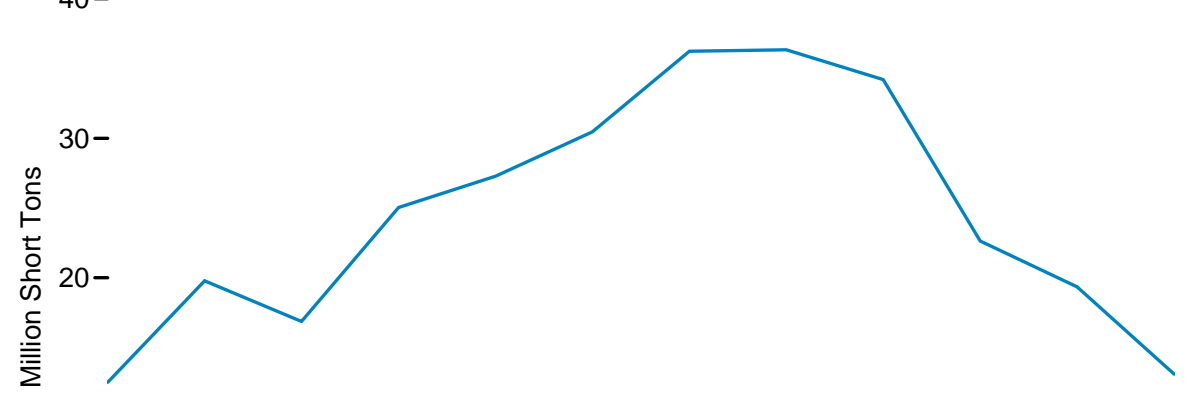

$10-$

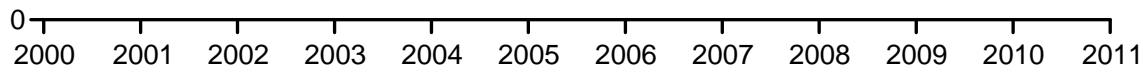

By Selected Country, 2000-2011

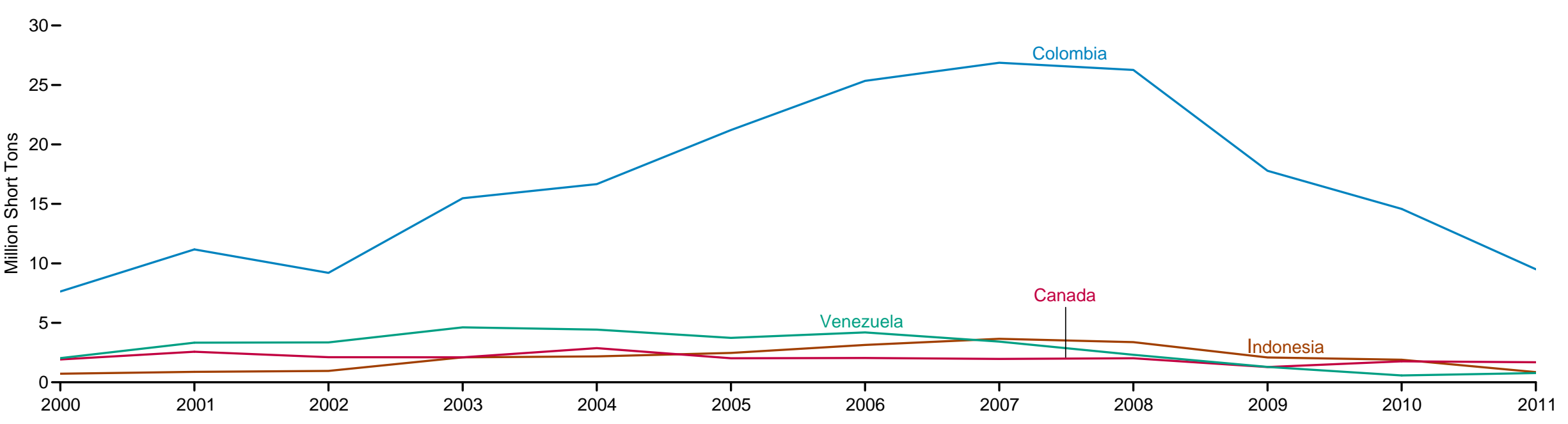

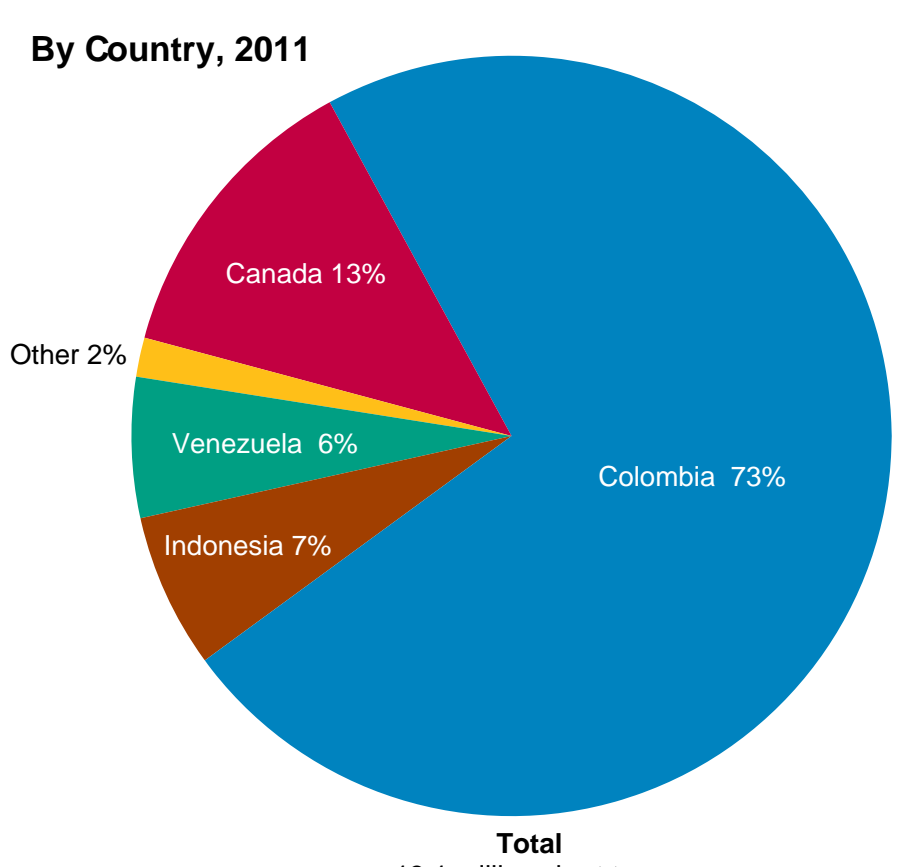

13.1 million short tons 
Table 7.4 Coal Imports by Country of Origin, 2000-2011

(Million Short Tons)

\begin{tabular}{|c|c|c|c|c|c|c|c|c|c|c|c|c|c|c|c|c|c|c|c|}
\hline \multirow[b]{2}{*}{ Year } & \multirow[b]{2}{*}{ Australia } & \multirow[b]{2}{*}{$\begin{array}{c}\text { New } \\
\text { Zealand }\end{array}$} & \multirow[b]{2}{*}{ Canada } & \multirow[b]{2}{*}{ Mexico } & \multirow[b]{2}{*}{ Colombia } & \multirow[b]{2}{*}{ Venezuela } & \multirow[b]{2}{*}{ China } & \multirow[b]{2}{*}{ India } & \multirow[b]{2}{*}{ Indonesia } & \multicolumn{7}{|c|}{ Europe } & \multirow[b]{2}{*}{$\begin{array}{l}\text { South } \\
\text { Africa }\end{array}$} & \multirow[b]{2}{*}{ Other } & \multirow[b]{2}{*}{ Total } \\
\hline & & & & & & & & & & Norway & Poland & Russia & Ukraine & $\begin{array}{c}\text { United } \\
\text { Kingdom }\end{array}$ & Other & Total & & & \\
\hline 2000 & 0.2 & 0.0 & 1.9 & (s) & 7.6 & 2.0 & (s) & (s) & 0.7 & 0.0 & 0.0 & (s) & 0.0 & (s) & 0.0 & (s) & 0.0 & (s) & 12.5 \\
\hline 2001 & .3 & (s) & 2.6 & (s) & 11.2 & 3.3 & .1 & (s) & .9 & (s) & .5 & .2 & .0 & .1 & (s) & .8 & .4 & .1 & 19.8 \\
\hline 2002 & .8 & .0 & 2.1 & (s) & 9.2 & 3.3 & .1 & (s) & 1.0 & .0 & .1 & .1 & .0 & (s) & (s) & .2 & .1 & (s) & 16.9 \\
\hline 2003 & .3 & .1 & 2.1 & .0 & 15.5 & 4.6 & .1 & (s) & 2.1 & .0 & .0 & .1 & .0 & (s) & (s) & .1 & .1 & .1 & 25.0 \\
\hline 2004 & .3 & .0 & 2.9 & (s) & 16.7 & 4.4 & .1 & (s) & 2.2 & .0 & .1 & .3 & .1 & (s) & .1 & .6 & (s) & (s) & 27.3 \\
\hline 2005 & .2 & (s) & 2.0 & (s) & 21.2 & 3.7 & (s) & .0 & 2.5 & .0 & .1 & .4 & (s) & (s) & .1 & .6 & .1 & .1 & 30.5 \\
\hline 2006 & .2 & .0 & 2.0 & .0 & 25.3 & 4.2 & (s) & .0 & 3.1 & (s) & .0 & .9 & .0 & (s) & .2 & 1.1 & .1 & (s) & 36.2 \\
\hline 2007 & .1 & .1 & 2.0 & .0 & 26.9 & 3.4 & .1 & (s) & 3.7 & (s) & .0 & .1 & (s) & (s) & (s) & .2 & .0 & .1 & 36.3 \\
\hline 2008 & .1 & .0 & 2.0 & .0 & 26.3 & 2.3 & (s) & .0 & 3.4 & .0 & .0 & (s) & (s) & .0 & (s) & (s) & (s) & (s) & 34.2 \\
\hline 2009 & .2 & .0 & 1.3 & (s) & 17.8 & 1.3 & (s) & (s) & 2.1 & .0 & .0 & .0 & (s) & (s) & (s) & (s) & .0 & (s) & 22.6 \\
\hline 2010 & .4 & (s) & 1.8 & .0 & 14.6 & .6 & .1 & (s) & 1.9 & .0 & .0 & .0 & (s) & (s) & (s) & (s) & .0 & (s) & 19.4 \\
\hline $2011^{P}$ & .1 & .0 & 1.7 & (s) & 9.5 & .8 & (s) & (s) & .9 & .0 & (s) & (s) & .1 & .0 & (s) & .1 & (s) & .1 & 13.1 \\
\hline
\end{tabular}

$P=$ Preliminary. $(s)=$ Less than 0.05 million short tons.

Note: Totals may not equal sum of components due to independent rounding

Web Page: For related information, see http://www.eia.gov/coal/.

Sources: • 2000-U.S. Department of Commerce, Bureau of the Census, "Monthly Report IM 145." - 2001 forward-U.S. Energy Information Administration, Quarterly Coal Report October-December, quarterly reports. 
Figure 7.5 Coal Exports by Country of Destination

\section{Total and Europe, 1960-2011}
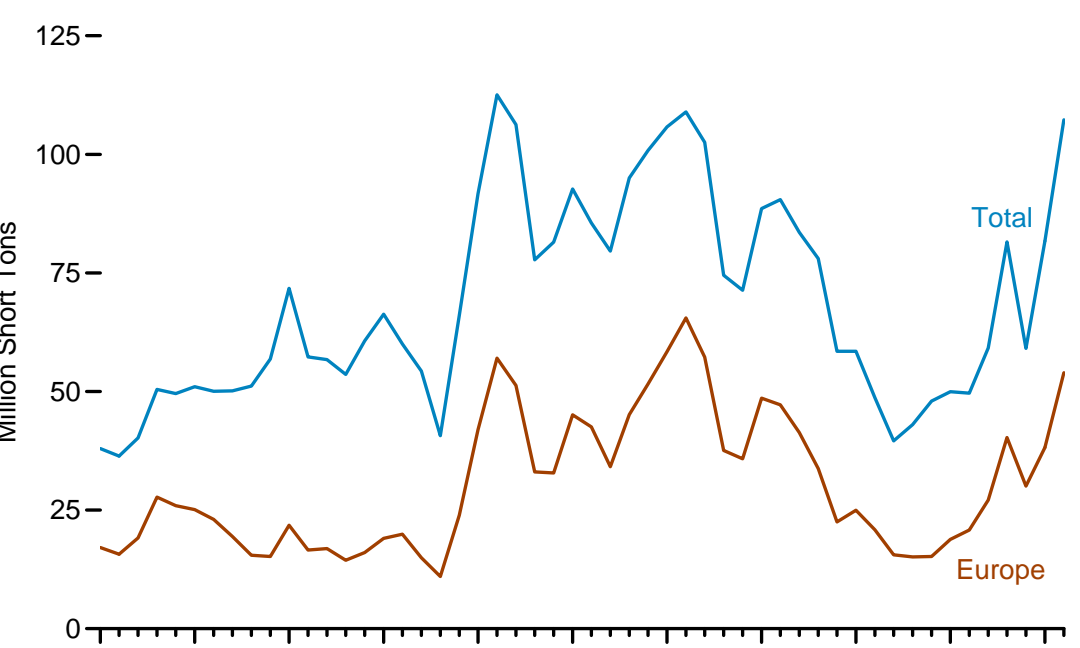

$\begin{array}{lllllllllllllllllllll}1960 & 1965 & 1970 & 1975 & 1980 & 1985 & 1990 & 1995 & 2000 & 2005 & 2010\end{array}$
By Selected Country, 2011

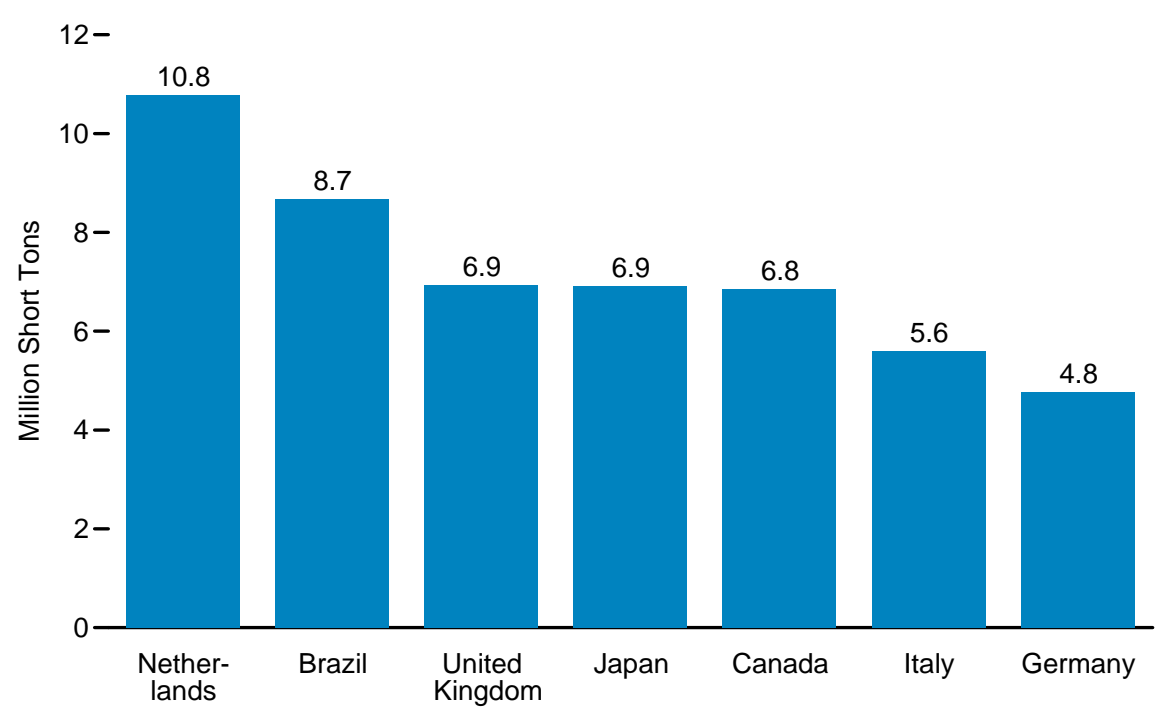

By Selected Country, 1960-2011

30-

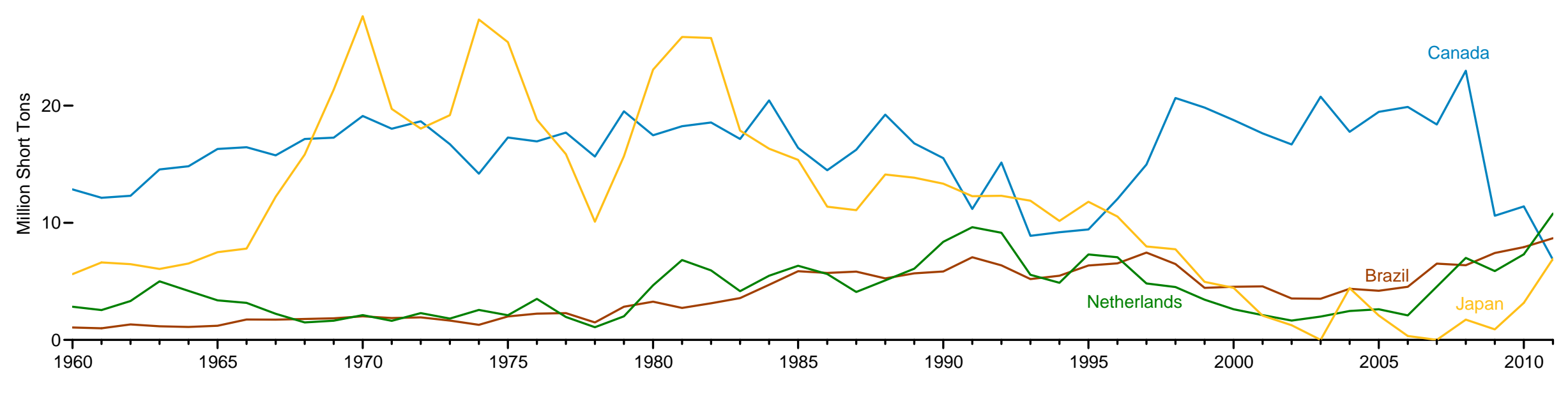

Source: Table 7.5 . 
Table 7.5 Coal Exports by Country of Destination, Selected Years, 1960-2011

(Million Short Tons)

\begin{tabular}{|c|c|c|c|c|c|c|c|c|c|c|c|c|c|c|c|c|}
\hline \multirow[b]{2}{*}{ Year } & \multirow[b]{2}{*}{ Canada } & \multirow[b]{2}{*}{ Brazil } & \multicolumn{11}{|c|}{ Europe } & \multirow[b]{2}{*}{ Japan } & \multirow[b]{2}{*}{ Other ${ }^{3}$} & \multirow[b]{2}{*}{ Total } \\
\hline & & & Belgium 1 & Denmark & France & Germany ${ }^{2}$ & Italy & $\begin{array}{l}\text { Nether- } \\
\text { lands }\end{array}$ & Spain & Turkey & $\begin{array}{c}\text { United } \\
\text { Kingdom }\end{array}$ & Other ${ }^{3}$ & Total & & & \\
\hline 1960 & 12.8 & 1.1 & 1.1 & 0.1 & 0.8 & 4.6 & 4.9 & 2.8 & 0.3 & NA & - & 2.4 & 17.1 & 5.6 & 1.3 & 38.0 \\
\hline 1965 & 16.3 & 1.2 & 2.2 & (s) & 2.1 & 4.7 & 9.0 & 3.4 & 1.4 & NA & (s) & 2.3 & 25.1 & 7.5 & .9 & 51.0 \\
\hline 1966 & 16.5 & 1.7 & 1.8 & (s) & 1.6 & 4.9 & 7.8 & 3.2 & 1.2 & NA & (s) & 2.5 & 23.1 & 7.8 & 1.0 & 50.1 \\
\hline 1967 & 15.8 & 1.7 & 1.4 & - & 2.1 & 4.7 & 5.9 & 2.2 & 1.0 & NA & - & 2.1 & 19.4 & 12.2 & 1.0 & 50.1 \\
\hline 1968 & 17.1 & 1.8 & 1.1 & - & 1.5 & 3.8 & 4.3 & 1.5 & 1.5 & NA & - & 1.9 & 15.5 & 15.8 & .9 & 51.2 \\
\hline 1969 & 17.3 & 1.8 & .9 & - & 2.3 & 3.5 & 3.7 & 1.6 & 1.8 & NA & - & 1.3 & 15.2 & 21.4 & 1.2 & 56.9 \\
\hline 1970 & 19.1 & 2.0 & 1.9 & - & 3.6 & 5.0 & 4.3 & 2.1 & 3.2 & NA & (s) & 1.8 & 21.8 & 27.6 & 1.2 & 71.7 \\
\hline 1971 & 18.0 & 1.9 & .8 & - & $\begin{array}{l}3.0 \\
3.2\end{array}$ & 2.9 & 2.7 & 1.6 & 2.6 & NA & 1.7 & 1.1 & $\begin{array}{l}1.0 \\
16.6\end{array}$ & 19.7 & 1.1 & 57.3 \\
\hline 1972 & 18.7 & 1.9 & 1.1 & - & 1.7 & 2.4 & 3.7 & 2.3 & 2.1 & NA & 2.4 & 1.1 & 16.9 & 18.0 & 1.2 & 56.7 \\
\hline 1973 & 16.7 & 1.6 & 1.2 & - & 2.0 & 1.6 & 3.3 & 1.8 & 2.2 & NA & .9 & 1.3 & 14.4 & 19.2 & 1.6 & 53.6 \\
\hline 1974 & 14.2 & 1.3 & 1.1 & - & 2.7 & 1.5 & 3.9 & 2.6 & 2.0 & NA & 1.4 & .9 & 16.1 & 27.3 & 1.8 & 60.7 \\
\hline 1975 & 17.3 & 2.0 & .6 & - & 3.6 & 2.0 & 4.5 & 2.1 & 2.7 & NA & 1.9 & 1.6 & 19.0 & 25.4 & $\begin{array}{l}2.0 \\
2.6\end{array}$ & 66.3 \\
\hline 1976 & 16.9 & 2.2 & 2.2 & (s) & 3.5 & $\begin{array}{l}1.0 \\
\text { S }\end{array}$ & 4.2 & 3.5 & 2.5 & NA & .8 & 2.1 & 19.9 & 18.8 & 2.1 & 60.0 \\
\hline 1977 & 17.7 & 2.3 & 1.5 & .1 & 2.1 & .9 & 4.1 & 2.0 & 1.6 & NA & .6 & 2.1 & 15.0 & 15.9 & 3.5 & 54.3 \\
\hline 1978 & 15.7 & 1.5 & 1.1 & - & 1.7 & .6 & 3.2 & $\begin{array}{l}1.1 \\
\text { S }\end{array}$ & .8 & NA & .4 & 2.2 & 11.0 & 10.1 & 2.5 & 40.7 \\
\hline 1979 & 19.5 & 2.8 & 3.2 & 2 & 3.9 & 2.6 & 5.0 & 2.0 & 1.4 & NA & 1.4 & 4.4 & 23.9 & 15.7 & 4.1 & 66.0 \\
\hline 1980 & 17.5 & 3.3 & 4.6 & 1.7 & 7.8 & 2.5 & $\begin{array}{l}.0 \\
7.1\end{array}$ & 4.7 & 3.4 & NA & 4.1 & 6.0 & 41.9 & 23.1 & 6.0 & 91.7 \\
\hline 1981 & 18.2 & 2.7 & 4.3 & 3.9 & 9.7 & 4.3 & 10.5 & 6.8 & $\begin{array}{l}6.4 \\
6.4\end{array}$ & .6 & 2.3 & 8.2 & 57.0 & 25.9 & 8.7 & 112.5 \\
\hline 1982 & 18.6 & 3.1 & 4.8 & 2.8 & 9.0 & 2.3 & 11.3 & 5.9 & 5.6 & 1.6 & 2.0 & 6.0 & 51.3 & 25.8 & 7.5 & 106.3 \\
\hline 1983 & $\begin{array}{l}17.0 \\
\end{array}$ & $\begin{array}{l}3.1 \\
3.6\end{array}$ & $\begin{array}{l}4.0 \\
2.5\end{array}$ & $\begin{array}{l}. .0 \\
1.7\end{array}$ & $\begin{array}{l}4.0 \\
4.2\end{array}$ & $\begin{array}{l}2.0 \\
1.5\end{array}$ & 8.1 & $\begin{array}{l}4.5 \\
4.2\end{array}$ & $\begin{array}{l}3.0 \\
3.3\end{array}$ & $\begin{array}{l}1.0 \\
\end{array}$ & $\begin{array}{l}1.0 \\
1.2\end{array}$ & $\begin{array}{l}4.0 \\
\end{array}$ & 33.1 & 17.9 & 6.1 & $\begin{array}{l}77.8 \\
\end{array}$ \\
\hline 1984 & 20.4 & 4.7 & 3.9 & .6 & 3.8 & .9 & 7.6 & 5.5 & 2.3 & 1.5 & 2.9 & 3.9 & 32.8 & 16.3 & 7.2 & 81.5 \\
\hline 1985 & 16.4 & 5.9 & 4.4 & 2.2 & 4.5 & 1.1 & 10.3 & 6.3 & 3.5 & 2.2 & 2.7 & 8.1 & 45.1 & 15.4 & 9.9 & 92.7 \\
\hline 1986 & 14.5 & 5.7 & 4.4 & 2.1 & 5.4 & .8 & 10.4 & 5.6 & 2.6 & 2.4 & 2.9 & 5.9 & 42.6 & 11.4 & 11.4 & 85.5 \\
\hline 1987 & $\begin{array}{l}16.2\end{array}$ & 5.8 & $\begin{array}{l}4.4 \\
4.6\end{array}$ & .9 & $\begin{array}{l}.4 \\
2.9\end{array}$ & .5 & 9.5 & $\begin{array}{l}4.0 \\
\end{array}$ & 2.5 & .8 & 2.6 & 5.8 & 34.2 & 11.1 & 12.3 & 79.6 \\
\hline 1988 & 19.2 & 5.3 & 6.5 & 2.8 & 4.3 & .7 & 11.1 & 5.1 & 2.5 & 2.0 & 3.7 & 6.4 & 45.1 & 14.1 & 11.3 & 95.0 \\
\hline 1989 & 16.8 & 5.7 & 7.1 & 3.2 & 6.5 & .7 & 11.2 & 6.1 & 3.3 & $\begin{array}{l}1.7 \\
\text { S }\end{array}$ & 4.5 & 7.2 & 51.6 & 13.8 & 12.9 & 100.8 \\
\hline 1990 & 15.5 & 5.8 & 8.5 & 3.2 & 6.9 & 1.1 & 11.9 & 8.4 & 3.8 & 2.1 & 5.2 & 7.4 & 58.4 & 13.3 & 12.7 & 105.8 \\
\hline 1991 & 11.2 & 7.1 & 7.5 & 4.7 & 9.5 & 1.7 & 11.3 & 9.6 & 4.7 & 2.2 & 6.2 & 8.2 & 65.5 & 12.3 & 13.0 & 109.0 \\
\hline 1992 & 15.1 & 6.4 & 7.2 & 3.8 & 8.1 & 1.0 & 9.3 & 9.1 & 4.5 & 2.0 & 5.6 & 6.6 & 57.3 & 12.3 & 11.4 & 102.5 \\
\hline 1993 & 8.9 & 5.2 & 5.2 & $\begin{array}{l}.0 \\
.3\end{array}$ & 4.0 & .5 & 6.9 & 5.6 & 4.1 & 1.6 & 4.1 & 5.3 & 37.6 & $\begin{array}{l}11.9 \\
\end{array}$ & 11.0 & 74.5 \\
\hline 1994 & 9.2 & 5.5 & 4.9 & .5 & 2.9 & .3 & 7.5 & 4.9 & 4.1 & 1.3 & 3.4 & 6.0 & 35.8 & 10.2 & 10.7 & 71.4 \\
\hline 1995 & 9.4 & 6.4 & 4.5 & 2.1 & 3.7 & 2.0 & 9.1 & $\begin{array}{l}7.5 \\
7.3\end{array}$ & 4.7 & 2.0 & $\begin{array}{l}4.4 \\
4.7\end{array}$ & $\begin{array}{l}8.0 \\
8.7\end{array}$ & $\begin{array}{l}48.0 \\
48.6\end{array}$ & $\begin{array}{l}11.8 \\
\end{array}$ & 12.4 & 88.5 \\
\hline 1996 & 12.0 & 6.5 & 4.6 & 1.3 & 3.9 & $\begin{array}{l}1.1\end{array}$ & 9.2 & 7.1 & 4.1 & 2.2 & 6.2 & 7.7 & 47.2 & 10.5 & $\begin{array}{l}14.2 \\
\end{array}$ & 90.5 \\
\hline 1997 & 15.0 & 7.5 & 4.3 & .4 & 3.4 & .9 & 7.0 & 4.8 & 4.1 & 2.1 & 7.2 & 7.1 & 41.3 & 8.0 & 11.8 & 83.5 \\
\hline 1998 & 20.7 & 6.5 & 3.2 & .3 & 3.2 & 1.2 & 5.3 & 4.5 & 3.2 & $\begin{array}{l}1.6 \\
\text { S }\end{array}$ & 5.9 & 5.3 & 33.8 & 7.7 & 9.4 & 78.0 \\
\hline 1999 & 19.8 & 4.4 & 2.1 & -0 & $\begin{array}{l}.24 \\
2.5\end{array}$ & .6 & $\begin{array}{l}.0 \\
4.0\end{array}$ & 3.4 & $\begin{array}{l}.4 \\
2.5\end{array}$ & .8 & 3.2 & 3.5 & 22.5 & 5.0 & $\begin{array}{l}3.4 \\
6.7\end{array}$ & 58.5 \\
\hline 2000 & 18.8 & 4.5 & 2.9 & .1 & 3.0 & 1.0 & 3.7 & 2.6 & 2.7 & 1.8 & 3.3 & 3.9 & 25.0 & 4.4 & 5.8 & 58.5 \\
\hline 2001 & 17.6 & 4.6 & 2.8 & - & 2.2 & .9 & 5.4 & 2.1 & $\begin{array}{l}1.6 \\
\text { S }\end{array}$ & .9 & 2.5 & 2.4 & 20.8 & 2.1 & 3.6 & 48.7 \\
\hline 2002 & 16.7 & 3.5 & 2.4 & - & $\begin{array}{l}1.3 \\
\end{array}$ & 1.0 & 3.1 & 1.7 & 1.9 & .6 & $\begin{array}{l}1.9 \\
\text { S }\end{array}$ & $\begin{array}{l}1.8 \\
\end{array}$ & $\begin{array}{l}15.6\end{array}$ & 1.3 & 2.6 & 39.6 \\
\hline 2003 & 20.8 & 3.5 & $\begin{array}{l}2.4 \\
1.8\end{array}$ & .3 & $\begin{array}{l}1.3 \\
1.3\end{array}$ & .5 & 2.8 & 2.0 & 1.8 & 1.1 & 1.5 & 2.1 & 15.1 & (s) & 3.6 & 43.0 \\
\hline 2004 & 17.8 & 4.4 & 1.7 & .1 & 1.1 & .6 & 2.1 & 2.5 & 1.5 & 1.3 & 2.0 & 2.3 & 15.2 & 4.4 & 6.2 & 48.0 \\
\hline 2005 & 19.5 & 4.2 & 2.1 & .1 & 1.3 & .7 & 2.5 & 2.6 & 1.9 & 1.9 & $\begin{array}{l}1.0 \\
\end{array}$ & 4.1 & 18.8 & $\begin{array}{l}4.4 \\
2.1\end{array}$ & 5.4 & 49.9 \\
\hline 2006 & 19.9 & 4.5 & 2.2 & .4 & 1.6 & 1.7 & 3.3 & 2.1 & 1.6 & 1.2 & 2.6 & 4.2 & 20.8 & .3 & 4.1 & 49.6 \\
\hline 2007 & 18.4 & 6.5 & 2.1 & $\begin{array}{l}.4 \\
.1\end{array}$ & 2.4 & 2.3 & $\begin{array}{l}.0 \\
3.5\end{array}$ & 4.6 & 1.5 & 1.4 & 3.4 & 5.8 & 27.1 & (s) & 7.1 & 59.2 \\
\hline 2008 & 23.0 & 6.4 & 3.1 & .4 & 3.5 & 2.5 & 3.2 & 7.0 & 2.4 & 1.9 & 5.8 & 10.6 & 40.3 & 1.7 & 10.1 & 81.5 \\
\hline 2009 & 10.6 & 7.4 & 2.7 & 3 & 3.4 & 2.5 & 2.3 & 5.9 & 1.7 & 1.4 & 4.6 & 5.3 & 30.1 & .9 & 10.1 & 59.1 \\
\hline 2010 & 11.4 & 7.9 & 2.3 & .1 & 3.2 & 2.7 & 3.3 & 7.3 & 1.9 & 2.5 & 4.4 & 10.5 & 38.2 & 3.2 & 21.0 & 81.7 \\
\hline $2011^{\mathrm{P}}$ & 6.8 & 8.7 & 3.1 & .2 & 4.0 & 4.8 & 5.6 & 10.8 & 1.8 & 2.9 & 6.9 & 13.9 & 53.9 & 6.9 & 30.9 & 107.3 \\
\hline
\end{tabular}

1 Through 1999, includes Luxembourg

2 Through 1990, data for Germany are for the former West Germany only. Beginning in 1991, data for Germany are for the unified Germany, i.e., the former East Germany and West Germany

See source publications for data for countries included in "Other."

$\mathrm{P}=$ Preliminary. $N A=$ Not Available. $-=$ No data reported. ( $\mathrm{s})=$ Less than 0.05 million short tons.
Note: Totals may not equal sum of components due to independent rounding.

Web Page: For all data beginning in 1960, see http://www.eia.gov/totalenergy/data/annual/\#coal.

Sources: - 1960-1988-U.S. Department of Commerce, Bureau of the Census, U.S. Exports by Schedule B Commodities, EM 522. - 1989-2000-U.S. Energy Information Administration (EIA), Coal Industry Annual, annual reports. - 2001 forward-EIA, Quarterly Coal Report October-December, 


\section{Figure 7.6 Coal Stocks, End of Year}

\section{Total and Electric Power Stocks, 1949-2011}

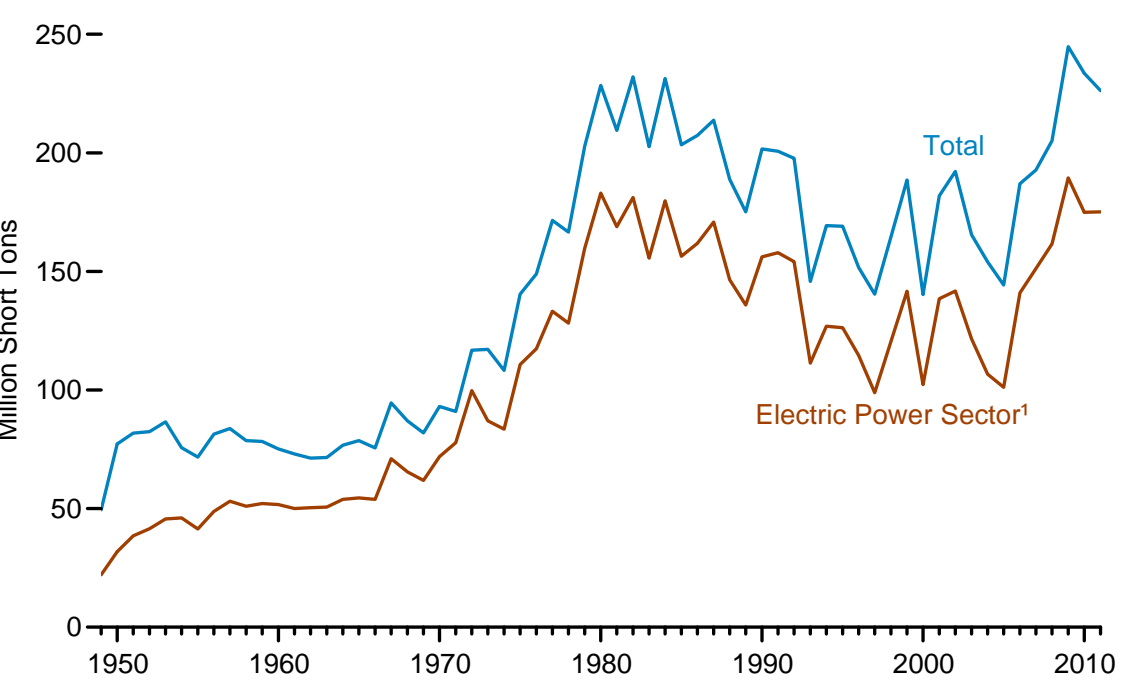

Electric Power, Producer/Distributor, and Industrial Stocks, 2011

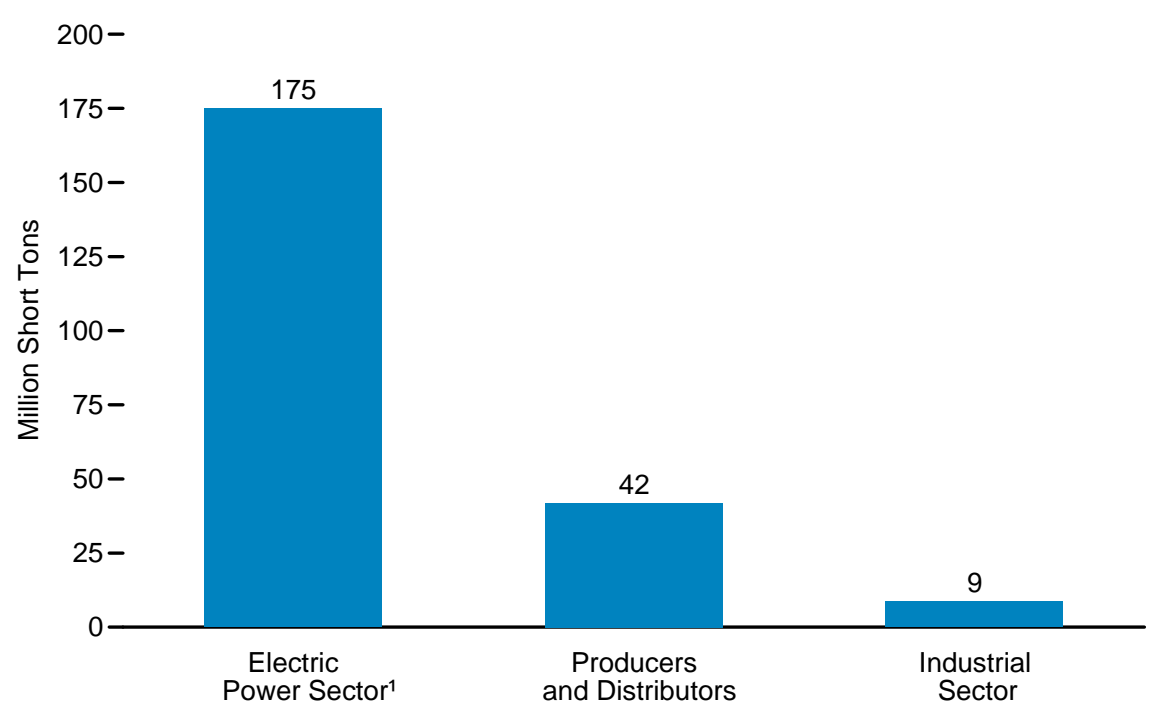

Electric Power, Producer/Distributor, and Industrial Stocks, 1949-2011

$200-$

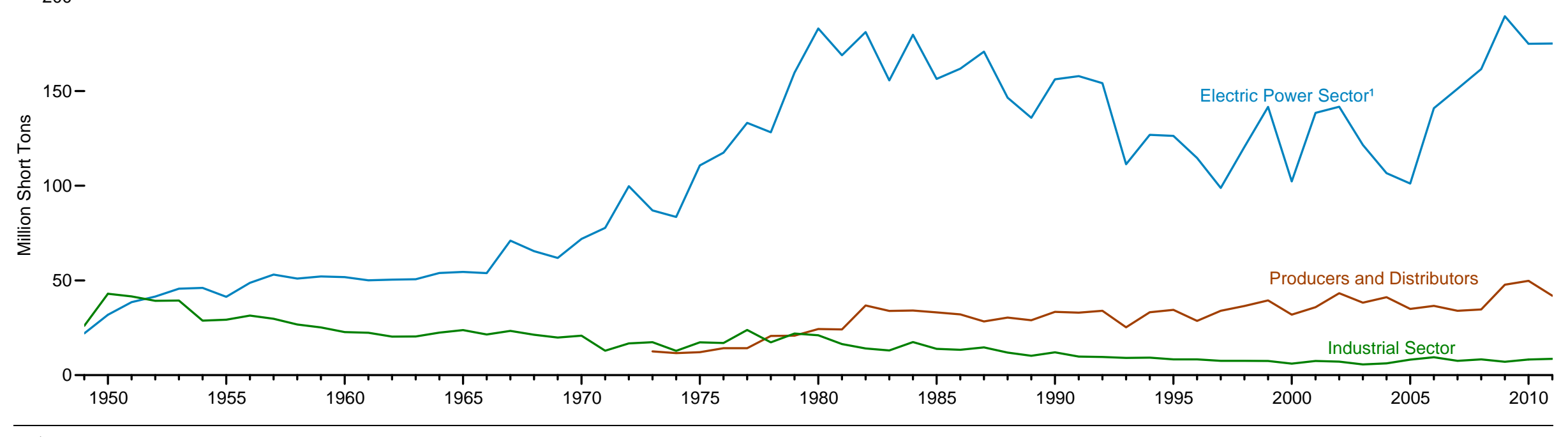

${ }^{1}$ Electricity-only and combined-heat-and-power (CHP) plants whose primary business is to sell electricity, or electricity and heat, to the public. 
Table 7.6 Coal Stocks by Sector, Selected Years, End of Year 1949-2011

(Million Short Tons)

\begin{tabular}{|c|c|c|c|c|c|c|c|c|c|}
\hline \multirow[b]{3}{*}{ Year } & \multirow{3}{*}{$\begin{array}{c}\text { Producers } \\
\text { and } \\
\text { Distributors }\end{array}$} & \multicolumn{7}{|c|}{ Consumers } & \multirow[b]{3}{*}{ Total } \\
\hline & & \multirow{2}{*}{$\begin{array}{c}\text { Residential } \\
\text { and Commercial } \\
\text { Sectors }\end{array}$} & \multicolumn{3}{|c|}{ Industrial Sector } & \multirow{2}{*}{$\begin{array}{l}\text { Transportation } \\
\text { Sector }\end{array}$} & \multirow{2}{*}{$\begin{array}{l}\text { Electric } \\
\text { Power } \\
\text { Sector }{ }^{2}\end{array}$} & \multirow[b]{2}{*}{ Total } & \\
\hline & & & Coke Plants & Other ${ }^{1}$ & Total & & & & \\
\hline $\begin{array}{l}1949 \\
1950\end{array}$ & $\begin{array}{l}\text { NA } \\
\text { NA }\end{array}$ & $\begin{array}{l}1.4 \\
2.5\end{array}$ & $\begin{array}{l}10.0 \\
16.8\end{array}$ & $\begin{array}{l}16.1 \\
26.2\end{array}$ & $\begin{array}{l}26.0 \\
43.0\end{array}$ & $\begin{array}{l}\left(\begin{array}{l}3 \\
(\end{array}\right) \\
\left(\begin{array}{l}3 \\
)\end{array}\right.\end{array}$ & $\begin{array}{l}22.1 \\
31.8\end{array}$ & $\begin{array}{l}49.5 \\
77.3\end{array}$ & $\begin{array}{l}49.5 \\
77.3\end{array}$ \\
\hline 1955 & NA & $\begin{array}{l}2.0 \\
1.0\end{array}$ & $\begin{array}{l}10.0 \\
13.4\end{array}$ & 15.9 & $\begin{array}{l}43.0 \\
29.3\end{array}$ & $\left({ }^{3}\right)$ & 41.4 & $\begin{array}{l}17.3 \\
71.7\end{array}$ & $\begin{array}{l}17.3 \\
71.7\end{array}$ \\
\hline 1960 & NA & .7 & 11.1 & 11.6 & 22.8 & $\left({ }^{3}\right)$ & 51.7 & 75.2 & 75.2 \\
\hline 1965 & NA & .4 & 10.6 & 13.1 & 23.8 & $(3)$ & 54.5 & 78.6 & 78.6 \\
\hline 1970 & NA & .3 & 9.0 & 11.8 & 20.8 & $\left({ }^{3}\right)$ & 71.9 & 93.0 & 93.0 \\
\hline 1975 & 12.1 & .2 & 8.8 & 8.5 & 17.3 & $\left({ }^{3}\right)$ & 110.7 & 128.3 & 140.4 \\
\hline 1976 & 14.2 & .2 & 9.9 & 7.1 & 17.0 & $\left({ }^{3}\right)$ & 117.4 & 134.7 & 148.9 \\
\hline 1977 & 14.2 & .2 & 12.8 & 11.1 & 23.9 & $\left(3^{\prime}\right)$ & 133.2 & 157.3 & 171.5 \\
\hline 1978 & 20.7 & .4 & 8.3 & 9.0 & 17.3 & NA' & 128.2 & 145.9 & 166.6 \\
\hline 1979 & 20.8 & .3 & 10.2 & 11.8 & 21.9 & NA & 159.7 & 182.0 & 202.8 \\
\hline 1980 & 24.4 & NA & 9.1 & 12.0 & 21.0 & NA & 183.0 & 204.0 & 228.4 \\
\hline 1981 & 24.1 & NA & 6.5 & 9.9 & 16.4 & NA & 168.9 & 185.3 & 209.4 \\
\hline 1982 & 36.8 & NA & 4.6 & 9.5 & 14.1 & NA & 181.1 & 195.3 & 232.0 \\
\hline 1983 & 33.9 & NA & 4.3 & 8.7 & 13.1 & NA & 155.6 & 168.7 & 202.6 \\
\hline 1984 & 34.1 & NA & 6.2 & 11.3 & 17.5 & NA & 179.7 & 197.2 & 231.3 \\
\hline 1985 & 33.1 & NA & 3.4 & 10.4 & 13.9 & NA & 156.4 & 170.2 & 203.4 \\
\hline 1986 & 32.1 & NA & 3.0 & 10.4 & 13.4 & NA & 161.8 & 175.2 & 207.3 \\
\hline 1987 & 28.3 & NA & 3.9 & 10.8 & 14.7 & NA & 170.8 & 185.5 & 213.8 \\
\hline 1988 & 30.4 & NA & 3.1 & 8.8 & 11.9 & NA & 146.5 & 158.4 & 188.8 \\
\hline 1989 & 29.0 & NA & 2.9 & 7.4 & 10.2 & NA & 135.9 & 146.1 & 175.1 \\
\hline 1990 & 33.4 & NA & 3.3 & 8.7 & 12.0 & NA & 156.2 & 168.2 & 201.6 \\
\hline 1991 & 33.0 & NA & 2.8 & 7.1 & 9.8 & NA & 157.9 & 167.7 & 200.7 \\
\hline 1992 & 34.0 & NA & 2.6 & 7.0 & 9.6 & NA & 154.1 & 163.7 & 197.7 \\
\hline 1993 & 25.3 & NA & 2.4 & 6.7 & 9.1 & NA & 111.3 & 120.5 & 145.7 \\
\hline 1994 & 33.2 & NA & 2.7 & 6.6 & 9.2 & NA & 126.9 & 136.1 & 169.4 \\
\hline 1995 & 34.4 & NA & 2.6 & 5.7 & 8.3 & NA & 126.3 & 134.6 & 169.1 \\
\hline 1996 & 28.6 & NA & 2.7 & 5.7 & 8.4 & NA & 114.6 & 123.0 & 151.6 \\
\hline 1997 & 34.0 & NA & 2.0 & 5.6 & 7.6 & NA & 98.8 & 106.4 & 140.4 \\
\hline 1998 & 36.5 & NA & 2.0 & 5.5 & 7.6 & NA & 120.5 & 128.1 & 164.6 \\
\hline 1999 & 39.5 & NA & 1.9 & 5.6 & 7.5 & NA & 141.6 & 149.1 & 188.6 \\
\hline 2000 & 31.9 & NA & 1.5 & 4.6 & 6.1 & NA & 102.3 & 108.4 & 140.3 \\
\hline 2001 & 35.9 & NA & 1.5 & 6.0 & 7.5 & NA & 138.5 & 146.0 & 181.9 \\
\hline 2002 & 43.3 & NA & 1.4 & 5.8 & 7.2 & NA & 141.7 & 148.9 & 192.1 \\
\hline 2003 & 38.3 & NA & .9 & 4.7 & 5.6 & NA & 121.6 & 127.2 & 165.5 \\
\hline 2004 & 41.2 & NA & 1.3 & 4.8 & 6.2 & NA & 106.7 & 112.9 & 154.0 \\
\hline 2005 & 35.0 & NA & 2.6 & 5.6 & 8.2 & NA & 101.1 & 109.3 & 144.3 \\
\hline 2006 & 36.5 & NA & 2.9 & 6.5 & 9.4 & NA & 141.0 & 150.4 & 186.9 \\
\hline 2007 & 34.0 & NA & 1.9 & 5.6 & 7.6 & NA & 151.2 & 158.8 & 192.8 \\
\hline 2008 & 34.7 & .5 & 2.3 & 6.0 & 8.3 & & 161.6 & 170.4 & 205.1 \\
\hline 2009 & 47.7 & .5 & 2.0 & 5.1 & 7.1 & NA & 189.5 & 197.1 & 244.8 \\
\hline 2010 & ${ }^{R} 49.8$ & .6 & 1.9 & ${ }^{R} 6.3$ & R8.3 & NA & $R_{174.9}$ & $\mathrm{R}_{183.7}$ & $R_{233.6}$ \\
\hline 2011 & $\mathrm{E}_{41.9}$ & P. 6 & $P_{2.6}$ & P6.0 & P8.6 & NA & $P_{175.1}$ & $P_{184.3}$ & $P_{226.2}$ \\
\hline
\end{tabular}

1 Through 1977, data are for stocks held by the manufacturing and transportation sectors. Beginning in 1978, data are for stocks held at manufacturing plants only.

2lectricty-only and combined-heat-and-power (CHP) plants within the NAICS 22 category whose primary business is to sell electricity, or electricity and heat, to the public. Through 1998, data are for electric utilities only; beginning in 1999, data are for electric utilities and independent power producers. 3 Included in "Industrial Sector Other."

Rented. PA=Preliminary. E=Estimate. NA available.

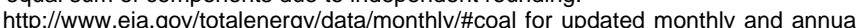

data. - See http://www.eia.gov/totalenergy/data/annual/\#coal for all annual data beginning in 1949. • See

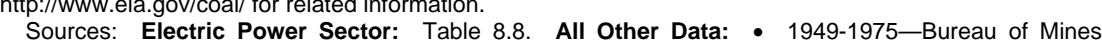
Minerals Yearbook, "Coal-Bituminous and Lignite" and "Coal-Pennsylvania Anthracite" chapters - 1976-U.S. Energy Information Administration (EIA), Energy Data Reports, Coal-Bituminous and Lignite in 1976 and Coal-Pennsylvania Anthracite 1976. - 1977 and 1978-EIA, Energy Data Reports, Coal Pennylvania Anthacle 1977; 1978, and Weekly Coal Report. 1978 - ElA, Energy Data Report, reports. • 2005 forward-EIA, QCR October-December 2011 (April 2012), Table 37. 


\section{Figure 7.7 Coal Mining Productivity}

\section{Total, 1949-2011}

8-

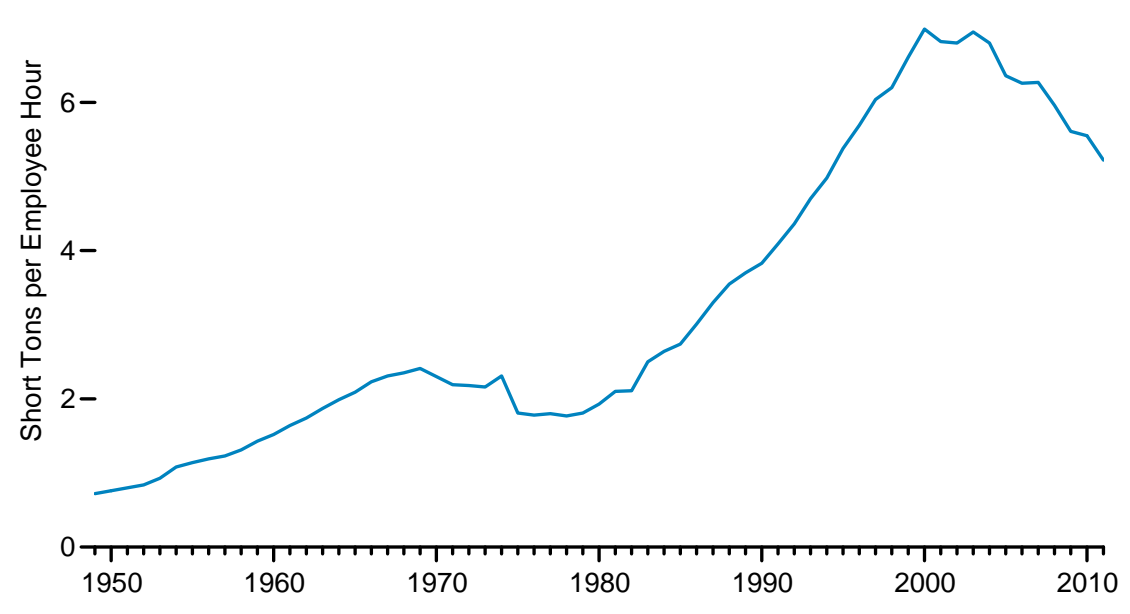

\section{By Mining Method, ${ }^{1}{ }^{1949-2011}$}

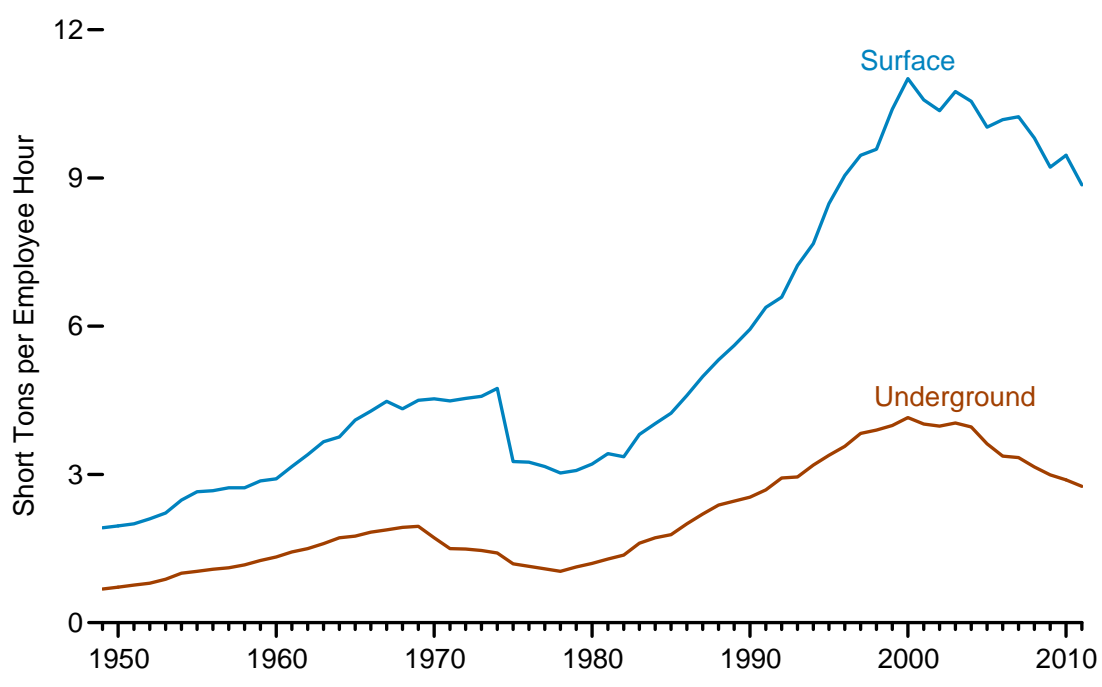

${ }^{1}$ For 1979 forward, includes all coal; prior to 1979 , excludes anthracite.
Note: Beginning in 2001, surface mining includes a small amount of refuse recovery.
By Mining Method, 2011

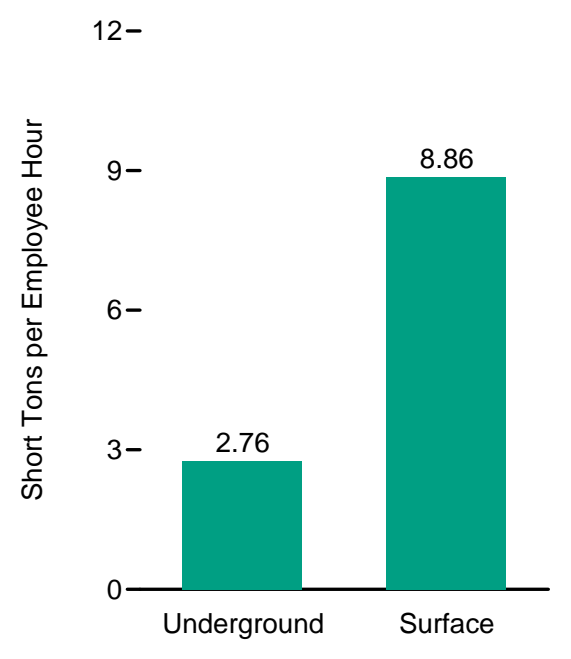

By Location, 2011

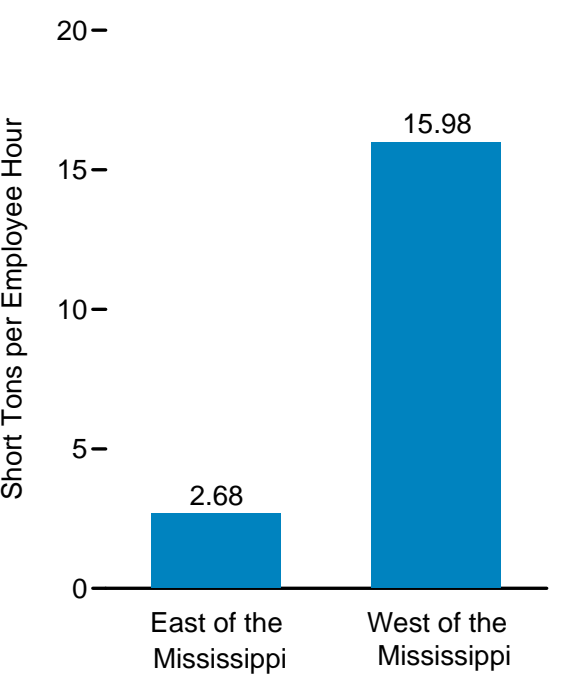

By Region and Mining Method, 2011

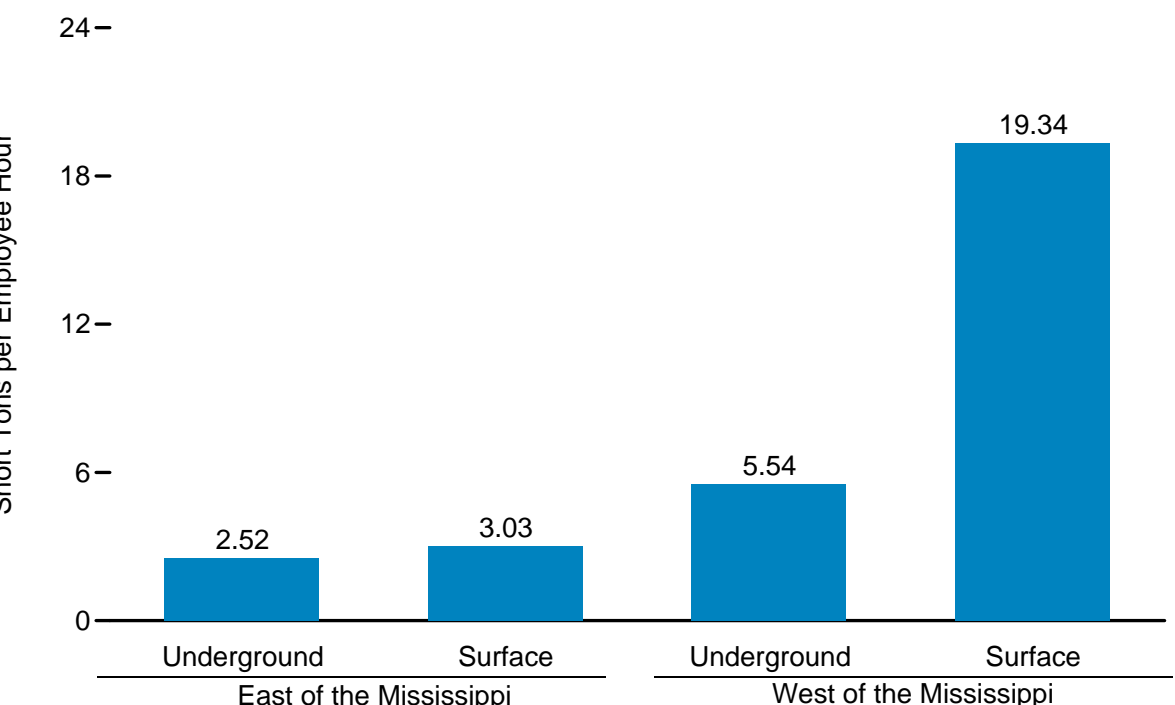
Source: Table 7.7 . 
Table 7.7 Coal Mining Productivity, Selected Years, 1949-2011

(Short Tons per Employee Hour ${ }^{1}$ )

\begin{tabular}{|c|c|c|c|c|c|c|c|c|c|}
\hline \multirow[b]{3}{*}{ Year } & \multicolumn{2}{|c|}{ Mining Method } & \multicolumn{6}{|c|}{ Location } & \multirow[b]{3}{*}{ Total $^{2}$} \\
\hline & \multirow[b]{2}{*}{ Underground } & \multirow[b]{2}{*}{ Surface $^{2}$} & \multicolumn{3}{|c|}{ East of the Mississippi } & \multicolumn{3}{|c|}{ West of the Mississippi } & \\
\hline & & & Underground & Surface $^{2}$ & Total $^{2}$ & Underground & Surface $^{2}$ & Total $^{2}$ & \\
\hline $\begin{array}{l}1949 \\
1950\end{array}$ & $\begin{array}{r}30.68 \\
3.72\end{array}$ & $\begin{array}{l}31.92 \\
31.96\end{array}$ & $\begin{array}{l}\text { NA } \\
\text { NA }\end{array}$ & $\begin{array}{l}\text { NA } \\
\text { NA }\end{array}$ & $\begin{array}{l}\text { NA } \\
\text { NA }\end{array}$ & $\begin{array}{l}\text { NA } \\
\text { NA }\end{array}$ & $\begin{array}{l}\text { NA } \\
\text { NA }\end{array}$ & $\begin{array}{l}\text { NA } \\
\text { NA }\end{array}$ & $\begin{array}{r}0.72 \\
.76\end{array}$ \\
\hline 1955 & 31.04 & 32.65 & NA & NA & NA & NA & NA & NA & 1.14 \\
\hline 1960 & 31.33 & 32.91 & NA & NA & NA & NA & NA & NA & 1.52 \\
\hline 1965 & 31.75 & 34.10 & NA & NA & NA & NA & NA & NA & 2.09 \\
\hline 1970 & 31.72 & 34.53 & NA & NA & NA & NA & NA & NA & 2.30 \\
\hline 1975 & 31.19 & 33.26 & NA & NA & NA & NA & NA & NA & 1.81 \\
\hline 1976 & 31.14 & 33.25 & NA & NA & NA & NA & NA & NA & 1.78 \\
\hline 1977 & 31.09 & 33.16 & NA & NA & NA & NA & NA & NA & 1.80 \\
\hline 1978 & ${ }^{31.04}$ & 33.03 & NA & NA & NA & NA & NA & NA & 1.77 \\
\hline 1979 & 1.13 & 3.08 & NA & NA & NA & NA & NA & NA & 1.81 \\
\hline 1980 & 1.20 & 3.21 & NA & NA & NA & NA & NA & NA & 1.93 \\
\hline 1981 & 1.29 & 3.42 & NA & NA & NA & NA & NA & NA & 2.10 \\
\hline 1982 & 1.37 & 3.36 & NA & NA & NA & NA & NA & NA & 2.11 \\
\hline 1983 & 1.61 & 3.81 & NA & NA & NA & NA & NA & NA & 2.50 \\
\hline 1984 & 1.72 & 4.03 & 1.69 & 2.56 & 1.98 & 2.49 & 8.15 & 7.07 & 2.64 \\
\hline 1985 & 1.78 & 4.24 & 1.75 & 2.52 & 2.00 & 2.45 & 8.61 & 7.40 & 2.74 \\
\hline 1986 & 2.00 & 4.60 & 1.96 & 2.75 & 2.21 & 2.80 & 9.02 & 7.90 & 3.01 \\
\hline 1987 & 2.20 & 4.98 & 2.16 & 2.97 & 2.42 & 3.39 & $\begin{array}{l}9.86 \\
\end{array}$ & 8.73 & 3.30 \\
\hline 1988 & 2.38 & 5.32 & 2.32 & 2.99 & 2.54 & 3.55 & 10.73 & 9.38 & 3.55 \\
\hline 1989 & 2.46 & 5.61 & 2.39 & 3.13 & 2.63 & 3.92 & 11.86 & 10.21 & 3.70 \\
\hline 1990 & 2.54 & 5.94 & 2.46 & 3.32 & 2.73 & 4.01 & 12.26 & 10.41 & 3.83 \\
\hline 1991 & 2.69 & 6.38 & 2.59 & 3.49 & 2.86 & 4.53 & 12.36 & 10.79 & 4.09 \\
\hline 1992 & 2.93 & 6.59 & 2.82 & 3.61 & 3.07 & 4.85 & 12.49 & 11.03 & 4.36 \\
\hline 1993 & 2.95 & 7.23 & 2.81 & 3.74 & 3.11 & 5.18 & 13.94 & 12.14 & 4.70 \\
\hline 1994 & 3.19 & 7.67 & 3.02 & 3.85 & 3.28 & 5.93 & 15.19 & 13.22 & 4.98 \\
\hline 1995 & 3.39 & 8.48 & 3.19 & 4.03 & 3.45 & 6.32 & 16.23 & 14.18 & 5.38 \\
\hline 1996 & 3.57 & 9.05 & 3.36 & 4.25 & 3.63 & 7.03 & 17.89 & 15.66 & 5.69 \\
\hline 1997 & 3.83 & 9.46 & 3.63 & 4.49 & 3.89 & 6.82 & 18.63 & 16.04 & 6.04 \\
\hline 1998 & 3.90 & 9.58 & 3.69 & 4.31 & 3.89 & 6.76 & 18.82 & 16.27 & 6.20 \\
\hline 1999 & 3.99 & 10.39 & 3.74 & 4.48 & 3.97 & 7.45 & 19.57 & 17.18 & 6.61 \\
\hline 2000 & 4.15 & 11.01 & 3.89 & 4.82 & 4.18 & 7.66 & 20.04 & 17.62 & 6.99 \\
\hline 2001 & 4.02 & 210.58 & 3.71 & ${ }^{2} 4.53$ & 23.98 & 8.39 & ${ }^{2} 20.63$ & 218.32 & ${ }^{2} 6.82$ \\
\hline 2002 & 3.98 & 10.36 & 3.67 & 4.22 & 3.86 & 7.80 & 20.67 & 18.06 & 6.80 \\
\hline 2003 & 4.04 & 10.75 & 3.68 & 4.18 & 3.85 & 8.33 & 21.42 & 18.67 & 6.95 \\
\hline 2004 & 3.96 & 10.55 & 3.59 & 3.95 & 3.72 & 8.22 & 22.04 & 19.00 & 6.80 \\
\hline 2005 & 3.62 & 10.03 & 3.28 & 3.75 & 3.44 & 7.48 & 21.98 & 18.50 & 6.36 \\
\hline 2006 & 3.37 & 10.18 & 3.06 & 3.74 & 3.29 & 6.62 & 22.26 & 18.33 & 6.26 \\
\hline 2007 & 3.34 & 10.24 & 3.03 & 3.74 & 3.27 & 6.52 & 22.35 & 18.23 & 6.27 \\
\hline 2008 & 3.15 & 9.81 & 2.87 & 3.58 & 3.12 & 6.07 & 21.85 & 17.77 & 5.96 \\
\hline 2009 & 2.99 & 9.22 & 2.74 & 3.33 & 2.94 & 5.51 & 19.85 & 16.15 & 5.61 \\
\hline 2010 & $R_{2.89}$ & R9.46 & $R_{2.66}$ & 3.24 & $\mathrm{R}_{2.84}$ & $R_{5} .54$ & $\mathrm{R}_{20.25}$ & 16.60 & $R_{5} .55$ \\
\hline $2011^{P}$ & 2.76 & 8.86 & 2.52 & 3.03 & 2.68 & 5.54 & 19.34 & 15.98 & 5.22 \\
\hline
\end{tabular}

1 Data through 1973 for bituminous coal, subbituminous coal, and lignite, and data through 1978 for anthracite, were originally reported in short tons per employee day-these data were converted to short tons per employee hour by assuming an eight-hour day. Through 1997, other data were calculated by dividing total production by total labor hours worked by all mine employees except office workers; beginning

in 1998, the calculation also includes office workers.

2 Beginning in 2001, includes a small amount of refuse recovery.

3 Through 1978, data for anthracite are not available by mining method, but are included in "Total."

R=Revised. P=Preliminary. NA=Not available. Web Pages: - For all data beginning in 1949 , see http://www.eia.gov/totalenergy/data/annual/\#coal.
- For related information, see http://www.eia.gov/coal/.

Sources: - 1949-1975-Bureau of Mines, Minerals Yearbook, "Coal-Bituminous and Lignite" and "Coal-Pennsylvania Anthracite" chapters. • 1976-U.S. Energy Information Administration (EIA), Energy Data Reports, Coal-Bituminous and Lignite in 1976 and Coal-Pennsylvania Anthracite 1976. • 1977 and 1978-EIA, Energy Data Reports, Bituminous Coal and Lignite Production and Mine Operations-1977; 1978 and Coal-Pennsylvania Anthracite 1977; 1978. • 1979-EIA, Energy Data Report, Coal Production-1979. • 1980-1988-EIA, Coal Production, annual reports. • 1989-2000-EIA, Coal Industry Annual, annual reports. - 2001-2010-EIA, Annual Coal Report, annual reports. ( 2011-EIA, Form EIA-7A, "Coal Production Report," and U.S. Department of Labor, Mine Safety and 
Figure 7.8 Coke Overview

\section{Production and Consumption, 1949-2011}

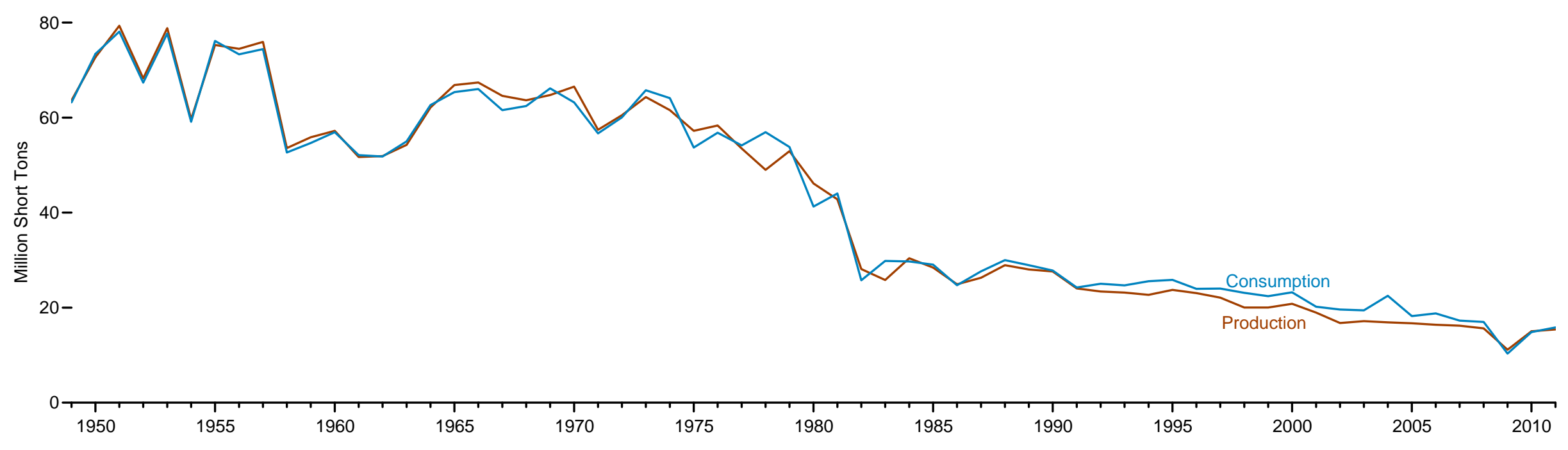

\section{Overview, 2011}

\section{Trade}

20-

8-
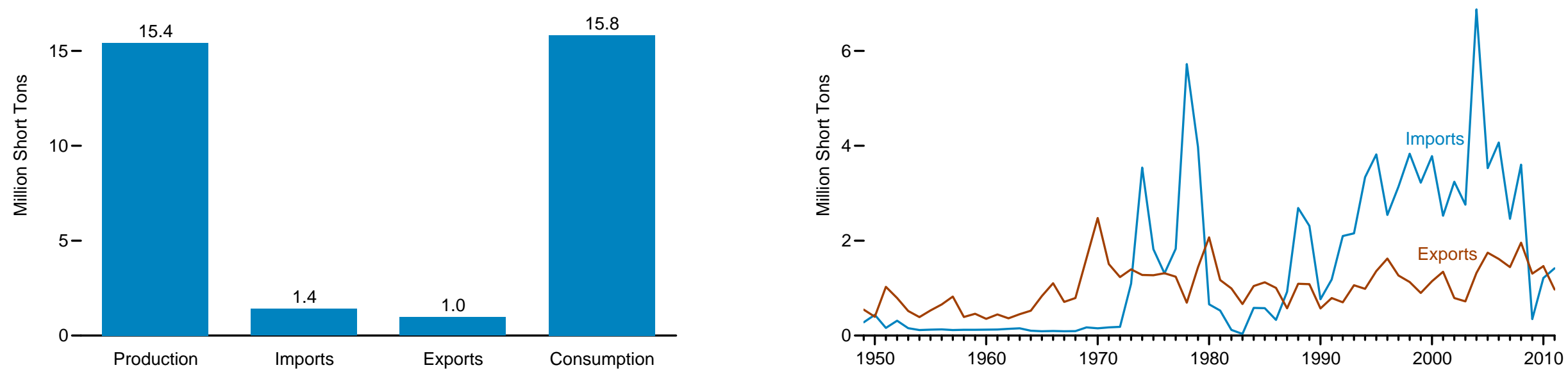

Source: Table 7.8 . 
Table 7.8 Coke Overview, Selected Years, 1949-2011

(Million Short Tons)

\begin{tabular}{|c|c|c|c|c|c|c|}
\hline \multirow[b]{2}{*}{ Year } & \multirow[b]{2}{*}{ Production } & \multicolumn{3}{|c|}{ Trade } & \multirow[b]{2}{*}{ Stock Change ${ }^{2}$} & \multirow[b]{2}{*}{ Consumption ${ }^{3}$} \\
\hline & & Imports & Exports & Net Imports ${ }^{1}$ & & \\
\hline 1949 & 63.6 & 0.3 & 0.5 & -0.3 & 0.2 & 63.2 \\
\hline 1950 & 72.7 & .4 & .4 & (s) & -.7 & 73.4 \\
\hline 1955 & 75.3 & .1 & .5 & -.4 & -1.2 & 76.1 \\
\hline 1960 & 57.2 & .1 & .4 & -.2 & .1 & 56.9 \\
\hline 1965 & 66.9 & .1 & .8 & -.7 & .7 & 65.4 \\
\hline 1970 & 66.5 & .2 & 2.5 & -2.3 & 1.0 & 63.2 \\
\hline 1975 & 57.2 & 1.8 & 1.3 & .5 & 4.1 & 53.7 \\
\hline 1976 & 58.3 & 1.3 & 1.3 & (s) & 1.5 & 56.8 \\
\hline 1977 & 53.5 & 1.8 & 1.2 & .6 & (s) & 54.1 \\
\hline 1978 & 49.0 & 5.7 & .7 & 5.0 & -2.9 & 56.9 \\
\hline 1979 & 52.9 & 4.0 & 1.4 & 2.5 & 1.7 & 53.8 \\
\hline 1980 & 46.1 & .7 & 2.1 & -1.4 & 3.4 & 41.3 \\
\hline 1981 & 42.8 & .5 & 1.2 & -.6 & -1.9 & 44.0 \\
\hline 1982 & 28.1 & .1 & 1.0 & -.9 & 1.5 & 25.8 \\
\hline 1983 & 25.8 & (s) & .7 & -.6 & -4.7 & 29.9 \\
\hline 1984 & 30.4 & .6 & 1.0 & -.5 & .2 & 29.7 \\
\hline 1985 & 28.4 & .6 & 1.1 & -.5 & -1.2 & 29.1 \\
\hline 1986 & 24.9 & .3 & 1.0 & -.7 & -.5 & 24.7 \\
\hline 1987 & 26.3 & .9 & .6 & .3 & -1.0 & 27.7 \\
\hline 1988 & 28.9 & 2.7 & 1.1 & 1.6 & .5 & 30.0 \\
\hline 1989 & 28.0 & 2.3 & 1.1 & 1.2 & .3 & 28.9 \\
\hline 1990 & 27.6 & .8 & .6 & .2 & (s) & 27.8 \\
\hline 1991 & 24.0 & 1.2 & .8 & .4 & .2 & 24.2 \\
\hline 1992 & 23.4 & 2.1 & .7 & 1.4 & -.2 & 25.0 \\
\hline 1993 & 23.2 & 2.2 & 1.1 & 1.1 & -.4 & 24.7 \\
\hline 1994 & 22.7 & 3.3 & 1.0 & 2.4 & -.5 & 25.6 \\
\hline 1995 & 23.7 & 3.8 & 1.4 & 2.5 & .4 & 25.8 \\
\hline 1996 & 23.1 & 2.5 & 1.6 & .9 & (s) & 24.0 \\
\hline 1997 & 22.1 & 3.1 & 1.3 & 1.9 & (s) & 24.0 \\
\hline 1998 & 20.0 & 3.8 & 1.1 & 2.7 & -.4 & 23.1 \\
\hline 1999 & 20.0 & 3.2 & .9 & 2.3 & -.1 & 22.4 \\
\hline 2000 & 20.8 & 3.8 & 1.1 & 2.6 & .2 & 23.2 \\
\hline 2001 & 18.9 & 2.5 & 1.3 & 1.2 & -.1 & 20.2 \\
\hline 2002 & 16.8 & 3.2 & .8 & 2.5 & -.4 & 19.6 \\
\hline 2003 & 17.2 & 2.8 & .7 & 2.0 & -.2 & 19.4 \\
\hline 2004 & 16.9 & 6.9 & 1.3 & 5.6 & (s) & 22.5 \\
\hline 2005 & 16.7 & 3.5 & 1.7 & 1.8 & .3 & 18.2 \\
\hline 2006 & 16.4 & 4.1 & 1.6 & 2.5 & .1 & 18.8 \\
\hline 2007 & 16.2 & 2.5 & 1.4 & 1.0 & -.1 & 17.3 \\
\hline 2008 & 15.6 & 3.6 & 2.0 & 1.6 & .3 & 17.0 \\
\hline 2009 & 11.1 & .3 & 1.3 & -1.0 & -.1 & 10.3 \\
\hline 2010 & 15.0 & 1.2 & 1.5 & -.2 & -.1 & 14.8 \\
\hline $2011^{P}$ & 15.4 & 1.4 & 1.0 & .4 & (s) & 15.8 \\
\hline
\end{tabular}

1 Net imports equal imports minus exports. Minus sign indicates exports are greater than imports. 2 Producer and distributor stocks at end of year. A negative value indicates a decrease in stocks; a positive value indicates an increase.

"Consumption" is calculated as the sum of production and imports minus exports and stock change. $\mathrm{P}=$ Preliminary. $(\mathrm{s})=$ Less than 0.05 million short tons.

Note: Totals may not equal sum of components due to independent rounding.

Web Pages: • For all data beginning in 1949, see http://www.eia.gov/totalenergy/data/annual/\#coal. - For related information, see $\mathrm{http}: / / \mathrm{ww} w$. eia.gov/coal/.

Sources: - 1949-1975-Bureau of Mines, Minerals Yearbook, "Coke and Coal Chemicals" chapter. - 1976-1980-U.S. Energy Information Administration (EIA), Energy Data Report, Coke and Coal Chemicals, annual reports. - 1981-2004-EIA, Quarterly Coal Report (QCR) October-December, quarterly reports. • 2005 forward-EIA, QCR October-December 2011 (April 2012), Table ES-2. 
Figure 7.9 Coal Prices

\section{Total, 1949-2011}

80-

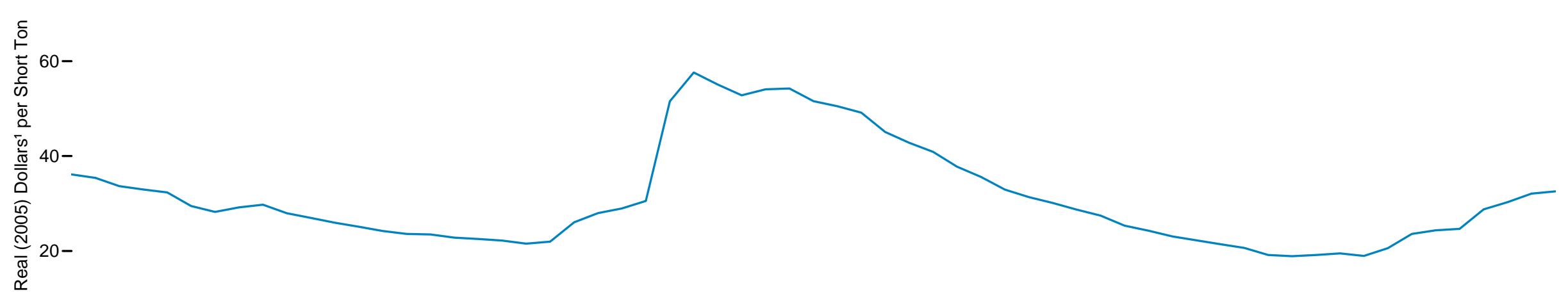

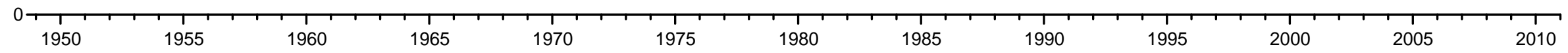

\section{Ву Tyрe, 1949-2011}

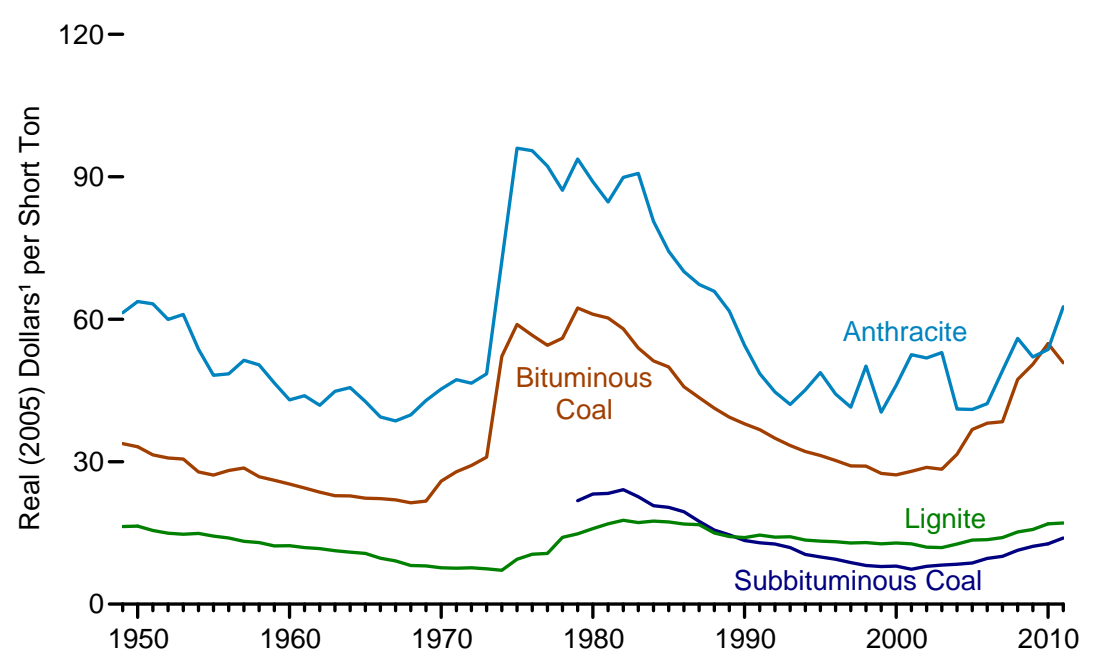

\section{By Type, 2011}

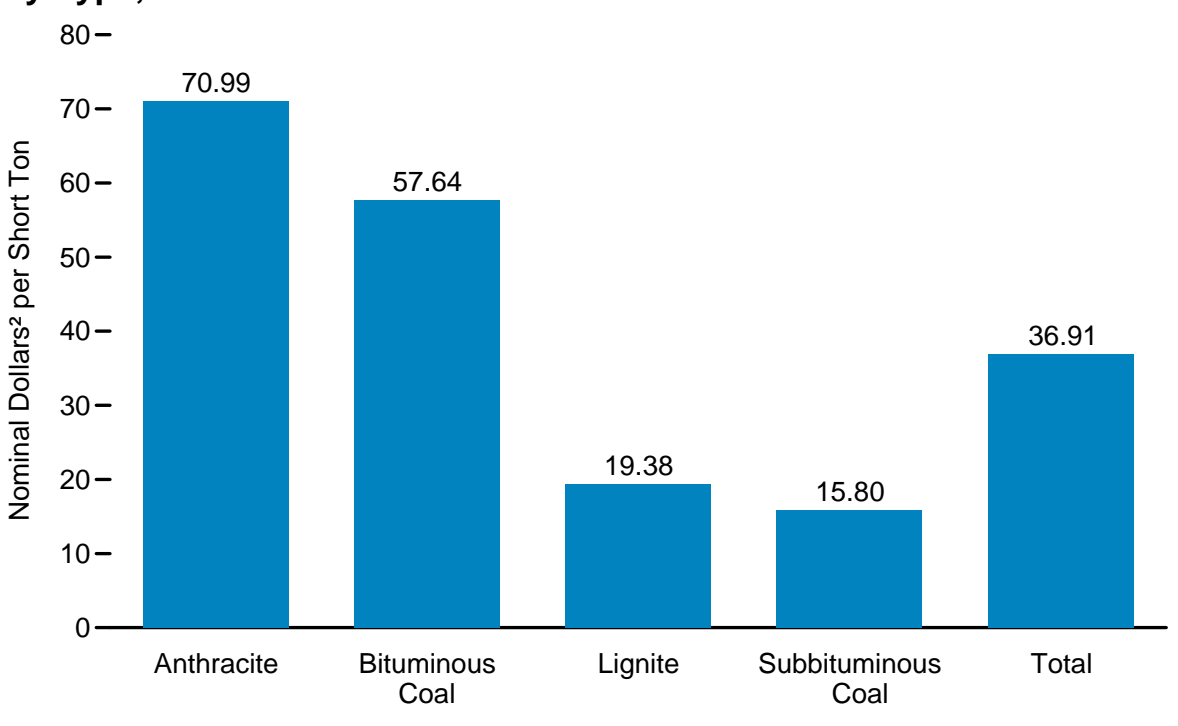

${ }^{1}$ In chained (2005) dollars, calculated by using gross domestic product implicit price defla-

2 See "Nominal Dollars" in Glossary. tors in Table D1. See "Chained Dollars" in Glossary. Source: Table 7.9. 
Table 7.9 Coal Prices, Selected Years, 1949-2011

(Dollars per Short Ton)

\begin{tabular}{|c|c|c|c|c|c|c|c|c|c|c|}
\hline \multirow[b]{2}{*}{ Year } & \multicolumn{2}{|c|}{ Bituminous Coal } & \multicolumn{2}{|c|}{ Subbituminous Coal } & \multicolumn{2}{|c|}{ Lignite 1} & \multicolumn{2}{|c|}{ Anthracite } & \multicolumn{2}{|c|}{ Total } \\
\hline & Nominal $^{2}$ & Real $^{3}$ & Nominal $^{2}$ & Real $^{3}$ & Nominal $^{2}$ & Real $^{3}$ & Nominal $^{2}$ & Real $^{3}$ & Nominal $^{2}$ & Real $^{3}$ \\
\hline 1949 & ${ }^{4} 4.90$ & $4, R_{33.80}$ & $\left({ }^{4}\right)$ & $\left({ }^{4}\right)$ & 2.37 & $\mathrm{R}_{16.35}$ & 8.90 & $\mathrm{R}_{61.38}$ & 5.24 & $R_{36.14}$ \\
\hline 1950 & 44.86 & 4,R33.16 & $\left({ }^{4}\right)$ & $\left({ }^{4}\right)$ & 2.41 & R16.44 & 9.34 & R63.73 & 5.19 & R35.41 \\
\hline 1955 & ${ }^{4} 4.51$ & 4,R 27.17 & $\left({ }^{4}\right)$ & $\left({ }^{4}\right)$ & 2.38 & $R_{14.34}$ & 8.00 & $\mathrm{R}_{48.19}$ & 4.69 & $\mathrm{R}_{28.25}$ \\
\hline 1960 & ${ }^{4} 4.71$ & $4, R_{25} .31$ & $\left(4^{\prime}\right)$ & $\left(4^{\prime}\right)$ & 2.29 & $\mathrm{R}_{12.30}$ & 8.01 & $\mathrm{R}_{43.04}$ & 4.83 & R25.95 \\
\hline 1965 & 44.45 & 4,R 22.32 & $\left(4^{\prime}\right)$ & $\left({ }^{4}\right)$ & 2.13 & $R_{10.68}$ & 8.51 & $\mathrm{R}_{42.69}$ & 4.55 & $\mathrm{R}_{22.82}$ \\
\hline 1970 & ${ }^{4} 6.30$ & 4,R R25.89 & $\left(4^{\prime}\right)$ & $\left({ }^{4}\right)$ & 1.86 & R7.64 & 11.03 & R45.32 & 6.34 & R26.05 \\
\hline 1975 & ${ }^{4} 19.79$ & 4,R58.91 & $\left({ }^{4}\right)$ & $\left({ }^{4}\right)$ & 3.17 & 9.44 & 32.26 & R96.04 & 19.35 & R57.60 \\
\hline 1976 & ${ }^{4} 20.11$ & 4,R56.62 & $\left(4^{\prime}\right)$ & $\left({ }^{\prime}\right)$ & 3.74 & $\mathrm{R}_{10.53}$ & 33.92 & R95.50 & 19.56 & R55.07 \\
\hline 1977 & 420.59 & $4, R_{54.50}$ & $\left(4^{\prime}\right)$ & $\left({ }^{4}\right)$ & 4.03 & $\mathrm{R}_{10.67}$ & 34.86 & R92.26 & 19.95 & $\mathrm{R}_{52.80}$ \\
\hline 1978 & ${ }^{4} 22.64$ & $4, R_{55.99}$ & $\left({ }^{4}\right)$ & $\left({ }^{4}\right)$ & 5.68 & $\mathrm{R}_{14.05}$ & 35.25 & R87.18 & 21.86 & R54.06 \\
\hline 1979 & 27.31 & $\mathrm{R}_{62.35}$ & 9.55 & $\mathrm{R}_{21.80}$ & 6.48 & $\mathrm{R}_{14.80}$ & 41.06 & R93.75 & 23.75 & R54.23 \\
\hline 1980 & 29.17 & $R_{61.04}$ & 11.08 & $\mathrm{R}_{23.18}$ & 7.60 & $\mathrm{R}_{15.90}$ & 42.51 & R88.95 & 24.65 & R51.58 \\
\hline 1981 & 31.51 & ${ }^{R} 60.28$ & 12.18 & $\mathrm{R}_{23.30}$ & 8.85 & $\mathrm{R}_{16.93}$ & 44.28 & R84.71 & 26.40 & $R_{50.51}$ \\
\hline 1982 & 32.15 & R57.97 & 13.37 & $\mathrm{R}_{24.11}$ & 9.79 & $\mathrm{R}_{17.65}$ & 49.85 & R89.89 & 27.25 & ${ }^{R} 49.14$ \\
\hline 1983 & 31.11 & $R_{53.96}$ & 13.03 & $\mathrm{R}_{22.60}$ & 9.91 & $\mathrm{R}_{17.19}$ & 52.29 & R90.70 & 25.98 & R45.06 \\
\hline 1984 & 30.63 & $\mathrm{R}_{51.21}$ & 12.41 & $\mathrm{R}_{20.75}$ & 10.45 & $\mathrm{R}_{17.47}$ & 48.22 & $\mathrm{R}_{80.61}$ & 25.61 & ${ }^{R} 42.81$ \\
\hline 1985 & 30.78 & $\mathrm{R}_{49.94}$ & 12.57 & $\mathrm{R}_{20.40}$ & 10.68 & $\mathrm{R}_{17.33}$ & 45.80 & $\mathrm{R}_{74.32}$ & 25.20 & R 40.89 \\
\hline 1986 & 28.84 & R45.78 & 12.26 & $\mathrm{R}_{19.46}$ & 10.64 & $\mathrm{R}_{16.89}$ & 44.12 & R70.04 & 23.79 & R37.77 \\
\hline 1987 & 28.19 & ${ }^{R} 43.49$ & 11.32 & $\mathrm{R}_{17.46}$ & 10.85 & $\mathrm{R}_{16.74}$ & 43.65 & R 67.34 & 23.07 & R35.59 \\
\hline 1988 & 27.66 & $\mathrm{R}_{41.26}$ & 10.45 & $\mathrm{R}_{15.59}$ & 10.06 & $\mathrm{R}_{15.00}$ & 44.16 & R65.87 & 22.07 & R32.92 \\
\hline 1989 & 27.40 & $R_{39.38}$ & 10.16 & $\mathrm{R}_{14.60}$ & 9.91 & $\mathrm{R}_{14.24}$ & 42.93 & $\mathrm{R}_{61.70}$ & 21.82 & $\mathrm{R}_{31.36}$ \\
\hline 1990 & 27.43 & R37.96 & 9.70 & $\mathrm{R}_{13.42}$ & 10.13 & $\mathrm{R}_{14.02}$ & 39.40 & R54.52 & 21.76 & ${ }^{R} 30.11$ \\
\hline 1991 & 27.49 & $\mathrm{R}_{36.74}$ & 9.68 & $\mathrm{R}_{12.94}$ & 10.89 & $\mathrm{R}_{14.55}$ & 36.34 & $\mathrm{R}_{48.57}$ & 21.49 & $\mathrm{R}_{28.72}$ \\
\hline 1992 & 26.78 & $\mathrm{R}_{34.96}$ & 9.68 & $\mathrm{R}_{12.64}$ & 10.81 & $\mathrm{R}_{14.11}$ & 34.24 & $\mathrm{R}_{44.70}$ & 21.03 & $\mathrm{R}_{27.46}$ \\
\hline 1993 & 26.15 & $\mathrm{R}_{33.40}$ & 9.33 & $\mathrm{R}_{11.92}$ & 11.11 & $\mathrm{R}_{14.19}$ & 32.94 & ${ }^{R} 42.07$ & 19.85 & R25.35 \\
\hline 1994 & 25.68 & ${ }^{R} 32.12$ & 8.37 & $\mathrm{R}_{10.47}$ & 10.77 & $\mathrm{R}_{13.47}$ & 36.07 & $\mathrm{R}_{45.12}$ & 19.41 & $\mathrm{R}_{24.28}$ \\
\hline 1995 & 25.56 & $\mathrm{R}_{31.32}$ & 8.10 & 9.93 & 10.83 & $\mathrm{R}_{13.27}$ & 39.78 & $\mathrm{R}_{48.75}$ & 18.83 & R23.07 \\
\hline 1996 & 25.17 & $\mathrm{R}_{30.27}$ & 7.87 & R9.46 & 10.92 & $\mathrm{R}_{13.13}$ & 36.78 & $\mathrm{R}_{44.23}$ & 18.50 & $\mathrm{R}_{22.25}$ \\
\hline 1997 & 24.64 & $\mathrm{R}_{29.12}$ & 7.42 & R8.77 & 10.91 & $\mathrm{R}_{12.89}$ & 35.12 & $\mathrm{R}_{41.50}$ & 18.14 & $\mathrm{R}_{21.43}$ \\
\hline 1998 & 24.87 & $\mathrm{R}_{29.06}$ & 6.96 & $\mathrm{R}_{8.13}$ & 11.08 & $\mathrm{R}_{12.95}$ & 42.91 & R50.14 & 17.67 & ${ }^{R} 20.65$ \\
\hline 1999 & 23.92 & $\mathrm{R}_{27.54}$ & 6.87 & R7.91 & 11.04 & $\mathrm{R}_{12.71}$ & 35.13 & $\mathrm{R}_{40.45}$ & 16.63 & $\mathrm{R}_{19.15}$ \\
\hline 2000 & 24.15 & $\mathrm{R}_{27.22}$ & 7.12 & $R_{8} .02$ & 11.41 & $\mathrm{R}_{12.86}$ & 40.90 & $\mathrm{R}_{46.10}$ & 16.78 & $\mathrm{R}_{18.91}$ \\
\hline 2001 & 25.36 & $\mathrm{R}_{27.95}$ & 6.67 & R7.35 & 11.52 & $\mathrm{R}_{12.70}$ & 47.67 & R52.54 & 17.38 & $\mathrm{R}_{19.16}$ \\
\hline 2002 & 26.57 & $\mathrm{R}_{28.82}$ & 7.34 & R7.96 & 11.07 & $\mathrm{R}_{12.01}$ & 47.78 & R51.82 & 17.98 & $\mathrm{R}_{19.50}$ \\
\hline 2003 & 26.73 & $\mathrm{R}_{28.40}$ & 7.73 & 8.21 & 11.20 & 11.90 & 49.87 & R52.98 & 17.85 & $\mathrm{R}_{18.96}$ \\
\hline 2004 & 30.56 & $\mathrm{R}_{31.57}$ & 8.12 & 8.39 & 12.27 & 12.68 & 39.77 & $\mathrm{R}_{41.09}$ & 19.93 & $\mathrm{R}_{20.59}$ \\
\hline 2005 & 36.80 & 36.80 & 8.68 & 8.68 & 13.49 & 13.49 & 41.00 & 41.00 & 23.59 & 23.59 \\
\hline 2006 & 39.32 & $\mathrm{R}_{38.09}$ & 9.95 & 9.64 & 14.00 & 13.56 & 43.61 & ${ }^{R} 42.25$ & 25.16 & 24.37 \\
\hline 2007 & 40.80 & $R_{38.41}$ & 10.69 & 10.06 & 14.89 & $\mathrm{R}_{14.02}$ & 52.24 & $\mathrm{R}_{49.18}$ & 26.20 & R24.66 \\
\hline 2008 & 51.39 & R47.33 & 12.31 & $\mathrm{R}_{11.34}$ & 16.50 & $\mathrm{R}_{15.20}$ & 60.76 & R55.96 & 31.25 & R28.78 \\
\hline 2009 & 55.44 & $R_{50.52}$ & 13.35 & $\mathrm{R}_{12.17}$ & 17.26 & $\mathrm{R}_{15.73}$ & 57.10 & $\mathrm{R}_{52.04}$ & 33.24 & $\mathrm{R}_{30.29}$ \\
\hline 2010 & $\mathrm{R}_{60.88}$ & $R_{54.85}$ & $\mathrm{R}_{14.11}$ & $\mathrm{R}_{12.71}$ & $\mathrm{R}_{18.76}$ & $\mathrm{R}_{16.90}$ & R59.51 & R53.62 & $R_{35.61}$ & $\mathrm{R}_{32.08}$ \\
\hline $2011^{\mathrm{E}}$ & 57.64 & 50.85 & 15.80 & 13.94 & 19.38 & 17.10 & 70.99 & 62.62 & 36.91 & 32.56 \\
\hline
\end{tabular}

Because of withholding to protect company confidentiality, lignite prices exclude Texas for 1955-1977 and Montana for 1974-1978. As a result, lignite prices for 1974-1977 are for North Dakota only.

See "Nominal Dollars" in Glossary.

3 In chained (2005) dollars, calculated by using gross domestic product implicit price deflators in Table D1. See "Chained Dollars" in Glossary.

Through 1978, subbituminous coal is included in "Bituminous Coal."

$\mathrm{R}=$ Revised. E=Estimate.

Note: Prices are free-on-board (F.O.B.) rail/barge prices, which are the F.O.B. prices of coal at the point of first sale, excluding freight or shipping and insurance costs. For 1949-2000, prices are for open market and captive coal sales; for 2001-2007, prices are for open market coal sales; for 2008 forward, prices are for open market and captive coal sale

Web Pages: - For all data beginning in 1949, see http://www.eia.gov/totalenergy/data/annual/\#coal. - For related information, see http://www.eia.gov/coal/.

Sources: • 1949-1975-Bureau of Mines (BOM), Minerals Yearbook. • 1976-U.S. Energy Information Administration (EIA), Energy Data Report, Coal-Bituminous and Lignite in 1976, and BOM, Minerals Yearbook. 1977 and 1978-EIA, Energy Data Reports, Bituminous Coal and Lignite Production and Report Coal-Pennsylvania Anthracite. - 1980-1992-EIA Coal Production, and Energy Data Report, Coal-Pennsylvania Anthracite. • 1980-1992-EIA, Coal Production, annual reports. Annual Coal Report, annual reports. - 2011-EIA, Form EIA-7A, "Coal Production Report," and U.S. Department of Labor, Mine Safety and Health Administration, Form 7000-2, "Quarterly Mine Employment 


\section{Coal}

Note 1. Coal Consumption. Data in this report on the consumption of bituminous coal, sub-bituminous coal, lignite, anthracite, and waste coal are developed primarily from consumption data reported in surveys. Included are data reported by all electric power companies and coke plant companies. Data on coal consumption by all industrial and manufacturing establishments are based on consumption data obtained quarterly from coal users. Beginning in 2008, data on coal consumption by the residential and commercial sectors are based on data received on Form EIA-3, "Quarterly Coal Consumption and Quality Report-Manufacturing and Transformation/Processing Coal Plants and Commercial and Institutional Users.” Prior to 2008, data on coal consumption by the residential and commercial sectors are based on distribution data obtained annually from coal distributors. Included in each sector's data are the following: Residential and Commercial Sectors-commercial and institutional establishments including military bases, universities, and various State facilities; Industrial Sector-consumption at manufacturing plants, coking plants, and coal preparation plants; Electric Power Sector (electric utilities and independent power producers) - consumption for electric generation and useful thermal output at electricity-only and CHP plants within the North American Industry Classification System (NAICS) 22 category, whose primary business is to sell electricity, or electricity and heat, to the public. There are no data for the Transportation Sector.
Note 2. Residential and Commercial Coal Consumption Estimates. Through 2007, coal consumption by the residential and commercial sectors was reported to the U.S. Energy Information Administration (EIA) for the two sectors combined; EIA estimates the amount consumed by the sectors individually. To create the estimates, it is first assumed that an occupied coal-heated housing unit consumes fuel at the same Btu rate as an oil-heated housing unit. Then, for the years in which data are available on the number of occupied housing units by heating source $(1950,1960,1970,1973-1981$, and subsequent odd-numbered years; see Table 2.7), residential consumption of coal is estimated by the following steps: a ratio is created of the number of occupied housing units heated by coal to the number of housing units heated by oil; that ratio is then multiplied by the Btu quantity of oil consumed by the residential sector to derive an estimate of the Btu quantity of coal consumed by the residential sector; and, finally, the amount estimated as the residential sector consumption is subtracted from the residential and commercial sectors' combined consumption to derive the commercial sector's estimated consumption. The 1950 share is applied to 1949, and the other missing years’ shares are interpolated.

Beginning in 2008, coal consumption is reported to EIA for commercial and institutional users. However, EIA continues to allocate a small portion of that consumption to the residential sector using the above methodology. 


\section{Electricity}




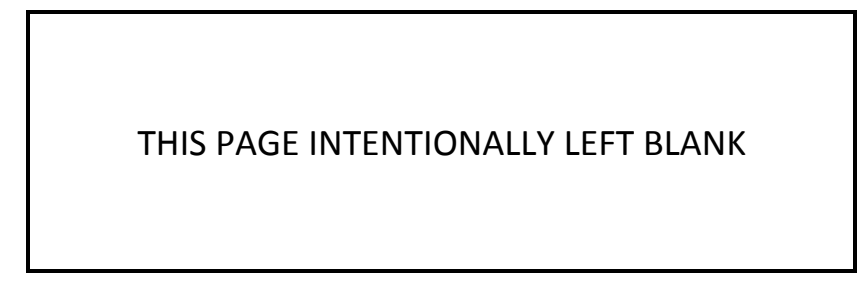


Figure 8.0 Electricity Flow, 2011

(Quadrillion Btu)

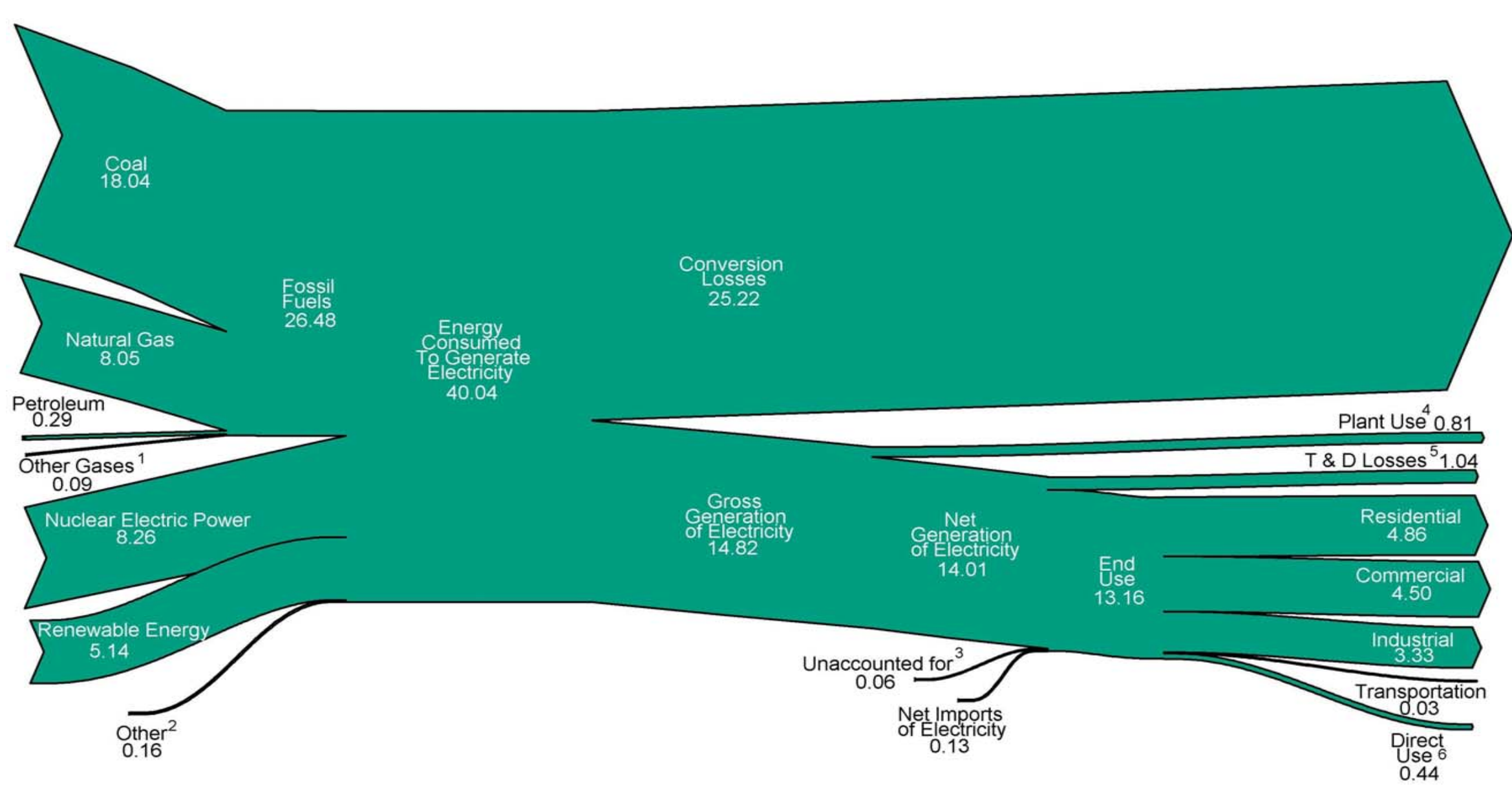

${ }^{1}$ Blast furnace gas, propane gas, and other manufactured and waste gases derived from fossil fuels.

${ }^{2}$ Batteries, chemicals, hydrogen, pitch, purchased steam, sulfur, miscellaneous technologies, and non-renewable waste (municipal solid waste from non-biogenic sources, and tire-derived fuels).

${ }^{3}$ Data collection frame differences and nonsampling error. Derived for the diagram by subtracting the "T \& D Losses" estimate from "T \& D Losses and Unaccounted for" derived from Table 8.1.

${ }^{4}$ Electric energy used in the operation of power plants.

${ }^{5}$ Transmission and distribution losses (electricity losses that occur between the point of

generation and delivery to the customer) are estimated as 7 percent of gross generation.

${ }^{6}$ Use of electricity that is 1 ) self-generated, 2) produced by either the same entity that consumes the power or an affiliate, and 3) used in direct support of a service or industrial process located within the same facility or group of facilities that house the generating equipment. Direct use is exclusive of station use.

Notes: • Data are preliminary. • See Note, "Electrical System Energy Losses," at the end of Section 2. - Net generation of electricity includes pumped storage facility production minus energy used for pumping. - Values are derived from source data prior to rounding for publication. - Totals may not equal sum of components due to independent rounding.

Sources: Tables 8.1, 8.4a, 8.9, A6 (column 7), and U.S. Energy Information Administration, Form EIA-923, "Power Plant Operations Report." 


\section{Overview, 2011}

$4,500-$

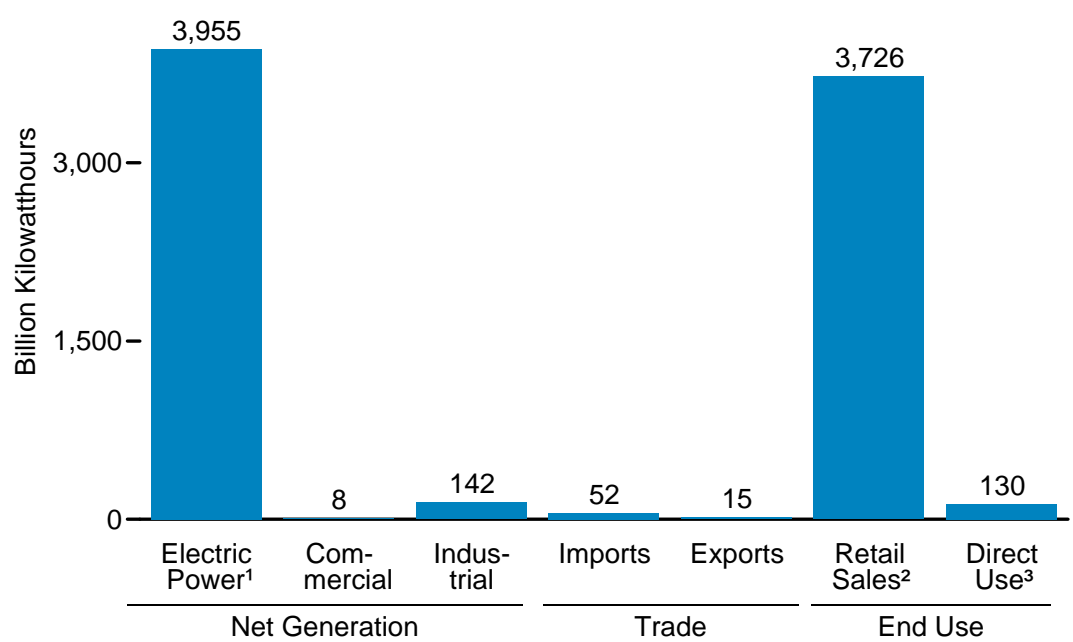

\section{Electricity Trade, 1949-2011}

$60-$

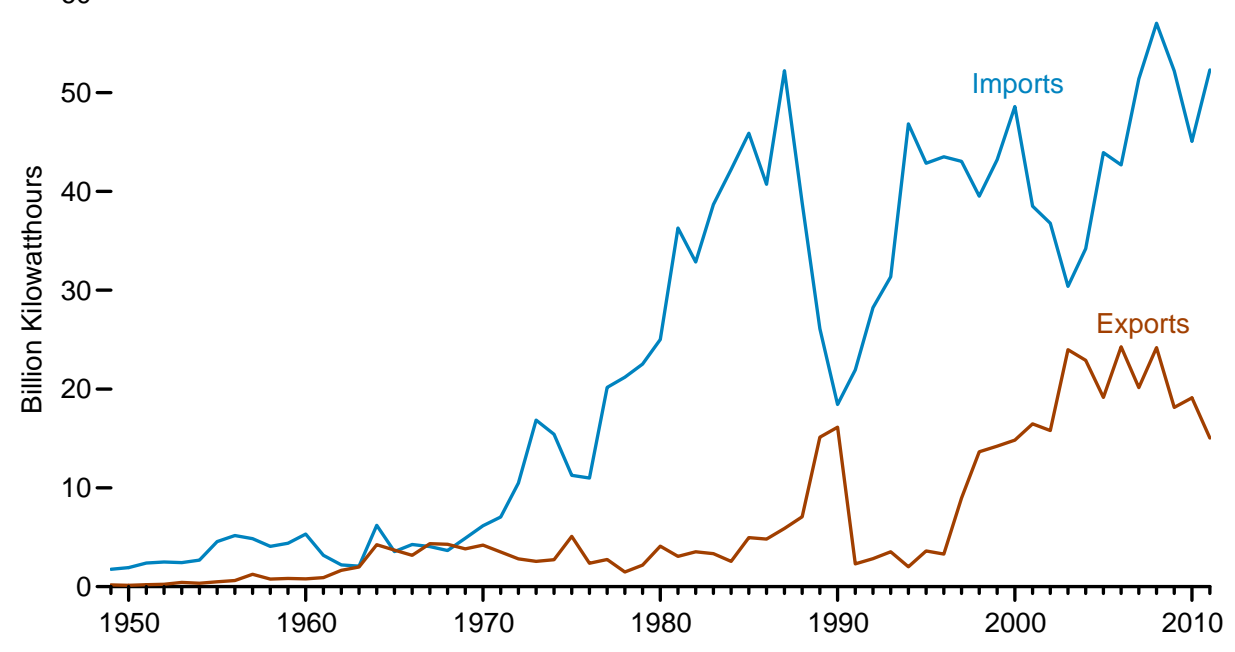

Net-Generation-to-End-Use Flow, 2011

(Billion Kilowatthours)

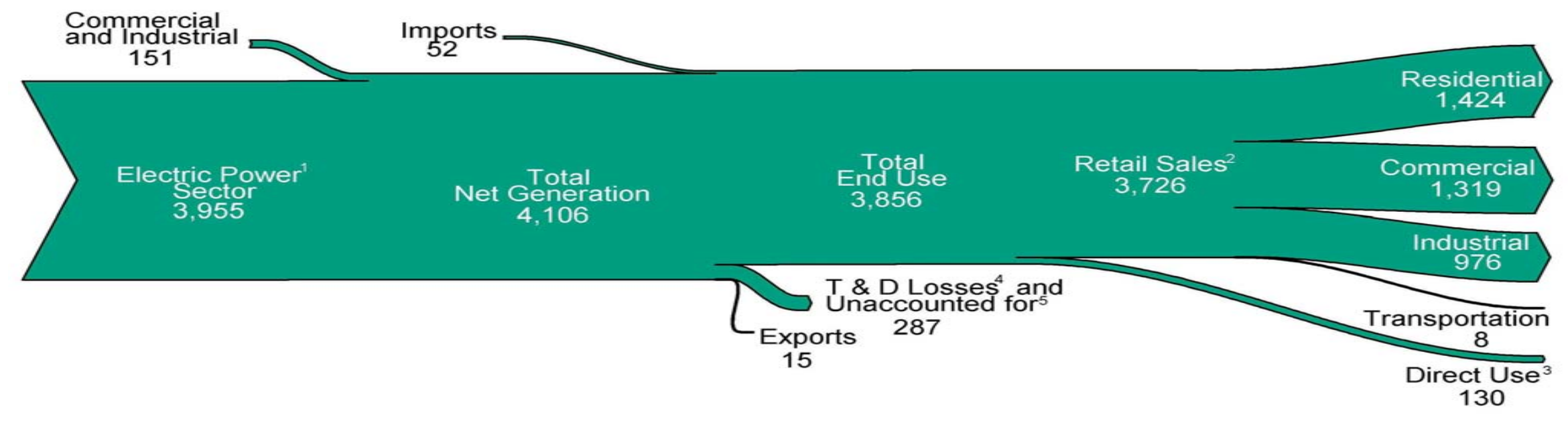

${ }^{1}$ Electricity-only and combined-heat-and-power plants within the NAICS 22 category whose primary business is to sell electricity, or electricity and heat, to the public.

${ }^{2}$ Electricity retail sales to ultimate customers reported by electric utilities and other energy service providers.

${ }^{3}$ See Table 8.1 , footnote 8.
${ }^{4}$ Transmission and distribution losses (electricity losses that occur between the point of generation and delivery to the customer). See Note, "Electrical System Energy Losses," at the end of Section 2.

${ }^{5}$ Data collection frame differences and nonsampling error.

Sources: Tables 8.1 and 8.9. 
Table 8.1 Electricity Overview, Selected Years, 1949-2011

(Billion Kilowatthours)

\begin{tabular}{|c|c|c|c|c|c|c|c|c|c|c|c|c|c|}
\hline \multirow[b]{3}{*}{ Year } & \multicolumn{4}{|c|}{ Net Generation } & \multicolumn{5}{|c|}{ Trade } & \multirow{3}{*}{$\begin{array}{c}T \& D \text { Losses } 5 \\
\text { and } \\
\text { Unaccounted } \\
\text { for } 6\end{array}$} & \multicolumn{3}{|c|}{ End Use } \\
\hline & \multirow[b]{2}{*}{$\begin{array}{l}\text { Electric Power } \\
\text { Sector }^{2}\end{array}$} & \multirow[b]{2}{*}{$\begin{array}{c}\text { Commercial } \\
\text { Sector }^{3}\end{array}$} & \multirow[b]{2}{*}{$\begin{array}{l}\text { Industrial } \\
\text { Sector }{ }^{4}\end{array}$} & \multirow[b]{2}{*}{ Total } & \multicolumn{2}{|c|}{ Imports ${ }^{1}$} & \multicolumn{2}{|c|}{ Exports ${ }^{1}$} & \multirow{2}{*}{$\begin{array}{c}\text { Net Imports }{ }^{1} \\
\text { Total }\end{array}$} & & \multirow[b]{2}{*}{$\begin{array}{l}\text { Retail } \\
\text { Sales } 7\end{array}$} & \multirow[b]{2}{*}{$\begin{array}{l}\text { Direct } \\
\text { Use }^{8}\end{array}$} & \multirow[b]{2}{*}{ Total } \\
\hline & & & & & From Canada & Total & To Canada & Total & & & & & \\
\hline 1949 & 291 & NA & 5 & 296 & NA & 2 & NA & (s) & 2 & 43 & 255 & NA & 255 \\
\hline 1950 & 329 & NA & 5 & 334 & NA & 2 & NA & (s) & 2 & 44 & 291 & NA & 291 \\
\hline 1955 & 547 & NA & 3 & 550 & NA & 5 & NA & (s) & 4 & 58 & 497 & NA & 497 \\
\hline 1960 & 756 & NA & 4 & 759 & NA & 5 & NA & 1 & 5 & 76 & 688 & NA & 688 \\
\hline 1965 & 1,055 & NA & $\begin{array}{l}4 \\
3\end{array}$ & 1,058 & NA & 4 & NA & 4 & (s) & 104 & 954 & NA & 954 \\
\hline 1970 & 1,532 & NA & 3 & 1,535 & NA & 6 & NA & 4 & 2 & 145 & 1,392 & NA & 1,392 \\
\hline 1975 & 1,918 & NA & 3 & 1,921 & NA & 11 & NA & 5 & 6 & 180 & 1,747 & NA & 1,747 \\
\hline 1976 & 2,038 & NA & 3 & 2,041 & NA & 11 & NA & 2 & 9 & 194 & 1,855 & NA & 1,855 \\
\hline 1977 & 2,124 & NA & 3 & 2,127 & NA & 20 & NA & 3 & 17 & 197 & 1,948 & NA & 1,948 \\
\hline 1978 & 2,206 & NA & 3 & 2,209 & NA & 21 & NA & 1 & 20 & 211 & 2,018 & NA & 2,018 \\
\hline 1979 & 2,247 & NA & 3 & 2,251 & NA & 23 & NA & 2 & 20 & 200 & 2,071 & NA & 2,071 \\
\hline 1980 & 2,286 & NA & 3 & 2,290 & NA & 25 & NA & 4 & 21 & 216 & 2,094 & NA & 2,094 \\
\hline 1981 & 2,295 & NA & 3 & 2,298 & NA & 36 & NA & 3 & 33 & 184 & 2,147 & NA & 2,147 \\
\hline 1982 & 2,241 & NA & 3 & 2,244 & NA & 33 & NA & 4 & 29 & 187 & 2,086 & NA & 2,086 \\
\hline 1983 & 2,310 & NA & 3 & 2,313 & NA & 39 & NA & $\begin{array}{l}4 \\
3\end{array}$ & 35 & 198 & 2,151 & NA & 2,151 \\
\hline 1984 & 2,416 & NA & 3 & 2,419 & NA & 42 & NA & 3 & 40 & 173 & 2,286 & NA & 2,286 \\
\hline 1985 & 2,470 & NA & 3 & 2,473 & NA & 46 & NA & 5 & 41 & 190 & 2,324 & NA & 2,324 \\
\hline 1986 & 2,487 & NA & 3 & 2,490 & NA & 41 & NA & 5 & 36 & 158 & 2,369 & NA & 2,369 \\
\hline 1987 & 2,572 & NA & 3 & 2,575 & NA & 52 & NA & 6 & 46 & 164 & 2,457 & NA & 2,457 \\
\hline 1988 & $\begin{array}{l}2,704 \\
\end{array}$ & NA & $\begin{array}{l}5 \\
3\end{array}$ & 2,707 & NA & 39 & NA & 7 & $\begin{array}{l}40 \\
32\end{array}$ & 161 & 2,578 & NA & $\begin{array}{l}\text {, } 2,451 \\
2,578\end{array}$ \\
\hline 1989 & $\begin{array}{r}22,848 \\
2,848\end{array}$ & 4 & 4115 & 2,967 & NA & 26 & NA & 15 & 11 & 222 & 2,647 & 109 & $\begin{array}{l}2,756 \\
2,\end{array}$ \\
\hline 1990 & 2,901 & 6 & 131 & 3,038 & 16 & 18 & 16 & 16 & 2 & 203 & 2,713 & 125 & 2,837 \\
\hline 1991 & 2,936 & 6 & 133 & 3,074 & 20 & 22 & 2 & 2 & 20 & 207 & 2,762 & 124 & 2,886 \\
\hline 1992 & 2,934 & 6 & 143 & 3,084 & 26 & 28 & 2 & 3 & 25 & 212 & 2,763 & 134 & 2,897 \\
\hline 1993 & $\begin{array}{l}3,044 \\
3,044\end{array}$ & 7 & 146 & $\begin{array}{l}3,004 \\
3,197\end{array}$ & 29 & 31 & 3 & 4 & 28 & 224 & 2,861 & $\begin{array}{l}134 \\
139\end{array}$ & $\begin{array}{l}3,001 \\
3,001\end{array}$ \\
\hline 1994 & 3,089 & 8 & 151 & 3,248 & 45 & 47 & 1 & 2 & 45 & 211 & 2,935 & 146 & $\begin{array}{l}3,081 \\
3,0\end{array}$ \\
\hline 1995 & 3,194 & 8 & 151 & 3,353 & 41 & 43 & 2 & 4 & 39 & 229 & 3,013 & 151 & 3,164 \\
\hline 1996 & 3,284 & 9 & 151 & 3,444 & 42 & 43 & 2 & 3 & 40 & 231 & 3,101 & 153 & 3,254 \\
\hline 1997 & 3,329 & 9 & 154 & 3,492 & 43 & 43 & 7 & 9 & 34 & 224 & 3,146 & 156 & 3,302 \\
\hline 1998 & 3,457 & 9 & 154 & $\begin{array}{l}3,620 \\
3,620\end{array}$ & 40 & 40 & 12 & 14 & 26 & 221 & 3,264 & 161 & $\begin{array}{l}3,425 \\
3,40\end{array}$ \\
\hline 1999 & 3,530 & 9 & 156 & 3,695 & 43 & 43 & 13 & 14 & 29 & 240 & 3,312 & 172 & 3,484 \\
\hline 2000 & $\begin{array}{l}3,638 \\
3,603\end{array}$ & 8 & 157 & $\begin{array}{l}3,090 \\
3,802\end{array}$ & 49 & $\begin{array}{l}40 \\
49\end{array}$ & 13 & 15 & 34 & 244 & $\begin{array}{l}3,421 \\
3,421\end{array}$ & 171 & $\begin{array}{l}3,404 \\
3,592\end{array}$ \\
\hline 2001 & 3,580 & 7 & 149 & 3,737 & 38 & 39 & 16 & 16 & 22 & 202 & 3,394 & 163 & 3,557 \\
\hline 2002 & 3,698 & 7 & 153 & 3,858 & 37 & 37 & 15 & 16 & 21 & 248 & 3,465 & 166 & 3,632 \\
\hline 2003 & 3,721 & 7 & 155 & 3,883 & 29 & 30 & 24 & 24 & 6 & 228 & 3,494 & 168 & 3,662 \\
\hline 2004 & 3,808 & 8 & 154 & $\begin{array}{l}\text {,.000 } \\
3,971\end{array}$ & 33 & 34 & $\begin{array}{l}24 \\
22\end{array}$ & $\begin{array}{l}24 \\
23\end{array}$ & 11 & 266 & $\begin{array}{l}3,494 \\
3,547\end{array}$ & 168 & $\begin{array}{l}3,002 \\
3,716\end{array}$ \\
\hline 2005 & 3,902 & 8 & 145 & 4,055 & 42 & 44 & 19 & 19 & 25 & 269 & 3,661 & 150 & 3,811 \\
\hline 2006 & 3,908 & 8 & 148 & 4,065 & 42 & 43 & 23 & 24 & 18 & 266 & 3,670 & 147 & $\begin{array}{l}3,817 \\
3\end{array}$ \\
\hline 2007 & 4,005 & 8 & 143 & 4,157 & 50 & 51 & 20 & 20 & 31 & 298 & 3,765 & 126 & 3,890 \\
\hline 2008 & 3,974 & 8 & 137 & 4,119 & 56 & 57 & 24 & 24 & 33 & 287 & 3,733 & 132 & 3,865 \\
\hline 2009 & 3,810 & 8 & 132 & 3,950 & 51 & 52 & $\begin{array}{l}24 \\
18\end{array}$ & $\begin{array}{l}24 \\
18\end{array}$ & 34 & 261 & 3,597 & 127 & $\begin{array}{l}3,724 \\
3,70\end{array}$ \\
\hline 2010 & $R_{3,972}$ & Rg & $R_{144}$ & $\mathrm{R}_{4,125}$ & 44 & 45 & $\mathrm{R}_{18}$ & 19 & 26 & $\mathrm{R}_{265}$ & $R_{3,754}$ & $\mathrm{R}_{132}$ & $\mathrm{R}_{3,886}$ \\
\hline 2011 & $P_{3,955}$ & P8 & P142 & $P_{4}, 106$ & $\begin{array}{l}\text { P51 } \\
\text { P }\end{array}$ & P52 & P14 & $P_{15}$ & P37 & P287 & $P_{3,726}$ & $E_{130}$ & $\mathrm{P}_{3,856}$ \\
\hline
\end{tabular}

1 Electricity transmitted across U.S. borders. Net imports equal imports minus exports.

2 Electricity-only and combined-heat-and-power (CHP) plants within the NAICS 22 category whose primary buticitic un only: beginning in 1989, data are for electric utilities and independent power producers.

3 Commercial combined-heat-and-power (CHP) and commercial electricity-only plants.

Industrial combined-heat-and-power (CHP) and industrial electricity-only plants. Through 1988, data are for industrial hydroelectric power only.

5 Transmission and distribution losses (electricity losses that occur between the point of generation and delivery to the customer). See Note, "Electrical System Energy Losses," at end of Section 2.

7 Electricity retail sales to ultimate customers by electric utilities and, beginning in 1996, other energy

8 Use of electricity that is 1) self-generated, 2) produced by either the same entity that consumes the power or an affiliate, and 3) used in direct support of a service or industrial process located within the same $\mathrm{R}=$ Revised. $\mathrm{P}=$ Preliminary. $\mathrm{E}=\mathrm{Estimate}$. NA=Not available. ( $\mathrm{s}$ ) =Less than 0.5 billion kilowatthours.

Notes: - See Note 1, "Coverage of Electricity Statistics," and Note 2, "Classification of Power Plants Into Energy-Use Sectors," at end of section. - Totals may not equal sum of components due to independent rounding.

Web Pages: - See http://www.eia.gov/totalenergy/data/monthly/\#electricity for updated monthly and annual data. - See http://www.eia.gov/totalenergy/data/annual/\#electricity for all annual data beginning in So. See hith:/ww.ela.gov/electricity/ for related information.

Sources: See end of section. 
Figure 8.2a Electricity Net Generation, Total (All Sectors)

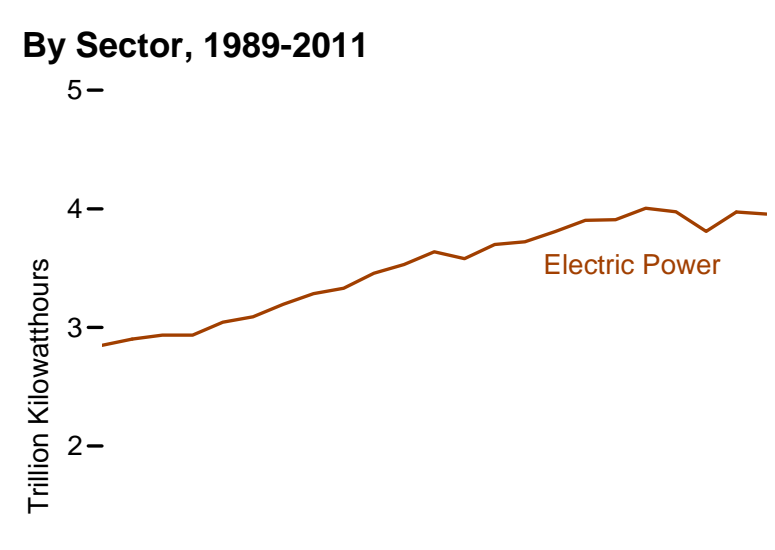

$1-$

Commercial and Industrial

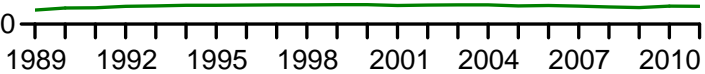

By Source, 1949-2011

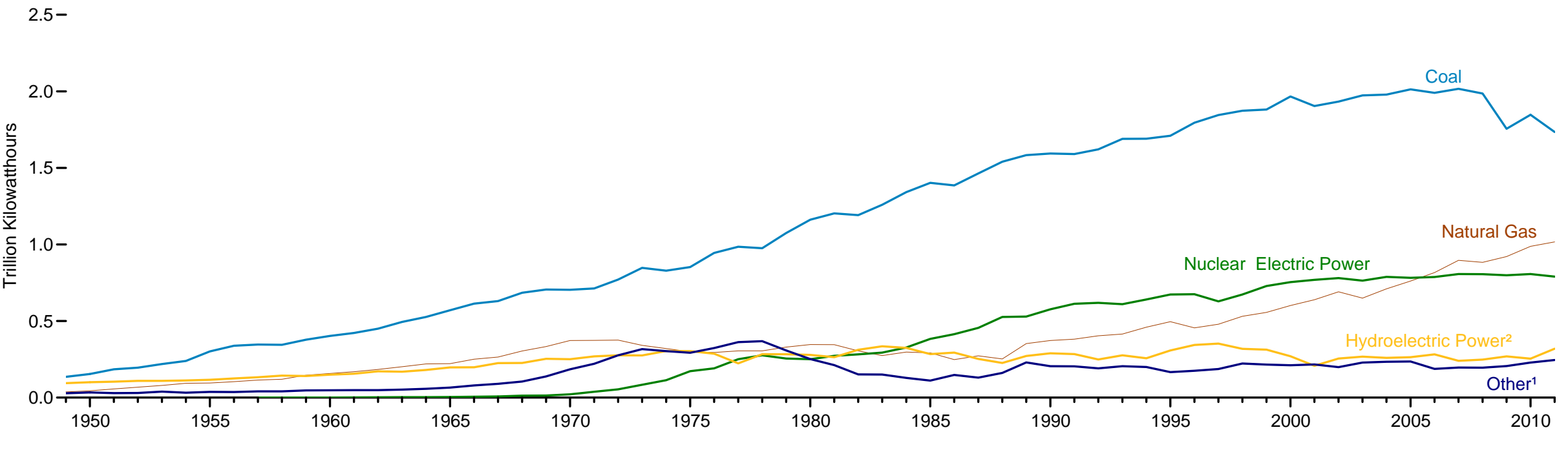

By Source Category, 2011

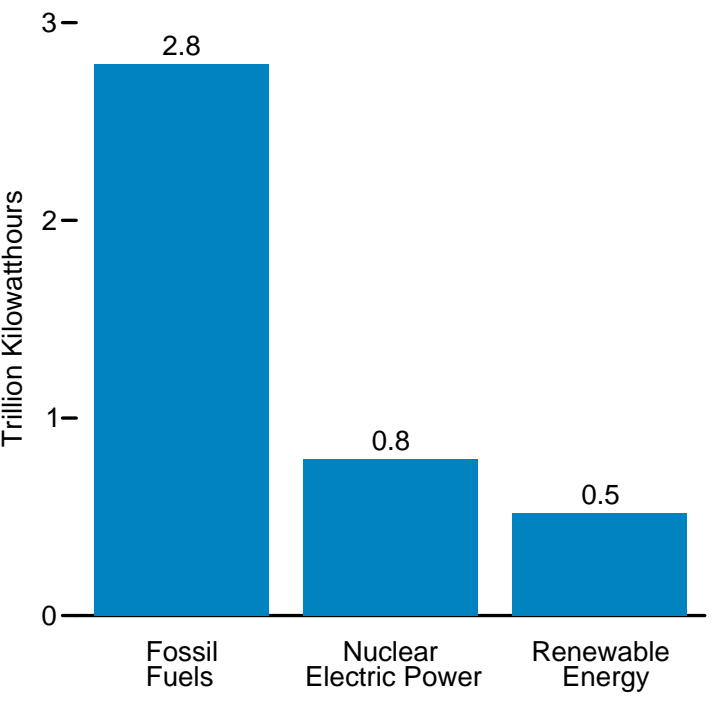

By Source, 2011

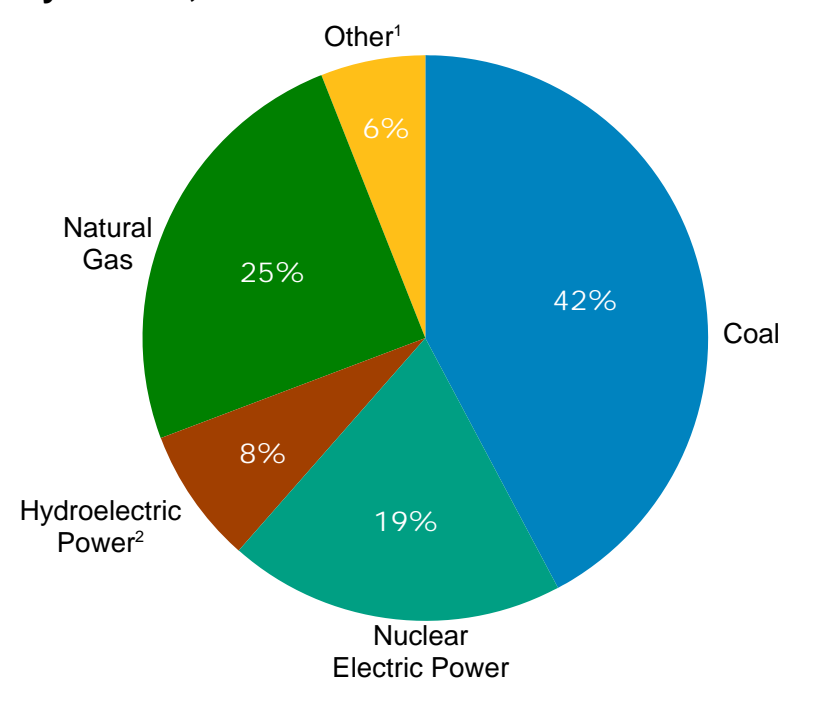

${ }^{1}$ Wind, petroleum, wood, waste, geothermal, other gases, solar thermal and photovoltaic, batteries, chemicals, hydrogen, pitch, purchased steam, sulfur, miscellaneous technologies, and non-renewable waste (municipal solid waste from non-biogenic sources, and tire-derived

${ }^{2}$ Conventional hydroelectric power and pumped storage.

Note: Sum of components may not equal 100 percent due to independent rounding fuels).

Sources: Tables 8.2a, 8.2b, and 8.2d. 


\section{By Sector, 2011}

$5-$

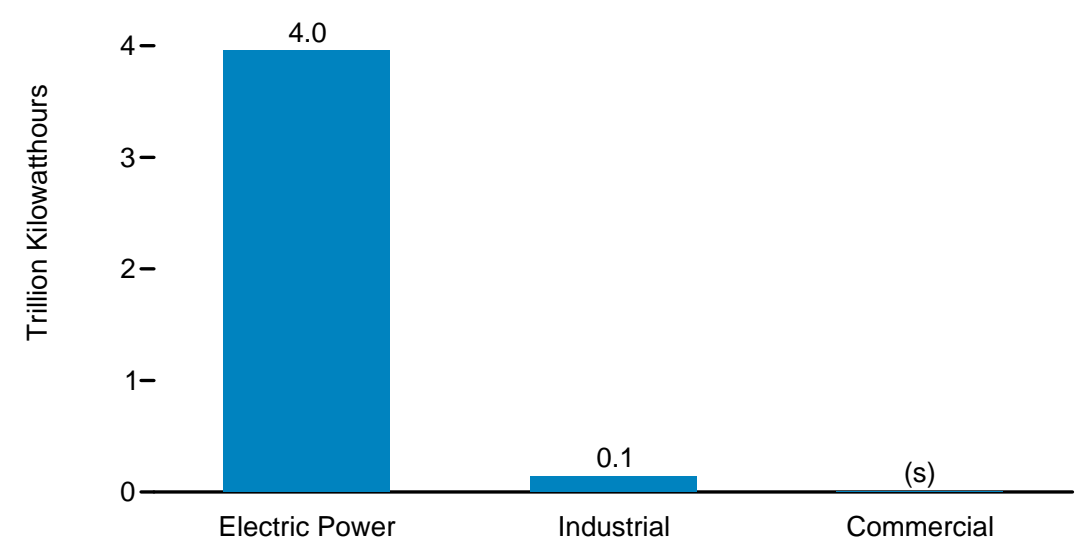

Electric Power Sector by Plant Type, 1989-2011

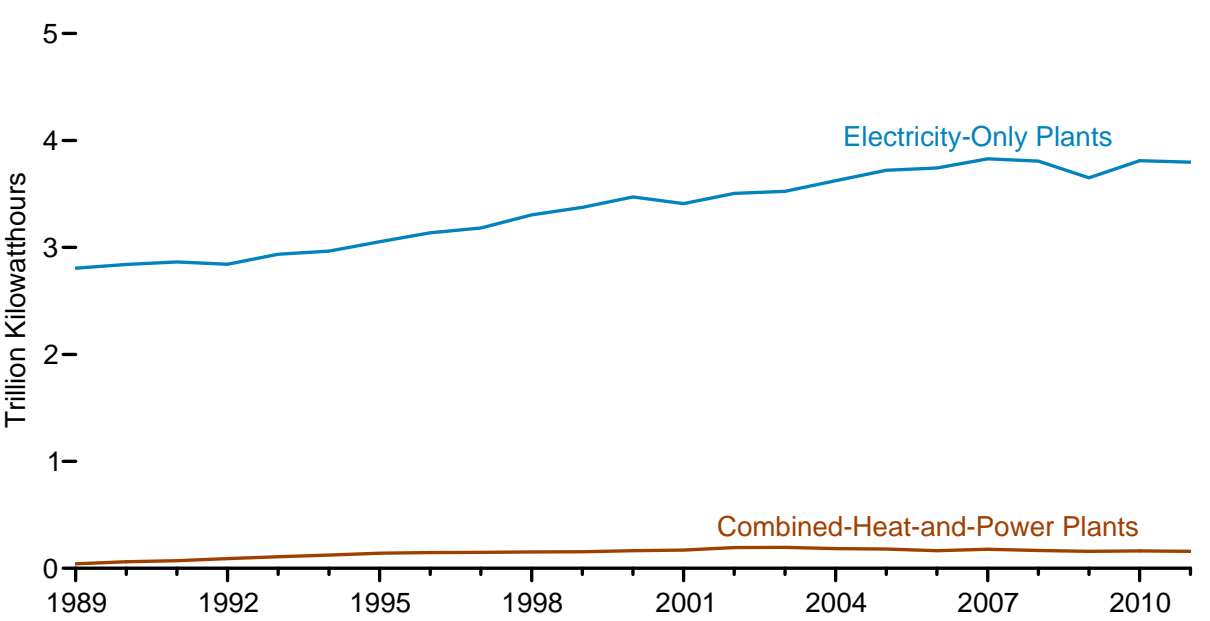

Industrial and Commercial Sectors, 2011

90-

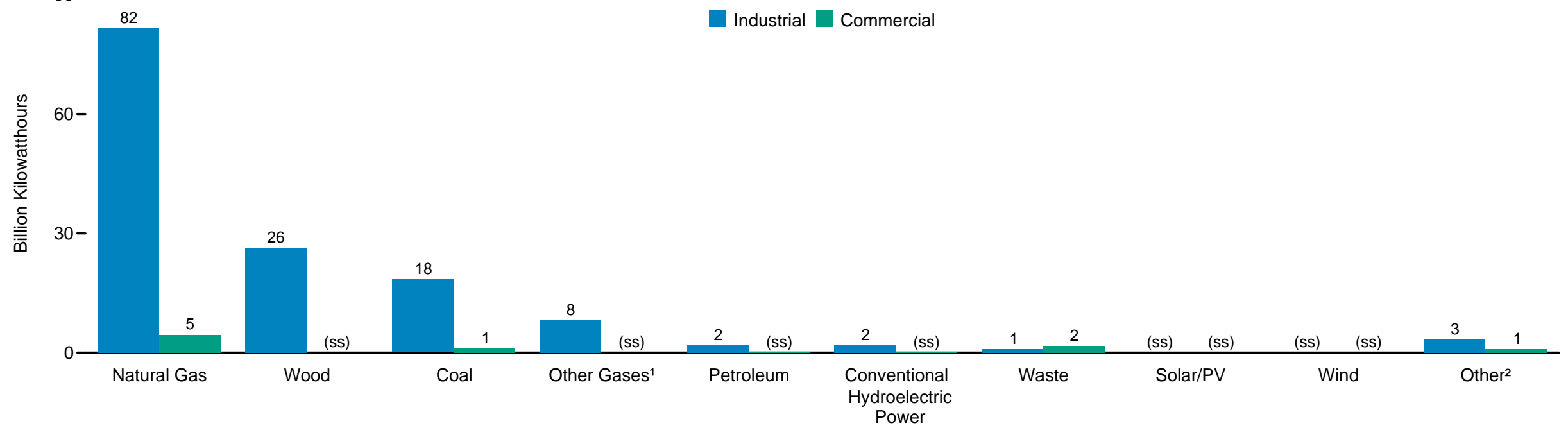

${ }^{1}$ Blast furnace gas, propane gas, and other manufactured and waste gases derived from fossil fuels.

${ }^{2}$ Batteries, chemicals, hydrogen, pitch, purchased steam, sulfur, miscellaneous technologies,

(s) = Less than 0.05 trillion kilowatthours. (ss) = Less than 0.5 billion kilowatthours. fuels).

Sources: Tables $8.2 \mathrm{~b}-8.2 \mathrm{~d}$. 
Table 8.2a Electricity Net Generation: Total (All Sectors), Selected Years, 1949-2011

(Sum of Tables 8.2b and 8.2d; Billion Kilowatthours)

\begin{tabular}{|c|c|c|c|c|c|c|c|c|c|c|c|c|c|c|c|c|}
\hline \multirow[b]{3}{*}{ Year } & \multicolumn{5}{|c|}{ Fossil Fuels } & \multirow{3}{*}{$\begin{array}{l}\text { Nuclear } \\
\text { Electric } \\
\text { Power }\end{array}$} & \multirow{3}{*}{$\begin{array}{l}\text { Hydro- } \\
\text { electric } \\
\text { Pumped } \\
\text { Storage } 5\end{array}$} & \multicolumn{7}{|c|}{ Renewable Energy } & \multirow[b]{3}{*}{ Other ${ }^{10}$} & \multirow[b]{3}{*}{ Total } \\
\hline & & & & & & & & \multirow{2}{*}{$\begin{array}{c}\text { Conventional } \\
\text { Hydroelectric } \\
\text { Power }^{6}\end{array}$} & \multicolumn{2}{|c|}{ Biomass } & \multirow{2}{*}{$\begin{array}{c}\text { Geo- } \\
\text { thermal }\end{array}$} & \multirow[b]{2}{*}{ Solar/PV 9} & \multirow[b]{2}{*}{ Wind } & \multirow[b]{2}{*}{ Total } & & \\
\hline & Coal 1 & Petroleum $^{2}$ & $\begin{array}{l}\text { Natural } \\
\text { Gas }^{3}\end{array}$ & $\begin{array}{l}\text { Uner } \\
\text { Gases }{ }^{4}\end{array}$ & Total & & & & Wood 7 & Waste $^{8}$ & & & & & & \\
\hline 1949 & 135.5 & 28.5 & 37.0 & NA & 201.0 & 0.0 & $\left({ }^{6}\right)$ & 94.8 & 0.4 & NA & NA & NA & NA & 95.2 & NA & 296.1 \\
\hline 1950 & 154.5 & 33.7 & 44.6 & NA & 232.8 & .0 & $\left({ }^{6}\right)$ & 100.9 & .4 & NA & NA & NA & NA & 101.3 & NA & 334.1 \\
\hline 1955 & 301.4 & 37.1 & 95.3 & NA & 433.8 & .0 & $\left({ }^{6}\right)$ & 116.2 & .3 & NA & NA & NA & NA & 116.5 & NA & 550.3 \\
\hline 1960 & 403.1 & 48.0 & 158.0 & NA & 609.0 & .5 & $\left({ }^{6}\right)$ & 149.4 & .1 & NA & (s) & NA & NA & 149.6 & NA & 759.2 \\
\hline 1965 & 570.9 & 64.8 & 221.6 & NA & 857.3 & 3.7 & $\left({ }^{6}\right)$ & 197.0 & .3 & NA & .2 & NA & NA & 197.4 & NA & $1,058.4$ \\
\hline 1970 & 704.4 & 184.2 & 372.9 & $\mathrm{NA}$ & $1,261.5$ & 21.8 & (6) & 251.0 & .1 & .2 & .5 & NA & NA & 251.8 & NA & $1,535.1$ \\
\hline 1975 & 852.8 & 289.1 & 299.8 & NA & $1,441.7$ & 172.5 & $(6)$ & 303.2 & (s) & 2 & 3.2 & NA & NA & 306.6 & NA & $1,920.8$ \\
\hline 1976 & 944.4 & 320.0 & 294.6 & $\mathrm{NA}$ & $1,559.0$ & 191.1 & $\left({ }^{6}\right)$ & 286.9 & .1 & .2 & $\begin{array}{l}3.6 \\
3.6\end{array}$ & NA & NA & 290.8 & NA & $2,040.9$ \\
\hline 1977 & 985.2 & 358.2 & 305.5 & NA & $1,648.9$ & 250.9 & $\left({ }^{6}\right)$ & 223.6 & .3 & .2 & 3.6 & NA & NA & 227.7 & NA & $2,127.4$ \\
\hline 1978 & 975.7 & 365.1 & 305.4 & NA & $1,646.2$ & 276.4 & $\left({ }^{6}\right)$ & 283.5 & .2 & .1 & 3.0 & NA & NA & 286.8 & NA & $2,209.4$ \\
\hline 1979 & $1,075.0$ & 303.5 & 329.5 & NA & $1,708.0$ & 255.2 & $\left({ }^{6}\right)$ & 283.1 & .3 & .2 & 3.9 & NA & NA & 287.5 & NA & $2,250.7$ \\
\hline 1980 & $1,161.6$ & 246.0 & 346.2 & NA & $1,753.8$ & 251.1 & $\left({ }^{6}\right)$ & 279.2 & .3 & .2 & 5.1 & NA & NA & 284.7 & NA & $2,289.6$ \\
\hline 1981 & $1,203.2$ & 206.4 & 345.8 & NA & $1,755.4$ & 272.7 & $(6)$ & 263.8 & .2 & .1 & 5.7 & NA & NA & 269.9 & NA & $2,298.0$ \\
\hline 1982 & $\begin{array}{l}1,003.2 \\
1,192.0\end{array}$ & $\begin{array}{l}20.4 \\
146.8\end{array}$ & $\begin{array}{l}45.0 \\
305.3\end{array}$ & NA & $\begin{array}{l}1,135.4 \\
1,644.1\end{array}$ & 282.8 & $(6)$ & $\begin{array}{l}312.0 \\
312.4\end{array}$ & .2 & .1 & $\begin{array}{l}.1 \\
4.8\end{array}$ & NA & NA & 317.5 & NA & $\begin{array}{l}2,244.4 \\
\end{array}$ \\
\hline 1983 & $\begin{array}{l}1,259.4 \\
\end{array}$ & $\begin{array}{l}144.5 \\
\end{array}$ & $\begin{array}{l}274.1 \\
274.5\end{array}$ & NA & $1,678.0$ & 293.7 & $(6)$ & 335.3 & .2 & .2 & $\begin{array}{l}6.0 \\
6.1\end{array}$ & NA & (s) & 341.7 & NA & $\begin{array}{l}2,313.4 \\
\end{array}$ \\
\hline 1984 & $1,341.7$ & 119.8 & 297.4 & NA & $1,758.9$ & 327.6 & $\left({ }^{6}\right)$ & 324.3 & .5 & .4 & 7.7 & (s) & (s) & 332.9 & NA & $2,419.5$ \\
\hline 1985 & $1,402.1$ & 100.2 & 291.9 & NA & $1,794.3$ & 383.7 & $\left({ }^{6}\right)$ & 284.3 & .7 & .6 & 9.3 & (s) & (s) & 295.0 & NA & $2,473.0$ \\
\hline 1986 & $1,385.8$ & 136.6 & 248.5 & NA & $1,770.9$ & 414.0 & $\left({ }^{6}\right)$ & 294.0 & .5 & .7 & 10.3 & (s) & (s) & 305.5 & NA & $2,490.5$ \\
\hline 1987 & $1,463.8$ & 118.5 & 272.6 & NA & $1,854.9$ & 455.3 & $\left(6^{\prime}\right)$ & 252.9 & .8 & .7 & 10.8 & (s) & (s) & 265.1 & NA & $2,575.3$ \\
\hline 1988 & $\begin{array}{r}1,400.0 \\
\end{array}$ & $\begin{array}{l}110.5 \\
148.9\end{array}$ & 252.8 & NA & $\begin{array}{r}1,054.5 \\
\end{array}$ & 527.0 & (6) & 226.1 & $\begin{array}{l}.0 \\
.9 \\
\end{array}$ & .7 & $\begin{array}{l}10.0 \\
10.3\end{array}$ & $\begin{array}{l}\text { (s) } \\
\text { (s) }\end{array}$ & $\begin{array}{l}\text { (s) } \\
\text { (s) }\end{array}$ & 238.1 & NA & $\begin{array}{l}2,707.4 \\
\end{array}$ \\
\hline $1989^{11}$ & $1,583.8$ & 164.4 & 352.6 & 7.9 & $2,108.6$ & 529.4 & $\left(6^{6}\right)$ & 272.0 & 27.2 & 9.2 & 14.6 & .3 & 2.1 & 325.3 & 3.8 & $2,967.1$ \\
\hline 1990 & $1,594.0$ & 126.5 & 372.8 & 10.4 & $2,103.6$ & 576.9 & $\begin{array}{l}1 \\
-3.5\end{array}$ & 292.9 & 32.5 & 13.3 & 15.4 & .4 & 2.8 & 357.2 & 3.6 & $3,037.8$ \\
\hline 1991 & $1,590.6$ & 119.8 & 381.6 & 11.3 & $2,103.3$ & 612.6 & -4.5 & 289.0 & 33.7 & 15.7 & $\begin{array}{l}16.4 \\
16.0\end{array}$ & .5 & $\begin{array}{l}3.0 \\
3.0\end{array}$ & 357.8 & $\begin{array}{l}3.0 \\
4.7\end{array}$ & $3,073.8$ \\
\hline 1992 & $1,621.2$ & 100.2 & 404.1 & 13.3 & $2,138.7$ & 618.8 & -4.2 & 253.1 & 36.5 & 17.8 & 16.1 & .4 & 2.9 & 326.9 & 3.7 & $3,083.9$ \\
\hline 1993 & $1,690.1$ & 112.8 & 414.9 & 13.0 & $2,230.7$ & 610.3 & -4.0 & 280.5 & 37.6 & 18.3 & 16.8 & .5 & 3.0 & 356.7 & 3.5 & $3,197.2$ \\
\hline 1994 & $1,690.7$ & $\begin{array}{l}105.9 \\
10.9\end{array}$ & 460.2 & 13.3 & $2,270.1$ & 640.4 & -3.4 & 260.1 & $\begin{array}{l}37.9 \\
37.0\end{array}$ & $\begin{array}{l}19.1 \\
19.1\end{array}$ & 15.5 & .5 & $\begin{array}{l}3.0 \\
3.4\end{array}$ & 336.7 & 3.7 & $3,247.5$ \\
\hline 1995 & $\begin{array}{l}1,0909.4 \\
\end{array}$ & 74.6 & 496.1 & $\begin{array}{l}13.0 \\
13.9\end{array}$ & $2,293.9$ & $\begin{array}{l}67.4 .4 \\
673.4\end{array}$ & $\begin{array}{l}-3.4 \\
-2.7\end{array}$ & 310.8 & 36.5 & 20.4 & $\begin{array}{l}13.4 \\
13.4\end{array}$ & .5 & $\begin{array}{l}.4 \\
3.2\end{array}$ & 384.8 & $\begin{array}{l}. .1 \\
4.1\end{array}$ & $\begin{array}{l}0,353.5 \\
3,5\end{array}$ \\
\hline 1996 & $\begin{array}{l}1,795.4 \\
\end{array}$ & $\begin{array}{l}81.4 \\
81.0\end{array}$ & 455.1 & 14.4 & $2,346.0$ & $\begin{array}{l}674.7 \\
6\end{array}$ & -3.1 & $\begin{array}{l}347.2 \\
34.2\end{array}$ & $\begin{array}{l}36.8 \\
36.8\end{array}$ & 20.9 & $\begin{array}{l}14.4 \\
14.3\end{array}$ & .5 & $\begin{array}{l}3.2 \\
3.2\end{array}$ & 423.0 & $\begin{array}{l}4.1 \\
3.6\end{array}$ & $3,444.2$ \\
\hline 1997 & $1,845.0$ & 92.6 & $\begin{array}{l}479.4 \\
479.1\end{array}$ & 13.4 & $2,430.3$ & 628.6 & -4.0 & 356.5 & 36.9 & 21.7 & 14.7 & .5 & 3.3 & 433.6 & $\begin{array}{l}3.0 \\
3.6\end{array}$ & $3,492.2$ \\
\hline 1998 & $1,873.5$ & 128.8 & 531.3 & 13.5 & $2,547.1$ & 673.7 & -4.5 & 323.3 & 36.3 & 22.4 & 14.8 & .5 & 3.0 & 400.4 & 3.6 & $3,620.3$ \\
\hline 1999 & $1,881.1$ & 118.1 & 556.4 & 14.1 & $2,569.7$ & 728.3 & -6.1 & 319.5 & 37.0 & 22.6 & 14.8 & .5 & 4.5 & 399.0 & 4.0 & $3,694.8$ \\
\hline 2000 & $1,966.3$ & 111.2 & 601.0 & 14.0 & $2,692.5$ & 753.9 & -5.5 & 275.6 & 37.6 & 23.1 & 14.1 & .5 & 5.6 & 356.5 & 4.8 & $3,802.1$ \\
\hline 2001 & $\begin{array}{l}1,904.0 \\
\end{array}$ & 124.9 & 639.1 & 9.0 & $2,677.0$ & 768.8 & -8.8 & 217.0 & $\begin{array}{l}35.2 \\
35.2\end{array}$ & 14.5 & 13.7 & .5 & $\begin{array}{l}0.0 \\
6.7\end{array}$ & 287.7 & $\begin{array}{r}4.0 \\
11.9\end{array}$ & $3,736.6$ \\
\hline 2002 & $\begin{array}{r}1,904.0 \\
1,933.1\end{array}$ & $\begin{array}{r}94.6 \\
\end{array}$ & 691.0 & 11.5 & $2,730.2$ & 780.1 & $\begin{array}{l}-0.0 \\
-8.7\end{array}$ & 264.3 & $\begin{array}{l}35.2 \\
38.7\end{array}$ & $\begin{array}{l}14.5 \\
15.0\end{array}$ & $\begin{array}{l}14.1 \\
14.5\end{array}$ & .6 & $\begin{array}{l}0.1 \\
10.4\end{array}$ & 343.4 & 13.5 & $\begin{array}{l}3,858.6 \\
3,858\end{array}$ \\
\hline 2003 & $1,973.7$ & 119.4 & 649.9 & 15.6 & $2,758.6$ & 763.7 & -8.5 & 275.8 & 37.5 & 15.8 & 14.4 & .5 & 11.2 & 355.3 & 14.0 & $3,883.2$ \\
\hline 2004 & $1,978.3$ & $\begin{array}{l}121.1 \\
121\end{array}$ & 710.1 & $\begin{array}{l}15.3 \\
15.3\end{array}$ & $2,824.8$ & 788.5 & -8.5 & 268.4 & 38.1 & $\begin{array}{l}15.4 \\
15.4\end{array}$ & $\begin{array}{l}14.8 \\
14.8\end{array}$ & .6 & 14.1 & 351.5 & 14.2 & $3,970.6$ \\
\hline 2005 & $2,012.9$ & 122.2 & 761.0 & 13.5 & $2,909.5$ & 782.0 & -6.6 & 270.3 & 38.9 & 15.4 & 14.7 & .6 & 17.8 & 357.7 & 12.8 & $4,055.4$ \\
\hline 2006 & $1,990.5$ & 64.2 & 816.4 & 14.2 & $2,885.3$ & 787.2 & -6.6 & 289.2 & 38.8 & 16.1 & 14.6 & .5 & 26.6 & 385.8 & 13.0 & $4,064.7$ \\
\hline 2007 & $2,016.5$ & 65.7 & 896.6 & 13.5 & $2,992.2$ & 806.4 & -6.9 & 247.5 & $\begin{array}{l}39.0 \\
39.0\end{array}$ & 16.5 & $\begin{array}{l}14.6 \\
\text {. }\end{array}$ & .6 & 34.4 & 352.7 & 12.2 & $4,156.7$ \\
\hline 2008 & $\begin{array}{l}2,985.8 \\
\end{array}$ & 46.2 & $\begin{array}{l}85.0 \\
883.0\end{array}$ & 11.7 & $\begin{array}{l}2,926.2 \\
\end{array}$ & $\begin{array}{l}000.4 \\
806.2\end{array}$ & $\begin{array}{l}-0.9 \\
-6.3\end{array}$ & 254.8 & 37.3 & $\begin{array}{l}17.7 \\
17.7\end{array}$ & $\begin{array}{l}14.0 \\
14.8\end{array}$ & $\begin{array}{l}.0 \\
.9\end{array}$ & $\begin{array}{l}55.4 \\
55.4\end{array}$ & 380.9 & 11.8 & $\begin{array}{r}4,119.4 \\
4,11\end{array}$ \\
\hline 2009 & $1,755.9$ & 38.9 & 921.0 & 10.6 & $2,726.5$ & 798.9 & -4.6 & 273.4 & 36.1 & 18.4 & 15.0 & .9 & 73.9 & 417.7 & 11.9 & $3,950.3$ \\
\hline 2010 & $\mathrm{R}_{1,847.3}$ & $\begin{array}{r}30.9 \\
\mathrm{R}_{37}\end{array}$ & R987.7 & $\mathrm{R}_{11.3}$ & $\mathrm{R}_{2,883.4}^{2, / 2}$ & 807.0 & $\mathrm{R}_{-5.5}^{-4.0}$ & $\begin{array}{r}\text { R } 260.4 \\
\end{array}$ & $\begin{array}{r}30.1 \\
R_{37.2}\end{array}$ & $\begin{array}{r}10.4 \\
R_{18.9}\end{array}$ & $\mathrm{R}_{15.2}$ & $\mathrm{R}_{1.2}^{.9}$ & $\begin{array}{r}\text { R94.9 } \\
\text { R94. }\end{array}$ & $\begin{array}{r}411.1 \\
R_{4} 227.4\end{array}$ & $\mathrm{R}_{12.9}$ & $\mathrm{R}_{4,125.1}$ \\
\hline $2011^{P}$ & $1,734.3$ & 28.2 & $1,016.6$ & 11.3 & $2,790.3$ & 790.2 & $\begin{array}{l}-5.3 \\
-5.9\end{array}$ & 325.1 & 36.9 & 19.8 & 16.7 & 1.8 & 119.7 & 520.1 & 11.1 & $4,105.7$ \\
\hline
\end{tabular}

1 Anthracite, bituminous coal, subbituminous coal, lignite, waste coal, and coal synfuel.

2 Distillate fuel oil, residual fuel oil, petroleum coke, jet fuel, kerosene, other petroleum, and waste oil Natural gas, plus a small amount of supplemental gaseous fuels.

tossil fuels.

Pumped storage facility production minus energy used for pumping

6 Through 1989, hydroelectric pumped storage is included in "Conventional Hydroelectric Power."

Municipal solid waste from biogenic sources, landfill gas, sludge waste, agricultural byproducts, and other biomass. Through 2000, also includes non-renewable waste (municipal solid waste from non-biogenic sources, and tire-derived fuels).

Solar thermal and photovoltaic (PV) energy.

(miscellaneous technologies, and fuels).

Through 1988, all data except hydroelectric are for electric utilities only; hydroelectric data through or electric utilities, independent power producers, commercial plants, and industrial plants.

Notes: - See Note 1, "Coverage of Electricity Statistics," at end of section. - Totals may not equal sum of components due to independent rounding.

Web Pages: - See http://www.eia.gov/totalenergy/data/monthly/\#electricity for updated monthly and annual data. - See http://www.eia.gov/totalenergy/data/annual/\#electricity for all annual data beginning in Sources: - 1949-1988- Table 8.2b for electric power sector, and Table 8.1 for industrial sector. - 1989
forward-Tables $8.2 \mathrm{~b}$ and $8.2 \mathrm{~d}$. 
Table 8.2b Electricity Net Generation: Electric Power Sector, Selected Years, 1949-2011

(Subset of Table 8.2a; Billion Kilowatthours)

\begin{tabular}{|c|c|c|c|c|c|c|c|c|c|c|c|c|c|c|c|c|}
\hline \multirow[b]{3}{*}{ Year } & \multicolumn{5}{|c|}{ Fossil Fuels } & \multirow{3}{*}{$\begin{array}{l}\text { Nuclear } \\
\text { Electric } \\
\text { Power }\end{array}$} & \multirow{3}{*}{$\begin{array}{l}\text { Hydro- } \\
\text { electric } \\
\text { Pumped } \\
\text { Storage }^{5}\end{array}$} & \multicolumn{7}{|c|}{ Renewable Energy } & \multirow[b]{3}{*}{ Other 10} & \multirow[b]{3}{*}{ Total } \\
\hline & \multirow[b]{2}{*}{ Coal 1} & \multirow[b]{2}{*}{ Petroleum ${ }^{2}$} & \multirow{2}{*}{$\begin{array}{c}\text { Natural } \\
\text { Gas }^{3}\end{array}$} & \multirow{2}{*}{$\begin{array}{c}\text { Other } \\
\text { Gases }{ }^{4}\end{array}$} & \multirow[b]{2}{*}{ Total } & & & \multirow{2}{*}{$\begin{array}{c}\text { Conventional } \\
\text { Hydroelectric } \\
\text { Power }^{6}\end{array}$} & \multicolumn{2}{|c|}{ Biomass } & \multirow{2}{*}{$\begin{array}{c}\text { Geo- } \\
\text { thermal }\end{array}$} & \multirow[b]{2}{*}{ Solar/PV 9} & \multirow[b]{2}{*}{ Wind } & \multirow[b]{2}{*}{ Total } & & \\
\hline & & & & & & & & & Wood $^{7}$ & Waste ${ }^{8}$ & & & & & & \\
\hline 1949 & 135.5 & 28.5 & 37.0 & NA & 201.0 & 0.0 & $\left(\begin{array}{l}6 \\
6\end{array}\right)$ & 89.7 & 0.4 & NA & NA & NA & NA & 90.1 & NA & 291.1 \\
\hline $\begin{array}{l}1950 \\
1955\end{array}$ & $\begin{array}{l}154.5 \\
3014\end{array}$ & 33.7 & $\begin{array}{l}44.6 \\
95.3\end{array}$ & NA & $\begin{array}{l}232.8 \\
433.8\end{array}$ & .0 & $\begin{array}{l}(6) \\
(6)\end{array}$ & 95.9 & .4 & NA & NA & NA & NA & 96.3 & NA & 329.1 \\
\hline 1960 & 403.1 & 48.0 & 158.0 & NA & $\begin{array}{l}409.0 \\
609.0\end{array}$ & .0 & $\begin{array}{l}(0) \\
(6)\end{array}$ & $\begin{array}{l}13.0 \\
145.8\end{array}$ & .3 & NA & NA & NA & $\begin{array}{l}\text { NA } \\
\text { NA }\end{array}$ & $\begin{array}{l}113.3 \\
1460\end{array}$ & NA & $\begin{array}{l}547.0 \\
755.5\end{array}$ \\
\hline 1965 & $\begin{array}{l}400.1 \\
570.9\end{array}$ & $\begin{array}{l}40.0 \\
64.8\end{array}$ & 221.6 & NA & 857.3 & 3.5 & $(6)$ & $\begin{array}{l}40.0 \\
1939\end{array}$ & 3 & NA & (1) & NA & NA & $\begin{array}{l}194.0 \\
1943\end{array}$ & NA & 1.0553 \\
\hline 1970 & 704.4 & 184.2 & 372.9 & NA & 1.261 .5 & 21.8 & $(6)$ & 247.7 & $\begin{array}{l}.3 \\
.1\end{array}$ & .2 & .5 & NA & NA & 2486 & NA & 1,5310 \\
\hline 1975 & $\begin{array}{l}04.4 \\
852.8\end{array}$ & $\begin{array}{l}184.2 \\
289.1\end{array}$ & $\begin{aligned} 299.8 & \end{aligned}$ & NA & $1,441.7$ & 172.5 & $(6)$ & 300.0 & (s) & .2 & 3.2 & NA & NA & $\begin{array}{l}240.0 \\
303.5\end{array}$ & NA & 1,9176 \\
\hline 1976 & $\begin{array}{l}852.8 \\
944.4\end{array}$ & 3200 & 2946 & NA & 1,5590 & 191.1 & $(6)$ & 2837 & (s) & 2 & 3.2 & NA & NA & 287.5 & NA & 2,037 \\
\hline 1977 & $\begin{array}{l}984.4 \\
985.2\end{array}$ & 358.2 & 305.5 & NA & $1,648.9$ & 250.9 & (6) & 220.5 & .3 & 2 & $\begin{array}{l}3.0 \\
3.6\end{array}$ & NA & NA & 224.5 & NA & $2,124.3$ \\
\hline 1978 & 975.7 & 365.1 & 305.4 & NA & $\begin{array}{l}1,046.2 \\
1,646 .\end{array}$ & 276.4 & $(6)$ & 280.4 & .2 & .1 & $\begin{array}{l}3.0 \\
3.0\end{array}$ & NA & NA & 283.7 & NA & $2,206.3$ \\
\hline 1979 & $1,075.0$ & 303.5 & 329.5 & NA & $\begin{array}{l}1,040.2 \\
1,708.0\end{array}$ & $\begin{array}{l}255.2 \\
25.2\end{array}$ & $(6)$ & $\begin{array}{l}279.8 \\
27.8\end{array}$ & .3 & .2 & $\begin{array}{l}3.0 \\
3.9\end{array}$ & NA & NA & $\begin{array}{l}284.1 \\
284.2\end{array}$ & NA & $2,247.4$ \\
\hline 1980 & $1,161.6$ & 246.0 & 346.2 & NA & $1,753.8$ & 251.1 & $(6)$ & 276.0 & .3 & 2 & 5.1 & NA & NA & $\begin{array}{l}284.2 \\
281.5\end{array}$ & NA & $2,286.4$ \\
\hline 1981 & $1,203.2$ & 206.4 & 345.8 & NA & $1,755.4$ & 272.7 & $(6)$ & 260.7 & .2 & .1 & 5.7 & NA & NA & 266.7 & NA & $2,294.8$ \\
\hline 1982 & $1,192.0$ & 146.8 & 305.3 & NA & $1,644.1$ & 282.8 & $(6)$ & 309.2 & .2 & .1 & 4.8 & NA & NA & 314.4 & NA & $2,241.2$ \\
\hline 1983 & $1,259.4$ & 144.5 & 274.1 & NA & $1,678.0$ & 293.7 & $(6)$ & 332.1 & .2 & .2 & 6.1 & NA & (s) & 338.6 & NA & $2,310.3$ \\
\hline 1984 & $1,341.7$ & 119.8 & 297.4 & NA & $1,758.9$ & 327.6 & $\left({ }^{6}\right)$ & 321.2 & .5 & .4 & 7.7 & (s) & (s) & 329.8 & NA & $2,416.3$ \\
\hline 1985 & $1,402.1$ & 100.2 & 291.9 & NA & $1,794.3$ & 383.7 & $(6)$ & 281.1 & .7 & 6 & 9.3 & (s) & (s) & 291.9 & $\mathrm{NA}$ & $2,469.8$ \\
\hline 1986 & $1,385.8$ & 136.6 & 248.5 & NA & $1,770.9$ & 414.0 & $\left({ }^{6}\right)^{\prime}$ & 290.8 & .5 & .7 & 10.3 & (s) & (s) & 302.3 & NA & $2,487.3$ \\
\hline 1987 & $1,463.8$ & 118.5 & 272.6 & NA & $1,854.9$ & 455.3 & $(6)$ & 249.7 & .8 & .7 & 10.8 & (s) & (s) & 262.0 & NA & $2,572.1$ \\
\hline 1988 & $1,540.7$ & 148.9 & 252.8 & NA & $1,942.4$ & 527.0 & (6) & 222.9 & .9 & .7 & 10.3 & (s) & (s) & 234.9 & NA & 2.704 .3 \\
\hline $1989^{11}$ & $1,562.4$ & 159.0 & 297.3 & .5 & $2,019.1$ & 529.4 & $(6)$ & 269.2 & 5.6 & 7.7 & 14.6 & .3 & 2.1 & 299.5 & .3 & $2,848.2$ \\
\hline 1990 & $1,572.1$ & 118.9 & 309.5 & .6 & $2,001.1$ & 576.9 & -3.5 & 289.8 & 7.0 & 11.5 & 15.4 & .4 & 2.8 & 326.9 & (s) & $2,901.3$ \\
\hline 1991 & $1,568.8$ & 112.8 & 317.8 & .7 & $2,000.1$ & 612.6 & -4.5 & 286.0 & 7.7 & 13.9 & 16.0 & .5 & 3.0 & 327.0 & .4 & $2,935.6$ \\
\hline 1992 & $1,597.7$ & 92.2 & 334.3 & 1.2 & $2,025.4$ & 618.8 & -4.2 & 250.0 & 8.5 & 15.9 & 16.1 & 4 & 2.9 & 293.9 & .5 & $2,934.4$ \\
\hline 1993 & $1,665.5$ & 105.4 & 342.2 & 1.0 & $2,114.1$ & 610.3 & -4.0 & 277.5 & $\begin{array}{l}9.2 \\
9.2\end{array}$ & 16.2 & 16.8 & .5 & 3.0 & 323.2 & .4 & $3,043.9$ \\
\hline 1994 & $1,666.3$ & 98.7 & 385.7 & 1.1 & $2,151.7$ & 640.4 & -3.4 & 254.0 & $\begin{array}{l}9.2 \\
9.2\end{array}$ & $\begin{array}{l}17.2 \\
17.0\end{array}$ & $\begin{array}{l}15.0 \\
15.5\end{array}$ & .5 & $\begin{array}{l}3.0 \\
3.4\end{array}$ & 299.7 & .2 & $3,088.7$ \\
\hline 1995 & $1,686.1$ & 68.1 & 419.2 & 1.9 & $2,175.3$ & 673.4 & $\begin{array}{l}-2.4 \\
-2.7\end{array}$ & $\begin{array}{l}305.4 \\
305\end{array}$ & $\begin{array}{l}9.2 \\
7.6\end{array}$ & 18.0 & $\begin{array}{l}13.5 \\
13.4\end{array}$ & .5 & $\begin{array}{l}3.4 \\
3.2\end{array}$ & 348.0 & .2 & $\begin{array}{l}3,0001 \\
3,194 ?\end{array}$ \\
\hline 1996 & $\begin{array}{l}1,000.1 \\
1,772.0\end{array}$ & 74.8 & 378.8 & 1.3 & $2,226.9$ & 674.7 & -3.1 & 341.2 & 8.4 & 17.8 & 14.3 & .5 & $\begin{array}{l}3.2 \\
3.2\end{array}$ & 385.4 & 2 & $3,284.1$ \\
\hline 1997 & $1,820.8$ & $\begin{array}{l}14.0 \\
86.5\end{array}$ & $\begin{array}{l}399.6 \\
399 .\end{array}$ & 1.5 & $2,308.4$ & 628.6 & -4.0 & 350.6 & $\begin{array}{l}.0 .4 \\
8.7\end{array}$ & 18.5 & 14.7 & .5 & $\begin{array}{l}.24 \\
3.3\end{array}$ & 396.3 & 1 & $3,329.4$ \\
\hline 1998 & $\begin{array}{l}1,0<0.0 \\
1,850.2\end{array}$ & $\begin{array}{r}00.5 \\
122.2\end{array}$ & 449.3 & $\begin{array}{l}1.0 \\
2.3\end{array}$ & $\begin{array}{l}2,000.4 \\
2,424.0\end{array}$ & $\begin{array}{l}60.0 \\
673.7\end{array}$ & -4.5 & $\begin{array}{l}350.6 \\
317.9\end{array}$ & $\begin{array}{l}8.1 \\
8.6\end{array}$ & $\begin{array}{l}18.5 \\
19.2\end{array}$ & $\begin{array}{l}14.7 \\
14.8\end{array}$ & .5 & $\begin{array}{l}3.3 \\
3.0\end{array}$ & $\begin{array}{l}39.3 \\
364.0\end{array}$ & $\begin{array}{l}.1 \\
2\end{array}$ & $\begin{array}{l}3,329.4 \\
3,457\end{array}$ \\
\hline $\begin{array}{l}1999 \\
1999\end{array}$ & $1,858.6$ & 111.5 & 473.0 & 1.6 & 2444.8 & 728.3 & $\begin{array}{l}-4.0 \\
-6.1\end{array}$ & 314.7 & $\begin{array}{l}\text {. } \\
9.0\end{array}$ & 19.5 & $\begin{array}{l}14.8 \\
14.8\end{array}$ & .5 & 4.5 & 362.9 & 1 & $3,530.0$ \\
\hline 2000 & $\begin{array}{l}1,858.6 \\
1,943.1\end{array}$ & $\begin{array}{l}111.5 \\
105.2\end{array}$ & $\begin{array}{l}418.0 \\
518.0\end{array}$ & $\begin{array}{l}1.6 \\
2.0\end{array}$ & $\begin{array}{l}2,444.8 \\
2,568.3\end{array}$ & 753.9 & $\begin{array}{l}-6.1 \\
-5.5\end{array}$ & $\begin{array}{l}271.1 \\
271.3\end{array}$ & $\begin{array}{l}.0 \\
8.9\end{array}$ & $\begin{array}{l}19.5 \\
20.3\end{array}$ & $\begin{array}{l}14.8 \\
14.1\end{array}$ & .5 & $\begin{array}{l}4.5 \\
5.6\end{array}$ & $\begin{array}{l}36.9 \\
320.7\end{array}$ & $\begin{array}{l}.1 \\
.1\end{array}$ & $\begin{array}{l}3,53.0 \\
3,637.5\end{array}$ \\
\hline 2001 & $\begin{array}{l}1,943.1 \\
1,882.8\end{array}$ & $\begin{array}{l}115.2 \\
119.1\end{array}$ & $\begin{array}{l}518.0 \\
554.9\end{array}$ & $\begin{array}{r}2.0 \\
.6\end{array}$ & $\begin{array}{l}2,568.3 \\
2,557.5\end{array}$ & $\begin{array}{l}563.9 \\
768.8\end{array}$ & $\begin{array}{l}-5.5 \\
-8.8\end{array}$ & $\begin{array}{l}217.3 \\
213.7\end{array}$ & $\begin{array}{l}8.9 \\
8.3\end{array}$ & $\begin{array}{l}20.3 \\
12.9\end{array}$ & $\begin{array}{l}14.1 \\
13.7\end{array}$ & .5 & $\begin{array}{l}5.6 \\
6.7\end{array}$ & $\begin{array}{l}320.1 \\
256.0\end{array}$ & 6.5 & $\begin{array}{l}3,63.5 \\
3,580.1\end{array}$ \\
\hline 2002 & $1,910.6$ & 89.7 & 607.7 & 2.0 & $2,610.0$ & 780.1 & $\begin{array}{l}-8.7 \\
-8.0\end{array}$ & 260.5 & $\begin{array}{l}9.0 \\
9.0\end{array}$ & 13.1 & 14.5 & 6 & 10.4 & 308.0 & 9.1 & $3,698.5$ \\
\hline 2003 & 1952.7 & 113.7 & 567.3 & 2.6 & $2,636.4$ & 763.7 & -8.5 & 271.5 & 9.5 & 13.8 & 14.4 & .0 & 11.2 & 321 & $\begin{array}{l}8.1 \\
8.6\end{array}$ & $3,721.2$ \\
\hline 2004 & $\begin{array}{l}1,957.1 \\
\end{array}$ & $\begin{array}{l}114.7 \\
114.7\end{array}$ & $\begin{array}{l}627.2 \\
62.2\end{array}$ & $\begin{array}{l}3.0 \\
3.6\end{array}$ & $2,702.6$ & 788.5 & $\begin{array}{l}-0.5 \\
-8.5\end{array}$ & 265.1 & 9.7 & 13. & 14. & .6 & $\begin{array}{l}11.2 \\
14.1\end{array}$ & 317.4 & 8.3 & 3.808 .4 \\
\hline 2005 & 19921 & 116.5 & 683.8 & $\begin{array}{l}3.0 \\
3.8\end{array}$ & 27961 & 782.0 & -0.5 & 2670 & 106 & 13 & 14 & .0 & 178 & 323 & 6.0 & $3,000.4$ \\
\hline 2006 & $1,969.7$ & 59.7 & 734.4 & $\begin{array}{l}3.0 \\
4.3\end{array}$ & $2,768.1$ & 787.2 & $\begin{array}{l}-0.0 \\
-6.6\end{array}$ & 286.3 & $\begin{array}{l}10.3 \\
10.3\end{array}$ & 13 & $\begin{array}{l}14.6 \\
14.6\end{array}$ & .5 & 26.6 & 352 & $\begin{array}{l}7.5 \\
7.1\end{array}$ & $3,908.1$ \\
\hline 2007 & $1,998.4$ & 61.3 & 814.8 & 4.0 & $2,878.5$ & 806.4 & -6.9 & $\begin{array}{l}245.0 \\
245.8\end{array}$ & 10.7 & 14. & 14 & 6 & 34.4 & 32 & 6.8 & $4,005.3$ \\
\hline 2008 & $1,968.8$ & 42.9 & 802.4 & 3.2 & $2,817.3$ & 806.2 & -6.3 & 253.1 & 10.6 & 15.4 & 14. & .9 & 55.4 & 35 & 7.0 & $3,974.3$ \\
\hline 2009 & $1,741.1$ & 35.8 & 841.0 & 3.1 & $2,621.0$ & 798.9 & -4.6 & 271.5 & 10.7 & 16.0 & $\begin{array}{l}14.0 \\
15.0\end{array}$ & .9 & 73.9 & 388.0 & 6.6 & $\begin{array}{l}3,9 / 4 . \\
3.809 .8\end{array}$ \\
\hline 2010 & $\mathrm{R}_{1,827.7}$ & R34.7 & R901.4 & $\mathrm{R}_{3.0}$ & $\mathrm{R}_{2,766.8}$ & 807.0 & $\mathrm{R}_{-5.5}$ & $\mathrm{R}_{258.5}$ & $\mathrm{R}_{11.4}$ & $\mathrm{R}_{16.4}$ & $R_{15} .2$ & $\mathrm{R}_{1.2}$ & 94.6 & R397.3 & $\mathrm{R}_{6.8}$ & $\begin{array}{r}3,009.8 \\
\text { R }\end{array}$ \\
\hline $2011^{P}$ & $1,714.9$ & 26.2 & 930.6 & 3.1 & $2,674.8$ & 790.2 & -5.9 & 323.1 & 10.5 & 17.2 & 16.7 & 1.8 & $\begin{array}{r}119.7 \\
\end{array}$ & 489.0 & 6.9 & $3,955.1$ \\
\hline
\end{tabular}

Anthracite, bituminous coal, subbituminous coal, lignite, waste coal, and coal synfuel.

Distillate fuel oil, residual fuel oil, petroleum coke, jet fuel, kerosene, other petroleum, and waste oil. Natural gas, plus a small amount of supplemental gaseous fuels.

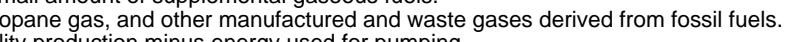

6 Through 1989, hydroelectric pumped storage is included in "Conventional Hydroelectric Power."

7 Wood and wood-derived fuels.

${ }^{8}$ Municipal solid waste from biogenic sources, landfill gas, sludge waste, agricultural byproducts, and other biomass. Through 2000, also includes non-renewable waste (municipal solid waste from non-biogenic sources, and tire-derived fuels).

10 Batteries, chemicals, hydrogen, pitch, purchased steam, sulfur, miscellaneous technologies, and beginning in 2001, non-renewable waste (municipal solid waste from non-biogenic sources, and tire-derived ${ }_{11}$ Through 1988, data are for electric utilities only. Beginning in 1989, data are for electric utilities and

independent power producers.
$R=$ Revised. $P=$ Preliminary. NA=Not available. $(s)=$ Less than 0.05 billion kilowatthours.

Notes: - The electric power sector comprises electricity-only and combined-heat-and-power (CHP) plants within the NAICS 22 category whose primary business is to sell electricity, or electricty and heat, to the public. - See Table 8.2d for commercial and industrial CHP and electricity-only data. • See Note 1, end of section. - Totals may not equal sum of components due to independent rounding. Web Pages: - See http://www.eia.gov/totalenergy/data/monthly/t\#electricity for updated monthly and annual data. - See http://www.eia.gov/totalenergy/data/annual/\#electricity for all annual data beginning in

Sources: 1949-September 1977-Federal Power Commission, Form FPC-4, "Monthly Power Plant Report." • October 1977-1981-Federal Energy Regulatory Commission, Form FPC-4, "Monthly Power Power Plant Report." - 1989-1997-EIA, Form EIA-759, "Monthly Power Plant Report," and Form EIA-867, "Annual Nonutility Power Producer Report." • 1998-2000—EIA, Form EIA-759, "Monthly Power Plant Report," and Form EIA-860B, "Annual Electric Generator Report-Nonutility." • 2001-2003-EIA, Form EIA-906, "Power Plant Report." • 2004-2007-EIA, Form EIA-906, "Power Plant Report," and Form EIA-920, "Combined Heat and Power Plant Report." • 2008 forward-EIA, Form EIA-923, "Power Plant 
Table 8.2c Electricity Net Generation: Electric Power Sector by Plant Type, Selected Years, 1989-2011

(Breakout of Table 8.2b; Billion Kilowatthours)

\begin{tabular}{|c|c|c|c|c|c|c|c|c|c|c|c|c|c|c|c|c|}
\hline \multirow[b]{3}{*}{ Year } & \multicolumn{5}{|c|}{ Fossil Fuels } & \multirow{3}{*}{$\begin{array}{l}\text { Nuclear } \\
\text { Electric } \\
\text { Power }\end{array}$} & \multirow{3}{*}{$\begin{array}{c}\text { Hydro- } \\
\text { electric } \\
\text { Pumped } \\
\text { Storage } 5\end{array}$} & \multicolumn{7}{|c|}{ Renewable Energy } & \multirow[b]{3}{*}{ Other ${ }^{10}$} & \multirow[b]{3}{*}{ Total } \\
\hline & & & & & & & & Conventional & & ass & & & & & & \\
\hline & Coal ${ }^{1}$ & Petroleum $^{2}$ & Gas $^{3}$ & Gases ${ }^{4}$ & Total & & & Power ${ }^{6}$ & Wood 7 & Waste ${ }^{8}$ & thermal & Solar/PV ${ }^{9}$ & Wind & Total & & \\
\hline
\end{tabular}

\begin{tabular}{|c|c|c|c|c|c|c|c|c|c|c|c|c|c|c|c|c|}
\hline \multirow[b]{2}{*}{1989} & \multicolumn{16}{|c|}{ Electricity-Only Plants 11} \\
\hline & $1,554.0$ & 158.3 & 266.9 & & $1,979.3$ & 529.4 & $\left({ }^{6}\right)$ & 269.2 & 4.2 & 6.9 & 14.6 & 0.3 & 2.1 & 297.3 & - & $2,805.9$ \\
\hline 1990 & $1,560.2$ & 117.6 & 264.7 & (s) & $1,942.4$ & 576.9 & -3.5 & 289.8 & 5.6 & 10.4 & 15.4 & .4 & 2.8 & 324.3 & - & $2,840.0$ \\
\hline 1995 & $1,658.0$ & 62.0 & 317.4 & (s) & $2,037.4$ & 673.4 & -2.7 & 305.4 & 5.9 & 16.3 & 13.4 & .5 & 3.2 & 344.7 & - & $3,052.8$ \\
\hline 1996 & $1,742.8$ & 68.5 & 272.8 & (s) & $2,084.1$ & 674.7 & -3.1 & 341.2 & 6.5 & 16.1 & 14.3 & .5 & 3.2 & 381.8 & - & $3,137.6$ \\
\hline 1997 & $1,793.2$ & 80.3 & 291.1 & (s) & $2,164.6$ & 628.6 & -4.0 & 350.6 & 6.5 & 16.4 & 14.7 & .5 & 3.3 & 392.0 & - & $3,181.3$ \\
\hline 1998 & $1,823.0$ & 115.7 & 335.9 & .1 & $2,274.6$ & 673.7 & -4.5 & 317.9 & $\begin{array}{l}6.5 \\
6.6\end{array}$ & $\begin{array}{l}17.0^{2} \\
17.0\end{array}$ & 14.8 & .5 & $\begin{array}{l}3.0 \\
3.0\end{array}$ & 359.8 & - & $3,303.6$ \\
\hline 1999 & $1,832.1$ & 104.8 & 356.6 & (s) & $2,293.6$ & 728.3 & -6.1 & 314.7 & 7.3 & 17.1 & 14.8 & .5 & 4.5 & 358.8 & - & $3,374.6$ \\
\hline 2000 & $1,910.6$ & 98.0 & 399.4 & 2 & $2,408.2$ & 753.9 & -5.5 & 271.3 & 7.3 & 17.6 & 14.1 & .5 & 5.6 & 316.4 & $=$ & $3,472.9$ \\
\hline 2001 & $1,851.8$ & 113.2 & 427.0 & (s) & $2,392.0$ & 768.8 & -8.8 & 213.7 & 6.6 & 11.3 & 13.7 & .5 & 6.7 & 252.6 & 5.9 & $3,410.5$ \\
\hline 2002 & $1,881.2$ & 83.3 & 456.8 & .2 & $2,421.5$ & 780.1 & -8.7 & 260.5 & 7.3 & 11.2 & 14.5 & .6 & 10.4 & 304.3 & 7.6 & $3,504.8$ \\
\hline 2003 & $1,915.8$ & 108.5 & 421.2 & .3 & $2,445.7$ & 763.7 & -8.5 & 271.5 & 7.4 & 11.9 & 14.4 & .5 & 11.2 & 317.0 & 7.6 & $3,525.5$ \\
\hline 2004 & $1,921.1$ & 109.4 & 491.2 & .4 & $2,522.0$ & 788.5 & -8.5 & 265.1 & 8.1 & 11.8 & 14.8 & .6 & 14.1 & 314.5 & 7.6 & $3,624.1$ \\
\hline 2005 & $1,955.5$ & 111.2 & 553.2 & (s) & $2,619.9$ & 782.0 & -6.6 & 267.0 & 8.5 & 11.7 & 14.7 & .6 & 17.8 & 320.3 & 6.2 & $3,721.8$ \\
\hline 2006 & $1,933.7$ & 55.2 & 618.0 & (s) & $2,607.0$ & 787.2 & -6.6 & 286.2 & 8.3 & 12.5 & 14.6 & .5 & 26.6 & 348.7 & 6.3 & $3,742.7$ \\
\hline 2007 & $\begin{array}{l}1,962.0 \\
\end{array}$ & 56.9 & $\begin{array}{l}686.3 \\
68.0\end{array}$ & (3) 1 & $2,705.3$ & 806.4 & -6.9 & 245.8 & $\begin{array}{l}8.0 \\
8.7\end{array}$ & 12.9 & $\begin{array}{l}14.6 \\
14.6\end{array}$ & .6 & 34.4 & 317.1 & $\begin{array}{l}6.0 \\
6.0\end{array}$ & $3,828.0$ \\
\hline 2008 & $1,932.0$ & $\begin{array}{l}39.3 \\
39.3\end{array}$ & $\begin{array}{l}680.0 \\
683.3\end{array}$ & $(\mathrm{~s})^{-1}$ & $2,654.6$ & $\begin{array}{l}800.4 \\
806.2\end{array}$ & $\begin{array}{l}-0.5 \\
-6.3\end{array}$ & 253.1 & $\begin{array}{l}.11 \\
8.6\end{array}$ & $\begin{array}{l}14.0 \\
14.0\end{array}$ & $\begin{array}{l}14.0 \\
14.8\end{array}$ & $\begin{array}{l}.0 \\
.9\end{array}$ & $\begin{array}{l}54.4 \\
55.4\end{array}$ & 346.8 & $\begin{array}{l}6.0 \\
6.2\end{array}$ & $\begin{array}{l}3,020.0 \\
3,807.4\end{array}$ \\
\hline 2009 & $1,711.9$ & 31.9 & 722.7 & .1 & $2,466.6$ & 798.9 & -4.6 & 271.5 & 8.5 & 14.3 & 15.0 & .9 & 73.9 & 384.0 & 5.8 & $\begin{array}{l}0,650.7 \\
3,6\end{array}$ \\
\hline 2010 & $\mathrm{R}_{1,797.5}$ & R32.4 & R779.4 & .1 & $\mathrm{R}_{2,609.3}$ & 807.0 & $\mathrm{R}_{-5.5}$ & R258.5 & $\begin{array}{r}8.5 \\
R_{9}\end{array}$ & $\mathrm{R}_{14.7}$ & $\begin{array}{l}R_{15} \\
\text { 15.2 }\end{array}$ & $\mathrm{R}_{1.2}$ & 94.6 & R393.6 & $\begin{array}{l}6.0 \\
6.0\end{array}$ & $\mathrm{R}_{3,810.3}$ \\
\hline $2011^{P}$ & $1,687.9$ & 24.1 & 809.2 & (s) & $2,521.2$ & 790.2 & -5.9 & 323.1 & 8.5 & 15.5 & 16.7 & 1.8 & 119.7 & 485.3 & 6.1 & $3,796.9$ \\
\hline
\end{tabular}

Combined-Heat-and-Power Plants ${ }^{12}$

\begin{tabular}{|c|c|c|c|c|c|c|c|c|c|c|c|c|c|c|c|c|}
\hline \multirow{3}{*}{$\begin{array}{l}1989 \\
1990\end{array}$} & \\
\hline & 8.4 & 0.7 & 30.4 & 0.5 & 39.9 & - & - & - & 1.3 & 0.9 & - & - & - & 2.2 & 0.3 & 42.3 \\
\hline & 11.9 & 1.3 & 44.8 & .6 & 58.7 & - & - & - & 1.4 & 1.1 & - & - & - & 2.6 & (s) & 61.3 \\
\hline 1995 & 28.1 & 6.1 & 101.7 & 1.9 & 137.9 & - & - & - & 1.7 & 1.7 & - & - & - & 3.4 & .2 & 141.5 \\
\hline 1996 & 29.2 & 6.3 & 105.9 & 1.3 & 142.7 & - & - & - & 1.9 & 1.7 & - & - & - & 3.6 & .2 & 146.6 \\
\hline 1997 & 27.6 & 6.2 & 108.5 & 1.5 & 143.7 & - & - & - & 2.2 & 2.1 & - & - & - & 4.3 & .1 & 148.1 \\
\hline 1998 & 27.2 & 6.6 & 113.4 & 2.3 & 149.4 & - & - & - & 2.0 & 2.3 & - & - & - & 4.2 & .2 & 153.8 \\
\hline 1999 & 26.6 & 6.7 & 116.4 & 1.6 & 151.2 & - & - & - & 1.7 & 2.4 & - & - & - & 4.1 & .1 & 155.4 \\
\hline 2000 & $\begin{array}{l}20.0 \\
32.5\end{array}$ & $\begin{array}{l}.01 \\
7.2\end{array}$ & $\begin{array}{l}118.4 \\
118.6\end{array}$ & $\begin{array}{l}1.0 \\
1.8\end{array}$ & 160.2 & - & - & - & 1.6 & $\begin{array}{l}2.4 \\
2.7\end{array}$ & - & - & - & $\begin{array}{l}.1 \\
4.3\end{array}$ & .1 & 164.6 \\
\hline 2001 & 31.0 & 6.0 & $\begin{array}{l}128.0 \\
128.0\end{array}$ & $\begin{array}{l}.0 \\
.6\end{array}$ & 165.5 & - & - & - & $\begin{array}{l}1.0 \\
1.7\end{array}$ & 1.7 & - & - & - & 3.4 & .6 & 169.5 \\
\hline 2002 & 29.4 & 6.5 & $\begin{array}{l}150.9 \\
\text { S }\end{array}$ & 1.7 & 188.5 & - & - & - & 1.7 & 2.0 & - & - & - & 3.7 & 1.4 & 193.7 \\
\hline 2003 & 36.9 & 5.2 & 146.1 & 2.4 & 190.6 & - & - & - & 2.1 & 1.9 & - & - & - & 4.0 & 1.1 & 195.7 \\
\hline 2004 & 36.1 & 5.3 & 136.0 & 3.2 & 180.6 & - & - & - & 1.6 & 1.3 & - & - & - & $\begin{array}{l}2.0 \\
2.9\end{array}$ & .7 & 184.3 \\
\hline 2005 & 36.5 & 5.3 & 130.7 & 3.8 & 176.2 & - & - & (s) & 2.1 & 1.3 & - & - & - & 3.4 & .7 & 180.4 \\
\hline 2006 & 36.0 & 4.5 & 116.4 & 4.2 & 161.1 & - & - & (s) & 2.0 & 1.4 & - & - & - & 3.5 & .8 & 165.4 \\
\hline 2007 & 36.4 & 4.4 & 128.4 & 3.9 & 173.2 & - & - & (s) & 2.0 & 1.4 & - & - & - & 3.5 & .7 & 177.4 \\
\hline 2008 & 36.9 & 3.6 & 119.0 & 3.2 & 162.7 & - & - & (s) & 2.0 & 1.4 & - & - & - & 3.4 & .8 & 166.9 \\
\hline 2009 & 29.2 & 3.9 & 118.3 & 3.0 & 154.4 & - & - & (s) & 2.3 & 1.7 & - & - & - & 3.9 & 8 & 159.1 \\
\hline 2010 & $\mathrm{R}_{30.3}$ & $\mathrm{R}_{2.3}$ & $R_{122.0}$ & $\mathrm{R}_{2.9}$ & $R_{157.5}$ & - & - & $R_{-}^{(3)}$ & $\mathrm{R}_{2.1}$ & 1.6 & - & - & - & $\begin{array}{r}\text { R. } \\
\mathrm{R}_{3.8}\end{array}$ & $\mathrm{R}_{.8}^{.0}$ & $\mathrm{R}_{162.0}$ \\
\hline $2011^{\mathrm{P}}$ & $\begin{array}{l}30.3 \\
26.9\end{array}$ & 2.1 & 121.4 & 3.1 & 153.6 & - & - & - & 2.0 & $\begin{array}{l}1.0 \\
1.7\end{array}$ & 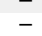 & - & - & $\begin{array}{l}3.0 \\
3.7\end{array}$ & $\begin{array}{l}.0 \\
.9\end{array}$ & 158.1 \\
\hline
\end{tabular}

1 Anthracite, bituminous coal, subbituminous coal, lignite, waste coal, and coal synfuel.
2 Distillate fuel oil, residual fuel oil, petroleum coke, jet fuel, kerosene, other petroleum, and waste oil. ${ }^{3}$ Natural gas, plus a small amount of supplemental gaseous fuels.

4 Blast furnace gas, propane gas, and other manufactured and waste gases derived from fossil fuels.

5 Pumped storage facility production minus energy used for pumping.

6 Through 1989, hydroelectric pumped storage is included in "Conventional Hydroelectric Power."

Wood and wood-derived fuels.

8 Municipal solid waste from biogenic sources, landfill gas, sludge waste, agricultural byproducts, and

9 Solar thermal and photovoltaic (PV) energy.

10 Batteries, chemicals, hydrogen, pitch, purchased steam, sulfur, miscellaneous technologies, and beginning in 2001, non-renewable waste (municipal solid waste from non-biogenic sources, and tire-derived fuels).

Electricity-only plants within the NAICS 22 category whose primary business is to sell electricity to the public. Data also include a small number of electric utility combined-heat-and-power (CHP) plants.

12 Combined-heat-and-power (CHP) plants within the NAICS 22 category whose primary business is to sell electricity and heat to the public. Data do not include electric utility CHP plants-these are included under "Electricity-Only Plants."

R=Revised. P=Preliminary. $-=$ No data reported. $(\mathrm{s})=$ Less than 0.05 billion kilowatthours.

Notes: - See Table 8.2d for commercial and industrial CHP and electricity-only data. - See Note "Coverage of Electricity Statistics," and Note 2, "Classification of Power Plants Into Energy-Use Sectors," a end of section. - Tolals may not equal sum of components due to independent rounding.

Web Pages: - See http://www.eia.gov/totalenergy/data/annual/\#electricity for all data beginning in 1989

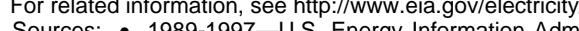

"Monthly Power EIA-759, "Monthly Power Plant Report," and Form EIA-860B, "Annual Electric Generato Report-Nonutility." • 2001-2003-EIA, Form EIA-906, "Power Plant Report." • 2004-2007-EIA, Form EIA-906, "Power Plant Report," and Form EIA-920, "Combined Heat and Power Plant Report." • 2008 
Table 8.2d Electricity Net Generation: Commercial and Industrial Sectors, Selected Years, 1989-2011

(Subset of Table 8.2a; Billion Kilowatthours)

\begin{tabular}{|c|c|c|c|c|c|c|c|c|c|c|c|c|c|c|c|c|}
\hline \multirow[b]{3}{*}{ Year } & \multicolumn{5}{|c|}{ Fossil Fuels } & \multirow{3}{*}{$\begin{array}{l}\text { Nuclear } \\
\text { Electric } \\
\text { Power }\end{array}$} & \multirow{3}{*}{$\begin{array}{c}\text { Hydro- } \\
\text { electric } \\
\text { Pumped } \\
\text { Storage } 5\end{array}$} & \multicolumn{7}{|c|}{ Renewable Energy } & \multirow[b]{3}{*}{ Other ${ }^{9}$} & \multirow[b]{3}{*}{ Tota } \\
\hline & & & & & & & & \multirow{2}{*}{$\begin{array}{c}\text { Conventional } \\
\text { Hydroelectric } \\
\text { Power }\end{array}$} & \multicolumn{2}{|c|}{ Biomass } & \multirow{2}{*}{$\begin{array}{c}\text { Geo- } \\
\text { themal }\end{array}$} & \multirow[b]{2}{*}{ Solar/PV 8} & \multirow[b]{2}{*}{ Wind } & \multirow[b]{2}{*}{ Total } & & \\
\hline & Coal ${ }^{1}$ & Petroleum ${ }^{2}$ & Gas $^{3}$ & Gases ${ }^{4}$ & Total & & & & Wood 6 & Waste $^{7}$ & & & & & & \\
\hline & \multicolumn{16}{|c|}{ Commercial Sector 10} \\
\hline 1989 & 0.7 & 0.6 & 2.2 & 0.1 & 3.6 & - & - & 0.1 & 0.1 & 0.5 & - & - & - & 0.7 & - & 4.3 \\
\hline 1990 & .8 & .6 & 3.3 & .1 & 4.8 & - & - & .1 & .1 & .8 & - & - & - & 1.1 & - & 5.8 \\
\hline 1995 & 1.0 & .4 & 5.2 & - & 6.5 & - & - & .1 & .1 & 1.5 & - & - & - & 1.7 & (s) & 8.2 \\
\hline 1996 & 1.1 & .4 & 5.2 & (s) & 6.7 & - & - & .1 & .1 & 2.2 & - & - & - & 2.4 & (s) & 9.0 \\
\hline 1997 & 1.0 & .4 & 4.7 & (s) & 6.2 & - & - & .1 & (s) & 2.3 & - & - & - & 2.5 & (s) & 8.7 \\
\hline 1998 & 1.0 & .4 & 4.9 & (s) & 6.3 & - & - & .1 & (s) & 2.3 & - & - & - & 2.5 & - & 8.7 \\
\hline 1999 & 1.0 & .4 & 4.6 & (s) & 6.0 & - & - & .1 & (s) & 2.4 & - & - & - & 2.5 & (s) & 8.6 \\
\hline 2000 & 1.1 & .4 & 4.3 & (s) & 5.8 & - & - & .1 & (s) & 2.0 & - & - & - & 2.1 & (s) & 7.9 \\
\hline 2001 & $\begin{array}{l}1.1 \\
1.0\end{array}$ & .4 & 4.4 & (s) & 5.9 & - & - & .1 & (s) & 1.0 & - & - & - & 1.1 & .5 & 7.4 \\
\hline 2002 & 1.0 & .4 & 4.3 & (s) & 5.7 & - & - & (s) & (s) & 1.1 & - & - & - & 1.1 & .6 & 7.4 \\
\hline 2003 & 1.2 & .4 & 3.9 & 101 & 5.5 & - & - & .1 & $\begin{array}{l}\text { (s) } \\
\text { (s) }\end{array}$ & 1.3 & - & - & - & 1.4 & .6 & 7.5 \\
\hline 2004 & 1.3 & .5 & 4.0 & - & 5.8 & - & - & .1 & (s) & 1.6 & - & - & - & 1.7 & .8 & 8.3 \\
\hline 2005 & 1.4 & .4 & 4.2 & - & 6.0 & - & - & .1 & (s) & 1.7 & - & - & - & 1.8 & .8 & 8.5 \\
\hline 2006 & 1.3 & .2 & 4.4 & (s) & 5.9 & - & - & .1 & (s) & 1.6 & - & - & - & 1.7 & .8 & 8.4 \\
\hline 2007 & 1.4 & .2 & 4.3 & - & 5.8 & - & - & .1 & (s) & 1.6 & - & - & - & 1.7 & .8 & 8.3 \\
\hline 2008 & 1.3 & .1 & 4.2 & - & 5.6 & - & - & .1 & (s) & 1.5 & - & (s) & - & 1.6 & .7 & 7.9 \\
\hline 2009 & $\begin{array}{l}1.0 \\
1.1\end{array}$ & .2 & 4.2 & - & $\begin{array}{l}5.0 \\
5.5\end{array}$ & - & - & .1 & $\begin{array}{l}\text { (s) } \\
\text { (s) }\end{array}$ & 1.7 & - & $\begin{array}{l}\text { (s) } \\
\text { (s) }\end{array}$ & (s) & $\begin{array}{l}1.0 \\
1.8\end{array}$ & .8 & 8.2 \\
\hline 2010 & 1.1 & .1 & $R_{4.7}$ & $\mathrm{R}(\mathrm{s})$ & $\mathrm{R}_{6.0}$ & - & - & .1 & (s) & 1.7 & - & (s) & $\begin{array}{l}\text { (s) } \\
\text { (s) }\end{array}$ & 1.8 & .8 & $\mathrm{R}_{8.6}$ \\
\hline \multirow{2}{*}{$2011^{P}$} & 1.0 & .1 & 4.5 & (s) & 5.6 & - & - & .1 & $\begin{array}{l}\text { (s) } \\
\text { (s) }\end{array}$ & 1.7 & - & (s) & (s) & 1.9 & .9 & 8.4 \\
\hline & \multicolumn{16}{|c|}{ Industrial Sector 11} \\
\hline 1989 & 20.7 & 4.8 & 53.2 & 7.3 & 85.9 & - & - & 2.7 & 21.6 & 0.9 & - & _- & - & 25.2 & 3.5 & 114.7 \\
\hline 1990 & 21.1 & 7.0 & 60.0 & 9.6 & 97.8 & - & - & 3.0 & 25.4 & .9 & - & - & - & 29.3 & 3.6 & 130.7 \\
\hline 1995 & 22.4 & 6.0 & 71.7 & 11.9 & 112.1 & - & - & 5.3 & 28.9 & .9 & - & - & - & 35.1 & 3.9 & 151.0 \\
\hline 1996 & 22.2 & 6.3 & 71.0 & 13.0 & 112.5 & - & - & 5.9 & 28.4 & .9 & - & - & - & 35.2 & 3.4 & 151.0 \\
\hline 1997 & 23.2 & 5.6 & 75.1 & 11.8 & 115.8 & - & - & 5.7 & 28.2 & .9 & - & - & - & 34.8 & 3.5 & 154.1 \\
\hline 1998 & 22.3 & 6.2 & 77.1 & 11.2 & 116.8 & - & - & 5.3 & 27.7 & .9 & - & - & - & 33.9 & 3.4 & 154.1 \\
\hline 1999 & 21.5 & 6.1 & 78.8 & 12.5 & 118.9 & - & - & 4.8 & 28.1 & .7 & - & - & - & 33.5 & 3.9 & 156.3 \\
\hline 2000 & 22.1 & 5.6 & 78.8 & 11.9 & 118.4 & - & - & 4.1 & 28.7 & .8 & - & - & - & 33.6 & 4.7 & 156.7 \\
\hline 2001 & 20.1 & 5.3 & 79.8 & 8.5 & 113.6 & - & - & 3.1 & 26.9 & .6 & - & - & - & 30.6 & 4.9 & 149.2 \\
\hline 2002 & 21.5 & 4.4 & 79.0 & 9.5 & 114.4 & - & - & 3.8 & 29.6 & .8 & - & - & - & 34.3 & 3.8 & 152.6 \\
\hline 2003 & $\begin{array}{l}19.8 \\
\text { a }\end{array}$ & $\begin{array}{l}5.4 \\
5.3\end{array}$ & 78.7 & $\begin{array}{r}1.0 \\
13.0\end{array}$ & 116.8 & - & - & 4.2 & 28.0 & .7 & - & - & - & 32.9 & 4.8 & 154.5 \\
\hline 2004 & 19.8 & 6.0 & 79.0 & 11.7 & 116.4 & - & - & 3.2 & 28.4 & .8 & - & - & - & 32.4 & 5.1 & 153.9 \\
\hline 2005 & 19.5 & 5.4 & 72.9 & 9.7 & 107.4 & - & - & 3.2 & 28.3 & .7 & - & - & - & 32.2 & 5.1 & 144.7 \\
\hline 2006 & 19.5 & 4.2 & 77.7 & 9.9 & 111.3 & - & - & 2.9 & 28.4 & .6 & - & - & - & 31.9 & 5.1 & 148.3 \\
\hline 2007 & 16.7 & 4.2 & 77.6 & 9.4 & 107.9 & - & - & 1.6 & 28.3 & .6 & - & - & - & 30.5 & 4.7 & 143.1 \\
\hline 2008 & 15.7 & 3.2 & 76.4 & 8.5 & 103.9 & - & - & 1.7 & 26.6 & .8 & - & - & - & 29.1 & 4.1 & 137.1 \\
\hline 2009 & 13.7 & 3.0 & 75.7 & 7.6 & 100.0 & - & - & 1.9 & 25.3 & .7 & - & - & - & 27.9 & 4.5 & 132.3 \\
\hline 2010 & 18.4 & $\mathrm{R}_{2.3}$ & R81. 6 & $\mathrm{R}_{8.3}$ & $R_{110.6}$ & - & - & $\mathrm{R}_{1.7}$ & $\mathrm{R}_{25.7}$ & R. & - & (s) & - & R28.2 & R5.2 & R144.1 \\
\hline $2011^{P}$ & 18.4 & 1.8 & 81.5 & 8.1 & 109.9 & - & - & 1.8 & 26.4 & .9 & - & $\begin{array}{l}\text { (s) } \\
\text { (s) }\end{array}$ & (s) & 29.1 & 3.3 & 142.3 \\
\hline
\end{tabular}

Anthracite, bituminous coal, subbituminous coal, lignite, waste coal, and coal synfuel.

Distillate fuel oil, residual fuel oil, petroleum coke, jet fuel, kerosene, other petroleum, and waste oil.

Natural gas, plus a small amount of supplemental gaseous fuels.

Blast furnace gas, propane gas, and other manufactured and waste gases derived from fossil fuels.

Pumped storage facility production minus energy used for pumping.

Municipal solid waste from bioge

7 , sources, landfill gas, sludge waste, agricultural byproducts, and sources, and tire-derived fuels).

Solar thermal and photovoltaic (PV) energy.

Batteries, chemicals, hydrogen, pitch, purchased steam, sulfur, miscellaneous technologies, and beginning in 2001, non-renewable waste (municipal solid waste from non-biogenic sources, and tire-derived fuels).

Commercial combined-heat-and-power (CHP) and commercial electricity-only plants.

Industrial combined-heat-and-power (CHP) and industrial electricity-only plants. $R=$ Revised. P=Preliminary. $-=$ No data reported. $(\mathrm{s})=$ Less than 0.05 billion kilowatthours.

Notes. - See Tables $8.2 \mathrm{~b}$ and $8.2 \mathrm{c}$ for electric power sector electricity-only and CHP data. - See Note " "Coverage of Electricly Statistics," and Nole 2, "Classifcation of Power Plants Into Energy-Use Sectors,"

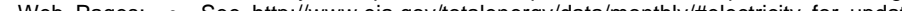

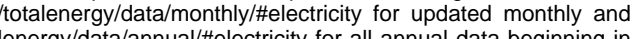
nnual data beginning in 1989. • See hittp://www.eia.gov/electricity/ for related information.
Sources: - 1989-1997-U.S. Energy Information Administration (EIA), Form EIA-867, "Annual Nonutility Power Producer Report." • 1998-2000-EIA, Form EIA-860B, "Annual Electric Generator Report-Nonutilty. " - 2001-2003-EIA, Form EIA-906, "Power Plant Report. • 2004-2007-EIA, Form " 2008 forward-EIA, Form EIA-923, "Power Plant Operations Report." 
Figure 8.3 Useful Thermal Output at Combined-Heat-and-Power Plants

\section{Total (All Sectors), 1989-2011}

$2.5-$

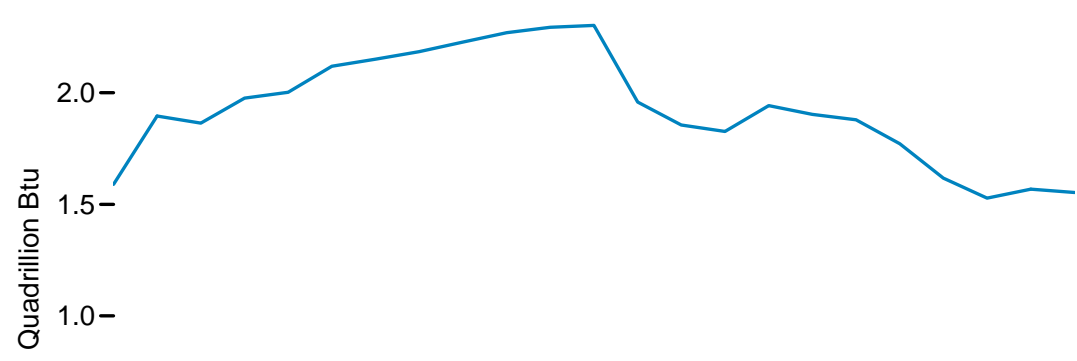

$0.5-$

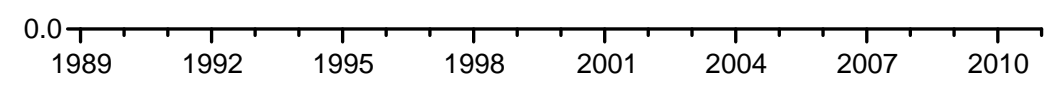

By Sector, 1989-2011

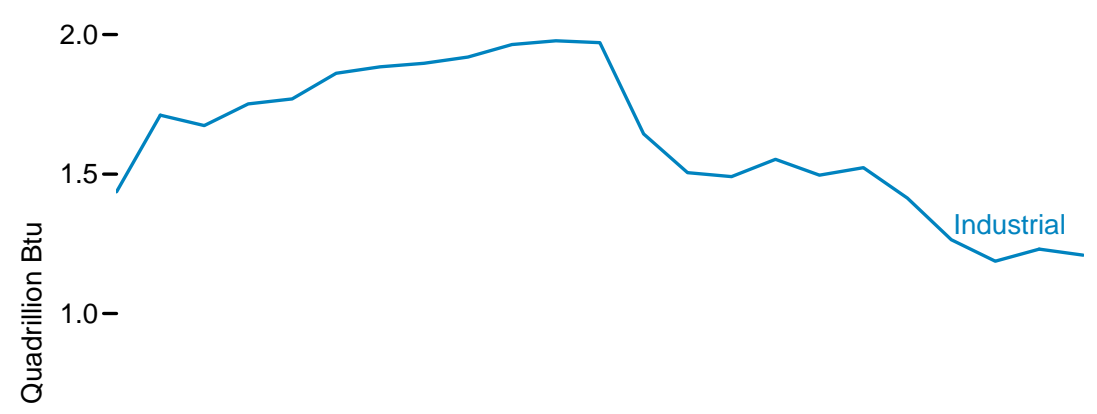

$0.5-$

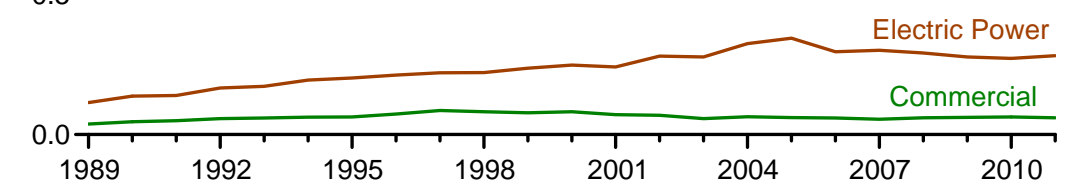

Total (All Sectors) by Source, 2011

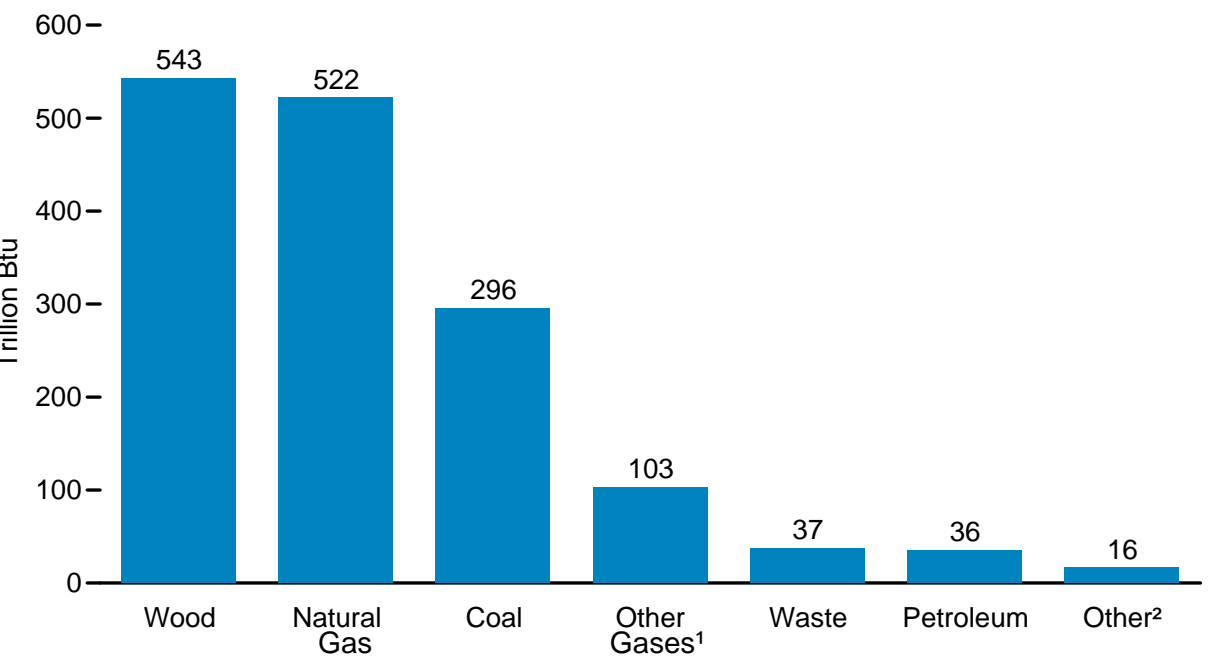

By Sector, 2011

$1.8-$
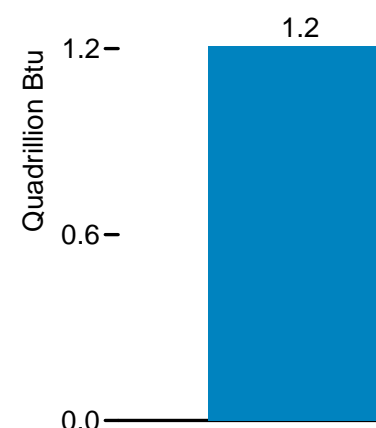

Industrial

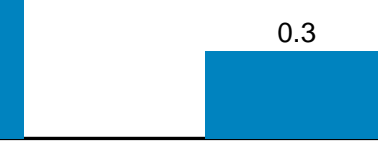

Electric Power

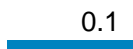

Commercial

${ }^{1}$ Blast furnace gas, propane gas, and other manufactured and waste gases derived from fossil fuels.

${ }^{2}$ Batteries, chemicals, hydrogen, pitch, purchased steam, sulfur, miscellaneous technologies, and non-renewable waste (municipal solid waste from non-biogenic sources, and tire-derived fuels).

Sources: Tables 8.3a-8.3c. 
Table 8.3a Useful Thermal Output at Combined-Heat-and-Power Plants: Total (All Sectors), 1989-2011

(Sum of Tables 8.3b and 8.3c; Trillion Btu)

\begin{tabular}{|c|c|c|c|c|c|c|c|c|c|c|}
\hline \multirow[b]{3}{*}{ Year } & \multicolumn{5}{|c|}{ Fossil Fuels } & \multicolumn{3}{|c|}{ Renewable Energy } & \multirow[b]{3}{*}{ Other ${ }^{7}$} & \multirow[b]{3}{*}{ Total } \\
\hline & \multirow[b]{2}{*}{ Coal ${ }^{1}$} & \multirow[b]{2}{*}{ Petroleum $^{2}$} & \multirow[b]{2}{*}{ Natural Gas ${ }^{3}$} & \multirow[b]{2}{*}{ Other Gases ${ }^{4}$} & \multirow[b]{2}{*}{ Total } & \multicolumn{2}{|c|}{ Biomass } & \multirow[b]{2}{*}{ Total } & & \\
\hline & & & & & & Wood $^{5}$ & Waste ${ }^{6}$ & & & \\
\hline 1989 & 323 & 96 & 462 & 93 & 973 & 546 & 30 & 577 & 39 & 1,589 \\
\hline 1990 & 363 & 127 & 538 & 141 & 1,168 & 651 & 36 & 687 & 40 & 1,896 \\
\hline 1991 & 352 & 112 & 547 & 148 & 1,159 & 623 & 37 & 660 & 44 & 1,863 \\
\hline 1992 & 367 & 117 & 592 & 160 & 1,236 & 658 & 40 & 698 & 42 & 1,976 \\
\hline 1993 & 373 & 129 & 604 & 142 & 1,248 & 668 & 45 & 713 & 41 & 2,002 \\
\hline 1994 & 388 & 133 & 646 & 144 & 1,309 & 722 & 45 & 767 & 42 & 2,119 \\
\hline 1995 & 386 & 121 & 686 & 145 & 1,338 & 721 & 47 & 768 & 44 & 2,151 \\
\hline 1996 & 392 & 133 & 711 & 150 & 1,385 & 701 & 55 & 756 & 43 & 2,184 \\
\hline 1997 & 389 & 137 & 713 & 150 & 1,389 & 731 & 55 & 785 & 53 & 2,227 \\
\hline 1998 & 382 & 136 & 782 & 167 & 1,466 & 700 & 57 & 757 & 46 & 2,269 \\
\hline 1999 & 386 & 125 & 811 & 179 & 1,501 & 690 & 55 & 744 & 48 & $\begin{array}{l}2,294 \\
\end{array}$ \\
\hline 2000 & 384 & 108 & 812 & 184 & 1,488 & 707 & 56 & 764 & 50 & 2,302 \\
\hline 2001 & 354 & 90 & 741 & 133 & 1,318 & 557 & 28 & 585 & 55 & 1,958 \\
\hline 2002 & 337 & 73 & 709 & 118 & 1,236 & 546 & 26 & 572 & 48 & 1,856 \\
\hline 2003 & 333 & 85 & 610 & 110 & 1,139 & 597 & 35 & 632 & 55 & 1,826 \\
\hline 2004 & 352 & 97 & 654 & 126 & 1,230 & 637 & 30 & 667 & 45 & 1,943 \\
\hline 2005 & 342 & 92 & 624 & 138 & 1,197 & 628 & 36 & 665 & 41 & 1,903 \\
\hline 2006 & 333 & 78 & 603 & 126 & 1,140 & 653 & 37 & 690 & 49 & 1,879 \\
\hline 2007 & 327 & 76 & 554 & 116 & 1,074 & 616 & 35 & 651 & 47 & 1,772 \\
\hline 2008 & 315 & 48 & 509 & 111 & 983 & 572 & 38 & 610 & 24 & 1,617 \\
\hline 2009 & 282 & 53 & 513 & 100 & 947 & 509 & 38 & 547 & 33 & 1,527 \\
\hline 2010 & $R_{300}$ & $\mathrm{R}_{41}$ & R524 & R91 & R958 & $R_{542}$ & $\mathrm{R}_{40}$ & R581 & $\mathrm{R}_{29}$ & $R_{1,568}$ \\
\hline $2011^{P}$ & 296 & 36 & 522 & 103 & 956 & 543 & 37 & 580 & 16 & 1,553 \\
\hline
\end{tabular}

1 Anthracite, bituminous coal, subbituminous coal, lignite, waste coal, and coal synfuel.

Distillate fuel oil, residual fuel oil, petroleum coke, jet fuel, kerosene, other petroleum, and waste oil.

Natural gas, plus a small amount of supplemental gaseous fuels.

Blast furnace gas, propane gas, and other manufactured and waste gases derived from fossil fuels.

5 Wood and wood-derived fuels.

Municipal solid waste from biogenic sources, landfill gas, sludge waste, agricultural byproducts, and other biomass. Through 2000, also includes non-renewable waste (municipal solid waste from non-biogenic sources, and tire-derived fuels).

Batteries, chemicals, hydrogen, pitch, purchased steam, sulfur, miscellaneous technologies, and,

beginning in 2001, non-renewable waste (municipal solid waste from non-biogenic sources, and tire-derived fuels).

$\mathrm{R}=$ Revised. $\mathrm{P}=$ Preliminary

Notes: - Data do not include electric utility combined-heat-and-power (CHP) plants. - See Note 1, "Coverage of Electricity Statistics," at end of section. • See "Useful Thermal Output" in Glossary. • Totals may not equal sum of components due to independent rounding.

Web Page: For related information, see http://www.eia.gov/electricity/.

Sources: Tables $8.3 \mathrm{~b}$ and $8.3 \mathrm{c}$. 
Table 8.3b Useful Thermal Output at Combined-Heat-and-Power Plants: Electric Power Sector, 1989-2011

(Subset of Table 8.3a; Trillion Btu)

\begin{tabular}{|c|c|c|c|c|c|c|c|c|c|c|}
\hline \multirow[b]{3}{*}{ Year } & \multicolumn{5}{|c|}{ Fossil Fuels } & \multicolumn{3}{|c|}{ Renewable Energy } & \multirow[b]{3}{*}{ Other ${ }^{7}$} & \multirow[b]{3}{*}{ Total } \\
\hline & \multirow[b]{2}{*}{ Coal ${ }^{1}$} & \multirow[b]{2}{*}{ Petroleum ${ }^{2}$} & \multirow[b]{2}{*}{ Natural Gas ${ }^{3}$} & \multirow[b]{2}{*}{ Other Gases 4} & \multirow[b]{2}{*}{ Total } & \multicolumn{2}{|c|}{ Biomass } & \multirow[b]{2}{*}{ Total } & & \\
\hline & & & & & & Wood $^{5}$ & Waste ${ }^{6}$ & & & \\
\hline 1989 & 13 & 8 & 67 & 2 & 90 & 19 & 5 & 24 & 1 & 114 \\
\hline 1990 & 21 & 9 & 80 & 4 & 114 & 18 & 6 & 25 & (s) & 138 \\
\hline 1991 & 21 & 6 & 82 & 4 & 113 & 17 & 9 & 26 & 1 & 140 \\
\hline 1992 & 28 & 6 & 102 & 5 & 140 & 17 & 8 & 25 & 2 & 167 \\
\hline 1993 & 30 & 8 & 107 & 3 & 147 & 16 & 8 & 24 & 1 & 173 \\
\hline 1994 & 37 & 9 & 119 & 5 & 170 & 15 & 10 & 24 & 1 & 195 \\
\hline 1995 & 40 & 13 & 118 & 4 & 176 & 15 & 12 & 27 & (s) & 203 \\
\hline 1996 & 43 & 12 & 121 & 4 & 180 & 16 & 16 & 33 & (s) & 213 \\
\hline 1997 & 39 & 12 & 132 & 8 & 191 & 16 & 14 & 30 & (s) & 221 \\
\hline 1998 & 43 & 6 & 142 & 5 & 196 & 10 & 16 & 26 & (s) & 222 \\
\hline 1999 & 52 & 7 & 146 & 4 & 208 & 10 & 20 & 30 & (s) & 238 \\
\hline 2000 & 53 & 7 & 158 & 5 & 223 & 6 & 19 & 26 & (s) & 249 \\
\hline 2001 & 52 & 6 & 164 & 5 & 226 & 8 & 4 & 13 & 3 & 243 \\
\hline 2002 & 40 & 4 & 214 & 6 & 264 & 8 & 5 & 13 & 5 & 281 \\
\hline 2003 & 38 & 7 & 200 & 9 & 255 & 9 & 11 & 20 & 3 & 278 \\
\hline 2004 & 39 & 8 & 239 & 18 & 305 & 9 & 9 & 17 & 4 & 326 \\
\hline 2005 & 40 & 8 & 239 & 37 & 323 & 10 & 8 & 18 & 4 & 346 \\
\hline 2006 & 38 & 7 & 207 & 23 & 275 & 10 & 7 & 17 & 4 & 297 \\
\hline 2007 & 38 & 7 & 213 & 20 & 279 & 11 & 8 & 19 & 4 & 302 \\
\hline 2008 & 37 & 7 & 204 & 22 & 270 & 9 & 8 & 17 & 5 & 292 \\
\hline 2009 & 38 & 7 & 191 & 20 & 256 & 9 & 8 & 18 & 5 & 278 \\
\hline 2010 & ${ }^{R} 38$ & 6 & $\mathrm{R}_{187}$ & $\mathrm{R}_{20}$ & $\mathrm{R}_{251}$ & $\mathrm{R}_{10}$ & 8 & $\mathrm{R}_{18}$ & 5 & $R_{273}$ \\
\hline $2011^{\mathrm{P}}$ & 37 & 6 & 197 & 22 & 262 & 9 & 7 & 16 & 6 & 283 \\
\hline
\end{tabular}

1 Anthracite, bituminous coal, subbituminous coal, lignite, waste coal, and coal synfuel.

Distillate fuel oil, residual fuel oil, petroleum coke, jet fuel, kerosene, other petroleum, and waste oil.

3 Natural gas, plus a small amount of supplemental gaseous fuels.

Blast furnace gas, propane gas, and other manufactured and waste gases derived from fossil fuels.

5 Wood and wood-derived fuels.

Municipal solid waste from biogenic sources, landfill gas, sludge waste, agricultural byproducts, and other biomass. Through 2000, also includes non-renewable waste (municipal solid waste from non-biogenic sources, and tire-derived fuels).

Batteries, chemicals, hydrogen, pitch, purchased steam, sulfur, miscellaneous technologies, and, beginning in 2001, non-renewable waste (municipal solid waste from non-biogenic sources, and tire-derived fuels)

$\mathrm{R}=$ Revised. $\mathrm{P}=$ Preliminary. ( $\mathrm{s}=$ =Less than 0.5 trillion Btu.

Notes: - Data are for combined-heat-and-power (CHP) plants within the NAICS 22 category whose primary business is to sell electricity and heat to the public. Data do not include electric utility CHP plants. - See Table 8.3c for commercial and industrial CHP data. - See Note 1, "Coverage of Electricity Statistics," and Note 2, "Classification of Power Plants Into Energy-Use Sectors," at end of section. • See "Useful Thermal Output" in Glossary. • Totals may not equal sum of components due to independent rounding.

Web Page: For related information, see http://www.eia.gov/electricity/.

Sources: • 1989-1997-U.S. Energy Information Administration (EIA), Form EIA-867, "Annual Nonutility Power Producer Report." • 1998-2000-EIA, Form EIA-860B, "Annual Electric Generator Report-Nonutility." • 2001-2003—EIA, Form EIA-906, "Power Plant Report." • 2004-2007—EIA, Form EIA-920, "Combined Heat and Power Plant Report." • 2008 forward-EIA, Form EIA-923, "Power Plant Operations Report." 
Table 8.3c Useful Thermal Output at Combined-Heat-and-Power Plants: Commercial and Industrial Sectors,

Selected Years, 1989-2011 (Subset of Table 8.3a; Trillion Btu)

\begin{tabular}{|c|c|c|c|c|c|c|c|c|c|c|}
\hline \multirow[b]{3}{*}{ Year } & \multicolumn{5}{|c|}{ Fossil Fuels } & \multicolumn{3}{|c|}{ Renewable Energy } & \multirow[b]{3}{*}{ Other ${ }^{7}$} & \multirow[b]{3}{*}{ Tota } \\
\hline & \multirow[b]{2}{*}{ Coal ${ }^{1}$} & \multirow[b]{2}{*}{ Petroleum $^{2}$} & \multirow[b]{2}{*}{ Natural Gas ${ }^{3}$} & \multirow[b]{2}{*}{ Other Gases ${ }^{4}$} & \multirow[b]{2}{*}{ Total } & \multicolumn{2}{|c|}{ Biomass } & \multirow[b]{2}{*}{ Total } & & \\
\hline & & & & & & Wood 5 & Waste 6 & & & \\
\hline & \multicolumn{10}{|c|}{ Commercial Sector 8} \\
\hline 1989 & 14 & 4 & 10 & (s) & 27 & (s) & 10 & 10 & - & 38 \\
\hline 1990 & 15 & $\begin{array}{l}4 \\
5\end{array}$ & 16 & $\begin{array}{l}\text { (s) } \\
\text { (s) }\end{array}$ & 36 & $\begin{array}{l}\text { (s) } \\
\text { (s) }\end{array}$ & 10 & 11 & - & 46 \\
\hline 1995 & 17 & 3 & 29 & - & 48 & (s) & 15 & 15 & (s) & 63 \\
\hline 1996 & 20 & 3 & 33 & $\mathrm{R}_{-}$ & 55 & 1 & 17 & 18 & - & 73 \\
\hline 1997 & 22 & 4 & 40 & (s) & 66 & 1 & 19 & 20 & - & 86 \\
\hline 1998 & 20 & $\begin{array}{l}4 \\
5\end{array}$ & 39 & (s) & 64 & 1 & 18 & 18 & - & 82 \\
\hline 1999 & 20 & 3 & 37 & $R_{-}^{(1)}-$ & 61 & 1 & 17 & 17 & - & 78 \\
\hline 2000 & 21 & 4 & $\begin{array}{l}31 \\
39\end{array}$ & $\mathrm{R}_{-}^{-}$ & 64 & 1 & 17 & 18 & - & 82 \\
\hline 2001 & 18 & 4 & 35 & - & 58 & 1 & 8 & 8 & 6 & 72 \\
\hline 2002 & 18 & 3 & 36 & - & 57 & 1 & 6 & 7 & 5 & 69 \\
\hline 2003 & 23 & 3 & 17 & - & 42 & 1 & 8 & 8 & 6 & 57 \\
\hline 2004 & 22 & 4 & 22 & - & 49 & (s) & 8 & 9 & 6 & 64 \\
\hline 2005 & 23 & 4 & 20 & - & 47 & (s) & 8 & 9 & 6 & 61 \\
\hline 2006 & 22 & 2 & 19 & (s) & 44 & (s) & 9 & 9 & 6 & 59 \\
\hline 2007 & 23 & 2 & 20 & - & 44 & 1 & 6 & 7 & 4 & 55 \\
\hline 2008 & 23 & 2 & 20 & - & 45 & (s) & 9 & 9 & 6 & 60 \\
\hline 2009 & 20 & 1 & 26 & - & 47 & (s) & 8 & 8 & 6 & 61 \\
\hline 2010 & $\mathrm{R}_{19}$ & 1 & $\mathrm{R}_{30}$ & ${ }^{R}(s)$ & R50 & $\begin{array}{l}\text { (1) } \\
\text { (s) }\end{array}$ & R8 & 8 & 5 & $\mathrm{R}_{63}$ \\
\hline \multirow[t]{2}{*}{$2011^{\mathrm{P}}$} & 17 & 1 & 28 & (s) & 46 & (s) & 8 & 8 & 6 & 60 \\
\hline & \multicolumn{10}{|c|}{ Industrial Sector 9} \\
\hline 1989 & 297 & 84 & 385 & 90 & 856 & 527 & 15 & 542 & 38 & 1.437 \\
\hline 1990 & 327 & $\begin{array}{r}04 \\
113\end{array}$ & 443 & 137 & $\begin{array}{r}1,019 \\
\end{array}$ & 632 & 20 & 652 & 40 & $\begin{array}{l}1,7011 \\
\end{array}$ \\
\hline 1995 & $\begin{array}{l}327 \\
329\end{array}$ & 105 & $\begin{array}{l}443 \\
540\end{array}$ & 140 & 1,114 & $\begin{array}{l}032 \\
706\end{array}$ & $\begin{array}{l}20 \\
20\end{array}$ & $\begin{array}{l}052 \\
726\end{array}$ & $\begin{array}{l}40 \\
44\end{array}$ & 1,884 \\
\hline 1996 & 329 & 118 & 557 & 146 & $\begin{array}{l}1,150 \\
\text { S }\end{array}$ & 684 & 21 & 705 & 43 & 1,897 \\
\hline 1997 & 328 & 121 & 541 & 142 & 1,132 & 713 & 22 & 735 & 53 & $\begin{array}{l}1,051 \\
1,920\end{array}$ \\
\hline 1998 & 318 & 124 & 601 & 162 & 1,206 & 689 & 24 & 713 & 46 & 1,965 \\
\hline 1999 & 313 & 115 & 629 & 175 & 1,233 & 679 & 18 & 697 & 48 & 1,978 \\
\hline 2000 & 309 & 98 & 615 & 179 & 1,201 & 700 & 20 & 720 & $\begin{array}{l}40 \\
50\end{array}$ & 1,971 \\
\hline 2001 & 284 & 80 & 542 & 128 & 1,034 & 548 & 16 & 564 & 46 & 1,644 \\
\hline 2002 & 278 & 66 & 458 & 112 & 914 & 537 & 15 & 552 & 39 & 1,505 \\
\hline 2003 & 272 & 75 & $\begin{array}{l}450 \\
393\end{array}$ & 101 & $\begin{array}{l}114 \\
842\end{array}$ & 588 & 16 & 604 & 46 & 1,491 \\
\hline 2004 & 290 & 85 & 393 & 108 & 876 & 628 & 13 & 641 & 35 & 1,553 \\
\hline 2005 & 280 & 81 & 364 & 102 & 827 & 618 & 20 & 638 & 32 & 1,496 \\
\hline 2006 & 272 & $\begin{array}{l}01 \\
69\end{array}$ & $\begin{array}{l}304 \\
377\end{array}$ & 103 & $\begin{array}{l}027 \\
821\end{array}$ & $\begin{array}{l}010 \\
642\end{array}$ & 21 & $\begin{array}{l}030 \\
663\end{array}$ & $\begin{array}{l}32 \\
39\end{array}$ & $\begin{array}{l}1,490 \\
1,523\end{array}$ \\
\hline 2007 & 266 & 67 & 322 & 96 & 751 & 605 & 21 & 625 & 38 & 1,414 \\
\hline 2008 & 255 & 39 & 285 & 89 & 668 & 563 & 21 & 584 & $\begin{array}{l}50 \\
13\end{array}$ & $\begin{array}{l}1,414 \\
1,265\end{array}$ \\
\hline 2009 & 223 & 45 & $\begin{array}{l}205 \\
296\end{array}$ & $\begin{array}{l}89 \\
80\end{array}$ & $\begin{array}{l}000 \\
644\end{array}$ & 500 & $\begin{array}{l}21 \\
21\end{array}$ & $\begin{array}{l}504 \\
521\end{array}$ & $\begin{array}{l}10 \\
22\end{array}$ & $\begin{array}{l}1,205 \\
1,188\end{array}$ \\
\hline 2010 & $R_{243}$ & $\mathrm{R}_{34}$ & $R_{308}$ & R72 & $R_{657}$ & R531 & $\mathrm{R}_{24}$ & $\mathrm{R}_{556}$ & $\mathrm{R}_{18}$ & $\mathrm{R}_{1,231}$ \\
\hline $2011^{\mathrm{P}}$ & 241 & 29 & 297 & 81 & 648 & 533 & 23 & 557 & 5 & 1,209 \\
\hline
\end{tabular}

1 Anthracite, bituminous coal, subbituminous coal, lignite, waste coal, and coal synfuel.

Distillate fuel oil, residual fuel oil, petroleum coke, jet fuel, kerosene, other petroleum, and waste oil.

4 Blast furnace gas, propane gas, and other manufactured and waste gases derived from fossil fuels.

6 Municipal wood-derived fuels. other biomass. Through 2000, also includes non-renewable waste (municipal solid waste from

non-biogenic sources, and tire-derived fuels).
7 Batteries, chemicals, hydrogen, pitch, purchased steam, sulfur, miscellaneous technologies, and, beginning in 2001, non-renewable waste (municipal solid waste from non-biogenic sources, and tire-derived fuels).

Commercial combined-heat-and-power (CHP) plants.

$R=$ Revised. P=Preliminary. $-=$ No data reported. $(\mathrm{s})=$ Less than 0.5 trillion Btu.

Notes: - See Table 8.3b for electric power sector CHP data. - See Note 1, "Coverage of Electricity "Useful Thermal Output" in Glossary. • Totals may not equal sum of components due to independent rounding. - For related information, see http://www.eia.gov/electricity/.

Sources: • 1989-1997-U.S. Energy Information Administration (EIA). Form EIA-867, "Annual Nonutility Power Producer Report." • 1998-2000-EIA, Form EIA-860B, "Annual Electric Generator Report-Nonutility. " 2001-2003-EIA, Form EIA-906, "Power Plant Report." • 2004-2007-EIA, Form EIA-920, "Combined Heat and Power Plant Report." • 2008 forward-EIA, Form EIA-923, "Power Plant

9 Industrial combined-heat-and-power (CHP) plants. 


\section{Figure 8.4 Consumption for Electricity Generation}

\section{By Major Category, 1949-2011}

$40-$

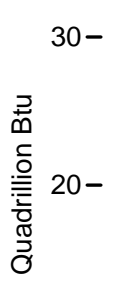

$30-$

$10-$

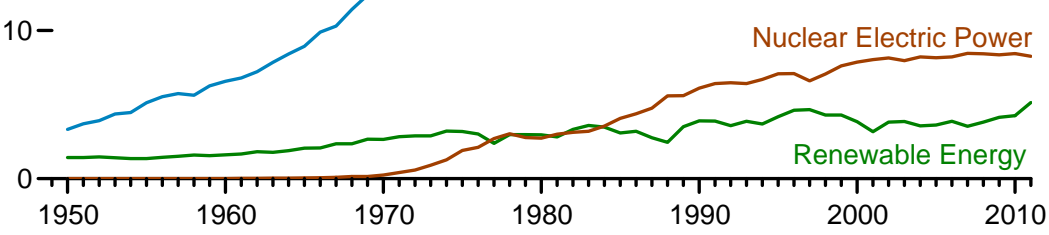

\section{By Major Source, 1949-2011}

24-

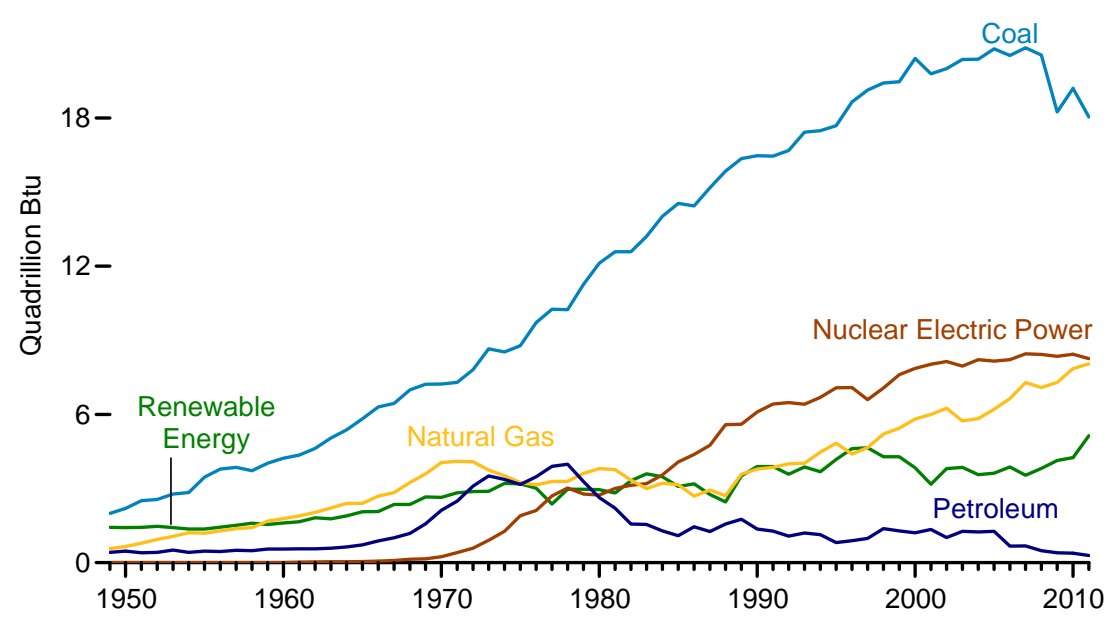

${ }^{1}$ Conventional hydroelectric power.

${ }^{2}$ Geothermal, other gases, electricity net imports, solar thermal and photovoltaic energy, batteries, chemicals, hydrogen, pitch, purchased steam, sulfur, miscellaneous technologies,

\section{By Major Fuel, 2011}

20-

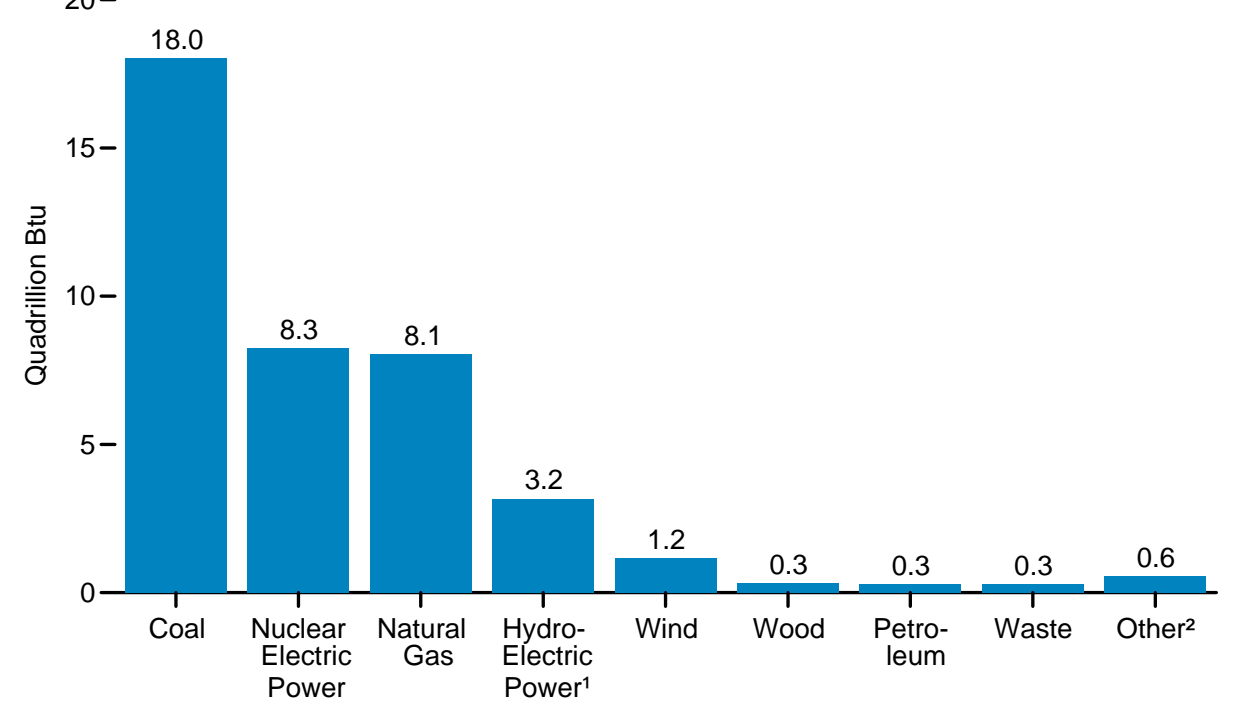

By Sector, 1989-2011

$45-$

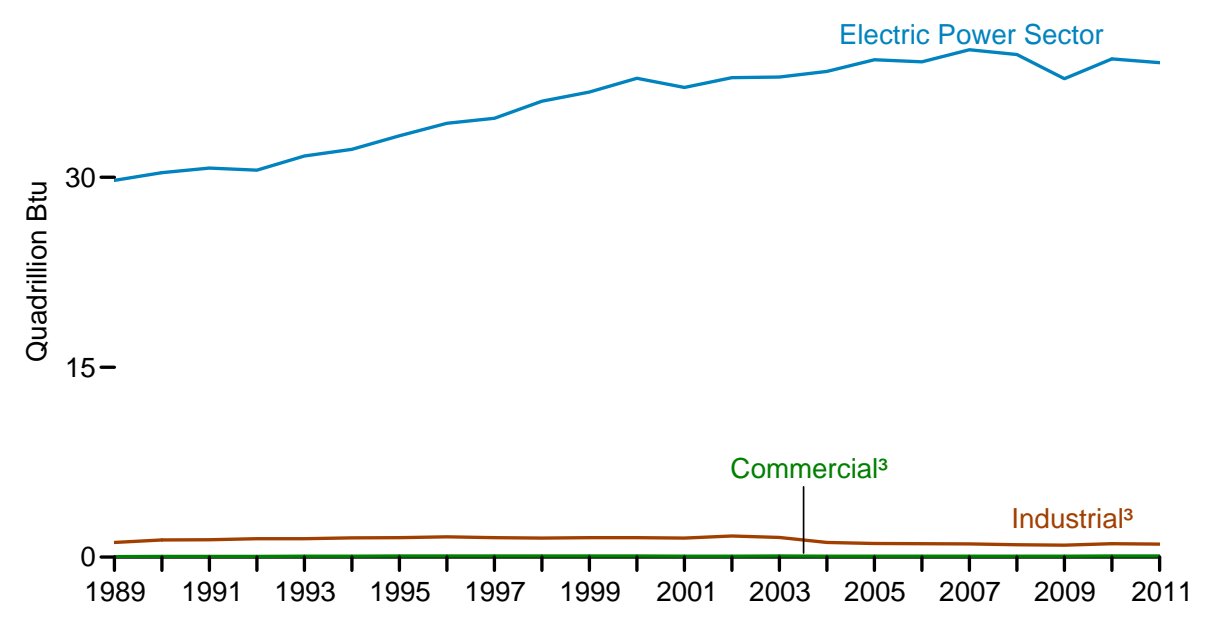

and non-renewable waste (municipal solid waste from non-biogenic sources, and tire-derived fuels).

${ }^{3}$ Combined-heat-and-power plants and a small number of electricity-only plants. Sources: Tables 8.4a-8.4c. 
Table 8.4a Consumption for Electricity Generation by Energy Source: Total (All Sectors), Selected Years, 1949-2011 (Sum of Tables 8.4b and 8.4c; Trillion Btu)

\begin{tabular}{|c|c|c|c|c|c|c|c|c|c|c|c|c|c|c|c|c|}
\hline \multirow[b]{3}{*}{ Year } & \multicolumn{5}{|c|}{ Fossil Fuels } & \multirow{3}{*}{$\begin{array}{l}\text { Nuclear } \\
\text { Electric } \\
\text { Power } 5\end{array}$} & \multicolumn{7}{|c|}{ Renewable Energy } & \multirow[b]{3}{*}{ Other $^{9}$} & \multirow{3}{*}{$\begin{array}{l}\text { Electricity } \\
\text { Net } \\
\text { Imports } 10\end{array}$} & \multirow[b]{3}{*}{ Total } \\
\hline & & & & & & & \multirow{2}{*}{\begin{tabular}{|c}
$\begin{array}{c}\text { Conventional } \\
\text { Hydroelectric } \\
\text { Power }^{5}\end{array}$ \\
\end{tabular}} & \multicolumn{2}{|c|}{ Biomass } & \multirow{2}{*}{$\begin{array}{c}\text { Geo- } \\
\text { thermal }\end{array}$} & \multirow[b]{2}{*}{ Solar/PV 5,8} & \multirow[b]{2}{*}{ Wind $^{5}$} & \multirow[b]{2}{*}{ Total } & & & \\
\hline & Coal $^{1}$ & Petroleum $^{2}$ & Gas $^{3}$ & Gases ${ }^{4}$ & Total & & & Wood 6 & Waste $^{7}$ & & & & & & & \\
\hline 1949 & 1,995 & 415 & 569 & NA & 2,979 & 0 & 1,425 & 6 & NA & NA & NA & NA & 1,431 & NA & 5 & 4,415 \\
\hline 1950 & 2,199 & 472 & 651 & NA & 3,322 & 0 & 1,415 & 5 & NA & NA & NA & NA & 1,421 & NA & 6 & 4,749 \\
\hline 1955 & 3,458 & 471 & 1,194 & NA & 5,123 & 0 & 1,360 & 3 & NA & NA & NA & NA & 1,363 & NA & 14 & 6,500 \\
\hline 1960 & 4,228 & 553 & 1,785 & NA & 6,565 & 6 & 1,608 & 2 & NA & (s) & NA & NA & 1,610 & NA & 15 & 8,197 \\
\hline 1965 & 5,821 & 722 & 2,395 & NA & 8,938 & 43 & 2,059 & 3 & NA & 2 & NA & NA & 2,064 & NA & (s) & 11,045 \\
\hline 1970 & 7,227 & 2,117 & 4,054 & NA & 13,399 & 239 & 2,634 & 1 & 2 & 6 & NA & NA & 2,643 & NA & 7 & 16,287 \\
\hline 1975 & 8,786 & 3,166 & 3,240 & NA & 15,191 & 1,900 & 3,155 & (s) & 2 & 34 & NA & NA & 3,190 & NA & 21 & 20,303 \\
\hline 1976 & 9,720 & 3,477 & 3,152 & NA & 16,349 & 2,111 & 2,976 & 1 & 2 & 38 & NA & NA & 3,017 & NA & 29 & 21,506 \\
\hline 1977 & 10,262 & 3,901 & 3,284 & NA & 17,446 & 2,702 & 2,333 & 3 & 2 & 37 & NA & NA & 2,376 & NA & 59 & 22,583 \\
\hline 1978 & 10,238 & 3,987 & 3,297 & NA & 17,522 & 3,024 & 2,937 & 2 & 1 & 31 & NA & NA & 2,971 & NA & 67 & 23,585 \\
\hline 1979 & 11,260 & 3,283 & 3,613 & NA & 18,156 & 2,776 & 2,931 & 3 & 2 & 40 & NA & NA & 2,976 & NA & 69 & 23,977 \\
\hline 1980 & 12,123 & 2,634 & 3,810 & NA & 18,567 & 2,739 & 2,900 & 3 & 2 & 53 & NA & NA & 2,957 & NA & 71 & 24,335 \\
\hline 1981 & 12,583 & 2,202 & 3,768 & NA & 18,553 & 3,008 & 2,758 & 3 & 1 & 59 & NA & NA & 2,821 & NA & 113 & 24,495 \\
\hline 1982 & 12,582 & 1,568 & $\begin{array}{l}3,342 \\
3,342\end{array}$ & NA & 17,491 & $\begin{array}{l}3,131 \\
3,130\end{array}$ & 3,266 & 2 & 1 & 51 & NA & NA & 3,320 & NA & 100 & 24,042 \\
\hline 1983 & 13,213 & $\begin{array}{l}1,500 \\
1,544\end{array}$ & $\begin{array}{l}2,042 \\
2,998\end{array}$ & NA & 17,754 & $\begin{array}{l}3,203 \\
3,203\end{array}$ & $\begin{array}{l}3,200 \\
3,527\end{array}$ & 2 & 2 & 64 & NA & (s) & $\begin{array}{l}3,0<0 \\
3,595\end{array}$ & NA & 121 & 24,673 \\
\hline 1984 & 14,019 & 1,286 & 3,220 & NA & 18,526 & 3,553 & 3,386 & 5 & 4 & 81 & (s) & (s) & 3,476 & NA & 135 & 25,690 \\
\hline 1985 & 14,542 & 1,090 & 3,160 & NA & 18,792 & 4,076 & 2,970 & 8 & 7 & 97 & (s) & (s) & 3,082 & NA & 140 & 26,090 \\
\hline 1986 & 14,444 & 1,452 & 2,691 & NA & 18,586 & 4,380 & 3,071 & 5 & 7 & 108 & (s) & (s) & 3,191 & NA & 122 & 26,280 \\
\hline 1987 & 15,173 & 1,257 & 2,935 & NA & 19,365 & 4,754 & 2,635 & 8 & 7 & 112 & (s) & (s) & 2,762 & NA & 158 & 27,040 \\
\hline 1988 & $\begin{array}{r}15,850 \\
\end{array}$ & $\begin{array}{r}1,563 \\
\end{array}$ & $\begin{array}{r}2,709 \\
\end{array}$ & NA & $\begin{array}{r}20,123 \\
\end{array}$ & $\begin{array}{r}5,587 \\
\end{array}$ & $\begin{array}{r}2,334 \\
\end{array}$ & 10 & 8 & $\begin{array}{r}106 \\
\end{array}$ & (s) & (s) & $\begin{array}{r}2,458 \\
\end{array}$ & NA & 108 & 28,276 \\
\hline 1989 & ${ }^{1116,359}$ & 111,756 & 113,582 & 90 & 1121,788 & 115,602 & 122,837 & 11345 & 11151 & ${ }^{11} 152$ & 113 & 1122 & 113,510 & 39 & 37 & 30,976 \\
\hline 1990 & 16,477 & 1,366 & 3,791 & 112 & 21,746 & 6,104 & 3,046 & 442 & 211 & 161 & 4 & 29 & 3,893 & 36 & 8 & 31,788 \\
\hline 1991 & 16,460 & 1,276 & 3,861 & 125 & 21,723 & 6,422 & 3,016 & 425 & 247 & 167 & 5 & 31 & 3,889 & 59 & 67 & 32,160 \\
\hline 1992 & 16,686 & 1,076 & 3,999 & 141 & 21,903 & 6,479 & 2,617 & 481 & 283 & 167 & 4 & 30 & 3,582 & 40 & 87 & 32,091 \\
\hline 1993 & 17,424 & 1,203 & 4,027 & 136 & 22,790 & 6,410 & 2,892 & 485 & 288 & 173 & 5 & 31 & 3,874 & 34 & 95 & 33,203 \\
\hline 1994 & 17,485 & 1,135 & 4,476 & 136 & 23,233 & 6,694 & 2,683 & 498 & 301 & 160 & 5 & 36 & 3,683 & 40 & 153 & 33,803 \\
\hline 1995 & 17,687 & 813 & 4,840 & 133 & 23,473 & 7,075 & 3,205 & 480 & 316 & 138 & 5 & 33 & 4,177 & 42 & 134 & 34,901 \\
\hline 1996 & 18,650 & 888 & 4,400 & 159 & $\begin{array}{l}24,097 \\
\end{array}$ & 7,087 & 3,590 & 513 & 324 & 148 & 5 & 33 & 4,613 & 37 & 137 & 35,971 \\
\hline 1997 & 19,128 & 985 & 4,658 & 119 & 24,890 & 6,597 & 3,640 & 484 & $\begin{array}{l}339 \\
339\end{array}$ & 150 & 5 & 34 & 4,653 & 36 & 116 & 36,293 \\
\hline 1998 & 19,417 & $\begin{array}{r}903 \\
1,378\end{array}$ & $\begin{array}{l}\text {, } 5000 \\
5,205\end{array}$ & 125 & $\begin{array}{l}26,124 \\
\end{array}$ & 7,068 & $\begin{array}{l}3,040 \\
3,297\end{array}$ & $\begin{array}{l}404 \\
475\end{array}$ & 332 & 151 & 5 & 31 & $\begin{array}{l}4,290 \\
4,00\end{array}$ & $\begin{array}{l}50 \\
36\end{array}$ & 88 & 37,607 \\
\hline $\begin{array}{l}1998 \\
1999\end{array}$ & $\begin{array}{r}19,417 \\
19,467\end{array}$ & 1,285 & $\begin{array}{l}5,200 \\
5,441\end{array}$ & 126 & $\begin{array}{l}20,124 \\
26,320\end{array}$ & $\begin{array}{l}7,060 \\
7,610\end{array}$ & $\begin{array}{l}3,297 \\
3,268\end{array}$ & 490 & $\begin{array}{l}302 \\
332\end{array}$ & $\begin{array}{l}151 \\
152\end{array}$ & $\begin{array}{l}5 \\
5\end{array}$ & 46 & $\begin{array}{l}4,290 \\
4,292\end{array}$ & $\begin{array}{l}30 \\
41\end{array}$ & $\begin{array}{l}00 \\
99\end{array}$ & 38,362 \\
\hline 2000 & 20,411 & 1,212 & 5,818 & 126 & 27,567 & 7,862 & 2,811 & 496 & 330 & 144 & 5 & 57 & 3,843 & 46 & 115 & 39,433 \\
\hline 2001 & 19,789 & 1,347 & 6,001 & 97 & 27,235 & 8,029 & 2,242 & 486 & 228 & 142 & 6 & 70 & 3,173 & 160 & 75 & 38,672 \\
\hline 2002 & 19,997 & 1,014 & 6,25 & 131 & 27,392 & 8,145 & 2,689 & 605 & 257 & 147 & 6 & 105 & 3,809 & 191 & 72 & 39,610 \\
\hline 2003 & 20,367 & 1,266 & 5,73 & 156 & 27,525 & 7,959 & 2,825 & 519 & 249 & 148 & 5 & 115 & 3,860 & 193 & 22 & 39,559 \\
\hline 2004 & 20,376 & 1,248 & 5,827 & 135 & 27,586 & 8,222 & 2,690 & 344 & 230 & 148 & 6 & 142 & 3,560 & 183 & 39 & 39,591 \\
\hline 2005 & 20,802 & 1,269 & 6,212 & 110 & 28,393 & 8,161 & 2,703 & 355 & 230 & 147 & 6 & 178 & 3,619 & 173 & 85 & 40,430 \\
\hline 2006 & 20,527 & 668 & 6,644 & 115 & 27,954 & 8,215 & 2,869 & 350 & 241 & 145 & 5 & 264 & 3,873 & 162 & 63 & 40,268 \\
\hline 2007 & 20,842 & 683 & 7,288 & 115 & 28,927 & 8,455 & 2,446 & 353 & 245 & 145 & 6 & 341 & 3,536 & 168 & 107 & 41,193 \\
\hline 2008 & 20,549 & 485 & 7,087 & 97 & 28,218 & 8,427 & 2,511 & 339 & 267 & 146 & 9 & 546 & 3,817 & 172 & 112 & 40,747 \\
\hline 2009 & 18,241 & 403 & 7,302 & 84 & 26,029 & 8,356 & 2,669 & 320 & 272 & 146 & 9 & 721 & 4,137 & 170 & 116 & $\begin{array}{l}40,41 \\
38,808\end{array}$ \\
\hline 2010 & $\mathrm{R}_{19,196}$ & $\mathrm{R}_{386}$ & R7,853 & R90 & R27,525 & R8,434 & $\mathrm{R}_{2,539}$ & R350 & $\mathrm{R}_{281}$ & R148 & $\mathrm{R}_{12}$ & R923 & $\mathrm{R}_{4,253}$ & $\mathrm{R}_{184}$ & R89 & R 40,485 \\
\hline $2011^{\mathrm{P}}$ & 18,044 & 291 & 8,051 & 91 & 26,477 & 8,259 & 3,171 & 333 & 287 & 163 & 18 & $\begin{array}{l}1,168 \\
\end{array}$ & 5,140 & 162 & 127 & 40,166 \\
\hline
\end{tabular}

1 Anthracite, bituminous coal, subbituminous coal, lignite, waste coal, and coal synfuel.
2 Distillate fuel oil, residual fuel oil, petroleum coke, jet fuel, kerosene, other petroleum, and waste oil. 3 Natural gas, plus a small amount of supplemental gaseous fuels.

Blast furnace gas, propane gas, and other manufactured and waste gases derived from fossil fuels. 6 Wood and wood-derived fuels.

Municipal solid waste from biogenic sources, landfill gas, sludge waste, agricultural byproducts, and other biomass. Through 2000 , also includes non-renewable waste (municipal solid waste from non-biogenic sources, and tire-derived fuels).

9 Batteries, chemicals, hydrogen, pitch, purchased steam, sulfur, miscellaneous technologies, and beginning in 2001, non-renewable waste (municipal solid waste from non-biogenic sources, and tire-derived ${ }_{10}$ Net imports equal imports minus exports. See Note 3, "Electricity Imports and Exports," at end of section.

Through 1988, data are for electric utilities only. Beginning in 1989, data are for electric utilities, independent power producers, commercial plants, and industrial plants.

Beginning in 1989, data are for electric utilities, independent power producers, commercial plants, and industrial plants.

Notes: - Data are for energy consumed to produce electricity 0.5 trillion Btu. produce useful thermal output at a small number of electric utility combined-heat-and-power (CHP) plants. - This table no longer shows energy consumption by hychoelectric pumped storage plants. The change was made because most of the electricity used to pump water into elevated storage reservoirs is generated the pla may not equal sum of components due to independent rounding. Web Pages: - See http://www.eia.gov/totalenergy/data/ann

- For related information, see http://wwW.eia.gov/electricity/.
Sources: $1949-1988-$ Table $8.4 \mathrm{~b}$ for electric power sector, and Tables 8.1 and A6 for industrial sector. - 1989 forward-Tables $8.4 \mathrm{~b}$ and $8.4 \mathrm{c}$ 
Table 8.4b Consumption for Electricity Generation by Energy Source: Electric Power Sector, Selected Years, 1949-2011 (Subset of Table 8.4a; Trillion Btu)

\begin{tabular}{|c|c|c|c|c|c|c|c|c|c|c|c|c|c|c|c|c|}
\hline \multirow[b]{3}{*}{ Year } & \multicolumn{5}{|c|}{ Fossil Fuels } & \multirow{3}{*}{$\begin{array}{l}\text { Nuclear } \\
\text { Electric } \\
\text { Power }^{5}\end{array}$} & \multicolumn{7}{|c|}{ Renewable Energy } & \multirow[b]{3}{*}{ Other $^{9}$} & \multirow{3}{*}{$\begin{array}{c}\text { Electricity } \\
\text { Net } \\
\text { Imports }\end{array}$} & \multirow[b]{3}{*}{ Total } \\
\hline & \multirow[b]{2}{*}{ Coal $^{1}$} & \multirow[b]{2}{*}{ Petroleum ${ }^{2}$} & \multirow{2}{*}{$\begin{array}{c}\text { Natural } \\
\text { Gas }^{3}\end{array}$} & \multirow{2}{*}{$\begin{array}{c}\text { Other } \\
\text { Gases }{ }^{4}\end{array}$} & \multirow[b]{2}{*}{ Total } & & \multirow{2}{*}{$\begin{array}{c}\text { Conventional } \\
\text { Hydroelectric } \\
\text { Power }^{5}\end{array}$} & \multicolumn{2}{|c|}{ Biomass } & \multirow{2}{*}{$\begin{array}{c}\text { Geo- } \\
\text { thermal }\end{array}$} & \multirow[b]{2}{*}{ Solar/PV 5,8 } & \multirow[b]{2}{*}{ Wind $^{5}$} & \multirow[b]{2}{*}{ Total } & & & \\
\hline & & & & & & & & Wood $^{6}$ & Waste $^{7}$ & & & & & & & \\
\hline 1949 & 1,995 & 415 & 569 & NA & 2,979 & 0 & 1,349 & 6 & NA & NA & NA & NA & 1,355 & NA & 5 & 4.339 \\
\hline 1950 & 2,199 & 472 & 651 & NA & 3,322 & 0 & 1,346 & $\begin{array}{l}0 \\
5\end{array}$ & NA & NA & NA & NA & $\begin{array}{l}1,350 \\
1,351\end{array}$ & NA & 6 & $\begin{array}{r}4,679 \\
4,679\end{array}$ \\
\hline 1955 & 3,458 & 471 & $\begin{array}{r}1,194 \\
\end{array}$ & NA & 5,123 & 0 & $\begin{array}{l}1,340 \\
1,322\end{array}$ & $\begin{array}{l}5 \\
3\end{array}$ & NA & NA & NA & NA & 1,325 & NA & 14 & $\begin{array}{l}\text {, } \\
6,461\end{array}$ \\
\hline 1960 & $\begin{array}{l}0,450 \\
4,228\end{array}$ & 553 & 1,785 & NA & 6,565 & 6 & 1,569 & $\begin{array}{l}0 \\
2\end{array}$ & NA & (s) & NA & NA & 1,571 & NA & 15 & 8,158 \\
\hline 1965 & $\begin{array}{l}4,2<0 \\
5,821\end{array}$ & 722 & 2,395 & NA & 8,938 & $\begin{array}{r}0 \\
43\end{array}$ & 2,026 & 3 & NA & (1) & NA & NA & 2,031 & NA & (s) & 11,012 \\
\hline 1970 & 7,227 & 2,117 & 4,054 & NA & 13,399 & 239 & 2,600 & 1 & 2 & 6 & NA & NA & $\begin{array}{l}2,609 \\
2,609\end{array}$ & NA & (5) & 16,253 \\
\hline 1975 & 8,786 & 3,166 & 3,240 & NA & 15,191 & 1,900 & 3,122 & (s) & 2 & 34 & NA & NA & 3,158 & NA & 21 & 20,270 \\
\hline 1976 & $\begin{array}{l}0,100 \\
9,720\end{array}$ & 3,477 & 3,152 & NA & 16,349 & 2,111 & 2,943 & (s) & 2 & $\begin{array}{l}54 \\
38\end{array}$ & NA & NA & 2,983 & NA & 29 & 21,473 \\
\hline 1977 & 10,262 & 3,901 & 3,284 & NA & 17,446 & 2,702 & 2,301 & 3 & 2 & 37 & NA & NA & 2,343 & NA & 59 & 22,551 \\
\hline 1978 & 10,238 & 3,987 & 3,297 & NA & 17,522 & 3,024 & 2,905 & 2 & 1 & 31 & NA & NA & $\begin{array}{l}2,940 \\
2,940\end{array}$ & NA & 67 & 23,553 \\
\hline 1979 & 11,260 & 3,283 & 3,613 & NA & 18,156 & $\begin{array}{l}2,776 \\
2,76\end{array}$ & 2,897 & 3 & 2 & 40 & NA & NA & 2,942 & NA & 69 & 23,943 \\
\hline 1980 & 12,123 & 2,634 & 3,810 & NA & 18,567 & 2,739 & 2,867 & 3 & 2 & 53 & NA & NA & $\begin{array}{r}2,925 \\
2,925\end{array}$ & NA & 71 & 24,302 \\
\hline 1981 & 12,583 & $\begin{array}{r}2,004 \\
2,202\end{array}$ & $\begin{array}{l}3,768 \\
3,768\end{array}$ & NA & 18,553 & 3,008 & $\begin{array}{l}2,725 \\
2,725\end{array}$ & 3 & 1 & 59 & NA & NA & 2,788 & NA & 113 & 24,462 \\
\hline $\begin{array}{l}1982 \\
\end{array}$ & $\begin{array}{l}12,582 \\
\end{array}$ & 1,568 & 3,342 & NA & 17,491 & 3,131 & 3,233 & 2 & 1 & 51 & NA & NA & 3,286 & NA & 100 & 24,009 \\
\hline 1983 & 13,213 & 1,544 & 2,998 & $\mathrm{NA}$ & 17,754 & 3,203 & 3,494 & 2 & 2 & 64 & NA & (s) & 3,562 & NA & 121 & 24,639 \\
\hline 1984 & 14,019 & 1,286 & 3,220 & NA & 18,526 & 3,553 & $\begin{array}{l}3,474 \\
3,353\end{array}$ & 5 & 4 & 81 & (s) & (s) & 3,443 & NA & 135 & 25,657 \\
\hline 1985 & 14,542 & 1,090 & 3,160 & NA & 18,792 & 4,076 & 2,937 & 8 & 7 & 97 & (s) & (s) & 3,049 & NA & 140 & 26,057 \\
\hline 1986 & 14,444 & 1,452 & 2,691 & NA & 18,586 & 4,380 & 3,038 & 5 & 7 & 108 & (s) & (s) & 3,158 & NA & 122 & 26,247 \\
\hline 1987 & 15,173 & 1,257 & 2,935 & NA & 19,365 & 4,754 & 2,602 & 8 & 7 & 112 & (s) & (s) & 2,729 & NA & 158 & 27,007 \\
\hline 1988 & $\begin{array}{r}15,850 \\
\end{array}$ & 1,563 & $\begin{array}{r}2,709 \\
\end{array}$ & $\mathrm{NA}$ & 20,123 & 5,587 & 2,302 & 10 & 8 & 106 & (s) & (s) & 2,425 & NA & 108 & 28,244 \\
\hline 1989 & 1116,121 & 111,697 & 113,107 & 7 & 1120,932 & 115,602 & 112,808 & 1175 & 11126 & 11152 & 113 & 1122 & 113,187 & 2 & 37 & 29,761 \\
\hline 1990 & 16,235 & 1,281 & 3,233 & 6 & 20,755 & 6,104 & 3,014 & 106 & 180 & 161 & 4 & 29 & 3,493 & (s) & 8 & 30,361 \\
\hline 1991 & 16,223 & 1,199 & 3,296 & 6 & 20,725 & 6,422 & 2,985 & 104 & 217 & 167 & 5 & 31 & 3,509 & $\begin{array}{r}101 \\
4\end{array}$ & 67 & 30,727 \\
\hline 1992 & 16,431 & 990 & 3,407 & 12 & 20,840 & 6,479 & 2,586 & 120 & 252 & 167 & 4 & 30 & 3,158 & 3 & 87 & 30,568 \\
\hline 1993 & 17,159 & 1,122 & 3,426 & 12 & 21,719 & 6,410 & 2,861 & 129 & 255 & 173 & 5 & 31 & 3,454 & 3 & 95 & 31,681 \\
\hline 1994 & 17,215 & 1,056 & 3,851 & 12 & 22,134 & 6,694 & 2,620 & 134 & 269 & 160 & 5 & 36 & 3,224 & 2 & 153 & 32,207 \\
\hline 1995 & 17,416 & 743 & 4,179 & 18 & 22,356 & 7,075 & 3,149 & 106 & 282 & 138 & 5 & 33 & 3,713 & 2 & 134 & 33,281 \\
\hline 1996 & 18,375 & 810 & 3,730 & 16 & 22,930 & 7,087 & 3,528 & 117 & 280 & 148 & 5 & 33 & 4,112 & 2 & 137 & 34,268 \\
\hline 1997 & 18,855 & 917 & 3,981 & 14 & 23,768 & 6,597 & 3,581 & 117 & 292 & 150 & 5 & 34 & 4,179 & 1 & 116 & 34,660 \\
\hline 1998 & 19,162 & 1,306 & 4,520 & 23 & 25,011 & 7,068 & 3,241 & 125 & 287 & 151 & 5 & 31 & 3,840 & 2 & 88 & 36,008 \\
\hline 1999 & 19,214 & 1,211 & 4,742 & 14 & 25,181 & 7,610 & 3,218 & 125 & 290 & 152 & 5 & 46 & 3,836 & 1 & 99 & 36,728 \\
\hline 2000 & 20,153 & 1,145 & 5,120 & 19 & 26,438 & 7,862 & 2,768 & 126 & 294 & 144 & 5 & 57 & 3,394 & 1 & 115 & 37,811 \\
\hline 2001 & 19,549 & 1,280 & 5,290 & 9 & 26,128 & 8,029 & 2,209 & 116 & 205 & 142 & 6 & 70 & 2,747 & 109 & 75 & 37,089 \\
\hline 2002 & 19,733 & 955 & 5,522 & 25 & 26,235 & 8,145 & 2,650 & 141 & 224 & 147 & 6 & 105 & 3,273 & 137 & 72 & \\
\hline 2003 & 20,137 & 1,199 & 5,009 & 30 & 26,374 & 7,959 & 2,781 & 156 & 216 & 148 & 5 & 115 & 3,421 & 136 & 22 & 37,912 \\
\hline 2004 & 20,217 & 1,202 & 5,209 & 27 & 26,655 & 8,222 & 2,656 & 150 & 206 & 148 & 6 & 142 & 3,308 & 131 & 39 & 38,355 \\
\hline 2005 & 20,649 & 1,227 & 5,643 & 24 & 27,543 & 8,161 & 2,670 & 166 & 205 & 147 & 6 & 178 & 3,372 & 116 & 85 & 39,276 \\
\hline 2006 & 20,377 & 635 & 6,055 & 28 & 27,095 & 8,215 & 2,839 & 163 & 216 & 145 & 5 & 264 & 3,632 & 117 & 63 & 39,122 \\
\hline 2007 & 20,723 & 651 & 6,681 & 27 & 28,083 & 8,455 & 2,430 & 165 & 221 & 145 & 6 & 341 & 3,307 & 117 & 107 & 40,068 \\
\hline 2008 & 20,431 & 463 & 6,516 & 23 & 27,434 & 8,427 & 2,494 & 159 & 242 & 146 & 9 & 546 & 3,596 & 122 & 112 & 39,691 \\
\hline 2009 & 18,135 & 382 & 6,731 & 21 & 25,270 & 8,356 & 2,650 & 160 & 244 & 146 & 9 & 721 & 3,931 & 115 & 116 & 37,788 \\
\hline 2010 & $\mathrm{R}_{19,043}$ & R371 & R7,242 & 20 & R26,675 & R8,434 & $R_{2,521}$ & R177 & R249 & $R_{148}$ & $\mathrm{R}_{12}$ & R923 & $\mathrm{R}_{4,031}$ & $\mathrm{R}_{116}$ & R89 & $\mathrm{R}_{39,345}$ \\
\hline $2011^{P}$ & 17,897 & 278 & 7,433 & 20 & 25,629 & 8,259 & 3,153 & 160 & 256 & 163 & 18 & 1,168 & 4,917 & 117 & 127 & 39,049 \\
\hline
\end{tabular}

1 Anthracite, bituminous coal, subbituminous coal, lignite, waste coal, and coal synfuel.
2 Distillate fuel oil, residual fuel oil, petroleum coke, jet fuel, kerosene, other petroleum, and waste oil. ${ }^{3}$ Natural gas, plus a small amount of supplemental gaseous fuels.

Blast furnace gas, propane gas, and other manufactured and waste gases derived from fossil fuels. 6 Wood and wood-derived fuels.

Municipal solid waste from biogenic sources, landfill gas, sludge waste, agricultural byproducts, and other biomass. Through 2000, also includes non-renewable waste (municipal solid waste from 8 Solar thermal and photovoltaic (PV) energy.

Batteries, chemicals, hydrogen, pitch, purchas beginning in 2001, non-renewable waste (municipal solid waste from non-biogenic sources, and tire-derived ${ }_{10} \mathrm{~N}$.

. imports equal imports minus exports. See Note 3, "Electricity Imports and Exports," at end of ${ }_{11}$ Through 1988, data are for electric utilities only. Beginning in 1989, data are for electric utilities and independent power producers.

NA $=$ Not available. $(\mathrm{s})=$ Less than 0.5 trillion Bt

Notes: produce useful thermal output at a small number of electric utility combined-heat-and-power (CHP) plants. the NAICS 22 cater secr whose primary business is to sell electricity or electricity and heat to the wuth - See Table 8.4c for commercial and industrial CHP and electricity-only data. - This table no longer shows energy consumption by hydroelectric pumped storage plants. The change was made because mos of the electricity used to pump water into elevated storage reservoirs is generated by plants other than . Energy-Use Sectors," at end of section. - Totals may not equal sum of components due to independent

Web Pages: • See http://www.eia.gov/totalenergy/data/annual/\#electricity for all data beginning in 1949 - For related information, see http://www.eia.gov/electricity/.

Sources: Electricity Net Imports: Tables 8.1 and A6. All Other Data: - 1949-1988-Tables 8.2b,
8.5b, A1, A4, A5, and A6. P. 1989-1997-U.S. Energy Information Administration (EIA), Form EIA-759 "Monthly Power Plant Report." and Form EIA-867, "Annual Nonutility Power Producer Report." - 1998-2000-EIA, Form ElA-759, "Monthly Power Plant Report," and Form ElA-860B, "Annual Electric Generator Report-Nonutility." " 2001-2003-EIA, Form EIA-906, "Power "Plant Report." - 2004-2007-EIA, Form EIA-906, "Power Plant Report," and Form EIA-920, "Combined Heat and Power 
Table 8.4c Consumption for Electricity Generation by Energy Source: Commercial and Industrial Sectors,

Selected Years, 1989-2011 (Subset of Table 8.4a; Trillion Btu)

\begin{tabular}{|c|c|c|c|c|c|c|c|c|c|c|c|c|c|c|c|c|}
\hline \multirow[b]{3}{*}{ Year } & \multicolumn{5}{|c|}{ Fossil Fuels } & \multirow{3}{*}{$\begin{array}{l}\text { Nuclear } \\
\text { Electric } \\
\text { Power }\end{array}$} & \multicolumn{7}{|c|}{ Renewable Energy } & \multirow[b]{3}{*}{ Other 9} & \multirow{3}{*}{$\begin{array}{c}\text { Electricity } \\
\text { Net } \\
\text { Imports }\end{array}$} & \multirow[b]{3}{*}{ Total } \\
\hline & \multirow[b]{2}{*}{ Coal ${ }^{1}$} & \multirow[b]{2}{*}{ Petroleum ${ }^{2}$} & \multirow{2}{*}{$\begin{array}{c}\text { Natural } \\
\text { Gas }^{3}\end{array}$} & \multirow{2}{*}{$\begin{array}{c}\text { Other } \\
\text { Gases }{ }^{4}\end{array}$} & \multirow[b]{2}{*}{ Total } & & \multirow{2}{*}{$\begin{array}{c}\text { Conventional } \\
\text { Hydroelectric } \\
\text { Power }^{5}\end{array}$} & \multicolumn{2}{|c|}{ Biomass } & \multirow{2}{*}{$\begin{array}{c}\text { Geo- } \\
\text { thermal }\end{array}$} & \multirow[b]{2}{*}{ Solar/PV 5,8 } & \multirow[b]{2}{*}{ Wind 5} & \multirow[b]{2}{*}{ Total } & & & \\
\hline & & & & & & & & Wood 6 & Waste ${ }^{7}$ & & & & & & & \\
\hline & \multicolumn{16}{|c|}{ Commercial Sector 10} \\
\hline 1989 & 9 & 7 & 18 & 1 & 36 & - & 1 & 2 & 9 & - & - & - & 12 & - & -- & 47 \\
\hline 1990 & 9 & 6 & 28 & 1 & 45 & - & 1 & 2 & 15 & - & - & - & 18 & - & -- & 63 \\
\hline 1995 & 12 & 4 & 44 & - & 60 & - & 1 & 1 & 21 & - & - & - & 23 & $(\mathrm{~s})$ & -- & 83 \\
\hline 1996 & 14 & 4 & 44 & (s) & 62 & - & 1 & 1 & 31 & - & - & - & 33 & (s) & -- & 95 \\
\hline 1997 & 14 & $\begin{array}{l}4 \\
5\end{array}$ & 40 & $\begin{array}{l}\text { (s) } \\
\text { (s) }\end{array}$ & 59 & - & 1 & 1 & 34 & - & - & - & 35 & (s) & -- & 94 \\
\hline 1998 & 11 & 5 & 42 & (s) & 57 & - & 1 & 1 & 32 & - & - & - & 34 & (s) & -- & 91 \\
\hline 1999 & 12 & 6 & 40 & (s) & 57 & - & 1 & (s) & 33 & - & - & - & 35 & (s) & -- & 92 \\
\hline 2000 & 12 & 5 & 38 & (s) & 55 & - & 1 & (s) & 26 & - & - & - & 28 & (s) & -- & 82 \\
\hline 2001 & 13 & 6 & 37 & (s) & 56 & - & 1 & $\begin{array}{l}\text { (s) } \\
\text { (s) }\end{array}$ & 15 & - & - & - & 16 & 7 & -- & 79 \\
\hline 2002 & 9 & 4 & 31 & (s) & 44 & - & (s) & (s) & 18 & - & - & - & 19 & 11 & -- & 73 \\
\hline 2003 & $\begin{array}{r}9 \\
13\end{array}$ & $\begin{array}{l}4 \\
5\end{array}$ & $\begin{array}{l}51 \\
39\end{array}$ & (S) & $\begin{array}{l}44 \\
58\end{array}$ & - & (S) 1 & $\begin{array}{l}\text { (s) } \\
\text { (s) }\end{array}$ & $\begin{array}{l}18 \\
19\end{array}$ & - & - & $\overline{-}$ & 21 & 11 & -- & $\begin{array}{l}13 \\
89\end{array}$ \\
\hline 2004 & 8 & 5 & 34 & - & 46 & - & 1 & (s) & 19 & - & - & - & 21 & 11 & -- & 78 \\
\hline 2005 & 8 & 4 & $\begin{array}{l}54 \\
35\end{array}$ & - & 46 & - & 1 & $\begin{array}{l}\text { (s) } \\
\text { (s) }\end{array}$ & 20 & - & - & - & 21 & 10 & -- & 78 \\
\hline 2006 & $\begin{array}{l}\circ \\
8\end{array}$ & $\begin{array}{l}4 \\
2\end{array}$ & 35 & (s) & $\begin{array}{l}40 \\
45\end{array}$ & $\overline{-}$ & 1 & $\begin{array}{l}\text { (s) } \\
\text { (s) }\end{array}$ & 21 & - & - & - & $\begin{array}{l}21 \\
22\end{array}$ & 10 & -- & 77 \\
\hline 2007 & 8 & 2 & 35 & - & 44 & - & 1 & (s) & 19 & - & - & - & 20 & 10 & -- & 75 \\
\hline 2008 & 8 & 1 & 34 & - & 43 & - & 1 & (s) & 20 & - & (s) & - & 21 & 11 & -- & 75 \\
\hline 2009 & 7 & 1 & 35 & - & 43 & - & 1 & (s) & 23 & - & (s) & (s) & 24 & 13 & -- & 80 \\
\hline 2010 & 7 & 1 & $\begin{array}{r}50 \\
\mathrm{R}_{4}\end{array}$ & $\mathrm{R}_{(\mathrm{s})}$ & $\begin{array}{r}43 \\
\mathrm{R}_{48}\end{array}$ & $\overline{-}$ & 1 & $\begin{array}{l}\text { (s) } \\
\text { (s) }\end{array}$ & $\begin{array}{l}20 \\
R_{24}\end{array}$ & $\overline{-}$ & $\begin{array}{l}\text { (s) } \\
\text { (s) }\end{array}$ & $\begin{array}{l}\text { (s) } \\
\text { (s) }\end{array}$ & $\begin{array}{r}24 \\
R_{25}\end{array}$ & $\mathrm{R}_{14}$ & -- & $\begin{array}{l}\text { R87 } \\
\text { R87 }\end{array}$ \\
\hline $2011^{P}$ & 6 & 1 & 39 & (s) & 45 & - & 1 & $\begin{array}{l}\text { (s) } \\
\text { (s) }\end{array}$ & 24 & - & $\begin{array}{l}\text { (1) } \\
\text { (s) }\end{array}$ & (s) & 25 & 14 & -- & 84 \\
\hline
\end{tabular}

Industrial Sector ${ }^{11}$

\begin{tabular}{|c|c|c|c|c|c|c|c|c|c|c|c|c|c|c|c|c|}
\hline 1989 & 229 & 52 & 456 & 83 & 820 & - & 28 & 267 & 15 & - & - & - & 311 & 37 & -- & 1,168 \\
\hline 1990 & 233 & 79 & 530 & 104 & 946 & - & 31 & 335 & 16 & - & - & - & 382 & 36 & -- & 1,364 \\
\hline 1995 & 259 & 66 & 617 & 114 & 1,057 & - & 55 & 373 & 13 & - & - & - & 440 & 40 & -- & 1,537 \\
\hline 1996 & 261 & 74 & 626 & 143 & 1,104 & - & 61 & 394 & 13 & - & - & - & 468 & 35 & -- & 1,607 \\
\hline 1997 & 260 & 63 & 637 & 105 & 1,064 & - & 58 & 367 & 14 & - & - & - & 439 & 36 & -- & 1,538 \\
\hline 1998 & 245 & 67 & 643 & 102 & 1,056 & - & 55 & 349 & 13 & - & - & - & 417 & 35 & -- & 1,508 \\
\hline 1999 & 242 & 68 & 660 & 112 & 1,081 & - & 49 & 364 & 8 & - & - & - & 422 & 39 & -- & 1,542 \\
\hline 2000 & 245 & 61 & 660 & 107 & 1,074 & - & 42 & 369 & 10 & - & - & - & 421 & 45 & -- & 1,540 \\
\hline 2001 & 227 & 62 & 674 & 88 & 1,051 & - & 33 & 370 & 7 & - & - & - & 410 & 44 & -- & 1,504 \\
\hline 2002 & 255 & 55 & 697 & 106 & 1,113 & - & 39 & 464 & 15 & - & - & - & 518 & 43 & -- & 1,675 \\
\hline 2003 & 217 & 61 & 687 & 127 & 1,093 & - & 43 & 362 & 13 & - & - & - & 419 & 46 & -- & 1,558 \\
\hline 2004 & 151 & 42 & 585 & 108 & 885 & - & 33 & 194 & 5 & - & - & - & 231 & 41 & -- & 1,158 \\
\hline 2005 & 145 & 39 & 534 & 85 & 804 & - & 32 & 189 & 5 & - & - & - & 226 & 46 & -- & $\begin{array}{l}1,076 \\
\text { S }\end{array}$ \\
\hline 2006 & 143 & 31 & 554 & 87 & 814 & - & 29 & 187 & 3 & - & - & - & 219 & 35 & -- & 1,068 \\
\hline 2007 & 111 & 30 & 572 & 88 & 800 & - & 16 & 188 & 4 & - & - & - & 208 & 41 & -- & 1,050 \\
\hline 2008 & 109 & 21 & 537 & 73 & 740 & - & 17 & 179 & 5 & - & - & - & 200 & 39 & -- & 980 \\
\hline 2009 & 99 & 20 & 535 & 62 & 716 & - & 18 & 160 & 4 & _- & - & _ & 182 & 42 & -- & 940 \\
\hline 2010 & $\begin{array}{r}\text { R146 } \\
\text { R }\end{array}$ & $\mathrm{R}_{14}$ & $\begin{array}{r}\text { R550 } \\
\text { R570 }\end{array}$ & R70 & R801 & $\overline{-}$ & $\begin{array}{l}10 \\
16\end{array}$ & R172 & $\begin{array}{l}R_{8}^{4} \\
\text { R }\end{array}$ & $\overline{-}$ & (s) & - & R197 & $\begin{array}{l}42 \\
\text { R55 }\end{array}$ & -- & $\mathrm{R}_{1,053}$ \\
\hline $2011^{\mathrm{P}}$ & 141 & 12 & 580 & 71 & 803 & - & 18 & 173 & 8 & - & (s) & (s) & 199 & 31 & -- & 1,033 \\
\hline
\end{tabular}

Anthracite, bituminous coal, subbituminous coal, lignite, waste coal, and coal synfuel.

Distillate fuel oil, residual fuel oil, petroleum coke, jet fuel, kerosene, other petroleum, and waste oil.

Natural gas, plus a small amount of supplemental gaseous fuels.

Blast furnace gas, propane gas, and other manufactured and waste gases derived from fossil fuels.

Wood and wood-derived fuels

7 Municipal solid waste from biogenic sources, landfill gas, sludge waste, agricultural byproducts, and other biomass. Through 2000, also includes non-renewable waste (municipal solid waste from non-biogenic sources, and tire-derived fuels).

Solar thermal and photovoltaic (PV) energy.

9 Batteries, chemicals, hydrogen, pitch, purchased steam, sulfur, miscellaneous technologies, and beginning in 2001, non-renewable waste (municipal solid waste from non-biogenic sources, and tire-derived fuels).

Commercial combined-heat-and-power (CHP) and commercial electricity-only plants.

11 Industrial combined-heat-and-power (CHP) and industrial electricity-only plants.

R=Revised. P=Preliminary. $--=$ Not applicable. $-=$ No data reported. $(\mathrm{s})=$ Less than 0.5 trillion Btu. Notes: - Data are for energy consumed to produce electricity. - See Table 8.4b for electric power "Classification of Power Plants Into Energy-Use Sectors," at end of o Electictly Stalstics," and Note 2 components due to independent rounding.

- For related information, see http://www.eia.gov/electricity/.

Sources: • 1989-1997-U.S. Energy Information Administration (EIA), Form EIA-867, "Annual Nonutility Power Producer Report." • 1998-2000-EIA, Form EIA-860B, "Annual Electric Generator Report-Nonutilty. " 2001-2003-EIA, Form EIA-906, "Power Plant Report. • 2004-2007-EIA, Form " "Comer Plant Repot." 0 and Power Plant Report." - 2008 forward-EIA, Form EIA-923, "Power Plant Operations Report." 
Figure 8.5a Consumption of Combustible Fuels for Electricity Generation (All Sectors), 1989-2011

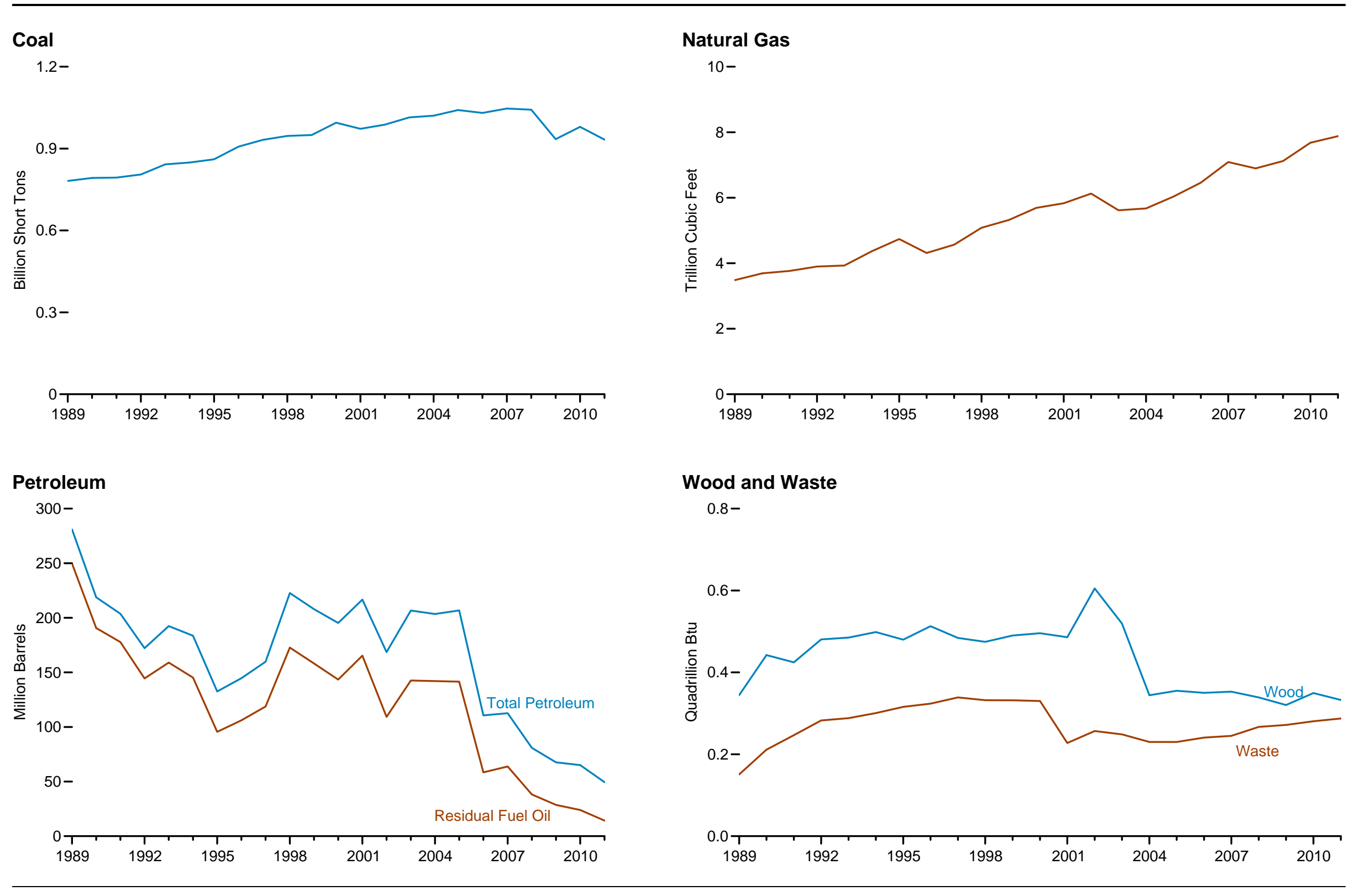

Source: Table $8.5 \mathrm{a}$. 


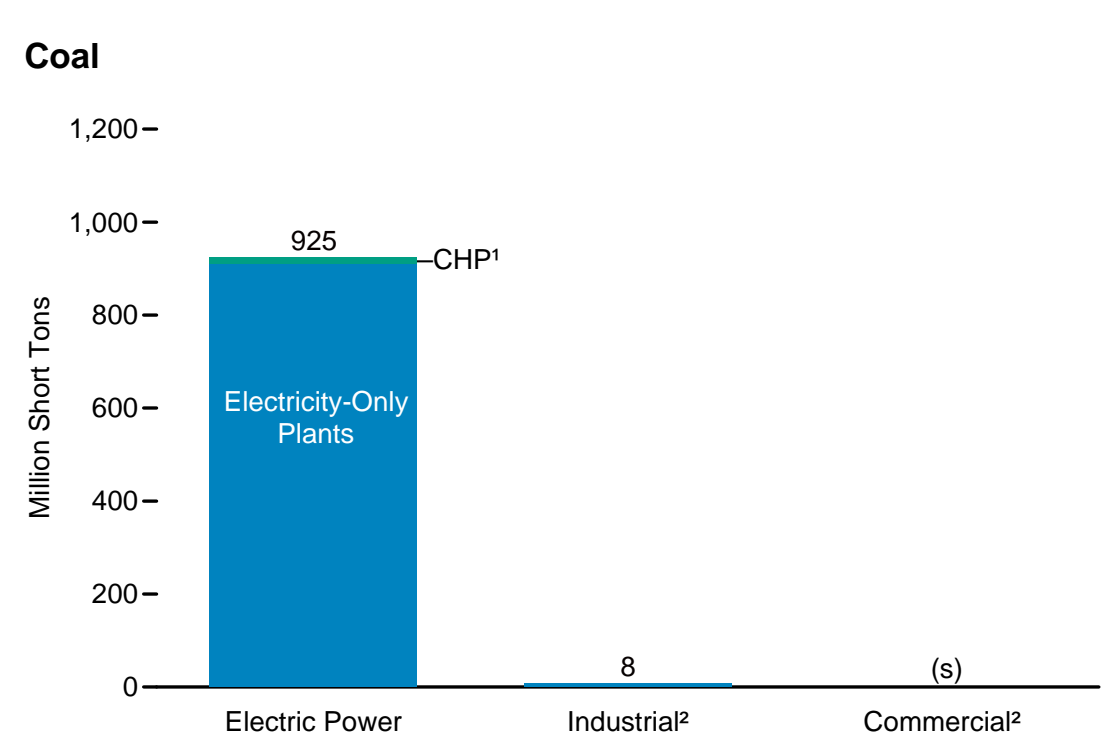

\section{Natural Gas}

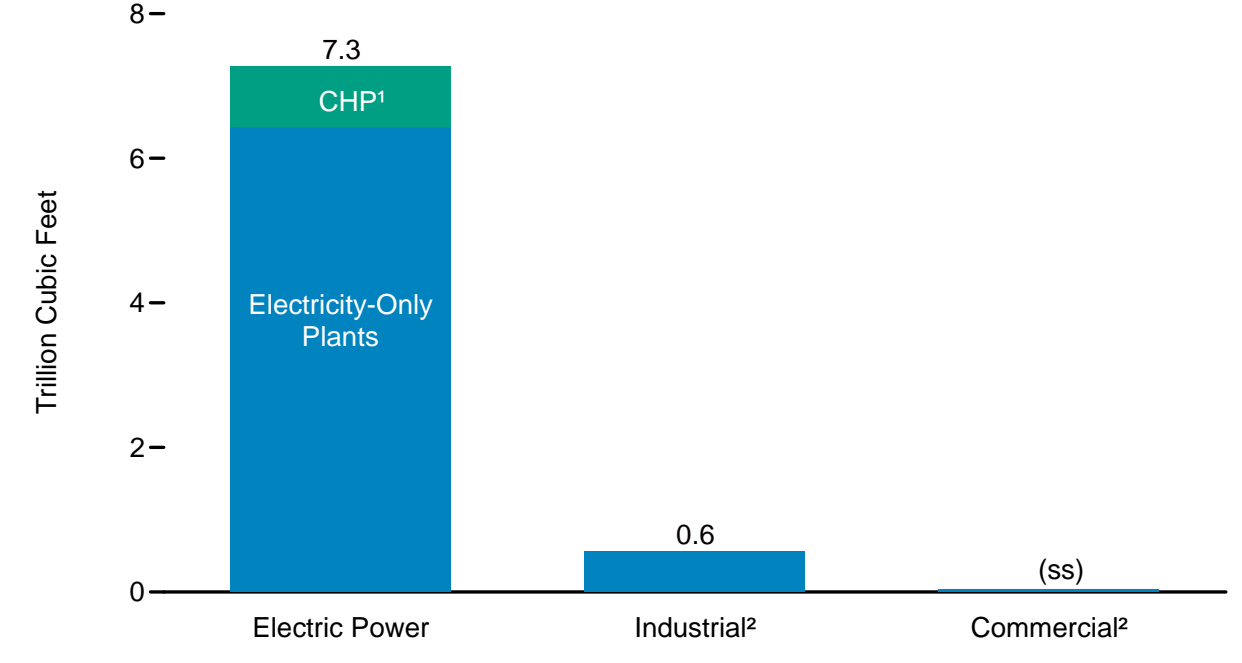

\section{Petroleum}

\section{Wood and Waste}

$60-$

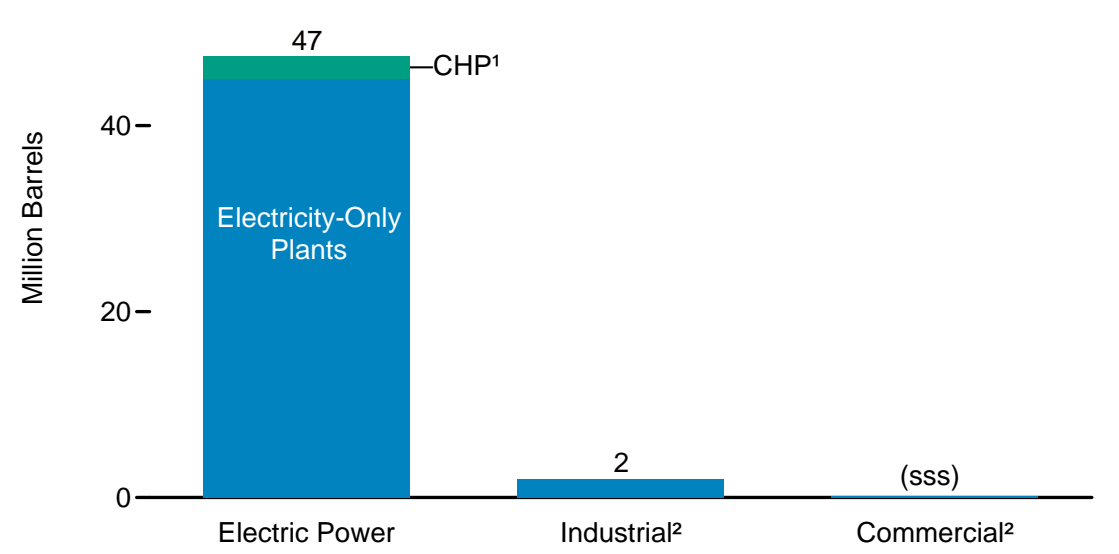

$500-$

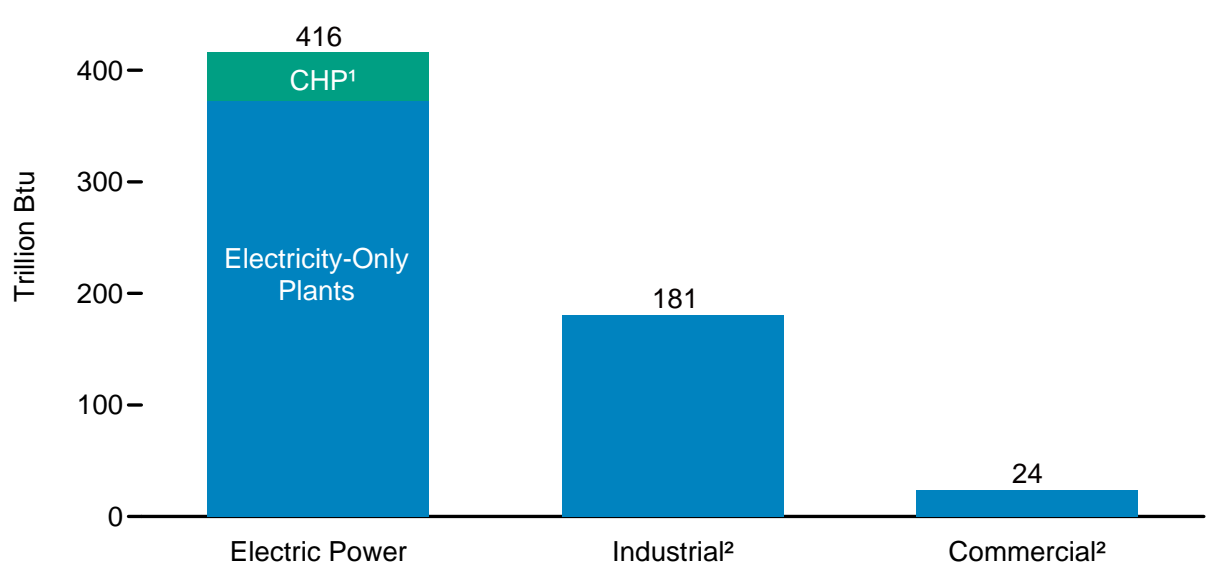


Table 8.5a Consumption of Combustible Fuels for Electricity Generation: Total (All Sectors), Selected Years, 1949-2011 (Sum of Tables 8.5b and 8.5d)

\begin{tabular}{|c|c|c|c|c|c|c|c|c|c|c|c|}
\hline \multirow[b]{3}{*}{ Year } & \multirow[b]{2}{*}{ Coal $^{1}$} & \multicolumn{5}{|c|}{ Petroleum } & \multirow[b]{2}{*}{ Natural Gas ${ }^{6}$} & \multirow[b]{2}{*}{ Other Gases ${ }^{7}$} & \multicolumn{2}{|c|}{ Biomass } & \multirow[b]{2}{*}{ Other ${ }^{10}$} \\
\hline & & Distillate Fuel Oil ${ }^{2}$ & Residual Fuel Oil ${ }^{3}$ & Other Liquids ${ }^{4}$ & Petroleum Coke 5 & Total $^{5}$ & & & Wood $^{8}$ & Waste $^{9}$ & \\
\hline & $\begin{array}{l}\text { Thousand } \\
\text { Short Tons }\end{array}$ & \multicolumn{3}{|c|}{ Thousand Barrels } & $\begin{array}{l}\text { Thousand } \\
\text { Short Tons }\end{array}$ & $\begin{array}{c}\text { Thousand } \\
\text { Barrels }\end{array}$ & $\begin{array}{c}\text { Million } \\
\text { Cubic Feet }\end{array}$ & $\begin{array}{c}\text { Trillion } \\
\text { Btu }\end{array}$ & \multicolumn{2}{|c|}{ Trillion Btu } & $\begin{array}{c}\text { Trillion } \\
\text { Btu }\end{array}$ \\
\hline $\begin{array}{l}1949 \\
1950\end{array}$ & $\begin{array}{l}83,963 \\
91,871\end{array}$ & $\begin{array}{l}4,767 \\
5423\end{array}$ & 61,534 & NA & NA & 66,301 & 550,121 & NA & 6 & NA & NA \\
\hline $\begin{array}{l}1950 \\
1955\end{array}$ & $\begin{array}{r}91,8 / 1 \\
143,759\end{array}$ & $\begin{array}{l}5,423 \\
5,412\end{array}$ & $\begin{array}{l}69,998 \\
69862\end{array}$ & $\begin{array}{l}\text { NA } \\
\text { NA }\end{array}$ & $\begin{array}{l}\text { NA } \\
\text { NA }\end{array}$ & $\begin{array}{l}75,421 \\
75,274\end{array}$ & $\begin{array}{r}628,919 \\
1.153280\end{array}$ & $\begin{array}{l}\text { NA } \\
\text { NA }\end{array}$ & $\begin{array}{l}5 \\
3\end{array}$ & $\begin{array}{l}\text { NA } \\
\text { NA }\end{array}$ & $\begin{array}{l}\text { NA } \\
\text { NA }\end{array}$ \\
\hline 1960 & 176,685 & 3,824 & 84,371 & NA & NA & 88,195 & $1,724,762$ & NA & 2 & NA & NA \\
\hline 1965 & 244,788 & 4,928 & 110,274 & NA & NA & 115,203 & $2,321,101$ & NA & 3 & NA & NA \\
\hline 1970 & 320,182 & 24,123 & 311,381 & NA & 636 & 338,686 & $3,931,860$ & NA & 1 & 2 & NA \\
\hline 1975 & 405,962 & 38,907 & 467,221 & NA & 70 & 506,479 & $3,157,669$ & NA & (s) & 2 & NA \\
\hline 1976 & 448,371 & 41,843 & 514,077 & NA & 68 & 556,261 & $3,080,868$ & NA & 1 & 2 & NA \\
\hline 1977 & 477,126 & 48,837 & 574,869 & NA & 98 & 624,193 & $3,191,200$ & NA & 3 & 2 & NA \\
\hline 1978 & 481,235 & 47,520 & 588,319 & NA & 398 & 637,830 & $3,188,363$ & NA & 2 & 1 & NA \\
\hline 1979 & 527,051 & 30,691 & 492,606 & NA & 268 & 524,636 & $3,490,523$ & NA & 3 & 2 & NA \\
\hline 1980 & 569,274 & 29,051 & 391,163 & NA & 179 & 421,110 & $3,681,595$ & NA & 3 & 2 & NA \\
\hline 1981 & 596,797 & 21,313 & 329,798 & NA & 139 & 351,806 & $3,640,154$ & NA & 3 & 1 & NA \\
\hline 1982 & 593,666 & 15,337 & 234,434 & NA & 149 & 250,517 & $3,225,518$ & NA & 2 & 1 & NA \\
\hline 1983 & 625,211 & 16,512 & 228,984 & NA & 261 & 246,804 & $2,910,767$ & NA & 2 & 2 & NA \\
\hline 1984 & 664,399 & 15,190 & 189,289 & NA & 252 & 205,736 & $3,111,342$ & NA & 5 & 4 & NA \\
\hline 1985 & 693,841 & 14,635 & 158,779 & NA & 231 & 174,571 & $3,044,083$ & NA & 8 & 7 & NA \\
\hline 1986 & 685,056 & 14,326 & 216,156 & NA & 313 & 232,046 & $2,602,370$ & NA & 5 & 7 & NA \\
\hline 1987 & 717,894 & 15,367 & 184,011 & NA & 348 & 201,116 & $2,844,051$ & NA & 8 & 7 & NA \\
\hline 1988 & 758,372 & 18,769 & 229,327 & NA & 409 & 250,141 & $2,635,613$ & NA & 10 & 8 & NA \\
\hline $1989^{11}$ & 781,672 & 27,733 & 249,614 & 303 & 667 & 280,986 & $3,485,429$ & 90 & 345 & 151 & 39 \\
\hline 1990 & 792,457 & 18,143 & 190,652 & 437 & 1,914 & 218,800 & $3,691,563$ & 112 & 442 & 211 & 36 \\
\hline 1991 & 793,666 & 16,564 & 177,780 & 380 & 1,789 & 203,669 & $3,764,778$ & 125 & 425 & 247 & 59 \\
\hline 1992 & 805,140 & 14,493 & 144,467 & 759 & 2,504 & 172,241 & $3,899,718$ & 141 & 481 & 283 & 40 \\
\hline 1993 & 842,153 & 16,845 & 159,059 & 715 & 3,169 & 192,462 & $3,928,653$ & 136 & 485 & 288 & 34 \\
\hline 1994 & 848,796 & 22,365 & 145,225 & 929 & 3,020 & 183,618 & $4,367,148$ & 136 & 498 & 301 & 40 \\
\hline 1995 & 860,594 & 19,615 & 95,507 & 680 & 3,355 & 132,578 & $4,737,871$ & 133 & 480 & 316 & 42 \\
\hline 1996 & 907,209 & 20,252 & 106,055 & 1,712 & 3,322 & 144,626 & $4,312,458$ & 159 & 513 & 324 & 37 \\
\hline 1997 & 931,949 & 20,309 & 118,741 & 237 & 4,086 & 159,715 & $4,564,770$ & 119 & 484 & 339 & 36 \\
\hline 1998 & 946,295 & 25,062 & 172,728 & 549 & 4,860 & 222,640 & $5,081,384$ & 125 & 475 & 332 & 36 \\
\hline 1999 & 949,802 & 25,951 & 158,187 & 974 & 4,552 & 207,871 & $5,321,984$ & 126 & 490 & 332 & 41 \\
\hline 2000 & 994,933 & 31,675 & 143,381 & 1,450 & 3,744 & 195,228 & $5,691,481$ & 126 & 496 & 330 & 46 \\
\hline 2001 & 972,691 & 31,150 & 165,312 & 855 & 3,871 & 216,672 & $5,832,305$ & 97 & 486 & 228 & 160 \\
\hline 2002 & 987,583 & 23,286 & 109,235 & 1,894 & 6,836 & 168,597 & $6,126,062$ & 131 & 605 & 257 & 191 \\
\hline 2003 & $1,014,058$ & 29,672 & 142,518 & 2,947 & 6,303 & 206,653 & $5,616,135$ & 156 & 519 & 249 & 193 \\
\hline 2004 & $1,020,523$ & 20,163 & 142,088 & 2,856 & 7,677 & 203,494 & $5,674,580$ & 135 & 344 & 230 & 183 \\
\hline 2005 & $1,041,448$ & 20,651 & 141,518 & 2,968 & 8,330 & 206,785 & $6,036,370$ & 110 & 355 & 230 & 173 \\
\hline 2006 & $1,030,556$ & 13,174 & 58,473 & 2,174 & 7,363 & 110,634 & $6,461,615$ & 115 & 350 & 241 & 162 \\
\hline 2007 & $1,046,795$ & 15,683 & 63,833 & 2,917 & 6,036 & 112,615 & $7,089,342$ & 115 & 353 & 245 & 168 \\
\hline 2008 & $1,042,335$ & 12,832 & 38,191 & 2,822 & 5,417 & 80,932 & $6,895,843$ & 97 & 339 & 267 & 172 \\
\hline 2009 & 934,683 & 12,658 & 28,576 & 2,328 & 4,821 & 67,668 & $7,121,069$ & 84 & 320 & 272 & 170 \\
\hline 2010 & R979,684 & $\mathrm{R}_{14,050}$ & $\mathrm{R}_{23,997}$ & $R_{2,056}$ & $\mathrm{R}_{4,994}$ & $\mathrm{R}_{65,071}$ & $\mathrm{R}_{7,680,185}$ & R90 & $\mathrm{R}_{350}$ & $\mathrm{R}_{281}$ & $\mathrm{R}_{184}$ \\
\hline $2011^{P}$ & 932.911 & 10,775 & 14,246 & 1,707 & 4.561 & 49.533 & $7,880,481$ & 91 & 333 & 287 & 162 \\
\hline
\end{tabular}

1 Anthracite, bituminous coal, subbituminous coal, lignite, waste coal, and coal synfuel.
2 Fuel oil nos. 1, 2, and 4. For 1949-1979, data are for gas turbine and internal combustion plant use of petroleum. For 1980-2000, electric utility data also include small amounts of kerosene and jet fuel. ${ }^{3}$ Fuel oil nos. 5 and 6. For 1949-1979, data are for steam plant use of petroleum. For 1980-2000, electric utility data also include a small amount of fuel oil no. 4.
4 Jet fuel, kerosene, other petroleum liquids, and waste oil.

5 Petroleum coke is converted from short tons to barrels by multiplying by 5 .

Natural gas, plus a small amount of supplemental gaseous fues.

Blast furnace gas, propane gas, and other manufactured and waste gases derived from fossil fuels.

7ood and wood-derived fuels. ther biomass. Through 2000 , also includes non-renewable waste (municipal solid waste from

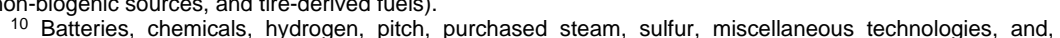

beginning in 2001, non-renewable waste (municipal solid waste from non-biogenic sources, and tire-derived ${ }_{11}$ Through 1988, data are for electric utilities only. Beginning in 1989, data are for electric utilities, independent power producers, commercial plants, and industrial plants.

$R=$ Revised. $P=$ Preliminary. $N A=N o t$ avallable. $(s)=$ Less than 0.5 trillion Btu.

Notes: - Data are for fuels consumed to produce electricity. Data also include fuels consumed to - See Note 1 "Coverage of Electricity Statistics," at end of section. - Totals may not equal sum of components due to independent rounding.

Web Pages: - See http://www.eia.gov/totalenergy/data/monthly/\#electricity for updated monthly and annual data. - See http://www.eia.gov/totalenergy/data/annual/\#electricity for all annual data beginning in

1949. - See $h$ ttp: $: / w w w . e i a . g o v / e l e c t r i c i t y /$ for related information.
Sources: Tables $8.5 \mathrm{~b}$ and $8.5 \mathrm{~d}$. 
Table 8.5b Consumption of Combustible Fuels for Electricity Generation: Electric Power Sector, Selected Years, 1949-2011 (Subset of Table 8.5a)

\begin{tabular}{|c|c|c|c|c|c|c|c|c|c|c|c|}
\hline \multirow[b]{3}{*}{ Year } & \multirow[b]{2}{*}{ Coal ${ }^{1}$} & \multicolumn{5}{|c|}{ Petroleum } & \multirow[b]{2}{*}{ Natural Gas ${ }^{6}$} & \multirow[b]{2}{*}{ Other Gases ${ }^{7}$} & \multicolumn{2}{|c|}{ Biomass } & \multirow[b]{2}{*}{ Other ${ }^{10}$} \\
\hline & & Distillate Fuel Oil ${ }^{2}$ & Residual Fuel Oil $^{3}$ & Other Liquids ${ }^{4}$ & Petroleum Coke ${ }^{5}$ & Total $^{5}$ & & & Wood $^{8}$ & Waste $^{9}$ & \\
\hline & $\begin{array}{l}\text { Thousand } \\
\text { Short Tons }\end{array}$ & \multicolumn{3}{|c|}{ Thousand Barrels } & $\begin{array}{l}\text { Thousand } \\
\text { Short Tons }\end{array}$ & $\begin{array}{c}\text { Thousand } \\
\text { Barrels }\end{array}$ & $\begin{array}{c}\text { Million } \\
\text { Cubic Feet } \\
\end{array}$ & $\begin{array}{c}\text { Trillion } \\
\text { Btu }\end{array}$ & \multicolumn{2}{|c|}{ Trillion Btu } & $\begin{array}{c}\text { Trillion } \\
\text { Btu }\end{array}$ \\
\hline 1949 & 83,963 & 4,767 & 61,534 & NA & NA & 66,301 & 550,121 & NA & 6 & NA & NA \\
\hline $\begin{array}{l}1950 \\
1955\end{array}$ & 91,871 & 5,423 & 69,998 & NA & NA & 75,421 & 628,919 & NA & 5 & NA & NA \\
\hline $\begin{array}{l}1955 \\
1960\end{array}$ & 143,759 & 5,412 & 69,862 & NA & NA & 75,274 & $1,153,280$ & NA & 3 & NA & NA \\
\hline $\begin{array}{l}1960 \\
1965\end{array}$ & 176,685 & 3,824 & 84,371 & NA & NA & 88,195 & $1,724,762$ & NA & 2 & NA & NA \\
\hline $\begin{array}{l}1965 \\
1970\end{array}$ & 244,788 & 4,928 & 110,274 & NA & NA & 115,203 & $2,321,101$ & NA & 3 & NA & NA \\
\hline $\begin{array}{l}1970 \\
1975\end{array}$ & $\begin{array}{l}320,182 \\
405962\end{array}$ & $\begin{array}{l}24,123 \\
38907\end{array}$ & $\begin{array}{l}311,381 \\
467221\end{array}$ & NA & 636 & 338,686 & $\begin{array}{l}3,931,860 \\
3157,669\end{array}$ & NA & 1 & 2 & NA \\
\hline $\begin{array}{l}1975 \\
1976\end{array}$ & $\begin{array}{l}405,962 \\
448,371\end{array}$ & $\begin{array}{l}38,907 \\
41,843\end{array}$ & $\begin{array}{l}467,221 \\
514,077\end{array}$ & $\begin{array}{l}\text { NA } \\
\text { NA }\end{array}$ & $\begin{array}{l}70 \\
68\end{array}$ & $\begin{array}{l}506,479 \\
556,261\end{array}$ & $\begin{array}{l}3,157,669 \\
3,080,868\end{array}$ & $\begin{array}{l}\text { NA } \\
\text { NA }\end{array}$ & (s) & ${ }_{2}^{2}$ & $\begin{array}{l}\text { NA } \\
\text { NA }\end{array}$ \\
\hline 1977 & 477,126 & 48,837 & 574,869 & NA & 98 & 624,193 & $3,191,200$ & NA & 3 & 2 & NA \\
\hline 1978 & 481,235 & 47,520 & 588,319 & NA & 398 & 637,830 & $3,188,363$ & NA & 2 & 1 & NA \\
\hline 1979 & 527,051 & 30,691 & 492,606 & NA & 268 & 524,636 & $3,490,523$ & NA & 3 & 2 & NA \\
\hline 1980 & 569,274 & 29,051 & 391,163 & NA & 179 & 421,110 & $3,681,595$ & NA & 3 & 2 & NA \\
\hline 1981 & 596,797 & 21,313 & 329,798 & NA & 139 & 351,806 & $3,640,154$ & NA & 3 & 1 & NA \\
\hline 1982 & 593,666 & 15,337 & 234,434 & NA & 149 & 250,517 & $3,225,518$ & NA & 2 & 1 & NA \\
\hline 1983 & 625,211 & 16,512 & 228,984 & NA & 261 & 246,804 & $2,910,767$ & NA & 2 & 2 & NA \\
\hline 1984 & 664,399 & 15,190 & 189,289 & NA & 252 & 205,736 & $3,111,342$ & NA & 5 & 4 & NA \\
\hline 1985 & 693,841 & 14,635 & 158,779 & NA & 231 & 174,571 & $3,044,083$ & NA & 8 & 7 & NA \\
\hline 1986 & 685,056 & 14,326 & 216,156 & NA & 313 & 232,046 & $2,602,370$ & NA & 5 & 7 & NA \\
\hline 1987 & 717,894 & 15,367 & 184,011 & NA & 348 & 201,116 & $2,844,051$ & NA & 8 & 7 & NA \\
\hline 1988 & 758,372 & 18,769 & 229,327 & NA & 409 & 250,141 & $2,635,613$ & NA & 10 & 8 & NA \\
\hline 198911 & 771,551 & 26,036 & 242,708 & 9 & 517 & 271,340 & $3,023,513$ & 7 & 75 & 126 & 2 \\
\hline 1990 & 781,301 & 16,394 & 183,285 & 25 & 1,008 & 204,745 & $3,147,289$ & 6 & 106 & 180 & (s) \\
\hline 1991 & 782,653 & 14,255 & 171,629 & 58 & 974 & 190,810 & $3,216,056$ & 6 & 104 & 217 & 4 \\
\hline 1992 & 793,390 & 12,469 & 137,681 & 118 & 1,490 & 157,719 & $3,324,963$ & 12 & 120 & 252 & 3 \\
\hline 1993 & 829,851 & 14,559 & 151,407 & 213 & 2,571 & 179,034 & $3,344,239$ & 12 & 129 & 255 & 3 \\
\hline 1994 & 836,113 & 20,241 & 137,198 & 667 & 2,256 & 169,387 & $3,758,484$ & 12 & 134 & 269 & 2 \\
\hline $\begin{array}{l}1995 \\
1996\end{array}$ & 847,854 & 18,066 & 88,895 & 441 & 2,452 & 119,663 & $4,093,773$ & 18 & 106 & 282 & 2 \\
\hline $\begin{array}{l}1996 \\
1997\end{array}$ & $\begin{array}{l}894,400 \\
919009\end{array}$ & $\begin{array}{l}18,472 \\
18646\end{array}$ & $\begin{array}{r}98,795 \\
112423\end{array}$ & $\begin{array}{l}567 \\
130\end{array}$ & $\begin{array}{l}2,467 \\
3201\end{array}$ & $\begin{array}{l}130,168 \\
147202\end{array}$ & $\begin{array}{r}3,659,810 \\
3903195\end{array}$ & $\begin{array}{l}16 \\
14\end{array}$ & $\begin{array}{l}117 \\
117\end{array}$ & 280 & 2 \\
\hline 1998 & 934,126 & $\begin{array}{r}10,040 \\
23,166\end{array}$ & $\begin{array}{l}165,875 \\
165\end{array}$ & 411 & $\begin{array}{l}3,2019 \\
3,999\end{array}$ & 209,447 & $4,415,813$ & $\begin{array}{l}14 \\
23\end{array}$ & 125 & 287 & $\frac{1}{2}$ \\
\hline 1999 & 937,888 & 23,875 & 151,921 & 514 & 3,607 & 194,345 & $4,643,775$ & 14 & 125 & 290 & 1 \\
\hline 2000 & $\begin{array}{l}93,000 \\
982,713\end{array}$ & $\begin{array}{l}23,722 \\
29,720\end{array}$ & 138,047 & $\begin{array}{l}503 \\
403\end{array}$ & $\begin{array}{l}3,155 \\
3,155\end{array}$ & $\begin{array}{l}183,946 \\
184,045\end{array}$ & $5,014,071$ & $\begin{array}{l}14 \\
19\end{array}$ & 126 & 294 & 1 \\
\hline 2001 & 961,523 & 29,056 & 159,150 & 374 & 3,308 & 205,119 & $5,142,493$ & 9 & 116 & 205 & 109 \\
\hline 2002 & 975,251 & 21,810 & 104,577 & $\begin{array}{r}1,243 \\
\end{array}$ & 5,705 & $\begin{array}{l}156,154 \\
\end{array}$ & $5,408,279$ & 25 & 141 & 224 & 137 \\
\hline 2003 & $1,003,036$ & 27,441 & 137,361 & $\begin{array}{l}1,937 \\
1,937\end{array}$ & 5,719 & $\begin{array}{l}195,336 \\
\end{array}$ & $4,909,248$ & 30 & 156 & 216 & 136 \\
\hline 2004 & $1,012,459$ & 18,793 & 138,831 & 2,511 & 7,135 & $\begin{array}{l}195,809 \\
\end{array}$ & $5,075,339$ & 27 & 150 & 206 & 131 \\
\hline 2005 & $1,033,567$ & 19,450 & $\begin{array}{l}138,337 \\
138,0\end{array}$ & 2,591 & 7,877 & 199,760 & $5,484,780$ & 24 & 166 & 205 & 116 \\
\hline 2006 & $1,022,802$ & 12,578 & 56,347 & 1,783 & 6,905 & 105,235 & $5,891,222$ & 28 & 163 & 216 & 117 \\
\hline 2007 & $1,041,346$ & 15,135 & 62,072 & 2,496 & 5,523 & 107,316 & $6,501,612$ & 27 & 165 & 221 & 117 \\
\hline 2008 & $1,036,891$ & 12,318 & 37,222 & 2,608 & 5,000 & 77,149 & $6,342,331$ & 23 & 159 & 242 & 122 \\
\hline 2009 & 929,692 & 11,848 & 27,768 & 2,110 & 4,485 & 64,151 & $6,566,991$ & 21 & 160 & 244 & 115 \\
\hline 2010 & R971,245 & R13,677 & $R_{23,560}$ & $R_{1,848}$ & R4,679 & $\mathrm{R}^{\mathrm{R}} 62,477$ & $R 7,085,416$ & 20 & R177 & $R_{249}$ & $\mathrm{R}_{116}$ \\
\hline $2011^{P}$ & 924,523 & 10,513 & 13,914 & 1,564 & 4,281 & 47,398 & $7,278,562$ & 20 & 160 & 256 & 117 \\
\hline
\end{tabular}

Anthracite, bituminous coal, subbituminous coal, lignite, waste coal, and coal synfuel. 2 Fuel oil nos. 1,2, and 4. For 1949-1979, data are for gas turbine and internal combustion plant use of petroleum. For 1980-2000, electric utility data also include small amounts of kerosene and jet fuel.
3 Fuel oil nos. 5 and 6 . For $1949-1979$, data are for steam plant electric utility data also include a small amount of fuel oil no. 4.

5 Jet fuel, kerosene, other petroleum liquids, and waste oil.

Natural gas, plus a small amount of supplemental gaseous fuels.

propane gas, and other manufactured and waste gases derived from fossil fuels. 9 Municipal solid waste from biogenic sources, landfill gas, sludge waste, agricultural byproducts, and
other biomass. Through 2000, also includes non-renewable waste (municipal solid waste from non-biogenic sources, and tire-derived fuels).

10 (municipal solid waste from non-biogenic sources, and tire-derived

${ }_{11}$ Through 1988, data are for electric utilities only. Beginning in 1989, data are for electric utilities and

independent power producers.
$R=$ Revised. $P=$ Preliminary. NA=Not available. $(\mathrm{s})=$ Less than 0.5 trillion Btu.

Notes: - Data are for fuels consumed to produce electricity. Data also include fuels consumed to produce useful thermal output at a small number of electric utility combined-heat-and-power (CHP) plants. the NAICS 22 category whose primary business is to sell electricity, or electricity and heat, to the public. - See Table 8.5d for commercial and industrial CHP and electricity-only data. - See Note 1, "Coverage of Electricity Statistics," and Note 2, "Classification of Power Plants Into Energy-Use Sectors," at end of section. - Totals may not equal sum or components due to independent rounding.

annual datas. 1949. - See http://www.eia.gov/electricity/ for related information. Sources: - 1949-September 1977-Federal Power Commission, Form FPC-4, "Monthly Power Plant Report." • October 1977-1981-Federal Energy Regulatory Commission, Form FPC-4, "Monthly Power Pont Power Plant Report." Plant Report," and Form EIA-860B, "Annual Electric Generator Report-Nonutility." • 2001-2003-EIA, Form EIA-906, "Power Plant Report." • 2004-2007-EIA, Form EIA-906, "Power Plant Report," and Form EIA-920, "Combined Heat and Power Plant Report." • 2008 forward-EIA, Form EIA-923, "Power Plan
Operations Report." 
Table 8.5c Consumption of Combustible Fuels for Electricity Generation: Electric Power Sector by Plant Type, Selected Years, 1989-2011 (Breakout of Table 8.5b)

\begin{tabular}{|c|c|c|c|c|c|c|c|c|c|c|c|}
\hline \multirow[b]{3}{*}{ Year } & \multirow[b]{2}{*}{ Coal 1} & \multicolumn{5}{|c|}{ Petroleum } & \multirow[b]{2}{*}{ Natural Gas 6} & \multirow[b]{2}{*}{ Other Gases ${ }^{7}$} & \multicolumn{2}{|c|}{ Biomass } & \multirow[b]{2}{*}{ Other 10} \\
\hline & & Distillate Fuel Oil ${ }^{2}$ & Residual Fuel Oil ${ }^{3}$ & Other Liquids ${ }^{4}$ & Petroleum Coke 5 & Total $^{5}$ & & & Wood $^{8}$ & Waste ${ }^{9}$ & \\
\hline & $\begin{array}{l}\text { Thousand } \\
\text { Short Tons }\end{array}$ & \multicolumn{3}{|c|}{ Thousand Barrels } & $\begin{array}{l}\text { Thousand } \\
\text { Short Tons }\end{array}$ & $\begin{array}{c}\text { Thousand } \\
\text { Barrels }\end{array}$ & $\begin{array}{c}\text { Million } \\
\text { Cubic Feet }\end{array}$ & $\begin{array}{c}\text { Trillion } \\
\text { Btu }\end{array}$ & \multicolumn{2}{|c|}{ Trillion Btu } & $\begin{array}{c}\text { Trillion } \\
\text { Btu }\end{array}$ \\
\hline & \multicolumn{11}{|c|}{ Electricity-Only Plants 11} \\
\hline 1989 & 767,378 & 25,574 & 241,960 & 3 & 517 & 270,125 & $2,790,567$ & - & 59 & 111 & - \\
\hline 1990 & 774,213 & 14,956 & 181,231 & 17 & 1,008 & 201,246 & $2,794,110$ & (s) & 87 & 162 & - \\
\hline 1995 & 832,928 & 16,169 & 86,584 & 133 & 1,082 & 108,297 & $3,287,571$ & (s) & 84 & 262 & - \\
\hline 1996 & 878,825 & 17,361 & 96,386 & 50 & 1,010 & 118,848 & $2,823,724$ & (s) & 94 & 258 & - \\
\hline 1997 & 904,245 & 17,702 & 109,989 & 30 & 1,687 & 136,156 & $3,039,227$ & $\begin{array}{c}101 \\
1\end{array}$ & 91 & 266 & - \\
\hline 1998 & 920,353 & 22,293 & 163,541 & 295 & 2,202 & 197,137 & $3,543,931$ & 1 & 95 & 263 & - \\
\hline 1999 & 924,692 & 22,877 & 149,193 & 380 & 1,891 & 181,905 & $3,729,175$ & 1 & 105 & 264 & - \\
\hline 2000 & 967,080 & 28,001 & 135,419 & 94 & 1,457 & 170,799 & $4,092,729$ & 2 & 105 & 267 & - \\
\hline 2001 & 946,068 & 27,695 & 157,090 & 26 & 1,827 & 193,945 & $4,163,930$ & (s) & 96 & 179 & 98 \\
\hline 2002 & 960,077 & 21,521 & 102,622 & 444 & 3,925 & 144212 & $4.258,467$ & 6 & 118 & 193 & 117 \\
\hline 2003 & 983,538 & 25,951 & 136,050 & 936 & 4.794 & 186,904 & $3,780,314$ & 6 & 127 & 185 & 120 \\
\hline 2004 & 994,774 & 17.944 & 137,736 & 1.441 & $\begin{array}{l}4,096 \\
6.096\end{array}$ & 187,601 & $4,141,535$ & 5 & 134 & 190 & 122 \\
\hline 2005 & $1.015,640$ & $\begin{array}{l}18,684 \\
18,689\end{array}$ & 137,082 & 1,676 & 6,876 & 191827 & 4592271 & (s) & 143 & 189 & 108 \\
\hline 2006 & 1.004 .769 & $\begin{array}{l}10,005 \\
12,375\end{array}$ & 55,192 & 991 & 5,988 & 98,497 & 5.091 .049 & (s) & 141 & 198 & 107 \\
\hline 2007 & $\begin{array}{l}1,004,, 69 \\
1,022,840\end{array}$ & $\begin{array}{l}12,315 \\
14,626\end{array}$ & $\begin{array}{l}55,192 \\
60,929\end{array}$ & $\begin{array}{r}9,709 \\
1,709\end{array}$ & $\begin{array}{l}5,988 \\
4,711\end{array}$ & $\begin{array}{r}98,497 \\
100,818\end{array}$ & $\begin{array}{l}5,091,049 \\
5,611,600\end{array}$ & (S) & $\begin{array}{l}141 \\
142\end{array}$ & $\begin{array}{l}198 \\
203\end{array}$ & $\begin{array}{l}107 \\
107\end{array}$ \\
\hline 2008 & $1.017,806$ & $\begin{array}{l}14,0<0 \\
11,950\end{array}$ & 36,059 & 2,478 & 4.254 & 71,760 & $5,520,491$ & 2 & 136 & 223 & 112 \\
\hline 2009 & $\begin{array}{r}1,011,806 \\
913,566\end{array}$ & $\begin{array}{l}11,950 \\
11,509\end{array}$ & $\begin{array}{l}36,059 \\
26,569\end{array}$ & $\begin{array}{l}2,4 / 8 \\
1,911\end{array}$ & $\begin{array}{l}4,254 \\
3,642\end{array}$ & $\begin{array}{l}71,100 \\
58,197\end{array}$ & $\begin{array}{l}5,520,491 \\
5,750,589\end{array}$ & 2 & $\begin{array}{l}136 \\
133\end{array}$ & $\begin{array}{l}223 \\
222\end{array}$ & $\begin{array}{l}112 \\
105\end{array}$ \\
\hline 2010 & $\begin{array}{r}\text { R954,514 } \\
\text { R95 }\end{array}$ & $\begin{aligned} 11,009 \\
\mathrm{R}_{13}, 337\end{aligned}$ & $\begin{array}{l}20,509 \\
R 22,470\end{array}$ & $\mathrm{R}_{1}, 777$ & $\begin{array}{r}r, 042 \\
\mathrm{R} 4,464\end{array}$ & R59, 902 & $\begin{array}{r}\text { R6, } \\
\text { R60, } 239,466\end{array}$ & $\mathrm{R}_{1}^{2}$ & R153 & R228 & $\begin{array}{l}105 \\
R_{105}\end{array}$ \\
\hline \multirow[t]{2}{*}{$2011^{\mathrm{P}}$} & 909,645 & 10,374 & 12,817 & 1,546 & 4,059 & 45,032 & $6,439,729$ & 1 & 137 & 235 & 107 \\
\hline & \multicolumn{11}{|c|}{ Combined-Heat-and-Power Plants ${ }^{12}$} \\
\hline 1989 & 4,173 & 462 & 747 & 6 & - & 1,215 & 232,946 & 7 & 16 & 16 & 2 \\
\hline 1990 & 7,088 & 1,438 & 2,054 & 7 & - & 3,499 & 353,179 & 6 & 18 & 18 & (s) \\
\hline 1995 & 14,926 & 1,898 & 2,311 & 307 & 1,370 & 11,366 & 806,202 & 18 & 22 & 20 & 2 \\
\hline 1996 & 15,575 & 1,111 & 2,410 & 517 & 1,456 & 11,320 & 836,086 & 15 & 24 & 22 & 2 \\
\hline 1997 & 14,764 & 944 & 2,434 & 100 & 1,514 & 11,046 & 863,968 & 14 & 26 & 26 & 1 \\
\hline 1998 & 13,773 & 872 & 2,334 & 117 & 1,797 & 12,310 & 871,881 & 21 & 30 & 24 & 2 \\
\hline 1999 & 13,197 & 998 & 2,728 & 134 & 1,716 & 12,440 & 914,600 & 14 & 20 & 26 & 1 \\
\hline 2000 & 15,634 & 1,721 & 2,627 & 310 & 1,698 & 13,147 & 921,341 & 17 & 21 & 28 & 1 \\
\hline 2001 & 15,455 & 1,360 & 2,059 & 347 & 1,482 & 11,175 & 978,563 & 9 & 20 & 26 & 11 \\
\hline 2002 & 15,174 & 289 & 1,955 & 800 & 1,780 & 11,942 & $1,149,812$ & 20 & 23 & 30 & 20 \\
\hline 2003 & 19,498 & 1,491 & 1,311 & 1,002 & 926 & 8,431 & $1,128,935$ & 23 & 29 & 31 & 16 \\
\hline 2004 & 17,685 & 850 & 1,095 & 1,070 & 1,039 & 8,209 & 933,804 & 22 & 16 & 16 & 9 \\
\hline 2005 & 17,927 & 760 & 1,254 & 915 & 1,001 & 7,933 & 892,509 & 24 & 22 & 17 & 9 \\
\hline 2006 & 18,033 & 203 & 1,155 & 792 & 918 & 6,738 & 800,173 & 27 & 22 & 18 & 10 \\
\hline 2007 & 18,506 & 509 & 1,144 & 787 & 812 & 6,498 & 890,012 & 25 & 23 & 18 & 9 \\
\hline 2008 & 19,085 & 368 & 1,162 & 130 & 746 & 5,389 & 821,839 & 22 & 23 & 18 & 10 \\
\hline 2009 & 16,126 & 340 & 1,199 & 199 & 843 & 5,953 & 816,402 & 19 & 27 & 22 & 11 \\
\hline 2010 & $\mathrm{R}_{16,731}$ & $\mathrm{R}_{340}$ & 1,090 & R71 & $\mathrm{R}_{215}$ & $R_{2,575}$ & R845,950 & $\mathrm{R}_{19}$ & $\mathrm{R}_{24}$ & $\mathrm{R}_{21}$ & $R_{10}$ \\
\hline $2011^{\mathrm{P}}$ & 14,878 & 139 & 1,097 & 18 & 223 & 2,366 & 838,833 & 19 & 23 & 21 & 10 \\
\hline
\end{tabular}

1 Anthracite, bituminous coal, subbituminous coal, lignite, waste coal, and coal synfuel.
2 Fuel oil nos. 1, 2, and 4. Through 2000, electric utility data also include small amounts of kerosene and jet fuel.

Fuel oil nos. 5 and 6 . Through 2000 , electric utility data also include a small amount of fuel oil no. 4.

4 Jet fuel, kerosene, other petroleum liquids, and waste oil.

Petroleum coke is converted from short tons to barrels by multiplying by 5 .

7 Blast furnace gas, propane gas, and other manufactured and waste gases derived from fossil fuels.

8 Wood and wood-derived fuels.

9 Municipal solid waste from biogenic sources, landfill gas, sludge waste, agricultural byproducts, and other bicmass.

10 Batteries, chemicals, hydrogen, pitch, purchased steam, sulfur, miscellaneous technologies, and,
beginning in 2001, non-renewable waste (municipal solid waste from non-biogenic sources, and tire-derived fuels).

${ }_{11}$ Electricity-only plants within the NAICS 22 category whose primary business is to sell electricity to the public. Data also include a small number of electric utility combined-heat-and-power (CHP) plants. 12 Combined-heat-and-power (CHP) plants within the NAICS 22 category whose primary business is to
sell electricity and heat to the public. Data do not include electric utility CHP plants-these are included

$\mathrm{R}=$ Revised. P=Preliminary. $-=$ No data reported. $(\mathrm{s})=$ Less than 0.5 .
Notes: - Data are for fuels consumed to produce electricity. Data also include fuels consumed to Notes: - Data are for fuels consumed to produce electricity. Data also include fuels consumed to
produce useful thermal output at a small number of electric utility combined-heat-and-power (CHP) plants. - See Table 8.5d for commercial and industrial CHP and electricity-only data. - See Note 1, "Coverage of Electricity Statistics," and Note 2, "Classification of Power Plants Into Energy-Use Sectors," at end of Web Pages: - See http://www.eia.gov/totalenergy/data/annual/\#electricity for all data beginning in 1989 - For related information, see http://www.eia.gov/electricity/. - Sources: - 1989-1997-U.S. Energy Information Administration (EIA), Form EIA-759, "Monthly Power Plant Report," and Form EIA-867, "Annual Nonutility Power Producer Report." • 1998-2000—EIA, Form EIA-759, "Monthly Power Plant Report," and Form EIA-860B, "Annual Electric Generato Report-Nonutility." • 2001-2003-EIA, Form EIA-906, "Power Plant Report." • 2004-2007-EIA, Form EIA-906, "Power Plant Report," and Form EIA-920, "Combined Heat and Power Plant Report." 
Table 8.5d Consumption of Combustible Fuels for Electricity Generation: Commercial and Industrial Sectors, Selected Years, 1989-2011 (Subset of Table 8.5a)

\begin{tabular}{|c|c|c|c|c|c|c|c|c|c|c|c|}
\hline \multirow[b]{3}{*}{ Year } & \multirow[b]{2}{*}{ Coal ${ }^{1}$} & \multicolumn{5}{|c|}{ Petroleum } & \multirow[b]{2}{*}{ Natural Gas ${ }^{6}$} & \multirow[b]{2}{*}{ Other Gases ${ }^{7}$} & \multicolumn{2}{|c|}{ Biomass } & \multirow[b]{2}{*}{ Other ${ }^{10}$} \\
\hline & & Distillate Fuel Oil ${ }^{2}$ & Residual Fuel Oil 3 & Other Liquids 4 & Petroleum Coke 5 & Total $^{5}$ & & & Wood 8 & Waste ${ }^{9}$ & \\
\hline & $\begin{array}{l}\text { Thousand } \\
\text { Short Tons }\end{array}$ & & Thousand Barrels & & $\begin{array}{l}\text { Thousand } \\
\text { Short Tons }\end{array}$ & $\begin{array}{c}\text { Thousand } \\
\text { Barrels }\end{array}$ & $\begin{array}{c}\text { Million } \\
\text { Cubic Feet }\end{array}$ & $\begin{array}{c}\text { Trillion } \\
\text { Btu }\end{array}$ & \multicolumn{2}{|c|}{ Trillion Btu } & $\begin{array}{c}\text { Trillion } \\
\text { Btu }\end{array}$ \\
\hline
\end{tabular}

\begin{tabular}{|c|c|c|c|c|c|c|c|c|c|c|c|}
\hline \multirow{3}{*}{$\begin{array}{l}1989 \\
1990\end{array}$} & \multicolumn{11}{|c|}{ Commercial Sector 11} \\
\hline & 414 & 882 & 282 & - & - & 1,165 & 17,987 & 1 & 2 & 9 & - \\
\hline & 417 & 580 & 372 & (s) & - & 953 & 27,544 & 1 & 2 & 15 & - \\
\hline 1995 & 569 & 493 & 152 & (s) & 1 & 649 & 42,700 & - & 1 & 21 & (s) \\
\hline 1996 & 656 & 422 & 218 & (s) & 1 & 645 & 42,380 & (s) & 1 & 31 & (s) \\
\hline 1997 & 630 & 583 & 200 & - & 1 & 790 & 38,975 & (s) & 1 & 34 & (s) \\
\hline 1998 & 440 & 436 & 359 & - & 1 & 802 & 40,693 & (s) & 1 & 32 & (1) \\
\hline 1999 & 481 & 506 & 421 & - & 1 & 931 & 39,045 & (s) & (s) & 33 & (s) \\
\hline 2000 & 514 & 505 & 310 & $\overline{1}$ & 1 & 823 & 37,029 & (s) & $\begin{array}{l}\text { (s) } \\
\text { (s) }\end{array}$ & 26 & $\begin{array}{l}\text { (s) } \\
\text { (s) }\end{array}$ \\
\hline 2001 & 532 & 520 & 469 & 2 & 6 & 1,023 & 36,248 & (s) & (s) & 15 & 7 \\
\hline 2002 & 477 & 524 & 292 & 10 & 2 & 834 & 32,545 & (s) & (s) & 18 & 11 \\
\hline 2003 & 582 & 553 & 326 & 3 & 2 & 894 & 38,480 & - & (s) & 19 & 11 \\
\hline 2004 & 377 & 545 & 214 & 1 & 1 & 766 & 32,839 & - & (s) & 19 & 11 \\
\hline 2005 & 377 & 377 & 201 & 1 & 1 & 585 & 33,785 & - & (s) & 20 & 10 \\
\hline 2006 & 347 & 211 & 116 & (s) & 1 & 333 & 34,623 & - & (s) & 21 & 10 \\
\hline 2007 & 361 & 156 & 94 & - & 2 & 258 & 34,087 & - & (s) & 19 & 10 \\
\hline 2008 & 369 & 131 & 29 & (s) & $\overline{1}$ & 166 & 33,403 & - & (s) & 20 & 11 \\
\hline 2009 & 317 & 145 & 39 & (s) & 1 & 190 & 34,279 & - & (s) & 23 & 13 \\
\hline 2010 & $R_{314}$ & $R_{143}$ & $\mathrm{R}_{21}$ & (s) & 2 & $\mathrm{R}_{172}$ & $\mathrm{R}_{39,462}$ & ${ }^{\mathrm{R}}(\mathrm{s})$ & (s) & $R_{24}$ & $\mathrm{R}_{14}$ \\
\hline \multirow[t]{2}{*}{$2011^{\mathrm{P}}$} & 297 & 94 & 15 & (s) & 1 & 112 & 37,773 & (s) & (s) & 24 & 14 \\
\hline & \multicolumn{11}{|c|}{ Industrial Sector ${ }^{12}$} \\
\hline 1989 & 9,707 & 815 & 6,624 & 294 & 150 & 8,482 & 443,928 & 83 & 267 & 15 & 37 \\
\hline 1990 & 10,740 & 1,169 & 6,995 & 412 & 905 & 13,103 & 516,729 & 104 & 335 & 16 & 36 \\
\hline 1995 & 12,171 & 1,056 & 6,460 & 239 & 902 & 12,265 & 601,397 & 114 & 373 & 13 & 40 \\
\hline 1996 & 12,153 & 1,359 & 7,042 & 1,145 & 853 & 13,813 & 610,268 & 143 & 394 & 13 & 35 \\
\hline 1997 & 12,311 & 1,079 & 6,118 & 107 & 884 & 11,723 & 622,599 & 105 & 367 & 14 & 36 \\
\hline 1998 & 11,728 & 1,461 & 6,494 & 137 & 860 & 12,392 & 624,878 & 102 & 349 & 13 & 35 \\
\hline 1999 & 11,432 & 1,571 & 5,845 & 460 & 944 & 12,595 & 639,165 & 112 & 364 & 8 & 39 \\
\hline 2000 & 11,706 & 1,448 & 5,024 & 1,046 & 588 & 10,459 & 640,381 & 107 & 369 & 10 & 45 \\
\hline 2001 & 10,636 & 1,574 & 5,693 & 479 & 557 & 10,530 & 653,565 & 88 & 370 & 7 & 44 \\
\hline 2002 & 11,855 & 952 & 4,366 & 640 & 1,130 & 11,608 & 685,239 & 106 & 464 & 15 & 43 \\
\hline 2003 & 10,440 & $\begin{array}{r}1,678 \\
\end{array}$ & 4,831 & $\begin{array}{r}0,006 \\
\end{array}$ & 582 & 10,424 & 668,407 & 127 & $\begin{array}{l}404 \\
362\end{array}$ & 13 & 46 \\
\hline 2004 & 7,687 & 825 & 3,043 & 344 & 541 & 6,919 & 566,401 & 108 & 194 & 5 & 41 \\
\hline 2005 & 7,504 & 824 & 2,980 & 377 & 452 & 6,440 & 517,805 & 85 & 189 & 5 & 46 \\
\hline 2006 & 7,408 & 385 & 2,010 & 391 & 456 & 5,066 & 535,770 & 87 & 187 & 3 & 35 \\
\hline 2007 & 5,089 & 392 & 1,666 & 421 & 512 & 5,041 & 553,643 & 88 & 188 & 4 & 41 \\
\hline 2008 & 5,075 & 383 & 941 & 214 & 416 & 3,617 & 520,109 & 73 & 179 & 5 & 39 \\
\hline 2009 & 4,674 & 664 & 769 & 218 & 335 & 3,328 & 519,799 & 62 & 160 & 4 & 42 \\
\hline 2010 & $R_{8,125}$ & $\mathrm{R}_{231}$ & R416 & $\mathrm{R}_{208}$ & $R_{313}$ & $R_{2,422}$ & R555,307 & R70 & $R_{172}$ & $R_{8}$ & R55 \\
\hline $2011^{P}$ & 8,091 & 168 & 318 & 144 & 279 & 2,023 & 564,146 & 71 & 173 & 8 & 31 \\
\hline
\end{tabular}

1 Anthracite, bituminous coal, subbituminous coal, lignite, waste coal, and coal synfuel. 2 Fuel oil nos. 1,2 , and 4 .

4 Jet fuel, kerosene, other petroleum liquids, and waste oil.

${ }_{6}^{5}$ Petroleum coke is converted from short tons to barrels by multiplying by 5 .

Natural gas, plus a small amount of supplemental gaseous fuels.

7 Blast furnace gas, propane gas, and other manufactured and waste gases derived from fossil fuels.

9 Municipal solid waste from biogenic sources, landfill gas, sludge waste agricultural byproducts, and other biomass. Through 2000, also includes non-renewable waste (municipal solid waste from non-biogenic sources, and tire-derived fuels).

${ }^{10}$ Batteries, chemicals, hydrogen, pitch, purchased steam, sulfur, miscellaneous technologies, and beginning in 2001, non-renewable waste (municipal solid waste from non-biogenic sources, and tire-derived fuels).

Commercial combined-heat-and-power (CHP) and commercial electricity-only plants.

12 Industrial combined-heat-and-power (CHP) and industrial electricity-only plants.

Notes: - Data are for fuels consumed to produce electricity. See Tables $8.5 \mathrm{~b}$ and $8.5 \mathrm{c}$ for electric power sector electricity-only and CHP data. - See Note 1, "Coverage of Electricity Statistics," and Note 2 , "Classification of Power Plants Into Energy-Use Sectors," at end of section. • Totals may not equal sum of components due to independent rounding.

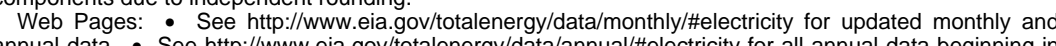
1989. See htrp//www eia gov/electricity/ for rgatdata/annual/\#electricity for all annual data beginning in Sources: • 1989-1997-U.S. Energy Information Administration (EIA), Form EIA-867, "Annual Nonutility Power Producer Report." - 1998-2000-EIA, Form EIA-860B, "Annual Electric Generato Report-Nonutility." • 2001-2003-EIA, Form EIA-906, "Power Plant Report. • 2004-2007_EIA, Form - 2008 forward-EIA, Form EIA-923, "Power Plant Operations Report." 
Figure 8.6 Estimated Consumption of Combustible Fuels for Useful Thermal Output at Combined-Heat-and-Power Plants by Sector, 1989-2011

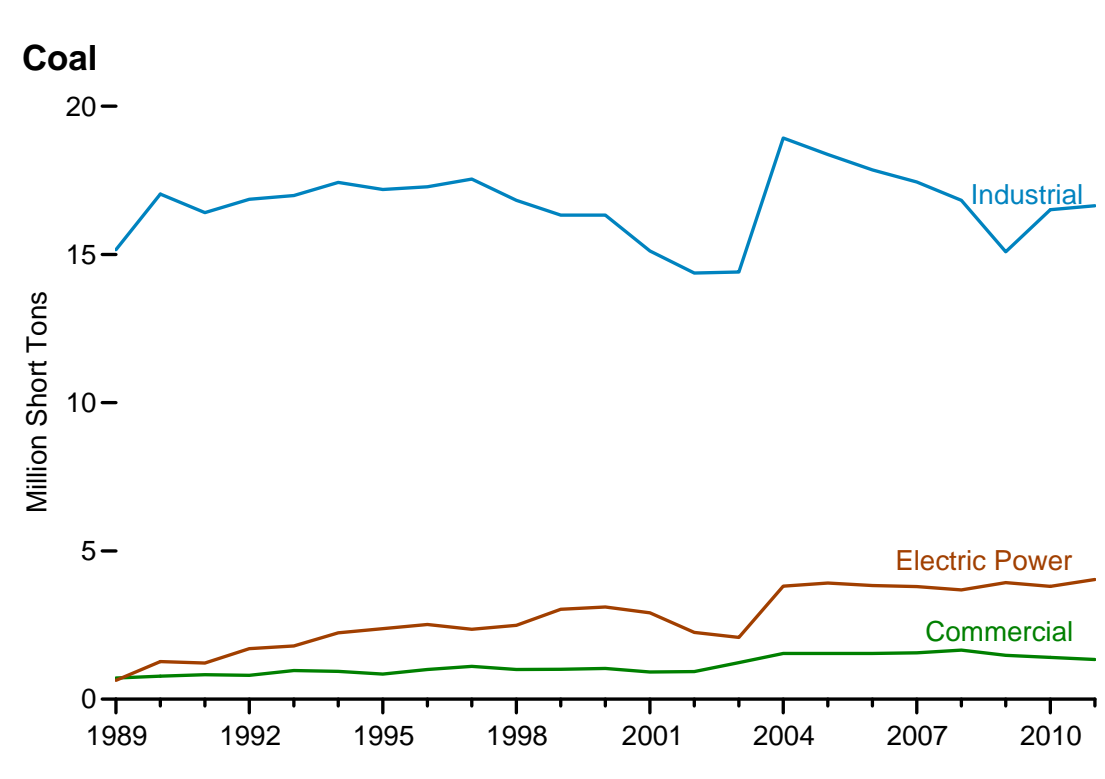

Natural Gas

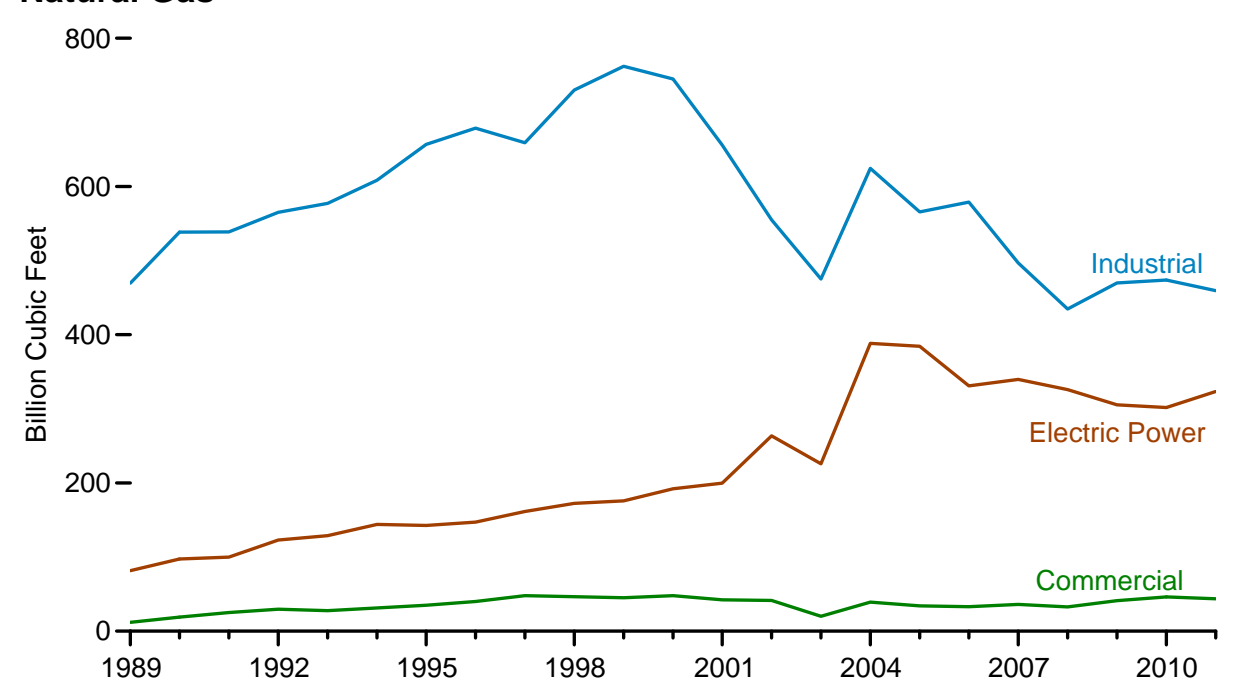

\section{Petroleum}

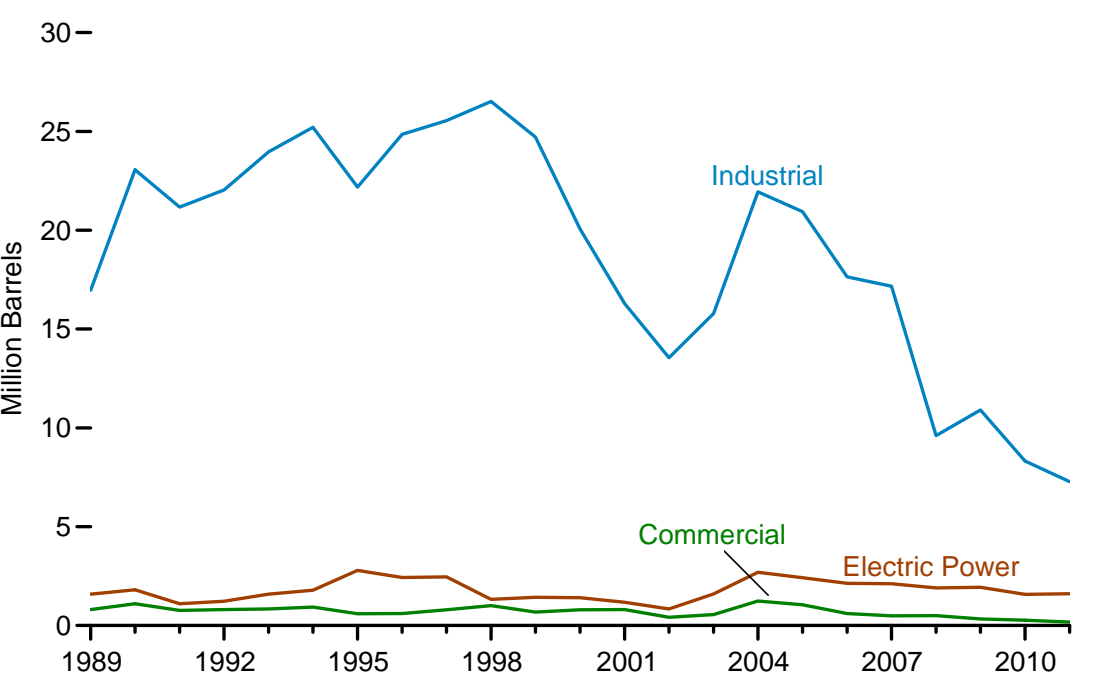

\section{Wood and Waste}

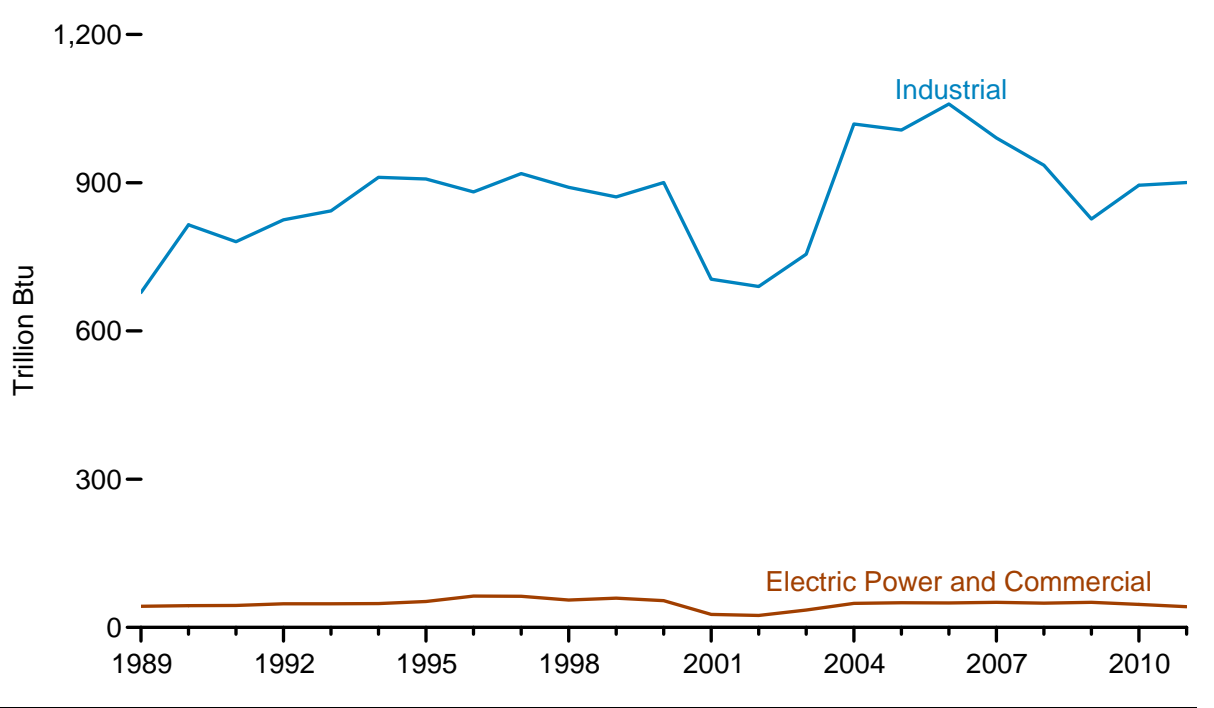

Sources: Tables 8.6b and 8.6c. 
Table 8.6a Estimated Consumption of Combustible Fuels for Useful Thermal Output

at Combined-Heat-and-Power Plants: Total (All Sectors), 1989-2011 (Sum of Tables 8.6b and 8.6c)

\begin{tabular}{|c|c|c|c|c|c|c|c|c|c|c|c|}
\hline \multirow[b]{3}{*}{ Year } & \multirow{3}{*}{$\begin{array}{c}\text { Coal }^{1} \\
\text { Thousand } \\
\text { Short Tons }\end{array}$} & \multicolumn{5}{|c|}{ Petroleum } & \multirow{3}{*}{$\begin{array}{c}\text { Natural Gas }{ }^{6} \\
\text { Million } \\
\text { Cubic Feet }\end{array}$} & \multirow{3}{*}{$\begin{array}{c}\text { Other Gases }^{7} \\
\text { Trillion } \\
\text { Btu }\end{array}$} & \multicolumn{2}{|c|}{ Biomass } & \multirow[b]{2}{*}{ Other 10} \\
\hline & & Distillate Fuel Oil 2 & Residual Fuel Oil 3 & Other Liquids ${ }^{4}$ & Petroleum Coke 5 & Total ${ }^{5}$ & & & Wood $^{8}$ & Waste $^{9}$ & \\
\hline & & \multicolumn{3}{|c|}{ Thousand Barrels } & $\begin{array}{l}\text { Thousand } \\
\text { Short Tons }\end{array}$ & $\begin{array}{c}\text { Thousand } \\
\text { Barrels }\end{array}$ & & & \multicolumn{2}{|c|}{ Trillion Btu } & $\begin{array}{c}\text { Trillion } \\
\text { Btu }\end{array}$ \\
\hline 1989 & 16,510 & 1,410 & 16,357 & 353 & 247 & 19,357 & 563,307 & 116 & 683 & 38 & 49 \\
\hline 1990 & 19,081 & 2,050 & 18,428 & 895 & 918 & 25,965 & 654,749 & 176 & 813 & 46 & 50 \\
\hline 1991 & 18,458 & 3,027 & 15,293 & 835 & 777 & 23,039 & 663,963 & 185 & 779 & 46 & 55 \\
\hline 1992 & 19,372 & 2,358 & 16,474 & 935 & 862 & 24,077 & 717,860 & 200 & 822 & 51 & 52 \\
\hline 1993 & 19,750 & 2,449 & 17,933 & 857 & 1,031 & 26,394 & 733,584 & 178 & 836 & 56 & 51 \\
\hline 1994 & 20,609 & 2,811 & 18,822 & 609 & 1,137 & 27,929 & 784,015 & 180 & 903 & 57 & 53 \\
\hline 1995 & 20,418 & 2,082 & 16,661 & 642 & 1,235 & 25,562 & 834,382 & 181 & 902 & 59 & 55 \\
\hline 1996 & 20,806 & 2,192 & 18,552 & 756 & 1,275 & 27,873 & 865,774 & 187 & 876 & 69 & 54 \\
\hline 1997 & 21,005 & 2,584 & 15,882 & 289 & 2,009 & 28,802 & 868,569 & 188 & 913 & 68 & 67 \\
\hline 1998 & 20,320 & 4,944 & 16,539 & 681 & 1,336 & 28,845 & 949,106 & 209 & 875 & 72 & 58 \\
\hline 1999 & 20,373 & 4,665 & 14,133 & 838 & 1,437 & 26,822 & 982,958 & 224 & 862 & 68 & 60 \\
\hline 2000 & 20,466 & 2,897 & 13,292 & 1,455 & 924 & 22,266 & 985,263 & 230 & 884 & 71 & 63 \\
\hline 2001 & 18,944 & 2,574 & 11,826 & 563 & 661 & 18,268 & 898,286 & 166 & 696 & 35 & 69 \\
\hline 2002 & 17,561 & 1,462 & 9,402 & 1,363 & 517 & 14,811 & 860,019 & 147 & 682 & 32 & 60 \\
\hline 2003 & 17,720 & 2,153 & 10,341 & 1,629 & 763 & 17,939 & 721,267 & 138 & 746 & 44 & 69 \\
\hline 2004 & 24,275 & 3,357 & 15,390 & 1,908 & 1,043 & 25,870 & $1,052,100$ & 218 & 1,016 & 51 & 70 \\
\hline 2005 & 23,833 & 3,795 & 15,397 & 1,302 & 783 & 24,408 & 984,340 & 238 & 997 & 59 & 64 \\
\hline 2006 & 23,227 & 1,481 & 11,373 & 1,222 & 1,259 & 20,371 & 942,817 & 226 & 1,049 & 60 & 75 \\
\hline 2007 & 22,810 & 1,359 & 10,783 & 1,320 & 1,262 & 19,775 & 872,579 & 214 & 982 & 59 & 71 \\
\hline 2008 & 22,168 & 1,305 & 5,285 & 943 & 897 & 12,016 & 793,537 & 203 & 924 & 61 & 39 \\
\hline 2009 & 20,507 & 2,142 & 5,097 & 890 & 1,007 & 13,161 & 816,787 & 176 & 816 & 61 & 58 \\
\hline 2010 & $\mathrm{R}_{21,727}$ & $\mathrm{R}_{1,197}$ & $\mathrm{R}_{2,947}$ & R722 & $R_{1,059}$ & $\mathrm{R}_{10,161}$ & R821,775 & 172 & R876 & ${ }^{R} 66$ & R52 \\
\hline $2011^{P}$ & 22,014 & 599 & 2,432 & 495 & 1,105 & 9,054 & 826,548 & 190 & 881 & 62 & 27 \\
\hline
\end{tabular}

1 Anthracite, bituminous coal, subbituminous coal, lignite, waste coal, and coal synfuel.

Fuel oil nos. 1, 2, and 4 .

3 Fuel oil nos. 5 and 6 .

4 Jet fuel, kerosene, other petroleum liquids, and waste oil.

Petroleum coke is converted from short tons to barrels by multiplying by 5 .

Natural gas, plus a small amount of supplemental gaseous fuels.

7 Blast furnace gas, propane gas, and other manufactured and waste gases derived from fossil fuels.

8 Wood and wood-derived fuels.

Municipal solid waste from biogenic sources, landfill gas, sludge waste, agricultural byproducts, and other biomass. Through 2000, also includes non-renewable waste (municipal solid waste from non-biogenic sources, and tire-derived fuels).

10 Batteries, chemicals, hydrogen, pitch, purchased steam, sulfur, miscellaneous technologies, and, beginning in 2001, non-renewable waste (municipal solid waste from non-biogenic sources, and tire-derived fuels)

$\mathrm{R}=$ Revised. P=Preliminary.

Notes: - Estimates are for fuels consumed to produce useful thermal output; they exclude fuels consumed to produce electricity. - Estimates do not include electric utility combined-heat-and-power (CHP) plants. • See Note 1, "Coverage of Electricity Statistics," at end of section. • See "Useful Thermal Output" in Glossary. - Totals may not equal sum of components due to independent rounding.

Web Page: For related information, see http://www.eia.gov/electricity/.

Sources: Tables $8.6 \mathrm{~b}$ and $8.6 \mathrm{c}$. 
Table 8.6b Estimated Consumption of Combustible Fuels for Useful Thermal Output

at Combined-Heat-and-Power Plants: Electric Power Sector, 1989-2011 (Subset of Table 8.6a)

\begin{tabular}{|c|c|c|c|c|c|c|c|c|c|c|c|}
\hline \multirow[b]{3}{*}{ Year } & \multirow{3}{*}{$\begin{array}{c}\text { Coal }^{1} \\
\text { Thousand } \\
\text { Short Tons }\end{array}$} & \multicolumn{5}{|c|}{ Petroleum } & \multirow[b]{2}{*}{ Natural Gas ${ }^{6}$} & \multirow[b]{2}{*}{ Other Gases ${ }^{7}$} & \multicolumn{2}{|c|}{ Biomass } & \multirow[b]{2}{*}{ Other ${ }^{10}$} \\
\hline & & Distillate Fuel Oil ${ }^{2}$ & Residual Fuel Oil $^{3}$ & Other Liquids ${ }^{4}$ & Petroleum Coke 5 & Total $^{5}$ & & & Wood 8 & Waste ${ }^{9}$ & \\
\hline & & \multicolumn{3}{|c|}{ Thousand Barrels } & $\begin{array}{l}\text { Thousand } \\
\text { Short Tons }\end{array}$ & $\begin{array}{c}\text { Thousand } \\
\text { Barrels }\end{array}$ & $\begin{array}{c}\text { Million } \\
\text { Cubic Feet }\end{array}$ & $\begin{array}{c}\text { Trillion } \\
\text { Btu }\end{array}$ & \multicolumn{2}{|c|}{ Trillion Btu } & $\begin{array}{c}\text { Trillion } \\
\text { Btu }\end{array}$ \\
\hline 1989 & 639 & 120 & 1,471 & 1 & - & 1,591 & 81,670 & 3 & 24 & 6 & 1 \\
\hline 1990 & 1,266 & 173 & 1,630 & 2 & - & 1,805 & 97,330 & 5 & 23 & 8 & (s) \\
\hline 1991 & 1,221 & 104 & 995 & 1 & - & 1,101 & 99,868 & 5 & 21 & 11 & 1 \\
\hline 1992 & 1,704 & 154 & 1,045 & 10 & 4 & 1,229 & 122,908 & 6 & 21 & 10 & 2 \\
\hline 1993 & 1,794 & 290 & 1,074 & 27 & 40 & 1,591 & 128,743 & 4 & 21 & 10 & 2 \\
\hline 1994 & 2,241 & 371 & 1,024 & 104 & 58 & 1,791 & 144,062 & 6 & 18 & 12 & 1 \\
\hline 1995 & 2,376 & 486 & 1,127 & 58 & 222 & 2,784 & 142,753 & 5 & 19 & 15 & (s) \\
\hline 1996 & 2,520 & 308 & 1,155 & 86 & 175 & 2,424 & 147,091 & 5 & 20 & 21 & (s) \\
\hline 1997 & 2,355 & 343 & 1,246 & 23 & 171 & 2,466 & 161,608 & 10 & 20 & 17 & (s) \\
\hline 1998 & 2,493 & 134 & 653 & 19 & 103 & 1,322 & 172,471 & 6 & 12 & 20 & (s) \\
\hline 1999 & 3,033 & 183 & 572 & 30 & 128 & 1,423 & 175,757 & 4 & 13 & 25 & (s) \\
\hline 2000 & 3,107 & 294 & 467 & 51 & 120 & 1,412 & 192,253 & 7 & 8 & 24 & (s) \\
\hline 2001 & 2,910 & 219 & 355 & 3 & 119 & 1,171 & 199,808 & 6 & 10 & 5 & 4 \\
\hline 2002 & 2,255 & 66 & 197 & 23 & 111 & 841 & 263,619 & 7 & 10 & 6 & 6 \\
\hline 2003 & 2,080 & 190 & 919 & 88 & 80 & 1,596 & 225,967 & 12 & 11 & 14 & 4 \\
\hline 2004 & 3,809 & 314 & 985 & 202 & 237 & 2,688 & 388,424 & 31 & 15 & 17 & 7 \\
\hline 2005 & 3,918 & 225 & 1,072 & 95 & 206 & 2,424 & 384,365 & 60 & 19 & 15 & 7 \\
\hline 2006 & 3,834 & 69 & 998 & 87 & 195 & 2,129 & 330,878 & 37 & 19 & 14 & 8 \\
\hline 2007 & 3,795 & 192 & 1,014 & 98 & 162 & 2,114 & 339,796 & 34 & 21 & 16 & 8 \\
\hline 2008 & 3,689 & 230 & 1,019 & 62 & 119 & 1,907 & 326,048 & 38 & 18 & 16 & 8 \\
\hline 2009 & 3,935 & 187 & 1,015 & 100 & 126 & 1,930 & 305,542 & 34 & 20 & 17 & 8 \\
\hline 2010 & $R_{3,808}$ & $R_{113}$ & 944 & $\mathrm{R}_{29}$ & R98 & $R_{1}, 578$ & $R_{301,769}$ & R33 & $R_{18}$ & $R_{15}$ & R8 \\
\hline $2011^{P}$ & 4,035 & 73 & 963 & 4 & 113 & 1,605 & 323,364 & 36 & 16 & 13 & 9 \\
\hline
\end{tabular}

1 Anthracite, bituminous coal, subbituminous coal, lignite, waste coal, and coal synfuel.

Fuel oil nos. 1, 2, and 4

3 Fuel oil nos. 5 and 6 .

4 Jet fuel, kerosene, other petroleum liquids, and waste oil.

Petroleum coke is converted from short tons to barrels by multiplying by 5 .

Natural gas, plus a small amount of supplemental gaseous fuels.

7 Blast furnace gas, propane gas, and other manufactured and waste gases derived from fossil fuels.

8 Wood and wood-derived fuels.

Municipal solid waste from biogenic sources, landfill gas, sludge waste, agricultural byproducts, and other biomass. Through 2000, also includes non-renewable waste (municipal solid waste from

non-biogenic sources, and tire-derived fuels).

10 Batteries, chemicals, hydrogen, pitch, purchased steam, sulfur, miscellaneous technologies, and, beginning in 2001, non-renewable waste (municipal solid waste from non-biogenic sources, and tire-derived fuels).

R=Revised. P=Preliminary. $-=$ No data reported. $(\mathrm{s})=$ Less than 0.5 .

Notes: - Estimates are for fuels consumed to produce useful thermal output; they exclude fuels consumed to produce electricity. - Estimates are for combined-heat-and-power (CHP) plants within the NAICS 22 category whose primary business is to sell electricity and heat to the public. Estimates do not include electric utility CHP plants. - See Table 8.6c for commercial and industrial CHP data. - See Note 1, "Coverage of Electricity Statistics," and Note 2, "Classification of Power Plants Into Energy-Use Sectors," at end of section. - See "Useful Thermal Output" in Glossary. • Totals may not equal sum of components due to independent rounding.

Web Page: For related information, see http://www.eia.gov/electricity/.

Sources: • 1989-1997-U.S. Energy Information Administration (EIA), Form EIA-867, "Annual Nonutility Power Producer Report." • 1998-2000-EIA, Form EIA-860B, "Annual Electric Generator Report-Nonutility." • 2001-2003—EIA, Form EIA-906, "Power Plant Report." • 2004-2007—EIA, Form EIA-920, "Combined Heat and Power Plant Report." • 2008 forward-EIA, Form EIA-923, "Power Plant Operations Report." 
Table 8.6c Estimated Consumption of Combustible Fuels for Useful Thermal Output at Combined-Heat-and-Power

Plants: Commercial and Industrial Sectors, Selected Years, 1989-2011 (Subset of Table 8.6a)

\begin{tabular}{|c|c|c|c|c|c|c|c|c|c|c|c|}
\hline & \multirow[b]{2}{*}{ Coal 1} & \multicolumn{5}{|c|}{ Petroleum } & \multirow[b]{2}{*}{ Natural Gas ${ }^{6}$} & \multirow[b]{2}{*}{ Other Gases ${ }^{7}$} & \multicolumn{2}{|c|}{ Biomass } & \multirow[b]{2}{*}{ Other 10} \\
\hline & & Distillate Fuel Oil 2 & Residual Fuel Oil ${ }^{3}$ & Other Liquids ${ }^{4}$ & Petroleum Coke 5 & Total 5 & & & Wood 8 & Waste ${ }^{9}$ & \\
\hline Year & $\begin{array}{l}\text { Thousand } \\
\text { Short Tons }\end{array}$ & & Thousand Barrels & & $\begin{array}{l}\text { Thousand } \\
\text { Short Tons }\end{array}$ & $\begin{array}{c}\text { Thousand } \\
\text { Barrels }\end{array}$ & $\begin{array}{c}\text { Million } \\
\text { Cubic Feet }\end{array}$ & $\begin{array}{c}\text { Trillion } \\
\text { Btu }\end{array}$ & \multicolumn{2}{|c|}{ Trillion Btu } & $\begin{array}{c}\text { Trillion } \\
\text { Btu }\end{array}$ \\
\hline
\end{tabular}

Commercial Sector 11

\begin{tabular}{|c|c|c|c|c|c|c|c|c|c|c|c|}
\hline $\begin{array}{l}1989 \\
1990\end{array}$ & $\begin{array}{l}711 \\
773\end{array}$ & $\begin{array}{l}202 \\
389\end{array}$ & $\begin{array}{l}601 \\
715\end{array}$ & $\overline{(s)}$ & - & $\begin{array}{r}803 \\
1104\end{array}$ & $\begin{array}{l}12,049 \\
18913\end{array}$ & (s) & $\begin{array}{l}\text { (s) } \\
\text { (s) }\end{array}$ & $\begin{array}{l}13 \\
13\end{array}$ & - \\
\hline 1995 & 850 & 319 & 261 & (s) & $\overline{3}$ & $\begin{array}{r}1,104 \\
596\end{array}$ & $\begin{array}{r}18,913 \\
34,964\end{array}$ & (s) & $\begin{array}{l}\text { (s) } \\
\text { (s) }\end{array}$ & 19 & $\overline{(s)}$ \\
\hline 1996 & $\begin{array}{r}1,005 \\
\end{array}$ & 260 & 328 & (s) & $\begin{array}{l}5 \\
3\end{array}$ & 601 & $\begin{array}{l}34,004 \\
40,075\end{array}$ & $\overline{-}$ & (I) & 22 & $\begin{array}{l}\text { (S) } \\
\text { (s) }\end{array}$ \\
\hline 1997 & 1,108 & 470 & 309 & - (1) & 3 & 794 & 47,941 & (s) & 1 & 24 & - \\
\hline 1998 & 1,002 & 418 & 573 & - & 3 & 1,006 & 46,527 & (s) & 1 & 22 & - \\
\hline 1999 & 1,009 & 254 & 412 & - & 3 & 682 & 44,991 & - & 1 & 21 & - \\
\hline 2000 & 1,034 & 403 & 366 & 2 & 4 & 792 & 47,844 & - & 1 & 21 & - \\
\hline 2001 & 916 & 505 & 304 & - & - & 809 & 42,407 & - & 1 & 10 & 7 \\
\hline 2002 & 929 & 248 & 108 & 28 & 6 & 416 & 41,430 & - & 1 & 8 & 6 \\
\hline 2003 & 1,234 & 119 & 381 & 12 & 9 & 555 & 19,973 & - & 1 & 10 & 8 \\
\hline 2004 & 1,540 & 570 & 613 & 20 & 8 & 1,243 & 39,233 & - & 1 & 15 & 11 \\
\hline 2005 & 1,544 & 417 & 587 & (s) & 8 & 1,045 & 34,172 & - & 1 & 14 & 10 \\
\hline 2006 & $\begin{array}{l}1,544 \\
1,539\end{array}$ & 155 & 404 & (s) & $\begin{array}{l}8 \\
9\end{array}$ & $\begin{array}{r}1,045 \\
601\end{array}$ & $\begin{array}{l}34,112 \\
33,112\end{array}$ & $(\bar{s})$ & 1 & $\begin{array}{l}14 \\
16\end{array}$ & 10 \\
\hline 2007 & 1,566 & 101 & 340 & - & 11 & 494 & 35,987 & - & 2 & 12 & 7 \\
\hline 2008 & 1,652 & 287 & 173 & - & 9 & 504 & 32,813 & - & 1 & 14 & 10 \\
\hline 2009 & $\begin{array}{l}1,481 \\
\end{array}$ & 120 & 173 & - & 8 & 331 & 41,275 & - & 1 & 13 & 9 \\
\hline 2010 & $\mathrm{R}_{1,406}$ & R90 & $R_{122}$ & - & 11 & $R_{265}$ & $\mathrm{R}_{46,324}$ & ${ }^{R}(s)$ & 1 & 12 & 8 \\
\hline $2011^{\mathrm{P}}$ & 1,336 & 53 & 88 & - & 6 & 169 & 43,661 & (s) & 1 & 12 & 9 \\
\hline
\end{tabular}

\begin{tabular}{|c|c|c|c|c|c|c|c|c|c|c|c|}
\hline \multirow{3}{*}{$\begin{array}{l}1989 \\
1990\end{array}$} & \multicolumn{11}{|c|}{ Industrial Sector ${ }^{12}$} \\
\hline & 15,160 & 1,088 & 14,285 & 352 & 247 & 16,963 & 469,588 & 113 & 659 & 19 & 48 \\
\hline & 17,041 & 1,488 & 16,084 & 893 & 918 & 23,056 & 538,506 & 171 & 790 & 25 & 50 \\
\hline 1995 & 17,192 & 1,277 & 15,272 & 584 & 1,010 & 22,182 & 656,665 & 175 & 882 & 25 & 55 \\
\hline 1996 & 17,281 & 1,624 & 17,069 & 670 & 1,097 & 24,848 & 678,608 & 182 & 855 & 26 & 53 \\
\hline 1997 & 17,542 & 1,772 & 14,328 & 267 & 1,835 & 25,541 & 659,021 & 178 & 892 & 27 & 67 \\
\hline 1998 & 16,824 & 4,391 & 15,313 & 662 & 1,230 & 26,518 & 730,108 & 202 & 862 & 29 & 58 \\
\hline 1999 & 16,330 & 4,228 & 13,148 & 808 & 1,307 & 24,718 & 762,210 & 219 & 849 & 23 & 60 \\
\hline 2000 & 16,325 & 2,200 & 12,459 & 1,402 & 800 & 20,062 & 745,165 & 223 & 875 & 25 & 63 \\
\hline 2001 & 15,119 & 1,850 & 11,167 & 560 & 542 & 16,287 & 656,071 & 160 & 685 & 20 & 58 \\
\hline 2002 & 14,377 & 1,149 & 9,097 & 1,312 & 399 & 13,555 & 554,970 & 139 & 672 & 18 & 48 \\
\hline 2003 & 14,406 & 1,844 & 9,041 & 1,529 & 675 & 15,788 & 475,327 & 126 & 735 & 21 & 57 \\
\hline 2004 & 18,926 & 2,473 & 13,791 & 1,686 & 798 & 21,939 & 624,443 & 187 & 1,000 & 19 & 53 \\
\hline 2005 & 18,371 & 3,153 & 13,738 & 1,207 & 568 & 20,940 & 565,803 & 179 & 977 & 30 & 48 \\
\hline 2006 & 17,854 & 1,258 & 9,971 & 1,136 & 1,055 & 17,640 & 578,828 & 190 & 1,029 & 30 & 57 \\
\hline 2007 & 17,449 & 1,066 & 9,429 & $\begin{array}{l}1,222 \\
\end{array}$ & $\begin{array}{l}1,090 \\
\text {. }\end{array}$ & 17,166 & $\begin{array}{l}496,796 \\
\end{array}$ & 180 & $\begin{array}{l}\text {, } \\
959\end{array}$ & 31 & 57 \\
\hline 2008 & 16,827 & 788 & 4,093 & 882 & 769 & 9,605 & 434,676 & 165 & 905 & 31 & 22 \\
\hline 2009 & 15,091 & 1,835 & 3,909 & 790 & 873 & 10,900 & 469,970 & 142 & 796 & 31 & 41 \\
\hline 2010 & $\mathrm{R}_{16,513}$ & R993 & $\mathrm{R}_{1,882}$ & $R_{692}$ & R950 & R8,318 & $\mathrm{R}_{473,683}$ & $\mathrm{R}_{139}$ & R857 & R38 & R36 \\
\hline $2011^{\mathrm{P}}$ & 16,643 & 473 & 1,381 & 491 & 987 & 7,279 & 459,524 & 154 & 864 & 36 & 9 \\
\hline
\end{tabular}

Anthracite, bituminous coal, subbituminous coal, lignite, waste coal, and coal synfuel. 2 Fuel oil nos. 1,2, and 4

Jet fuel, kerosene, other petroleum liquids, and waste oil.

Petroleum coke is converted trom short tons to barrels by multiplying by 5 .

Natural gas, plus a small amount of supplemental gaseous fuels.

manufactured and waste gases derived from fossil fuels.

9 Municipal solid waste from biogenic sources, landfill gas, sludge waste, agricultural byproducts, and other biomass. Through 2000, also includes non-renewable waste (municipal solid waste from non-biogenic sources, and tire-derived fuels).

${ }^{10}$ Batteries, chemicals, hydrogen, pitch, purchased steam, sulfur, miscellaneous technologies, and beginning in 2001, non-renewable waste (municipal solid waste from non-biogenic sources, and tire-derived 11 .

Commercial combined-heat-and-power (CHP) plants.

12 Industrial combined-heat-and-power (CHP) plants.

R=Revised. P=Preliminary. - = No data reported. (s)=Less than 0.5

Notes: - Estimates are for fuels consumed to produce useful thermal output; they exclude fuels consumed to produce electricity. - See Table $8.6 \mathrm{~b}$ for electric power sector CHP data. - See Note 1 "Coverage of Electricity Statistics," and Note 2, "Classification of Power Plants Into Energy-Use Sectors," a end of section. - See "Useful Thermal Output" in Glossary. - Totals may not equal sum of components Web Pages:

Pages: - See http://www.eia.gov/totalenergy/data/annual/\#electricity for all data beginning in 1989 . Wor related information, see http://www eia gov/electricity/.

Sources: • 1989-1997-U.S. Energy Information Administration (EIA), Form EIA-867, "Annual Nonutility Power Producer Report." - 1998-2000-EIA, Form EIA-860B, "Annual Electric Generator Report-Nonutility." • 2001-2003-EIA, Form EIA-906, "Power Plant Report." • 2004-2007-EIA, Form EIA-920, "Combined Heat and Power Plant Report." • 2008 forward-EIA, Form ElA-923, "Power Plan
Operations Report." 


\section{Figure 8.7 Consumption of Combustible Fuels for Electricity Generation and Useful Thermal Output,}

1989-2011
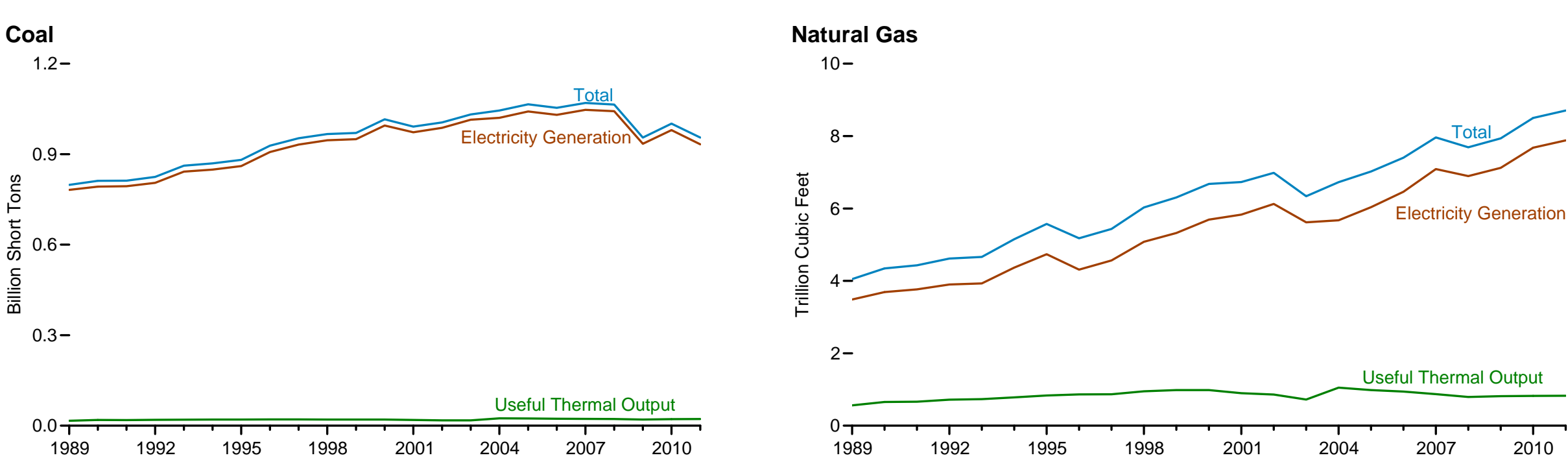

\section{Petroleum}

$350-$

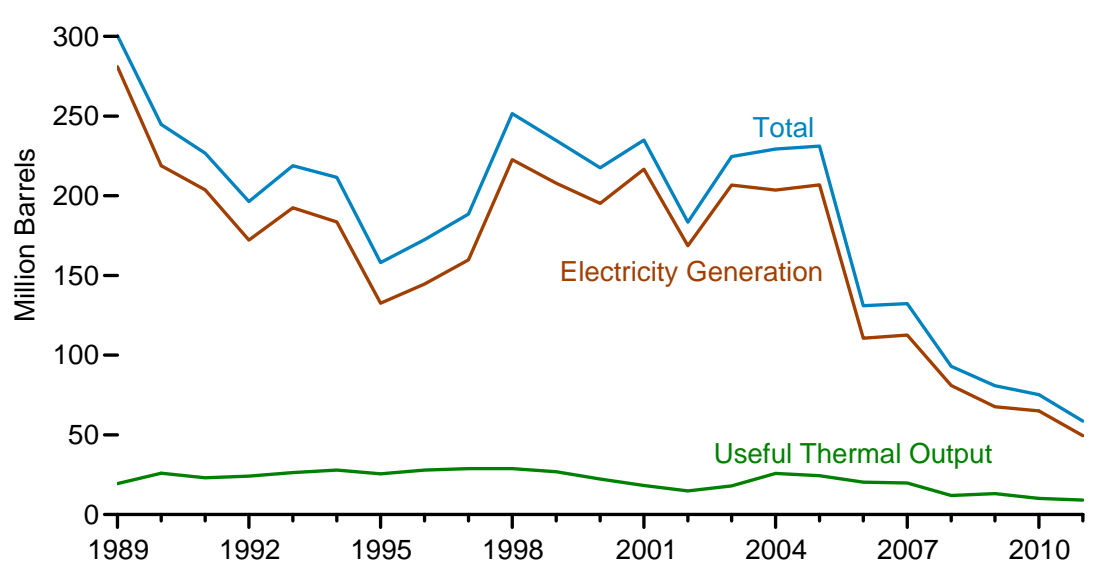

\section{Wood and Waste}

$2.0-$
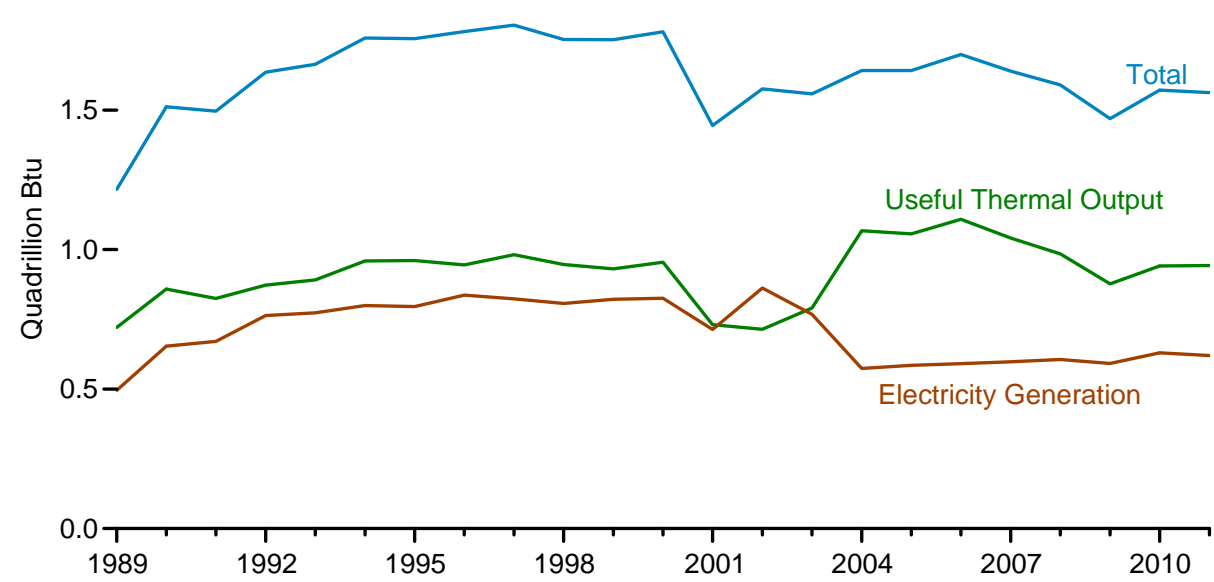

Sources: Tables 8.5a, 8.6a, and 8.7a. 
Table 8.7a Consumption of Combustible Fuels for Electricity Generation and Useful Thermal Output:

Total (All Sectors), 1989-2011 (Sum of Tables 8.7b and 8.7c)

\begin{tabular}{|c|c|c|c|c|c|c|c|c|c|c|c|}
\hline \multirow[b]{3}{*}{ Year } & \multirow[b]{2}{*}{ Coal ${ }^{1}$} & \multicolumn{5}{|c|}{ Petroleum } & \multirow[b]{2}{*}{ Natural Gas ${ }^{6}$} & \multirow[b]{2}{*}{ Other Gases ${ }^{7}$} & \multicolumn{2}{|c|}{ Biomass } & \multirow[b]{2}{*}{ Other ${ }^{10}$} \\
\hline & & Distillate Fuel Oil ${ }^{2}$ & Residual Fuel Oil 3 & Other Liquids ${ }^{4}$ & Petroleum Coke 5 & Total 5 & & & Wood $^{8}$ & Waste $^{9}$ & \\
\hline & $\begin{array}{l}\text { Thousand } \\
\text { Short Tons }\end{array}$ & \multicolumn{3}{|c|}{ Thousand Barrels } & $\begin{array}{l}\text { Thousand } \\
\text { Short Tons }\end{array}$ & $\begin{array}{c}\text { Thousand } \\
\text { Barrels }\end{array}$ & $\begin{array}{l}\text { Million } \\
\text { Cubic Feet }\end{array}$ & $\begin{array}{c}\text { Trillion } \\
\text { Btu }\end{array}$ & \multicolumn{2}{|c|}{ Trillion Btu } & $\begin{array}{c}\text { Trillion } \\
\text { Btu }\end{array}$ \\
\hline 1989 & 798,181 & 29,143 & 265,970 & 656 & 915 & 300,342 & $4,048,736$ & 206 & 1,028 & 189 & 88 \\
\hline 1990 & 811,538 & 20,194 & 209,081 & 1,332 & 2,832 & 244,765 & $4,346,311$ & 288 & 1,256 & 257 & 86 \\
\hline 1991 & 812,124 & 19,590 & 193,073 & 1,215 & 2,566 & 226,708 & $4,428,742$ & 311 & 1,204 & 292 & 114 \\
\hline 1992 & 824,512 & 16,852 & 160,941 & 1,695 & 3,366 & 196,318 & $4,617,578$ & 341 & 1,303 & 333 & 92 \\
\hline 1993 & 861,904 & 19,293 & 176,992 & 1,571 & 4,200 & 218,855 & $4,662,236$ & 314 & 1,321 & 344 & 85 \\
\hline 1994 & 869,405 & 25,177 & 164,047 & 1,539 & 4,157 & 211,547 & $5,151,163$ & 316 & 1,401 & 357 & 92 \\
\hline 1995 & 881,012 & 21,697 & 112,168 & 1,322 & 4,590 & 158,140 & $5,572,253$ & 313 & 1,382 & 374 & 97 \\
\hline 1996 & 928,015 & 22,444 & 124,607 & 2,468 & 4,596 & 172,499 & $5,178,232$ & 346 & 1,389 & 392 & 91 \\
\hline 1997 & 952,955 & 22,893 & 134,623 & 526 & 6,095 & 188,517 & $5,433,338$ & 307 & 1,397 & 407 & 103 \\
\hline 1998 & 966,615 & 30,006 & 189,267 & 1,230 & 6,196 & 251,486 & $6,030,490$ & 334 & 1,349 & 404 & 95 \\
\hline 1999 & 970,175 & 30,616 & 172,319 & 1,812 & 5,989 & 234,694 & $6,304,942$ & 350 & 1,352 & 400 & 101 \\
\hline 2000 & $1,015,398$ & 34,572 & 156,673 & 2,904 & 4,669 & 217,494 & $6,676,744$ & 356 & 1,380 & 401 & 109 \\
\hline 2001 & 991,635 & 33,724 & 177,137 & 1,418 & 4,532 & 234,940 & $6,730,591$ & 263 & 1,182 & 263 & 229 \\
\hline 2002 & $1,005,144$ & 24,748 & 118,637 & 3,257 & 7,353 & 183,408 & $6,986,081$ & 278 & 1,287 & 289 & 252 \\
\hline 2003 & $1,031,778$ & 31,825 & 152,859 & 4,576 & 7,067 & 224,593 & $6,337,402$ & 294 & 1,266 & 293 & 262 \\
\hline 2004 & $1,044,798$ & 23,520 & 157,478 & 4,764 & 8,721 & 229,364 & $6,726,679$ & 353 & 1,360 & 282 & 254 \\
\hline 2005 & $1,065,281$ & 24,446 & 156,915 & 4,270 & 9,113 & 231,193 & $7,020,709$ & 348 & 1,353 & 289 & 237 \\
\hline 2006 & $1,053,783$ & 14,655 & 69,846 & 3,396 & 8,622 & 131,005 & $7,404,432$ & 341 & 1,399 & 300 & 237 \\
\hline 2007 & $1,069,606$ & 17,042 & 74,616 & 4,237 & 7,299 & 132,389 & $7,961,922$ & 329 & 1,336 & 304 & 239 \\
\hline 2008 & $1,064,503$ & 14,137 & 43,477 & 3,765 & 6,314 & 92,948 & $7,689,380$ & 300 & 1,263 & 328 & 212 \\
\hline 2009 & 955,190 & 14,800 & 33,672 & 3,218 & 5,828 & 80,830 & $7,937,856$ & 259 & 1,137 & 333 & 228 \\
\hline 2010 & $\mathrm{R}_{1,001,411}$ & $R_{15,247}$ & $R_{26,944}$ & $R_{2,777}$ & $\mathrm{R}_{6,053}$ & R 75,231 & $R_{8,501,960}$ & $\mathrm{R}_{262}$ & $R_{1,226}$ & $R_{346}$ & $\mathrm{R}_{237}$ \\
\hline $2011^{P}$ & 954,925 & 11,374 & 16,678 & 2,203 & 5,666 & 58,586 & $8,707,029$ & 281 & 1,214 & 349 & 189 \\
\hline
\end{tabular}

1 Anthracite, bituminous coal, subbituminous coal, lignite, waste coal, and coal synfuel.

Fuel oil nos. 1, 2, and 4. Through 2000, electric utility data also include small amounts of kerosene and jet fuel.

3 Fuel oil nos. 5 and 6. Through 2000, electric utility data also include a small amount of fuel oil no. 4 .

4 Jet fuel, kerosene, other petroleum liquids, and waste oil.

5 Petroleum coke is converted from short tons to barrels by multiplying by 5 .

Natural gas, plus a small amount of supplemental gaseous fuels.

Blast furnace gas, propane gas, and other manufactured and waste gases derived from fossil fuels.

8 Wood and wood-derived fuels.

Municipal solid waste from biogenic sources, landfill gas, sludge waste, agricultural byproducts, and other biomass. Through 2000, also includes non-renewable waste (municipal solid waste from

non-biogenic sources, and tire-derived fuels).

${ }_{10}$ Batteries, chemicals, hydrogen, pitch, purchased steam, sulfur, miscellaneous technologies, and, beginning in 2001, non-renewable waste (municipal solid waste from non-biogenic sources, and tire-derived fuels).

Notes: - See Note 1, "Coverage of Electricity Statistics," at end of section. • See "Useful Thermal Output" in Glossary. - Totals may not equal sum of components due to independent rounding.

Web Pages: - See http://www.eia.gov/totalenergy/data/monthly/\#electricity for updated monthly and annual data. - See http://www.eia.gov/electricity/ for related information.

Sources: Tables $8.7 \mathrm{~b}$ and $8.7 \mathrm{c}$ 
Table 8.7b Consumption of Combustible Fuels for Electricity Generation and Useful Thermal Output:

Electric Power Sector, 1989-2011 (Subset of Table 8.7a)

\begin{tabular}{|c|c|c|c|c|c|c|c|c|c|c|c|}
\hline \multirow[b]{3}{*}{ Year } & \multirow[b]{2}{*}{ Coal ${ }^{1}$} & \multicolumn{5}{|c|}{ Petroleum } & \multirow[b]{2}{*}{ Natural Gas ${ }^{6}$} & \multirow[b]{2}{*}{ Other Gases ${ }^{7}$} & \multicolumn{2}{|c|}{ Biomass } & \multirow[b]{2}{*}{ Other 10} \\
\hline & & Distillate Fuel Oil ${ }^{2}$ & Residual Fuel Oil 3 & Other Liquids ${ }^{4}$ & Petroleum Coke ${ }^{5}$ & Total 5 & & & Wood $^{8}$ & Waste $^{9}$ & \\
\hline & $\begin{array}{l}\text { Thousand } \\
\text { Short Tons }\end{array}$ & \multicolumn{3}{|c|}{ Thousand Barrels } & $\begin{array}{l}\text { Thousand } \\
\text { Short Tons }\end{array}$ & $\begin{array}{c}\text { Thousand } \\
\text { Barrels }\end{array}$ & $\begin{array}{c}\text { Million } \\
\text { Cubic Feet }\end{array}$ & $\begin{array}{c}\text { Trillion } \\
\text { Btu }\end{array}$ & \multicolumn{2}{|c|}{ Trillion Btu } & $\begin{array}{c}\text { Trillion } \\
\text { Btu }\end{array}$ \\
\hline 1989 & 772,190 & 26,156 & 244,179 & 10 & 517 & 272,931 & $3,105,183$ & 9 & 100 & 132 & 3 \\
\hline 1990 & 782,567 & 16,567 & 184,915 & 26 & 1,008 & 206,550 & $3,244,619$ & 11 & 129 & 188 & (s) \\
\hline 1991 & 783,874 & 14,359 & 172,625 & 59 & 974 & 191,911 & $3,315,925$ & 11 & 126 & 229 & 4 \\
\hline 1992 & 795,094 & 12,623 & 138,726 & 128 & 1,494 & 158,948 & $3,447,871$ & 18 & 140 & 262 & 5 \\
\hline 1993 & 831,645 & 14,849 & 152,481 & 239 & 2,611 & 180,625 & $3,472,982$ & 16 & 150 & 265 & 5 \\
\hline 1994 & 838,354 & 20,612 & 138,222 & 771 & 2,315 & 171,178 & $3,902,546$ & 19 & 152 & 282 & 3 \\
\hline 1995 & 850,230 & 18,553 & 90,023 & 499 & 2,674 & 122,447 & $4,236,526$ & 24 & 125 & 296 & 2 \\
\hline 1996 & 896,921 & 18,780 & 99,951 & 653 & 2,642 & 132,593 & $3,806,901$ & 20 & 138 & 300 & 2 \\
\hline 1997 & 921,364 & 18,989 & 113,669 & 152 & 3,372 & 149,668 & $4,064,803$ & 24 & 137 & 309 & 1 \\
\hline 1998 & 936,619 & 23,300 & 166,528 & 431 & 4,102 & 210,769 & $4,588,284$ & 29 & 137 & 308 & 2 \\
\hline 1999 & 940,922 & 24,058 & 152,493 & 544 & 3,735 & 195,769 & $4,819,531$ & 19 & 138 & 315 & 1 \\
\hline 2000 & 985,821 & 30,016 & 138,513 & 454 & 3,275 & 185,358 & $5,206,324$ & 25 & 134 & 318 & 1 \\
\hline 2001 & 964,433 & 29,274 & 159,504 & 377 & 3,427 & 206,291 & $5,342,301$ & 15 & 126 & 211 & 113 \\
\hline 2002 & 977,507 & 21,876 & 104,773 & 1,267 & 5,816 & 156,995 & $5,671,897$ & 33 & 150 & 230 & 143 \\
\hline 2003 & $1,005,116$ & 27,632 & 138,279 & 2,026 & 5,799 & 196,932 & $5,135,215$ & 41 & 167 & 230 & 140 \\
\hline 2004 & $1,016,268$ & 19,107 & 139,816 & 2,713 & 7,372 & 198,498 & $5,463,763$ & 58 & 165 & 223 & 138 \\
\hline 2005 & $1,037,485$ & 19,675 & 139,409 & 2,685 & 8,083 & 202,184 & $5,869,145$ & 84 & 185 & 221 & 123 \\
\hline 2006 & $1,026,636$ & 12,646 & 57,345 & 1,870 & 7,101 & 107,365 & $6,222,100$ & 65 & 182 & 231 & 125 \\
\hline 2007 & $1,045,141$ & 15,327 & 63,086 & 2,594 & 5,685 & 109,431 & $6,841,408$ & 61 & 186 & 237 & 124 \\
\hline 2008 & $1,040,580$ & 12,547 & 38,241 & 2,670 & 5,119 & 79,056 & $6,668,379$ & 61 & 177 & 258 & 131 \\
\hline 2009 & 933,627 & 12,035 & 28,782 & 2,210 & 4,611 & 66,081 & $6,872,533$ & 55 & 180 & 261 & 124 \\
\hline 2010 & R975,052 & $\mathrm{R}_{13,790}$ & $\mathrm{R}_{24,503}$ & $R_{1,877}$ & $\mathrm{R}_{4,777}$ & $R_{64,055}$ & R $7,387,184$ & 52 & $R_{196}$ & $\mathrm{R}_{264}$ & 124 \\
\hline $2011^{P}$ & 928,558 & 10,586 & 14,876 & 1,568 & 4,394 & 49,003 & $7,601,926$ & 56 & 175 & 269 & 126 \\
\hline
\end{tabular}

1 Anthracite, bituminous coal, subbituminous coal, lignite, waste coal, and coal synfuel.

2 Fuel oil nos. 1, 2, and 4. Through 2000, electric utility data also include small amounts of kerosene and jet fuel.

3 Fuel oil nos. 5 and 6. Through 2000, electric utility data also include a small amount of fuel oil no. 4.

4 Jet fuel, kerosene, other petroleum liquids, and waste oil.

5 Petroleum coke is converted from short tons to barrels by multiplying by 5 .

6 Natural gas, plus a small amount of supplemental gaseous fuels.

Blast furnace gas, propane gas, and other manufactured and waste gases derived from fossil fuels.

Wood and wood-derived fuels.

9 Municipal solid waste from biogenic sources, landfill gas, sludge waste, agricultural byproducts, and other biomass. Through 2000, also includes non-renewable waste (municipal solid waste from non-biogenic sources, and tire-derived fuels).

10 Batteries, chemicals, hydrogen, pitch, purchased steam, sulfur, miscellaneous technologies, and, beginning in 2001, non-renewable waste (municipal solid waste from non-biogenic sources, and tire-derived fuels).

R=Revised. P=Preliminary. ( $(\mathrm{s})=$ Less than 0.5 .

Notes: - The electric power sector comprises electricity-only and combined-heat-and-power (CHP) plants within the NAICS 22 category whose primary business is to sell electricity, or electricity and heat, to the public. - See Table 8.7c for commercial and industrial CHP and electricity-only data. - See Note 1, "Coverage of Electricity Statistics," and Note 2, "Classification of Power Plants Into Energy-Use Sectors," at end of section. - See "Useful Thermal Output" in Glossary. • Totals may not equal sum of components due to independent rounding.

Web Pages: - See http://www.eia.gov/totalenergy/data/monthly/\#electricity for updated monthly and annual data. - See http://www.eia.gov/electricity/ for related information.

Sources: • 1989-1997-U.S. Energy Information Administration (EIA), Form EIA-759, "Monthly Power Plant Report," and Form EIA-867, "Annual Nonutility Power Producer Report." • 1998-2000—EIA, Form EIA-759, "Monthly Power Plant Report," and Form EIA-860B, "Annual Electric Generator Report-Nonutility." • 2001-2003-EIA, Form EIA-906, "Power Plant Report." • 2004-2007-EIA, Form EIA-906, "Power Plant Report," and Form EIA-920, "Combined Heat and Power Plant Report." - 2008 forward-EIA, Form EIA-923, "Power Plant Operations Report." 
Table 8.7c Consumption of Combustible Fuels for Electricity Generation and Useful Thermal Output:

Commercial and Industrial Sectors, Selected Years, 1989-2011 (Subset of Table 8.7a)

\begin{tabular}{|c|c|c|c|c|c|c|c|c|c|c|c|}
\hline \multirow[b]{3}{*}{ Year } & \multirow[b]{2}{*}{ Coal ${ }^{1}$} & \multicolumn{5}{|c|}{ Petroleum } & \multirow[b]{2}{*}{ Natural Gas ${ }^{6}$} & \multirow[b]{2}{*}{ Other Gases ${ }^{7}$} & \multicolumn{2}{|c|}{ Biomass } & \multirow[b]{2}{*}{ Other 10} \\
\hline & & Distillate Fuel Oil 2 & Residual Fuel Oil ${ }^{3}$ & Other Liquids ${ }^{4}$ & Petroleum Coke 5 & Total 5 & & & Wood 8 & Waste 9 & \\
\hline & $\begin{array}{l}\text { Thousand } \\
\text { Short Tons }\end{array}$ & & Thousand Barrels & & $\begin{array}{l}\text { Thousand } \\
\text { Short Tons }\end{array}$ & $\begin{array}{c}\text { Thousand } \\
\text { Barrels }\end{array}$ & $\begin{array}{c}\text { Million } \\
\text { Cubic Feet }\end{array}$ & $\begin{array}{c}\text { Trillion } \\
\text { Btu }\end{array}$ & \multicolumn{2}{|c|}{ Trillion Btu } & $\begin{array}{c}\text { Trillion } \\
\text { Btu }\end{array}$ \\
\hline
\end{tabular}

Commercial Sector ${ }^{11}$

\begin{tabular}{|c|c|c|c|c|c|c|c|c|c|c|c|}
\hline \\
\hline 1989 & 1,125 & 1,085 & 883 & - & - & 1,967 & 30,037 & 1 & 2 & 22 & - \\
\hline 1990 & 1,191 & 969 & 1,087 & (s) & - & 2,056 & 46,458 & 1 & 2 & 28 & - \\
\hline 1995 & 1,419 & 812 & 413 & (s) & 4 & 1,245 & 77,664 & - & 1 & 40 & (s) \\
\hline 1996 & 1,660 & 682 & 545 & (s) & 4 & 1,246 & 82,455 & (s) & 2 & 53 & (s) \\
\hline 1997 & 1,738 & 1,053 & 509 & - & 4 & 1,584 & 86,915 & (s) & 2 & 58 & (s) \\
\hline 1998 & 1,443 & 854 & 932 & - & 4 & 1,807 & 87,220 & (s) & 2 & 54 & - \\
\hline 1999 & 1,490 & 759 & 834 & - & 4 & 1,613 & 84,037 & (s) & 1 & 54 & (s) \\
\hline 2000 & 1,547 & 908 & 676 & 3 & 6 & 1,615 & 84,874 & (s) & 1 & 47 & (s) \\
\hline 2001 & 1,448 & 1,026 & 773 & 2 & 6 & 1,832 & 78,655 & (s) & 1 & 25 & 15 \\
\hline 2002 & 1,405 & 771 & 400 & 38 & 8 & 1,250 & 73,975 & (s) & 1 & 26 & 17 \\
\hline 2003 & 1,816 & 671 & 708 & 16 & 11 & 1,449 & 58,453 & - & 1 & 29 & 18 \\
\hline 2004 & 1,917 & 1,115 & 827 & 21 & 9 & 2.009 & 72.072 & - & 2 & 34 & 21 \\
\hline 2005 & 1,922 & 794 & 789 & 1 & 9 & 1,630 & 67,957 & - & 1 & 34 & 20 \\
\hline 2006 & 1,886 & 366 & $\begin{array}{l}189 \\
520\end{array}$ & (s) & 10 & $\begin{array}{r}1,630 \\
935\end{array}$ & $\begin{array}{l}67,7351 \\
67,735\end{array}$ & $\bar{s})$ & 1 & $\begin{array}{l}34 \\
36\end{array}$ & 21 \\
\hline 2007 & 1,927 & 257 & 434 & - & 12 & 752 & 70,074 & - & 2 & 31 & 17 \\
\hline 2008 & 2,021 & 418 & 202 & (s) & 10 & 671 & 66,216 & - & 1 & 34 & 21 \\
\hline 2009 & 1,798 & 266 & 212 & (s) & 9 & 521 & 75,555 & - & 1 & $\begin{array}{l}54 \\
36\end{array}$ & 22 \\
\hline 2010 & $\mathrm{R}_{1,720}$ & $R_{233}$ & $R_{143}$ & (s) & 12 & ${ }^{R} 437$ & $R_{85,786}$ & $\begin{array}{ll}{ }^{R} & (s)\end{array}$ & 1 & $R_{36}$ & $\mathrm{R}_{22}$ \\
\hline $2011^{\mathrm{P}}$ & 1,633 & 147 & 103 & (s) & 6 & 282 & 81,433 & (s) & 1 & 36 & 23 \\
\hline & \multicolumn{11}{|c|}{ Industrial Sector ${ }^{12}$} \\
\hline 1989 & 24,867 & 1,903 & 20,909 & 646 & 397 & 25,444 & 913,516 & 195 & 926 & 35 & 85 \\
\hline 1990 & 27,781 & 2,657 & 23,079 & 1,305 & 1,824 & 36,159 & $1,055,235$ & 275 & 1,125 & 41 & 86 \\
\hline 1995 & 29,363 & 2,333 & $\begin{array}{l}21,732 \\
21,70\end{array}$ & 823 & $\begin{array}{l}1,024 \\
1,912\end{array}$ & 34,448 & $1,258,063$ & 290 & 1,255 & $\begin{array}{l}41 \\
38\end{array}$ & $\begin{array}{l}00 \\
95\end{array}$ \\
\hline 1996 & $\begin{array}{l}29,030 \\
29,434\end{array}$ & $\begin{array}{l}2,033 \\
2,983\end{array}$ & 24,111 & $\begin{array}{r}0<3 \\
1,815\end{array}$ & 1,950 & $\begin{array}{l}34,440 \\
38,661\end{array}$ & $1,288,876$ & 325 & $\begin{array}{l}1,250 \\
1,249\end{array}$ & $\begin{array}{l}30 \\
39\end{array}$ & $\begin{array}{l}50 \\
89\end{array}$ \\
\hline 1997 & $\begin{array}{l}29,434 \\
29,853\end{array}$ & $\begin{array}{l}2,903 \\
2,851\end{array}$ & $\begin{array}{l}24,111 \\
20,445\end{array}$ & $\begin{array}{r}1,815 \\
374\end{array}$ & $\begin{array}{l}1,950 \\
2,719\end{array}$ & $\begin{array}{l}30,001 \\
37,265\end{array}$ & $\begin{array}{l}1,281,620 \\
1,281,620\end{array}$ & $\begin{array}{l}320 \\
283\end{array}$ & $\begin{array}{l}1,249 \\
1,259\end{array}$ & $\begin{array}{l}39 \\
41\end{array}$ & $\begin{array}{r}89 \\
102\end{array}$ \\
\hline 1998 & 28,553 & 5,852 & 21,807 & 800 & 2,090 & 38,910 & $1,354,986$ & 305 & 1,211 & 42 & 93 \\
\hline 1999 & 27,763 & 5,799 & 18,993 & 1,268 & 2,251 & 37,312 & $1,401,374$ & 331 & 1,213 & 31 & 99 \\
\hline 2000 & 28,031 & 3,648 & 17,483 & 2,448 & 1,388 & 30,520 & $1,385,546$ & 331 & 1,244 & 35 & 108 \\
\hline 2001 & $\begin{array}{l}20,011 \\
25,755\end{array}$ & $\begin{array}{l}3,040 \\
3,424\end{array}$ & $\begin{array}{l}11,800 \\
16,860\end{array}$ & $\begin{array}{l}1,039 \\
1,039\end{array}$ & $\begin{array}{l}1,090 \\
1,099\end{array}$ & 26,817 & $1,309,636$ & 248 & $\begin{array}{l}1,244 \\
1,054\end{array}$ & 27 & 101 \\
\hline 2002 & 26,232 & 2,101 & 13,463 & 1,953 & 1,529 & 25,163 & $1,240,209$ & 245 & 1,136 & 34 & 92 \\
\hline 2003 & 24,846 & 3,522 & 13,872 & 2,535 & 1,257 & 26,212 & $1,143,734$ & 253 & 1,097 & 34 & 103 \\
\hline 2004 & 26,613 & 3,298 & 16,835 & 2,030 & 1,339 & 28,857 & $1,190,844$ & 295 & 1,193 & 24 & 94 \\
\hline 2005 & 25,875 & 3,977 & 16,718 & 1,583 & 1,020 & 27,380 & $1,083,607$ & 264 & 1,166 & 34 & 94 \\
\hline 2006 & 25,262 & 1,643 & 11,981 & 1,526 & 1,511 & 22,706 & $1,114,597$ & 277 & 1,216 & 33 & 92 \\
\hline 2007 & 22,537 & 1,458 & 11,096 & 1,643 & 1,602 & 22,207 & $1,050,439$ & 268 & 1,148 & 36 & 98 \\
\hline 2008 & 21,902 & 1,171 & 5,034 & 1,095 & 1,184 & 13,222 & 954,785 & 239 & $\begin{array}{l}1,084 \\
1,040\end{array}$ & 35 & 60 \\
\hline 2009 & 19,766 & 2.499 & 4,678 & 1,008 & 1,209 & 14,228 & 989,769 & 204 & 955 & 35 & 82 \\
\hline 2010 & $\begin{array}{r}r, 100 \\
R 24,638\end{array}$ & $\mathrm{R}_{1}^{2,424}$ & $\begin{array}{r}4,010 \\
R_{2}, 298\end{array}$ & $\begin{array}{l}\text { R900 } \\
\text { R900 }\end{array}$ & $\mathrm{R}_{1}, 264$ & $\begin{array}{r}1,<20 \\
R_{10,740}\end{array}$ & $\mathrm{R}_{1}, 028,990$ & $\mathrm{R}_{210}$ & $R_{1} .029$ & $\begin{array}{r}35 \\
R 47\end{array}$ & R91 \\
\hline $2011^{\mathrm{P}}$ & $\begin{array}{r}24,638 \\
24,733\end{array}$ & 641 & $\begin{array}{l}2,698 \\
1,699\end{array}$ & 635 & $\begin{array}{r}1,264 \\
1,265\end{array}$ & $\begin{array}{r}1, / 40 \\
9,302\end{array}$ & $\begin{array}{r}1,023,670 \\
1,028,90\end{array}$ & 224 & $\begin{array}{l}1,037 \\
1,037\end{array}$ & 44 & 40 \\
\hline
\end{tabular}

1 Anthracite, bituminous coal, subbituminous coal, lignite, waste coal, and coal synfuel.

2 Fuel oil nos. 1,2 , and 4 .
3 Fuel oil nos. 5 and 6 .

Jet fuel, kerosene, other petroleum liquids, and waste oil.

Petroleum coke is converted from short tons to barrels by multiplying by 5 .

Natural gas, plus a small amount of supplemental gaseous fuels.

Blast furnace gas, propane gas, and other manufactured and waste gases derived from fossil fuels.

9 Municipal solid waste from biogenic sources, landfill gas, sludge waste, agricultural byproducts, and other biomass. Through 2000, also includes non-renewable waste (municipal solid waste from non-biogenic sources, and tire-derived fuels).

${ }^{10}$ Batteries, chemicals, hydrogen, pitch, purchased steam, sulfur, miscellaneous technologies, and, beginning in 2001, non-renewable waste (municipal solid waste from non-biogenic sources, and tire-derived fuels).

Commercial combined-heat-and-power (CHP) and commercial electricity-only plants.

12 Industrial combined-heat-and-power (CHP) and industrial electricity-only plants.

$\mathrm{R}=$ Revised. P=Preliminary. - = No data reported. (s)=Less than 0.5

Notes: - See Table $8.7 \mathrm{~b}$ for electric power sector electricity-only and CHP data. - See Note 1 "Coverage of Electricity Statistics," and Note 2, "Classification of Power Plants Into Energy-Use Sectors," at end of section. - See "Useful Thermal Output" in Glossary. - Totals may not equal sum of components due to independent rounding.

Whe 1989. See http//www://www.eia.gov/totalenergy/data/annual/\#electricity for all annual data beginning in Sources: - 1989-1997-U.S. Energy Information Administration (EIA), Form EIA-867, "Annual Nonutility Power Producer Report." - 1998-2000-EIA, Form EIA-860B, "Annual Electric Generator Report-Nonutility." • 2001-2003-EIA, Form EIA-906, "Power Plant Report." • 2004-2007-EIA, Form EIA-906, "Power Plant Report," and Form EIA-920, "Combined Heat and Power Plant Report." 2008 forward-EIA, Form EIA-923, "Power Plant Operations Report." 
Figure 8.8 Stocks of Coal and Petroleum: Electric Power Sector, End of Year

\section{Coal, 1949-2011}

$200-$
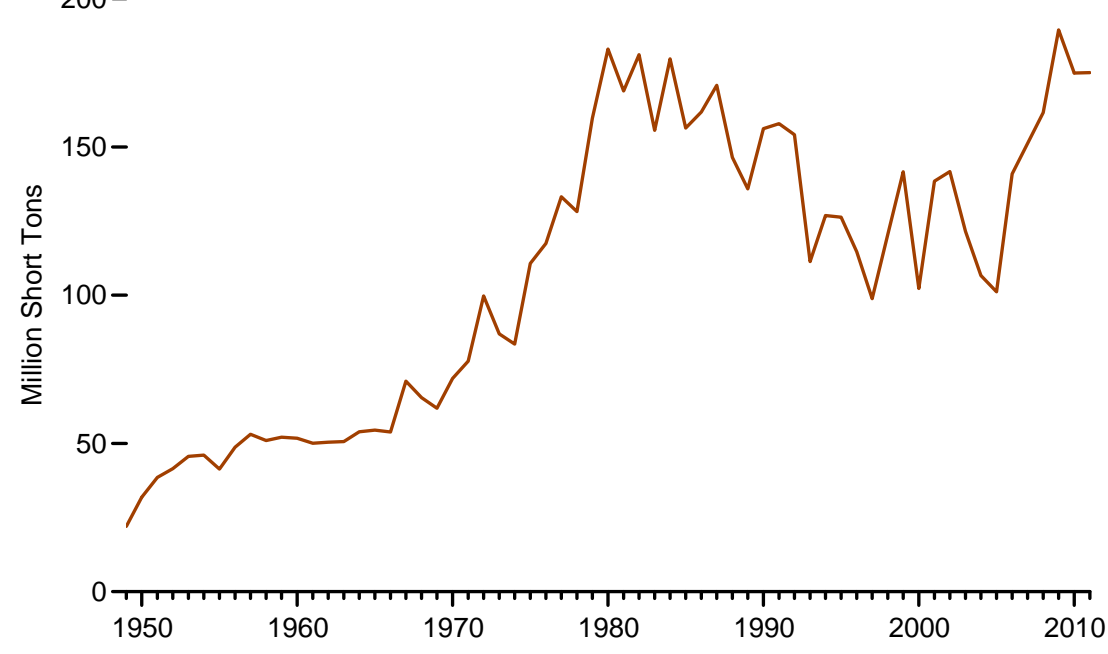

Petroleum, 1949-2011

$150-$

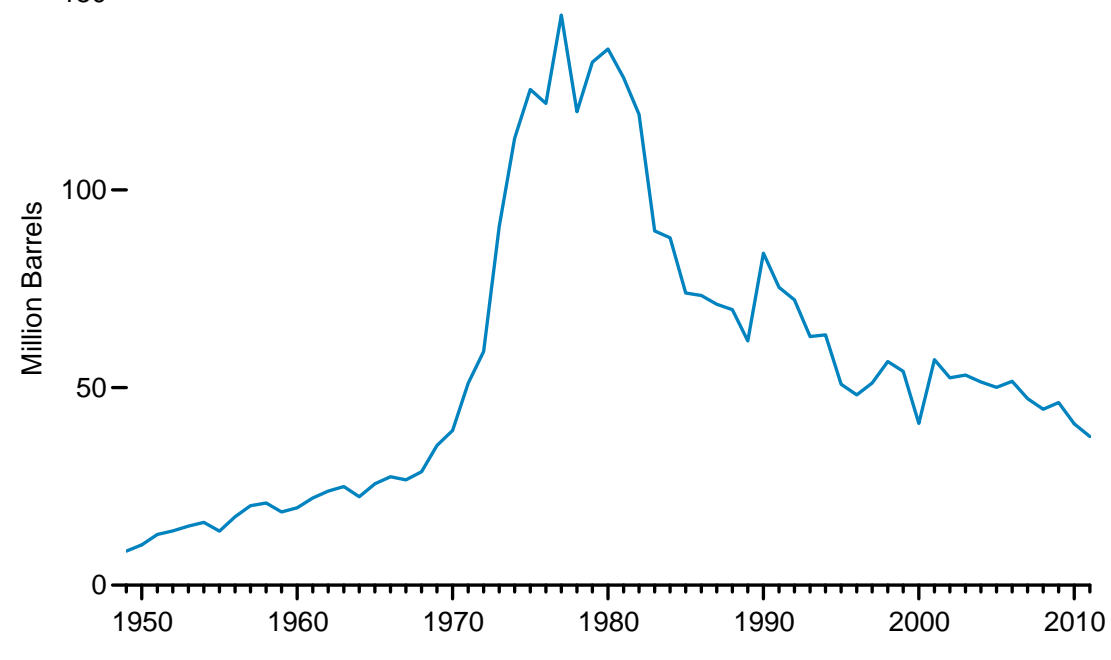

Coal and Petroleum, 1973-2011

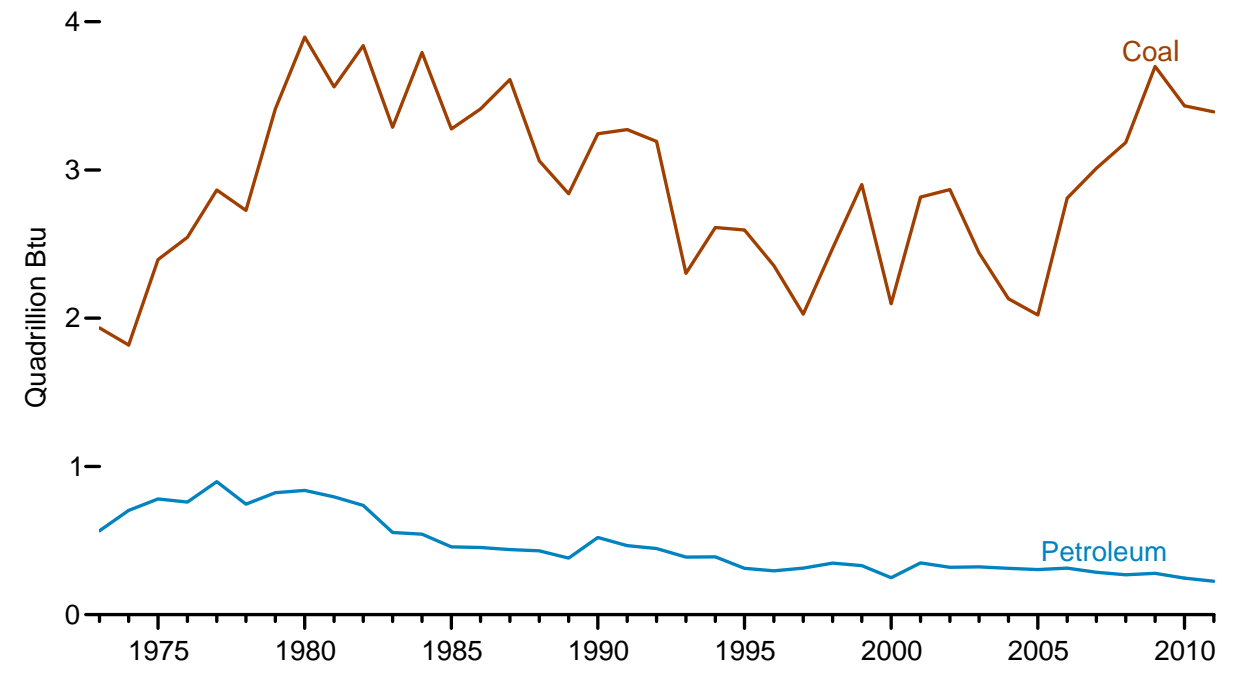

Petroleum Products, 2011

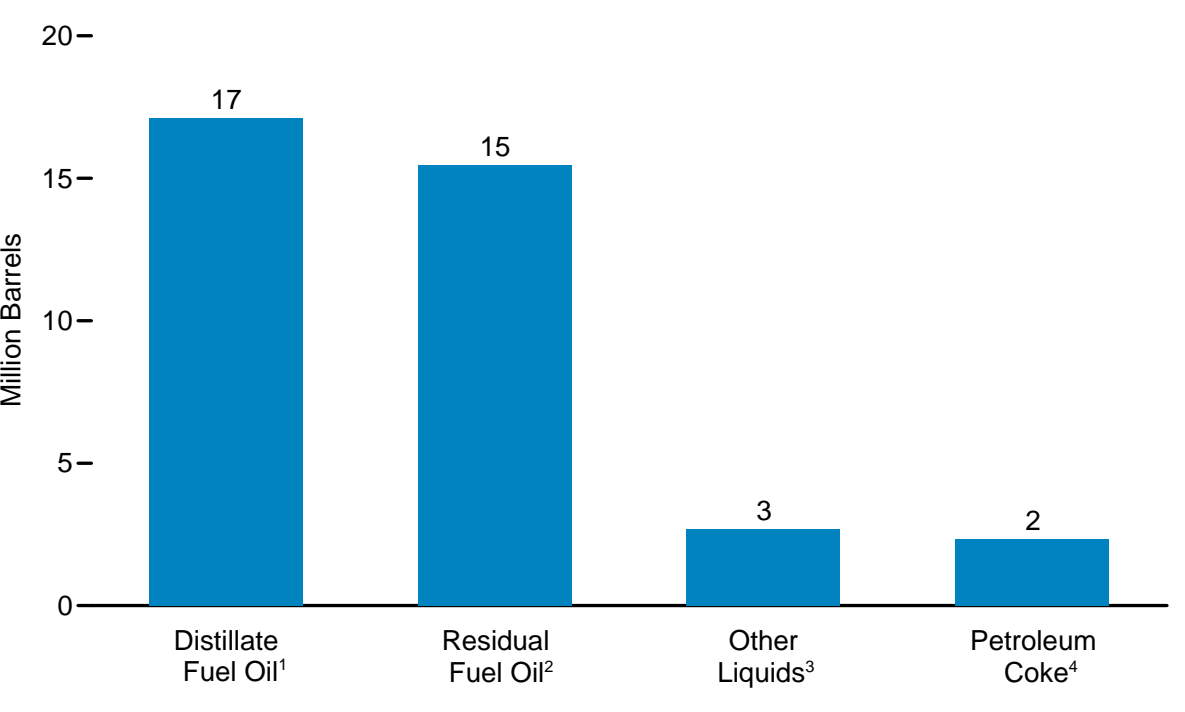

${ }^{4}$ Petroleum coke, which is reported in short tons, is converted at a rate of 5 barrels per short ton.

Sources: Tables 8.8, A3, and A5. 
Table 8.8 Stocks of Coal and Petroleum: Electric Power Sector, Selected Years, End of Year 1949-2011

\begin{tabular}{|c|c|c|c|c|c|c|}
\hline \multirow[b]{3}{*}{ Year } & \multirow[b]{2}{*}{ Coal ${ }^{1}$} & \multicolumn{5}{|c|}{ Petroleum } \\
\hline & & Distillate Fuel Oil ${ }^{2}$ & Residual Fuel Oil ${ }^{3}$ & Other Liquids ${ }^{4}$ & Petroleum Coke 5 & Total 5,6 \\
\hline & Thousand Short Tons & \multicolumn{3}{|c|}{ Thousand Barrels } & Thousand Short Tons & Thousand Barrels \\
\hline 1949 & 22,054 & NA & NA & NA & NA & 8,604 \\
\hline 1950 & 31,842 & NA & NA & NA & NA & 10,201 \\
\hline 1955 & 41,391 & NA & NA & NA & NA & 13,671 \\
\hline 1960 & 51,735 & NA & NA & NA & NA & 19,572 \\
\hline 1965 & 54,525 & NA & NA & NA & NA & 25,647 \\
\hline 1970 & 71,908 & NA & NA & NA & 239 & 39,151 \\
\hline 1975 & 110,724 & 16,432 & 108,825 & NA & 31 & 125,413 \\
\hline 1976 & 117,436 & 14,703 & 106,993 & NA & 32 & 121,857 \\
\hline 1977 & 133,219 & 19,281 & 124,750 & NA & 44 & 144,252 \\
\hline 1978 & 128,225 & 16,386 & 102,402 & NA & 198 & 119,778 \\
\hline 1979 & 159,714 & 20,301 & 111,121 & NA & 183 & 132,338 \\
\hline 1980 & 183,010 & 30,023 & 105,351 & NA & 52 & 135,635 \\
\hline 1981 & 168,893 & 26,094 & 102,042 & NA & 42 & 128,345 \\
\hline 1982 & 181,132 & 23,369 & 95,515 & NA & 41 & 119,090 \\
\hline 1983 & 155,598 & 18,801 & 70,573 & NA & 55 & 89,652 \\
\hline 1984 & 179,727 & 19,116 & 68,503 & NA & 50 & 87,870 \\
\hline 1985 & 156,376 & 16,386 & 57,304 & NA & 49 & 73,933 \\
\hline 1986 & 161,806 & 16,269 & 56,841 & NA & 40 & 73,313 \\
\hline 1987 & 170,797 & 15,759 & 55,069 & NA & 51 & 71,084 \\
\hline 1988 & 146,507 & 15,099 & 54,187 & NA & 86 & 69,714 \\
\hline 1989 & 135,860 & 13,824 & 47,446 & NA & 105 & 61,795 \\
\hline 1990 & 156,166 & 16,471 & 67,030 & NA & 94 & 83,970 \\
\hline 1991 & 157,876 & 16,357 & 58,636 & NA & 70 & 75,343 \\
\hline 1992 & 154,130 & 15,714 & 56,135 & NA & 67 & 72,183 \\
\hline 1993 & 111,341 & 15,674 & 46,770 & NA & 89 & 62,890 \\
\hline 1994 & 126,897 & 16,644 & 46,344 & NA & 69 & 63,333 \\
\hline 1995 & 126,304 & 15,392 & 35,102 & NA & 65 & 50,821 \\
\hline 1996 & 114,623 & 15,216 & 32,473 & NA & 91 & 48,146 \\
\hline 1997 & 98,826 & 15,456 & 33,336 & NA & 469 & 51,138 \\
\hline 1998 & 120,501 & 16,343 & 37,451 & NA & 559 & 56,591 \\
\hline $1999^{7}$ & 141,604 & 17,995 & 34,256 & NA & 372 & 54,109 \\
\hline 2000 & 102,296 & 15,127 & 24,748 & NA & 211 & 40,932 \\
\hline 2001 & 138,496 & 20,486 & 34,594 & NA & 390 & 57,031 \\
\hline 2002 & 141,714 & 17,413 & 25,723 & 800 & 1,711 & 52,490 \\
\hline 2003 & 121,567 & 19,153 & 25,820 & 779 & 1,484 & 53,170 \\
\hline 2004 & 106,669 & 19,275 & 26,596 & 879 & 937 & 51,434 \\
\hline 2005 & 101,137 & 18,778 & 27,624 & 1,012 & 530 & 50,062 \\
\hline 2006 & 140,964 & 18,013 & 28,823 & 1,380 & 674 & 51,583 \\
\hline 2007 & 151,221 & 18,395 & 24,136 & 1,902 & 554 & 47,203 \\
\hline 2008 & 161,589 & 17,761 & 21,088 & 1,955 & 739 & 44,498 \\
\hline 2009 & 189,467 & 17,886 & 19,068 & 2,257 & 1,394 & 46,181 \\
\hline 2010 & R174,917 & R16,758 & $\mathrm{R}_{1} 16,629$ & $\mathrm{R}_{2,319}$ & $R_{1,019}$ & $\mathrm{R}_{40,800}$ \\
\hline $2011^{P}$ & 175,100 & 17,101 & 15,469 & 2,690 & 470 & 37,608 \\
\hline
\end{tabular}

Anthracite, bituminous coal, subbituminous coal, and lignite.

2 Fuel oil nos. 1, 2, and 4. For 1973-1979, data are for gas turbine and internal combustion plant stocks

of petroleum. For 1980-2000, electric uttity data also include small amounts of kerosene and jet fuel.

Into Energy-Use Sectors," at end of section. - Totals may not equal sum of components due to independent rounding.

Web Pages: - See http://www.eia.gov/totalenergy/data/monthly/\#electricity for updated monthly and annual data. - See $\mathrm{http}: / / \mathrm{www}$.eia.gov/totalenergy/data/annual/\#electricity for all annual data beginning in 949. - See http://www.eia.gov/electricity/ for related information.

electric utility data also include a small amount of fuel oil no. 4

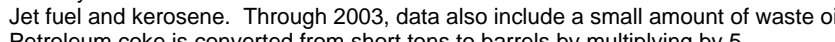

6 Distillate fuel oil and residual fuel oil; beginning in 1970, also includes petroleum coke; and beginning in 2002 , also includes other liquids.

Through 1998, data are for electric utilities only. Beginning in 1999, data are for electric utilities and independent power producers.

Notes: - The electric power sector comprises electricity-only and combined-heat-and-power (CHP) plants within the NAICS 22 category whose primary business is to sell electricity, or electricity and heat, to

Sources: • 1949-September 1977-Federal Power Commission, Form FPC-4, "Monthly Power Plant Report." • October 1977-1981—Federal Energy Regulatory Commission, Form FPC-4, "Monthly Power Power Plant Report." - 1989-1997-EIA, Form EIA-759, "Monthly Power Plant Report," and Form EIA-867, "Annual Nonutility Power Producer Report." • 1998-2000—EIA, Form EIA-759, "Monthly Power Plant Report," and Form EIA-860B, "Annual Electric Generator Report-Nonutility." • 2001-2003-EIA, Form EIA-906, "Power Plant Report." - 2004-2007-EIA, Form EIA-906, "Power Plant Report," and Form EIA-920, "Combined Heat and Power Plant Report." • 2008 forward-EIA, Form EIA-923, "Power Plan Operations Report." 


\title{
Figure 8.9 Electricity End Use
}

\section{Overview, 1989-2011}

$5-$

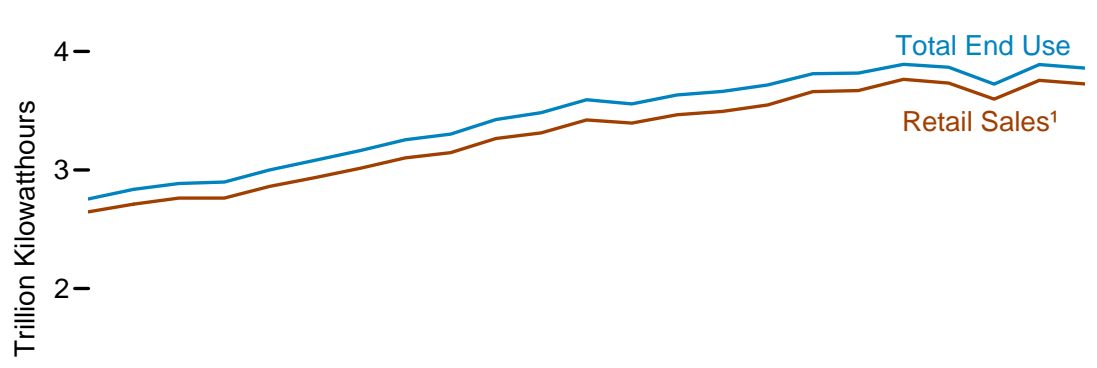

$1-$

Direct Use ${ }^{2}$

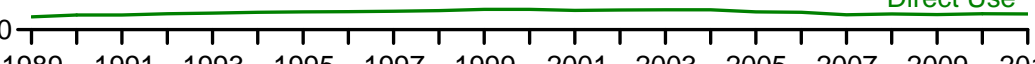

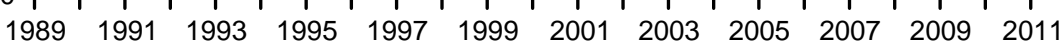

\section{Retail Sales ${ }^{1}$ by Sector, 2011}

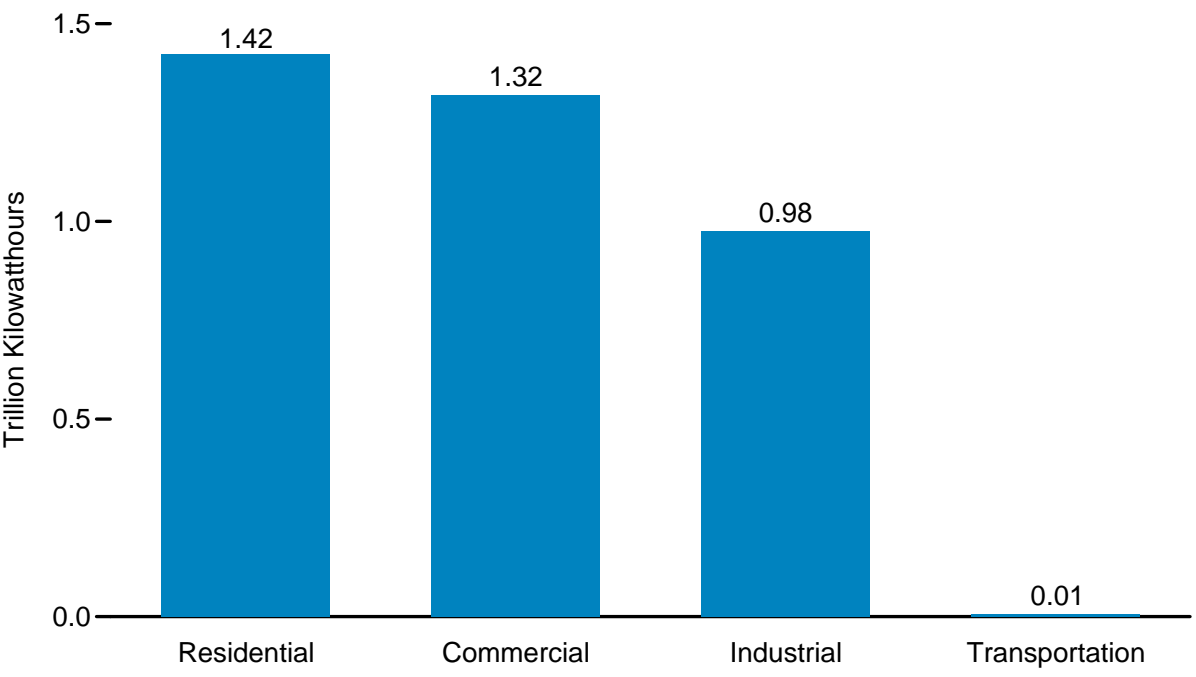

\section{Retail Sales ${ }^{1}$ by Sector, $1949-2011$}

$$
1.5-
$$

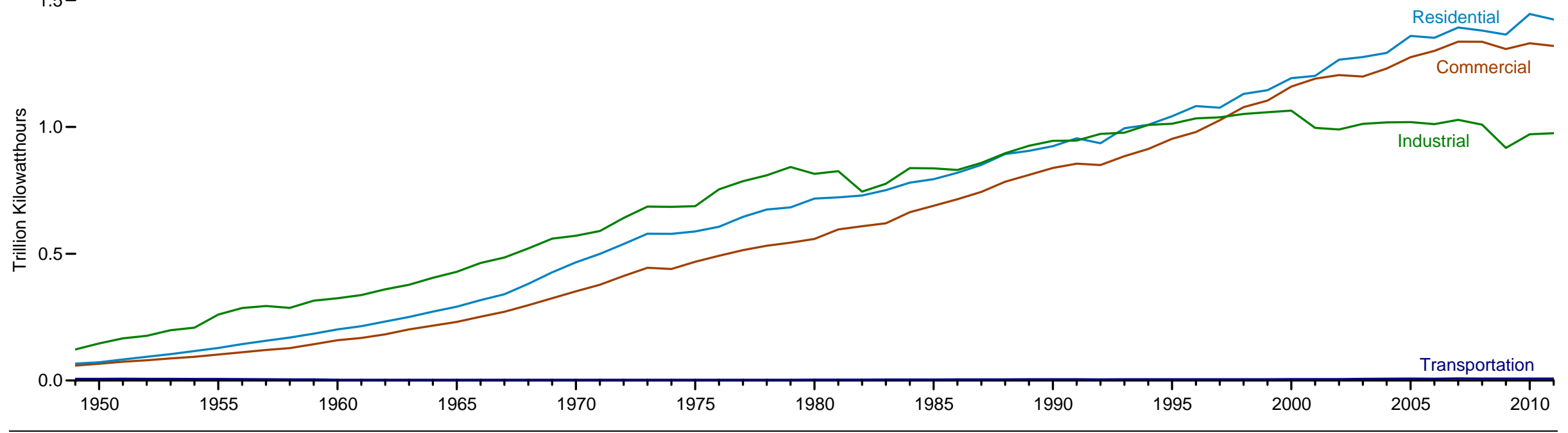

${ }^{1}$ Electricity retail sales to ultimate customers reported by electric utilities and, beginning in 1996, other energy service providers.

${ }^{2}$ Use of electricity that is 1 ) self-generated, 2) produced by either the same entity that process located within the same facility or group of facilities that house the generating equipment. Direct use is exclusive of station use.

consumes the power or an affiliate, and 3) used in direct support of a service or industrial

\author{
Source: Table 8.9 .
}


Table 8.9 Electricity End Use, Selected Years, 1949-2011

(Billion Kilowatthours)

\begin{tabular}{|c|c|c|c|c|c|c|c|c|c|}
\hline \multirow[b]{2}{*}{ Year } & \multicolumn{5}{|c|}{ Retail Sales ${ }^{1}$} & \multirow[b]{2}{*}{ Direct Use 6} & \multirow[b]{2}{*}{ Total End Use ${ }^{7}$} & \multicolumn{2}{|c|}{ Discontinued Retail Sales Series } \\
\hline & Residential & Commercial $^{2}$ & Industrial ${ }^{3}$ & Transportation 4 & Total Retail Sales 5 & & & Commercial (Old) 8 & Other $(\text { Old })^{9}$ \\
\hline $\begin{array}{l}1949 \\
1950\end{array}$ & $\begin{array}{l}67 \\
72\end{array}$ & $\begin{array}{l}\text { E59 } \\
E_{66}\end{array}$ & $\begin{array}{l}123 \\
146\end{array}$ & $\begin{array}{l}\text { E6 } \\
\text { E7 }\end{array}$ & $\begin{array}{l}255 \\
291\end{array}$ & NA & $\begin{array}{l}255 \\
259\end{array}$ & $\begin{array}{l}45 \\
51\end{array}$ & $\begin{array}{l}20 \\
20\end{array}$ \\
\hline $\begin{array}{l}1950 \\
1955\end{array}$ & 128 & $\begin{array}{l}566 \\
E_{103}\end{array}$ & 260 & $\mathrm{E}_{6}$ & 297 & NA & 291 & 57 & 22 \\
\hline 1960 & 201 & $\mathrm{E}_{159}$ & 324 & $\mathrm{E}_{3}$ & 688 & NA & 688 & 131 & 32 \\
\hline 1965 & 291 & $E_{231}$ & 429 & $E_{3}$ & 954 & NA & 954 & 200 & 34 \\
\hline 1970 & 466 & E352 & 571 & $E_{3}$ & 1,392 & NA & 1,392 & 307 & 48 \\
\hline 1975 & 588 & E468 & 688 & E3 & 1,747 & NA & 1,747 & 403 & 68 \\
\hline 1976 & 606 & E492 & 754 & $E_{3}$ & 1,855 & NA & 1,855 & 425 & 70 \\
\hline 1977 & 645 & E514 & 786 & $E_{3}$ & 1,948 & NA & 1,948 & 447 & 71 \\
\hline 1978 & 674 & E531 & 809 & $\mathrm{E}_{3}$ & 2,018 & NA & 2,018 & 461 & 73 \\
\hline 1979 & 683 & 543 & 842 & 3 & 2,071 & NA & 2,071 & 473 & 73 \\
\hline 1980 & 717 & 559 & 815 & 3 & 2,094 & NA & 2,094 & 488 & 74 \\
\hline 1981 & 722 & 596 & 826 & 3 & 2,147 & $\mathrm{NA}$ & 2,147 & 514 & 85 \\
\hline 1982 & 730 & 609 & 745 & 3 & 2,086 & NA & 2,086 & 526 & 86 \\
\hline 1983 & 751 & 620 & 776 & 4 & 2,151 & NA & 2,151 & 544 & 80 \\
\hline 1984 & 780 & 664 & 838 & 4 & 2,286 & NA & 2,286 & 583 & 85 \\
\hline 1985 & 794 & 689 & 837 & 4 & 2,324 & $\mathrm{NA}$ & 2,324 & 606 & 87 \\
\hline 1986 & 819 & 715 & 831 & 4 & 2,369 & NA & 2,369 & 631 & 89 \\
\hline 1987 & 850 & 744 & 858 & 5 & 2,457 & $\mathrm{NA}$ & 2,457 & 660 & 88 \\
\hline 1988 & 893 & 784 & 896 & 5 & 2,578 & NA & 2,578 & 699 & 90 \\
\hline 1989 & 906 & 811 & 926 & 5 & 2,647 & 109 & 2,756 & 726 & 90 \\
\hline 1990 & 924 & 838 & 946 & 5 & 2,713 & 125 & 2,837 & 751 & 92 \\
\hline 1991 & 955 & 855 & 947 & 5 & 2,762 & 124 & 2,886 & 766 & 94 \\
\hline 1992 & 936 & 850 & 973 & 5 & 2,763 & 134 & 2,897 & 761 & 93 \\
\hline 1993 & 995 & 885 & 977 & 5 & 2,861 & 139 & 3,001 & 795 & 95 \\
\hline 1994 & 1,008 & 913 & 1,008 & 5 & 2,935 & 146 & 3,081 & 820 & 98 \\
\hline 1995 & 1,043 & 953 & 1,013 & 5 & 3,013 & 151 & 3,164 & 863 & 95 \\
\hline 1996 & 1,083 & 980 & 1,034 & 5 & 3,101 & 153 & 3,254 & 887 & 98 \\
\hline 1997 & 1,076 & 1,027 & 1,038 & 5 & 3,146 & 156 & 3,302 & 929 & 103 \\
\hline 1998 & 1,130 & 1,078 & 1,051 & 5 & 3,264 & 161 & 3,425 & 979 & 104 \\
\hline 1999 & 1,145 & 1,104 & 1,058 & 5 & 3,312 & 172 & 3,484 & 1.002 & 107 \\
\hline 2000 & 1,192 & 1,159 & 1,064 & 5 & 3,421 & 171 & 3,592 & 1,055 & 109 \\
\hline 2001 & 1,202 & 1,191 & 997 & 6 & 3,394 & 163 & 3,557 & 1,083 & 113 \\
\hline 2002 & 1,265 & 1,205 & 990 & 6 & 3,465 & 166 & 3,632 & 1,104 & 106 \\
\hline 2003 & 1,276 & 1,199 & 1,012 & 7 & 3,494 & 168 & 3,662 & -- & -- \\
\hline 2004 & 1,292 & 1,230 & 1,018 & 7 & 3,547 & 168 & 3,716 & -- & -- \\
\hline 2005 & 1,359 & 1,275 & 1,019 & 8 & 3,661 & 150 & 3.811 & -- & -- \\
\hline 2006 & 1,352 & 1,300 & 1,011 & 7 & 3,670 & 147 & 3,817 & -- & -- \\
\hline 2007 & 1,392 & 1,336 & 1,028 & 8 & 3.765 & 126 & 3,890 & -- & -- \\
\hline 2008 & 1,380 & 1,336 & 1,009 & 8 & 3,733 & 132 & 3.865 & -- & -- \\
\hline 2009 & $\begin{array}{l}1,364 \\
1,30\end{array}$ & 1,307 & 917 & 8 & 3,597 & 127 & 3,724 & -- & -- \\
\hline 2010 & $\mathrm{R}_{1,446}$ & $\mathrm{R}_{1}, 330$ & R971 & 8 & $R_{3,754}$ & R132 & $R_{3,886}$ & -- & -- \\
\hline 2011 & $\mathrm{P}_{1,424}$ & $\mathrm{P}_{1,319}$ & P976 & P8 & $P_{3,726}$ & $\mathrm{E}_{130}$ & $P_{3,856}$ & -- & -- \\
\hline
\end{tabular}

1 Electricity retail sales to ultimate customers reported by electric utilities and, beginning in 1996, other 2

ind hublic street and highway lighting, interdepartmental sales, and other 3 Industrial sector. Through 2002, excludes agriculture and irrigation; beginning in 2003, includes
4 sali agriculture and irrigatio

4 Transportation sector, including sales to railroads and railways.

6 Use of electricity that is 1) self-generated, 2) produced by either the same entity that consumes the , and 3) used in direct support of a service or industrial process located within the same

The sum of "Total Retail Sales" and "Direct Use."

8 "Commercial (Old)" is a discontinued series-data are for the commercial sector, excluding public street and highway lighting, interdepartmental sales, and other sales to public authorities.

to public authorities, agriculture and irrigation, and tray lighting, including railroads and railways.

$R=$ Revised. $P=$ Preliminary. E=Estimate. NA=Not available. $--=$ Not applicable.

Web Pages: annual data. - See http://www.eia.gov/totalenergy/data/annual/\#electricity for all annual data beginning in Sources: Residential and Industrial: - 1949-September 1977-Federal Power Commission, Form FPC-5, "Monthly Statement of Electric Operating Revenue and Income." • October 1977-February 1980-Federal Energy Regulatory Commission (FERC), Form FPC-5, "Monthly Statement of Electric Operating Revenue and Income." - March 1980-1982-FERC, Form FPC-5, "Electric Utility Company Monthly Statement." • 1983-U.S. Energy Information Administration (EIA), Form EIA-826, "Electric Utility (EPM) (Fobruary "2012), Table 5.1. Commercial: - 1949-2002-Estimated by ElA as the sum of "Commercial (Old)" and the non-transportation portion of "Other (Old)." See estimation methodology at http://www.eia.gov/state/seds/sep_use/notes/use elec.pdf. - 2003 forward-EIA, EPM (February 2012), Table 5.1. Transportation: • 1949-2002-Estimated by EIA as the transportation portion of "Other (Old)." See estimation methodology a Tol6 5. Direct Use: - 1989-1997-E/A Form ElA-867. "Annual Nonutility Power Producer Report" - 1998-EIA, Form EIA-860B, "Annual Electric Generator Report-Nonutility." • 1999-2010-EIA, Electric the percentage change in commercial and industrial net generation on Table 8.1. Commercial (Old) and
Other (OId): - 1949-2002-See sources for "Residential" and "Industrial." 


\section{Figure 8.10 Average Retail Prices of Electricity}

\section{Total, 1960-2011}

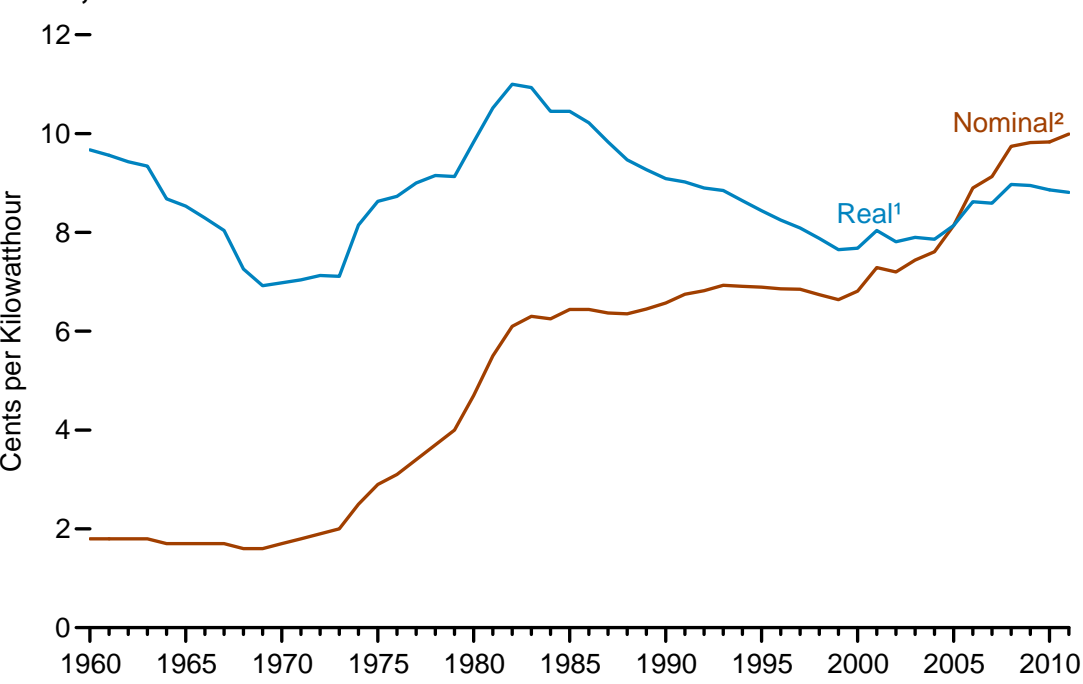

\section{By Sector, Nominal² Prices, 1960-2011}

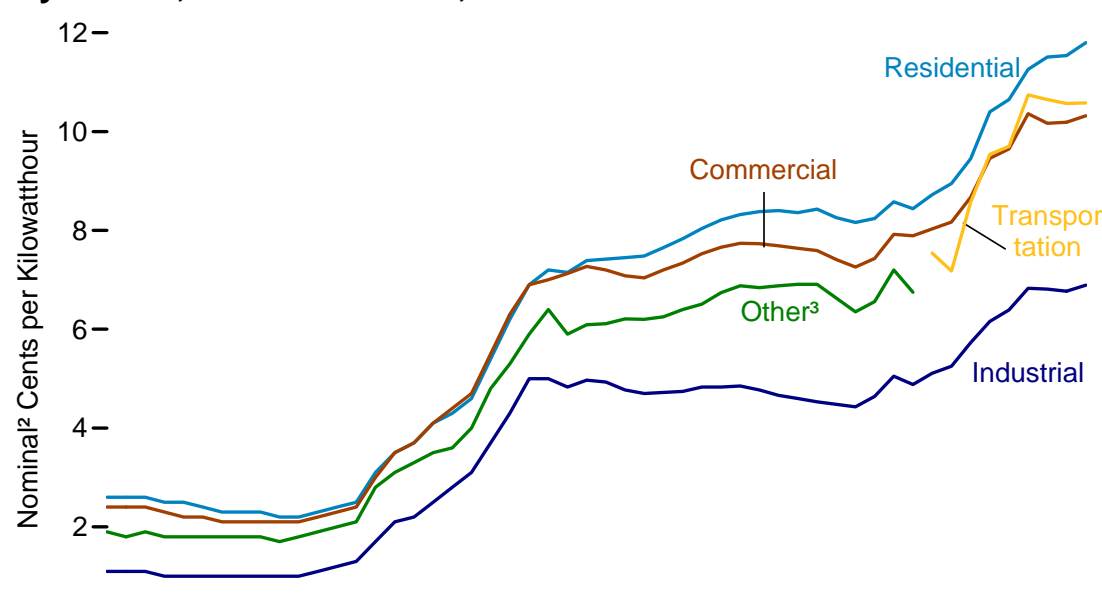

\section{By Sector, 2011}

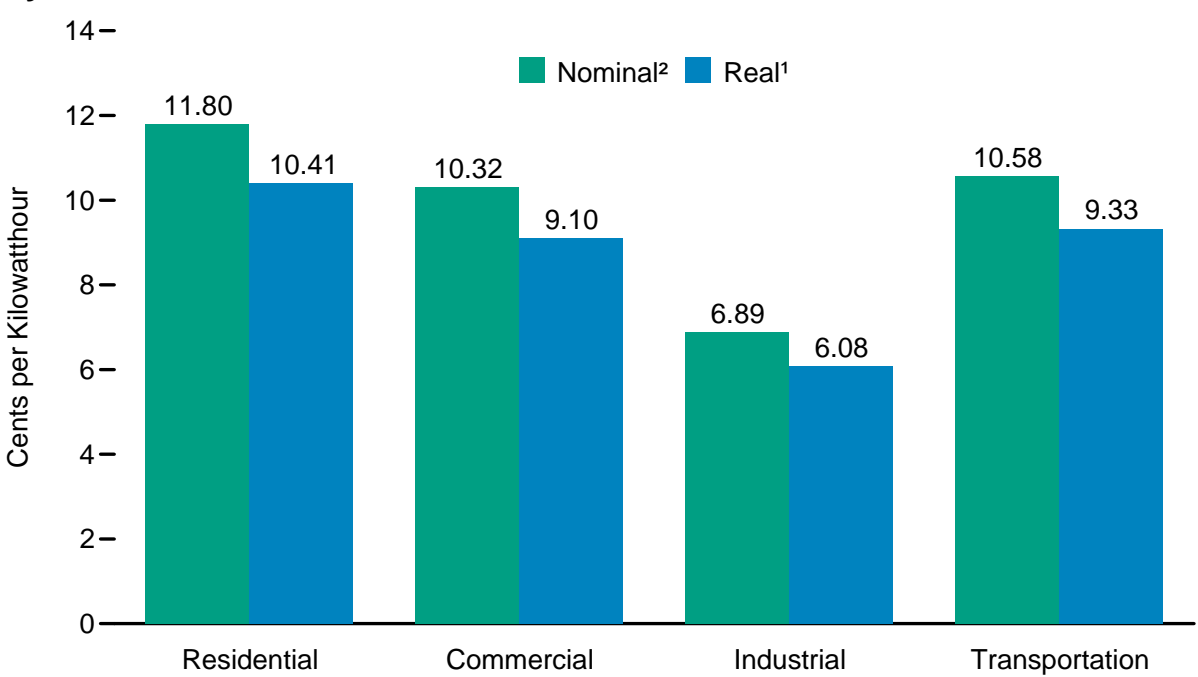

\section{By Sector, Real ${ }^{1}$ Prices, $1960-2011$}

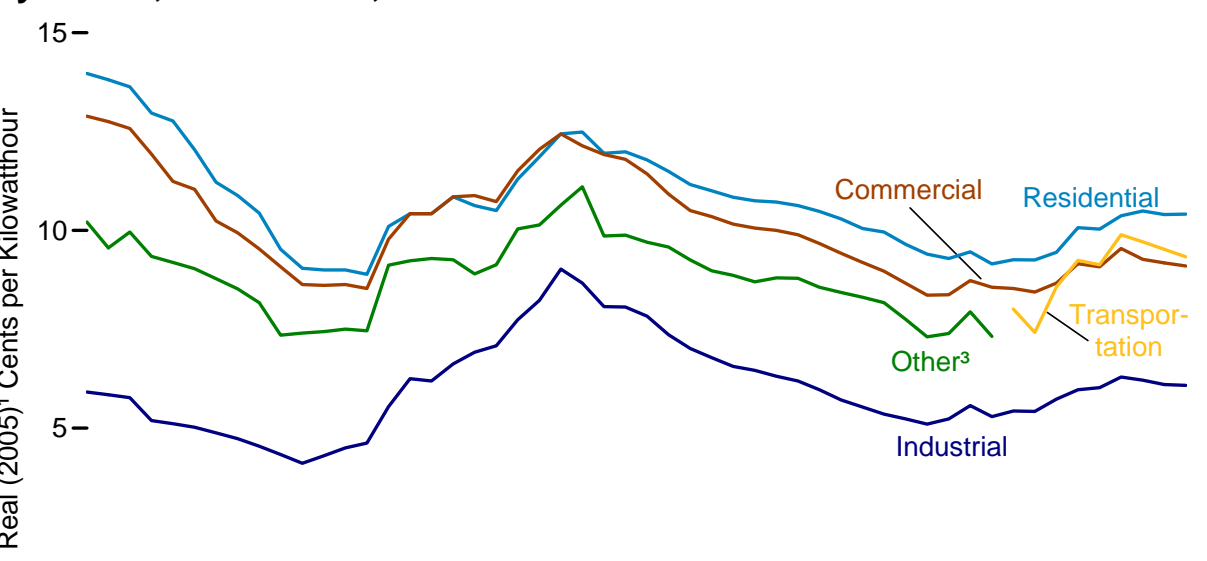

$\begin{array}{lllllllllll}0 & 1 \\ 1960 & 1965 & 1970 & 1975 & 1980 & 1985 & 1990 & 1995 & 2000 & 2005 & 2010\end{array}$

${ }^{3}$ Public street and highway lighting, interdepartmental sales, other sales to public authorities, agriculture and irrigation, and transportation including railroads and railways.

Note: Taxes are included.

Source: Table 8.10 
Table 8.10 Average Retail Prices of Electricity, Selected Years, 1960-2011

(Cents per Kilowatthour, Including Taxes)

\begin{tabular}{|c|c|c|c|c|c|c|c|c|c|c|c|c|}
\hline \multirow[b]{2}{*}{ Year } & \multicolumn{2}{|c|}{ Residential } & \multicolumn{2}{|c|}{ Commercial ${ }^{1}$} & \multicolumn{2}{|c|}{ Industrial $^{2}$} & \multicolumn{2}{|c|}{ Transportation $^{3}$} & \multicolumn{2}{|c|}{ Other ${ }^{4}$} & \multicolumn{2}{|c|}{ Total } \\
\hline & Nominal 5 & Real 6 & Nominal 5 & Real 6 & Nominal 5 & Real 6 & Nominal 5 & Real 6 & Nominal 5 & Real ${ }^{6}$ & Nominal 5 & Real 6 \\
\hline 1960 & 2.6 & 14.0 & 2.4 & 12.9 & 1.1 & 5.9 & NA & NA & 1.9 & 10.2 & 1.8 & 9.7 \\
\hline 1965 & 2.4 & $R_{12} .0$ & 2.2 & 11.0 & 1.0 & 5.0 & NA & NA & 1.8 & 9.0 & 1.7 & 8.5 \\
\hline 1966 & 2.3 & $\begin{array}{l}11.2 \\
109\end{array}$ & 2.1 & $\begin{array}{r}10.2 \\
899\end{array}$ & 1.0 & 4.9 & NA & NA & 1.8 & 8.8 & 1.7 & $\begin{array}{r}8.3 \\
\mathrm{R} 80\end{array}$ \\
\hline $\begin{array}{l}1967 \\
1968\end{array}$ & $\begin{array}{l}2.3 \\
2.3\end{array}$ & $\begin{array}{r}10.9 \\
R 10.4\end{array}$ & $\begin{array}{l}2.1 \\
2.1\end{array}$ & $\begin{array}{l}39.9 \\
9.5\end{array}$ & $\begin{array}{l}1.0 \\
1.0\end{array}$ & $\begin{array}{l}4.7 \\
4.5\end{array}$ & $\begin{array}{l}\text { NA } \\
\text { NA }\end{array}$ & $\begin{array}{l}\text { NA } \\
\text { NA }\end{array}$ & $\begin{array}{l}1.8 \\
1.8\end{array}$ & $\begin{array}{l}8.5 \\
82\end{array}$ & 1.7 & $\begin{array}{l}\mathrm{R} 8.0 \\
7.3\end{array}$ \\
\hline 1969 & 2.2 & 9.5 & 2.1 & 9.1 & 1.0 & $\begin{array}{l}4.5 \\
4.3\end{array}$ & NA & NA & $\begin{array}{l}1.8 \\
1.7 \\
-1\end{array}$ & 7.4 & $\begin{array}{l}1.6 \\
1.6\end{array}$ & $\begin{array}{l}1.3 \\
6.9\end{array}$ \\
\hline 1970 & 2.2 & R9.0 & 2.1 & 8.6 & 1.0 & 4.1 & NA & NA & 1.8 & 7.4 & 1.7 & 7.0 \\
\hline 1971 & 2.3 & 9.0 & 2.2 & 8.6 & 1.1 & 4.3 & NA & NA & 1.9 & 7.4 & 1.8 & R7.0 \\
\hline 1972 & 2.4 & 9.0 & 2.3 & 8.6 & 1.2 & 4.5 & NA & NA & 2.0 & 7.5 & 1.9 & 7.1 \\
\hline 1973 & 2.5 & 8.9 & 2.4 & 8.5 & 1.3 & 4.6 & NA & NA & 2.1 & 7.5 & 2.0 & 7.1 \\
\hline 1974 & 3.1 & 10.1 & 3.0 & 9.8 & 1.7 & 5.5 & NA & NA & 2.8 & 9.1 & 2.5 & 8.2 \\
\hline 1975 & 3.5 & 10.4 & 3.5 & 10.4 & 2.1 & 6.3 & NA & NA & 3.1 & 9.2 & 2.9 & 8.6 \\
\hline 1976 & 3.7 & 10.4 & 3.7 & 10.4 & 2.2 & 6.2 & NA & NA & 3.3 & 9.3 & 3.1 & 8.7 \\
\hline 1977 & 4.1 & 10.9 & 4.1 & 10.9 & 2.5 & 6.6 & $\mathrm{NA}$ & $\mathrm{NA}$ & 3.5 & 9.3 & 3.4 & 9.0 \\
\hline 1978 & 4.3 & 10.6 & 4.4 & 10.9 & 2.8 & 6.9 & NA & NA & 3.6 & 8.9 & 3.7 & 9.2 \\
\hline 1979 & & 10.5 & 4.7 & 10.7 & 3.1 & 7.1 & NA & NA & 4.0 & 9.1 & 4.0 & 9.1 \\
\hline 1980 & 5.4 & 11.3 & 5.5 & 11.5 & 3.7 & R7.7 & $\mathrm{NA}$ & NA & 4.8 & $R_{10.0}$ & 4.7 & 9.8 \\
\hline 1981 & 6.2 & 11.9 & 6.3 & 12.1 & 4.3 & 8.2 & $\mathrm{NA}$ & $\mathrm{NA}$ & 5.3 & $\mathrm{R}_{10.1}$ & 5.5 & 10.5 \\
\hline 1982 & 6.9 & R12.4 & 6.9 & $R_{12.4}$ & 5.0 & $\begin{array}{l}0.4 \\
9.0\end{array}$ & NA & NA & 5.9 & $\mathrm{R}_{10.6}$ & 6.1 & 11.0 \\
\hline 1983 & $\begin{array}{l}.9 .9 \\
7.2\end{array}$ & 12.5 & $\begin{array}{l}.9 \\
7.0\end{array}$ & $\mathrm{R}_{12.1}$ & $\begin{array}{l}.0 \\
5.0\end{array}$ & $\begin{array}{l}9.0 \\
8.7\end{array}$ & NA & NA & $\begin{array}{l}5.9 \\
6.4\end{array}$ & 11.1 & $\begin{array}{l}.1 \\
6.3\end{array}$ & 10.9 \\
\hline 1984 & 7.15 & $R_{11.95}$ & 7.13 & $R_{11.92}$ & 4.83 & $\begin{array}{r}8.1 \\
\mathrm{R}_{8} .07\end{array}$ & NA & NA & $\begin{array}{l}\text { l.4 } \\
5.90\end{array}$ & R9.86 & $\begin{array}{l}.3 .3 \\
6.25\end{array}$ & R10.45 \\
\hline 1985 & $\begin{array}{l}.10 \\
7.39\end{array}$ & R11.99 & $\begin{array}{l}.13 \\
7.27\end{array}$ & $R_{11.80}$ & $\begin{array}{l}4.03 \\
4.97\end{array}$ & R8.06 & NA & NA & $\begin{array}{l}6.09 \\
6.09\end{array}$ & $\begin{array}{r}\mathrm{R} 9.86 \\
\end{array}$ & $\begin{array}{l}\text {. } 65 \\
6.44\end{array}$ & R10.45 \\
\hline 1986 & $\begin{array}{l}.3 .39 \\
7.42\end{array}$ & $R_{11.78}$ & $\begin{array}{l}.7 .27 \\
7.20\end{array}$ & $R_{11.43}$ & $\begin{array}{l}4.97 \\
4.93\end{array}$ & $\begin{array}{l}8.06 \\
7.83\end{array}$ & NA & NA & $\begin{array}{l}6.09 \\
6.11\end{array}$ & $\begin{array}{r}\text { R9. } \\
\text { Ro }\end{array}$ & $\begin{array}{l}.44 \\
6.44\end{array}$ & $\begin{array}{l}\text { R10.25 } \\
\text { R }\end{array}$ \\
\hline 1987 & $\begin{array}{l}7.42 \\
7.45\end{array}$ & $R_{11.49}$ & $\begin{array}{l}1.20 \\
7.08\end{array}$ & $\mathrm{R}_{10.92}$ & $\begin{array}{l}4.75 \\
4.77\end{array}$ & $\begin{array}{r}8.03 \\
87.36\end{array}$ & NA & NA & $\begin{array}{l}6.11 \\
6.21\end{array}$ & R9.58 & $\begin{array}{l}. .44 \\
6.37\end{array}$ & R9.83 \\
\hline 1988 & 7.48 & $R_{11.16}$ & 7.04 & $\mathrm{R}_{10.50}$ & 4.70 & R7.01 & NA & $\mathrm{NA}$ & 6.20 & R9. 25 & 6.35 & R9. 47 \\
\hline 1989 & 7.65 & 11.00 & 7.20 & $\mathrm{R}_{10.35}$ & 4.72 & $\mathrm{R}_{6} .78$ & $\mathrm{NA}$ & $\mathrm{NA}$ & 6.25 & R8.98 & 6.45 & $\begin{array}{r}R 9.27 \\
\text { R }\end{array}$ \\
\hline 1990 & 7.83 & 10.84 & 7.34 & $R_{10.16}$ & 4.74 & $R_{6} .56$ & $\mathrm{NA}$ & $\mathrm{NA}$ & 6.40 & 8.86 & 6.57 & R9.09 \\
\hline 1991 & 8.04 & 10.75 & 7.53 & $R_{10.06}$ & 4.83 & 6.46 & NA & $\mathrm{NA}$ & 6.51 & R8.70 & 6.75 & R9. 02 \\
\hline 1992 & 8.21 & $\mathrm{R}_{10.72}$ & 7.66 & $\mathrm{R}_{10.00}$ & 4.83 & 6.31 & $\mathrm{NA}$ & $\mathrm{NA}$ & 6.74 & R8.80 & 6.82 & R8.90 \\
\hline 1993 & $\begin{array}{l}0.21 \\
8.32\end{array}$ & R10.63 & 7.74 & 9.89 & 4.85 & $\begin{array}{r}8.31 \\
R_{6} .19\end{array}$ & NA & NA & $\begin{array}{l}0.14 \\
6.88\end{array}$ & $\begin{array}{l}0.00 \\
88.79\end{array}$ & $\begin{array}{l}6.02 \\
6.93\end{array}$ & R8.85 \\
\hline 1994 & 8.38 & $\mathrm{R}_{10.48}$ & 7.73 & R. 9.67 & 4.77 & 5.97 & NA & $\mathrm{NA}$ & 6.84 & 8.56 & 6.91 & R8.64 \\
\hline 1995 & 8.40 & $R_{10.29}$ & 7.69 & R9. 42 & 4.66 & R5.71 & NA & NA & 6.88 & R8.43 & 6.89 & R8.44 \\
\hline 1996 & 8.36 & $R_{10.05}$ & 7.64 & R9. 19 & 4.60 & R5.53 & $\mathrm{NA}$ & NA & 6.91 & R8.31 & 6.86 & R8.25 \\
\hline 1997 & 8.43 & R9.96 & 7.59 & R8.97 & 4.53 & R5.35 & NA & NA & 6.91 & 8.17 & 6.85 & R8.09 \\
\hline 1998 & 8.26 & R9.65 & 7.41 & R8.66 & 4.48 & $\mathrm{R}_{5} .23$ & NA & NA & 6.63 & 7.75 & 6.74 & 7.88 \\
\hline 1999 & 8.16 & 9.40 & 7.26 & R8. 86 & 4.43 & R5.10 & NA & NA & $\begin{array}{l}6.00 \\
6.35\end{array}$ & R7.31 & $\begin{array}{l}6.64 \\
6.64\end{array}$ & $\begin{array}{l}7.60 \\
7.65\end{array}$ \\
\hline 2000 & 8.24 & R9. 29 & 7.43 & R8.37 & 4.64 & 5.23 & NA & NA & 6.56 & R7.39 & 6.81 & 7.68 \\
\hline 2001 & 8.58 & 9.46 & 7.92 & R8.73 & 5.05 & 5.57 & NA & NA & $\begin{array}{l}7.00 \\
7.20\end{array}$ & 7.94 & $\begin{array}{l}7.01 \\
7.29\end{array}$ & 8.04 \\
\hline 2002 & 8.44 & $\begin{array}{r}\text { R9. } 15 \\
\text {. }\end{array}$ & 7.89 & R8.56 & 4.88 & $\begin{array}{r}\text { R5. } \\
\text { (1) }\end{array}$ & NA & NA & 6.75 & R7.32 & 7.20 & R7. 81 \\
\hline 2003 & 8.72 & $\begin{array}{r}\text { R9. } 26 \\
\text { la }\end{array}$ & 8.03 & 8.53 & 5.11 & 5.43 & 7.54 & 8.01 & -1 & -- & 7.44 & R7. \\
\hline 2004 & 8.95 & 9.25 & 8.17 & 8.44 & 5.25 & $\begin{array}{r}R 5.42 \\
\text { R }\end{array}$ & 7.18 & 7.42 & -- & -- & 7.61 & 7.86 \\
\hline 2005 & 9.45 & 9.45 & 8.67 & 8.67 & 5.73 & 5.73 & 8.57 & 8.57 & -- & -- & 8.14 & 8.14 \\
\hline 2006 & 10.40 & 10.07 & 9.46 & 9.16 & 6.16 & 5.97 & 9.54 & 9.24 & -- & -- & 8.90 & 8.62 \\
\hline 2007 & 10.65 & $\mathrm{R}_{10} .03$ & 9.65 & 9.08 & 6.39 & R6.02 & 9.70 & 9.13 & -- & -- & 9.13 & 8.59 \\
\hline 2008 & 11.26 & $\begin{array}{r}10.37 \\
\end{array}$ & 10.36 & 9.54 & 6.83 & 6.29 & 10.74 & 9.89 & -- & -- & 9.74 & 8.97 \\
\hline 2009 & 11.51 & $R_{10} .49$ & 10.17 & R9. 27 & 6.81 & 6.21 & 10.65 & R9.71 & -- & -- & 9.82 & R8.95 \\
\hline 2010 & R11.54 & $\mathrm{R}_{10.40}$ & $R_{10.19}$ & R9. 18 & $\mathrm{R}_{6} .77$ & $R_{6.10}$ & $R_{10.57}$ & R9.52 & -- & -- & R9.83 & R8.86 \\
\hline $2011^{P}$ & 11.80 & 10.41 & 10.32 & 9.10 & 6.89 & 6.08 & 10.58 & 9.33 & -- & -- & 9.99 & 8.81 \\
\hline
\end{tabular}

1 Commercial sector. For 1960-2002, prices exclude public street and highway lighting, interdepartmental sales, and other sales to public authorities.

Industrial sector. For 1960-2002, prices exclude agriculture and irrigation.

Transportation sector, including railroads and railways.

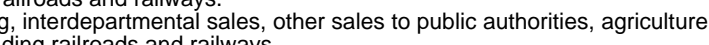
5 See "Nominal Price" in Glossary.

6 In chained (2005) dollars, calculated by using gross domestic product implicit price deflators in Table

D1. See "Chained Dollars" in Glossary.
R=Revised. P=Preliminary. NA=Not available. $--=$ =Not applicable.
Notes: - Beginning in 2003 the category "Other" has been replaced by "Transportation," and the categories "Commercial" and "Industrial" have been redefined. - Data represent revenue from electricity retail sales divided by electricity retail sales. - Prices include State and local taxes, energy or demand charges, customer service charges, environmental surcharges, franchise fees, fuel adjustments, and other miscellaneous charges applied to end-use customers during normal billing operations. Prices do not include

previous reporting periods. - Through 1979 , data are for Classes A and B privately owned electric utilities with between $\$ 1$ million and $\$ 2.5$ million.) For $1980-1982$, data are for selected Class A utilities whose

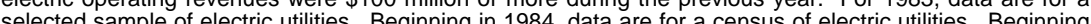
in 1996, data also include energy service providers selling to retail customers. Web Pages: - See http://www.eia.gov/totalenergy/data/monthly/\#prices for updated monthly and annual data. - See $h$ ttp:///www.eia.gov/totalenergy/data/annual/\#electricity for all annual data beginning in 1960. See http://www.eia.gov/electricity/ for related information.

ssion, Form FPC-5, "Monthly Statement of Commission (FERC) Form FPC-5, "Monthly Statement of Electric Operating Revenues and Income" - March 1980-1982-FERC, Form FERC-5, "Electric Utility Company Monthly Statement. 1983-U.S. Energy Information Administration (EIA), Form EIA-826, "Electric Utility Company Monthly Statement." • 1984-1996-EIA, Form EIA-861, "Annual Electric Utility Report." • 1997 forward-EIA, 
Figure 8.11a Electric Net Summer Capacity, Total (All Sectors)

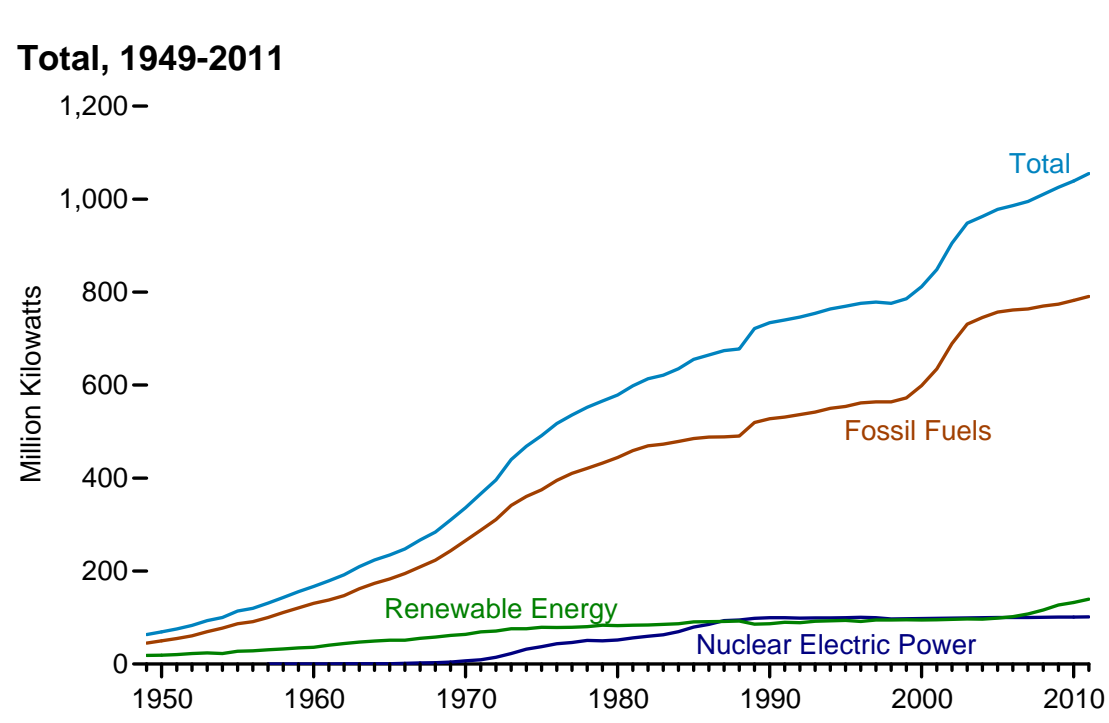

By Major Category, 2011

900-

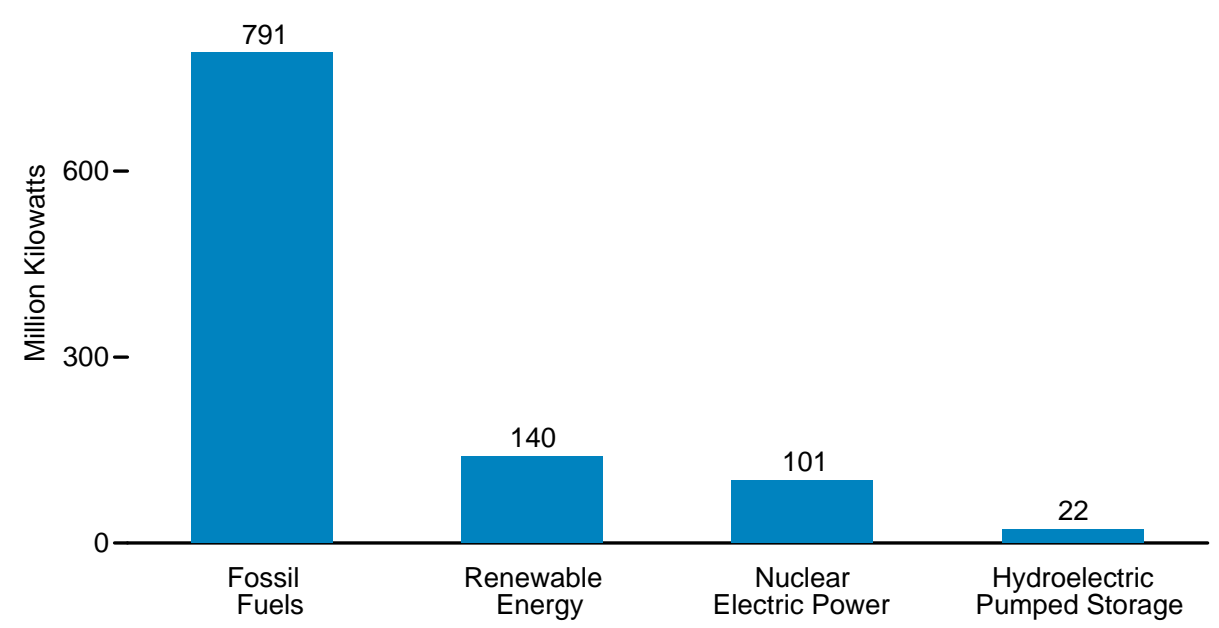

By Source, 2011

$500-$

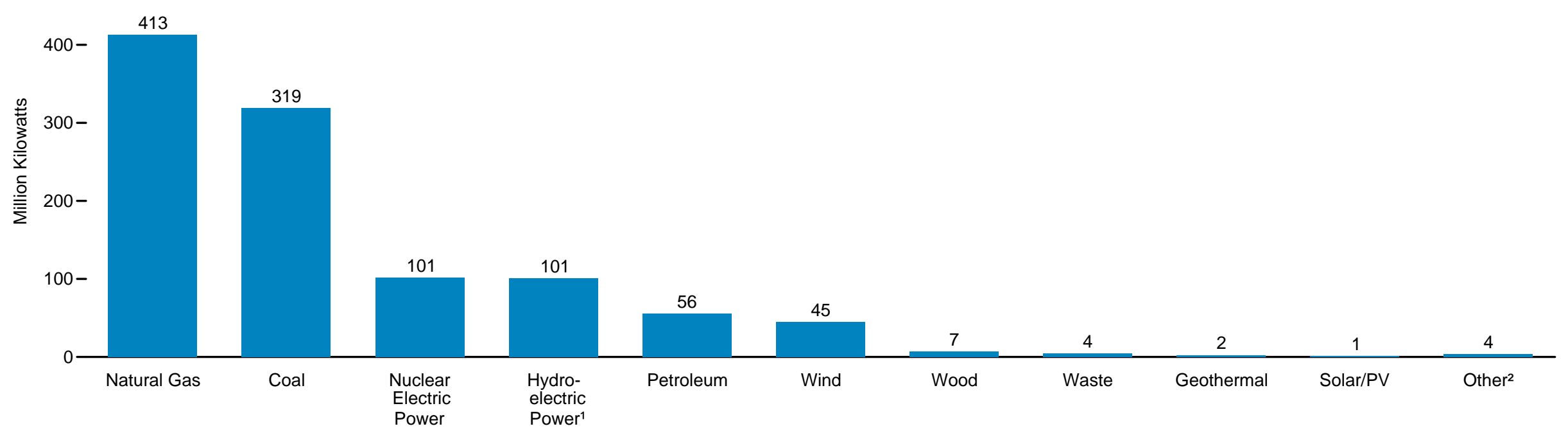

${ }^{1}$ Conventional and pumped storage.

${ }^{2}$ Blast furnace gas, propane gas, other manufactured and waste gases derived from fossil fuels, batteries, chemicals, hydrogen, pitch, purchased steam, sulfur, and miscellaneous technologies.

Source: Table 8.11a. 


\section{Figure 8.11b Electric Net Summer Capacity by Sector}

\section{Total (All Sectors) and Sectors, 1989-2011}

$1,200-$

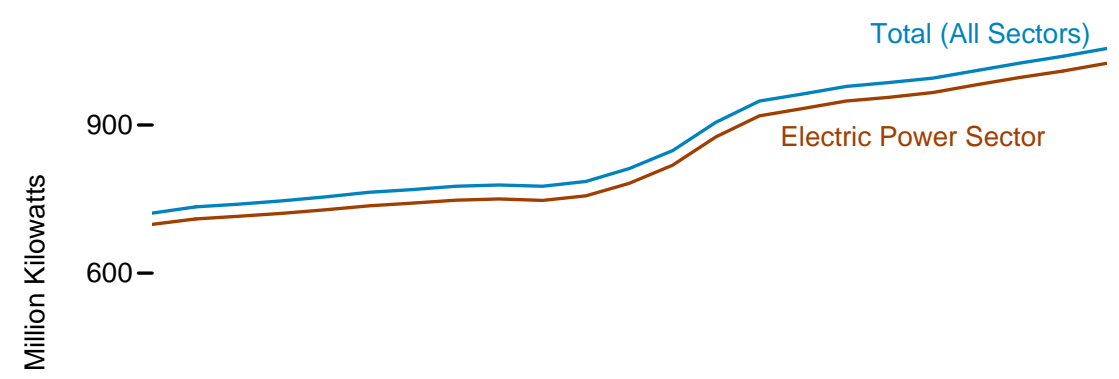

$300-$

Commercial and Industrial

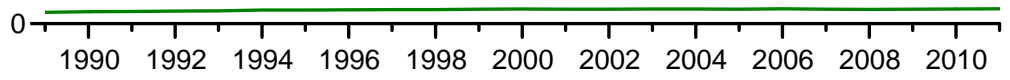

\section{Commercial Sector, 2011}

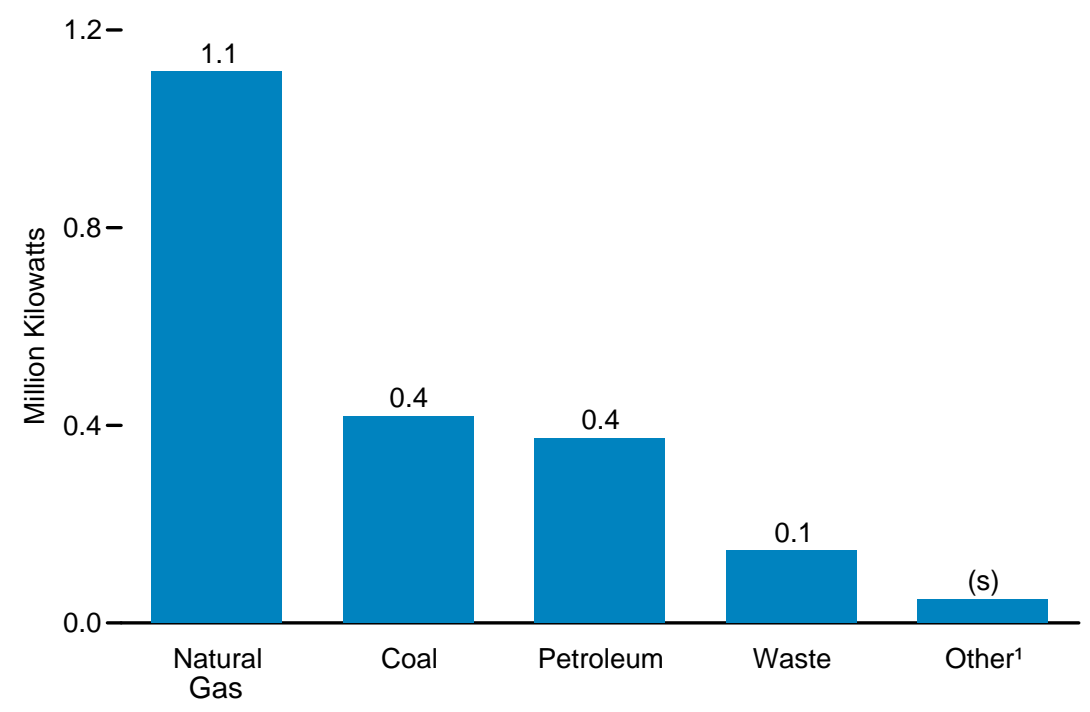

Electric Power Sector by Plant Type, 1989-2011

$1,250-$

$1,000-$

Electricity-Only Plants

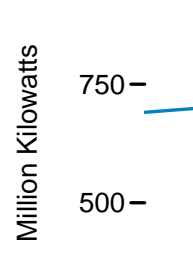

$250-$

Combined-Heat-and-Power Plants

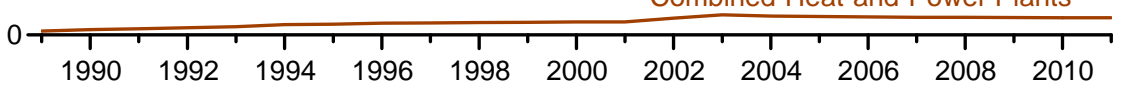

Industrial Sector, 2011

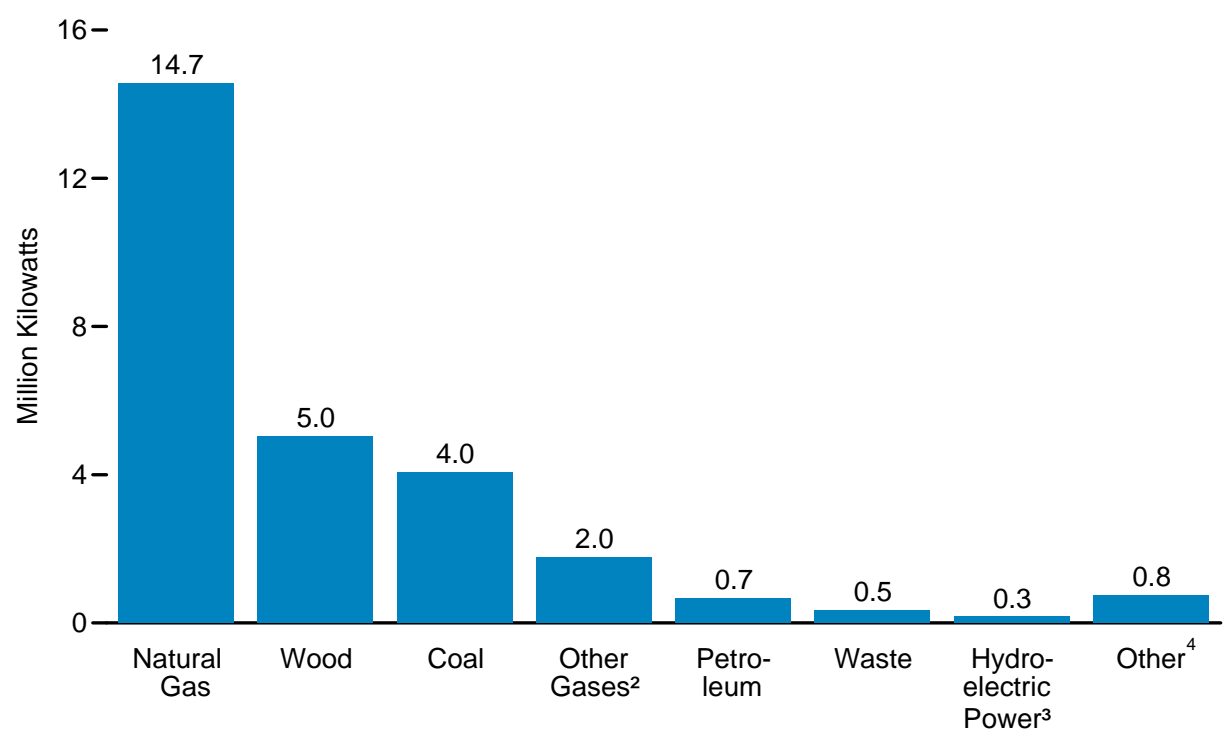

${ }^{1}$ Conventional hydroelectric power, solar/PV, wood, wind, blast furnace gas, propane gas, and other manufactured and waste gases derived from fossil fuels, batteries, chemicals, hydrogen, pitch, purchased steam, sulfur, and miscellaneous technologies.

${ }^{2}$ Blast furnace gas, propane gas, and other manufactured and waste gases derived from

${ }^{3}$ Conventional hydroelectric power.

${ }^{4}$ Solar/PV, wind, batteries, chemicals, hydrogen, pitch, purchased steam, sulfur, and miscellaneous technologies.

(s)=Less than 0.05 million kilowatts fossil fuels 
Table 8.11a Electric Net Summer Capacity: Total (All Sectors), Selected Years, 1949-2011

(Sum of Tables $8.11 \mathrm{~b}$ and $8.11 \mathrm{~d}$; Million Kilowatts)

\begin{tabular}{|c|c|c|c|c|c|c|c|c|c|c|c|c|c|c|c|c|}
\hline \multirow[b]{3}{*}{ Year } & \multicolumn{5}{|c|}{ Fossil Fuels } & \multirow{3}{*}{$\begin{array}{l}\text { Nuclear } \\
\text { Electric } \\
\text { Power }\end{array}$} & \multirow{3}{*}{$\begin{array}{l}\text { Hydro- } \\
\text { electric } \\
\text { Pumped } \\
\text { Storage }\end{array}$} & \multicolumn{7}{|c|}{ Renewable Energy } & \multirow[b]{3}{*}{ Other ${ }^{9}$} & \multirow[b]{3}{*}{ Total } \\
\hline & & & & & & & & \multirow{2}{*}{\begin{tabular}{|c} 
Conventional \\
Hydroelectric \\
Power $^{5}$
\end{tabular}} & \multicolumn{2}{|c|}{ Biomass } & \multirow{2}{*}{$\begin{array}{c}\text { Geo- } \\
\text { thermal }\end{array}$} & \multirow[b]{2}{*}{ Solar/PV ${ }^{8}$} & \multirow[b]{2}{*}{ Wind } & \multirow[b]{2}{*}{ Total } & & \\
\hline & Coal ${ }^{1}$ & Petroleum ${ }^{2}$ & Gas $^{3}$ & Gases ${ }^{4}$ & Total & & & & Wood $^{6}$ & Waste ${ }^{7}$ & & & & & & \\
\hline 1949 & NA & NA & NA & NA & 44.9 & 0.0 & $\left({ }^{5}\right)$ & 18.5 & (s) & $\left({ }^{10}\right)$ & NA & NA & NA & 18.5 & NA & 63.4 \\
\hline 1950 & NA & NA & NA & NA & 50.0 & .0 & $\left({ }^{5}\right)$ & 19.2 & (s) & $(10)$ & NA & NA & NA & 19.2 & NA & 69.2 \\
\hline 1955 & NA & NA & NA & NA & 86.8 & .0 & $(5)$ & 27.4 & (s) & $(10)$ & NA & NA & NA & 27.4 & NA & 114.2 \\
\hline 1960 & NA & NA & NA & NA & 130.8 & .4 & $(5)$ & 35.8 & .1 & $(10)$ & (s) & NA & NA & 35.9 & NA & 167.1 \\
\hline 1965 & NA & NA & NA & NA & $\begin{array}{l}182.9 \\
182.9\end{array}$ & .8 & $(5)$ & $\begin{array}{l}51.0 \\
51.0\end{array}$ & .1 & (10) & (s) & NA & NA & 51.1 & NA & 234.8 \\
\hline 1970 & NA & NA & NA & NA & 265.4 & 7.0 & $(5)$ & 63.8 & .1 & $(10)$ & .1 & NA & NA & 63.9 & NA & 336.4 \\
\hline 1975 & NA & NA & NA & NA & 375.1 & 37.3 & $(5)$ & 78.4 & .1 & $\left({ }^{10}\right)$ & .5 & NA & NA & 79.0 & NA & 491.3 \\
\hline 1976 & NA & NA & NA & NA & 394.8 & 43.8 & $\left({ }^{5}\right)$ & 78.0 & .1 & $(10)$ & .5 & NA & NA & 78.6 & NA & 517.2 \\
\hline 1977 & NA & NA & NA & NA & 410.4 & 46.3 & $\left({ }^{5}\right)$ & 78.6 & .1 & $(10)$ & .5 & NA & NA & 79.2 & NA & 535.9 \\
\hline 1978 & NA & NA & NA & NA & 420.8 & 50.8 & $(5)$ & $\begin{array}{l}79.9 \\
\end{array}$ & .1 & $(10)$ & .5 & NA & NA & 80.5 & NA & 552.1 \\
\hline 1979 & NA & NA & NA & NA & 432.1 & 49.7 & $\left(5^{5}\right)$ & 82.9 & .1 & $(10)$ & .7 & NA & NA & 83.6 & NA & 565.5 \\
\hline 1980 & NA & NA & NA & NA & 444.1 & 51.8 & $(5)$ & 81.7 & .1 & $\left({ }^{(10}\right)$ & .9 & NA & NA & 82.7 & NA & 578.6 \\
\hline 1981 & NA & NA & NA & NA & 458.9 & 56.0 & $\left({ }^{5}\right)$ & 82.4 & .1 & $\left({ }^{10}\right)$ & .9 & NA & (s) & 83.4 & NA & 598.3 \\
\hline 1982 & NA & NA & NA & NA & 469.6 & 60.0 & $\left(5^{5}\right)$ & 83.0 & .1 & $(10)$ & 1.0 & NA & (s) & 84.1 & NA & 613.7 \\
\hline 1983 & NA & NA & NA & NA & $\begin{array}{l}47.0 \\
472.8\end{array}$ & 63.0 & (5) & $\begin{array}{l}8.0 \\
83.9\end{array}$ & .2 & (10) & 1.2 & NA & $\begin{array}{l}\text { (s) } \\
\text { (s) }\end{array}$ & $\begin{array}{l}8.1 \\
85.3\end{array}$ & NA & 621.1 \\
\hline 1984 & NA & NA & NA & NA & 478.6 & $\begin{array}{l}69.0 \\
69.7\end{array}$ & $(5)$ & $\begin{array}{l}\text { o.j } \\
85.3\end{array}$ & .3 & (10) & $\begin{array}{l}1.2 \\
1.2\end{array}$ & $(11)$ & $\begin{array}{l}\text { (s) } \\
\text { (s) }\end{array}$ & $\begin{array}{l}86.0 \\
86.9\end{array}$ & NA & 635.1 \\
\hline 1985 & NA & NA & NA & NA & 485.0 & 79.4 & $(5)$ & 88.9 & .2 & $\begin{array}{l}1 \\
1 \\
\end{array}$ & 1.6 & (11) & (s) & 90.8 & NA & 655.2 \\
\hline 1986 & NA & NA & NA & NA & 488.3 & 85.2 & $\left({ }^{5}\right)$ & 89.3 & .2 & .2 & 1.6 & $\left({ }^{(11)}\right)$ & (s) & 91.2 & NA & 664.8 \\
\hline 1987 & NA & NA & NA & NA & 488.8 & 93.6 & $(5)$ & 89.7 & .2 & .2 & 1.5 & $(11)$ & (s) & 91.7 & NA & 674.1 \\
\hline 1988 & NA & NA & NA & NA & $\begin{array}{r}40.0 \\
490.6\end{array}$ & $\begin{array}{l}94.0 \\
94.7\end{array}$ & (5) & $\begin{array}{l}90.1 \\
90.3\end{array}$ & .2 & .2 & $\begin{array}{l}1.5 \\
\end{array}$ & (11) & $\begin{array}{l}\text { (s) } \\
\text { (s) }\end{array}$ & 92.4 & NA & 677.7 \\
\hline $1989^{12}$ & 303.1 & 79.1 & 135.7 & 1.5 & 519.4 & 98.2 & 18.1 & 74.1 & 5.2 & 2.1 & 2.1 & $\begin{array}{l}1 \\
1\end{array}$ & 1.5 & 85.7 & .5 & 721.8 \\
\hline 1990 & 307.4 & 77.9 & 140.8 & 1.6 & 527.8 & 99.6 & 19.5 & 73.9 & 5.5 & 2.5 & 2.7 & .3 & 1.8 & 86.8 & .5 & 734.1 \\
\hline 1991 & 307.4 & 74.2 & 147.6 & 2.1 & 531.4 & 99.6 & 18.4 & 76.0 & 6.1 & 2.9 & 2.6 & .3 & 1.9 & 89.9 & .5 & 739.9 \\
\hline 1992 & 309.4 & 73.1 & $\begin{array}{l}152.2 \\
\end{array}$ & 2.1 & 536.7 & 99.0 & 21.2 & 74.8 & 6.2 & 3.0 & 2.9 & .3 & 1.8 & 89.1 & .5 & 746.5 \\
\hline 1993 & 310.1 & 71.1 & 158.6 & 1.9 & 541.8 & 99.0 & 21.1 & 77.4 & 6.5 & 3.1 & 2.9 & .3 & 1.8 & 92.1 & .5 & 754.6 \\
\hline 1994 & 311.4 & 71.7 & 164.8 & 2.1 & 550.0 & 99.1 & 21.2 & 78.0 & 6.7 & 3.3 & 3.0 & .3 & 1.7 & 93.1 & .5 & 764.0 \\
\hline 1995 & 311.4 & 66.6 & 174.5 & 1.7 & 554.2 & 99.5 & 21.4 & 78.6 & 6.7 & 3.5 & $\begin{array}{l}3.0 \\
3.0\end{array}$ & .3 & 1.7 & 93.9 & .5 & 769.5 \\
\hline 1996 & 313.4 & 72.5 & 174.1 & 1.7 & 561.7 & 100.8 & 21.1 & 76.4 & 6.8 & 3.6 & 2.9 & .3 & 1.7 & 91.7 & .5 & 775.9 \\
\hline 1997 & 313.6 & 72.5 & 176.5 & 1.5 & 564.1 & 99.7 & 19.3 & 79.4 & 6.9 & 3.6 & 2.9 & .3 & 1.6 & 94.8 & .8 & 778.6 \\
\hline 1998 & 315.8 & 66.3 & 180.3 & 1.5 & 563.9 & 97.1 & 19.5 & $\begin{array}{l}79.4 \\
79.2\end{array}$ & 6.8 & 3.7 & 2.9 & .3 & 1.7 & 94.6 & .0 & 775.9 \\
\hline 1999 & 315.5 & $\begin{array}{l}0.0 \\
60.1\end{array}$ & 195.1 & $\begin{array}{l}1.5 \\
1.9\end{array}$ & 572.6 & 97.4 & 19.6 & 79.4 & $\begin{array}{l}.0 .0 \\
6.8\end{array}$ & $\begin{array}{l}3.1 \\
3.7\end{array}$ & $\begin{array}{l}.9 \\
2.8\end{array}$ & .4 & 2.3 & $\begin{array}{l}45.0 \\
95.3\end{array}$ & $\begin{array}{l}.0 \\
1.0\end{array}$ & 785.9 \\
\hline 2000 & 315.1 & 61.8 & 219.6 & 2.3 & 598.9 & 97.9 & 19.5 & 79.4 & 6.1 & 3.9 & 2.8 & .4 & 2.4 & 94.9 & .5 & 811.7 \\
\hline 2001 & 314.2 & 66.2 & 252.8 & 1.7 & 634.9 & 98.2 & 19.7 & 78.9 & 5.9 & $\begin{array}{l}3.5 \\
3.7\end{array}$ & 2.2 & .4 & $\begin{array}{l}3.4 \\
3.9\end{array}$ & 95.0 & .5 & 848.3 \\
\hline 2002 & 315.4 & 59.7 & 312.5 & 2.0 & 689.5 & 98.7 & 20.4 & 79.4 & 5.8 & 3.8 & 2.3 & .4 & 4.4 & 96.1 & .7 & 905.3 \\
\hline 2003 & 313.0 & 60.7 & 355.4 & 2.0 & 731.2 & 99.2 & 20.5 & 78.7 & 5.9 & 3.8 & 2.1 & .4 & 6.0 & 96.8 & .7 & 948.4 \\
\hline 2004 & 313.0 & 59.1 & $\begin{array}{l}371.0 \\
371.0\end{array}$ & 2.3 & $\begin{array}{l}745.4 \\
\text {. }\end{array}$ & 99.6 & 20.8 & $\begin{array}{l}7.1 \\
77.6\end{array}$ & 6.2 & $\begin{array}{l}3.0 \\
3.5\end{array}$ & 2.2 & .4 & 6.5 & 96.4 & .7 & 962.9 \\
\hline 2005 & 313.4 & 58.5 & 383.1 & 2.1 & 757.1 & 100.0 & 21.3 & 77.5 & $\begin{array}{l}6.2 \\
6.2\end{array}$ & 3.6 & 2.3 & .4 & 8.7 & 98.7 & .9 & 978.0 \\
\hline 2006 & 313.0 & 58.1 & 388.3 & 2.3 & 761.6 & 100.3 & 21.5 & 77.8 & 6.4 & $\begin{array}{l}3.0 \\
3.7\end{array}$ & 2.3 & .4 & 11.3 & 101.9 & .9 & 986.2 \\
\hline 2007 & 312.7 & 56.1 & 392.9 & 2.3 & 764.0 & 100.3 & 21.9 & 77.9 & 6.7 & 4.1 & 2.2 & .5 & 16.5 & 108.0 & .8 & 994.9 \\
\hline 2008 & 313.3 & 57.4 & 397.5 & 2.0 & 770.2 & 100.8 & 21.9 & 77.9 & 6.9 & 4.2 & 2.2 & .5 & 24.7 & 116.4 & .9 & $1,010.2$ \\
\hline 2009 & 314.3 & 56.8 & 4013 & 19 & 7743 & 1010 & 22.2 & 78.5 & 6.9 & 4.3 & 24 & 6 & 343 & 1271 & 9 & 1.025 \\
\hline 2010 & $\begin{array}{r}r 14.3 \\
\text { R316.8 }\end{array}$ & $\begin{array}{r}50.0 \\
\text { R55.6 }\end{array}$ & $\begin{array}{r}401.0 \\
\text { R } 407.0\end{array}$ & $\begin{array}{r}r .9 \\
R_{2.7}\end{array}$ & $\begin{array}{r}\text { R782. } \\
\end{array}$ & R101.2 & $\mathrm{R}_{22.2}^{22.2}$ & $\begin{array}{r}10.5 \\
\text { R78.8 }\end{array}$ & $\begin{array}{l}0.9 \\
7.0\end{array}$ & $\begin{array}{l}4.3 \\
4.4\end{array}$ & $\begin{array}{l}2.4 \\
2.4\end{array}$ & $\begin{array}{l}.0 \\
.9\end{array}$ & $\begin{array}{r}3.3 \\
\mathrm{R}_{39.1}\end{array}$ & $\mathrm{R} 132.6$ & R.9 & $\begin{array}{r}1,0<5.1 \\
R_{1}, 039.1\end{array}$ \\
\hline $2011^{P}$ & 319.2 & 55.6 & 413.1 & 2.7 & 790.7 & 101.4 & 22.3 & 78.9 & 7.1 & 4.4 & 2.4 & 1.5 & 45.2 & 139.6 & .9 & $1,054.8$ \\
\hline
\end{tabular}

1 Anthracite, bituminous coal, subbituminous coal, lignite, waste coal, and coal synfuel.

Distillate fuel oil, residual fuel oil, petroleum coke, jet fuel, kerosene, other petroleum, and waste oil.

11 Included in "Wind."

12 Through 1988, data are for electric utilities only. Beginning in 1989, data are for electric utilities, R=Revised. P=Preliminary. NA =Not available, and industrial plants.

Notes: - Data are at end of year. - For plants that use multiple sources of energy, capacity is assigned to the energy source reported as the predominant one. - See Note 1, "Coverage of Electricity Statistics," at end of section. See "Generator Net Summer Capacity" in Glossary. - Totals may not equal sum of

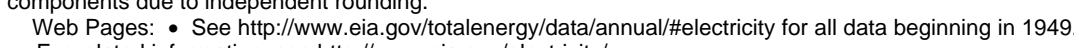
For related information, see http://www.eia.gov/electricity/.

7 Municipal solid waste from biogenic sources, landfill gas, sludge waste, agricultural byproducts, and other biomass. For all years, also includes non-renewable waste (municipal solid waste from non-biogenic sources, and tire-derived fuels).

Solar thermal and photovoltaic (PV) energy.

9 Batteries, chemicals, hydrogen, pitch, purchased steam, sulfur, and miscellaneous technologies. 
Table 8.11b Electric Net Summer Capacity: Electric Power Sector, Selected Years, 1949-2011

(Subset of Table 8.11a; Million Kilowatts)

\begin{tabular}{|c|c|c|c|c|c|c|c|c|c|c|c|c|c|c|c|c|}
\hline \multirow[b]{3}{*}{ Year } & \multicolumn{5}{|c|}{ Fossil Fuels } & \multirow{3}{*}{$\begin{array}{l}\text { Nuclear } \\
\text { Electric } \\
\text { Power }\end{array}$} & \multirow{3}{*}{$\begin{array}{l}\text { Hydro- } \\
\text { electric } \\
\text { Pumped } \\
\text { Storage }\end{array}$} & \multicolumn{7}{|c|}{ Renewable Energy } & \multirow[b]{3}{*}{ Other ${ }^{9}$} & \multirow[b]{3}{*}{ Total } \\
\hline & \multirow[b]{2}{*}{ Coal ${ }^{1}$} & \multirow[b]{2}{*}{ Petroleum ${ }^{2}$} & \multirow{2}{*}{$\begin{array}{c}\text { Natural } \\
\text { Gas }^{3}\end{array}$} & \multirow{2}{*}{$\begin{array}{c}\text { Other } \\
\text { Gases }{ }^{4}\end{array}$} & \multirow[b]{2}{*}{ Total } & & & \multirow{2}{*}{$\begin{array}{c}\text { Conventional } \\
\text { Hydroelectric } \\
\text { Power }^{5}\end{array}$} & \multicolumn{2}{|c|}{ Biomass } & \multirow{2}{*}{$\begin{array}{c}\text { Geo- } \\
\text { thermal }\end{array}$} & \multirow[b]{2}{*}{ Solar/PV 8} & \multirow[b]{2}{*}{ Wind } & \multirow[b]{2}{*}{ Total } & & \\
\hline & & & & & & & & & Wood 6 & Waste ${ }^{7}$ & & & & & & \\
\hline 1949 & NA & NA & NA & NA & 44.9 & 0.0 & $\left({ }^{5}\right)$ & 18.5 & (s) & $\left({ }^{10}\right)$ & NA & NA & NA & 18.5 & NA & 63.4 \\
\hline 1950 & NA & NA & NA & NA & 50.0 & .0 & $(5)$ & 19.2 & (s) & $(10)$ & NA & NA & NA & 19.2 & NA & 69.2 \\
\hline 1955 & NA & NA & NA & NA & 86.8 & .0 & $(5)$ & 27.4 & (s) & $(10)$ & NA & NA & NA & 27.4 & NA & 114.2 \\
\hline 1960 & NA & NA & NA & NA & 130.8 & .4 & $(5)$ & 35.8 & .1 & $(10)$ & (s) & NA & NA & 35.9 & NA & 167.1 \\
\hline 1965 & NA & NA & NA & NA & 182.9 & .8 & $(5)$ & 51.0 & .1 & $(10)$ & (s) & NA & NA & 51.1 & $\mathrm{NA}$ & 234.8 \\
\hline 1970 & NA & NA & NA & NA & 265.4 & 7.0 & $\left({ }^{5}\right)$ & 63.8 & .1 & $(10)$ & .1 & NA & NA & 63.9 & NA & 336.4 \\
\hline 1975 & NA & NA & NA & NA & 375.1 & 37.3 & $\left(5^{5}\right)$ & 78.4 & .1 & $(10)$ & .5 & NA & NA & 79.0 & NA & 491.3 \\
\hline 1976 & NA & NA & NA & NA & 394.8 & 43.8 & $\left({ }^{5}\right)$ & 78.0 & .1 & $\left({ }^{10}\right)^{\prime}$ & .5 & NA & NA & 78.6 & NA & 517.2 \\
\hline 1977 & NA & NA & NA & NA & 410.4 & 46.3 & $(5)$ & 78.6 & .1 & $(10)$ & .5 & NA & NA & 79.2 & NA & 535.9 \\
\hline 1978 & NA & NA & NA & NA & 420.8 & 50.8 & $\left({ }^{5}\right)$ & 79.9 & .1 & $(10)^{\prime}$ & .5 & NA & NA & 80.5 & NA & 552.1 \\
\hline 1979 & NA & NA & NA & NA & 432.1 & 49.7 & $\left({ }^{5}\right)$ & 82.9 & .1 & $(10)$ & .7 & NA & NA & 83.6 & $\mathrm{NA}$ & 565.5 \\
\hline 1980 & NA & NA & NA & NA & 444.1 & 51.8 & $\left({ }^{5}\right)$ & 81.7 & .1 & $(10)$ & .9 & NA & NA & 82.7 & NA & 578.6 \\
\hline 1981 & NA & NA & NA & NA & 458.9 & 56.0 & $\left({ }^{5}\right)$ & 82.4 & .1 & $(10)$ & .9 & NA & (s) & 83.4 & NA & 598.3 \\
\hline 1982 & NA & $\mathrm{NA}$ & $\mathrm{NA}$ & NA & 469.6 & 60.0 & (5) & 83.0 & .1 & $(10)$ & 1.0 & NA & (s) & 84.1 & NA & 613.7 \\
\hline 1983 & NA & $\mathrm{NA}$ & $\mathrm{NA}$ & $\mathrm{NA}$ & 472.8 & 63.0 & (5) & $\begin{array}{l}80.0 \\
83.9\end{array}$ & .2 & (10) & 1.2 & NA & (s) & $\begin{array}{l}8.1 \\
85.3\end{array}$ & NA & 621.1 \\
\hline 1984 & NA & NA & $\mathrm{NA}$ & NA & 478.6 & 69.7 & (5) & 85.3 & .3 & (10) & 1.2 & (11) & $\begin{array}{l}\text { (s) } \\
\text { (s) }\end{array}$ & $\begin{array}{l}0.5 \\
86.9\end{array}$ & NA & 6351 \\
\hline $\begin{array}{l}1984 \\
1985\end{array}$ & NA & NA & NA & NA & $\begin{array}{l}48.0 \\
485.0\end{array}$ & $\begin{array}{l}79.1 \\
79.4\end{array}$ & (5) & $\begin{array}{l}5.3 \\
88.9\end{array}$ & .2 & . & $\begin{array}{l}1.2 \\
1.6\end{array}$ & (11) & $\begin{array}{l}\text { (s) } \\
\text { (s) }\end{array}$ & 90.8 & NA & 655.2 \\
\hline 1986 & NA & NA & NA & NA & 488.3 & 85.2 & (5) & 89.3 & .2 & .2 & 1.6 & $(11)^{\prime}$ & (s) & 91.2 & NA & 664.8 \\
\hline 1987 & NA & $\mathrm{NA}$ & NA & NA & 488.8 & 93.6 & (5) & 89.7 & 2 & 2 & $\begin{array}{l}1.0 \\
1.5\end{array}$ & (11) & (s) & 91.7 & NA & $\begin{array}{l}674.1 \\
\end{array}$ \\
\hline 1988 & NA & NA & NA & NA & $\begin{array}{l}40.0 \\
490.6\end{array}$ & 94.7 & (5) & $\begin{array}{l}9.1 \\
90.3\end{array}$ & 2 & $\begin{array}{l}.2 \\
2\end{array}$ & 1.7 & (11) & $\begin{array}{l}\text { (s) } \\
\text { (s) }\end{array}$ & 92.4 & $\mathrm{NA}$ & 677.7 \\
\hline $1989^{12}$ & 298.0 & 78.1 & $\begin{array}{l}1 N A \\
125.4\end{array}$ & .4 & 501.9 & $\begin{array}{l}4.1 .1 \\
98.2\end{array}$ & 18.1 & 73.6 & 1.1 & 1.2 & 2.1 & .2 & 1.5 & 80.4 & INA & 698.8 \\
\hline 1990 & 302.3 & $\begin{array}{l}1.1 \\
76.8\end{array}$ & $\begin{array}{l}129.9 \\
129 .\end{array}$ & .4 & 509.3 & $\begin{array}{l}99.6 \\
99.6\end{array}$ & 19.5 & 73.3 & $\begin{array}{l}1.1 \\
1.2\end{array}$ & 2.1 & $\begin{array}{l}2.0 \\
2.7\end{array}$ & .3 & 1.8 & 81.4 & (s) & 709.9 \\
\hline 1991 & 302.5 & 73.0 & 137.1 & .7 & 513.3 & 99.6 & 18.4 & 75.4 & 1.3 & 2.5 & 2.6 & .3 & 1.9 & 84.0 & - & 715.3 \\
\hline 1992 & 304.3 & 71.8 & 141.0 & .7 & 517.9 & 99.0 & 21.2 & 74.2 & 1.4 & 2.5 & 2.9 & .3 & 1.8 & 83.1 & - & 721.2 \\
\hline 1993 & 305.0 & 69.9 & 146.9 & .7 & 522.5 & 99.0 & 21.1 & 76.8 & 1.5 & 2.6 & 2.9 & .3 & 1.8 & 85.9 & - & 728.6 \\
\hline 1994 & 306.1 & 70.5 & 152.5 & .7 & 529.8 & 99.1 & 21.2 & 76.9 & 1.7 & 2.7 & 3.0 & .3 & 1.7 & 86.4 & - & 736.5 \\
\hline 1995 & 306.0 & 65.4 & 161.9 & .3 & 533.7 & 99.5 & 21.4 & 77.4 & 1.8 & 3.0 & 3.0 & 3 & 1.7 & 87.3 & - & 741.8 \\
\hline 1996 & 308.1 & 71.3 & 161.4 & .1 & 540.9 & 100.8 & 21.1 & 75.3 & 1.7 & 2.9 & 2.9 & .3 & 1.7 & 84.9 & - & 747.7 \\
\hline 1997 & 308.5 & 71.0 & 163.4 & .2 & 543.1 & 99.7 & 19.3 & 78.3 & 1.8 & 2.9 & 2.9 & .3 & 1.6 & 87.8 & .2 & 750.1 \\
\hline 1998 & 310.9 & 65.0 & $\begin{array}{l}167.1 \\
\text { S }\end{array}$ & .1 & 543.0 & 97.1 & 19.5 & $\begin{array}{l}1.0 \\
78.0\end{array}$ & $\begin{array}{l}1.0 \\
1.8\end{array}$ & 3.0 & 2.9 & .3 & $\begin{array}{l}1.0 \\
1.7\end{array}$ & 87.8 & .2 & 747.6 \\
\hline 1999 & 310.7 & 58.6 & 181.1 & .2 & 550.7 & 97.4 & 19.6 & 78.3 & $\begin{array}{l}1.0 \\
1.8\end{array}$ & $\begin{array}{l}3.0 \\
3.0\end{array}$ & $\begin{array}{l}2.9 \\
2.8\end{array}$ & .4 & 2.3 & $\begin{array}{l}8.0 \\
88.6\end{array}$ & .2 & 756.5 \\
\hline 2000 & $\begin{array}{l}310.2 \\
\end{array}$ & $\begin{array}{l}00.0 \\
60.7\end{array}$ & 204.7 & .3 & 575.9 & $\begin{array}{l}97.4 \\
97.9\end{array}$ & 19.5 & $\begin{array}{l}1.03 \\
78.2\end{array}$ & $\begin{array}{l}1.0 \\
1.7\end{array}$ & 3.3 & $\begin{array}{l}. .0 \\
2.8\end{array}$ & .4 & $\begin{array}{l}2.5 \\
2.4\end{array}$ & $\begin{array}{l}0.0 \\
88.8\end{array}$ & $(\mathrm{~s})^{2}$ & 782.1 \\
\hline 2001 & 309.8 & 64.7 & $\begin{array}{l}23.1 .8 \\
236.8\end{array}$ & .3 & 611.6 & 98.2 & 19.7 & $\begin{array}{l}10.2 \\
77.9\end{array}$ & 1.6 & $\begin{array}{l}\text {.5 } \\
3.3\end{array}$ & $\begin{array}{l}.8 .8 \\
2.2\end{array}$ & .4 .4 & $\begin{array}{l}3.4 \\
3.9\end{array}$ & $\begin{array}{l}8.8 \\
89.2\end{array}$ & (s) .1 & 818.8 \\
\hline 2002 & 311.0 & 58.6 & 296.6 & .3 & 666.5 & 98.7 & 20.4 & 78.3 & 1.6 & 3.3 & 2.3 & .4 & 4.4 & 90.2 & .1 & 875.8 \\
\hline 2003 & 308.5 & 59.6 & 339.1 & 3 & 707.6 & 99.2 & 20.5 & 77.9 & 1.6 & 3.3 & 2.1 & .4 & 6.0 & 91.3 & .1 & 918.6 \\
\hline 2004 & 308.8 & 58.0 & 355.2 & .4 & 722. & 99.6 & 20.8 & 77.0 & 1.6 & 2.9 & 2. & .4 & 6.5 & 90. & .1 & 933.4 \\
\hline 2005 & 309.0 & 57.4 & 367.5 & .3 & 734.3 & 100 & 21.3 & 76.9 & 1.6 & 3.0 & 2. & .4 & 8.7 & 92.9 & .1 & 948.6 \\
\hline 2006 & 309.2 & 56.8 & 372.0 & .4 & 738.4 & 100.3 & 21.5 & 77.1 & 1.7 & 3.1 & 2.3 & .4 & $\begin{array}{l}0.1 \\
11.3\end{array}$ & 95.9 & .1 & 956.2 \\
\hline 2007 & 309.1 & 54.8 & 377.1 & .5 & 741.5 & 100.3 & 21.9 & 77.5 & 1.7 & 3.5 & 2.2 & .5 & 16.5 & 102.0 & .1 & 965 \\
\hline 2008 & 309.6 & 56.4 & 381.8 & .2 & 748.1 & 100.8 & 21.9 & 77.6 & 1.8 & 3.6 & 2.2 & .5 & 24.7 & 110.5 & .1 & 981.3 \\
\hline 2009 & 310.5 & 55.7 & 385.5 & .2 & 751.9 & 101.0 & 22.2 & 78.2 & 1.9 & 3.7 & 2.4 & .6 & 34.3 & 121.1 & .1 & 996.2 \\
\hline 2010 & $\mathrm{R}_{312.4}$ & $R_{54} 6$ & $R_{391.4}$ & R.7 & R759.1 & $\mathrm{R}_{101.2}$ & $\mathrm{R}_{22.2}$ & R78.5 & $\mathrm{R}_{2.1}$ & $\mathrm{R}_{3.7}$ & 2.4 & .9 & $R_{39.1}$ & $R_{126.6}$ & $R_{.1}$ & $1,009.2$ \\
\hline $2011^{P}$ & 314.8 & 54.6 & 397.3 & .7 & 767.4 & 101.4 & 22.3 & 78.6 & 2.1 & 3.8 & 2.4 & 1.5 & 45.2 & 133.5 & .1 & $1,024.8$ \\
\hline
\end{tabular}

1 Anthracite, bituminous coal, subbituminous coal, lignite, waste coal, and coal synfuel.

Distillate fuel oil, residual fuel oil, petroleum coke, jet fuel, kerosen, other petroleum, and waste oil.

Natural gas, plus a small amount of supplemental gaseous fuels.

5 Bhrough 1988, hydroelectric pumped and storage is included in "Conventiones derived from fossil fuels.

Wood and wood-derived fuels.

7 Municipal solid waste from biogenic sources, landfill gas, sludge waste, agricultural byproducts, and other biomass. For all years, also includes non-renewable waste (municipal solid waste from non-biogenic sources, and tire-derived fuels).

9 Batteries, chemicals, hydrogen, pitch, purchased steam, sulfur, and miscellaneous technologies.

10 Included in "Wood."

1 Included in "Wind." dependent power producers.

kilowatts.

Notes: - Data are at end of year. - For plants that use multiple sources of energy, capacity is assigned to the energy source reported as the predominant one. - The electric power sector comprises business is to sell electricity industrial CHP and electricity-only data. - See Note 1, "Coverage of Electricity Statistics," and Note 2, "Classification of Power Plants Into Energy-Use Sectors," at end of section. - See "Generator Net Summer "Capacity" in Glossary. • Totals may not equal sum of components due to independent rounding. - For related information, see http://www.eia.gov/electricity/. Sources: - 1949-1984-U.S. Energy Information Administration (EIA) estimates. - 1985-1988-EIA Form EIA-860, "Annual Electric Generator Report." • 1989-1997-EIA, Form EIA-860, "Annual Electric Generator Report," and Form EIA-867, "Annual Nonutility Power Producer Report." • 1998-2000-EIA, Form EIA-860A, "Annual Electric Generator Report-Utility," and Form ElA-860B, "Annual Electric
Generator Report-Nonutility." • 2001 forward-EIA, Form EIA-860, "Annual Electric Generator Report." 
Table 8.11c Electric Net Summer Capacity: Electric Power Sector by Plant Type, Selected Years, 1989-2011

(Breakout of Table 8.11b; Million Kilowatts)

\begin{tabular}{|c|c|c|c|c|c|c|c|c|c|c|c|c|c|c|c|c|}
\hline \multirow[b]{3}{*}{ Year } & \multicolumn{5}{|c|}{ Fossil Fuels } & \multirow{3}{*}{$\begin{array}{c}\text { Nuclear } \\
\text { Electric } \\
\text { Power }\end{array}$} & \multirow{3}{*}{$\begin{array}{l}\text { Hydro- } \\
\text { electric } \\
\text { Pumped } \\
\text { Storage }\end{array}$} & \multicolumn{7}{|c|}{ Renewable Energy } & \multirow[b]{3}{*}{ Other ${ }^{8}$} & \multirow[b]{3}{*}{ Total } \\
\hline & & & & & & & & \multirow{2}{*}{$\begin{array}{c}\text { Conventional } \\
\text { Hydroelectric } \\
\text { Power }\end{array}$} & \multicolumn{2}{|c|}{ Biomass } & \multirow{2}{*}{$\begin{array}{c}\text { Geo- } \\
\text { thermal }\end{array}$} & \multirow[b]{2}{*}{ Solar/PV 7} & \multirow[b]{2}{*}{ Wind } & \multirow[b]{2}{*}{ Total } & & \\
\hline & Coal ${ }^{1}$ & Petroleum ${ }^{2}$ & $\begin{array}{c}\text { Natural } \\
\text { Gas }^{3}\end{array}$ & $\begin{array}{l}\text { Other } \\
\text { Gases }{ }^{4}\end{array}$ & Total & & & & Wood $^{5}$ & Waste ${ }^{6}$ & & & & & & \\
\hline & \multicolumn{16}{|c|}{ Electricity-Only Plants ${ }^{9}$} \\
\hline 1989 & 296.5 & 78.0 & 119.3 & 0.4 & 494.2 & 98.2 & 18.1 & 73.6 & 0.9 & 1.5 & 2.6 & 0.2 & 1.5 & 80.3 & - & 690.7 \\
\hline 1990 & 299.9 & 76.6 & $\begin{array}{l}121.8 \\
\end{array}$ & .4 & 498.6 & 99.6 & 19.5 & 73.3 & $\begin{array}{l}1.0 \\
1.0\end{array}$ & 1.9 & $\begin{array}{l}2.0 \\
2.7\end{array}$ & .3 & $\begin{array}{l}1.8 \\
1.8\end{array}$ & 80.9 & (s) & 698.6 \\
\hline 1995 & 301.3 & 64.7 & 145.3 & .3 & 511.5 & 99.5 & 21.4 & 77.4 & 1.5 & 2.7 & 3.0 & .3 & 1.7 & 86.6 & - & 719.1 \\
\hline 1996 & 303.1 & 70.6 & 143.1 & .0 & 516.9 & 100.8 & 21.1 & 75.3 & 1.4 & 2.6 & $\begin{array}{l}3.0 \\
2.9\end{array}$ & .3 & 1.7 & $\begin{array}{l}8.0 \\
84.2\end{array}$ & - & 723.0 \\
\hline 1997 & 303.6 & 70.2 & 144.7 & .2 & 518.7 & 99.7 & 19.3 & 78.3 & 1.5 & 2.5 & 2.9 & .3 & 1.6 & 87.1 & .2 & 725.0 \\
\hline 1998 & 305.9 & 64.2 & 147.5 & .1 & 517.5 & 97.1 & 19.5 & 78.0 & 1.4 & 2.6 & 2.9 & .3 & 1.7 & 87.0 & .2 & 721.4 \\
\hline 1999 & 305.5 & 57.5 & 161.7 & .2 & 525.0 & 97.4 & 19.6 & 78.3 & 1.5 & 2.6 & 2.8 & .4 & 2.3 & 87.8 & .2 & 730.0 \\
\hline 2000 & 305.2 & 59.8 & 184.0 & .1 & 549.0 & 97.9 & 19.5 & $\begin{array}{l}10.0 \\
78.2\end{array}$ & 1.5 & $\begin{array}{l}2.0 \\
2.8\end{array}$ & $\begin{array}{l}2.0 \\
2.8\end{array}$ & .4 & 2.4 & $\begin{array}{l}8.0 \\
88.1\end{array}$ & (s) & 754.5 \\
\hline 2001 & 305.2 & $\begin{array}{l}53.8 \\
63.8\end{array}$ & $\begin{array}{l}18.0 \\
215.5\end{array}$ & .1 & 584.5 & 98.2 & 19.7 & 77.9 & $\begin{array}{l}1.5 \\
1.5\end{array}$ & $\begin{array}{l}.8 \\
2.9\end{array}$ & $\begin{array}{l}2.8 \\
2.2\end{array}$ & .4 & $\begin{array}{l}2.4 \\
3.9\end{array}$ & $\begin{array}{l}8.1 \\
88.7\end{array}$ & (s) & $\begin{array}{l}794.5 \\
791.1\end{array}$ \\
\hline 2002 & 305.8 & 57.5 & 268.1 & .1 & 631.5 & 98.7 & 20.4 & 78.3 & 1.4 & 2.9 & 2.3 & .4 & 4.4 & 89.7 & .1 & 840.3 \\
\hline 2003 & 303.0 & 58.6 & 304.2 & .1 & 665.9 & 99.2 & 20.5 & 77.9 & 1.4 & 2.8 & 2.1 & .4 & 6.0 & 90.6 & .1 & 876.3 \\
\hline 2004 & 303.2 & 57.3 & 322.6 & .1 & 683.2 & 99.6 & 20.8 & 77.0 & 1.5 & 2.6 & 2.2 & .4 & 6.5 & 90.0 & .1 & 893.7 \\
\hline 2005 & 303.4 & 56.9 & 335.8 & (s) & 696.2 & 100.0 & 21.3 & 76.9 & 1.4 & 2.6 & 2.3 & 4 & 8.7 & 92.3 & .1 & 909.8 \\
\hline 2006 & 303.4 & 55.8 & 341.9 & .1 & 701.2 & 100.3 & 21.5 & 77.1 & 1.5 & 2.7 & 2.3 & .4 & 11.3 & 95.3 & 1 & 918.4 \\
\hline 2007 & 303.2 & 53.9 & 347.6 & .1 & 704.9 & 100.3 & 21.9 & 77.5 & $\begin{array}{l}1.5 \\
1.5\end{array}$ & 3.1 & $\begin{array}{l}2.0 \\
2.2\end{array}$ & .5 & 16.5 & $\begin{array}{r}10.3 \\
101.3\end{array}$ & .1 & 928.5 \\
\hline 2008 & $\begin{array}{l}303.2 \\
303.7\end{array}$ & $\begin{array}{l}53.9 \\
55.5\end{array}$ & $\begin{array}{l}34.6 \\
352.3\end{array}$ & .1 & 711.5 & 100.8 & $\begin{array}{l}21.9 \\
21.9\end{array}$ & $\begin{array}{l}77.5 \\
77.6\end{array}$ & $\begin{array}{l}1.5 \\
1.6\end{array}$ & $\begin{array}{l}.1 \\
3.2\end{array}$ & $\begin{array}{l}2.2 \\
2.2\end{array}$ & .5 & $\begin{array}{l}1.5 \\
24.7\end{array}$ & $\begin{array}{l}109.8 \\
109.8\end{array}$ & $\begin{array}{l}1 \\
.1\end{array}$ & $\begin{array}{l}98.5 \\
944.0\end{array}$ \\
\hline 2009 & 304.5 & 54.8 & 356.6 & (s) & 716.0 & 101.0 & 22.2 & 78.2 & 1.7 & 3.2 & 2.4 & .6 & 34.3 & 120.3 & .1 & 959.5 \\
\hline 2010 & $\mathrm{R}_{306.9}$ & $\begin{array}{r}r .0 \\
R_{53} .8\end{array}$ & R362.4 & $\begin{array}{l}\text { R. } \\
\text {. }\end{array}$ & R723.7 & $R_{101.2}$ & $\mathrm{R}_{22.2}$ & R78.5 & 1.7 & 3.3 & $\begin{array}{l}2.4 \\
2.4\end{array}$ & $\begin{array}{l}.0 \\
.9\end{array}$ & $\begin{array}{l}\text { R39.1 } \\
\text { R }\end{array}$ & $R_{125.8}$ & $R_{.1}^{-1}$ & R973.0 \\
\hline $2011^{\mathrm{P}}$ & 309.4 & 53.8 & 368.3 & .5 & 732.0 & 101.4 & 22.3 & 78.6 & 1.7 & 3.3 & 2.4 & 1.5 & 45.2 & 132.6 & .1 & 988.5 \\
\hline
\end{tabular}

Combined-Heat-and-Power Plants 10

\begin{tabular}{|c|c|c|c|c|c|c|c|c|c|c|c|c|c|c|c|c|}
\hline 1989 & 1.5 & 0.2 & 6.1 & - & 7.7 & - & - & - & 0.2 & 0.2 & - & - & - & 0.4 & - & 8.1 \\
\hline 1990 & 2.4 & .2 & 8.1 & - & 10.7 & - & - & - & .2 & .2 & - & - & - & .5 & - & 11.2 \\
\hline 1995 & 4.8 & .8 & 16.6 & - & 22.1 & - & - & - & .4 & .2 & - & - & - & .6 & - & 22.7 \\
\hline 1996 & 5.0 & .7 & 18.4 & - & 24.0 & - & - & - & .3 & .3 & - & - & - & .6 & - & 24.6 \\
\hline 1997 & 4.9 & .8 & 18.7 & (s) & 24.4 & - & - & - & .3 & .4 & - & - & - & .7 & - & 25.1 \\
\hline 1998 & 5.0 & .8 & 19.6 & - & 25.5 & - & - & - & .4 & .4 & - & 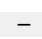 & 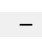 & .7 & 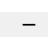 & 26.2 \\
\hline 1999 & 5.2 & 1.1 & 19.4 & - & 25.7 & - & - & - & .4 & .4 & - & - & - & .7 & - & 26.5 \\
\hline 2000 & 5.0 & .9 & 20.7 & .3 & 26.9 & - & - & - & .2 & .5 & - & - & - & .7 & - & 27.7 \\
\hline 2001 & 4.6 & 1.0 & 21.2 & .3 & 27.1 & - & - & (s) & .1 & .4 & - & - & - & .5 & (s) & 27.6 \\
\hline 2002 & 5.2 & 1.1 & 28.5 & .2 & 34.9 & - & - & - & .1 & .4 & - & - & - & .6 & - & 35.5 \\
\hline 2003 & 5.5 & 1.1 & 34.9 & .2 & 41.7 & - & - & (s) & .2 & .5 & - & - & - & .7 & - & 42.3 \\
\hline 2004 & $\begin{array}{l}.50 \\
5.6\end{array}$ & $\begin{array}{l}.1 \\
.7\end{array}$ & $\begin{array}{l}34.5 \\
32.6\end{array}$ & .3 & 39.2 & 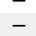 & 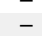 & (s) & .2 & 4 & 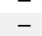 & 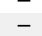 & 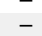 & .6 & 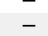 & 39.7 \\
\hline 2005 & 5.6 & .5 & 31.7 & .3 & 38.1 & - & - & (s) & .2 & .4 & - & - & - & .6 & - & 38.7 \\
\hline 2006 & 5.8 & 1.0 & 30.0 & .3 & 37.2 & - & - & (s) & .2 & .4 & - & - & - & .6 & - & 37.8 \\
\hline 2007 & 5.9 & .9 & 29.5 & .3 & 36.6 & - & - & - & .2 & .4 & - & - & - & .7 & - & 37.3 \\
\hline 2008 & 5.9 & .9 & 29.6 & .2 & 36.6 & - & - & - & .2 & .5 & - & - & - & .7 & - & 37.3 \\
\hline 2009 & 5.9 & .9 & 28.9 & .2 & 35.9 & - & - & - & .2 & .5 & - & - & - & .7 & - & 36.7 \\
\hline 2010 & R5.5 & R. 8 & $\mathrm{R}_{29.0}$ & .2 & R35.4 & - & - & - & $R_{.4}^{.2}$ & .5 & 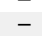 & - & 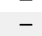 & .8 & - & R36.1 \\
\hline $2011^{\mathrm{P}}$ & 5.5 & $\begin{array}{l}.0 \\
.8\end{array}$ & 29.0 & .2 & 35.4 & - & 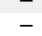 & 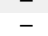 & $\begin{array}{l}.4 \\
.4\end{array}$ & .5 & 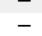 & 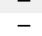 & 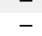 & $\begin{array}{l}.0 \\
.9\end{array}$ & 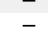 & $\begin{array}{l}30.3 \\
36.3\end{array}$ \\
\hline
\end{tabular}

1 Anthracite, bituminous coal, subbituminous coal, lignite, waste coal, and coal synfuel.

Distillate fuel oil, residual fuel oil, petroleum coke, jet fuel, kerosene, other petroleum, and waste oil.

Natural gas, plus a small amount of supplemental gaseous fuels.

Blast furnace gas, propane gas, and other manulactured and waste gases derived from fossil fuels.

Municipal solid waste from biogen

(andfill gas, sludge waste, agricultural byproducts, and sources, and tire-derived fuels.

Solar thermal and photovoltaic (PV) energy.

Batteries, chemicals, hydrogen, pitch, purchased steam, sulfur, and miscellaneous technologies. Electricity-only plants within the NACS 22 category whose primary business is to sell electricity to the public. Data also include a small number of electric utility combined-heat-and-power (CHP) plants.

sell Cecricity and heat to the (Cublic. plata do not include electric ulity CHP plents - these ariess is to under "Electricity-Only Plants."

R=Revised. P=Preliminary. $-=$ No data reported. (s)=Less than 0.05 million kilowatts.

Notes: - Data are at end of year. - For plants that use multiple sources of energy, capacity is assigned to the energy source reported as the predominant one. - See Table $8.11 \mathrm{~d}$ for commercial and industria of Power Plants Into Energy-Use Sectors" "at end of section. - See "Generator Net Summer Capacity" in Glossary. - Totals may no

- For related information, see http://www.eia.gov/electricity/. Generator: Report," and Form EIA-867, "Annual Nonutility Power Producer Report." • "1998-2000-EIA, GeneriA 
Table 8.11d Electric Net Summer Capacity: Commercial and Industrial Sectors, Selected Years, 1989-2011

(Subset of Table 8.11a; Million Kilowatts)

\begin{tabular}{|c|c|c|c|c|c|c|c|c|c|c|c|c|c|c|c|c|}
\hline \multirow[b]{3}{*}{ Year } & \multicolumn{5}{|c|}{ Fossil Fuels } & \multirow{3}{*}{$\begin{array}{l}\text { Nuclear } \\
\text { Electric } \\
\text { Power }\end{array}$} & \multirow{3}{*}{$\begin{array}{l}\text { Hydro- } \\
\text { electric } \\
\text { Pumped } \\
\text { Storage }\end{array}$} & \multicolumn{7}{|c|}{ Renewable Energy } & \multirow[b]{3}{*}{ Other ${ }^{8}$} & \multirow[b]{3}{*}{ Total } \\
\hline & \multirow[b]{2}{*}{ Coal ${ }^{1}$} & \multirow[b]{2}{*}{ Petroleum ${ }^{2}$} & \multirow{2}{*}{$\begin{array}{c}\text { Natural } \\
\text { Gas }^{3}\end{array}$} & \multirow{2}{*}{$\begin{array}{l}\text { Other } \\
\text { Gases }{ }^{4}\end{array}$} & \multirow[b]{2}{*}{ Total } & & & \multirow{2}{*}{$\begin{array}{c}\text { Conventional } \\
\text { Hydroelectric } \\
\text { Power }\end{array}$} & \multicolumn{2}{|c|}{ Biomass } & \multirow{2}{*}{$\begin{array}{l}\text { Geo- } \\
\text { thermal }\end{array}$} & \multirow[b]{2}{*}{ Solar/PV 7} & \multirow[b]{2}{*}{ Wind } & \multirow[b]{2}{*}{ Total } & & \\
\hline & & & & & & & & & Wood 5 & Waste ${ }^{6}$ & & & & & & \\
\hline & \multicolumn{16}{|c|}{ Commercial Sector 9} \\
\hline 1989 & 0.3 & 0.2 & 0.6 & - & 1.0 & - & - & (s) & (s) & 0.2 & - & - & - & 0.2 & - & 1.2 \\
\hline 1990 & .3 & .2 & .7 & - & 1.2 & - & - & (s) & (s) & .2 & - & - & - & .2 & - & 1.4 \\
\hline 1995 & .3 & .2 & 1.2 & - & 1.8 & - & - & (s) & (s) & .3 & - & - & - & .3 & - & 2.1 \\
\hline 1996 & .3 & .3 & 1.2 & - & 1.8 & - & - & (s) & (s) & .4 & - & - & - & .5 & - & 2.3 \\
\hline 1997 & .3 & .4 & 1.2 & - & 1.9 & - & - & (s) & (s) & .4 & - & - & - & .5 & - & 2.3 \\
\hline 1998 & .3 & 3 & 1.2 & - & 1.8 & - & - & (s) & (s) & .5 & - & - & - & .5 & - & 2.3 \\
\hline 1999 & .3 & .4 & 1.1 & - & 1.8 & - & - & (s) & (s) & .5 & - & - & - & .5 & - & 2.3 \\
\hline 2000 & .3 & 3 & 1.2 & - & 1.8 & - & - & (s) & (s) & .4 & - & - & - & .4 & - & 2.2 \\
\hline 2001 & .3 & .3 & 1.9 & - & 2.5 & - & - & (s) & (s) & .3 & - & - & - & .4 & - & 2.9 \\
\hline 2002 & .3 & .3 & 1.2 & - & 1.8 & - & - & (s) & (s) & .4 & - & - & - & .4 & - & 2.2 \\
\hline 2003 & .3 & 3 & 1.0 & - & 1.7 & - & - & (s) & (s) & .4 & - & - & - & .4 & - & 2.1 \\
\hline 2004 & .4 & .3 & 1.1 & (s) & 1.8 & - & - & $\begin{array}{l}\text { (s) } \\
\text { (s) }\end{array}$ & (s) & .4 & - & - & - & .4 & - & 2.2 \\
\hline 2005 & .4 & .3 & 1.0 & (s) & 1.8 & - & - & (s) & (s) & .4 & - & - & - & .5 & - & 2.2 \\
\hline 2006 & .4 & .3 & 1.0 & (s) & 1.8 & - & - & (s) & (s) & .4 & - & - & - & .5 & - & 2.3 \\
\hline 2007 & .4 & .3 & 1.1 & (s) & 1.8 & - & - & (s) & (s) & .4 & - & - & - & .5 & (s) & 2.3 \\
\hline 2008 & .4 & .4 & 1.1 & (s) & 1.8 & - & - & (s) & (s) & .4 & - & (s) & - & .5 & (s) & 2.3 \\
\hline 2009 & .4 & .3 & 1.1 & (s) & 1.9 & - & - & (s) & (s) & .5 & - & $\begin{array}{l}\text { (10) } \\
\text { (s) }\end{array}$ & (s) & .5 & (s) & 2.4 \\
\hline 2010 & .4 & R. & 1.2 & (s) & 1.9 & - & - & $\begin{array}{l}\text { (s) } \\
\text { (s) }\end{array}$ & (s) & .5 & - & $\begin{array}{l}\text { (s) } \\
\text { (s) }\end{array}$ & (s) & .5 & (s) & 2.5 \\
\hline $2011^{\mathrm{P}}$ & .4 & .4 & 1.1 & (s) & 1.9 & - & - & (s) & (s) & .1 & - & (s) & (s) & .2 & (s) & 2.1 \\
\hline
\end{tabular}

Industrial Sector ${ }^{10}$

\begin{tabular}{|c|c|c|c|c|c|c|c|c|c|c|c|c|c|c|c|c|}
\hline 1989 & 4.8 & 0.7 & 9.7 & 1.2 & 16.5 & - & - & 0.5 & 4.1 & 0.2 & - & - & - & 4.8 & 0.5 & 21.8 \\
\hline 1990 & 4.8 & .9 & 10.3 & 1.3 & 17.3 & - & - & .6 & 4.3 & .2 & - & - & - & 5.1 & .5 & 22.9 \\
\hline 1995 & 5.0 & 1.0 & 11.3 & 1.4 & 18.7 & - & - & 1.1 & 4.9 & .2 & - & - & - & 6.3 & .5 & 25.5 \\
\hline 1996 & 5.0 & .9 & 11.5 & 1.6 & 19.0 & - & - & 1.1 & 5.1 & .2 & - & - & - & 6.4 & .5 & 25.9 \\
\hline 1997 & 4.8 & 1.1 & 11.9 & 1.3 & 19.2 & - & - & 1.1 & 5.1 & .2 & - & - & - & 6.5 & .6 & 26.2 \\
\hline 1998 & 4.6 & 1.0 & 12.0 & 1.5 & 19.1 & - & - & 1.1 & 5.0 & .2 & - & - & - & 6.3 & .6 & 26.0 \\
\hline 1999 & 4.4 & 1.1 & 12.9 & 1.7 & 20.1 & - & - & 1.1 & 5.0 & .2 & - & - & - & 6.2 & .8 & 27.1 \\
\hline 2000 & 4.6 & .8 & 13.7 & 2.0 & 21.2 & - & - & 1.1 & 4.4 & .2 & - & - & - & 5.7 & .5 & 27.3 \\
\hline 2001 & 4.2 & 1.1 & 14.1 & 1.3 & 20.7 & - & - & 1.0 & 4.2 & .1 & - & - & - & 5.4 & .4 & 26.6 \\
\hline 2002 & 4.0 & .7 & 14.7 & 1.8 & 21.2 & - & - & 1.0 & 4.3 & .1 & - & - & - & 5.5 & .6 & 27.3 \\
\hline 2003 & 4.1 & .7 & 15.3 & 1.7 & 21.9 & - & - & .8 & 4.3 & .1 & - & - & - & 5.2 & .6 & 27.7 \\
\hline 2004 & 3.8 & 8 & 14.8 & 1.9 & 21.3 & - & - & .6 & 4.5 & .2 & - & - & - & 5.4 & .7 & 27.4 \\
\hline 2005 & 4.0 & .8 & 14.5 & 1.8 & 21.0 & - & - & .7 & 4.5 & .2 & - & - & - & 5.4 & 8 & 27.2 \\
\hline 2006 & 3.3 & 1.0 & 15.3 & 1.8 & 21.4 & - & - & .7 & 4.7 & .2 & - & - & - & 5.6 & .8 & 27.8 \\
\hline 2007 & 3.2 & .9 & 14.7 & 1.9 & 20.6 & - & - & .3 & 5.0 & .2 & - & (s) & - & 5.5 & .7 & 26.8 \\
\hline 2008 & 3.2 & .7 & 14.6 & 1.8 & 20.3 & - & - & .3 & 5.0 & .1 & - & (s) & - & 5.4 & .9 & 26.6 \\
\hline 2009 & 3.4 & .7 & 14.7 & 1.7 & 20.5 & - & - & .3 & 5.0 & .1 & - & (s) & - & 5.5 & .8 & 26.8 \\
\hline 2010 & 4.0 & .7 & $\mathrm{R}_{14.4}$ & $\mathrm{R}_{2} .0$ & $\mathrm{R}_{21.1}$ & - & - & .3 & $\mathrm{R}_{4} .9$ & R.2 & - & (s) & ${ }^{R}(s)$ & 5.5 & .8 & $\mathrm{R}_{27.4}$ \\
\hline $2011^{\mathrm{P}}$ & 4.0 & .7 & 14.7 & 2.0 & 21.3 & - & - & .3 & 5.0 & .5 & - & (s) & (s) & 5.9 & .7 & 28.0 \\
\hline
\end{tabular}

Anthracite, bituminous coal, subbituminous coal, lignite, waste coal, and coal synfuel.
2 Distillate fuel oil, residual fuel oil, petroleum coke, jet fuel, kerosene, other petroleum, and waste oil.

R=Revised. $P=$ Preliminary. $-=$ No data reported. $(\mathrm{s})=$ Less than 0.05 million kilowatts.

${ }_{4}$ Blast furnace gas, propane gas, and other manufactured and waste gases derived from fossil fuels.

5 Wood and wood-derived fuels.

6 Municipal solid waste from biogenic sources, landfill gas, sludge waste, agricultural byproducts, and other biomass. For all years, also includes non-renewable waste (municipal solid waste from non-biogenic

sources, and tire-derived fuels).

Solar thermal and photovoltaic (PV) energy.

Batteries, chemicals, hydrogen, pitch, purchased steam, sulfur, and miscellaneous technologies.

90 Commercial combined-heat-and-power (CHP) and commercial electricity-only plants. to the energy source reported as the predominant one. - See Tables $8.11 \mathrm{~b}$ and $8.11 \mathrm{c}$ for electric power sector electricity-only and CHP data. - See Note 1, "Coverage of Electricity Statistics," and Note 2, "Classification of Power Plants Into Energy-Use Sectors," at end of section. • See "Generator Net Summer Capacity" in Glossary. - Totals may not equal sum of components due to independent rounding.

Web Pages: - See http://www.eia.gov/totalenergy/data/annual/\#electricity for all data beginning in 1989.

- For related information, see http://www.eia.gov/electricity/.
Sources: - 1989-1997-U.S. Energy Information Administration (EIA), Form EIA-867, "Annual Nonutility Power Producer Report." • $1998-2000$-EIA, Form EIA-860B, "Annual Electric Generator
Report-Nonutility." • 2001 forward-EIA, Form EIA-860, "Annual Electric Generator Report." 
Figure 8.12a Electric Noncoincident Peak Load and Capacity Margin: Summer Peak Period

\section{U.S. ${ }^{1}$ Summer Peak Load, ${ }^{2}$ All Interconnections, 1986-2011}

900-

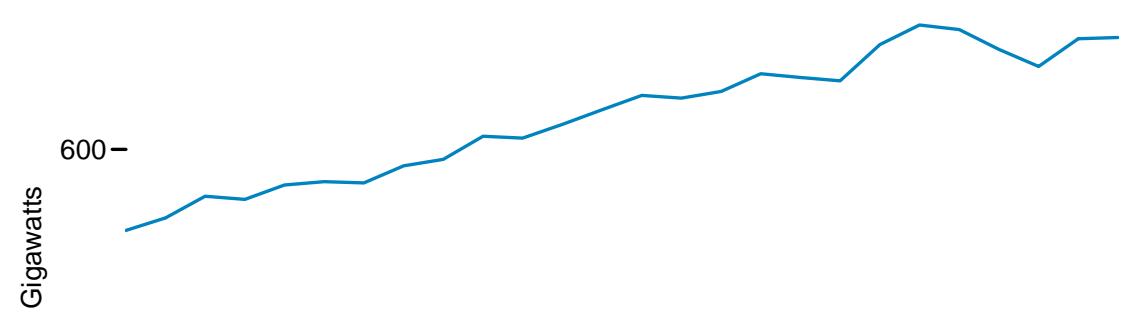

$300-$

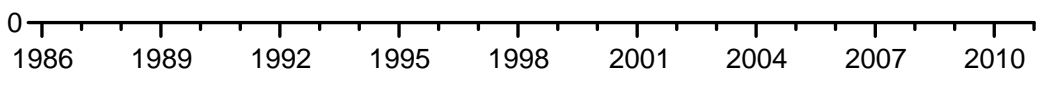

Summer Capacity Margin, 1996-2011

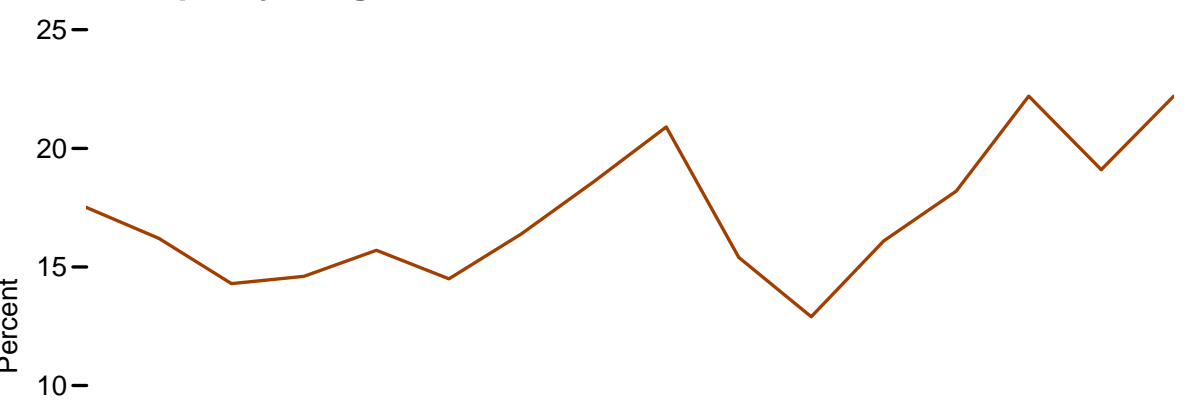

5-

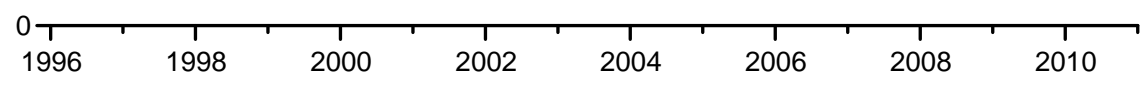

U.S. ${ }^{1}$ Summer Peak Load ${ }^{2}$ by NERC ${ }^{3}$ Regional Assessment Area, 2011 200-

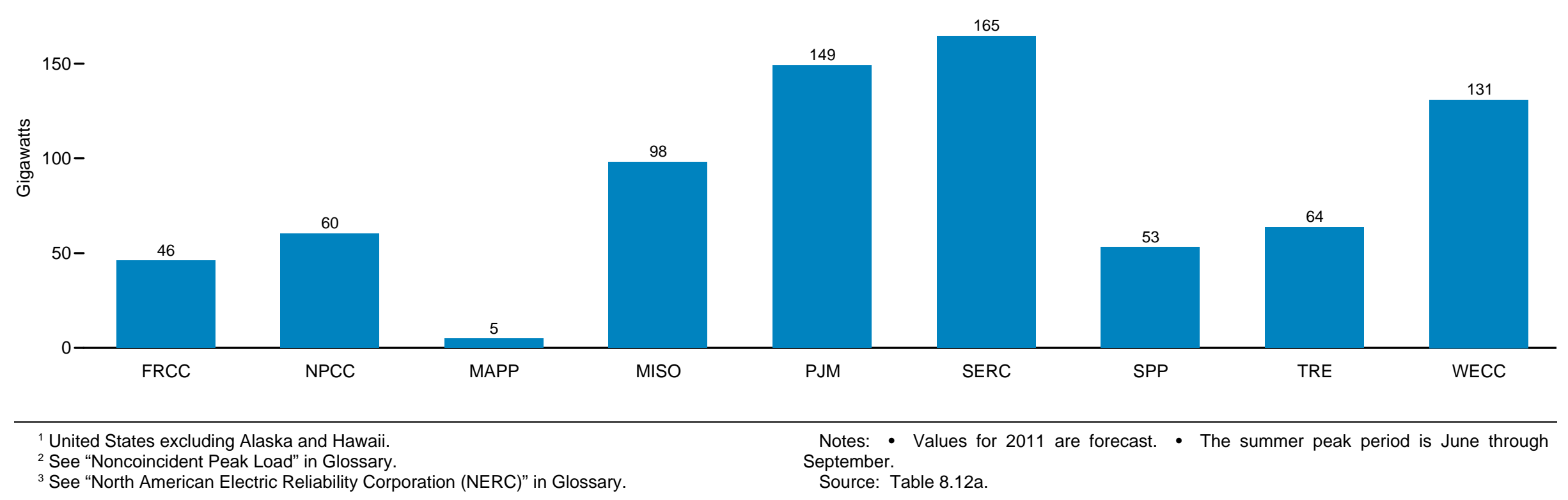




\section{Figure 8.12b Electric Noncoincident Peak Load and Capacity Margin: Winter Peak Period}

\section{U.S. ${ }^{1}$ Winter Peak Load, ${ }^{2}$ All Interconnections, 1986-2011}

$700-$

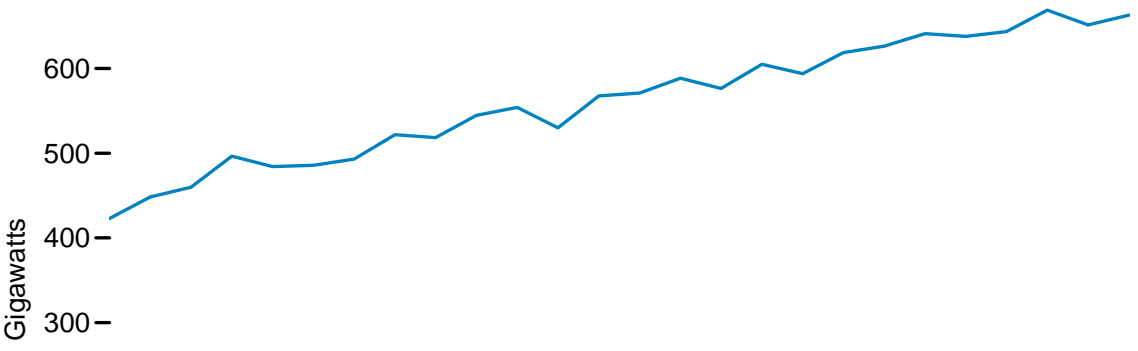

$200-$

$100-$

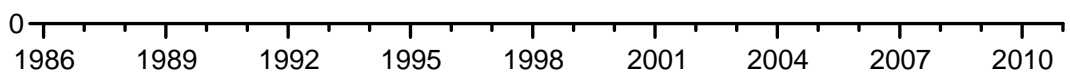

Winter Capacity Margin, 1996-2011
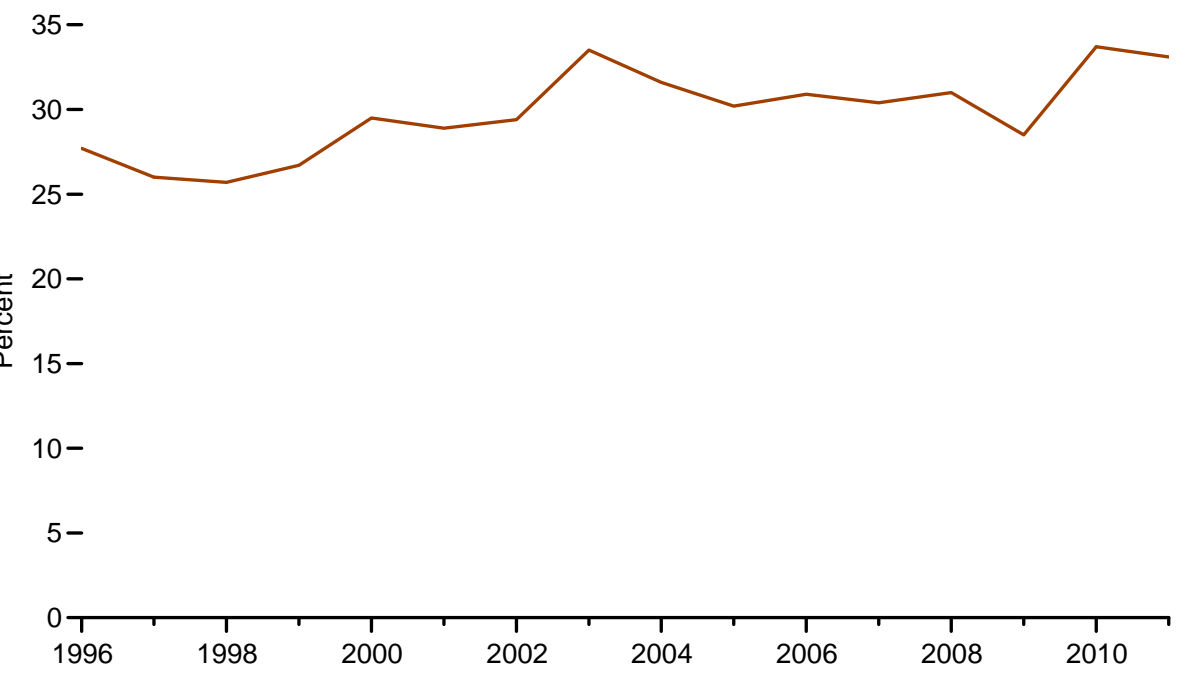

U.S. ${ }^{1}$ Winter Peak Load ${ }^{2}$ by NERC ${ }^{3}$ Regional Assessment Area, 2011

$200-$

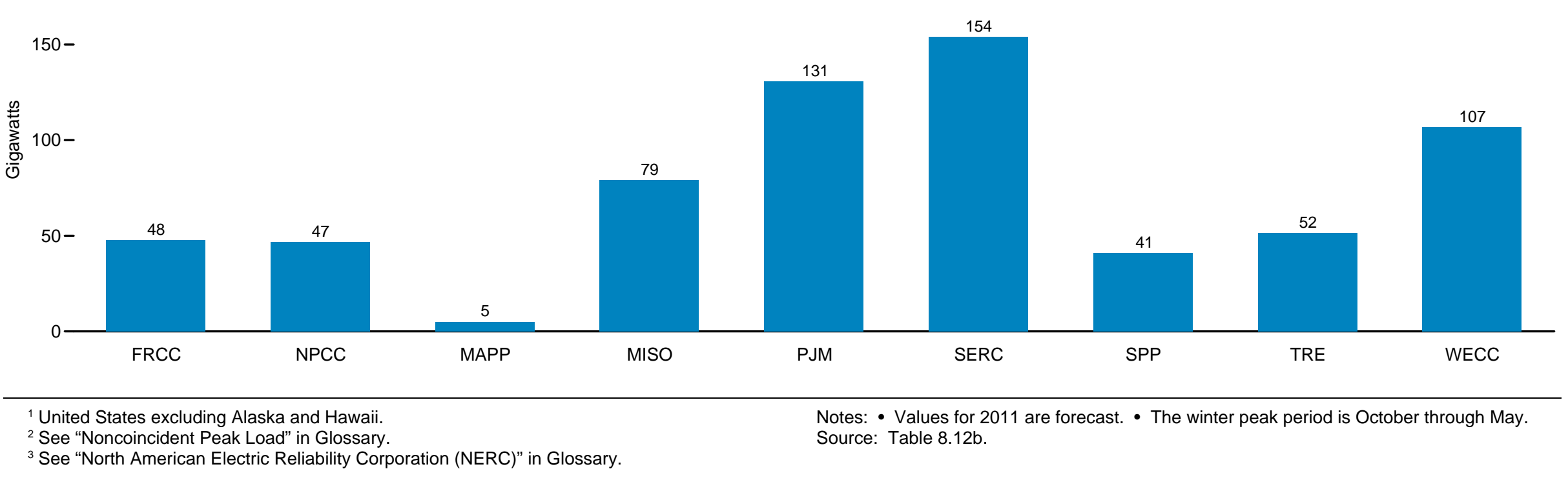


Table 8.12a Electric Noncoincident Peak Load and Capacity Margin: Summer Peak Period, 1986-2011 (Megawatts, Except as Noted)

\begin{tabular}{|c|c|c|c|c|c|c|c|c|c|c|c|c|c|c|c|c|c|}
\hline \multirow[b]{4}{*}{ Year } & \multicolumn{16}{|c|}{ Noncoincident Peak Load ${ }^{1}$ by North American Electric Reliability Corporation (NERC) ${ }^{2}$ Regional Assessment Area } & \multirow{4}{*}{$\begin{array}{c}\text { Capacity } \\
\text { Margin 2 } \\
\text { (percent) }\end{array}$} \\
\hline & \multicolumn{13}{|c|}{ Eastern Interconnection } & \multirow{3}{*}{\begin{tabular}{|c|} 
ERCOT $^{4}$ \\
TRE ${ }^{18}$
\end{tabular}} & \multirow{3}{*}{\begin{tabular}{|c|}
$\begin{array}{c}\text { Western } \\
\text { Inter- } \\
\text { connection }\end{array}$ \\
WECC 19
\end{tabular}} & \multirow{3}{*}{\begin{tabular}{|c|}
$\begin{array}{c}\text { All } \\
\text { Inter- } \\
\text { connections }\end{array}$ \\
Total 20 \\
\end{tabular}} & \\
\hline & \multirow[b]{2}{*}{ FRCC 5} & \multirow[b]{2}{*}{ NPCC ${ }^{6}$} & \multicolumn{11}{|c|}{ Balance of Eastern Region ${ }^{3}$} & & & & \\
\hline & & & $\operatorname{ECAR}^{7,8}$ & MAAC 8,9 & MAIN 8,10 & MAPP 11 & MISO 12 & MRO ${ }^{13}$ & PJM 14 & $\mathbf{R F C}^{8,15}$ & SERC ${ }^{16}$ & SPP 17 & Subtotal & & & & \\
\hline 1986 & -- & 39,026 & 69,606 & 37,564 & 35,943 & -- & -- & 21,029 & -- & -- & 105,570 & 47,123 & 316,835 & 39,335 & 81,787 & 476,983 & NA \\
\hline 1987 & -- & 42,651 & 72,561 & 40,526 & 37,446 & -- & -- & 23,162 & -- & -- & 109,798 & 47,723 & 331,216 & 39,339 & 82,967 & 496,173 & NA \\
\hline 1988 & -- & 45,245 & 79,149 & 43,110 & 41,139 & -- & -- & 24,899 & -- & -- & 115,168 & 49,356 & 352,821 & 40,843 & 90,551 & 529,460 & NA \\
\hline 1989 & -- & 45,031 & 75,442 & 41,614 & 39,460 & -- & -- & 24,336 & -- & -- & 117,729 & 49,439 & 348,020 & 40,402 & 90,657 & 524,110 & NA \\
\hline 1990 & -- & 44,116 & 79,258 & 42,613 & 40,740 & -- & -- & 24,994 & -- & -- & 121,943 & 52,541 & 362,089 & 42,737 & 97,389 & 546,331 & 21.6 \\
\hline 1991 & -- & 46,594 & 81,224 & 45,937 & 41,598 & -- & -- & 25,498 & -- & -- & 124,716 & 51,885 & 370,858 & 41,870 & 92,096 & 551,418 & 20.9 \\
\hline 1992 & -- & 43,658 & 78,550 & 43,658 & 38,819 & -- & -- & 22,638 & -- & -- & 128,236 & 51,324 & 363,225 & 42,619 & 99,205 & 548,707 & 20.5 \\
\hline 1993 & -- & 46,706 & 80,930 & 46,494 & 41,956 & -- & -- & 24,396 & -- & -- & 135,704 & 57,106 & 386,586 & 44,255 & 97,809 & 575,356 & 19.9 \\
\hline 1994 & -- & 47,581 & 87,165 & 46,019 & 42,562 & -- & -- & 27,000 & -- & -- & 132,584 & 56,035 & 391,365 & 44,162 & 102,212 & 585,320 & 18.7 \\
\hline 1995 & -- & 47,705 & 92,619 & 48,577 & 45,782 & -- & -- & 29,192 & -- & -- & 146,569 & 59,595 & 422,334 & 46,618 & 103,592 & 620,249 & 18.9 \\
\hline 1996 & -- & 45,094 & 90,798 & 44,302 & 46,402 & -- & -- & 28,253 & -- & -- & 145,650 & 60,072 & 415,477 & 47,480 & 108,739 & 616,790 & 17.5 \\
\hline 1997 & 35,375 & 49,269 & 93,492 & 49,464 & 45,887 & -- & -- & 29,787 & -- & -- & 137,382 & 36,479 & 392,491 & 50,541 & 110,001 & 637,677 & 16.2 \\
\hline 1998 & 38,730 & 49,566 & 93,784 & 48,445 & 47,509 & -- & -- & 30,722 & -- & -- & 143,226 & 37,724 & 401,410 & 54,666 & 115,921 & 660,293 & 14.3 \\
\hline 1999 & 37,493 & 52,855 & 99,239 & 51,645 & 51,535 & -- & -- & 31,903 & -- & -- & 149,685 & 38,609 & 422,616 & 55,529 & 113,629 & 682,122 & 14.6 \\
\hline 2000 & 37,194 & 50,057 & 92,033 & 49,477 & 52,552 & -- & -- & 28,605 & -- & -- & 156,088 & 40,199 & 418,954 & 57,606 & 114,602 & 678,413 & 15.7 \\
\hline 2001 & 39,062 & 55,949 & 100,235 & 54,015 & 56,344 & -- & -- & 28,321 & -- & -- & 149,293 & 40,273 & 428,481 & 55,201 & 109,119 & 687,812 & 14.5 \\
\hline 2002 & 40,696 & 56,012 & 102,996 & 55,569 & 56,396 & -- & -- & 29,119 & -- & -- & 158,767 & 39,688 & 442,535 & 56,248 & 119,074 & 714,565 & 16.4 \\
\hline 2003 & 40,475 & 55,018 & 98,487 & 53,566 & 56,988 & -- & -- & 28,831 & -- & -- & 153,110 & 40,367 & 431,349 & 59,996 & 122,537 & 709,375 & 18.6 \\
\hline 2004 & 42,383 & 52,549 & 95,300 & 52,049 & 53,439 & -- & -- & 29,351 & -- & -- & 157,615 & 40,106 & 427,860 & 58,531 & 123,136 & 704,459 & 20.9 \\
\hline 2005 & 46,396 & 58,960 & -- & -- & -- & -- & -- & 39,918 & -- & 190,200 & 190,705 & 41,727 & 462,550 & 60,210 & 130,760 & 758,876 & 15.4 \\
\hline 2006 & 45,751 & 63,241 & -- & -- & -- & -- & -- & 42,194 & -- & 191,920 & 199,052 & 42,882 & 476,048 & 62,339 & 142,096 & 789,475 & 12.9 \\
\hline 2007 & 46,676 & 58,314 & -- & -- & -- & -- & -- & 41,684 & -- & 181,700 & 209,109 & 43,167 & 475,660 & 62,188 & 139,389 & 782,227 & 16.1 \\
\hline 2008 & 44,836 & 58,543 & -- & -- & -- & -- & -- & 39,677 & -- & 169,155 & 199,779 & 43,476 & 452,087 & 62,174 & 134,829 & 752,470 & 18.2 \\
\hline 2009 & 46,550 & 55,944 & -- & -- & -- & -- & -- & 37,963 & -- & 161,241 & 191,032 & 41,465 & 431,701 & 63,518 & 128,245 & 725,958 & 22.2 \\
\hline 2010 & $\mathrm{R}_{45,722}$ & ${ }^{R} 60,554$ & -- & -- & -- & 4,598 & 108,346 & $R_{--}$ & 136,465 & $R_{--}$ & $R_{164,058}$ & $\mathrm{R}_{53,077}$ & 466,543 & $R_{65,776}$ & $R_{129,352}$ & $\mathrm{R}_{767,948}$ & $\mathrm{R}_{19.1}$ \\
\hline $2011^{F}$ & 46,091 & 60,262 & -- & -- & -- & 4,810 & 98,068 & -- & 148,941 & -- & 164,510 & 53,084 & 469,412 & 63,770 & 130,962 & 770,497 & 22.2 \\
\hline
\end{tabular}

1 Noncoincident peak load is the sum of two or more peak loads on individual systems that do not occur at the same time interval. Peak load represents one hour of a day during the associated peak period. See "Noncoincident Peak Load" in Glossary.

See "North American Electric Reliablility Corporation (NERC)" in Glossary. Data include the U.S. portion of NERC only.

Historically, the MRO, RFC, SERC, and SPP regional boundaries were altered as utilities changed reliability organizations. The historical data series for these regions have not been adjusted. Instead, the "Balance of Eastern Region" category was introduced to provide a consistent trend of the Eastern Interconnection.

Electric Reliability Council of Texas (ERCOT).

Florida Reliability Coordinating Council (FRCC).

Northeast Power Coordinating Council (NPCC).

East Central Area Reliability Coordination Agreement (ECAR).

8 ECAR, MAAC, and MAIN dissolved at the end of 2005. Many of the former utility members joined RFC, which came into existence on January 1, 2006. RFC submitted a consolidated filing covering the historical NERC regions of ECAR, MAAC, and MAIN.

Mid-Atlantic Area Council (MAAC).

10 Mid-America Interconnected Network (MAIN)

1 Mid-Continent Area Power Pool (MAPP).
12 Midwest Independent Transmission System Operator (MISO)

13 Midwest Reliability Organization (MRO).

14 PJM Interconnection (PJM).

15 ReliabilityFirst Corporation (RFC).

16 SERC Reliability Corporation (SERC)

17 Southwest Power Pool (SPP).

18 Texas Reliability Entity (TRE).

19 Western Electricity Coordinating Council (WECC).

20 United States excluding Alaska and Hawail.

${ }_{21}$ Capacity margin is the amount of unused available capability of an electric power system at peak load as a percentage of capacity resources. Data are for the United States excluding Alaska and Hawaii.

$\mathrm{R}=$ Revised. F=Forecast. NA=Not available. $--=$ Not applicable.

Notes: - The summer peak period is June through September. - Totals may not equal sum of

components due to independent rounding.

Web Page: For related information, see http://www.eia.gov/electricity/.

Sources: U.S. Energy Information Administration (EIA), Electric Power Annual 2010 (November 2011), Tables 4.1.A., 4.1.B., 4.3.A., and 4.3.B.; and EIA, Form EIA-411, "Coordinated Bulk Power Supply and Demand Program Report," and predecessor forms. 
Table 8.12b Electric Noncoincident Peak Load and Capacity Margin: Winter Peak Period, 1986-2011 (Megawatts, Except as Noted)

\begin{tabular}{|c|c|c|c|c|c|c|c|c|c|c|c|c|c|c|c|c|c|}
\hline \multirow[b]{4}{*}{ Year } & \multicolumn{16}{|c|}{ Noncoincident Peak Load ${ }^{1}$ by North American Electric Reliability Corporation (NERC) ${ }^{2}$ Regional Assessment Area } & \multirow{4}{*}{$\begin{array}{c}\text { Capacity } \\
\text { Margin 2 } \\
\text { (percent) }\end{array}$} \\
\hline & \multicolumn{13}{|c|}{ Eastern Interconnection } & \multirow{3}{*}{\begin{tabular}{|c|} 
ERCOT $^{4}$ \\
TRE ${ }^{18}$
\end{tabular}} & \multirow{3}{*}{\begin{tabular}{|c|}
$\begin{array}{c}\text { Western } \\
\text { Inter- } \\
\text { connection }\end{array}$ \\
WECC 19
\end{tabular}} & \multirow{3}{*}{\begin{tabular}{|c|c}
$\begin{array}{c}\text { All } \\
\text { Inter- } \\
\text { connections }\end{array}$ \\
Total 20
\end{tabular}} & \\
\hline & \multirow[b]{2}{*}{ FRCC 5} & \multirow[b]{2}{*}{ NPCC ${ }^{6}$} & \multicolumn{11}{|c|}{ Balance of Eastern Region ${ }^{3}$} & & & & \\
\hline & & & $\operatorname{ECAR}^{7,8}$ & MAAC 8,9 & MAIN 8,10 & MAPP 11 & MISO 12 & MRO ${ }^{13}$ & PJM 14 & $\mathbf{R F C}^{8,15}$ & SERC ${ }^{16}$ & SPP 17 & Subtotal & & & & \\
\hline 1986 & -- & 37,976 & 64,561 & 32,807 & 28,036 & -- & -- & 18,850 & -- & -- & 101,849 & 33,877 & 279,980 & 28,730 & 76,171 & 422,857 & NA \\
\hline 1987 & -- & 41,902 & 68,118 & 35,775 & 30,606 & -- & -- & 19,335 & -- & -- & 105,476 & 34,472 & 293,782 & 31,399 & 81,182 & 448,265 & NA \\
\hline 1988 & -- & 42,951 & 67,771 & 36,363 & 30,631 & -- & -- & 20,162 & -- & -- & 108,649 & 35,649 & 299,225 & 34,621 & 82,937 & 459,734 & NA \\
\hline 1989 & -- & 42,588 & 73,080 & 38,161 & 33,770 & -- & -- & 21,360 & -- & -- & 121,995 & 42,268 & 330,634 & 38,388 & 84,768 & 496,378 & NA \\
\hline 1990 & -- & 40,545 & 67,097 & 36,551 & 32,461 & -- & -- & 21,113 & -- & -- & 117,448 & 38,949 & 313,619 & 35,815 & 94,252 & 484,231 & NA \\
\hline 1991 & -- & 41,866 & 71,181 & 37,983 & 33,420 & -- & -- & 21,432 & -- & -- & 119,575 & 38,759 & 322,350 & 35,448 & 86,097 & 485,761 & NA \\
\hline 1992 & -- & 41,125 & 72,885 & 37,915 & 31,289 & -- & -- & 21,866 & -- & -- & 121,250 & 39,912 & 325,117 & 35,055 & 91,686 & 492,983 & NA \\
\hline 1993 & -- & 42,063 & 81,846 & 41,406 & 34,966 & -- & -- & 21,955 & -- & -- & 133,635 & 41,644 & 355,452 & 35,407 & 88,811 & 521,733 & NA \\
\hline 1994 & -- & 42,547 & 75,638 & 40,653 & 33,999 & -- & -- & 23,033 & -- & -- & 132,661 & 42,505 & 348,489 & 36,180 & 91,037 & 518,253 & NA \\
\hline 1995 & -- & 42,755 & 83,465 & 40,790 & 35,734 & -- & -- & 23,429 & -- & -- & 142,032 & 44,624 & 370,074 & 36,965 & 94,890 & 544,684 & NA \\
\hline 1996 & -- & 41,208 & 84,534 & 40,468 & 37,162 & -- & -- & 24,251 & -- & -- & 143,060 & 49,095 & 378,570 & 38,868 & 95,435 & 554,081 & 27.7 \\
\hline 1997 & 33,076 & 41,338 & 75,670 & 37,217 & 34,973 & -- & -- & 25,390 & -- & -- & 122,649 & 27,437 & 323,336 & 37,966 & 94,158 & 529,874 & 26.0 \\
\hline 1998 & 39,975 & 44,199 & 84,401 & 36,532 & 37,410 & -- & -- & 26,080 & -- & -- & 127,416 & 27,847 & 339,686 & 41,876 & 101,822 & 567,558 & 25.7 \\
\hline 1999 & 40,178 & 45,227 & 86,239 & 40,220 & 39,081 & -- & -- & 25,200 & -- & -- & 128,563 & 27,963 & 347,266 & 39,164 & 99,080 & 570,915 & 26.7 \\
\hline 2000 & 38,606 & 43,852 & 84,546 & 43,256 & 41,943 & -- & -- & 24,536 & -- & -- & 139,146 & 30,576 & 364,003 & 44,641 & 97,324 & 588,426 & 29.5 \\
\hline 2001 & 40,922 & 42,670 & 85,485 & 39,458 & 40,529 & -- & -- & 21,815 & -- & -- & 135,182 & 29,614 & 352,083 & 44,015 & 96,622 & 576,312 & 28.9 \\
\hline 2002 & 45,635 & 46,009 & 87,300 & 46,551 & 42,412 & -- & -- & 23,645 & -- & -- & 141,882 & 30,187 & 371,977 & 45,414 & 95,951 & 604,986 & 29.4 \\
\hline 2003 & 36,841 & 48,079 & 86,332 & 45,625 & 41,719 & -- & -- & 24,134 & -- & -- & 137,972 & 28,450 & 364,232 & 42,702 & 102,020 & 593,874 & 33.5 \\
\hline 2004 & 44,839 & 48,176 & 91,800 & 45,905 & 42,929 & -- & -- & 24,526 & -- & -- & 144,337 & 29,490 & 378,987 & 44,010 & 102,689 & 618,701 & 31.6 \\
\hline 2005 & 42,657 & 46,828 & -- & -- & -- & -- & -- & 33,748 & -- & 151,600 & 164,638 & 31,260 & 381,246 & 48,141 & 107,493 & 626,365 & 30.2 \\
\hline 2006 & 42,526 & 46,697 & -- & -- & -- & -- & -- & 34,677 & -- & 149,631 & 175,163 & 30,792 & 390,263 & 50,402 & 111,093 & 640,981 & 30.9 \\
\hline 2007 & 41,701 & 46,795 & -- & -- & -- & -- & -- & 33,191 & -- & 141,900 & 179,888 & 31,322 & 386,301 & 50,408 & 112,700 & 637,905 & 30.4 \\
\hline 2008 & 45,275 & 46,043 & -- & -- & -- & -- & -- & 36,029 & -- & 142,395 & 179,596 & 32,809 & 390,829 & 47,806 & 113,605 & 643,557 & 31.0 \\
\hline 2009 & 53,022 & 44,864 & -- & -- & -- & -- & -- & 35,351 & -- & 143,827 & 193,135 & 32,863 & 405,176 & 56,191 & 109,565 & 668,818 & 28.5 \\
\hline 2010 & $\mathrm{R}_{46,135}$ & $\mathrm{R}_{45,712}$ & -- & -- & -- & 5,069 & 86,728 & $R_{--}$ & 115,535 & $R_{--}$ & $R_{152,030}$ & $\mathrm{R}_{41,226}$ & 400,589 & R57,315 & $R_{101,668}$ & R651,418 & R33.7 \\
\hline $2011^{F}$ & 47,613 & 46,788 & -- & -- & -- & 5,118 & 79,052 & -- & 130,711 & -- & 154,150 & 41,138 & 410,168 & 51,642 & 106,717 & 662,928 & 33.1 \\
\hline
\end{tabular}

1 Noncoincident peak load is the sum of two or more peak loads on individual systems that do not occur at the same time interval. Peak load represents one hour of a day during the associated peak period. See "Noncoincident Peak Load" in Glossary.

See "North American Electric Reliablility Corporation (NERC)" in Glossary. Data include the U.S. portion of NERC only.

Historically, the MRO, RFC, SERC, and SPP regional boundaries were altered as utilities changed reliability organizations. The historical data series for these regions have not been adjusted. Instead, the "Balance of Eastern Region" category was introduced to provide a consistent trend of the Eastern Interconnection.

Electric Reliability Council of Texas (ERCOT).

Florida Reliability Coordinating Council (FRCC).

Northeast Power Coordinating Council (NPCC).

East Central Area Reliability Coordination Agreement (ECAR).

$\triangle$ ECAR, MAAC, and MAIN dissolved at the end of 2005. Many of the former utility members joined RFC, which came into existence on January 1, 2006. RFC submitted a consolidated filing covering the historical NERC regions of ECAR, MAAC, and MAIN.

Mid-Atlantic Area Council (MAAC).

10 Mid-America Interconnected Network (MAIN)

11 Mid-Continent Area Power Pool (MAPP).
12 Midwest Independent Transmission System Operator (MISO)

13 Midwest Reliability Organization (MRO).

14 PJM Interconnection (PJM).

15 ReliabilityFirst Corporation (RFC).

${ }^{16}$ SERC Reliability Corporation (SERC).

17 Southwest Power Pool (SPP)

18 Texas Reliability Entity (TRE).

19 Western Electricity Coordinating Council (WECC).

20 United States excluding Alaska and Hawail.

${ }_{21}$ Capacity margin is the amount of unused available capability of an electric power system at peak load as a percentage of capacity resources. Data are for the United States excluding Alaska and Hawaii.

R=Revised. F=Forecast. NA=Not available. $--=$ Not applicable.

Notes: - The winter peak period is October through May of the following year. In this table, data years correspond to the beginning of the winter peak period; for example, data year 2011 represents October 2011-May 2012. - Totals may not equal sum of components due to independent rounding.

Web Page: For related information, see http://wwW.eia.gov/electricity/. Tables 4.1.A., 4.1.B., 4.4.A., and 4.4.B.; and EIA, Form EIA-411, "Coordinated Bulk Power Supply and Demand Program Report," and predecessor forms. 
Figure 8.13 Electric Utility Demand-Side Management Programs

\section{Actual Peakload Reductions Total, 1989-2010}

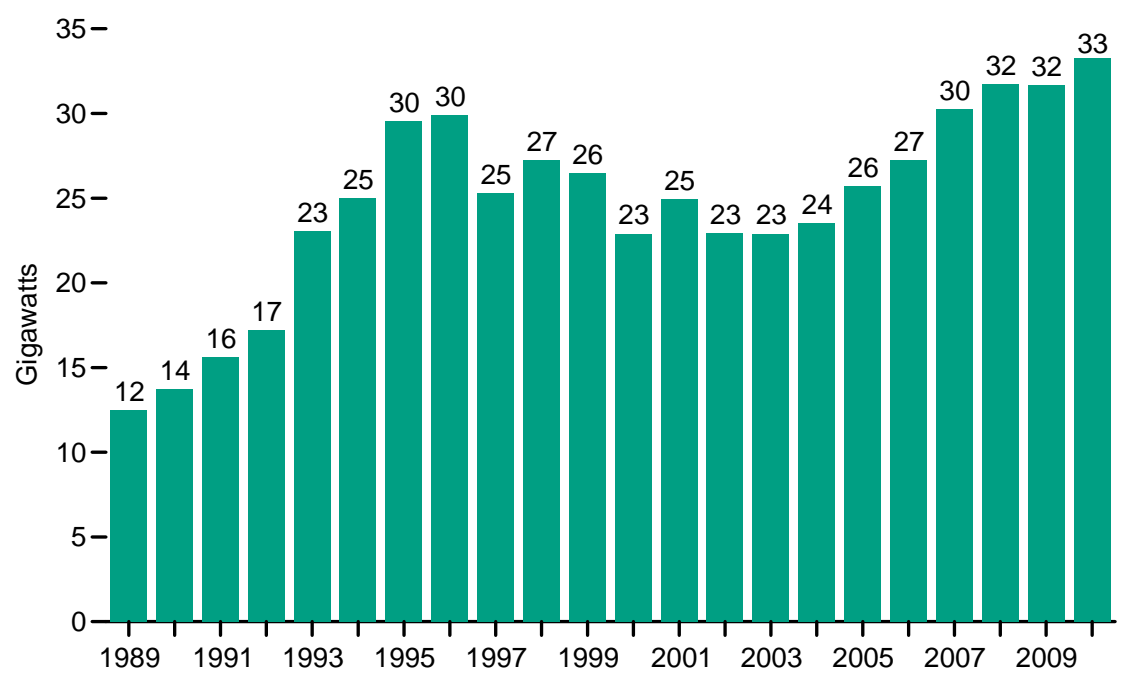

Energy Savings, 1989-2010

$100-$

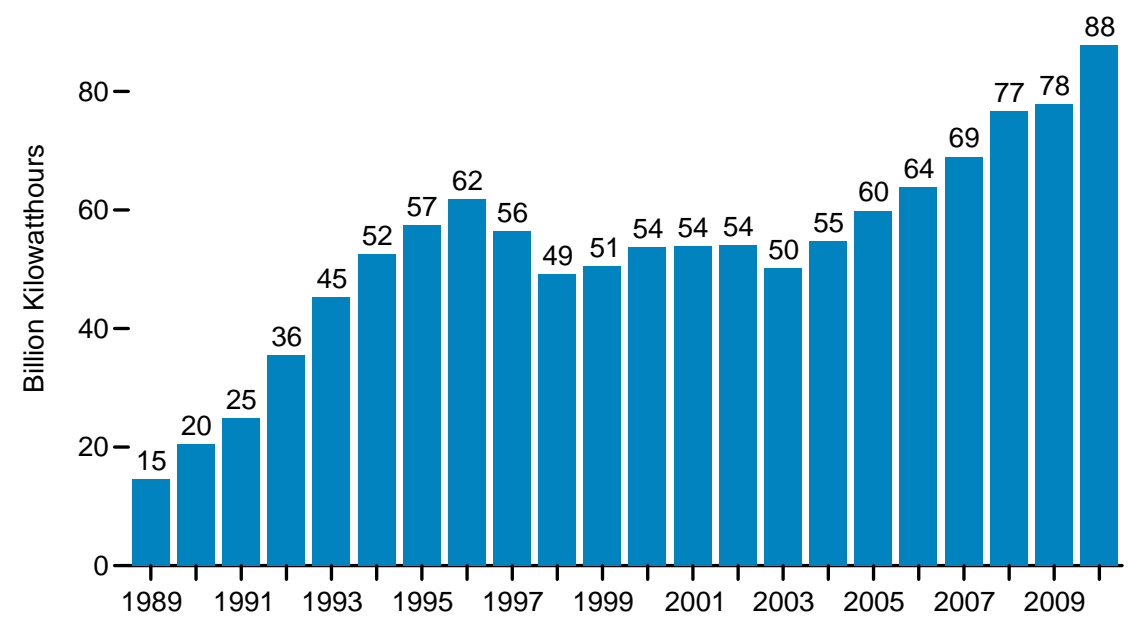

Actual Peakload Reductions, 2010

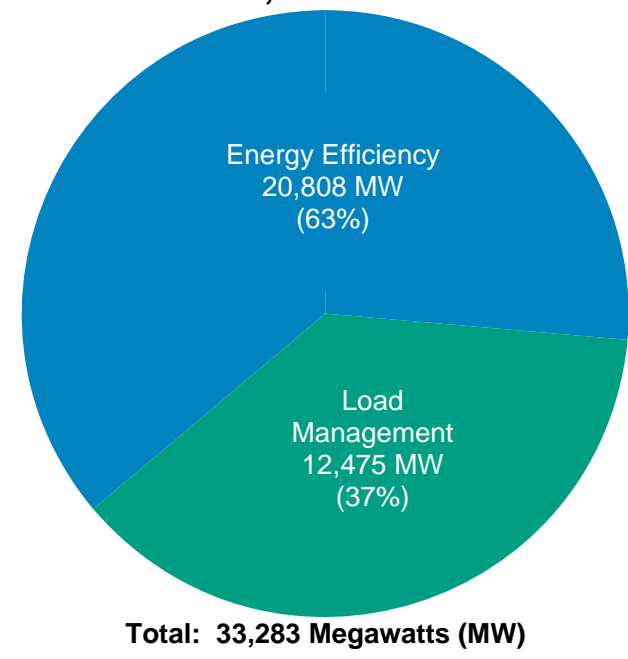

\section{Electric Utility Costs, ${ }^{1}$ 1989-2010}

$$
5-
$$

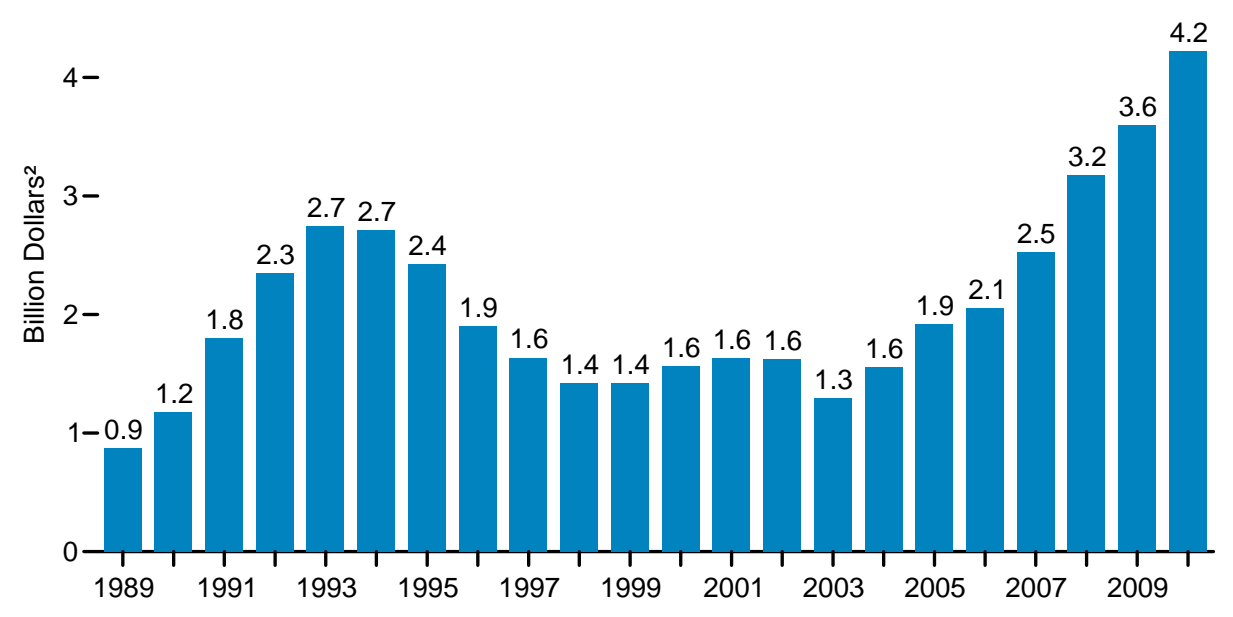

${ }^{1}$ Program costs consist of all costs associated with providing the various Demand-Side Management (DSM) programs or measures. The costs of DSM programs fall into these major categories: customer rebates/incentives, administration/marketing/training, performance incentives, research and evaluation, and other (most likely indirect) costs.
${ }^{2}$ Prices are not adjusted for inflation. See "Nominal Dollars" in Glossary. Source: Table 8.13. 
Table 8.13 Electric Utility Demand-Side Management Programs, 1989-2010

\begin{tabular}{|c|c|c|c|c|c|}
\hline \multirow[b]{3}{*}{ Year } & \multicolumn{3}{|c|}{ Actual Peakload Reductions 1} & \multirow[b]{2}{*}{ Energy Savings } & \multirow[b]{2}{*}{ Electric Utility Costs ${ }^{4}$} \\
\hline & Energy Efficiency ${ }^{2}$ & Load Management ${ }^{3}$ & Total & & \\
\hline & \multicolumn{3}{|c|}{ Megawatts } & Million Kilowatthours & Thousand Dollars ${ }^{5}$ \\
\hline 1989 & NA & NA & 12,463 & 14,672 & 872,935 \\
\hline 1990 & NA & NA & 13,704 & 20,458 & $1,177,457$ \\
\hline 1991 & NA & NA & 15,619 & 24,848 & $1,803,773$ \\
\hline 1992 & 7,890 & 9,314 & 17,204 & 35,563 & $2,348,094$ \\
\hline 1993 & 10,368 & 12,701 & 23,069 & 45,294 & $2,743,533$ \\
\hline 1994 & 11,662 & 13,340 & 25,001 & 52,483 & $2,715,657$ \\
\hline 1995 & 13,212 & 16,347 & 29,561 & 57,421 & $2,421,284$ \\
\hline 1996 & 14,243 & 15,650 & 29,893 & 61,842 & $1,902,197$ \\
\hline 1997 & 13,327 & 11,958 & 25,284 & 56,406 & $1,636,020$ \\
\hline 1998 & 13,591 & 13,640 & 27,231 & 49,167 & $1,420,920$ \\
\hline 1999 & 13,452 & 13,003 & 26,455 & 50,563 & $1,423,644$ \\
\hline 2000 & 12,873 & 10,027 & 22,901 & 53,701 & $1,564,901$ \\
\hline 2001 & 13,027 & 11,928 & 24,955 & 53,936 & $1,630,286$ \\
\hline 2002 & 13,420 & 9,516 & 22,936 & 54,075 & $1,625,537$ \\
\hline 2003 & 13,581 & 9,323 & 22,904 & 50,265 & $1,297,210$ \\
\hline 2004 & 14,272 & 9,260 & 23,532 & 54,710 & $1,557,466$ \\
\hline 2005 & 15,351 & 10,359 & 25,710 & 59,897 & $1,921,352$ \\
\hline 2006 & 15,959 & 11,281 & 27,240 & 63,817 & $2,051,394$ \\
\hline 2007 & 17,710 & 12,543 & 30,253 & 68,992 & $2,523,117$ \\
\hline 2008 & 19,707 & 12,028 & 31,735 & 76,674 & $3,175,410$ \\
\hline 2009 & 19,766 & 11,916 & 31,682 & 77,907 & $3,593,750$ \\
\hline 2010 & 20,808 & 12,475 & 33,283 & 87,839 & $4,220,064$ \\
\hline
\end{tabular}

1 The actual reduction in peak load reflects the change in demand for electricity that results from a utility demand-side management (DSM) program that is in effect at the time that the utility experiences its actual peak load as opposed to the potential installed peakload reduction capacity. Differences between actual and potential peak reduction result from changes in weather, economic activity, and other variable conditions.

conditions.
2 "Energy Efficiency" refers to programs that are aimed at reducing the energy used by specific end-use devices and systems, typically without affecting the services provided. These programs reduce overall electricity consumption, often without explicit consideration for the timing of program-induced savings. Such savings are generally achieved by substituting technically more advanced equipment to produce the same level of end-use services (e g. lighting, heating, motor drive) with less electricity. Examples include high-efficiency appliances, efficient lighting programs, high-efficiency heating ventilating and air conditioning systems or control modifications, efficient building design, advanced electric motor drives, and heat recovery systems.

3 "Load Management" includes programs such as "Direct Load Control," "Interruptible Load Control," and "Other Types" of DSM programs. "Direct Load Control" refers to program activities that can interrupt consumer load at the time of annual peak load by direct control of the utility system operator by interrupting power supply to individual appliances or equipment on consumer premises. This type of control usually involves residential consumers. "Interruptible Load Control" refers to program activities that, in accordance with contractual arrangements, can interrupt consumer load at times of seasonal peak load by direct control of the utility system operator or by action of the consumer at the direct request of the system operator. It

usually involves commercial and industrial consumers. In some instances, the load reduction may be affected by direct action of the system operator (remote tripping) after notice to the consumer in accordance with contractual provisions. "Other Types" are programs that limit or shift peak loads from on-peak to off-peak time periods, such as space heating and water heating storage systems.

4 Program costs consist of all costs associated with providing the various DSM programs or measures. The costs of DSM programs fall into these major categories: customer rebates/incentives, administration/marketing/training, performance, incentives, research and evaluation, and other (most likely indirect) costs.

5 Prices are not adjusted for inflation. See "Nominal Dollars" in Glossary.

Note: This table reports on the results of DSM programs operated by electric utilities. The decrease since 1998 in peakload reductions from DSM programs can be attributed in part to utilities cutting back or terminating these programs due to industry deregulation. Some State governments have created new programs to promote DSM. Examples include the "Energy \$mart Loan Fund" administered by the New York Energy Research and Development Authority and the "Efficiency Vermont" program of the Vermont Public Service Board. Data on energy savings attributable to these non-utility programs are not collected by the U.S. Energy Information Administration (EIA).

Web Page: For related information, see http://www.eia.gov/electricity/.

Sources: • 1989-1998-EIA, Form EIA-861, "Annual Electric Utility Report." • 1999 forward-EIA, Electric Power Annual 2010 (November 2011), Tables 9.1, 9.6, and 9.7. 


\section{Electricity}

Note 1. Coverage of Electricity Statistics. Through 1984, data for electric utilities also include institutions (such as universities) and military facilities that generated electricity primarily for their own use; beginning in 1985, data for electric utilities exclude institutions and military facilities. Data for independent power producers, commercial plants, and industrial plants include plants with a generator nameplate capacity of 1 megawatt or greater; they exclude plants with a generator nameplate capacity less than 1 megawatt. Also excluded from the electricity statistics in Section 8 are data for residential and commercial selfgeneration from solar energy, except for the small amount sold to the grid and included in data for the electric power sector.

Note 2. Classification of Power Plants Into Energy-Use Sectors. The U.S. Energy Information Administration (EIA) classifies power plants (both electricityonly and combined-heat-and-power plants) into energy-use sectors based on the North American Industry Classification System (NAICS), which replaced the Standard Industrial Classification (SIC) system in 1997. Plants with a NAICS code of 22 are assigned to the Electric Power Sector. Those with NAICS codes beginning with 11 (agriculture, forestry, fishing, and hunting); 21 (mining, including oil and gas extraction); 23 (construction); 31-33 (manufacturing); 2212 (natural gas distribution); and 22131 (water supply and irrigation systems) are assigned to the Industrial Sector. Those with all other codes are assigned to the Commercial Sector. Form EIA860, "Annual Electric Generator Report," asks respondents to indicate the primary purpose of the facility by assigning a NAICS code from the list at: http://www.eia.gov/cneaf/electricity/forms/eia860.doc.

Note 3. Electricity Imports and Exports. Through the Annual Energy Review (AER) 2001, EIA estimated the proportions of traded electricity from fossil fuels and hydropower (and applied the fossil-fuel steam-electric-plant heat rate to convert from kilowatthours to Btu) and from geothermal (and applied the heat rate for geothermal energy plants). Beginning with the AER 2002, because of inadequate data, EIA is applying an overall rate of 3,412 Btu per kilowatthour to all traded electricity. In addition, electricity net imports derived from hydroelectric power and geothermal energy are no longer included in renewable energy consumption data. They continue to be included in total U.S. energy consumption as components of electricity net imports, with energy sources unspecified (see Tables 1.3 and 2.1f). This change between AER 2001 and AER 2002 resulted in a 0.0-to-0.5 quadrillion Btu drop in total renewable energy consumption from 1949 forward.

Table 8.1 Sources: Net Generation, Electric Power Sector: Table 8.2b. Net Generation, Commercial Sector: Table 8.2d. Net Generation, Industrial Sector: • 1949-September 1977-Federal Power Commission (FPC), Form FPC-4, "Monthly Power Plant Report," for plants with generating capacity exceeding 10 megawatts, and FPC, Form FPC-12C, "Industrial Electric Generating Capacity," for all other plants. • October 1977-1978-Federal Energy Regulatory Commission (FERC), Form FPC-4, "Monthly Power Plant Report," for plants with generating capacity exceeding 10 megawatts, and FERC, Form FPC-12C, "Industrial Electric Generating Capacity," for all other plants. • 1979—FERC, Form FPC-4, "Monthly Power Plant Report," for plants with generating capacity exceeding 10 megawatts, and EIA estimates for all other plants. • 1980-1988-Estimated by U.S. Energy Information Administration (EIA) as the average generation over the 6-year period of 1974-1979. • 1989 forward-Table 8.2d. Net Generation, Total: Table 8.2a. Imports and Exports: - 1949-September 1977-Unpublished FPC data. • October 1977-1980-Unpublished Economic Regulatory Administration (ERA) data. • 1981-U.S. Department of Energy (DOE), Office of Energy Emergency Operations, "Report on Electric Energy Exchanges with Canada and Mexico for Calendar Year 1981," April 1982 (revised June 1982). • 1982 and 1983-DOE, ERA, Electricity Exchanges Across International Borders. • 19841986-DOE, ERA, Electricity Transactions Across International Borders. • 1987 and 1988-DOE, ERA, Form ERA-781R, "Annual Report of International Electrical Export/Import Data." • 1989—DOE, Fossil Energy, Form FE-781R, "Annual Report of International Electrical Export/Import Data." • 1990 forward-National Energy Board of Canada, and DOE, Office of Electricity Delivery and Energy Reliability, Form OE-781R, "Monthly Electricity Imports and Exports Report," and predecessor form. For 2001 forward, data from the California Independent System Operator are used in combination with the Form OE-781 values to estimate electricity trade with Mexico. T \& D Losses and Unaccounted for: Calculated as the sum of total net generation and imports minus total end use and exports. End Use: Table 8.9. 


\section{Nuclear Energy}




\section{Figure 9.1 Nuclear Generating Units}

\section{Operable Units, ${ }^{1} 1957-2011$}

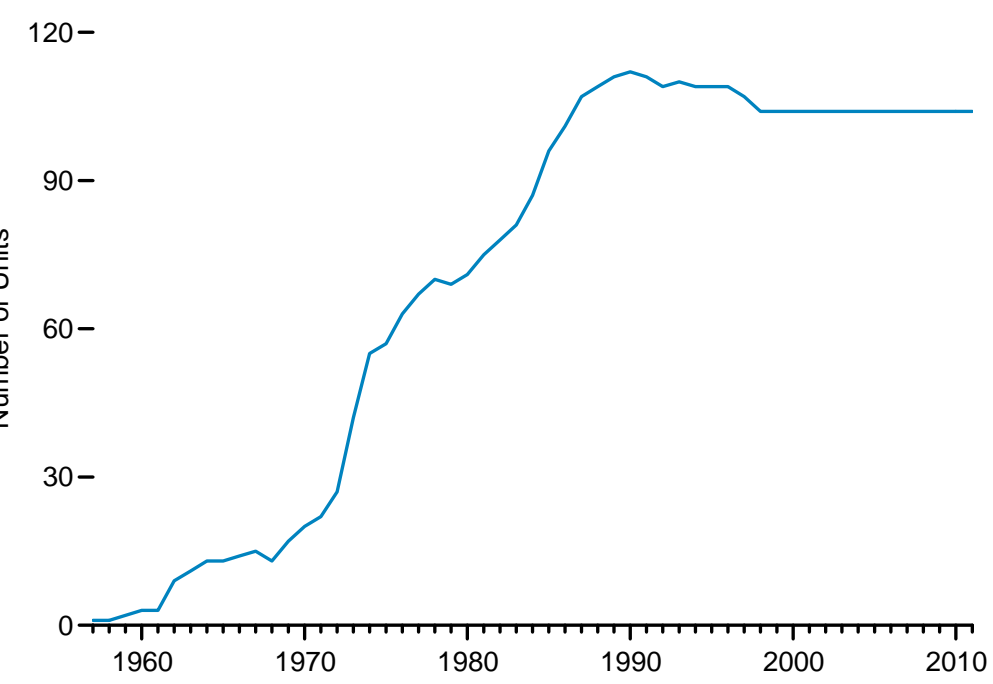

Status of All Nuclear Generating Units, 2011

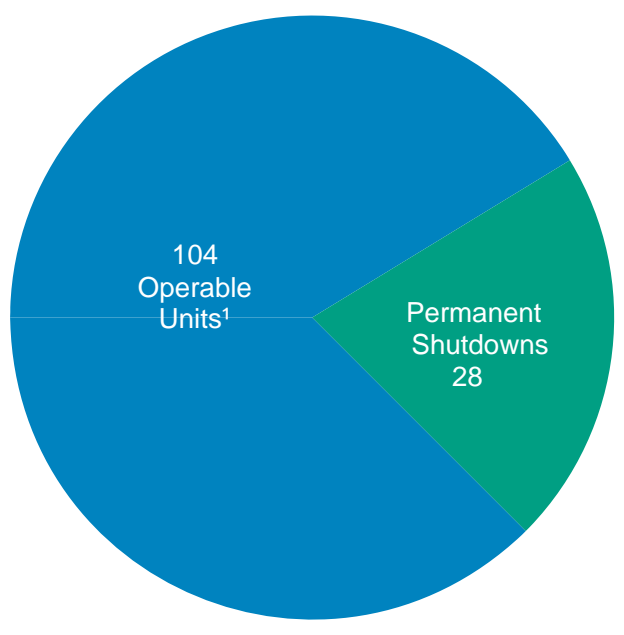

Nuclear Net Summer Capacity Change, 1950-2011

$12-$

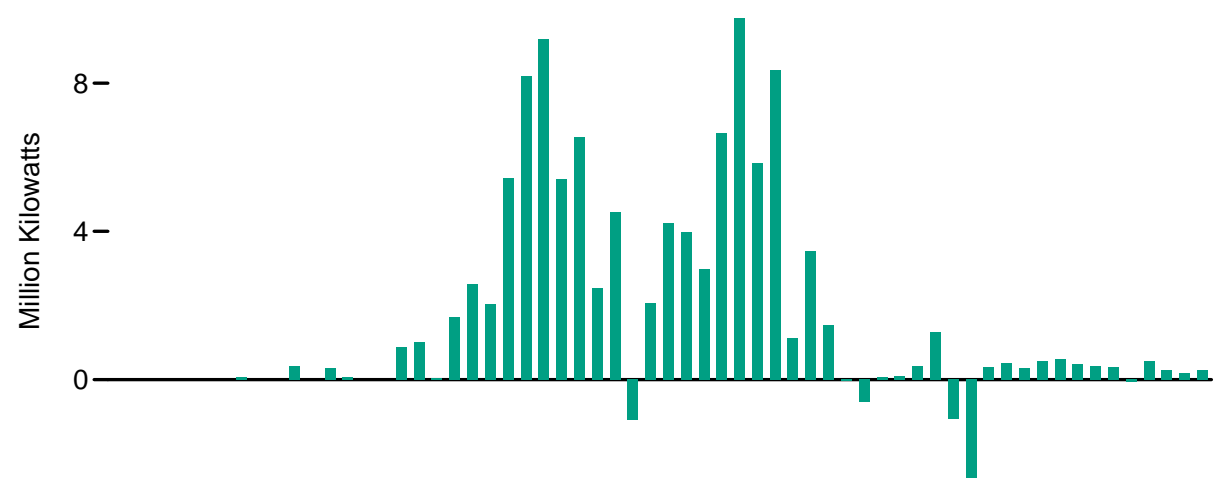

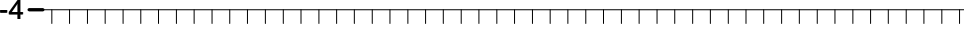

$\begin{array}{lllllllllllll}1950 & 1955 & 1960 & 1965 & 1970 & 1975 & 1980 & 1985 & 1990 & 1995 & 2000 & 2005 & 2010\end{array}$
Permanent Shutdowns by Year, 1955-2011

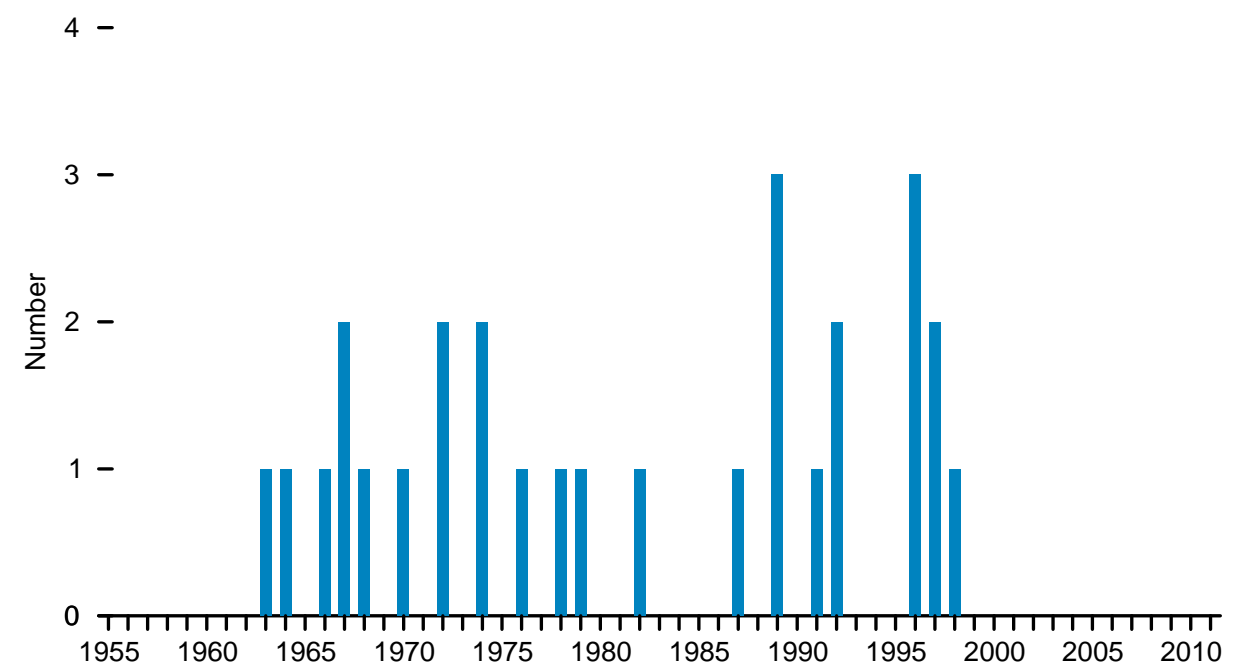

${ }^{1}$ Units holding full-power operating licenses, or equivalent permission to operate, at the end of the year.

Sources: Tables 9.1 and 8.11 a. 
Table 9.1 Nuclear Generating Units, 1955-2011

\begin{tabular}{|c|c|c|c|c|c|c|c|c|}
\hline \multirow[b]{2}{*}{ Year } & \multicolumn{3}{|c|}{ Original Licensing Regulations (10 CFR Part 50) ${ }^{1}$} & \multicolumn{3}{|c|}{ Current Licensing Regulations (10 CFR Part 52) ${ }^{1}$} & \multirow[b]{2}{*}{$\begin{array}{l}\text { Permanent } \\
\text { Shutdowns }\end{array}$} & \multirow[b]{2}{*}{ Operable Units } \\
\hline & $\begin{array}{l}\text { Construction } \\
\text { Permits } \\
\text { Issued } 2,3\end{array}$ & $\begin{array}{l}\text { Low-Power } \\
\text { Operating Licenses } \\
\text { Issued } 3,4\end{array}$ & $\begin{array}{l}\text { Full-Power } \\
\text { Operating Licenses } \\
\text { Issued } 3,5\end{array}$ & $\begin{array}{l}\text { Early Site } \\
\text { Permits } \\
\text { Issued }^{3}\end{array}$ & $\begin{array}{c}\text { Combined } \\
\text { License Applications } \\
\text { Received } 6\end{array}$ & $\begin{array}{l}\text { Combined } \\
\text { Licenses } \\
\text { Issued }^{3}\end{array}$ & & \\
\hline 1955 & 1 & 0 & 0 & -- & -- & -- & 0 & 0 \\
\hline $\begin{array}{l}1956 \\
1957\end{array}$ & $\begin{array}{l}3 \\
1\end{array}$ & 0 & 0 & -- & -- & -- & 0 & 0 \\
\hline 1958 & 0 & 0 & $\begin{array}{l}1 \\
0\end{array}$ & -- & -- & -- & $\begin{array}{l}0 \\
0\end{array}$ & 1 \\
\hline 1959 & 3 & 1 & 1 & -- & -- & -- & 0 & 2 \\
\hline 1960 & 7 & 1 & 1 & -- & -- & -- & 0 & 3 \\
\hline 1961 & $\begin{array}{l}0 \\
1\end{array}$ & 0 & $\begin{array}{l}0 \\
6\end{array}$ & -- & -- & -- & $\begin{array}{l}0 \\
0\end{array}$ & $\begin{array}{l}3 \\
9\end{array}$ \\
\hline 1963 & 1 & 3 & $\begin{array}{l}0 \\
2\end{array}$ & -- & -- & -- & $\mathrm{R}_{1}$ & 99 \\
\hline 1964 & 3 & 2 & 3 & -- & -- & -- & 1 & 13 \\
\hline 1965 & 1 & 0 & 0 & -- & -- & -- & 0 & 13 \\
\hline 1966 & 5 & 1 & 2 & -- & -- & -- & 1 & 14 \\
\hline 1967 & 14 & 3 & 3 & -- & -- & -- & 2 & 15 \\
\hline 1968 & 23 & 0 & 0 & -- & -- & -- & $\mathrm{R}_{1}^{\mathrm{C}}$ & 13 \\
\hline 1969 & $\begin{array}{r}0 \\
7\end{array}$ & 4 & 4 & -- & -- & -- & 0 & 17 \\
\hline 1970 & 10 & 4 & 3 & -- & -- & -- & $R_{1}$ & 20 \\
\hline 1971 & 4 & 5 & 2 & -- & -- & -- & 0 & 22 \\
\hline 1972 & 8 & 6 & 6 & -- & -- & -- & $\mathrm{R}_{2}$ & 27 \\
\hline 1973 & 14 & 12 & 15 & -- & -- & -- & $\overline{0}$ & 42 \\
\hline 1974 & 23 & 14 & 15 & -- & -- & -- & 2 & 55 \\
\hline 1975 & 9 & 3 & 2 & -- & -- & -- & 0 & 57 \\
\hline 1976 & 9 & 7 & 7 & -- & -- & -- & 1 & 63 \\
\hline 1977 & 15 & 4 & 4 & -- & -- & -- & 0 & 67 \\
\hline 1978 & 13 & 3 & 4 & -- & -- & -- & 1 & 70 \\
\hline 1979 & 2 & 0 & 0 & -- & -- & -- & 1 & 69 \\
\hline 1980 & 0 & 5 & 2 & -- & -- & -- & 0 & 71 \\
\hline 1981 & 0 & 3 & 4 & -- & -- & -- & 0 & 75 \\
\hline 1982 & 0 & 6 & 4 & -- & -- & -- & 1 & 78 \\
\hline 1983 & $\begin{array}{l}0 \\
0\end{array}$ & 3 & 3 & -- & -- & -- & 0 & 81 \\
\hline $\begin{array}{l}1984 \\
1985\end{array}$ & $\begin{array}{l}0 \\
0 \\
0\end{array}$ & 7 & 6 & -- & -- & -- & 0 & 87 \\
\hline $\begin{array}{l}1985 \\
1986\end{array}$ & $\begin{array}{l}0 \\
0\end{array}$ & 7 & $\begin{array}{l}9 \\
5\end{array}$ & -- & -- & -- & $\begin{array}{l}0 \\
0\end{array}$ & $\begin{array}{r}96 \\
101\end{array}$ \\
\hline 1987 & 0 & 6 & 8 & -- & -- & -- & $R_{1}$ & 107 \\
\hline 1988 & 0 & 1 & 2 & -- & -- & -- & 0 & 109 \\
\hline 1989 & 0 & 3 & 4 & -- & -- & -- & $\mathrm{R}_{3}$ & 111 \\
\hline 1990 & 0 & 1 & 2 & -- & -- & -- & $\mathrm{R}_{0}$ & 112 \\
\hline 1991 & 0 & 0 & 0 & -- & -- & -- & 1 & 111 \\
\hline 1992 & 0 & 0 & 0 & -- & -- & -- & 2 & 109 \\
\hline 1993 & 0 & 1 & 1 & -- & -- & -- & 0 & 110 \\
\hline 1994 & 0 & 0 & 0 & -- & -- & -- & $R_{0}$ & 109 \\
\hline 1995 & 0 & 1 & 0 & -- & -- & -- & 0 & 109 \\
\hline 1996 & 0 & 0 & 1 & -- & -- & -- & $\mathrm{R}_{3}$ & 109 \\
\hline 1997 & 0 & 0 & 0 & 0 & 0 & 0 & ${ }_{81}^{2}$ & 107 \\
\hline 1998 & 0 & 0 & 0 & 0 & 0 & 0 & $\mathrm{R}_{1}$ & 104 \\
\hline 1999-2006 & 0 & 0 & 0 & 0 & 0 & 0 & 0 & 104 \\
\hline 2007 & $\begin{array}{l}0 \\
0\end{array}$ & $\begin{array}{l}0 \\
0\end{array}$ & $\begin{array}{l}0 \\
0\end{array}$ & $\begin{array}{l}3 \\
0\end{array}$ & $\begin{array}{r}5 \\
\mathrm{R}_{12}\end{array}$ & $\begin{array}{l}0 \\
0\end{array}$ & $\begin{array}{l}0 \\
0\end{array}$ & $\begin{array}{l}104 \\
104\end{array}$ \\
\hline 2009 & 0 & 0 & 0 & 1 & 1 & 0 & 0 & $\begin{array}{l}104 \\
\end{array}$ \\
\hline $\begin{array}{l}2010 \\
2011\end{array}$ & $\begin{array}{l}0 \\
0\end{array}$ & $\begin{array}{l}0 \\
0\end{array}$ & $\begin{array}{l}0 \\
0\end{array}$ & $\begin{array}{l}0 \\
0\end{array}$ & $\begin{array}{l}0 \\
0\end{array}$ & $\begin{array}{l}0 \\
0\end{array}$ & $\begin{array}{l}0 \\
0\end{array}$ & $\begin{array}{l}104 \\
104\end{array}$ \\
\hline Total & 177 & 132 & 132 & 4 & R18 & 0 & 28 & -- \\
\hline
\end{tabular}

1 Data in columns $1-3$ are based on the U.S. Nuclear Regulatory Commission (NRC) regulation 10 CFR
Part 50 . Data in columns $4-6$ are based on the NRC regulation 10 CFR Part 52 . See Note 1 , "Pending Actions on Nuclear Generating Units," at end of section. 2 Issuance by regulatory authority of a permit, or equivalent permission, to begin construction. Under
current licensing regulations, the construction permit is no longer issued separately from the operating 3 Nimb

Issuance by regulatory authority of license, or equivalent permission, to conduct testing but not to

5 Issuance by regulatory authority of full-power operating license, or equivalent permission (note that some units receive full-power licenses the same year they receive low-power licenses). Units initially

was Number of applications received for combined construction and operating licenses, including one that applications. See Note 1, "Pending Actions on Nuclear Generating Units," at end of Section.

was the end of the year (the number of operable units equals the cumulative number of uniss hold operate, at licenses minus the cumulative number of permanent shutdowns).

Note: See Note 2, "Coverage of Nuclear Energy Statistics," at end of section.

Web Page: For related information, see http://www.eia.gov/nuclear/.

Sources: See end of section. 


\section{Figure 9.2 Nuclear Power Plant Operations}

Total Electricity and Nuclear Electricity Net Generation, 1957-2011 $5-$

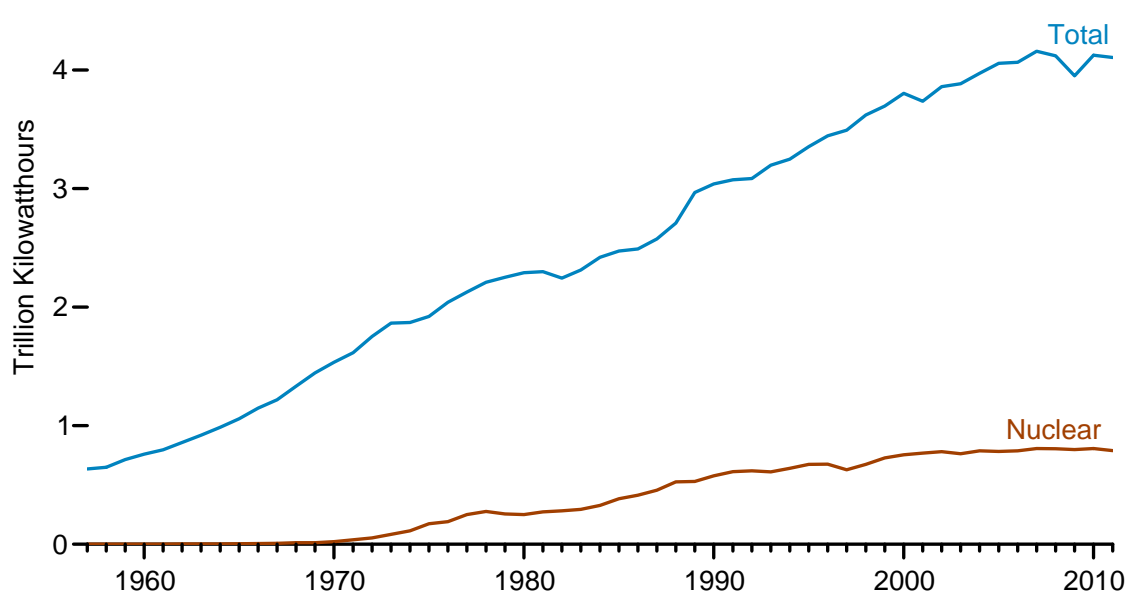

Net Summer Capacity of Operable Units, 1957-2011 $120-$

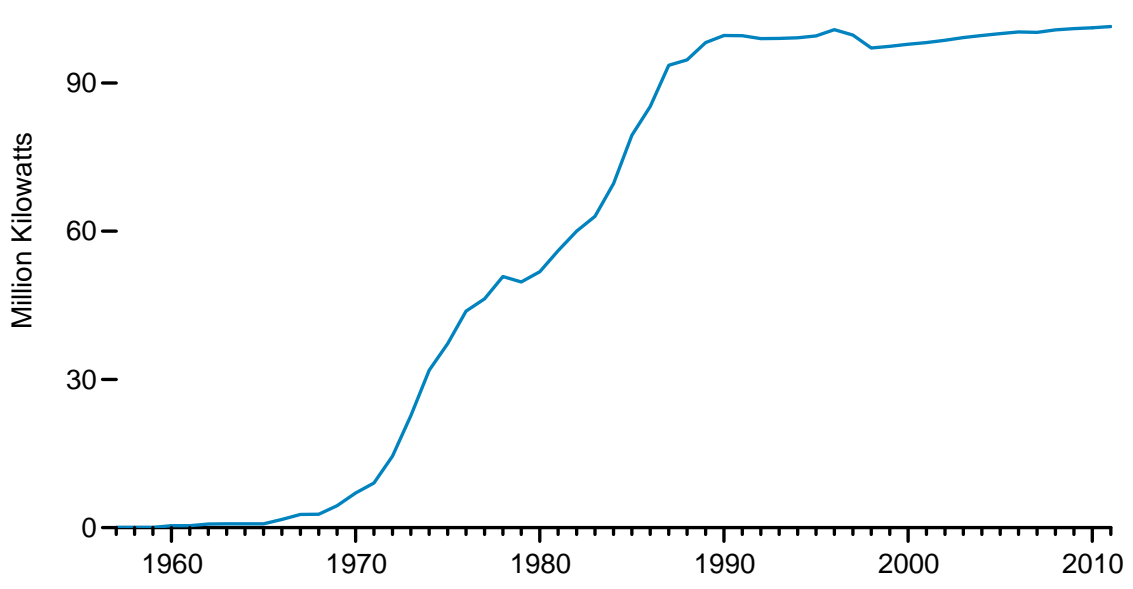

Nuclear Share of Total Electricity Net Generation, 1957-2011

$25-$

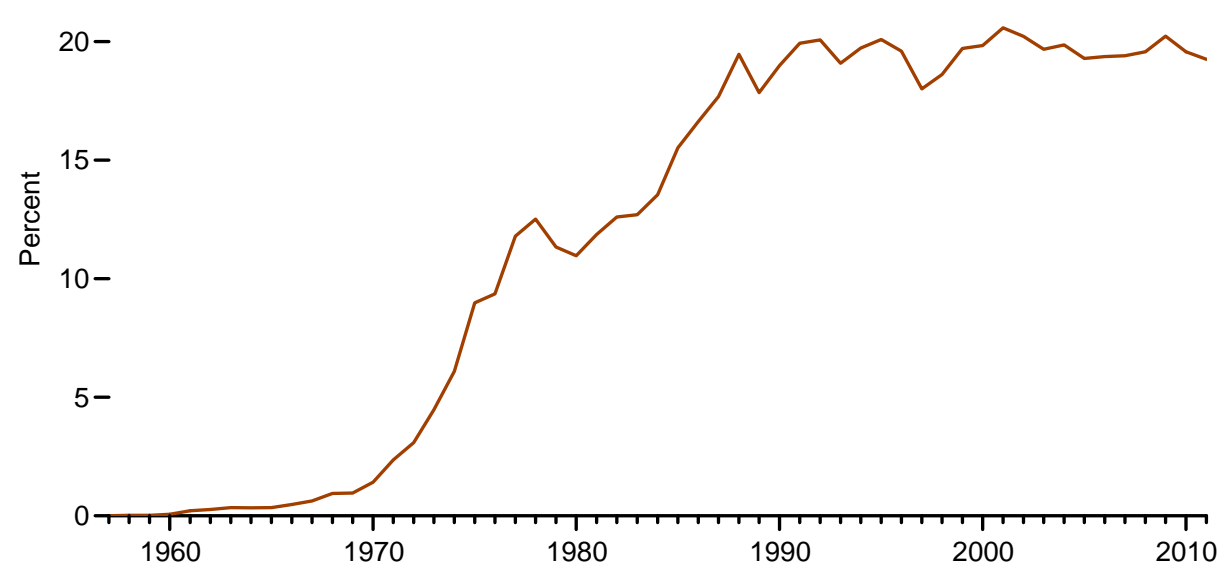

Capacity Factor, 1973-2011

$100-$

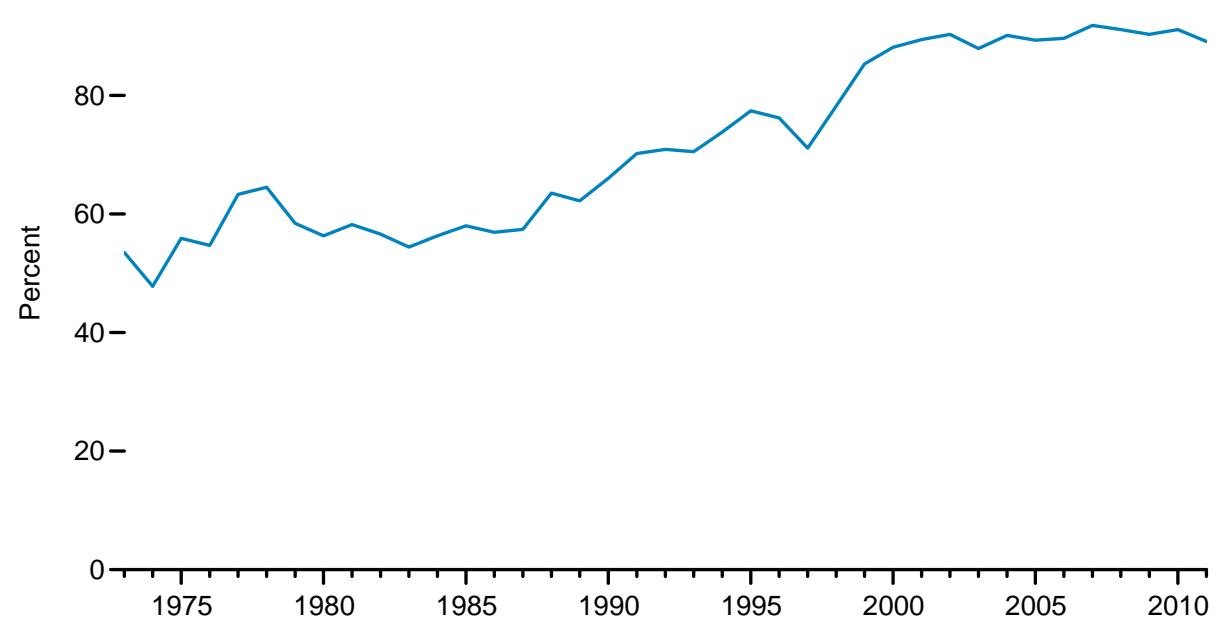

Sources: Tables 8.1 and 9.2. 
Table 9.2 Nuclear Power Plant Operations, 1957-2011

\begin{tabular}{|c|c|c|c|c|}
\hline & Nuclear Electricity Net Generation & Nuclear Share of Total Electricity Net Generation & Net Summer Capacity of Operable Units ${ }^{1}$ & Capacity Factor $^{2}$ \\
\hline Year & Billion Kilowatthours & Percent & Million Kilowatts & Percent \\
\hline 1957 & (s) & (s) & 0.1 & NA \\
\hline 1958 & .2 & (s) & .1 & NA \\
\hline 1959 & .2 & (s) & .1 & NA \\
\hline 1960 & .5 & .1 & .4 & NA \\
\hline 1961 & 1.7 & .2 & .4 & NA \\
\hline 1962 & 2.3 & .3 & .7 & NA \\
\hline 1963 & 3.2 & .3 & .8 & NA \\
\hline 1964 & 3.3 & .3 & .8 & NA \\
\hline 1965 & 3.7 & .3 & .8 & NA \\
\hline 1966 & 5.5 & .5 & 1.7 & NA \\
\hline 1967 & 7.7 & .6 & 2.7 & NA \\
\hline 1968 & 12.5 & .9 & 2.7 & NA \\
\hline 1969 & 13.9 & 1.0 & 4.4 & NA \\
\hline 1970 & 21.8 & 1.4 & 7.0 & NA \\
\hline 1971 & 38.1 & 2.4 & 9.0 & NA \\
\hline 1972 & 54.1 & 3.1 & 14.5 & NA \\
\hline 1973 & 83.5 & 4.5 & 22.7 & 53.5 \\
\hline 1974 & 114.0 & 6.1 & 31.9 & 47.8 \\
\hline 1975 & 172.5 & 9.0 & 37.3 & 55.9 \\
\hline 1976 & 191.1 & 9.4 & 43.8 & 54.7 \\
\hline 1977 & 250.9 & 11.8 & 46.3 & 63.3 \\
\hline 1978 & 276.4 & 12.5 & 50.8 & 64.5 \\
\hline 1979 & 255.2 & 11.3 & 49.7 & 58.4 \\
\hline 1980 & 251.1 & 11.0 & 51.8 & 56.3 \\
\hline 1981 & 272.7 & 11.9 & 56.0 & 58.2 \\
\hline 1982 & 282.8 & 12.6 & 60.0 & 56.6 \\
\hline 1983 & 293.7 & 12.7 & 63.0 & 54.4 \\
\hline 1984 & 327.6 & 13.5 & 69.7 & 56.3 \\
\hline 1985 & 383.7 & 15.5 & 79.4 & 58.0 \\
\hline 1986 & 414.0 & 16.6 & 85.2 & 56.9 \\
\hline 1987 & 455.3 & 17.7 & 93.6 & 57.4 \\
\hline 1988 & 527.0 & 19.5 & 94.7 & 63.5 \\
\hline 1989 & 529.4 & 17.8 & 98.2 & 62.2 \\
\hline 1990 & 576.9 & 19.0 & 99.6 & 66.0 \\
\hline 1991 & 612.6 & 19.9 & 99.6 & 70.2 \\
\hline 1992 & 618.8 & 20.1 & 99.0 & 70.9 \\
\hline 1993 & 610.3 & 19.1 & 99.0 & 70.5 \\
\hline 1994 & 640.4 & 19.7 & 99.1 & 73.8 \\
\hline 1995 & 673.4 & 20.1 & 99.5 & 77.4 \\
\hline 1996 & 674.7 & 19.6 & 100.8 & 76.2 \\
\hline 1997 & 628.6 & 18.0 & 99.7 & 71.1 \\
\hline 1998 & 673.7 & 18.6 & 97.1 & 78.2 \\
\hline 1999 & 728.3 & 19.7 & 97.4 & 85.3 \\
\hline 2000 & 753.9 & 19.8 & 97.9 & 88.1 \\
\hline 2001 & 768.8 & 20.6 & 98.2 & 89.4 \\
\hline 2002 & 780.1 & 20.2 & 98.7 & 90.3 \\
\hline 2003 & 763.7 & 19.7 & 99.2 & 87.9 \\
\hline 2004 & 788.5 & 19.9 & 99.6 & 90.1 \\
\hline 2005 & 782.0 & 19.3 & 100.0 & 89.3 \\
\hline 2006 & 787.2 & 19.4 & 100.3 & 89.6 \\
\hline 2007 & 806.4 & 19.4 & 100.3 & 91.8 \\
\hline 2008 & 806.2 & 19.6 & 100.8 & 91.1 \\
\hline 2009 & 798.9 & 20.2 & 101.0 & 90.3 \\
\hline 2010 & 807.0 & 19.6 & $R_{101.2}$ & R91.1 \\
\hline $2011^{P}$ & 790.2 & 19.2 & 101.4 & 89.1 \\
\hline
\end{tabular}

At end of year. See "Generator Net Summer Capacity" in Glossary. See "Generator Capacity Factor" in Glossary.

annual data. - See $h$ ttp: //www.eia.gov/nuclear/ for related information.

Sources. Nuclear Electricity Net Generation and Nuclear Share of Electricity Net Generation Table 8.2a. Net Summer Capacity of Operable Units: Table 8.11a. Capacity Factor: U.S. Energy Information Administration, Monthly Energy Review (April 2012), Table 8.1. Annual capacity factors ar

Nete See Note 2 "Covy. NA=Not avalable. (s)=Less than 0.05 .

We. See Note 2, "Coverage of Nuclear Energy Statistics," at end of section. for updated monthly and 


\section{Figure 9.3 Uranium Overview}

\section{Production and Trade, 1949-2011} 75-

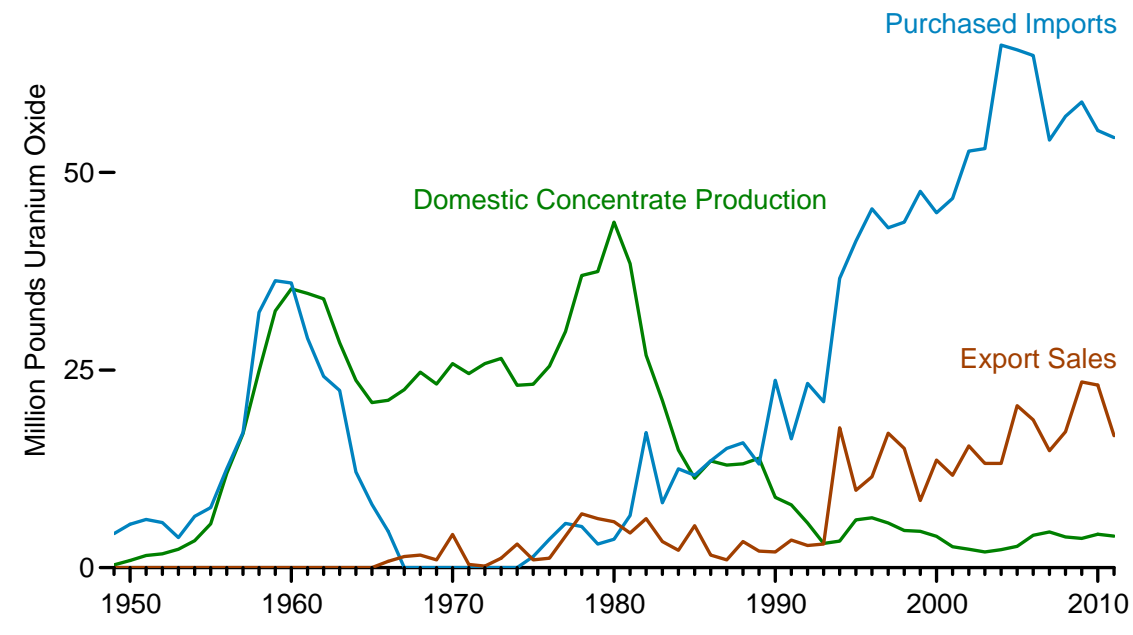

\section{Inventories, End of Year 1981-2011}

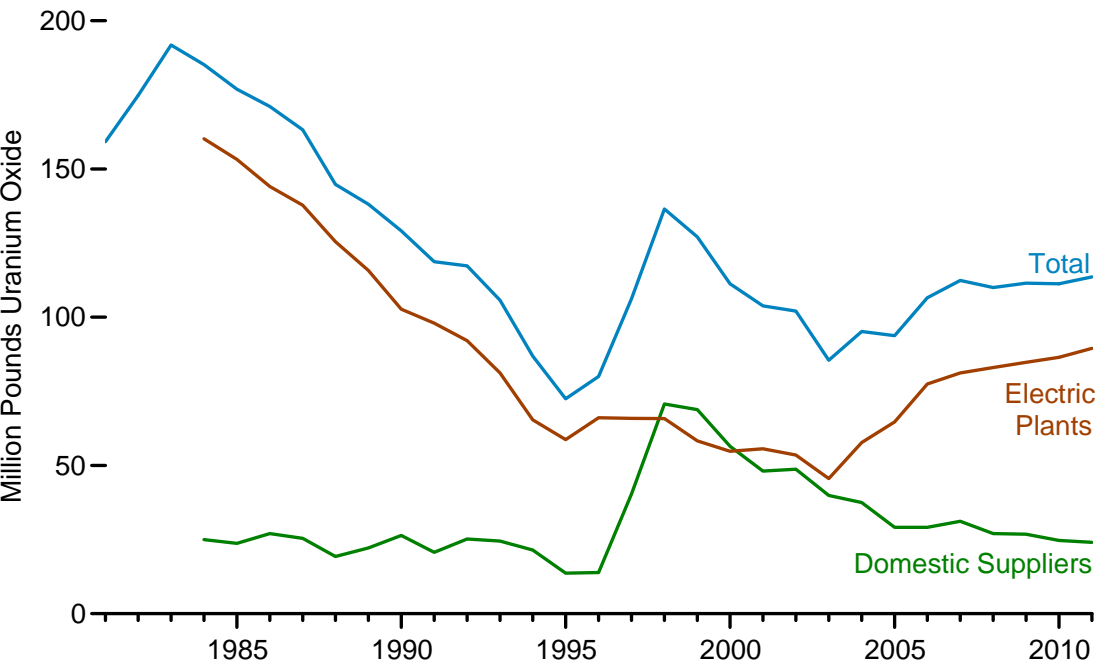

\section{Production and Trade, 2011}

$60-$

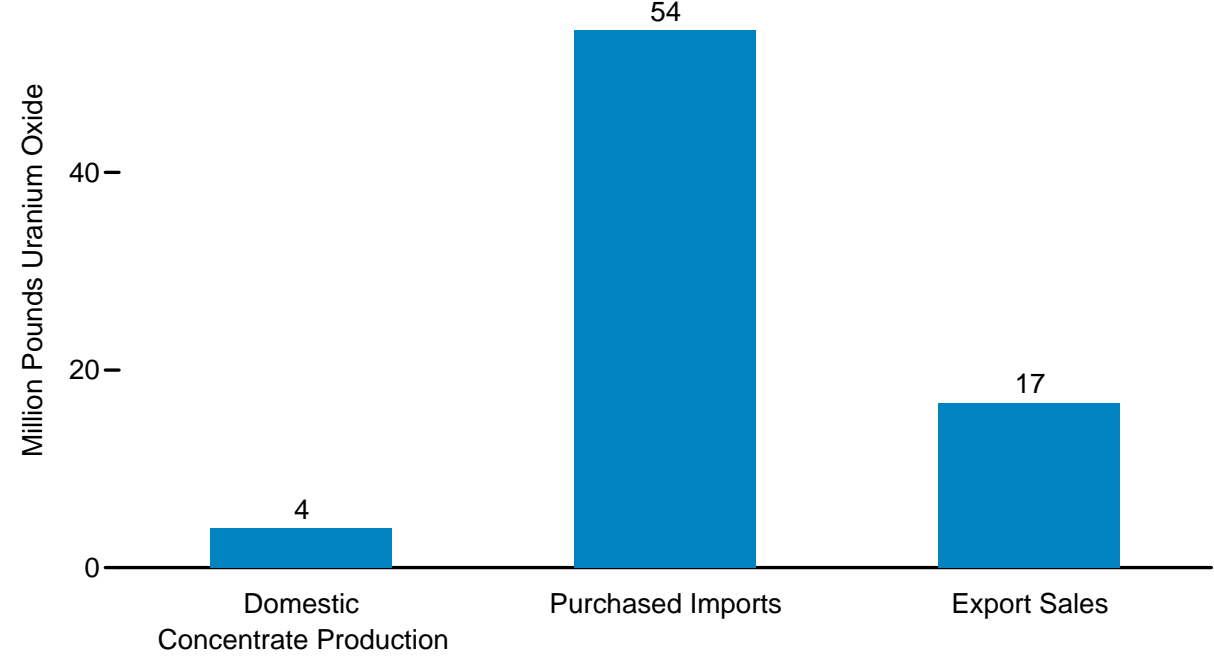

Average Prices, 1981-2011

$60-$

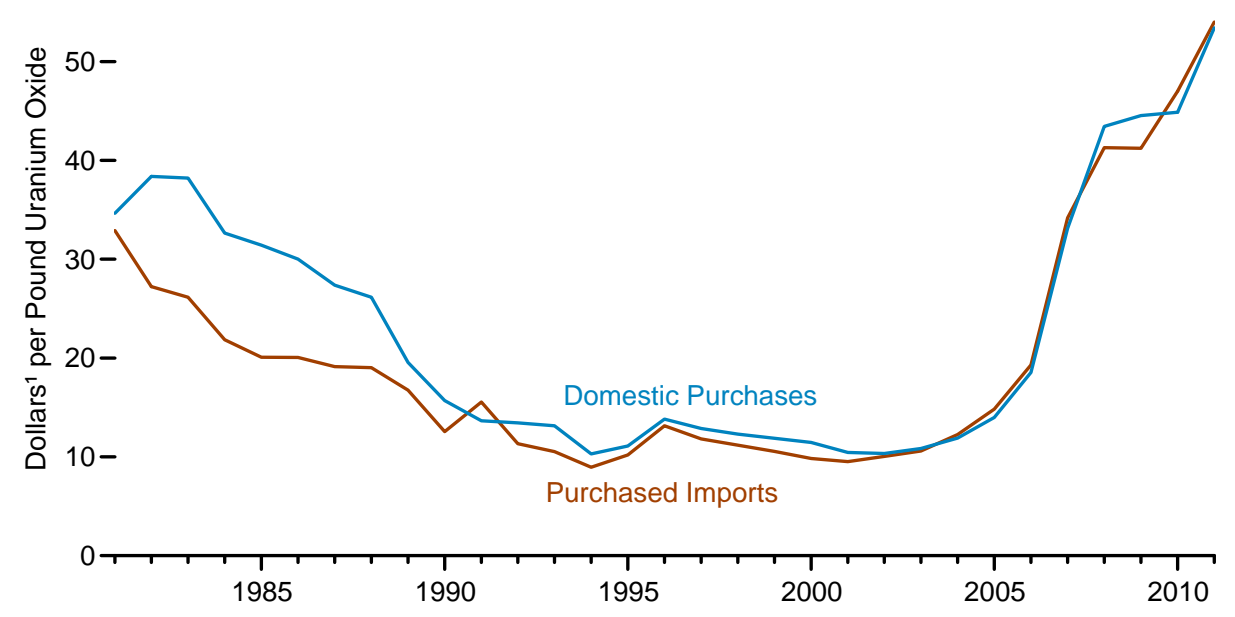

${ }^{1}$ Prices are not adjusted for inflation. See "Nominal Dollars" in Glossary. Note: See "Uranium Oxide" in Glossary. 
Table 9.3 Uranium Overview, Selected Years, 1949-2011

\begin{tabular}{|c|c|c|c|c|c|c|c|c|c|c|}
\hline \multirow[b]{3}{*}{ Year } & \multirow{2}{*}{$\begin{array}{l}\text { Domestic } \\
\text { Concentrate } \\
\text { Production } 1\end{array}$} & \multirow{2}{*}{$\begin{array}{l}\text { Purchased } \\
\text { Imports }^{2}\end{array}$} & \multirow{2}{*}{$\begin{array}{l}\text { Export }^{2} \\
\text { Sales }\end{array}$} & \multirow{2}{*}{$\begin{array}{c}\text { Electric Plant } \\
\text { Purchases From } \\
\text { Domestic Suppliers }\end{array}$} & \multirow{2}{*}{$\begin{array}{l}\text { Loaded Into } \\
\text { U.S. Nuclear } \\
\text { Reactors }{ }^{3}\end{array}$} & \multicolumn{3}{|c|}{ Inventories } & \multicolumn{2}{|c|}{ Average Price } \\
\hline & & & & & & Domestic Suppliers & Electric Plants & Total & Purchased Imports & Domestic Purchases \\
\hline & \multicolumn{8}{|c|}{ Million Pounds Uranium Oxide } & \multicolumn{2}{|c|}{ Dollars ${ }^{4}$ per Pound Uranium Oxide } \\
\hline 1949 & 0.36 & 4.3 & 0.0 & NA & NA & NA & NA & NA & NA & NA \\
\hline 1950 & .92 & 5.5 & .0 & NA & NA & NA & NA & NA & NA & NA \\
\hline 1955 & 5.56 & 7.6 & .0 & NA & NA & NA & NA & NA & NA & NA \\
\hline 1960 & 35.28 & 36.0 & .0 & NA & NA & NA & NA & NA & NA & NA \\
\hline 1965 & 20.88 & 8.0 & .0 & NA & NA & NA & NA & NA & NA & NA \\
\hline 1970 & 25.81 & .0 & 4.2 & NA & NA & NA & NA & NA & -- & NA \\
\hline 1975 & 23.20 & 1.4 & 1.0 & NA & NA & NA & NA & NA & NA & NA \\
\hline 1976 & 25.49 & 3.6 & 1.2 & NA & NA & NA & NA & NA & NA & NA \\
\hline 1977 & 29.88 & 5.6 & 4.0 & NA & NA & NA & NA & NA & NA & NA \\
\hline 1978 & 36.97 & 5.2 & 6.8 & NA & NA & NA & NA & NA & NA & NA \\
\hline 1979 & 37.47 & 3.0 & 6.2 & NA & NA & NA & NA & NA & NA & NA \\
\hline 1980 & 43.70 & 3.6 & 5.8 & NA & NA & NA & NA & NA & NA & NA \\
\hline 1981 & 38.47 & 6.6 & 4.4 & 32.6 & NA & NA & NA & 159.2 & 32.90 & 34.65 \\
\hline 1982 & 26.87 & 17.1 & 6.2 & 27.1 & NA & NA & NA & 174.8 & 27.23 & 38.37 \\
\hline 1983 & 21.16 & 8.2 & 3.3 & 24.2 & NA & NA & NA & 191.8 & 26.16 & 38.21 \\
\hline 1984 & 14.88 & 12.5 & 2.2 & 22.5 & NA & 25.0 & 160.2 & 185.2 & 21.86 & 32.65 \\
\hline 1985 & 11.31 & 11.7 & 5.3 & 21.7 & NA & 23.7 & 153.2 & 176.9 & 20.08 & 31.43 \\
\hline 1986 & 13.51 & 13.5 & 1.6 & 18.9 & NA & 27.0 & 144.1 & 171.1 & 20.07 & 30.01 \\
\hline 1987 & 12.99 & 15.1 & 1.0 & 20.8 & NA & 25.4 & 137.8 & 163.2 & 19.14 & 27.37 \\
\hline 1988 & 13.13 & 15.8 & 3.3 & 17.6 & NA & 19.3 & 125.5 & 144.8 & 19.03 & 26.15 \\
\hline 1989 & 13.84 & 13.1 & 2.1 & 18.4 & NA & 22.2 & 115.8 & 138.1 & 16.75 & 19.56 \\
\hline 1990 & 8.89 & 23.7 & 2.0 & 20.5 & NA & 26.4 & 102.7 & 129.1 & 12.55 & 15.70 \\
\hline 1991 & 7.95 & 16.3 & 3.5 & 26.8 & 34.6 & 20.7 & 98.0 & 118.7 & 15.55 & 13.66 \\
\hline 1992 & 5.65 & 23.3 & 2.8 & 23.4 & 43.0 & 25.2 & 92.1 & 117.3 & 11.34 & 13.45 \\
\hline 1993 & 3.06 & 21.0 & 3.0 & 15.5 & 45.1 & 24.5 & 81.2 & 105.7 & 10.53 & 13.14 \\
\hline 1994 & 3.35 & 36.6 & 17.7 & 22.7 & 40.4 & 21.5 & 65.4 & 86.9 & 8.95 & 10.30 \\
\hline 1995 & 6.04 & 41.3 & 9.8 & 22.3 & 51.1 & 13.7 & 58.7 & 72.5 & 10.20 & 11.11 \\
\hline 1996 & 6.32 & 45.4 & 11.5 & 23.7 & 46.2 & 13.9 & 66.1 & 80.0 & 13.15 & 13.81 \\
\hline 1997 & 5.64 & 43.0 & 17.0 & 19.4 & 48.2 & 40.4 & 65.9 & 106.2 & 11.81 & 12.87 \\
\hline 1998 & 4.71 & 43.7 & 15.1 & 21.6 & 38.2 & 70.7 & 65.8 & 136.5 & 11.19 & 12.31 \\
\hline 1999 & 4.61 & 47.6 & 8.5 & 21.4 & 58.8 & 68.8 & 58.3 & 127.1 & 10.55 & 11.88 \\
\hline 2000 & 3.96 & 44.9 & 13.6 & 24.3 & 51.5 & 56.5 & 54.8 & 111.3 & 9.84 & 11.45 \\
\hline 2001 & 2.64 & 46.7 & 11.7 & 27.5 & 52.7 & 48.1 & 55.6 & 103.8 & 9.51 & 10.45 \\
\hline 2002 & 2.34 & 52.7 & 15.4 & 22.7 & 57.2 & 48.7 & 53.5 & 102.1 & 10.05 & 10.35 \\
\hline 2003 & 5, E2.00 & 53.0 & 13.2 & 21.7 & 62.3 & 39.9 & 45.6 & 85.5 & 10.59 & 10.84 \\
\hline 2004 & 2.28 & 66.1 & 13.2 & 28.2 & 50.1 & 37.5 & 57.7 & 95.2 & 12.25 & 11.91 \\
\hline 2005 & 2.69 & 65.5 & 20.5 & 27.3 & 58.3 & 29.1 & 64.7 & 93.8 & 14.83 & 13.98 \\
\hline 2006 & 4.11 & 64.8 & 18.7 & 27.9 & 51.7 & 29.1 & 77.5 & 106.6 & 19.31 & 18.54 \\
\hline 2007 & 4.53 & 54.1 & 14.8 & 18.5 & 45.5 & 31.2 & 81.2 & 112.4 & 34.18 & 33.13 \\
\hline 2008 & 3.90 & 57.1 & 17.2 & 20.4 & 51.3 & 27.0 & 83.0 & 110.0 & 41.30 & 43.43 \\
\hline 2009 & 3.71 & 58.9 & 23.5 & 17.6 & 49.4 & 26.8 & 84.8 & 111.5 & 41.23 & 44.53 \\
\hline 2010 & 4.23 & 55.3 & 23.1 & 16.2 & 44.3 & $R_{24.7}$ & 86.5 & $\mathrm{R}_{111.3}$ & 47.01 & 44.88 \\
\hline 2011 & 3.99 & 54.4 & 16.7 & 19.8 & P 52.0 & P 24.1 & P 89.5 & P 113.6 & 54.00 & 53.41 \\
\hline
\end{tabular}

1 See "Uranium Concentrate" in Glossary.

2 Import quantities through 1970 are reported for fiscal years. Prior to 1968, the Atomic Energy Commission was the sole purchaser of all imported uranium oxide. Trade data prior to 1982 were for transactions conducted by uranium suppliers only. For 1982 forward, transactions by uranium buyers (consumers) have been included. Buyer imports and exports prior to 1982 are believed by unall.

4 Prics not ine any fuel rods removed "

5 Vaces are not adjusted tor inlation. See "No in hidual in Glossary.

$R=$ Revised. $P=$ Preliminary. $E=$ Estimate. $N A=$ Not available. $--=$ Not applicable.

Note: See "Uranium Oxide" in Glossary

Web Pages: • For all data beginning in 1949, see http://www.eia.gov/totalenergy/data/annual/\#nuclear. For related information, see http://www.eia.gov/nuclear/.

Sources: - 1949-1966-U.S. Department of Energy, Grand Junction Office, Statistical Data of the Uranium Industry, Report No. GJO-100, annual reports. • 1967-2002-U.S. Energy Information Administration (EIA), Uranium Industry Annual, annual reports. - 2003-2006-EIA, "Uranium Marketing 2012), Tapl, "EIA, "2011 Uranium Marketing Annual Report" (May 2012), Tables 5, 18, 19, 21, and 22; 2012), Table 3; EIA, "2011 Uranium Marketing Annual Report" (May 2012), Tables 5, 18, 19, 21, and 22 


\section{Nuclear Energy}

Note 1. Pending Actions on Nuclear Generating Units. Much of Table 9.1 is based on the U.S. Nuclear Regulatory Commission (NRC) regulation 10 CFR Part 50, which has in most instances been supplanted by 10 CFR Part 52 following the passage of the Energy Policy Act of 1992 and procedural reforms initiated in 1989 by the NRC. (This statement applies to permit and license procedures only.)

The NRC did not issue any Early Site Permits (ESP) during 2011. Two ESP applications are currently under review; one to Victoria County Station and the other to PSEG Site.

As of December 31, 2011, the NRC had received 18 Combined License (COL) applications representing 28 nuclear generating units. The following $14 \mathrm{COL}$ applications are under review: Bell Bend (Pennsylvania); Bellefonte Units 3 and 4 (Alabama); Calvert Cliffs Unit 3 (Maryland); Comanche Peak Units 3 and 4 (Texas); Fermi Unit 3 (Michigan); Levy County Units 1 and 2 (Florida); Nine Mile Point Unit 3 (New York); North Anna Unit 3 (Virginia); Shearon Harris Units 2 and 3 (North Carolina); South Texas Units 3 and 4 (Texas); Turkey Point Units 6 and 7 (Florida); Virgil C. Summer Units 2 and 3 (South Carolina); Vogtle Units 3 and 4 (Georgia); and William States Lee III Units 1 and 2 (South Carolina). At the request of the applicants, review has been suspended for three COL applications: Callaway Unit 2 (Missouri), Grand Gulf Unit 3 (Mississippi), and River Bend Unit 3 (Louisiana). The Victoria County Units 1 and 2 COL application was withdrawn in 2010 following the announcement that the applicant intends to apply instead for an ESP with the reactor choice unspecified. In addition to the COL applications currently under review, Watts Bar Unit 2 is currently under construction. Watts Bar Unit 2 was issued a construction permit in 1973, and the U.S. Energy Information Administration projects that it will be brought on line in 2013. This is the only reactor that is anticipated to apply for the license separate of construction permit. TVA has also requested that Bellefonte Units 1 and 2, two partially completed units, be moved to 'deferred plan' status as the Agency considers completing one or both.

As of December 31, 2011, 11 applications for license extensions were under review by the NRC. The NRC granted 20-year license extensions in 2011 to: Kewaunee Power Station on February 24, Vermont Yankee Nuclear Power Station on March 21, Palo Verde Units 1, 2, and 3 on April 21, Prairie Island Units 1 and 2 on June 27, Salem Units 1 and 2 on June 30, and Hope Creek on July 20.

For more information on nuclear reactors, see http://www.nrc.gov/reactors.html.

Note 2. Coverage of Nuclear Energy Statistics. In 1997, the U.S. Energy Information Administration undertook a major revision of Table 9.1 to more fully describe the history of the U.S. commercial nuclear power industry. The time frame was extended back to the birth of the industry in 1953 and the data categories were revised for greater relevance to current industry conditions and trends. To acquire the data for the revised categories, it was necessary to develop a reactor unit database employing different sources than those used previously for Table 9.1 and still used for Table 9.2.
The data in Table 9.1 apply to commercial nuclear power units, meaning that the units contributed power to the commercial electricity grid. A total of 259 units have been ordered over the lifetime of the nuclear industry. Although most orders were placed by electric utilities, several units were ordered, owned, and operated wholly or in part by the Federal Government, including BONUS (Boiling Nuclear Superheater Power Station), Elk River, Experimental Breeder Reactor 2, Hallam, Hanford N, Piqua, and Shippingport.

A reactor is generally defined as operable in Table 9.1 if it possesses a fullpower license, or an equivalent, from the NRC or its predecessor, the Atomic Energy Commission, at the end of the year. The definition is liberal in that it does not exclude units retaining full-power licenses during long, non-routine shutdowns.

For example:

- In 1985, the five Tennessee Valley Authority units (Browns Ferry 1, 2, and 3 and Sequoyah 1 and 2) were shut down under a regulatory forced outage. Browns Ferry 1 was authorized by the NRC to restart in 2007, while the other units restarted in 1991, 1995, 1988, and 1988, respectively. All five units were counted as operable during the shutdowns.

- $\quad$ Shippingport was shut down from 1974 through 1976 for conversion to a lightwater breeder reactor, but is counted as operable until its retirement in 1982.

- $\quad$ Calvert Cliffs 2 was shut down in 1989 and 1990 for replacement of pressurizer heater sleeves but is counted as operable during those years.

Exceptions to the rule are Shoreham and Three Mile Island 2. Shoreham was granted a full-power license in April 1989, but was shut down two months later and never restarted. In 1991, the license was changed to Possession Only. Although not operable at the end of the year, Shoreham is treated as operable during 1989 and shut down in 1990, because counting it as operable and shut down in the same year would introduce a statistical discrepancy in the tallies. A major accident closed Three Mile Island 2 in 1979, and although the unit retained its full-power license for several years, it is considered permanently shut down since that year.

Table 9.1 Sources: Operable Units: • 1955-1982-Compiled from various sources, primarily U.S. Department of Energy (DOE), Office of Nuclear Reactor Programs, "U.S. Central Station Nuclear Electric Generating Units: Significant Milestones." • 1983 forward-U.S. Energy Information Administration (EIA), Form EIA-860, "Annual Electric Generator Report," and predecessor forms. All Other Data: • 1955-1997-U.S. Atomic Energy Commission, 1973 Annual Report to Congress, Volume 2, Regulatory Activities; Nuclear Energy Institute, Historical Profile of U.S. Nuclear Power Development (1988); EIA, Commercial Nuclear Power 1991 (September 1991); DOE, Nuclear Reactors Built, Being Built, and Planned: 1995; U.S. Nuclear Regulatory Commission (NRC), Information Digest (1997 and 1998) and "Plant Status Report"; and various utility, Federal, and contractor officials. • 1998 forward-NRC, Information Digest, annual reports. 


\section{Renewable Energy}


Figure 10.1 Renewable Energy Consumption by Major Source

\section{Renewable Energy as Share of Total Primary Energy Consumption, 2011}

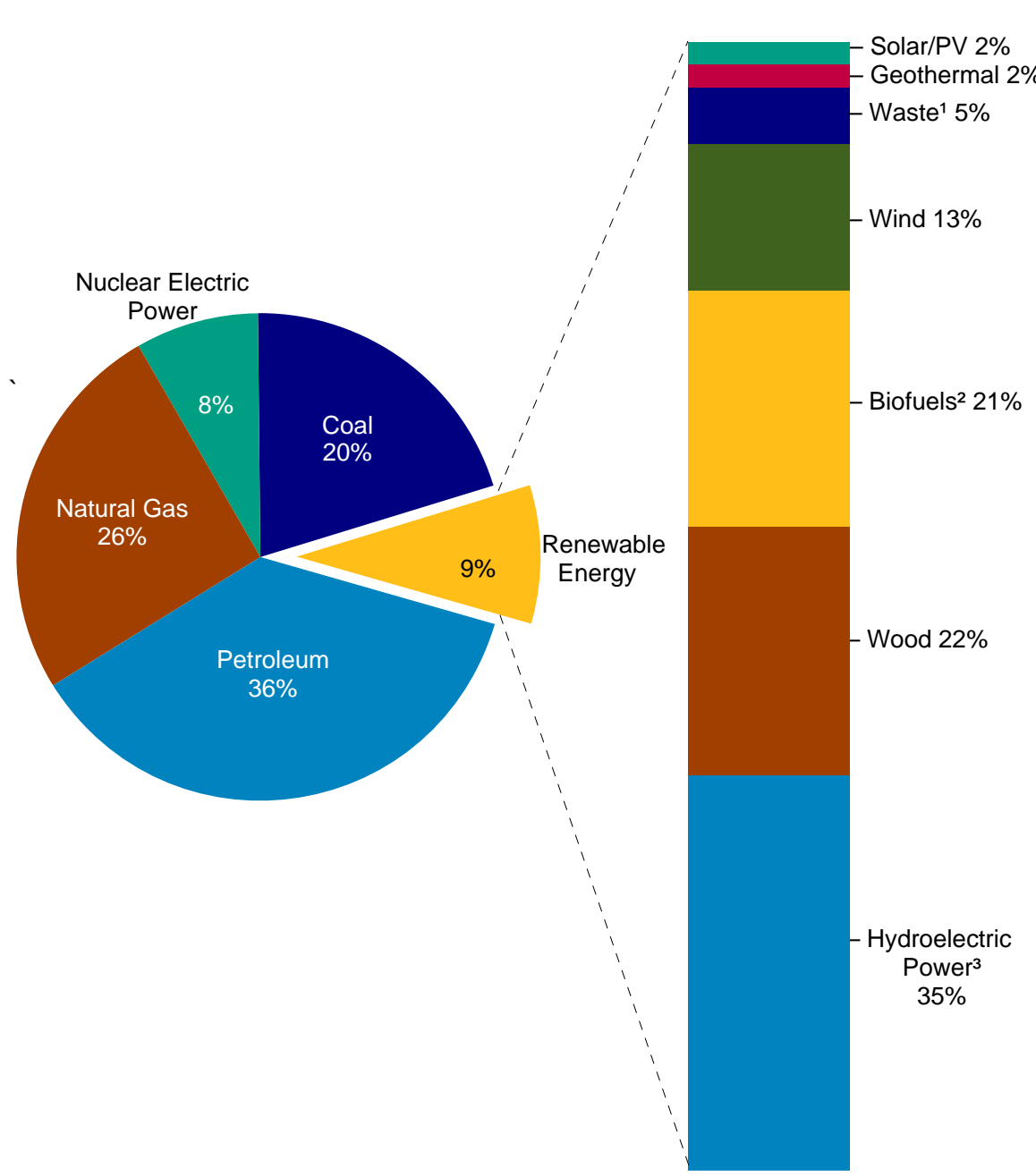

Renewable Energy Consumption by Source, 1949-2011

4-

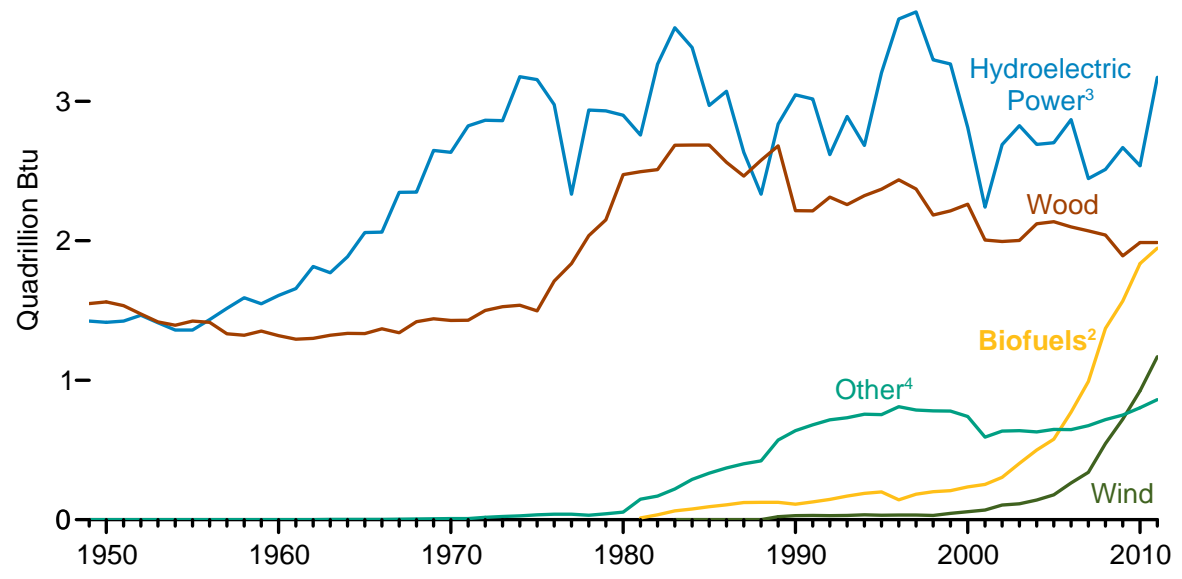

Renewable Energy Consumption by Source, 2011

4-

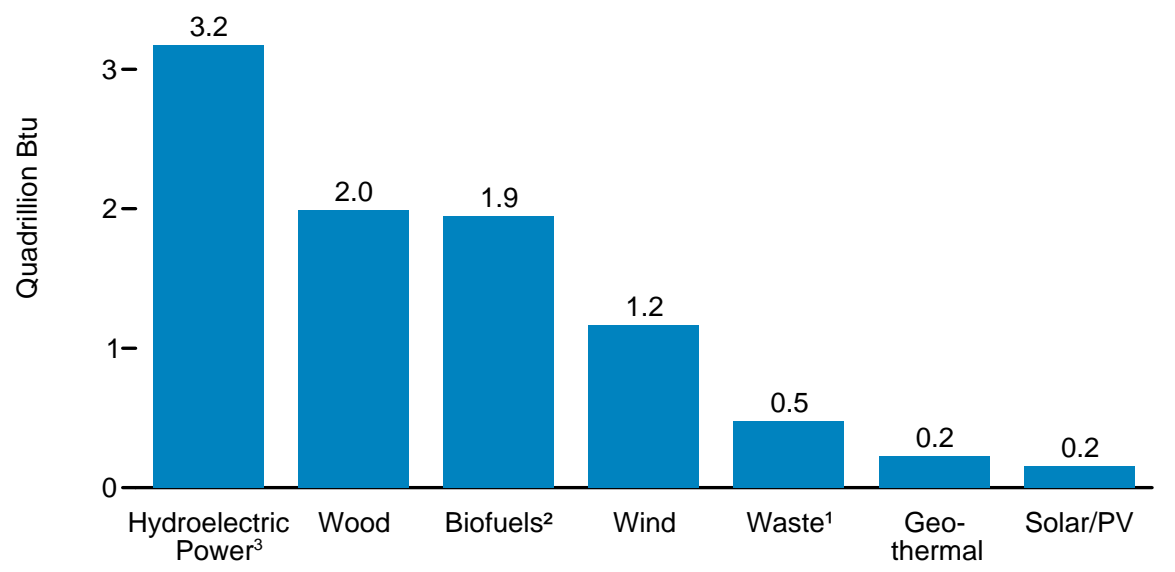

\footnotetext{
${ }^{1}$ Municipal solid waste from biogenic sources, landfill gas, sludge waste, agricultural byprod ucts, and other biomass.

${ }^{2}$ Fuel ethanol (minus denaturant) and biodiesel consumption, plus losses and co-products from the production of fuel ethanol and biodiesel.
}

${ }^{3}$ Conventional hydroelectric power.

${ }^{4}$ Geothermal, solar/PV, and waste.

Notes: Sum of components may not equal 100 percent due to independent rounding.

Sources: Tables 1.3 and 10.1. 
Table 10.1 Renewable Energy Production and Consumption by Primary Energy Source, Selected Years, $1949-2011$

(Trillion Btu)

\begin{tabular}{|c|c|c|c|c|c|c|c|c|c|c|c|c|}
\hline \multirow[b]{3}{*}{ Year } & \multicolumn{3}{|c|}{ Production ${ }^{1}$} & \multicolumn{9}{|c|}{ Consumption } \\
\hline & \multicolumn{2}{|c|}{ Biomass } & \multirow{2}{*}{$\begin{array}{c}\text { Total } \\
\text { Renewable } \\
\text { Energy }{ }^{4}\end{array}$} & \multirow{2}{*}{$\begin{array}{l}\text { Hydro- } \\
\text { electric } \\
\text { Power } 5\end{array}$} & \multirow{2}{*}{$\begin{array}{c}\text { Geo- } \\
\text { thermal } 6\end{array}$} & \multirow[b]{2}{*}{ Solar/PV ${ }^{7}$} & \multirow[b]{2}{*}{ Wind 8} & \multicolumn{4}{|c|}{ Biomass } & \multirow{2}{*}{$\begin{array}{c}\text { Total } \\
\text { Renewable } \\
\text { Energy }\end{array}$} \\
\hline & Biofuels 2 & Total ${ }^{3}$ & & & & & & Wood $^{9}$ & Waste ${ }^{10}$ & Biofuels ${ }^{11}$ & Total & \\
\hline 1949 & NA & 1,549 & 2,974 & 1,425 & NA & NA & NA & 1,549 & NA & NA & 1,549 & 2,974 \\
\hline 1950 & NA & 1,562 & 2,978 & 1,415 & NA & NA & NA & 1,562 & NA & NA & 1,562 & 2,978 \\
\hline 1955 & NA & 1,424 & $\begin{array}{l}2,784 \\
2,78\end{array}$ & 1,360 & NA & NA & NA & 1,424 & NA & NA & 1,424 & 2,784 \\
\hline 1960 & NA & 1,320 & 2,928 & 1,608 & $(\mathrm{~s})$ & NA & NA & 1,320 & NA & NA & 1,320 & 2,928 \\
\hline 1965 & NA & 1,335 & 3,396 & $\begin{array}{l}2,059 \\
2,050\end{array}$ & 2 & NA & NA & 1,335 & NA & NA & 1,335 & 3,396 \\
\hline 1970 & NA & $\begin{array}{r}1,431 \\
1,430\end{array}$ & 4,070 & $\begin{array}{l}2,034 \\
2,634\end{array}$ & 6 & NA & NA & $\begin{array}{l}1,030 \\
1,429\end{array}$ & 2 & NA & $\begin{array}{l}1,431 \\
1,435\end{array}$ & $\begin{array}{l}4,070 \\
4,070\end{array}$ \\
\hline 1975 & NA & 1.499 & 4,687 & 3,155 & 34 & NA & NA & $\begin{array}{l}1,4<9 \\
1,497\end{array}$ & 2 & NA & $\begin{array}{l}1,401 \\
1,499\end{array}$ & 4,687 \\
\hline 1976 & NA & 1,713 & 4,727 & 2,976 & 38 & NA & NA & 1,711 & 2 & NA & 1,713 & 4,727 \\
\hline 1977 & NA & $\begin{array}{l}1,838 \\
1,83\end{array}$ & 4,209 & 2,333 & 37 & NA & NA & 1,837 & 2 & NA & $\begin{array}{l}1,838 \\
1,80\end{array}$ & 4,209 \\
\hline 1978 & NA & 2,038 & 5,005 & 2,937 & 31 & NA & NA & $\begin{array}{l}2,036 \\
2,036\end{array}$ & 1 & NA & $\begin{array}{l}2,030 \\
2,038\end{array}$ & $\begin{array}{l}4,209 \\
5,005\end{array}$ \\
\hline 1979 & NA & 2,152 & 5,123 & 2,931 & 40 & NA & NA & 2,150 & 2 & NA & $\begin{array}{l}2,152 \\
2,150\end{array}$ & 5,123 \\
\hline 1980 & NA & 2,476 & 5,428 & 2,900 & 53 & NA & NA & 2,474 & 2 & NA & 2,476 & 5,428 \\
\hline 1981 & 13 & 2,596 & 5,414 & 2,758 & 59 & NA & NA & 2,496 & 88 & 13 & 2,596 & 5,414 \\
\hline 1982 & 34 & 2,663 & 5,980 & 3,266 & 51 & NA & NA & 2,510 & 119 & 34 & 2,663 & 5,980 \\
\hline 1983 & 63 & 2,904 & 6,496 & 3,527 & 64 & NA & (s) & 2,684 & 157 & 63 & 2,904 & 6,496 \\
\hline 1984 & 77 & 2,971 & 6,438 & 3,386 & 81 & (s) & (s) & 2,686 & 208 & 77 & 2,971 & 6,438 \\
\hline 1985 & 93 & 3,016 & $\begin{array}{l}6,084 \\
6\end{array}$ & 2,970 & 97 & (s) & (s) & 2,687 & 236 & 93 & 3,016 & 6,084 \\
\hline 1986 & 107 & 2,932 & 6,111 & 3,071 & 108 & (s) & (s) & 2,562 & 263 & 107 & 2,932 & 6,111 \\
\hline 1987 & 123 & 2,875 & 5,622 & 2,635 & 112 & (s) & (s) & 2,463 & 289 & 123 & 2,875 & 5,622 \\
\hline 1988 & 124 & 3,016 & 5,457 & 2,334 & 106 & $\begin{array}{l}\text { (s) } \\
\text { (s) }\end{array}$ & $\begin{array}{l}\text { (s) } \\
\text { (s) }\end{array}$ & $\begin{array}{l}2,400 \\
2,577\end{array}$ & 315 & 124 & 3,016 & 5,457 \\
\hline 1989 & 125 & $\begin{array}{l}3,159 \\
3,10\end{array}$ & $\begin{array}{l}6,235 \\
6,235\end{array}$ & $\begin{array}{l}2,837 \\
2,837\end{array}$ & 162 & 55 & 22 & 2,680 & 354 & 125 & $\begin{array}{l}3,159 \\
3,0\end{array}$ & $\begin{array}{l}6,235 \\
6,40\end{array}$ \\
\hline 1990 & 111 & $\begin{array}{l}2,159 \\
2,735\end{array}$ & $\begin{array}{l}l, 2030 \\
6,041\end{array}$ & $\begin{array}{l}2,031 \\
3,046\end{array}$ & 171 & $\begin{array}{l}50 \\
59\end{array}$ & $\begin{array}{l}22 \\
29\end{array}$ & $\begin{array}{l}2,080 \\
2,216\end{array}$ & $\begin{array}{l}504 \\
408\end{array}$ & 111 & $\begin{array}{l}3,139 \\
2,735\end{array}$ & $\begin{array}{l}6,041 \\
6,041\end{array}$ \\
\hline 1991 & 128 & $\begin{array}{l}\text {, } \\
2,785\end{array}$ & $\begin{array}{l}\begin{array}{l}6,041 \\
6,069\end{array}\end{array}$ & $\begin{array}{l}3,046 \\
3,016\end{array}$ & 178 & $\begin{array}{l}59 \\
62\end{array}$ & 31 & $\begin{array}{r}2,210 \\
2,214\end{array}$ & 440 & 128 & $\begin{array}{l}2,785 \\
2,782\end{array}$ & $\begin{array}{l}\text {, } 041 \\
6,069\end{array}$ \\
\hline 1992 & 145 & 2,932 & 5,821 & 2,617 & 179 & 64 & 30 & 2,313 & 473 & 145 & 2,932 & 5,821 \\
\hline 1993 & 169 & 2,908 & 6,083 & 2,892 & 186 & 66 & 31 & 2,260 & 479 & 169 & 2,908 & 6,083 \\
\hline 1994 & 188 & 3,028 & 5,988 & 2,683 & 173 & 68 & 36 & 2,324 & 515 & 188 & 3,028 & 5,988 \\
\hline 1995 & 198 & 3,099 & 6,558 & 3,205 & 152 & 69 & 33 & 2,370 & 531 & 200 & 3,101 & 6,560 \\
\hline 1996 & 141 & 3,155 & 7,012 & 3,590 & 163 & 70 & 33 & 2,437 & 577 & 143 & 3,157 & 7,014 \\
\hline 1997 & 186 & 3,108 & 7,018 & 3,640 & 167 & 70 & 34 & 2,371 & 551 & 184 & 3,105 & 7,016 \\
\hline 1998 & 202 & 2,929 & 6,494 & 3,297 & 168 & 69 & 31 & 2,184 & 542 & 201 & 2,927 & 6,493 \\
\hline 1999 & 211 & 2,965 & 6,517 & 3,268 & 171 & 68 & 46 & 2,214 & 540 & 209 & 2,963 & 6,516 \\
\hline 2000 & 233 & 3,006 & 6,104 & 2,811 & 164 & R66 & 57 & 2,262 & 511 & 236 & 3,008 & 6,106 \\
\hline 2001 & 254 & 2,624 & 5,164 & 2,242 & 164 & 64 & 70 & 2,006 & 364 & 253 & 2,622 & 5,163 \\
\hline 2002 & 308 & 2,705 & 5,734 & 2,689 & 171 & 63 & 105 & 1,995 & 402 & 303 & 2,701 & 5,729 \\
\hline 2003 & 402 & 2,805 & 5,982 & 2,825 & 175 & 62 & 115 & 2,002 & 401 & 404 & 2,807 & 5,983 \\
\hline 2004 & 487 & $\begin{array}{l}\text { 2,ovj } \\
\text { 2,998 }\end{array}$ & $\begin{array}{l}6,9070 \\
6,070\end{array}$ & $\begin{array}{l}2,0<5 \\
2,690\end{array}$ & 178 & 63 & 142 & $\begin{array}{l}2,00< \\
2,121\end{array}$ & 389 & $\begin{array}{l}404 \\
499\end{array}$ & $\begin{array}{l}2,001 \\
3,010\end{array}$ & $\begin{array}{l}6,903 \\
6,082\end{array}$ \\
\hline 2005 & $\begin{array}{l}401 \\
564\end{array}$ & $\begin{array}{l}2,950 \\
3,104\end{array}$ & $\begin{array}{l}0,229 \\
6,229\end{array}$ & $\begin{array}{l}2,0903 \\
2,703\end{array}$ & 181 & $\begin{array}{l}00 \\
63\end{array}$ & 178 & $R_{2,137}$ & 403 & 577 & $\mathrm{R}_{3,117}$ & $\begin{array}{l}0,002 \\
6,242\end{array}$ \\
\hline 2006 & $\begin{array}{l}504 \\
720\end{array}$ & $\begin{array}{r}3,104 \\
R_{3,216}\end{array}$ & $\begin{array}{r}0,2<99 \\
R 6,599\end{array}$ & $\begin{array}{l}2,803 \\
2,869\end{array}$ & 181 & $\begin{array}{l}03 \\
68\end{array}$ & 264 & $R_{2,099}$ & $\begin{array}{l}403 \\
397\end{array}$ & 771 & $\mathrm{R}_{3,267}$ & $\begin{array}{r}0,62 \\
R 6,649\end{array}$ \\
\hline 2007 & $\begin{array}{l}120 \\
978\end{array}$ & $\begin{array}{l}R_{3,461} \\
\text { R }\end{array}$ & $\begin{array}{l}0,595 \\
R 6,509\end{array}$ & $\begin{array}{l}2,869 \\
2,446\end{array}$ & $\begin{array}{l}181 \\
186\end{array}$ & $\begin{array}{l}68 \\
76\end{array}$ & $\begin{array}{l}264 \\
341\end{array}$ & $\begin{array}{l}2,099 \\
R_{2}, 070\end{array}$ & 413 & 991 & $\begin{array}{l}3,474 \\
\mathrm{R}_{3}, 40\end{array}$ & $\begin{array}{r}0,649 \\
\text { R6,523 }\end{array}$ \\
\hline 2008 & 1,387 & $\mathrm{R}_{3,864}$ & R7,202 & 2,511 & $\begin{array}{l}192 \\
\end{array}$ & $\begin{array}{l}10 \\
89\end{array}$ & 546 & $R_{2,040}$ & 436 & $\begin{array}{r}1,372 \\
\end{array}$ & $\begin{array}{r}\mathrm{R} 3,849 \\
\mathrm{R}\end{array}$ & R7,186 \\
\hline 2009 & $\begin{array}{r}1,584 \\
R_{1}\end{array}$ & $\begin{array}{r}3,004 \\
R_{3,928}\end{array}$ & $\mathrm{R}_{7,616}$ & 2,669 & 200 & $\begin{array}{l}09 \\
98\end{array}$ & $\begin{array}{l}340 \\
721\end{array}$ & $\mathrm{R}_{1,891}$ & $\begin{array}{r}400 \\
R_{453}\end{array}$ & $\begin{array}{r}R_{1,568}\end{array}$ & $\begin{array}{r}0,945 \\
\mathrm{R}_{3}, 912\end{array}$ & R7,600 \\
\hline 2010 & $\begin{array}{r}1,584 \\
R_{1}, 884\end{array}$ & $\mathrm{R}_{4,341}$ & $\begin{array}{l}\text { R8,136 } \\
\text { R }\end{array}$ & $\begin{array}{l}R_{2,539}^{2,569}\end{array}$ & $\mathrm{R}_{208}$ & $\mathrm{R}_{126}$ & $\begin{array}{l}r 21 \\
R_{923}\end{array}$ & $\begin{array}{l}1,891 \\
\mathrm{R}_{1}, 988\end{array}$ & $\begin{array}{r}453 \\
\mathrm{R}_{4}\end{array}$ & $\begin{array}{l}1,568 \\
R_{1}, 837\end{array}$ & $\mathrm{R}_{4,294}$ & $\mathrm{R}_{8,090}$ \\
\hline $2011^{P}$ & $\begin{array}{l}1,004 \\
2,047\end{array}$ & $\begin{array}{r}4,541 \\
4,511\end{array}$ & $\begin{array}{l}8,136 \\
9,236\end{array}$ & $\begin{array}{l}2,539 \\
3,171\end{array}$ & 226 & 158 & 1,168 & $\begin{array}{r}1,988 \\
1,987\end{array}$ & 477 & $\begin{array}{r}1,831 \\
1,947\end{array}$ & 4,411 & 9,135 \\
\hline
\end{tabular}

1 Production equals consumption for all renewable energy sources except biofuels.

${ }^{3}$ Wood and wood-derived fuels, biomass waste, and total biomass inputs to the production of fuel ethanol and biodiesel.

${ }_{5}^{4}$ Hydroelectric power, geothermal, solar thermal/photovoltaic, wind, and biomass.

5 Conventional hydroelectricity net generation (converted to Btu using the fossil-fuels heat rate- see Table A6).

6 Geothermal electricity net generation (converted to Btu using the fossil-fuels heat rate-see Table A6), and geothermal heat pump and direct use energy.

(t) heat rate- -see Table A6), and solar thermal direct use energy.

${ }^{8}$ Wind electricity net generation (converted to Btu using the fossil-fuels heat rate-see Table A6).

9 Wood and wood-derived fuels.

Municipal solid waste from biogenic sources, landfill gas, sludge waste, agricultural byproducts, and other biomass. Through 2000, also includes non-renewable waste (municipal solid waste from

${ }^{11}$ Fuel ethanol (minus denaturant) and biodiesel consumption, plus losses and co-products from the production of fuel ethanol and biodiesel.

Notes: - Most data for the residential, commercial industrial 0.5 trilion But. See notes and sources for Tables 10.2a and 10.2b. - See Tables 8.2a-8.2d and 8.3a-8.3c for electricity net generation and useful thermal output from renewable energy sources; Tables 8.4a-8.4c, 8.5a-8.5d, 8.6a-8.6c, and 8.7a-8.7c for renewable energy consumption for electricity generation and useful therma "Rutput; and Tables 8.11a-8.11d for renewable energy electric net summer capacity. $\bullet$ See Note "enewable energy consumption for 1635-1945. • Totals may not equal sum of components due to independent rounding.

Web Pages: - See http://www.eia.gov/totalenergy/data/monthly/\#renewable for updated monthly and annual data. - See http://www.eia.gov/totalenergy/data/annual/\#renewable for all annual data beginning in

Sources: Biofuels: Tables 10.3 and 10.4. All Other Data: Tables 10.2a-10.2c. 


\section{Figure 10.2a Renewable Energy Consumption: End-Use Sectors, 1989-2011}

\section{Residential Sector}

$1.0-$

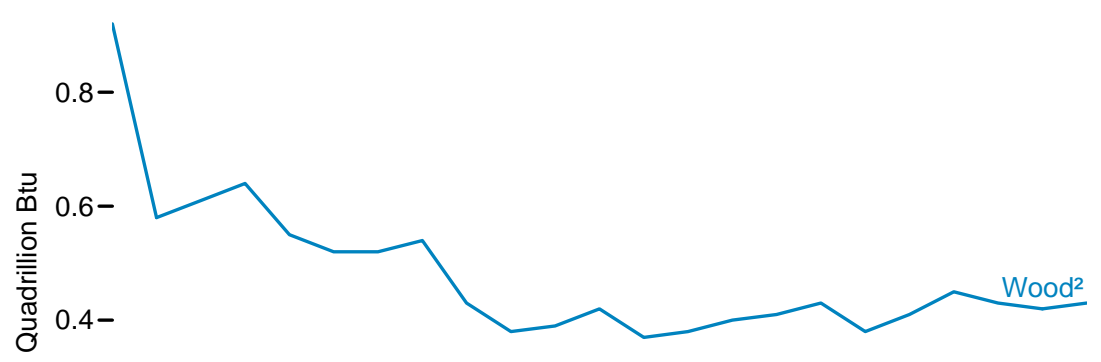

$0.2-$

Solar/PV 3 and Geothermal ${ }^{4}$

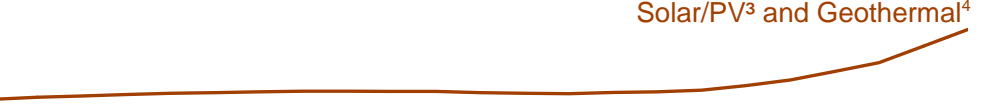

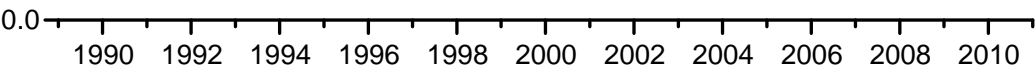

Industrial $^{1}$ Sector, Major Sources

$2.0-$

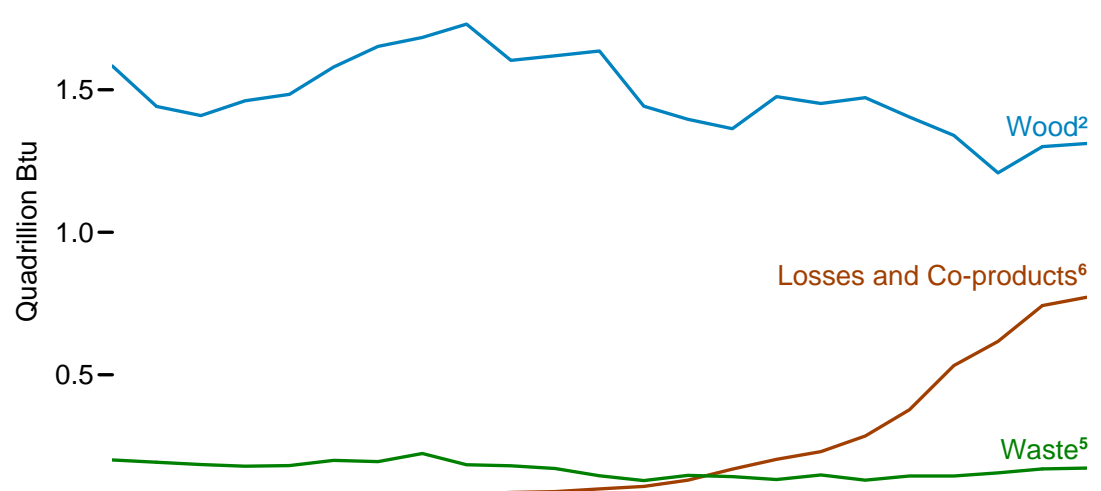

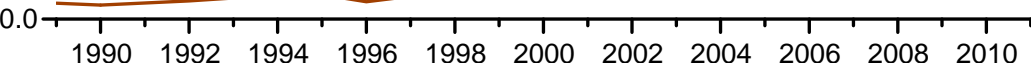

\section{Commercial ${ }^{1}$ Sector, Major Sources}

$90-$

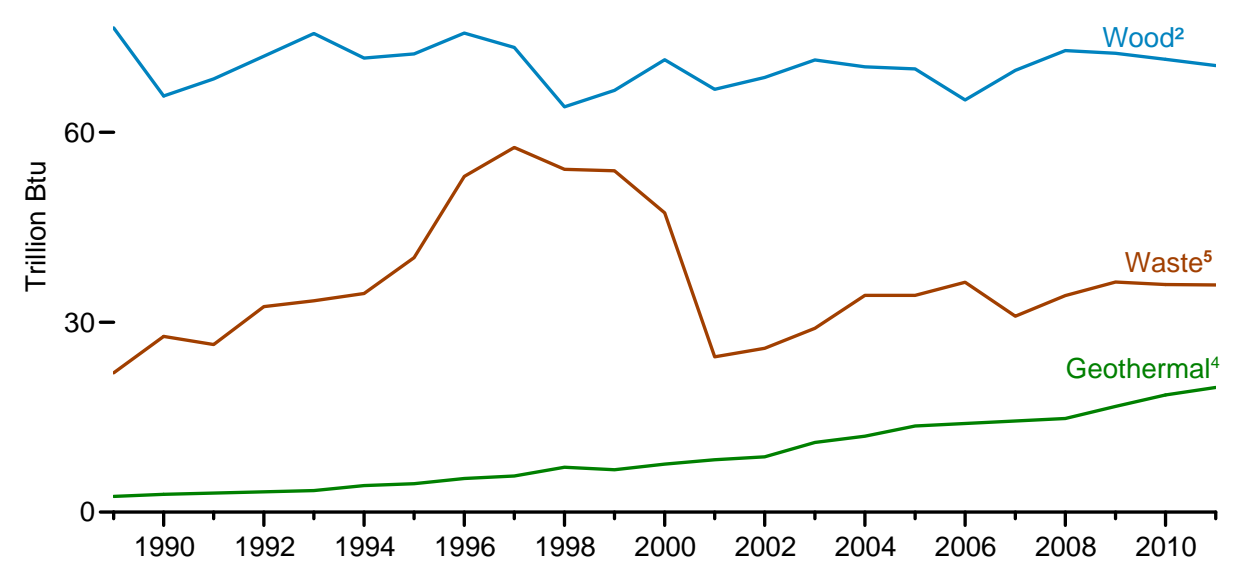

Transportation Sector

$1,400-$

$1,200-$

$1,000-$

苗 $800-$

亭 $600-$

$400-$

$200-$

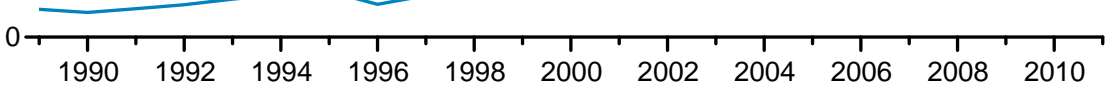

${ }^{1}$ Includes fuel used at combined-heat-and-power (CHP) plants and a small number of electricityonly plants.

2 Wood and wood-derived fuels.

${ }^{3}$ Solar thermal direct use energy, and photovoltaic (PV) electricity net generation. Includes small amounts of distributed solar thermal and PV energy used in the commercial, industrial, and electric power sectors.

${ }^{4}$ Geothermal heat pump and direct use energy.

${ }^{5}$ Municipal solid waste from biogenic sources, landfill gas, sludge waste, agricultural byproducts, and other biomass. Through 2000 , also includes non-renewable waste (municipal solid waste from non-biogenic sources, and tire-derived fuels).

${ }^{6}$ From the production of fuel ethanol and biodiesel.

7 The fuel ethanol (minus denaturant) portion of motor fuels (such as E10 and E85), and biodiesel. See "Biodiesel" in Glossary.

Note: See related Figures $10.2 \mathrm{~b}$ and $10.2 \mathrm{c}$

Sources: Tables $10.2 \mathrm{a}$ and $10.2 \mathrm{~b}$. 


\section{Figure 10.2b Renewable Energy Consumption: End-Use Sectors and Electric Power Sector}

\section{End-Use Sectors, 1949-2011}

$$
2.5-
$$

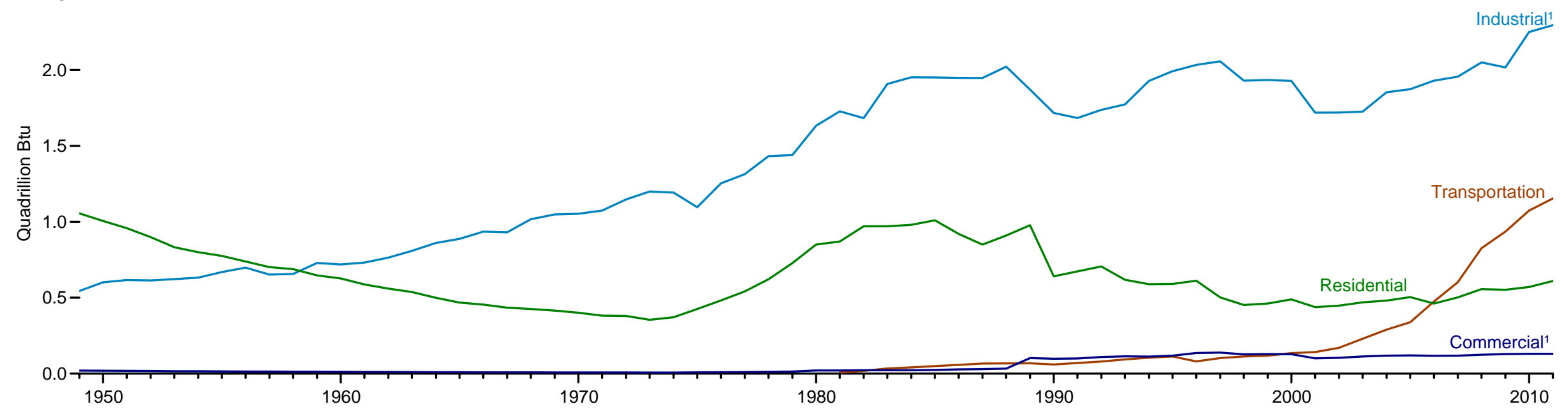

End-Use Sectors and Electric Power Sector, 2011

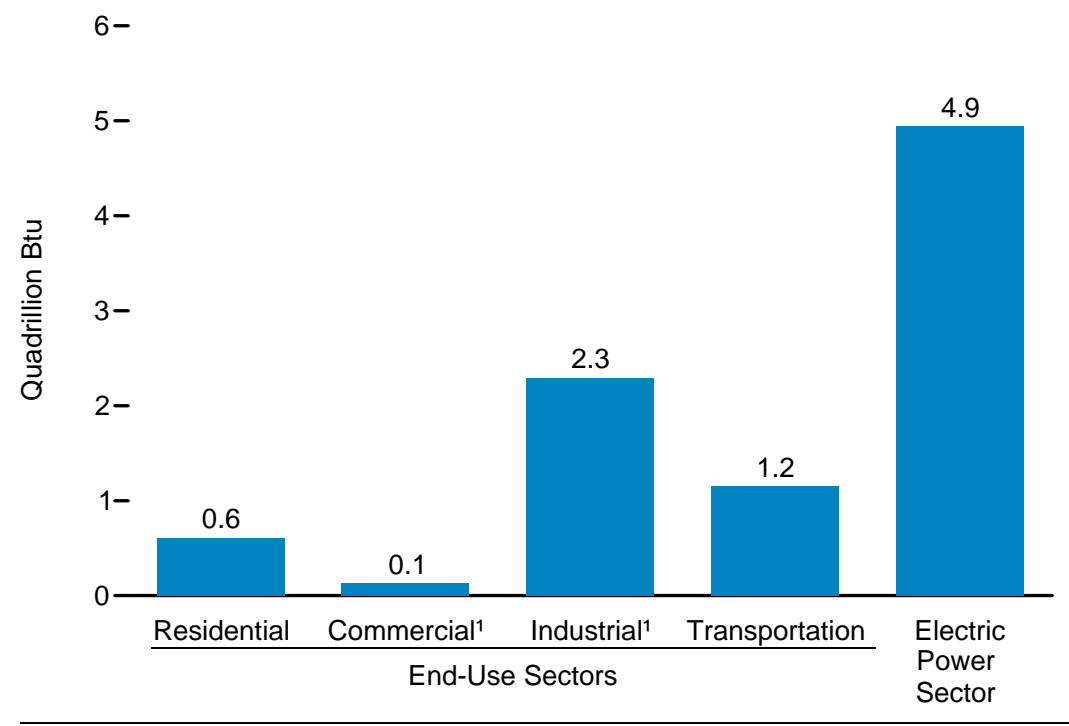

${ }^{1}$ Includes fuel use at combined-heat-and-power (CHP) plants and a small number of electricity-only plants.
End-Use Sectors and Electric Power Sector

Shares of Total Renewable Energy Consumption, 2011

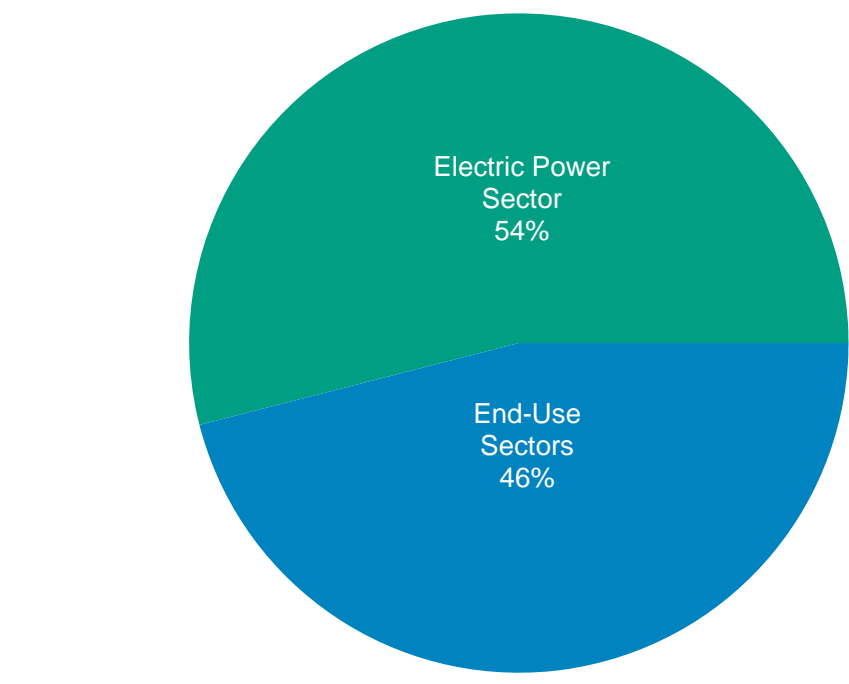

Note: See related Figures 10.2a and 10.2c.

Sources: Tables 10.2a-10.2c. 
Table 10.2a Renewable Energy Consumption: Residential and Commercial Sectors, Selected Years, $1949-2011$

(Trillion Btu)

\begin{tabular}{|c|c|c|c|c|c|c|c|c|c|c|c|c|c|}
\hline \multirow[b]{3}{*}{ Year } & \multicolumn{4}{|c|}{ Residential Sector } & \multicolumn{9}{|c|}{ Commercial Sector ${ }^{1}$} \\
\hline & \multirow{2}{*}{$\begin{array}{c}\text { Geo- } \\
\text { thermal }{ }^{2}\end{array}$} & \multirow[b]{2}{*}{ Solar/PV ${ }^{3}$} & \multirow{2}{*}{$\begin{array}{c}\text { Biomass } \\
\text { Wood }^{4}\end{array}$} & \multirow[b]{2}{*}{ Total } & \multirow{2}{*}{$\begin{array}{l}\text { Hydro- } \\
\text { electric } \\
\text { Power }^{5}\end{array}$} & \multirow{2}{*}{$\begin{array}{c}\text { Geo- } \\
\text { thermal }\end{array}$} & \multirow[b]{2}{*}{ Solar/PV 6} & \multirow[b]{2}{*}{ Wind $^{7}$} & \multicolumn{4}{|c|}{ Biomass } & \multirow[b]{2}{*}{ Total } \\
\hline & & & & & & & & & Wood $^{4}$ & Waste $^{8}$ & Fuel Ethanol ${ }^{9}$ & Total & \\
\hline 1949 & NA & NA & 1,055 & 1,055 & NA & NA & NA & NA & 20 & NA & NA & 20 & 20 \\
\hline 1950 & NA & NA & 1,006 & 1,006 & NA & NA & NA & NA & 19 & NA & $\mathrm{NA}$ & 19 & 19 \\
\hline 1955 & NA & NA & 775 & 775 & NA & NA & NA & NA & 15 & NA & NA & 15 & 15 \\
\hline 1960 & NA & NA & 627 & 627 & NA & NA & NA & NA & 12 & NA & NA & 12 & 12 \\
\hline 1965 & NA & NA & 468 & 468 & NA & NA & $\mathrm{NA}$ & NA & 9 & NA & $\mathrm{NA}$ & 9 & 9 \\
\hline 1970 & NA & NA & 401 & 401 & NA & NA & NA & NA & 8 & NA & $\mathrm{NA}$ & 8 & 8 \\
\hline 1975 & NA & NA & 425 & 425 & NA & NA & NA & NA & 8 & NA & NA & 8 & 8 \\
\hline 1976 & NA & NA & 482 & 482 & NA & NA & NA & NA & 9 & NA & NA & 9 & 9 \\
\hline 1977 & NA & NA & 542 & 542 & NA & NA & NA & NA & 10 & NA & $\mathrm{NA}$ & 10 & 10 \\
\hline 1978 & NA & NA & 622 & 622 & NA & NA & NA & NA & 12 & NA & NA & 12 & 12 \\
\hline 1979 & NA & NA & 728 & 728 & NA & NA & $\mathrm{NA}$ & NA & 14 & $\mathrm{NA}$ & $\mathrm{NA}$ & 14 & 14 \\
\hline 1980 & NA & NA & 850 & 850 & NA & NA & NA & NA & 21 & NA & NA & 21 & 21 \\
\hline 1981 & NA & NA & 870 & 870 & NA & NA & NA & NA & 21 & NA & (s) & 21 & 21 \\
\hline 1982 & NA & NA & 970 & 970 & NA & NA & NA & NA & 22 & NA & (s) & 22 & 22 \\
\hline 1983 & NA & NA & 970 & 970 & $\mathrm{NA}$ & $\mathrm{NA}$ & NA & NA & 22 & NA & $\begin{array}{l}\text { (s) } \\
\text { (s) }\end{array}$ & 22 & 22 \\
\hline 1984 & NA & NA & 980 & 980 & NA & NA & NA & NA & 22 & NA & (s) & 22 & 22 \\
\hline 1985 & NA & NA & 1,010 & $\begin{array}{r}1,010 \\
\end{array}$ & $\mathrm{NA}$ & NA & NA & NA & 24 & NA & (s) & 24 & 24 \\
\hline 1986 & NA & NA & 920 & 920 & NA & NA & NA & NA & 27 & NA & (s) & 27 & 27 \\
\hline 1987 & NA & NA & 850 & 850 & NA & NA & NA & NA & 29 & NA & 1 & 30 & 30 \\
\hline 1988 & NA & NA & 910 & 910 & $\mathrm{NA}$ & NA & NA & NA & 32 & NA & 1 & 33 & 33 \\
\hline 1989 & 5 & 52 & 920 & 977 & 1 & 3 & - & - & 76 & 22 & 1 & 99 & 102 \\
\hline 1990 & 6 & 56 & 580 & 641 & 1 & 3 & - & - & 66 & 28 & (s) & 94 & 98 \\
\hline 1991 & $\begin{array}{l}6 \\
6\end{array}$ & $\begin{array}{l}56 \\
57\end{array}$ & $\begin{array}{l}580 \\
610\end{array}$ & $\begin{array}{l}641 \\
673\end{array}$ & 1 & $\begin{array}{l}3 \\
3\end{array}$ & $\overline{-}$ & $\overline{-}$ & $\begin{array}{l}66 \\
68\end{array}$ & $\begin{array}{l}28 \\
26\end{array}$ & $\begin{array}{l}\text { (s) } \\
\text { (s) }\end{array}$ & $\begin{array}{l}94 \\
95\end{array}$ & 100 \\
\hline 1992 & 6 & $\mathrm{R}_{60}$ & 640 & 706 & 1 & 3 & - & - & 72 & 32 & (s) & 105 & 109 \\
\hline 1993 & 7 & 61 & 550 & 618 & 1 & 3 & - & - & 76 & 33 & $\begin{array}{l}\text { (1) } \\
\text { (s) }\end{array}$ & 109 & 114 \\
\hline 1994 & 6 & 63 & 520 & 589 & 1 & 4 & - & - & 72 & 35 & $\begin{array}{l}\text { (s) } \\
\text { (s) }\end{array}$ & 106 & 112 \\
\hline 1995 & $\begin{array}{l}0 \\
7\end{array}$ & $\begin{array}{l}00 \\
64\end{array}$ & 520 & 591 & 1 & $\begin{array}{l}4 \\
5\end{array}$ & $\overline{-}$ & $\overline{-}$ & 72 & 40 & $\begin{array}{l}\text { (s) } \\
\text { (s) }\end{array}$ & 113 & 118 \\
\hline 1996 & 7 & $\begin{array}{l}04 \\
65\end{array}$ & 540 & 612 & 1 & $\begin{array}{l}5 \\
5\end{array}$ & $\overline{-}$ & $\overline{-}$ & 76 & $\begin{array}{l}40 \\
53\end{array}$ & $\begin{array}{l}\text { (s) } \\
\text { (s) }\end{array}$ & 129 & 135 \\
\hline 1997 & 8 & 64 & 430 & 502 & 1 & 6 & - & - & 73 & 58 & (s) & 131 & 138 \\
\hline 1998 & 8 & 64 & 380 & 452 & 1 & 7 & - & - & 64 & 54 & (s) & 118 & 127 \\
\hline 1999 & 9 & 63 & 390 & 461 & 1 & 7 & - & - & 67 & 54 & (s) & 121 & 129 \\
\hline 2000 & 9 & $\mathrm{R}_{61}$ & 420 & 489 & 1 & 8 & - & - & 71 & 47 & (s) & 119 & 128 \\
\hline 2001 & 9 & 59 & 370 & 438 & 1 & 8 & _- & - & 67 & 25 & (s) & 92 & 101 \\
\hline 2002 & 10 & 57 & 380 & 448 & (s) & $\begin{array}{l}0 \\
9\end{array}$ & - & - & $\begin{array}{l}01 \\
69\end{array}$ & $\begin{array}{l}25 \\
26\end{array}$ & (s) & 95 & 104 \\
\hline 2003 & $\begin{array}{l}10 \\
13\end{array}$ & $\begin{array}{l}51 \\
57\end{array}$ & $\begin{array}{l}380 \\
400\end{array}$ & $\begin{array}{l}448 \\
470\end{array}$ & $\begin{array}{l}\text { (S) } \\
1\end{array}$ & $\begin{array}{r}9 \\
11\end{array}$ & - & - & $\begin{array}{l}69 \\
71\end{array}$ & $\begin{array}{l}26 \\
29\end{array}$ & $\begin{array}{l}\text { (S) } \\
1\end{array}$ & $\begin{array}{r}95 \\
101\end{array}$ & $\begin{array}{l}104 \\
113\end{array}$ \\
\hline 2004 & 14 & 57 & 410 & 481 & 1 & 12 & - & - & 70 & 34 & 1 & 105 & 118 \\
\hline 2005 & 16 & 58 & 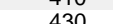 & $\begin{array}{l}401 \\
504\end{array}$ & 1 & 14 & - & - & 70 & 34 & 1 & 105 & $\begin{array}{l}110 \\
\text { R120 }\end{array}$ \\
\hline 2006 & $\begin{array}{l}10 \\
18\end{array}$ & $\begin{array}{l}50 \\
63\end{array}$ & $\begin{array}{r}4300 \\
\mathrm{R}_{3}\end{array}$ & $\begin{array}{r}504 \\
\mathrm{R}_{4}\end{array}$ & 1 & $\begin{array}{l}14 \\
14\end{array}$ & - & $\overline{-}$ & 65 & $\begin{array}{l}34 \\
36\end{array}$ & 1 & R103 & $\mathrm{R}_{118}$ \\
\hline 2007 & $\begin{array}{l}18 \\
22\end{array}$ & $\begin{array}{l}63 \\
70\end{array}$ & $\mathrm{R}_{410}$ & $\begin{array}{r}462 \\
\mathrm{R}_{502}\end{array}$ & 1 & $\begin{array}{l}14 \\
14\end{array}$ & $\overline{-}$ & $\overline{-}$ & $\begin{array}{r}65 \\
\text { R70 }\end{array}$ & $\begin{array}{l}36 \\
31\end{array}$ & $\begin{array}{l}1 \\
2\end{array}$ & $\mathrm{R}_{103}$ & 118 \\
\hline 2008 & 26 & 80 & 450 & R557 & 1 & 15 & (s) & - & 73 & 34 & 2 & 109 & 125 \\
\hline 2009 & 33 & 89 & 430 & 552 & 1 & 17 & (s) & $(\mathrm{s})$ & 72 & 36 & 3 & 112 & 129 \\
\hline 2010 & 37 & $\mathrm{R}_{114}$ & 420 & R571 & 1 & 19 & (s) & (s) & R72 & R36 & 3 & $\mathrm{R}_{111}$ & $\mathrm{R}_{130}$ \\
\hline $2011^{P}$ & 40 & 140 & 430 & 610 & 1 & 20 & $\begin{array}{l}\text { (s) } \\
\text { (s) }\end{array}$ & $\begin{array}{l}\text { (s) } \\
\text { (s) }\end{array}$ & 71 & 36 & $\begin{array}{l}5 \\
3\end{array}$ & 110 & 131 \\
\hline
\end{tabular}

1 Commercial sector, including commercial combined-heat-and-power (CHP) and commercial electricity-only plants. See Note 2, "Classification of Power Plants Into Energy-Use Sectors," at end of Section 8.

Geothermal heat pump and direct use energy.

Solar thermal direct use energy, and photovoltaic (PV) electricity net generation (converted to Btu using the fossil-fuels heat rate-see Table A6). Includes distributed solar thermal and PV energy used in the commercial, industrial, and electric power sectors.

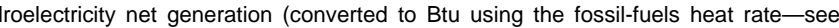
Table A6).

Phosiltaic (PV) electricity net generation (converted to Btu using the fossil-fuels heat rate-see Table A6) at commercial plants with capacity of 1 megawatt or greater.

. Wha electicily net generalion (converted to Blu using the lossil-tuels heat rate-see Table A6).

Municipal solid waste from biogenic sources, landfill gas, sludge waste, agricultural byproducts, and non-biogenic sources, and tire-derived fuels)

9 The fuel ethanol (minus denaturant) portion of motor fuels, such as E10, consumed by the commercial $R=$ Revised. P=Preliminary. NA=Not available. $-=$ No data reported. $(\mathrm{s})=$ Less than 0.5 trillion Btu. Notes: - Data are estimates, except for commercial sector solar/PV, hydroelectric power, wind, and waste. - See Tables $8.2 a-8.22$ and $8.3 a-8.3 c$ for electrictly net generation and useful thermal output from consumption for electricity energy electric net summer capacity. - Totals may not equal sum of components due to independent rounding.

Web Pages: - See http://www.eia.gov/totalenergy/data/monthly/\#renewable for updated monthly and annual data. - See http://www.eia.gov/totalenergy/data/annual/\#renewable for all annual data beginning in 449. - See http://www.eia.gov/renewable/ for related information. 
Table 10.2b Renewable Energy Consumption: Industrial and Transportation Sectors, Selected Years, 1949-2011 (Trillion Btu)

\begin{tabular}{|c|c|c|c|c|c|c|c|c|c|c|c|c|c|}
\hline \multirow[b]{3}{*}{ Year } & \multicolumn{10}{|c|}{ Industrial Sector ${ }^{1}$} & \multirow{2}{*}{\multicolumn{3}{|c|}{$\begin{array}{c}\text { Transportation Sector } \\
\text { Biomass }\end{array}$}} \\
\hline & \multirow{2}{*}{$\begin{array}{l}\text { Hydro- } \\
\text { electric } \\
\text { Power }^{2} \\
\end{array}$} & \multirow[b]{2}{*}{$\begin{array}{c}\text { Geo- } \\
\text { thermal }^{3}\end{array}$} & \multirow[b]{2}{*}{ Solar/PV ${ }^{4}$} & \multirow[b]{2}{*}{ Wind 5} & \multicolumn{5}{|c|}{ Biomass } & \multirow[b]{2}{*}{ Total } & & & \\
\hline & & & & & Wood 6 & Waste ${ }^{7}$ & $\begin{array}{c}\text { Fuel } \\
\text { Ethanol } 8\end{array}$ & $\begin{array}{l}\text { Losses and } \\
\text { Co-products }{ }^{9}\end{array}$ & Total & & $\begin{array}{c}\text { Fuel } \\
\text { Ethanol } 10\end{array}$ & Biodiesel & Total \\
\hline 1949 & 76 & NA & NA & NA & 468 & NA & NA & NA & 468 & 544 & NA & NA & NA \\
\hline 1950 & 69 & NA & NA & NA & 532 & NA & NA & NA & 532 & 602 & NA & NA & NA \\
\hline 1955 & 38 & NA & NA & NA & 631 & NA & NA & NA & 631 & 669 & NA & NA & NA \\
\hline 1960 & 39 & NA & NA & NA & 680 & NA & NA & NA & 680 & 719 & NA & NA & NA \\
\hline 1965 & 33 & NA & NA & NA & 855 & NA & NA & NA & 855 & 888 & NA & NA & NA \\
\hline 1970 & 34 & NA & NA & NA & 1,019 & NA & NA & NA & 1,019 & 1,053 & NA & NA & NA \\
\hline 1975 & 32 & NA & NA & NA & 1,063 & NA & NA & NA & 1,063 & 1,096 & NA & NA & NA \\
\hline 1976 & 33 & NA & NA & NA & 1,220 & NA & NA & NA & 1,220 & 1,253 & NA & NA & NA \\
\hline 1977 & 33 & NA & NA & NA & 1,281 & NA & NA & NA & 1,281 & 1,314 & NA & NA & NA \\
\hline 1978 & 32 & NA & NA & NA & 1,400 & NA & NA & NA & 1,400 & 1,432 & NA & NA & NA \\
\hline 1979 & 34 & NA & NA & NA & 1,405 & NA & NA & NA & 1,405 & 1,439 & NA & NA & NA \\
\hline 1980 & 33 & NA & NA & NA & 1,600 & NA & NA & NA & 1,600 & 1,633 & NA & NA & NA \\
\hline 1981 & 33 & NA & NA & NA & 1,602 & 87 & (s) & 6 & 1,695 & 1,728 & 7 & $\mathrm{NA}$ & 7 \\
\hline 1982 & 33 & NA & NA & NA & 1,516 & 118 & (s) & 16 & 1,650 & $\begin{array}{l}1,683 \\
\text { S }\end{array}$ & 18 & NA & 18 \\
\hline 1983 & 33 & NA & NA & NA & 1,690 & 155 & (s) & 29 & 1,874 & 1,908 & 34 & NA & 34 \\
\hline 1984 & 33 & NA & NA & NA & 1,679 & 204 & 1 & 35 & 1,918 & 1,951 & 41 & NA & 41 \\
\hline 1985 & 33 & $\mathrm{NA}$ & NA & NA & 1,645 & 230 & 1 & 42 & 1,918 & 1,951 & 50 & NA & 50 \\
\hline 1986 & 33 & NA & NA & NA & 1,610 & 256 & 1 & 48 & 1,915 & 1,948 & 57 & NA & 57 \\
\hline 1987 & 33 & NA & NA & NA & 1,576 & 282 & 1 & 55 & 1,914 & 1,947 & 66 & NA & 66 \\
\hline 1988 & 33 & NA & NA & NA & 1,625 & 308 & 1 & 55 & 1,989 & 2,022 & 67 & NA & 67 \\
\hline 1989 & 28 & 2 & - & - & 1,584 & 200 & 1 & 56 & 1,841 & 1,871 & 68 & NA & 68 \\
\hline 1990 & 31 & 2 & - & - & 1,442 & 192 & 1 & 49 & $\begin{array}{l}1,684 \\
\text {. }\end{array}$ & 1,717 & 60 & NA & 60 \\
\hline 1991 & 30 & 2 & - & - & 1,410 & 185 & 1 & 56 & 1,652 & 1,684 & 70 & NA & 70 \\
\hline 1992 & 31 & 2 & - & - & 1,461 & 179 & 1 & 64 & 1,705 & 1,737 & 80 & NA & 80 \\
\hline 1993 & 30 & 2 & - & - & 1,484 & 181 & 1 & 74 & 1,741 & 1,773 & 94 & NA & 94 \\
\hline 1994 & 62 & 3 & - & - & 1,580 & 199 & 1 & 82 & 1,862 & 1,927 & 105 & NA & 105 \\
\hline 1995 & 55 & 3 & - & - & 1,652 & 195 & 2 & 86 & 1,934 & 1,992 & 112 & NA & 112 \\
\hline 1996 & 61 & 3 & - & - & 1,683 & 224 & 1 & 61 & 1,969 & 2,033 & 81 & NA & 81 \\
\hline 1997 & 58 & 3 & - & - & 1,731 & 184 & 1 & 80 & 1,996 & 2,057 & 102 & NA & 102 \\
\hline 1998 & 55 & 3 & - & - & 1,603 & 180 & 1 & 86 & 1,872 & 1,929 & 113 & NA & 113 \\
\hline 1999 & 49 & 4 & - & - & 1,620 & 171 & 1 & 90 & 1,882 & 1,934 & 118 & NA & 118 \\
\hline 2000 & 42 & 4 & - & - & 1,636 & 145 & 1 & 99 & 1,881 & 1,928 & 135 & NA & 135 \\
\hline 2001 & 33 & 5 & - & - & 1,443 & $\begin{array}{l}129 \\
129\end{array}$ & 3 & 108 & 1,681 & 1,719 & 141 & 1 & 142 \\
\hline 2002 & $\begin{array}{l}50 \\
39\end{array}$ & 5 & - & - & 1,396 & 146 & 3 & 130 & 1,676 & 1,720 & 168 & 2 & 170 \\
\hline 2003 & 43 & 3 & - & - & 1,363 & $\begin{array}{l}140 \\
142\end{array}$ & 4 & $\begin{array}{l}160 \\
169\end{array}$ & 1,679 & 1,726 & $\begin{array}{l}2280 \\
228\end{array}$ & 2 & 230 \\
\hline 2004 & 33 & 4 & - & - & 1,476 & 132 & 6 & 203 & 1,817 & 1,853 & 286 & 3 & 290 \\
\hline 2005 & 32 & 4 & - & - & 1,452 & 148 & 7 & 230 & 1,837 & 1,873 & 327 & 12 & 339 \\
\hline 2006 & 29 & 4 & - & - & 1,472 & 130 & 10 & 285 & 1,897 & 1,930 & 442 & 33 & 475 \\
\hline 2007 & 16 & 5 & - & - & $\mathrm{R}_{1,405}$ & 144 & 10 & 377 & $\mathrm{R}_{1}, 936$ & $\mathrm{R}_{1,956}$ & 557 & 46 & 602 \\
\hline 2008 & 17 & 5 & - & - & $\mathrm{R}_{1,340}$ & 144 & 12 & 532 & $\mathrm{R}_{2,028}$ & $\mathrm{R}_{2,049}$ & 786 & 40 & 826 \\
\hline 2009 & 18 & 4 & - & - & $\mathrm{R}_{1,208}$ & $\mathrm{R}_{155}$ & 13 & 617 & $\mathrm{R}_{1}, 994$ & $\mathrm{R}_{2,016}$ & 894 & $\mathrm{R}_{42}$ & $\begin{array}{l}\text { R935 } \\
\text { R935 }\end{array}$ \\
\hline 2010 & 16 & 4 & (s) & - & $\mathrm{R}_{1,301}$ & $\mathrm{R}_{169}$ & $R_{17}$ & R742 & $\mathrm{R}_{2,230}$ & $\mathrm{R}_{2,250}$ & $\mathrm{R}_{1,040}$ & $\mathrm{R}_{34}$ & $\mathrm{R}_{1,074}$ \\
\hline $2011^{\mathrm{P}}$ & 18 & 4 & (s) & (s) & 1,311 & 172 & 17 & 772 & 2,273 & 2,295 & 1,042 & 112 & 1,154 \\
\hline
\end{tabular}

1 Industrial sector, including industrial combined-heat-and-power (CHP) and industrial electricity-only plants. See Note 2, "Classification of Power Plants Into Energy-Use Sectors," at end of Section 8.

2 Convent
Table A6)

4 Photovoltaic (PV) electricity net generation (converted to Btu using the fossil-fuels heat rate-see Table A6) at industrial plants with capacity of 1 megawatt or greater

Iossil-fuels heat rate-see Table A6).

7 Municipal solid waste from biogenic sources, landfill gas, sludge waste, agricultural byproducts, and other biomass. Through 2000, also includes non-renewable waste (municipal solid waste from non-biogenic sources, and tire-derived fuels).

portion of motor fuels, such as E10, consumed by the industria sector.

Losses and co-products from the production of fuel ethanol and biodiesel. Does not include natural gas, electricity, and other non-biomass energy used in the production of fuel ethanol and biodiesel-these 10 The fuel ethanol (minus denaturant) portion of motics for the appropriate energy source.

transportation sector

R=Revised. P=Preliminary. NA=Not available. $-=$ No data reported. ( $s)=$ Less than 0.5 trillion Btu.
Notes: - Data are estimates, except for industrial sector hydroelectric power in 1949-1978 and 1989 forward, solar/PV, and wind. - See Tables 8.2a-8.2d and 8.3a-8.3c for electricity net generation and $8.7 a-8.7 \mathrm{c}$ for renes Tables $8.11 \mathrm{a}-8.11 \mathrm{~d}$ for renewable energy electric net summer capacity. - Totals may not equal sum of components due to independent rounding.

Web Pages: - See http://www.eia.gov/totalenergy/data/monthly/\#renewable for updated monthly and

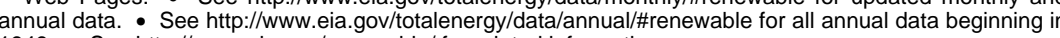
Source: See end of section. 


\title{
Figure 10.2c Renewable Energy Consumption: Electric Power Sector
}

\section{Electric Power Sector Total and Hydroelectric Power, 1949-2011}

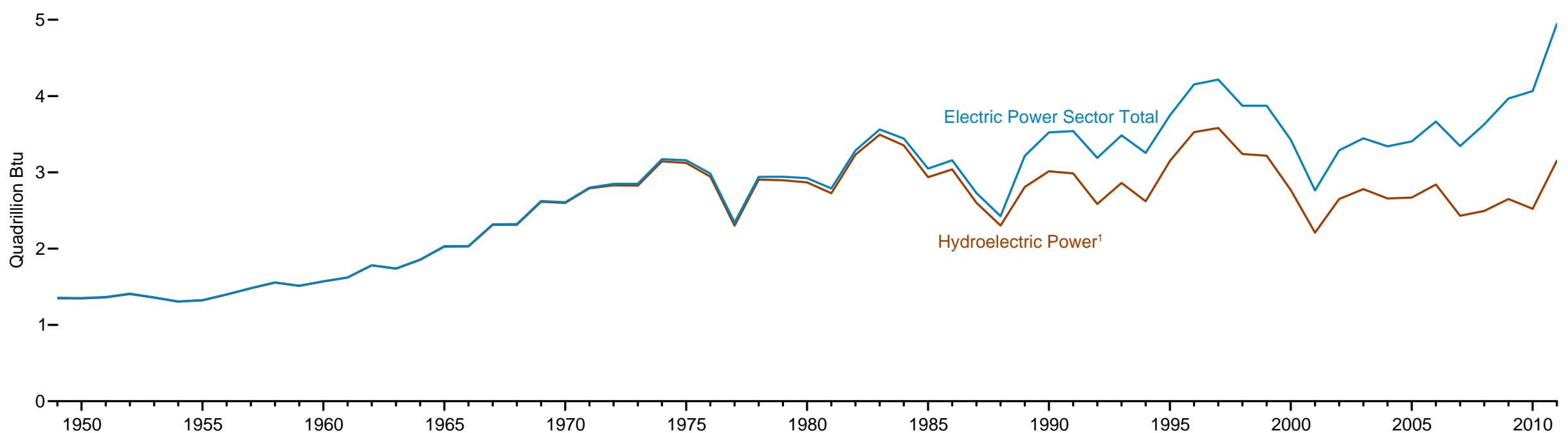

Non-Hydroelectric Power Sources, 1989-2011

$1,400-$

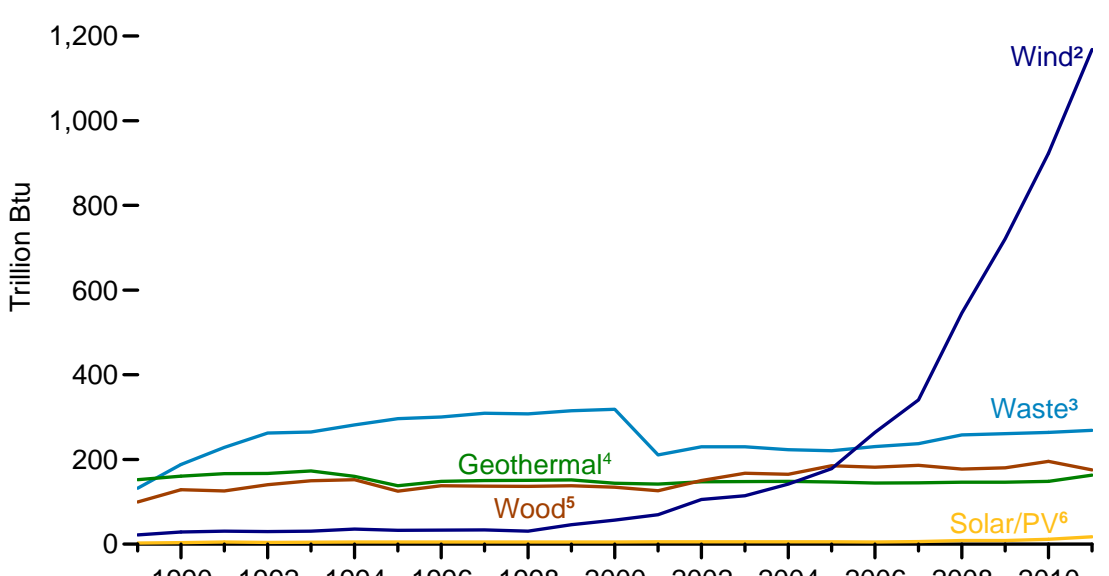

\section{Non-Hydroelectric Power Sources, 2011}

\author{
$1,400-$
}

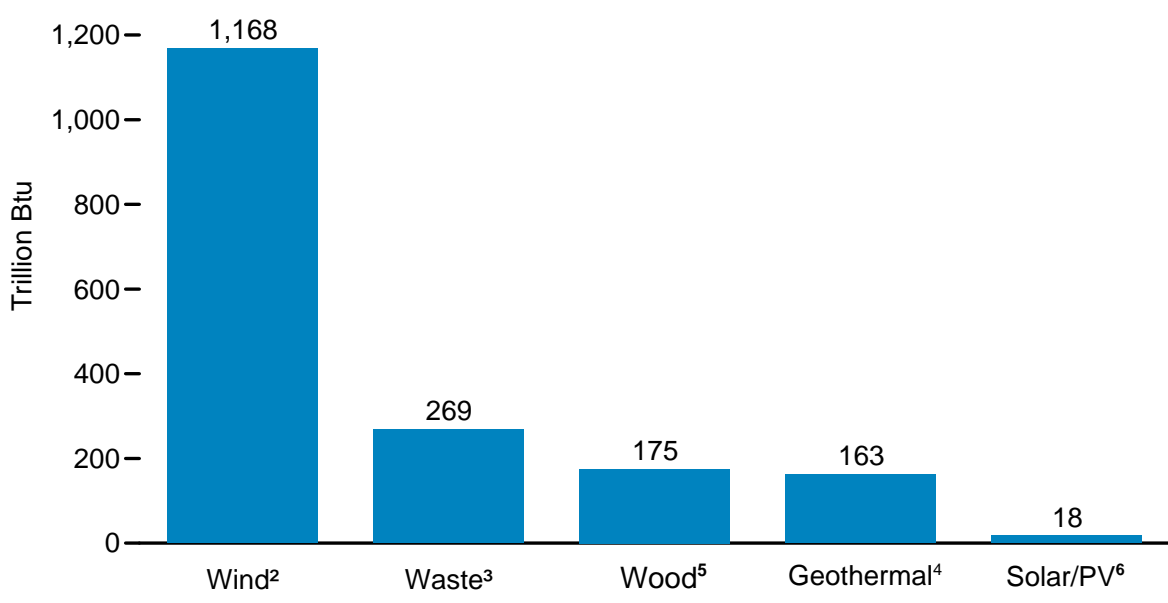

${ }^{4}$ Geothermal electricity net generation.

${ }^{5}$ Wood and wood-derived fuels.

${ }^{1}$ Conventional hydroelectricity net generation.

${ }^{2}$ Wind electricity net generation.

${ }^{3}$ Municipal solid waste from biogenic sources, landfill gas, sludge waste, agricultural byproducts, and other biomass. Through 2000 , also includes non-renewable waste (municipal solid waste from non-biogenic sources, and tire-derived fuels).

${ }^{6}$ Solar thermal and photovoltaic (PV) electricity net generation.

Note: See related Figures $10.2 \mathrm{a}$ and $10.2 \mathrm{~b}$ on the end-use sectors.

Source: Table 10.2c. 
Table 10.2c Renewable Energy Consumption: Electric Power Sector, Selected Years, 1949-2011

(Trillion Btu)

\begin{tabular}{|c|c|c|c|c|c|c|c|c|}
\hline \multirow[b]{2}{*}{ Year } & \multirow{2}{*}{$\begin{array}{l}\text { Hydroelectric } \\
\text { Power }^{1}\end{array}$} & \multirow[b]{2}{*}{ Geothermal $^{2}$} & \multirow[b]{2}{*}{ Solar/PV ${ }^{3}$} & \multirow[b]{2}{*}{ Wind ${ }^{4}$} & \multicolumn{3}{|c|}{ Biomass } & \multirow[b]{2}{*}{ Total } \\
\hline & & & & & Wood $^{5}$ & Waste ${ }^{6}$ & Total & \\
\hline 1949 & 1,349 & NA & NA & NA & 6 & NA & 6 & 1,355 \\
\hline 1950 & 1,346 & NA & NA & NA & 5 & NA & 5 & 1,351 \\
\hline 1955 & 1,322 & NA & NA & NA & 3 & NA & 3 & 1,325 \\
\hline 1960 & 1,569 & (s) & NA & NA & 2 & NA & 2 & 1,571 \\
\hline 1965 & 2,026 & 2 & NA & NA & 3 & NA & 3 & 2,031 \\
\hline 1970 & 2,600 & 6 & NA & NA & 1 & 2 & 4 & 2,609 \\
\hline 1975 & 3,122 & 34 & NA & NA & (s) & 2 & 2 & 3,158 \\
\hline 1976 & 2,943 & 38 & NA & NA & 1 & 2 & 3 & 2,983 \\
\hline 1977 & 2,301 & 37 & NA & NA & 3 & 2 & 5 & 2,343 \\
\hline 1978 & 2,905 & 31 & NA & NA & 2 & 1 & 3 & 2,940 \\
\hline 1979 & 2,897 & 40 & NA & NA & 3 & 2 & 5 & 2,942 \\
\hline 1980 & 2,867 & 53 & NA & NA & 3 & 2 & 5 & 2,925 \\
\hline 1981 & 2,725 & 59 & NA & NA & 3 & 1 & 4 & 2,788 \\
\hline 1982 & 3,233 & 51 & NA & NA & 2 & 1 & 3 & 3,286 \\
\hline 1983 & 3,494 & 64 & NA & (s) & 2 & 2 & 4 & 3,562 \\
\hline 1984 & 3,353 & 81 & (s) & (s) & 5 & 4 & 9 & 3,443 \\
\hline 1985 & 2,937 & 97 & (s) & (s) & 8 & 7 & 14 & 3,049 \\
\hline 1986 & 3,038 & 108 & (s) & (s) & 5 & 7 & 12 & 3,158 \\
\hline 1987 & 2,602 & 112 & (s) & (s) & 8 & 7 & 15 & 2,729 \\
\hline 1988 & 2,302 & 106 & (s) & (s) & 10 & 8 & 17 & 2,425 \\
\hline $1989^{7}$ & 2,808 & 152 & 3 & 22 & 100 & 132 & 232 & 3,217 \\
\hline 1990 & 3,014 & 161 & 4 & 29 & 129 & 188 & 317 & 3,524 \\
\hline 1991 & 2,985 & 167 & 5 & 31 & 126 & 229 & 354 & 3,542 \\
\hline 1992 & 2,586 & 167 & 4 & 30 & 140 & 262 & 402 & 3,189 \\
\hline 1993 & 2,861 & 173 & 5 & 31 & 150 & 265 & 415 & 3,484 \\
\hline 1994 & 2,620 & 160 & 5 & 36 & 152 & 282 & 434 & 3,255 \\
\hline 1995 & 3,149 & 138 & 5 & 33 & 125 & 296 & 422 & 3,747 \\
\hline 1996 & 3,528 & 148 & 5 & 33 & 138 & 300 & 438 & 4,153 \\
\hline 1997 & 3,581 & 150 & 5 & 34 & 137 & 309 & 446 & 4,216 \\
\hline 1998 & 3,241 & 151 & 5 & 31 & 137 & 308 & 444 & 3,872 \\
\hline 1999 & 3,218 & 152 & 5 & 46 & 138 & 315 & 453 & 3,874 \\
\hline 2000 & 2,768 & 144 & 5 & 57 & 134 & 318 & 453 & 3,427 \\
\hline 2001 & 2,209 & 142 & 6 & 70 & 126 & 211 & 337 & 2,763 \\
\hline 2002 & 2,650 & 147 & 6 & 105 & 150 & 230 & 380 & 3,288 \\
\hline 2003 & 2,781 & 148 & 5 & 115 & 167 & 230 & 397 & 3,445 \\
\hline 2004 & 2,656 & 148 & 6 & 142 & 165 & 223 & 388 & 3,340 \\
\hline 2005 & 2,670 & 147 & 6 & 178 & 185 & 221 & 406 & 3,406 \\
\hline 2006 & 2,839 & 145 & 5 & 264 & 182 & 231 & 412 & 3,665 \\
\hline 2007 & 2,430 & 145 & 6 & 341 & 186 & 237 & 423 & 3,345 \\
\hline 2008 & 2,494 & 146 & 9 & 546 & 177 & 258 & 435 & 3,630 \\
\hline 2009 & 2,650 & 146 & 9 & 721 & 180 & 261 & 441 & 3,967 \\
\hline 2010 & $\mathrm{R}_{2,521}$ & $\mathrm{R}_{148}$ & $\mathrm{R}_{12}$ & R923 & $\mathrm{R}_{196}$ & $\mathrm{R}_{264}$ & R459 & $\mathrm{R}_{4,064}$ \\
\hline $2011^{P}$ & 3,153 & 163 & 18 & 1,168 & 175 & 269 & 444 & 4,945 \\
\hline
\end{tabular}

Conventional hydroelectricity net generation (converted to Btu using the fossil-fuels heat rate-see Table A6).

2 Geothermal electricity net generation (converted to Btu using the fossil-fuels heat rate-see Table A6). ${ }_{3}^{3}$ Solar thermal and photovoltaic (PV) electricity net generation (converted to Btu using the fossil-fuels heat rate- see Table A6).

Wind electricity net generation (converted to Btu using the fossil-fuels heat rate-see Table A6).

Wood and wood-derived fuels.

Municipal solid waste from biogenic sources, landfill gas, sludge waste, agricultural byproducts, and other biomass. Through 2000, also includes non-renewable waste (municipal solid waste from non-biogenic sources, and tire-derived fuels).

Beginning in 1989, data are for electric utilities and independent power producers.
$\mathrm{R}=$ Revised. $\mathrm{P}=$ Preliminary. $\mathrm{NA}=$ Not available. $(\mathrm{s})=$ Less than 0.5 trillion Btu.

Notes: - The electric power sector comprises electricity-only and combined-heat-and-power (CHP) plants within the NAICS 22 category whose primary business is to sell electricity, or electricity and heat, to the public. - See Tables $8.2 \mathrm{a}-8.2 \mathrm{~d}$ and $8.3 \mathrm{a}-8.3 \mathrm{c}$ for electricity net generation and useful thermal output from renewable energy sources; Tables 8.4a-8.4c, 8.5a-8.5d, 8.6a-8.6c, and 8.7a-8.7c for renewable

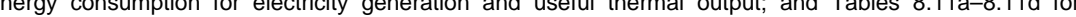
renewable energy electric net summer capacity. - See Note 3, "Electricity Imports and Exports," at end of Wection 8. - Totals may not equal sum of components due to independent rounding

for updated monthly and annual data. - See http://www.eia.gov/totalenergy/data/annual/\#renewable for all annual data beginning in ( See 


\section{Figure 10.3 Fuel Ethanol Overview}

\section{Overview, 2011}

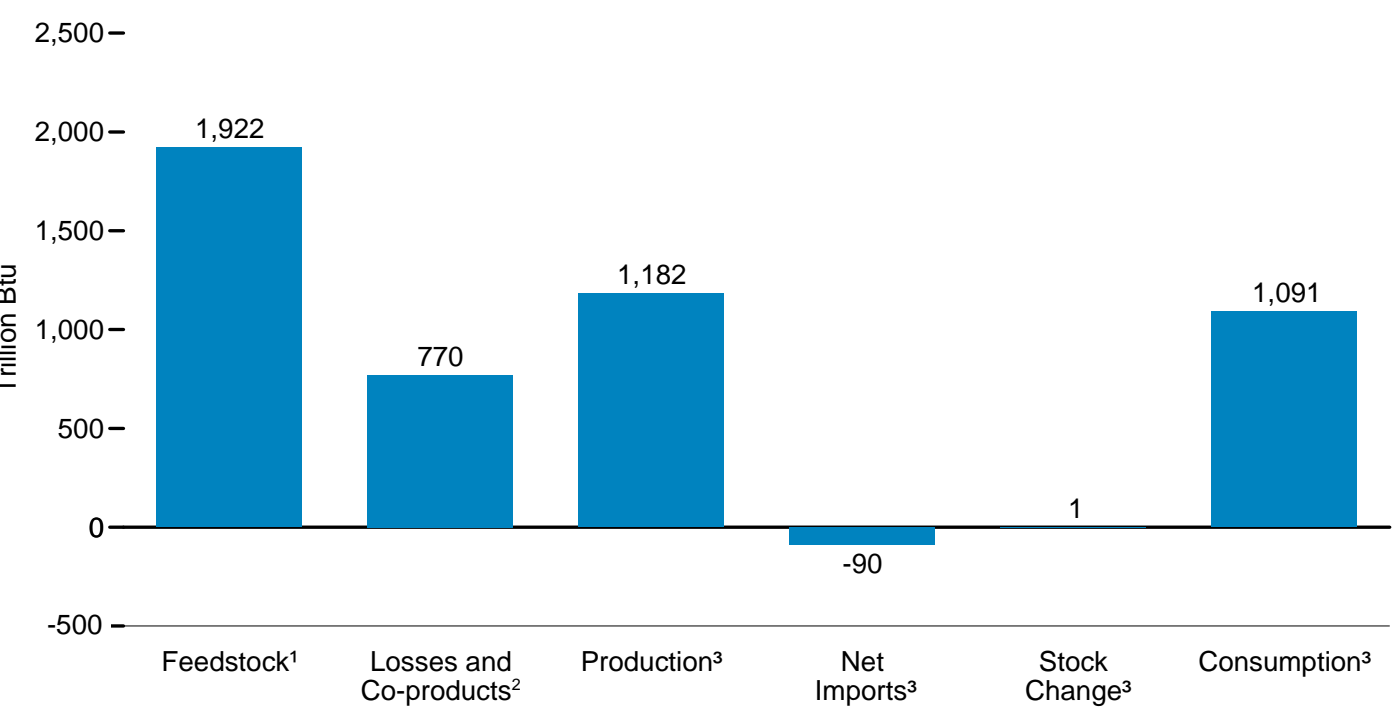

Consumption, ${ }^{3}$ 1981-2011

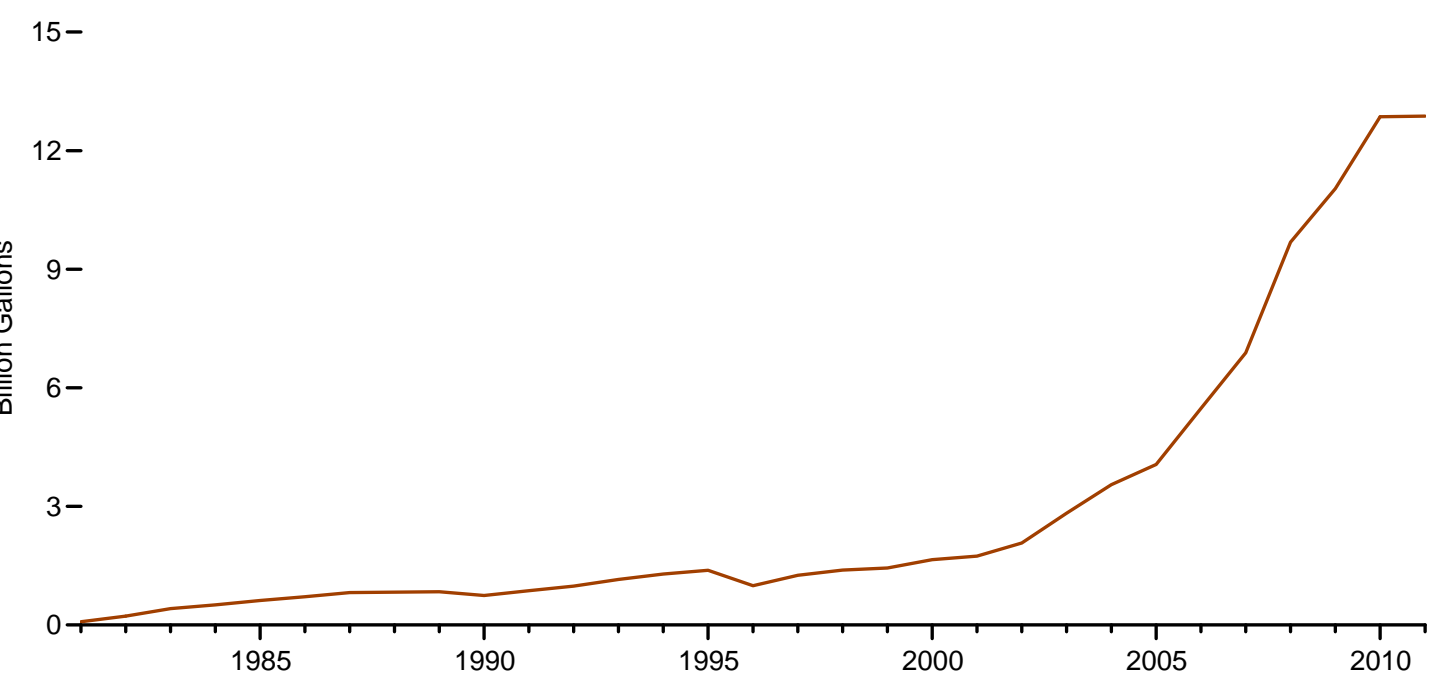

\section{Stocks, ${ }^{3}$ End of Year 1992-2011}

$20-$

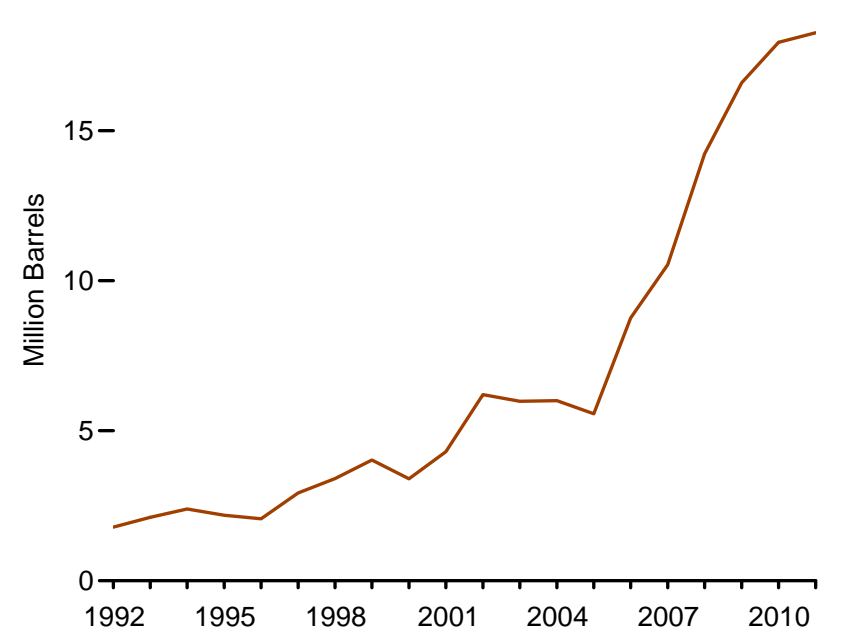

Fuel Ethanol and Biodiesel Production, 2011

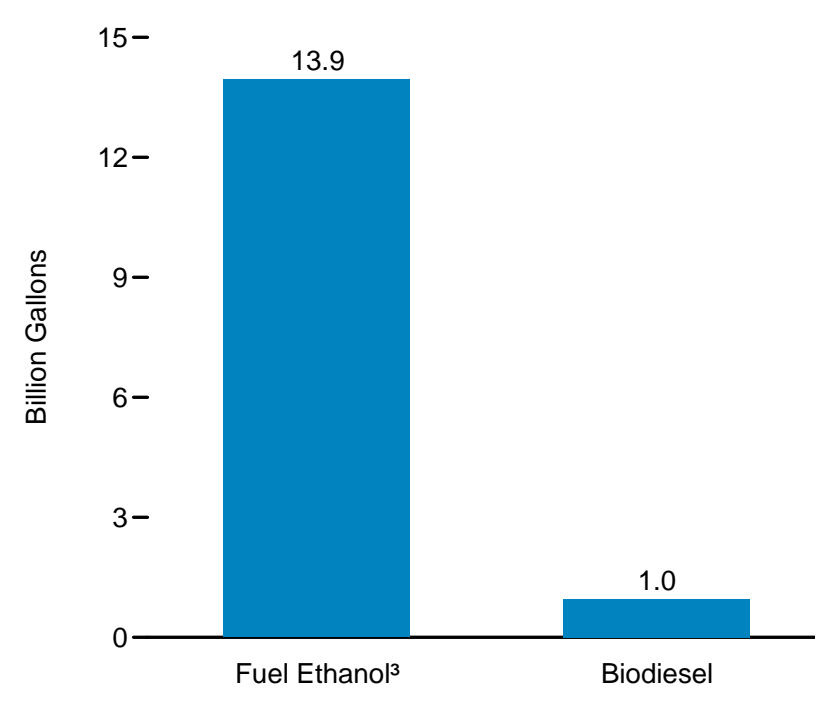

\footnotetext{
${ }^{1}$ Total corn and other biomass inputs to the production of undenatured ethanol used for fuel ethanol.

${ }^{2}$ Losses and co-products from the production of fuel ethanol. 
Table 10.3 Fuel Ethanol Overview, 1981-2011

\begin{tabular}{|c|c|c|c|c|c|c|c|c|c|c|c|c|c|c|c|}
\hline \multirow[b]{3}{*}{ Year } & \multirow{3}{*}{$\begin{array}{c}\begin{array}{c}\text { Feed- } \\
\text { stock }\end{array} \\
\begin{array}{c}\text { Trillion } \\
\text { Btu }\end{array}\end{array}$} & \multirow{3}{*}{$\begin{array}{c}\begin{array}{c}\text { Losses } \\
\text { and Co- } \\
\text { products }\end{array} \\
\begin{array}{c}\text { Trillion } \\
\text { Btu }\end{array}\end{array}$} & \multirow{3}{*}{$\begin{array}{c}\begin{array}{c}\text { Dena- } \\
\text { turant }\end{array}{ }^{3} \\
\text { Thousand } \\
\text { Barrels }\end{array}$} & \multirow{2}{*}{\multicolumn{3}{|c|}{ Production 4}} & \multicolumn{3}{|c|}{ Trade $^{4}$} & \multirow{3}{*}{\begin{tabular}{|c|}
$\begin{array}{c}\text { Stocks, } \\
\text { End of Year }\end{array}$ \\
$\begin{array}{c}\text { Thousand } \\
\text { Barrels }\end{array}$ \\
\end{tabular}} & \multirow{3}{*}{$\begin{array}{c}\begin{array}{c}\text { Stock } \\
\text { Change }^{4,6}\end{array} \\
\begin{array}{c}\text { Thousand } \\
\text { Barrels }\end{array} \\
\end{array}$} & \multirow{2}{*}{\multicolumn{3}{|c|}{ Consumption ${ }^{4}$}} & \multirow{3}{*}{\begin{tabular}{|c}
$\begin{array}{c}\text { Consumption } \\
\text { Minus } \\
\text { Denaturant }\end{array}$ \\
$\begin{array}{c}\text { Trillion } \\
\text { Btu }\end{array}$ \\
\end{tabular}} \\
\hline & & & & & & & \multirow{2}{*}{$\begin{array}{c}\text { Imports } \\
\begin{array}{c}\text { Thousand } \\
\text { Barrels }\end{array}\end{array}$} & \multirow{2}{*}{$\begin{array}{c}\text { Exports } \\
\begin{array}{c}\text { Thousand } \\
\text { Barrels }\end{array}\end{array}$} & \multirow{2}{*}{$\begin{array}{c}\begin{array}{c}\text { Net } \\
\text { Imports }\end{array} \\
\begin{array}{c}\text { Thousand } \\
\text { Barrels }\end{array}\end{array}$} & & & & & & \\
\hline & & & & $\begin{array}{c}\text { Thousand } \\
\text { Barrels }\end{array}$ & $\begin{array}{l}\text { Million } \\
\text { Gallons }\end{array}$ & $\begin{array}{c}\text { Trillion } \\
\text { Btu }\end{array}$ & & & & & & $\begin{array}{c}\text { Thousand } \\
\text { Barrels }\end{array}$ & $\begin{array}{l}\text { Million } \\
\text { Gallons }\end{array}$ & $\begin{array}{c}\text { Trillion } \\
\text { Btu }\end{array}$ & \\
\hline 1981 & 13 & 6 & 40 & 1,978 & 83 & 7 & NA & NA & NA & NA & NA & 1,978 & 83 & 7 & 7 \\
\hline 1982 & 34 & 16 & 107 & 5,369 & 225 & 19 & NA & NA & NA & NA & NA & 5,369 & 225 & 19 & 19 \\
\hline 1983 & 63 & 29 & 198 & 9,890 & 415 & 35 & NA & NA & NA & NA & NA & 9,890 & 415 & 35 & 34 \\
\hline 1984 & 77 & 35 & 243 & 12,150 & 510 & 43 & NA & NA & NA & NA & NA & 12,150 & 510 & 43 & 42 \\
\hline 1985 & 93 & 42 & 294 & 14,693 & 617 & 52 & NA & NA & NA & NA & NA & 14,693 & 617 & 52 & 51 \\
\hline 1986 & 107 & 48 & 339 & 16,954 & 712 & 60 & NA & NA & NA & NA & NA & 16,954 & 712 & 60 & 59 \\
\hline 1987 & 123 & 55 & 390 & 19,497 & 819 & 69 & NA & NA & NA & NA & NA & 19,497 & $81 \overline{9}$ & 69 & 68 \\
\hline 1988 & 124 & 55 & 396 & 19,780 & 831 & 70 & NA & NA & NA & NA & NA & 19,780 & 831 & 70 & 69 \\
\hline 1989 & 125 & 56 & 401 & 20,062 & 843 & 71 & NA & NA & NA & NA & NA & 20,062 & 843 & 71 & 70 \\
\hline 1990 & 111 & 49 & 356 & 17,802 & 748 & 63 & NA & NA & NA & NA & NA & 17,802 & 748 & 63 & 62 \\
\hline 1991 & 128 & 56 & 413 & 20,627 & 866 & 73 & NA & NA & NA & NA & NA & 20,627 & 866 & 73 & 72 \\
\hline 1992 & 145 & 64 & 469 & 23,453 & 985 & 84 & NA & NA & NA & 1,791 & NA & 23,453 & 985 & 84 & 81 \\
\hline 1993 & 169 & 74 & 550 & 27,484 & 1,154 & 98 & 244 & NA & 244 & 2,114 & 323 & 27,405 & 1,151 & 98 & 95 \\
\hline 1994 & 188 & 82 & 614 & 30,689 & 1,289 & 109 & 279 & NA & 279 & 2,393 & 279 & 30,689 & 1,289 & 109 & 106 \\
\hline 1995 & 198 & 86 & 647 & 32,325 & 1,358 & 115 & 387 & NA & 387 & 2,186 & -207 & 32,919 & $\begin{array}{l}1,383 \\
\end{array}$ & 117 & 114 \\
\hline 1996 & 141 & 61 & 464 & 23,178 & 973 & 83 & 313 & NA & 313 & 2,065 & -121 & 23,612 & 992 & 84 & 82 \\
\hline 1997 & $\begin{array}{l}186 \\
186\end{array}$ & 80 & 613 & 30,674 & $\begin{array}{r}1,288 \\
\end{array}$ & 109 & 85 & NA & 85 & $\begin{array}{l}2,925 \\
2,90\end{array}$ & 860 & 29,899 & $\begin{array}{l}1,256 \\
\end{array}$ & $\begin{array}{c}04 \\
107\end{array}$ & 104 \\
\hline 1998 & 202 & 86 & 669 & 33,453 & $\begin{array}{l}1,405 \\
1\end{array}$ & 119 & 66 & NA & $\begin{array}{l}00 \\
66\end{array}$ & 3,406 & 481 & 33,038 & $\begin{array}{l}1,388 \\
\text {. }\end{array}$ & 118 & 115 \\
\hline 1999 & 211 & 90 & 698 & 34,881 & 1,465 & 124 & 87 & NA & 87 & 4,024 & 618 & 34,350 & 1,443 & 122 & 119 \\
\hline 2000 & 233 & 99 & 773 & 38,627 & 1,622 & 138 & 116 & NA & 116 & 3,400 & -624 & 39,367 & 1,653 & 140 & 137 \\
\hline 2001 & 253 & 108 & 841 & 42,028 & 1,765 & 150 & 315 & NA & 315 & 4,298 & 898 & 41,445 & 1,741 & 148 & 144 \\
\hline 2002 & 307 & 130 & 1,019 & 50,956 & 2,140 & 182 & 306 & NA & 306 & 6,200 & 1,902 & 49,360 & 2,073 & 176 & 171 \\
\hline 2003 & 400 & 169 & 1,335 & 66,772 & 2,804 & 238 & 292 & NA & 292 & 5,978 & -222 & 67,286 & 2,826 & 240 & 233 \\
\hline 2004 & 484 & 203 & 1,621 & 81,058 & 3,404 & 289 & 3,542 & NA & 3,542 & 6,002 & 24 & 84,576 & 3,552 & 301 & 293 \\
\hline 2005 & 552 & 230 & 1,859 & 92,961 & 3,904 & 331 & 3,234 & NA & 3,234 & 5,563 & -439 & 96,634 & 4,059 & 344 & 335 \\
\hline 2006 & 688 & 285 & 2,326 & 116,294 & 4,884 & 414 & 17,408 & NA & 17,408 & 8,760 & 3,197 & 130,505 & 5,481 & 465 & 453 \\
\hline 200 & 914 & 376 & 3,1 & & 6,521 & 55 & 10,4 & $\mathrm{~N}$ & 10,45 & 10,535 & 1,77 & & 6,886 & & 569 \\
\hline 2008 & 1,300 & 531 & 4,433 & 221,637 & 9,309 & 790 & $\begin{array}{l}12,610 \\
\end{array}$ & NA & 12,6 & 14,226 & 3,691 & 230,556 & $\begin{array}{l}9,683 \\
9\end{array}$ & 21 & 800 \\
\hline 2009 & 1,517 & 616 & 5,688 & 260,424 & 10,938 & 928 & 4,720 & NA & 4,720 & 16,594 & $\begin{array}{l}2,368 \\
\end{array}$ & 262,776 & 11,037 & 36 & 910 \\
\hline 2010 & $\mathrm{R}_{1,839}$ & R742 & $\mathrm{R}_{6,506}$ & R316,617 & $R_{13,298}$ & $\mathrm{R}_{1,127}$ & R373 & R9,488 & $R_{-}-115$ & $\mathrm{R}_{17,941}$ & $\mathrm{R}_{1,347}$ & $\mathrm{R}_{306,155}$ & $R_{12,858}$ & $\mathrm{R}_{1}, 090$ & $\mathrm{R}_{1,061}$ \\
\hline $2011^{P}$ & 1,922 & 770 & 6,636 & 332,107 & 13,948 & 1,182 & 3,135 & 28,457 & $-25,322$ & 18,261 & 321 & 306,464 & 12,871 & 1,091 & 1,063 \\
\hline
\end{tabular}

1 Total corn and other biomass inputs to the production of undenatured ethanol used for fuel ethanol. 2 Losses and co-products from the production of fuel ethanol. Does not include natural gas, electricity,
and other non-biomass energy used in the production of fuel ethanol-these are included in the industrial sector consumption statistics for the appropriate energy source.

3 sector consumption statistics for the appropriate energy
The amount of denaturant in fuel ethanol produced.

4 Includes denaturant.

Net imports equal imports minus exports.

A negative value indicates a decrease in stocks and a positive value indicates an increase Consumption of fuel ethanol minus denaturant. Data for fuel ethanol minus denaturant are used to 8 Derived from the preliminary 2010 stocks value (17.940 thousand barrels), not the final 2010 $\mathrm{R}=$ Revised. P=Preliminary. NA=Not available.

Notes: - Fuel ethanol data in thousand barrels are converted to million gallons by multiplying by 0.042 , and are converted to Btu by multiplying by the approximate heat content of fuel ethanol-see Table A3 fedrock, only data for feedstock, losses and co-products, and denaturant are estimates. Beginning in 2009, only data for
feedstock, and losses and co-products, are estimates. " See "Denaturant," "Ethanol," "Fuel Ethanol," and "Fuel Ethanol Minus Denaturant" in Glossary. - Totals may not equal sum of components due to

independent rounding.
Web Pages: See htp://www.eia.gov/totalenergy/data/monthly/\#renewable for updated monthly and nnual data. - See http://www.eia.gov/petroleum/supply/monthly/ for related information

Sources. Feedstock: Calculated as fuel ethanol production (in thousand barrels) minus denaturant, and

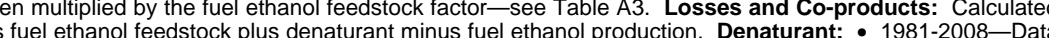
in thousand barrels for petroleum denaturant in fuel ethanol produced are estimated as 2 percent of fuel (the estimated quantity-weighted factor of pentanes plus and conventional motor gasoline used as denaturant).

report, Table 1, and Petroleum Supply Monthly (PSM), monthly reports, Table 1. Data in thousand barrels for net production of pentanes plus at renewable fuels and oxygenate plants are multiplied by -1 ; these data are convered to Bu by thlily ang by 4.620 mon gasoline blending components at renewable fuels and oxygenate plants are multiplied by -1: these data are converted to Btu by multiplying by 5.253 million Btu per barrel (the approximate heat content of conventional motor gasoline). Total denaturant is the sum of the values for pentanes plus, conventional motor gasoline, and motor gasoline blending components. Production: - 1981-1992-Fuel ethanol production is assumed to equal fuel enthanol consumption-see sources for "Consumption." net imports. These data differ slightly from the original production data from EIA, Form EIA-819, "Monthly the final balance. - 2005-2008-EIA, Form EIA-819, "Monthly Oxygenate Report." • 2009 and 2010-EIA, PSA, Table 1, data tor net production of fuel ethanol at renewable fuels and oxygenate plants. ( 2011 EIA PSM (Fobrary 2012) Tabl 1. Consumption: 1981-1989, EIA Estimates of US. Biofuels Consumption 1990. Table 10; and interpolated values for 1982, 1983, 1985, 1986, and 1988. - 1990-1992-EIA, Estimates of U.S. Biomass Energy Consumption 1992, Table D2; and interpolated value for 1991. - 1993-2004-EIA, PSA, annual reports, Tables 2 and 16. Calculated as 10 percent of - 2005-2008-EIA, PSA, annual reports, Tables 1 and 15 . Calculated as motor gasoline blending components adjustments (Table 1), plus finished motor gasoline adjustments (Table 1), plus fuel ethanol . 2011-EIA, PSM (February 2012), Table 1. Calculated as fuel ethanol refinery and blender net inputs minus fuel ethanol adjustments. Consumption Minus Denaturant: Calculated as fuel ethanol consumption minus the amount of denaturant in fuel ethanol consumed. Denaturant in fuel ethanol consumed is estimated by multiplying 
Figure 10.4 Biodiesel Overview

\section{Overview, 2011}

$150-$

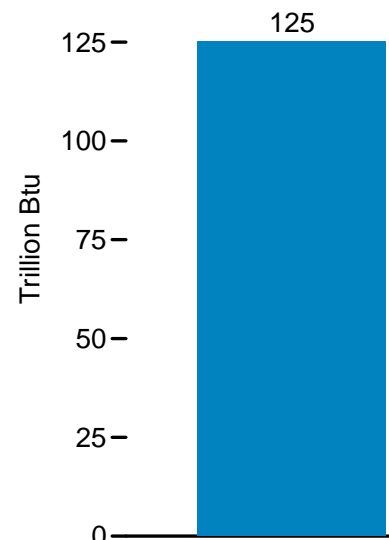

Feedstock $^{1}$
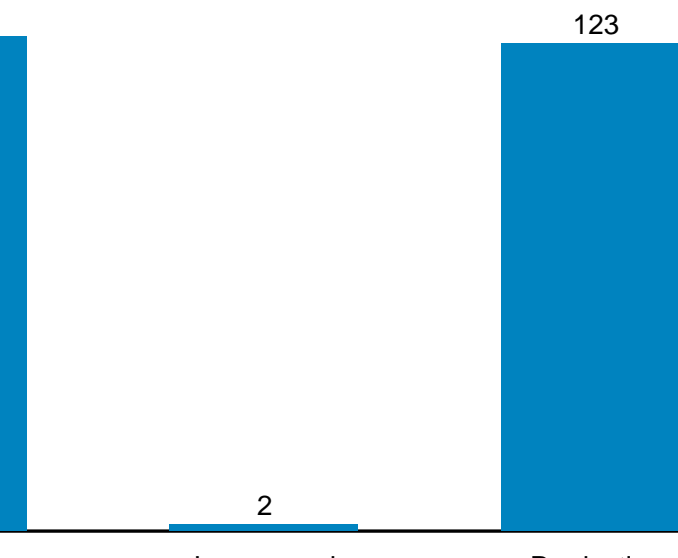

Losses and

Production
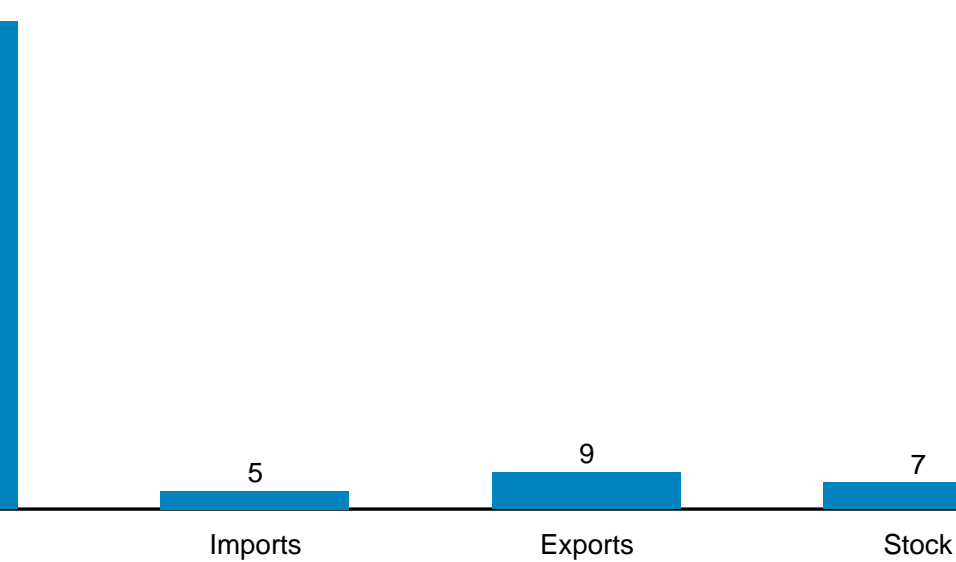

Change

\section{Consumption, 2001-2011}

$1,200-$

$900-$

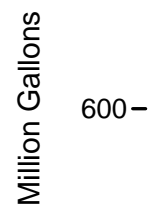

$300-$

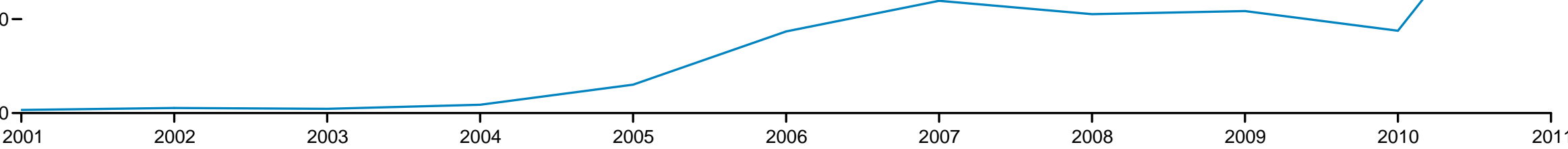

${ }^{1}$ Total vegetable oil and other biomass inputs to the production of biodiesel.

Sources: Tables 10.4 and A3.

Total vegetable oil and other biomass inputs to the production
Losses and co-products from the production of biodiesel. 
Table 10.4 Biodiesel Overview, 2001-2011

\begin{tabular}{|c|c|c|c|c|c|c|c|c|c|c|c|c|c|c|}
\hline \multirow[b]{3}{*}{ Year } & \multirow{3}{*}{$\begin{array}{c}\text { Feedstock }{ }^{1} \\
\begin{array}{c}\text { Trillion } \\
\text { Btu }\end{array}\end{array}$} & \multirow{3}{*}{\begin{tabular}{|c}
$\begin{array}{c}\text { Losses and } \\
\text { Co-products }\end{array}$ \\
Trillion \\
Btu
\end{tabular}} & \multirow{2}{*}{\multicolumn{3}{|c|}{ Production }} & \multicolumn{3}{|c|}{ Trade } & \multirow{3}{*}{$\begin{array}{c}\begin{array}{c}\text { Stocks, } \\
\text { End of Year }\end{array} \\
\begin{array}{c}\text { Thousand } \\
\text { Barrels }\end{array}\end{array}$} & \multirow{3}{*}{$\begin{array}{c}\begin{array}{c}\text { Stock } \\
\text { Change } \\
\end{array} \\
\text { Thousand } \\
\text { Barrels }\end{array}$} & \multirow{3}{*}{$\begin{array}{c}\begin{array}{c}\text { Balancing } \\
\text { Item } 5\end{array} \\
\text { Thousand } \\
\text { Barrels }\end{array}$} & \multirow{2}{*}{\multicolumn{3}{|c|}{ Consumption }} \\
\hline & & & & & & \multirow{2}{*}{$\begin{array}{c}\text { Imports } \\
\begin{array}{c}\text { Thousand } \\
\text { Barrels }\end{array}\end{array}$} & \multirow{2}{*}{$\begin{array}{c}\text { Exports } \\
\begin{array}{c}\text { Thousand } \\
\text { Barrels }\end{array}\end{array}$} & \multirow{2}{*}{$\begin{array}{c}\begin{array}{c}\text { Net } \\
\text { Imports }{ }^{3}\end{array} \\
\begin{array}{c}\text { Thousand } \\
\text { Barrels }\end{array}\end{array}$} & & & & & & \\
\hline & & & $\begin{array}{c}\text { Thousand } \\
\text { Barrels }\end{array}$ & $\begin{array}{l}\text { Million } \\
\text { Gallons }\end{array}$ & $\begin{array}{c}\text { Trillion } \\
\text { Btu }\end{array}$ & & & & & & & $\begin{array}{c}\text { Thousand } \\
\text { Barrels }\end{array}$ & $\begin{array}{l}\text { Million } \\
\text { Gallons }\end{array}$ & $\begin{array}{c}\text { Trillion } \\
\text { Btu }\end{array}$ \\
\hline 2001 & 1 & (s) & 204 & 9 & 1 & 78 & 39 & 39 & NA & NA & NA & 243 & 10 & 1 \\
\hline 2002 & 1 & (s) & 250 & 10 & 1 & 191 & 56 & 135 & NA & NA & NA & 385 & 16 & 2 \\
\hline 2003 & 2 & (s) & 338 & 14 & 2 & 94 & 110 & -16 & NA & NA & NA & 322 & 14 & 2 \\
\hline 2004 & 4 & (s) & 666 & 28 & 4 & 97 & 124 & -26 & NA & NA & NA & 640 & 27 & 3 \\
\hline 2005 & 12 & (s) & 2,162 & 91 & 12 & 207 & 206 & 1 & NA & NA & NA & 2,163 & 91 & 12 \\
\hline 2006 & 32 & (s) & 5,963 & 250 & 32 & 1,069 & 828 & 242 & NA & NA & NA & 6,204 & 261 & 33 \\
\hline 2007 & 63 & 1 & 11,662 & 490 & 62 & 3,342 & 6,477 & $-3,135$ & NA & NA & NA & 8,528 & 358 & 46 \\
\hline 2008 & 88 & 1 & 16,145 & 678 & 87 & 7,502 & 16,128 & $-8,626$ & NA & NA & NA & 7,519 & 316 & 40 \\
\hline 2009 & $\mathrm{R}_{67}$ & 1 & $\mathrm{R}_{12,281}$ & R516 & $R_{66}$ & 1,844 & 6,332 & $-4,489$ & 711 & 711 & $R_{669}$ & $\mathrm{R}_{7,750}$ & $R_{326}$ & $R_{42}$ \\
\hline 2010 & R44 & 1 & $\mathrm{R}_{8,177}$ & R343 & R44 & 546 & 2,503 & $-1,958$ & ${ }^{\mathrm{R}} 672$ & R-39 & 0 & $\mathrm{R}_{6,258}$ & $R_{263}$ & R34 \\
\hline $2011^{P}$ & 125 & 2 & 23,034 & 967 & 123 & 861 & 1,740 & -879 & 1,902 & 61,240 & 0 & 20,915 & 878 & 112 \\
\hline
\end{tabular}

1 Total vegetable oil and other biomass inputs to the production of biodiesel.

${ }_{2}$ Losses and co-products from the production of biodiesel. Does not include natural gas, electricity, and other non-biomass energy used in the production of biodiesel-these are included in the industrial sector consumption statistics for the appropriate energy source.

3 Net imports equal imports minus exports.

in stocks and a positive value indicates an increase.

5 Beginning in 2009, because of incomplete data coverage and different data sources, "Balancing Item" is used to balance biodiesel supply and disposition.

${ }^{6}$ Derived from the preliminary 2010 stocks value (662 thousand barrels), not the final 2010 value (672 thousand barrels) that is shown under "Stocks."

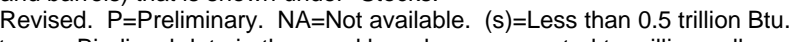

Notes: - Biodiesel data in thousand barrels are converted to million gallons by multiplying by 0.042 , and are converted to Btu by multiplying by 5.359 million Btu per barrel (the approximate heat content of ( Through 2000, data are not available. Beginning in 2001, data not from U.S. - Totals may not equal sum of Pependent rounding.

Pages: - See http://www.eia.gov/totalenergy/data/monthly/\#renewable for updated monthly and annual data. - See http://www.eia.gov/biofuels/biodiesel/production/ for related information.

Sources: Feedstock: Calculated as biodiesel production in thousand barrels multiplied by 5.433 million Btu per barrel (the biodiesel feedstock factor-see Table A3). Losses and Co-products: Calculated as biodiesel feedstock minus biodiesel production. Production: - 2001-2005-U.S. Department of Agriculture, Commodity Credit Corporation, Bioenergy Program records. Annual data are derived from quarterly data. • 2006-U.S. Department of Commerce, Bureau of the Census, "M311K - Fats and Oils:

Production, Consumption, and Stocks," data for soybean oil consumed in methyl esters (biodiesel). In addition, EIA estimates that 14.4 million gallons of yellow grease were consumed in methyl esters

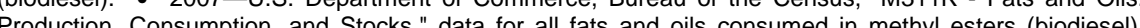
- 2008 - EIA, Monthly Biodiesel Production Report, Decemer 2009 (rease date October 2010), Table 11. 2009 forward BIA, Monthly Biodiesel Production Report (May 2012), Table 1. Trade: US.

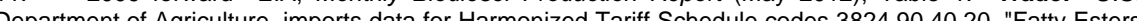

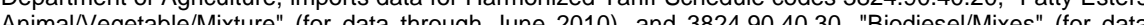
AnimalVega 2011). Althoug the (2017) biodiesel trade. EIA sees these data as good estimates. Stocks and Stock Change: 2009 and 2010-EIA Petroleum Supply Annual, annual reports, Table 1 data for renewable fuels except fuel - 2011-EIA, Petroleum Supply Monthly (PSM), monthly reports, Table 1, data for renewable fuels except fuel ethanol. Balancing Item: • 2009 forward-Calculated as biodiesel consumption and biodiesel stock change minus biodiesel production and biodiesel net imports Consumption: - 2001-2008-Calculated as biodiesel production plus biodiesel net imports. • 2009-Calculated as the sum of the monthly consumption data. Data for January and February 2009 are from EIA, PSM, monthly reports, Table 1 refinery and blender net inputs of renewable fuels except fuel ethanol. Data for March-December 2009 are calculated as biodiesel production plus biodiesel net imports minus biodiesel stock change. - 2010 and 2011-Calculated as biodiesel production plus biodiesel net imports minus biodiesel stock change. 


\section{Vehicles in Use, 1995-2010}

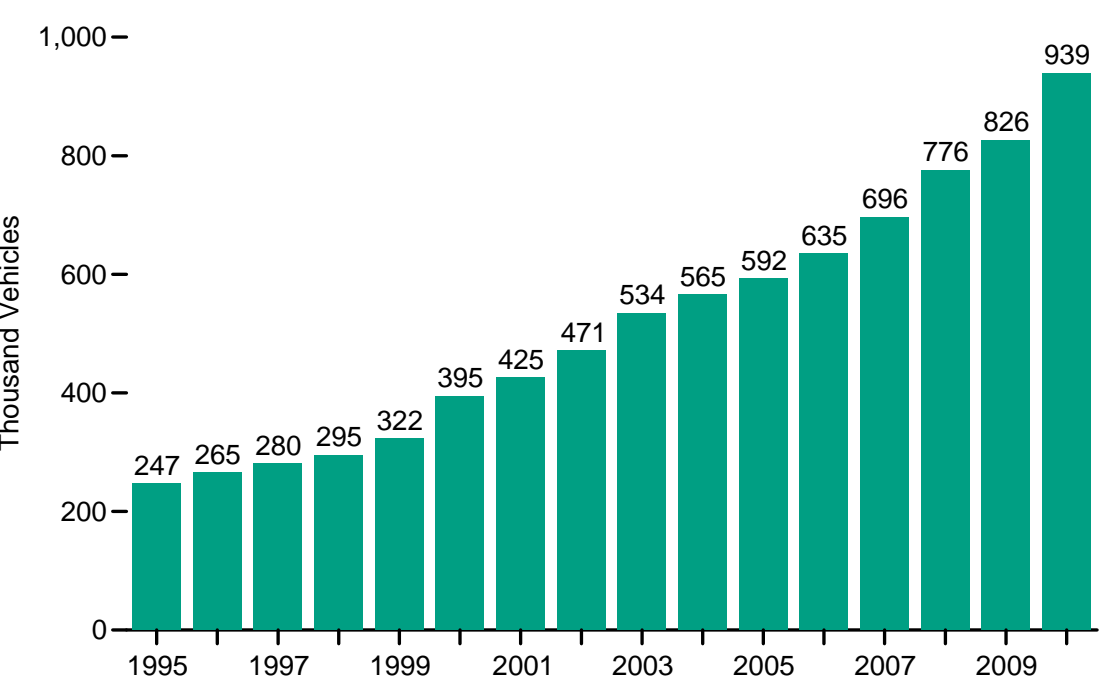

\section{Fuel Consumption, ${ }^{5}$ 1995-2010}

$500-$

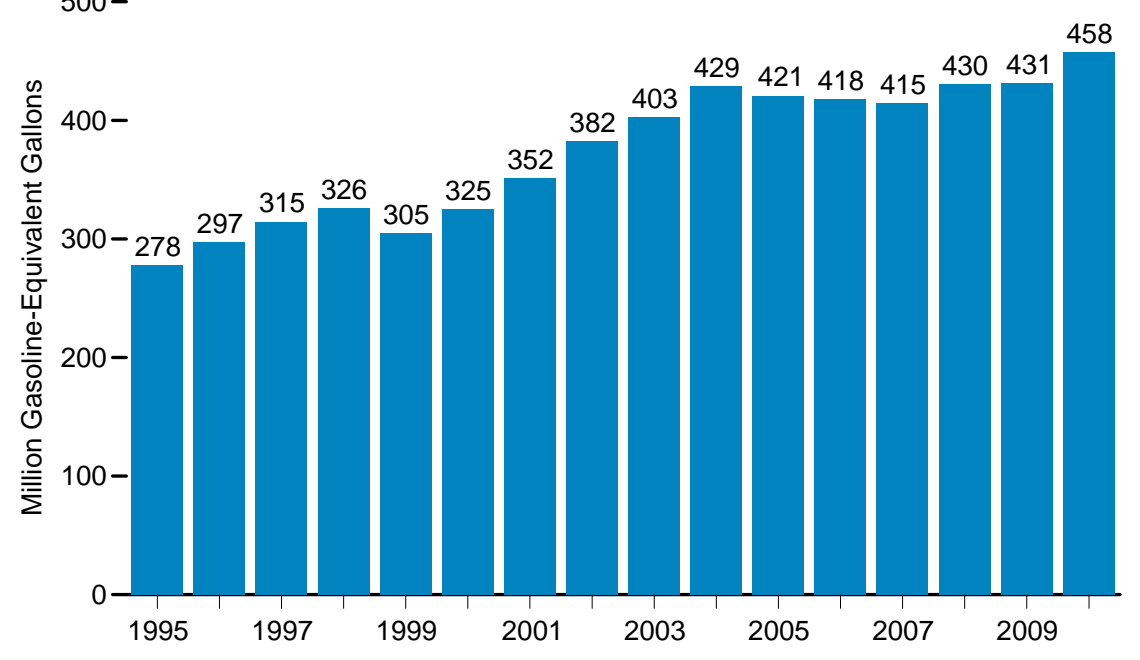

${ }^{1}$ Ethanol, 85 percent (E85). Includes only those E85 vehicles believed to be used as alternative-fueled vehicles, primarily fleet-operated vehicles; excludes other vehicles with E85fueling capability.

${ }^{2}$ Liquefied petroleum gases.

${ }^{3}$ Compressed natural gas.
Vehicles in Use by Fuel Type, 2010

$700-$

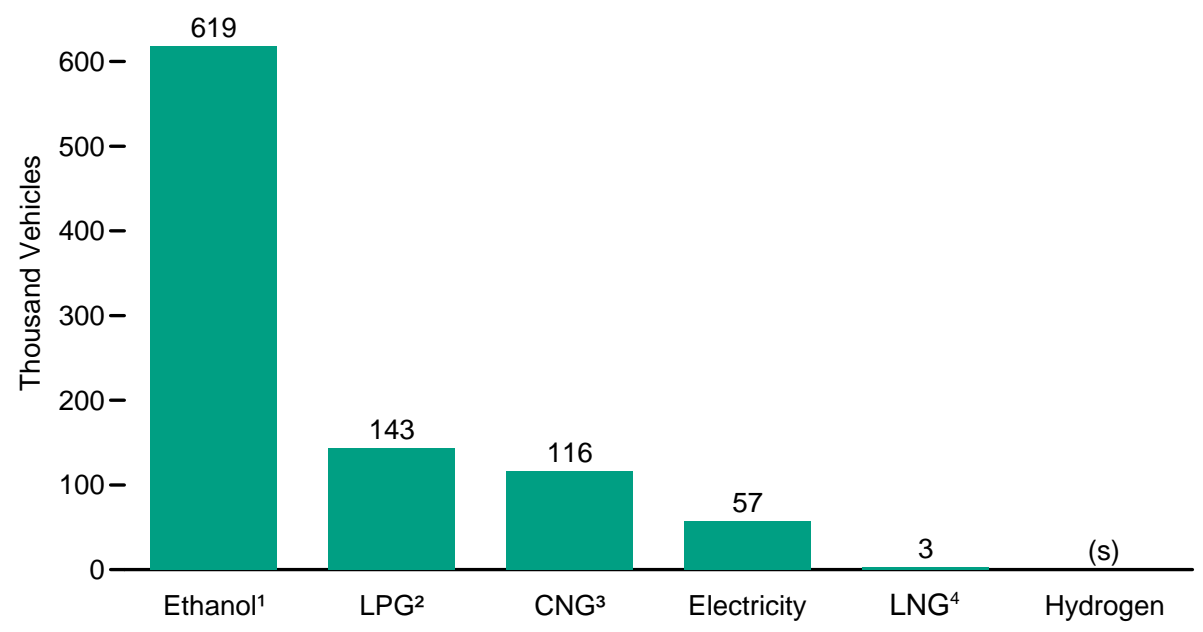

Fuel Consumption by Type, 2010

$250-$

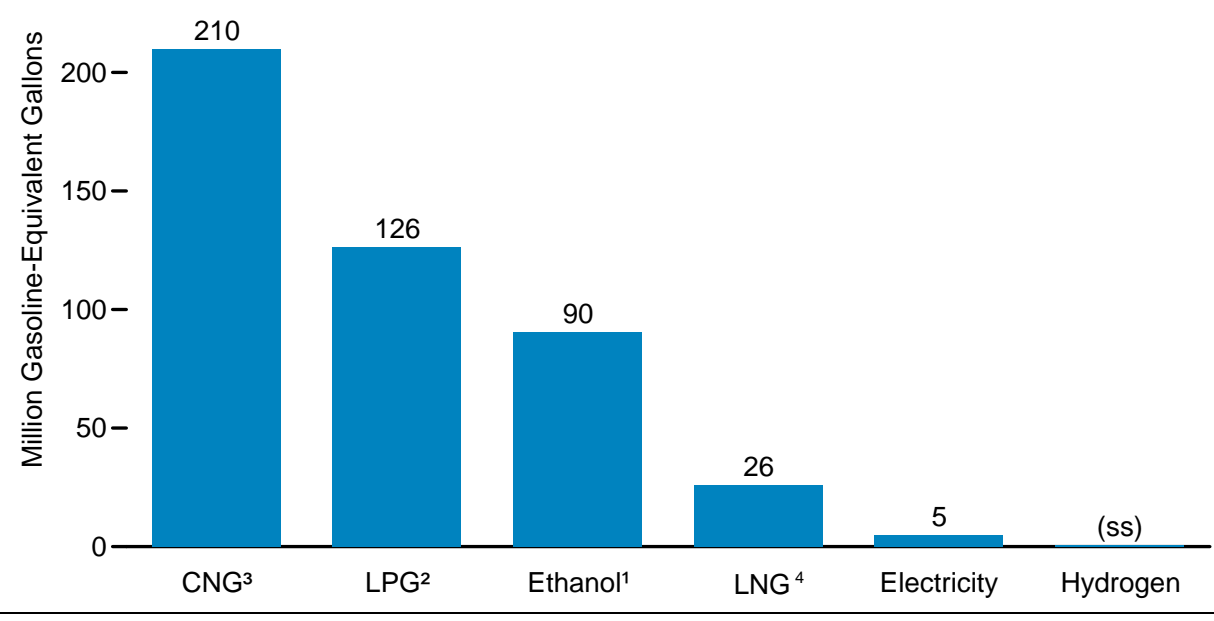

${ }^{4}$ Liquefied natural gas.

${ }^{5}$ Excludes oxygenates and biodiesel.

(s)=Fewer than 0.5 thousand vehicles.

(ss)=Less than 0.5 million gasoline-equivalent gallons.

Source: Table 10.5 . 
Table 10.5 Estimated Number of Alternative-Fueled Vehicles in Use and Fuel Consumption, 1992-2010

\begin{tabular}{|c|c|c|c|c|c|c|c|c|c|c|c|c|c|c|c|c|}
\hline \multirow[b]{3}{*}{ Year } & \multicolumn{16}{|c|}{ Alternative and Replacement Fuels ${ }^{1}$} \\
\hline & \multirow[b]{2}{*}{$\begin{array}{l}\text { Liquefied } \\
\text { Petroleum } \\
\text { Gases }\end{array}$} & \multirow[b]{2}{*}{$\underset{\text { Natural }}{\text { Gas }}$} & \multirow[b]{2}{*}{$\begin{array}{l}\text { Liquefied } \\
\text { Natural } \\
\text { Gas }\end{array}$} & \multirow[b]{2}{*}{$\begin{array}{l}\text { Methanol, } \\
85 \text { Percent } \\
\text { (M85) }^{3}\end{array}$} & \multirow[b]{2}{*}{$\begin{array}{c}\text { Methanol, } \\
\text { Neat } \\
\text { (M100) }{ }^{4}\end{array}$} & \multirow[b]{2}{*}{$\begin{array}{c}\text { Ethanol, } \\
\text { 85 Percent } \\
\text { (E85) } \text { (E), }^{3,5}\end{array}$} & \multirow[b]{2}{*}{$\begin{array}{l}\text { Ethanol, } \\
\text { 95 Percent } \\
\text { (E95) }^{3}\end{array}$} & \multirow[b]{2}{*}{$\begin{array}{l}\text { Elec- } \\
\text { tricity } 6\end{array}$} & \multirow[b]{2}{*}{$\begin{array}{l}\text { Hydro- } \\
\text { gen }\end{array}$} & \multirow[b]{2}{*}{$\begin{array}{l}\text { Other } \\
\text { Fuels }\end{array}$} & \multirow[b]{2}{*}{ Subtotal } & \multicolumn{3}{|c|}{ Oxygenates $^{2}$} & \multirow[b]{2}{*}{$\begin{array}{l}\text { Bio- } \\
\text { diesel } 10\end{array}$} & \multirow[b]{2}{*}{ Total } \\
\hline & & & & & & & & & & & & $\begin{array}{l}\text { Methyl Tertiary } \\
\text { Butyl Ether }{ }^{8}\end{array}$ & $\begin{array}{l}\text { Ethanol in } \\
\text { Gasohol } 9\end{array}$ & Total & & \\
\hline & \multicolumn{16}{|c|}{ Alternative-Fueled Vehicles in Use ${ }^{11}$ (number) } \\
\hline 1992 & NA & 23,191 & 90 & 4,850 & 404 & 172 & 38 & 1,607 & NA & NA & NA & NA & NA & NA & NA & NA \\
\hline 1993 & NA & 32,714 & 299 & 10,263 & 414 & 441 & 27 & 1,690 & NA & NA & NA & NA & NA & NA & NA & NA \\
\hline 1994 & NA & 41,227 & 484 & 15,484 & 415 & 605 & 33 & 2,224 & NA & NA & NA & NA & NA & NA & NA & NA \\
\hline 1995 & 172,806 & 50,218 & 603 & 18,319 & 386 & 1,527 & 136 & 2,860 & 0 & 0 & 246,855 & NA & NA & NA & NA & NA \\
\hline 1996 & 175,585 & 60,144 & 663 & 20,265 & 172 & 4,536 & 361 & 3,280 & 0 & 0 & 265,006 & NA & NA & NA & NA & NA \\
\hline 1997 & 175,679 & 68,571 & 813 & 21,040 & 172 & 9,130 & 347 & 4,453 & 0 & 0 & 280,205 & NA & NA & NA & NA & NA \\
\hline 1998 & 177,183 & 78,782 & 1,172 & 19,648 & 200 & 12,788 & 14 & 5,243 & 0 & 0 & 295,030 & NA & NA & NA & NA & NA \\
\hline 1999 & 178,610 & 91,267 & 1,681 & 18,964 & 198 & 24,604 & 14 & 6,964 & 0 & 0 & 322,302 & NA & NA & NA & NA & NA \\
\hline 2000 & 181,994 & 100,750 & 2,090 & 10,426 & 0 & 87,570 & 4 & 11,830 & 0 & 0 & 394,664 & NA & NA & NA & NA & NA \\
\hline 2001 & 185,053 & 111,851 & 2,576 & 7,827 & 0 & 100,303 & 0 & 17,847 & 0 & 0 & 425,457 & NA & NA & NA & NA & NA \\
\hline 2002 & 187,680 & 120,839 & 2,708 & 5,873 & 0 & 120,951 & 0 & 33,047 & 0 & 0 & 471,098 & NA & NA & NA & NA & NA \\
\hline 2003 & 190,369 & 114,406 & 2,640 & 0 & 0 & 179,090 & 0 & 47,485 & 9 & 0 & 533,999 & NA & NA & NA & NA & NA \\
\hline 2004 & 182,864 & 118,532 & 2,717 & 0 & 0 & 211,800 & 0 & 49,536 & 43 & 0 & 565,492 & NA & NA & NA & NA & NA \\
\hline 2005 & 173,795 & 117,699 & 2,748 & 0 & 0 & 246,363 & 0 & 51,398 & 119 & 3 & 592,125 & NA & NA & NA & NA & NA \\
\hline 2006 & 164,846 & 116,131 & 2,798 & 0 & 0 & 297,099 & 0 & 53,526 & 159 & 3 & 634,562 & NA & NA & NA & NA & NA \\
\hline 2007 & 158,254 & 114,391 & 2,781 & 0 & 0 & 364,384 & 0 & 55,730 & 223 & 3 & 695,766 & NA & NA & NA & NA & NA \\
\hline 200 & 151,049 & 113,973 & 3,101 & 0 & 0 & 450,327 & 0 & 56,901 & 313 & 3 & 775,667 & $\mathrm{NA}$ & $\mathrm{NA}$ & $\mathrm{NA}$ & NA & NA \\
\hline 2009 & 147,030 & 114,270 & 3,176 & 0 & 0 & 504,297 & 0 & 57,185 & 357 & 3 & 826,318 & NA & NA & NA & NA & NA \\
\hline \multirow[t]{2}{*}{2010} & 143,037 & 115,863 & 3,354 & 0 & 0 & 618,505 & 0 & 57,462 & 421 & 0 & 938,643 & NA & NA & NA & NA & NA \\
\hline & \multicolumn{16}{|c|}{ Fuel Consumption ${ }^{12}$ (thousand gasoline-equivalent gallons) } \\
\hline 1992 & NA & 17,159 & 598 & 1,121 & 2,672 & 22 & 87 & 359 & NA & NA & NA & $1,175,964$ & 719,408 & $1,895,372$ & NA & NA \\
\hline 1993 & NA & 22,035 & 1,944 & 1,671 & 3,321 & 49 & 82 & 288 & NA & NA & NA & & & & NA & NA \\
\hline 1994 & NA & 24,643 & 2,398 & 2,455 & 3,347 & 82 & 144 & 430 & NA & NA & NA & $2,020,455$ & 868,113 & $2,888,5$ & NA & NA \\
\hline 1995 & 233,178 & 35,865 & 2,821 & 2,122 & 2,255 & 195 & 1,021 & 663 & 0 & 0 & 278,121 & $2,693,407$ & 934,615 & & NA & $3,906,142$ \\
\hline 1996 & 239,648 & 47,861 & 3,320 & 1,862 & 364 & 712 & 2,770 & 773 & 0 & 0 & 297,310 & $2,751,955$ & 677,5 & $3,429,4$ & NA & $3,726,802$ \\
\hline 1997 & 238,845 & 66,495 & 3,798 & 1,630 & 364 & 1,314 & 1,166 & 1,010 & 0 & 0 & 314,621 & $3,106,745$ & 852,514 & $3,959,2$ & NA & $4,273,880$ \\
\hline 1998 & 241,881 & 73,859 & 5,463 & 1,271 & 471 & 1,772 & 61 & & 0 & 0 & 325,98 & $2,905,781$ & 912,858 & & NA & $4,144,620$ \\
\hline 1999 & 210,247 & 81,21 & 5,95 & 1,126 & 469 & 4,019 & 64 & 1,524 & 0 & 0 & 304,618 & $3,405,390$ & 975,255 & 4,380 & NA & $4,685,263$ \\
\hline 2000 & 213,012 & 88,4 & 7,423 & 614 & 0 & 12,388 & 13 & 3,058 & 0 & 0 & 324,98 & $3,298,803$ & $1,114,313$ & $4,413,1$ & 6,828 & $4,744,930$ \\
\hline 2001 & 216,319 & 106,584 & 9,12 & 461 & 0 & 15,007 & 0 & 4,0 & 0 & 0 & 351,558 & $3,354,949$ & $1,173,323$ & $4,528,2$ & 10,627 & $4,890,457$ \\
\hline 2002 & 223,600 & 3,08 & 9,59 & 354 & 0 & 18,25 & 0 & 7,274 & 0 & 0 & 382,152 & $3,122,859$ & $1,450,721$ & $4,573,5$ & 16,824 & $4,972,556$ \\
\hline 2003 & 224,697 & 3,222 & 13,50 & 0 & 0 & 26,37 & 0 & 5,141 & 2 & 0 & 402,941 & $2,368,400$ & $1,919,572$ & 4,287, & 14,082 & $4,704,995$ \\
\hline 2004 & 211,883 & 158,903 & 20,888 & 0 & 0 & 31,5 & 0 & 5,2 & 8 & 0 & 428,532 & $1,877,300$ & $2,414,167$ & $4,291,4$ & 27,616 & $4,747,615$ \\
\hline 2005 & 188,171 & 16 & 22,4 & 0 & 0 & 38,07 & 0 & 5,21 & 25 & 2 & 420,778 & $1,654,500$ & $2,756,663$ & 4,411, & 93,281 & $4,925,222$ \\
\hline 2006 & 173,130 & 172,011 & 23,4 & 0 & 0 & 44,04 & 0 & 5,10 & 41 & 2 & 417,803 & 435,000 & $3,729,168$ & $4,164,1$ & 267,623 & $4,849,594$ \\
\hline 2007 & 152,360 & 178,565 & 24,59 & 0 & 0 & 54,091 & 0 & 5,037 & 66 & 2 & 414,715 & & $4,694,304$ & $4,694,304$ & 367,764 & $5,476,783$ \\
\hline 2008 & 147,784 & 189,358 & 25,554 & 0 & 0 & 62,464 & 0 & 5,050 & 117 & 2 & 430,329 & 0 & $6,442,781$ & $6,442,7$ & 324,329 & $7,197,439$ \\
\hline 2009 & 129,631 & 199,513 & 25,652 & 0 & 0 & 71,213 & 0 & 4,956 & 140 & 2 & 431,107 & 0 & $7,343,133$ & $7,343,133$ & 325,102 & $8,099,342$ \\
\hline & 126,354 & 210,007 & 26,072 & 0 & 0 & 90,323 & 0 & 4,847 & 152 & 0 & 457,755 & & $8,527,431$ & $8,527,431$ & 235,188 & $9,220,374$ \\
\hline
\end{tabular}

1 See "Alternative Fuel" and "Replacement Fuel" in Glossary.

See "Oxygenates" in Glossary.

5 Includes only those E85 vehicles believed to be used as alternative-fuels vehicles (AFVs), primarily fleet-operated vehicles; excludes other vehicles with E85-fueling capability. In 1997, some vehicle

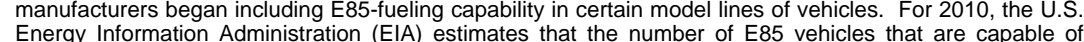
operating on E85, motor gasoline, or both, is about 10 million. Many of these AFVs are sold and used as traditional gasoline-powered vehicles.

Excludes gasoline-electric hybrids.
May include P-Series fuel or any other fuel designated by the Secretary of Energy as an alternative fuel in acordance with the Energy Policy Act of 1995

8 In addition to methyl tertiary butyl ether (MTBE), includes a very small amount of other ethers, primarily tertiary amyl methyl ether (TAME) and ethyl tertiary butyl ether (ETBE).

10 "Biodiesel" may be used as a diesel fuel substitute or diesel fuel additive or extender. See "Biodiesel" in Glossary.

11 "Vehicles in Use" data represent accumulated acquisitions, less retirements, as of the end of each calendar year; data do not include concept and demonstration vehicles that are not ready for delivery to end users. See "Alternative-Fuel Vehicle" in Glossary.

Fuel consumption quantities are expressed in a common base unit of gasoline-equivalent gallons to displacement. Gasoline equivalent is computed by dividing the gross heat content of the replacement fue by the gross heat content of gasoline (using an approximate heat content of 122,619 Btu per gallon) and multiplying the result by the replacement fuel consumption value. See "Heat Content" in Glossary.

Note: Totals may not equal sum of components due to independent rounding

Sources: - 1992-1994-Science Applications International Corporation, "Alternative Transportation Fuels and Vehicles Data Development," unpublished final report prepared for the EIA, (McLean, VA, July 1996), and U.S. Department of "Energy, Office of Energy Efficiency and Renewable Energy. Data were revised by using gross instead of net heat contens. For a table of gross and net heat contents, see EIA, Alternatives to Traditional Transportation Fuels: An Overview (June 2003 Estimated Data" (February 2004), Tables 1 and 10 . Data were revised by using gross instead of net heat contents. - 2003 forward-EIA, Alternative-Fuel Vehicle Interactive Data Viewer (see http://www.eia.gov/renewable/afv/users.cfm\#tabs_charts-2 and http://wWw.eia.gov/renewable/afv//x/s/New\%20C1\%20GEGs.xls); and "Alternatives to Traditional 
Figure 10.6 Solar Thermal Collector Shipments by Type, Price, and Trade

\section{Total Shipments, 1974-2009}

24-

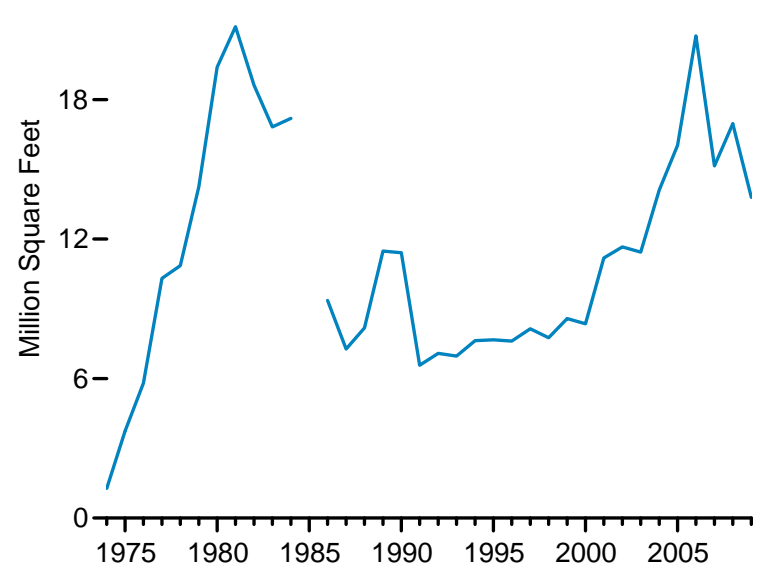

Trade, 1978-2009

$6-$

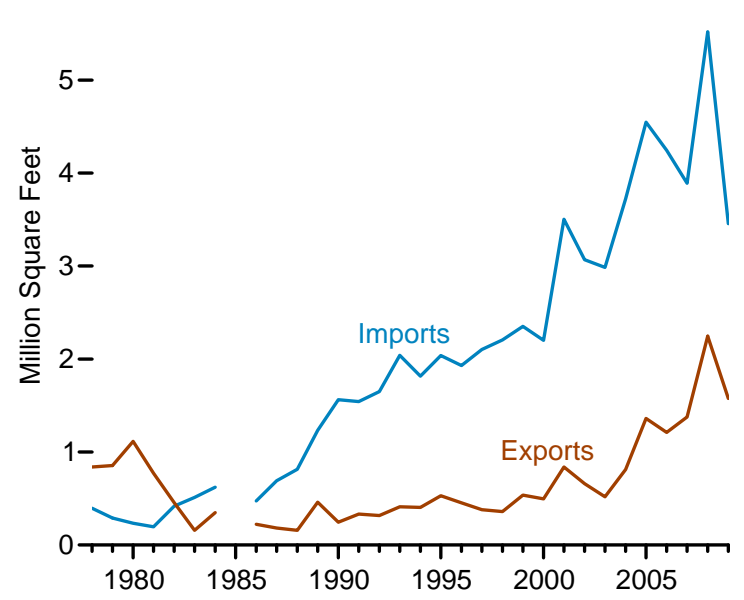

Price of Total Shipments, 1986-2009

$12-$

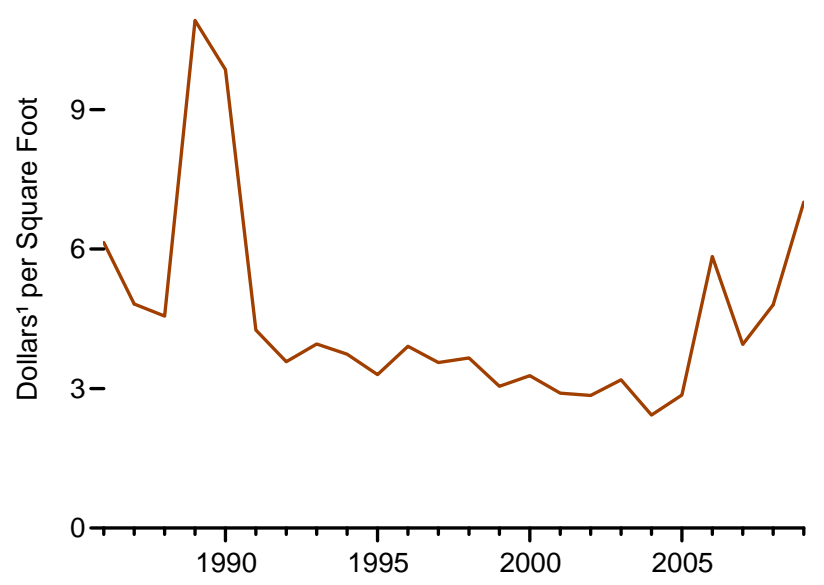

Number of U.S. Manufacturers by Type of Collector, 1974-2009

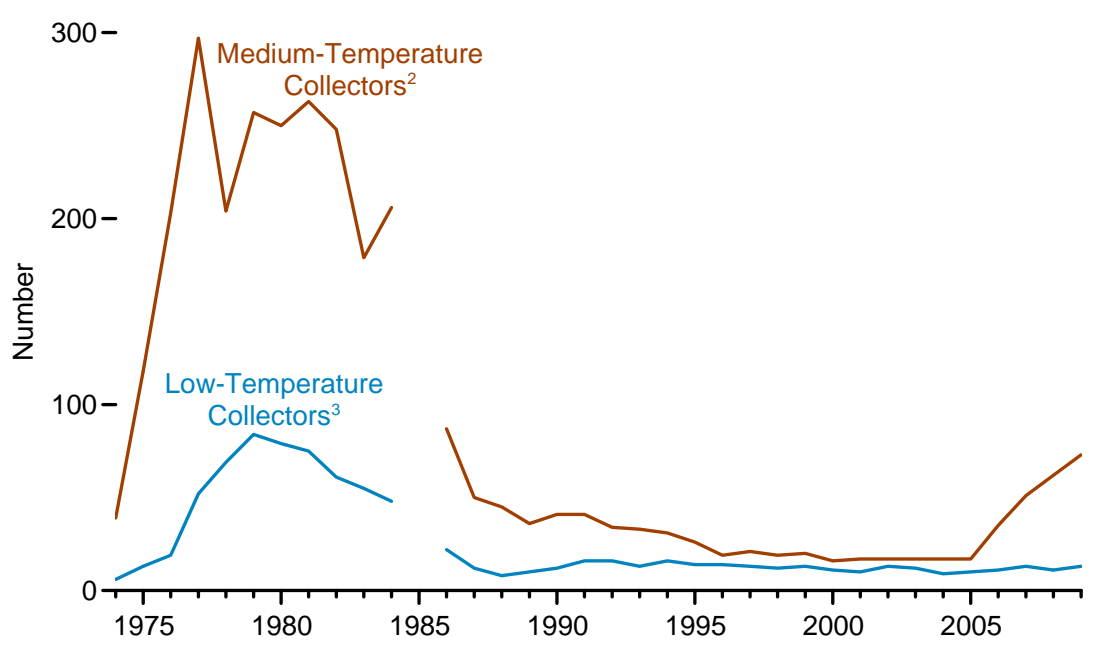

Average Annual Shipments per Manufacturer, 1974-2009

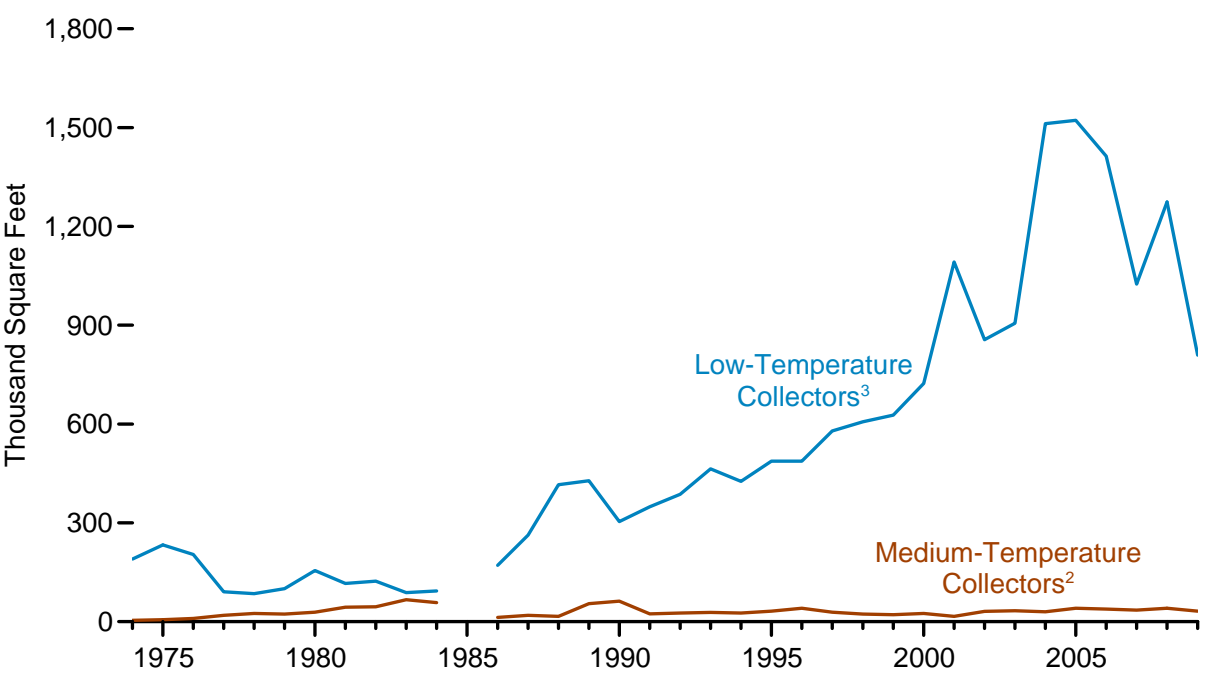

1 Prices are not adjusted for inflation. See "Nominal Dollars" in Glossary.

${ }^{2}$ Collectors that generally operate in the temperature range of 140 degrees Fahrenheit to 180 degrees Fahrenheit but can also operate at temperatures as low as 110 degrees Fahrenheit. Special collectors-evacuated tube collectors or concentrating (focusing) collectors-are included in the medium-temperature category.
${ }^{3}$ Collectors that generally operate at temperatures below 110 degrees Fahrenheit. Notes: - Shipments are for domestic and export shipments, and may include imports that subsequently were shipped to domestic or foreign customers. - Data were not collected for 1985.

Source: Table 10.6 
Table 10.6 Solar Thermal Collector Shipments by Type, Price, and Trade, 1974-2009

(Thousand Square Feet, Except as Noted)

\begin{tabular}{|c|c|c|c|c|c|c|c|c|c|c|c|c|c|c|}
\hline \multirow[b]{2}{*}{ Year } & \multicolumn{4}{|c|}{ Low-Temperature Collectors ${ }^{1}$} & \multicolumn{4}{|c|}{ Medium-Temperature Collectors ${ }^{2}$} & \multicolumn{2}{|c|}{ High-Temperature Collectors ${ }^{3}$} & \multicolumn{2}{|c|}{ Total Shipments } & \multicolumn{2}{|c|}{ Trade } \\
\hline & $\begin{array}{l}\text { Number } \\
\text { of U.S. } \\
\text { Manu- } \\
\text { facturers }\end{array}$ & $\begin{array}{l}\text { Quantity } \\
\text { Shipped }\end{array}$ & $\begin{array}{c}\text { Shipments } \\
\text { per } \\
\text { Manu- } \\
\text { facturer }\end{array}$ & $\begin{array}{c}\text { Price }^{4} \\
\text { (dollars } 5 \text { per } \\
\text { square foot) }\end{array}$ & $\begin{array}{c}\text { Number } \\
\text { of U.S. } \\
\text { Manu- } \\
\text { facturers }\end{array}$ & $\begin{array}{l}\text { Quantity } \\
\text { Shipped }\end{array}$ & $\begin{array}{c}\text { Shipments } \\
\text { per } \\
\text { Manu- } \\
\text { facturer }\end{array}$ & $\begin{array}{c}\text { Price }{ }^{4} \\
\text { (dollars } 5 \text { per } \\
\text { square foot) }\end{array}$ & $\begin{array}{l}\text { Quantity } \\
\text { Shipped }\end{array}$ & $\begin{array}{c}\text { Price } 4 \\
\text { (dollars }{ }^{5} \text { per } \\
\text { square foot) }\end{array}$ & $\begin{array}{l}\text { Quantity } \\
\text { Shipped }\end{array}$ & $\begin{array}{c}\text { Price }{ }^{4} \\
\text { (dollars } 5 \text { per } \\
\text { square foot) }\end{array}$ & Imports & Exports \\
\hline 1974 & 6 & 1,137 & 190 & NA & 39 & 137 & 4 & NA & NA & NA & 1,274 & NA & NA & NA \\
\hline 1975 & 13 & 3,026 & 233 & NA & 118 & 717 & 6 & NA & NA & NA & 3,743 & NA & NA & NA \\
\hline 1976 & 19 & 3,876 & 204 & NA & 203 & 1,925 & 10 & NA & NA & NA & 5,801 & NA & NA & NA \\
\hline 1977 & 52 & 4,743 & 91 & NA & 297 & 5,569 & 19 & NA & NA & NA & 10,312 & NA & NA & NA \\
\hline 1978 & 69 & 5,872 & 85 & NA & 204 & 4,988 & 25 & NA & NA & NA & 10,860 & NA & 396 & 840 \\
\hline 1979 & 84 & 8,394 & 100 & NA & 257 & 5,856 & 23 & NA & NA & NA & 14,251 & NA & 290 & 855 \\
\hline 1980 & 79 & 12,233 & 155 & NA & 250 & 7,165 & 29 & NA & NA & NA & 19,398 & NA & 235 & 1,115 \\
\hline 1981 & 75 & 8,677 & 116 & NA & 263 & 11,456 & 44 & NA & NA & NA & 21,133 & NA & 196 & 771 \\
\hline 1982 & 61 & 7,476 & 123 & NA & 248 & 11,145 & 45 & NA & NA & NA & 18,621 & NA & 418 & 455 \\
\hline 1983 & 55 & 4,853 & 88 & NA & 179 & 11,975 & 67 & NA & NA & NA & 16,828 & NA & 511 & 159 \\
\hline 1984 & 48 & 4,479 & 93 & NA & 206 & 11,939 & 58 & NA & 773 & NA & 17,191 & NA & 621 & 348 \\
\hline $1985^{6}$ & NA & NA & NA & NA & NA & NA & NA & NA & NA & NA & NA & NA & NA & NA \\
\hline 1986 & 22 & 3,751 & 171 & 2.30 & 87 & 1,111 & 13 & 18.30 & 4,498 & NA & 9,360 & 6.14 & 473 & 224 \\
\hline 1987 & 12 & 3,157 & 263 & 2.18 & 50 & 957 & 19 & 13.50 & 3,155 & NA & 7,269 & 4.82 & 691 & 182 \\
\hline 1988 & 8 & 3,326 & 416 & 2.24 & 45 & 732 & 16 & 14.88 & 4,116 & NA & 8,174 & 4.56 & 814 & 158 \\
\hline 1989 & 10 & 4,283 & 428 & 2.60 & 36 & 1,989 & 55 & 11.74 & 5,209 & 17.76 & 11,482 & 10.92 & 1,233 & 461 \\
\hline 1990 & 12 & 3,645 & 304 & 2.90 & 41 & 2,527 & 62 & 7.68 & 5,237 & 15.74 & 11,409 & 9.86 & 1,562 & 245 \\
\hline 1991 & 16 & 5,585 & 349 & 2.90 & 41 & 989 & 24 & 11.94 & 1 & 31.94 & 6,574 & 4.26 & 1,543 & 332 \\
\hline 1992 & 16 & 6,187 & 387 & 2.50 & 34 & 897 & 26 & 10.96 & 2 & 75.66 & 7,086 & 3.58 & 1,650 & 316 \\
\hline 1993 & 13 & 6,025 & 464 & 2.80 & 33 & 931 & 28 & 11.74 & $1 \overline{2}$ & 22.12 & 6,968 & 3.96 & 2,039 & 411 \\
\hline 1994 & 16 & 6,823 & 426 & 2.54 & 31 & 803 & 26 & 13.54 & 2 & 177.00 & 7,627 & 3.74 & 1,815 & 405 \\
\hline 1995 & 14 & 6,813 & 487 & 2.32 & 26 & 840 & 32 & 10.48 & 13 & 53.26 & 7,666 & 3.30 & 2,037 & 530 \\
\hline 1996 & 14 & 6,821 & 487 & 2.67 & 19 & 785 & 41 & 14.48 & 10 & 18.75 & 7,616 & 3.91 & 1,930 & 454 \\
\hline 1997 & 13 & 7,524 & 579 & 2.60 & 21 & 606 & 29 & 15.17 & 7 & 25.00 & 8,138 & 3.56 & 2,102 & 379 \\
\hline 1998 & 12 & 7,292 & 607 & 2.83 & 19 & 443 & 23 & 15.17 & 21 & 53.21 & 7,756 & 3.66 & 2,206 & 360 \\
\hline 1999 & 13 & 8,152 & 627 & 2.08 & 20 & 427 & 21 & 19.12 & 4 & 286.49 & 8,583 & 3.05 & 2,352 & 537 \\
\hline 2000 & 11 & 7,948 & 723 & 2.09 & 16 & 400 & 25 & W & 5 & W & 8,354 & 3.28 & 2,201 & 496 \\
\hline 2001 & 10 & 10,919 & 1,092 & 2.15 & 17 & 268 & 16 & W & 2 & W & 11,189 & 2.90 & 3,502 & 840 \\
\hline 2002 & 13 & 11,126 & 856 & 1.97 & 17 & 535 & 31 & W & 2 & W & 11,663 & 2.85 & 3,068 & 659 \\
\hline 2003 & 12 & 10,877 & 906 & 2.08 & 17 & 560 & 33 & W & 7 & W & 11,444 & 3.19 & 2,986 & 518 \\
\hline 2004 & 9 & 13,608 & 1,512 & 1.80 & 17 & 506 & 30 & 19.30 & - & -- & 14,114 & 2.43 & 3,723 & 813 \\
\hline 2005 & 10 & 15,224 & 1,522 & 2.00 & 17 & 702 & 41 & W & 115 & W & 16,041 & 2.86 & 4,546 & 1,361 \\
\hline 2006 & 11 & 15,546 & 1,413 & 1.95 & 35 & 1,346 & 38 & W & 3,852 & W & 20,744 & 5.84 & 4,244 & 1,211 \\
\hline 2007 & 13 & 13,323 & 1,025 & 1.97 & 51 & 1,797 & 35 & W & 33 & W & 15,153 & 3.95 & 3,891 & 1,376 \\
\hline 2008 & 11 & 14,015 & 1,274 & 1.89 & 62 & 2,560 & 41 & 19.57 & 388 & 11.96 & 16,963 & 4.80 & 5,517 & 2,247 \\
\hline 2009 & 13 & 10,511 & 809 & 1.94 & 73 & 2,307 & 32 & 27.32 & 980 & 25.32 & 13,798 & 7.01 & 3,456 & 1,577 \\
\hline
\end{tabular}

Low-temperature collectors are solar thermal collectors that generally operate at temperatures below $110^{\circ} \mathrm{F}$

Medium-temperature collectors are solar thermal collectors that generally operate in the temperature range of $140^{\circ} \mathrm{F}$ to $180^{\circ} \mathrm{F}$ but can also operate at temperatures as low as $110^{\circ} \mathrm{F}$. Special collectors are included in this category. Special collectors are evacuated tube collectors or concentrating (focusing) colloctors. They operate in the temperature range from just above ambientemperature (low concentration for pool heating) to several hundred degrees Fahrenheit (high concentration for air conditioning and specialized industrial processes).

High-temperature collectors are solar thermal collectors that generally operate at temperatures above $180^{\circ} \mathrm{F}$. High-temperature collector shipments are dominated by one manufacturer, and the collectors are used by the electric power sector to build new central station solar thermal power plants and generate
electricity. Year-to-year fluctations depend on how much new capacity is brought online. 4 Pric. Year-lo-year tuctallons depend on how much new capacily is brought online.

advertising and

5 Prices are not adjusted for inflation. See "Nominal Dollars" in Glossary. No data are available for 1985 .

$\mathrm{NA}=$ Not available. $\quad-$ =No data reported. $--=$ =Not applicable. W=Value withheld to avoid disclosure of proprietary company data.

Notes: - Data for this table are not available for 2010. - Shipments data are for domestic and expor shipments, and may include imports that subsequently were shipped to domestic or foreign customers. Manufacturers producing more than one type of collector are accounted for in both groups.

Web Page: For related information, see http://www.eia.gov/renewable/.

Sources: - 1974-1992-U.S. Energy Information Administration (EIA), Solar Collector Manufacturing Activity, annual reports, and Form CE-63A, "Annual Solar Thermal Collector Manufacturers Survey," and "Annul "Annual Solar The SIA T3A, "Annual Solar Thermat Collector Manufacturers Survey." warranties. Excluded are excise taxes and the cost of freight or transportation for the shipments. 
Figure 10.7 Solar Thermal Collector Domestic Shipments by Market Sector, End-Use, and Type, 2009

\section{End Use}

$16-$
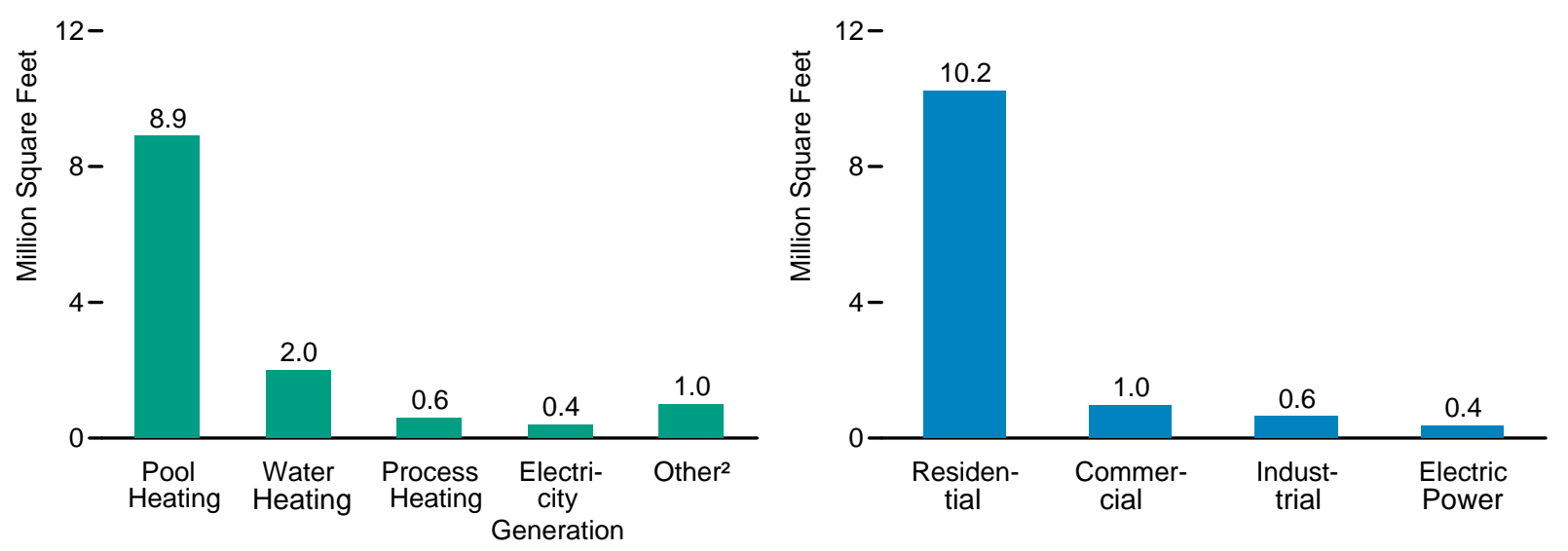

\section{ket Sector}

$16-$

tial

\section{Type of Collector}

$16-$

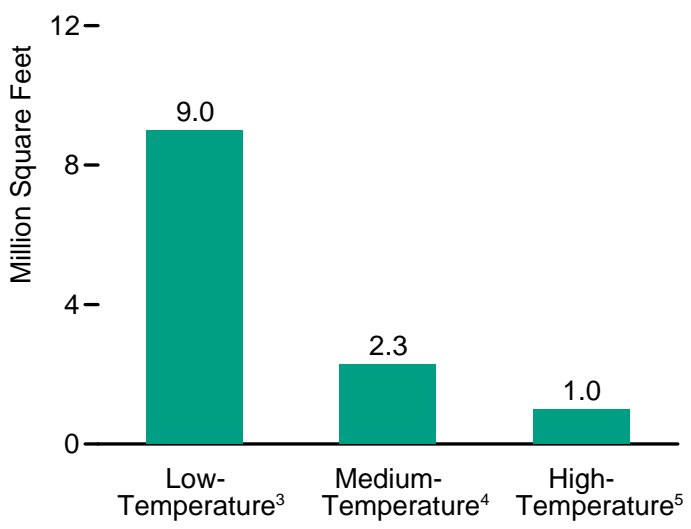

$$
\text { Low- }
$$

\section{End Use by Type of Collector}

$16-$

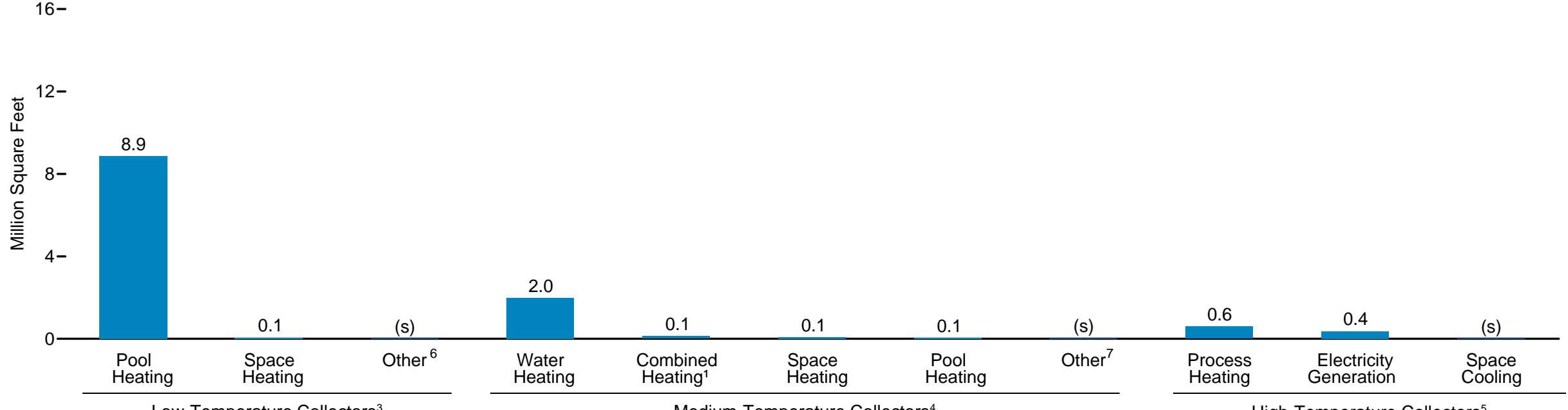

Low-Temperature Collectors ${ }^{3}$

${ }^{1}$ Combined space and water heating.

2 Space heating, combined heating, and space cooling.

${ }^{3}$ Collectors that generally operate at temperatures below 110 degrees Fahrenheit.

Medium-Temperature Collectors ${ }^{4}$

High-Temperature Collectors ${ }^{5}$

${ }^{4}$ Collectors that generally operate in the temperature range of 140 degrees Fahrenheit to 180 degrees Fahrenheit but can also operate at temperatures as low as 110 degrees

${ }^{5}$ Collectors that generally operate at temperatures above 180 degrees Fahrenheit.

${ }^{6}$ Water heating and combined heating.

${ }^{7}$ Space cooling, process heating, and electricity generation. Fahrenheit.

$(\mathrm{s})=$ Less than 0.05 million square feet. 
Table 10.7 Solar Thermal Collector Shipments by Market Sector, End Use, and Type, 2001-2009

(Thousand Square Feet)

\begin{tabular}{|c|c|c|c|c|c|c|c|c|c|c|c|c|c|}
\hline \multirow[b]{2}{*}{$\begin{array}{c}\begin{array}{c}\text { Year } \\
\text { and Type }\end{array} \\
\end{array}$} & \multicolumn{5}{|c|}{ By Market Sector } & \multicolumn{7}{|c|}{ By End Use } & \multirow[b]{2}{*}{ Total } \\
\hline & Residential & Commercial ${ }^{1}$ & Industrial ${ }^{2}$ & $\begin{array}{l}\text { Electric } \\
\text { Power }{ }^{3}\end{array}$ & Other ${ }^{4}$ & $\begin{array}{l}\text { Pool } \\
\text { Heating }\end{array}$ & $\begin{array}{l}\text { Water } \\
\text { Heating }\end{array}$ & $\begin{array}{l}\text { Space } \\
\text { Heating }\end{array}$ & $\begin{array}{c}\text { Space } \\
\text { Cooling }\end{array}$ & $\begin{array}{l}\text { Combined } \\
\text { Heating }{ }^{5}\end{array}$ & $\begin{array}{l}\text { Process } \\
\text { Heating }\end{array}$ & $\begin{array}{l}\text { Electricity } \\
\text { Generation }\end{array}$ & \\
\hline & \multicolumn{13}{|c|}{ Total Shipments ${ }^{6}$} \\
\hline $\begin{array}{l}2001 \text { Total ..... } \\
\text { Low } 7 \text {........... }\end{array}$ & $\begin{array}{r}10,125 \\
9,885\end{array}$ & $\begin{array}{r}1,012 \\
987\end{array}$ & $\begin{array}{l}17 \\
12\end{array}$ & $\begin{array}{l}1 \\
0\end{array}$ & $\begin{array}{l}35 \\
34\end{array}$ & $\begin{array}{l}\mathbf{1 0 , 7 9 7} \\
10782\end{array}$ & $\begin{array}{r}274 \\
42\end{array}$ & $\begin{array}{l}70 \\
61\end{array}$ & $\stackrel{0}{0}$ & 12 & $\begin{array}{l}34 \\
34\end{array}$ & 2 & $\begin{array}{l}11,189 \\
10919\end{array}$ \\
\hline Medium $8 \ldots . .$. & $\begin{array}{r}9,885 \\
240\end{array}$ & $\begin{array}{r}981 \\
24\end{array}$ & $\begin{array}{r}12 \\
5\end{array}$ & 0 & $\begin{array}{r}34 \\
1\end{array}$ & $\begin{array}{r}10, / 82 \\
16\end{array}$ & $\begin{array}{l}42 \\
232\end{array}$ & $\begin{array}{c}61 \\
9\end{array}$ & 0 & $\begin{array}{r}0 \\
12\end{array}$ & $\begin{array}{r}34 \\
0\end{array}$ & $\begin{array}{l}0 \\
0\end{array}$ & $\begin{array}{r}10,919 \\
268\end{array}$ \\
\hline $\operatorname{High}^{9} \ldots \ldots \ldots . . . .$. & 0 & 1 & 0 & 1 & 0 & 0 & 0 & 0 & 0 & 0 & 0 & 2 & 2 \\
\hline 2002 Total .... & 11,000 & 595 & 62 & 4 & 1 & 11,073 & 423 & 146 & (s) & 17 & 4 & 0 & 11,663 \\
\hline Medium $8 \ldots . . .$. & $\begin{array}{r}10,519 \\
481\end{array}$ & 524 & 60 & $\begin{array}{l}0 \\
4\end{array}$ & 0 & $\begin{array}{l}11,045 \\
28\end{array}$ & $\begin{array}{r}1 \\
422\end{array}$ & $\begin{array}{r}0 \\
146\end{array}$ & 0 & $\begin{array}{r}0 \\
15\end{array}$ & 0 & 0 & 11,046 \\
\hline $\begin{array}{l}\text { Medium } \\
\text { High }^{9} \ldots \ldots . . . . .\end{array}$ & & $\begin{array}{r}69 \\
2\end{array}$ & $\begin{array}{r}60 \\
0\end{array}$ & $\begin{array}{l}4 \\
0\end{array}$ & 0 & & $\begin{array}{r}422 \\
0\end{array}$ & $\begin{array}{r}146 \\
0\end{array}$ & $\begin{array}{l}\text { (S) } \\
0\end{array}$ & $\begin{array}{r}15 \\
2\end{array}$ & $\begin{array}{l}4 \\
0\end{array}$ & $\begin{array}{l}0 \\
0\end{array}$ & $\begin{array}{r}615 \\
2\end{array}$ \\
\hline 2003 Total .... & $\begin{array}{r}10,506 \\
9993\end{array}$ & $\begin{array}{l}864 \\
813\end{array}$ & 71 & $\begin{array}{l}0 \\
0\end{array}$ & 2 & $\begin{array}{l}10,800 \\
10778\end{array}$ & 511 & $\begin{array}{l}76 \\
65\end{array}$ & (s) & 23 & $\begin{array}{l}34 \\
34\end{array}$ & $\begin{array}{l}0 \\
0 \\
0\end{array}$ & $\begin{array}{l}11,444 \\
10877\end{array}$ \\
\hline Medium $8 \ldots$ & $\begin{array}{l}9,993 \\
513\end{array}$ & 44 & 0 & 0 & 2 & $\begin{array}{r}10,7 / 8 \\
22\end{array}$ & 511 & $\begin{array}{l}65 \\
11\end{array}$ & (s) & $\begin{array}{r}0 \\
16\end{array}$ & $\begin{array}{r}34 \\
0\end{array}$ & $\begin{array}{l}0 \\
0\end{array}$ & $\begin{array}{l}10,8 / 7 \\
560\end{array}$ \\
\hline High 9 .................. & & 7 & 0 & 0 & 0 & 0 & 0 & 0 & 0 & 7 & 0 & 0 & 7 \\
\hline 2004 Total .... & 12,864 & 1,178 & 70 & 0 & 3 & 13,634 & 452 & 13 & 0 & 16 & 0 & 0 & 14,115 \\
\hline Low & 12,386 & 1,178 & 44 & 0 & $\begin{array}{l}0 \\
3\end{array}$ & $\begin{array}{l}13,600 \\
33\end{array}$ & $\begin{array}{r}0 \\
452\end{array}$ & 8 & 0 & 0 & 0 & ${ }_{0}^{0}$ & 13,608 \\
\hline High $^{9} \ldots \ldots \ldots . . . . . . . . .$. & & 0 & 0 & 0 & 0 & $\begin{array}{r}30 \\
0\end{array}$ & $\begin{array}{r}452 \\
0\end{array}$ & $\begin{array}{l}5 \\
0\end{array}$ & $\begin{array}{l}0 \\
0\end{array}$ & $\begin{array}{r}16 \\
0\end{array}$ & $\begin{array}{l}0 \\
0\end{array}$ & $\begin{array}{l}0 \\
0\end{array}$ & $\begin{array}{r}506 \\
0\end{array}$ \\
\hline 2005 Total .... & 14,681 & 1,160 & 31 & 114 & 56 & 15,041 & 640 & 228 & 2 & 16 & 0 & 114 & 16.041 \\
\hline Low 7 ............... & 14,045 & 1,099 & 30 & 0 & 50 & 15,022 & 12 & 190 & 0 & 0 & 0 & 0 & 15,224 \\
\hline Medium 8 ..... & & & 1 & & 6 & & 628 & 38 & 0 & 16 & 0 & 0 & 702 \\
\hline High 9 ............... & & 2 & 0 & 114 & 0 & 0 & 0 & 0 & 2 & 0 & 0 & 114 & 115 \\
\hline 2006 Total .... & 15,123 & 1,626 & 42 & 3,845 & 107 & 15,362 & 1,136 & 330 & 3 & 66 & 0 & 3,847 & 20,744 \\
\hline $\operatorname{Low}^{7} \ldots \ldots \ldots . .$. & 13,906 & 1,500 & 40 & 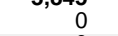 & 100 & 15,225 & 10 & 290 & 0 & 21 & 0 & 0 & 15,546 \\
\hline Medium $8 \ldots$. & 1,217 & 120 & 2 & & 7 & 137 & 1,126 & 40 & 3 & 38 & 0 & 2 & 1,346 \\
\hline \multirow[t]{2}{*}{ High $^{9} \ldots \ldots \ldots . . . . . .}$. & 0 & 7 & 0 & 3,845 & 0 & 0 & 0 & 0 & 0 & 7 & 0 & 3,845 & 3,852 \\
\hline & \multicolumn{13}{|c|}{ Domestic Shipments ${ }^{6}$} \\
\hline 2007 Total .... & 12,799 & 931 & 46 & 1 & - & 12,076 & 1,393 & 189 & 13 & 73 & 27 & 6 & 13,777 \\
\hline & 11,352 & 633 & - & 1 & - & 11,917 & 4 & 63 & - & - & - & 1 & 11,986 \\
\hline Medium 8 .... & 1,447 & 298 & 18 & - & - & 158 & 1,389 & 126 & 13 & 73 & - & 5 & 1,764 \\
\hline $\operatorname{High}^{9} \ldots \ldots \ldots . . .$. & & (s) & 27 & - & - & & (s) & - & - & - & 27 & - & \\
\hline 2008 Total .... & 13,000 & 1,294 & 128 & 294 & - & 11,973 & 1,978 & 186 & 18 & 148 & 50 & 361 & 14,716 \\
\hline $\operatorname{Low}^{7} \ldots \ldots \ldots . .$. & 10,983 & 918 & - & - & - & 11,880 & 8 & 10 & & 2 & & & 11,900 \\
\hline Medium 8 .... & 2,017 & 376 & 33 & 6 & - & 93 & 1,971 & 176 & 18 & 141 & 21 & 12 & 2,432 \\
\hline High 9 ........... & & - & 95 & 289 & - & - & - & - & - & 5 & 29 & 349 & 383 \\
\hline 2009 Total .... & 10,239 & 974 & 634 & 374 & - & 8,934 & 1,992 & 150 & 10 & 137 & 608 & 389 & 12,221 \\
\hline Low 7 .............. & 8,423 & 526 & $\begin{array}{l}11 \\
20\end{array}$ & - & - & 8,882 & 7 & 61 & - & $\begin{array}{r}9 \\
90\end{array}$ & - & $-\overline{1}$ & 8,959 \\
\hline Medium $^{8} \ldots$ & 1,816 & 439 & 29 & $\overline{374}$ & - & 52 & 1,985 & 89 & (s) & 128 & 14 & 15 & 2,284 \\
\hline High $^{9} \ldots \ldots \ldots . .$. & & 10 & 594 & 374 & - & - & - & - & 10 & - & 594 & 374 & 978 \\
\hline
\end{tabular}

1 Through 2006, data are for the commercial sector, excluding government, which is included in "Other." Beginning in 2007, data are for the commercial sector, including government.

2 Through 2006, data are for the industrial sector and independent power producers. Beginning in 2007, data are for the industrial sector only; independent power producers are included in "Electric Power." "Industrial.". Beginning in 2007, data are for electric utilities and independent power producers.
Thcluded in space applications. Beginning in 2007, data are for the transportation sector.

6 Through 2006, data are for domestic and export shipments, and may include imports that subsequently were 7 Low-temperature collectors are solar thermal collectors that generally operate at temperatures below $110^{\circ} \mathrm{F}$. 8 Medium-temperature collectors are solar thermal collectors that generally operate in the temperature
range of $140^{\circ} \mathrm{F}$ to $180^{\circ} \mathrm{F}$, but can also operate at temperatures as low as $110^{\circ} \mathrm{F}$. Special collectors are

included in this category. Special collectors are evacuated tube collectors or concentrating (focusing) collectors. They operate in the temperature range from just above ambient temperature (low concentration
for pool heating) to several hundred degrees Fahrenheit (high concentration for air conditioning and specialized industrial processes).

High-temperature collectors are solar thermal collectors that generally operate at temperatures above

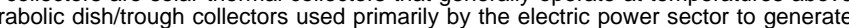
electricity for the electric grid.
$-=$ No data reported. $(\mathrm{s})=$ Less than 0.5 thousand square feet.

Notes: - Data for this table are not available for 2010. - Totals may not equal sum of components due to independent rounding.

Sources: For related information, see http://www.eia.gov/renewable/. annual reports, and Form EIA-63A, "Annual Solar Thermal Collector Manufacturers Survey." • 2003 forward-EIA, Solar Thermal Collector Manufacturing Activities (and predecessor reports), annual reports, and Form EIA-63A, "Annual Solar Thermal Collector Manufacturers Survey." 
Figure 10.8 Photovoltaic Cell and Module Shipments, Trade, and Prices

\section{Number of U.S. Companies Reporting Shipments, 1982-2010}

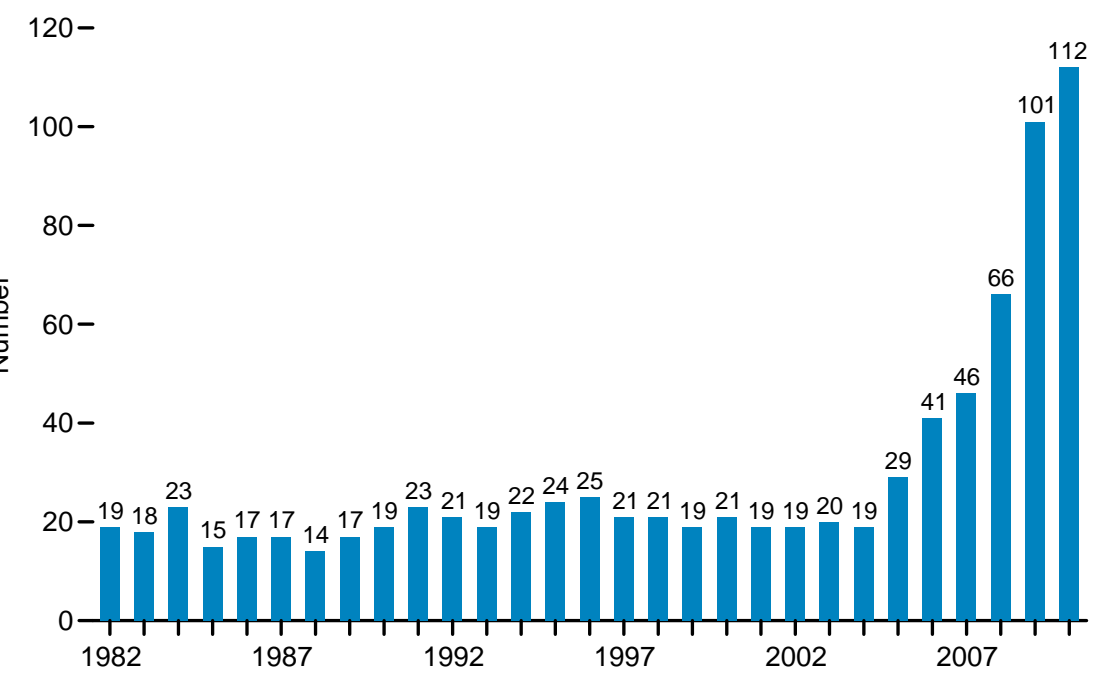

Trade, Modules Only, 1996-2010

$2,000-$

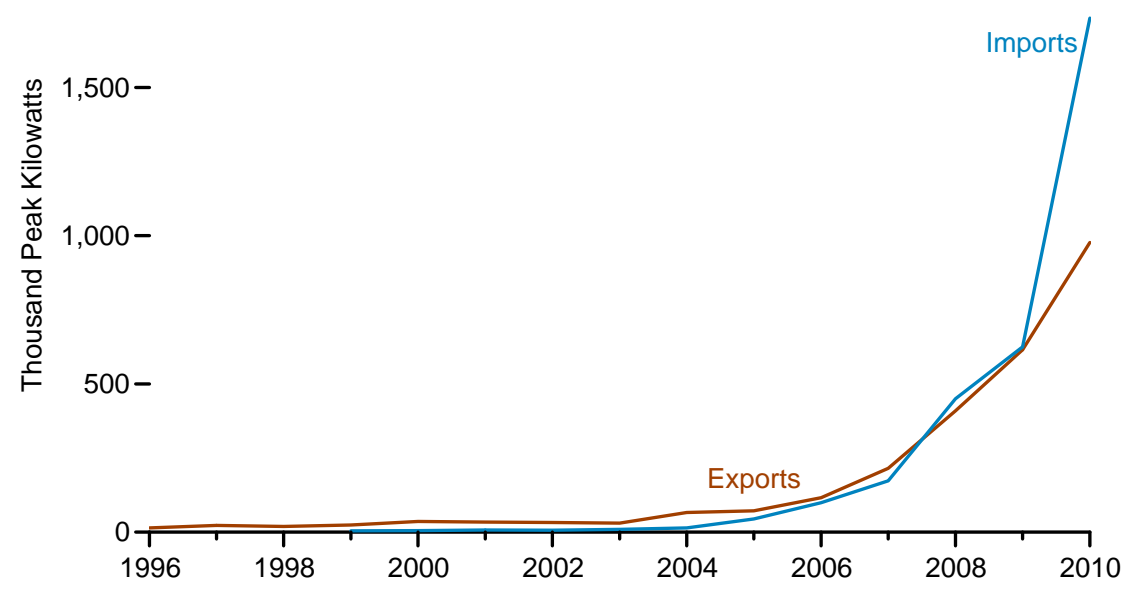

Prices are not adjusted for inflation. See "Nominal Dollars" in Glossary.

Note: Shipments are for domestic and export shipments, and may include imports that subsequently were shipped to domestic or foreign customers.

\section{Total Shipments, 1982-2010}

\section{$3,000-$}

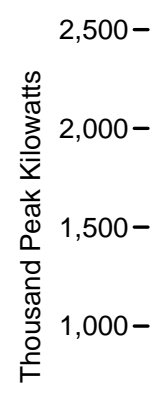

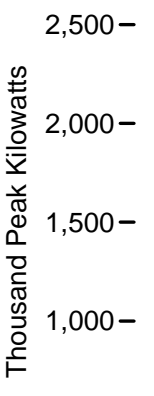

$500-$

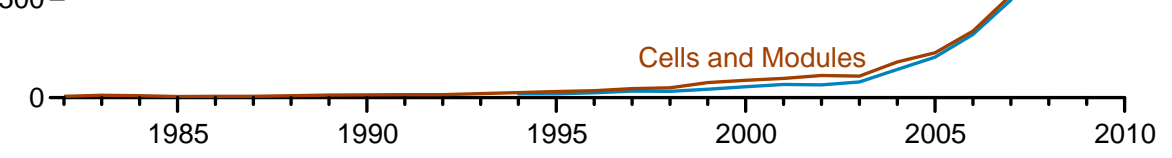

Prices, 1989-2010

$$
7-
$$

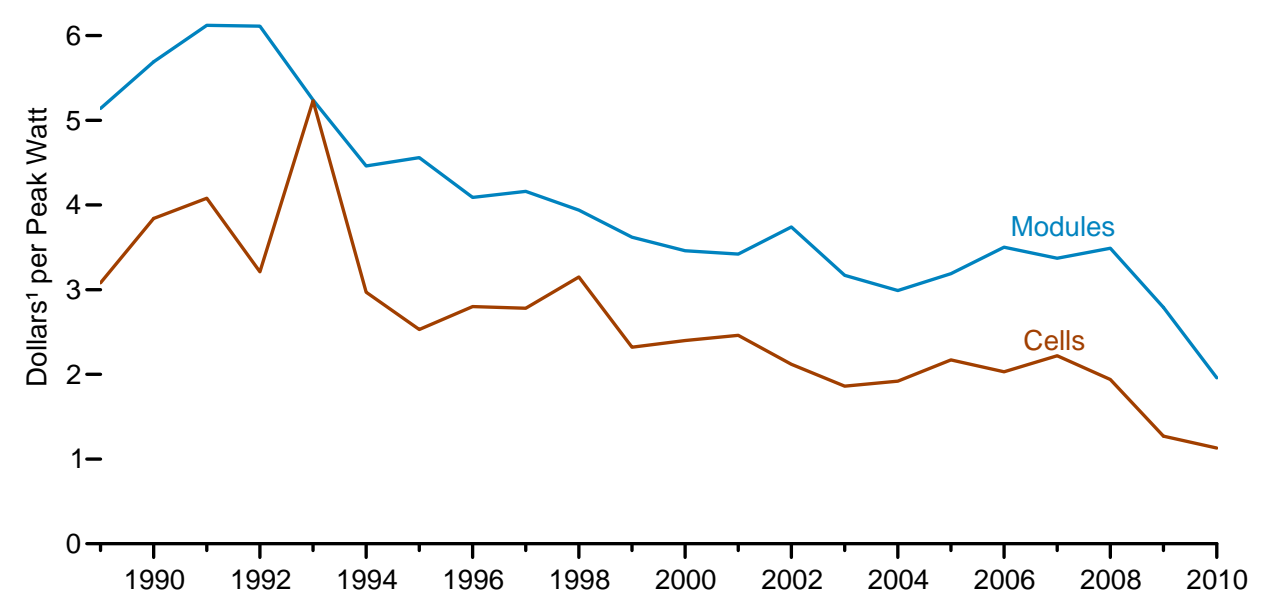

Source: Table 10.8 . 
Table 10.8 Photovoltaic Cell and Module Shipments by Type, Trade, and Prices, 1982-2010

\begin{tabular}{|c|c|c|c|c|c|c|c|c|c|c|c|c|c|}
\hline \multirow[b]{4}{*}{ Year } & \multirow{4}{*}{$\begin{array}{c}\text { U.S. } \\
\text { Companies } \\
\text { Reporting } \\
\text { Shipments } \\
\text { Number }\end{array}$} & \multicolumn{6}{|c|}{ Shipments } & \multicolumn{4}{|c|}{ Trade } & \multicolumn{2}{|c|}{ Prices 1} \\
\hline & & \multicolumn{2}{|c|}{ Crystalline Silicon } & \multicolumn{2}{|c|}{ Thin-Film } & \multicolumn{2}{|c|}{ Total $^{2}$} & \multicolumn{2}{|c|}{ Imports } & \multicolumn{2}{|c|}{ Exports } & \multirow[b]{2}{*}{ Cells } & \multirow[b]{2}{*}{ Modules } \\
\hline & & $\begin{array}{l}\text { Cells } \\
\text { and Modules }\end{array}$ & $\begin{array}{l}\text { Modules } \\
\text { Only }\end{array}$ & $\begin{array}{l}\text { Cells } \\
\text { and Modules }\end{array}$ & $\begin{array}{l}\text { Modules } \\
\text { Only }\end{array}$ & $\begin{array}{l}\text { Cells } \\
\text { and Modules }\end{array}$ & $\begin{array}{l}\text { Modules } \\
\text { Only }\end{array}$ & $\begin{array}{l}\text { Cells } \\
\text { and Modules }\end{array}$ & $\begin{array}{c}\text { Modules } \\
\text { Only }\end{array}$ & $\begin{array}{l}\text { Cells } \\
\text { and Modules }\end{array}$ & $\begin{array}{l}\text { Modules } \\
\text { Only }\end{array}$ & & \\
\hline & & \multicolumn{10}{|c|}{ Peak Kilowatts ${ }^{3}$} & \multicolumn{2}{|c|}{ Dollars ${ }^{4}$ per Peak Watt ${ }^{3}$} \\
\hline 1982 & 19 & NA & NA & NA & NA & 6,897 & NA & NA & NA & NA & NA & NA & NA \\
\hline 1983 & 18 & NA & NA & NA & NA & 12,620 & NA & NA & NA & 1,903 & NA & NA & NA \\
\hline 1984 & 23 & NA & NA & NA & NA & 9,912 & NA & NA & NA & 2,153 & NA & NA & NA \\
\hline 1985 & 15 & 5,461 & NA & 303 & NA & 5,769 & NA & 285 & NA & 1,670 & NA & NA & NA \\
\hline 1986 & 17 & 5,806 & NA & 516 & NA & 6,333 & NA & 678 & NA & 3,109 & NA & NA & NA \\
\hline 1987 & 17 & 5,613 & NA & 1,230 & NA & 6,850 & NA & 921 & NA & 3,821 & NA & NA & NA \\
\hline 1988 & 14 & 7,364 & NA & 1,895 & NA & 9,676 & NA & 1,453 & NA & 5,358 & NA & NA & NA \\
\hline 1989 & 17 & 10,747 & NA & 1,628 & NA & 12,825 & NA & 826 & NA & 7,363 & NA & 3.08 & 5.14 \\
\hline 1990 & ${ }^{5} 19$ & 12,492 & NA & 1,321 & NA & 513,837 & NA & 1,398 & NA & 7,544 & NA & 3.84 & 5.69 \\
\hline 1991 & 23 & 14,205 & NA & 723 & NA & 14,939 & NA & 2,059 & NA & 8,905 & NA & 4.08 & 6.12 \\
\hline 1992 & 21 & 14,457 & NA & 1,075 & NA & 15,583 & NA & 1,602 & NA & 9,823 & NA & 3.21 & 6.11 \\
\hline 1993 & 19 & 20,146 & NA & 782 & NA & 20,951 & NA & 1,767 & NA & 14,814 & NA & 5.23 & 5.24 \\
\hline 1994 & 22 & 24,785 & NA & 1,061 & NA & 26,077 & 19,064 & 1,960 & NA & 17,714 & NA & 2.97 & 4.46 \\
\hline 1995 & 24 & 29,740 & NA & 1,266 & NA & 31,059 & 19,627 & 1,337 & NA & 19,871 & NA & 2.53 & 4.56 \\
\hline 1996 & 25 & 33,996 & NA & 1,445 & NA & 35,464 & 24,534 & 1,864 & NA & 22,448 & 14,128 & 2.80 & 4.09 \\
\hline 1997 & 21 & 44,314 & NA & 1,886 & NA & 46,354 & 33,645 & 1,853 & NA & 33,793 & 22,956 & 2.78 & 4.16 \\
\hline 1998 & 21 & 47,186 & NA & 3,318 & NA & 50,562 & 32,313 & 1,931 & NA & 35,493 & 19,015 & 3.15 & 3.94 \\
\hline 1999 & 19 & 73,461 & NA & 3,269 & NA & 76,787 & 43,073 & 4,784 & 4,630 & 55,585 & 24,545 & 2.32 & 3.62 \\
\hline 2000 & 21 & 85,155 & NA & 2,736 & NA & 88,221 & 55,007 & 8,821 & 5,016 & 68,382 & 36,277 & 2.40 & 3.46 \\
\hline 2001 & 19 & 84,651 & NA & 12,541 & NA & 97,666 & 67,033 & 10,204 & 7,029 & 61,356 & 34,282 & 2.46 & 3.42 \\
\hline 2002 & 19 & 104,123 & NA & 7,396 & NA & 112,090 & 64,413 & 7,297 & 6,378 & 66,778 & 32,559 & 2.12 & 3.74 \\
\hline 2003 & 20 & 97,940 & NA & 10,966 & NA & 109,357 & 80,062 & 9,731 & 9,289 & 60,693 & 30,229 & 1.86 & 3.17 \\
\hline 2004 & 19 & 159,138 & NA & 21,978 & NA & 181,116 & 143,274 & 47,703 & 14,096 & 102,770 & 66,278 & 1.92 & 2.99 \\
\hline 2005 & 29 & 172,965 & NA & 53,826 & NA & 226,916 & 204,996 & 90,981 & 44,443 & 92,451 & 72,017 & 2.17 & 3.19 \\
\hline 2006 & 41 & 233,518 & NA & 101,766 & NA & 337,268 & 320,208 & 173,977 & 99,687 & 130,757 & 116,561 & 2.03 & 3.50 \\
\hline 2007 & 46 & 310,330 & NA & 202,519 & NA & 517,684 & 494,148 & 238,018 & 173,165 & 237,209 & 215,364 & 2.22 & 3.37 \\
\hline 2008 & 66 & 665,795 & NA & 293,182 & NA & 986,504 & 920,693 & 586,558 & 449,813 & 462,252 & 409,261 & 1.94 & 3.49 \\
\hline 2009 & 101 & 984,161 & NA & 266,547 & NA & $1,282,560$ & $1,188,879$ & 743,414 & 625,182 & 681,427 & 615,094 & 1.27 & 2.79 \\
\hline 2010 & 112 & $\left({ }^{6}\right)$ & $2,114,881$ & $\left({ }^{6}\right)$ & 519,516 & $\left({ }^{6}\right)$ & $2,644,498$ & $\left({ }^{6}\right)$ & $1,734,149$ & $\left({ }^{6}\right)$ & 976,955 & 1.13 & 1.96 \\
\hline
\end{tabular}

1 Prices equal shipment value divided by quantity shipped. Value includes charges for advertising and warranties. Excluded are excise taxes and the cost of freight or transportation for the shipments.

${ }^{2}$ Includes all types of photovoltaic cells and modules (single-crystal silicon, cast silicon, ribbon silicon, thin-film silicon, and concentrator silicon). Excludes cells and modules for space and satellite applications.

4 See "Peak Kilowatt" and "Peak Watt" in Glossary.

" cannot be summed.

NA=Not available.

Note: Shipments data are for domestic and export shipments, and may include imports that subsequently were shipped to domestic or foreign customers.

Web Page: For related information, see http://www.eia.gov/renewable/.

Sources: - 1982-1992-U.S. Energy Information Administration (EIA), Solar Collector Manufacturing Activity, annual reports. - 1993-2002-EIA, Renewable Energy Annual, annual reports. - 2003 forward-EIA, Solar Photovoltaic Cell/Module Shipments Report (and predecessor reports), annual reports. 


\section{By End Use}

Grid-Connected ${ }^{1}$

$1,500-$

$1,408.5$

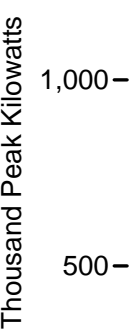

243.9

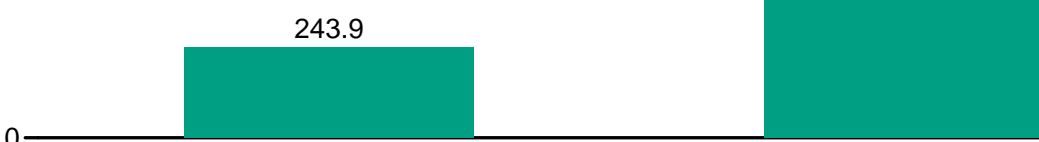

Centralized $^{2}$

Distributed $^{3}$

10.9

4.2

Domestic

Non-Domestic ${ }^{5}$

\section{By Sector}

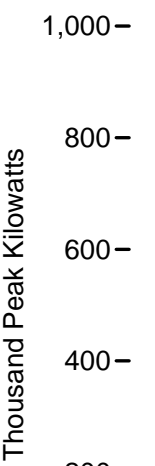

908.2

$200-$
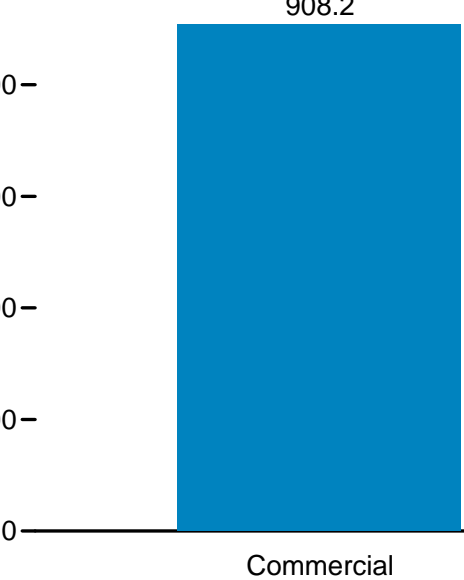

Residential

467.2

${ }^{1}$ See "Electric Power Grid" in Glossary.

2 Photovoltaic modules that are connected to the electric power grid, and whose output is fed directly into the grid.

${ }^{3}$ Photovoltaic modules that are connected to the electric power grid, and whose output is consumed mainly onsite.

${ }^{4}$ Photovoltaic modules that are not connected to the electric power grid, and that are used to provide electric power to remote households or communities.

\section{2}

Industrial $^{7}$

Photovoltaic modules that are not connected to the electric power grid, and that are used to provide electric power for a variety of non-domestic applications.

${ }^{6}$ Electric utilities and independent power producers.

${ }^{7}$ Industrial sector only; independent power producers are included in "Electric Power."

Source: Table 10.9 
Table 10.9 Photovoltaic Cell and Module Shipments by Sector and End Use, 1989-2010

(Peak Kilowatts ${ }^{1}$ )

\begin{tabular}{|c|c|c|c|c|c|c|c|c|c|c|}
\hline \multirow[b]{3}{*}{ Year } & \multicolumn{5}{|c|}{ By Sector } & \multicolumn{4}{|c|}{ By End Use } & \multirow[b]{3}{*}{ Total } \\
\hline & \multirow[b]{2}{*}{ Residential } & \multirow[b]{2}{*}{ Commercial $^{3}$} & \multirow[b]{2}{*}{ Industrial 4} & \multirow{2}{*}{$\begin{array}{l}\text { Electric } \\
\text { Power } 5\end{array}$} & \multirow[b]{2}{*}{ Other ${ }^{6}$} & \multicolumn{2}{|c|}{ Grid-Connected $^{2}$} & \multicolumn{2}{|c|}{ Off-Grid ${ }^{2}$} & \\
\hline & & & & & & Centralized $^{7}$ & Distributed $^{8}$ & Domestic ${ }^{9}$ & Non-Domestic ${ }^{10}$ & \\
\hline & \multicolumn{10}{|c|}{ Total Shipments of Photovoltaic Cells and Modules ${ }^{11}$} \\
\hline 1989 & 1,439 & $\mathrm{R}_{6,057}$ & 3,993 & 785 & 551 & $\left({ }^{12}\right)$ & 121,251 & 2,620 & 8,954 & 12,825 \\
\hline 1990 & 1,701 & $\mathrm{R}_{8,062}$ & 2,817 & 826 & 432 & $(12)$ & 12469 & 3,097 & 10,271 & 13,837 \\
\hline 1991 & 3,624 & $\mathrm{R}_{5,715}$ & 3,947 & 1,275 & 377 & $\left({ }^{12}\right)$ & ${ }^{12} 856$ & 3,594 & 10,489 & 14,939 \\
\hline 1992 & 4,154 & $R_{5,122}$ & 4,279 & 1,553 & 477 & $\left({ }^{12}\right)$ & 121,227 & 4,238 & 10,118 & 15,583 \\
\hline 1993 & 5,237 & $R_{8,004}$ & 5,352 & 1,503 & 856 & $\left({ }^{12}\right)$ & 121,096 & 5,761 & 14,094 & 20,951 \\
\hline 1994 & 6,632 & $\mathrm{R} 9,717$ & 6,855 & 2,364 & 510 & $(12)$ & 122,296 & 9,253 & 14,528 & 26,077 \\
\hline 1995 & 6,272 & $R_{12,483}$ & 7,198 & 3,759 & 1,347 & $\left({ }^{12}\right)$ & 124,585 & 8,233 & 18,241 & 31,059 \\
\hline 1996 & 8,475 & $\mathrm{R}_{12,297}$ & 8,300 & 4,753 & 1,639 & $(12)$ & 124,844 & 10,884 & 19,736 & 35,464 \\
\hline 1997 & 10,993 & $R_{15,594}$ & 11,748 & 5,651 & 2,367 & $\left({ }^{12}\right)$ & ${ }^{12} 8,273$ & 8,630 & 29,451 & 46,354 \\
\hline 1998 & 15,936 & $\mathrm{R}_{14,708}$ & 13,232 & 3,965 & 2,720 & $(12)$ & 1214,193 & 8,634 & 27,735 & 50,562 \\
\hline 1999 & 19,817 & $\mathrm{R}_{24,731}$ & 24,972 & 5,876 & 1,392 & $\left({ }^{12}\right)$ & 1224,782 & 10,829 & 41,176 & 76,787 \\
\hline 2000 & 24,814 & $R_{23,611}$ & 28,808 & 6,298 & 4,690 & $\left({ }^{12}\right)$ & 1221,713 & 14,997 & 51,511 & 88,221 \\
\hline 2001 & 33,262 & $R_{29,924}$ & 28,063 & 5,846 & 571 & $\left({ }^{12}\right)$ & 1227,226 & 21,447 & 48,993 & 97,666 \\
\hline 2002 & 29,315 & R 42,075 & 32,218 & 7,640 & 841 & $(12)$ & 1233,983 & 21,693 & 56,414 & 112,090 \\
\hline 2003 & 23,389 & $\mathrm{R}_{49,231}$ & 27,951 & 8,474 & 313 & $\left({ }^{12}\right)$ & ${ }^{12} 42,485$ & 15,025 & 51,847 & 109,357 \\
\hline 2004 & 53,928 & R79,146 & 30,493 & 3,233 & 14,316 & $(12)$ & 12129,265 & 18,371 & 33,480 & 181,116 \\
\hline 2005 & 75,040 & $R_{119,763}$ & 22,199 & 143 & 9,772 & $(12)$ & 12168,474 & 24,958 & 33,484 & 226,916 \\
\hline \multirow[t]{2}{*}{2006} & 95,815 & $\mathrm{R}_{190,998}$ & 28,618 & 3,981 & 17,857 & $\left({ }^{12}\right)$ & ${ }^{12} 274,197$ & 18,003 & 45,068 & 337,268 \\
\hline & \multicolumn{10}{|c|}{ U.S. Shipments of Photovoltaic Cells and Modules ${ }^{11}$} \\
\hline 2007 & 68,417 & $R_{144,061}$ & 32,702 & 35,294 & -- & $(12)$ & 12253,101 & 10,867 & 16,507 & 280,475 \\
\hline 2008 & 173,989 & $R_{262,952}$ & 51,493 & 35,819 & -- & $(12)$ & 12500,854 & 15,527 & 7,871 & 524,252 \\
\hline \multirow[t]{2}{*}{2009} & 221,245 & $\mathrm{R}_{282,807}$ & 43,445 & 53,636 & -- & $\left({ }^{12}\right)$ & ${ }^{12} 585,189$ & 8,119 & 7,825 & 601,133 \\
\hline & \multicolumn{10}{|c|}{ U.S. Shipments of Photovoltaic Modules Only 11} \\
\hline 2010 & 467,165 & 908,224 & 48,208 & 243,947 & -- & 243,947 & $1,408,462$ & 10,941 & 4,193 & $1,667,543$ \\
\hline
\end{tabular}

1 See "Peak Kilowatt" in Glossary.
2 See "Electric Power Grid" in Glossa

3 Includes data that were previously shown in the "Commercial," "Government," and "Transportation" sector categories.

4 Through 2006, data are for the industrial sector and independent power producers. Beginning in 2007, data are for the industrial sector only; independent power producers are included in "Electric Power." 5 Through 2006, data are for electric utilities only; independent power producers are included in "Industrial." Beginning in 2007, data are for electric utilities and independent power producers.

6 Through 2006, data are for specialty purposes such as research.

7 Photovoltaic cells/modules that are connected to the electric power grid, and whose output is fed directly into the grid.

consumed mainly onsite. provide electric power to remote households or communities. the "Remote" end-use category.

10 Photovoltaic cells/modules that are not connected to the electric power grid, and that are used to provide electric power for a variety of non-domestic applications. Includes data that were previously shown in the following end-use categories: "Communications," "Consumer Goods," "Health," "Original Equipment Manufacturers" (non-photovoltaic manufacturers that combine photovoltaic technology into existing newly developed product lines), "Transportation," "Water Pumping," and "Other" (applications such as cooking food, desalinization, and distilling).

11 Through 2006, data are for domestic and export shipments of photovoltaic cells and modules, and may include imports that subsequently were shipped to domestic or foreign customers. For 2007-2009, data are for domestic shipments of photovoltaic cells and modules. For 2010, data are for domestic shipments of photovoltaic modules only.

12 Through 2009, data for "Centralized" are included in "Distributed."

R=Revised $---=$ Not applicable.

Notes: • See "Photovoltaic Cell (PVC)" and "Photovoltaic Module" in Glossary. • Totals may not equal sum of components due to independent rounding.

Web Page: For related information, see http://www.eia.gov/fuelrenewable.html.

Sources: - 1989-1992-U.S. Energy Information Administration (EIA), Solar Collector Manufacturing Activity, annual reports. - 1993-2002-EIA, Renewable Energy Annual, annual reports. - 2003 forward-EIA, Solar Photovoltaic Cell/Module Shipments Report (and predecessor reports), annual reports. 


\section{Renewable Energy}

Note. Renewable Energy Production and Consumption. In Tables 1.1, 1.3, and 10.1, renewable energy consumption consists of: conventional hydroelectricity net generation (converted to Btu using the fossil-fuels heat rate-see Table A6); geothermal electricity net generation (converted to Btu using the fossil-fuels heat rate-see Table A6), and geothermal heat pump and geothermal direct use energy; solar thermal and photovoltaic electricity net generation (converted to Btu using the fossil-fuels heat rate- see Table A6), and solar thermal direct use energy; wind electricity net generation (converted to Btu using the fossil-fuels heat rate-see Table A6); wood and woodderived fuels consumption; biomass waste (municipal solid waste from biogenic sources, landfill gas, sludge waste, agricultural byproducts, and other biomass) consumption; fuel ethanol (minus denaturant) and biodiesel consumption; and losses and co-products from the production of fuel ethanol and biodiesel. In Tables 1.1, 1.2, and 10.1, renewable energy production is assumed to equal consumption for all renewable energy sources except biofuels (biofuels production comprises biomass inputs to the production of fuel ethanol and biodiesel).

\section{Table 10.2a Sources}

Residential Sector, Geothermal: Oregon Institute of Technology, Geo-Heat Center. Residential Sector, Solar/PV: • 1989-2009: U.S. Energy Information Administration (EIA) estimates based on Form EIA-63A, “Annual Solar Thermal Collector Manufacturers Survey,” and Form EIA-63B, “Annual Photovoltaic Cell/Module Shipments Report.” • 2010 and 2011: EIA estimates based on Form EIA-63B, “Annual Photovoltaic Cell/Module Shipments Report”; Form EIA-63A, “Annual Solar Thermal Collector Manufacturers Survey” (pre-2010 data); and SEIA/GTM Research, U.S. Solar Market Insight: 2010 Year in Review.

Residential Sector, Wood: • 1949-1979: EIA, Estimates of U.S. Wood Energy Consumption from 1949 to 1981, Table A2. • 1980 forward: EIA, Form EIA-457, "Residential Energy Consumption Survey"; and EIA estimates based on Form EIA457 and regional heating degree-day data.

Commercial Sector, Hydroelectric Power: EIA, Annual Energy Review (AER), Tables 8.2d and A6.

Commercial Sector, Geothermal: Oregon Institute of Technology, Geo-Heat Center. Commercial Sector, Solar/PV: EIA, AER, Tables 8.2d and A6.

Commercial Sector, Wind: 2009 forward: EIA, AER, Tables 8.2d and A6.

Commercial Sector, Wood: • 1949-1979: EIA, Estimates of U.S. Wood Energy Consumption from 1949 to 1981, Table A2. • 1980-1983: EIA, Estimates of U.S. Wood Energy Consumption 1980-1983, Table ES1. • 1984: EIA estimate based on the 1983 value. • 1985-1988: Values interpolated. • 1989 forward: EIA, AER, Table 8.7c; and EIA estimates based on Form EIA-871, "Commercial Buildings Energy Consumption Survey."

Commercial Sector, Biomass Waste: EIA, AER, Table 8.7c.
Commercial Sector, Fuel Ethanol (Minus Denaturant): EIA, AER, Tables 5.11, 5.13a, and 10.3. Calculated as commercial sector motor gasoline consumption (Table 5.13a) divided by total motor gasoline product supplied (Table 5.11), and then multiplied by fuel ethanol (minus denaturant) consumption (Table 10.3).

\section{Table 10.2b Sources}

Industrial Sector, Hydroelectric Power: • 1949-1988: U.S. Energy Information Administration (EIA), Annual Energy Review (AER), Tables 8.1 and A6. • 1989 forward: EIA, AER, Tables 8.2d and A6.

Industrial Sector, Geothermal: Oregon Institute of Technology, Geo-Heat Center. Industrial Sector, Solar/PV: 2010 and 2011: EIA, AER, Tables 8.2d and A6.

Industrial Sector, Wind: 2011: EIA, AER, Tables 8.2d and A6.

Industrial Sector, Wood: - 1949-1979: EIA, Estimates of U.S. Wood Energy Consumption from 1949 to 1981, Table A2. • 1980-1983: EIA, Estimates of U.S. Wood Energy Consumption 1980-1983, Table ES1. • 1984: EIA, Estimates of U.S. Biofuels Consumption 1990, Table 1. • 1985 and 1986: Values interpolated. - 1987: EIA, Estimates of Biofuels Consumption in the United States During 1987, Table 2. • 1988: Value interpolated. • 1989 forward: EIA, AER, Table 8.7c; and EIA estimates based on Form EIA-846, "Manufacturing Energy Consumption Survey." Industrial Sector, Biomass Waste: - 1981: EIA, Estimates of U.S. Biofuels Consumption 1990, Table 8, total waste consumption minus electric power sector waste consumption (see AER, Table 10.2c). • 1982 and 1983: EIA estimates for total waste consumption based on Estimates of U.S. Biofuels Consumption 1990, Table 8, minus electric power waste consumption (see AER, Table 10.2c). • 1984: EIA, Estimates of U.S. Biofuels Consumption 1990, Table 8, total waste consumption minus electric power sector waste consumption (see AER, Table 10.2c). • 1985 and 1986: Values interpolated. • 1987: EIA, Estimates of U.S. Biofuels Consumption 1990, Table 8, total waste consumption minus electric power sector waste consumption (see AER, Table 10.2c). • 1988: Value interpolated. • 1989 forward: EIA, AER, Table 8.7c; and EIA, estimates based on information presented in Government Advisory Associates, Resource Recovery Yearbook and Methane Recovery Yearbook, and information provided by the U.S. Environmental Protection Agency, Landfill Methane Outreach Program.

Industrial Sector, Fuel Ethanol (Minus Denaturant): EIA, AER, Tables 5.11, 5.13b, and 10.3. Calculated as industrial sector motor gasoline consumption (Table $5.13 \mathrm{~b}$ ) divided by total motor gasoline product supplied (Table 5.11), and then multiplied by fuel ethanol (minus denaturant) consumption (Table 10.3).

Industrial Sector, Losses and Co-products: EIA, AER, Tables 10.3 and 10.4 Calculated as fuel ethanol losses and co-products (Table 10.3) plus biodiesel losses and co-products (Table 10.4).

Transportation Sector, Fuel Ethanol (Minus Denaturant): EIA, AER, Tables 5.11, 5.13c, and 10.3. Calculated as transportation sector motor gasoline consumption (Table 5.13c) divided by total motor gasoline product supplied (Table 5.11), and then multiplied by fuel ethanol (minus denaturant) consumption (Table 10.3).

Transportation Sector, Biodiesel: EIA, AER, Table 10.4. 


\section{Environment}




\section{Figure 11.1 Carbon Dioxide Emissions From Energy Consumption}

\section{Total $^{1} 1949-2011$}
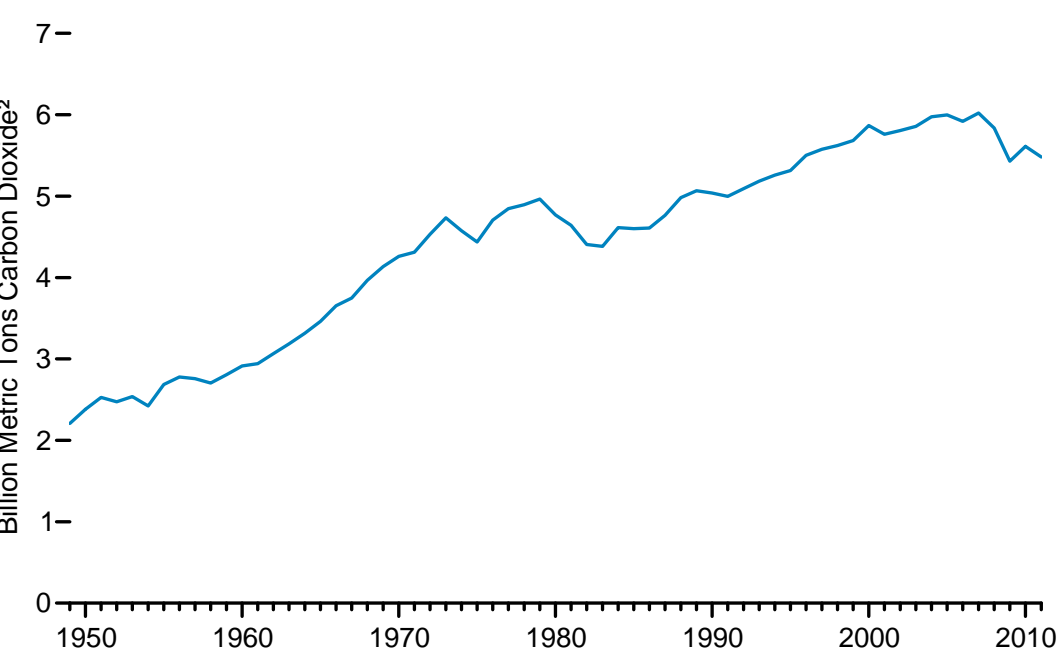

By Major Source, 1949-2011

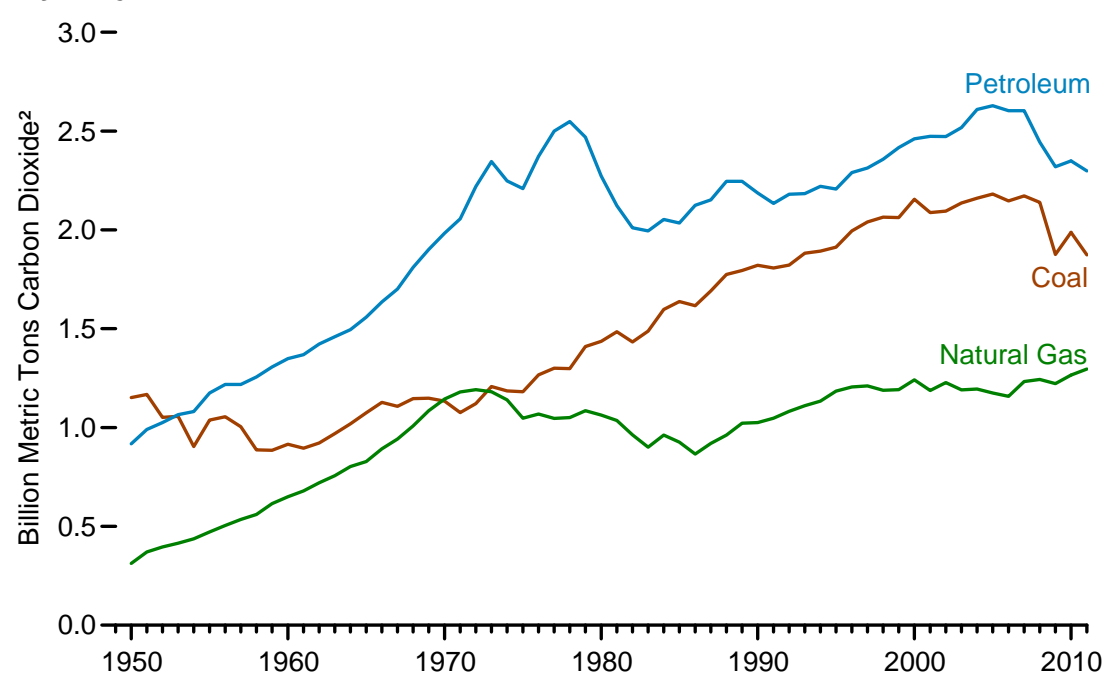

${ }^{1}$ Carbon dioxide emissions from biomass energy consumption are excluded from total emissions. See Note, "Accounting for Carbon Dioxide Emissions From Biomass Energy Combustion," at end of section.

\section{Economic Growth and Carbon Dioxide Emissions, 1949-2011} $800-$

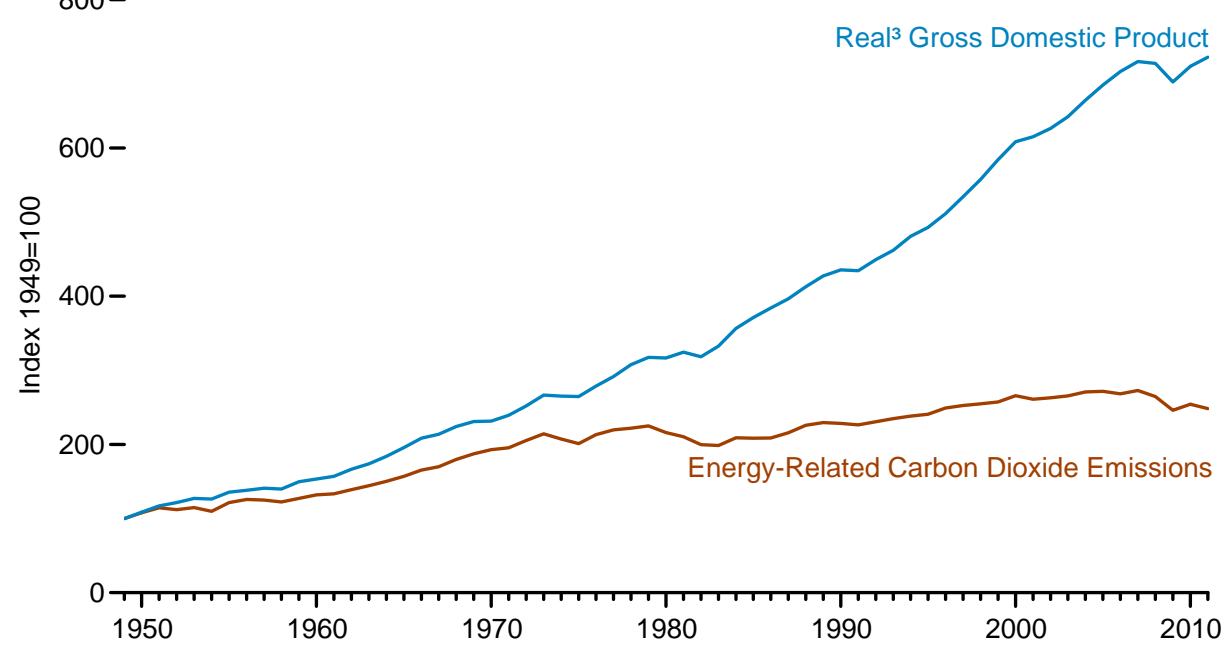

By Biomass $^{1}$ Source, 2011

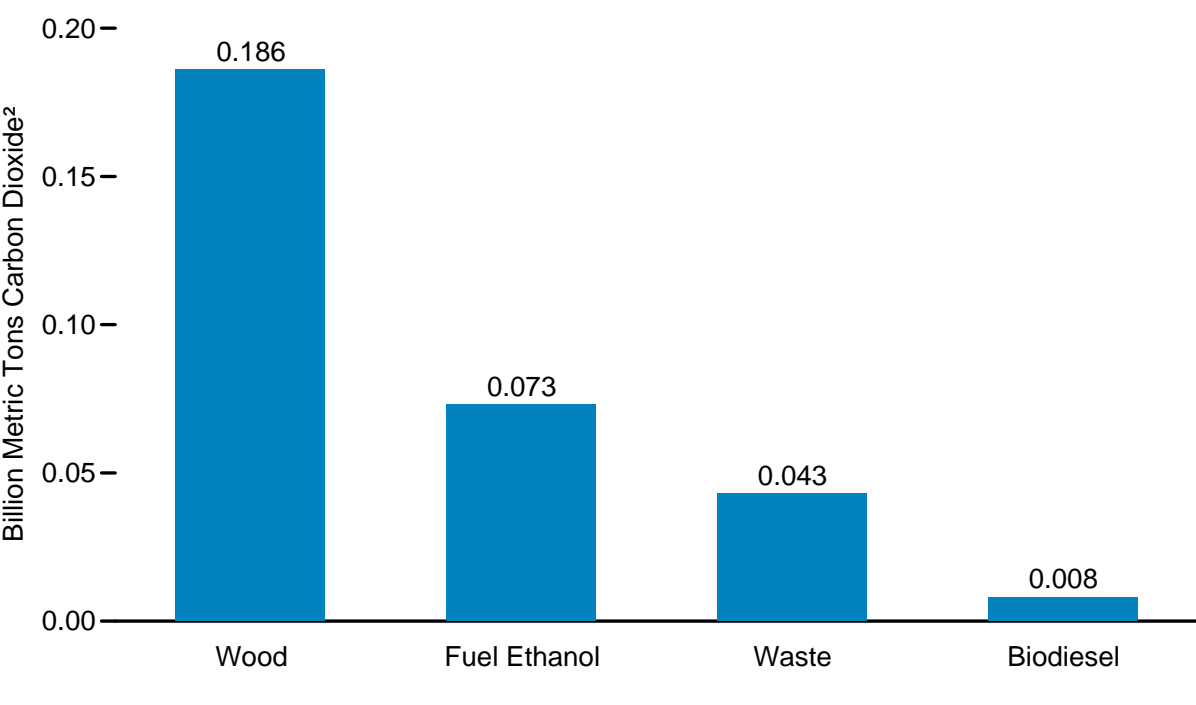

${ }^{2}$ Metric tons of carbon dioxide can be converted to metric tons of carbon equivalent by multiplying by $12 / 44$.

${ }^{3}$ Based on chained (2005) dollars.

Sources: Tables 1.5, 11.1, and 11.2a-11.2e. 
Table 11.1 Carbon Dioxide Emissions From Energy Consumption by Source, Selected Years, 1949-2011

(Million Metric Tons of Carbon Dioxide ${ }^{1}$ )

\begin{tabular}{|c|c|c|c|c|c|c|c|c|c|c|c|c|c|c|c|c|c|c|c|}
\hline \multirow[b]{2}{*}{ Year } & \multirow[b]{2}{*}{$\mathrm{Coal}^{3}$} & \multirow[b]{2}{*}{$\begin{array}{c}\text { Natural } \\
\text { Gas }{ }^{4}\end{array}$} & \multicolumn{11}{|c|}{ Petroleum } & \multirow[b]{2}{*}{ Total 2,9 } & \multicolumn{5}{|c|}{ Biomass $^{2}$} \\
\hline & & & $\begin{array}{l}\text { Aviation } \\
\text { Gasoline }\end{array}$ & $\begin{array}{l}\text { Distillate } \\
\text { Fuel Oil } 5\end{array}$ & $\begin{array}{l}\text { Jet } \\
\text { Fuel }\end{array}$ & $\begin{array}{l}\text { Kero- } \\
\text { sene }\end{array}$ & LPG 6 & $\begin{array}{l}\text { Lubri- } \\
\text { cants }\end{array}$ & $\begin{array}{c}\text { Motor } \\
\text { Gasoline }\end{array}$ & $\begin{array}{c}\text { Petroleum } \\
\text { Coke }\end{array}$ & $\begin{array}{c}\text { Residual } \\
\text { Fuel Oil }\end{array}$ & Other 8 & Total & & Wood $^{10}$ & Waste ${ }^{11}$ & $\begin{array}{c}\text { Fuel } \\
\text { Ethanol }^{12}\end{array}$ & $\begin{array}{c}\text { Bio- } \\
\text { diesel }\end{array}$ & Total \\
\hline 1949 & 1,118 & 270 & 12 & 140 & NA & 42 & 13 & 7 & 329 & 8 & 244 & 25 & 820 & 2,207 & 145 & $N A$ & $N A$ & $N A$ & 145 \\
\hline 1950 & 1,152 & 313 & 14 & 168 & NA & 48 & 16 & 9 & 357 & 8 & 273 & 26 & 918 & 2,382 & 147 & $N A$ & $N A$ & $N A$ & 147 \\
\hline 1955 & 1,038 & 472 & 24 & 247 & 21 & 48 & 27 & 10 & 473 & 13 & 274 & 38 & 1,175 & 2,685 & 134 & $N A$ & $N A$ & $N A$ & 134 \\
\hline 1960 & 915 & 650 & 21 & 291 & 53 & 41 & 42 & 10 & 543 & 29 & 275 & 45 & 1,349 & 2,914 & 124 & $N A$ & $N A$ & $N A$ & 124 \\
\hline 1965 & 1,075 & 828 & 15 & 330 & 87 & 40 & 57 & 11 & 627 & 39 & 289 & 65 & 1,559 & 3,462 & 125 & NA & $N A$ & $N A$ & 125 \\
\hline 1970 & 1,134 & 1,144 & 7 & 394 & 141 & 39 & 78 & 11 & 789 & 41 & 396 & 85 & 1,983 & 4,261 & 134 & $(s)$ & $N A$ & $N A$ & 134 \\
\hline 1975 & 1,181 & 1,047 & 5 & 443 & 146 & 24 & 82 & 11 & 911 & 48 & 443 & 97 & 2,209 & 4,437 & 140 & (s) & $N A$ & $N A$ & 141 \\
\hline 1976 & 1,266 & 1,068 & 5 & 488 & 144 & 25 & 86 & 13 & 955 & 47 & 506 & 103 & 2,372 & 4,705 & 161 & (s) & $N A$ & $N A$ & 161 \\
\hline 1977 & 1,300 & 1,046 & 5 & 520 & 152 & 26 & 85 & 13 & 979 & 52 & 553 & 115 & 2,500 & 4,846 & 172 & (s) & $N A$ & $N A$ & 172 \\
\hline 1978 & 1,298 & 1,050 & 5 & 533 & 154 & 26 & 83 & 14 & 1,011 & 50 & 544 & 127 & 2,548 & 4,896 & 191 & (s) & $N A$ & $N A$ & 191 \\
\hline 1979 & 1,410 & 1,085 & 5 & 514 & 157 & 28 & 95 & 15 & 960 & 48 & 509 & 139 & 2,469 & 4,964 & 202 & $\begin{array}{l}\text { (D) } \\
\text { (s) }\end{array}$ & $N A$ & $N A$ & 202 \\
\hline 1980 & 1,436 & 1,063 & 4 & 446 & 156 & 24 & 87 & 13 & 900 & 46 & 453 & 142 & 2,272 & 4,770 & 232 & $\begin{array}{l}\text { (D) } \\
\text { (s) }\end{array}$ & $N A$ & $N A$ & 232 \\
\hline 1981 & 1,485 & 1,036 & 4 & 439 & 147 & 19 & 85 & 13 & 899 & 48 & 376 & 93 & 2,122 & 4,642 & 234 & (3) & $(s)$ & $N A$ & 240 \\
\hline 1982 & 1,433 & 963 & 3 & 415 & 148 & 19 & 85 & 11 & 892 & 49 & 309 & 80 & 2,011 & 4,406 & 235 & 7 & 1 & $N A$ & 244 \\
\hline 1983 & 1,488 & 901 & 3 & 418 & 153 & 19 & 85 & 12 & 904 & 48 & 255 & 98 & 1,995 & 4,383 & 252 & 10 & 2 & $N A$ & 264 \\
\hline 1984 & 1,598 & 962 & 3 & 443 & 172 & 17 & 88 & 13 & 914 & 51 & 247 & 106 & 2,053 & 4,613 & 252 & 13 & 3 & $N A$ & 267 \\
\hline $\begin{array}{l}1984 \\
1985\end{array}$ & $\begin{array}{l}1,038 \\
1,638\end{array}$ & 926 & $\begin{array}{l}5 \\
3\end{array}$ & $\begin{array}{l}443 \\
445\end{array}$ & 178 & 17 & $\begin{array}{l}80 \\
86\end{array}$ & 12 & $\begin{array}{l}914 \\
930\end{array}$ & 55 & $\begin{array}{l}247 \\
216\end{array}$ & 93 & $\begin{array}{l}2,035 \\
2,035\end{array}$ & $\begin{array}{r}4,0 \\
4,600\end{array}$ & $\begin{array}{l}252 \\
252\end{array}$ & 14 & $\begin{array}{l}3 \\
3\end{array}$ & $N A$ & $\begin{array}{l}201 \\
270\end{array}$ \\
\hline $\begin{array}{l}1985 \\
1986\end{array}$ & $\begin{array}{l}1,6317 \\
1,617\end{array}$ & $\begin{array}{l}926 \\
866\end{array}$ & 4 & $\begin{array}{l}445 \\
453\end{array}$ & $\begin{array}{l}178 \\
191\end{array}$ & $\begin{array}{l}17 \\
15\end{array}$ & $\begin{array}{l}86 \\
83\end{array}$ & $\begin{array}{l}12 \\
12\end{array}$ & $\begin{array}{l}930 \\
958\end{array}$ & $\begin{array}{l}55 \\
56\end{array}$ & $\begin{array}{l}210 \\
255\end{array}$ & $\begin{array}{l}93 \\
98\end{array}$ & $\begin{array}{l}2,035 \\
2,125\end{array}$ & $\begin{array}{l}4,600 \\
4,608\end{array}$ & $\begin{array}{l}252 \\
240\end{array}$ & $\begin{array}{l}14 \\
16\end{array}$ & 4 & NA & 260 \\
\hline 1987 & 1,691 & 920 & 3 & 463 & 202 & 14 & 82 & 13 & 982 & 60 & 227 & $\begin{array}{r}50 \\
106\end{array}$ & 2,152 & 4,764 & 231 & 18 & 5 & $N A$ & 253 \\
\hline 1988 & $\begin{array}{l}1,091 \\
1,775\end{array}$ & $\begin{array}{l}y<0 \\
962\end{array}$ & 3 & 487 & 212 & 14 & $\begin{array}{l}02 \\
83\end{array}$ & 13 & $\begin{array}{r}1,003 \\
\end{array}$ & 63 & 249 & 119 & 2,246 & $\begin{array}{l}4,1,982 \\
4,9\end{array}$ & 242 & $\begin{array}{l}10 \\
19\end{array}$ & 5 & $N A$ & $\begin{array}{l}200 \\
266\end{array}$ \\
\hline 1989 & 1,795 & 1,022 & 3 & 491 & 218 & 13 & 82 & 13 & 1,000 & 62 & 246 & 118 & 2,246 & 5,067 & 251 & 22 & 5 & $N A$ & 278 \\
\hline 1990 & 1,821 & 1,025 & 3 & 470 & 223 & 6 & 69 & 13 & 988 & 67 & 220 & 127 & 2,187 & 5,039 & 208 & 24 & 4 & $N A$ & 237 \\
\hline 1991 & 1.807 & 1,047 & 3 & 454 & 215 & 7 & 71 & 12 & 982 & 66 & 207 & 117 & 2,134 & 4,996 & 208 & 26 & 5 & $N A$ & 239 \\
\hline 1992 & $\begin{array}{l}1,827 \\
1,822\end{array}$ & $\begin{array}{l}1,047 \\
1,082\end{array}$ & $\begin{array}{l}5 \\
3\end{array}$ & $\begin{array}{l}454 \\
464\end{array}$ & 213 & 6 & 77 & $\begin{array}{l}12 \\
12\end{array}$ & $\begin{array}{l}902 \\
999\end{array}$ & 74 & 196 & 135 & $\begin{array}{l}2,134 \\
2,180\end{array}$ & $\begin{array}{l}\text {, } 5,909 \\
5,0\end{array}$ & 217 & $\begin{array}{l}20 \\
27\end{array}$ & $\begin{array}{l}5 \\
6\end{array}$ & $N A$ & 250 \\
\hline 1993 & $\begin{array}{l}1,882 \\
\end{array}$ & 1,110 & 3 & 473 & 215 & 7 & 76 & 12 & 1.015 & 76 & 193 & 114 & 2,184 & 5,185 & 212 & 28 & 7 & $N A$ & 246 \\
\hline 1994 & 1,893 & 1,134 & 3 & 492 & 224 & 7 & 79 & 13 & 1,022 & 74 & 183 & 124 & 2,221 & 5,258 & 218 & $\begin{array}{l}20 \\
29\end{array}$ & 7 & $N A$ & 255 \\
\hline 1995 & 1,913 & 1,184 & 3 & 498 & 222 & 8 & 78 & 13 & 1,044 & 75 & 152 & 114 & 2,207 & 5,314 & 222 & 30 & 8 & $N A$ & 260 \\
\hline 1996 & 1,995 & 1,205 & 3 & 524 & 232 & 9 & 84 & 12 & 1,063 & 78 & 152 & 132 & 2,290 & 5,501 & 229 & 32 & 6 & $N A$ & 266 \\
\hline 1997 & 2,040 & 1,211 & 3 & 534 & 234 & 10 & 85 & 13 & 1,075 & 79 & 142 & 138 & 2,313 & 5,575 & 222 & 30 & 7 & $N A$ & 259 \\
\hline 1998 & $\begin{array}{l}2,064 \\
2,064\end{array}$ & 1,189 & 2 & 538 & 238 & 12 & 75 & 14 & 1,107 & 89 & 158 & $\begin{array}{l}100 \\
125\end{array}$ & 2,358 & 5,622 & 205 & 30 & 8 & $N A$ & 242 \\
\hline $\begin{array}{l}1998 \\
1999\end{array}$ & $\begin{array}{l}2,064 \\
2,062\end{array}$ & $\begin{array}{l}1,182 \\
1,192\end{array}$ & $\begin{array}{l}2 \\
3\end{array}$ & $\begin{array}{l}538 \\
555\end{array}$ & $\begin{array}{l}38 \\
245\end{array}$ & $\begin{array}{l}12 \\
11\end{array}$ & 91 & $\begin{array}{l}14 \\
14\end{array}$ & $\begin{array}{l}1,107 \\
1,127\end{array}$ & $\begin{array}{l}89 \\
93\end{array}$ & $\begin{array}{l}158 \\
148\end{array}$ & $\begin{array}{l}125 \\
130\end{array}$ & $\begin{array}{l}2,350 \\
2,47\end{array}$ & $\begin{array}{l}5,622 \\
5,682\end{array}$ & $\begin{array}{l}205 \\
208\end{array}$ & $\begin{array}{l}30 \\
29\end{array}$ & $\begin{array}{l}8 \\
8\end{array}$ & NA & $\begin{array}{l}242 \\
245\end{array}$ \\
\hline 2000 & $\begin{array}{l}\text {, }, 155 \\
2,155\end{array}$ & 1241 & 3 & 580 & 254 & 10 & 102 & 14 & 1.135 & 84 & $\begin{array}{l}140 \\
163\end{array}$ & 117 & $\begin{array}{l}2,411 \\
2,461\end{array}$ & 5867 & $\begin{array}{l}200 \\
212\end{array}$ & 27 & $\begin{array}{l}0 \\
9\end{array}$ & $N A$ & 248 \\
\hline 2001 & 2.088 & 1,187 & 2 & 598 & 243 & 11 & 92 & 13 & 1,151 & 88 & 145 & 132 & 2,473 & 5,759 & 188 & 33 & 10 & (s) & 231 \\
\hline 2002 & 2,095 & $\mathrm{R}_{1,227}$ & 2 & 587 & 237 & 6 & 98 & 12 & 1,183 & 94 & 125 & 127 & 2,472 & $\mathrm{R}_{5,806}$ & 187 & 36 & 12 & (s) & 235 \\
\hline 2003 & 2,136 & 1,191 & 2 & 610 & 231 & 8 & 95 & 11 & 1,188 & 94 & 138 & 140 & 2,518 & 5,857 & 188 & 36 & 16 & (s) & 240 \\
\hline 2004 & 2,160 & $R_{1,195}$ & 2 & 632 & 240 & 10 & 98 & 12 & 1,214 & $\begin{array}{r}54 \\
105\end{array}$ & 155 & 142 & 2,609 & 5,975 & 199 & 35 & 20 & $\begin{array}{l}\text { (3) } \\
\text { (s) }\end{array}$ & 255 \\
\hline 2005 & 2.182 & 1.175 & 2 & 640 & 246 & 10 & 94 & 12 & 1.214 & 105 & 164 & 141 & 2,628 & R5 997 & 200 & 37 & 23 & 1 & 261 \\
\hline 2006 & $\begin{array}{l}2,182 \\
2,147\end{array}$ & $\begin{array}{r}1,115 \\
\mathrm{R}_{1,158}\end{array}$ & 2 & $\begin{array}{l}640 \\
648\end{array}$ & $\begin{array}{l}246 \\
240\end{array}$ & $\begin{array}{r}10 \\
8\end{array}$ & $\begin{array}{l}94 \\
93\end{array}$ & $\begin{array}{l}12 \\
11\end{array}$ & $\begin{array}{l}1,214 \\
1,224\end{array}$ & $\begin{array}{l}105 \\
104\end{array}$ & $\begin{array}{l}164 \\
122\end{array}$ & $\begin{array}{l}141 \\
150\end{array}$ & $\begin{array}{l}2,628 \\
2,603\end{array}$ & $\begin{array}{r}5,997 \\
\mathrm{R}_{5}, 919\end{array}$ & $\begin{array}{r}200 \\
\mathrm{R}_{197}\end{array}$ & 36 & $\begin{array}{l}23 \\
31\end{array}$ & $\begin{array}{l}7 \\
2\end{array}$ & $\begin{array}{r}267 \\
\text { R266 }\end{array}$ \\
\hline 2007 & 2,172 & $R_{1,233}$ & 2 & 652 & 238 & $\begin{array}{l}0 \\
5\end{array}$ & 94 & 12 & 1,227 & 98 & 129 & 148 & 2,603 & $\mathrm{R}_{6,020}$ & R194 & 37 & 39 & 3 & R274 \\
\hline 2008 & 2,139 & 1,243 & 2 & 615 & 226 & 2 & $\begin{array}{l}89 \\
89\end{array}$ & 11 & 1.166 & 92 & 111 & 130 & $\begin{array}{l}2,60 \\
2,44\end{array}$ & $\begin{array}{l}0,0 \\
5,8\end{array}$ & R 191 & 40 & 55 & 3 & 289 \\
\hline 2009 & 1,876 & $\mathrm{R}_{1}, 222$ & 2 & 564 & 204 & 3 & 91 & 10 & 1,157 & 87 & 91 & 111 & 2,320 & $\mathrm{R}_{5}, 429$ & R177 & 41 & 62 & 3 & $\begin{array}{r}209 \\
R_{2} 284\end{array}$ \\
\hline 2010 & $R_{1,988}$ & $R_{1,265}$ & 2 & R590 & $\mathrm{R}_{210}$ & 3 & R94 & 11 & $R_{1,146}$ & 77 & R96 & $\mathrm{R}_{120}$ & $R_{2,349}$ & $\begin{array}{r}r, 612 \\
\mathrm{R}_{5}\end{array}$ & 186 & $\begin{array}{r}41 \\
\mathrm{R}_{4}\end{array}$ & $\mathrm{R}_{73}$ & 2 & 304 \\
\hline $2011^{P}$ & $\begin{array}{r}1,900 \\
1,874\end{array}$ & $\begin{array}{l}1,205 \\
1,296\end{array}$ & 2 & 596 & 209 & $\begin{array}{l}3 \\
2\end{array}$ & $\begin{array}{l}94 \\
92\end{array}$ & 10 & $\begin{array}{r}1,111 \\
\end{array}$ & 75 & 86 & 116 & $\begin{array}{r}2,249 \\
2,299\end{array}$ & 5,481 & $\begin{array}{l}180 \\
186\end{array}$ & 43 & 73 & 8 & 311 \\
\hline
\end{tabular}

${ }_{1}^{1}$ Metric tons of carbon dioxide can be converted to metric tons of carbon equivalent by multiplying by $12 / 44$.

2 Carbon dioxide emissions from biomass energy consumption are excluded from total emissions in this
ble. See Note, "Accounting for Carbon Dioxide Emissions From Biomass Energy Combustion," at end o table. S

3 Includes coal coke net imports.

${ }^{4}$ Natural gas, excluding supplemental gaseous fuels.

5 Distillate fuel oil, excluding biodiesel.

6 Liquefied petroleum gases.

Finished motor gasoline, excluding fuel ethanol.

Aviation gasoline blending components, crude oil, motor gasoline blending components, pentanes plus, petrochemical feedstocks, special naphthas, still gas, unfinished oils, waxes, and miscellaneous

petroleum products.
9 Includes electric power sector use of geothermal energy and non-biomass waste. See Table 11.3e.
${ }_{11}^{10}$ Wood and wood-derived fuels.

Municipal solid waste from biogenic sources, landfill gas, sludge waste, agricultural byproducts, and other biomass.

R=Revised. P=Preliminary. NA=Not available. (s)=Less than 0.5 million metric tons of carbon dioxide. Rotev: non-combustion use of fossil fuels. - See "Carbon Dioxide" in Glossary. - Totals may not equal sum of components due to independent rounding.

Web Pages: - See http://www.eia.gov/totalenergy/data/monthly/\#environment for updated monthly and annual data. - See http://www.eia.gov/totalenergy/data/annual/\#environment for all annual data beginning

Sources: - 1949-1972-U.S. Energy Information Administration (EIA) estimates based on data in Annual Energy Review Tables 2.1b-2.1f, 5.12, 7.3, 7.8, 10.2a-10.2c, and A5. • 1973 forward-EIA, 


\section{Figure 11.2 Carbon Dioxide Emissions From Energy Consumption by Sector, 1949-2011}

\section{Residential and Commercial, by Major Source}

$2,000-$

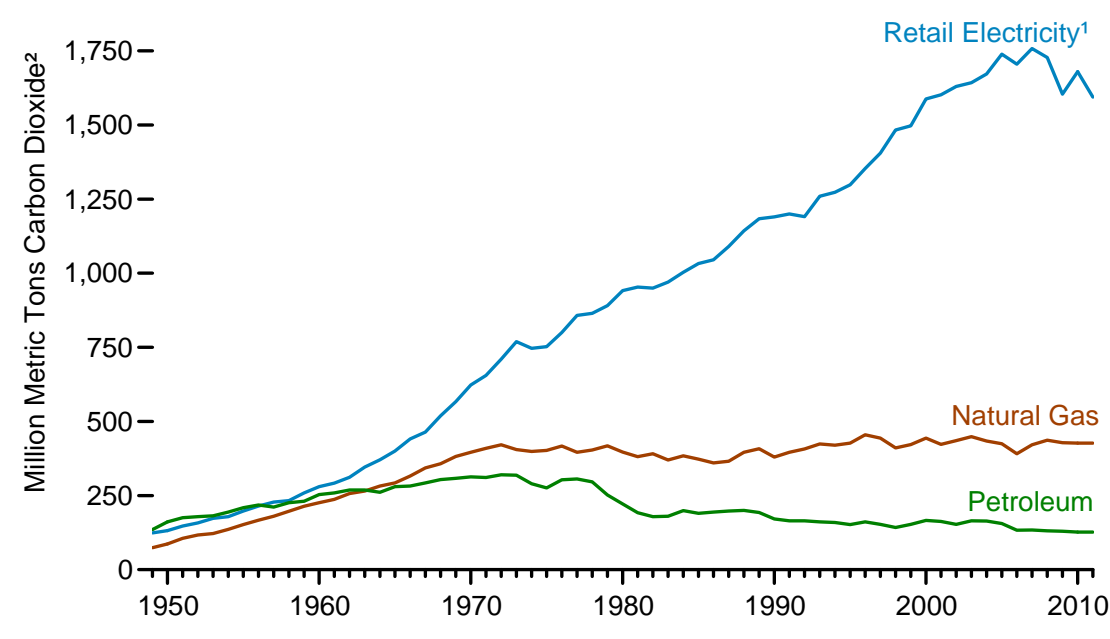

\section{Transportation, by Major Source}

$2,500-$

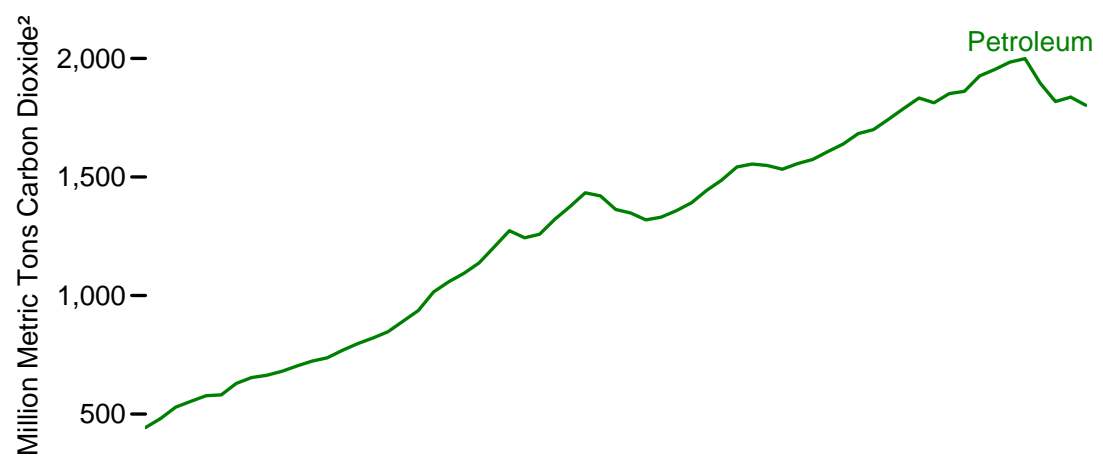

Natural Gas

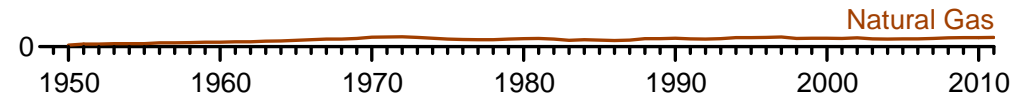

${ }^{1}$ Emissions from energy consumption in the electric power sector are allocated to the enduse sectors in proportion to each sector's share of total electricity retail sales (see Tables 8.9 and 11.2e).

\section{Industrial, by Major Source}

$1,000-$

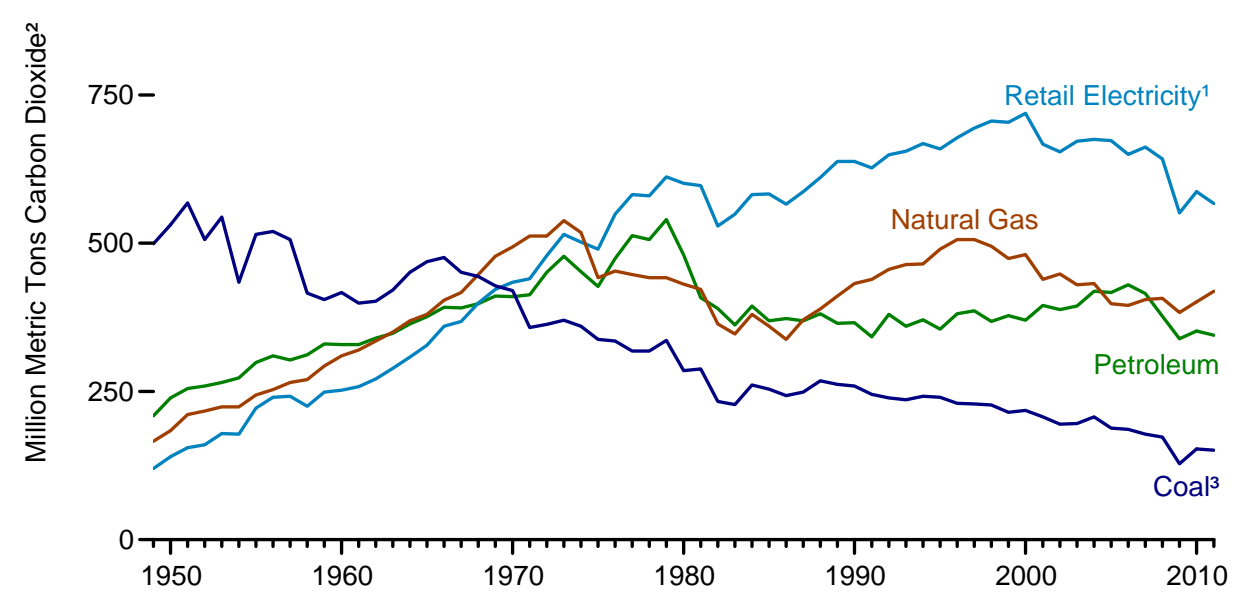

\section{Electric Power, by Major Source}

$2,500-$

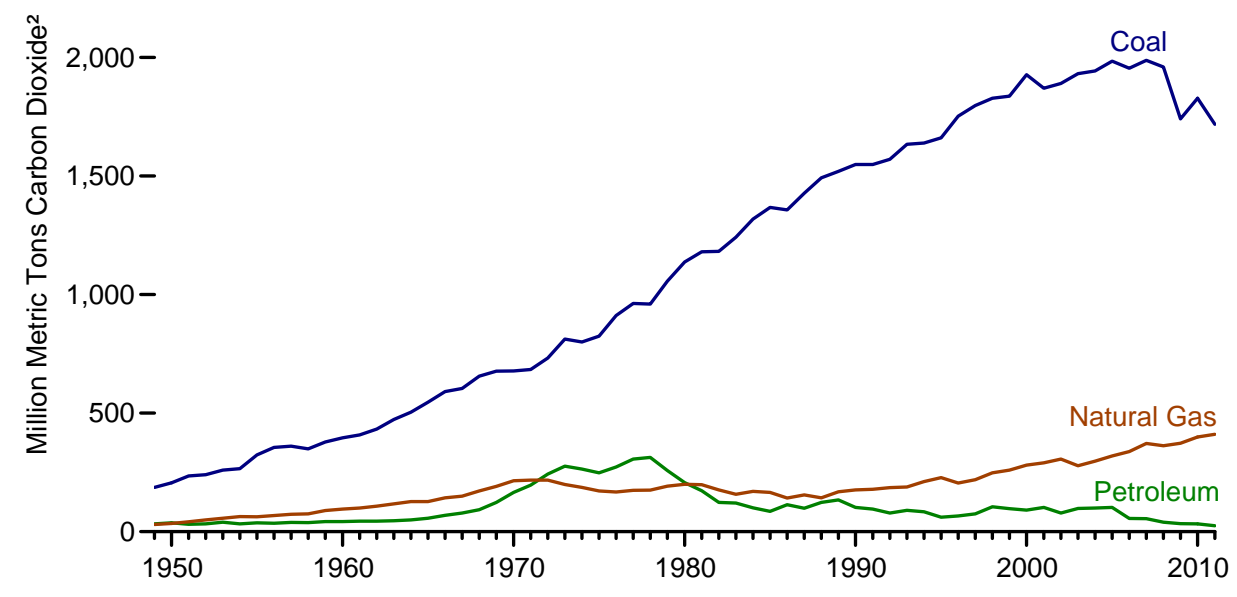

${ }^{2}$ Metric tons of carbon dioxide can be converted to metric tons of carbon equivalent by multiplying by $12 / 44$.

${ }^{3}$ Includes coal coke net imports.

Source: Tables 11.2a-11.2e. 
Table 11.2a Carbon Dioxide Emissions From Energy Consumption: Residential Sector, Selected Years, 1949-2011 (Million Metric Tons of Carbon Dioxide ${ }^{1}$ )

\begin{tabular}{|c|c|c|c|c|c|c|c|c|c|c|}
\hline \multirow[b]{2}{*}{ Year } & \multirow[b]{2}{*}{ Coal } & \multirow[b]{2}{*}{$\begin{array}{c}\text { Natural } \\
\text { Gas }^{3}\end{array}$} & \multicolumn{4}{|c|}{ Petroleum } & \multirow[b]{2}{*}{$\begin{array}{c}\text { Retail } \\
\text { Electricity } 5\end{array}$} & \multirow[b]{2}{*}{ Total $^{2}$} & \multicolumn{2}{|c|}{ Biomass $^{2}$} \\
\hline & & & $\begin{array}{l}\text { Distillate } \\
\text { Fuel Oil }^{4}\end{array}$ & Kerosene & $\begin{array}{l}\text { Liquefied } \\
\text { Petroleum Gases }\end{array}$ & Total & & & Wood $^{6}$ & Total $^{6}$ \\
\hline 1949 & 121 & 55 & 51 & 21 & 7 & 80 & 66 & 321 & 99 & 99 \\
\hline 1950 & 120 & 66 & 61 & 25 & 9 & 95 & 69 & 350 & 94 & 94 \\
\hline 1955 & 83 & 117 & 87 & 27 & 13 & 127 & 110 & 436 & 73 & 73 \\
\hline 1960 & 56 & 170 & 115 & 26 & 19 & 160 & 156 & 542 & 59 & 59 \\
\hline 1965 & 34 & 214 & 125 & 24 & 24 & 174 & 223 & 644 & 44 & 44 \\
\hline 1970 & 20 & 265 & 137 & 22 & 35 & 194 & 355 & 833 & 38 & 38 \\
\hline 1975 & 6 & 266 & 132 & 12 & 32 & 176 & 419 & 867 & 40 & 40 \\
\hline 1976 & 6 & 273 & 145 & 13 & 34 & 192 & 442 & 913 & 45 & 45 \\
\hline 1977 & 5 & 261 & 146 & 12 & 33 & 191 & 478 & 935 & 51 & 51 \\
\hline 1978 & 5 & 264 & 143 & 11 & 32 & 186 & 484 & 938 & 58 & 58 \\
\hline 1979 & 4 & 268 & 119 & 10 & 21 & 150 & 496 & 918 & 68 & 68 \\
\hline 1980 & 3 & 256 & 96 & 8 & 20 & 124 & 529 & 911 & 80 & 80 \\
\hline 1981 & 3 & 245 & 84 & 6 & 19 & 109 & 522 & 878 & 82 & 82 \\
\hline 1982 & 3 & 250 & 77 & 7 & 18 & 102 & 518 & 873 & 91 & 91 \\
\hline 1983 & 3 & 238 & 68 & 6 & 22 & 95 & 531 & 867 & 91 & 91 \\
\hline 1984 & 4 & 247 & 80 & 12 & 18 & 109 & 542 & 902 & 92 & 92 \\
\hline 1985 & 4 & 241 & 80 & 11 & 20 & 111 & 553 & 909 & 95 & 95 \\
\hline 1986 & 4 & 234 & 81 & 9 & 19 & 109 & 558 & 905 & 86 & 86 \\
\hline 1987 & 4 & 234 & 85 & 9 & 22 & 115 & 581 & 934 & 80 & 80 \\
\hline 1988 & 4 & 251 & 87 & 10 & 22 & 119 & 609 & 982 & 85 & 85 \\
\hline 1989 & 3 & 260 & 85 & 8 & 24 & 117 & 625 & 1,005 & 86 & 86 \\
\hline 1990 & 3 & 238 & 72 & 5 & 22 & 98 & 624 & 963 & 54 & 54 \\
\hline 1991 & 2 & 248 & 68 & 5 & 24 & 97 & 633 & 980 & 57 & 57 \\
\hline 1992 & 2 & 255 & 72 & 5 & 23 & 100 & 624 & 981 & 60 & 60 \\
\hline 1993 & 2 & 269 & 71 & 5 & 25 & 101 & 667 & 1,040 & 52 & 52 \\
\hline 1994 & 2 & 263 & 70 & 5 & 24 & 99 & 668 & 1,032 & 49 & 49 \\
\hline 1995 & 2 & 263 & 66 & 5 & 25 & 96 & 678 & 1,039 & 49 & 49 \\
\hline 1996 & 2 & 284 & 68 & 6 & 30 & 104 & 710 & 1,099 & 51 & 51 \\
\hline 1997 & 2 & 270 & 64 & 7 & 29 & 99 & 719 & 1,090 & 40 & 40 \\
\hline 1998 & 1 & 247 & 56 & 8 & 27 & 91 & 759 & 1,097 & 36 & 36 \\
\hline 1999 & 1 & 257 & 61 & 8 & 33 & 102 & 762 & 1,122 & 37 & 37 \\
\hline 2000 & 1 & 271 & 66 & 7 & 35 & 108 & 805 & 1,185 & 39 & 39 \\
\hline 2001 & 1 & 259 & 66 & 7 & 33 & 106 & 805 & 1,172 & 35 & 35 \\
\hline 2002 & 1 & $R_{265}$ & 63 & 4 & 34 & 101 & 835 & $R_{1,203}$ & 36 & 36 \\
\hline 2003 & 1 & 276 & 66 & 5 & 34 & 106 & 847 & 1,230 & 38 & 38 \\
\hline 2004 & 1 & 264 & 68 & 6 & 32 & 106 & 856 & 1,228 & 38 & 38 \\
\hline 2005 & 1 & 262 & 62 & 6 & 32 & 101 & 897 & 1,261 & 40 & 40 \\
\hline 2006 & 1 & 237 & 52 & 5 & 28 & 85 & 869 & 1,192 & $\mathrm{R}_{36}$ & R36 \\
\hline 2007 & 1 & 257 & 53 & 3 & 31 & 87 & 897 & $\mathrm{R}_{1,241}$ & $\mathrm{R}_{38}$ & ${ }^{R} 38$ \\
\hline 2008 & 1 & 266 & 49 & 2 & 35 & 85 & 878 & 1,229 & 42 & 42 \\
\hline 2009 & 1 & 259 & 44 & 2 & 35 & 81 & 819 & 1,159 & 40 & 40 \\
\hline 2010 & 1 & $\mathrm{R}_{259}$ & $\mathrm{R}_{43}$ & 2 & $\mathrm{R}_{33}$ & R78 & R875 & $\mathrm{R}_{1,212}$ & 39 & 39 \\
\hline $2011^{P}$ & 1 & 256 & 43 & 1 & 33 & 78 & 827 & 1,162 & 40 & 40 \\
\hline
\end{tabular}

1 Metric tons of carbon dioxide can be converted to metric tons of carbon equivalent by multiplying by $12 / 44$.

${ }^{2}$ Carbon dioxide emissions from biomass energy consumption are excluded from total emissions in this table. See Note, "Accounting for Carbon Dioxide Emissions From Biomass Energy Combustion," at end of section.

Natural gas, excluding supplemental gaseous fuels.

Distillate fuel oil, excluding biodiesel.

Emissions from energy consumption (for electricity and a small amount of useful thermal output) in the electric power sector are allocated to the end-use sectors in proportion to each sector's share of tota
${ }^{6}$ Wood and wood-derived fuels

$\mathrm{R}=$ Revised. P=Preliminary.

Notes: - Data are estimates for carbon dioxide emissions from energy consumption. - See "Carbon Dioxide" in Glossary. - Totals may not equal sum of components due to independent rounding.

Web Pages: - See http://www.eia.gov/totalenergy/data/monthly/\#environment for updated monthly and annual data. - See http://www.eia.gov/totalenergy/data/annual/\#environment for all annual data beginning in 1949. - See http://www.eia.gov/environment/ for related information.

Annul Energed on data in Annual Energy Review Tables 2.1b, 5.14a, 8.9, 10.2a, and 11.2e. • 1973 forward-EIA, Monthly Energy 
Table 11.2b Carbon Dioxide Emissions From Energy Consumption: Commercial Sector, Selected Years, 1949-2011 (Million Metric Tons of Carbon Dioxide ${ }^{1}$ )

\begin{tabular}{|c|c|c|c|c|c|c|c|c|c|c|c|c|c|c|c|}
\hline \multirow[b]{2}{*}{ Year } & \multirow[b]{2}{*}{ Coal } & \multirow[b]{2}{*}{$\begin{array}{l}\text { Natural } \\
\text { Gas }^{3}\end{array}$} & \multicolumn{7}{|c|}{ Petroleum } & \multirow[b]{2}{*}{$\begin{array}{c}\text { Retail } \\
\text { Electricity }{ }^{7}\end{array}$} & \multirow[b]{2}{*}{ Total $^{2}$} & \multicolumn{4}{|c|}{ Biomass $^{2}$} \\
\hline & & & $\begin{array}{l}\text { Distillate } \\
\text { Fuel Oil }{ }^{4}\end{array}$ & Kerosene & LPG 5 & $\begin{array}{c}\text { Motor } \\
\text { Gasoline }\end{array}$ & $\begin{array}{l}\text { Petroleum } \\
\text { Coke }\end{array}$ & $\begin{array}{l}\text { Residual } \\
\text { Fuel Oil }\end{array}$ & Total & & & Wood $^{8}$ & Waste $^{9}$ & $\begin{array}{c}\text { Fuel } \\
\text { Ethanol }{ }^{10}\end{array}$ & Total \\
\hline 1949 & 148 & 19 & 16 & 3 & 2 & 7 & NA & 28 & 55 & 58 & 280 & 2 & $N A$ & $N A$ & 2 \\
\hline 1950 & 147 & 21 & 19 & 3 & 2 & 7 & NA & 33 & 66 & 63 & 297 & 2 & $N A$ & $N A$ & 2 \\
\hline 1955 & 76 & 35 & 28 & 4 & 3 & 9 & NA & 38 & 82 & 88 & 281 & 1 & NA & $N A$ & 1 \\
\hline 1960 & 39 & 56 & 36 & 3 & 5 & 5 & NA & 44 & 93 & 124 & 312 & 1 & $N A$ & $N A$ & 1 \\
\hline 1965 & 25 & 79 & $\begin{array}{l}50 \\
39\end{array}$ & 4 & 6 & 5 & NA & $\begin{array}{l}44 \\
51\end{array}$ & $\begin{array}{r}50 \\
106\end{array}$ & 177 & 387 & 1 & $N A$ & $N A$ & 1 \\
\hline 1970 & 16 & 131 & 43 & 4 & 9 & 6 & NA & 56 & 119 & 268 & 534 & 1 & NA & $N A$ & 1 \\
\hline 1975 & 14 & 136 & 43 & 4 & 8 & 6 & NA & 39 & 100 & 333 & 583 & 1 & $N A$ & $N A$ & 1 \\
\hline 1976 & 14 & 144 & 48 & 3 & 9 & 7 & NA & 45 & 111 & 358 & 627 & 1 & $N A$ & $N A$ & 1 \\
\hline 1977 & 14 & 135 & 49 & 4 & 9 & 7 & NA & 46 & 115 & 380 & 645 & 1 & $N A$ & $N A$ & 1 \\
\hline 1978 & 16 & 140 & 49 & 4 & 8 & 8 & NA & 42 & 110 & 381 & 648 & 1 & $N A$ & $N A$ & 1 \\
\hline 1979 & 14 & 150 & 43 & 6 & 6 & 7 & NA & 40 & 102 & 395 & 661 & 1 & $N A$ & $N A$ & 1 \\
\hline 1980 & 11 & 141 & 38 & 3 & 6 & 8 & NA & 44 & 98 & 412 & 662 & 2 & $N A$ & $N A$ & 2 \\
\hline 1981 & 13 & 136 & 33 & 5 & 5 & 7 & NA & 33 & 83 & 431 & 663 & 2 & $N A$ & (s) & 2 \\
\hline 1982 & 15 & 141 & 32 & 2 & 5 & 6 & NA & 31 & 77 & 432 & 665 & 2 & $N A$ & (s) & 2 \\
\hline 1983 & 15 & 132 & 48 & 8 & 6 & 7 & NA & 16 & 85 & 439 & 671 & 2 & $N A$ & (s) & 2 \\
\hline 1984 & 16 & 137 & 54 & 3 & 5 & 8 & NA & 21 & 90 & 461 & 704 & 2 & $N A$ & (s) & 2 \\
\hline $\begin{array}{l}1904 \\
1985\end{array}$ & 13 & 132 & 46 & $\begin{array}{l}5 \\
2\end{array}$ & 6 & $\begin{array}{l}\circ \\
7\end{array}$ & NA & 18 & 79 & 480 & 704 & 2 & $N A$ & (s) & 2 \\
\hline 1986 & 13 & 126 & 46 & 4 & 6 & 8 & NA & 23 & 85 & 487 & 711 & 3 & $N A$ & (s) & 3 \\
\hline 1987 & 12 & 132 & 44 & 4 & 6 & 8 & NA & 21 & 83 & 509 & 736 & 3 & $N A$ & (s) & 3 \\
\hline 1988 & 12 & 145 & 44 & 2 & 6 & 8 & NA & 21 & 81 & 534 & 772 & 3 & $N A$ & (s) & 3 \\
\hline $\begin{array}{l}1900 \\
1989\end{array}$ & 11 & 148 & $\begin{array}{l}44 \\
42\end{array}$ & 2 & 7 & $\begin{array}{l}0 \\
7\end{array}$ & 0 & 18 & 76 & 559 & 794 & 7 & 1 & (s) & 9 \\
\hline 1990 & 12 & 142 & 39 & 1 & 6 & 8 & 0 & 18 & 73 & 566 & 793 & 6 & 2 & (s) & 8 \\
\hline 1991 & 11 & 148 & 38 & 1 & 7 & 6 & 0 & 17 & 68 & 567 & 794 & 6 & 2 & (s) & 8 \\
\hline 1992 & 11 & 152 & 37 & 1 & 7 & 6 & (s) & 15 & 65 & 567 & 796 & 7 & 2 & (s) & 9 \\
\hline 1993 & 11 & 155 & 36 & 1 & 7 & 2 & (s) & 14 & 60 & 593 & 819 & 7 & 2 & (s) & 9 \\
\hline $\begin{array}{l}1994 \\
\end{array}$ & 11 & 157 & 37 & 1 & 7 & 2 & $\begin{array}{l}\text { (s) } \\
\text { (s) }\end{array}$ & 14 & 60 & 605 & 833 & 7 & 2 & (s) & 9 \\
\hline $\begin{array}{l}1994 \\
1995\end{array}$ & 11 & 164 & 35 & 2 & 7 & 1 & $\begin{array}{l}\text { (s) } \\
\text { (s) }\end{array}$ & $\begin{array}{l}14 \\
11\end{array}$ & 56 & 620 & $\begin{array}{l}833 \\
851\end{array}$ & 7 & 2 & (s) & 9 \\
\hline 1996 & 12 & 171 & 35 & 2 & 8 & 2 & $\begin{array}{l}\text { (s) } \\
\text { (s) }\end{array}$ & 11 & 57 & 643 & 883 & 7 & 3 & (s) & 10 \\
\hline 1997 & 12 & 174 & 32 & 2 & $\begin{array}{l}\circ \\
8\end{array}$ & 3 & $\begin{array}{l}\text { (s) } \\
\text { (s) }\end{array}$ & 9 & 54 & $\begin{array}{l}040 \\
686\end{array}$ & $\begin{array}{l}000 \\
926\end{array}$ & 7 & 3 & (s) & 10 \\
\hline 1998 & 9 & 164 & 31 & 2 & 7 & 3 & (s) & 7 & 51 & 724 & 947 & 6 & 3 & (s) & 9 \\
\hline 1999 & 10 & 165 & 32 & 2 & 9 & 2 & (s) & 6 & 51 & 735 & 960 & 6 & 3 & (s) & 9 \\
\hline 2000 & 9 & $\begin{array}{l}105 \\
173\end{array}$ & 36 & 2 & 9 & 3 & $\begin{array}{l}\text { (s) } \\
\text { (s) }\end{array}$ & 7 & 58 & 783 & $\begin{array}{r}500 \\
1,022\end{array}$ & $\begin{array}{l}0 \\
7\end{array}$ & 2 & (s) & 9 \\
\hline 2001 & 9 & 164 & 37 & 2 & 9 & 3 & (s) & 6 & 57 & 797 & 1,027 & 6 & 2 & (s) & 9 \\
\hline 2002 & 9 & $\mathrm{R}_{170}$ & 32 & 1 & 9 & 3 & (s) & 6 & 52 & 795 & $\mathrm{R}_{1,026}$ & 6 & 2 & (s) & 9 \\
\hline 2003 & 8 & 173 & 35 & 1 & 10 & 4 & (s) & 9 & 59 & 796 & 1,036 & 7 & 3 & (s) & 9 \\
\hline 2004 & 10 & 170 & 34 & 1 & 10 & 3 & (s) & 10 & 58 & 816 & 1.054 & 7 & 3 & (s) & 10 \\
\hline 2005 & 9 & 163 & 33 & 2 & 8 & 3 & (s) & 9 & 55 & 842 & $\begin{array}{l}1,069 \\
1.09\end{array}$ & 7 & 3 & (s) & 10 \\
\hline 2006 & 6 & $\begin{array}{l}163 \\
154\end{array}$ & $\begin{array}{l}33 \\
29\end{array}$ & $\begin{array}{l}2 \\
1\end{array}$ & $\begin{array}{l}8 \\
8\end{array}$ & $\begin{array}{l}3 \\
3\end{array}$ & $\begin{array}{l}\text { (s) } \\
\text { (s) }\end{array}$ & 6 & $\begin{array}{l}55 \\
48\end{array}$ & $\begin{array}{l}842 \\
836\end{array}$ & $\begin{array}{l}1,069 \\
1,043\end{array}$ & 6 & $\begin{array}{l}3 \\
3\end{array}$ & $\begin{array}{l}\text { (s) } \\
\text { (s) }\end{array}$ & 9 \\
\hline 2007 & 7 & 164 & 28 & 1 & 8 & 4 & $\begin{array}{l}\text { (s) } \\
\text { (s) }\end{array}$ & 6 & 47 & 861 & $R_{1,078}$ & 7 & 3 & (s) & 9 \\
\hline 2008 & 7 & 171 & 27 & (s) & 10 & 3 & (s) & 6 & 46 & 850 & 1,074 & 7 & 3 & (s) & 10 \\
\hline 2009 & 6 & 169 & 30 & (s) & 9 & 4 & (s) & 6 & 49 & 785 & 1,008 & 7 & 3 & (s) & 10 \\
\hline 2010 & $R_{6}$ & $\mathrm{R}_{168}$ & $\mathrm{R}_{30}$ & $\begin{array}{l}\text { (s) } \\
\text { (s) }\end{array}$ & 9 & 4 & $\begin{array}{l}\text { (s) } \\
\text { (s) }\end{array}$ & $\mathrm{R}_{6}$ & $\mathrm{R}_{49}$ & 805 & $\mathrm{R}_{1,027}$ & 7 & 3 & $\begin{array}{l}\text { (s) } \\
\text { (s) }\end{array}$ & 10 \\
\hline $2011^{P}$ & $\begin{array}{r}0 \\
5\end{array}$ & 171 & 30 & $\begin{array}{l}\text { (s) } \\
\text { (s) }\end{array}$ & 9 & $\begin{array}{l}4 \\
4\end{array}$ & $\begin{array}{l}\text { (s) } \\
\text { (s) }\end{array}$ & 6 & $\begin{array}{r}49 \\
49\end{array}$ & $\begin{array}{l}805 \\
767\end{array}$ & $\begin{array}{r}1,021 \\
992\end{array}$ & 7 & $\begin{array}{l}3 \\
3\end{array}$ & $\begin{array}{l}\text { (s) } \\
\text { (s) }\end{array}$ & 10 \\
\hline
\end{tabular}

1 Metric tons of carbon dioxide can be converted to metric tons of carbon equivalent by multiplying by $2 / 44$.

Carbon dioxide emissions from biomass energy consumption are excluded from total emissions in this table. See Note, "Accounting for Carbon Dioxide Emissions From Biomass Energy Combustion," at end of

3 Natural gas, excluding supplemental gaseous fuels.

Listillate fuel oil, excluding

Finished motor gasoline, excluding fuel ethanol.

Emissions from energy consumption (for electricity and a small amount of useful thermal output) in the electric power sector are allocated to the end-use sectors in proportion to each sector's share of total 8 woth and wos. See Tables 8.9 and $11.2 \mathrm{e}$.

${ }^{9}$ Municipal solid waste from biogenic sources, landfill gas, sludge waste, agricultural byproducts, and ther biomass.

R=Revised. P=Preliminary. NA=Not available ( $(\mathrm{s})=$ Less than 0.5 million metric tons of carbon dioxide. Notes: - Data are estimates for carbon dioxide emissions from energy consumption. - See "Carbo Dioxide" in Glossary. - Totals may not equal sum of components due to independent rounding.

Web Pages: - See http://www.eia.gov/totalenergy/data/monthly/\#environment for updated monthly and annual data. - See http://www.eia.gov/totalenergy/data/annual/\#environment for all annual data beginning in 1949. - See $h$ ttp: $: / / w w w . e i a . g o v / e n v i r o n m e n t /$ for related information.

Sources: - 1949-1972-U.S. Energy Information Administration (EIA) estimates based on data in Annual Energy Review Tables 2.1c, 5.14a, 8.9, 10.2a, and 11.2e. • 1973 forward-EIA, Monthly Energy Review (MER) (May 2012), Tables 12.3 and 12.7, and MER data system calculations. 
Table 11.2c Carbon Dioxide Emissions From Energy Consumption: Industrial Sector, Selected Years, 1949-2011 (Million Metric Tons of Carbon Dioxide ${ }^{1}$ )

\begin{tabular}{|c|c|c|c|c|c|c|c|c|c|c|c|c|c|c|c|c|c|c|}
\hline \multirow[b]{2}{*}{ Year } & \multirow[b]{2}{*}{ Coal } & \multirow{2}{*}{$\begin{array}{c}\text { Coal } \\
\text { Coke } \\
\text { Net } \\
\text { Imports }\end{array}$} & \multirow[b]{2}{*}{$\begin{array}{c}\text { Natural } \\
\text { Gas }^{3}\end{array}$} & \multicolumn{9}{|c|}{ Petroleum } & \multirow{2}{*}{$\begin{array}{l}\text { Retail } \\
\text { Elec- } \\
\text { tricity } 8\end{array}$} & \multirow[b]{2}{*}{ Total $^{2}$} & \multicolumn{4}{|c|}{ Biomass $^{2}$} \\
\hline & & & & $\begin{array}{l}\text { Distillate } \\
\text { Fuel Oil } 4\end{array}$ & $\begin{array}{l}\text { Kero- } \\
\text { sene }\end{array}$ & LPG 5 & $\begin{array}{l}\text { Lubri- } \\
\text { cants }\end{array}$ & $\begin{array}{c}\text { Motor } \\
\text { Gasoline } 6\end{array}$ & $\begin{array}{c}\text { Petroleum } \\
\text { Coke }\end{array}$ & $\begin{array}{l}\text { Residual } \\
\text { Fuel Oil }\end{array}$ & Other ${ }^{7}$ & Total & & & Wood $^{9}$ & Waste 10 & $\begin{array}{c}\text { Fuel } \\
\text { Ethanol }{ }^{11}\end{array}$ & Total \\
\hline 1949 & 500 & -1 & 166 & 41 & 18 & 3 & 3 & 16 & 8 & 95 & 25 & 209 & 120 & 995 & 44 & $N A$ & $N A$ & 44 \\
\hline 1950 & 531 & (s) & 184 & 51 & 20 & 4 & 3 & 18 & 8 & 110 & 26 & 239 & 140 & 1,095 & 50 & $N A$ & $N A$ & 50 \\
\hline 1955 & 516 & -1 & 244 & 72 & 17 & 10 & 4 & 24 & 13 & 122 & 38 & 299 & 222 & 1,281 & 59 & $N A$ & $N A$ & 59 \\
\hline 1960 & 418 & -1 & 310 & 74 & 12 & 17 & 4 & 27 & 29 & 123 & 45 & 329 & 252 & 1,308 & 64 & $N A$ & $N A$ & 64 \\
\hline 1965 & 471 & -2 & 380 & 83 & 12 & 24 & 5 & 24 & 39 & 123 & 65 & 376 & 328 & 1,553 & 80 & $N A$ & $N A$ & 80 \\
\hline 1970 & 427 & -7 & 494 & 89 & 13 & 31 & 6 & 21 & 39 & 126 & 85 & 410 & 434 & 1,759 & 96 & $N A$ & $N A$ & 96 \\
\hline 1975 & 336 & 2 & 442 & 97 & 9 & 39 & 6 & 16 & 48 & 117 & 97 & 427 & 490 & 1,696 & 100 & $N A$ & $N A$ & 100 \\
\hline 1976 & 335 & (s) & 453 & 111 & 9 & 41 & 6 & 15 & 47 & 141 & 103 & 474 & 549 & 1,811 & 114 & $N A$ & $N A$ & 114 \\
\hline 1977 & 316 & 2 & 447 & 125 & 10 & 40 & 7 & 14 & 52 & 150 & 115 & 513 & 582 & 1,860 & 120 & $N A$ & $N A$ & 120 \\
\hline 1978 & 304 & $1 \overline{4}$ & 442 & 127 & 11 & 40 & 7 & 13 & 48 & 133 & 127 & 506 & 580 & 1,846 & 131 & $N A$ & $N A$ & 131 \\
\hline 1979 & 329 & 7 & 442 & 128 & 13 & 66 & 8 & 11 & 47 & 128 & 139 & 540 & 612 & 1,931 & 132 & $N A$ & $N A$ & 132 \\
\hline 1980 & 289 & -4 & 431 & 96 & 13 & 61 & 7 & 11 & 45 & 105 & 142 & 480 & 601 & $\begin{array}{l}1,797\end{array}$ & 150 & $N A$ & $N A$ & 150 \\
\hline 1981 & 290 & -2 & 422 & 101 & 8 & 58 & 6 & 11 & 47 & 83 & 93 & 408 & 597 & 1,715 & 150 & 5 & (s) & 156 \\
\hline 1982 & 235 & -2 & 364 & 95 & 10 & 60 & 6 & 10 & 48 & 81 & 80 & 390 & 529 & 1,515 & 142 & 7 & (s) & 149 \\
\hline 1983 & 230 & -2 & 347 & 83 & 5 & 55 & 6 & 8 & 48 & 61 & 98 & 362 & 549 & 1,486 & 159 & 9 & (s) & 168 \\
\hline 1984 & 262 & -1 & 380 & 87 & 3 & 62 & 7 & 11 & 50 & 68 & 106 & 394 & 582 & 1,617 & 157 & 12 & (s) & 170 \\
\hline 1985 & 256 & -2 & 360 & 81 & 3 & 58 & 6 & 15 & 54 & 57 & 93 & 369 & 583 & 1,566 & 154 & 14 & (s) & 168 \\
\hline 1986 & 245 & -2 & 338 & 84 & 2 & 56 & 6 & 15 & 55 & 57 & 98 & 373 & 566 & 1,520 & 151 & 16 & (s) & 167 \\
\hline 1987 & 248 & 1 & 371 & 83 & 2 & 53 & 7 & 15 & 59 & 45 & 106 & 369 & 587 & 1,575 & 148 & 17 & (s) & 165 \\
\hline 1988 & 263 & 5 & 389 & 82 & 2 & 54 & 7 & 14 & 61 & 42 & 119 & 381 & 611 & 1,648 & 152 & 19 & (s) & 171 \\
\hline 1989 & 259 & 3 & 411 & 83 & 2 & 49 & 7 & 14 & 60 & 31 & 118 & 365 & 638 & 1,677 & 149 & 12 & (s) & 161 \\
\hline 1990 & 258 & 1 & 432 & 84 & 1 & 39 & 7 & 13 & 64 & 31 & 127 & 366 & 638 & 1,695 & 135 & 12 & (s) & 147 \\
\hline 1991 & 244 & 1 & 439 & 79 & 1 & 39 & 6 & 14 & 63 & 24 & 117 & 342 & 627 & 1,653 & 132 & 11 & (s) & 143 \\
\hline 1992 & 235 & 4 & 456 & 81 & 1 & 45 & 6 & 14 & 70 & 28 & 135 & 380 & 649 & 1,724 & 137 & 10 & (s) & 148 \\
\hline 1993 & 233 & 3 & 464 & 81 & 1 & 43 & 6 & 13 & 68 & 33 & 114 & 360 & 655 & 1,715 & 139 & 11 & (s) & 150 \\
\hline 1994 & 235 & 7 & 465 & 81 & 1 & 46 & 7 & 14 & 67 & 31 & 124 & 371 & 668 & 1,745 & 148 & 11 & (s) & 160 \\
\hline 1995 & 233 & 7 & 490 & 82 & 1 & 45 & 7 & 14 & 67 & 24 & 114 & 355 & 659 & $\begin{array}{l}1,743 \\
\end{array}$ & 155 & 11 & (s) & 166 \\
\hline 1996 & 227 & 3 & 506 & 86 & 1 & 46 & 6 & 14 & 70 & 24 & 132 & 381 & 678 & 1,795 & 158 & 12 & (s) & 170 \\
\hline 1997 & 224 & 5 & 506 & 88 & 1 & 48 & 7 & 15 & 68 & 21 & 138 & 386 & 694 & 1,815 & 162 & 10 & (s) & 172 \\
\hline 1998 & 219 & 8 & 495 & 88 & 2 & 39 & 7 & 14 & 77 & 16 & 125 & 368 & 706 & 1,796 & 150 & 10 & (s) & 160 \\
\hline 1999 & 208 & 7 & 474 & 86 & 1 & 48 & 7 & 11 & 81 & 14 & 130 & 378 & 704 & $\begin{array}{l}1,772 \\
\end{array}$ & 152 & 9 & (s) & 161 \\
\hline 2000 & 211 & 7 & 481 & 87 & 1 & 56 & 7 & 11 & 74 & 17 & 117 & 370 & 719 & 1,788 & 153 & 8 & (s) & 161 \\
\hline 2001 & 204 & 3 & 439 & 95 & 2 & 49 & 6 & 21 & 77 & 14 & 132 & 395 & 667 & 1,709 & 135 & 12 & (s) & 147 \\
\hline 2002 & 188 & 7 & R448 & 88 & $\overline{1}$ & 54 & 6 & 22 & 76 & 13 & 127 & 388 & 654 & $\mathrm{R}_{1,685}$ & 131 & 13 & (s) & 144 \\
\hline 2003 & 190 & 6 & 430 & 83 & 2 & 50 & 6 & 23 & 76 & 15 & 140 & 394 & 672 & 1,692 & 128 & 13 & (s) & 141 \\
\hline 2004 & 191 & 16 & ${ }^{R} 432$ & 88 & 2 & 55 & 6 & 26 & 82 & 17 & 142 & 419 & 675 & $R_{1}, 732$ & 138 & 12 & (s) & 151 \\
\hline 2005 & 183 & 5 & 398 & 92 & 3 & 51 & 6 & 25 & 80 & 20 & 141 & 417 & 673 & 1,675 & 136 & 13 & (s) & 150 \\
\hline 2006 & 179 & 7 & $\mathrm{R}_{395}$ & 92 & 2 & 56 & 6 & 26 & 82 & 16 & 150 & 430 & 650 & $\mathrm{R}_{1,662}$ & 138 & 12 & 1 & 151 \\
\hline 2007 & 175 & 3 & $\mathrm{R}_{405}$ & 92 & 1 & 54 & 6 & 21 & 80 & 13 & 148 & 415 & 662 & $\mathrm{R}_{1,661}$ & R132 & 13 & 1 & 146 \\
\hline 2008 & 168 & 5 & 407 & 93 & (s) & 42 & 6 & 17 & 76 & 14 & 130 & 377 & 642 & $\begin{array}{r}R \\
R\end{array}$ & 126 & 13 & 1 & 140 \\
\hline 2009 & 131 & -3 & 383 & 80 & (s) & 46 & 5 & 17 & 73 & 7 & 111 & 339 & 551 & 1,401 & R113 & 14 & 1 & $\mathrm{R}_{128}$ \\
\hline 2010 & R154 & -1 & R401 & R86 & $R_{1}$ & R50 & 6 & $\mathrm{R}_{19}$ & 62 & 8 & $R_{120}$ & R352 & R587 & $\mathrm{R}_{1,494}$ & $R_{122}$ & 15 & 1 & 139 \\
\hline $2011^{P}$ & 150 & 1 & 419 & 88 & (s) & 48 & 5 & 18 & 62 & 8 & 116 & 345 & 567 & 1,482 & 123 & 16 & 1 & 140 \\
\hline
\end{tabular}

${ }_{1}^{1}$ Metric tons of carbon dioxide can be converted to metric tons of carbon equivalent by multiplying by $12 / 44$

2 Carbon dioxide emissions from biomass energy consumption are excluded from total emissions in this table. See Note, "Accounting for Carbon Dioxide Emissions From Biomass Energy Combustion," at end o section.

Natural gas, excluding supplemental gaseous fuels.

4 Distillate fuel oil, excluding biodiesel.

5 Liquefied petroleum gases.

Aviation gasoline blending components, crude oil, motor gasoline blending components, pentanes plus, petrochemical feedstocks, special naphthas, still gas, unfinished oils, waxes, and miscellaneous petroleum products.

Emissions from energy consumption (for electricity and a small amount of useful thermal output) in the electric power sector are allocated to the end-use sectors in proportion to each sector's share of tota
9 Wood and wood-derived fuels.

10 Municipal solid waste from biogenic sources, landfill gas, sludge waste, agricultural byproducts, and other biomass.

Ruel ethanol minus denaturant.

$R=$ Revised. $P=$ Preliminary. $N A=$ Not available. $(\mathrm{s})=$ Less than 0.5 and greater than -0.5 million metric ons of carbon dioxide.

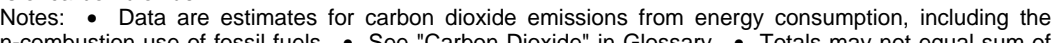
of fossil fuels. - See "Carbon Dioxide" in Glossary. • Totals may not equal sum of (c)

Web Pages: - See http://www.eia.gov/totalenergy/data/monthly/\#environment for updated monthly and annual data. - See http://www.eia.gov/totalenergy/data/annual/\#environment for all annual data beginning

in 1949. - See http://Www.eia. gov/environment/ for related information.
Sources: - 1949-1972- U.S. Energy Information Administration (EIA) estimates based on data in Annual Energy Review Tables 2.1d, 5.14b, 8.9, 10.2b, and 11.2e. • 1973 forward-ElA, Monthly Energy 
Table 11.2d Carbon Dioxide Emissions From Energy Consumption: Transportation Sector, Selected Years, 1949-2011 (Million Metric Tons of Carbon Dioxide ${ }^{1}$ )

\begin{tabular}{|c|c|c|c|c|c|c|c|c|c|c|c|c|c|c|c|}
\hline \multirow[b]{2}{*}{ Year } & \multirow[b]{2}{*}{ Coal } & \multirow[b]{2}{*}{$\begin{array}{c}\text { Natural } \\
\text { Gas }^{3}\end{array}$} & \multicolumn{8}{|c|}{ Petroleum } & \multirow{2}{*}{$\begin{array}{c}\text { Retail } \\
\text { Elec- } \\
\text { tricity } 7\end{array}$} & \multirow[b]{2}{*}{ Total $^{2}$} & \multicolumn{3}{|c|}{ Biomass $^{2}$} \\
\hline & & & $\begin{array}{l}\text { Aviation } \\
\text { Gasoline }\end{array}$ & $\begin{array}{l}\text { Distillate } \\
\text { Fuel Oil }{ }^{4}\end{array}$ & $\begin{array}{l}\text { Jet } \\
\text { Fuel }\end{array}$ & LPG 5 & Lubricants & $\begin{array}{c}\text { Motor } \\
\text { Gasoline }\end{array}$ & $\begin{array}{l}\text { Residual } \\
\text { Fuel Oil }\end{array}$ & Total & & & $\begin{array}{c}\text { Fuel } \\
\text { Ethanol }^{8}\end{array}$ & Biodiesel & Total \\
\hline 1949 & 161 & NA & 12 & 30 & NA & (s) & 4 & 306 & 91 & 443 & 6 & 611 & $N A$ & $N A$ & $N A$ \\
\hline 1950 & 146 & 7 & 14 & 35 & NA & (s) & 5 & 332 & 95 & 481 & 6 & 640 & $N A$ & $N A$ & $N A$ \\
\hline 1955 & 39 & 13 & 24 & 58 & 21 & 1 & 6 & 439 & 80 & 629 & 5 & 687 & $N A$ & $N A$ & $N A$ \\
\hline 1960 & 7 & 19 & 21 & 65 & 53 & 1 & 6 & 511 & 66 & 723 & 2 & 751 & $N A$ & $N A$ & $N A$ \\
\hline 1965 & 1 & 27 & 15 & 80 & 87 & 2 & 6 & 597 & 61 & 847 & 2 & 878 & $N A$ & $N A$ & $N A$ \\
\hline 1970 & 1 & 40 & 7 & 115 & 141 & 3 & 5 & 763 & 60 & 1,093 & 2 & 1,136 & $N A$ & $N A$ & $N A$ \\
\hline 1975 & (s) & 32 & 5 & 155 & 145 & 3 & 6 & 889 & 56 & 1,258 & 2 & 1,292 & $N A$ & $N A$ & $N A$ \\
\hline 1976 & (s) & 30 & 5 & 167 & 143 & 3 & 6 & 933 & 65 & 1,322 & 2 & 1,354 & $N A$ & $N A$ & $N A$ \\
\hline 1977 & (s) & 29 & 5 & 182 & 149 & 3 & 6 & 958 & 72 & 1,375 & 2 & 1,406 & $N A$ & $N A$ & $N A$ \\
\hline 1978 & $\left({ }^{9}\right)$ & 29 & 5 & 196 & 153 & 3 & 7 & 991 & 78 & 1,433 & 2 & 1,464 & $N A$ & $N A$ & $N A$ \\
\hline 1979 & $\left({ }^{9}\right)$ & 32 & 5 & 213 & 156 & 1 & 7 & 941 & 97 & 1,420 & 2 & 1,454 & $N A$ & $N A$ & $N A$ \\
\hline 1980 & $\left({ }^{9}\right)$ & 34 & 4 & 204 & 155 & 1 & 6 & 881 & 110 & 1,363 & 2 & 1,400 & $N A$ & $N A$ & $N A$ \\
\hline 1981 & $\left({ }^{9}\right)$ & 35 & 4 & 212 & 147 & 2 & 6 & 881 & 96 & 1,348 & 2 & 1,385 & (s) & $N A$ & (s) \\
\hline 1982 & $\left(9^{\prime}\right)$ & 32 & 3 & 204 & 148 & 2 & 6 & 876 & 80 & 1,319 & 2 & 1,354 & 1 & $N A$ & 1 \\
\hline 1983 & $\left({ }^{9}\right)$ & 27 & 3 & 213 & 153 & 3 & 6 & 888 & 65 & 1,330 & 3 & 1,359 & 2 & $N A$ & 2 \\
\hline 1984 & $\left({ }^{9}\right)$ & 29 & 3 & 216 & 172 & 3 & 6 & 895 & 64 & 1,358 & 3 & 1,390 & 3 & $N A$ & 3 \\
\hline 1985 & $\left.\left({ }^{9}\right)^{\prime}\right)$ & 28 & 3 & 232 & 178 & 2 & 6 & 908 & 62 & 1,391 & 3 & 1,421 & 3 & $N A$ & 3 \\
\hline 1986 & $\left({ }^{9}\right)$ & 26 & 4 & 235 & 191 & 2 & 6 & 936 & 69 & 1,443 & 3 & 1,472 & 4 & $N A$ & 4 \\
\hline 1987 & $\left({ }^{9}\right)$ & 28 & 3 & 244 & 202 & 1 & 6 & 959 & 71 & 1,487 & 3 & 1,519 & 5 & $N A$ & 5 \\
\hline 1988 & $\left({ }^{9}\right)$ & 34 & 3 & 265 & 212 & 1 & 6 & 981 & 72 & 1,542 & 3 & 1,579 & 5 & $N A$ & 5 \\
\hline 1989 & $\left({ }^{9}\right)$ & 34 & 3 & 270 & 218 & 1 & 6 & 979 & 77 & 1,554 & 3 & 1,591 & 5 & $N A$ & 5 \\
\hline 1990 & $\left({ }^{9}\right)$ & 36 & 3 & 268 & 223 & 1 & 7 & 967 & 80 & 1,548 & 3 & 1,588 & 4 & $N A$ & 4 \\
\hline 1991 & $\left({ }^{9}\right)$ & 33 & 3 & 263 & 215 & 1 & 6 & 962 & 81 & 1,532 & 3 & 1,568 & 5 & $N A$ & 5 \\
\hline 1992 & $\left({ }^{9}\right)$ & 32 & 3 & 269 & 213 & 1 & 6 & 979 & 84 & 1,556 & 3 & 1,592 & 5 & $N A$ & 5 \\
\hline 1993 & $\left({ }^{9}\right)$ & 34 & 3 & 278 & 215 & 1 & 6 & 1,000 & 71 & 1,574 & 3 & 1,611 & 6 & $N A$ & 6 \\
\hline 1994 & $\left({ }^{9}\right)$ & 38 & 3 & 295 & 224 & 2 & 6 & 1,007 & 70 & 1,607 & 3 & 1,647 & 7 & $N A$ & 7 \\
\hline 1995 & $\left({ }^{9}\right)$ & 38 & 3 & 307 & 222 & 1 & 6 & 1,029 & 72 & 1,639 & 3 & 1,681 & 8 & $N A$ & 8 \\
\hline 1996 & $\left({ }^{9}\right)$ & 39 & 3 & 327 & 232 & 1 & 6 & 1,047 & 67 & 1,683 & 3 & 1,725 & 6 & $N A$ & 6 \\
\hline 1997 & $\left({ }^{9}\right)$ & 41 & 3 & 342 & 234 & 1 & 6 & 1,057 & 56 & 1,699 & 3 & 1,744 & 7 & $N A$ & 7 \\
\hline 1998 & $\left({ }^{9}\right)$ & 35 & 2 & 352 & 238 & 1 & 7 & 1,090 & 53 & 1,743 & 3 & 1,782 & 8 & $N A$ & 8 \\
\hline 1999 & $\left({ }^{9}\right)$ & 36 & 3 & 366 & 245 & 1 & 7 & 1,115 & 52 & 1,789 & 3 & 1,828 & 8 & $N A$ & 8 \\
\hline 2000 & $\left({ }^{9}\right)$ & 36 & 3 & 378 & 254 & 1 & 7 & 1,121 & 70 & 1,833 & 4 & 1,872 & 9 & $N A$ & 9 \\
\hline 2001 & $\left({ }^{9}\right)$ & 35 & 2 & 387 & 243 & 1 & 6 & 1,127 & 46 & 1,813 & 4 & 1,852 & 10 & $(s)$ & 10 \\
\hline 2002 & $\left({ }^{9}\right)$ & 37 & 2 & 394 & 237 & 1 & 6 & 1,158 & 53 & 1,851 & 4 & 1,892 & 11 & (s) & 12 \\
\hline 2003 & $\left({ }^{9}\right)$ & 33 & 2 & 414 & 231 & 1 & 6 & 1,161 & 45 & 1,861 & 5 & 1,899 & 16 & (s) & 16 \\
\hline 2004 & $\left({ }^{9}\right)$ & 32 & 2 & 434 & 240 & 1 & 6 & 1,185 & 58 & 1,926 & 5 & 1,962 & 20 & (s) & 20 \\
\hline 2005 & $\left({ }^{9}\right)$ & 33 & 2 & 444 & 246 & 2 & 6 & 1,186 & 66 & 1,953 & 5 & 1,991 & 22 & 1 & 23 \\
\hline 2006 & $\left({ }^{9}\right)^{\prime}$ & 33 & 2 & 469 & 240 & 2 & 5 & 1,194 & 71 & 1,984 & 5 & 2,022 & 30 & 2 & 33 \\
\hline 2007 & $\left({ }^{9}\right)$ & 35 & 2 & 472 & 238 & 1 & 6 & 1,201 & 78 & 1,999 & 5 & 2,040 & 38 & 3 & ${ }^{R} 41$ \\
\hline 2008 & $\left({ }^{9}\right)$ & 37 & 2 & 440 & 226 & 3 & 5 & 1,146 & 72 & 1,895 & 5 & 1,937 & 54 & 3 & 57 \\
\hline 2009 & $\left({ }^{9}\right)$ & $\mathrm{R}_{38}$ & 2 & 404 & 204 & 2 & 5 & 1,137 & 64 & 1,818 & 5 & $\mathrm{R}_{1,860}$ & 61 & 3 & 64 \\
\hline 2010 & $\left({ }^{9}\right)$ & $\mathrm{R}_{38}$ & 2 & $\mathrm{R}_{4} 25$ & $\mathrm{R}_{210}$ & 2 & 5 & $R_{1,124}$ & $\begin{array}{l}R_{69} \\
\text { R }\end{array}$ & $R_{1,836}$ & 5 & $\mathrm{R}_{1,879}$ & $\mathrm{R}_{71}$ & 2 & R74 \\
\hline $2011^{\mathrm{P}}$ & $(9)$ & 39 & 2 & 430 & 209 & 2 & 5 & 1,089 & 65 & 1,802 & 4 & 1,845 & 71 & 8 & 80 \\
\hline
\end{tabular}

${ }_{1}^{1}$ Metric tons of carbon dioxide can be converted to metric tons of carbon equivalent by multiplying by $2 / 44$.

2 Carbon dioxide emissions from biomass energy consumption are excluded from total emissions in this table. See Note, "Accounting for Carbon Dioxide Emissions From Biomass Energy Combustion," at end of

Natural gas, excluding supplemental gaseous fuels.

Listillate fuel oil, excluding biod

Finished motor gasoline, excluding fuel ethanol.

Emissions from energy consumplion (for electricity and a small amount of useful thermal output) in the electric power sector are allocated to the end-use sectors in proportion to each sector's share of total 8 Fuet ethan sales. See Tables 8.9 and $11.2 \mathrm{e}$.
${ }^{9}$ Beginning in 1978, the small amounts of coal consumed for transportation are reported as industria sector consumption.

Preliminary. NA=Not available. (s)=Less than 0.5 million metric tons of carbon dioxide. non-combustion use of fossil fuels. - See "Carbon Dioxide" in Glossary. - Totals may not equal sum of components due to independent rounding.

Web Pages: - See http://www.eia.gov/totalenergy/data/monthly/\#environment for updated monthly and annual data. - See http://www.eia.gov/totalenergy/data/annual/\#environment for all annual data beginning in 1949. - See $h$ ttp: $: / / w w w . e i a . g o v / e n v i r o n m e n t /$ for related information.

Sources: - 1949-1972-U.S. Energy Information Administration (EIA) estimates based on data in Annual Energy Review Tables 2.1e, 5.14c, 8.9, 10.2b, and 11.2e. - 1973 forward-EIA, Monthly Energy
Review (MER) (May 2012), Tables 12.5 and 12.7, and MER data system calculations. 
Table 11.2e Carbon Dioxide Emissions From Energy Consumption: Electric Power Sector, Selected Years, 1949-2011 (Million Metric Tons of Carbon Dioxide ${ }^{1}$ )

\begin{tabular}{|c|c|c|c|c|c|c|c|c|c|c|c|c|}
\hline \multirow[b]{2}{*}{ Year } & \multirow[b]{2}{*}{ Coal } & \multirow[b]{2}{*}{$\begin{array}{c}\text { Natural } \\
\text { Gas }^{3}\end{array}$} & \multicolumn{4}{|c|}{ Petroleum } & \multirow[b]{2}{*}{$\begin{array}{c}\text { Geo- } \\
\text { thermal }\end{array}$} & \multirow{2}{*}{$\begin{array}{c}\text { Non- } \\
\text { Biomass } \\
\text { Waste }{ }^{5}\end{array}$} & \multirow[b]{2}{*}{ Total $^{2}$} & \multicolumn{3}{|c|}{ Biomass $^{2}$} \\
\hline & & & $\begin{array}{l}\text { Distillate } \\
\text { Fuel Oil }{ }^{4}\end{array}$ & $\begin{array}{l}\text { Petroleum } \\
\text { Coke }\end{array}$ & $\begin{array}{c}\text { Residual } \\
\text { Fuel Oil }\end{array}$ & Total & & & & Wood $^{6}$ & Waste $^{7}$ & Total \\
\hline 1949 & 187 & 30 & 2 & NA & 30 & 33 & NA & NA & 250 & 1 & $N A$ & 1 \\
\hline 1950 & 206 & 35 & 2 & NA & 35 & 37 & NA & NA & 278 & 1 & $N A$ & 1 \\
\hline 1955 & 324 & 63 & 2 & NA & 35 & 37 & NA & NA & 424 & (s) & $N A$ & (s) \\
\hline 1960 & 396 & 95 & 2 & NA & 42 & 43 & NA & NA & 535 & (s) & $N A$ & (s) \\
\hline 1965 & 546 & 127 & 2 & NA & 55 & 57 & NA & NA & 730 & (s) & $N A$ & (s) \\
\hline 1970 & 678 & 215 & 10 & 2 & 154 & 166 & NA & NA & 1,059 & (s) & (s) & (s) \\
\hline 1975 & 824 & 172 & 17 & (s) & 231 & 248 & NA & NA & 1,244 & (s) & (s) & (s) \\
\hline 1976 & 911 & 167 & 18 & (s) & 255 & 273 & NA & NA & 1,351 & (s) & (s) & (s) \\
\hline 1977 & 962 & 174 & 21 & (s) & 285 & 306 & NA & NA & 1,442 & (s) & (s) & (s) \\
\hline 1978 & 960 & 175 & 20 & 1 & 291 & 313 & NA & NA & 1,448 & (s) & (s) & (s) \\
\hline 1979 & 1,056 & 192 & 13 & 1 & 244 & 258 & NA & NA & 1,505 & $\begin{array}{l}\text { (s) } \\
\text { (s) }\end{array}$ & $\begin{array}{l}\text { (o) } \\
\text { (s) }\end{array}$ & (s) \\
\hline 1980 & 1,137 & 200 & 12 & 1 & 194 & 207 & NA & NA & 1,544 & (s) & (s) & (s) \\
\hline 1981 & 1,180 & 198 & 9 & (s) & 163 & 173 & NA & NA & 1,551 & (s) & (s) & (s) \\
\hline 1982 & 1,182 & 176 & 7 & (s) & 116 & 123 & NA & NA & 1,481 & (s) & (s) & (s) \\
\hline 1983 & 1,242 & 158 & 7 & 1 & 113 & 121 & NA & NA & 1,521 & (s) & (s) & (s) \\
\hline 1984 & 1,318 & 170 & 6 & 1 & 94 & 101 & NA & NA & 1,588 & (s) & (s) & 1 \\
\hline 1985 & 1,367 & 166 & 6 & 1 & 79 & 86 & NA & NA & 1,619 & 1 & (s) & 1 \\
\hline 1986 & 1,357 & 142 & 6 & 1 & 107 & 114 & NA & NA & 1,613 & (s) & $\begin{array}{l}\text { (D) } \\
\text { (s) }\end{array}$ & 1 \\
\hline 1987 & 1,427 & 155 & 7 & 1 & 91 & 99 & NA & NA & 1,680 & 1 & (s) & 1 \\
\hline 1988 & 1,492 & 143 & 8 & 1 & 114 & 123 & NA & NA & 1,758 & 1 & (s) & 1 \\
\hline 1989 & 1,519 & 168 & 11 & 2 & 121 & 134 & (s) & 4 & 1,826 & 9 & 8 & 17 \\
\hline 1990 & 1,548 & 176 & 7 & 3 & 92 & 102 & (s) & 6 & $\begin{array}{l}1,831 \\
\end{array}$ & 12 & 11 & 23 \\
\hline $\begin{array}{l}1991 \\
\end{array}$ & $\begin{array}{l}1,548 \\
\end{array}$ & 179 & 6 & 3 & 86 & 95 & (s) & 7 & 1,830 & 12 & 13 & 25 \\
\hline 1992 & 1,570 & 186 & 5 & 5 & 69 & 79 & (s) & 8 & 1,843 & 13 & 15 & 28 \\
\hline 1993 & 1,633 & 188 & 6 & 8 & 76 & 90 & (s) & 9 & 1.919 & 14 & 15 & 29 \\
\hline 1994 & 1,639 & 211 & 9 & 7 & 68 & 84 & (s) & 9 & 1,944 & 14 & 16 & 30 \\
\hline 1995 & 1,661 & 228 & 8 & 8 & 45 & 61 & (s) & 10 & 1,960 & 12 & 17 & 28 \\
\hline 1996 & 1,752 & 205 & 8 & 8 & 50 & 66 & (s) & 10 & 2,033 & 13 & 17 & 30 \\
\hline 1997 & 1,797 & 219 & 8 & 10 & 56 & 75 & (s) & 10 & 2,101 & 13 & 17 & 30 \\
\hline 1998 & $\begin{array}{l}1,828 \\
\end{array}$ & 248 & 10 & 13 & 82 & 105 & (s) & 10 & 2,192 & 13 & 17 & 30 \\
\hline 1999 & 1,836 & 260 & 10 & 11 & 76 & 97 & (s) & 10 & 2,204 & 13 & 17 & 30 \\
\hline 2000 & 1,927 & 281 & 13 & 10 & 69 & 91 & (s) & 10 & 2,310 & 13 & 17 & 29 \\
\hline 2001 & 1,870 & 290 & 12 & 11 & 79 & 102 & (s) & 11 & 2,273 & 12 & 19 & 31 \\
\hline 2002 & 1,890 & 306 & 9 & 18 & 52 & 79 & (s) & 13 & 2,288 & 14 & 21 & 35 \\
\hline 2003 & 1,931 & 278 & 12 & 18 & 69 & 98 & (s) & 11 & 2,319 & 16 & 21 & 37 \\
\hline 2004 & 1,943 & 297 & 8 & 23 & 69 & 100 & (s) & 11 & 2,352 & 15 & 20 & 36 \\
\hline 2005 & 1,984 & 319 & 8 & 25 & 69 & 102 & (s) & 11 & 2,417 & 17 & 20 & 37 \\
\hline 2006 & 1,954 & 338 & 5 & 22 & 28 & 56 & (s) & 12 & 2,359 & 17 & 21 & 38 \\
\hline 2007 & $\begin{array}{l}1,987 \\
\end{array}$ & 372 & 7 & 17 & 31 & 55 & (s) & 11 & 2,426 & 17 & 22 & 39 \\
\hline 2008 & 1,959 & 362 & 5 & 16 & 19 & 40 & (s) & 12 & 2,374 & 17 & 23 & 40 \\
\hline 2009 & 1,741 & 373 & 5 & 14 & 14 & 34 & (s) & 11 & 2,159 & 17 & 24 & 41 \\
\hline 2010 & 1,828 & 399 & 6 & 15 & 12 & 33 & (s) & 11 & 2,271 & 18 & R24 & $\mathrm{R}_{42}$ \\
\hline $2011^{P}$ & $\begin{array}{l}1,718 \\
\end{array}$ & 411 & 5 & 14 & 7 & 25 & (s) & 11 & 2,166 & 16 & 24 & 41 \\
\hline
\end{tabular}

1 Metric tons of carbon dioxide can be converted to metric tons of carbon equivalent by multiplying by $12 / 44$.

2 Carbon dioxide emissions from biomass energy consumption are excluded from total emissions in this table. See Note, "Accounting for Carbon Dioxide Emissions From Biomass Energy Combustion," at end o section.

Natural gas, excluding supplemental gaseous fuels.

Distillate fuel oil, excluding biodiesel.

Municipal solid waste from non-biogenic sources, and tire-derived fuels.

other biomass.

$\mathrm{R}=$ Revised. $\mathrm{P}=$ Preliminary. $\mathrm{NA}=\mathrm{Not}$ available. $(\mathrm{s})=$ Less than 0.5 million metric tons of carbon dioxide. Notes: - Data are estimates for carbon dioxide emissions from energy consumption. - See "Carbon Dioxide" in Glossary. - Totals may not equal sum of components due to independent rounding.

Web Pages: - See http://www.eia.gov/totalenergy/data/monthly/\#environment for updated monthly and annual data. - See http://www.eia.gov/totalenergy/data/annual/\#environment for all annual data beginning in 1949. - See http://www.eia.gov/environment/ for related information.

Sources: - 1949-1972-U.S. Energy Information Administration (EIA) estimates based on data in Annual Energy Review Tables 2.1f, 5.14c, and 10.2c. • 1973 forward-EIA, Monthly Energy Review 


\section{Figure 11.3 Methane Emissions}

\section{Total, 1980-2009}

$40-$
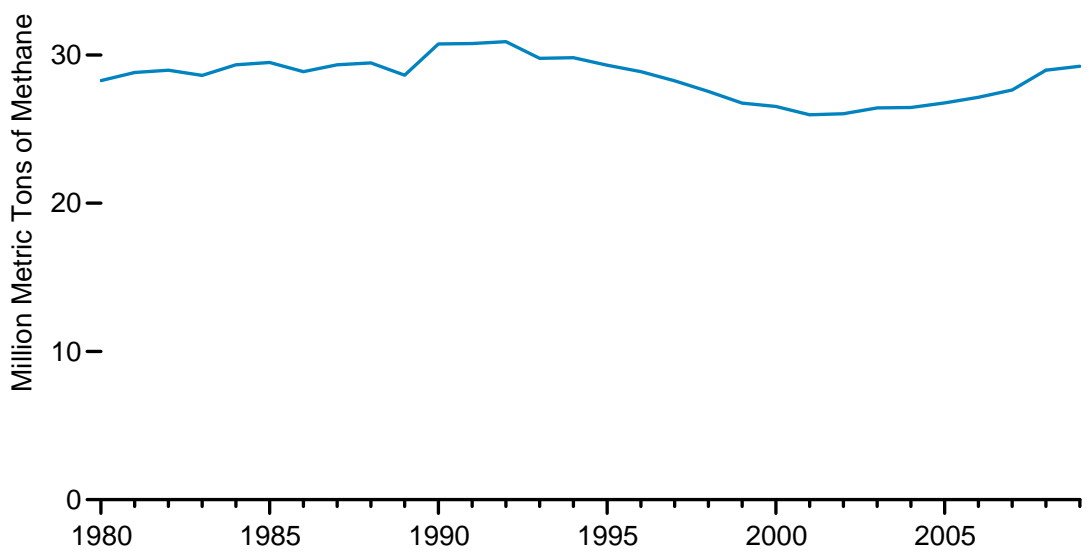

\section{Energy Sources by Type 1980-2009}

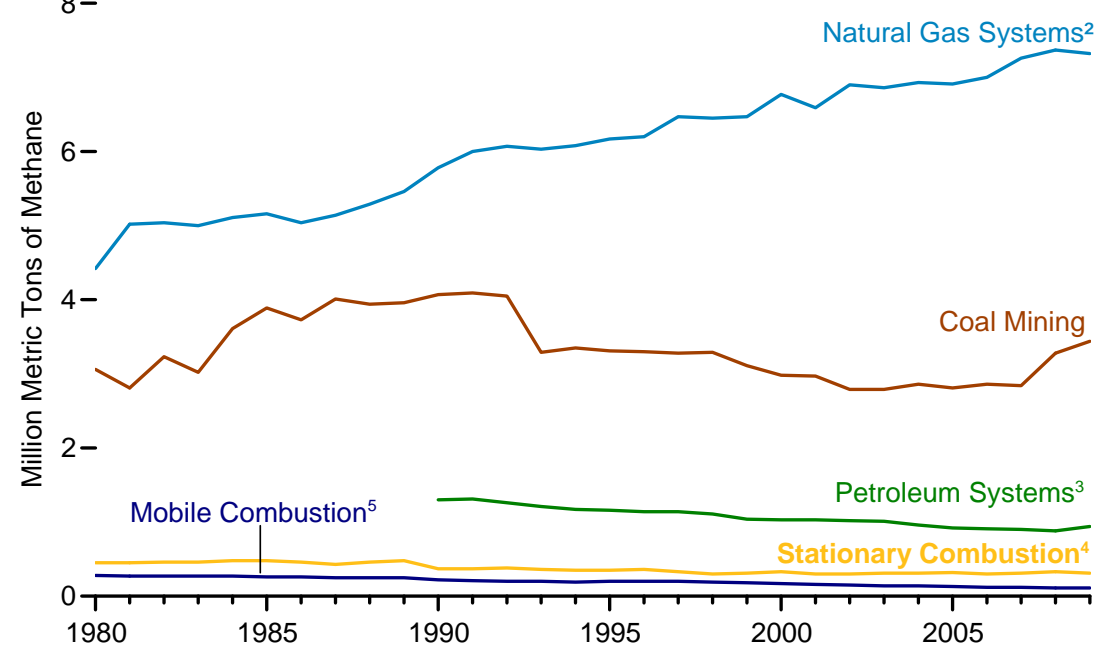

${ }^{1}$ Chemical production, and iron and steel production

${ }^{2}$ Natural gas production, processing, and distribution.

${ }^{3}$ Petroleum production, refining, and distribution.

${ }^{4}$ Consumption of coal, petroleum, natural gas, and wood for heat or electricity.

\section{By Source, 2009}

$15-$

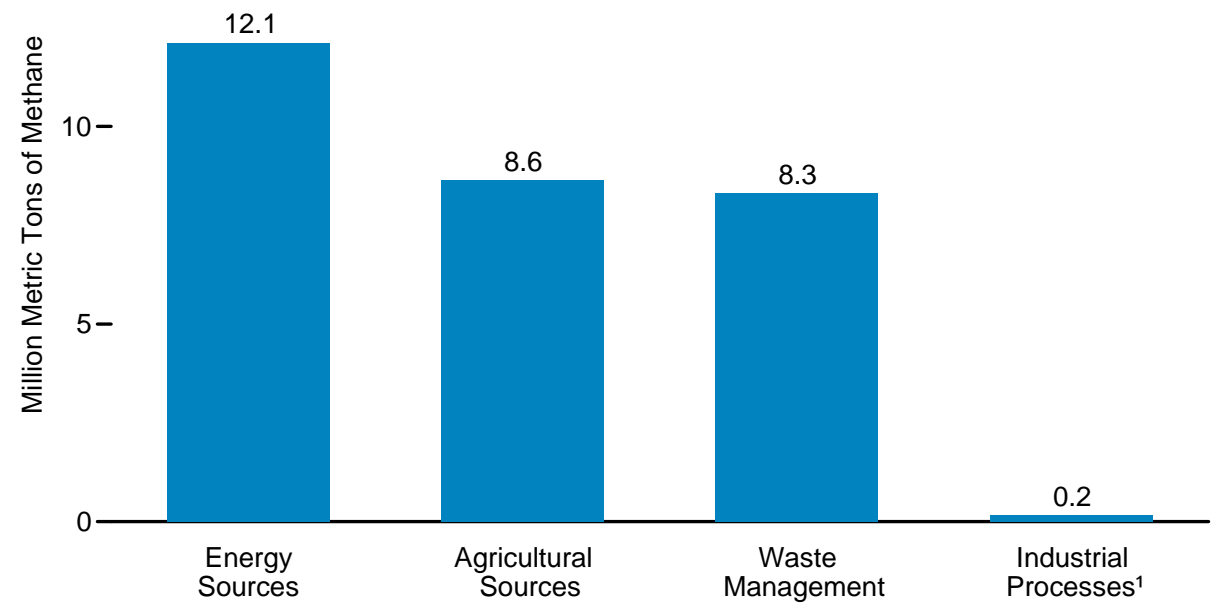

\section{Agricultural Sources by Major Type, 1980-2009}

8-

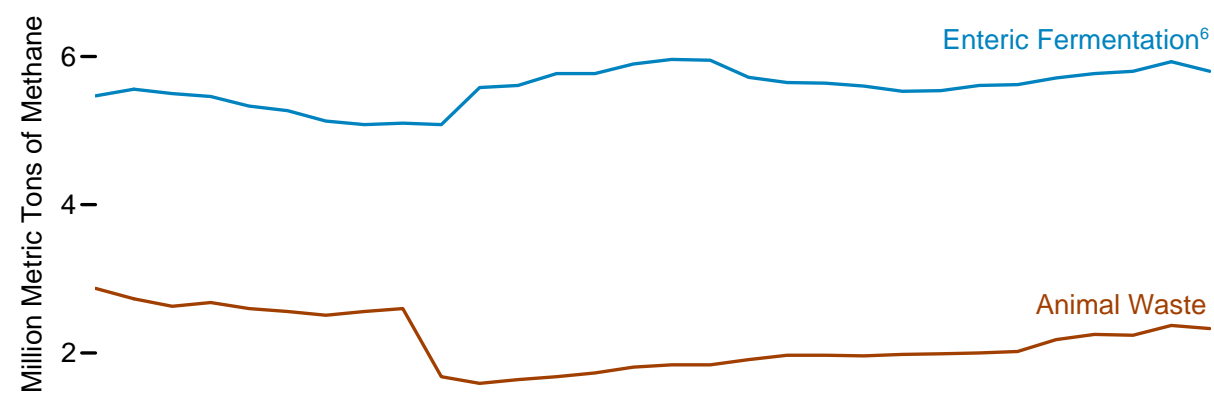

Rice Cultivation

\begin{tabular}{|c|c|c|c|c|c|}
\hline 1980 & 1985 & 1990 & 1995 & 2000 & 2005 \\
\hline
\end{tabular}

${ }^{5}$ Emissions from passenger cars, trucks, buses, motorcycles, and other transport.

${ }^{6}$ Methane emitted as a product of digestion in animals such as cattle, sheep, goats, and swine.

Source: Table 11.3 
Table 11.3 Methane Emissions, 1980-2009

(Million Metric Tons of Methane)

\begin{tabular}{|c|c|c|c|c|c|c|c|c|c|c|c|c|c|c|c|c|}
\hline \multirow[b]{2}{*}{ Year } & \multicolumn{6}{|c|}{ Energy Sources } & \multicolumn{3}{|c|}{ Waste Management } & \multicolumn{5}{|c|}{ Agricultural Sources } & \multirow[b]{2}{*}{$\begin{array}{c}\text { Industrial } \\
\text { Processes }^{9}\end{array}$} & \multirow[b]{2}{*}{ Total $^{5}$} \\
\hline & $\begin{array}{l}\text { Coal } \\
\text { Mining }\end{array}$ & $\begin{array}{c}\text { Natural } \\
\text { Gas } \\
\text { Systems }{ }^{1}\end{array}$ & $\begin{array}{l}\text { Petroleum } \\
\text { Systems }{ }^{2}\end{array}$ & $\begin{array}{c}\text { Mobile } \\
\text { Com- } \\
\text { bustion }{ }^{3}\end{array}$ & $\begin{array}{c}\text { Stationary } \\
\text { Com- } \\
\text { bustion }{ }^{4}\end{array}$ & Total $^{5}$ & Landfills & $\begin{array}{c}\text { Waste- } \\
\text { water } \\
\text { Treatment }{ }^{6}\end{array}$ & Total 5 & $\begin{array}{c}\text { Enteric } \\
\text { Fermen- } \\
\text { tation }^{7}\end{array}$ & $\begin{array}{l}\text { Animal } \\
\text { Waste }^{8}\end{array}$ & $\begin{array}{c}\text { Rice } \\
\text { Cultivation }\end{array}$ & $\begin{array}{c}\text { Crop } \\
\text { Residue } \\
\text { Burning }\end{array}$ & Total $^{5}$ & & \\
\hline 1980 & 3.06 & 4.42 & NA & 0.28 & 0.45 & 8.20 & 10.52 & 0.52 & 11.04 & 5.47 & 2.87 & 0.48 & 0.04 & 8.86 & 0.17 & 28.27 \\
\hline 1981 & 2.81 & 5.02 & NA & .27 & .45 & 8.55 & 10.69 & .53 & 11.22 & 5.56 & 2.73 & .54 & .05 & 8.88 & .18 & 28.82 \\
\hline 1982 & 3.23 & 5.04 & NA & .27 & .46 & 9.01 & 10.63 & .54 & 11.17 & 5.50 & 2.63 & .47 & .05 & 8.65 & .13 & 28.97 \\
\hline 1983 & 3.02 & 5.00 & NA & .27 & .46 & 8.76 & 10.67 & .54 & 11.21 & 5.46 & 2.68 & .31 & .04 & 8.49 & .15 & 28.62 \\
\hline 1984 & 3.61 & 5.11 & NA & .27 & .48 & 9.46 & 10.68 & .66 & 11.33 & 5.33 & 2.60 & .40 & .05 & 8.38 & .16 & 29.34 \\
\hline 1985 & 3.89 & 5.16 & NA & .26 & .48 & 9.79 & 10.65 & .67 & 11.32 & 5.27 & 2.56 & .36 & .05 & 8.23 & .15 & 29.49 \\
\hline 1986 & 3.73 & 5.04 & NA & .26 & .46 & 9.48 & 10.53 & .67 & 11.20 & 5.13 & 2.51 & .34 & .04 & 8.02 & .16 & 28.87 \\
\hline 1987 & 4.01 & 5.14 & NA & .25 & .43 & 9.85 & 10.63 & .68 & 11.31 & 5.08 & 2.56 & .33 & .04 & 8.02 & .17 & 29.34 \\
\hline 1988 & 3.94 & 5.29 & NA & .25 & .46 & 9.95 & 10.51 & .69 & 11.20 & 5.10 & 2.60 & .41 & .05 & 8.14 & .18 & 29.47 \\
\hline 1989 & 3.96 & 5.46 & NA & .25 & .48 & 10.15 & 10.43 & .70 & $\begin{array}{l}11.13 \\
\end{array}$ & 5.08 & 1.68 & .38 & .05 & 7.18 & .18 & 28.64 \\
\hline 1990 & 4.07 & 5.78 & 1.30 & .22 & .37 & 11.72 & 10.31 & .91 & 11.23 & 5.58 & 1.59 & .40 & .05 & 7.62 & .18 & 30.75 \\
\hline 1991 & 4.09 & 6.00 & 1.31 & .21 & .37 & 11.98 & 10.00 & .93 & 10.93 & 5.61 & 1.64 & .40 & .05 & 7.69 & .19 & 30.78 \\
\hline 1992 & 4.05 & 6.07 & 1.26 & .20 & .38 & 11.97 & 9.84 & .95 & 10.79 & 5.77 & 1.68 & .45 & .05 & 7.95 & .19 & 30.90 \\
\hline 1993 & 3.29 & 6.03 & 1.21 & .20 & .36 & 11.08 & 9.58 & .96 & 10.54 & 5.77 & 1.73 & .41 & .04 & 7.96 & .20 & 29.77 \\
\hline 1994 & 3.35 & 6.08 & 1.17 & .19 & .35 & 11.15 & 9.25 & .98 & 10.23 & 5.90 & 1.81 & .48 & .05 & 8.23 & .21 & 29.82 \\
\hline 1995 & 3.31 & 6.17 & 1.16 & .20 & .35 & 11.20 & 8.62 & 1.00 & 9.61 & 5.96 & 1.84 & .44 & .05 & 8.28 & .22 & 29.31 \\
\hline 1996 & 3.30 & 6.20 & 1.14 & .20 & .36 & 11.20 & 8.19 & 1.01 & 9.19 & 5.95 & 1.84 & .41 & .05 & 8.25 & .22 & 28.87 \\
\hline 1997 & 3.28 & 6.47 & 1.14 & .20 & .33 & 11.42 & 7.45 & 1.02 & 8.47 & 5.72 & 1.91 & .45 & .05 & 8.13 & .23 & 28.26 \\
\hline 1998 & 3.29 & 6.45 & 1.11 & .19 & .30 & 11.34 & 6.80 & 1.03 & 7.83 & 5.65 & 1.97 & .47 & .05 & 8.14 & .23 & 27.54 \\
\hline 1999 & 3.11 & 6.47 & 1.04 & .18 & .31 & 11.11 & 6.21 & 1.05 & 7.25 & 5.64 & 1.97 & .50 & .05 & 8.16 & .24 & 26.76 \\
\hline 2000 & 2.98 & 6.77 & 1.03 & .17 & .33 & 11.27 & 5.93 & 1.05 & 6.98 & 5.60 & 1.96 & .44 & .05 & 8.05 & .22 & 26.53 \\
\hline 2001 & 2.97 & 6.59 & 1.03 & .16 & .30 & 11.05 & 5.65 & 1.05 & 6.70 & 5.53 & 1.98 & .47 & .05 & 8.02 & .20 & 25.97 \\
\hline 2002 & 2.79 & 6.90 & 1.02 & .15 & .30 & 11.16 & 5.58 & 1.06 & 6.64 & 5.54 & 1.99 & .44 & .05 & 8.03 & .21 & 26.03 \\
\hline 2003 & 2.79 & 6.86 & 1.01 & .14 & .31 & 11.11 & 5.97 & 1.06 & 7.03 & 5.61 & 2.00 & .43 & .05 & 8.08 & .20 & 26.43 \\
\hline 2004 & 2.86 & 6.93 & .96 & .14 & .31 & 11.20 & 5.80 & 1.07 & 6.88 & 5.62 & 2.02 & .47 & .05 & 8.16 & .22 & 26.46 \\
\hline 2005 & 2.81 & 6.91 & .92 & .13 & .32 & 11.08 & 6.02 & 1.08 & 7.09 & 5.71 & 2.18 & .46 & .05 & 8.40 & .20 & 26.77 \\
\hline 2006 & 2.86 & 7.00 & .91 & .12 & .30 & 11.19 & 6.18 & 1.10 & 7.27 & 5.77 & 2.25 & .39 & .05 & 8.47 & .20 & 27.14 \\
\hline 2007 & 2.84 & 7.26 & .90 & .12 & .31 & 11.43 & 6.40 & 1.11 & 7.51 & 5.80 & 2.24 & .39 & .05 & 8.49 & .21 & 27.64 \\
\hline 2008 & 3.28 & 7.37 & .88 & .11 & .33 & 11.97 & 6.90 & 1.12 & 8.02 & 5.93 & 2.37 & .43 & .05 & 8.79 & .18 & 28.97 \\
\hline 2009 & 3.44 & 7.32 & .94 & .11 & .31 & 12.12 & 7.19 & 1.12 & 8.31 & 5.80 & 2.33 & .45 & .06 & 8.64 & .17 & 29.24 \\
\hline
\end{tabular}

1 Natural gas production, processing, and distribution; processing is not included in 1980 and is incompletely covered in 1981-1989.

Petroleum production, refining, and distribution.

Emissions from passenger cars, trucks, buses, motorcycles, and other transport

Consumption of coal, petroleum, natural gas, and wood for heat or electricity.

See notes on components for specific coverage, which is inconsistent prior to 1990 in some cases.

1980-1983, domestic wastewater only; 1984 forward, industrial and domestic wastewater.

Methane emitted as a product of digestion in animals such as cattle, sheep, goats, and swine.

8 Estimation methods for 1990 forward reflect a shift in waste management away from liquid systems to

dry-lot systems, thus lowering emissions.

Chemical production, and iron and steel production.

NA=Not available.
Notes: - Data for this table are not available for 2010. - Emissions are from anthropogenic sources

"Anthropogenic" means produced as the result of human activities, including emissions from agricultural teck. Emissions from natural sources, such as wetlands and wild animals, are not included. - Under certain conditions, methane may be produced via anaerobic decomposition of organic materials in landfills, animal wastes, and rice paddies. - Totals may not equal sum of components due to independent rounding.

Web Page: For related information, see http://www.eia.gov/environment/.

Sources: U.S. Energy Information Administration (EIA), Emissions of Greenhouse Gases in the United States 2009 (March 2011), Tables 17-21; and ElA estimates based on the Intergovernmental Panel on Climate Change's Guidelines for National Greenhouse Gas Inventories (2006 and revised 1996 guidelines)—see http://www.ipcc-nggip.iges.or.jp/public/gl/invs6.html; and the U.S. Environmental Protection Agency's Inventory of U.S. Greenhouse Gas Emissions and Sinks: 1990-2008 (April 2010)-see http://www.epa.gov/climatechange/emissions/usinventoryreport.html. 


\section{Figure 11.4 Nitrous Oxide Emissions}

\section{Total, 1980-2009}

$0.9-$
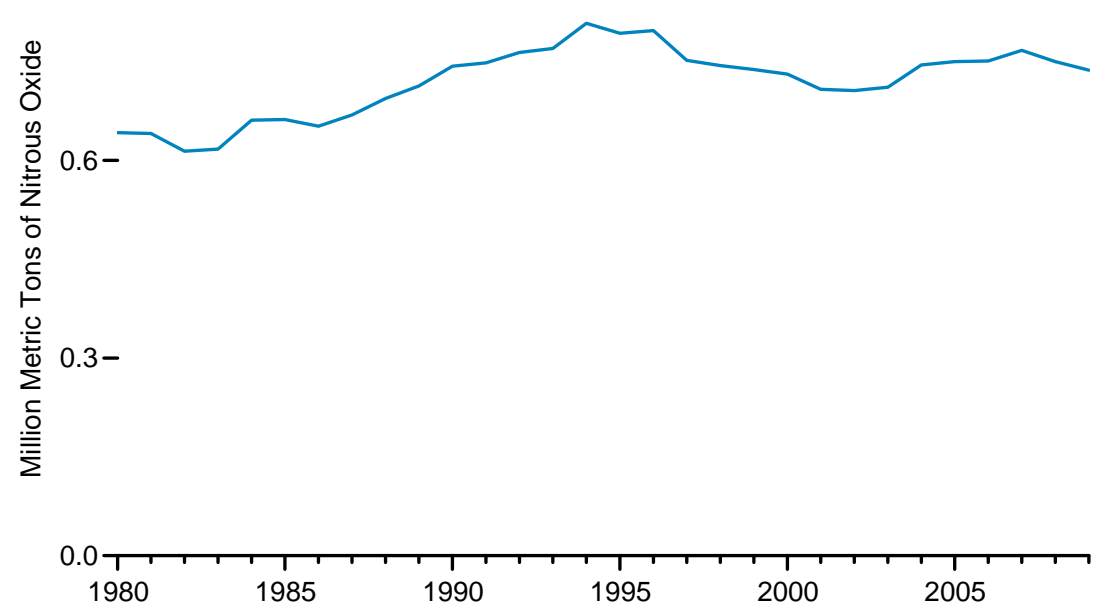

\section{Energy Sources by Type, 1980-2009}

$$
200-
$$
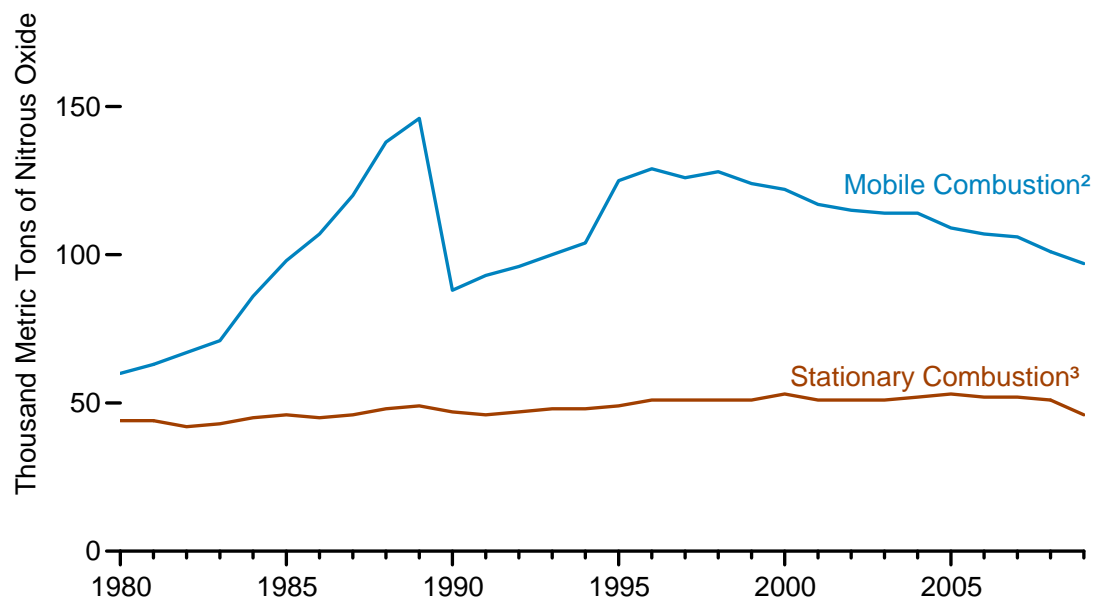

${ }^{1}$ Adipic acid production (primarily for the manufacture of nylon fibers and plastics) and nitric acid production (primarily for fertilizers).

${ }^{2}$ Emissions from passenger cars and trucks; air, rail, and marine transportation; and farm and construction equipment.

\section{By Source, 2009}

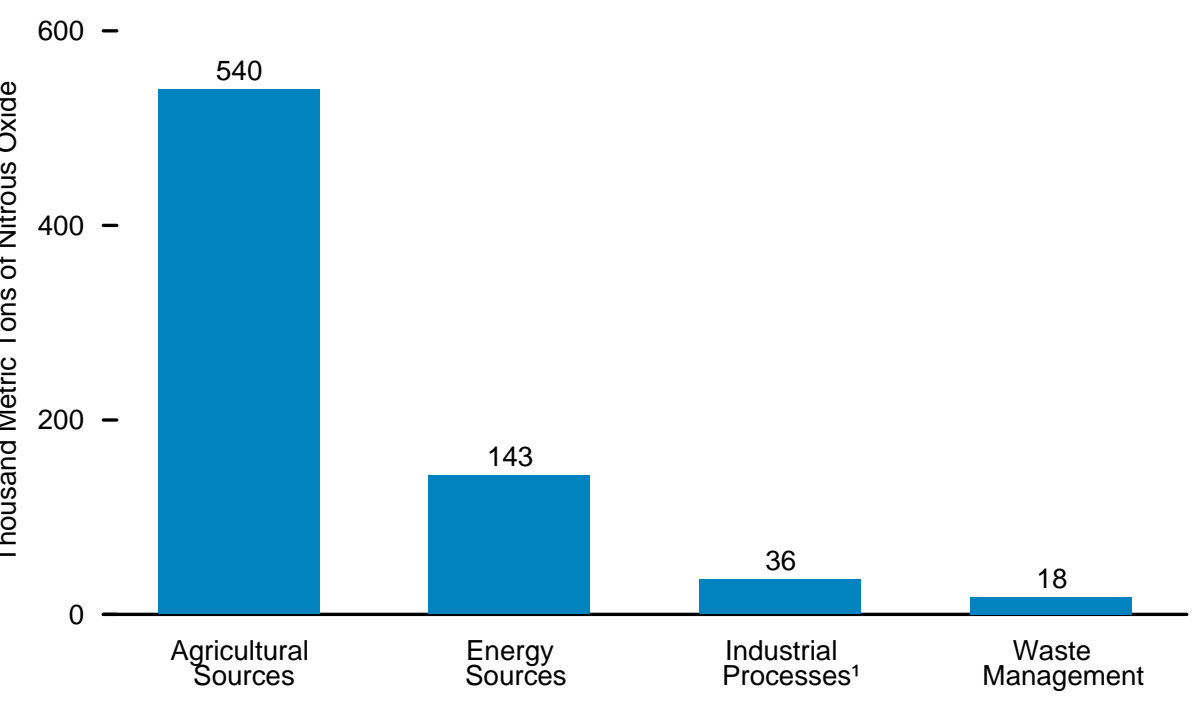

Agricultural Sources by Major Type, 1980-2009

$800-$

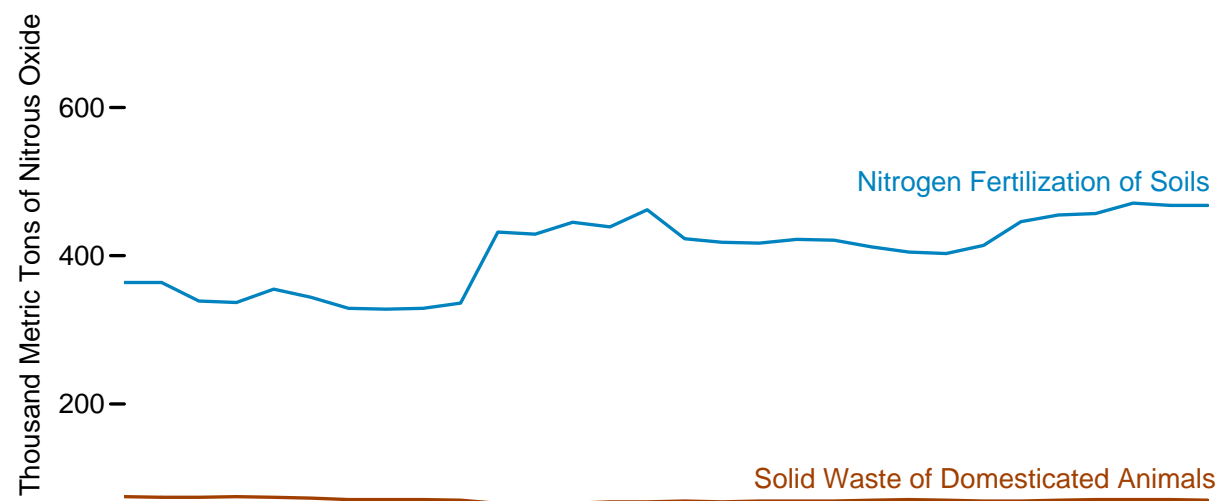

\begin{tabular}{|c|c|c|c|c|c|}
\hline$u_{\top}$ & 1 & 1 & 1 & 1 & 1 \\
\hline 1980 & 1985 & 1990 & 1995 & 2000 & 2005 \\
\hline
\end{tabular}

${ }^{3}$ Consumption of coal, petroleum, natural gas, and wood for heat or electricity. Source: Table 11.4 . 
Table 11.4 Nitrous Oxide Emissions, 1980-2009

(Thousand Metric Tons of Nitrous Oxide)

\begin{tabular}{|c|c|c|c|c|c|c|c|c|c|c|c|c|}
\hline \multirow[b]{2}{*}{ Year } & \multicolumn{3}{|c|}{ Energy Sources } & \multicolumn{3}{|c|}{ Waste Management } & \multicolumn{4}{|c|}{ Agricultural Sources } & \multirow[b]{2}{*}{$\begin{array}{c}\text { Industrial } \\
\text { Processes }^{3}\end{array}$} & \multirow[b]{2}{*}{ Total } \\
\hline & $\begin{array}{c}\text { Mobile } \\
\text { Combustion } 1\end{array}$ & $\begin{array}{c}\text { Stationary } \\
\text { Combustion } 2\end{array}$ & Total & $\begin{array}{l}\text { Waste } \\
\text { Combustion }\end{array}$ & $\begin{array}{l}\text { Human } \\
\text { Sewage in } \\
\text { Wastewater }\end{array}$ & Total & $\begin{array}{l}\text { Nitrogen } \\
\text { Fertilization } \\
\text { of Soils }\end{array}$ & $\begin{array}{l}\text { Crop } \\
\text { Residue } \\
\text { Burning }\end{array}$ & $\begin{array}{l}\text { Solid Waste } \\
\text { of Domesticated } \\
\text { Animals }\end{array}$ & Total & & \\
\hline 1980 & 60 & 44 & 104 & 1 & 10 & 11 & 364 & 1 & 75 & 440 & 88 & 642 \\
\hline 1981 & 63 & 44 & 106 & 1 & 10 & 11 & 364 & 2 & 74 & 440 & 84 & 641 \\
\hline 1982 & 67 & 42 & 108 & 1 & 10 & 11 & 339 & 2 & 74 & 414 & 80 & 614 \\
\hline 1983 & 71 & 43 & 114 & 1 & 11 & 11 & 337 & 1 & 75 & 413 & 79 & 617 \\
\hline 1984 & 86 & 45 & 132 & 1 & 11 & 11 & 355 & 2 & 74 & 431 & 87 & 661 \\
\hline 1985 & 98 & 46 & 143 & 1 & 11 & 12 & 344 & 2 & 73 & 419 & 88 & 662 \\
\hline 1986 & 107 & 45 & 152 & 1 & 11 & 12 & 329 & 2 & 71 & 402 & 86 & 652 \\
\hline 1987 & 120 & 46 & 166 & 1 & 12 & 13 & 328 & 1 & 71 & 400 & 90 & 669 \\
\hline 1988 & 138 & 48 & 185 & 1 & 12 & 13 & 329 & 1 & 71 & 401 & 95 & 694 \\
\hline 1989 & 146 & 49 & 195 & 1 & 12 & 13 & 336 & 1 & 70 & 407 & 98 & 713 \\
\hline 1990 & 88 & 47 & 135 & 1 & 12 & 13 & 432 & 1 & 66 & 499 & 96 & 743 \\
\hline 1991 & 93 & 46 & 139 & 1 & 13 & 14 & 429 & 1 & 66 & 497 & 98 & 748 \\
\hline 1992 & 96 & 47 & 143 & 1 & 13 & 14 & 445 & 2 & 66 & 512 & 95 & 764 \\
\hline 1993 & 100 & 48 & 148 & 1 & 13 & 14 & 439 & 1 & 68 & 508 & 100 & 770 \\
\hline 1994 & 104 & 48 & 152 & 1 & 13 & 15 & 462 & 2 & 68 & 532 & 110 & 808 \\
\hline 1995 & 125 & 49 & 174 & 1 & 13 & 15 & 423 & 1 & 69 & 494 & 110 & 793 \\
\hline 1996 & 129 & 51 & 180 & 1 & 14 & 15 & 418 & 2 & 68 & 487 & 115 & 797 \\
\hline 1997 & 126 & 51 & 178 & 1 & 14 & 15 & 417 & 2 & 69 & 487 & 72 & 752 \\
\hline 1998 & 128 & 51 & 179 & 1 & 14 & 15 & 422 & 2 & 69 & 493 & 57 & 744 \\
\hline 1999 & 124 & 51 & 175 & 1 & 15 & 16 & 421 & 2 & 69 & 492 & 56 & 738 \\
\hline 2000 & 122 & 53 & 175 & 1 & 15 & 16 & 412 & 2 & 70 & 484 & 56 & 731 \\
\hline 2001 & 117 & 51 & 168 & 1 & 15 & 16 & 405 & 2 & 71 & 477 & 46 & 708 \\
\hline 2002 & 115 & 51 & 166 & 1 & 15 & 16 & 403 & 2 & 70 & 474 & 50 & 706 \\
\hline 2003 & 114 & 51 & 165 & 1 & 15 & 16 & 414 & 2 & 69 & 485 & 45 & 711 \\
\hline 2004 & 114 & 52 & 167 & 1 & 15 & 17 & 446 & 2 & 69 & 517 & 45 & 745 \\
\hline 2005 & 109 & 53 & 162 & 1 & 16 & 17 & 455 & 2 & 70 & 526 & 45 & 750 \\
\hline 2006 & 107 & 52 & 159 & 1 & 16 & 17 & 457 & 2 & 71 & 530 & 46 & 751 \\
\hline 2007 & 106 & 52 & 159 & 1 & 16 & 17 & 471 & 2 & 71 & 544 & 47 & 767 \\
\hline 2008 & 101 & 51 & 151 & 1 & 16 & 17 & 468 & 2 & 71 & 541 & 41 & 750 \\
\hline 2009 & 97 & 46 & 143 & 1 & 16 & 18 & 468 & 2 & 70 & 540 & 36 & 737 \\
\hline
\end{tabular}

1 Emissions from passenger cars and trucks; air, rail, and marine transportation; and farm and construction equipment

Consumption of coal, petroleum, natural gas, and wood for heat or electricity.

Adipic acid production (primarily for the manufacture of nylon fibers and plastics), and nitric acid production (primarily for fertilizers).

Notes: - Data for this table are not available for 2010. - Emissions are from anthropogenic sources "Anthropogenic" means produced as the result of human activities, including emissions from agricultural activity and domestic livestock. Emissions from natural sources, such as wetlands and wild animals, are

not included. - Totals may not equal sum of components due to independent rounding.

Web Page: For related information, see http://www.eia.gov/environment/. States 2009 (March 2011), Table 22; and ElA estimates based on the Intergovernmental Panel on Climate Change's Guldelines for National Greenhouse Gas Inventories (2006 and revised 1996 guidelines)-see http://www.ipcc-nggip.iges.or.jp/public/gl/invs6.html, and the U.S. Environmental Protection Agency's Inventory of U.S. Greenhouse Gas Emissions and Sinks: 1990-2008 (April 2010)-see http://www.epa.gov/climatechange/emissions/usinventoryreport.html. 
Emissions by Type of Generating Unit, 2010
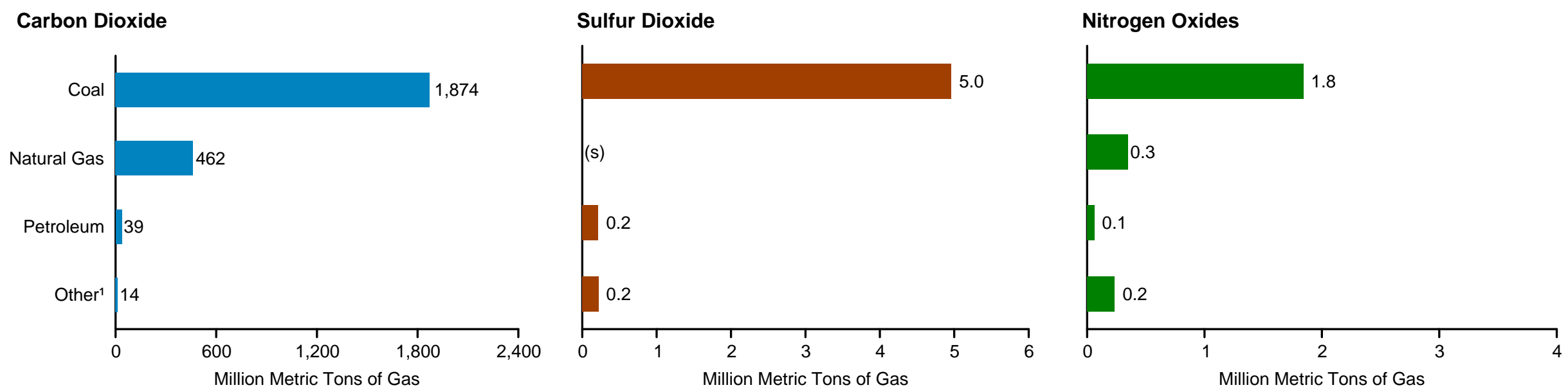

Emissions by Sector, 1989-2010

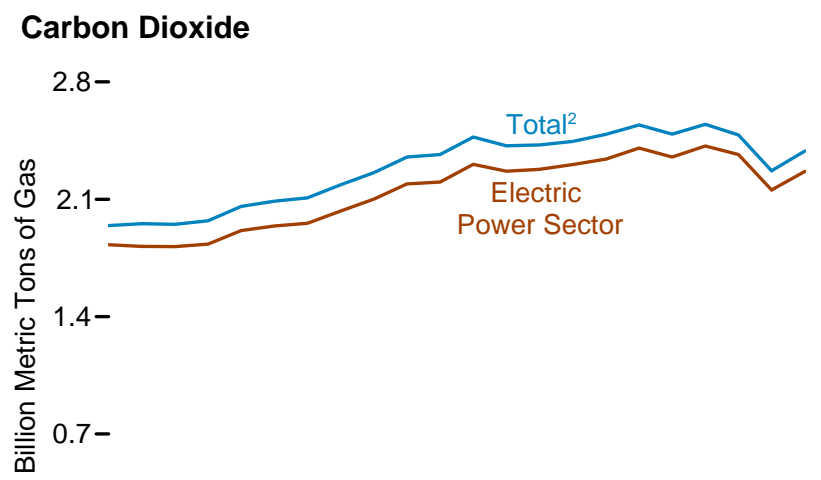

\section{Sulfur Dioxide}

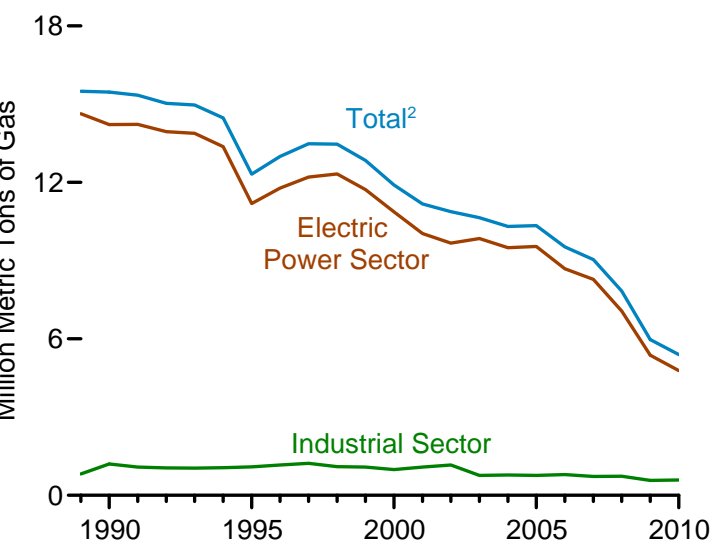

Nitrogen Oxides

$10-$
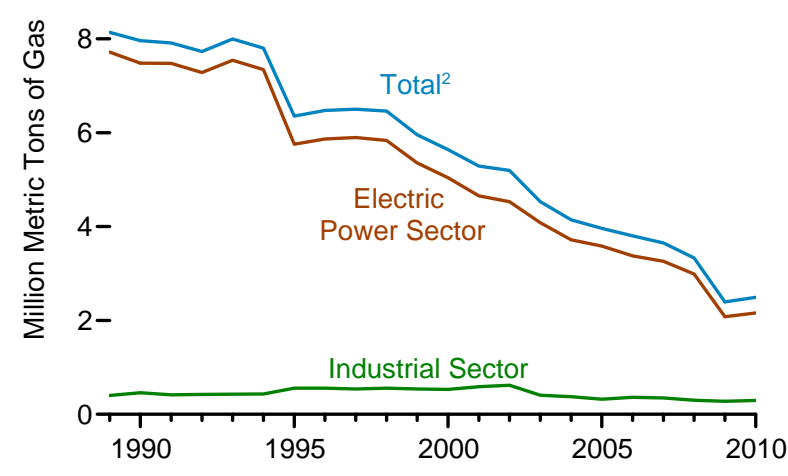

${ }^{1}$ For carbon dioxide: municipal solid waste from non-biogenic sources; tire-derived fuel, and geothermal. For sulfur dioxide and nitrogen oxides: blast furnace gas, propane gas, and other manufactured and waste gases derived from fossil fuels; wood and wood-derived fuels;

${ }^{2}$ Includes Commercial Sector manufactured and waste gases derived from fossil fuels; wood and wood-derived fuels;
municipal solid waste, landfill gas, sludge waste, tires, agricultural byproducts, and other biomass; and chemicals, hydrogen, pitch, sulfur, and tar coal. 
Table 11.5a Emissions From Energy Consumption for Electricity Generation and Useful Thermal Output: Total (All Sectors), 1989-2010 (Sum of Tables 11.5b and 11.5c; Thousand Metric Tons of Gas)

\begin{tabular}{|c|c|c|c|c|c|c|c|c|c|c|c|c|c|c|c|c|}
\hline \multirow[b]{2}{*}{ Year } & \multicolumn{6}{|c|}{ Carbon Dioxide ${ }^{1}$} & \multicolumn{5}{|c|}{ Sulfur Dioxide } & \multicolumn{5}{|c|}{ Nitrogen Oxides } \\
\hline & Coal $^{2}$ & $\begin{array}{c}\text { Natural } \\
\text { Gas }^{3}\end{array}$ & Petroleum 4 & $\begin{array}{c}\text { Geo- } \\
\text { thermal } 5\end{array}$ & $\begin{array}{c}\text { Non- } \\
\text { Biomass } \\
\text { Waste }{ }^{6}\end{array}$ & Total & Coal $^{2}$ & $\begin{array}{c}\text { Natural } \\
\text { Gas }^{3}\end{array}$ & Petroleum ${ }^{4}$ & Other ${ }^{7}$ & Total & Coal $^{2}$ & $\begin{array}{c}\text { Natural } \\
\text { Gas }^{3}\end{array}$ & Petroleum 4 & Other ${ }^{7}$ & Total \\
\hline 1989 & $1,573,566$ & 218,384 & 145,399 & 363 & 5,590 & $1,943,302$ & 14,469 & 1 & 984 & 39 & 15,493 & 7,281 & 495 & 269 & 93 & 8,136 \\
\hline 1990 & $1,592,395$ & 233,852 & 119,580 & 384 & 7,488 & $1,953,699$ & 14,281 & 1 & 937 & 243 & 15,462 & 7,119 & 513 & 208 & 122 & 7,961 \\
\hline 1991 & $1,592,186$ & 238,084 & 111,351 & 398 & 8,447 & $1,950,466$ & 14,240 & 1 & 856 & 246 & 15,342 & 7,109 & 498 & 193 & 113 & 7,913 \\
\hline 1992 & $1,617,034$ & 248,149 & 96,638 & 400 & 10,053 & $1,972,275$ & 14,060 & 1 & 704 & 264 & 15,030 & 6,975 & 477 & 158 & 119 & 7,728 \\
\hline 1993 & $1,687,623$ & 250,411 & 108,164 & 415 & 10,439 & $2,057,053$ & 13,843 & 1 & 851 & 271 & 14,966 & 7,225 & 475 & 173 & 124 & 7,997 \\
\hline 1994 & $1,697,416$ & 276,308 & 102,844 & 384 & 11,186 & $2,088,138$ & 13,398 & 1 & 794 & 279 & 14,472 & 7,005 & 513 & 159 & 124 & 7,801 \\
\hline 1995 & $1,720,062$ & 298,601 & 77,032 & 329 & 11,982 & $2,108,006$ & 11,188 & 2 & 826 & 298 & 12,314 & 5,136 & 653 & 332 & 234 & 6,355 \\
\hline 1996 & $1,812,022$ & 277,856 & 84,024 & 360 & 12,718 & $2,186,980$ & 11,811 & 1 & 876 & 304 & 12,991 & 5,307 & 577 & 352 & 238 & 6,474 \\
\hline 1997 & $1,858,944$ & 293,139 & 93,497 & 374 & 13,368 & $2,259,322$ & 12,211 & 1 & 965 & 303 & 13,480 & 5,322 & 619 & 326 & 233 & 6,500 \\
\hline 1998 & $1,887,335$ & 327,456 & 123,542 & 375 & 12,891 & $2,351,600$ & 12,012 & 1 & 1,162 & 289 & 13,464 & 5,123 & 700 & 395 & 241 & 6,459 \\
\hline 1999 & $1,894,211$ & 343,090 & 115,677 & 381 & 12,943 & $2,366,302$ & 11,453 & 1 & 1,101 & 288 & 12,843 & 4,687 & 632 & 391 & 245 & 5,955 \\
\hline 2000 & $1,986,100$ & 363,526 & 108,407 & 362 & 12,440 & $2,470,834$ & $R_{10,669}$ & 1 & 933 & 300 & $R_{11,904}$ & 4,370 & 614 & 404 & 250 & 5,638 \\
\hline 2001 & $1,920,901$ & 367,146 & 117,196 & 353 & 13,010 & $2,418,607$ & 9,905 & 2 & 1,002 & 265 & 11,174 & 4,096 & 631 & 294 & 268 & 5,290 \\
\hline 2002 & $1,938,613$ & 378,950 & 91,110 & 372 & 14,918 & $2,423,963$ & 9,786 & 2 & 773 & 321 & 10,881 & 4,057 & 625 & 225 & 287 & 5,194 \\
\hline 2003 & $1,973,597$ & 345,119 & 112,065 & 371 & 13,943 & $2,445,094$ & 9,688 & 2 & 717 & 239 & 10,646 & 3,607 & 453 & 240 & 232 & 4,532 \\
\hline 2004 & $1,989,580$ & 367,112 & 115,726 & 381 & 14,183 & $2,486,982$ & 9,437 & 2 & 633 & 237 & 10,309 & 3,286 & 416 & 225 & 217 & 4,143 \\
\hline 2005 & $2,028,614$ & 383,461 & 117,086 & 377 & 14,299 & $2,543,838$ & 9,499 & 2 & 587 & 251 & 10,340 & 3,135 & 383 & 221 & 222 & 3,961 \\
\hline 2006 & $2,001,085$ & 404,278 & 67,988 & 374 & 15,193 & $2,488,918$ & 8,867 & 2 & 427 & 227 & 9,524 & 2,996 & 399 & 164 & 240 & 3,799 \\
\hline 2007 & $2,029,804$ & 434,536 & 67,769 & 376 & 14,548 & $2,547,032$ & 8,389 & 3 & 422 & 227 & 9,042 & 2,870 & 382 & 157 & 242 & 3,650 \\
\hline 2008 & $2,001,806$ & 419,599 & 47,855 & 381 & 14,370 & $2,484,012$ & 7,351 & 3 & 250 & 225 & 7,830 & 2,680 & 351 & 75 & 225 & 3,330 \\
\hline 2009 & $1,781,278$ & 432,206 & 41,474 & 386 & 14,163 & $2,269,508$ & 5,535 & 2 & 210 & 223 & 5,970 & 1,769 & 336 & 66 & 225 & 2,395 \\
\hline 2010 & $1,873,813$ & 461,723 & 38,793 & 391 & 13,875 & $2,388,596$ & 4,961 & 3 & 217 & 219 & 5,400 & 1,843 & 349 & 63 & 236 & 2,491 \\
\hline
\end{tabular}

1 Metric tons of carbon dioxide can be converted to metric tons of carbon equivalent by multiplying by $12 / 44$

Anthracite, bituminous coal, subbituminous coal, lignite, waste coal, and synthetic coal.

3 Natural gas, plus a small amount of supplemental gaseous fuels.

Distillate fuel oil, residual fuel oil, petroleum coke, jet fuel, kerosene, other petroleum, and waste oil.

5 Carbon dioxide in geothermal steam.

Municipal solid waste from non-biogenic sources, and tire-derived fuel.

Blast furnace gas, propane gas, and other manufactured and waste gases derived from fossil fuels; wood and wood-derived fuels; municipal solid waste, landfill gas, sludge waste, tires, agricultural byproducts, an
Revised.

Notes: - Data are for emissions from energy consumption for electricity generation and useful thermal output. - See "Useful Thermal Output" in Glossary. - Totals may not equal sum of components due to independent rounding.

Web Page: For related information, see http://www.eia.gov/electricity/.

Sources: Tables $11.5 \mathrm{~b}$ and $11.5 \mathrm{c}$ 
Table 11.5b Emissions From Energy Consumption for Electricity Generation and Useful Thermal Output: Electric Power Sector, 1989-2010 (Subset of Table 11.5a; Thousand Metric Tons of Gas)

\begin{tabular}{|c|c|c|c|c|c|c|c|c|c|c|c|c|c|c|c|c|}
\hline \multirow[b]{2}{*}{ Year } & \multicolumn{6}{|c|}{ Carbon Dioxide ${ }^{1}$} & \multicolumn{5}{|c|}{ Sulfur Dioxide } & \multicolumn{5}{|c|}{ Nitrogen Oxides } \\
\hline & Coal $^{2}$ & $\begin{array}{c}\text { Natural } \\
\text { Gas }^{3}\end{array}$ & Petroleum ${ }^{4}$ & $\begin{array}{c}\text { Geo- } \\
\text { thermal } 5\end{array}$ & $\begin{array}{c}\text { Non- } \\
\text { Biomass } \\
\text { Waste }{ }^{6}\end{array}$ & Total & Coal $^{2}$ & $\begin{array}{l}\text { Natural } \\
\text { Gas }^{3}\end{array}$ & Petroleum ${ }^{4}$ & Other ${ }^{7}$ & Total & Coal $^{2}$ & $\begin{array}{c}\text { Natural } \\
\text { Gas }^{3}\end{array}$ & Petroleum 4 & Other ${ }^{7}$ & Total \\
\hline 1989 & $1,520,230$ & 169,653 & 133,546 & 363 & 4,366 & $1,828,158$ & 13,815 & 1 & 810 & 7 & 14,633 & 7,055 & 390 & 246 & 25 & 7,717 \\
\hline 1990 & $1,534,141$ & 177,232 & 101,800 & 384 & 5,795 & $1,819,351$ & 13,576 & 1 & 628 & 13 & 14,218 & 6,878 & 390 & 175 & 36 & 7,480 \\
\hline 1991 & $1,534,559$ & 180,541 & 95,149 & 398 & 7,207 & $1,817,854$ & 13,590 & 1 & 621 & 15 & 14,227 & 6,886 & 384 & 165 & 42 & 7,476 \\
\hline 1992 & $1,556,741$ & 187,730 & 79,153 & 400 & 8,476 & $1,832,501$ & 13,375 & 1 & 559 & 12 & 13,946 & 6,749 & 359 & 128 & 46 & 7,282 \\
\hline 1993 & $1,626,161$ & 188,291 & 90,400 & 415 & 8,592 & $1,913,860$ & 13,133 & 1 & 735 & 13 & 13,882 & 6,996 & 357 & 143 & 49 & 7,544 \\
\hline 1994 & $1,634,282$ & 211,154 & 85,005 & 384 & 9,323 & $1,940,148$ & 12,695 & 1 & 665 & 11 & 13,373 & 6,777 & 390 & 128 & 47 & 7,343 \\
\hline 1995 & $1,656,743$ & 228,675 & 61,057 & 329 & 10,015 & $1,956,819$ & 10,573 & 1 & 581 & 34 & 11,189 & 4,974 & 402 & 282 & 95 & 5,754 \\
\hline 1996 & $1,747,945$ & 205,250 & 66,113 & 360 & 9,932 & $2,029,599$ & 11,129 & 1 & 617 & 32 & 11,779 & 5,144 & 326 & 301 & 96 & 5,866 \\
\hline 1997 & $1,794,629$ & 220,174 & 75,079 & 374 & 10,372 & $2,100,628$ & 11,515 & 1 & 653 & 36 & 12,205 & 5,157 & 370 & 269 & 98 & 5,894 \\
\hline 1998 & $1,825,027$ & 249,836 & 105,539 & 375 & 10,264 & $2,191,041$ & 11,373 & 1 & 911 & 37 & 12,321 & 4,965 & 431 & 337 & 103 & 5,836 \\
\hline 1999 & $1,831,670$ & 262,455 & 97,892 & 381 & 10,312 & $2,202,710$ & 10,843 & 1 & 836 & 42 & 11,722 & 4,535 & 381 & 332 & 109 & 5,357 \\
\hline 2000 & $1,923,054$ & 283,034 & 92,226 & 362 & 10,178 & $2,308,855$ & $\mathrm{R}_{10,081}$ & 1 & 746 & 45 & $\mathrm{R}_{10,872}$ & 4,225 & 338 & 367 & 111 & 5,040 \\
\hline 2001 & $1,862,800$ & 291,101 & 102,900 & 353 & 10,900 & $2,268,054$ & 9,281 & 2 & 754 & 5 & 10,041 & 3,878 & 425 & 253 & 96 & 4,652 \\
\hline 2002 & $1,878,923$ & 307,455 & 78,820 & 372 & 12,758 & $2,278,328$ & 9,106 & 2 & 549 & 16 & 9,672 & 3,813 & 425 & 187 & 104 & 4,528 \\
\hline 2003 & $1,917,303$ & 279,300 & 98,208 & 371 & 11,453 & $2,306,635$ & 9,255 & 2 & 579 & 13 & 9,849 & 3,496 & 282 & 207 & 98 & 4,082 \\
\hline 2004 & $1,929,818$ & 297,782 & 100,236 & 381 & 11,177 & $2,339,394$ & 8,991 & 2 & 493 & 9 & 9,495 & 3,183 & 241 & 193 & 101 & 3,717 \\
\hline 2005 & $1,970,908$ & 320,545 & 102,537 & 377 & 11,257 & $2,405,625$ & 9,071 & 2 & 461 & 10 & 9,543 & 3,051 & 243 & 189 & 103 & 3,585 \\
\hline 2006 & $1,944,759$ & 339,557 & 55,358 & 374 & 11,544 & $2,351,592$ & 8,416 & 2 & 264 & 8 & 8,690 & 2,902 & 230 & 135 & 107 & 3,374 \\
\hline 2007 & $1,977,528$ & 373,268 & 55,545 & 376 & 11,304 & $2,418,022$ & 8,002 & 3 & 265 & 9 & 8,279 & 2,781 & 236 & 130 & 112 & 3,259 \\
\hline 2008 & $1,951,138$ & 363,749 & 40,442 & 381 & 11,620 & $2,367,331$ & 6,909 & 2 & 146 & 8 & 7,065 & 2,578 & 230 & 58 & 124 & 2,990 \\
\hline 2009 & $1,736,284$ & 374,082 & 33,700 & 386 & 11,256 & $2,155,707$ & 5,253 & 2 & 110 & 9 & 5,374 & 1,688 & 214 & 50 & 128 & 2,080 \\
\hline 2010 & $1,821,497$ & 400,974 & 32,667 & 391 & 11,034 & $2,266,563$ & 4,662 & 2 & 111 & 8 & 4,783 & 1,751 & 224 & 49 & 135 & 2,159 \\
\hline
\end{tabular}

1 Metric tons of carbon dioxide can be converted to metric tons of carbon equivalent by multiplying by $12 / 44$

Anthracite, bituminous coal, subbituminous coal, lignite, waste coal, and synthetic coal.

3 Natural gas, plus a small amount of supplemental gaseous fuels.

Distillate fuel oil, residual fuel oil, petroleum coke, jet fuel, kerosene, other petroleum, and waste oil.

5 Carbon dioxide in geothermal steam.

Municipal solid waste from non-biogenic sources, and tire-derived fuel.

7 Blast furnace gas, propane gas, and other manufactured and waste gases derived from fossil fuels; wood and wood-derived fuels; municipal solid waste, landfill gas, sludge waste, tires, agricultural byproducts, and other biomass; and chemicals, hydrogen, pitch, sulfur, and tar coal.

R=Revised.

Notes: - There are small differences in carbon dioxide emissions values between this table and Table

11.2e due to differences in the methodologies for calculating the data. - Data are for emissions from energy consumption for electricity generation and useful thermal output. - The electric power sector comprises electricity-only and combined-heat-and-power (CHP) plants within the NAICS 22 category whose primary business is to sell electricity, or electricity and heat, to the public. - See Table 11.5c for commercial and industrial CHP and electricity-only data. - See Note 2, "Classification of Power Plants Into Energy-Use Sectors," at end of Section 8. • See "Useful Thermal Output" in Glossary. • Totals may not equal sum of components due to independent rounding.

Sources: Carbon Dioxide: U.S. Energy Information Administration (EIA) estimates based on Form EIA-923, "Power Plant Operations Report" (and predecessor forms). Sulfur Dioxide and Nitrogen Oxides: EIA estimates based on Form EIA-923, "Power Plant Operations Report" (and predecessor forms). Data were adjusted by the U.S. Environmental Protection Agency's Continuous Emissions Monitoring System. 
Table 11.5c Emissions From Energy Consumption for Electricity Generation and Useful Thermal Output:

Commercial and Industrial Sectors, 1989-2010 (Subset of Table 11.5a; Thousand Metric Tons of Gas)

\begin{tabular}{|c|c|c|c|c|c|c|c|c|c|c|c|c|c|c|c|c|}
\hline \multirow[b]{2}{*}{ Year } & \multicolumn{6}{|c|}{ Carbon Dioxide 1} & \multicolumn{5}{|c|}{ Sulfur Dioxide } & \multicolumn{5}{|c|}{ Nitrogen Oxides } \\
\hline & Coal $^{2}$ & $\begin{array}{c}\text { Natural } \\
\text { Gas }^{3}\end{array}$ & Petroleum 4 & $\begin{array}{c}\text { Geo- } \\
\text { thermal } 5\end{array}$ & $\begin{array}{c}\text { Non- } \\
\text { Biomass } \\
\text { Waste }\end{array}$ & Total & Coal $^{2}$ & $\begin{array}{c}\text { Natural } \\
\text { Gas }^{3}\end{array}$ & Petroleum 4 & Other ${ }^{7}$ & Total & Coal $^{2}$ & $\begin{array}{c}\text { Natural } \\
\text { Gas }^{3}\end{array}$ & Petroleum 4 & Other 7 & Total \\
\hline & \multicolumn{16}{|c|}{ Commercial Sector 8} \\
\hline 1989 & 2,320 & 1,542 & 637 & - & 804 & 5,303 & 37 & (s) & 5 & 1 & 43 & 9 & 3 & 2 & 3 & 17 \\
\hline 1990 & 2,418 & 2,294 & 706 & - & 959 & 6,377 & 39 & (s) & 4 & 1 & 45 & 10 & 6 & 1 & 4 & 21 \\
\hline 1991 & 2,680 & 2,287 & 544 & - & 1,014 & 6,526 & 32 & (s) & 3 & 1 & 35 & 10 & 6 & 1 & 4 & 21 \\
\hline 1992 & 2,552 & 2,787 & 474 & - & 1,258 & 7,070 & 32 & (s) & 3 & 1 & 35 & 10 & 7 & 1 & 4 & 21 \\
\hline 1993 & 2,988 & 3,315 & 616 & - & 1,285 & 8,205 & 40 & (s) & 3 & 1 & 44 & 12 & 7 & 1 & 4 & 24 \\
\hline 1994 & 2,932 & 3,722 & 654 & - & 1,292 & 8,601 & 39 & (s) & $\begin{array}{l}5 \\
3\end{array}$ & (s) & $\begin{array}{l}44 \\
42\end{array}$ & 11 & 8 & 1 & $\begin{array}{l}4 \\
4\end{array}$ & 24 \\
\hline 1995 & 3,106 & 4,070 & 509 & - & 1,462 & 9,147 & 30 & (s) & 3 & 3 & 35 & 8 & 20 & 6 & 11 & 45 \\
\hline 1996 & 3,639 & 4,369 & 534 & - & 2,023 & 10,565 & 40 & (s) & $\begin{array}{l}5 \\
3\end{array}$ & 4 & 47 & $\begin{array}{l}0 \\
9\end{array}$ & 23 & $\begin{array}{l}0 \\
4\end{array}$ & 14 & $\begin{array}{l}45 \\
50\end{array}$ \\
\hline 1997 & $\begin{array}{l}3,0031 \\
3,871\end{array}$ & 4,654 & 716 & - & 2,277 & 11,518 & 43 & $\begin{array}{l}\text { (s) } \\
\text { (s) }\end{array}$ & 3 & $\begin{array}{l}4 \\
6\end{array}$ & 51 & 10 & 34 & $\begin{array}{l}4 \\
7\end{array}$ & 14 & 65 \\
\hline 1998 & 3,341 & 4.707 & 829 & - & 2.081 & 10,958 & 37 & $\begin{array}{l}\text { (1) } \\
\text { (s) }\end{array}$ & 5 & 4 & 45 & 10 & 35 & 5 & 16 & 66 \\
\hline 1999 & $\begin{array}{l}3,041 \\
3,468\end{array}$ & 4,535 & $\begin{array}{l}029 \\
742\end{array}$ & - & 2,008 & 10,752 & 34 & (s) & 4 & $\begin{array}{l}4 \\
4\end{array}$ & 42 & 9 & 28 & 4 & 17 & 57 \\
\hline 2000 & $\begin{array}{l}3,468 \\
3,635\end{array}$ & $\begin{array}{l}4,535 \\
4,605\end{array}$ & 740 & 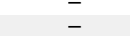 & $\begin{array}{l}2,008 \\
1,684\end{array}$ & 10,665 & $\begin{array}{l}34 \\
33\end{array}$ & $\begin{array}{l}\text { (s) } \\
\text { (s) }\end{array}$ & $\begin{array}{l}4 \\
4\end{array}$ & $\begin{array}{l}4 \\
7\end{array}$ & $\begin{array}{l}42 \\
43\end{array}$ & 8 & $\begin{array}{l}28 \\
38\end{array}$ & $\begin{array}{l}4 \\
4\end{array}$ & 16 & $\begin{array}{l}51 \\
65\end{array}$ \\
\hline 2001 & $\begin{array}{l}3,000 \\
3,366\end{array}$ & 4,280 & 839 & - & $\begin{array}{l}1,004 \\
1.418\end{array}$ & 9,903 & 43 & $\begin{array}{l}\text { (s) } \\
\text { (s) }\end{array}$ & $\begin{array}{l}4 \\
4\end{array}$ & 2 & $\begin{array}{l}43 \\
48\end{array}$ & $\begin{array}{r}8 \\
13\end{array}$ & $\begin{array}{l}38 \\
19\end{array}$ & $\begin{array}{l}4 \\
2\end{array}$ & $\begin{array}{l}16 \\
16\end{array}$ & $\begin{array}{l}65 \\
50\end{array}$ \\
\hline 2002 & $\begin{array}{l}3,366 \\
3,025\end{array}$ & $\begin{array}{l}4,280 \\
4,035\end{array}$ & $\begin{array}{l}839 \\
571\end{array}$ & $\begin{array}{ll}- \\
-\end{array}$ & $\begin{array}{l}1,418 \\
1,520\end{array}$ & $\begin{array}{l}9,903 \\
9,151\end{array}$ & $\begin{array}{l}43 \\
41\end{array}$ & $\begin{array}{l}\text { (s) } \\
\text { (s) }\end{array}$ & $\begin{array}{l}4 \\
2\end{array}$ & 2 & $\begin{array}{l}48 \\
46\end{array}$ & $\begin{array}{l}13 \\
13\end{array}$ & $\begin{array}{l}19 \\
20\end{array}$ & 2 & $\begin{array}{l}16 \\
13\end{array}$ & $\begin{array}{l}50 \\
48\end{array}$ \\
\hline 2003 & 3,904 & 3,222 & 683 & - & 1,706 & 9,515 & 32 & (s) & 3 & 1 & 36 & 9 & 16 & 5 & 15 & 45 \\
\hline 2004 & $\begin{array}{l}3,904 \\
4018\end{array}$ & $\begin{array}{l}3,2<2 \\
3,916\end{array}$ & $\begin{array}{l}003 \\
920\end{array}$ & - & 1962 & $\begin{array}{r} \\
10,810\end{array}$ & $\begin{array}{l}32 \\
30\end{array}$ & $\begin{array}{l}\text { (s) } \\
\text { (s) }\end{array}$ & $\begin{array}{l}5 \\
3\end{array}$ & 2 & $\begin{array}{l}30 \\
35\end{array}$ & 8 & $\begin{array}{l}10 \\
18\end{array}$ & $\begin{array}{l}5 \\
8\end{array}$ & $\begin{array}{l}15 \\
16\end{array}$ & $\begin{array}{l}45 \\
49\end{array}$ \\
\hline 2005 & 4,031 & 3,701 & 759 & - & $\begin{array}{l}1,897 \\
\end{array}$ & 10,387 & 33 & (s) & 3 & 1 & 36 & 9 & 24 & 6 & 15 & 54 \\
\hline 2006 & 3,908 & 3,686 & 445 & - & $\begin{array}{l}1,946 \\
\end{array}$ & 9,984 & 33 & (s) & 3 & 1 & 36 & 9 & 35 & 3 & 17 & 64 \\
\hline 2007 & 3,994 & 3,800 & 363 & - & 1,635 & 9,792 & 33 & (s) & 3 & 1 & 3 & 10 & 16 & 2 & 16 & 44 \\
\hline 2008 & 4,155 & 3,589 & 310 & - & 1,953 & 10,006 & 32 & (s) & 1 & (s) & 33 & 9 & 14 & 1 & 16 & 40 \\
\hline \multirow{3}{*}{2010} & 3,727 & 4,093 & 245 & - & 2,084 & 10,149 & 26 & (s) & 1 & (s) & 27 & 8 & 13 & 1 & 16 & 39 \\
\hline & 3,530 & 4,639 & 206 & - & 2,063 & 10,437 & 25 & (s) & 1 & (s) & 27 & 7 & 14 & 1 & 15 & 38 \\
\hline & \multicolumn{16}{|c|}{ Industrial Sector ${ }^{9}$} \\
\hline 1989 & 51,017 & 47,188 & 11,216 & - & 420 & 109,842 & 616 & (s) & 169 & 32 & 817 & 218 & 100 & 21 & 63 & 403 \\
\hline 1990 & 55,837 & 54,326 & 17,074 & - & 734 & 127,971 & 666 & (s) & 304 & 229 & 1,199 & 233 & 116 & 31 & 80 & 461 \\
\hline 1991 & 54,947 & 55,255 & 15,659 & - & 225 & 126,086 & 618 & (s) & 232 & 230 & 1,080 & 215 & 108 & 27 & 66 & 416 \\
\hline 1992 & 57,742 & 57,632 & 17,010 & - & 319 & 132,704 & 655 & (s) & 143 & 251 & 1,049 & 218 & 110 & 29 & 67 & 425 \\
\hline 1993 & 58,474 & 58,805 & 17,148 & - & 562 & 134,988 & 671 & (s) & 113 & 257 & 1,041 & 219 & 110 & 29 & 70 & 429 \\
\hline 1994 & 60,202 & 61,431 & 17,186 & - & 571 & 139,390 & 664 & (s) & 126 & 267 & 1,057 & 219 & 114 & 30 & 71 & 435 \\
\hline 1995 & 60,212 & 65,856 & 15,466 & - & 505 & 142,040 & 585 & (s) & 243 & 262 & 1,090 & 154 & 231 & 43 & 128 & 556 \\
\hline 1996 & 60,438 & 68,237 & 17,377 & - & 763 & 146,815 & 642 & (s) & 256 & 268 & 1,166 & 154 & 228 & 48 & 128 & 558 \\
\hline 1997 & 60,444 & 68,311 & 17,701 & - & 719 & 147,175 & 653 & (s) & 309 & 261 & 1,223 & 155 & 215 & 50 & 121 & 541 \\
\hline 1998 & 58,967 & 72,914 & 17,174 & - & 546 & 149,601 & 603 & (s) & 247 & 248 & $\begin{array}{l}1,099 \\
\end{array}$ & 148 & 234 & 53 & 121 & 557 \\
\hline 1999 & 59,073 & 76,100 & 17,043 & - & 624 & 152,840 & 576 & (s) & 260 & 243 & 1,080 & 144 & 223 & 55 & 120 & 541 \\
\hline 2000 & 59,410 & 75,887 & 15,440 & - & 577 & 151,315 & 556 & (s) & 184 & 248 & 988 & 138 & 238 & 34 & 123 & 533 \\
\hline 2001 & 54,735 & 71,765 & 13,457 & - & 693 & 140,650 & 581 & (s) & 245 & 259 & 1,085 & 206 & 187 & 39 & 156 & 587 \\
\hline 2002 & 56,665 & 67,460 & 11,719 & - & 640 & 136.484 & 639 & (s) & 221 & 303 & 1,163 & 231 & 181 & 36 & 170 & 618 \\
\hline 2003 & 52,390 & 62,598 & 13,173 & - & 783 & 128,944 & 401 & (s) & 135 & 224 & 761 & 102 & 155 & 28 & 119 & 404 \\
\hline 2004 & 55,744 & 65,413 & 14,570 & - & 1,044 & 136,771 & 415 & (s) & 136 & 227 & 779 & 95 & 157 & 25 & 100 & 376 \\
\hline 2005 & 53,675 & 59,216 & 13,791 & - & 1,145 & 127.826 & 395 & (s) & 124 & 241 & 760 & 75 & 117 & 27 & 104 & 322 \\
\hline 2006 & 52,418 & 61.035 & 12,185 & - & 1,703 & 127,341 & 419 & (s) & 161 & 218 & 798 & 86 & 134 & 26 & 117 & 362 \\
\hline 2007 & 48,282 & 57,467 & 11,860 & - & 1,609 & 119,218 & 353 & 1 & 154 & 217 & 726 & 79 & 129 & 26 & 113 & 346 \\
\hline 2008 & $\begin{array}{l}40,502 \\
46,514\end{array}$ & $\begin{array}{l}52,261 \\
52,261\end{array}$ & 7,103 & - & $\begin{array}{l}1,009 \\
798\end{array}$ & 106,675 & 411 & 1 & 103 & 217 & 731 & 93 & 107 & $\begin{array}{l}20 \\
16\end{array}$ & 84 & $\begin{array}{l}340 \\
300\end{array}$ \\
\hline 2009 & $\begin{array}{l}40,268 \\
41,268\end{array}$ & 54,031 & $\begin{array}{l}7,105 \\
7,529\end{array}$ & $\overline{-}$ & 824 & $\begin{array}{l}03,651 \\
0,65\end{array}$ & 256 & (s) & 98 & 214 & 569 & 73 & 108 & $\begin{array}{l}10 \\
15\end{array}$ & $\begin{array}{l}84 \\
81\end{array}$ & 277 \\
\hline 2010 & 48,786 & 56,110 & $\begin{array}{l}5,920 \\
5,929\end{array}$ & - & $\begin{array}{l}024 \\
779\end{array}$ & 111,596 & 274 & $\begin{array}{l}\text { (s) } \\
\text { (s) }\end{array}$ & $\begin{array}{r}50 \\
105\end{array}$ & $\begin{array}{l}214 \\
210\end{array}$ & 590 & 85 & 110 & 14 & 86 & 294 \\
\hline
\end{tabular}

Metric tons of carbon dioxide can be converted to metric tons of carbon equivalent by multiplying by

- = No data reported. (s)=Less than 0.5 thousand metric tons.

${ }_{2}^{2 / 44}$ Anthracite, bituminous coal, subbituminous coal, lignite, waste coal, and synthetic coal.

Natural gas, plus a small amount of supplemental gaseous fuels.

4 Distillate fuel oil, residual fuel oil, petroleum coke, jet fuel, kerosene, other petroleum, and waste oil.

Municipal solid waste from non-biog.

Blast furnace gas, propane gas, and other manufactured and waste gases derived from fossil fuels: wood and wood-derived fuels; municipal solid waste, landfill gas, sludge waste, tires, agricultura

byproducts, and other biomass; and chemicals, hydrogen, pitch, sulfur, and tar coal.

8 Commercial combined-heat-and-power (CHP) and commercial electricity-only plants.

Notes: output. - See Table 11.5b for electric power sector data. - See Note 2, "Classification of Power Plants Into Energy-Use Sectors," at end of Section 8. • See "Useful Thermal Output" in Glossary. • Totals may not equal sums of components due to independent rounding.

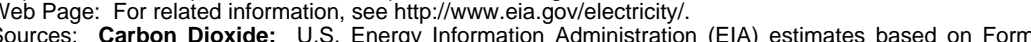
EIA-923, "Power Plant Operations Report" (and predecessor forms). Sulfur Dioxide and Nitrogen Oxides: EIA estimates based on Form EIA-923, "Power Plant Operations Report" (and predecesso forms). Data were adjusted by the U.S. Environmental Protection Agency's Continuous Emissions
Monitoring System. 
Figure 11.6 Installed Nameplate Capacity of Fossil-Fuel Steam-Electric Generators With Environmental Equipment

By Fuel and Equipment Type, 2010

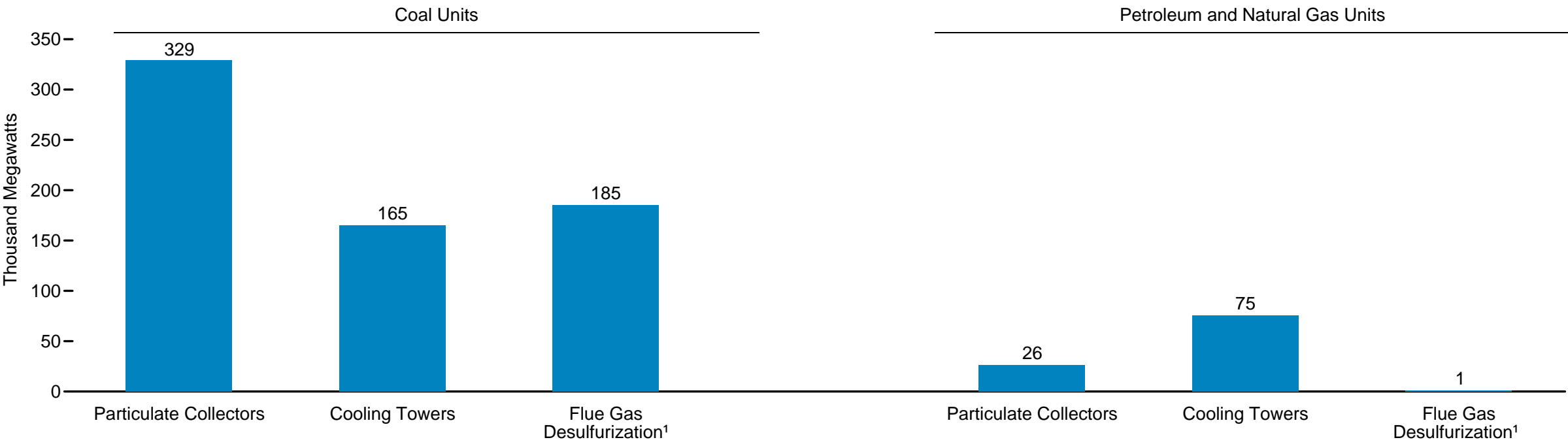

Total Units by Equipment Type, 1985-2010²

$400-$

Particulate Collectors 00000000000000000

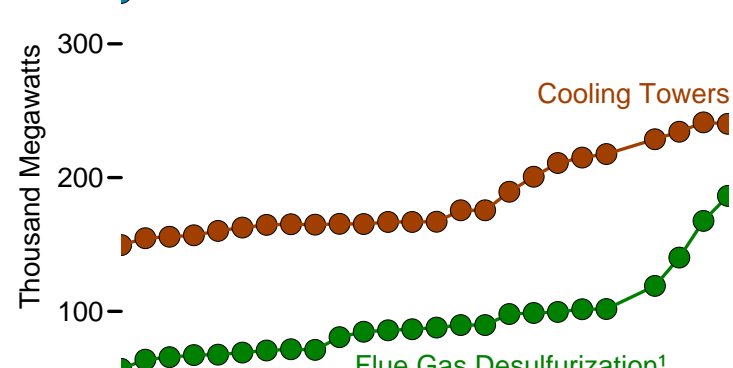

Flue Gas Desulfurization ${ }^{1}$

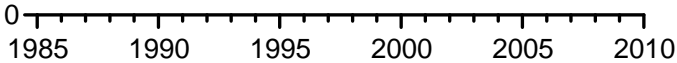

Coal Units by Equipment Type, 1985-2010²

$400-$
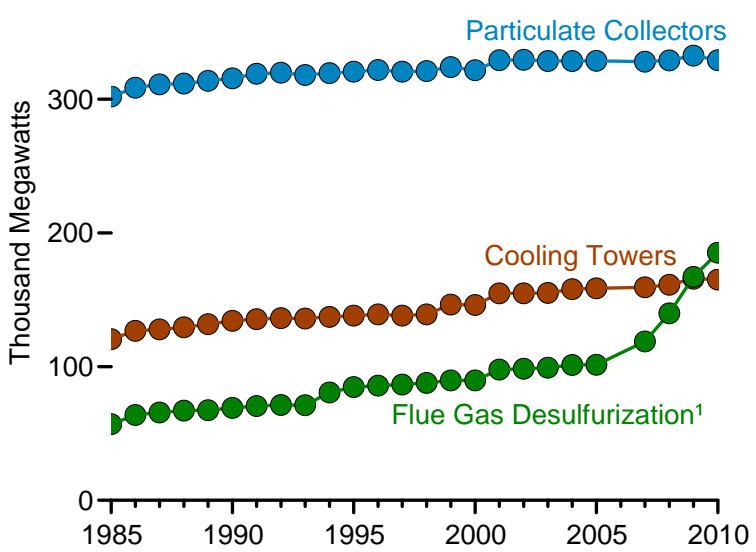

Petroleum and Natural Gas Units by Equipment Type, 1985-2010²

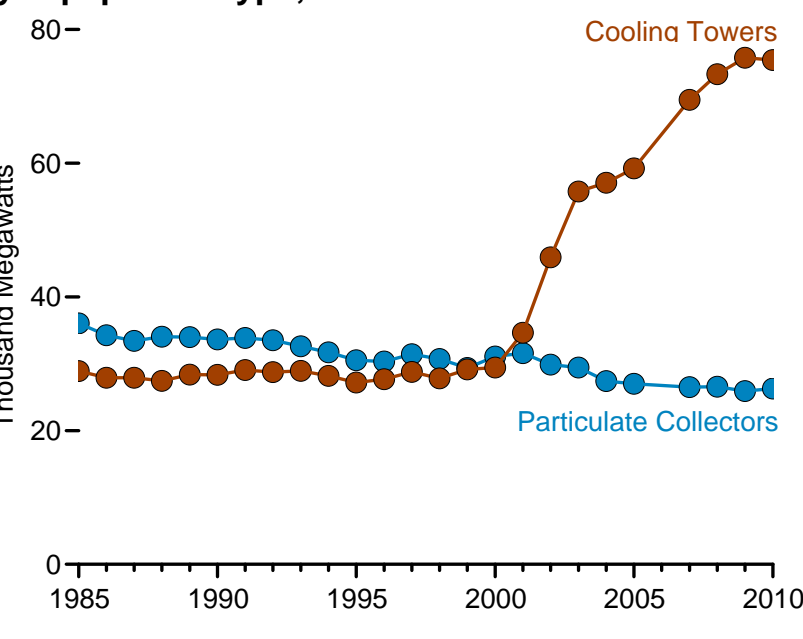

1 Also called "scrubbers."

2 Through 2000, data are for electric utility plants with fossil-fueled steam-electric capacity of 100 megawatts or greater. Beginning in 2001, data are for electric utility and unregulated operating or standby status, with fossil-fueled steam-electric capacity of 100 megawatts or greater, or combustible-renewable steam electric capacity of 10 megawatts or greater. generating plants (independent power producers, commercial plants, and industrial plants) in one category.

Source: Table 11.6 
Table 11.6 Installed Nameplate Capacity of Fossil-Fuel Steam-Electric Generators With Environmental Equipment, 1985-2010 (Megawatts)

\begin{tabular}{|c|c|c|c|c|c|c|c|c|c|c|c|c|}
\hline \multirow[b]{2}{*}{ Year } & \multicolumn{4}{|c|}{ Coal } & \multicolumn{4}{|c|}{ Petroleum and Natural Gas } & \multicolumn{4}{|c|}{ Total $^{1}$} \\
\hline & $\begin{array}{l}\text { Particulate } \\
\text { Collectors }\end{array}$ & $\begin{array}{l}\text { Cooling } \\
\text { Towers }\end{array}$ & $\begin{array}{c}\text { Flue Gas } \\
\text { Desulfurization } \\
\text { (Scrubbers) }\end{array}$ & Total $^{2}$ & $\begin{array}{l}\text { Particulate } \\
\text { Collectors }\end{array}$ & $\begin{array}{l}\text { Cooling } \\
\text { Towers }\end{array}$ & $\begin{array}{c}\text { Flue Gas } \\
\text { Desulfurization } \\
\text { (Scrubbers) }\end{array}$ & Total $^{2}$ & $\begin{array}{l}\text { Particulate } \\
\text { Collectors }\end{array}$ & $\begin{array}{l}\text { Cooling } \\
\text { Towers }\end{array}$ & $\begin{array}{c}\text { Flue Gas } \\
\text { Desulfurization } \\
\text { (Scrubbers) }\end{array}$ & Total $^{2}$ \\
\hline 1985 & 302,056 & 120,591 & 56,955 & 304,706 & 36,054 & 28,895 & 65 & 62,371 & 338,110 & 149,486 & 57,020 & 367,078 \\
\hline 1986 & 308,566 & 126,731 & 63,735 & 311,217 & 34,258 & 27,919 & 65 & 59,618 & 342,825 & 154,650 & 63,800 & 370,835 \\
\hline 1987 & 311,043 & 127,875 & 65,688 & 312,885 & 33,431 & 27,912 & 65 & 58,783 & 344,474 & 155,786 & 65,753 & 371,668 \\
\hline 1988 & 311,776 & 129,366 & 67,156 & 313,618 & 34,063 & 27,434 & 65 & 58,937 & 345,839 & 156,800 & 67,221 & 372,555 \\
\hline 1989 & 313,680 & 131,701 & 67,469 & 315,521 & 33,975 & 28,386 & 65 & 59,736 & 347,655 & 160,087 & 67,534 & 375,257 \\
\hline 1990 & 315,681 & 134,199 & 69,057 & 317,522 & 33,639 & 28,359 & 65 & 59,372 & 349,319 & 162,557 & 69,122 & 376,894 \\
\hline 1991 & 319,046 & 135,565 & 70,474 & 319,110 & 33,864 & 29,067 & 260 & 59,773 & 352,910 & 164,632 & 70,734 & 378,883 \\
\hline 1992 & 319,856 & 136,266 & 71,336 & 319,918 & 33,509 & 28,764 & 195 & 59,116 & 353,365 & 165,030 & 71,531 & 379,034 \\
\hline 1993 & 318,188 & 135,885 & 71,106 & 318,251 & 32,620 & 28,922 & - & 58,580 & 350,808 & 164,807 & 71,106 & 376,831 \\
\hline 1994 & 319,485 & 137,266 & 80,617 & 319,776 & 31,695 & 28,186 & - & 57,123 & 351,180 & 165,452 & 80,617 & 376,899 \\
\hline 1995 & 320,685 & 138,108 & 84,677 & 320,749 & 30,513 & 27,187 & - & 54,942 & 351,198 & 165,295 & 84,677 & 375,691 \\
\hline 1996 & 321,805 & 139,065 & 85,842 & 321,869 & 30,349 & 27,685 & - & 55,275 & 352,154 & 166,749 & 85,842 & 377,144 \\
\hline 1997 & 320,646 & 138,120 & 86,605 & 320,710 & 31,422 & 28,766 & - & 56,485 & 352,068 & 166,886 & 86,605 & 377,195 \\
\hline 1998 & 321,082 & 139,082 & 87,783 & 321,353 & 30,708 & 27,814 & - & 55,764 & 351,790 & 166,896 & 87,783 & 377,117 \\
\hline 1999 & 324,109 & 146,377 & 89,666 & 331,379 & 29,371 & 29,142 & - & 55,812 & 353,480 & 175,520 & 89,666 & 387,192 \\
\hline 2000 & 321,636 & 146,093 & 89,675 & 328,741 & 31,090 & 29,427 & - & 57,697 & 352,727 & 175,520 & 89,675 & 386,438 \\
\hline $2001^{3}$ & 329,187 & 154,747 & 97,804 & 329,187 & 31,575 & 34,649 & 184 & 61,634 & 360,762 & 189,396 & 97,988 & 390,821 \\
\hline 2002 & 329,459 & 154,750 & 98,363 & 329,459 & 29,879 & 45,920 & 310 & 72,008 & 359,338 & 200,670 & 98,673 & 401,341 \\
\hline 2003 & 328,587 & 155,158 & 99,257 & 328,587 & 29,422 & 55,770 & 310 & 81,493 & 358,009 & 210,928 & 99,567 & 409,954 \\
\hline 2004 & 328,506 & 157,968 & 101,182 & 328,506 & 27,402 & 57,082 & 310 & 81,450 & 355,782 & 214,989 & 101,492 & 409,769 \\
\hline 2005 & 328,720 & 158,493 & 101,338 & 328,720 & 27,005 & 59,214 & 310 & 83,307 & 355,599 & 217,646 & 101,648 & 411,840 \\
\hline 2006 & NA & NA & NA & NA & NA & NA & NA & NA & NA & NA & NA & NA \\
\hline 2007 & 328,029 & 159,388 & 118,739 & 328,336 & 26,496 & 69,497 & 285 & 93,066 & 354,407 & 228,704 & 119,024 & 421,120 \\
\hline 2008 & 329,099 & 161,234 & 139,877 & 329,513 & 26,565 & 73,315 & 346 & 96,984 & 355,517 & 234,254 & 140,223 & 426,073 \\
\hline 2009 & 332,546 & 165,795 & 167,172 & 332,546 & 25,925 & 75,770 & 346 & 98,756 & 358,342 & 241,347 & 167,517 & 430,956 \\
\hline 2010 & 329,248 & 165,030 & 185,217 & 329,608 & 26,289 & 75,444 & 1,049 & 98,946 & 355,407 & 240,257 & 186,266 & 428,207 \\
\hline
\end{tabular}

1 Totals may not equal sum of components due to independent rounding.

Components are not additive because some generators are included in more than one category.

3 Through 2000, data are for electric utility plants with fossil-fueled steam-electric capacity of 100 megawatts or greater. Beginning in 2001, data are for electric utility and unregulated generating plants (independent power producers, commercial plants, and industrial plants) in operating or standby status, with fossil-fueled steam-electric capacity of 10 megawatts or greater.

$\mathrm{NA}=$ Not available. $-=$ No data reported.

Note: See "Cooling Tower," "Flue Gas Desulfurization," and "Particulate Collectors" in Glossary.

Web Page: For related information, see http://www.eia.gov/electricity/.

Sources: • 1985-1996-U.S. Energy Information Administration (EIA), Form EIA-767, "Steam-Electric Plant Operation and Design Report." • 1997-2005-EIA, Electric Power Annual 2008 (January 2010), Table 3.10, and Form EIA-767, "Steam-Electric Plant Operation and Design Report." • 2007 forward-EIA, Electric Power Annual 2010 (November 2011), Table 3.10, and Form EIA-860, "Annual Electric Generator Report." 


\section{Environment}

Note. Accounting for Carbon Dioxide Emissions From Biomass Energy Combustion. Carbon dioxide $\left(\mathrm{CO}_{2}\right)$ emissions from the combustion of biomass to produce energy are excluded from the total energy-related $\mathrm{CO}_{2}$ emissions reported in the Annual Energy Review Section 11, but appear separately in Tables 11.1-11.2e. According to current international convention (see the Intergovernmental Panel on Climate Change’s “2006 IPCC Guidelines for National Greenhouse Gas Inventories"), carbon released through biomass combustion is excluded from reported energy-related emissions. The release of carbon from biomass combustion is assumed to be balanced by the uptake of carbon when the feedstock is grown, resulting in zero net emissions over some period of time. (This is not to say that biomass energy is carbon-neutral. Energy inputs are required in order to grow, fertilize, and harvest the feedstock and to produce and process the biomass into fuels.)

However, analysts have debated whether increased use of biomass energy may result in a decline in terrestrial carbon stocks, leading to a net positive release of carbon rather than the zero net release assumed by its exclusion from reported energy-related emissions. For example, the clearing of forests for biofuel crops could result in an initial release of carbon that is not fully recaptured in subsequent use of the land for agriculture.

To reflect the potential net emissions, the international convention for greenhouse gas inventories is to report biomass emissions in the category "agriculture, forestry, and other land use," usually based on estimates of net changes in carbon stocks over time.

This indirect accounting of $\mathrm{CO}_{2}$ emissions from biomass can potentially lead to confusion in accounting for and understanding the flow of $\mathrm{CO}_{2}$ emissions within energy and non-energy systems. In recognition of this issue, reporting of $\mathrm{CO}_{2}$ emissions from biomass combustion alongside other energy-related $\mathrm{CO}_{2}$ emissions offers an alternative accounting treatment. It is important, however, to avoid misinterpreting emissions from fossil energy and biomass energy sources as necessarily additive. Instead, the combined total of direct $\mathrm{CO}_{2}$ emissions from biomass and energy-related $\mathrm{CO}_{2}$ emissions implicitly assumes that none of the carbon emitted was previously or subsequently reabsorbed in terrestrial sinks or that other emissions sources offset any such sequestration. 


\section{Appendix A}

\section{British Thermal Unit Conversion Factors}

\section{Using Thermal Conversion Factors}

The thermal conversion factors presented in the following tables can be used to estimate the heat content in British thermal units (Btu) of a given amount of energy measured in physical units, such as barrels or cubic feet. For example, 10 barrels of asphalt has a heat content of approximately 66.36 million Btu (10 barrels x 6.636 million Btu per barrel $=66.36$ million Btu).

The heat content rates (i.e., thermal conversion factors) provided in this section represent the gross (or higher or upper) energy content of the fuels. Gross heat content rates are applied in all Btu calculations for the Annual Energy Review and are commonly used in energy calculations in the United States; net (or lower) heat content rates are typically used in European energy calculations. The difference between the two rates is the amount of energy that is consumed to vaporize water that is created during the combustion process. Generally, the difference ranges from 2 percent to 10 percent, depending on the specific fuel and its hydrogen content. Some fuels, such as unseasoned wood, can be more than 40 percent different in their gross and net heat content rates. See "Heat Content" and "British thermal unit (Btu)" in the Glossary for more information.

Thermal conversion factors for hydrocarbon mixes (Table A1) are weighted averages of the thermal conversion factors for each hydrocarbon included in the mix. For example, in calculating the thermal conversion factor for a 60-40 butanepropane mixture, the thermal conversion factor for butane is weighted 1.5 times the thermal conversion factor for propane.

In general, the annual thermal conversion factors presented in Tables A2 through A6 are computed from final annual data, or are from the best available data and labeled "preliminary." Often, the previous year's factor is used as the preliminary value until data become available to calculate the factor appropriate to the year. The source of each factor is described in the section entitled "Thermal Conversion Factor Source Documentation,” which follows Table A6 in this appendix.

\begin{tabular}{|c|c|}
\hline $\begin{array}{ll}\text { Table A1. } & \begin{array}{l}\text { Approximate Heat Content of Petroleum Products } \\
\text { (Million Btu per Barrel) }\end{array} \\
\end{array}$ & \\
\hline Asphalt $\ldots .$. & 6.636 \\
\hline …… & 5.048 \\
\hline 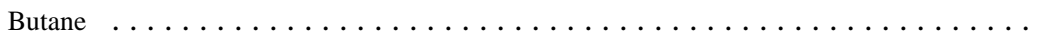 & 4.326 \\
\hline Butane-Propane Mixture (60 percent- 40 percent) & 4.130 \\
\hline 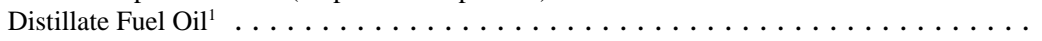 & 5.825 \\
\hline 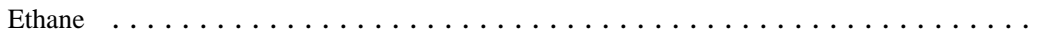 & 3.082 \\
\hline Ethane-Propane Mixture $(70$ percent-30 percent) $\ldots \ldots \ldots \ldots \ldots \ldots \ldots$ & 3.308 \\
\hline Isobutane $\quad \ldots \ldots \ldots \ldots \ldots \ldots \ldots \ldots$ & 3.974 \\
\hline Jet Fuel, Kerosene-Type .. & 5.670 \\
\hline Jet Fuel, Naphtha-Type $\ldots \ldots \ldots \ldots \ldots$ & 5.355 \\
\hline Kerosene $\quad \ldots \ldots \ldots \ldots \ldots \ldots$ & 5.670 \\
\hline$\ldots \ldots \ldots \ldots \ldots \ldots \ldots$ & 6.065 \\
\hline \multicolumn{2}{|l|}{ Motor Gasoline ${ }^{2}$} \\
\hline Conventional & 5.253 \\
\hline Oxygenated & 5.150 \\
\hline Reformulated & 5.150 \\
\hline Natural Gasoline ..... . & 4.620 \\
\hline Pentanes Plus . . & 4.620 \\
\hline \multicolumn{2}{|l|}{ Petrochemical Feedstocks } \\
\hline Naphtha less than $401^{\circ} \mathrm{F}$ & 5.248 \\
\hline Other Oils equal to or greater than $401^{\circ} \mathrm{F}$ & 5.825 \\
\hline Still Gas $\ldots \ldots \ldots \ldots \ldots \ldots$ & 6.000 \\
\hline Petroleum Coke & 6.024 \\
\hline Plant Condensate & 5.418 \\
\hline Propane $\ldots . .$. & 3.836 \\
\hline Residual Fuel Oil & 6.287 \\
\hline Road Oil $\ldots \ldots \ldots \ldots \ldots$ & 6.636 \\
\hline Special Naphthas $\quad$. & 5.248 \\
\hline Still Gas $\ldots . .$. & 6.000 \\
\hline Unfinished Oils & 5.825 \\
\hline Unfractionated Stream & 5.418 \\
\hline Waxes $\ldots \ldots \ldots \ldots$ & 5.537 \\
\hline 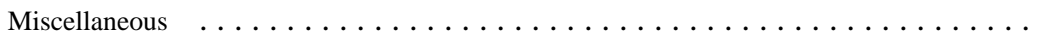 & 5.796 \\
\hline
\end{tabular}

${ }^{1}$ Does not include biodiesel. See Table A3 for biodiesel heat contents.

${ }^{2}$ See Table A3 for motor gasoline weighted heat contents beginning in 1994, and for fuel ethanol heat contents.

Web Page: For related information, see http://www.eia.gov/totalenergy/data/annual/\#appendices. Note: The values in this table are for gross heat contents. See "Heat Content" in Glossary.

Sources: See “Thermal Conversion Factor Source Documentation,” which follows Table A6. 
Table A2. Approximate Heat Content of Petroleum Production, Imports, and Exports, Selected Years, 1949-2011 (Million Btu per Barrel)

\begin{tabular}{|c|c|c|c|c|c|c|c|c|}
\hline \multirow[b]{2}{*}{ Year } & \multicolumn{2}{|c|}{ Production } & \multicolumn{3}{|c|}{ Imports } & \multicolumn{3}{|c|}{ Exports } \\
\hline & Crude Oil ${ }^{1}$ & $\begin{array}{l}\text { Natural Gas } \\
\text { Plant Liquids }\end{array}$ & Crude Oil $^{1}$ & $\begin{array}{c}\text { Petroleum } \\
\text { Products }\end{array}$ & Total & Crude Oil ${ }^{1}$ & $\begin{array}{l}\text { Petroleum } \\
\text { Products }\end{array}$ & Total \\
\hline 1949 & 5.800 & 4.544 & 5.952 & 6.261 & 6.059 & 5.800 & 5.651 & 5.692 \\
\hline 1950 & 5.800 & 4.522 & 5.943 & 6.263 & 6.080 & 5.800 & 5.751 & 5.766 \\
\hline 1955 & 5.800 & 4.406 & 5.924 & 6.234 & 6.040 & 5.800 & 5.765 & 5.768 \\
\hline 1960 & 5.800 & 4.295 & 5.911 & 6.161 & 6.021 & 5.800 & 5.835 & 5.834 \\
\hline 1965 & 5.800 & 4.264 & 5.872 & 6.123 & 5.997 & 5.800 & 5.742 & 5.743 \\
\hline 1970 & 5.800 & 4.146 & 5.822 & 6.088 & 5.985 & 5.800 & 5.811 & 5.810 \\
\hline 1975 & 5.800 & 3.984 & 5.821 & 5.935 & 5.858 & 5.800 & 5.747 & 5.748 \\
\hline 1976 & 5.800 & 3.964 & 5.808 & 5.980 & 5.856 & 5.800 & 5.743 & 5.745 \\
\hline 1977 & 5.800 & 3.941 & 5.810 & 5.908 & 5.834 & 5.800 & 5.796 & 5.797 \\
\hline 1978 & 5.800 & 3.925 & 5.802 & 5.955 & 5.839 & 5.800 & 5.814 & 5.808 \\
\hline 1979 & 5.800 & 3.955 & 5.810 & 5.811 & 5.810 & 5.800 & 5.864 & 5.832 \\
\hline 1980 & 5.800 & 3.914 & 5.812 & 5.748 & 5.796 & 5.800 & 5.841 & 5.820 \\
\hline 1981 & 5.800 & 3.930 & 5.818 & 5.659 & 5.775 & 5.800 & 5.837 & 5.821 \\
\hline 1982 & 5.800 & 3.872 & 5.826 & 5.664 & 5.775 & 5.800 & 5.829 & 5.820 \\
\hline 1983 & 5.800 & 3.839 & 5.825 & 5.677 & 5.774 & 5.800 & 5.800 & 5.800 \\
\hline 1984 & 5.800 & 3.812 & 5.823 & 5.613 & 5.745 & 5.800 & 5.867 & 5.850 \\
\hline 1985 & 5.800 & 3.815 & 5.832 & 5.572 & 5.736 & 5.800 & 5.819 & 5.814 \\
\hline 1986 & 5.800 & 3.797 & 5.903 & 5.624 & 5.808 & 5.800 & 5.839 & 5.832 \\
\hline 1987 & 5.800 & 3.804 & 5.901 & 5.599 & 5.820 & 5.800 & 5.860 & 5.858 \\
\hline 1988 & 5.800 & 3.800 & 5.900 & 5.618 & 5.820 & 5.800 & 5.842 & 5.840 \\
\hline 1989 & 5.800 & 3.826 & 5.906 & 5.641 & 5.833 & 5.800 & 5.869 & 5.857 \\
\hline 1990 & 5.800 & 3.822 & 5.934 & 5.614 & 5.849 & 5.800 & 5.838 & 5.833 \\
\hline 1991 & 5.800 & 3.807 & 5.948 & 5.636 & 5.873 & 5.800 & 5.827 & 5.823 \\
\hline 1992 & 5.800 & 3.804 & 5.953 & 5.623 & 5.877 & 5.800 & 5.774 & 5.777 \\
\hline 1993 & 5.800 & 3.801 & 5.954 & 5.620 & 5.883 & 5.800 & 5.777 & 5.779 \\
\hline 1994 & 5.800 & 3.794 & 5.950 & 5.534 & 5.861 & 5.800 & 5.777 & 5.779 \\
\hline 1995 & 5.800 & 3.796 & 5.938 & 5.483 & 5.855 & 5.800 & 5.740 & 5.746 \\
\hline 1996 & 5.800 & 3.777 & 5.947 & 5.468 & 5.847 & 5.800 & 5.728 & 5.736 \\
\hline 1997 & 5.800 & 3.762 & 5.954 & 5.469 & 5.862 & 5.800 & 5.726 & 5.734 \\
\hline 1998 & 5.800 & 3.769 & 5.953 & 5.462 & 5.861 & 5.800 & 5.710 & 5.720 \\
\hline 1999 & 5.800 & 3.744 & 5.942 & 5.421 & 5.840 & 5.800 & 5.684 & 5.699 \\
\hline 2000 & 5.800 & 3.733 & 5.959 & 5.432 & 5.849 & 5.800 & 5.651 & 5.658 \\
\hline 2001 & 5.800 & 3.735 & 5.976 & 5.443 & 5.862 & 5.800 & 5.751 & 5.752 \\
\hline 2002 & 5.800 & 3.729 & 5.971 & 5.451 & 5.863 & 5.800 & 5.687 & 5.688 \\
\hline 2003 & 5.800 & 3.739 & 5.970 & 5.438 & 5.857 & 5.800 & 5.739 & 5.740 \\
\hline 2004 & 5.800 & 3.724 & 5.981 & 5.475 & 5.863 & 5.800 & 5.753 & 5.754 \\
\hline 2005 & 5.800 & 3.724 & 5.977 & 5.474 & 5.845 & 5.800 & 5.741 & 5.743 \\
\hline 2006 & 5.800 & 3.712 & 5.980 & 5.454 & 5.842 & 5.800 & 5.723 & 5.724 \\
\hline 2007 & 5.800 & 3.701 & 5.985 & 5.503 & 5.862 & 5.800 & 5.749 & 5.750 \\
\hline 2008 & 5.800 & 3.706 & 5.990 & 5.479 & 5.866 & 5.800 & 5.762 & 5.762 \\
\hline 2009 & 5.800 & 3.692 & 5.988 & 5.525 & 5.882 & 5.800 & 5.737 & 5.738 \\
\hline 2010 & 5.800 & $\mathrm{R}_{3.674}$ & 5.989 & $\mathrm{R}_{5.557}$ & $\mathrm{R}_{5.894}$ & 5.800 & $R_{5} .670$ & $\mathrm{R}_{5.672}$ \\
\hline $2011^{P}$ & 5.800 & 3.675 & 6.007 & 5.555 & 5.910 & 5.800 & 5.619 & 5.622 \\
\hline
\end{tabular}


Table A3. Approximate Heat Content of Petroleum Consumption and Biofuels Production, Selected Years, 1949-2011 (Million Btu per Barrel)

\begin{tabular}{|c|c|c|c|c|c|c|c|c|c|c|c|c|}
\hline \multirow[b]{2}{*}{ Year } & \multicolumn{6}{|c|}{ Total Petroleum ${ }^{1}$ Consumption by Sector } & \multirow{2}{*}{$\begin{array}{c}\text { Liquefied } \\
\text { Petroleum } \\
\text { Gases } \\
\text { Consumption } 6\end{array}$} & \multirow{2}{*}{$\begin{array}{c}\text { Motor } \\
\text { Gasoline } \\
\text { Consumption } 7\end{array}$} & \multirow[b]{2}{*}{$\begin{array}{c}\text { Fuel } \\
\text { Ethanol } 8\end{array}$} & \multirow{2}{*}{$\begin{array}{c}\text { Fuel } \\
\text { Ethanol } \\
\text { Feedstock } \\
\text { Factor }^{9}\end{array}$} & \multirow[b]{2}{*}{ Biodiesel } & \multirow{2}{*}{$\begin{array}{c}\text { Biodiesel } \\
\text { Feedstock } \\
\text { Factor }{ }^{10}\end{array}$} \\
\hline & Residential & Commercial $^{2}$ & Industrial ${ }^{2}$ & $\begin{array}{c}\text { Trans- } \\
\text { portation }\end{array}$ & $\begin{array}{l}\text { Electric } \\
\text { Power }{ }^{4,5}\end{array}$ & Total $^{2}$ & & & & & & \\
\hline 1949 & 5.484 & 5.813 & 5.957 & 5.465 & 6.254 & 5.649 & 4.011 & 5.253 & NA & $N A$ & NA & $N A$ \\
\hline 1950 & 5.473 & 5.817 & 5.953 & 5.461 & 6.254 & 5.649 & 4.011 & 5.253 & NA & $N A$ & NA & $N A$ \\
\hline 1955 & 5.469 & 5.781 & 5.881 & 5.407 & $\begin{array}{l}6.254 \\
6.254\end{array}$ & 5.591 & 4.011 & 5.253 & NA & $N A$ & NA & $N A$ \\
\hline 1960 & 5.417 & 5.781 & $\begin{array}{l}5.818 \\
5.018\end{array}$ & 5.387 & 6.267 & 5.555 & 4.011 & 5.253 & NA & $N A$ & NA & $N A$ \\
\hline 1965 & 5.364 & 5.760 & 5.748 & 5.386 & 6.267 & 5.532 & 4.011 & 5.253 & NA & $N A$ & NA & $N A$ \\
\hline 1970 & 5.260 & 5.708 & 5.595 & 5.393 & 6.252 & 5.503 & 63.779 & 5.253 & NA & $N A$ & NA & $N A$ \\
\hline 1975 & 5.253 & 5.649 & 5.513 & 5.392 & 6.250 & 5.494 & 3.715 & 5.253 & NA & $N A$ & NA & $N A$ \\
\hline 1976 & 5.277 & 5.672 & 5.523 & 5.396 & 6.251 & 5.504 & 3.711 & 5.253 & NA & $N A$ & NA & $N A$ \\
\hline 1977 & 5.285 & 5.682 & 5.539 & 5.401 & 6.249 & 5.518 & 3.677 & 5.253 & NA & $N A$ & NA & $N A$ \\
\hline 1978 & 5.287 & 5.665 & 5.536 & 5.405 & 6.251 & 5.519 & 3.669 & 5.253 & NA & $N A$ & NA & $N A$ \\
\hline 1979 & 5.365 & 5.717 & 5.409 & 5.429 & 6.258 & 5.494 & 3.680 & 5.253 & NA & $N A$ & $\mathrm{NA}$ & $N A$ \\
\hline 1980 & 5.321 & 5.751 & 5.366 & 5.441 & 6.254 & 5.479 & 3.674 & 5.253 & 3.563 & 6.586 & NA & $N A$ \\
\hline 1981 & 5.283 & 5.693 & 5.299 & 5.433 & 6.258 & 5.448 & 3.643 & 5.253 & 3.563 & 6.562 & NA & $N A$ \\
\hline 1982 & 5.266 & 5.698 & 5.247 & 5.423 & 6.258 & 5.415 & 3.615 & 5.253 & 3.563 & 6.539 & NA & $N A$ \\
\hline 1983 & 5.140 & 5.591 & 5.254 & 5.416 & 6.255 & 5.406 & 3.614 & 5.253 & 3.563 & 6.515 & NA & $N A$ \\
\hline 1984 & 5.307 & 5.657 & 5.207 & 5.418 & 6.251 & 5.395 & 3.599 & 5.253 & 3.563 & 6.492 & NA & $N A$ \\
\hline 1985 & 5.263 & 5.598 & 5.199 & 5.423 & 6.247 & 5.387 & 3.603 & 5.253 & 3.563 & 6.469 & NA & $N A$ \\
\hline 1986 & 5.268 & 5.632 & 5.269 & 5.426 & 6.257 & 5.418 & 3.640 & 5.253 & 3.563 & 6.446 & NA & $N A$ \\
\hline 1987 & 5.239 & 5.594 & 5.233 & 5.429 & 6.249 & 5.403 & 3.659 & 5.253 & 3.563 & 6.423 & NA & $N A$ \\
\hline $\begin{array}{l}1988 \\
1989\end{array}$ & $\begin{array}{l}5.257 \\
5.194\end{array}$ & $\begin{array}{l}5.597 \\
5549\end{array}$ & $\begin{array}{l}5.228 \\
5.219\end{array}$ & $\begin{array}{l}5.433 \\
5.438\end{array}$ & $\begin{array}{r}6.250 \\
46.240\end{array}$ & $\begin{array}{l}5.410 \\
5.410\end{array}$ & $\begin{array}{l}3.652 \\
3.683\end{array}$ & $\begin{array}{l}5.253 \\
5.253\end{array}$ & $\begin{array}{l}3.563 \\
3.563\end{array}$ & $\begin{array}{l}6.400 \\
6.377\end{array}$ & $\begin{array}{l}\text { NA } \\
\text { NA }\end{array}$ & $\begin{array}{l}N A \\
N A\end{array}$ \\
\hline 1990 & 5.145 & 5.553 & 5.253 & $\begin{array}{l}.400 \\
5.442\end{array}$ & 6.244 & 5.411 & $\begin{array}{l}3.683 \\
3.625\end{array}$ & $\begin{array}{l}.253 \\
5.253\end{array}$ & $\begin{array}{l}3.563 \\
3.563\end{array}$ & $\begin{array}{l}6.371 \\
6.355\end{array}$ & NA & $N A$ \\
\hline 1991 & $\begin{array}{l}5.145 \\
5.094\end{array}$ & $\begin{array}{l}5.553 \\
5.528\end{array}$ & $\begin{array}{l}5.253 \\
5.167\end{array}$ & $\begin{array}{l}5.442 \\
5.441\end{array}$ & $\begin{array}{l}.0 .244 \\
6.246\end{array}$ & $\begin{array}{l}5.411 \\
5.384\end{array}$ & $\begin{array}{l}3.625 \\
3.614\end{array}$ & $\begin{array}{l}.253 \\
5.253\end{array}$ & $\begin{array}{l}\text {.563 } \\
3.563\end{array}$ & $\begin{array}{l}6.355 \\
6.332\end{array}$ & NA & NA \\
\hline 1992 & $\begin{array}{l}5.094 \\
5.124\end{array}$ & $\begin{array}{l}5.528 \\
5.513\end{array}$ & $\begin{array}{l}5.16 / \\
5.168\end{array}$ & $\begin{array}{l}5.441 \\
5.443\end{array}$ & $\begin{array}{l}6.246 \\
6.238\end{array}$ & $\begin{array}{l}5.384 \\
5.378\end{array}$ & $\begin{array}{l}3.614 \\
3.624\end{array}$ & $\begin{array}{l}.253 \\
5.253\end{array}$ & $\begin{array}{l}3.563 \\
3.563\end{array}$ & $\begin{array}{l}6.332 \\
6.309\end{array}$ & NA & NA \\
\hline 1993 & $\begin{array}{l}5.124 \\
5.102\end{array}$ & $\begin{array}{r}5.513 \\
25.505\end{array}$ & $\begin{array}{r}5.168 \\
25.178\end{array}$ & $\begin{array}{r}5.443 \\
25.436\end{array}$ & $\begin{array}{l}6.238 \\
6.230\end{array}$ & $\begin{array}{r}5.378 \\
25.379\end{array}$ & $\begin{array}{l}.024 \\
3.606\end{array}$ & $\begin{array}{l}5.253 \\
5.253\end{array}$ & $\begin{array}{l}\text { S.563 } \\
3.563\end{array}$ & $\begin{array}{l}6.309 \\
6.287\end{array}$ & NA & $N A$ \\
\hline 1994 & $\begin{array}{l}5.102 \\
5.098\end{array}$ & 5.515 & 5.150 & 5.424 & $\begin{array}{l}.230 \\
6.213\end{array}$ & 5.361 & $\begin{array}{l}3.606 \\
3.635\end{array}$ & $\begin{array}{l}55.235 \\
75.230\end{array}$ & $\begin{array}{l}\text { S.563 } \\
3.563\end{array}$ & $\begin{array}{l}0.281 \\
6.264\end{array}$ & NA & NA \\
\hline 1995 & 5.063 & 5.478 & 5.121 & $\begin{array}{l}5.424 \\
5.417\end{array}$ & $\begin{array}{l}6.188 \\
6.188\end{array}$ & $\begin{array}{l}5.341 \\
5.361\end{array}$ & $\begin{array}{l}.035 \\
3.623\end{array}$ & 5.215 & $\begin{array}{l}3.563 \\
3.563\end{array}$ & $\begin{array}{l}\begin{array}{l}6.204 \\
6.242\end{array}\end{array}$ & NA & $N A$ \\
\hline 1996 & $\begin{array}{l}.0003 \\
4.998\end{array}$ & $\begin{array}{l}.0418 \\
5.433\end{array}$ & 5.114 & $\begin{array}{l}5.411 \\
5.420\end{array}$ & $\begin{array}{l}.180 \\
6.195\end{array}$ & $\begin{array}{l}\text { S.J41 } \\
5.336\end{array}$ & $\begin{array}{l}.025 \\
3.613\end{array}$ & 5.216 & $\begin{array}{l}3.563 \\
3.563\end{array}$ & $\begin{array}{l}\text { l. } \\
6.2220\end{array}$ & NA & $N A$ \\
\hline 1997 & 4.989 & 5.391 & 5.120 & 5.416 & 6.199 & 5.336 & $\begin{array}{l}.015 \\
3.616\end{array}$ & $\begin{array}{l}.210 \\
5.213\end{array}$ & $\begin{array}{l}\text {.503 } \\
3.563\end{array}$ & $\begin{array}{l}\text { l. } \\
6.198\end{array}$ & NA & $N A$ \\
\hline 1998 & 4.975 & 5.365 & 5.137 & 5.413 & 6.210 & 5.349 & $\begin{array}{l}.010 \\
3.614\end{array}$ & 5.212 & $\begin{array}{l}.500 \\
3.563\end{array}$ & $\begin{array}{l}0.170 \\
6.176\end{array}$ & NA & $N A$ \\
\hline 1999 & 4.902 & 5.291 & 5.092 & 5.413 & 6.205 & 5.328 & $\begin{array}{l}.014 \\
3.616\end{array}$ & 5.211 & $\begin{array}{l}3.500 \\
3.563\end{array}$ & $\begin{array}{l}0.167 \\
6.167\end{array}$ & NA & $N A$ \\
\hline 2000 & 4.908 & 5.316 & 5.057 & 5.422 & 6.189 & 5.326 & 3.607 & 5.210 & 3.563 & 6.159 & NA & $N A$ \\
\hline 2001 & 4.937 & 5.325 & 5.142 & 5.412 & 6.199 & 5.345 & 3.614 & 5.210 & 3.563 & 6.151 & 5.359 & 5.433 \\
\hline 2002 & 4.886 & 5.293 & 5.093 & 5.411 & 6.173 & 5.324 & 3.613 & 5.208 & 3.563 & 6.143 & 5.359 & 5.433 \\
\hline 2003 & 4.907 & 5.307 & 5.142 & 5.409 & 6.182 & 5.340 & $\begin{array}{l}3.629 \\
3.0\end{array}$ & 5.207 & 3.563 & 6.116 & 5.359 & 5.433 \\
\hline 2004 & 4.953 & 5.328 & 5.144 & 5.421 & 6.192 & 5.350 & 3.618 & 5.215 & 3.563 & 6.089 & 5.359 & 5.433 \\
\hline 2005 & 4.916 & 5.364 & 5.178 & 5.427 & 6.188 & 5.365 & 3.620 & 5.218 & 3.563 & 6.063 & 5.359 & 5.433 \\
\hline 2006 & 4.894 & 5.310 & 5.160 & 5.431 & 6.143 & 5.353 & 3.605 & 5.218 & 3.563 & 6.036 & 5.359 & 5.433 \\
\hline 2007 & 4.850 & 5.298 & 5.127 & 5.434 & 6.151 & 5.346 & 3.591 & 5.219 & 3.563 & 6.009 & 5.359 & 5.433 \\
\hline 2008 & 4.732 & 5.175 & 5.149 & 5.426 & 6.123 & 5.339 & 3.600 & 5.218 & 3.563 & 5.983 & 5.359 & 5.433 \\
\hline 2009 & 4.691 & 5.266 & 5.018 & 35.414 & 6.105 & 35.301 & 3.558 & 5.218 & 3.563 & 5.957 & 5.359 & 5.433 \\
\hline 2010 & R4.692 & R5.263 & R4.988 & R5.421 & R6.084 & R5.297 & $R_{3} .557$ & 5.218 & 3.561 & R 5.931 & 5.359 & 5.433 \\
\hline 2011 & $\mathrm{E}_{4.692}$ & E5.261 & $\mathrm{E}_{4} .964$ & E5.425 & P6.062 & P5.291 & P3.529 & P5.218 & P3.560 & 5.905 & 5.359 & 5.433 \\
\hline
\end{tabular}
1 Petroleum products supplied, including natural gas plant liquids and crude oil burned directly as fuel.
Quantity-weighted averages of the petroleum products included in each category are calculated by using heat content values shown in Table A1

Beginning in 1993, includes fuel ethanol blended into motor gasoline.

Beginning in 2009, includes renewable diesel fuel (including biodiesel) blended into distillate fuel oil. 4 Electricity-only and combined-heat-and-power (CHP) plants within the NAICS 22 category whose

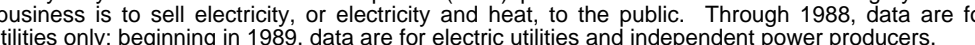
5 Electric power sector factors are weighted average heat contents for distillate fuel oil, petroleum coke, and residual fuel oil; they exclude other liquids.

6 There is a discerity in this time series between 1966 and 1967; beginning in 1967, the single conter major 7 There is a discontinuity in this time series between 1993 and 1994; beginning in 1994, the single constant factor is replaced by a quantity-weighted factor-quantity-weighted averages of the majo components of motor gasoline, incluaing fuel ethanol, are calculated by using heat content values shown in
Table A1.

8 Includes denaturant (petroleum added to ethanol to make it undrinkable). Fuel ethanol factors are weighted average heat contents for undenatured ethanol (3.539 million Btu per barrel), pentanes plus used as denaturant (4.620 million Btu per barrel), and conventional motor gasoline and motor gasoline blending
components used as denaturant ( 5.253 million Btu per barrel). The factor for 2009 is used as the estimated

factor for 1980-2008.

Corn input to the production of undenatured ethanol (million Btu corn per barrel undenatured ethanol) used as the factor to estimate total biomass inputs to the production of undenatured ethanol. Observed
ethanol yields (gallons undenatured ethanol per bushel of corn) are 2.5 in 1980, 2.666 in 1998, 2.68 in ethanol yields (gallons undenatured ethanol per bushel of corn) are 2.5 in 1980, 2.666 in 1998, 2.68 in 2002, and 2.764 in 2009; yields in other years are estimated. Corn is assumed to have a gross heat 3.539 million Btu per barrel. 10 Soybean oil input to the production of biodiesel (million Btu soybean oil per barrel biodiesel), used as the factor to estimate total biomass inputs to the production of biodiesel. It is assumed that 7.65 pounds of
soybean oil are needed to produce one gallon of biodiesel, and 5.433 million Btu of soybean oil are needed soybean oil are needed to produce one gallon of biodiesel, and 5.433 million Btu of soybean oil are needed
to produce one barrel of biodiesel. Soybean oil is assumed to have a gross heat content of 16,909 Btu per
pound, or 5.483 million Btu per barrel. Biodiesel is assumed to have a gross heat content of 17,253 Btu per pound, or 5.359 million Btu per barre.

R=Revised. P=Preliminary. E=Estimate. NA=Not available.

Notes: - Residential, commercial, industrial, and transportation petroleum heat contents are revised beginning in 1949 due to a change in the estimation methodology. - The heat content values in this table are gros geat contents. See
Web Pages: See htpp://www.eia.gov//totalenergy//data/monthly/\#appendices for updated annual
conversion factors. - See http://www.eia.gov/totalenergy/data/annual/\#appendices for all annual data

beginning in 1949 .Thermal Conversion Factor Source Documentation," which follows Table A6. 
Table A4. Approximate Heat Content of Natural Gas, Selected Years, 1949-2011

(Btu per Cubic Foot)

\begin{tabular}{|c|c|c|c|c|c|c|c|}
\hline \multirow[b]{2}{*}{ Year } & \multicolumn{2}{|c|}{ Production } & \multicolumn{3}{|c|}{ Consumption ${ }^{1}$} & \multirow[b]{2}{*}{ Imports } & \multirow[b]{2}{*}{ Exports } \\
\hline & Marketed & Dry & End-Use Sectors ${ }^{2}$ & Electric Power Sector ${ }^{3}$ & Total & & \\
\hline 1949 & 1,120 & 1,035 & 1,035 & 1,035 & 1,035 & -- & 1,035 \\
\hline 1950 & 1,119 & 1,035 & 1,035 & 1,035 & 1,035 & -- & 1,035 \\
\hline 1955 & 1,120 & 1,035 & 1,035 & 1,035 & 1,035 & 1,035 & 1,035 \\
\hline 1960 & 1,107 & 1,035 & 1,035 & 1,035 & 1,035 & 1,035 & 1,035 \\
\hline 1965 & 1,101 & 1,032 & 1,032 & 1,032 & 1,032 & 1,032 & 1,032 \\
\hline 1970 & 1,102 & 1,031 & 1,031 & 1,031 & 1,031 & 1,031 & 1,031 \\
\hline 1975 & 1,095 & 1,021 & 1,020 & 1,026 & 1,021 & 1,026 & 1,014 \\
\hline 1976 & 1,093 & 1,020 & 1,019 & 1,023 & 1,020 & 1,025 & 1,013 \\
\hline 1977 & 1,093 & 1,021 & 1,019 & 1,029 & 1,021 & 1,026 & 1,013 \\
\hline 1978 & 1,088 & 1,019 & 1,016 & 1,034 & 1,019 & 1,030 & 1,013 \\
\hline 1979 & 1,092 & 1,021 & 1,018 & 1,035 & 1,021 & 1,037 & 1,013 \\
\hline 1980 & 1,098 & 1,026 & 1,024 & 1,035 & 1,026 & 1,022 & 1,013 \\
\hline 1981 & 1,103 & 1,027 & 1,025 & 1,035 & 1,027 & 1,014 & 1,011 \\
\hline 1982 & 1,107 & 1,028 & 1,026 & 1,036 & 1,028 & 1,018 & 1,011 \\
\hline 1983 & 1,115 & 1,031 & 1,031 & 1,030 & 1,031 & 1,024 & 1,010 \\
\hline 1984 & 1,109 & 1,031 & 1,030 & 1,035 & 1,031 & 1,005 & 1,010 \\
\hline 1985 & 1,112 & 1,032 & 1,031 & 1,038 & 1,032 & 1,002 & 1,011 \\
\hline 1986 & 1,110 & 1,030 & 1,029 & 1,034 & 1,030 & 997 & 1,008 \\
\hline 1987 & 1,112 & 1,031 & 1,031 & 1,032 & 1,031 & 999 & 1,011 \\
\hline 1988 & 1,109 & 1,029 & 1,029 & 1,028 & 1,029 & 1,002 & 1,018 \\
\hline 1989 & 1,107 & 1,031 & 1,031 & 31,028 & 1,031 & 1,004 & 1,019 \\
\hline 1990 & 1,105 & 1,029 & 1,030 & 1,027 & 1,029 & 1,012 & 1,018 \\
\hline 1991 & 1,108 & 1,030 & 1,031 & 1,025 & 1,030 & 1,014 & 1,022 \\
\hline 1992 & 1,110 & 1,030 & 1,031 & 1,025 & 1,030 & 1,011 & 1,018 \\
\hline 1993 & 1,106 & 1,027 & 1,028 & 1,025 & 1,027 & 1,020 & 1,016 \\
\hline 1994 & 1,105 & 1,028 & 1,029 & 1,025 & 1,028 & 1,022 & 1,011 \\
\hline 1995 & 1,106 & 1,026 & 1,027 & 1,021 & 1,026 & 1,021 & 1,011 \\
\hline 1996 & 1,109 & 1,026 & 1,027 & 1,020 & 1,026 & 1,022 & 1,011 \\
\hline 1997 & 1,107 & 1,026 & 1,027 & 1,020 & 1,026 & 1,023 & 1,011 \\
\hline 1998 & 1,109 & 1,031 & 1,033 & 1,024 & 1,031 & 1,023 & 1,011 \\
\hline 1999 & 1,107 & 1,027 & 1,028 & 1,022 & 1,027 & 1,022 & 1,006 \\
\hline 2000 & 1,107 & 1,025 & 1,026 & 1,021 & 1,025 & 1,023 & 1,006 \\
\hline 2001 & 1,105 & 1,028 & 1,029 & 1,026 & 1,028 & 1,023 & 1,010 \\
\hline 2002 & $R_{1,103}$ & $R_{1,024}$ & $R_{1,025}$ & 1,020 & $R_{1,024}$ & 1,022 & 1,008 \\
\hline 2003 & $\mathrm{R}_{1,103}$ & 1,028 & 1,029 & 1,025 & 1,028 & 1,025 & 1,009 \\
\hline 2004 & 1,104 & 1,026 & 1,026 & 1,027 & 1,026 & 1,025 & 1,009 \\
\hline 2005 & 1,104 & 1,028 & 1,028 & 1,028 & 1,028 & 1,025 & 1,009 \\
\hline 2006 & 1,103 & 1,028 & 1,028 & 1,028 & 1,028 & 1,025 & 1,009 \\
\hline 2007 & $R_{1,102}$ & $R_{1,027}$ & $R_{1,027}$ & 1,027 & $R_{1,027}$ & 1,025 & 1,009 \\
\hline 2008 & 1,100 & 1,027 & 1,027 & 1,027 & 1,027 & 1,025 & 1,009 \\
\hline 2009 & 1,101 & 1,025 & 1,025 & 1,025 & 1,025 & 1,025 & 1,009 \\
\hline 2010 & $\mathrm{R}_{1,097}$ & $\mathrm{R}_{1,023}$ & $\mathrm{R}_{1,023}$ & 1,022 & $\mathrm{R}_{1,023}$ & 1,025 & 1,009 \\
\hline 2011 & $E_{1,097}$ & $E_{1,022}$ & $E_{1,023}$ & $\mathrm{P}_{1,021}$ & $E_{1,022}$ & $E_{1,025}$ & $E_{1,009}$ \\
\hline
\end{tabular}

1 Consumption factors are for natural gas, plus a small amount of supplemental gaseous fuels.

2 Residential, commercial, industrial, and transportation sectors.

Note: The values in this table are for gross heat contents. See "Heat Content" in Glossary.

3 Electricity-only and combined-heat-and-power (CHP) plants within the NAICS 22 category whose primary business is to sell electricity, or electricity and heat, to the public. Through 1988, data are for electric utilities only; beginning in 1989, data are for electric utilities and independent power producers.

Web Pages: - See http://www.eia.gov/totalenergy/data/monthly/\#appendices for updated annual beginning in 1949 .

Sources: See "Thermal Conversion Factor Source Documentation," which follows Table A6. 
Table A5. Approximate Heat Content of Coal and Coal Coke, Selected Years, 1949-2011

(Million Btu per Short Ton)

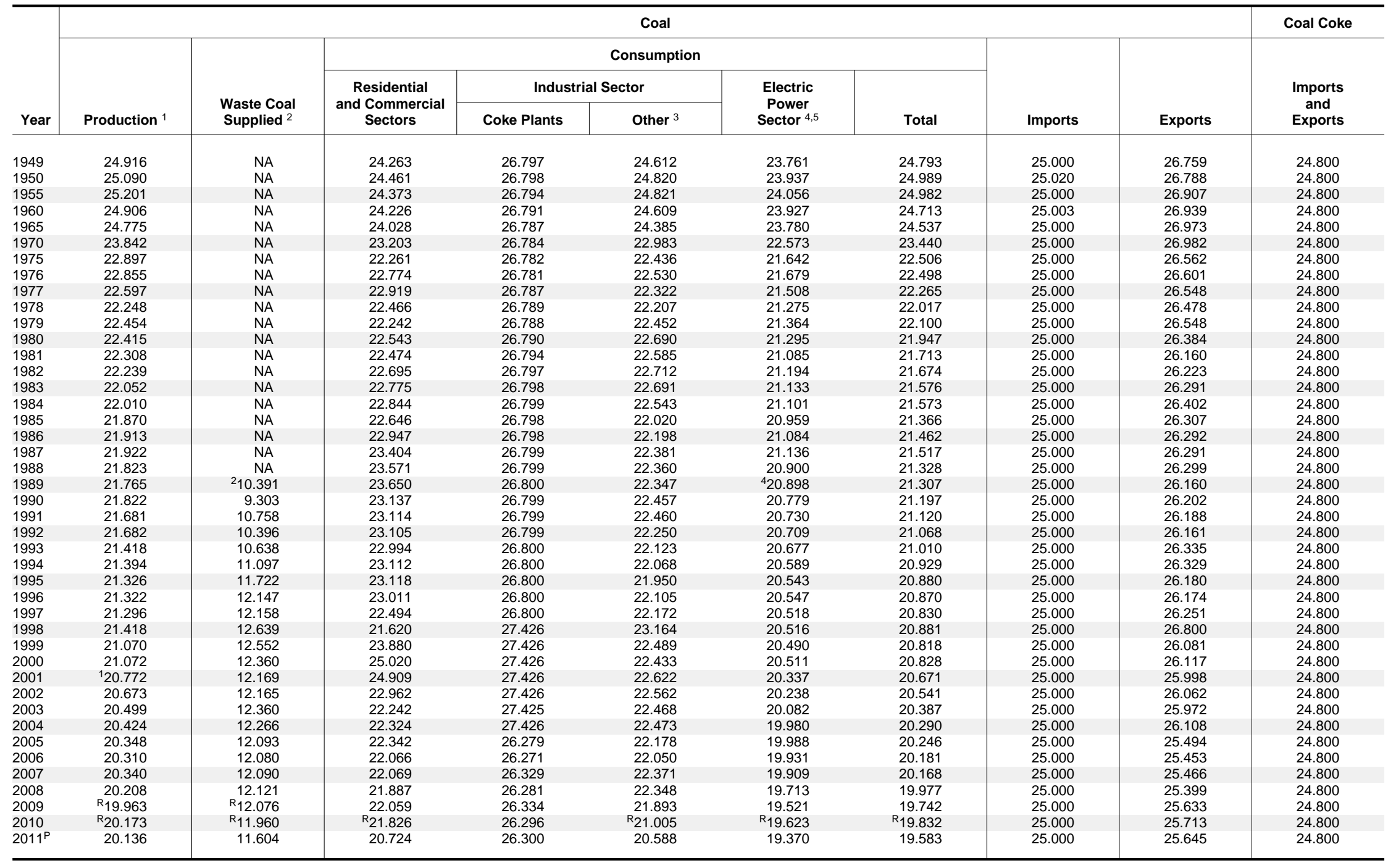

1 Beginning in 2001, includes a small amount of refuse recovery (coal recaptured from a refuse mine, and cleaned to reduce the concentration of noncombustible materials).

Waste coal (including fine coal, coal obtained from a refuse bank or slurry dam, anthracite culm, bituminous gob, and lignite waste) consumed by the electric power and industrial sectors. Beginning in 1989, waste coal supplied is counted as a supply-side item to balance the same amount of waste coal included in "Consumption."

Includes transportation. Excludes coal synfuel plants.

Electricity-only and combined-heat-and-power (CHP) plants within the NAICS 22 category whose primary business is to sell electricity, or electricity and heat, to the public. Through 1988, data are for

electric utilities only; beginning in 1989, data are for electric utilities and independent power producers. 5 Electric power sector factors are for anthracite, bituminous coal, subbituminous coal, lignite, waste coal, and, beginning in 1998, coal synfue

Note: The values in this table are for gross heat contents. See "Heat Content" in Glossary.

Web Pages: - See http://www.eia.gov/totalenergy/data/monthly/\#appendices for updated annual conversion factors. - See http://www.eia.gov/totalenergy/data/annual/\#appendices for all annual data

beginning in 1949. 
Table A6. Approximate Heat Rates for Electricity, and Heat Content of Electricity, Selected Years, 1949-2011

(Btu per Kilowatthour)

\begin{tabular}{|c|c|c|c|c|c|c|c|}
\hline \multirow[b]{3}{*}{ Year } & \multicolumn{6}{|c|}{ Approximate Heat Rates ${ }^{1}$ for Electricity Net Generation } & \multirow{3}{*}{$\begin{array}{l}\text { Heat Content }{ }^{10} \text { of } \\
\text { Electricity }{ }^{11}\end{array}$} \\
\hline & \multicolumn{4}{|c|}{ Fossil Fuels ${ }^{2}$} & \multirow[b]{2}{*}{ Nuclear 8} & \multirow{2}{*}{$\begin{array}{c}\text { Noncombustible } \\
\text { Renewable } \\
\text { Energy } 7,9\end{array}$} & \\
\hline & Coal $^{3}$ & Petroleum ${ }^{4}$ & Natural Gas 5 & Total Fossil Fuels 6,7 & & & \\
\hline 1949 & NA & NA & NA & 15,033 & -- & 15,033 & 3,412 \\
\hline 1950 & NA & NA & NA & 14,030 & -- & 14,030 & $\begin{array}{l}3,412 \\
3,412\end{array}$ \\
\hline 1955 & NA & NA & NA & 11,699 & -- & 11,699 & 3,412 \\
\hline 1960 & NA & NA & NA & 10,760 & 11,629 & 10,760 & 3,412 \\
\hline 1965 & NA & NA & NA & 10,453 & 11,804 & 10,453 & 3,412 \\
\hline 1970 & NA & NA & NA & 10,494 & 10,977 & 10,494 & 3,412 \\
\hline 1975 & NA & NA & NA & $\begin{array}{l}10,406 \\
10,406\end{array}$ & 11,013 & $\begin{array}{l}10,406 \\
10,406\end{array}$ & $\begin{array}{l}r, 412 \\
3,412\end{array}$ \\
\hline 1976 & NA & NA & NA & 10,373 & 11,047 & 10,373 & $\begin{array}{l}3,412 \\
3,412\end{array}$ \\
\hline 1977 & NA & NA & NA & 10,435 & 10,769 & 10,435 & 3,412 \\
\hline 1978 & NA & NA & NA & 10,361 & 10,941 & 10,361 & 3,412 \\
\hline 1979 & NA & NA & NA & 10,353 & 10,879 & 10,353 & 3,412 \\
\hline 1980 & NA & NA & NA & 10,388 & 10,908 & 10,388 & 3,412 \\
\hline 1981 & NA & NA & NA & 10,453 & 11,030 & 10,453 & 3.412 \\
\hline 1982 & NA & NA & NA & 10,454 & 11,073 & 10,454 & $\begin{array}{l}3,412 \\
3,412\end{array}$ \\
\hline 1983 & NA & NA & NA & 10,520 & 10,905 & 10,520 & 3,412 \\
\hline 1984 & NA & NA & NA & 10,440 & 10,843 & 10,440 & 3,412 \\
\hline 1985 & $\mathrm{NA}$ & NA & NA & 10,447 & 10,622 & 10,447 & 3,412 \\
\hline 1986 & NA & NA & NA & 10,446 & 10,579 & 10,446 & 3,412 \\
\hline 1987 & NA & NA & NA & 10,419 & 10,442 & 10,419 & 3.412 \\
\hline 1988 & NA & NA & NA & 10,324 & $\begin{array}{l}10,602 \\
\end{array}$ & 10,324 & $\begin{array}{l}3,412 \\
3,412\end{array}$ \\
\hline 1989 & NA & NA & NA & 10,432 & 10,583 & 10,432 & 3,412 \\
\hline 1990 & NA & NA & NA & 10,402 & 10,582 & 10,402 & 3,412 \\
\hline 1991 & $\mathrm{NA}$ & NA & NA & 10,436 & 10,484 & 10,436 & 3,412 \\
\hline 1992 & NA & NA & NA & 10,342 & 10,471 & 10,342 & 3,412 \\
\hline 1993 & NA & NA & NA & 10,309 & 10,504 & 10,309 & 3.412 \\
\hline 1994 & NA & NA & NA & 10,316 & 10,452 & 10,316 & $\begin{array}{l}3,412 \\
3,412\end{array}$ \\
\hline 1995 & NA & NA & NA & 10,312 & 10,507 & 10,312 & 3,412 \\
\hline 1996 & NA & NA & NA & 10,340 & 10,503 & 10,340 & 3,412 \\
\hline 1997 & NA & NA & NA & 10,213 & 10,494 & 10,213 & 3,412 \\
\hline 1998 & NA & NA & NA & 10,197 & 10,491 & 10,197 & 3,412 \\
\hline 1999 & NA & NA & NA & 10,226 & 10,450 & 10,226 & 3.412 \\
\hline 2000 & NA & NA & NA & 10,201 & 10,429 & 10,201 & $\begin{array}{l}3,412 \\
3,412\end{array}$ \\
\hline 2001 & 10,378 & 10,742 & 10,051 & 10,333 & 10,443 & 10,333 & $\begin{array}{l}3,412 \\
3\end{array}$ \\
\hline 2002 & 10,314 & 10,641 & 9,533 & 10,173 & 10,442 & 10,173 & 3,412 \\
\hline 2003 & 10,297 & 10,610 & 9,207 & 10,241 & 10,421 & 10,241 & 3,412 \\
\hline 2004 & 10,331 & 10,571 & 8,647 & 10,022 & 10,427 & 10,022 & 3,412 \\
\hline 2005 & 10,373 & 10,631 & 8.551 & $\begin{array}{r}9,999 \\
\end{array}$ & 10,436 & 9,999 & $\begin{array}{l}3,412 \\
3,412\end{array}$ \\
\hline 2006 & 10,351 & 10,809 & 8,471 & 9,919 & 10,436 & 9,919 & $\begin{array}{l}3,412 \\
3,412\end{array}$ \\
\hline 2007 & 10,375 & 10,794 & 8,403 & 9,884 & 10,485 & 9,884 & $\begin{array}{l}3,412 \\
3\end{array}$ \\
\hline 2008 & 10,378 & 11,015 & 8,305 & 9,854 & 10,453 & 9,854 & 3,412 \\
\hline 2009 & 10,414 & 10,923 & 8,160 & 9,760 & 10,460 & 9,760 & 3,412 \\
\hline 2010 & 10,415 & 10,984 & 8,185 & Rg,756 & $\mathrm{R}_{10,452}$ & R9,756 & 3,412 \\
\hline 2011 & $\mathrm{E}_{10,415}$ & $\mathrm{E}_{10,984}$ & $E_{8,185}$ & E9,756 & $\mathrm{E}_{10,452}$ & E9,756 & 3,412 \\
\hline
\end{tabular}

1 The values in columns $1-6$ of this table are for net heat rates. See "Heat Rate" in Glossary.

2 Through 2000, heat rates are for fossil-fueled steam-electric plants at electric utilities. Beginning in 2001, heat rates are for all fossil-fueled plants at electric utilities and electricity-only independent powe

3 Includes anthracite, bituminous coal, subbituminous coal, lignite, and, beginning in 2002, waste coal
produces. and coal synfue
4 Includes dis
6 .

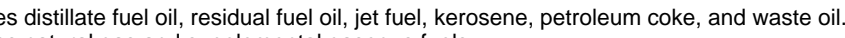

Includes natural gas and supplemental gaseous fuels.

Includes coal, petroleum, natural gas, and, beginning in 2001, other gases (blast furnace gas, propane

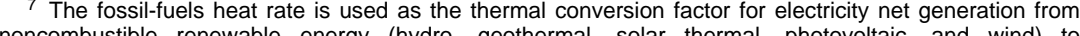
noncombustible renewable energy (hydro, geothermal, solar thermal, photovoltaic, and wind) to
approximate the quantity of fossil fuels replaced by these sources. Through 2000, also used as the thermal

conversion factor for wood and waste electricity net generation at electric utilities; beginning in 2001, Btu data for wood and waste at electric utilities are available from surveys

9 Technology-based geothermal heat rates are no longer used in Btu calculations in this report. For technology-based geothermal heat rates for 1960-2010, see the Annual Energy Review 2010, Table A6.

10 See "Heat Content" in Glossary. 11 The value of 3,412 Btu per kilowatthour is a conorts.
ctricity retail sales, and electricity imports and export

$\mathrm{R}=$ Revised. E=Estimate. NA=Not available. $--=$ Not applicable.

Web Pages: - See http://www.eia.gov/totalenergy/data/monthly/\#appendices for updated annual conversion factors. - See http://www.eia.gov/totalenergy/data/annual/\#appendices for all annual data

Sources: See "Thermal Conversion Factor Source Documentation," which follows this table. 


\section{Thermal Conversion Factor Source Documentation}

\section{Approximate Heat Content of Petroleum and Natural Gas Plant Liquids}

Asphalt. The U.S. Energy Information Administration (EIA) adopted the thermal conversion factor of 6.636 million British thermal units (Btu) per barrel as estimated by the Bureau of Mines and first published in the Petroleum Statement, Annual, 1956.

Aviation Gasoline. EIA adopted the thermal conversion factor of 5.048 million Btu per barrel as adopted by the Bureau of Mines from the Texas Eastern Transmission Corporation publication Competition and Growth in American Energy Markets 1947-1985, a 1968 release of historical and projected statistics.

Butane. EIA adopted the Bureau of Mines thermal conversion factor of 4.326 million Btu per barrel as published in the California Oil World and Petroleum Industry, First Issue, April 1942.

Butane-Propane Mixture. EIA adopted the Bureau of Mines calculation of 4.130 million Btu per barrel based on an assumed mixture of 60 percent butane and 40 percent propane. See Butane and Propane.

Crude Oil Exports. Assumed by EIA to be 5.800 million Btu per barrel or equal to the thermal conversion factor for crude oil produced in the United States. See Crude Oil Production.

Crude Oil Imports. Calculated annually by EIA as the average of the thermal conversion factors for each type of crude oil imported weighted by the quantities imported. Thermal conversion factors for each type were calculated on a foreign country basis, by determining the average American Petroleum Institute (API) gravity of crude oil imported from each foreign country from Form ERA-60 in 1977 and converting average API gravity to average Btu content by using National Bureau of Standards, Miscellaneous Publication No. 97, Thermal Properties of Petroleum Products, 1933.

Crude Oil Production. EIA adopted the thermal conversion factor of 5.800 million Btu per barrel as reported in a Bureau of Mines internal memorandum, "Bureau of Mines Standard Average Heating Values of Various Fuels, Adopted January 3, 1950.”

Distillate Fuel Oil. EIA adopted the Bureau of Mines thermal conversion factor of 5.825 million Btu per barrel as reported in a Bureau of Mines internal memorandum, "Bureau of Mines Standard Average Heating Values of Various Fuels, Adopted January 3, 1950.”

Ethane. EIA adopted the Bureau of Mines thermal conversion factor of 3.082 million Btu per barrel as published in the California Oil World and Petroleum Industry, First Issue, April 1942.

Ethane-Propane Mixture. EIA calculation of 3.308 million Btu per barrel based on an assumed mixture of 70 percent ethane and 30 percent propane. See Ethane and Propane.

Isobutane. EIA adopted the Bureau of Mines thermal conversion factor of 3.974 million Btu per barrel as published in the California Oil World and Petroleum Industry, First Issue, April 1942.

Jet Fuel, Kerosene-Type. EIA adopted the Bureau of Mines thermal conversion factor of 5.670 million Btu per barrel for "Jet Fuel, Commercial" as published by the Texas Eastern Transmission Corporation in the report Competition and Growth in American Energy Markets 1947-1985, a 1968 release of historical and projected statistics.

Jet Fuel, Naphtha-Type. EIA adopted the Bureau of Mines thermal conversion factor of 5.355 million Btu per barrel for "Jet Fuel, Military" as published by the Texas Eastern Transmission Corporation in the report Competition and Growth in American Energy Markets 1947-1985, a 1968 release of historical and projected statistics.

Kerosene. EIA adopted the Bureau of Mines thermal conversion factor of 5.670 million Btu per barrel as reported in a Bureau of Mines internal memorandum, "Bureau of Mines Standard Average Heating Values of Various Fuels, Adopted January 3, 1950.”

Liquefied Petroleum Gases Consumption. • 1949-1966: U.S. Department of the Interior, Bureau of Mines, Mineral Industry Surveys, "Crude Petroleum and Petroleum Products, 1956," Table 4 footnote, constant value of 4.011 million Btu per barrel. • 1967 forward: Calculated annually by EIA as the average of the thermal conversion factors for all liquefied petroleum gases consumed (see Table A1) weighted by the quantities consumed. The component products of liquefied petroleum gases are ethane (including ethylene), propane (including propylene), normal butane (including butylene), butane-propane mixtures, ethane-propane mixtures, and isobutane. For 1967-1980, quantities consumed are from EIA, Energy Data Reports, "Petroleum Statement, Annual," Table 1. For 1981 forward, quantities consumed are from EIA, Petroleum Supply Annual, Table 2. 
Lubricants. EIA adopted the thermal conversion factor of 6.065 million Btu per barrel as estimated by the Bureau of Mines and first published in the Petroleum Statement, Annual, 1956.

Miscellaneous Products. EIA adopted the thermal conversion factor of 5.796 million Btu per barrel as estimated by the Bureau of Mines and first published in the Petroleum Statement, Annual, 1956.

Motor Gasoline Consumption. • 1949-1993: EIA adopted the Bureau of Mines thermal conversion factor of 5.253 million Btu per barrel for "Gasoline, Motor Fuel” as published by the Texas Eastern Transmission Corporation in Appendix V of Competition and Growth in American Energy Markets 1947-1985, a 1968 release of historical and projected statistics. - 1994 forward: EIA calculated national annual quantity-weighted average conversion factors for conventional, reformulated, and oxygenated motor gasolines (see Table A3). The factor for conventional motor gasoline is 5.253 million Btu per barrel, as used for previous years. The factors for reformulated and oxygenated gasolines, both currently 5.150 million Btu per barrel, are based on data published in Environmental Protection Agency, Office of Mobile Sources, National Vehicle and Fuel Emissions Laboratory report EPA 420-F-95-003, "Fuel Economy Impact Analysis of Reformulated Gasoline.” See Fuel Ethanol (Denatured).

Natural Gas Plant Liquids Production. Calculated annually by EIA as the average of the thermal conversion factors for each natural gas plant liquid produced weighted by the quantities produced.

Natural Gasoline. EIA adopted the thermal conversion factor of 4.620 million Btu per barrel as estimated by the Bureau of Mines and first published in the Petroleum Statement, Annual, 1956.

Pentanes Plus. EIA assumed the thermal conversion factor to be 4.620 million Btu or equal to that for natural gasoline. See Natural Gasoline.

Petrochemical Feedstocks, Naphtha less than $401^{\circ}$ F. Assumed by EIA to be 5.248 million Btu per barrel or equal to the thermal conversion factor for special naphthas. See Special Naphthas.

Petrochemical Feedstocks, Other Oils equal to or greater than $401^{\circ} \mathrm{F}$. Assumed by EIA to be 5.825 million Btu per barrel or equal to the thermal conversion factor for distillate fuel oil. See Distillate Fuel Oil.

Petrochemical Feedstocks, Still Gas. Assumed by EIA to be 6.000 million Btu per barrel or equal to the thermal conversion factor for still gas. See Still Gas.
Petroleum Coke. EIA adopted the thermal conversion factor of 6.024 million Btu per barrel as reported in Btu per short ton in the Bureau of Mines internal memorandum, "Bureau of Mines Standard Average Heating Values of Various Fuels, Adopted January 3, 1950.” The Bureau of Mines calculated this factor by dividing 30.120 million Btu per short ton, as given in the referenced Bureau of Mines internal memorandum, by 5.0 barrels per short ton, as given in the Bureau of Mines Form 6-1300-M and successor EIA forms.

Petroleum Consumption, Commercial Sector. Calculated annually by EIA as the average of the thermal conversion factors for all petroleum products consumed by the commercial sector weighted by the estimated quantities consumed by the commercial sector. The quantities of petroleum products consumed by the commercial sector are estimated in the State Energy Data System — see documentation at http://www.eia.gov/emeu/states/sep_use/notes/use_petrol.pdf.

Petroleum Consumption, Electric Power Sector. Calculated annually by EIA as the average of the thermal conversion factors for all petroleum products consumed by the electric power sector weighted by the quantities consumed by the electric power sector. Data are from Form EIA-923, "Power Plant Operations Report,” and predecessor forms.

Petroleum Consumption, Industrial Sector. Calculated annually by EIA as the average of the thermal conversion factors for all petroleum products consumed by the industrial sector weighted by the estimated quantities consumed by the industrial sector. The quantities of petroleum products consumed by the industrial sector are estimated in the State Energy Data System-see documentation at http://www.eia.gov/emeu/states/sep_use/notes/use_petrol.pdf.

Petroleum Consumption, Residential Sector. Calculated annually by EIA as the average of the thermal conversion factors for all petroleum products consumed by the residential sector weighted by the estimated quantities consumed by the residential sector. The quantities of petroleum products consumed by the residential sector are estimated in the State Energy Data System-see documentation at http://www.eia.gov/emeu/states/sep_use/notes/use_petrol.pdf.

Petroleum Consumption, Total. Calculated annually by EIA as the average of the thermal conversion factors for all petroleum products consumed weighted by the quantities consumed.

Petroleum Consumption, Transportation Sector. Calculated annually by EIA as the average of the thermal conversion factors for all petroleum products consumed by the transportation sector weighted by the estimated quantities consumed by the transportation sector. The quantities of petroleum products consumed by the 
transportation sector are estimated in the State Energy Data System-see documentation at http://www.eia.gov/emeu/states/sep_use/notes/use_petrol.pdf.

Petroleum Products Exports. Calculated annually by EIA as the average of the thermal conversion factors for each petroleum product exported weighted by the quantities exported.

Petroleum Products Imports. Calculated annually by EIA as the average of the thermal conversion factors for each petroleum product imported weighted by the quantities imported.

Plant Condensate. Estimated to be 5.418 million Btu per barrel by EIA from data provided by McClanahan Consultants, Inc., Houston, Texas.

Propane. EIA adopted the Bureau of Mines thermal conversion factor of 3.836 million Btu per barrel as published in the California Oil World and Petroleum Industry, First Issue, April 1942.

Residual Fuel Oil. EIA adopted the thermal conversion factor of 6.287 million Btu per barrel as reported in the Bureau of Mines internal memorandum, "Bureau of Mines Standard Average Heating Values of Various Fuels, Adopted January 3, 1950."

Road Oil. EIA adopted the Bureau of Mines thermal conversion factor of 6.636 million Btu per barrel, which was assumed to be equal to that of asphalt (see Asphalt) and was first published by the Bureau of Mines in the Petroleum Statement, Annual, 1970.

Special Naphthas. EIA adopted the Bureau of Mines thermal conversion factor of 5.248 million Btu per barrel, which was assumed to be equal to that of the total gasoline (aviation and motor) factor and was first published in the Petroleum Statement, Annual, 1970.

Still Gas. EIA adopted the Bureau of Mines estimated thermal conversion factor of 6.000 million Btu per barrel, first published in the Petroleum Statement, Annual, 1970.

Total Petroleum Exports. Calculated annually by EIA as the average of the thermal conversion factors for crude oil and each petroleum product exported weighted by the quantities exported. See Crude Oil Exports and Petroleum Products Exports.

Total Petroleum Imports. Calculated annually by EIA as the average of the thermal conversion factors for each type of crude oil and petroleum product imported weighted by the quantities imported. See Crude Oil Imports and Petroleum Products Imports.
Unfinished Oils. EIA assumed the thermal conversion factor to be 5.825 million Btu per barrel or equal to that for distillate fuel oil (see Distillate Fuel Oil) and first published it in EIA’s Annual Report to Congress, Volume 3, 1977.

Unfractionated Stream. EIA assumed the thermal conversion factor to be 5.418 million Btu per barrel or equal to that for plant condensate (see Plant Condensate) and first published it in EIA’s Annual Report to Congress, Volume 2, 1981.

Waxes. EIA adopted the thermal conversion factor of 5.537 million Btu per barrel as estimated by the Bureau of Mines and first published in the Petroleum Statement, Annual, 1956.

\section{Approximate Heat Content of Biofuels}

Biodiesel. EIA estimated the thermal conversion factor for biodiesel to be 5.359 million Btu per barrel, or 17,253 Btu per pound.

Biodiesel Feedstock. EIA used soybean oil input to the production of biodiesel (million Btu soybean oil per barrel biodiesel) as the factor to estimate total biomass inputs to the production of biodiesel. EIA assumed that 7.65 pounds of soybean oil are needed to produce one gallon of biodiesel, and 5.433 million Btu of soybean oil are needed to produce one barrel of biodiesel. EIA also assumed that soybean oil has a gross heat content of 16,909 Btu per pound, or 5.483 million Btu per barrel.

Ethanol (Undenatured). EIA adopted the thermal conversion factor of 3.539 million Btu per barrel published in "Oxygenate Flexibility for Future Fuels," a paper presented by William J. Piel of the ARCO Chemical Company at the National Conference on Reformulated Gasolines and Clean Air Act Implementation, Washington, D.C., October 1991.

Fuel Ethanol (Denatured). • 1981-2008: EIA used the 2009 factor. • 2009 forward: Calculated by EIA as the annual quantity-weighted average of the thermal conversion factors for undenatured ethanol (3.539 million Btu per barrel), pentanes plus used as denaturant (4.620 million Btu per barrel), and conventional motor gasoline used as denaturant (5.253 million Btu per barrel). The quantity of ethanol consumed is from EIA's Petroleum Supply Annual (PSA) and Petroleum Supply Monthly (PSM), Table 1, data for renewable fuels and oxygenate plant net production of fuel ethanol. The quantity of pentanes plus used as denaturant is from PSA/PSM, Table 1, data for renewable fuels and oxygenate plant net production of pentanes plus, multiplied by -1 . The quantity of conventional motor gasoline and motor gasoline blending components used as denaturant is from PSA/PSM, Table 1, data for renewable fuels and oxygenate plant net production of conventional motor gasoline and motor gasoline blending components, multiplied by -1 . 
Fuel Ethanol Feedstock. EIA used corn input to the production of undenatured ethanol (million Btu corn per barrel undenatured ethanol) as the annual factor to estimate total biomass inputs to the production of undenatured ethanol. U.S. Department of Agriculture observed ethanol yields (gallons undenatured ethanol per bushel of corn) were 2.5 in 1980, 2.666 in 1998, 2.68 in 2002, and 2.764 in 2009; EIA estimated the ethanol yields in other years. EIA also assumed thatcorn has a gross heat content of 0.392 million Btu per bushel.

\section{Approximate Heat Content of Natural Gas}

Natural Gas Consumption, Electric Power Sector. Calculated annually by EIA by dividing the heat content of natural gas consumed by the electric power sector by the quantity consumed. Data are from Form EIA-923, "Power Plant Operations Report,” and predecessor forms.

Natural Gas Consumption, End-Use Sectors. Calculated annually by EIA by dividing the heat content of natural gas consumed by the end-use sectors (residential, commercial, industrial, and transportation) by the quantity consumed. Data are from Form EIA-176, “Annual Report of Natural and Supplemental Gas Supply and Disposition.”

Natural Gas Consumption, Total. • 1949-1962: EIA adopted the thermal conversion factor of 1,035 Btu per cubic foot as estimated by the Bureau of Mines and first published in the Petroleum Statement, Annual, 1956. • 1963-1979: EIA adopted the thermal conversion factor calculated annually by the American Gas Association (AGA) and published in Gas Facts, an AGA annual publication. • 1980 forward: Calculated annually by EIA by dividing the total heat content of natural gas consumed by the total quantity consumed.

Natural Gas Exports. 1949-1972: Assumed by EIA to be equal to the thermal conversion factor for dry natural gas consumed (see Natural Gas Consumption, Total). • 1973 forward: Calculated annually by EIA by dividing the heat content of natural gas exported by the quantity exported. For 1973-1995, data are from Form FPC-14, “Annual Report for Importers and Exporters of Natural Gas.” Beginning in 1996, data are from U.S. Department of Energy, Office of Fossil Energy, Natural Gas Imports and Exports.

Natural Gas Imports. • 1949-1972: Assumed by EIA to be equal to the thermal conversion factor for dry natural gas consumed (see Natural Gas Consumption, Total). - 1973 forward: Calculated annually by EIA by dividing the heat content of natural gas imported by the quantity imported. For 1973-1995, data are from Form FPC-14, “Annual Report for Importers and Exporters of Natural Gas.” Beginning in 1996, data are from U.S. Department of Energy, Office of Fossil Energy, Natural Gas Imports and Exports.

Natural Gas Production, Dry. Assumed by EIA to be equal to the thermal conversion factor for dry natural gas consumed. See Natural Gas Consumption, Total.

Natural Gas Production, Marketed. Calculated annually by EIA by dividing the heat content of dry natural gas produced (see Natural Gas Production, Dry) and natural gas plant liquids produced (see Natural Gas Plant Liquids Production) by the total quantity of marketed natural gas produced.

\section{Approximate Heat Content of Coal and Coal Coke}

Coal Coke Imports and Exports. EIA adopted the Bureau of Mines estimate of 24.800 million Btu per short ton.

Coal Consumption, Electric Power Sector. Calculated annually by EIA by dividing the heat content of coal consumed by the electric power sector by the quantity consumed. Data are from Form EIA-923, "Power Plant Operations Report,” and predecessor forms.

Coal Consumption, Industrial Sector, Coke Plants. Calculated annually by EIA by dividing the heat content of coal consumed by coke plants by the quantity consumed. Data are from Form EIA-5, “Quarterly Coal Consumption and Quality Report-Coke Plants.”

Coal Consumption, Industrial Sector, Other. Calculated annually by EIA by dividing the heat content of coal consumed by manufacturing plants by the quantity consumed. Data are from Form EIA-3, "Quarterly Coal Consumption and Quality Report-Manufacturing Plants.”

Coal Consumption, Residential and Commercial Sectors. Calculated annually by EIA by dividing the heat content of coal consumed by the residential and commercial sectors by the quantity consumed. Through 1999, data are from Form EIA-6, “Coal Distribution Report.” Beginning in 2000, data are for commercial combinedheat-and-power (CHP) plants from Form EIA-923, "Power Plant Operations Report," and predecessor forms.

Coal Consumption, Total. Calculated annually by EIA by dividing the total heat content of coal consumed by all sectors by the total quantity consumed. 
Coal Exports. Calculated annually by EIA by dividing the heat content of steam coal and metallurgical coal exported by the quantity exported. Data are from U.S. Department of Commerce, Bureau of the Census, "Monthly Report EM 545.”

Coal Imports. • 1949-1963: Calculated annually by EIA by dividing the heat content of coal imported by the quantity imported. • 1963 forward: Assumed by EIA to be 25.000 million Btu per short ton.

Coal Production. Calculated annually by EIA to balance the heat content of coal supply (production and imports) and the heat content of coal disposition (exports, stock change, and consumption).

Waste Coal Supplied. Calculated annually by EIA by dividing the total heat content of waste coal supplied by the quantity supplied. For 1989-1997, data are from Form EIA-867, “Annual Nonutility Power Producer Report.” For 1998-2000, data are from Form EIA-860B, “Annual Electric Generator Report-Nonutility.” For 2001 forward, data are from Form EIA-3, "Quarterly Coal Consumption and Quality Report-Manufacturing Plants”; Form EIA-923, "Power Plant Operations Report”; and predecessor forms.

\section{Approximate Heat Rates for Electricity}

Electricity Net Generation, Coal. 2001 forward: Calculated annually by EIA by using fuel consumption and net generation data reported on Form EIA-923, "Power Plant Operations Report," and predecessor forms. The computation includes data for all electric utilities and electricity-only independent power producers using anthracite, bituminous coal, subbituminous coal, lignite, and beginning in 2002, waste coal and coal synfuel.

Electricity Net Generation, Natural Gas. 2001 forward: Calculated annually by EIA by using fuel consumption and net generation data reported on Form EIA-923, "Power Plant Operations Report," and predecessor forms. The computation includes data for all electric utilities and electricity-only independent power producers using natural gas and supplemental gaseous fuels.

Electricity Net Generation, Noncombustible Renewable Energy. There is no generally accepted practice for measuring the thermal conversion rates for power plants that generate electricity from hydro, geothermal, solar thermal, photovoltaic, and wind energy sources. Therefore, EIA calculates a rate factor that is equal to the annual average heat rate factor for fossil-fueled power plants in the United States. (see "Electricity Net Generation, Total Fossil Fuels"). By using that factor it is possible to evaluate fossil fuel requirements for replacing those sources during periods of interruption, such as droughts.

Electricity Net Generation, Nuclear. • 1957-1984: Calculated annually by dividing the total heat content consumed in nuclear generating units by the total (net) electricity generated by nuclear generating units. The heat content and electricity generation were reported on Form FERC-1, “Annual Report of Major Electric Utilities, Licensees, and Others”; Form EIA-412, “Annual Report of Public Electric Utilities”; and predecessor forms. For 1982, the factors were published in EIA, Historical Plant Cost and Annual Production Expenses for Selected Electric Plants 1982, page 215. For 1983 and 1984, the factors were published in EIA, Electric Plant Cost and Power Production Expenses 1991, Table 13. 1985 forward: Calculated annually by EIA by using the heat rate data reported on Form EIA-860, “Annual Electric Generator Report,” and predecessor forms.

Electricity Net Generation, Petroleum. 2001 forward: Calculated annually by EIA by using fuel consumption and net generation data reported on Form EIA-923, "Power Plant Operations Report," and predecessor forms. The computation includes data for all electric utilities and electricity-only independent power producers using distillate fuel oil, residual fuel oil, jet fuel, kerosene, petroleum coke, and waste oil.

Electricity Net Generation, Total Fossil Fuels. • 1949-1955: The weighted annual average heat rate for fossil-fueled steam-electric power plants in the United States, as published by EIA in Thermal-Electric Plant Construction Cost and Annual Production Expenses-1981 and Steam-Electric Plant Construction Cost and Annual Production Expenses-1978. • 1956-1988: The weighted annual average heat rate for fossil-fueled steam-electric power plants in the United States, as published in EIA, Electric Plant Cost and Power Production Expenses 1991, Table 9. - 1989-2000: Calculated annually by EIA by using heat rate data reported on Form EIA-860, “Annual Electric Generator Report,” and predecessor forms; and net generation data reported on Form EIA-759, "Monthly Power Plant Report.” The computation includes data for all electric utility steam-electric plants using fossil fuels. • 2001 forward: Calculated annually by EIA by using fuel consumption and net generation data reported on Form EIA-923, "Power Plant Operations Report," and predecessor forms. The computation includes data for all electric utilities and electricity-only independent power producers using coal, petroleum, natural gas, and other gases (blast furnace gas, propane gas, and other manufactured and waste gases derived from fossil fuels). 


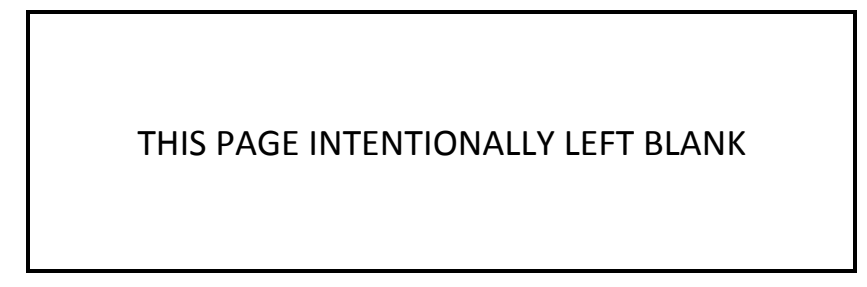




\section{Appendix B. Metric Conversion Factors, Metric Prefixes, and Other Physical Conversion Factors}

Data presented in the Annual Energy Review and in other U.S. Energy Information Administration publications are expressed predominately in units that historically have been used in the United States, such as British thermal units, barrels, cubic feet, and short tons.

The metric conversion factors presented in Table B1 can be used to calculate the metric-unit equivalents of values expressed in U.S. customary units. For example, 500 short tons are the equivalent of 453.6 metric tons (500 short tons x 0.9071847 metric tons/short ton $=453.6$ metric tons).
In the metric system of weights and measures, the names of multiples and subdivisions of any unit may be derived by combining the name of the unit with prefixes, such as deka, hecto, and kilo, meaning, respectively, 10, 100, 1,000, and deci, centi, and milli, meaning, respectively, one-tenth, one-hundredth, and one-thousandth. Common metric prefixes can be found in Table B2.

The conversion factors presented in Table B3 can be used to calculate equivalents in various physical units commonly used in energy analyses. For example, 10 barrels are the equivalent of 420 U.S. gallons (10 barrels x 42 gallons/barrel $=420$ gallons). 
Table B1. Metric Conversion Factors

\begin{tabular}{|c|c|c|c|c|}
\hline Type of Unit & U.S. Unit & & Equivalent in & Metric Units \\
\hline Mass & $\begin{array}{l}1 \text { short ton }(2,000 \mathrm{lb}) \\
1 \text { long ton } \\
1 \text { pound }(\mathrm{lb}) \\
1 \text { pound uranium oxide }\left(\mathrm{lb} \mathrm{U}_{3} \mathrm{O}_{8}\right) \\
1 \text { ounce, avoirdupois (avdp oz) }\end{array}$ & $\begin{array}{l}= \\
= \\
= \\
= \\
=\end{array}$ & $\begin{array}{l}0.9071847 \\
1.016047 \\
0.45359237^{\mathrm{a}} \\
0.384647^{\mathrm{b}} \\
28.34952\end{array}$ & $\begin{array}{l}\text { metric tons }(\mathrm{t}) \\
\text { metric tons }(\mathrm{t}) \\
\text { kilograms }(\mathrm{kg}) \\
\text { kilograms uranium }(\mathrm{kgU}) \\
\text { grams }(\mathrm{g})\end{array}$ \\
\hline Volume & $\begin{array}{l}1 \text { barrel of oil }(\mathrm{bbl}) \\
1 \text { cubic yard }\left(\mathrm{yd}^{3}\right) \\
1 \text { cubic foot }\left(\mathrm{ft}^{3}\right) \\
1 \text { U.S. gallon }(\mathrm{gal}) \\
1 \text { ounce, fluid (fl oz) } \\
1 \text { cubic inch }\left(\mathrm{in}^{3}\right)\end{array}$ & $\begin{array}{l}= \\
= \\
= \\
= \\
= \\
=\end{array}$ & $\begin{array}{l}0.1589873 \\
0.764555 \\
0.02831685 \\
3.785412 \\
29.57353 \\
16.38706\end{array}$ & $\begin{array}{l}\text { cubic meters }\left(\mathrm{m}^{3}\right) \\
\text { cubic meters }\left(\mathrm{m}^{3}\right) \\
\text { cubic meters }\left(\mathrm{m}^{3}\right) \\
\text { liters }(\mathrm{L}) \\
\text { milliliters }(\mathrm{mL}) \\
\text { milliliters }(\mathrm{mL})\end{array}$ \\
\hline Length & $\begin{array}{l}1 \text { mile }(\mathrm{mi}) \\
1 \text { yard }(\mathrm{yd}) \\
1 \text { foot }(\mathrm{ft}) \\
1 \text { inch (in) }\end{array}$ & $\begin{array}{l}= \\
= \\
= \\
=\end{array}$ & $\begin{array}{c}1.609344^{\mathrm{a}} \\
0.9144^{\mathrm{a}} \\
0.3048^{\mathrm{a}} \\
2.54^{\mathrm{a}}\end{array}$ & $\begin{array}{l}\text { kilometers }(\mathrm{km}) \\
\text { meters }(\mathrm{m}) \\
\text { meters }(\mathrm{m}) \\
\text { centimeters }(\mathrm{cm})\end{array}$ \\
\hline Area & $\begin{array}{l}1 \text { acre } \\
1 \text { square mile }\left(\mathrm{mi}^{2}\right) \\
1 \text { square yard }\left(\mathrm{yd}^{2}\right) \\
1 \text { square foot }\left(\mathrm{ft}^{2}\right) \\
1 \text { square inch }\left(\mathrm{in}^{2}\right)\end{array}$ & $\begin{array}{l}= \\
= \\
= \\
= \\
=\end{array}$ & $\begin{array}{l}0.40469 \\
2.589988 \\
0.8361274 \\
0.09290304^{a} \\
6.4516^{a}\end{array}$ & $\begin{array}{l}\text { hectares }(\text { ha) } \\
\text { square kilometers }\left(\mathrm{km}^{2}\right) \\
\text { square meters }\left(\mathrm{m}^{2}\right) \\
\text { square meters }\left(\mathrm{m}^{2}\right) \\
\text { square centimeters }\left(\mathrm{cm}^{2}\right)\end{array}$ \\
\hline Energy & $\begin{array}{l}1 \text { British thermal unit }(\mathrm{Btu})^{\mathrm{c}} \\
1 \text { calorie }(\mathrm{cal}) \\
1 \text { kilowatthour }(\mathrm{kWh})\end{array}$ & $\begin{array}{l}= \\
= \\
=\end{array}$ & $\begin{array}{c}1,055.05585262^{\mathrm{a}} \\
4.1868^{\mathrm{a}} \\
3.6^{\mathrm{a}}\end{array}$ & $\begin{array}{l}\text { joules }(\mathrm{J}) \\
\text { joules }(\mathrm{J}) \\
\text { megajoules }(\mathrm{MJ})\end{array}$ \\
\hline Temperature $^{d}$ & $\begin{array}{l}32 \text { degrees Fahrenheit }\left({ }^{\circ} \mathrm{F}\right) \\
212 \text { degrees Fahrenheit }\left({ }^{\circ} \mathrm{F}\right)\end{array}$ & $\begin{array}{l}= \\
=\end{array}$ & $\begin{array}{r}0^{\mathrm{a}} \\
100^{\mathrm{a}}\end{array}$ & $\begin{array}{l}\text { degrees Celsius }\left({ }^{\circ} \mathrm{C}\right) \\
\text { degrees Celsius }\left({ }^{\circ} \mathrm{C}\right)\end{array}$ \\
\hline
\end{tabular}

\footnotetext{
${ }^{a}$ Exact conversion.
}

${ }^{b}$ Calculated by the U.S. Energy Information Administration.

${ }^{\mathrm{c}}$ The Btu used in this table is the International Table Btu adopted by the Fifth International Conference on Properties of Steam, London, 1956.

${ }^{\mathrm{d}}$ To convert degrees Fahrenheit $\left({ }^{\circ} \mathrm{F}\right)$ to degrees Celsius $\left({ }^{\circ} \mathrm{C}\right)$ exactly, subtract 32 , then multiply by $5 / 9$.

Notes: - Spaces have been inserted after every third digit to the right of the decimal for ease of reading. • Most metric units belong to the International System of Units (SI), and the liter, hectare, and metric ton are accepted for use with the SI units. For more information about the SI units, see http://physics.nist.gov/cuu/Units/index.html.

Web Page: For related information, see http://www.eia.gov/totalenergy/data/annual/\#appendices.

Sources: • General Services Administration, Federal Standard 376B, Preferred Metric Units for General Use by the Federal Government (Washington, DC, January 1993), pp. 9-11, 13, and 16. - U.S. Department of Commerce, National Institute of Standards and Technology, Special Publications 330,811, and 814. • American National Standards Institute/Institute of Electrical and Electronic Engineers, ANSI/IEEE Std 268-1992, pp. 28 and 29. 
Table B2. Metric Prefixes

\begin{tabular}{clcclc}
\hline Unit Multiple & Prefix & Symbol & Unit Subdivision & Prefix & Symbol \\
\hline $10^{1}$ & deka & da & $10^{-1}$ & deci & $\mathrm{d}$ \\
$10^{2}$ & hecto & $\mathrm{h}$ & $10^{-2}$ & centi & $\mathrm{c}$ \\
$10^{3}$ & kilo & $\mathrm{k}$ & $10^{-3}$ & milli & $\mathrm{m}$ \\
$10^{6}$ & mega & $\mathrm{M}$ & $10^{-6}$ & micro & $\mathrm{\mu}$ \\
$10^{9}$ & giga & $\mathrm{G}$ & $10^{-9}$ & nano & $\mathrm{n}$ \\
$10^{12}$ & tera & $\mathrm{T}$ & $10^{-12}$ & pico & $\mathrm{p}$ \\
$10^{15}$ & peta & $\mathrm{P}$ & $10^{-15}$ & femto & $\mathrm{f}$ \\
$10^{18}$ & exa & $\mathrm{E}$ & $10^{-18}$ & atto & $\mathrm{a}$ \\
$10^{21}$ & zetta & $\mathrm{Z}$ & $10^{-21}$ & zepto & $\mathrm{z}$ \\
$10^{24}$ & yotta & $\mathrm{Y}$ & $10^{-24}$ & yocto & $\mathrm{y}$ \\
\end{tabular}

Web Page: For related information, see http://www.eia.gov/totalenergy/data/annual/\#appendices.

Source: U.S. Department of Commerce, National Institute of Standards and Technology, The International System of Units (SI), NIST Special Publication 330, 1991 Edition (Washington, DC, August 1991), p.10.

\section{Table B3. Other Physical Conversion Factors}

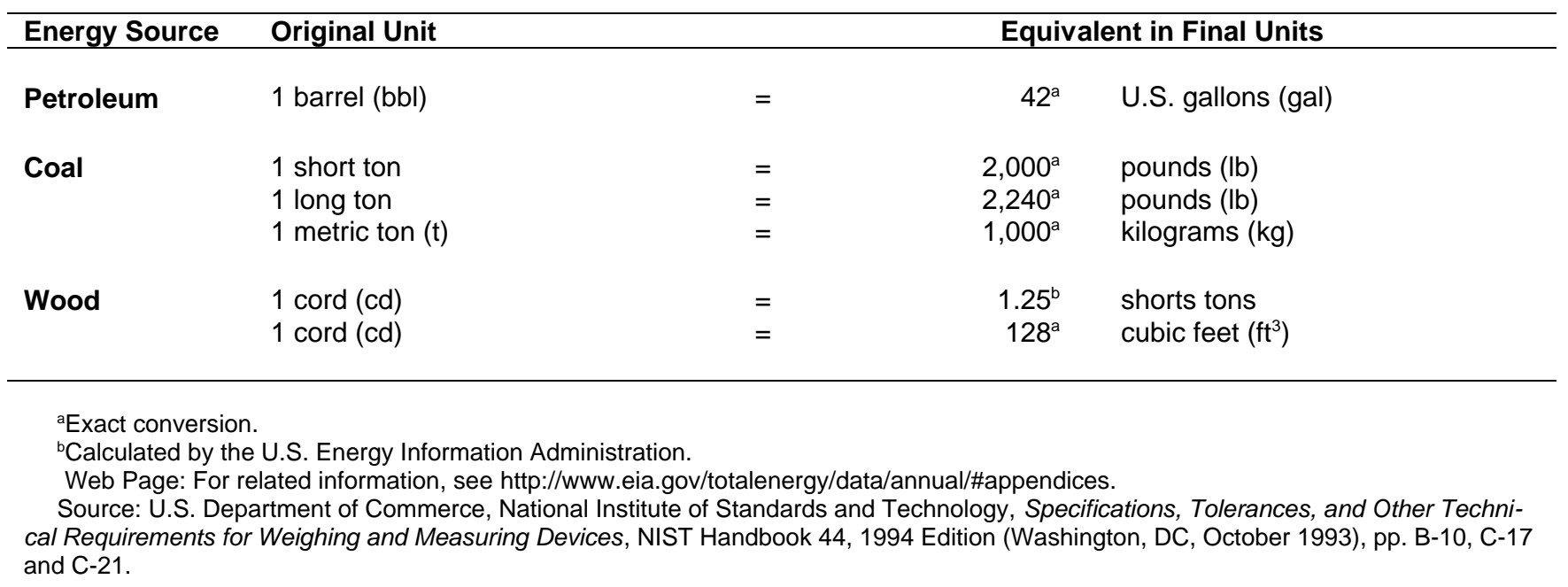




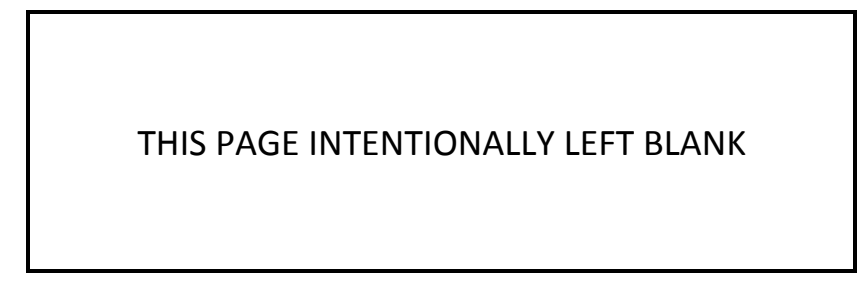




\section{Appendix C}

Figure C1. U.S. Census Regions and Divisions

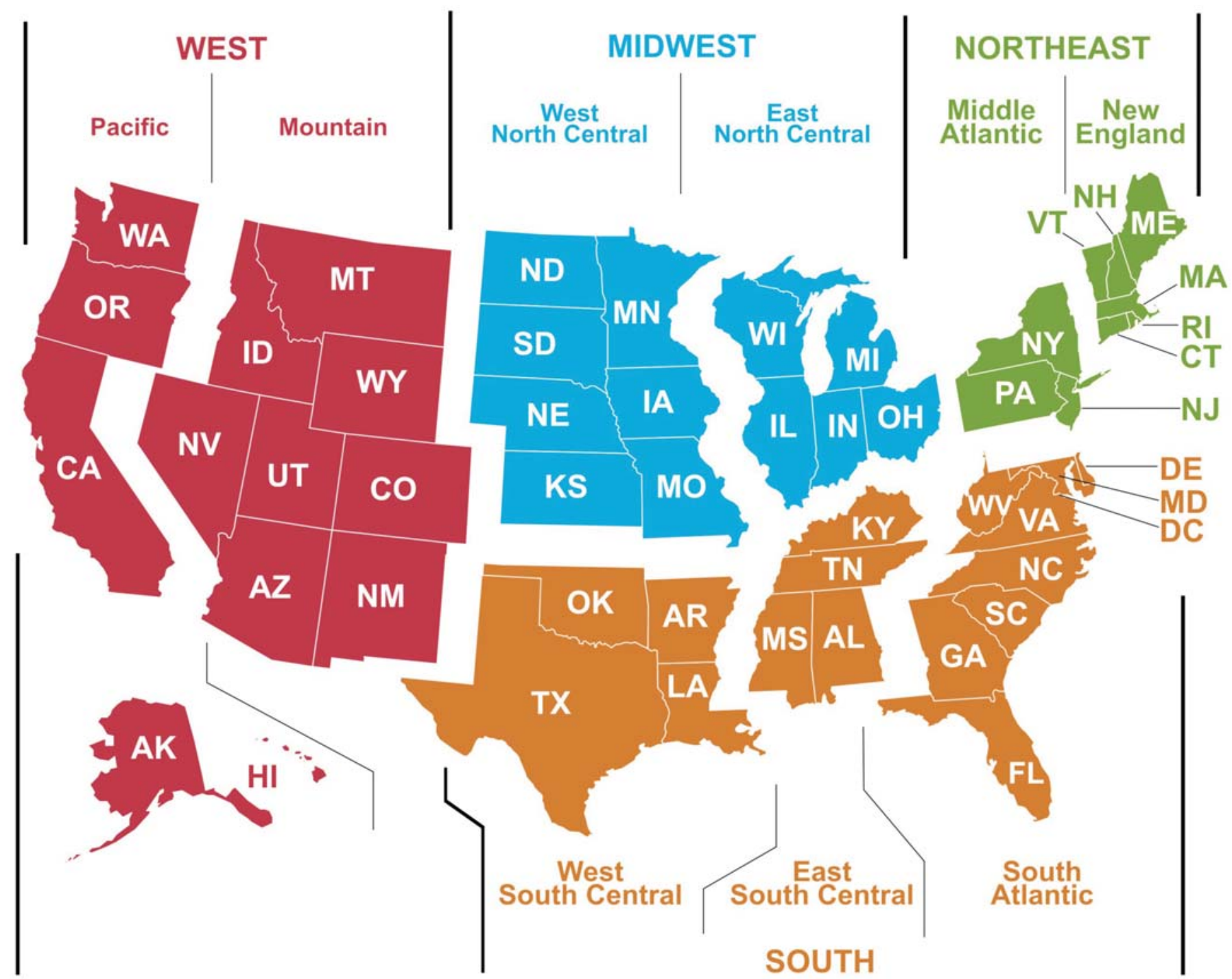




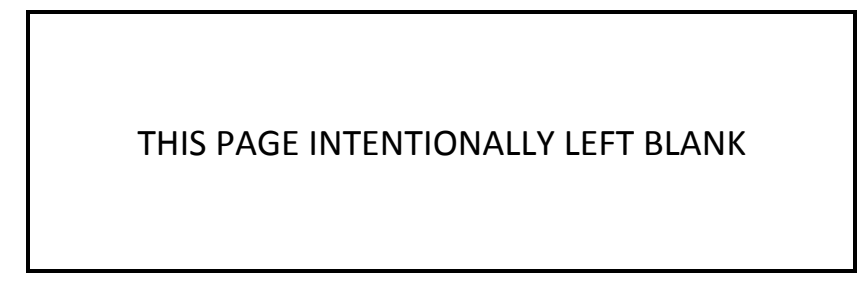




\section{Appendix D}

Table D1. Population, U.S. Gross Domestic Product, and Implicit Price Deflator, Selected Years, 1949-2011

\begin{tabular}{|c|c|c|c|c|c|c|}
\hline \multirow[b]{3}{*}{ Year } & \multicolumn{3}{|c|}{ Population } & \multicolumn{3}{|c|}{ U.S. Gross Domestic Product } \\
\hline & United States ${ }^{1}$ & World & \multirow{2}{*}{$\begin{array}{c}\begin{array}{c}\text { United States } \\
\text { as Share of World }\end{array} \\
\text { Percent }\end{array}$} & \multirow{2}{*}{$\begin{array}{c}\text { Billion } \\
\text { Nominal Dollars }{ }^{2}\end{array}$} & \multirow{2}{*}{$\begin{array}{c}\text { Billion } \\
\text { Real (2005) Dollars }{ }^{3}\end{array}$} & \multirow{2}{*}{$\begin{array}{l}\text { Implicit Price Deflator }{ }^{4} \\
(2005=1.00000)\end{array}$} \\
\hline & \multicolumn{2}{|c|}{ Million People } & & & & \\
\hline 1949 & 149.2 & NA & NA & 267.2 & $\mathrm{R}_{1,843.1}$ & $\mathrm{R}_{0} .14499$ \\
\hline 1950 & 152.3 & $2,556.5$ & 6.0 & 293.7 & $R_{2,004.2}$ & R. 14656 \\
\hline 1955 & 165.9 & $2,781.2$ & 6.0 & 414.7 & $R_{2,498.2}$ & R. 16601 \\
\hline 1960 & 180.7 & $3,042.4$ & 5.9 & 526.4 & $R_{2,828.5}$ & R. 18612 \\
\hline 1965 & 194.3 & $R_{3,350.3}$ & 5.8 & 719.1 & $\mathrm{R}_{3,607.0}$ & R.19936 \\
\hline 1970 & 205.1 & $3,713.0$ & 5.5 & $1,038.3$ & $\begin{aligned} \mathrm{R} \\
\mathrm{R}\end{aligned}$ & R. 24338 \\
\hline 1975 & 216.0 & $\begin{array}{ll}8,090.6 \\
\mathrm{R} 4,0\end{array}$ & 5.3 & $1,637.7$ & $\begin{aligned} 4,20.0 \\
R_{4}, 875.4\end{aligned}$ & R. 33591 \\
\hline 1976 & 218.0 & $R_{4,161.9}$ & 5.2 & $1,824.6$ & $\mathrm{R} 5,136.9$ & R. 35519 \\
\hline 1977 & 220.2 & $\mathrm{R} 4,233.9$ & 5.2 & $2,030.1$ & $\mathrm{R} 5,373.1$ & R. .37783 \\
\hline 1978 & 222.6 & $\mathrm{R}_{4,306.1}$ & 5.2 & $2,293.8$ & $\mathrm{R}_{5,672.8}$ & R. .40435 \\
\hline 1979 & 225.1 & $\mathrm{R}_{4,381.1}$ & 5.1 & $2,562.2$ & $\mathrm{R} 5,850.1$ & R. 43798 \\
\hline 1980 & 227.2 & $\mathrm{R}_{4,453.5}$ & 5.1 & $2,788.1$ & $\mathrm{R} 5,834.0$ & R.47791 \\
\hline 1981 & 229.5 & $\mathrm{R}_{4,536.3}$ & 5.1 & $3,126.8$ & $\mathrm{R}_{5}, 982.1$ & R. 52270 \\
\hline 1982 & 231.7 & $\mathrm{R}_{4,616.4}$ & 5.0 & $3,253.2$ & $\mathrm{R}_{5,865.9}$ & R.55459 \\
\hline 1983 & 233.8 & $\mathrm{R}_{4,697.6}$ & 5.0 & $3,534.6$ & $\mathrm{R}_{6,130.9}$ & R. .57652 \\
\hline 1984 & 235.8 & $\mathrm{R}_{4,777.8}$ & 4.9 & $3,930.9$ & $\mathrm{R}_{6,571.5}$ & R. 59817 \\
\hline $\begin{array}{l}1984 \\
1985\end{array}$ & $\begin{array}{l}235.8 \\
237.9\end{array}$ & $\begin{array}{r}\mathrm{R}_{4}, 859.5 \\
\end{array}$ & $\begin{array}{l}4.9 \\
4.9\end{array}$ & $\begin{array}{l}3,930.9 \\
4,217.5\end{array}$ & $\begin{array}{r}0,543.4 \\
\mathrm{R}_{6}, 843\end{array}$ & $\begin{array}{l}.59817 \\
\text { R.61628 }\end{array}$ \\
\hline 1986 & 240.1 & $\mathrm{R}_{4,943.4}$ & 4.9 & $4,460.1$ & $\mathrm{R} 7,080.5$ & R. 62991 \\
\hline 1987 & 242.3 & $\begin{array}{r}\mathrm{R} 5,029.9 \\
\mathrm{R}\end{array}$ & 4.8 & $4,736.4$ & $\mathrm{R} 7,307.0$ & R. 64819 \\
\hline 1988 & 244.5 & $\mathrm{R}_{5,117.0}$ & 4.8 & $5,100.4$ & $\mathrm{R}_{7,607.4}$ & R. 67046 \\
\hline 1989 & 246.8 & $\mathrm{R}_{5,203.7}$ & 4.7 & $5,482.1$ & $\mathrm{R}_{7,879.2}$ & R. 69577 \\
\hline 1990 & 249.6 & $\mathrm{R}_{5,291.1}$ & 4.7 & $5,800.5$ & $R_{8}, 027.1$ & R. 72262 \\
\hline 1991 & 253.0 & $\mathrm{R}_{5,374.1}$ & 4.7 & $5,992.1$ & $\mathrm{R}_{8,008.3}$ & R. 74824 \\
\hline 1992 & 256.5 & R5,459.0 & 4.7 & $6,342.3$ & $\mathrm{R}_{8,280.0}$ & $\begin{array}{r}.170698 \\
\text { R. }\end{array}$ \\
\hline 1993 & 259.9 & $\mathrm{R}_{5,541.4}$ & 4.7 & $6,667.4$ & $\mathrm{R}_{8}, 516.2$ & R. 78290 \\
\hline $\begin{array}{l}1993 \\
1994\end{array}$ & 263.1 & $\begin{array}{r}5,51.4 \\
\mathrm{R}_{5}, 622.4\end{array}$ & 4.7 & $\begin{array}{l}6,661.4 \\
7,085.2\end{array}$ & $\begin{array}{r}8,563.2 \\
R_{8}, 863.1\end{array}$ & R. 79940 \\
\hline 1995 & 266.3 & $\mathrm{R} 5,703.5$ & 4.7 & $7,414.7$ & $\mathrm{Rg}, 086.0$ & R. 81606 \\
\hline 1996 & 269.4 & R5,783.8 & 4.7 & $7,838.5$ & R9,425.8 & R. .83159 \\
\hline 1997 & 272.6 & $\mathrm{R}_{5,862.7}$ & 4.7 & $8,332.4$ & $\mathrm{Rg}, 845.9$ & R. 84628 \\
\hline 1998 & 275.9 & R5,940.6 & 4.6 & $8,793.5$ & $\mathrm{R}_{10,274.7}$ & R. 85584 \\
\hline 1999 & 279.0 & $\mathrm{R}_{6,017.9}$ & 4.6 & $9,353.5$ & $\mathrm{R}_{10,770.7}$ & R. 86842 \\
\hline 2000 & 282.2 & $\mathrm{R}_{6,094.7}$ & 4.6 & $9,951.5$ & $R_{11,216.4}$ & R. .88723 \\
\hline 2001 & 285.0 & $\mathrm{R}_{6,171.9}$ & $\begin{array}{l}4.6 \\
4.6\end{array}$ & $10,286.2$ & $R_{11,337.5}$ & $\begin{array}{l}.000727 \\
\text { R.907 }\end{array}$ \\
\hline 2002 & R287.6 & $\mathrm{R}_{6,249.1}$ & 4.6 & $10,642.3$ & R115431 & R. 92196 \\
\hline 2003 & $\mathrm{R}_{2} 290.1$ & $\mathrm{R}_{6,325.7}$ & $\begin{array}{l}4.6 \\
4.6\end{array}$ & $\mathrm{R}_{11,142.2}$ & $\mathrm{R}_{11,836.4}$ & R. 94135 \\
\hline 2004 & R292.8 & $\mathrm{R}_{6,402.7}$ & 4.6 & $\mathrm{R}_{11,853.3}$ & $\mathrm{R}_{12,246.9}$ & R. .96786 \\
\hline 2005 & R295.5 & $\mathrm{R}_{6,480.0}$ & 4.6 & $\mathrm{R}_{12,623.0}$ & $R_{12,623.0}$ & 1.00000 \\
\hline 2006 & 298.4 & $\mathrm{R}_{6,558.1}$ & $\mathrm{R}_{4.5}$ & $\mathrm{R}_{13,377.2}$ & $\mathrm{R}_{12,958.5}$ & $\mathrm{R}_{1.03231}$ \\
\hline 2007 & $R_{301.2}$ & $\mathrm{R}_{6,636.8}$ & $\mathrm{R}_{4.5}$ & $\mathrm{R}_{14,028.7}$ & $\mathrm{R}_{13,206.4}$ & $\mathrm{R}_{1.06227}$ \\
\hline 2008 & R304.1 & $\mathrm{R}_{6,715.2}$ & 4.5 & $R_{14,291.5}$ & $\mathrm{R}_{13,161.9}$ & $\mathrm{R}_{1.08582}$ \\
\hline 2009 & R306.8 & $\mathrm{R}_{6,792.9}$ & 4.5 & $R_{13}, 939.0$ & $\mathrm{R}_{12,703.1}$ & $\mathrm{R}_{1.09729}$ \\
\hline 2010 & $R_{309.3}$ & $\mathrm{R}_{6,868.5}$ & 4.5 & $\mathrm{R}_{14,526.5}$ & $\mathrm{R}_{13,088.0}$ & $\mathrm{R}_{1.10992}$ \\
\hline 2011 & 311.6 & $6,946.0$ & 4.5 & $15,094.0$ & $13,315.1$ & 1.13361 \\
\hline \multicolumn{4}{|c|}{$\begin{array}{l}1 \text { Resident population of the } 50 \text { States and the District of Columbia estimated for July } 1 \text { of each year. } \\
2 \text { See "Nominal Dollars" in Glossary. } \\
3 \text { In chained (2005) dollars. See "Chained Dollars" in Glossary. } \\
4 \text { The gross domestic product implicit price deflator is used to convert nominal dollars to chained (2005) } \\
\text { dollars. } \\
\text { R=Revised. NA=Not available. } \\
\text { Web Pages: . See http://www.eia.gov/totalenergy/data/annual/\#appendices for all data beginning in } \\
\text { 1949. - For related information, see http://www.census.gov/ and http://www.bea.gov/. } \\
\text { Sources: U.S. Population: - 1949-1989-U.S. Department of Commerce (DOC), U.S. Bureau of the }\end{array}$} & \multicolumn{3}{|c|}{ 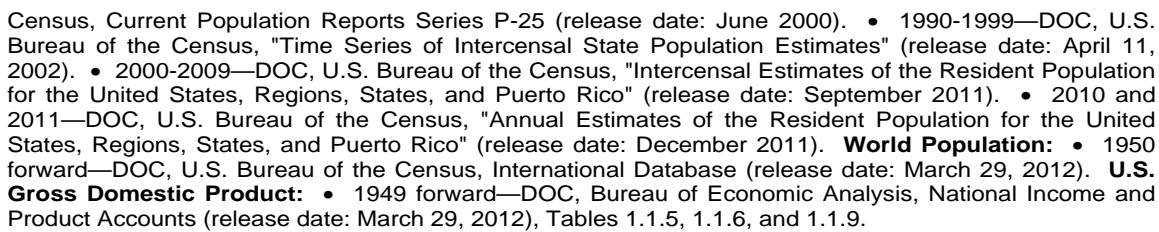 } \\
\hline
\end{tabular}




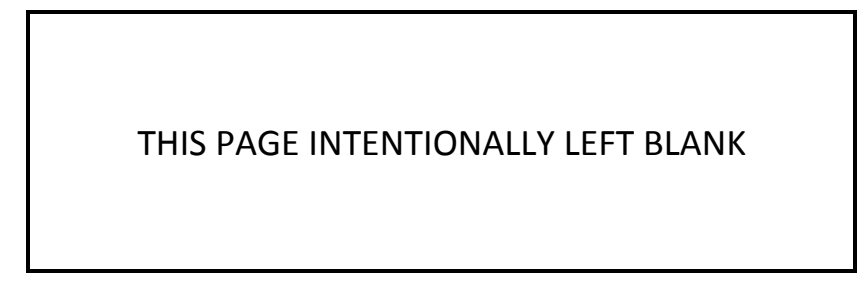




\section{Appendix E}

Table E1. Estimated Primary Energy Consumption in the United States, Selected Years, 1635-1945

(Quadrillion Btu)

\begin{tabular}{|c|c|c|c|c|c|c|c|c|c|}
\hline \multirow[b]{3}{*}{ Year } & \multicolumn{4}{|c|}{ Fossil Fuels } & \multicolumn{3}{|c|}{ Renewable Energy } & \multirow{3}{*}{$\begin{array}{l}\text { Electricity } \\
\text { Net } \\
\text { Imports }\end{array}$} & \multirow[b]{3}{*}{ Total } \\
\hline & \multirow[b]{2}{*}{ Coal } & \multirow{2}{*}{$\begin{array}{c}\text { Natural } \\
\text { Gas }\end{array}$} & \multirow[b]{2}{*}{ Petroleum } & \multirow[b]{2}{*}{ Total } & \multirow{2}{*}{$\begin{array}{l}\text { Conventional } \\
\text { Hydroelectric } \\
\text { Power }\end{array}$} & Biomass & \multirow[b]{2}{*}{ Total } & & \\
\hline & & & & & & Wood ${ }^{1}$ & & & \\
\hline 1635 & NA & -- & -- & NA & -- & (s) & (s) & -- & (s) \\
\hline 1645 & NA & -- & -- & NA & -- & 0.001 & 0.001 & -- & 0.001 \\
\hline 1655 & NA & -- & -- & NA & -- & .002 & .002 & -- & .002 \\
\hline 1665 & NA & -- & -- & NA & -- & .005 & .005 & -- & .005 \\
\hline 1675 & NA & -- & -- & NA & -- & .007 & .007 & -- & .007 \\
\hline 1685 & NA & -- & -- & NA & -- & .009 & .009 & -- & .009 \\
\hline 1695 & NA & -- & -- & NA & -- & .014 & .014 & -- & .014 \\
\hline 1705 & NA & -- & -- & NA & -- & .022 & .022 & -- & .022 \\
\hline 1715 & NA & -- & -- & NA & -- & .037 & .037 & -- & .037 \\
\hline 1725 & NA & -- & -- & NA & -- & .056 & .056 & -- & .056 \\
\hline 1735 & NA & -- & -- & NA & -- & .080 & .080 & -- & .080 \\
\hline 1745 & NA & -- & -- & NA & -- & .112 & .112 & -- & .112 \\
\hline 1755 & NA & -- & -- & NA & -- & .155 & .155 & -- & .155 \\
\hline 1765 & NA & -- & -- & NA & -- & .200 & .200 & -- & 200 \\
\hline 1775 & NA & -- & -- & NA & -- & 249 & .249 & -- & 249 \\
\hline 1785 & NA & -- & -- & NA & -- & .310 & .310 & -- & .310 \\
\hline 1795 & NA & -- & -- & NA & -- & 402 & .402 & -- & .402 \\
\hline 1805 & NA & -- & -- & NA & -- & .537 & .537 & -- & .537 \\
\hline 1815 & NA & -- & -- & NA & -- & .714 & .714 & -- & .714 \\
\hline 1825 & NA & -- & -- & NA & -- & .960 & .960 & -- & .960 \\
\hline 1835 & NA & -- & -- & NA & -- & 1.305 & 1.305 & -- & 1.305 \\
\hline 1845 & NA & -- & -- & NA & -- & 1.757 & 1.757 & -- & 1.757 \\
\hline 1850 & 0.219 & -- & -- & 0.219 & -- & 2.138 & 2.138 & -- & 2.357 \\
\hline 1855 & .421 & -- & -- & .421 & -- & 2.389 & 2.389 & -- & 2.810 \\
\hline 1860 & .518 & -- & 0.003 & .521 & -- & 2.641 & 2.641 & -- & 3.162 \\
\hline 1865 & .632 & -- & .010 & .642 & -- & 2.767 & 2.767 & -- & 3.409 \\
\hline 1870 & 1.048 & -- & .011 & 1.059 & -- & 2.893 & 2.893 & -- & 3.952 \\
\hline 1875 & 1.440 & -- & .011 & 1.451 & -- & 2.872 & 2.872 & -- & 4.323 \\
\hline 1880 & 2.054 & -- & .096 & 2.150 & -- & 2.851 & 2.851 & -- & 5.001 \\
\hline 1885 & 2.840 & 0.082 & .040 & 2.962 & -- & 2.683 & 2.683 & -- & 5.645 \\
\hline 1890 & 4.062 & .257 & .156 & 4.475 & 0.022 & 2.515 & 2.537 & -- & 7.012 \\
\hline 1895 & 4.950 & .147 & .168 & 5.265 & .090 & 2.306 & 2.396 & -- & 7.661 \\
\hline 1900 & 6.841 & .252 & $\begin{array}{l}.100 \\
.229\end{array}$ & $\begin{array}{l}.205 \\
7.322\end{array}$ & .250 & $\begin{array}{l}2.000 \\
2.015\end{array}$ & $\begin{array}{l}\text { 2.0390 } \\
2.265\end{array}$ & -- & 9.587 \\
\hline 1905 & $\begin{array}{r}10.001 \\
\end{array}$ & .372 & .610 & 10.983 & .386 & 1.843 & 2.229 & -- & 13.212 \\
\hline 1910 & 12.714 & .540 & 1.007 & 14.261 & .539 & 1.765 & 2.304 & -- & 16.565 \\
\hline 1915 & 13.294 & .673 & 1.418 & 15.385 & .659 & 1.688 & 2.347 & 0.002 & 17.734 \\
\hline 1920 & $\begin{array}{l}15.594 \\
1504\end{array}$ & $\begin{array}{l}.015 \\
.813\end{array}$ & $\begin{array}{l}2.410 \\
2.676\end{array}$ & 18.993 & .738 & $\begin{array}{l}1.000 \\
1.610\end{array}$ & $\begin{array}{l}2.341 \\
2.348\end{array}$ & .003 & $\begin{array}{l}21.344 \\
21.344\end{array}$ \\
\hline 1925 & $\begin{array}{l}1.504 \\
14.706\end{array}$ & $\begin{array}{l}.0010 \\
1.191\end{array}$ & 4.280 & 20.177 & . 668 & 1.533 & $\begin{array}{l}2.040 \\
2.201\end{array}$ & .004 & $\begin{array}{l}1.544 \\
22.382\end{array}$ \\
\hline 1930 & 13.639 & 1.932 & 5.897 & 21.468 & .752 & 1.455 & 2.207 & .005 & 23.680 \\
\hline 1935 & 10.634 & 1.919 & 5.675 & 18.228 & .806 & $\begin{array}{l}1.397 \\
\end{array}$ & 2.203 & .005 & 20.436 \\
\hline 1940 & $\begin{array}{l}12.534 \\
\end{array}$ & 2.665 & 7.760 & 22.960 & $\begin{array}{l}.000 \\
.880\end{array}$ & 1.358 & 2.238 & .007 & 25.205 \\
\hline 1945 & 15.972 & 3.871 & 10.110 & 29.953 & 1.442 & 11.261 & 2.703 & .009 & 32.665 \\
\hline
\end{tabular}

1 There is a discontinuity in the "Wood" time series between 1945 and 1949. Through 1945, data are for fuelwood only; beginning in 1949, data are for wood and wood-derived fuels (see Table 10.1).

$\mathrm{NA}=$ Not available. $--=$ Not applicable. (s)=Less than 0.0005 quadrillion Btu.

Notes: - For years not shown, there are no data available. - See Tables 1.3 and 10.1 for continuation of these data series from 1949 forward. - See Note, "Geographic Coverage of Statistics for 1635-1945," at end of section.

Sources: Coal, Natural Gas, and Petroleum: Energy in the American Economy, 1850-1975, Table VII.

Conventional Hydroelectric Power: Energy in the American Economy, 1850-1975, Table II. Wood:

- 1635-1845: U.S. Department of Agriculture Circular No. 641, Fuel Wood Used in the United States

1630-1930, February 1942. This source estimates fuelwood consumption in cords per decade, which were converted to Btu using the conversion factor of 20 million Btu per cord. The annual average value for were decade was assigned to the fifth year of the decade on the assumption that annual use was likely to increase during any given decade and the average annual value was more likely to reflect mid-decade yearly consumption than use at either the beginning or end of the decade. Values thus begin in 1635 and are plotted at 10-year intervals. - 1850-1945: Energy in the American Economy, 1850-1975, Table VII. Electricity Net Imports: Energy in the American Economy, 1850-1975, Tables I and VI. Calculated as the difference between hydroelectric consumption and hydroelectric production times 3,412 Btu per
kilowatthour. 


\section{Appendix E}

Note: Geographic Coverage of Statistics for 1635-1945. Table E1 presents estimates of U.S. energy consumption by energy source for a period that begins a century and a half before the original 13 colonies formed a political union and continues through the decades during which the United States was still expanding territorially. The question thus arises, what exactly is meant by "U.S. consumption" of an energy source for those years when the United States did not formally exist or consisted of less territory than is now encompassed by the 50 States and the District of Columbia?

The documents used to assemble the estimates, and (as far as possible) the sources of those documents, were reviewed carefully for clues to geographic coverage. For most energy sources, the extent of coverage expanded more rapidly than the Nation, defined as all the official States and the District of Columbia. Estimates or measurements of consumption of each energy source generally appear to follow settlement patterns. That is, they were made for areas of the continent that were settled enough to have economically significant consumption even though those areas were not to become States for years. The wood data series, for example, begins in 1635 and includes 12 of the original colonies (excepting Georgia), as well as Maine, Vermont, and the area that would become the District of Columbia. By the time the series reaches 1810 , the rest of the continental States are all included, although the last of the 48 States to achieve statehood did not do so until 1912. Likewise, the coal data series begins in 1850 but includes consumption in areas, such as Utah and Washington (State), which were significant coal-producing regions but had not yet attained statehood. (Note: No data were available on State-level historical coal consumption. The coal data shown in Table E1 through 1945 describe apparent consumption, i.e., production plus imports minus exports. The geographic coverage for coal was therefore based on a tally of coal-producing States listed in various historical issues of Minerals Yearbook. It is likely that coal was consumed in States where it was not mined in significant quantities.)

By energy source, the extent of coverage can be summarized as follows: • Coal—35 coal-producing States by 1885 . - Natural Gas-All 48 contiguous States, the District of Columbia, and Alaska by 1885. • Petroleum-All 48 contiguous States, the District of Columbia, and Alaska by 1885 . Conventional Hydroelectric Power-Coverage for 1890 and 1895 is uncertain, but probably the 48 contiguous States and the District of Columbia. Coverage for 1900 through 1945 is the 48 contiguous States, and the District of Columbia. - Wood-All 48 contiguous States and the District of Columbia by 1810 . 


\section{Appendix F}

\section{Alternatives for Estimating Energy Consumption}

\section{This appendix is reprinted from the Annual Energy Review 2010. EIA continues to review alternative options for accounting for energy consumption and related losses, such as those associated with the generation and distribution of electricity.}

\section{Introduction}

This year, the U.S. Energy Information Administration (EIA) has examined different ways to represent energy consumption in the Annual Energy Review (AER). This examination centered on two methods for representing related aspects of energy consumption and losses. The first is an alternative method for deriving the energy content of noncombustible renewable resources, which has been implemented in AER 2010 (Table 1.3). The second is a new representation of delivered total energy and energy losses.

This appendix provides an explanation of these alternative methods. Section II provides a background discussion of the alternatives and the reasons for considering these changes to the energy balance presentation. Section III identifies the specific changes incorporated in AER 2010.

\section{Background}

\section{Alternative Approaches for Deriving Energy Contents for Noncombustible Renewables}

EIA compiles data on most energy sources in physical units, such as barrels and cubic feet, in order to calculate total primary energy consumption. Before aggregation, EIA converts data for these energy sources to the common unit of British thermal units (Btu), a measure that is based on the thermal conversion of energy resources to heat and power.

Noncombustible renewables are resources from which energy is extracted without the burning or combustion of a fuel. They include hydroelectric, geothermal, solar, and wind energy. Because power from noncombustible renewables is produced without fuel combustion, there are no set Btu conversion factors for these energy sources.

In the past, EIA has represented hydroelectric, solar, and wind energy consumed for electric generation as the amount of energy it would require, on average, to produce an equivalent number of kilowatthours (kWh) of electricity using fossil fuels. In this appendix, this approach is referred to as the "fossil-fuel equivalency" approach. For the remaining noncombustible renewable resource, geothermal energy, energy consumed for electricity generation has been based on estimates of plant efficiencies in converting geothermal energy to electricity.

The fossil-fuel equivalency approach evolved in an era when the primary goal of U.S. energy policy was reducing dependence on imported petroleum and when a significant amount of electricity was generated using fuel oil. It was intended to indicate the amount of fossil energy displaced by the renewable energy source. But fuel oil is no longer used to generate electricity to a substantial degree and the international community largely uses a different approach, applying the constant conversion factor of 3,412 Btu/kWh. In addition, using a separate approach for geothermal generation may distort the analysis of the relative share of this generation resource. EIA also has a desire to better account for energy losses and efficiency. For these reasons, EIA considered three alternative methods for deriving the energy contents for noncombustible renewables, designated here as the fossil-fuel equivalency, captured energy, and incident energy approaches.

\section{Fossil-Fuel Equivalency Approach}

With this approach, EIA would continue to apply the fossil-fuel equivalent conversion factor to hydroelectric, solar, and wind energy and would begin applying it to geothermal energy. This approach would eliminate the inconsistency between geothermal and other noncombustibles, enable fuel displacement analysis, and 
maintain the continuity of a data series with which users are familiar. However, the fossil-fuel equivalency approach does not represent any real market quantity. It measures neither primary energy consumed nor fossil fuel actually displaced. Additionally, its use will likely become increasingly problematic if renewables begin to displace other renewables instead of fossil fuels.

\section{Captured Energy Approach}

With this approach, EIA would apply the fixed factor of 3,412 Btu/kWh the Btu value of electric energy generated) to measure the renewable energy consumed for electric generation for all noncombustible renewables. Using this approach would effectively count as primary energy only that noncombustible renewable energy that is captured for economic use.

EIA will use the term captured energy in referring to the energy actually "captured" by a noncombustible renewable energy system for final use. Thus, it is the net energy available for consumption after transformation of a noncombustible renewable resource into a usable energy carrier (such as electricity) or energy that is directly used. Another way of stating it is that captured energy is the energy measured as the "output" of the device, such as electricity from a wind turbine or solar plant.

This approach would not require EIA to make generalized assumptions regarding the actual conversion of these resources (wind, sunshine, falling water) into electricity. It would move U.S. reporting standards closer to international norms, which have been vetted by the International Energy Agency (IEA) and the international energy statistical community through years of actual use. Additionally, this approach better shows the economically significant energy transformations in the United States because the "lost" noncombustible renewable energy does not incur any significant economic cost (there is no market for the resource-specific energy apart from its immediate, site-specific energy conversion, and there is no substantive opportunity cost to its continued exploitation. ${ }^{1}$ ) On the other hand, this approach implies that conversion of noncombustible renewable energy is 100 -percent efficient. In other words, it implies that there is no physical energy loss from the conversion of noncombustible renewables to electricity. In fact, renewable energy conversion can be very inefficient (largely because of the lack of alternative economic uses discussed above). Thus, this approach does not provide an accurate measure of the physical consumption of energy to produce electricity from these resources.

\section{Incident Energy Approach}

With this approach, EIA would use actual or estimated energy efficiencies of renewable conversion technologies to determine the Btu value of the input energy used to produce reported renewable generation. For example, rather than treating the electricity generated at a solar plant as primary energy, an empirical estimate of the actual portion of solar radiation incident on the solar panel that is converted to electricity would be used.

EIA will define "incident energy" for noncombustible renewable resources as the gross energy that first strikes an energy conversion device. In contrast to captured energy, incident energy is the mechanical, radiation, or thermal energy that is measurable at the "input" of the device. For wind, this would be the energy contained in the wind that passes through the rotor disc; for solar, the energy contained in the sunlight that strikes the panel or collector mirror; for hydroelectric, the energy contained in the water passing through the penstock (a closed conduit for carrying water to the turbines); and, for geothermal, the energy contained in the hot fluid at the surface of the wellbore.

This approach lends itself to a view of showing the physical reality of energy transformations in the United States. However, few renewable energy plants track cumulative input energy because of its lack of economic significance. Therefore, it would be difficult to obtain accurate estimates of efficiency without creating undue burden on survey respondents. Furthermore, this approach has not been vetted in the energy statistics community and its use would be inconsistent with IEA and other international statistics.

Table F1 shows factors that could be used to estimate the energy incident on the primary energy collection device of a noncombustible renewable power plant. These factors represent energy output as a percent of energy input. The conversion efficiency of renewable generation equipment is generally specified by the manufacturer, although this specification may differ from realized efficiencies for several reasons, including: the effects of balance-of-plant factors; environmental conditions that are different than conditions that the equipment was rated for; and variability in operating conditions for equipment that is rated under fixed conditions The efficiencies shown in this table are not estimates of the actual, operational efficiency of the technologies indicated. Rather they are notional indications of the efficiencies that each technology may be able to achieve with typical equipment operating within the normal operating range for that technology.

${ }^{1}$ There is an initial opportunity cost when first building such a facility: the water behind a dam might inundate land with alternative uses or a solar panel might shade some area that could otherwise use the sunlight. But that is a "fixed" opportunity cost that does not effectively change by normal operation of the plant. 


\begin{tabular}{|c|c|}
\hline $\begin{array}{c}\text { Table F1. Conversion Efficiencies of Noncombustible } \\
\text { Renewable Energy Sources } \\
\text { (Percent) }\end{array}$ \\
\hline Source & Notional Efficiency $^{\mathbf{1}}$ \\
\hline Geothermal & 16 \\
\hline Conventional Hydroelectric & 90 \\
\hline Solar Photovoltaic & 12 \\
\hline Solar Thermal Power & 21 \\
\hline Wind & 26 \\
\hline
\end{tabular}

${ }^{1}$ Efficiencies may vary significantly for each technology based on site-specific

technology and environmental factors. Factors shown represent engineering estimates for typical equipment under specific operational conditions.

Sources: Geothermal: Estimated by EIA on the basis of an informal survey of relevant plants. Conventional Hydroelectric: Based on published estimates for the efficiency of large-scale hydroelectric plants. See

http://www.usbr.gov/power/edu/pamphlet.pdf. Solar Photovoltaic: Based on the average rated efficiency for a sample of commercially available modules. Rated efficiency is the conversion efficiency under standard test conditions, which represents a fixed, controlled operating point for the equipment; efficiency can vary with temperature and the strength of incident sunlight. Rated efficiencies are based on the direct current (DC) output of the module; since grid-tied applications require alternating current (AC) output, efficiencies are adjusted to account for a 20 percent reduction in output when converting from DC to

AC. Solar Thermal Power: Estimated by dividing the rated maximum power available from the generator by the power available under standard solar conditions $(1,000 \mathrm{~W} / \mathrm{m} 2)$ from the aperture area of solar collectors. Wind: Based on the average efficiency at rated wind speed for a sample of commercially available wind turbines. The rated wind speed is the minimum wind speed at which a turbine achieves its nameplate rated output under standard atmospheric conditions. Efficiency is calculated by dividing the nameplate rated power by the power available from the wind stream intercepted by the rotor disc at the rated wind speed.

\section{Conclusion}

After review of the three options, EIA has elected to follow a hybrid of the first two approaches for the AER 2010. The primary energy value of noncombustible renewables consumed for electricity generation will be measured using the fossil-fuel equivalent factor. However, this value will be reported as the sum of captured energy and an "Adjustment for Fossil Fuel Equivalence," which is the difference between the fossil-fuel equivalent value and the value obtained using the $3,412 \mathrm{Btu} / \mathrm{kWh}$ factor. This adjustment value represents the energy loss that would have been incurred if the electricity had been generated by fossil fuels. For solar and geothermal energy used directly, EIA will continue to use the factors currently employed.
This method will not cause a change to total primary energy consumption of hydro, solar, or wind energy, but it will allow users to easily distinguish actual economic energy consumption from the imputed displacement value, which is retained both to provide backward compatibility for data users accustomed to this measure and to allow for easier analysis of certain energy efficiency and production trends. The separate reporting of captured energy will also facilitate comparisons with international data sets.

For geothermal energy consumed to generate electricity, EIA will recalculate current and historical values using the fossil-fuel equivalent factor. This recalculation will change the following values presented in the AER 2010: the primary consumption of total energy (Tables 1.1 and 1.3); the consumption of geothermal for electricity generation (Tables 8.4a and b); and the consumption of renewable energy (Tables 10.1 and $10.2 \mathrm{c})$.

\section{New Representation of Delivered Total Energy and Energy Losses}

The examination of heat rates for noncombustible fuels led EIA to also consider alternative methods of accounting for final energy consumption and energy losses. Final energy consumption differs from primary energy consumption in that it represents the amount (in terms of Btu) of energy actually consumed, in its final form, by an end user. For example, primary energy consumption of coal includes all the heat content in the coal consumed, while final energy consumption will include only the heat content of any coal consumed in its original form and the heat content of any products transformed from coal, such as electricity generated from coal.

EIA analyzed energy transformation in the United States. In all transformation processes, some useful energy is lost in achieving the conversion from one energy form to another. The most significant losses, by far, occur when electricity is generated from primary energy resources. Figure F1 illustrates an alternate method of accounting for energy consumption, based on the concept of delivered total energy.

In the AER 2010, as in previous AERs, the electric power sector is viewed as an energy-consuming sector. For each of the end-use sectors - residential, commercial, industrial, and transportation - total energy consumption is made up of the primary energy source consumed plus electricity retail sales and electrical system energy losses. Electrical system energy losses include transformation losses, the adjustment for fossil fuel equivalence (as discussed above), power plant use of electricity, transmission and distribution losses, and unaccounted for electricity. They are allocated to the end-use demand sectors in proportion to each sector's share of total electricity sales. 
In the alternative representation (Figure F1), the electric power sector is not treated as an energy-consuming sector but as a sector that transforms and redistributes energy to final users. In order to better represent the amount of energy actually consumed by the final user, this method eliminates the allocation of electrical system energy losses to consuming sectors. Electricity retail sales to each sector, as reported by energy service providers, continue to be viewed as end-use consumption and, thus, are included in Delivered Total Energy. In Figure F1, delivered total energy represents the gross energy that enters an end-use facility (home, business, factory, and so forth). In some cases, there are conversion or transformation processes within the facility that create additional losses before the final consumption of the energy, so that the net energy consumed for useful application will be less than shown in the figure. For example, natural gas furnaces typically lose some amount of heat in the chimney, energy which then does not go toward heating the building.

Table F2 provides a comparison of Primary Energy Consumption and Delivered Total Energy by energy-use sector. Sources for Primary Energy Consumption by sector are AER Tables 5.14, 6.5, 7.3, 8.9, and 10.2. Data from those tables are converted from physical units to Btu using heat contents given in Appendix A. Sources for Delivered Total Energy are AER Tables $2.1 \mathrm{~b}$ through e.

\section{Changes to the AER 2010}

The major change to AER 2010 is the modification of Table 1.3 to incorporate the new treatment of noncombustible renewable energy consumption. The value of geothermal energy consumption and, consequently, total primary energy consumption is slightly lower than previously published for all years due to the use of a new geothermal conversion factor (the fossil-fuels heat rate from Table A6). See Section II of this appendix for further explanation.

The sum of hydroelectric, geothermal, solar, and wind primary consumption is now shown as total primary energy consumption for noncombustible renewables. That total includes: geothermal heat pump and direct use of geothermal energy; solar thermal direct use energy; and noncombustible resources that are transformed into electricity. Noncombustible resources transformed into electricity are equal to electricity generation from all noncombustible renewables converted to Btu using the fossil-fuels heat rate. Direct final consumption of geothermal and solar energy is obtained from AER Tables 10.2a and 10.2b.
Total primary consumption for noncombustible renewables is the sum of captured energy (or energy produced) and the "adjustment for fossil fuel equivalence." Like total primary consumption, captured energy includes: geothermal heat pump and direct use of geothermal energy; solar thermal direct use energy; and noncombustible resources that are transformed into electricity. However, electricity generation for all noncombustible renewables is converted to Btu using the energy content of electricity, 3,412 Btu per kWh.

The "adjustment for fossil fuel equivalence" is equal to the difference between total primary consumption of noncombustibles in Btu (calculated using the fossil-fuels heat rate) and captured energy. There is no adjustment for fossil fuel equivalence associated with direct consumption of geothermal and solar energy.

In order to prevent any inconsistency between data presented in the modified Table 1.3 and the AER Section 10, "Renewable Energy," EIA will show data for the individual noncombustible renewables (hydroelectricity, wind, etc.,) in Section 10 only. In the AER 2010, total primary energy consumed for individual noncombustible renewables can be found in Table 10.1. A detailed breakout of the noncombustible renewable consumption components summarized in Table 1.3 is provided in Table F3. Table F3 shows the components of captured energy and the adjustment for fossil fuel equivalence (regarded as a loss), by individual energy source, for 2010. The columns labeled "Transformed into Electricity" represent the energy value of electricity generated from each type of noncombustible renewable resource. These values are calculated by multiplying net generation in Table 8.2 by 3,412 Btu/kWh.

For each noncombustible renewable, the adjustment for fossil fuel equivalence is calculated as the difference between the fossil fuel equivalent value of electricity generated and the value of "Transformed into Electricity." ${ }^{2}$ For geothermal, direct consumption is the heat either captured and used directly from thermal ground water sources or extracted by ground-source heat pump. Values are from Tables 10.2a and 10.2b. Solar/PV direct consumption includes solar thermal energy used directly in the residential and electric power sectors. These values are from Tables 10.2a and 10.2c. Captured energy is equal to energy "transformed into electricity" for conventional hydroelectricity and wind. For geothermal and solar/PV, captured energy equals the sum of direct consumption and energy transformed into electricity.

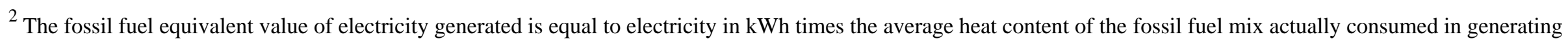
electricity for a given year. 
Figure F1. Primary Energy Consumption and Delivered Total Energy, 2010

(Quadrillion Btu)

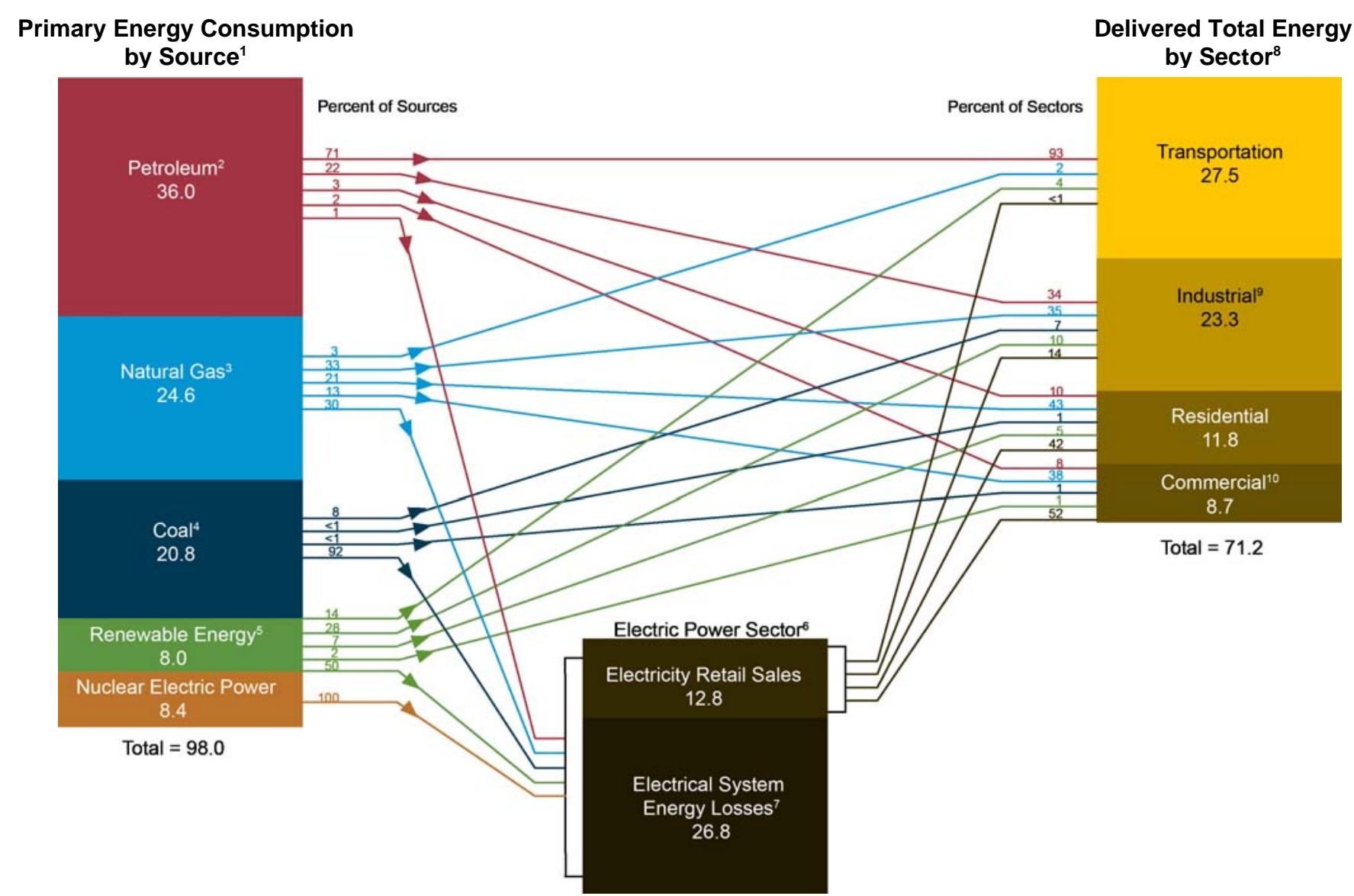

${ }^{1}$ Includes electricity net imports, not shown separately.

2 Does not include biofuels that have been blended with petroleum-biofuels are included in "Renewable Energy."

${ }^{3}$ Excludes supplemental gaseous fuels.

${ }^{4}$ Includes less than 0.1 quadrillion Btu of coal coke net exports.

${ }^{5}$ Conventional hydroelectric power, geothermal, solar/PV, wind, and biomass.

${ }^{6}$ Electricity-only and combined-heat-and-power (CHP) plants whose primary business is to sell electricity, or electricity and heat, to the public.

${ }^{7}$ Calculated as the primary energy consumed by the electric power sector minus the energy content of electricity retail sales. See Note, "Electrical System Energy Losses," at end of Section 2 .
${ }^{8}$ Includes transformation losses other than electrical system energy losses. For example, see notes 9 and 10 on this page.

${ }^{9}$ Includes industrial combined-heat-and-power (CHP) and industrial electricity-only plants.

${ }_{10}$ Includes commercial combined-heat-and-power (CHP) and commercial electricity-only plants.

Note: Sum of components may not equal total due to independent rounding.

Sources: U.S. Energy Information Administration, Annual Energy Review 2010, Tables

$1.3,2.1 \mathrm{~b}-\mathrm{f}, 10.3$, and 10.4 . 


\section{Table F2. Energy Consumption by Sector, 2010}

(Quadrillion Btu)

\begin{tabular}{|c|c|c|c|c|c|c|c|c|c|c|c|c|}
\hline \multirow{3}{*}{$\begin{array}{l}\text { Year } \\
2010\end{array}$} & \multicolumn{6}{|c|}{ Primary Energy Consumption $^{1}$} & \multicolumn{5}{|c|}{ Delivered Total Energy $^{2}$} & \multirow[b]{2}{*}{$\begin{array}{c}\text { Electrical System } \\
\text { Energy } \\
\text { Losses }^{4} \\
\end{array}$} \\
\hline & $\begin{array}{l}\text { Residen- } \\
\text { tial }\end{array}$ & $\begin{array}{l}\text { Commer- } \\
\text { cial }\end{array}$ & $\begin{array}{l}\text { Indus- } \\
\text { trial }^{3}\end{array}$ & $\begin{array}{c}\text { Transporta- } \\
\text { tion }^{3}\end{array}$ & $\begin{array}{c}\text { Electric } \\
\text { Power }\end{array}$ & Total & $\begin{array}{l}\text { Residen- } \\
\text { tial }\end{array}$ & $\begin{array}{l}\text { Commer- } \\
\text { cial }\end{array}$ & $\begin{array}{c}\text { Indust- } \\
\text { rial }\end{array}$ & $\begin{array}{c}\text { Transporta- } \\
\text { tion }\end{array}$ & Total & \\
\hline & 6,841 & 4,175 & 19,984 & 27,425 & 39,579 & 98,004 & 11,791 & 8,711 & 23,267 & 27,451 & 71,220 & 26,784 \\
\hline
\end{tabular}

${ }^{1}$ Includes Adjustment for Fossil Fuel Equivalence. See "Primary Energy Consumption" in Glossary.

${ }^{2}$ Includes electricity sales to each sector in addition to Primary Energy consumed in the sector.

${ }^{3}$ Small amounts of coal consumed for transportation are reported as industrial sector consumption. Includes net imports of supplemental liquids and coal coke.

${ }^{4}$ Calculated as the primary energy consumed by the electric power sector minus the energy content of electricity retail sales.

\section{Table F3. Noncombustible Renewable Primary Energy Consumption by Energy Source, 2010} (Trillion Btu)

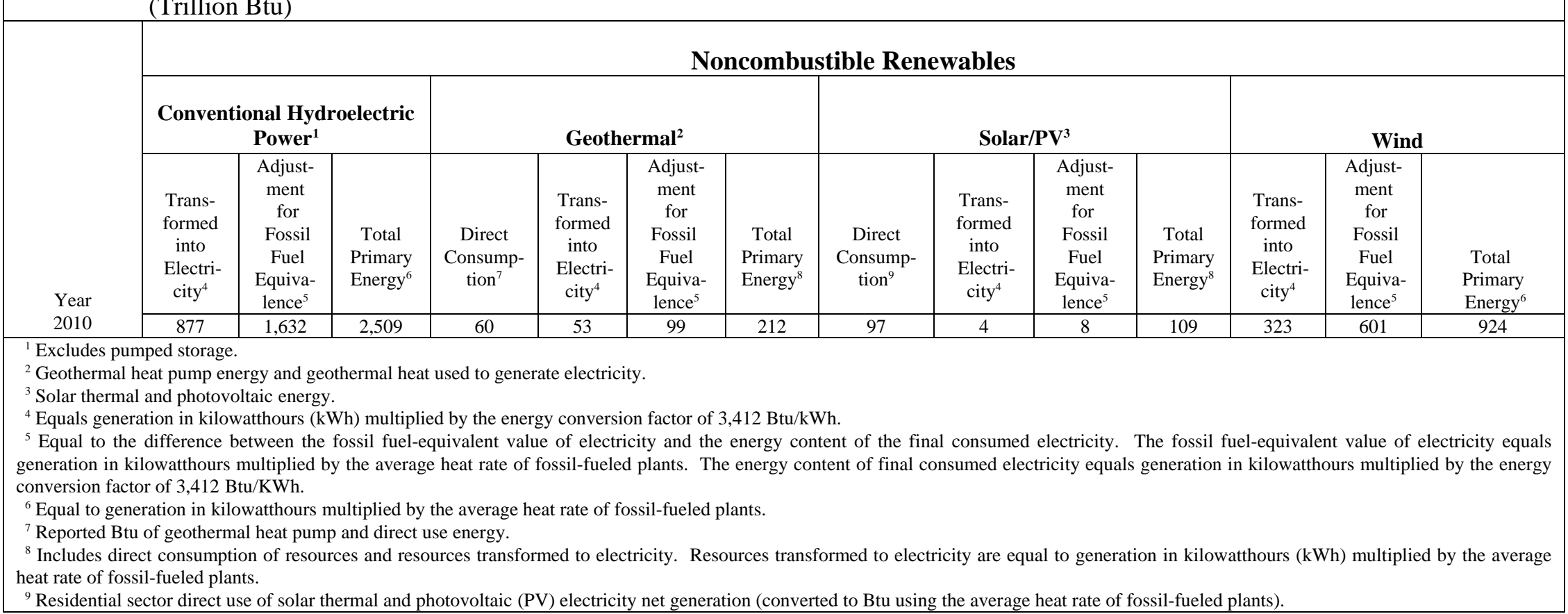




\section{Glossary}

Alcohol: The family name of a group of organic chemical compounds composed of carbon, hydrogen, and oxygen. The series of molecules vary in chain length and are composed of a hydrocarbon plus a hydroxyl group: $\mathrm{CH}_{3}-\left(\mathrm{CH}_{2}\right) \mathrm{n}-\mathrm{OH}$ (e.g., methanol, ethanol, and tertiary butyl alcohol). See Fuel Ethanol.

Alternative Fuel: Alternative fuels, for transportation applications, include the following: methanol; denatured ethanol, and other alcohols; fuel mixtures containing 85 percent or more by volume of methanol, denatured ethanol, and other alcohols with motor gasoline or other fuels; natural gas; liquefied petroleum gas (propane); hydrogen; coal-derived liquid fuels; fuels (other than alcohol) derived from biological materials (biofuels such as soy diesel fuel); electricity (including electricity from solar energy); and "... any other fuel the Secretary determines, by rule, is substantially not petroleum and would yield substantial energy security benefits and substantial environmental benefits." The term "alternative fuel" does not include alcohol or other blended portions of primarily petroleum-based fuels used as oxygenates or extenders, i.e. MTBE, ETBE, other ethers, and the 10 -percent ethanol portion of gasohol.

Alternative-Fuel Vehicle (AFV): A vehicle designed to operate on an alternative fuel (e.g., compressed natural gas, methane blend, or electricity). The vehicle could be either a dedicated vehicle designed to operate exclusively on alternative fuel or a nondedicated vehicle designed to operate on alternative fuel and/or a traditional fuel.

Anthracite: The highest rank of coal; used primarily for residential and commercial space heating. It is a hard, brittle, and black lustrous coal, often referred to as hard coal, containing a high percentage of fixed carbon and a low percentage of volatile matter. The moisture content of fresh-mined anthracite generally is less than 15 percent. The heat content of anthracite ranges from 22 to 28 million Btu per short ton on a moist, mineral-matter-free basis. The heat content of anthracite consumed in the United States averages 25 million Btu per short ton, on the as-received basis (i.e., containing both inherent moisture and mineral matter). Note: Since the 1980's, anthracite refuse or mine waste has been used for steamelectric power generation. This fuel typically has a heat content of 15 million Btu per short ton or less. See Coal Rank.

Anthracite Culm: Waste from Pennsylvania anthracite preparation plants, consisting of coarse rock fragments containing as much as 30 percent small-sized coal; sometimes defined as including very fine coal particles called silt. Its heat value ranges from 8 to 17 million Btu per short ton.
Anthropogenic: Made or generated by a human or caused by human activity. The term is used in the context of global climate change to refer to gaseous emissions that are the result of human activities, as well as other potentially climate-altering activities, such as deforestation.

API: The American Petroleum Institute, a trade association.

API Gravity: American Petroleum Institute measure of specific gravity of crude oil or condensate in degrees. An arbitrary scale expressing the gravity or density of liquid petroleum products. The measuring scale is calibrated in terms of degrees API; it is calculated as follows: Degrees API = (141.5 / sp.gr.60 deg.F/60 deg.F $)$ 131.5 .

Asphalt: A dark-brown to black cement-like material obtained by petroleum processing and containing bitumens as the predominant component; used primarily for road construction. It includes crude asphalt as well as the following finished products: cements, fluxes, the asphalt content of emulsions (exclusive of water), and petroleum distillates blended with asphalt to make cutback asphalts. Note: The conversion factor for asphalt is 5.5 barrels per short ton.

ASTM: The American Society for Testing and Materials.

Aviation Gasoline Blending Components: Naphthas that will be used for blending or compounding into finished aviation gasoline (e.g., straight run gasoline, alkylate, reformate, benzene, toluene, and xylene). Excludes oxygenates (alcohols, ethers), butane, and pentanes plus. Oxygenates are reported as other hydrocarbons, hydrogen, and oxygenates.

Aviation Gasoline, Finished: A complex mixture of relatively volatile hydrocarbons with or without small quantities of additives, blended to form a fuel suitable for use in aviation reciprocating engines. Fuel specifications are provided in ASTM Specification D910 and Military Specification MIL-G-5572. Note: Data on blending components are not counted in data on finished aviation gasoline. See Jet Fuel; Jet Fuel, Kerosene-Type; and Jet Fuel, Naphtha-Type.

Barrel (Petroleum): A unit of volume equal to 42 U.S. Gallons.

Barrels per Calendar Day: The amount of input that a distillation facility can process under usual operating conditions. The amount is expressed in terms of capacity during a 24-hour period and reduces the maximum processing capability of 
all units at the facility under continuous operation to account for the following limitations that may delay, interrupt, or slow down production: 1) the capability of downstream processing units to absorb the output of crude oil processing facilities of a given refinery (no reduction is necessary for intermediate streams that are distributed to other than downstream facilities as part of a refinery's normal operation); 2) the types and grades of inputs to be processed; 3) the types and grades of products expected to be manufactured; 4) the environmental constraints associated with refinery operations; 5) the reduction of capacity for scheduled downtime due to such conditions as routine inspection, maintenance, repairs, and turnaround; and 6) the reduction of capacity for unscheduled downtime due to such conditions as mechanical problems, repairs, and slowdowns.

Base Gas: The volume of gas needed as a permanent inventory to maintain adequate underground storage reservoir pressures and deliverability rates throughout the withdrawal season. All native gas is included in the base gas volume.

Biodiesel: A fuel typically made from soybean, canola, or other vegetable oils; animal fats; and recycled grease. It can serve as a substitute for petroleum-derived diesel fuel or distillate fuel oil. For U.S. Energy Information Administration reporting, it is a fuel composed of mono-alkyl esters of long chain fatty acids derived from vegetable oils or animal fats, designated B100, and meeting the requirements of ASTM (American Society for Testing \& Materials) D 6751.

Biofuels: Liquid fuels and blending components produced from biomass (plant) feedstocks, used primarily for transportation. See Biodiesel and Fuel Ethanol.

Biogenic: Produced by biological processes of living organisms. Note: EIA uses the term "biogenic" to refer only to organic nonfossil material of biological origin.

Biomass: Organic nonfossil material of biological origin constituting a renewable energy source. See Biodiesel, Biofuels, Biomass Waste, Fuel Ethanol, and Wood and Wood-Derived Fuels.

Biomass Waste: Organic nonfossil material of biological origin that is a byproduct or a discarded product. "Biomass waste" includes municipal solid waste from biogenic sources, landfill gas, sludge waste, agricultural crop byproducts, straw, and other biomass solids, liquids, and gases; but excludes wood and woodderived fuels (including black liquor), biofuels feedstock, biodiesel, and fuel ethanol. Note: EIA "biomass waste" data also include energy crops grown specifically for energy production, which would not normally constitute waste.

Bituminous Coal: A dense coal, usually black, sometimes dark brown, often with well-defined bands of bright and dull material, used primarily as fuel in steam-electric power generation, with substantial quantities also used for heat and power applications in manufacturing and making coke. Bituminous coal is the most abundant coal in active U.S. mining regions. Its moisture content usually is less than 20 percent. The heat content of bituminous coal ranges from 21 to 30 million Btu per short ton on a moist, mineral-matter-free basis. The heat content of bituminous coal consumed in the United States averages 24 million Btu per short ton, on the as-received basis (i.e., containing both inherent moisture and mineral matter). See Coal Rank.

Black Liquor: A byproduct of the paper production process, alkaline spent liquor, that can be used as a source of energy. Alkaline spent liquor is removed from the digesters in the process of chemically pulping wood. After evaporation, the residual "black" liquor is burned as a fuel in a recovery furnace that permits the recovery of certain basic chemicals.

Breeze: The fine screenings from crushed coke. Usually breeze will pass through a $1 / 2$-inch or 3/4-inch screen opening. It is most often used as a fuel source in the process of agglomerating iron ore.

British Thermal Unit (Btu): The quantity of heat required to raise the temperature of 1 pound of liquid water by 1 degree Fahrenheit at the temperature at which water has its greatest density (approximately 39 degrees Fahrenheit). See Heat Content.

\section{Btu: See British Thermal Unit.}

Btu Conversion Factor: A factor for converting energy data between one unit of measurement and British thermal units (Btu). Btu conversion factors are generally used to convert energy data from physical units of measure (such as barrels, cubic feet, or short tons) into the energy-equivalent measure of Btu. (See http://www.eia.gov/emeu/mer/append_a.html for further information on Btu conversion factors.)

Bunker Fuels: Fuel supplied to ships and aircraft, both domestic and foreign, consisting primarily of residual fuel oil and distillate fuel oil for ships and kerosene-type jet fuel for aircraft. The term "international bunker fuels” is used to denote the consumption of fuel for international transport activities. Note: For the purposes of greenhouse gas emissions inventories, data on emissions from combustion of international bunker fuels are subtracted from national emissions totals. Historically, bunker fuels have meant only ship fuel.

Butane: A normally gaseous straight-chain or branched-chain hydrocarbon $\left(\mathrm{C}_{4} \mathrm{H}_{10}\right)$ extracted from natural gas or refinery gas streams. It includes isobutane and normal butane and is designated in ASTM Specification D1835 and Gas Processors Association Specifications for commercial butane. 
Isobutane: A normally gaseous branched-chain hydrocarbon. It is a colorless paraffinic gas that boils at a temperature of 10.9 degrees Fahrenheit. It is extracted from natural gas or refinery gas streams.

Normal Butane: A normally gaseous straight-chain hydrocarbon. It is a colorless paraffinic gas that boils at a temperature of 31.1 degrees Fahrenheit. It is extracted from natural gas or refinery gas streams.

Butylene: An olefinic hydrocarbon $\left(\mathrm{C}_{4} \mathrm{H}_{8}\right)$ recovered from refinery processes.

\section{Capacity: See Generator Capacity.}

\section{Capacity Factor: See Generator Capacity Factor.}

Captured Energy: The net energy available for consumption after transformation of a noncombustible renewable resource into electricity and noncombustible renewable energy that is directly used. For example, it is the energy measured at the "output" of a conversion device, such as electricity from a wind turbine or solar plant.

Captive Coal: Coal produced to satisfy the needs of the mine owner, or of a parent, subsidiary, or other affiliate of the mine owner (for example, steel companies and electricity generators), rather than for open market sale. See Open Market Coal.

Carbon Dioxide: A colorless, odorless, non-poisonous gas $\left(\mathrm{CO}_{2}\right)$ that is a normal part of Earth's atmosphere. Carbon dioxide is a product of fossil-fuel combustion as well as other processes. It is considered a greenhouse gas as it traps heat (infrared energy) radiated by the Earth into the atmosphere and thereby contributes to the potential for global warming. The global warming potential (GWP) of other greenhouse gases is measured in relation to that of carbon dioxide, which by international scientific convention is assigned a value of one (1).

Carbon Dioxide Equivalent: The amount of carbon dioxide by weight emitted into the atmosphere that would produce the same estimated radiative forcing as a given weight of another radiatively active gas. Carbon dioxide equivalents are computed by multiplying the weight of the gas being measured (for example, methane) by its estimated global warming potential (which is 21 for methane). "Carbon equivalent units" are defined as carbon dioxide equivalents multiplied by the carbon content of carbon dioxide (i.e., 12/44).

Chained Dollars: A measure used to express real prices. Real prices are those that have been adjusted to remove the effect of changes in the purchasing power of the dollar; they usually reflect buying power relative to a reference year. Prior to 1996, real prices were expressed in constant dollars, a measure based on the weights of goods and services in a single year, usually a recent year. In 1996, the
U.S. Department of Commerce introduced the chained-dollar measure. The new measure is based on the average weights of goods and services in successive pairs of years. It is "chained" because the second year in each pair, with its weights, becomes the first year of the next pair. The advantage of using the chained-dollar measure is that it is more closely related to any given period covered and is therefore subject to less distortion over time.

Chlorofluorocarbon (CFC): Any of various compounds consisting of carbon, hydrogen, chlorine, and flourine used as refrigerants. CFCs are now thought to be harmful to the Earth's atmosphere.

Citygate: A point or measuring station at which a distribution gas utility receives gas from a natural gas pipeline company or transmission system.

Climate Change: A term used to refer to all forms of climatic inconsistency, but especially to significant change from one prevailing climatic condition to another. In some cases, "climate change" has been used synonymously with the term "global warming"; scientists, however, tend to use the term in a wider sense to include natural changes in climate as well as climatic cooling.

Coal: A readily combustible black or brownish-black rock whose composition, including inherent moisture, consists of more than 50 percent by weight and more than 70 percent by volume of carbonaceous material. It is formed from plant remains that have been compacted, hardened, chemically altered, and metamorphosed by heat and pressure over geologic time. See Coal Rank.

Coalbed methane: Methane is generated during coal formation and is contained in the coal microstructure. Typical recovery entails pumping water out of the coal to allow the gas to escape. Methane is the principal component of natural gas. Coalbed methane can be added to natural gas pipelines without any special treatment.

\section{Coal Coke: See Coke, Coal.}

Coal Rank: The classification of coals according to their degree of progressive alteration from lignite to anthracite. In the United States, the standard ranks of coal include lignite, subbituminous coal, bituminous coal, and anthracite and are based on fixed carbon, volatile matter, heating value, and agglomerating (or caking) properties.

Coal Stocks: Coal quantities that are held in storage for future use and disposition. Note: When coal data are collected for a particular reporting period (month, quarter, or year), coal stocks are commonly measured as of the last day of this period. 
Coal Synfuel: Coal-based solid fuel that has been processed by a coal synfuel plant; and coal-based fuels such as briquettes, pellets, or extrusions, which are formed from fresh or recycled coal and binding materials.

Coal Synfuel Plant: A plant engaged in the chemical transformation of coal into coal synfuel.

Coke, Coal: A solid carbonaceous residue derived from low-ash, low-sulfur bituminous coal from which the volatile constituents are driven off by baking in an oven at temperatures as high as 2,000 degrees Fahrenheit so that the fixed carbon and residual ash are fused together. Coke is used as a fuel and as a reducing agent in smelting iron ore in a blast furnace. Coke from coal is gray, hard, and porous and has a heating value of 24.8 million Btu per short ton.

Coke, Petroleum: A residue high in carbon content and low in hydrogen that is the final product of thermal decomposition in the condensation process in cracking. This product is reported as marketable coke or catalyst coke. The conversion is 5 barrels (of 42 U.S. gallons each) per short ton. Coke from petroleum has a heating value of 6.024 million Btu per barrel.

Combined-Heat-and-Power (CHP) Plant: A plant designed to produce both heat and electricity from a single heat source. Note: This term is being used in place of the term "cogenerator" that was used by EIA in the past. CHP better describes the facilities because some of the plants included do not produce heat and power in a sequential fashion and, as a result, do not meet the legal definition of cogeneration specified in the Public Utility Regulatory Polices Act (PURPA). See Electricity-Only Plant.

Commercial Building: A building with more than 50 percent of its floorspace used for commercial activities. Commercial buildings include, but are not limited to, stores, offices, schools, churches, gymnasiums, libraries, museums, hospitals, clinics, warehouses, and jails. Government buildings are included, except buildings on military bases or reservations.

Commercial Sector: An energy-consuming sector that consists of service-providing facilities and equipment of: businesses; Federal, State, and local governments; and other private and public organizations, such as religious, social, or fraternal groups. The commercial sector includes institutional living quarters. It also includes sewage treatment facilities. Common uses of energy associated with this sector include space heating, water heating, air conditioning,lighting, refrigeration, cooking, and running a wide variety of other equipment. Note: This sector includes generators that produce electricity and/or useful thermal output primarily to support the activities of the above-mentioned commercial establishments. Various EIA programs differ in sectoral coverage-for more information see
http://www.eia.gov/neic/datadefinitions/Guideforwebcom.htm. Sectors and Energy-Use Sectors.

Completion (Crude Oil/Natural Gas Production): The term refers to the installation of permanent equipment for the production of crude oil or natural gas. If a well is equipped to produce only crude oil or natural gas from one zone or reservoir, the definition of a "well" (classified as a crude oil well or natural gas well) and the definition of a "completion" are identical. However, if a well is equipped to produce crude oil and/or natural gas separately from more than one reservoir, a "well” is not synonymous with a "completion."

Compressed Natural Gas (CNG): Natural gas compressed to a pressure at or above 200-248 bar (i.e., 2900-3600 pounds per square inch) and stored in highpressure containers. It is used as a fuel for natural gas-powered vehicles.

\section{Conventional Hydroelectric Power: See Hydroelectric Power, Conventional.}

\section{Conventional Motor Gasoline: See Motor Gasoline, Conventional.}

Conversion Factor: A factor for converting data between one unit of measurement and another (such as between short tons and British thermal units, or between barrels and gallons). (See http://www.eia.gov/emeu/mer/append_a.html and http://www.eia.gov/emeu/mer/append_b.html for further information on conversion factors.) See Btu Conversion Factor and Thermal Conversion Factor.

Cooling Tower: A common type of environmental equipment installed at electric power plants used to transfer heat, produced by burning fuel, to the atmosphere Cooling towers are installed where there is insufficient cooling water available or where waste heat discharged into cooling water would affect marine life.

Criteria Pollutant: A pollutant determined to be hazardous to human health and regulated under the Environmental Protection Agency's (EPA) National Ambient Air Quality Standards. The 1970 amendments to the Clean Air Act require EPA to describe the health and welfare impacts of a pollutant as the "criteria" for inclusion in the regulatory regime.

Crude Oil: A mixture of hydrocarbons that exists in liquid phase in natural underground reservoirs and remains liquid at atmospheric pressure after passing through surface separating facilities. Depending upon the characteristics of the crude stream, it may also include: 1) small amounts of hydrocarbons that exist in gaseous phase in natural underground reservoirs but are liquid at atmospheric pressure after being recovered from oil well (casinghead) gas in lease separators and are subsequently commingled with the crude stream without being separately measured. Lease condensate recovered as a liquid from 
natural gas wells in lease or field separation facilities and later mixed into the crude stream is also included; 2) small amounts of nonhydrocarbons produced with the oil, such as sulfur and various metals; and 3) drip gases, and liquid hydrocarbons produced from tar sands, oil sands, gilsonite, and oil shale. Liquids produced at natural gas processing plants are excluded. Crude oil is refined to produce a wide array of petroleum products, including heating oils; gasoline, diesel and jet fuels; lubricants; asphalt; ethane, propane, and butane; and many other products used for their energy or chemical content.

Crude Oil Domestic First Purchase Price: The price for domestic crude oil reported by the company that owns the crude oil the first time it is removed from the lease boundary.

Crude Oil Landed Cost: The price of crude oil at the port of discharge, including charges associated with purchasing, transporting, and insuring a cargo from the purchase point to the port of discharge. The cost does not include charges incurred at the discharge port (e.g., import tariffs or fees, wharfage charges, and demurrage).

Crude Oil Refiner Acquisition Cost: The cost of crude oil to the refiner, including transportation and other fees. The composite cost is the weighted average of domestic and imported crude oil costs. The refiner acquisition cost does not include the cost of crude oil purchased for the Strategic Petroleum Reserve.

Crude Oil Refinery Input: The total crude oil put into processing units at refineries.

Crude Oil Stocks: Stocks of crude oil and lease condensate held at refineries, in petroleum pipelines, at pipeline terminals, and on leases.

Crude Oil Used Directly: Crude oil consumed as fuel by petroleum pipelines and on crude oil leases.

Crude Oil Well: A well completed for the production of crude oil from one or more crude oil zones or reservoirs. Wells producing both crude oil and natural gas are classified as crude oil wells.

Cubic Foot (Natural Gas) The amount of natural gas contained at standard temperature and pressure (60 degrees Fahrenheit and 14.73 pounds standard per square inch) in a cube whose edges are one foot long.

Degree-Day Normal: Simple arithmetic averages of monthly or annual degree-days over a long period of time (usually the 30-year period 1971-2000). The averages may be simple degree-day normals or population-weighted degree-day normals.
Degree-Days, Cooling (CDD): A measure of how warm a location is over a period of time relative to a base temperature, most commonly specified as 65 degrees Fahrenheit. The measure is computed for each day by subtracting the base temperature (65 degrees) from the average of the day's high and low temperatures, with negative values set equal to zero. Each day's cooling degreedays are summed to create a cooling degree-day measure for a specified reference period. Cooling degree-days are used in energy analysis as an indicator of air conditioning energy requirements or use.

Degree-Days, Heating (HDD): A measure of how cold a location is over a period of time relative to a base temperature, most commonly specified as 65 degrees Fahrenheit. The measure is computed for each day by subtracting the average of the day's high and low temperatures from the base temperature (65 degrees), with negative values set equal to zero. Each day's heating degree-days are summed to create a heating degree-day measure for a specified reference period. Heating degree-days are used in energy analysis as an indicator of space heating energy requirements or use.

Degree-Days, Population-Weighted: Heating or cooling degree-days weighted by the population of the area in which the degree-days are recorded. To compute State population-weighted degree-days, each State is divided into from one to nine climatically homogeneous divisions, which are assigned weights based on the ratio of the population of the division to the total population of the State. Degree-day readings for each division are multiplied by the corresponding population weight for each division and those products are then summed to arrive at the State population-weighted degree-day figure. To compute national population-weighted degree-days, the Nation is divided into nine Census regions, each comprising from three to eight States, which are assigned weights based on the ratio of the population of the region to the total population of the Nation. Degree-day readings for each region are multiplied by the corresponding population weight for each region and those products are then summed to arrive at the national population-weighted degree-day figure.

Demand-Side Management: The planning, implementation, and monitoring of electric utility activities designed to encourage consumers to modify patterns of electricity usage, including the timing and level of electricity demand.

Demonstrated Reserve Base (Coal): A collective term for the sum of coal in both measured and indicated resource categories of reliability, representing 100 percent of the in-place coal in those categories as of a certain date. Includes beds of bituminous coal and anthracite 28 or more inches thick and beds of subbituminous coal 60 or more inches thick that can occur at depths of as much as 1,000 feet. Includes beds of lignite 60 or more inches thick that can be surface mined. Includes also thinner and/or deeper beds that currently are being mined or for 
which there is evidence that they could be mined commercially at a given time. Represents that portion of the identified coal resource from which reserves are calculated.

Denaturant: Petroleum, typically pentanes plus or conventional motor gasoline, added to fuel ethanol to make it unfit for human consumption. Fuel ethanol is denatured, usually prior to transport from the ethanol production facility, by adding 2 to 5 volume percent denaturant. See Fuel Ethanol and Fuel Ethanol Minus Denaturant.

Development Well: A well drilled within the proved area of a crude oil or natural gas reservoir to the depth of a stratigraphic horizon known to be productive.

Diesel Fuel: A fuel composed of distillate fuel oils obtained in petroleum refining operation or blends of such distillate fuel oils with residual fuel oil used in motor vehicles. The boiling point and specific gravity are higher for diesel fuels than for gasoline.

Direct Use: Use of electricity that 1) is self-generated, 2) is produced by either the same entity that consumes the power or an affiliate, and 3) is used in direct support of a service or industrial process located within the same facility or group of facilities that house the generating equipment. Direct use is exclusive of station use.

Distillate Fuel Oil: A general classification for one of the petroleum fractions produced in conventional distillation operations. It includes diesel fuels and fuel oils. Products known as No. 1, No. 2, and No. 4 diesel fuel are used in on-highway diesel engines, such as those found in cars and trucks, as well as off-highway engines, such as those in railroad locomotives and agricultural machinery. Products known as No. 1, No. 2, and No. 4 fuel oils are used primarily for space heating and electricity generation.

Distillation Unit (Atmospheric): The primary distillation unit that processes crude oil (including mixtures of other hydrocarbons) at approximately atmospheric conditions. It includes a pipe still for vaporizing the crude oil and a fractionation tower for separating the vaporized hydrocarbon components in the crude oil into fractions with different boiling ranges. This is done by continuously vaporizing and condensing the components to separate higher boiling point material. The selected boiling ranges are set by the processing scheme, the properties of the crude oil, and the product specifications.

District Heat: Steam or hot water from an outside source used as an energy source in a building. The steam or hot water is produced in a central plant and is piped into the building. District heat may be purchased from a utility or provided by a physical plant in a separate building that is part of the same facility (for example, a hospital complex or university).
Dry Hole: An exploratory well or development well found to be incapable of producing either crude oil or natural gas in sufficient quantities to justify completion as a crude oil well or natural gas well.

Dry Natural Gas: See Natural Gas, Dry.

\section{Dry Natural Gas Production: See Natural Gas (Dry) Production.}

E85: A fuel containing a mixture of 85 percent ethanol and 15 percent motor gasoline.

Electric Energy: The ability of an electric current to produce work, heat, light, or other forms of energy. It is measured in kilowatthours

Electric Non-Utility: Any entity that generates, transmits, or sells electricity, or sells or trades electricity services and products, where costs are not established and recovered by regulatory authority. Examples of these entities include, but are not limited to, independent power producers, power marketers and aggregators (both wholesale and retail), merchant transmission service providers, selfgeneration entities, and cogeneration firms with Qualifying Facility Status. See Electric Utility.

Electric Power Grid: A system of synchronized power providers and consumers connected by transmission and distribution lines and operated by one or more control centers. In the continental United States, the electric power grid consists of three systems: the Eastern Interconnect, the Western Interconnect, and the Texas Interconnect. In Alaska and Hawaii, several systems encompass areas smaller than the State (e.g., the interconnect serving Anchorage, Fairbanks, and the Kenai Peninsula; and individual islands)

Electric Power Plant: A station containing prime movers, electric generators, and auxiliary equipment for converting mechanical, chemical, and/or fission energy into electric energy.

Electric Power Sector: An energy-consuming sector that consists of electricityonly and combined-heat-and-power (CHP) plants within the NAICS (North American Industry Classification System) 22 category whose primary business is to sell electricity, or electricity and heat, to the public. Note: This sector includes electric utilities and independent power producers. See Energy-Use Sectors

Electric Utility: Any entity that generates, transmits, or distributes electricity and recovers the cost of its generation, transmission or distribution assets and 
operations, either directly or indirectly, through cost-based rates set by a separate regulatory authority (e.g., State Public Service Commission), or is owned by a governmental unit or the consumers that the entity serves. Examples of these entities include: investor-owned entities, public power districts, public utility districts, municipalities, rural electric cooperatives, and State and Federal agencies. Electric utilities may have Federal Energy Regulatory Commission approval for interconnection agreements and wholesale trade tariffs covering either costof-service and/or market-based rates under the authority of the Federal Power Act. See Electric Non-Utility.

Electrical System Energy Losses: The amount of energy lost during generation, transmission, and distribution of electricity, including plant and unaccounted-for uses.

Electricity: A form of energy characterized by the presence and motion of elementary charged particles generated by friction, induction, or chemical change.

Electricity Generation: The process of producing electric energy, or the amount of electric energy produced by transforming other forms of energy; commonly expressed in kilowatthours (kWh) or megawatthours (MWh). See Electricity Generation, Gross and Electricity Generation, Net.

Electricity Generation, Gross: The total amount of electric energy produced by generating units and measured at the generating terminal.

Electricity Generation, Net: The amount of gross electricity generation less station use (the electric energy consumed at the generating station(s) for station service or auxiliaries). Note: Electricity required for pumping at hydroelectric pumped-storage plants is regarded as electricity for station service and is deducted from gross generation.

Electricity Retail Sales: The amount of electricity sold by electric utilities and other energy service providers to customers purchasing electricity for their own use and not for resale.

Emissions: Anthropogenic releases of gases to the atmosphere. In the context of global climate change, they consist of radiatively important greenhouse gases (e.g., the release of carbon dioxide during fuel combustion).

End-Use Sectors: The residential, commercial, industrial, and transportation sectors of the economy. See Energy-Use Sectors.

Energy: The capacity for doing work as measured by the capability of doing work (potential energy) or the conversion of this capability to motion (kinetic energy). Energy has several forms, some of which are easily convertible and can be changed to another form useful for work. Most of the world's convertible energy comes from fossil fuels that are burned to produce heat that is then used as a transfer medium to mechanical or other means in order to accomplish tasks. Electric energy is usually measured in kilowatthours, while heat energy is usually measured in British thermal units.

Energy Consumption: The use of energy as a source of heat or power or as an input in the manufacturing process.

Energy Expenditures: The money spent directly by consumers to purchase energy. Expenditures equal the amount of energy used by the consumer times the price per unit paid by the consumer.

Energy Service Provider: An energy entity that provides service to a retail or enduse customer.

Energy Source: Any substance or natural phenomenon that can be consumed or transformed to supply heat or power. Examples include petroleum, coal, natural gas, nuclear, wood, waste, electricity, wind, geothermal, sunlight (solar energy), water movement, and hydrogen in fuel cells.

Energy-Use Sectors: A group of major energy-consuming components of U.S. society developed to measure and analyze energy use. The sectors most commonly referred to in EIA are: residential, commercial, industrial, transportation, and electric power.

Ethane: A normally gaseous straight-chain hydrocarbon $\left(\mathrm{C}_{2} \mathrm{H}_{6}\right)$. It is a colorless, paraffinic gas that boils at a temperature of -127.48 degrees Fahrenheit. It is extracted from natural gas and refinery gas streams.

Ether: The family name applied to a group of organic chemical compounds composed of carbon, hydrogen, and oxygen, and which are characterized by an oxygen atom attached to two carbon atoms (for example, methyl tertiary butyl ether).

Ethanol $\left(\mathbf{C}_{2} \mathbf{H}_{5} \mathbf{O H}\right)$ : A clear, colorless, flammable alcohol. Ethanol is typically produced biologically from biomass feedstocks such as agricultural crops and cellulosic residues from agricultural crops or wood. Ethanol can also be produced chemically from ethylene. See Biomass, Fuel Ethanol, and Fuel Ethanol Minus Denaturant.

Ethyl Tertiary Butyl Ether (ETBE): A colorless, flammable, oxygenated hydrocarbon blend stock, $\left(\mathbf{C H}_{3}\right)_{3} \mathbf{C O C}_{2} \mathbf{H}_{5}$, formed by the catalytic etherification of isobutylene with ethanol. See Oxygenates. 
Ethylene: An olefinic hydrocarbon recovered from refinery processes or petrochemical processes. Ethylene is used as a petrochemical feedstock for numerous chemical applications and the production of consumer goods.

Eurasia: The physical land mass containing the continents of Europe and Asia. For U.S. Energy Information Administration reporting, it includes the former parts of the

Union of Soviet Socialist Republics (U.S.S.R.): Armenia, Azerbaijan, Belarus, Estonia, Georgia, Kazakhstan, Kyrgyzstan, Latvia, Lithuania, Moldova, Russia, Tajikistan, Turkmenistan, Ukraine, and Uzbekistan.

Exploratory Well: A well drilled to find and produce crude oil or natural gas in an area previously considered unproductive, to find a new reservoir in a known field (i.e., one previously producing crude oil or natural gas in another reservoir), or to extend the limit of a known crude oil or natural gas reservoir.

Exports: Shipments of goods from within the 50 States and the District of Columbia to U.S. possessions and territories or to foreign countries.

Extraction Loss: The reduction in volume of natural gas due to the removal of natural gas liquid constituents such as ethane, propane, and butane at natural gas processing plants.

Federal Energy Administration (FEA): A predecessor of the U.S. Energy Information Administration.

Federal Energy Regulatory Commission (FERC): The Federal agency with jurisdiction over interstate electricity sales, wholesale electric rates, hydroelectric licensing, natural gas pricing, petroleum pipeline rates, and natural gas pipeline certification. FERC is an independent regulatory agency within the U.S. Department of Energy and is the successor to the Federal Power Commission.

Federal Power Commission (FPC): The predecessor agency of the Federal Energy Regulatory Commission. The Federal Power Commission was created by an Act of Congress under the Federal Water Power Act on June 10, 1920. It was charged originally with regulating the electric power and natural gas industries. It was abolished on September 30, 1977, when the U.S. Department of Energy was created. Its functions were divided between the U.S. Department of Energy and the Federal Energy Regulatory Commission, an independent regulatory agency.

Financial Reporting System (FRS): The U.S. Energy Information Administration's statutory requirement to identify major energy-producing companies and develop and implement a data-reporting program for energy financial and operating information from these companies. Companies are selected if they are within the top 50 publiclyowned U.S. crude oil producers that have at least 1 percent of either production or reserves of crude oil, natural gas, coal, or uranium in the United States, or 1 percent of either refining capacity or petroleum product sales in the United States.

\section{Finished Motor Gasoline: See Motor Gasoline, Finished.}

\section{First Purchase Price: See Crude Oil Domestic First Purchase Price.}

First Use: Manufacturing establishments' consumption of the energy that was originally produced offsite or was produced onsite from input materials not classified as energy.

Fiscal Year: The U.S. Government's fiscal year runs from October 1 through September 30. The fiscal year is designated by the calendar year in which it ends; e.g., fiscal year 2002 began on October 1, 2001, and ended on September 30, 2002

\section{Flared Natural Gas: See Natural Gas, Flared.}

Flue Gas Desulfurization: Equipment used to remove sulfur oxides from the combustion gases of a boiler plant before discharge to the atmosphere. Also referred to as scrubbers. Chemicals such as lime are used as scrubbing media.

\section{F.O.B.: See Free on Board.}

Footage Drilled: Total footage for wells in various categories, as reported for any specified period, includes (1) the deepest total depth (length of well bores) of all wells drilled from the surface, (2) the total of all bypassed footage drilled in connection with reported wells, and (3) all new footage drilled for directional sidetrack wells. Footage reported for directional sidetrack wells does not include footage in the common bore, which is reported as footage for the original well. In the case of old wells drilled deeper, the reported footage is that which was drilled below the total depth of the old well.

\section{Former U.S.S.R.: See Union of Soviet Socialist Republics (U.S.S.R.).}

Forward Costs (Uranium): The operating and capital costs that will be incurred in any future production of uranium from in-place reserves. Included are costs for labor, materials, power and fuel, royalties, payroll taxes, insurance, and general and administrative costs that are dependent upon the quantity of production and, thus, applicable as variable costs of production. Excluded from forward costs are prior expenditures, if any, incurred for property acquisition, exploration, mine development, and mill construction, as well as income taxes, profit, and the cost of money. 
Note: By use of forward costing, estimates of reserves for uranium ore deposits in differing geological settings can be aggregated and reported as the maximum amount that can theoretically be extracted to recover the specified costs of uranium oxide production under the listed forward cost categories.

Fossil Fuel: An energy source formed in the Earth's crust from decayed organic material, such as petroleum, coal, and natural gas.

Fossil-Fueled Steam-Electric Power Plant: An electric power plant in which the prime mover is a turbine rotated by high-pressure steam produced in a boiler by heat from burning fossil fuels.

Fractionation: The process by which saturated hydrocarbons are removed from natural gas and separated into distinct parts, or "fractions" such as propane, butane, and ethane.

Free Alongside Ship (F.A.S.): The value of a commodity at the port of exportation, generally including the purchase price plus all charges incurred in placing the commodity alongside the carrier at the port of exportation.

Free on Board (F.O.B.): A sales transaction in which the seller makes the product available for pick up at a specified port or terminal at a specified price and the buyer pays for the subsequent transportation and insurance.

Free on Board (F.O.B.) Rail/Barge Price: The free on board price of coal at the point of first sale. It excludes freight or shipping and insurance costs.

Fuel Ethanol: Ethanol intended for fuel use. Fuel ethanol in the United States must be anhydrous (less than 1 percent water). Fuel ethanol is denatured (made unfit for human consumption), usually prior to transport from the ethanol production facility, by adding 2 to 5 volume percent petroleum, typically pentanes plus or conventional motor gasoline. Fuel ethanol is used principally for blending in low concentrations with motor gasoline as an oxygenate or octane enhancer. In high concentrations, it is used to fuel alternative-fuel vehicles specially designed for its use. See Alternative-Fuel Vehicle, Denaturant, E85, Ethanol, Fuel Ethanol Minus Denaturant, and Oxygenates.

Fuel Ethanol Minus Denaturant: An unobserved quantity of anhydrous, biomass-derived, undenatured ethanol for fuel use. The quantity is obtained by subtracting the estimated denaturant volume from fuel ethanol volume. Fuel ethanol minus denaturant is counted as renewable energy, while denaturant is counted as nonrenewable fuel. See Denaturant, Ethanol, Fuel Ethanol, Nonrenewable Fuels, Oxygenates, and Renewable Energy.

Full-Power Operation: Operation of a nuclear generating unit at 100 percent of its design capacity. Full-power operation precedes commercial operation.

Gasohol: A blend of finished motor gasoline containing alcohol (generally ethanol but sometimes methanol) at a concentration between 5.7 percent and 10 percent by volume. See Oxygenates.

Generating Unit: Any combination of physically connected generators, reactors, boilers, combustion turbines, or other prime movers operated together to produce electric power.

Generator: A machine that converts mechanical energy into electric energy.

Generator Capacity: The maximum output, commonly expressed in megawatts (MW), that generating equipment can supply to system load, adjusted for ambient conditions. See Generator Nameplate (Installed) Capacity and Generator Net Summer Capacity.

Generator Capacity Factor: The ratio of the electric energy produced by a generating unit for a given period of time to the electric energy that could have been produced at continuous full-power operation during the same period.

Generator Nameplate (Installed) Capacity: The maximum rated output of a generator, prime mover, or other electric power production equipment under specific conditions designated by the manufacturer. Installed generator nameplate capacity is commonly expressed in megawatts (MW) and is usually indicated on a nameplate physically attached to the generator.

Generator Net Summer Capacity: The maximum output, commonly expressed in megawatts (MW), that generating equipment can supply to system load, as demonstrated by a multi-hour test, at the time of summer peak demand (period of June 1 through September 30). This output reflects a reduction in capacity due to electricity use for station service or auxiliaries.

Geothermal Energy: Hot water or steam extracted from geothermal reservoirs in the Earth's crust and used for geothermal heat pumps, water heating, or electricity generation.

Global Warming: An increase in the near-surface temperature of the Earth. Global warming has occurred in the distant past as the result of natural influences, but the term is today most often used to refer to the warming some scientists 
predict will occur as a result of increased anthropogenic emissions of greenhouse gases. See Climate Change.

Global Warming Potential (GWP): An index used to compare the relative radiative forcing of different gases without directly calculating the changes in atmospheric concentrations. GWPs are calculated as the ratio of the radiative forcing that would result from the emission of one kilogram of a greenhouse gas to that from the emission of one kilogram of carbon dioxide over a period of time, such as 100 years.

Greenhouse Gases: Those gases, such as water vapor, carbon dioxide, nitrous oxide, methane, hydrofluorocarbons (HFCs), perfluorocarbons (PFCs), and sulfur hexafluoride, that are transparent to solar (short-wave) radiation but opaque to long-wave radiation, thus preventing long-wave radiant energy from leaving the Earth's atmosphere. The net effect is a trapping of absorbed radiation and a tendency to warm the planet's surface.

Gross Domestic Product (GDP): The total value of goods and services produced by labor and property located in the United States. As long as the labor and property are located in the United States, the supplier (that is, the workers and, for property, the owners) may be either U.S. residents or residents of foreign countries.

Gross Domestic Product (GDP) Implicit Price Deflator: A measure used to convert nominal prices to real prices. See Chained Dollars.

\section{Gross Electricity Generation: See Electricity Generation, Gross.}

Gross Withdrawals: See Natural Gas Gross Withdrawals.

Gross Input to Atmospheric Crude Oil Distillation Units: Total input to atmospheric crude oil distillation units. Includes all crude oil, lease condensate, natural gas plant liquids, unfinished oils, liquefied refinery gases, slop oils, and other liquid hydrocarbons produced from tar sands, gilsonite, and oil shale.

Heat Content: The amount of heat energy available to be released by the transformation or use of a specified physical unit of an energy form (e.g., a short ton of coal, a barrel of crude oil, a kilowatthour of electricity, a cubic foot of natural gas, or a pound of steam). The amount of heat energy is commonly expressed in British thermal units (Btu). Note: Heat content of combustible energy forms can be expressed in terms of either gross heat content (higher or upper heating value) or net heat content (lower heating value), depending upon whether or not the available heat energy includes or excludes the energy used to vaporize water (contained in the original energy form or created during the combustion process). The U.S. Energy Information Administration typically uses gross heat content values.

Heat Rate: A measure of generating station thermal efficiency commonly stated as Btu per kilowatthour. Note: Heat rates can be expressed as either gross or net heat rates, depending whether the electricity output is gross or net generation. Heat rates are typically expressed as net heat rates.

Household: A family, an individual, or a group of up to nine unrelated persons occupying the same housing unit. "Occupy" means the housing unit was the person's usual or permanent place of residence.

Housing Unit: A house, an apartment, a group of rooms, or a single room if it is either occupied or intended for occupancy as separate living quarters by a family, an individual, or a group of one to nine unrelated persons. Separate living quarters means the occupants (1) live and eat separately from other persons in the house or apartment and (2) have direct access from the outside of the buildings or through a common hall- that is, they can get to it without going through someone else's living quarters. Housing units do not include group quarters such as prisons or nursing homes where ten or more unrelated persons live. A common dining area used by residents is an indication of group quarters. Hotel and motel rooms are considered housing units if occupied as the usual or permanent place of residence.

Hydrocarbon: An organic chemical compound of hydrogen and carbon in the gaseous, liquid, or solid phase. The molecular structure of hydrocarbon compounds varies from the simplest (methane, a constituent of natural gas) to the very heavy and very complex.

Hydroelectric Power: The production of electricity from the kinetic energy of falling water. See Hydroelectric Power, Conventional and Hydroelectric Pumped Storage.

Hydroelectric Power, Conventional: Hydroelectric power generated from flowing water that is not created by hydroelectric pumped storage.

Hydroelectric Pumped Storage: Hydroelectric power that is generated during peak load periods by using water previously pumped into an elevated storage reservoir during off-peak periods when excess generating capacity is available to do so. When additional generating capacity is needed, the water can be released from the reservoir through a conduit to turbine generators located in an electric power plant at a lower level.

Hydrofluorocarbons (HFCs): A group of man-made chemicals composed of one 
or two carbon atoms and varying numbers of hydrogen and fluorine atoms. Most HFCs have 100-year global warming potentials in the thousands.

Hydrogen (H): The lightest of all gases, hydrogen occurs chiefly in combination with oxygen in water. It also exists in acids, bases, alcohols, petroleum, and other hydrocarbons.

Implicit Price Deflator: The implicit price deflator, published by the U.S. Department of Commerce, Bureau of Economic Analysis, is used to convert nominal prices to real prices.

Imports: Receipts of goods into the 50 States and the District of Columbia from U.S. possessions and territories or from foreign countries.

Independent Power Producer: A corporation, person, agency, authority, or other legal entity or instrumentality that owns or operates facilities for the generation of electricity for use primarily by the public, and that is not an electric utility. Independent power producers are included in the electric power sector.

Indicated Resources, Coal: Coal for which estimates of the coal rank, quality, and quantity are based partly on sample analyses and measurements and partly on reasonable geologic projections. Indicated resources are computed partly from specified measurements and partly from projection of visible data for a reasonable distance on the basis of geologic evidence. The points of observation are $1 / 2$ to $1 \frac{1}{2}$ miles apart. Indicated coal is projected to extend as a $1 / 2$-mile-wide belt that lies more than $1 / 4$ mile from the outcrop or points of observation or measurement.

Industrial Sector: An energy-consuming sector that consists of all facilities and equipment used for producing, processing, or assembling goods. The industrial sector encompasses the following types of activity: manufacturing (NAICS codes 31-33); agriculture, forestry, fishing and hunting (NAICS code 11); mining, including oil and gas extraction (NAICS code 21); and construction (NAICS code 23). Overall energy use in this sector is largely for process heat and cooling and powering machinery, with lesser amounts used for facility heating, air conditioning, and lighting. Fossil fuels are also used as raw material inputs to manufactured products. Note: This sector includes generators that produce electricity and/or useful thermal output primarily to support the above-mentioned industrial activities. Various EIA programs differ in sectoral coverage-for more information see http://www.eia.gov/neic/datadefinitions/Guideforwebind.htm. See End-Use Sectors and Energy-Use Sectors.

Isobutane: See Butane.
Isobutylene: An olefinic hydrocarbon recovered from refinery processes or petrochemical processes.

Isopentane: A saturated branched-chain hydrocarbon obtained by fractionation of natural gasoline or isomerization of normal pentane.

Jet Fuel: A refined petroleum product used in jet aircraft engines. See Jet Fuel, Kerosene-Type and Jet Fuel, Naphtha-Type.

Jet Fuel, Kerosene-Type: A kerosene-based product with a maximum distillation temperature of 400 degrees Fahrenheit at the 10-percent recovery point and a final maximum boiling point of 572 degrees Fahrenheit and meeting ASTM Specification 1655 and Military Specifications MIL-T-5624P and MIL-T-83133D (Grades JP-5 and JP-8). It is used for commercial and military turbojet and turboprop aircraft engines.

Jet Fuel, Naphtha-Type: A fuel in the heavy naphtha boiling range, with an average gravity of 52.8 degrees API, 20 to 90 percent distillation temperature of 290 to 470 degrees Fahrenheit, and meeting Military Specification MIL-T-5624L (Grade JP-4). It is used primarily for military turbojet and turboprop aircraft engines because it has a lower freeze point than other aviation fuels and meets engine requirements at high altitudes and speeds.

Kerosene: A light petroleum distillate that is used in space heaters, cook stoves, and water heaters and is suitable for use as a light source when burned in wick-fed lamps. Kerosene has a maximum distillation temperature of 400 degrees Fahrenheit at the 10-percent recovery point, a final boiling point of 572 degrees Fahrenheit, and a minimum flash point of 100 degrees Fahrenheit. Included are No. 1-K and No. 2-K, the two grades recognized by ASTM Specification D3699 as well as all other grades of kerosene called range or stove oil, which have properties similar to those of No. 1 fuel oil. See Jet Fuel, Kerosene-Type.

\section{Kerosene-Type Jet Fuel: See Jet Fuel, Kerosene-Type.}

Kilowatt: A unit of electrical power equal to 1,000 watts.

Kilowatthour (kWh): A measure of electricity defined as a unit of work or energy, measured as 1 kilowatt (1,000 watts) of power expended for 1 hour. One kilowatthour is equivalent to 3,412 Btu. See Watthour.

\section{Landed Cost: See Crude Oil Landed Cost.}

Lease and Plant Fuel: Natural gas used in well, field, and lease operations (such as natural gas used in drilling operations, heaters, dehydrators, and field 
compressors) and used as fuel in natural gas processing plants.

Lease Condensate: A mixture consisting primarily of pentanes and heavier hydrocarbons which is recovered as a liquid from natural gas in lease separation facilities. This category excludes natural gas plant liquids, such as butane and propane, which are recovered at downstream natural gas processing plants or facilities.

Lignite: The lowest rank of coal, often referred to as brown coal, used almost exclusively as fuel for steam-electric power generation. It is brownish-black and has a high inherent moisture content, sometimes as high as 45 percent. The heat content of lignite ranges from 9 to 17 million Btu per short ton on a moist, mineral-matterfree basis. The heat content of lignite consumed in the United States averages 13 million Btu per short ton, on the as-received basis (i.e., containing both inherent moisture and mineral matter). See Coal Rank.

Liquefied Natural Gas (LNG): Natural gas (primarily methane) that has been liquefied by reducing its temperature to -260 degrees Fahrenheit at atmospheric pressure.

Liquefied Petroleum Gases (LPG): A group of hydrocarbon-based gases derived from crude oil refining or natural gas fractionation. They include ethane, ethylene, propane, propylene, normal butane, butylene, isobutane, and isobutylene. For convenience of transportation, these gases are liquefied through pressurization.

Liquefied Refinery Gases (LRG): Liquefied petroleum gases fractionated from refinery or still gases. Through compression and/or refrigeration, they are retained in the liquid state. The reported categories are ethane/ethylene, propane/propylene, normal butane/butylene, and isobutane. Excludes still gas.

\section{Losses: See Electrical System Energy Losses.}

Low-Power Testing: The period of time between a nuclear generating unit's initial fuel loading date and the issuance of its operating (full-power) license. The maximum level of operation during that period is 5 percent of the unit's design thermal rating.

Lubricants: Substances used to reduce friction between bearing surfaces or incorporated into other materials used as processing aids in the manufacture of other products, or used as carriers of other materials. Petroleum lubricants may be produced either from distillates or residues. Lubricants include all grades of lubricating oils, from spindle oil to cylinder oil, and those used in greases.

Manufacturing: An energy-consuming subsector of the industrial sector that consists of all facilities and equipment engaged in the mechanical, physical, chemical, or electronic transformation of materials, substances, or components into new products. Assembly of component parts of products is included, except for that which is included in construction.

\section{Marketed Production (Natural Gas): See Natural Gas Marketed Production.}

Measured Resources, Coal: Coal resources for which estimates of the coal rank, quality, and quantity have been computed, within a margin of error of less than 20 percent, from sample analyses and measurements from closely spaced and geologically well known sample sites. Measured resources are computed from dimensions revealed in outcrops, trenches, mine workings, and drill holes. The points of observation and measurement are so closely spaced and the thickness and extent of coals are so well defined that the tonnage is judged to be accurate within 20 percent. Although the spacing of the point of observation necessary to demonstrate continuity of the coal differs from region to region, according to the character of the coalbeds, the points of observation are no greater than $1 / 2$ mile apart. Measured coal is projected to extend as a belt $1 / 4$ mile wide from the outcrop or points of observation or measurement.

Methane: A colorless, flammable, odorless hydrocarbon gas $\left(\mathrm{CH}_{4}\right)$, which is the major component of natural gas. It is also an important source of hydrogen in various industrial processes.

Methanol: A light, volatile alcohol $\left(\mathrm{CH}_{3} \mathrm{OH}\right)$ eligible for motor gasoline blending See Oxygenates.

Methyl Tertiary Butyl Ether (MTBE): An ether, $\left(\mathrm{CH}_{3}\right)_{3} \mathrm{COCH}_{3}$, intended for motor gasoline blending. See Oxygenates.

Miscellaneous Petroleum Products: All finished petroleum products not classified elsewhere-for example, petrolatum, lube refining byproducts (aromatic extracts and tars), absorption oils, ram-jet fuel, petroleum rocket fuels, synthetic natural gas feedstocks, and specialty oils.

Motor Gasoline Blending: Mechanical mixing of motor gasoline blending components and oxygenates as required, to produce finished motor gasoline. Finished motor gasoline may be further mixed with other motor gasoline blending components or oxygenates, resulting in increased volumes of finished motor gasoline and/or changes in the formulation of finished motor gasoline (e.g., conventional motor gasoline mixed with MTBE to produce oxygenated motor gasoline).

Motor Gasoline Blending Components: Naphthas (e.g., straight-run gasoline, alkylate, reformate, benzene, toluene, xylene) used for blending or compounding into finished motor gasoline. These components include reformulated gasoline blendstock 
for oxygenate blending (RBOB) but exclude oxygenates (alcohols, ethers), butane, and pentanes plus. Note: Oxygenates are reported as individual components and are included in the total for other hydrocarbons, hydrogen, and oxygenates.

Motor Gasoline, Conventional: Finished motor gasoline not included in the oxygenated or reformulated motor gasoline categories. Note: This category excludes reformulated gasoline blendstock for oxygenate blending (RBOB) as well as other blendstock. Conventional motor gasoline can be leaded or unleaded; regular, midgrade, or premium. See Motor Gasoline Grades.

Motor Gasoline, Finished: A complex mixture of relatively volatile hydrocarbons with or without small quantities of additives, blended to form a fuel suitable for use in spark-ignition. Motor gasoline, as defined in ASTM Specification D-4814 or Federal Specification VV-G-1690C, is characterized as having a boiling range of 122 to 158 degrees Fahrenheit at the 10-percent recovery point to 365 to 374 degrees Fahrenheit at the 90-percent recovery point. "Motor gasoline" includes conventional motor gasoline, all types of oxygenated motor gasoline including gasohol, and reformulated motor gasoline, but excludes aviation gasoline. Note: Volumetric data on motor gasoline blending components, as well as oxygenates, are not counted in data on finished motor gasoline until the blending components are blended into the gasoline.

Motor Gasoline Grades: The classification of gasoline by octane ratings. Each type of gasoline (conventional, oxygenated, and reformulated; leaded or unleaded) is classified by three grades: regular, midgrade, and premium. Note: Motor gasoline sales are reported by grade in accordance with their classification at the time of sale. In general, automotive octane requirements are lower at high altitudes. Therefore, in some areas of the United States, such as the Rocky Mountain States, the octane ratings for the gasoline grades may be 2 or more octane points lower.

Regular Gasoline: Gasoline having an antiknock index, i.e., octane rating, greater than or equal to 85 and less than 88 .

Midgrade Gasoline: Gasoline having an antiknock index, i.e., octane rating, greater than or equal to 88 and less than or equal to 90 .

Premium Gasoline: Gasoline having an antiknock index, i.e., octane rating, greater than 90 .

Motor Gasoline, Oxygenated: Finished motor gasoline other than reformulated motor gasoline, having an oxygen content of 2.7 percent or higher by weight and required by the U.S. Environmental Protection Agency (EPA) to be sold in areas designated by EPA as carbon monoxide (CO) nonattainment areas. Note: Oxygenated gasoline excludes oxygenated fuels program reformulated gasoline (OPRG) and reformulated gasoline blendstock for oxygenate blending (RBOB). Data on gasohol that has at least 2.7 percent oxygen, by weight, and is intended for sale inside $\mathrm{CO}$ nonattainment areas are included in data on oxygenated gasoline. Other data on gasohol (for use outside of nonattainment areas) are included in data on conventional gasoline.

Motor Gasoline, Reformulated: Finished motor gasoline formulated for use in motor vehicles, the composition and properties of which meet the requirements of the reformulated gasoline regulations promulgated by the U.S. Environmental Protection Agency under Section 211(k) of the Clean Air Act. It includes gasoline produced to meet or exceed emissions performance and benzene content standards of federal-program reformulated gasoline even though the gasoline may not meet all of the composition requirements (e.g. oxygen content) of federal-program reformulated gasoline. Note: This category includes oxygenated fuels program reformulated gasoline (OPRG). Reformulated gasoline excludes reformulated blendstock for oxygenate blending (RBOB) and gasoline treated as blendstock (GTAB).

\section{MTBE: See Methyl Tertiary Butyl Ether.}

\section{NAICS: See North American Industry Classification System.}

Naphtha: A generic term applied to a petroleum fraction with an approximate boiling range between 122 and 400 degrees Fahrenheit.

\section{Naphtha-Type Jet Fuel: See Jet Fuel, Naphtha-Type.}

Natural Gas: A gaseous mixture of hydrocarbon compounds, primarily methane, used as a fuel for electricity generation and in a variety of ways in buildings, and as raw material input and fuel for industrial processes.

Natural Gas, Dry: Natural gas which remains after: 1) the liquefiable hydrocarbon portion has been removed from the gas stream (i.e., gas after lease, field, and/or plant separation); and 2) any volumes of nonhydrocarbon gases have been removed where they occur in sufficient quantity to render the gas unmarketable. Note: Dry natural gas is also known as consumer-grade natural gas. The parameters for measurement are cubic feet at 60 degrees Fahrenheit and 14.73 pounds per square inch absolute.

Natural Gas (Dry) Production: The process of producing consumer-grade natural gas. Natural gas withdrawn from reservoirs is reduced by volumes used at the production (lease) site and by processing losses. Volumes used at the production site include 1) the volume returned to reservoirs in cycling, repressuring of oil 
reservoirs, and conservation operations; and 2) vented natural gas and flared natural gas. Processing losses include 1) nonhydrocarbon gases (e.g., water vapor, carbon dioxide, helium, hydrogen sulfide, and nitrogen) removed from the gas stream; and 2) gas converted to liquid form, such as lease condensate and natural gas plant liquids. Volumes of dry gas withdrawn from gas storage reservoirs are not considered part of production. Dry natural gas production equals natural gas marketed production less extraction loss.

Natural Gas, Flared: Natural gas burned in flares on the base site or at gas processing plants.

Natural Gas Gross Withdrawals: Full well stream volume of produced natural gas, excluding lease condensate separated at the lease.

Natural Gas Liquids (NGL): Those hydrocarbons in natural gas that are separated from the gas as liquids through the process of absorption, condensation, adsorption, or other methods in gas processing or cycling plants. Generally such liquids consist of propane and heavier hydrocarbons and are commonly referred to as lease condensate, natural gasoline, and liquefied petroleum gases. Natural gas liquids include natural gas plant liquids (primarily ethane, propane, butane, and isobutane) and lease condensate (primarily pentanes produced from natural gas at lease separators and field facilities).

Natural Gas Marketed Production: Natural gas gross withdrawals from production reservoirs, less gas used for reservoir repressuring; nonhydrocarbon gases removed in treating or processing operations; and quantities of vented natural gas and flared natural gas. Includes all quantities of natural gas used in field and processing operations.

Natural Gas Pipeline: A continuous pipe conduit, complete with such equipment as valves, compressor stations, communications systems, and meters, for transporting natural gas and/or supplemental gaseous fuels from one point to another, usually from a point in or beyond the producing field or processing plant to another pipeline or to points of utilization. Also refers to a company operating such facilities.

Natural Gas Plant Liquids (NGPL): Those hydrocarbons in natural gas that are separated as liquids at natural gas processing plants, fractionating and cycling plants, and, in some instances, field facilities. Lease condensate is excluded. Products obtained include ethane; liquefied petroleum gases (propane, butanes, propane-butane mixtures, ethane-propane mixtures); isopentane; and other small quantities of finished products, such as motor gasoline, special naphthas, jet fuel, kerosene, and distillate fuel oil. See Natural Gas Liquids.
Natural Gas Processing Plant: A surface installation designed to separate and recover natural gas liquids from a stream of produced natural gas through the processes of condensation, absorption, refrigeration, or other methods, and to control the quality of natural gas marketed or returned to oil or gas reservoirs for pressure maintenance, repressuring, or cycling.

Natural Gas, Vented: Natural gas released into the air on the production site or at processing plants.

Natural Gas Well: A well completed for the production of natural gas from one or more natural gas zones or reservoirs. (Wells producing both crude oil and natural gas are classified as crude oil wells.)

Natural Gas Wellhead Price: Price of natural gas calculated by dividing the total reported value at the wellhead by the total quantity produced as reported by the appropriate agencies of individual producing States and the U.S. Mineral Management Service. The price includes all costs prior to shipment from the lease, including gathering and compression costs, in addition to State production, severance, and similar charges.

Natural Gasoline: A mixture of hydrocarbons (mostly pentanes and heavier) extracted from natural gas that meets vapor pressure, end-point, and other specifications for natural gasoline set by the Gas Processors Association. Includes isopentane, which is a saturated branch-chain hydrocarbon obtained by fractionation of natural gasoline or isomerization of normal pentane.

\section{NERC: See North American Electric Reliability Corporation.}

Net Electricity Generation: See Electricity Generation, Net.

Net Summer Capacity: See Generator Net Summer Capacity.

Neutral Zone: A 6,200 square-mile area shared equally between Kuwait and Saudi Arabia under a 1992 agreement.

Nitrogen Oxides (NO $\mathbf{N}_{\mathbf{x}}$ ): Compounds of nitrogen and oxygen produced by the burning of fossil fuels.

Nominal Dollars: A measure used to express nominal price.

Nominal Price: The price paid for a product or service at the time of the transaction. Nominal prices are those that have not been adjusted to remove the 
effect of changes in the purchasing power of the dollar; they reflect buying power in the year in which the transaction occurred.

Non-Biomass Waste: Material of non-biological origin that is a byproduct or a discarded product. "Non-biomass waste" includes municipal solid waste from nonbiogenic sources, such as plastics, and tire-derived fuels.

Noncoincident Peak Load: The sum of two or more peak loads on individual systems that do not occur in the same time interval. Meaningful only in the context of loads within a limited period of time, such as day, week, month, a heating or cooling season, and usually for not more than 1 year.

Nonhydrocarbon Gases: Typical nonhydrocarbon gases that may be present in reservoir natural gas, such as carbon dioxide, helium, hydrogen sulfide, and nitrogen.

Nonrenewable Fuels: Fuels that cannot be easily made or "renewed," such as crude oil, natural gas, and coal.

\section{Normal Butane: See Butane.}

North American Electric Reliability Corporation (NERC): A nonprofit corporation formed in 2006 as the successor to the North American Electric Reliability Council established to develop and maintain mandatory reliability standards for the bulk electric system, with the fundamental goal of maintaining and improving the reliability of that system. NERC consists of regional reliability entities covering the interconnected power regions of the contiguous United States, Canada, and Mexico. See the NERC regions at http://www.eia.gov/cneaf/electricity/chg_str_fuel/html/fig02.html.

North American Industry Classification System (NAICS): A classification scheme, developed by the Office of Management and Budget to replace the Standard Industrial Classification (SIC) System, that categorizes establishments according to the types of production processes they primarily use.

Nuclear Electric Power (Nuclear Power): Electricity generated by the use of the thermal energy released from the fission of nuclear fuel in a reactor.

Nuclear Electric Power Plant: A single-unit or multi-unit facility in which heat produced in one or more reactors by the fissioning of nuclear fuel is used to drive one or more steam turbines.

Nuclear Reactor: An apparatus in which a nuclear fission chain reaction can be initiated, controlled, and sustained at a specific rate. A reactor includes fuel (fissionable material), moderating material to control the rate of fission, a heavywalled pressure vessel to house reactor components, shielding to protect personnel, a system to conduct heat away from the reactor, and instrumentation for monitoring and controlling the reactor's systems.

Octane Rating: A number used to indicate gasoline's antiknock performance in motor vehicle engines. The two recognized laboratory engine test methods for determining the antiknock rating, i.e., octane rating, of gasolines are the Research method and the Motor method. To provide a single number as guidance to the consumer, the antiknock index $(\mathrm{R}+\mathrm{M}) / 2$, which is the average of the Research and Motor octane numbers, was developed.

\section{OECD: See Organization for Economic Cooperation and Development.}

Offshore: That geographic area that lies seaward of the coastline. In general, the coastline is the line of ordinary low water along with that portion of the coast that is in direct contact with the open sea or the line marking the seaward limit of inland water. If a State agency uses a different basis for classifying onshore and offshore areas, the State classification is used (e.g., Cook Inlet in Alaska is classified as offshore; for Louisiana, the coastline is defined as the Chapman Line, as modified by subsequent adjudication).

\section{Oil: See Crude Oil.}

\section{OPEC: See Organization of the Petroleum Exporting Countries.}

Open Market Coal: Coal sold in the open market, i.e., coal sold to companies other than the reporting company's parent company or an operating subsidiary of the parent company. See Captive Coal.

Operable Nuclear Unit: In the United States, a nuclear generating unit that has completed low-power testing and is in possession of a full-power operating license issued by the Nuclear Regulatory Commission.

Operable Refineries: Refineries that were in one of the following three categories at the beginning of a given year: in operation; not in operation and not under active repair, but capable of being placed into operation within 30 days; or not in operation, but under active repair that could be completed within 90 days.

Operating Income: Operating revenues less operating expenses. Excludes items of other revenue and expense, such as equity in earnings of unconsolidated affiliates, dividends, interest income and expense, income taxes, extraordinary items, and cumulative effect of accounting changes. 
Organization for Economic Cooperation and Development (OECD): An international organization helping governments tackle the economic, social and governance challenges of a globalized economy. Its membership comprises about 30 member countries. With active relationships with some 70 other countries, nongovernmental organizations (NGOs) and civil society, it has a global reach. For details about the organization, see http://www.oecd.org.

Organization of the Petroleum Exporting Countries (OPEC): An intergovernmental organization whose stated objective is to "coordinate and unify the petroleum policies of member countries." It was created at the Baghdad Conference on September 10-14, 1960. Current members (with years of membership) include Algeria (1969-present), Angola (2007-present), Ecuador (1973-1992 and 2007-present), Iran (1960-present), Iraq (1960-present), Kuwait (1960-present), Libya (1962-present), Nigeria (1971-present), Qatar (1961-present), Saudi Arabia (1960-present), United Arab Emirates (1967-present), and Venezuela (1960-present). Countries no longer members of OPEC include Gabon (1975-1994) and Indonesia (1962-2008).

\section{Oxygenated Motor Gasoline: See Motor Gasoline, Oxygenated.}

Oxygenates: Substances which, when added to motor gasoline, increase the amount of oxygen in that gasoline blend. Ethanol, methyl tertiary butyl ether (MTBE), ethyl tertiary butyl ether (ETBE), and methanol are common oxygenates. See Motor Gasoline, Oxygenated.

Ozone: A molecule made up of three atoms of oxygen. Occurs naturally in the stratosphere and provides a protective layer shielding the Earth from harmful ultraviolet radiation. In the troposphere, it is a chemical oxidant, a greenhouse gas, and a major component of photochemical smog.

PAD Districts: Petroleum Administration for Defense Districts. Geographic aggregations of the 50 States and the District of Columbia into five districts for the Petroleum Administration for Defense in 1950. The districts were originally instituted for economic and geographic reasons as Petroleum Administration for War (PAW) Districts, which were established in 1942.

Particulate Collectors: Equipment used to remove fly ash from the combustion gases of a boiler plant before discharge to the atmosphere. Particulate collectors include electrostatic precipitators, mechanical collectors (cyclones, fabric filters [baghouses]), and wet scrubbers.

Peak Kilowatt: Thousand peak watts.
Peak Watt: A manufacturer's unit indicating the amount of power a photovoltaic cell or module will produce at standard test conditions (normally 1,000 watts per square meter and 25 degrees Celsius).

Pentanes Plus: A mixture of hydrocarbons, mostly pentanes and heavier, extracted from natural gas. Includes isopentane, natural gasoline, and plant condensate.

Perfluorocarbons (PFCs): A group of man-made chemicals composed of one or two carbon atoms and four to six flourine atoms, containing no chlorine. PFCs have no commercial uses and are emitted as a byproduct of aluminum smelting and semiconductor manufacturing. PFCs have very high 100-year global warming potentials and are very long-lived in the atmosphere.

Petrochemical Feedstocks: Chemical feedstocks derived from petroleum principally for the manufacture of chemicals, synthetic rubber, and a variety of plastics.

Petroleum: A broadly defined class of liquid hydrocarbon mixtures. Included are crude oil, lease condensate, unfinished oils, refined products obtained from the processing of crude oil, and natural gas plant liquids. Note: Volumes of finished petroleum products include nonhydrocarbon compounds, such as additives and detergents, after they have been blended into the products.

\section{Petroleum Coke: See Coke, Petroleum.}

\section{Petroleum Consumption: See Products Supplied (Petroleum).}

Petroleum Imports: Imports of petroleum into the 50 States and the District of Columbia from foreign countries and from Puerto Rico, the Virgin Islands, and other U.S. territories and possessions. Included are imports for the Strategic Petroleum Reserve and withdrawals from bonded warehouses for onshore consumption, offshore bunker use, and military use. Excluded are receipts of foreign petroleum into bonded warehouses and into U.S. territories and U.S. Foreign Trade Zones.

Petroleum Pipeline: Crude oil and product pipelines used to transport crude oil and petroleum products, respectively (including interstate, intrastate, and intracompany pipelines), within the 50 States and the District of Columbia.

Petroleum Products: Petroleum products are obtained from the processing of crude oil (including lease condensate), natural gas, and other hydrocarbon compounds. Petroleum products include unfinished oils, liquefied petroleum gases, pentanes plus, aviation gasoline, motor gasoline, naphtha-type jet fuel, kerosene-type jet fuel, kerosene, distillate fuel oil, residual fuel oil 
petrochemical feedstocks, special naphthas, lubricants, waxes, petroleum coke, asphalt, road oil, still gas, and miscellaneous petroleum products.

Petroleum Stocks, Primary: For individual petroleum products, quantities that are held at refineries, in petroleum pipelines, and at bulk terminals that have a capacity of 50,000 barrels or more, or that are in transit thereto. Stocks held by product retailers and resellers, as well as tertiary stocks held at the point of consumption, are excluded. Stocks of individual products held at gas processing plants are excluded from individual product estimates but are included in other oil estimates and total.

Photovoltaic Cell (PVC): An electronic device consisting of layers of semiconductor materials fabricated to form a junction (adjacent layers of materials with different electronic characteristics) and electrical contacts and being capable of converting incident light directly into electricity (direct current).

Photovoltaic Energy: Direct-current electricity generated from sunlight through solid-state semiconductor devices that have no moving parts.

Photovoltaic Module: An integrated assembly of interconnected photovoltaic cells designed to deliver a selected level of working voltage and current at its output terminals, packaged for protection against environmental degradation, and suited for incorporation in photovoltaic power systems.

Pipeline Fuel: Natural gas consumed in the operation of pipelines, primarily in compressors.

Plant Condensate: One of the natural gas liquids, mostly pentanes and heavier hydrocarbons, recovered and separated as liquids at gas inlet separators or scrubbers in processing plants.

Primary Energy: Energy in the form that it is first accounted for in a statistical energy balance, before any transformation to secondary or tertiary forms of energy. For example, coal can be converted to synthetic gas, which can be converted to electricity; in this example, coal is primary energy, synthetic gas is secondary energy, and electricity is tertiary energy. See Primary Energy Production and Primary Energy Consumption.

Primary Energy Consumption: Consumption of primary energy. (Energy sources that are produced from other energy sources, e.g., coal coke from coal, are included in primary energy consumption only if their energy content has not already been included as part of the original energy source. Thus, U.S. primary energy consumption does include net imports of coal coke, but not the coal coke produced from domestic coal.) The U.S. Energy Information Administration includes the following in U.S. primary energy consumption: coal consumption; coal coke net imports; petroleum consumption (petroleum products supplied, including natural gas plant liquids and crude oil burned as fuel); dry natural gas excluding supplemental gaseous fuels consumption; nuclear electricity net generation (converted to Btu using the nuclear plants heat rates); conventional hydroelectricity net generation (converted to Btu using the fossil-fuels plant heat rates); geothermal electricity net generation (converted to Btu using the fossil-fuels plant heat rates), and geothermal heat pump energy and geothermal direct use energy; solar thermal and photovoltaic electricity net generation (converted to Btu using the fossil-fuels plant heat rates), and solar thermal direct use energy; wind electricity net generation (converted to Btu using the fossil-fuels plant heat rates); wood and wood-derived fuels consumption; biomass waste consumption; fuel ethanol and biodiesel consumption; losses and co-products from the production of fuel ethanol and biodiesel; and electricity net imports (converted to Btu using the electricity heat content of 3,412 Btu per kilowatthour).

Primary Energy Production: Production of primary energy. The U.S. Energy Information Administration includes the following in U.S. primary energy production: coal production, waste coal supplied, and coal refuse recovery; crude oil and lease condensate production; natural gas plant liquids production; dry natural gas excluding supplemental gaseous fuels production; nuclear electricity net generation (converted to Btu using the nuclear plant heat rates); conventional hydroelectricity net generation (converted to Btu using the fossil-fuels plant heat rates); geothermal electricity net generation (converted to Btu using the fossil-fuels plant heat rates), and geothermal heat pump energy and geothermal direct use energy; solar thermal and photovoltaic electricity net generation (converted to Btu using the fossil-fuels plant heat rates), and solar thermal direct use energy; wind electricity net generation (converted to Btu using the fossil-fuels plant heat rates); wood and woodderived fuels consumption; biomass waste consumption; and biofuels feedstock.

Prime Mover: The engine, turbine, water wheel, or similar machine that drives an electric generator; or, for reporting purposes, a device that converts energy to electricity directly.

Process Fuel: All energy consumed in the acquisition, processing, and transportation of energy. Quantifiable process fuel includes three categories: natural gas lease and plant operations, natural gas pipeline operations, and oil refinery operations.

Processing Gain: The volumetric amount by which total output is greater than input for a given period of time. This difference is due to the processing of crude oil into petroleum products which, in total, have a lower specific gravity than the crude oil processed.

Processing Loss: The volumetric amount by which total refinery output is less than input for a given period of time. This difference is due to the processing of crude 
oil into petroleum products which, in total, have a higher specific gravity than the crude oil processed.

Products Supplied (Petroleum): Approximately represents consumption of petroleum products because it measures the disappearance of these products from primary sources, i.e., refineries, natural gas processing plants, blending plants, pipelines, and bulk terminals. In general, product supplied of each product in any given period is computed as follows: field production, plus refinery production, plus imports, plus unaccounted-for crude oil (plus net receipts when calculated on a PAD District basis) minus stock change, minus crude oil losses, minus refinery inputs, and minus exports.

Propane: A normally gaseous straight-chain hydrocarbon $\left(\mathrm{C}_{3} \mathrm{H}_{8}\right)$. It is a colorless paraffinic gas that boils at a temperature of -43.67 degrees Fahrenheit. It is extracted from natural gas or refinery gas streams. It includes all products designated in ASTM Specification D1835 and Gas Processors Association Specifications for commercial propane and HD-5 propane.

Propylene: An olefinic hydrocarbon $\left(\mathrm{C}_{3} \mathrm{H}_{6}\right)$ recovered from refinery processes or petrochemical processes.

Proved Reserves, Crude Oil: The estimated quantities of all liquids defined as crude oil that geological and engineering data demonstrate with reasonable certainty to be recoverable in future years from known reservoirs under existing economic and operating conditions.

Proved Reserves, Lease Condensate: The volumes of lease condensate expected to be recovered in future years in conjunction with the production of proved reserves of natural gas based on the recovery efficiency of lease and/or field separation facilities installed.

Proved Reserves, Natural Gas: The estimated quantities of natural gas that analysis of geological and engineering data demonstrates with reasonable certainty to be recoverable in future years from known reservoirs under existing economic and operating conditions.

Proved Reserves, Natural Gas Liquids: Those volumes of natural gas liquids (including lease condensate) demonstrated with reasonable certainty to be separable in the future from proved natural gas reserves, under existing economic and operating conditions.

Pumped Storage: See Hydroelectric Pumped Storage.
Real Price: A price that has been adjusted to remove the effect of changes in the purchasing power of the dollar. Real prices, which are expressed in constant dollars, usually reflect buying power relative to a base year. See Chained Dollars.

\section{Refiner Acquisition Cost of Crude Oil: See Crude Oil Refiner Acquisition Cost.}

Refinery Gas: See Still Gas.

Refinery and Blender Net Inputs: Raw materials, unfinished oils, and blending components processed at refineries, or blended at refineries or petroleum storage terminals to produce finished petroleum products. Included are gross inputs of crude oil, natural gas plant liquids, other hydrocarbon raw materials, hydrogen, oxygenates (excluding fuel ethanol), and renewable fuels (including fue ethanol). Also included are net inputs of unfinished oils, motor gasoline blending components, and aviation gasoline blending components. Net inputs are calculated as gross inputs minus gross production. Negative net inputs indicate gross inputs are less than gross production. Examples of negative net inputs include reformulated gasoline blendstock for oxygenate blending (RBOB) produced at refineries for shipment to blending terminals, and unfinished oils produced and added to inventory in advance of scheduled maintenance of a refinery crude oil distillation unit.

Refinery and Blender Net Production: Liquefied refinery gases, and finished petroleum products produced at a refinery or petroleum storage terminal blending facility. Net production equals gross production minus gross inputs. Negative net production indicates gross production is less than gross inputs for a finished petroleum product. Examples of negative net production include reclassification of one finished product to another finished product, or reclassification of a finished product to unfinished oils or blending components.

Refinery (Petroleum): An installation that manufactures finished petroleum products from crude oil, unfinished oils, natural gas liquids, other hydrocarbons, and alcohol.

\section{Reformulated Motor Gasoline: See Motor Gasoline, Reformulated.}

Refuse Mine: A surface mine where coal is recovered from previously mined coal. It may also be known as a silt bank, culm bank, refuse bank, slurry dam, or dredge operation.

Refuse Recovery: The recapture of coal from a refuse mine or the coal recaptured by that process. The resulting product has been cleaned to reduce the concentration of noncombustible materials. 
Renewable Energy: Energy obtained from sources that are essentially inexhaustible (unlike, for example, fossil fuels, which are in finite supply). Renewable sources of energy include conventional hydroelectric power, geothermal, solar, wind, and biomass.

Replacement Fuel: The portion of any motor fuel that is methanol, ethanol, or other alcohols, natural gas, liquefied petroleum gases, hydrogen, coal-derived liquid fuels, electricity (including electricity from solar energy), ethers, biodiesel, or any other fuel the Secretary of Energy determines, by rule, is substantially not petroleum and would yield substantial energy security benefits and substantial environmental benefits.

Repressuring: The injection of gas into crude oil or natural gas formations to effect greater ultimate recovery.

Residential Sector: An energy-consuming sector that consists of living quarters for private households. Common uses of energy associated with this sector include space heating, water heating, air conditioning, lighting, refrigeration, cooking, and running a variety of other appliances. The residential sector excludes institutional living quarters. Note: Various EIA programs differ in sectoral coverage—-for further explanation see http://www.eia.gov/neic/datadefinitions/Guideforwebres.htm. See End-Use Sectors and Energy-Use Sectors.

Residual Fuel Oil: The heavier oils, known as No. 5 and No. 6 fuel oils, that remain after the distillate fuel oils and lighter hydrocarbons are distilled away in refinery operations. It conforms to ASTM Specifications D396 and D975 and Federal Specification VV-F-815C. No. 5, a residual fuel oil of medium viscosity, is also known as Navy Special and is defined in Military Specification MIL-F-859E, including Amendment 2 (NATO Symbol F-770). It is used in steam-powered vessels in government service and inshore electric power plants. No. 6 fuel oil includes Bunker C fuel oil and is used for electricity generation, space heating, vessel bunkering, and various industrial purposes.

Road Oil: Any heavy petroleum oil, including residual asphaltic oil, used as a dust palliative and surface treatment on roads and highways. It is generally produced in six grades, from 0 , the most liquid, to 5 , the most viscous.

Rotary Rig: A machine used for drilling wells that employs a rotating tube attached to a bit for boring holes through rock.

Royalty Interest: An interest in a mineral property provided through a royalty contract.
Shale gas: Natural gas produced from organic (black) shale formations. See Natural gas.

Short Ton (Coal): A unit of weight equal to 2,000 pounds.

\section{Solar Energy: See Solar Thermal Energy and Photovoltaic Energy.}

Solar Thermal Collector: A device designed to receive solar radiation and convert it to thermal energy. Normally, a solar thermal collector includes a frame, glazing, and an absorber, together with appropriate insulation. The heat collected by the solar thermal collector may be used immediately or stored for later use. Solar collectors are used for space heating, domestic hot water heating, and heating swimming pools, hot tubs, or spas.

Solar Thermal Energy: The radiant energy of the sun that can be converted into other forms of energy, such as heat or electricity.

Space Heating: The use of energy to generate heat for warmth in housing units using space-heating equipment. The equipment could be the main space-heating equipment or secondary space-heating equipment. It does not include the use of energy to operate appliances (such as lights, televisions, and refrigerators) that give off heat as a byproduct.

Special Naphthas: All finished petroleum products within the naphtha boiling range that are used as paint thinners, cleaners, or solvents. Those products are refined to a specified flash point. Special naphthas include all commercial hexane and cleaning solvents conforming to ASTM Specifications D1836 and D484, respectively. Naphthas to be blended or marketed as motor gasoline or aviation gasoline or that are to be used as petrochemical feedstocks or synthetic natural gas (SNG) feedstocks are excluded.

Spent Liquor: The liquid residue left after an industrial process; can be a component of waste materials used as fuel.

\section{Spot Market Price: See Spot Price.}

Spot Price: The price for a one-time open market transaction for immediate delivery of the specific quantity of product at a specific location where the commodity is purchased "on the spot" at current market rates.

Station Use: Energy that is used to operate an electric power plant. It includes energy consumed for plant lighting, power, and auxiliary facilities, regardless of whether the energy is produced at the plant or comes from another source. 
Steam-Electric Power Plant: An electric power plant in which the prime mover is a steam turbine. The steam used to drive the turbine is produced in a boiler where fossil fuels are burned.

Still Gas (Refinery Gas): Any form or mixture of gases produced in refineries by distillation, cracking, reforming, and other processes. The principal constituents are methane, ethane, ethylene, normal butane, butylene, propane, propylene, etc. Still gas is used as a refinery fuel and a petrochemical feedstock. The conversion factor is 6 million Btu per fuel oil equivalent barrel.

Stocks: Inventories of fuel stored for future use. See Crude Oil Stocks, Coal Stocks, and Petroleum Stocks, Primary.

Strategic Petroleum Reserve (SPR): Petroleum stocks maintained by the Federal Government for use during periods of major supply interruption.

Subbituminous Coal: A coal whose properties range from those of lignite to those of bituminous coal and used primarily as fuel for steam-electric power generation. It may be dull, dark brown to black, soft and crumbly, at the lower end of the range, to bright, jet black, hard, and relatively strong, at the upper end. Subbituminous coal contains 20 to 30 percent inherent moisture by weight. The heat content of subbituminous coal ranges from 17 to 24 million Btu per short ton on a moist, mineral-matter-free basis. The heat content of subbituminous coal consumed in the United States averages 17 to 18 million Btu per short ton, on the as-received basis (i.e., containing both inherent moisture and mineral matter). See Coal Rank.

Sulfur Dioxide ( $\left.\mathrm{SO}_{2}\right)$ : A toxic, irritating, colorless gas soluble in water, alcohol, and ether. Used as a chemical intermediate, in paper pulping and ore refining, and as a solvent.

Sulfur Hexafluoride $\left(\mathbf{S F}_{6}\right)$ : A colorless gas soluble in alcohol and ether, and slightly less soluble in water. It is used as a dielectric in electronics. It possesses the highest 100 -year global warming potential of any gas $(23,900)$.

Supplemental Gaseous Fuels: Any gaseous substance introduced into or commingled with natural gas that increases the volume available for disposition. Such substances include, but are not limited to, propane-air, refinery gas, coke-oven gas, manufactured gas, biomass gas, or air or inerts added for Btu stabilization.

Synthetic Natural Gas (SNG): (Also referred to as substitute natural gas.) A manufactured product, chemically similar in most respects to natural gas, resulting from the conversion or reforming of hydrocarbons that may easily be substituted for or interchanged with pipeline-quality natural gas.

Thermal Conversion Factor: A factor for converting data between physical units of measure (such as barrels, cubic feet, or short tons) and thermal units of measure (such as British thermal units, calories, or joules); or for convertingdata between different thermal units of measure. See Btu Coversion Factor.

Total Energy Consumption: Primary energy consumption in the end-use sectors, plus electricity retail sales and electrical system energy losses.

Transportation Sector: An energy-consuming sector that consists of all vehicles whose primary purpose is transporting people and/or goods from one physical location to another. Included are automobiles; trucks; buses; motorcycles; trains, subways, and other rail vehicles; aircraft; and ships, barges, and other waterborne vehicles. Vehicles whose primary purpose is not transportation (e.g., construction cranes and bulldozers, farming vehicles, and warehouse tractors and forklifts) are classified in the sector of their primary use. Note: Various EIA programs differ in sectoral coverage-for more information see http://www.eia.gov/neic/datadefinitions/Guideforwebtrans.htm. See End-Use Sectors and Energy-Use Sectors.

Unaccounted-for Crude Oil: Represents the arithmetic difference between the calculated supply and the calculated disposition of crude oil. The calculated supply is the sum of crude oil production plus imports minus changes in crude oil stocks. The calculated disposition of crude oil is the sum of crude oil input to refineries, crude oil exports, crude oil burned as fuel, and crude oil losses.

Unaccounted-for Natural Gas: Represents differences between the sum of the components of natural gas supply and the sum of components of natural gas disposition. These differences may be due to quantities lost or to the effects of data reporting problems. Reporting problems include differences due to the net result of conversions of flow data metered at varying temperatures and pressure bases and converted to a standard temperature and pressure base; the effect of variations in company accounting and billing practices; differences between billing cycle and calendar-period time frames; and imbalances resulting from the merger of data reporting systems that vary in scope, format, definitions, and type of respondents.

Underground Natural Gas Storage: The use of sub-surface facilities for storing natural gas that has been transferred from its original location. The facilities are usually hollowed-out salt domes, geological reservoirs (depleted crude oil or natural gas fields) or water-bearing sands topped by an impermeable cap rock (aquifer). 
Undiscovered Recoverable Reserves (Crude Oil and Natural Gas): Those economic resources of crude oil and natural gas, yet undiscovered, that are estimated to exist in favorable geologic settings.

Unfinished Oils: All oils requiring further processing, except those requiring only mechanical blending. Unfinished oils are produced by partial refining of crude oil and include naphthas and lighter oils, kerosene and light gas oils, heavy gas oils, and residuum.

Unfractionated Stream: Mixtures of unsegregated natural gas liquid components, excluding those in plant condensate. This product is extracted from natural gas.

Union of Soviet Socialist Republics (U.S.S.R.): A political entity that consisted of 15 constituent republics: Armenia, Azerbaijan, Belarus, Estonia, Georgia, Kazakhstan, Kyrgyzstan, Latvia, Lithuania, Moldova, Russia, Tajikistan, Turkmenistan, Ukraine, and Uzbekistan. The U.S.S.R. ceased to exist as of December 31, 1991.

United States: The 50 States and the District of Columbia. Note: The United States has varying degrees of jurisdiction over a number of territories and other political entities outside the 50 States and the District of Columbia, including Puerto Rico, the U.S. Virgin Islands, Guam, American Samoa, Johnston Atoll, Midway Islands, Wake Island, and the Northern Mariana Islands. EIA data programs may include data from some or all of these areas in U.S. totals. For these programs, data products will contain notes explaining the extent of geographic coverage included under the term "United States."

Uranium: A heavy, naturally radioactive, metallic element (atomic number 92). Its two principally occurring isotopes are uranium-235 and uranium-238. Uranium-235 is indispensable to the nuclear industry because it is the only isotope existing in nature, to any appreciable extent, that is fissionable by thermal neutrons. Uranium238 is also important because it absorbs neutrons to produce a radioactive isotope that subsequently decays to the isotope plutonium-239, which also is fissionable by thermal neutrons.

Uranium Concentrate: A yellow or brown powder obtained by the milling of uranium ore, processing of in situ leach mining solutions, or as a byproduct of phosphoric acid production. See Uranium Oxide.

Uranium Ore: Rock containing uranium mineralization in concentrations that can be mined economically, typically one to four pounds of U3O8 (uranium oxide) per ton or 0.05 percent to 0.2 percent $\mathrm{U}_{3} \mathrm{O}_{8}$

Uranium Oxide: Uranium concentrate or yellowcake. Abbreviated as $\mathrm{U}_{3} \mathrm{O}_{8}$.
Uranium Resource Categories: Three categories of uranium resources defined by the international community to reflect differing levels of confidence in the existence of the resources. Reasonably assured resources (RAR), estimated additional resources (EAR), and speculative resources (SR) are described below.

Reasonably assured resources (RAR): Uranium that occurs in known mineral deposits of such size, grade, and configuration that it could be recovered within the given production cost ranges, with currently proven mining and processing technology. Estimates of tonnage and grade are based on specific sample data and measurements of the deposits and on knowledge of deposit characteristics. Note: RAR corresponds to DOE's uranium reserves category.

Estimated additional resources (EAR): Uranium in addition to RAR that is expected to occur, mostly on the basis of geological evidence, in extensions of well-explored deposits, in little-explored deposits, and in undiscovered deposits believed to exist along well-defined geological trends with known deposits. This uranium can subsequently be recovered within the given cost ranges. Estimates of tonnage and grade are based on available sampling data and on knowledge of the deposit characteristics, as determined in the best-known parts of the deposit or in similar deposits. Note: EAR corresponds to DOE's probable potential resources category.

Speculative resources (SR): Uranium in addition to EAR that is thought to exist, mostly on the basis of indirect evidence and geological extrapolations, in deposits discoverable with existing exploration techniques. The location of deposits in this category can generally be specified only as being somewhere within given regions or geological trends. The estimates in this category are less reliable than estimates of RAR and EAR. Note: SR corresponds to the combination of DOE's possible potential resources and speculative potential resources categories.

Useful Thermal Output: The thermal energy made available in a combinedheat-and-power system for use in any industrial or commercial process, heating or cooling application, or delivered to other end users, i.e., total thermal energy made available for processes and applications other than electricity generation.

\section{Vented Natural Gas: See Natural Gas, Vented.}

Vessel Bunkering: Includes sales for the fueling of commercial or private boats, such as pleasure craft, fishing boats, tugboats, and ocean-going vessels, including vessels operated by oil companies. Excluded are volumes sold to the U.S. Armed Forces. 


\section{Waste: See Biomass Waste and Non-Biomass Waste.}

Waste Coal: Usable material that is a byproduct of previous coal processing operations. Waste coal is usually composed of mixed coal, soil, and rock (mine waste). Most waste coal is burned as-is in unconventional fluidized-bed combustors. For some uses, waste coal may be partially cleaned by removing some extraneous noncombustible constituents. Examples of waste coal include fine coal, coal obtained from a refuse bank or slurry dam, anthracite culm, bituminous gob, and lignite waste.

Watt (W): The unit of electrical power equal to one ampere under a pressure of one volt. A watt is equal to $1 / 746$ horsepower.

Watthour (Wh): The electric energy unit of measure equal to one watt of power supplied to, or taken from, an electric circuit steadily for one hour.

Wax: A solid or semi-solid material at 77 degrees Fahrenheit consisting of a mixture of hydrocarbons obtained or derived from petroleum fractions, or through a Fischer-Tropsch type process, in which the straight chained paraffin series predominates. This includes all marketable wax, whether crude or refined, with a congealing point (ASTM D 938) between 80 (or 85) and 240 degrees Fahrenheit and a maximum oil content (ASTM D 3235) of 50 weight percent.

Well: A hole drilled in the Earth for the purpose of (1) finding or producing crude oil or natural gas; or (2) producing services related to the production of crude oil or natural gas. See Completion (Crude Oil/Natural Gas Production), Crude Oil Well, Development Well, Dry Hole, Exploratory Well, and Natural Gas Well.

Wellhead: The point at which the crude oil (and/or natural gas) exits the ground. Following historical precedent, the volume and price for crude oil production are labeled as "wellhead," even though the cost and volume are now generally measured at the lease boundary. In the context of domestic crude price data, the term "wellhead" is the generic term used to reference the production site or lease property.

Wellhead Price: The value of crude oil or natural gas at the mouth of the well. See Natural Gas Wellhead Price.

Western Europe: Includes Austria, Belgium, Bosnia and Herzegovina, Croatia, Denmark, Finland, France, Germany, Greece, Iceland, Ireland, Italy, Luxembourg, Macedonia (The Former Yugoslav Republic of), Malta, Netherlands, Norway, Portugal, Serbia and Montenegro, Slovenia, Spain, Sweden, Switzerland, Turkey, and the United Kingdom.

Wind Energy: Kinetic energy present in wind motion that can be converted to mechanical energy for driving pumps, mills, and electric power generators.

Wood and Wood-Derived Fuels: Wood and products derived from wood that are used as fuel, including round wood (cord wood), limb wood, wood chips, bark, sawdust, forest residues, charcoal, paper pellets, railroad ties, utility poles, black liquor, red liquor, sludge wood, spent sulfite liquor, and other wood-based solids and liquids.

Working Gas: The volume of gas in the reservoir that is in addition to the cushion or base gas. It may or may not be completely withdrawn during any particular withdrawal season. Conditions permitting, the total working capacity could be used more than once during any season.

Yellowcake: A natural uranium concentrate that takes its name from its color and texture. Yellowcake typically contains 70 to 90 percent $\mathrm{U}_{3} \mathrm{O}_{8}$ (uranium oxide) by weight. It is used as feedstock for uranium fuel enrichment and fuel pellet fabrication. 